Printed November 1992

\title{
A Method for Determining the Spent-Fuel Contribution to Transport Cask Containment Requirements
}

Thomas L. Sanders, Kevin D. Seager, Yusef R. Rashid, Peter R. Barrett, Anthony P. Malinauskas, Robert E. Einziger, Hans Jordan, Thomas A. Duffey, Stephen H. Sutherland, Philip C. Reardon

Prepared by

Sandia National Laboratories

Albuquerque, New Mexico 87185 and Livermore, California 94550

for the United States Department of Energy

under Contract DE-AC04-76DP00789 


\section{DISCLAIMER}

This report was prepared as an account of work sponsored by an agency of the United States Government. Neither the United States Government nor any agency Thereof, nor any of their employees, makes any warranty, express or implied, or assumes any legal liability or responsibility for the accuracy, completeness, or usefulness of any information, apparatus, product, or process disclosed, or represents that its use would not infringe privately owned rights. Reference herein to any specific commercial product, process, or service by trade name, trademark, manufacturer, or otherwise does not necessarily constitute or imply its endorsement, recommendation, or favoring by the United States Government or any agency thereof. The views and opinions of authors expressed herein do not necessarily state or reflect those of the United States Government or any agency thereof. 


\section{DISCLAIMER}

Portions of this document may be illegible in electronic image products. Images are produced from the best available original document. 
Issued by Sandia National Laboratories, operated for the United States Department of Energy by Sandia Corporation.

NOTICE: This report was prepared as an account of work sponsored by an agency of the United States Government. Neither the United States Government nor any agency thereof, nor any of their employees, nor any of their contractors, subcontractors, or their employees, makes any warranty, express or implied, or assumes any legal liability or responsibility for the accuracy, completeness, or usefulness of any information, apparatus, product, or process disclosed, or represents that its use would not infringe privately owned rights. Reference herein to any specific commercial product, process, or service by trade name, trademark, manufacturer, or otherwise, does not necessarily constitute or imply its endorsement, recommendation, or favoring by the United States Government, any agency thereof or any of their contractors or subcontractors. The views and opinions expressed herein do not necessarily state or reflect those of the United States Government, any agency thereof or any of their contractors.

Printed in the United States of America. This report has been reproduced directly from the best available copy.

Available to DOE and DOE contractors from

Office of Scientific and Technical Information

PO Box 62

Oak Ridge, TN 37831

Prices available from (615) 576-8401, FTS 626-8401

Available to the public from

National Technical Information Service

US Department of Commerce

5285 Port Royal Rd

Springfield, VA 22161

NTIS price codes

Printed copy: A24

Microfiche copy: A01 
Unlimited Release

November 1992

\title{
A METHOD FOR DETERYINING THE SPENT-FUEL CONTRIBUTION TO TRANSPORT CASR CONTAINMENT REQUIREMENTS*
}

\author{
Thomas L. Sanders \\ Kevin D. Seager \\ Sandia National Laboratories** \\ P.O. Box 5800 \\ Albuquerque, NM 87185 \\ Yusef R. Rashid \\ Peter R. Barrett \\ ANATECH Research Corporation \\ P.0. Box 9165 \\ La Jolla, CA 92038 \\ Anthony P. Malinauskas \\ Oak Ridge National Laboratory \\ P.0. Box 2008 \\ Oak Ridge, TN 37831-6135 \\ Robert E. Einziger \\ Pacific Northwest Laboratory \\ P.0. Box 999 \\ Richland, WA 99352 \\ Hans Jordan \\ EG\&G Rocky Flats Inc. \\ Rocky Flats Plant \\ P.0. Box 464 \\ Golden, CO 80402-0464 \\ Thomas A. Duffey \\ Stephen H. Sutherland \\ APTEK Incorporated \\ Colorado Springs, CO 80906 \\ Philip C. Reardon \\ GRAM Incorporated \\ 8500 Menual Blvd. NE \\ Suite $B-370$ \\ Albuquerque, NM 87112
}

*This work was performed at Sandia National Laboratories, Albuquerque, New Mexico, supported by the U.S. Department of Energy under Contract DE - AC04-76DP00789.

$* *$ A U.S. Department of Energy facility. 
This work was performed under contract to the office of Civilian Radioactive Waste Management (OCRWM) of the U.S. Department of Energy (DOE). The authors thank N. Burre11, M. Fisher, K. Golliher, C. Kouts, W. Lake, and $M$. Pellechi of the DOE for supporting this work. In addition, special thanks go to D. Ammerman and R. Glass of Sandia National

Laboratories and the staff of Creative Computer Services for their review efforts and assistance in preparing this manuscript. 
This report examines containment requirements for spent-fuel transport containers that are transported under normal and hypothetical accident conditions. A methodology is described that estimates the probability of rod failure and the quantity of radioactive material released from breached rods. This methodology characterizes the dynamic environment of the cask and its contents and deterministically models the peak stresses that are induced in spent-fuel cladding by the mechanical and thermal dynamic environments. The peak stresses are evaluated in relation to probabilistic failure criteria for generated or preexisting ductile tearing and material fractures at cracks partially through the wall in fuel rods. Activity concentrations in the cask cavity are predicted from estimates of the fraction of gases, volatiles, and fuel fines that are released when the rod cladding is breached. Containment requirements based on the source term are calculated in terms of maximum permissible volumetric leak rates from the cask. Calculations are included for representative cask designs. 


\section{QUALITY ASSURANCE}

This work was performed under a quality level of QL-3. The applicable level-three requirements of the Sandia National Laboratories' Transportation Systems Development Department Quality Assurance Program Plan were implemented for all phases of this activity. 


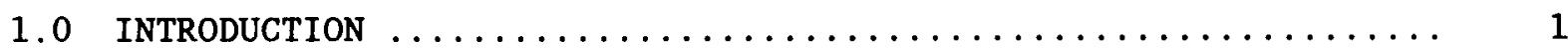

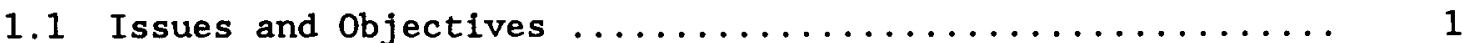

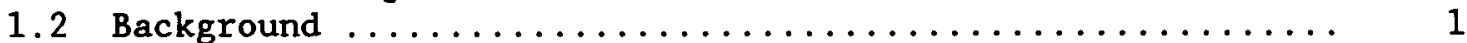

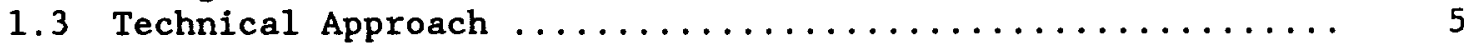

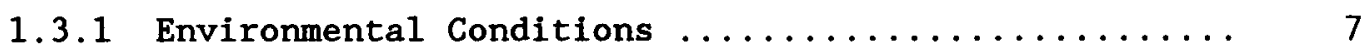

1.3.2 Release Mechanisms .................... 10

1.4 Summary and Analysis Sequence $\ldots \ldots \ldots \ldots \ldots \ldots \ldots \ldots \ldots \ldots$

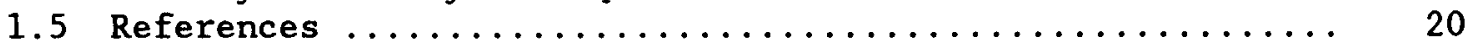

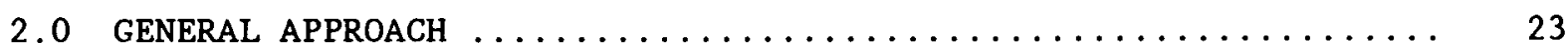

2.1 Fuel Geometric and Initial Conditions .............. 25

2.2 Definition of Regulatory Transport and Associated

2.3 Determination of Fuel Rod/Assembly Mechanical Response .... 28

2.4 Radioactive Material Release Estimates ............... 33

2.5 References ............................. 34

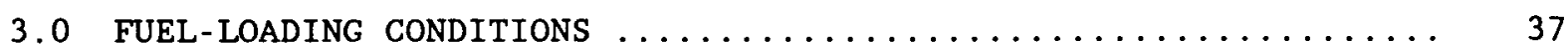

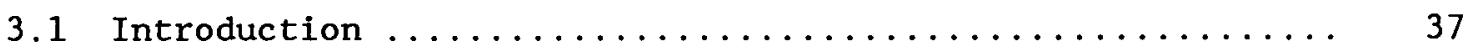

3.2 Normal Transport Environments ................. 38

3.2 .1 Shock and Vibration .................... 38

3.2.2 Accelerations for the 0.3 -m Drop .............. 40

3.2.3 Normal Thermal Environment ................ 40

3.3 Accident Environments ...................... 43

3.3.1 Nine-Meter-Drop Impact Environment ............. 44

3.3.2 Example Oblique Impact .................... 46

3.3.3 One-Meter Drop onto a Mild Steel Bar ........... 48

3.3.4 Thirty-Minute Engulfing Fire .............. 50

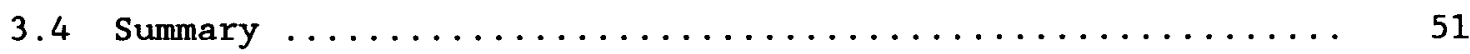

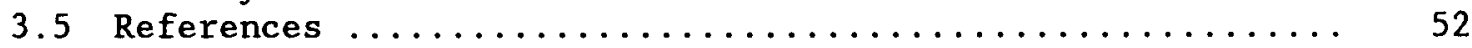

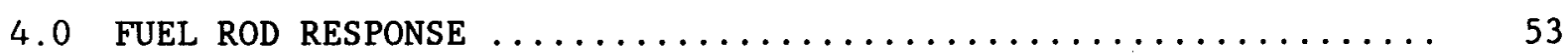

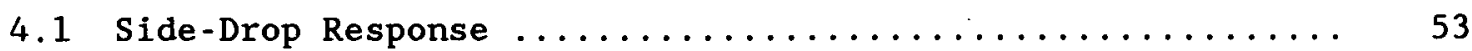

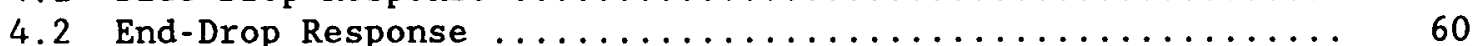

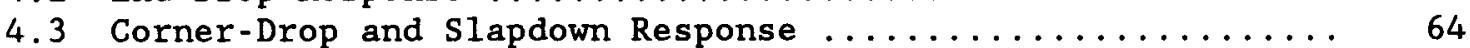

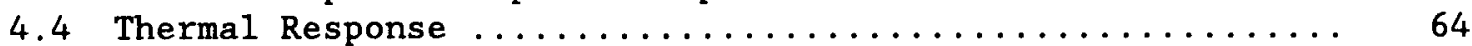

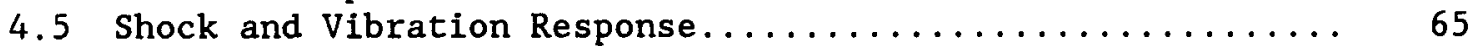

4.6 Failure Criteria and Failure Modes ............... 68

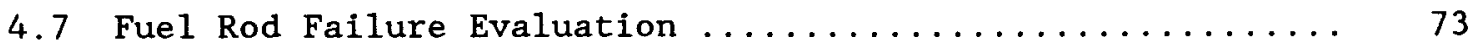

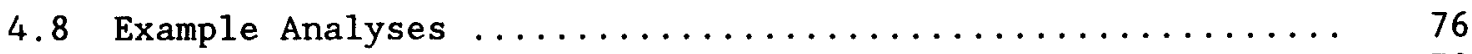

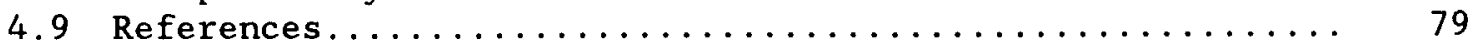


TABLE OF CONTENTS

(Continued)

Page

5.0 EVALUATION OF POTENTIAL RADIONUCLIDE RELEASES $\ldots \ldots \ldots \ldots \ldots \ldots \ldots$

5.1 Estimate of Total Rod Activity ................ 81

5.2 Determination of Gap Inventories ............... 81

5.3 Radionuclide Release from Fuel Rods ............... 84

5.4 Releases to a Cask Cavity at $530^{\circ} \mathrm{C} \ldots \ldots \ldots \ldots \ldots \ldots \ldots \ldots$

5.5 Example Containment Criteria .................... 85

5.6 References .............................. 89

6.0 SENSITIVITIES AND UNCERTAINTIES $\ldots \ldots \ldots \ldots \ldots \ldots \ldots \ldots \ldots \ldots \ldots$

6.1 Cask and Limiter Design Parameters ................ 91

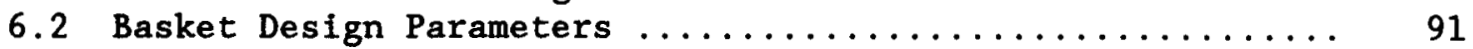

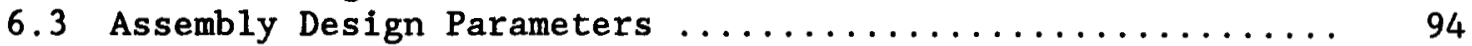

6.4 Initial State Parameters of Fuel Rods ............... 96

6.5 In-Reactor Operational Parameters .................. 100

6.6 In-Transport Operational Parameters ............... 103

6.7 Summary of Sensitive Parameters and Sources of

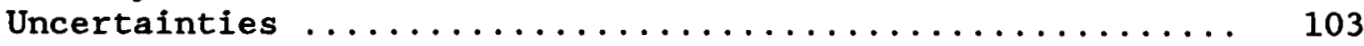

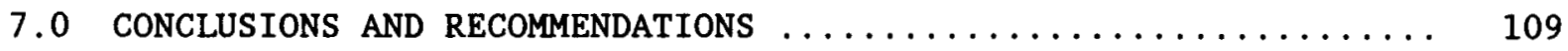

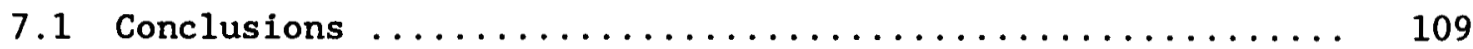

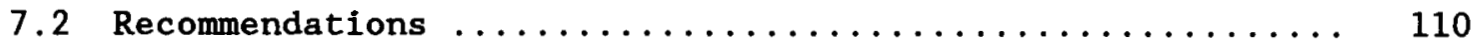

APPENDIX I INITIAL CONDITIONS OF SPENT FUEL BEFORE TRANSPORT ...... I-1

I.1 Introduction $\ldots \ldots \ldots \ldots \ldots \ldots \ldots \ldots \ldots \ldots \ldots \ldots \ldots \ldots \ldots \ldots \ldots \ldots$

I. 2 Physical Conditions of As-Manufactured Fuel Components .... I-4

I. 3 Pretransport Spent-Fuel Characteristics ............ I-27

I.3.1 Fuel Rod Breach Behavior $\ldots \ldots \ldots \ldots \ldots \ldots \ldots \ldots$ I-27

I.3.1.1 In-Reactor Fuel Rod Cladding Breaches .... I-27

I.3.1.2 Damage During Handling and Storage ...... I-31

I.3.1.3 Defective Fuel in Storage ........... I-34

I.3.2 Postirradiation Fuel Pellet Condition .......... I-34

I.3.3 Intact Rod Cladding Damage ................ I-39

I.3.3.1 Hydride Distribution and Degradation ..... I-41

I.3.3.2 In-Reactor External Cladding Oxidation .... I I-49

I.3.3.3 Determination of Incipient Breaches ...... I-51 
TABLE OF CONTENTS

(Continued)

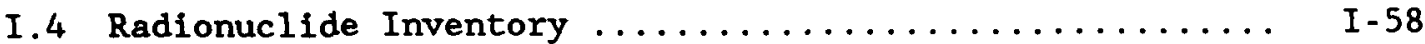

I.5 Gap Inventory of an Irradiated Fuel Rod ........... I-61

I.6 Conclusions and Recommendations ................ I 66

I.7 References ............................. I -67

APPENDIX II CHARACTERISTICS OF SPENT-FUEL TRANSPORTATION

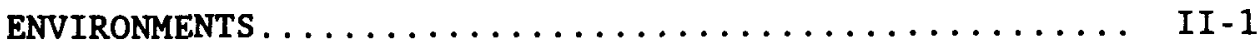

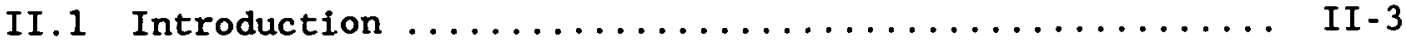

II.2 Cask Concepts and Spent-Fue1 Descriptions........... II-5

II. 3 Generic Cask Impact Analyses .................. II-8

II.3.1 Analytical Modeling Methods ............ II-9

II.3.2 Lumped Parameter Modeling .............. II-17

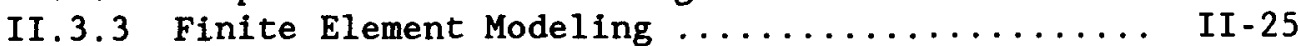

II.3.4 Rigid-Body Kinematics Analysis of Impact ..... II-27

II.3.5 Predicted Decelerations of Currently

Certified U.S. Casks ............... II-70

II.3.6 Summary Remarks $\ldots \ldots \ldots \ldots \ldots \ldots \ldots \ldots \ldots$ II -70

II.4 Shock and Vibration Characterization ............ II -74

II.4.1 Introduction ..................... II

II.4.2 Summary and Discussion of Available

Shock/Vibration Data ................ II 75

II.4.3 Simplified Bounding Input Loading Curves...... II-99

II.5 Cask Thermal Analyses $\ldots \ldots \ldots \ldots \ldots \ldots \ldots \ldots \ldots \ldots$ II-107

II.5.1 Introduction..................... II-107

II.5.2 Cask Thermal Analysis Models............ II-109

II.5.3 Cask Material Models.................. II -120

II.5.4 Spent-Fuel Effective Conductivity........... II-122

II.5.5 Cask Thermal Response Results............. II-138

II.5.6 Thermal Analyses Conclusions.............. II-146

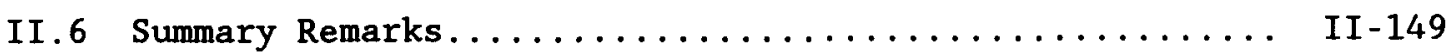

II. 7 References ............................ II -150

APPENDIX III SPENT-FUEL RESPONSE TO TRANSPORT ENVIRONMENTS ....... III-1

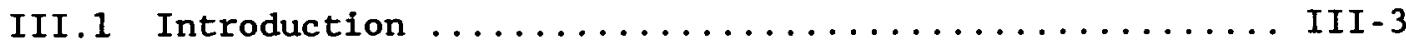

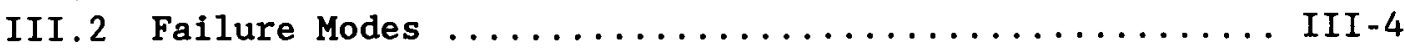

III.2.1 Burst Failure $\ldots \ldots \ldots \ldots \ldots \ldots \ldots \ldots \ldots$ III-6

III.2.2 Breaking Failure .................. III-10 
TABLE OF CONTENTS

(Concluded)

Page

III.3 Structural Modeling

III -11

III.3.1 Modeling Assumptions .............. III-11

III.3.2 Assembly Mechanical Loading Conditions ..... III-18

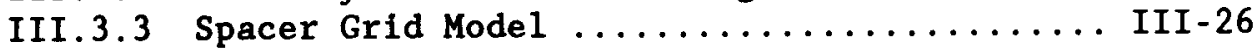

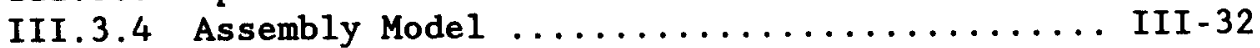

III.3.5 Method of Analysis ................ III-43

III.4 Failure Criteria $\ldots \ldots \ldots \ldots \ldots \ldots \ldots \ldots \ldots \ldots \ldots \ldots$ III -48

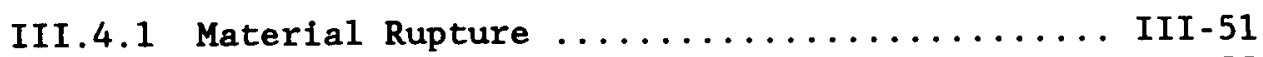

III.4.2 Material Fracture ................. III-55

III.4.3 Scoping Calculations .............. III-74

III.5 Example Structural Analysis Results ........... III-76

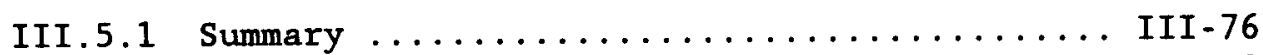

III.5.2 Example Assemblies $\ldots \ldots \ldots \ldots \ldots \ldots \ldots \ldots$ III-78

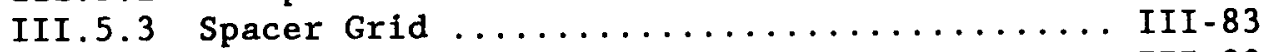

III.5.4 Hypothetical Accident Side Drop .......... III-90

III.5.5 Hypothetical Accident End Drop .......... III-109

III.5.6 Hypothetical Accident Corner Drop ......... III-124

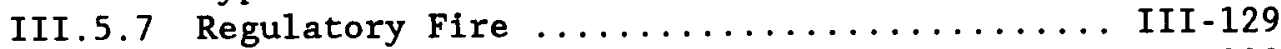

III.5.8 Normal Transport ................ III-129

III.6 Example Failure Evaluation $\ldots \ldots \ldots \ldots \ldots \ldots \ldots \ldots$ III-132

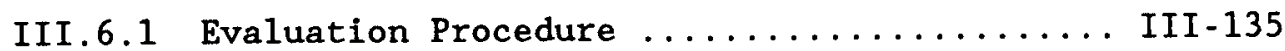

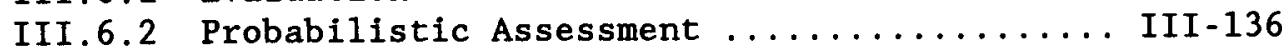

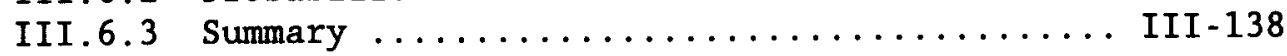

III.7 Summary and Conclusions $\ldots \ldots \ldots \ldots \ldots \ldots \ldots \ldots$ III-138

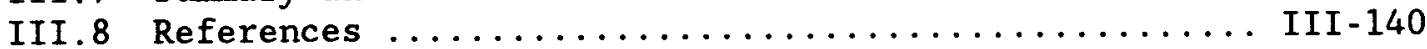

APPENDIX IV RADIONUCLIDE RELEASE MODELS $\ldots \ldots \ldots \ldots \ldots \ldots \ldots \ldots$ IV-I

IV. 1 Introduction $\ldots \ldots \ldots \ldots \ldots \ldots \ldots \ldots \ldots \ldots \ldots \ldots \ldots \ldots \ldots \ldots \ldots$ IV -3

IV. 2 Release of Gaseous and Volatile Species .......... IV -3

IV. 3 Escape of Nonvolatile Species .............. IV -6

IV. 4 Generation of Aerosol from Fracturing Fuel . . . . . . IV 9

IV. 5 Impact Energy Correlation Function ............. IV-16

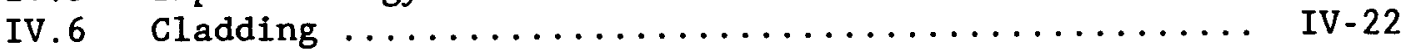

IV. 7 Air Oxidation of Rods Having Defected Claddings ..... IV-23

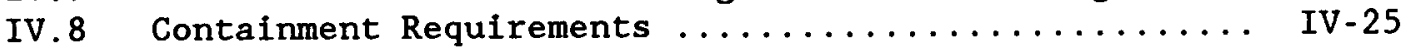

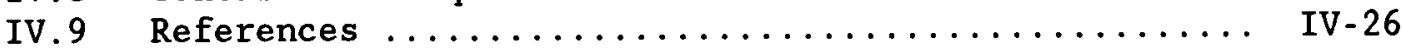




\section{LIST OF FIGURES}

Figure

Page

1 Limiting Average Leak Rates Versus Activity Concentration .....

2 Projected Burnup Characteristics for the Spent-Fuel Inventory to Be Transported During the First $25 \mathrm{Yr}$ of Repository

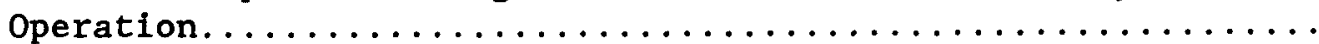

3 Release Scenarios Affecting the Source Term in a Spent-Fuel

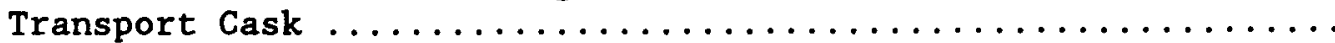

4 Processes and Events Affecting Radionuclide Release from Spent Fuel During Mechanical Impact Conditions

5 Processes and Events Affecting Radionuclide Release from Spent Fuel During Severe Thermal Conditions ............

6 Processes and Events Required for Leaching Release of

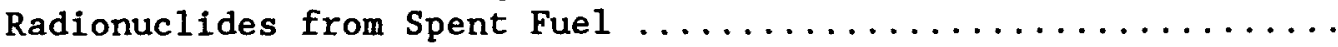

7 Processes and Events Required for Diffusion Release of

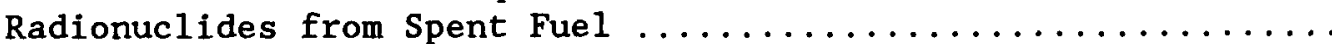

8 Processes and Events Required for Oxidation Release of

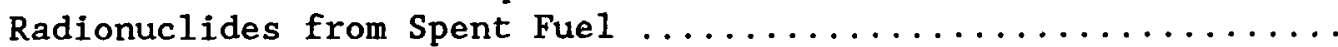

9 Factors Affecting the Releasable Source Term in a Spent-Fuel Transport Cask ............................. 19

10 Geometric Models for Spent-Fuel Assemblies in Transport Casks .. 29

11 Fuel Rod Failure Modes $\ldots \ldots \ldots \ldots \ldots \ldots \ldots \ldots \ldots \ldots \ldots \ldots \ldots$

12 Bounding Acceseration Shock Response Spectrum for a Truck Cask

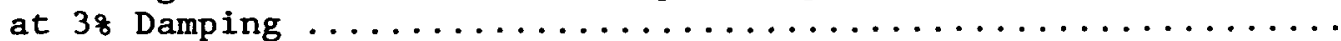

13 Bounding Acceleration Shock Response Spectrum for 38 Damping for All Three Axes.

14 Bounding Acceleration Shock Response Spectrum for 38 Damping for All Three Axes, Including a Recommended Linear Bounding Response Spectrum for Rail-Coupling Events...............

15 Bounding Truck Vibration Data for All Three Axes .......... 41

16 Bounding Rail Vibration Data $\ldots \ldots \ldots \ldots \ldots \ldots \ldots \ldots \ldots \ldots \ldots \ldots$

17 Vertical Acceleration in $g$ Versus the Impact Angle for the

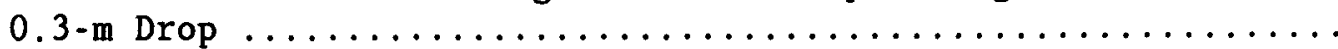

18 Predicted Peak Fuel Temperatures in a Truck Cask as a Function of Assembly Decay Heat Generation Rate .............. 


\section{LIST OF FIGURES}

(Continued)

Figure

19 Predicted Peak Fuel Temperatures in a Rail Cask as a Function of Assembly Decay Heat Generation Rate ..................

20 Vertical Acceleration Histories; Slippage with No Friction and a $30^{\circ}$ Impact Angle Assumed ..................... 46

21 Vertical Acceleration Histories; No Slippage and a $30^{\circ}$ Impact Angle Assumed $\ldots \ldots \ldots \ldots \ldots \ldots \ldots \ldots \ldots \ldots \ldots \ldots \ldots \ldots \ldots \ldots$

22 Peak Vertical Accelerations as a Function of Impact Angle ..... 48

23 Peak Horizontal Accelerations as a Function of Impact Angle ... 49

24 Peak Angular Accelerations as a Function of Impact Angle ..... 50

25 Maximum Punch-Drop Accelerations $\ldots \ldots \ldots \ldots \ldots \ldots \ldots \ldots \ldots \ldots \ldots \ldots \ldots \ldots$

26 Cask Impact Orientations for the 9.0 -m Drop $\ldots \ldots \ldots \ldots \ldots \ldots \ldots$

27 Schematic Diagram of Spacer Grid Response $\ldots \ldots \ldots \ldots \ldots \ldots \ldots$

28 Two-Dimensional Assembly Mode1, the Spacer Grid Spring Elements, and the Force-Displacement Relationship ......... 57

29 Example Computational Model for Side-Drop Analysis ......... 58

30 Assembly Side-Drop Loading Condition and Free-Body Diagram .... 59

31 Lateral Displacement of Assembly Limited by Rod-to-Rod Contact and Basket-Assembly Gaps .................. 61

32 Example Single Rod End-Drop Assembly Mode1 $\ldots \ldots \ldots \ldots \ldots \ldots \ldots$

33 Assembly End-Drop Loading Condition and Free-Body Diagram ..... 63

34 Illustration of End-Drop and Initial Velocity Loading....... 65

35 Assembly Corner Drop, Initial Impact Loading Condition, and

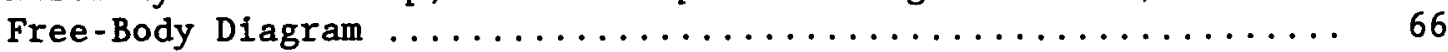

36 Assembly Corner Drop, Slapdown Loading Condition, and Free-

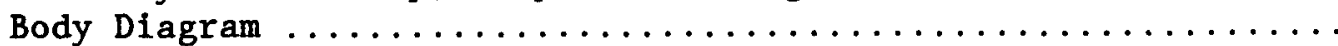

37 Probability Distribution for Cladding Rupture Strain as a

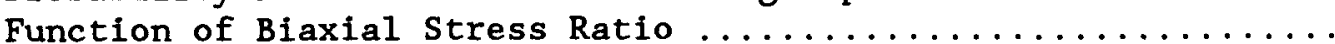




\section{LIST OF FIGURES}

(Continued)

Figure

Page

38 Probability Density Function for Cladding Fracture

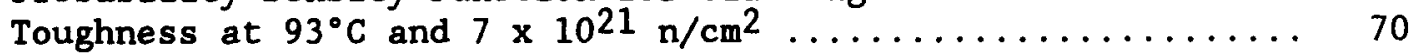

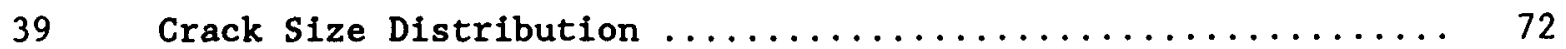

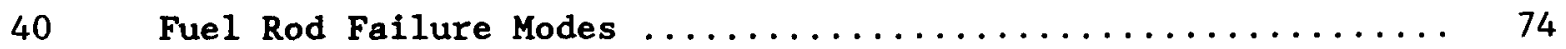

41 Cladding Material Fracture Fallure Mode .............. 75

42 Cladding Material Rupture Failure Mode $\ldots \ldots \ldots \ldots \ldots \ldots \ldots$

43 Typical Cask Design Components ................. 92

$44 \quad$ IF-300 BWR Fuel Basket Detail ...................... 94

45 Lateral Displacement of Assembly Limited by Rod-to-Rod

Contact and Basket-Assembly Gaps ................. 95

46 Material Fracture Failure Probability Versus the Number of Part-Wall Cracks for Example BWR and PWR Assemblies ...... 97

47 Exponential Distribution Material Fracture Failure

Probability Versus the Frequency of Critical Crack Size

for Example BWR and PWR Assemblies ..................

48 Weibull Distribution Material Fracture Failure Probability Versus the Frequency of Critical Crack Size for Example BWR

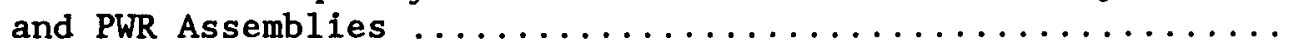

49 Lowest Buckling Mode Shape of a Fuel Rod ............. 101

50 Cumulative Probability Distributions for Cladding Fracture Toughness at Various Temperatures and Fast Fluences ........ 102

51 Example BWR Assembly and the Sensitivity of Material Fracture Failure Probability.......................... 104

52 Example PWR Assembly and the Sensitivity of Material Fracture Failure Probability......................... 105

I-1 Fuel Rod Handling Paths $\ldots \ldots \ldots \ldots \ldots \ldots \ldots \ldots \ldots \ldots \ldots \ldots \ldots \ldots \ldots \ldots \ldots$

I-2 Typical LWR Fuel Rod: GE BWR Rod $\ldots \ldots \ldots \ldots \ldots \ldots \ldots \ldots \ldots$

I-3 CE $16 \times 16$ PWR Fuel $\operatorname{Rod} \ldots \ldots \ldots \ldots \ldots \ldots \ldots \ldots \ldots \ldots \ldots \ldots \ldots \ldots \ldots \ldots \ldots \ldots$

I-4 Typical PWR Assemblies: (a) CE $14 \times 14$; (b) WE $15 \times 15 \ldots \ldots$ I-13 
LIST OF FIGURES

(Continued)

Figure

Page

I-5 Typical BWR Assembly $\ldots \ldots \ldots \ldots \ldots \ldots \ldots \ldots \ldots \ldots \ldots \ldots \ldots \ldots \ldots \ldots \ldots$

I-6 Control Rod Guide Tube and Tie Rod Locations:

(a) WE $15 \times 15$, (b) CE $14 \times 14$, (c) WE $14 \times 14 \mathrm{ZCA}$,

(d) B\&W $15 \times 15$, (e) GE $7 \times 7$, and (f) GE $8 \times 8 \ldots \ldots \ldots$ I-17

I-7 Photograph of GE $7 \times 7$ BWR Spacer Grid $\ldots \ldots \ldots \ldots \ldots \ldots \ldots \ldots$

I-8 Description of B\&W $15 \times 15$ PWR Spacer Grid $\ldots \ldots \ldots \ldots \ldots \ldots \ldots$ I-20

I-9 Overall Significance of Fuel Breaches in PWR Plants ....... I-35

I-10 Overall Significance of Fuel Breaches in BWR Plants ....... I-35

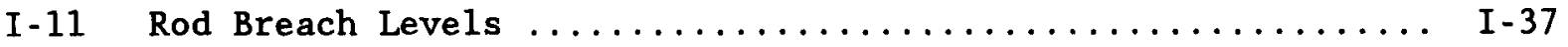

I-12 Cross Section of As-Irradiated Turkey Point PWR Fuel Rod .... I-38

I-13 Longitudinal Cross Section of a Turkey Point Fuel Rod

After Irradiation .......................... I-39

I-14 Mosaic of a Longitudinal Cross Section of a Turkey Point Fuel Rod at the Spacer Grid Location of Sample G9-18 (2.0 m)

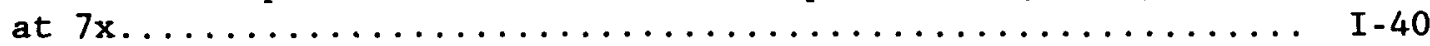

I-15 Profilometry Scan of Rod DO1-19 Showing Continuous

Localized Ridging at Pellet Interfaces Between 1.65 and

$1.78 \mathrm{~m}$ from the Rod Bottom (Neg. 8007580-6) .............

$I-41$

I-16 Photomacrograph of an As-Polished Transverse Sample of Cooper

BWR Fuel from the Peak Power Region ( $10 x)$ Burnup of

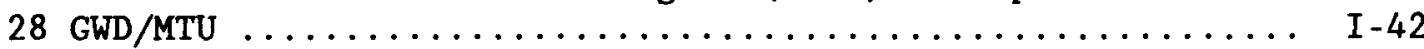

I-17 Photomacrograph of an As-Polished Longitudinal Sample of Cooper BWR Fuel from the Peak Power Region (-10x) Burnup of

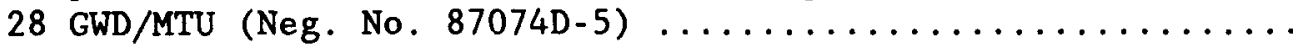

I-18 Hydrogen Solubility in Zircaloy $\ldots \ldots \ldots \ldots \ldots \ldots \ldots \ldots \ldots \ldots \ldots \ldots \ldots \ldots$

I-19 Hydrogen Content of Cladding at Various Locations from the

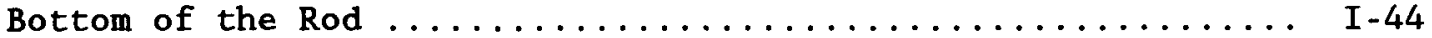

I-20 (a) Pre- and (b) Post-test Hydride Structure $\ldots \ldots \ldots \ldots \ldots \ldots$ I-45

I-21 Reduction of the Area of a Short Transverse Tensile Specimen from a Rolled Zircaloy-2 S1ab as a Function of Temperature and Hydrogen Content 


\section{LIST OF FIGURES}

(Continued)

Figure

Page

I-22 Influence of Hydrogen on the Fracture Energy Transition

Temperature ........................... I 47

I-23 Variations in Tensile Properties of Zircaloy with the

Effective Hydrogen Content .................... I-48

I-24 Reoriented Hydrides After a Slow Cooling of PWR Cladding

Under an Internal Pressure Hoop Stress ............... I-48

I-25 Maximum Oxide Layer Thickness of Non-KWU Fuel Rods Versus

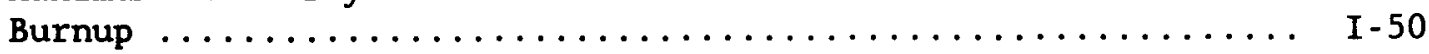

I-26 Linear Heat Rating ( $\mathrm{kW} / \mathrm{m}$ ) Versus Critical Incipient Crack

Depth (Percentage of Cladding Thickness) ............. I-53

I-27 Comparison of Exponential, Weibul1, and Manufacturing

Crack (Flaw) Size Exceedence Probability Distributions....... I-57

I-28 Fission Gas Release for BWR Rods $\ldots \ldots \ldots \ldots \ldots \ldots \ldots \ldots \ldots \ldots \ldots$

I-29 Fission Gas Release for PWR Rods $\ldots \ldots \ldots \ldots \ldots \ldots \ldots \ldots \ldots$ I 65

II-1 Representative Lead-Shielded Cask Dimensions (cm) $\ldots \ldots \ldots$. II-7

II-2 Representative Cask Acceleration Predictions as a Function

of Crush Strength for End-On Impact ................. II -11

II-3 Assumed Impact Limiter Deformation During Side Impact....... II-12

II-4 Lead-Shielded Cask Decelerations Versus Limiter Length for

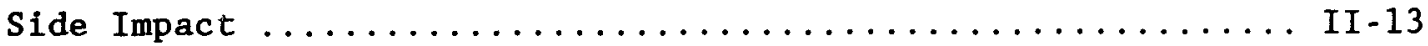

II-5 Schematic of CMC Impact Showing the Impact Limiter Only ..... II-14

II-6 Simplified End-On Impact Mode1 (left) and Crush Strength

Versus Deformation Characteristics of Impact Limiter

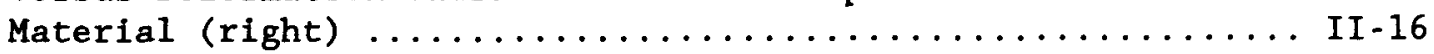

II-7 Average Deceleration Versus Limiter Crush Depth for the 9.0 -m Drop .......................... II 18

II-8 Outline of a Steel Truck Cask (top drawing) and Its Lumped

Parameter Model (bottom drawing) $\ldots \ldots \ldots \ldots \ldots \ldots \ldots$ II -19

II-9 Displacement History of the All-Steel Truck Cask End for Three Discretizations for End-On Impact ............... II-22 
(Continued)

Figure

II-10 Velocity History of the Al1-Steel Truck Cask End for Three Discretizations for End-On Impact .................. II-23

II-11 Acceleration History of the A11-Steel Truck Cask End for Three Discretizations for End-On Impact ............... II-23

II-12 Acceleration History of the All-Steel Cask End for Assumed Levels of Damping Using Seven-Mass Points for the Fuel Assembly ............................. II 24

II-13 Acceleration History of the All-Steel Truck Cask End and PWR Fuel Assembly for End-On Impact $\ldots \ldots \ldots \ldots \ldots \ldots \ldots$ II -25

II-14 Finite Element Model for the All-Steel Truck Cask ....... II-26

II-15 Displacement Histories for Three Al1-Steel Truck Cask Nodes Predicted by the Finite Element Analysis .......... II-28

II-16 Acceleration History Predicted by Finite Element Analysis of the All-Steel Truck Cask During End Impact .............. II-28

II-17 Rigid-Body Cask Degrees of Freedom ................. II-29

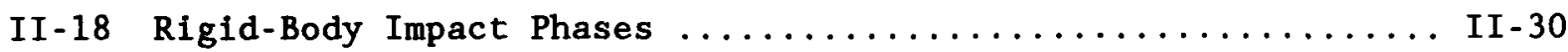

II-19 Stress and Strain Behavior of the Impact Limiter ......... II-33

II-20 Rigid-Body Model Parameters $\ldots \ldots \ldots \ldots \ldots \ldots \ldots \ldots \ldots \ldots \ldots \ldots \ldots \ldots \ldots \ldots$

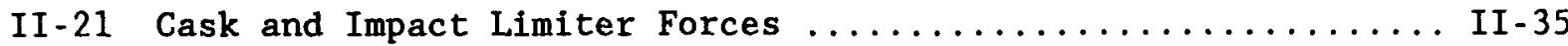

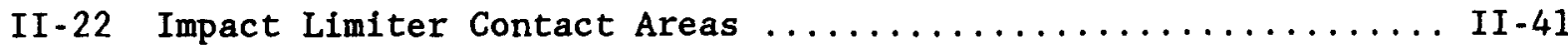

II-23 Impact Limiter Contact Areas for End-On and Side-On

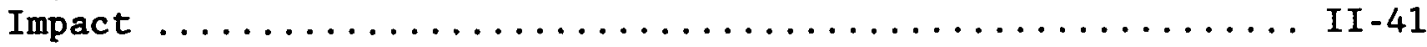

II-24 Impact Limiter Contact Area for Oblique Impact .......... II-43

II-25 End-Drop Analytical Accelerations for the NUPAC 125-B Mode1... II-46

II-26 Side-Drop Analytical Accelerations for the NUPAC 125-B

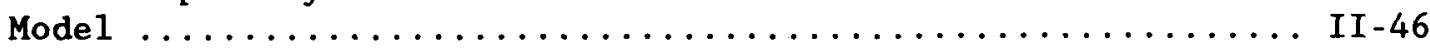

II-27 Oblique-Drop Analytical Accelerations for the NUPAC 125-B

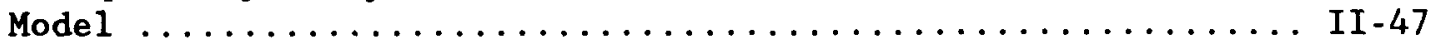


LIST OF FIGURES

(Continued)

Figure

II-28 End-Drop Analytical and Experimental Accelerations for the

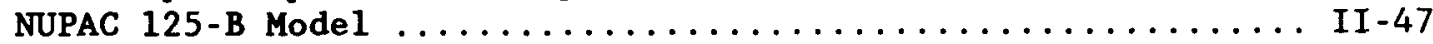

II-29 Side-Drop Analytical and Experimental Accelerations for the

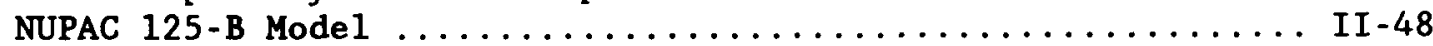

II-30 Oblique-Drop Analytical and Experimental Accelerations for

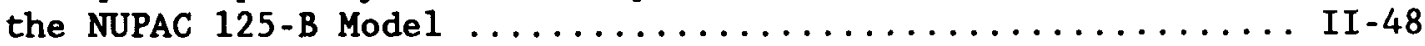

II-31 Comparison of SLAM and Closed Form Side-On Impact Accelerations ........................... II -50

II-32 SLAM End-Drop Conservation of Energy Check $\ldots \ldots \ldots \ldots \ldots \ldots$ II-5I

II-33 SLAM Side-Drop Conservation of Energy Check $\ldots \ldots \ldots \ldots \ldots \ldots$ II-51

II-34 Horizontal Center-of-Mass Position for Oblique Impact on a Frictionless Surface ...................... II -52

II-35 Vertical Acceleration Histories for the 0.3- and 9.0-m

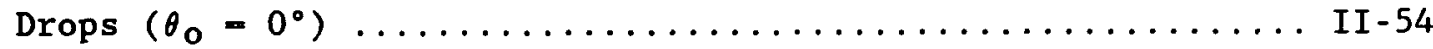

II-36 Vertical Acceleration Histories for the 0.3- and 9.0-m

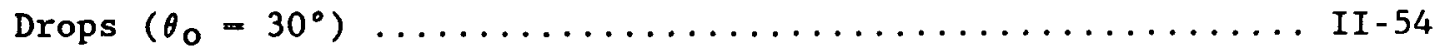

II-37 Vertical Acceleration Histories for the 0.3- and 9.0-m

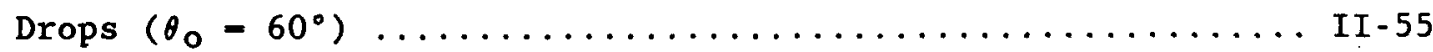

II-38 Vertical Acceleration Histories for the 0.3 - and 9.0-m

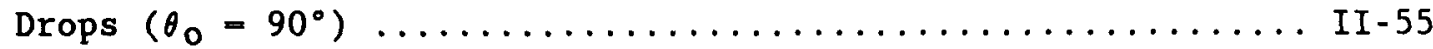

II-39 Angular Acceleration Histories for the 0.3- and 9.0-m

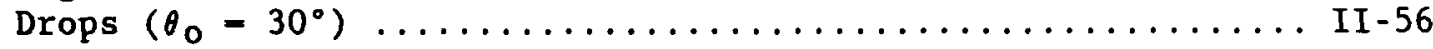

II-40 Angular Acceleration Histories for the $0.3-$ and $9.0-\mathrm{m}$

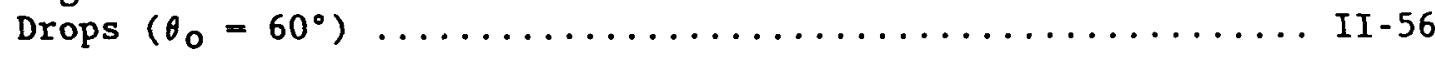

II-41 Peak Accelerations for the 0.3- and 9.0-m Drops ......... II-57

II-42 Impact Durations for the $0.3-$ and 9.0 -m Drops $\ldots \ldots \ldots \ldots \ldots$ II-58

II-43 Vertical Acceleration Histories for the End Drop $\left(\theta=90^{\circ}\right) \ldots$ II-59

II-44 Vertical Acceleration Histories for the Side Drop $\left(\theta=0^{\circ}\right) \ldots$ II-59

II-45 Vertical Acceleration Histories Where $\theta=30^{\circ}$ (no slip) .... II-60

II-46 Horizonta1 Acceleration Histories Where $\theta=30^{\circ}$ (no slip) ... II-60 


\section{LIST OF FIGURES}

(Continued)

Figure

$\underline{\text { Page }}$

II-47 Angular Acceleration Histories Where $\theta=30^{\circ}$ (no s1ip) $\ldots \ldots$ II-61

II-48 Vertical Acceleration Histories Where $\theta-30^{\circ}$ (frictionless). II-61

II-49 Angular Acceleration Histories Where $\theta=30^{\circ}$ (frictionless) .. II-62

II-50 Vertical Acceleration Histories Where $\theta=60^{\circ}$ (no slip) .... II-62

II-51 Horizontal Acceleration Histories Where $\theta=60^{\circ}$ (no s1ip) $\ldots$ II-63

II-52 Angular Acceleration Histories Where $\theta=60^{\circ}$ (no slip) $\ldots .$. II-63

II-53 Vertical Acceleration Histories Where $\theta=60^{\circ}$ (frictionless). II-64

II-54 Angular Acceleration Histories Where $\theta=60^{\circ}$ (frictionless) .. II-64

II-55 Peak Vertical Accelerations for Initial Impact .......... II-65

II-56 Peak Vertical Accelerations for Slapdown $\ldots \ldots \ldots \ldots \ldots \ldots \ldots$ II-66

II-57 Peak Vertical Accelerations for All Phases ............. II-66

II-58 Peak Horizontal Accelerations for Initial Impact ......... II-67

II-59 Peak Horizontal Accelerations for Slapdown $\ldots \ldots \ldots \ldots \ldots \ldots \ldots$ II-67

II-60 Peak Horizontal Accelerations for All Phases ........... II-68

II-61 Peak Angular Accelerations for Initial Impact $\ldots \ldots \ldots \ldots \ldots \ldots$ II-68

II-62 Peak Angular Accelerations for Slapdown .............. II -69

II-63 Peak Angular Accelerations for A11 Phases ............. II-69

II-64 Typical Truck and Rail Transport Configurations for SpentFuel Casks ........................... II 76

II-65 Spring-Mass Mode1 for Input Excitation to the Truck Trailer Body from Prescribed Road Surface Roughness, $X(t) \ldots \ldots \ldots$. II -77

II-66 Acceleration-Time History Illustrating the Presence of Discrete Shock Signals Superimposed on Continuous Vibration ............................ II -77

II-67 Comparison of Two Rail Shock Spectrum Envelopes ........... II-79

II-68 Shock Response Spectrum Envelopes, at A11 Three Axes for Truck Transport Events, at High- and Low-Encounter Speeds .... II-80 


\section{LIST OF FIGURES}

(Continued)

Figure

$\underline{\text { Page }}$

II-69 Rail Vibration Summary at 998 Level $\ldots \ldots \ldots \ldots \ldots \ldots \ldots \ldots$ II -80

II -70 Truck Vibration Summary at 998 Level $\ldots \ldots \ldots \ldots \ldots \ldots \ldots$ II-81

II-71 Truck Superimposed Shock Response Envelopes with 38 Damping .. II-83

II-72 Superimposed Shock Response Envelopes for Rail Transport

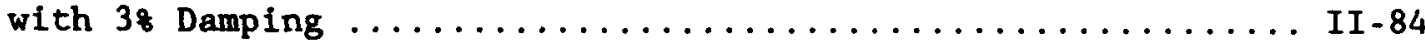

II-73 Test Data Response Spectra Enveloped by Simple Pulse Response Spectrum ........................ II -84

II-74 Superimposed Shock Response Spectra with 38 Damping for the Longitudinal Axis ...................... II 87

II-75 Superimposed Shock Response Spectra with 38 Damping for the Transverse Axis .......................... II -88

II-76 Superimposed Shock Response Spectra with 38 Damping for the Vertical Axis .......................... II 89

II-77 Comparison of Truck Vibration Data for the Longitudinal Axis ............................. II 90

II-78 Comparison of Truck Vibration Data for the Transverse Axis ... II-90

II-79 Comparison of Truck Vibration Data for the Vertical Axis .... II-91

II-80 Comparison of Response Spectra from Truck Data with 3 Damping for the Longitudinal Axis ................ II-91

II-81 Comparison of Response Spectra from Truck Data with 38 Damping for the Transverse Axis ................. II 92

II-82 Comparison of Response Spectra from Truck Data with 38 Damping for the Vertical Axis .................. II-92

II-83 Composite Response Spectra of Rail Car Structure for the Longitudinal Axis with Standard Draft Gear, 36- and 64-Tonne Cargo, 12.91 to $17.98 \mathrm{~km} / \mathrm{hr}$ Impact Velocity, and 38 Damping .. II-94

II-84 Composite Response Spectra of Rail Car Structure for the Transverse Axis with Standard Draft Gear, 36- and 64-Tonne Cargo, 12.91 to $17.98 \mathrm{~km} / \mathrm{hr}$ Impact Velocity, and 38 Damping .. II -95

II-85 Composite Response Spectra of Rail Car Structure for the Vertical Axis with Standard Draft Gear, 36- and 64-Tonne Cargo, 12.91 to $17.98 \mathrm{~km} / \mathrm{hr}$ Impact Velocity, and 38 Damping .. II-96 


\section{LIST OF FIGURES}

(Continued)

Figure

II-86 Input to Cargo of Rail Vibration in (g) including the 99 Percentile Level of Zero-to-Peak Amplitudes ............ II-97

II-87 Mean-Plus-Three Standard Deviation Amplitude Envelopes of Shock Response Spectra with 38 Damping ............... II-98

II-88 Bounding Acceleration Shock Response Spectrum for 38 Damping on All Three Axes for Truck ................ II-102

II-89 Bounding Acceleration Shock Response Spectrum for 38 Damping on All Three Axes for Rail ................. II-103

II-90 Bounding Acceleration Shock Response Spectrum for 38 Damping on All Three Axes for Rail Coupling .............. II-104

II-91 Bounding Half-Sine Pulse Response Spectrum for Truck Shock ................................. II 104

II-92 Bounding Half-Sine Pulse Response Spectrum for Rail Shock ... II-106

II-93 Bounding Half-Sine Pulse Response Spectrum for RailCoupling Shock ............................ II-106

II-94 Bounding Truck Vibration Data for All Three Axes ......... II-107

II-95 Bounding Rail Vibration Data for Al1 Three Axes .......... II-108

II-96 Nonlinear Slab Thermal Analysis Problem ............. II-110

II-97 Layered Slabs with Internal Heat Generation ........... II-112

II-98 Radiative Exchange Between Conducting Fins ............ II-114

II-99 Finite Element Mesh (top) and Material Zones (bottom)

for the Lead-Shielded Truck Cask .................. II-115

II-100 Finite Element Mesh (top) and Material Zones (bottom)

for the Lead-Shielded Rail Cask .................. II-116

II-101 Cross Section of the Surry $15 \times 15$ PWR Fuel Assembly ...... II-123

II-102 Simplified Model of an Actual Fuel Pin ............. II-124

II-103 A Problem in Heat Conduction Similar to an Enclosed Array of Fuel Pins ......................... II 28 


\section{LIST OF FIGURES \\ (Continued)}

Figure

II-104 Finite Element Mesh of the Steel-Tube-Enclosed $9 \times 9$ Simplified Fuel Pin Model Array ................. II -130

II-105 Predicted Temperature Contours in the $9 \times 9$ Pin Model for $327^{\circ} \mathrm{C}$ Boundary Temperatures ...................... II -131

II-106 Actual and Homogenized Fuel Pin Cells ............. II-132

II-107 Finite Element Mesh of the Steel-Tube-Enclosed Homogenized Smeared $9 \times 9$ Fuel Pin Array .................... II-134

II-108 Predicted Temperature Contours for the Homogenized $9 \times 9$ Fuel Pin Array $\left(327^{\circ} \mathrm{C}\right.$ boundary temperature) $\ldots \ldots \ldots \ldots$ II -135

II-109 Thermocouple Lance Locations in Castor-V/21 Spent-Fuel

Temperature Measurement Tests .................. II-136

II-110 Measured Radial Temperature Profiles of Horizontal HeliumBackfilled Castor-V/21 Casks ................... II-136

II-111 Finite Element Mesh of Assembly A1 and Its Basket Tube

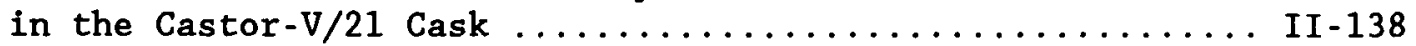

II-112 Predicted Temperature Contours for Assembly A1 in the Castor-v $/ 21$ Cask ............................. II -139

II-113 Predicted Peak Fuel Temperature in Truck Casks as a Function of Assembly Decay Heat Generation Rate .......... II-140

II-114 Predicted Temperature Contours in Fuel (top) and Wall (bottom) of the Truck Cask for $3.0-\mathrm{kW}$ Fuel Under Normal Transport Conditions ......................... II-141

II-115 Predicted Peak Fuel Temperature in the Rail Cask as a Function of Assembly Decay Heat Generation Rate .......... II-142

II-116 Predicted Temperature Contours in Fuel (top) and Wall (bottom) of the Rail Cask for 1.0-kW Fuel Under Normal Transport Conditions ........................ II -143

II-117 Truck Cask Node Locations for Which Fire Accident Temperature Histories Are Presented .................. II - 144

II-118 Predicted Regulatory Fire Accident Temperature $\left({ }^{\circ} \mathrm{C}\right)$ Histories of Several Truck Cask Nodes ................ II-145

II-119 Rail Cask Node Locations for Which Fire Accident Temperature Histories Are Presented II -147 


\section{LIST OF FIGURES}

(Continued)

Figure

II-120 Predicted Regulatory Fire Accident Temperature $\left({ }^{\circ} \mathrm{C}\right)$

Histories of Several Rail Cask Nodes ................ II-148

III-1 Fuel Rod, Assembly, and Cask Component Definitions ....... III-5

III-2 Example Analysis of a Truck Cask Regulatory Fire--

Assembly Temperature History ................. III 7

III-3 Comparison of Posttransition Corrosion Models with Data ... III-9

III-4 Lowest Buckling Mode Shape of the Fue1 Rod............ III-13

III-5 Longitudinal sliding of Spacer Grids $\ldots \ldots \ldots \ldots \ldots \ldots \ldots$ III-14

III-6 Lateral Displacement of Assembly Limited by Rod-to-Rod

Contact and Assembly-Basket Gaps ................. III-16

III-7 Cask Drop Impact Orientations $\ldots \ldots \ldots \ldots \ldots \ldots \ldots \ldots$ III -19

III-8 Free-Body Diagram: Assembly Side-Drop Loading Condition... III-20

III-9 Free-Body Diagram: Assembly End-Drop Loading Condition... III-21

III-10 Free-Body Diagram: Assembly Corner Drop, Initial Impact

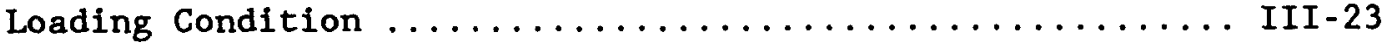

III-11 Free-Body Diagram: Assembly Corner Drop, Slapdown

Loading Condition .......................... III 24

III-12 Illustration of End Drop: Initial Velocity Loading ...... III-25

III-13 Cask-Assembly End Drop: Initial Velocity Loading

Schematic Diagram......................... III-27

III-14 Development of Cask-Rod Vertical Gap Deceleration

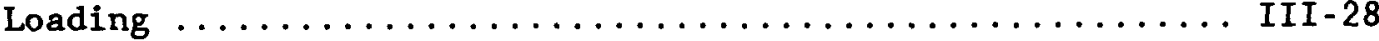

III-15 Schematic Diagram of Spacer Grid Response ............ III-29

III-16 Single-Cell Spacer Grid Analysis Model $\ldots \ldots \ldots \ldots \ldots \ldots$ III-31

III-17 Two-Dimensional Assembly Mode1: Spacer Grid Spring

Element, Force-Displacement Relationship ............ III-32

III-18 Spacer Grid Mode1: Typical Single Rod End-Drop Assembly... III-33

III-19 Example BWR GE $7 \times 7$ Bundle Side Drop: Two-Dimensional

Assembly Model Description ................... III -35 
III-20 Example BWR Side Drop: Two-Dimensional Assembly Model, Detail A .............................. III -36

III-21 BWR Side Drop: Two-Dimensional Assembly Mode1 Cross Sections ................................ III -37

III-22 Example PWR Side Drop: Two-Dimensional Assembly Model Description. . . . . . . . . . . . . . . . . . . . . . . III 38

III-23 PWR Side Drop: Two-Dimensional Assembly Slice Mode1 Cross Sections ........................... III-39

III-24 Example BWR and PWR End-Drop Single-Rod Models ........ III-41

III-25 Example BWR and PWR Corner Drop: Initial Impact SingleRod Models ............................. III -44

III-26 Computational Model for Slapdown Analysis .......... III-45

III-27 Cladding Material Rupture Failure Mode .............. III-49

III-28 Cladding Material Fracture Failure Mode ............. III-50

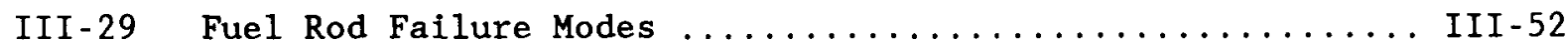

III-30 Probability Distribution for Cladding Rupture Strain as a Function of Biaxial Stress Ratios ................. III-53

III-31 Three Basic Fracture Mechanics Modes of Crack Surface Displacements ........................ III -57

III-32 Probability Density Function for Cladding Fracture

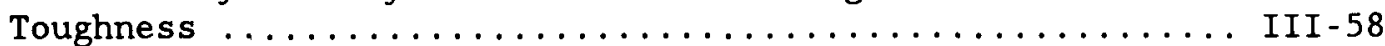

III-33 Cumulative Probability Distributions for Cladding Fracture Toughness at Various Temperatures and Fast Fluences....... III-59

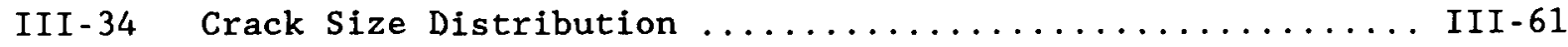

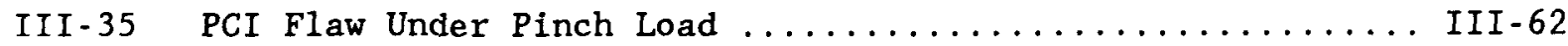

III-36 Example Stress Intensity Values Versus Part-Wa11 Cracks Under Pinch Loads ........................... III -63

III-37 Locations of Potential Part-Wall Cracks .............. III-65 
LIST OF FIGURES

(Continued)

Figure

Page

III-38 Stress Intensity Magnification Factors for Axial

Crack in a Cylinder $(b / r=0.1) \ldots \ldots \ldots \ldots \ldots$ III -67

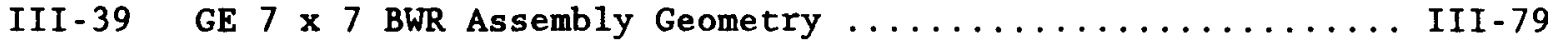

III-40 B\&W $15 \times 15$ PWR Assembly Geometry $\ldots \ldots \ldots \ldots \ldots \ldots \ldots$ III-81

III-41 Example BWR Spacer Grid Geometry $\ldots \ldots \ldots \ldots \ldots \ldots \ldots \ldots$ III-84

III-42 BWR One-Half Spacer Grid Model: Deflected Shape Plot ... III-85

III-43 Geometry and Boundary Conditions of the BWR Spacer Grid

S1ice Mode1 ........................... III-86

III-44 BWR Spacer Grid S1ice Mode1: Deflected Shape P1ot ...... III-88

III-45 GE $7 \times 7$ BWR Assembly Spacer and Grid Elements......... III-89

III-46 Example PWR Spacer Grid Geometry .............. III-90

III-47 PWR Spacer Grid Slice Model: Deflected Shape Plot ...... III-91

III-48 B\&W $15 \times 15$ PWR Assembly Spacer Grid Elements.......... III-92

III-49 PWR End Drop Mode1: Top and Bottom Spacer Grid Spring

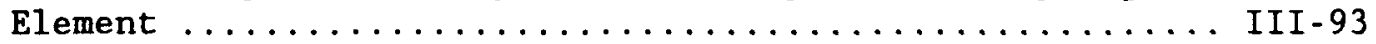

III-50 GE $7 \times 7$ BWR Side-Drop Two-Dimensional Assembly, 100-g

Quasi-static Analyses: Deflected Shape Plots .......... III-95

III-51 GE $7 \times 7$ BWR Side-Drop Two-Dimensional Assembly, 100-g

Quasi-static Analysis: Longitudinal Outer Fiber Strain

Profile Plots, Slice Model with Interior Tie Rods ....... III-96

III-52 GE $7 \times 7$ BWR Side-Drop Two-Dimensional Assembly, 100-g

Quasi-static Analysis: Longitudinal Outer Fiber Strain

Profile Plots, Slice Model with Top and Bottom Tie Rods... III-97

III-53 GE $7 \times 7$ BWR Side-Drop Two-Dimensional Assembly, 100-g

Quasi-static Analysis: Longitudinal Outer Fiber Strain

Profile Plots, Slice Model with No Tie Rods............ III-98

III-54 GE $7 \times 7$ BWR Side-Drop Two-Dimensional Assembly, 100-g

Analysis: Distribution of Spacer Grid Pinch Loads,

Slice Model with Tie Rods Top and Bottom.............. III-99

III-55 B\&W $15 \times 15$ PWR Side-Drop, Two-Dimensional Assembly,

100-g Quasi-static Analysis: Deflected Shape Plots ...... III-101 


\section{LIST OF FIGURES}

(Continued)

\section{Figure}

III-56 B\&W $15 \times 15$ PWR Side Drop, Two-Dimensional Assembly, 100-g Static Analysis: Distribution of Spacer Grid Pinch Loads, Slice Model with Tie Rods Top and Bottom .... III-102

III-57 GE $7 \times 7$ BWR 9.0-m Side-Drop Dynamic Analysis: TwoDimensional S1ice Model Deflected Shapes ............. III-104

III-58 GE $7 \times 7$ BWR 9.0-m Side-Drop Dynamic Analysis: Maximum Tensile Strain Time History ..................... III-105

III-59 GE $7 \times 7$ BWR 9.0-m Side-Drop Dynamic Analysis: Maximum Pinch Force Time History .................... III-106

III-60 BWR Example Assembly GE $7 \times 7$ Slice Mode1 with Two Interior Tie Rods ......................... III-107

III-61 B\&W $15 \times 15$ PWR 9.0-m Side-Drop Dynamic Analysis: TwoDimensional Slice Model Deflected Shapes ............. III-108

III-62 B\&W $15 \times 15$ PWR 9.0-m Side-Drop Dynamic Analysis:

Maximum Pinch Force Time History ............... III-110

III-63 B\&W $15 \times 15$ PWR 9.0-m Side-Drop Dynamic Analysis:

Maximum Tensile Strain Time History ............... III-111

III-64 PWR Example Assembly B\&W $15 \times 15$ Slice Model with Four Interior Control Rods ...................... III-112

III-65 B\&W $15 \times 15$ PWR and GE $7 \times 7$ BWR End-Drop Buckling Mode

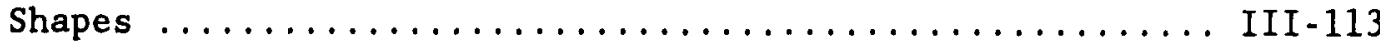

III-66 Example Assemblies: Sensitivity of Buckling Load Versus Percentage of Fuel Mass Active ................... III-114

III-67 End-Drop Quasi-static Collapse Analyses: Deflected Shape Plots ............................... III-116

III-68 End-Drop Quasi-static Collapse Analyses: Maximum Tensile Strain and Displacement Versus Load ............... III-117

III-69 B\&W $15 \times 15$ PWR End-Drop Analysis: Maximum Tensile and Compressive Rod Surface Strain Versus Maximum Lateral Displacement

III-70 B\&W $15 \times 15$ PWR 9.0-m End-Drop Analysis: Deflected Shape Plot 
LIST OF FIGURES

(Concluded)

Figure

Page

III-71 B\&W $15 \times 15$ PWR 9.0-m End-Drop Analysis: Maximum Strain

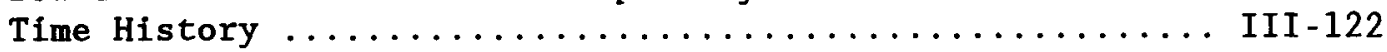

III-72 B\&W $15 \times 15$ PWR 9.0-m End-Drop Analysis: Maximum Lateral

Displacement Time History .................... III -123

III-73 Example Corner-Drop Slapdown Load Distribution:

9.0-m Drop of the Lead-Shielded Truck Cask........... III-126

III-74 Regulatory 9.0-m Corner-Drop at 2-Degree Initial Impact

Angle: Slapdown Impact Velocity Profile ............ III-127

III-75 B\&W $15 \times 15$ PWR and GE $7 \times 7$ BWR 9.0-m Corner-Drop

Slapdown Analysis: Deformed Shape Plot ............. III-128

III-76 Fire Analysis: Two-Dimensional Fuel Rod S1ice

Computational Model with Part-Wall Crack ............. III-130

III-77 Regulatory Fire: Maximum Hoop Strain History at a

Part-Wal1 Crack in PWR Cladding............... III-131

III-78 B\&W $15 \times 15$ PWR Normal Transport Analysis: Deflected

Shape ............................. III -133

III-79 GE $7 \times 7$ BWR and B\&W $15 \times 15$ PWR Summary of 0.3-m Side-

Drop Dynamic Analysis ...................... III-134

IV-1 Lognormal Plot of Particle-Size Data for Fracture

Particulates of Seven Materials ............... IV -12

IV-2 Fracture Particle Size Distribution for Depleted $\mathrm{UO}_{2} \ldots \ldots$ IV-13

IV-3 Summary of Fracture-Surface Energy Data from Impact

Tests and Slow-Compression Tests ................ IV-15

IV-4 Size Distribution of Depleted $\mathrm{UO}_{2}$ Fragments After

Impact with Various Energy Densities ............. IV-16

IV-5 Size Distribution of 33 GWD/MTU KWU Fuel Fragments After Impact with Various Energy Densities ............. IV -17

IV-6 Specific Surface Area of 33 GWD/MTU Fuel Fragments After

Impact with Various Energy Densities............... IV-18 
Table

1 Engineering Tests Performed to Simulate Expected Conditions

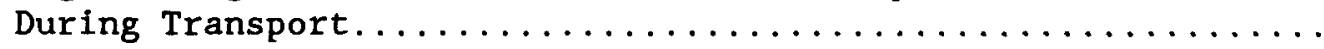

2 Summary of Example Assembly Fallure Probabilities Under Regulatory Transport Loading of Spent Fuel ........... 78

3 Radionuclide Contents of Selected LWR Fuel Rods .......... 82

4 Gap Concentrations of Selected Radionuclides in PWR and BWR Fue1 Rods ............................. 83

5 Radionuclide Releases from Selected LWR Fuel Rods Due to

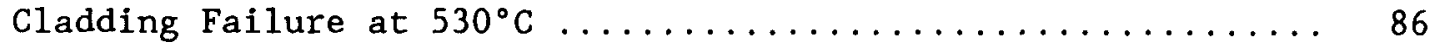

6 Radionuclide Concentrations in an NLI-1/2 Transport Cask on Cladding Failure of a Single Fuel Rod ............ 87

7 Containment Requirements for the NLI-1/2 Transport Cask for Selected LWR Spent-Fuel Assemblies with a Single Rod

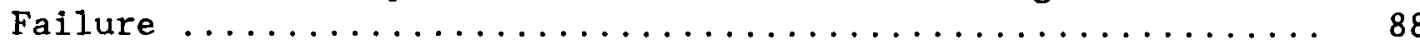

8 Summary of Sensitivity Parameters $\ldots \ldots \ldots \ldots \ldots \ldots \ldots \ldots \ldots$

I-1 BWR Assembly Discharges as of December $1985 \ldots \ldots \ldots \ldots \ldots$ I-6

I-2 PWR Assembly Discharges as of December $1985 \ldots \ldots \ldots \ldots \ldots$ I-7

I-3 As-Manufactured Physical Characteristics of LWR Fuel Rods .... I-10

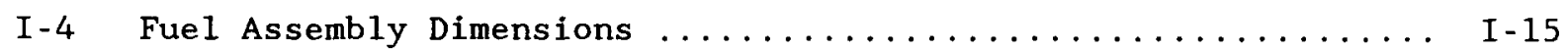

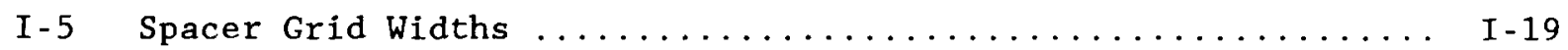

I-6 Guide Tube and Spacer Grid Data for LWR Assemblies ......... I-21

I-7 Breaches Due to Debris-Induced Fretting in PWRs $\ldots \ldots \ldots \ldots \ldots$ I-28

I-8 PWR Fuel Rods Breached Due to Baffle Jetting $\ldots \ldots \ldots \ldots \ldots \ldots$ I-29

I-9 PWR Baffle Jetting Breach Statistics $\ldots \ldots \ldots \ldots \ldots \ldots \ldots \ldots$ I-30

I-10 Fuel Rod Breach Levels in BWRs and PWRs $\ldots \ldots \ldots \ldots \ldots \ldots \ldots$ I-36

I-11 PCI Incipient Defects from Maine Yankee Core $1 \ldots \ldots \ldots \ldots \ldots$ I-55

I-12 Ratio of Measured to Predicted Values of Selected Nuclides ... I-59

I-13 Radionuclide Contents of Selected LWR Fuel Rods .......... I-60 


\section{LIST OF TABLES}

(Continued)

$\underline{\text { Table }}$

I-15 Calculated Fission Gas Production and Gap Inventory in

Irradiated PWR and BWR Fuel Rods $\ldots \ldots \ldots \ldots \ldots \ldots \ldots \ldots \ldots \ldots \ldots$ I- 63

I-16 Tritium Distribution as a Percentage of the Inventory in

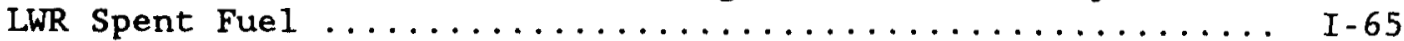

II-1 Physical and Mechanical Properties of Cask Materials ....... II-8

II-2 Characteristics of Representative BWR and PWR Fue1 Assemblies ......................... II 8

II-3 Representative Casks Mass Summaries (kg) $\ldots \ldots \ldots \ldots \ldots \ldots \ldots$ II-9

II-4 Lumped Parameter Model Masses (kg) $\ldots \ldots \ldots \ldots \ldots \ldots \ldots \ldots$ II -20

II-5 Lumped Parameter Model Stiffnesses $\left(10^{9} \mathrm{~N} / \mathrm{m}\right) \ldots \ldots \ldots \ldots$ II -21

II-6 Rigid-Body Mode1 Capabilities and Limitations ........... II-31

II-7 Rigid-Body Cask Boundary Conditions $\ldots \ldots \ldots \ldots \ldots \ldots \ldots \ldots$ II-37

II-8 Equations of Motion for the Rigid-Body Cask Model ........ II-39

II-9 Input Parameters for the Quarter-Scale NUPAC 125-B Cask .... II-45

II-10 Representative Cask Input Parameters $\ldots \ldots \ldots \ldots \ldots \ldots \ldots \ldots$ II-53

II-11 Peak Acceleration Loadings for Certified Casks for the Regulatory 9.0-m-Drop Impact Test (from SARPs) .......... II-71

II-12 Accelerations for Certified Casks for the Regulatory 1.0-m Free-Drop Puncture Condition (from SARPs) ............ II -73

II-13 Qualitative Comparison of Analytical Methods for Cask Drop Events ....................... II -73

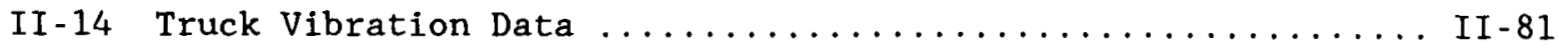

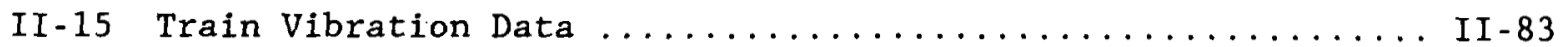

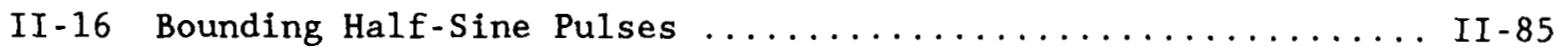

II-17 Truck Vibration Data for a 20-Tonne Cargo ............. II-86

II-18 Bounding Half-Sine Pulses for Truck Vibration Data ........ II-89

II-19 Bounding Truck Half-Sine Pulses $\ldots \ldots \ldots \ldots \ldots \ldots \ldots \ldots \ldots \ldots$ II-93 


\section{LIST OF TABLES \\ (Continued)}

$\underline{\text { Table }}$

II-20 Bounding Half-Sine Pulses for Rail-Coupling Shocks ....... II-97

II-21 Summary of Recent Shock and Vibration Studies ........... II-100

II-22 Nonlinear Slab Temperature Predictions $\ldots \ldots \ldots \ldots \ldots \ldots \ldots$ II-111

II-23 Slab with Heat Source-Temperature Predictions .......... II-113

II-24 Temperature Predictions of Radiative Heat Exchange

Between Fins............................. II 114

II-25 Temperature-Dependent Properties of the Basic Cask Model

Materials .............................. II-121

II-26 Equivalent Thermal Material Properties for the Simplified

Fuel Pin Mode1 .......................... II-127

II-27 Effective Conductivity Estimates for the $9 \times 9$ Simplified

Fuel Pin Array .......................... II-132

II-28 Effective Thermal Material Properties of Homogenized

Smeared Spent Fue1 ......................... II-133

III-1 Zircaloy Oxidation During Transport Conditions.......... III-10

III-2 Influence Coefficients, Gj, for Semielliptical Surface Cracks .................................. III-69

III- 3 Normalized Stress-Intensity Factors for Surface Cracks in Pipes Under Tension and Bending Loads .............. III-73

III-4 Example Structural Analysis Results: Regulatory Transport Loading of Spent Fuel .................. III-77

III-5 Geometric and Material Properties of the GE $7 \times 7$ BWR Fuel $\operatorname{Rod} \ldots \ldots \ldots \ldots \ldots \ldots \ldots \ldots \ldots \ldots \ldots \ldots \ldots \ldots$ III $-8 \ldots$

III- 6 Geometric and Material Properties of the B\&W $15 \times 15$ Mark B

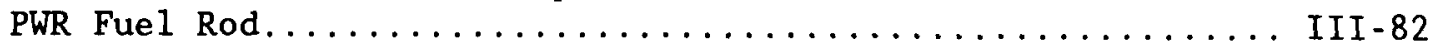

III-7 Summary of Rod-Cask Gap Rigid-Surface Impact Loadings ...... III-118

III-8 Results for the PWR Rod Models with Lateral Gaps and Both Maximum and One-Half Maximum Bowing.................. III-119

III-9 Results for the Initial Impact Analyses Using a Single-Rod Assembly Model to Measure the Effect of Angle on the Maximum Strains of Fuel Rods. 
Table

III-10 Summary of Example Assemblies Failure Probabilities Under Regulatory Transport Loading of Spent Fuel........... III-137

IV-1 Comparison of the Releases of $137 \mathrm{Cs}$ from a PWR Fuel Rod Irradiated to $38.2 \mathrm{MWD} / \mathrm{kg}$ U Burnup After 5 -yr Decay ...... IV-5

IV-2 Radioactive Material Release from a PWR Fuel Rod as

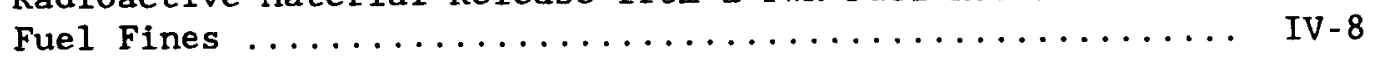

IV-3 Correlation Parameter Values for Depleted $\mathrm{UO}_{2} \ldots \ldots \ldots$ IV-20

IV-4 Correlation Parameter Values for Spent UO 2 (22 GWD/MTU) .. IV-20

IV-5 Correlation Parameter Values for Spent UO 2 (33 GWD/MTU) ... IV-21

IV-6 Concentrations of Airborne Radionuclides Resulting from the Failure of One PWR Fuel Rod in a Representative Truck Cask volume ....................... IV -26 


\section{ACRONYMS}

\begin{tabular}{|c|c|}
\hline ANF & $\begin{array}{l}\text { Advanced Nuclear Fuels Corporation (formerly Exxon Nuclear } \\
\text { Company) }\end{array}$ \\
\hline $\mathrm{AC}$ & Allis Chalmers \\
\hline ANSI & American National Standards Institute \\
\hline B\&W & Babcock and Wilcox \\
\hline BCL & Battelle Columbus Laboratories \\
\hline BET & Becker-Emmet-Teller \\
\hline BWR & boiling water reactor \\
\hline CMC & center-of-mass-over-corner \\
\hline$C E$ & Combustion Engineering, Inc. \\
\hline CIT & Cranfield Institute of Technology \\
\hline CILC & CRUD-induced localized corrosion \\
\hline DOE & Department of Energy \\
\hline EPRI & Electric Power Research Institute \\
\hline FCMI & fuel-clad mechanical interaction \\
\hline FPDB & fuel performance database \\
\hline GE & General Electric Company \\
\hline HEDL & Hanford Engineering Development Laboratory \\
\hline INEL & Idaho National Engineering Laboratory \\
\hline IAEA & International Atomic Energy Agency \\
\hline ICRP & International Commission on Radiological Protection \\
\hline LLNL & Lawrence Livermore National Laboratory \\
\hline LWR & light water reactor \\
\hline LEFM & linear elastic fracture mechanics \\
\hline MCC & Materials Characterization Center \\
\hline MNOP & maximum normal operating pressure \\
\hline MARRSS & $\begin{array}{l}\text { Model for the Analysis of Radioactivity Releases in Spent-Fuel } \\
\text { Shipments }\end{array}$ \\
\hline MCC & Materials Characterization Center \\
\hline MRS & monitored retrievable storage \\
\hline NFS & Nuclear Fuel Service \\
\hline NRC & Nuclear Regulatory Commission \\
\hline ORNL & Oak Ridge National Laboratory \\
\hline OCRWM & Office of Civilian Radioactive Waste Management \\
\hline PNL & Pacific Northwest Laboratory \\
\hline PCI & pellet-clad interaction \\
\hline PIE & postirradiation examination \\
\hline PWR & pressurized water reactor \\
\hline QA & quality assurance \\
\hline RAM & radioactive material \\
\hline SARs & Safety Analysis Reports \\
\hline SARPs & Safety Analysis Reports for Packaging \\
\hline SNL & Sandia National Laboratories \\
\hline SFDB & Spent-Fuel Database \\
\hline UN NUC & United Nuclear \\
\hline US NRC & U.S. Nuclear Regulatory Commission \\
\hline WE & Westinghouse Electric Corp. \\
\hline
\end{tabular}




\section{NOMENCLATURE}

A

$\mathrm{A}_{2}$

$a_{0}$

b

$C_{1}$

$\mathrm{C}_{\mathrm{N}}, \mathrm{C}_{\mathrm{A}}$

$\varepsilon f$

$\mathrm{FP}_{\mathrm{P}}$

Keff

$\mathrm{K}_{\text {IC }}$

$\mathrm{K}_{\mathrm{I}}$

$\mathrm{L}_{\mathbf{i}}$

$\mathrm{L}_{\mathbf{R}}$

N

$\mathrm{R}_{\mathbf{i}}$

$\mathrm{R}_{\mathrm{N}}, \mathrm{R}_{\mathrm{A}}$

$\sigma_{c}$

$\delta t$

W accident conditions

assigned limit on the activity of particular radionuclides amplitude of half-sine pulse

hoop-to-axial stress ratio

source concentration

activity concentrations under normal and accident conditions material ductility

maximum bar failure force

thermal conductivity

fracture toughness

stress intensity factor

leak rate

reference air leak rate

normal conditions

instantaneous release rate

release rates under normal and accident conditions

crush stress

time step

cask weight 


\subsection{INTRODUCTION}

\subsection{Issues and Objectives}

A cask containment system can generally be designed from two perspectives: the cask and its associated hardware are either assumed to provide containment alone or the cask contents (in this case, the spent fuel) are also considered part of the containment system. The approach in which no containment benefits, or credit, are calculated based on the behavior of cask contents is known as a leak-tight design basis. A sourceterm methodology includes in the cask containment assessment the material, physical, or chemical properties of the cask contents that tend to limit or inhibit radionuclide release.

The use of a source-term methodology in the analysis, design, and operation of a spent-fuel cask containment system is expected to result in safety benefits and cost savings: (1) occupational exposure can be reduced if the time required to perform containment assessment before transport is reduced, and (2) fabrication and maintenance expenses can be reduced and cask service life can be extended.

The radionuclides in a spent-fuel transport cask originate from three distinct media:

- The loaded spent fuel,

- Activated corrosion and free fission products adhering to the surface of spent-fuel rods (CRUD), and

- Residual loose contamination that may build up in the cavity of a cask over time.

The development of a source-term methodology must consider the individual contributions of each of these media. Preliminary assessments of the CRUD and residual contamination contributions were performed [SA91a, SA91b].

The purpose of these analyses is to evaluate the applicability of using a source-term methodology to review cask containment system designs. Models are developed for evaluating the response of spent-fuel assemblies to the normal and hypothetical accident conditions defined by regulations, and example cases are evaluated. The report is limited to describing and evaluating the developed methodologies. The report does not provide limiting cases, nor should the provided examples be considered as such.

\subsection{Background}

Containment of cask contents by a transport cask is a function of the cask body, one or more closure lids, and various bolting, hardware, and seals associated with the cavity closure and other containment 
penetrations. In addition, characteristics of cask contents that impede the ability of radionuclides to move from an origin to the external environment provide containment. In essence, multiple release barriers exist in a series in a shipping cask and the magnitude of the releasable activity available in the cask is considerably lower than the total activity of its contents. A source-term approach credits the magnitude of the releasable activity available in the cask by assessing the degree of barrier resistance to release provided by material characteristics and inherent barriers that impede the release of radioactive contents.

The containment requirements for radioactive material in transport casks are defined by both International Atomic Energy Agency (IAEA) and U.S. Nuclear Regulatory Commission (NRC) regulations [IA90, US90]. Both IAEA and NRC regulations allow a source-term approach in containment evaluations. The U.S. Code of Federal Regulations, Title 10, Part 71 (10 CFR 71), requires that irradiated nuclear reactor fuel be transported in transport casks that are "designed, constructed, and prepared for shipment so that . . . (under specified normal conditions of transport) . . there would be no loss or dispersal of radioactive contents, as demonstrated to a sensitivity of $10^{-6}$ A2 per hour, no significant increase in external radiation levels, and no substantial reduction in the effectiveness of the packaging; and . . . [under specified hypothetical accident conditions] . . . there would be no escape of krypton-85 exceeding 10,000 curies in one week, no escape of other radioactive material exceeding a total amount of $A_{2}$ in one week, and no external radiation dose rate exceeding one rem per hour at one meter from the external surface of the [transport cask]" [US90]. The quantity $A_{2}$ is an activity limit that, under specific release scenarios, would prevent radiological effects from exceeding a specified level consistent with radiological protection standards of the International Commission on Radiological Protection (ICRP). Values of $\mathrm{A}_{2}$ (e.g., $\mathrm{A}_{2}=7 \mathrm{Ci}$ for ${ }^{60} \mathrm{Co} ; \mathrm{A}_{2}=10 \mathrm{Ci}$ for ${ }^{137} \mathrm{Cs}$ ) are tabulated in Appendix A of 10 CFR 71; see also Safety Series 6 and Safety Series 7 by the IAEA [IA90, IA87].

Procedures generally acceptable to the NRC for assessing compliance with these provisions are identified in Regulatory Guide 7.4 [US75]. This guide endorses the containment and leak test procedures that are specified in the American National Standards Institute (ANSI) standard ANSI N14.5 [AN87].

The ANSI standard permits time-integrated values for both $R_{N}$ and $R_{A}$; that is, if $r_{i}(t)$ ( $i=N$ or $A$ ) represents the instantaneous release rate under the appropriate transport conditions, the release limits are

$$
\mathrm{R}_{\mathrm{N}}=\mathrm{R}_{\mathrm{N}}(\tau)=\int_{0}^{\tau} \mathrm{r}_{\mathrm{N}}(t) \mathrm{dt} \leq 10-6 \mathrm{~A}_{2}
$$

where

$i=N$ for normal conditions

$\tau=1 \mathrm{hr}$ 


$$
\mathrm{R}_{\mathrm{A}}=\mathrm{R}_{\mathrm{A}}(r)=\int_{0}^{r} \mathrm{r}_{\mathrm{A}}(t) \mathrm{d} t \leq \mathrm{A}_{2},
$$

where

$i=A$ for accident conditions

$\tau=1 \mathrm{wk}$.

Compliance with these release limits may be demonstrated by direct measurement of $R_{i}$. If this is not practical, the standard provides an alternative method for demonstrating containment adequacy that evolves from the definition of $R_{N}$ and $R_{A}$. The alternate approach relates leak rates, $L_{i}$ $\left(\mathrm{cm}^{3} / \mathrm{s}\right)$, to the appropriate release rates, $R_{i}$, through the expression

$$
L_{i}=R_{i} / C_{i}
$$

where $i=N$ for normal conditions or $A$ for accident conditions and $C_{i}$ represents the time-averaged volumetric concentration of suspended particulate, liquid, or gaseous radioactivity (in $\mathrm{Ci} / \mathrm{cm}^{3}$ of the transport cask medium) that could escape from the containment system during transport.

To account for cask atmosphere differences (e.g., air, helium, nitrogen, or water) and different sets of pressure and temperature conditions, ANSI N14.5 further defines a reference air leak rate, $\mathrm{L}_{R}$, expressed in standard cubic centimeters per second (std $\mathrm{cm}^{3} / \mathrm{s}$ ). Through this definition, all leak rates are referred to the standard conditions of dry air at $1 \mathrm{~atm}$ absolute pressure and $25^{\circ} \mathrm{C}$. In the event $L_{R}$ is $\geq 10$ std $\mathrm{cm}^{3} / \mathrm{s}$, ANSI N14.5 exempts the package from leak testing. Otherwise, the fabricated package must be shown to have adequate containment before its initial use, after a shakedown period (normally after the first three uses), and annually thereafter. Demonstration that the fabricated package is in compliance with $10 \mathrm{CFR} 71$ requires that the assembled system, which includes the cask and fuel, be shown to release material at rates $\leq R_{N}(\tau)$ and $\mathrm{R}_{\mathrm{A}}(\tau)$ under normal and accident transport conditions, or that the reference air leak rates are $\leq L_{R}$. When $L_{R}$ is $\leq 10^{-7}$ std $\mathrm{cm}^{3} / \mathrm{s}$ for either normal or accident conditions, it is only necessary to demonstrate that the leak rate does not exceed $10^{-7} \mathrm{std} \mathrm{cm}^{3} / \mathrm{s}$.

Although ANSI N14.5 is quite prescriptive, little guidance is given regarding the determination of the activity concentrations $C_{N}$ and $C_{A}$. When $C_{N}$ and $C_{A}$ cannot be definitively established, the leak-tight design criterion is required.

The objective of this analysis is twofold: (1) to develop methodologies for defining $C_{N}$ and $C_{A}$, and (2) to estimate appropriate containment requirements (as demonstrated by corresponding maximum 
permissible leak rates) for values of $C_{N}$ and $C_{A}$ expected during loading conditions associated with normal and accident transport environments.

The allowable release limit for normal transport, $R_{N}$, was defined in ANSI N14.5 for the most adverse expected conditions. These conditions are simulated by a series of engineering tests known as normal conditions of transport, which are prescribed by regulations. These regulatory normal transport conditions define an environment that represents the cask performance requirements and must not result in exceeding the technical safety limit, $R_{N}$. This limit is a constraint to a safety objective, not the objective per se, and should therefore be interpreted as the lower boundary of a "forbidden region." The original derivation of $R_{N}$, given in detail in Appendix II of the IAEA Safety Series 7 [IA87], is based on ensuring that the exposure of transport workers during normal transport conditions and under most adverse conditions is highly unlikely to exceed limits recommended by the International Commission on Radiological Protection (ICRP) [IA90]. Analyses subsequent to the original derivation (described in Safety Series 7 ) indicate that more realistic scenarios for worker exposure result in conservative estimates of doses of 20 and 0.28 of annual ICRP limits for indoor and outside scenarios, respectively.

The accident release limit, $R_{A}$, is also conservative in terms of both radiological implications and the likelihood of a cask undergoing conditions comparable to the performance standards defined by regulatory accident conditions. The limits on $R_{A}$ ensure that the incidental exposure of persons near an accident involving a Type $B$ transport cask does not exceed ICRP annual dose limits for radiation workers. Thus $R_{A}$ is an upperbound technical limit or safety criterion, not a specific design objective.

The radiological implications of $R_{A}$ during accident conditions are also discussed in Appendix II of the IAEA Safety Series 7 [IA87]. The corresponding dose limit is based on the integrated exposure of a person remaining continuously downwind of the releasing cask throughout the 1 -wk period. In addition, Safety Series 7 [IA87] states that (1) it is highly unlikely that such a release would persist for a full week and (2) emergency actions would limit the exposure of individuals in the immediate vicinity of the releasing cask. The maximum expected dose is an estimated 0.1 to $1 \mathrm{rem}$, or 2 to 208 of the allowable annual dose for radiation workers.

The release limits $R_{N}$ and $R_{A}$ are thus quite small and are designed to restrict the exposure of radiation workers during normal and accident transport conditions within the limits of the ICRP [IN80] and the U.S. Code of Federal Regulations (10 CFR 20) [US88]. These limits are based on very conservative assumptions regarding the time a worker remains in the vicinity of the source and characteristics of the source release, which together are expected to provide "the most adverse expected conditions" [ IA90].

Safety Series 7 [IA87] further states the following:

"The design principle embodied in the Regulations is that intentional leakage of activity from a Type $B$ package should be 
avoided. However, because absolute containment cannot be guaranteed, the purpose of specifying maximum allowable activity leak rates is to enable the specification of appropriate and practical test procedures, which are related to acceptable radiological protection criteria."

The use of the $R_{N}$ and $R_{A}$ criteria is the technical basis for the source-term criterion that was developed. This analysis approach evaluates and defines acceptable containment on the basis of regulatory material retention requirements to minimize exposure of the general public and radiation workers.

\subsection{Technical Approach}

Figure 1 illustrates the effect of the activity available for release from the cask on cask containment requirements in normal and accident transport conditions. The diagonal lines represent technical limit lines based on $R_{N}$ and $R_{A}$ for various combinations of $L_{i}$ on the vertical axis and $\mathrm{C}_{i}$ on the horizontal axis. These lines are essentially constant release lines corresponding to the lower limits of unacceptable release regions to the right of each line. Limiting leak rates $L_{N}$ and $L_{A}$ are plotted for $R_{N}$ and $R_{A}$ and for varying specific source concentrations in the cask cavity, $\mathrm{C}_{\mathrm{N}}$ and $\mathrm{C}_{\mathrm{A}}$, in $\mathrm{A}_{2} / \mathrm{cm}^{3}$. The leak-tight criterion shown by the horizontal line is bounding for concentrations exceeding $C_{N}=3 \times 10^{-3} \mathrm{~A}_{2} / \mathrm{cm}^{3}$ and $\mathrm{C}_{\mathrm{A}}=16.5 \mathrm{~A} 2 / \mathrm{cm}^{3}$. For smaller concentrations, ANSI N14.5 permits average gas leak rates below the sloping lines.

A radiological consequence can occur in the external environment of the cask only if radionuclides are first released from the spent fuel to the cask cavity and then from the cask cavity to the external environment. The first phase is governed by the multibarrier characteristics of the spent fuel and the second by the characteristics of both the material released to the cask cavity and the pathway through the cask to the external environment.

To realistically estimate $L_{i}$, a mechanistic description of fission product release mechanisms and transport behavior in the cask is required. The concentration of fission and activation product radionuclides available for release is a function of the following:

1. The environmental conditions that could initiate a release;

2. The total fission and activation product inventory;

3. The physical, chemical, and radioactive characteristics of the radionuclide products contained in the spent fuel;

4. The initial conditions of the spent fuel that affect its response to the transport environment; 


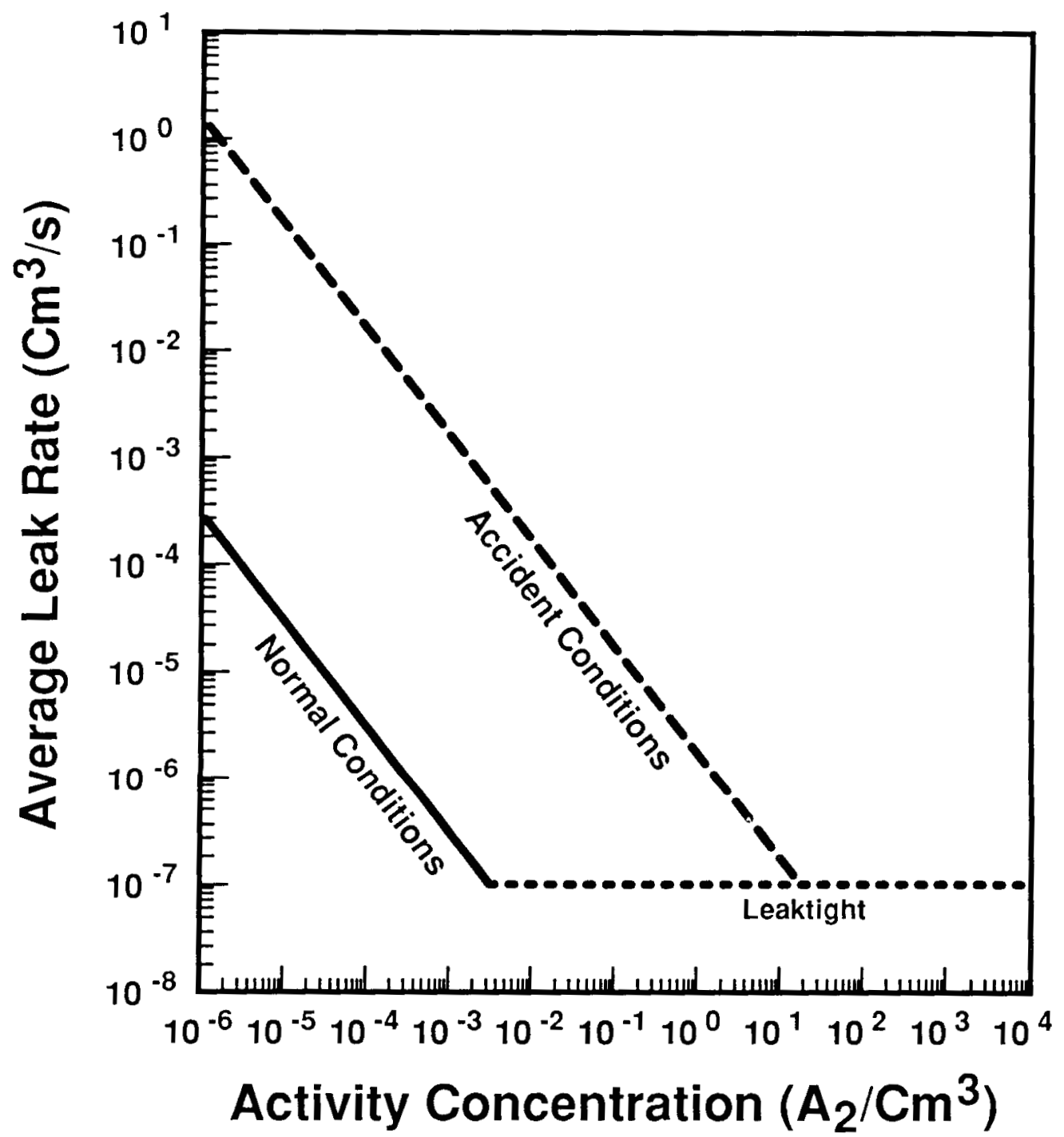

Figure 1. Limiting Average Leak Rates Versus Activity Concentration

5. The mechanisms and pathways for release of radionuclides from fuel pellets to the void space in the fuel rod (commonly called the fuel cladding gap and/or plenum regions), and from this gap through a cladding breach to the cask cavity;

6. The mobility or mass transport characteristics of the fission products released to the cask cavity; and

7. The geometry of the internal cask configuration, including void volume and internal surface areas. 


\subsubsection{Environmental Conditions}

Because the release-initiating environmental conditions are defined here by the regulatory tests for normal and accident transport conditions, the fuel response to these conditions must be determined. The fuel response, in turn, largely depends on the cask response. The engineering tests designed to simulate expected conditions during normal and accident transport conditions are described in Table 1 [US90].

The response behavior of a cask subjected to this hypothetical accident sequence has been demonstrated to be more severe than the insults of forces experienced by the casks in more than $98 \%$ of real-world accident scenarios [FI87]. The probability of a real accident producing responses less than the accident conditions is basically 0.98 , and the allowable consequence of that event is limited to a release $\leq R_{A}$. Thus, the risk of such events is within the ICRP guidelines for radiation workers as previously described. The probabilities of real-world accidents producing cask conditions more severe than the regulatory accident conditions are approximately 0.02 per event [FI87], and the limiting consequences in terms of $R_{A}$ can be increased accordingly while still maintaining risk within the ICRP guidelines.

The radionuclide inventory in the spent fuel that is to be transported is a function of the fuel type (pressurized water reactor [PWR] or boiling water reactor [BWR]), fuel burnup, power history, initial enrichment, and decay time (age) since discharge from an operating nuclear reactor. Figure 2 illustrates a prediction of burnup characteristics of the spent fuel to be transported in next-generation transport casks [SA87]. The two distributions shown in this figure illustrate the differences between the two reactor systems, particularly in the higher burnup range projected. The age of all fuel in this inventory is $\geq 10 \mathrm{yr}$. As indicated, burnups as high as 60 gigawatt days per metric ton of uranium (GWD/MTU) and 40 GWD/MTU are predicted for PWR and BWR fuel, respectively.

The physical, chemical, and radioactive characteristics of the fission products contained in the spent fuel are important because they affect the releasability of particular species. Also, the individual biological effects are factored into the $A_{2}$ values for each radionuclide. Releasability is a qualitative measure of the ease with which a particular species can migrate across pellet and cladding barriers to a release point under varying pressure, temperature, and/or mechanical driving forces. Physical and chemical characteristics can affect both the initiation of species transport and the transport process. For example, while some fission products are of gaseous or volatile form, their bonding within the fuel matrix is such that only a small fraction may be transported across the cladding in the event of a cladding failure.

The physical condition of the spent fuel before transport significantly determines fuel response to the transport environment. Fuel response depends on the mechanical properties of the constituents of the spent-fuel assemblies and the effect of the following assembly conditions on these properties: irradiation, temperature, and the presence of flaws induced during the in-reactor and storage portions of the fuel cycle. 
Table 1

\section{Engineering Tests Performed to Simulate Expected Conditions During Transport}

\section{Norma1 Transport}

1. Initial conditions prior to test sequence:

A. Ambient temperature: -29 to $38^{\circ} \mathrm{C}$.

B. Pressure: maximum normal operating pressure (MNOP) unless ambient is more unfavorable.

2. Final conditions after the test sequence and prior to evaluation: A. Ambient temperature: -29 and $38^{\circ} \mathrm{C}$.

3. Heat test: ambient temperature of $38^{\circ} \mathrm{C}$ combined with maximum solar insolation.

4. Cold test: ambient temperature of $-40^{\circ} \mathrm{C}$ in still air and shade.

5. Reduced external pressure: $24.5 \mathrm{kPa}$.

6. Increased external pressure: $140 \mathrm{kPa}$.

7. Vibration: normal transport vibration.

8. Water spray: $5 \mathrm{~cm}$ per hr for $1 \mathrm{hr}$.

9. Free drop: $0.3 \mathrm{~m}$ on a nonyielding horizontal surface oriented for maximum expected damage. (Cask weight is greater than 15 tonne.)

10. Corner drop: not required for spent-fuel casks.

11. Compression: not required for spent-fuel casks.

12. Penetration: drop of a $3.2-\mathrm{cm}$ diameter, $6-\mathrm{kg}$ right circular mild steel cylinder from a height of $1.0 \mathrm{~m}$ onto the most vulnerable cask surface. 
Table 1

Engineering Tests Performed to Simulate Expected Conditions During Transport (Concluded)

\section{Accident Transport}

1. Initial conditions prior to test sequence:

A. Ambient temperature: value between -29 and $38^{\circ} \mathrm{C}$, which is most unfavorable for the feature being tested.

B. Pressure: MNOP or other lower pressure if it is more unfavorable.

2. Final conditions after test sequence:

A. Ambient temperature: value between -29 and $38^{\circ} \mathrm{C}$. which is most unfavorable.

3. The following tests must be performed in sequence:

A. Free drop: 9.0-m drop onto a flat unyielding target oriented for maximum expected damage.

B. Puncture: $1.0-\mathrm{m}$ drop onto the upper end of a vertical steel bar mounted on an unyielding surface, with the cask oriented for maximum expected damage.

C. Thermal: exposure to a fully engulfing radiation environment corresponding to $800^{\circ} \mathrm{C}$ with an emissivity coefficient of at least 0.9 for $30 \mathrm{~min}$ minimum.

4. Immersion: a separate, undamaged cask must experience external pressure equivalent to immersion under a 15 -m head of water for at least $8 \mathrm{hr}$.

These flaws could appear as actual pinhole breaches or part-wall cracks caused by mechanisms such as pellet-clad interactions (PCI) or hydriding. A certain percentage of the fuel may contain absorbed water that could lead to alternative release mechanisms.

The spent-fuel-cask configuration is a multibarrier confinement and containment system. For a release to occur, radionuclides contained in the spent-fuel pellets must first be in a dispersible condition, a driving force is necessary to initiate and maintain radionuclide transport, and some form of barrier release pathway must exist or be initiated. 


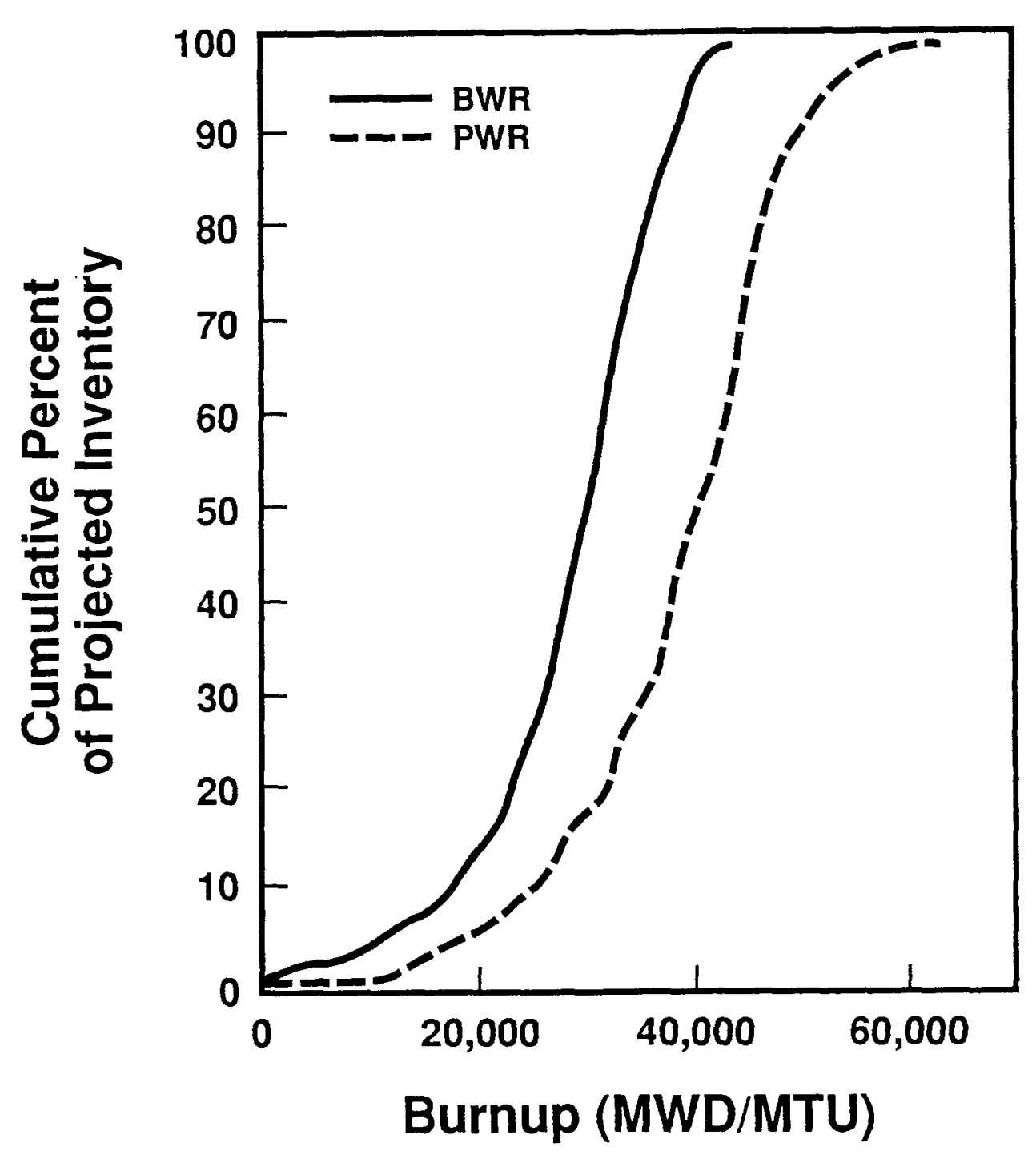

Figure 2. Projected Burnup Characteristics for the Spent-Fuel Inventory to Be Transported During the First 25 Yr of Repository Operation. The fue 1 age is $\geq 10 \mathrm{yr}$.

\subsubsection{Release Mechanisms}

The fault tree in Figure 3 traces the potential consequences of an initiating event. Radioactive material released to the cask cavity from spent fuel can occur as a result of one of five different mechanisms. Each mechanism, in turn, is instigated by a particular initiating event brought about by some environmental condition. The physical mechanisms that can cause release of radionuclides from the spent fuel are (1) impact failure of fuel cladding; (2) burst rupture of intact fuel cladding; (3) leaching of soluble fission products from rods with failed cladding when in contact with water, or from waterlogged rods; (4) diffusion of vaporized fission products from rods with defected cladding; or (5) rapid fuel oxidation in severely failed fuel rods [WI84]. 

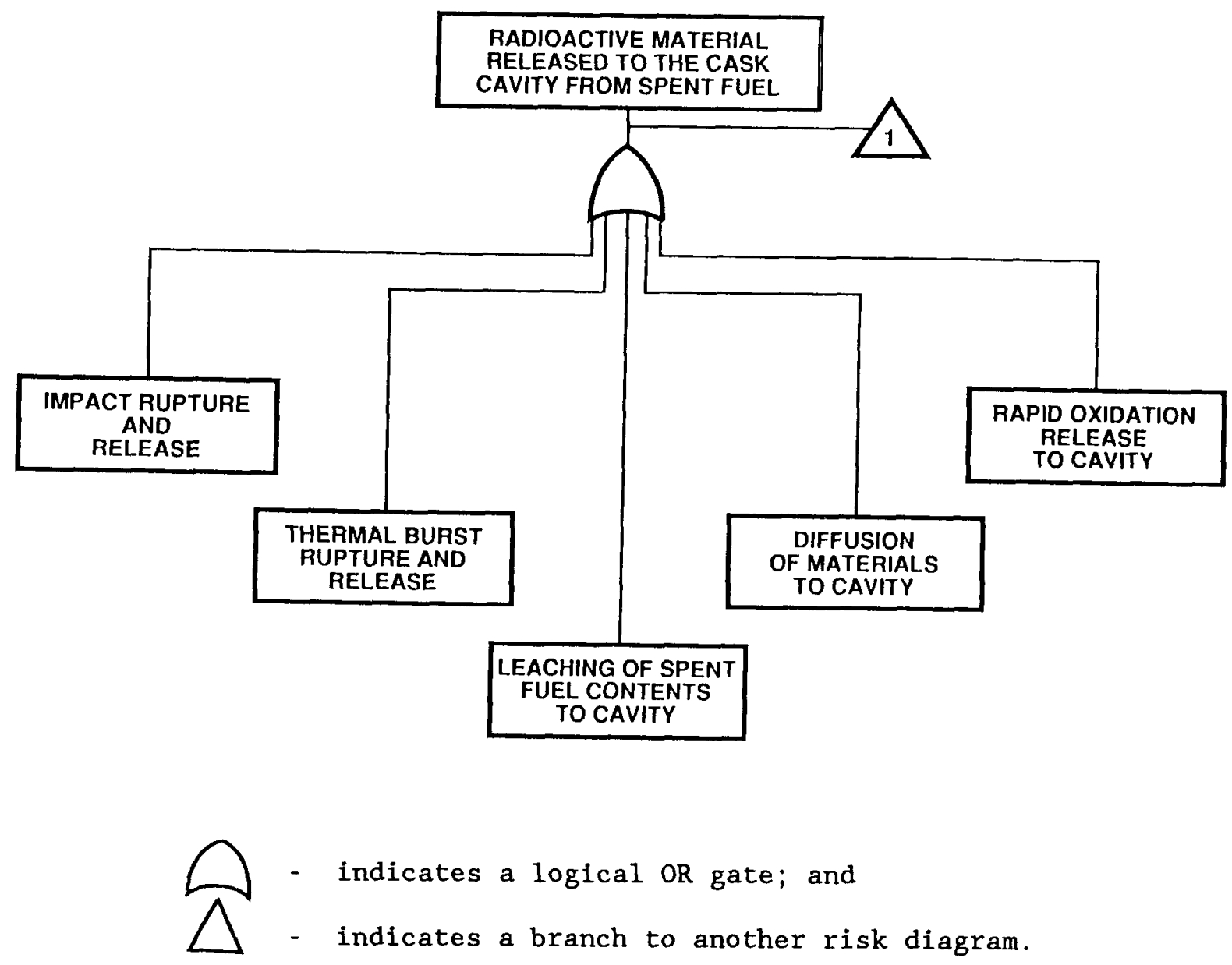

Figure 3. Release Scenarios Affecting the Source Term in a Spent-Fuel Transport Cask

These mechanisms can be further investigated by using fault-tree logic to identify subevents and parameters that may influence the initiation and maintenance of each mechanism. The fault tree in Figure 4 describes the sequence of events involved in an impact failure of an intact (i.e., pressurized) spent-fuel rod or group of rods. The release of radioactive material occurs as a result of mechanical disruption of the cladding and subsequent depressurization of the fuel rod. As a result of the impact, small particles, referred to as fuel fines, may be generated with a size and mass distribution such that some particles are transported to the cask cavity by venting gases. Depending on temperature, fuel rod venting also releases fission-product gases and volatile species contained in the fuelcladding gap.

Both the cladding mode and threshold depend on the initial cladding conditions, the loading condition of the assembly, and internal pressure. The cladding may exhibit ductile or brittle behavior, and rupture may occur as a result of crack initiation followed by through-the-wall propagation or extension of a preexisting crack. The response behavior depends on several 


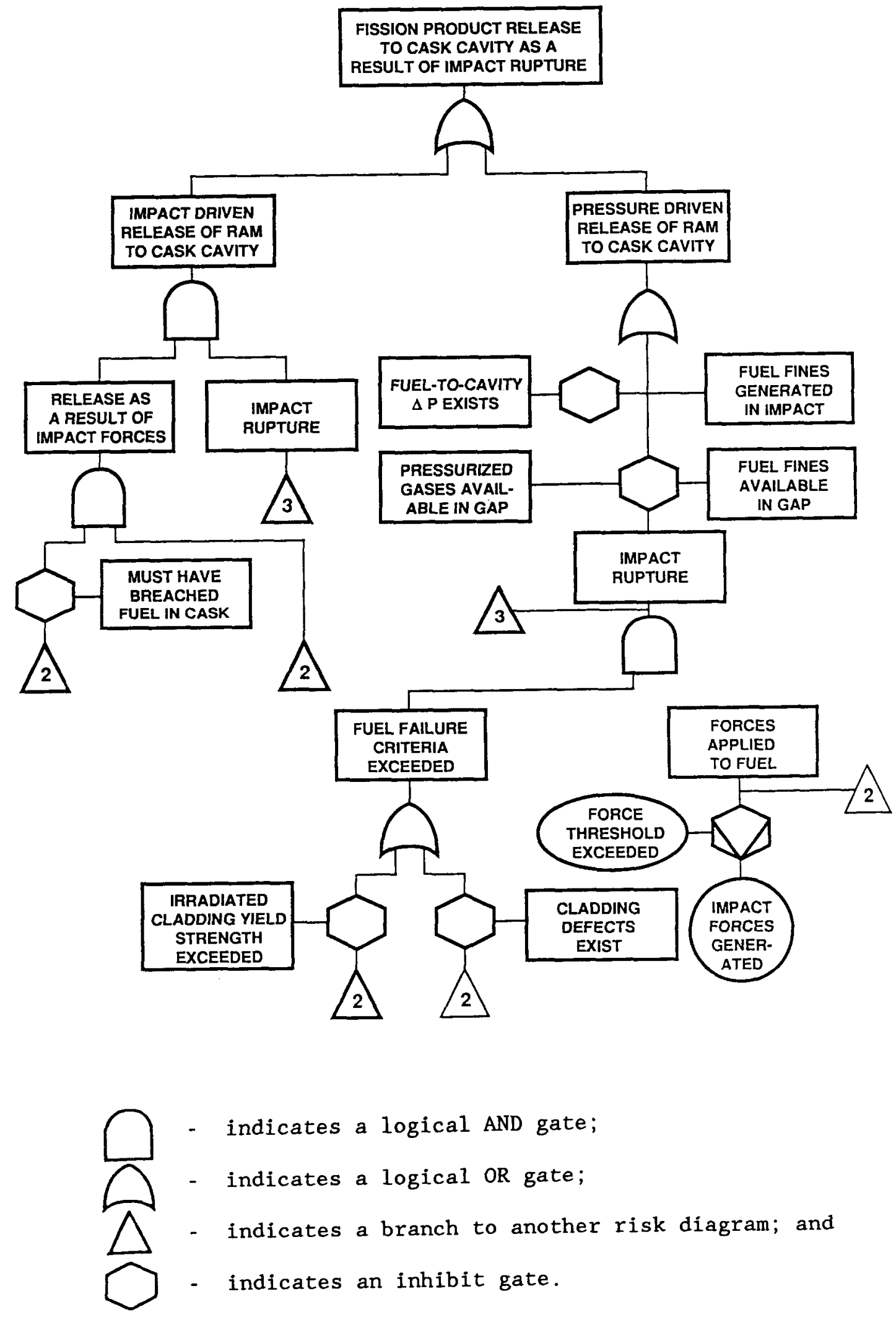

Figure 4. Processes and Events Affecting Radionuclide Release from Spent Fuel During Mechanical Impact Conditions 
fuel geometry and initial condition variables. These include fuel assembly geometry, temperature, irradiation history, and the presence of preexisting partial through-the-wall flaws.

The loading conditions that affect the contaminants depend on the cask and impact limiter design; that is, the way the cask responds to the impact event and the process by which forces involved are transferred through the cask to the fuel. The loads can range from severe impact conditions over short-time durations to the long-duration time-varying accelerations undergone during normal transport.

Some percentage of the rods may have failed or breached during inreactor service, thereby venting internal pressures to equilibrium with reactor or storage system pressures. Releasability from a source-term perspective is diminished in this instance because (1) much of the highly mobile gaseous fission products are gone, and (2) the potential highpressure source for transporting products to the cask cavity is no longer available. Thus, in an impact condition, the dispersion can only occur as a result of local mechanical forces generated by the impact.

Sometimes previously breached fuel may become waterlogged; that is, the differential pressure between the rod and reactor system coolant is sufficient to force some amount of coolant into the pellet-clad gap regions. Cladding hydriding can occur when hydrogen gas is liberated from the moisture in the fuel (or during corrosion) and reacts with cladding material to form zirconium hydrides, resulting in localized embrittlement. Secondary hydriding can occur in waterlogged fuel. While such embrittlement in waterlogged fuel can significantly reduce the impact failure threshold for the rod, failure consequences are offset by the loss of the high-pressure mobilizing force available in intact rods.

Burst rupture is initiated by high internal rod pressures generated by a severe thermal environment. As a rod is heated by either excessive internal heat generation or as a result of an external heat source (such as a fire), the internal pressure of the rod will increase until a local failure threshold is exceeded. The cladding then yields, and burst rupture occurs at the location of maximum stress concentration or at a preexisting flaw.

The sequence and governing conditions for burst rupture failure are illustrated by Figure 5. As in the impact case, failure criteria depend on initial fuel conditions. The temperature of the cladding must reach some corresponding threshold condition. The effect of heat load on the cask ranges from the extreme accident fire environment to the normal heat load during transport. The effect of temperatures on the fuel depends on ambient conditions, internal heat generation rates, and the heat transfer design of the cask.

Burst rupture typically results in a localized hole that has a diameter approaching a few millimeters [L080]. The release mechanism (pressure-driven) is the same as the impact release from a mechanically breached rod. The release products, however, will likely include more volatile species. 


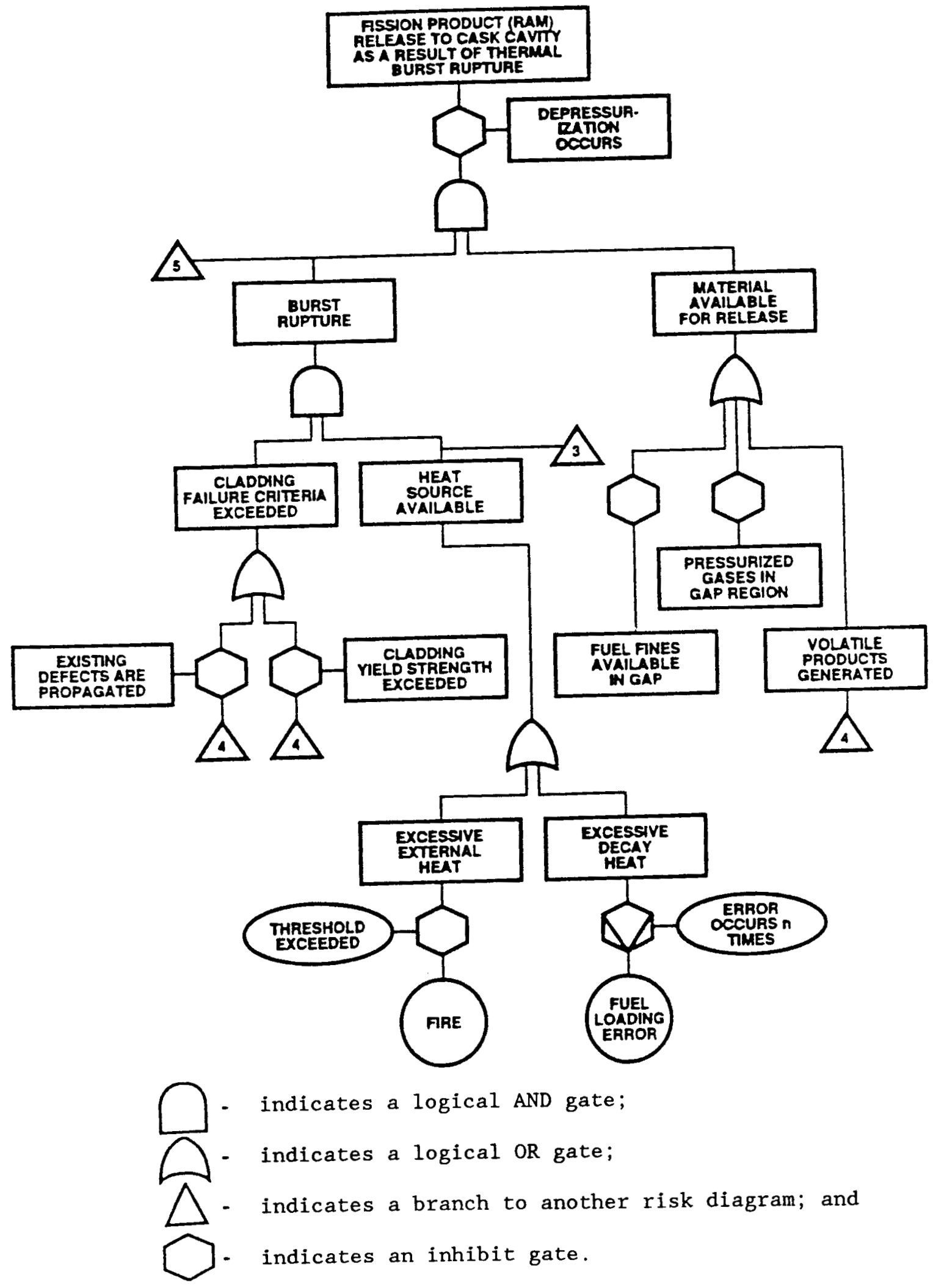

Figure 5. Processes and Events Affecting Radionuclide Release from Spent Fuel During Severe Thermal Conditions 
Leaching of contaminants by water or steam is another mechanism for radionuclide release. Its importance for current transport operations in the United States is less than that of the scenarios previously discussed because a dry, inert gas atmosphere is required in cask cavities. Figure 6
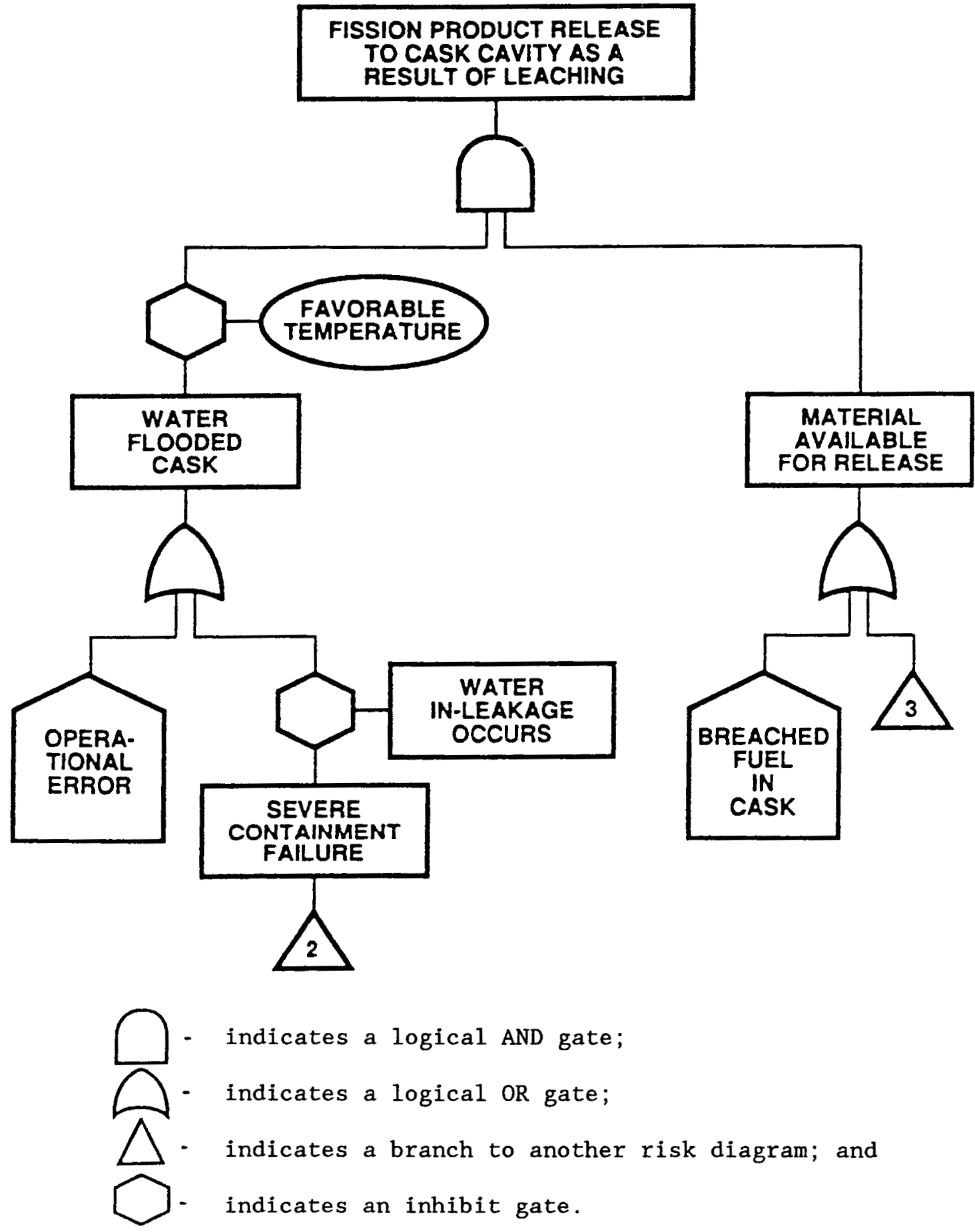

Figure 6. Processes and Events Required for Leaching Release of Radionuclides from Spent Fuel 
illustrates the events and processes required for leaching to occur, Water may be present as a result of operational error before transport or through gross containment failure, or it may be contained in fuel. In the event of a high heat input, sufficiently waterlogged fuel could generate and release steam that would carry fuel products. Direct contact between water and spent-fuel pellets can result in contact leaching of fission and activation products that can then be carried directly throughout the cask cavity in the event of gross water in-leakage.

Previously breached fuel rods can release radionuclides by diffusion. The events and conditions necessary for diffusion to occur are illustrated by Figure 7 . When a spent-fuel rod ruptures, remaining vaporized radionuclides can diffuse through the fuel to the gap region and out of the breach point after the initial release transient (if applicable). The likelihood of diffusion occurring increases with temperature.

As illustrated by Figure 8 , the events and sequences required for fue 1 oxidation to occur as a release mechanism have a low probability of occurrence. These extra-severe events lie beyond the range of the regulatory conditions and thus beyond the scope of this analysis. For cask cavities filled with inert gases, rapid oxidation is credible only if (1) gross operational error occurs before transport, or (2) gross containment failure results in replacement of the inert cavity gas with air, (3) mechanical failure of the fuel occurs, and (4) a high-temperature environment exists.

As illustrated in Figures 6 through 8 , leaching, diffusion, and oxidation are relevant only to extreme accident conditions and appear as secondary release mechanisms following severe impact or burst rupture events. For these processes to occur in normal transport, such gross loading and/or other pretransport errors are required that the particular scenario cannot be construed as normal. For the purpose of this analysis, the applicability of these second-order release mechanisms to regulatory transport conditions is investigated only as necessary to demonstrate or refute their pertinence.

The mass transport characteristics of released material from spent fuel govern the dispersal process from within the cask cavity to a containment release point. Internal movement of the material occurs under the influence of temperature, pressure, or concentration gradients. Influencing factors and conditions are illustrated by Figure 9.

The size of released particles must also be considered. To reach the external environment through a containment leak pathway, radioactive gases and particles must diffuse through the cavity gas. For particles, diffusive deposition and gravitational settling processes may significantly reduce the particle source term exiting the containment. The attenuation capabilities of these processes depend on the aerosol residence time, particle-size distributions, and the fuel and cask designs. Cask design parameters that are influential include the total surface area in the cask cavity, the cask orientation and gravitational settling area described by that orientation, and the gas-void volume of the cask and fuel. 


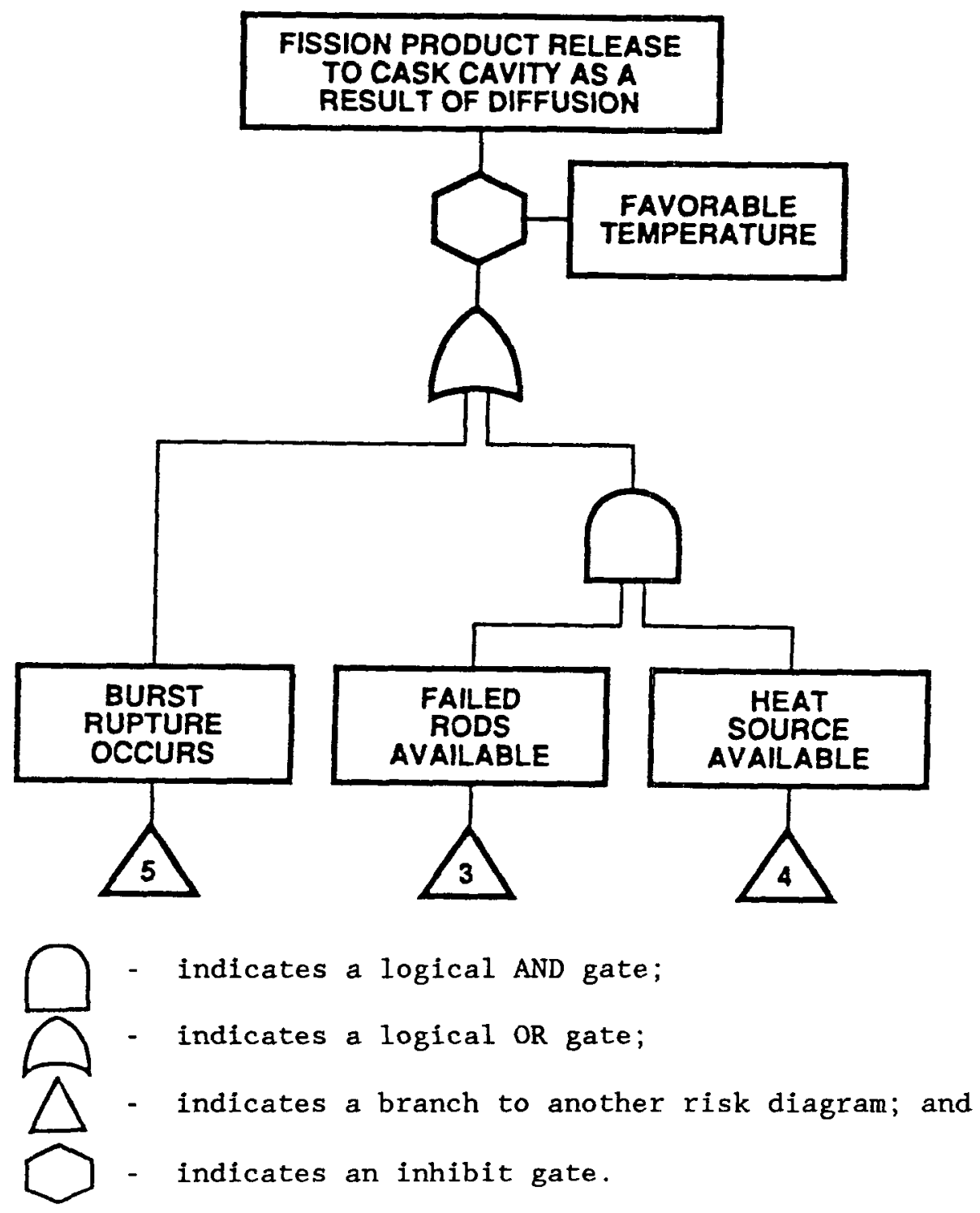

Figure 7. Processes and Events Required for Diffusion Release of Radionuclides from Spent Fuel

\subsection{Summary and Analys is Sequence}

This analysis also describes the development of a physical model of the release mechanisms and physical factors that affect those scenarios previously discussed. A detailed review of the technical approach is presented in Section 2.0. Results of analyses performed to evaluate cask response to the various transport environment conditions are presented in Section 3.0. Expected pretransport fuel conditions based on available spent-fuel data are summarized in Section 4.0. The analytical models developed to evaluate fuel response are described in section 5.0. 


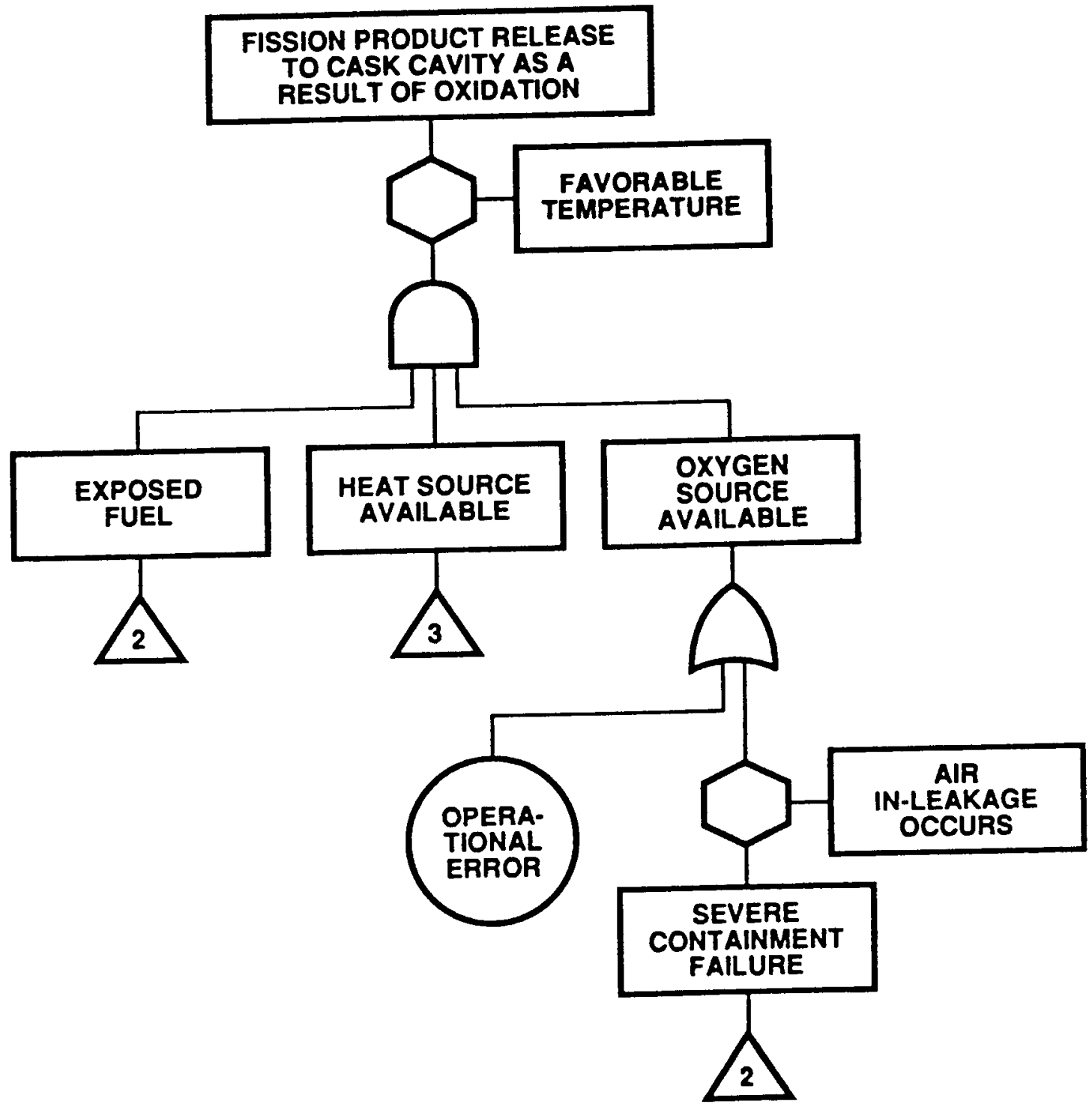

Figure 8. Processes and Events Required for Oxidation Release of Radionuclides from Spent Fuel 


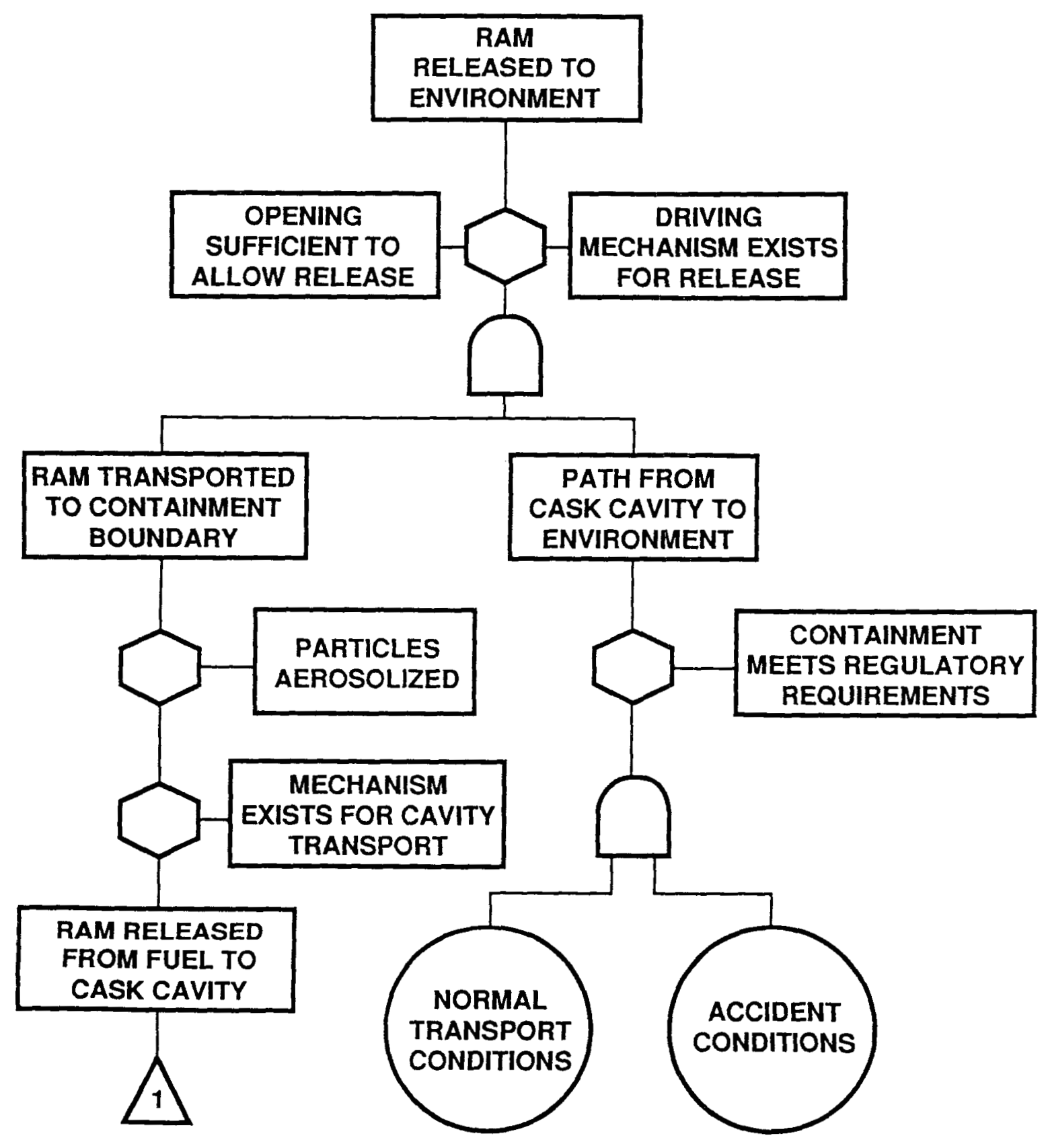

Figure 9. Factors Affecting the Releasable Source Term in a Spent-Fuel Transport Cask 
In Sections 4.0 and 5.0 the integrated analysis method was used to evaluate release scenarios for two representative assemblies: PWR and BWR. These results illustrate the attenuating effects of numerous factors that considerably reduce the potential for radioactive material release.

Factors evaluated include spent-fuel response mechanisms, breached cladding release phenomena, and processes that result in gas-borne dispersion and settling of particulates during movements from an originating cladding defect to the cask containment boundary.

In Section 6.0 the results of sensitivity analyses that were performed are discussed, as well as identifiable uncertainties in data that were used and the effects of assumptions that were made necessary by absent data. Section 7.0 discusses the results of this analysis effort and presents conclusions and recommendations derived therefrom. Detailed discussion of expected pretransport fuel conditions based on available spent-fuel data is provided in Appendix I. Detailed analyses and descriptions of the analytical models used to develop ranges of possible environmental loading conditions are discussed in Appendix II. Complete derivations of the fuel response models used are provided in Appendix III. Radionuclide generation, release, and transport models are described in Appendix IV.

\subsection{References}

[AN87] American Nationa1 Standards Institute, "American National Standards for Radioactive Materials Leakage Tests on Packages for Shipment," ANSI Standard N14.5, January 16, 1987.

[FI87] Fischer, L. E., C. K. Chou, M. A. Gerhard, C. Y. Kimura, R. W. Martin, R. W. Mensing, M. E. Mount, and M. C. Witte, "Transport Container Response to Severe Highway and Railway Accident Conditions," Lawrence Livermore National Laboratory, Livermore, CA, NUREG/CR-4829, 1987.

[IA87] IAEA Safety Standards, Safety Series 7, International Atomic Energy Agency, Vienna, Austria, 1987.

[IA90] IAEA Safety Standards, Safety Series 6, International Atomic Energy Agency, Vienna, Austria, 1990.

[IN80] International Commission on Radiological Protection, Limits for Intake of Radionuclides by Workers, Publication 30, Parts 1-3, (New York: Pergamon Press, 1980).

[L080] Lorenz, R. A., J. L. Collins, A. P. Malinauskas, "Fission Product Source Terms for the LWR Loss-of-Coolant Accident," Oak Ridge National Laboratory, Oak Ridge, TN, NUREG/CR-1288, July 1980.

[SA87] Sanders, T. L., "Feasibility and Incentives for the Consideration of Spent Fuel Operating Histories in the Criticality Analysis of Spent Fuel Transport Casks," Sandia National Laboratories, Albuquerque, NM, SAND87-0151, August 1987. 
[SA91a] Sanders, T. L., H. Jordan, V. Pasupathi, W. J. Mings, and P. C. Reardon, "A Methodology for Estimating the Residual Contamination Contribution to the Source Term in a Spent-Fuel Transport Cask," Sandia National Laboratories, Albuquerque, NM, SAND90-2407, TTC1020, September 1991.

[SA91b] Sandoval, R. P., R. E. Einziger, H. Jordan, A. P. Malinauskas, and W. J. Mings, "Estimate of CRUD Contribution to Shipping Cask Containment Requirements," Sandia National Laboratories, Albuquerque, NM, SAND88-1358, TTC-0811, January 1991.

[US75] U.S. Nuclear Regulatory Commission, "Leakage Tests on Packages for Shipment of Radioactive Materials," in Regulatory Guide 7.4, U.S. Nuclear Regulatory Commission, Washington, D.C., June 1975.

[US88] "Standards for Protection Against Radiation," in U.S. Code of Federa1 Regulations, Title 10, Part 20, 1988.

[US90] "Packaging and Transportation of Radioactive Material," in U.S. Code of Federal Regulations, Title 10, Part 71 , revised January 1 , 1990 .

[WI84] Wilmot, E. L., "Transportation Accident Scenarios for Commercial Spent Fue1," Sandia National Laboratories, Albuquerque, NM, SAND80-2124, July 1984. 
This page is intentionally left blank. 


\subsection{GENERAL APPROACH}

The three sources of potentially releasable radioactivity contained within a loaded spent-fuel transport cask are fuel, CRUD, and residual contamination. Fuel has by far the largest potentially releasable radioactivity of these three sources. The contribution of fuel to the overall release rate largely depends on its initial pretransport condition and on subsequent fuel rod response to transportation loading events. The type and amount of radioactive materials that may be released from the fuel rod to the cask cavity are governed by cladding failure behavior. Cladding failure behavior is a function of cask and assembly designs, transportloading conditions, fuel irradiation histories, and other initial conditions, making cladding failures highly statistical events. Hence, criteria for evaluating fuel contribution to release estimates must be probabilistic, although they may depend on deterministically derived response characteristics. For these reasons, the general approach combines a detailed deterministic mechanical response of fuel rods and assemblies with probabilistic failure evaluations and release estimates.

From detailed computational models of fuel rods and assemblies, the postfailure geometry of a cladding breach (i.e., failure modes) can be determined. This is an essential prerequisite for defining release mechanisms because the physical composition of fuel rod contents that could be released through a cladding breach is strongly dependent on the geometry of the breach. A pinhole failure, for example, could result in the release of fission gases and volatile species and finely dispersed fuel, whereas a guillotine break could generate fragments and particles, and further permit the release of fuel fragments. Other failure modes, such as longitudinal or transverse slits, fall in between these two release mechanisms, depending on the size and location of the failure.

Detailed geometric models combined with knowledge of accurate material constitutive behavior and well-defined loading conditions permit a mechanistic description of the cladding failure modes and postfailure geometries of cladding breaches. Furthermore, the detailed models are capable of treating all such possible failure states and associated release mechanisms. Sufficient data must be available to define the various failure modes, and these data can be translated into specific failure criteria. Typically, failure data are in the form of statistical distributions. Other parameters of fuel rod response analysis, such as design-basis loading conditions, are essentially deterministic. From this basis of statistical variation of initial conditions and failure property data, a probabilistic failure evaluation procedure is developed and applied to the deterministically calculated fuel rod response.

Because of the wide range of fuel conditions involved, a single totally generic analysis model applicable to all cases is not feasible. Furthermore, detailed analysis of special cases may be required to address 
case-specific issues. The general methodology must be sufficiently broadbased to permit both generic and case-specific analyses.

To accomplish this, detailed response and failure evaluation calculations were developed. Then several representative cases were analyzed that were carefully chosen to encompass the conditions of spent fuel in transport. From these analyses, simplified models were derived that are specially constructed to capture the necessary characteristics of each detailed response mode and release mechanism. These simplified models combined with similar models for CRUD and residual contamination were used to develop an overall radionuclide release methodology.

Two representative test cases were used to illustrate the utility of a spent-fuel methodology for determining the contribution of fuel to the source term. General Electric (GE) 7 × 7 BWR and Babcock \& Wilcox (B\&W) $15 \times 15$ PWR fuel designs, with known irradiation histories, were loaded in a generic lead-shielded truck cask and subjected to the service and accident environments specified in Title 10, Part 71, of the U.S. Code of Federal Regulations (10 CFR 71) [US90]. These two test cases provide useful preliminary estimates of expected radioactivity releases under regulatory transport conditions. Although the results of this analysis strictly apply to the two cases analyzed, the general approach is sufficiently developed so that its application to other cases requires no basic changes in procedure or models.

The complete fuel-response model includes the following component databases and analysis routines:

- The U.S. Department of Energy's (DOE) database for spent fuel and high-level waste developed at Oak Ridge National Laboratory (ORNL) [N087]. A subset of this database, referred to as DOE-SFDB (U.S. Department of Energy-Spent Fuel Database), was constructed to provide mainly geometric and design data;

- Idaho National Engineering Laboratory's (INEL) MATPRO package, which contains fuel and cladding material properties [HA81];

- Selected portions of Electric Power Research Institute's (EPRI) FPDB (fuel performance database), which contributes irradiation data such as power histories and burnup [MI88];

- ORNL's ORIGEN2 code, which is used to calculate radionuclide inventories [CR83];

- EPRI's FREY code, which is used to calculate PCI cracking characteristics and cladding response to regulatory fire conditions [RA89]; and

- The ANS-5.4 fission gas release model, which, together with its host code, FREY, is used to calculate the fission gas release [TU82]. 
The detailed deterministic element of the spent-fuel mechanical response is calculated using the ABAQUS general-purpose finite element code [HI89]. ABAQUS is used extensively in the nuclear industry. By applying these probabilistic failure criteria to deterministic response parameters (e.g., stresses, strains, stress intensity factors) in combination with the probabilistic characterization of the cladding initial cracking state, failure frequencies (the number of failed rods) are obtained for a given cask design under a given transport loading.

This methodology and its implementation into an integrated computational form consists of five basic processes:

- Identify geometric and initial fuel conditions,

- Define the regulatory transport environment and associated loading conditions,

- Determine fuel rod/assembly mechanical response,

- Estimate the release of radioactivity, and

- Develop a computer program for the fuel response analysis and evaluation of radioactive material releases in spent-fuel transport.

\subsection{Fuel Geometric and Initial Conditions}

Approximately 98,000 light water reactor (LWR) fuel assemblies either have been discharged or are in operation at U.S. commercial plants; 628 are BWRs and $38 \%$ are PWRs (see Appendix $I$ ). These assemblies vary in size from $6 \times 6$ to $11 \times 11$ for BWR assemblies, and $14 \times 14$ to $17 \times 17$ for PWR assemblies. (The $\mathrm{N} \times \mathrm{N}$ notation indicates the number of rods along each side of a square assembly.) The rod dimensions, initial fill gas pres. sures, and configurations of spacer grids and other assembly hardware vary widely among fuel vendors and from design to design (see Appendix I). Furthermore, some of the older fuel assemblies were disassembled and consolidated in canisters to allow for larger storage capacity in spentfuel pools. Other variables include cask design variations in limiter and basket designs. Data for this first group of variables, which have a major effect on spent-fuel response and potential releases, are referred to as the geometric fuel variables. Data for these variables have been compiled predominantly from the DOE's spent-fuel database (DOE-SFDB) or from other available sources [N087]. Therefore, a generic set of fuel parameters has been established based on prevailing assembly and basket/cask characteristics, structural vulnerabilities, and potential releases. Accounting for differences in geometric fuel variables is nearly impossible; therefore, representative cases that encompass prevailing geometric conditions were selected for analyses.

The second group of variables having a major effect on spent-fuel response and potential releases are in-reactor fuel operational variables; that is, power history, burnup, and fission gas release. Fission gas release is the release from fuel to the fuel cladding gap and plenum 
region. Power history burnup and fission gas release are interdependent and can vary widely between BWRs and PWRs, from assembly to assembly, and from rod to rod in each assembly. The impact of these variables on fuel mechanical response is primarily the result of the effect of irradiation history on material properties and, to a much lesser extent, the result of internal gas as a loading source, which is of minor concern except possibly under regulatory fire conditions.

The main information source for this group of variables is the EPRI FPDB [MI88], which provides a basis for power history and burnup variations, but not for fission gas release. Because release characteristics of the fuel rod gap inventory of volatile species depend on release characteristics of the fission gases in the gap, accurate data on fission gas release must be compiled.

The available literature on fuel performance contains extensive information on fission gas release. However, much of the data are not consistent and show large scatter because of the inclusion of problem fuels and ill-defined irradiation histories. More importantly, the available fission gas data cannot be extrapolated to all fuel types and burnup ranges in a systematic and reliable manner without the use of a model. Therefore, a consistent and conservative method for quantifying fission gas was developed for release using accepted models and computational procedures. The ANSI standard ANS-5.4 fission gas release model [TU82] was used and was incorporated in EPRI's FREY [RA89] fuel behavior code. Power histories from the EPRI-FPDB were used as input for FREY/ANS-5.4 models for each of the representative assemblies selected for analysis to generate complete fission gas release data.

The third group of initial condition variables are those associated with the damage state of the fuel cladding at the time of cask loading and transport. There are two possible sources of damage: in-reactor or operational damage, and handling damage. Typical handling damage that may occur when spent fuel is loaded in transport casks has a minor effect on in-transport fuel response because heavily damaged assemblies are and will continue to be subject to special transport considerations. In-reactor damage, however, has the greatest potential to affect release because it has a direct impact on cladding response and failure rates, and subsequent radionuclide release. The most significant in-reactor damage is induced by PCI. PCI incipient failures (partway-through-the-wall cracks) can be of any depth, with a maximum depth or thickness ratio in the range of 25 to 308--a median estimate for current burnup levels (see Appendices I and II). Other possible types of in-reactor damage are waterside oxidation, corrosion, hydriding, and debris erosion fretting from flow-induced vibrations. Hydriding affects cladding response through cladding embrittlement; therefore, special allowance is made for treating this type of damage by modifying the cladding failure criteria. Waterside damage is also of minor importance except for some of the CRUD-induced localized corrosion (CILC). This type of BWR fuel affected by damage is nongeneric and can be treated in the response analysis on a case-specific basis by locally reducing cladding thickness. Partial PCI cracks, however, are generic and can be treated statistically (see Tasooji et al. [TA84]). 
PCI cracks are assumed to initiate from manufacturing flaws and thus can be considered to have similar statistical characteristics. Specifically, the relative frequency distribution of crack size is assumed to be the same as that for manufacturing flaws, although the absolute sizing may be greater. Thus, with statistical information on manufacturing flaws as a basis, a probabilistic crack characterization methodology was developed to establish the initial conditions for PCI-affected spent-fuel cladding.

Experimental data are very limited on irradiated flaw distributions in spent fuel to verify these assumptions. The technical basis for this methodology consists of five main elements:

- Fuel failure statistics reported in LWR fuel performance literature,

- Flaw population analysis based on established probabilistic estimation procedures,

- PCI analysis using the FREY fuel behavior code,

- Independent prior analysis of probable crack depth based on a chemical/mechanical stress corrosion cracking model [TA84], and

- Available data from measurements.

This crack analysis procedure and the associated probabilistic fracture mechanics failure criterion are major elements in the mechanical response analysis of spent fuel in transport.

\subsection{Definition of Regulatory Transport and Associated Loading Conditions}

Spent-fuel loading environments can be characterized for generic or representative cask designs. General loading parameters provide estimates of expected load levels for a cask under normal and accident transport conditions. Design-basis transport loads that are important to spent-fuel response consist of the following:

- Accelerations associated with the shock and vibration incurred by truck or rail transported casks, including rail-coupling events;

- The peak rigid-body accelerations incurred by a cask during 0.3 - and 9.0-m drop events; and

- The thermal environment imposed by a hypothetical fire event.

Modeling of the force transfer from the cask to the fuel assemblies ignores deformability of the cask, which contributes a high-frequency response with little or no effect on fuel rod response (see Appendix II). Also, because of large stiffness differences between the highly flexible fuel assemblies and the very stiff cask, the two systems can be decoupled. 
Thus, fuel assemblies are estimated to contribute only mass in the cask response analysis model. For impact considerations, the cask is treated as a rigid body governed by rigid-body kinematics as it impacts the regulatory unyielding target. The cask impact limiter, however, is modeled as a crushable material with an energy absorption capacity that is based on the crush strength and geometry of the limiter. The rigid-body cask model and limiter model are included in the SLAM computer code (see Appendix II). The forcing functions on the fuel can also be described by direct input or another analysis tool such as SCANS [GE88].

The rigid-body kinematics analysis method contained in the SLAM code treats a cask as a two-dimensional rigid body with three degrees of freedom: two translational and one rotational. This method provides acceleration histories for any impact orientation and excludes the highfrequency response associated with deformable casks that has little effect on fuel assembly response. The SLAM model is sufficiently detailed to capture all three phases of impact: initial impact, rotation, and secondary impact. Acceleration time histories are produced for all three phases of cask motion and are subsequently used in calculating the deterministic response of fuel rods and assemblies. The effect of gaps between the fuel assembly and the cask is explicitly treated in the dynamic analyzing. Such gaps act to increase the peak mechanical loads on the assemblies.

Part of the design basis transport loading is a regulatory fire accident condition in which the cask is subjected to a totally engulfing $800^{\circ} \mathrm{C}$ fire of half-hour duration. The TOPAZ2D code [SH86] is used to calculate temperature distribution in the cask and fuel assemblies. A smearing technique, verified through detailed analysis (see Appendix II), is used for geometric modeling of the various materials and derivation of equivalent thermal properties. The resulting temperature history for the hottest point in the fuel is used in the mechanical response analysis.

\subsection{Determination of Fuel Rod/Assembly Mechanical Response}

A two-step process was used to evaluate spent-fuel mechanical response. The first step was to develop detailed geometric and computational models and to use these models to obtain the deterministic mechanical response of the fuel rods and assemblies. The second step involved a probabilistic failure evaluation.

A detailed response analysis of a fully loaded transport cask and its entire contents is computationally intractable unless substructural decomposition is performed where smaller substructures can be isolated from the total system and analyzed separately. However, such a decomposition must take place at naturally identifiable interfaces so that force or displacement boundary conditions can be properly defined. The smallest structural element that can be isolated is a single fuel rod, as shown in Figure 10A. The force transfer interfaces for the rod are tie plates, spacer grids, and to a lesser extent, adjacent rods. Under certain conditions (e.g., end impact), the single rod model response can be used adequately to represent the response of the whole assembly, assuming that 


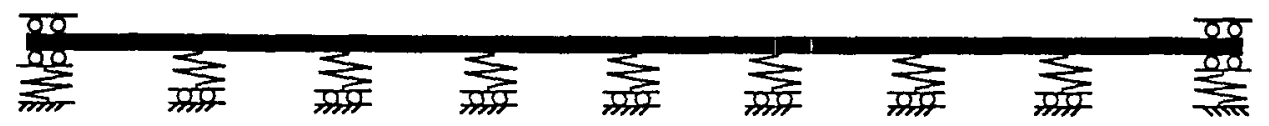

A. SINGLE ROD MODEL

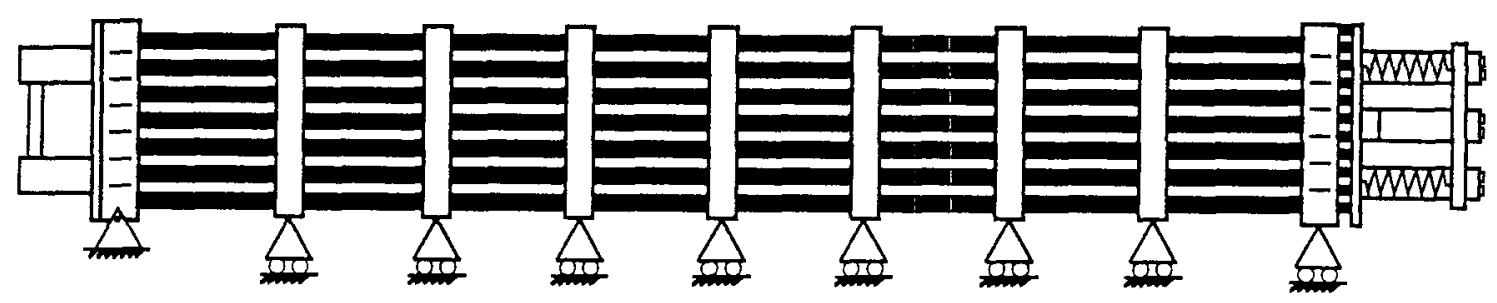

B. SINGLE ASSEMBLY MODEL

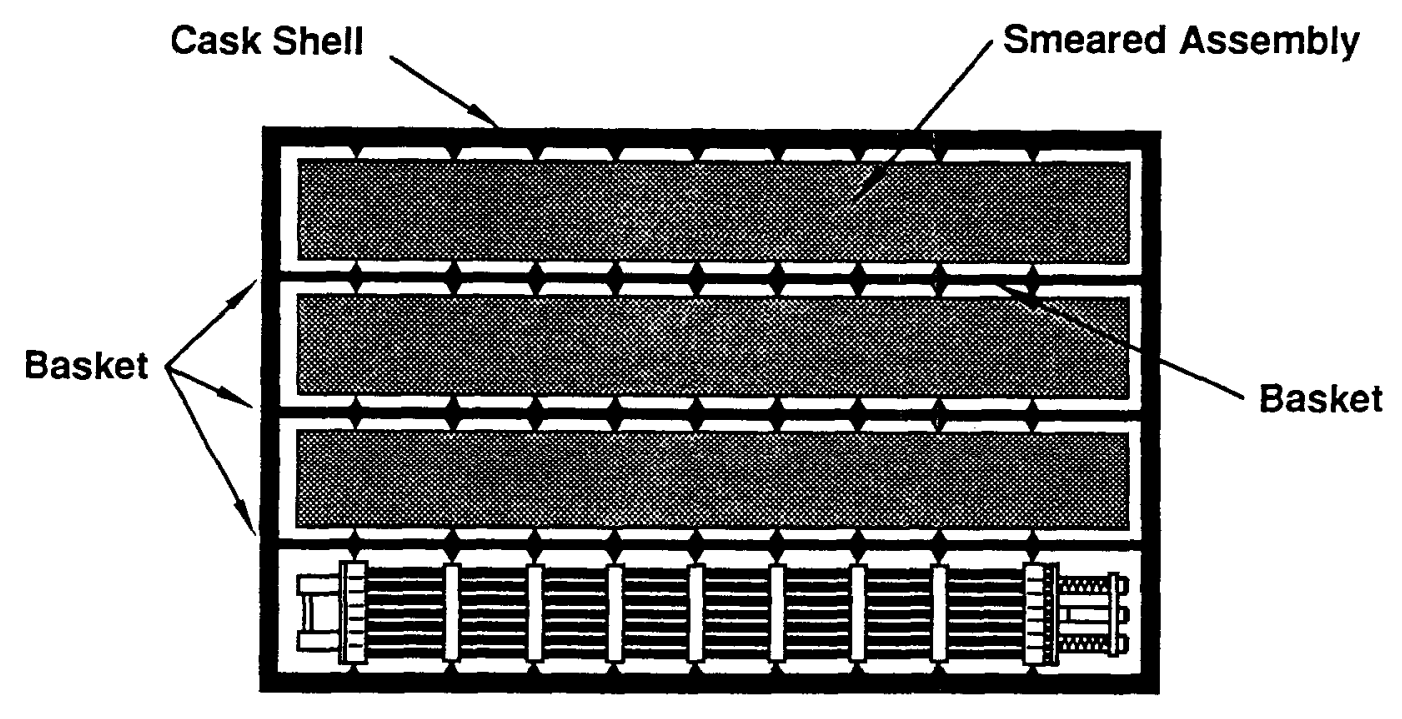

C. MULTI-ASSEMBLY MODEL

WORST CASE POSITION OF OBJECT ASSEMBLY

Figure 10. Geometric Models for Spent-Fuel Assemblies in Transport Casks 
all rods in the assembly have similar deformation patterns. This assumption is conservative because the predominant end-drop failure mode is caused by high tensile bending strains produced by lateral displacement. However, a single rod model is clearly inapplicable to other impact orientations, such as side drop and slapdown. For these conditions, a higher level of structural decomposition is required.

This higher-level structural element is a single assembly, shown in Figure 10B. In this case, the force transfer interface is the basket structure. Depending on the structural design of the basket, force transfer between assemblies may be replaced by displacement boundary conditions that isolate the object assembly from surrounding assemblies. This type of structural decomposition permits detailed geometric modeling of individual rods, spacer grids, and end-tie plates. The basket-assembly interface is replaced by point supports for a basket design of the openframe type, and by line support for a continuous-plate basket design. In either case, the basket structure is not part of the structural model.

The detailed geometric model of the isolated assembly consists of several hundred beam-column elements that represent individual rods and spring elements that represent spacer grids at each interface. To capture the detailed deformation characteristics of the fuel rods, each rod is represented by no fewer than 30 beam-column elements with elastic-plastic and large displacement and strain capabilities. Rod-to-rod interaction is simulated by nonlinear contact spring elements with contact/release capabilities.

The most critical response behavior in the single assembly model is exhibited by spacer grid springs. Although sparsely positioned in the assembly, the spacer grid structures provide significant flexural stiffness to the fuel assembly. This is the dominant structural property that enables the assembly to resist deformations. The spacer grids are highly complex structures, and proper simulation of their behavior in the single assembly model is critical to make correct predictions of cladding failure states. Thus, a spacer spring model is necessary that simulates the complex behavior of spacers over the total deformation range. This model was combined with material models for beam-column elements that represent individual fuel rods and contact elements that simulate rod-to-rod interaction. The cladding material constitutive relations were provided by the MATPRO property data package [HA81]. The contribution of fuel pellets to rod stiffness was not assessed; the mass contribution, however, was included.

Computations for the single assembly model described above were carried out in two dimensions. This implies that only a single row of rods was considered; however, several geometries were analyzed to treat the different rod fixtures (e.g., tie rods and guide tubes) at the tie plates. The general response of the fuel assembly is three dimensional because of the articulated three-dimensional geometry of the assembly and arbitrary impact orientation that is relative to the assembly cross section. This requires consideration of all rods, thus increasing the number of computational model degrees of freedom by factors of 15 to 30 , depending on assembly design. Such an increase in model size would be expensive to 
compute because of the large demand on computer storage requirements and run time. Therefore, two-dimensional approximation is desirable as well as technically justifiable. The two-dimensional simulation is conservative because failure is directly proportional to lateral displacement and the two-dimensional model conservatively neglects all out-of-plane stiffness from the spacer grids and interaction with the basket structure. Moreover, the two-dimensional model does not take credit for the outer frame of the spacer grid, which in some designs is significantly stiffer than the internal cells.

The single assembly model is strictly valid only if the basket structure is sufficiently stiff and remains elastic under all transport loading events and impact orientations. If the basket structure deforms plastically or collapses, it must be included in the assembly response analysis. The adjacent assemblies and, most certainly, the entire contents of the cask must then be further considered.

This requires a higher level of structural decomposition, in which two levels of modeling details are considered: a detailed model for the object assembly, as described earlier for the single assembly model, and additional beam-column models for the remaining assemblies. A detailed model of the basket structure is also included. Each of the surrounding assemblies was replaced by a single beam with equivalent flexural and axial stiffnesses and mass. This multi-assembly model is shown in Figure 10C. The purpose of the super beam-column model is to simulate the force transfer to the object assembly. Therefore, the position of the object assembly in the cask becomes a parameter in the analysis.

The single rod and single assembly models were used to analyze the results for the $\mathrm{GE} 7 \times 7$ and the $B \& W 15 \times 15$ representative cases. The dynamic mechanical response was obtained using the ABAQUS general-purpose finite element code (see Appendix III). Acceleration time histories for the governing impact orientations, that is, end drop, side drop, and corner drop (initial impact and slapdown), were obtained using the SLAM code, described in Section 2.2. Deterministic response parameters, cladding stresses and strains, and rod interaction forces were obtained at critical points along each length of the rod. These quantities were then used in the failure evaluation procedure described below.

The first step in failure evaluation identifies the fuel failure mode from the deterministic mechanical response analysis. The first identifiable failure mode, a transverse crack, could result when strain exceeds material ductility limits. This failure mode can occur during all four impact conditions, that is, end drop, side drop, and corner drop (initial impact and slapdown); it is most probable with the side drop because during side impact plastic bending at the end plates dominates the response. It is assumed that once a crack is initiated, it will immediately extend through the wall, forming a pinhole or narrow transverse crack. The geometry of this failure mode is shown in Figure 11A. The probabilistic failure criterion applied to this type of failure was the material ductility expressed in distribution form (see Appendix III). The deterministic response parameters used to evaluate the conditional failure probability for this mode were the cladding strains and stresses. 


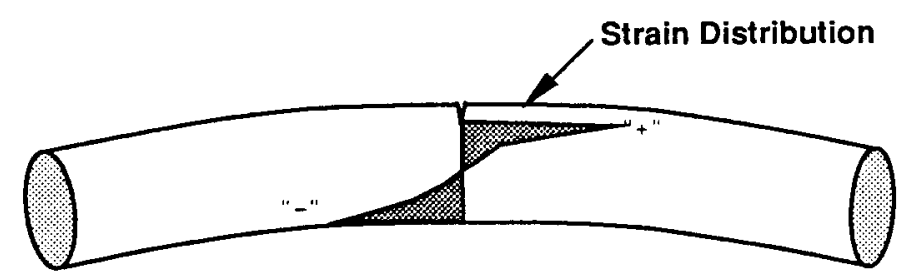

A. TRANSVERSE TEARING
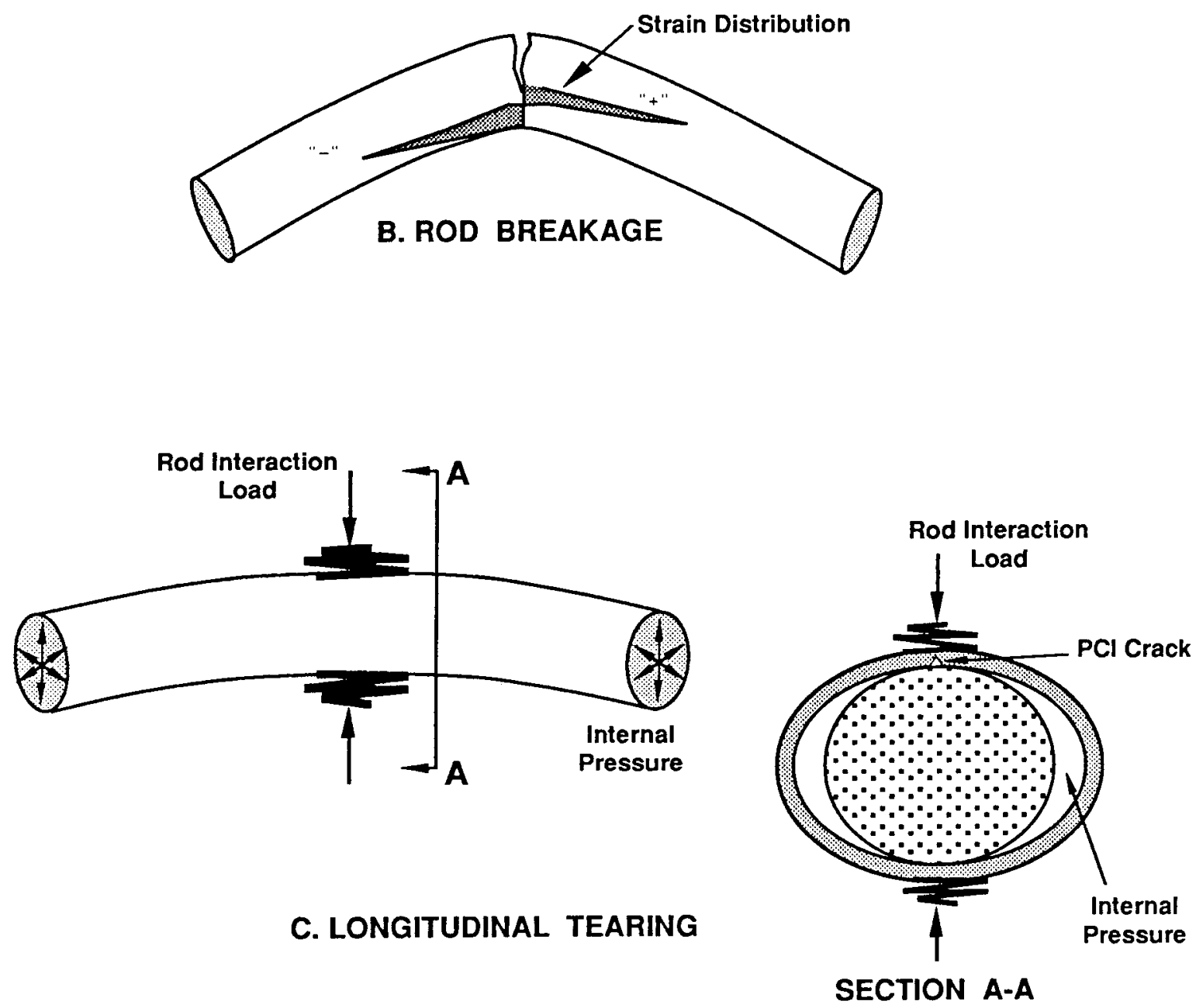

Figure 11. Fuel Rod Failure Modes 
The second failure mode is an extension of the transverse crack failure mode discussed above. Depending on the amount of available energy, a narrow transverse tear could extend through a large portion of the cross section or even result in a guillotine break. The geometry of this failure mode is shown in Figure 11B. First-mode rupture geometry determined the conditional failure probability for this mode to be an initial crack, for which the stress intensity factor $\mathrm{K}_{\mathrm{I}}$ was calculated and compared to the probabilistic criterion of fracture toughness, $\mathrm{K}_{I C}$.

The third failure mode, shown in Figure $11 \mathrm{C}$, is a longitudinal rupture that initiates at the inner surface from a PCI-induced crack or a manufacturing flaw. This failure mode is dominant for side-drop and slapdown impact loadings and regulatory fire conditions. The deterministic response parameter used to evaluate this type of failure was the stress intensity factor $\mathrm{K}_{\mathrm{I}}$ that was calculated for crack sizes in the distributions characterizing the cladding initial state. The probability density function for fracture toughness, $\mathrm{K}_{I C}$, and $\mathrm{K}_{\mathrm{I}}$ were used to obtain a failure probability density function for the crack sizes in the distribution. This probability density function was then integrated with the crack distribution function (relative frequency versus crack size) to obtain the conditional failure probability for the PCI crack locations.

The conditional failure probabilities estimated for the three failure modes were calculated for all strain and stress concentration locations in the rod. These were then added to obtain the total failure probability for the rod and, subsequently, the assembly.

The proposed failure modes have been created in the laboratory [GE80] or have occurred during reactor operations due to PCI failures [FU76]. Similar failure modes have been predicted for nonreactor handling accidents [AB91].

\subsection{Radioactive Material Release Estimates}

Calculation of radionuclide releases following cladding failure requires a three-step process. In the first step, the total radionuclide content contained within a fuel rod is determined. Then the fraction of each radionuclide present that is in a potentially releasable form is quantified. Finally, the quantity of each radionuclide actually released through a cladding breach is estimated.

Many radionuclides are produced within fuel rods during reactor operation. The specific nuclide composition depends on the initial enrichment, irradiation history, and length of time since reactor shutdown. Estimates of the nuclide composition were obtained for each of the representative assemblies using the ORIGEN2 code and irradiation histories from the EPRI-FPDB, as described in Section 2.1 .

ORIGEN2 calculations were carried out for several burnup levels to represent current and projected (extended) burnup limits for BWR and PWR fuel assemblies. These assemblies comprised the detailed analysis cases selected as bounding. In addition, for each burnup level, the radionuclide compositions were obtained for several time periods after removal from the 
reactor. These results were compiled in a radionuclide inventory database; input data for case-specific analyses were obtained from this database by interpolation for the specified fuel type, burnup level, and time of transport after discharge.

After quantification of the radionuclide inventory of fuel assemblies prepared for transport, the amount of this source released to the cask cavity during cladding breach was determined. The fuel release source term includes those radionuclides released from the fuel matrix to the fuel-clad gap in gaseous or vapor form and/or gas-borne particulate species (fines). A model for gap inventory was developed with the aid of the ANSI/ANS-5.4 standard. This standard was used to account for the buildup of xenon and krypton isotopes in the pellet-cladding gap. To determine the buildup of moderately volatile species (iodine, cesium, and tellurium) in the gap, it was assumed that they have the same mobility and diffusion characteristics as the noble gases, thereby establishing relationships for magnitude and distribution between the long-lived isotopes of volatile species and fission gases (see Appendix I). Gas-borne fuel fines and their long-lived radionuclide contents were estimated using available data. This particulate matter was then added to the gap inventory.

The entire gap inventory was conservatively assumed to be readily available for release in the event of cladding rupture irrespective of rupture location or size. This inventory must be accurately estimated. Therefore, a fission gas release calculational procedure was developed using representative irradiation histories for the selected cases. This was accomplished using the ANSI/ANS-5.4 fission gas release model and its host fuel behavior code, FREY.

The final step in release analysis was to characterize radionuclide releases to the cask cavity in the event that cladding rupture occurs. The geometric shapes of cladding breaches were identified from deterministically calculated postfailure geometries. Therefore, the source and physical composition of radioactivity releases were deterministically based. However, because cladding failure was determined probabilistically, the magnitude of radioactivity release estimates was also probabilistic.

The models developed to describe the characteristics of radionuclide releases account for temperature-dependent physical compositions and volatility. A distinction was made between the types of radionuclides that can be released in the initial burst release mode and the later, slower diffusion release mode.

\subsection{References}

[AB91] ABB Combustion Engineering, "Fuel-Assembly Behavior Under Dynamic Impact Loads Due to Dry-Storage Cask Mishandling," Electric Power Research Institute, Palo Alto, CA, EPRI NP-7419, RP-2813-9, -28, July 1991.

[CR83] Croff, A. G., "ORIGEN2: A Versatile Computer Code for Calculating the Nuclide Compositions and Characteristics of Nuclear Materials," Nuclear Technology, 62, p. 335 (1983). 
[FU76] Fuhrman, N., V. Pasupathi, D. B. Scott, S. M. Temple, S. R. Pati, and T. E. Hollowell, "Evaluation of Fuel Rod Performance in Maine Yankee Core I," Electric Power Research Institute, Palo Alto, CA, EPRI NP-218, November 1976.

[GE80] General Electric Company, "Final Safety Analysis Report: GE IF-300 Spent Fuel Shipping Cask," NEDO-10084-2, December 1980.

[GE88] Gerhardt, M. A., D. J. Trummer, G. L. Johnson, and G. C. Mok, "SCANS Microcomputer-Based Analysis System for Transport Cask Design Review," Lawrence Livermore National Laboratory, Livermore, CA, NUREG/CR-4554, December 1988.

[HA81] Hagrman, D. L., "MATPRO--Version II (Revision 2): A Handbook of Material Properties for Use in the Analysis of Light Water Reactor Fue1 Rod Behavior," EG\&G Idaho Inc., Idaho Falls, ID, NUREG/CR0479, TREE-1280, August 1981.

[HI89] Hibbitt, Karlsson \& Sorensen, Inc., "ABAQUS," A General Purpose Finite Element Computer Program (Providence, RI: Hibbitt, Karlsson \& Sorensen, Inc., 1989).

[MI88] Michae1s, W. J., M. I. McKenna, S. P. Nguyen, and B. D. Paulson, "Fuel Performance Data Base," Electric Power Research Institute, Palo Alto, CA, EPRI NP-4068, January 1988.

[N087]. Notz, K., "Characteristics of Spent Fuel, High-Level Waste and Other Radioactive Wastes Which May Require Long-Term Isolation," U.S. Department of Energy, Richland, WA, DOE/RW-0184, December 1987.

[RA89] Rashid, Y. R., R. O. Montgomery, and A. J. Zangari, "FREY-01: Fue1 Rod Evaluation System," Electric Power Research Institute, Palo A1to, CA, EPRI NP-3277-CCM, revised March 1989.

[SH86] Shapiro, A. B., "TOPAZ2D--Two-Dimensional Finite Element Code for Heat Transfer Analysis of Electrostatic and Magnetostatic Problems," Lawrence Livermore National Laboratory, Livermore, CA, UCID-20824, July 1986.

[TA84] Tasooji, A., R. E. Einziger, and A. K. Miller, "Modeling of Zircaloy Stress-Corrosion Cracking: Texture Effects and Dry Storage Spent Fuel Behavior," in "Zirconium in the Nuclear Industry: Sixth International Symposium," American Society of Testing Materials, ASTM-STP-824, pp. 595-626, 1984.

[TU82] Turner, S. E., "Background and Derivation of ANS-5.4 Standard Fission Product Release Model," U.S. Nuclear Regulatory Commission, Washington, D.C., NUREG/CR-2507, January 1982.

[US90] "Packaging and Transportation of Radioactive Material," in U.S. Code of Federal Regulations, Title 10, Part 71 (10 CFR 71), revised January 1, 1990. 
This page is intentionally left blank. 


\subsection{FUEL-LOADING CONDITIONS}

\subsection{Introduction}

The spent-fuel loading environment is characterized by normal and hypothetical accident transport conditions specified by regulations. Normal transport conditions are characterized by the following:

- Shock and vibration normally incident to over-the-road and overthe-rail transportation,

- Cask impact on a rigid target following a free fall of $0.3 \mathrm{~m}$, and

- Thermal response of spent fuel when the cask is subjected to full solar heat input and an ambient temperature of $38^{\circ} \mathrm{C}$ with maximum decay heat assumed.

Hypothetical accident conditions are characterized by the following:

- Cask impact on a rigid target following a free fall of $9.0 \mathrm{~m}$,

- Cask impact onto a mild steel bar following a free fall of $1.0 \mathrm{~m}$, and

- Thermal response of spent fuel when the cask is subjected to a fully engulfing $800^{\circ} \mathrm{C}$ fire of half-hour duration (maximum decay heat assumed).

Other normal and hypothetical accident transport conditions are specified in the regulations (see Section 1.3), but none of these result in spentfuel environments more severe than those conditions characterized above.

The environmental analyses that were performed are described in detail in Appendix II. Two main approaches were used to define the transport environment characteristics: (1) test data for and analyses of the response of existing spent-fuel casks to the above regulatory conditions were reviewed and assessed for their applicability to a generic characterization of the spent-fuel environments, and (2) methodologies were developed to evaluate the response of generic casks.

Thermal and mechanical response analyses were then performed for four representative generic cask designs to demonstrate the methodologies: an all-steel truck cask, an all-steel rail cask, a lead-shielded truck cask, and a lead-shielded rail cask. The response analyses were based on concepts defined by Fischer as being representative of typical cask designs [FI87]. The resulting spent-fuel environments are briefly described in the following two sections. See Appendix II for additional details. 


\subsection{Norma1 Transport Environments}

\subsubsection{Shock and Vibration}

A large quantity of experimental data has been derived from various sources to quantify the shock and vibration environment of cargo during truck and rail transport. The data usually were collected from instrumentation located at the interface between the packaging or cargo and the transporter, and generally consist of acceleration-response spectra as a function of frequency. The total acceleration response measured for a cargo includes response to superimposed shock and vibration. The vibration component is usually identified as a continuous excitation comprised of all responses lower than or equal to 998 of the peaks in the acceleration response records. The remaining higher intensity, infrequently occurring acceleration peaks correspond to sporadic shock events.

An extensive survey of relevant shock and vibration data is provided in Appendix II. Appropriate data from this survey were used in this analysis to specify representative, simplified shock response curves and equivalent pulses that conservatively bound the relevant data and are meaningful in assessing shock response of spent fuel. Bounding truck and rail vibration data were also derived in a similar manner.

The bounding acceleration shock response spectrum for spent fuel in truck casks was based on the union of triaxial data (longitudinal, transverse, and vertical axis accelerations) for 20- and 25-tonne cargoes reported in [MA77] and [MA78]. These data are shown in Figure 12. The suggested bilinear curve (in the log-log plane) that bounds these data from above consists of a linearly increasing portion up to a frequency of approximately $3.5 \mathrm{~Hz}$, followed by a constant segment at $4.4-\mathrm{g}$ acceleration, up to a maximum frequency of $300 \mathrm{~Hz}$. For even greater simplicity, the dashed line indicated on the figure could be used at low frequencies, but this may be overly conservative because low-frequency response may be of dominant importance for the fuel assembly system.

The bounding rail shock spectrum is based on the union of measured triaxial data for a 45-tonne cargo reported by Magnuson [MA82]. The data and the linear (in the $\log -\log$ plane) bounding response spectrum are shown in Figure 13. The measured data include responses to typical shockgenerating events (e.g., crossing of bridges and switches), but do not include coupling event shocks resulting from "humping" or train makeup or switching.

The acceleration response to rail-car-coupling events is typically more severe than response to other rail-shock-generating events. Figure 14 shows the union of coupling response spectra for all three axes for 36- and 64-tonne casks [MA80], together with a recommended linear bounding response spectrum for rail-coupling events. 


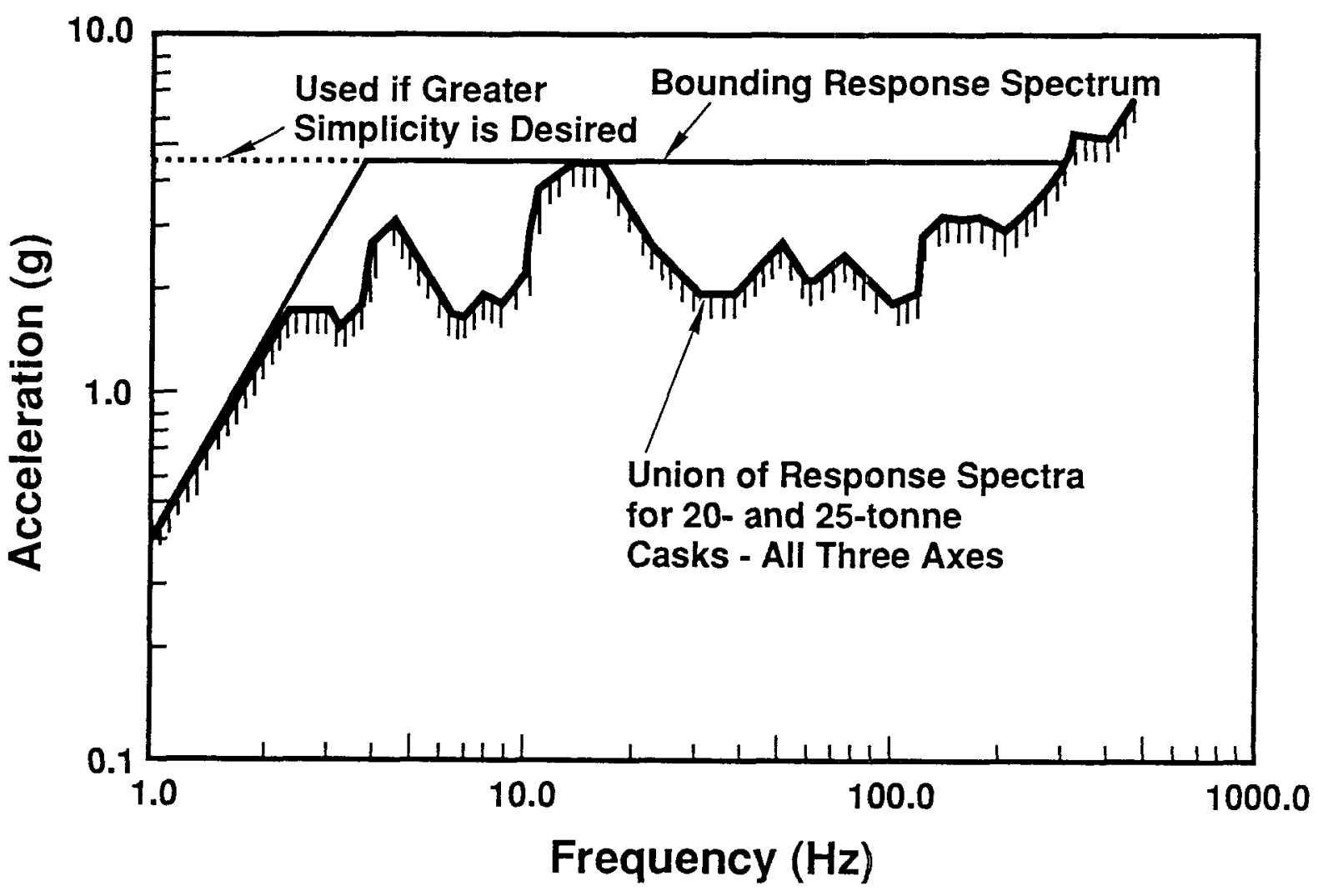

Figure 12. Bounding Acceleration Shock Response Spectrum for a Truck Cask at 38 Damping

These high accelerations resulted from very short duration loads during rail-coupling events at speeds of 12.9 to $17.7 \mathrm{~km} / \mathrm{hr}$ and may not be representative of normal transport conditions. Rail-coupling events at 3.2 to $8.0 \mathrm{~km} / \mathrm{hr}$ reduced peak accelerations by approximately $10 \mathrm{~g}$.

Because the impact duration was very short and amplitude of acceleration was very high, static analyses are not applicable for these loading conditions. The graphical superposition procedure described by Magnuson [MA77] was used to determine half-wave pulses that bound the truck and rail shock response spectra from above (see Appendix II). These halfwave pulses were used as input for dynamic time history analysis.

Bounding truck vibration data for all three response directions are shown in Figure 15. This bounding environment was based on data reported by Magnuson [MA77, MA78] for 20- and 25-tonne casks, respectively. Similar bounding rail vibration data are presented in Figure 16 based on data from Magnuson for a 45-tonne cask mass [MA82]. 


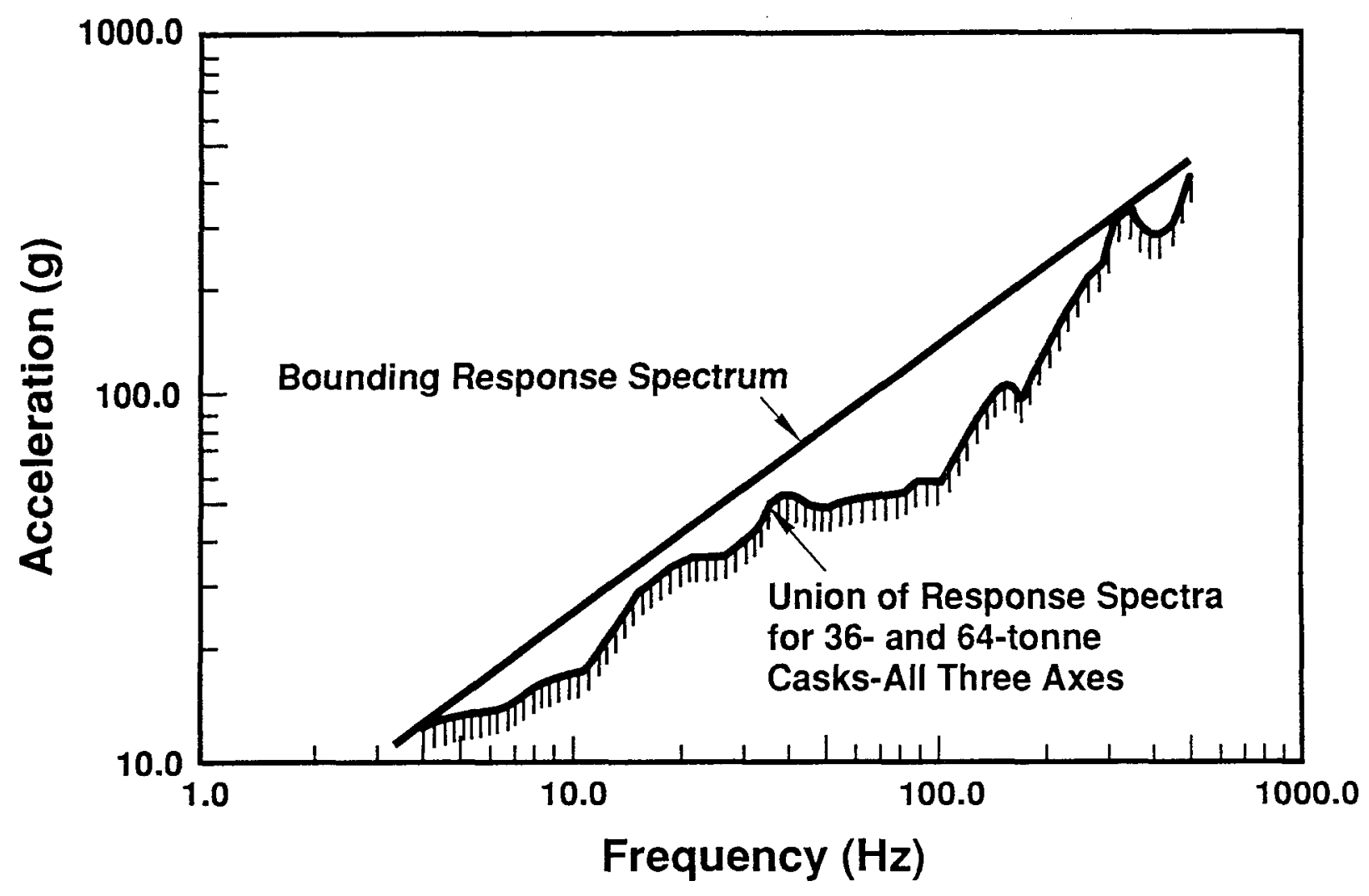

Figure 13. Bounding Acceleration Shock Response Spectrum for 38 Damping for All Three Axes

\subsubsection{Accelerations for the $0.3-\mathrm{m}$ Drop}

An additional regulatory condition for normal transport requires that a spent-fuel cask withstand the impact of a $0.3-\mathrm{m}$ drop onto an unyielding target. Figure 17 shows the accelerations predicted by rigid-body analysis of a $0.3-\mathrm{m}$ drop of a lead-shielded truck cask with impact limiters in place. As illustrated, the accelerations produced by the initial impact exceeded $100 \mathrm{~g}$ at impact angles of $90^{\circ}$ (end drop), but were small for most other angles. This cask was chosen because it was the lightest of the generic cask designs considered and so would undergo the largest accelerations. The accelerations predicted for the $0.3-\mathrm{m}$ drop are considered conservative because of assumptions concerning impact limiter stresses and contact area geometries (see Appendix II).

\subsubsection{Normal Thermal Environment}

The cask normal thermal environment defined by $10 \mathrm{CFR} 71$ includes both a heat test at $38^{\circ} \mathrm{C}$ combined with a solar insolation and a cold test at an ambient temperature of $-40^{\circ} \mathrm{C}$ in still air and shade. The peak fuel rod temperatures under these normal transport conditions are discussed in detail in Appendix II. 


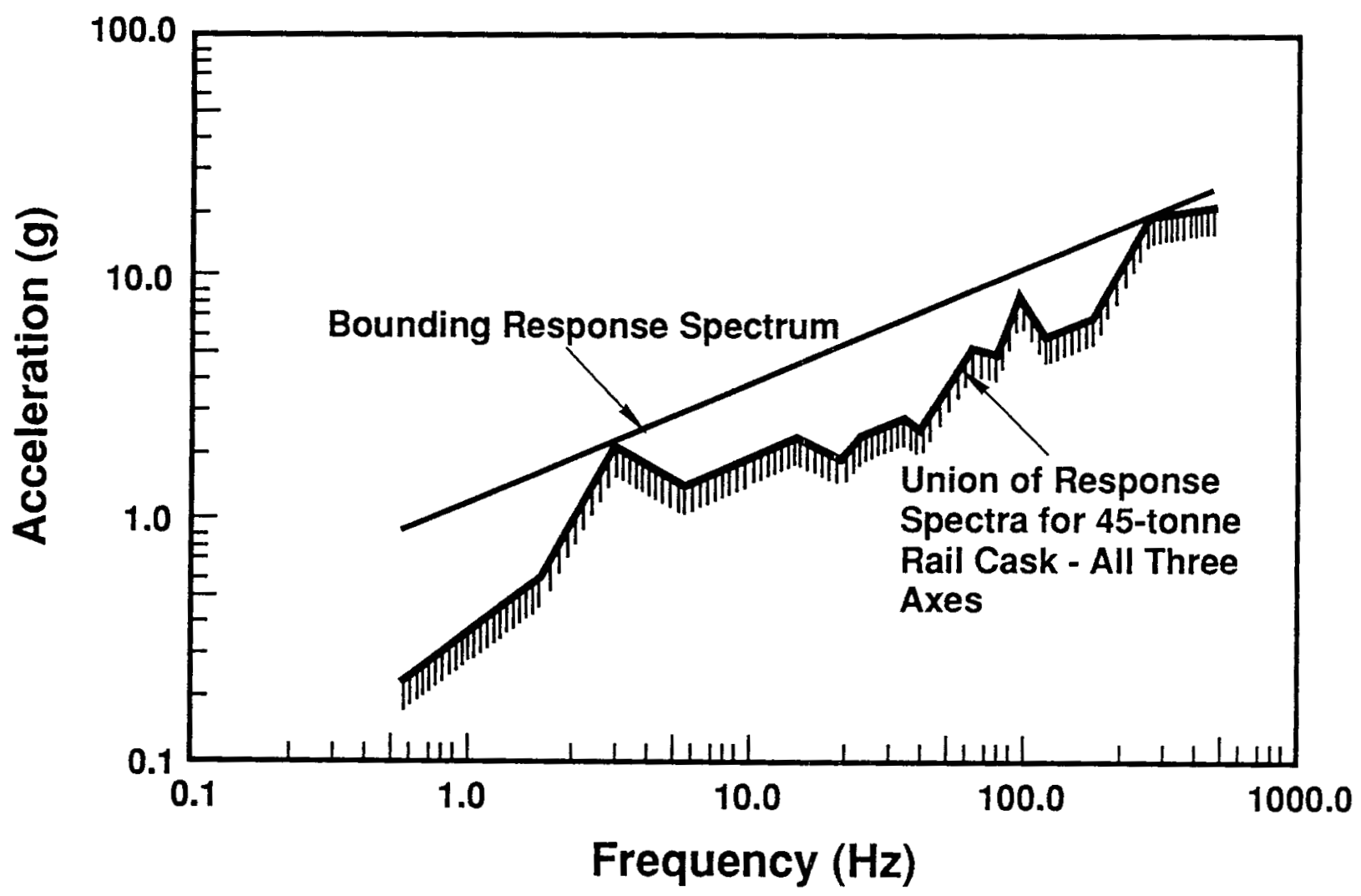

Figure 14. Bounding Acceleration Shock Response Spectrum for $3 \%$ Damping for All Three Axes, Including a Recommended Linear Bounding Response Spectrum for Rail-Coupling Events

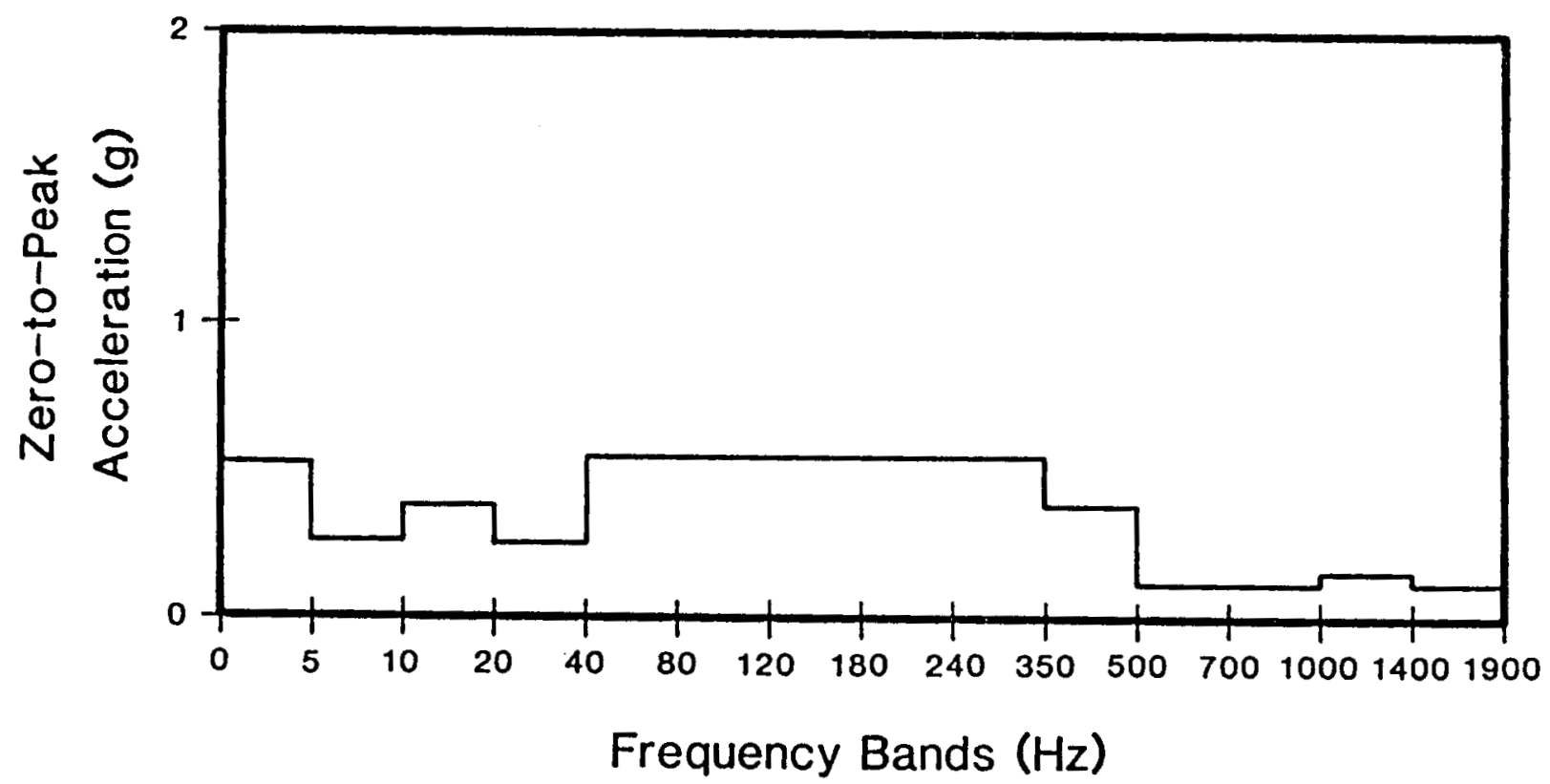

Figure 15. Bounding Truck Vibration Data for All Three Axes 


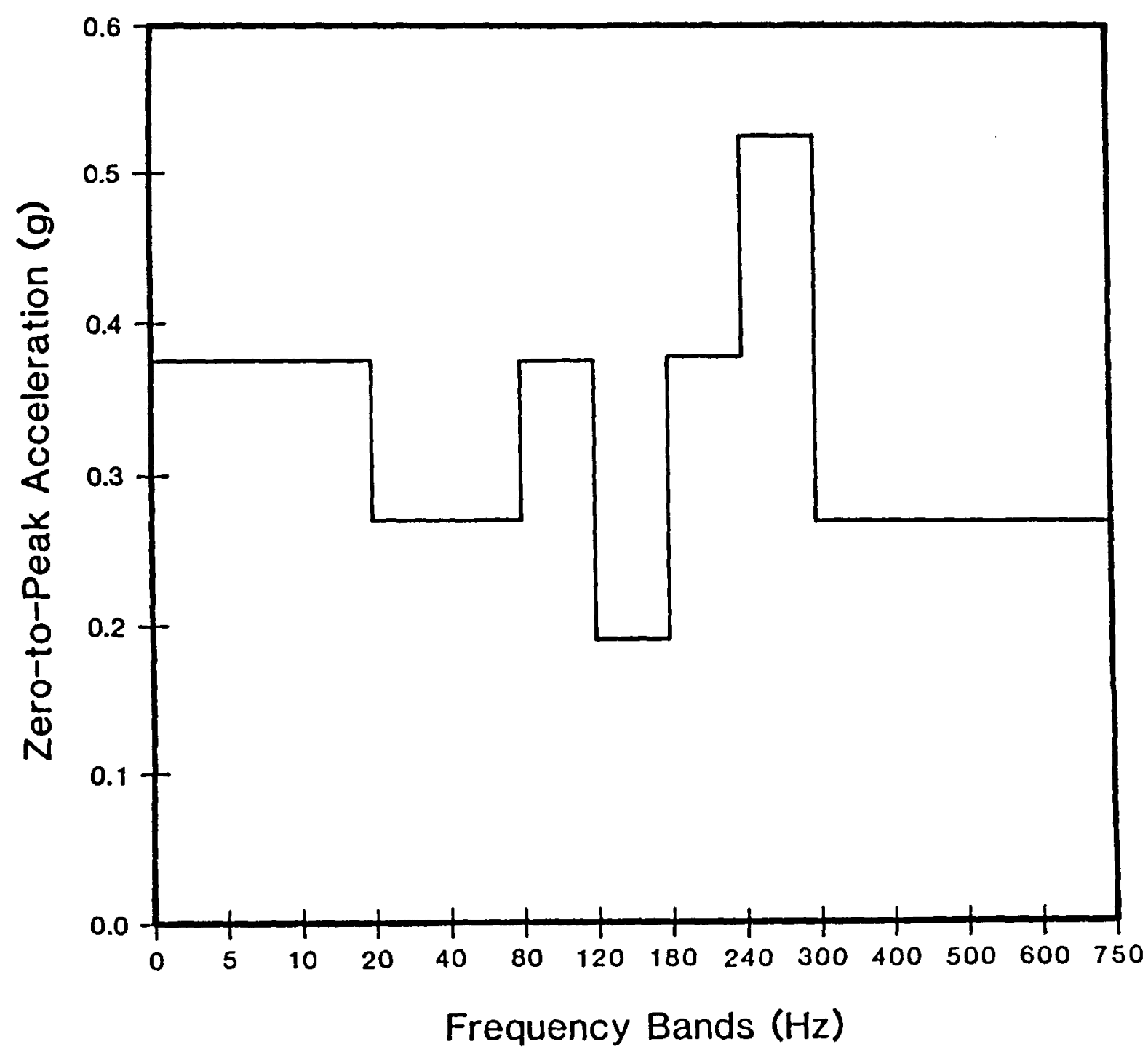

Figure 16. Bounding Rail Vibration Data

An analysis of representative lead-shielded truck and rail casks is discussed in Appendix II. This analysis was performed to determine peak fuel temperatures expected during normal transport. The truck cask was assumed to have a payload capacity of 1 PWR assembly, while the rail cask payload was assumed to have 21 PWR assemblies. The predicted peak fuel rod temperatures as a function of fuel assembly decay heat generation for the truck and rail casks are provided in Figures 18 and 19, respectively.

The peak temperatures of the example analyses were well below the acceptable peak fuel temperature of $380^{\circ} \mathrm{C}$ recommended for storage [US85]. The $380^{\circ} \mathrm{C}$ temperature was derived from tests conducted at INEL with the Castor-V21 PWR spent-fuel storage cask [EP86]. In these tests, the Castor cask was loaded with thirteen $1.0-\mathrm{kW}$ spent-fuel assemblies and eight 


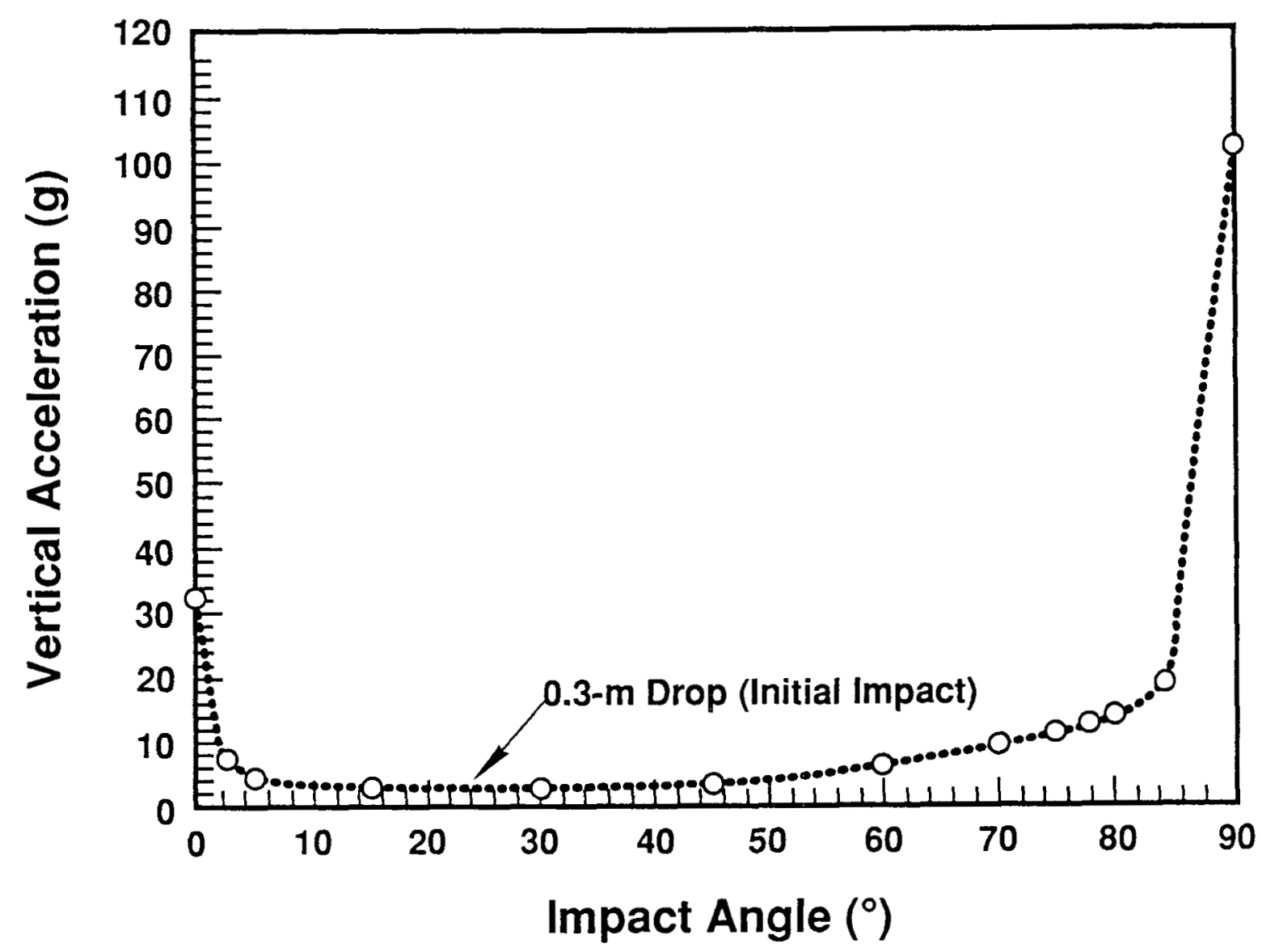

Figure 17. Vertical Acceleration in $g$ Versus the Impact Angle for the $0.3-\mathrm{m}$ Drop

$1.8-\mathrm{kW}$ assemblies. Steady-state temperatures were measured within several of the fuel assemblies for horizontal and vertical cask orientations and three different cavity gases (nitrogen, helium, vacuum).

The cold temperature specified for a normal environment, $-40^{\circ} \mathrm{C}$, does not pose a threat to the spent-fuel assembly without additional environmental loading. However, cold fuel temperatures degrade the ductile strength of cladding material such that subsequent external mechanical loading could lead to fuel rod failures at considerably lower loads than fuel at higher temperatures. The lower-bound spent-fuel temperature is a function of age and fuel burnup along with the cask design. The effects of temperature on the degradation of spent-fuel material properties are provided in detail in Appendix III.

\subsection{Accident Environments}

The regulatory accident conditions of transport include a $9.0 \mathrm{~m}$ drop impact, a $1.0-\mathrm{m}$ drop onto a mild steel bar, and a $800^{\circ} \mathrm{C}$ fully engulfing fire environment of a 30 -min duration. Each of these accident conditions is addressed in the following sections. 


\section{Lead-Shielded Truck Cask - 1 PWR - MAX Fuel Temperature}

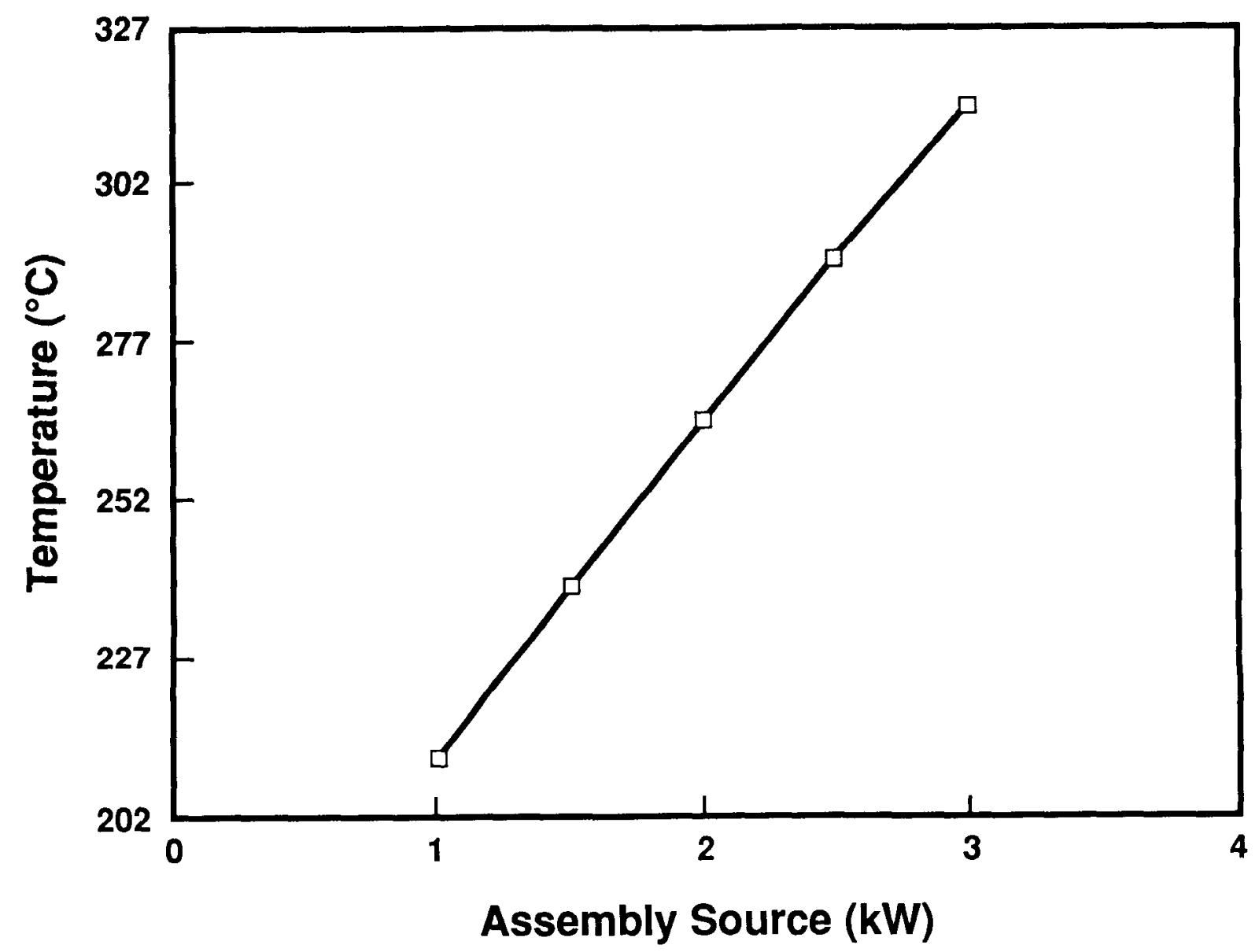

Figure 18. Predicted Peak Fuel Temperatures in a Truck Cask as a Function of Assembly Decay Heat Generation Rate

\subsubsection{Nine-Meter-Drop Impact Environment}

Several analysis techniques were considered to characterize the spentfuel environment in transport casks subjected to the regulatory 9.0 -m drop. After the available test and analysis data for existing casks had been surveyed, a rigid-body kinematics analysis of the impact was chosen as the technique most suitable for the purposes of a spent-fuel response model.

Rigid-body kinematic analysis of the impact treats the cask as a twodimensional rigid body with three degrees of freedom: two translational and one rotational. Three response phases during impact were considered: (1) rigid-body deceleration during the initial deformation of an impact limiter on the impacting end of the cask, (2) rigid-body rotation about the 


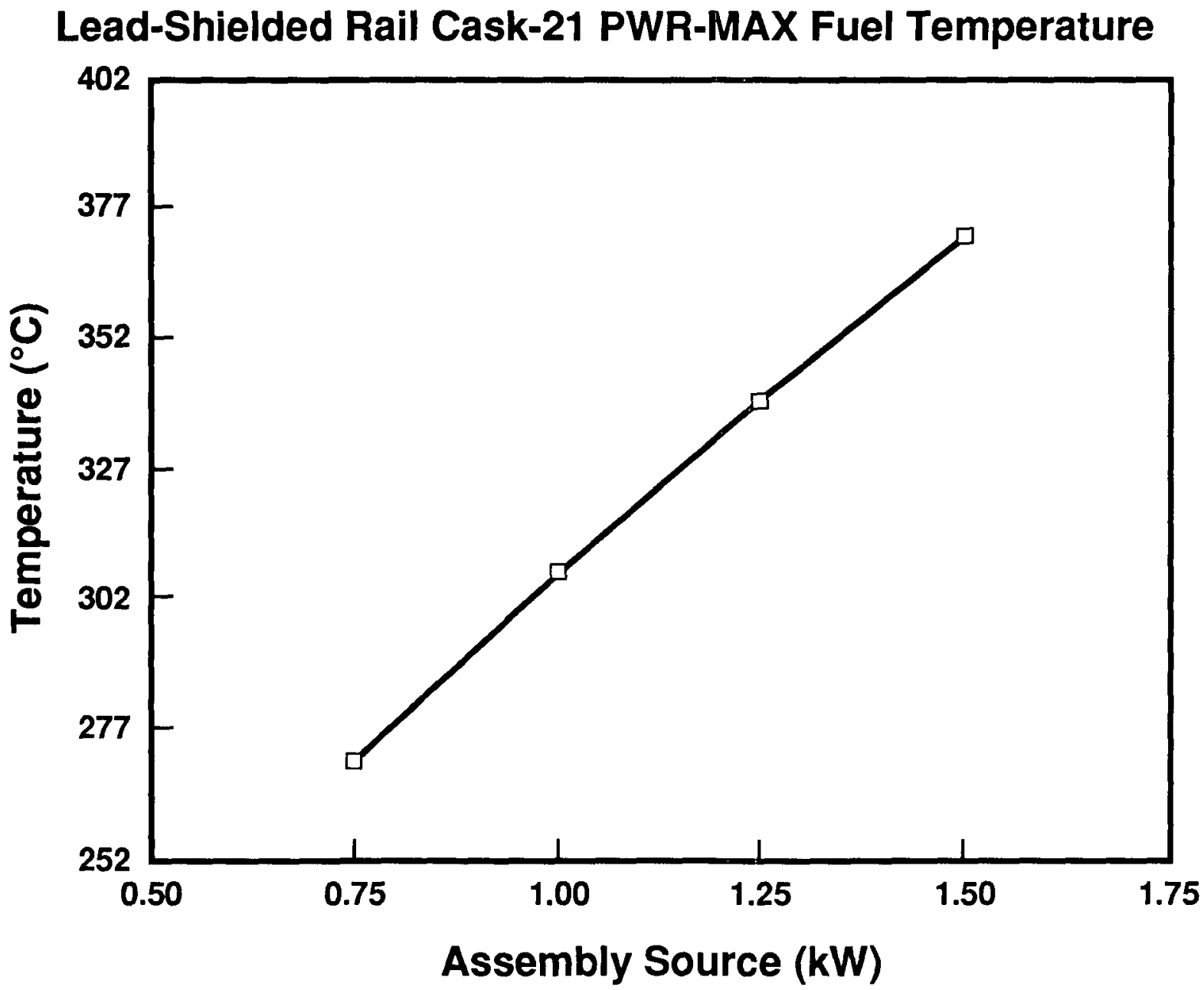

Figure 19. Predicted Peak Fuel Temperatures in a Rail Cask as a Function of Assembly Decay Heat Generation Rate

impacting end for oblique impact angles, and (3) additional cask deceleration during slapdown as the other end of the cask impacts the target surface. The impact limiter material was assumed to have a constant crush stress; details of the impacting surface shape are described in Appendix II. Both horizontal slipping and nonslipping of the impacting end of the cask were considered. The computer code SLAM was developed to solve the coupled equations of motion for the cask during each phase of the impact. This code was validated using accelerometer data available from drop tests of a quarter-scale model of the NUPAC 125-B cask [NU85]. Complete analysis results for four generic cask concepts are presented in Appendix II; the results are summarized in the following sections. 


\subsubsection{Example Oblique Impact}

Figure 20 shows predicted vertical acceleration histories for each of the four generic casks for a $9.0-\mathrm{m}$ drop at an inclination of $30^{\circ}\left(90^{\circ}\right.$ is an end-on impact with no rotation). The curves shown are in the event of no horizontal frictional force acting at the impact surface (100\% slip). The first negative swing in each of the four curves corresponds to the cask acceleration as the limiter at the initial impact end deforms. The flat portions in the curves correspond to the rotational phase of the response during which the vertical acceleration component is small. The second peak in the results is the predicted acceleration as the second impact limiter deforms during the slapdown phase. Figure 20 illustrates that peak vertical accelerations may occur during the slapdown phase rather than during initial cask impact.

\section{Vertical Accelerations (frictionless) Impact Angle $\mathbf{=} \mathbf{3 0} . \mathbf{0}$ degrees}

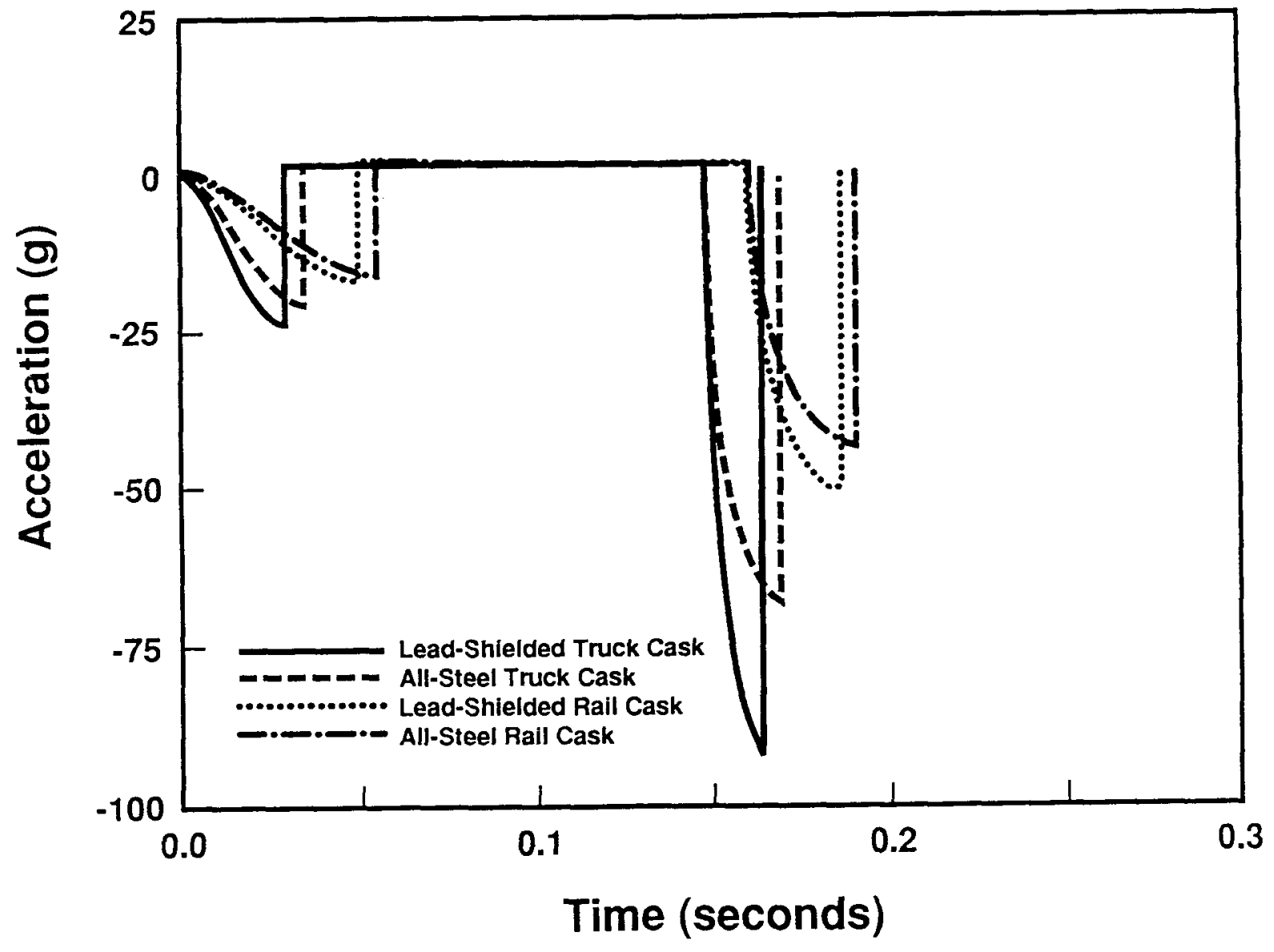

Figure 20. Vertical Acceleration Histories; Slippage with No Friction and a $30^{\circ}$ Impact Angle Assumed 
When the end of a cask was assumed not to slip horizontally during initial impact (termed infinite friction), the vertical acceleration histories differed, as illustrated in Figure 21. A comparison with the frictionless results indicates that friction results in somewhat higher vertical accelerations during the initial phase of impact and slightly decreased and delayed accelerations during the slapdown phase.

\section{Vertical Accelerations (no slip) Impact Angle $=\mathbf{3 0} .0$ degrees}

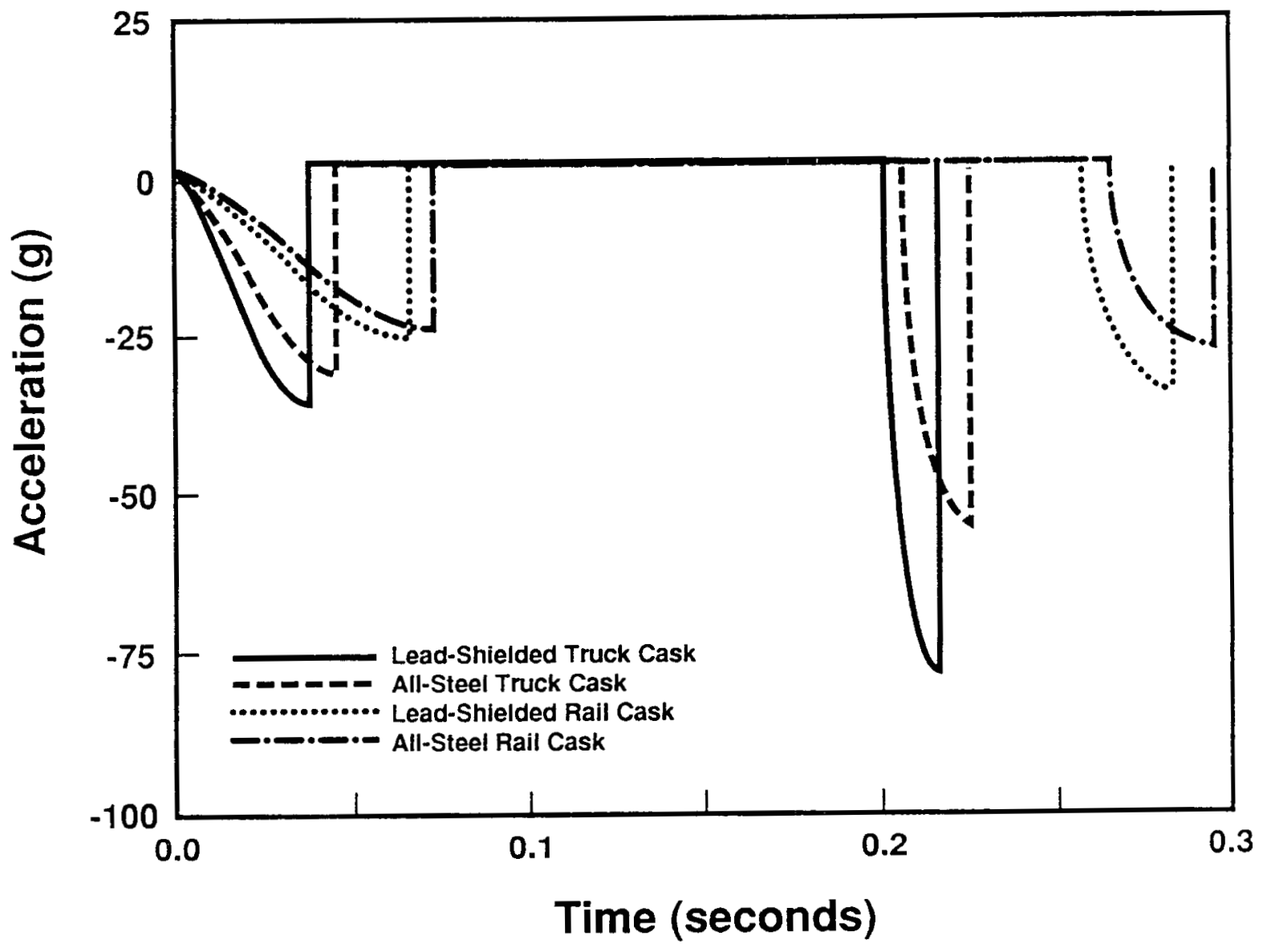

Figure 21. Vertical Acceleration Histories; No Slippage and a $30^{\circ}$ Impact Angle Assumed

The peak vertical accelerations predicted for all four representative casks are shown in Figure 22 as a function of impact angle. As indicated, the peak accelerations may occur either during the initial impact phase or during the slapdown phase. The largest peak vertical acceleration for each cask concept was approximately $100 \mathrm{~g}$ or less, which is typical for a soft impact limiter design. 


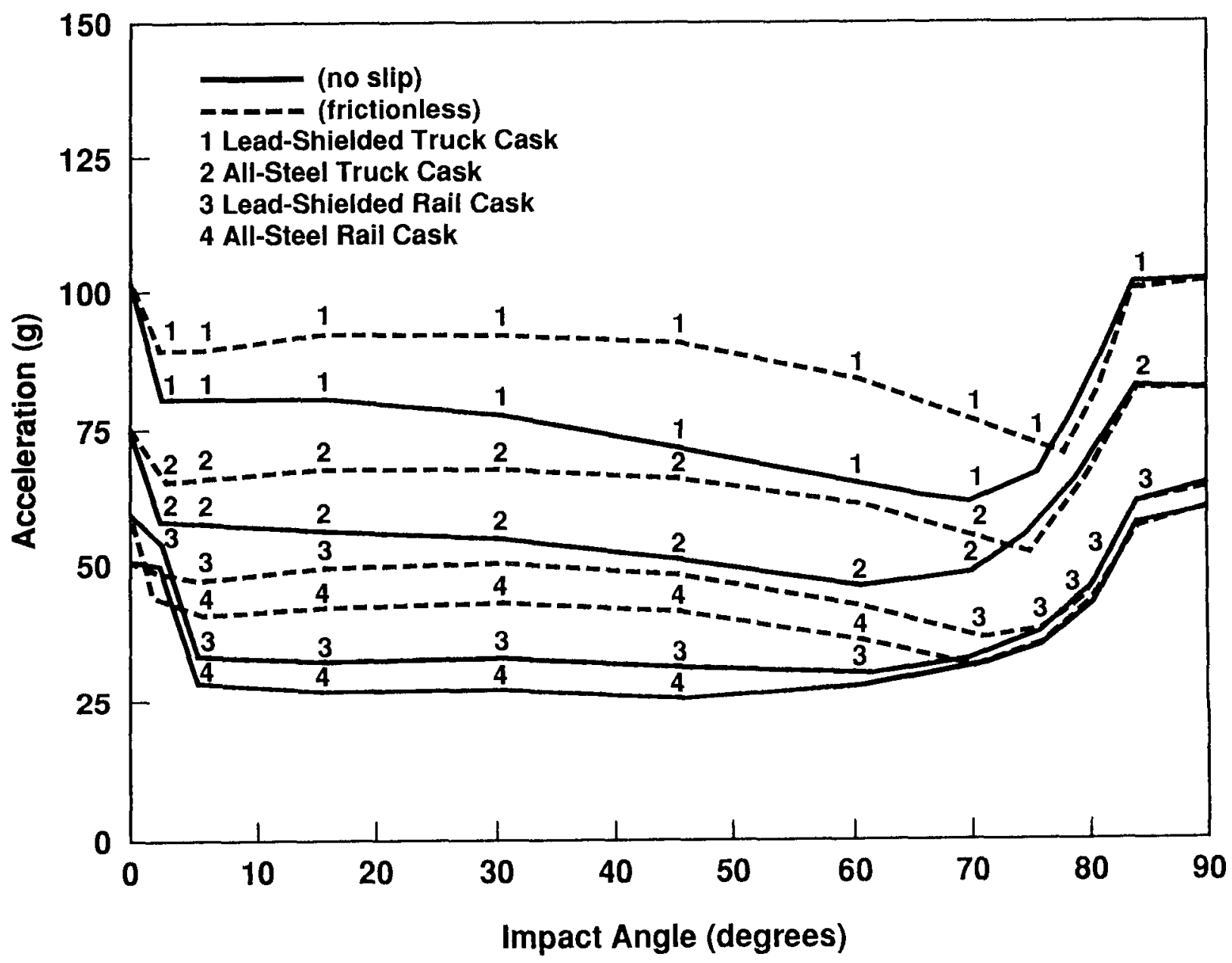

Figure 22. Peak Vertical Accelerations as a Function of Impact Angle

As illustrated in Figures 23 and 24, the predicted peak horizontal and angular accelerations for the representative casks are a function of impact angle. Slippage at the impact point enhances the cask rotation during the second phase of the response.

\subsubsection{One-Meter Drop onto a Mild Steel Bar}

In addition to the $9.0-\mathrm{m}$-drop accident condition, a cask must further pass a 1.0-m-drop test onto a $15-\mathrm{cm}$-diameter mild steel bar. As discussed in Appendix II, the accelerations occurring during this event are bounded by the characteristics of the bar. In particular, the maximum force that the bar is capable of exerting on the cask or impact limiter is equal to the product of the dynamic flow stress of the mild steel and the crosssectional area of the bar. 


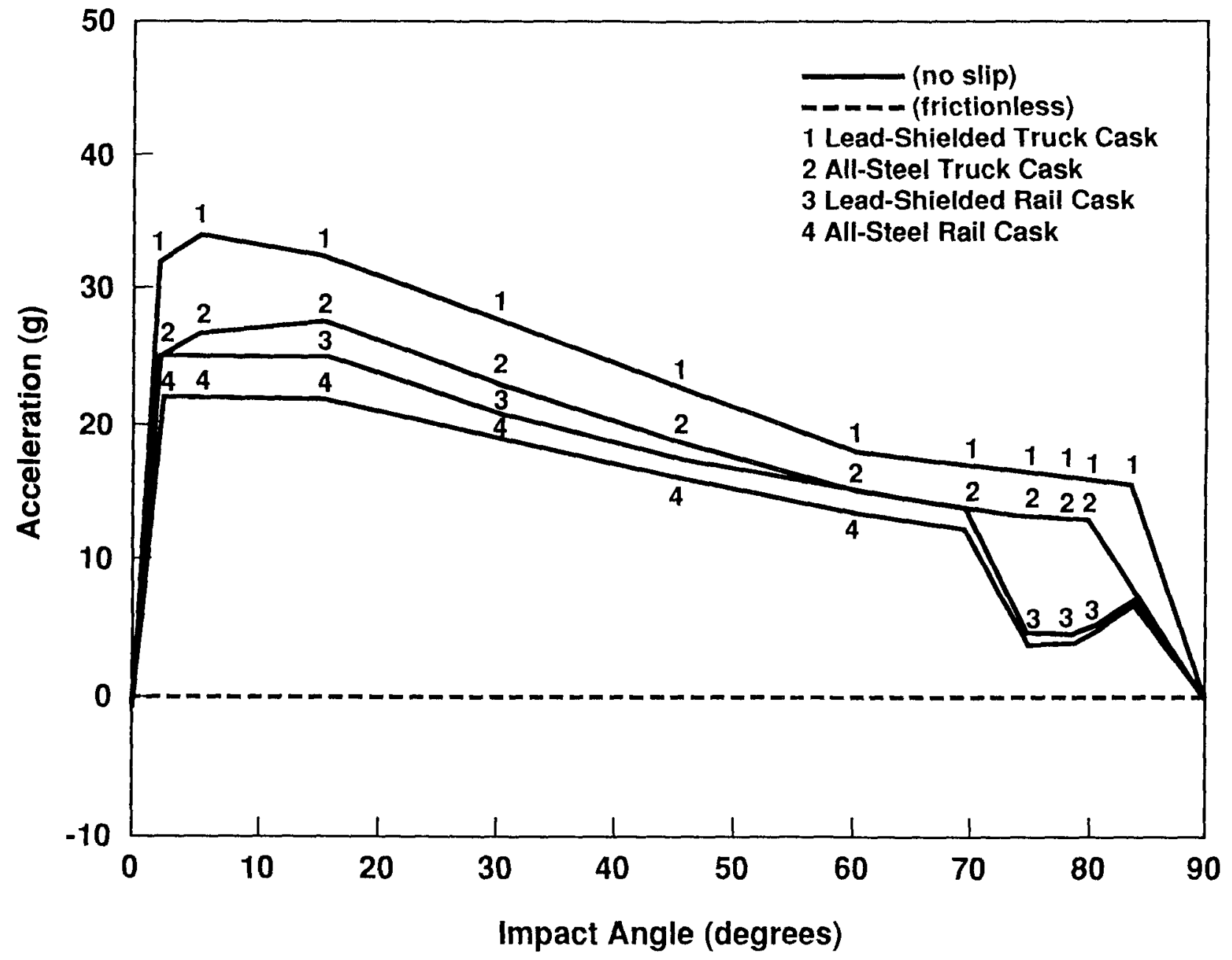

Figure 23. Peak Horizontal Accelerations as a Function of Impact Angle

The maximum resulting cask acceleration that can occur is then

$$
\mathrm{a}=\mathrm{F}_{\mathrm{P}} / \mathrm{W},
$$

where

$$
\begin{aligned}
\mathrm{F}_{\mathrm{P}} & =\text { the maximum bar failure force } \\
\mathrm{W} & =\text { the cask weight. }
\end{aligned}
$$

Maximum bounding accelerations are shown in Figure 25 for a range of cask weights. Also plotted are results from the Safety Analysis Reports (SARs) for a number of existing casks, all of which are in general agreement with this upper-bound expression. 


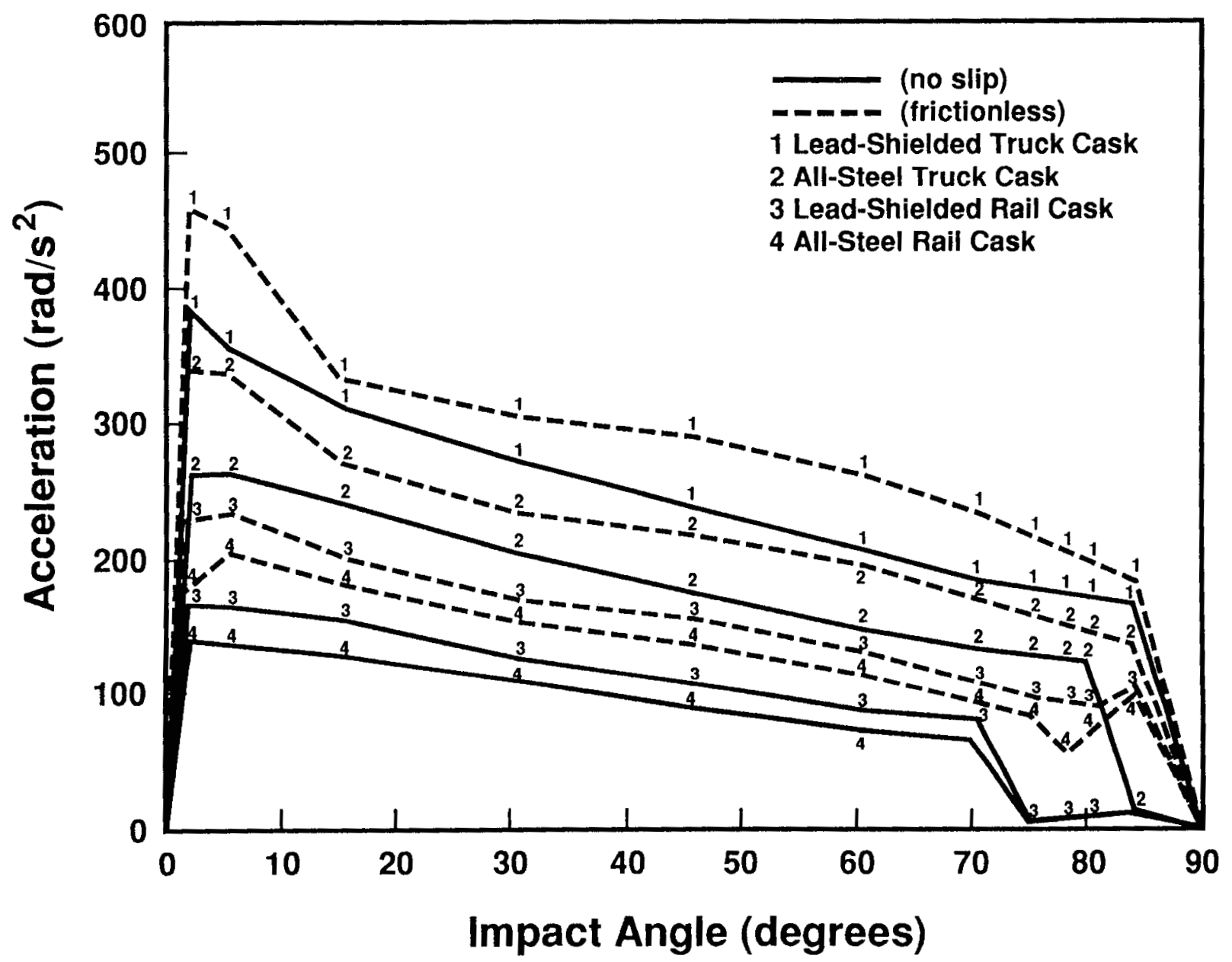

Figure 24. Peak Angular Accelerations as a Function of Impact Angle

\subsubsection{Thirty-Minute Engulfing Fire}

Transient thermal response analyses of two representative cask concepts during 30 -min regulatory fire conditions were used to quantify maximum expected fuel temperatures during transport. The thermal analyses were performed using two-dimensional planar models of one-eighth pie sections of the casks taken from their axial midlength. Finite element models of the casks were used to evaluate the effects of conduction, convection, and radiation heat transfer (see Appendix II). The results indicate that a peak fuel temperature of $402^{\circ} \mathrm{C}$ could occur in a truck cask during the regulatory fire. For the rail cask, the peak fuel temperature is $347^{\circ} \mathrm{C}$. 


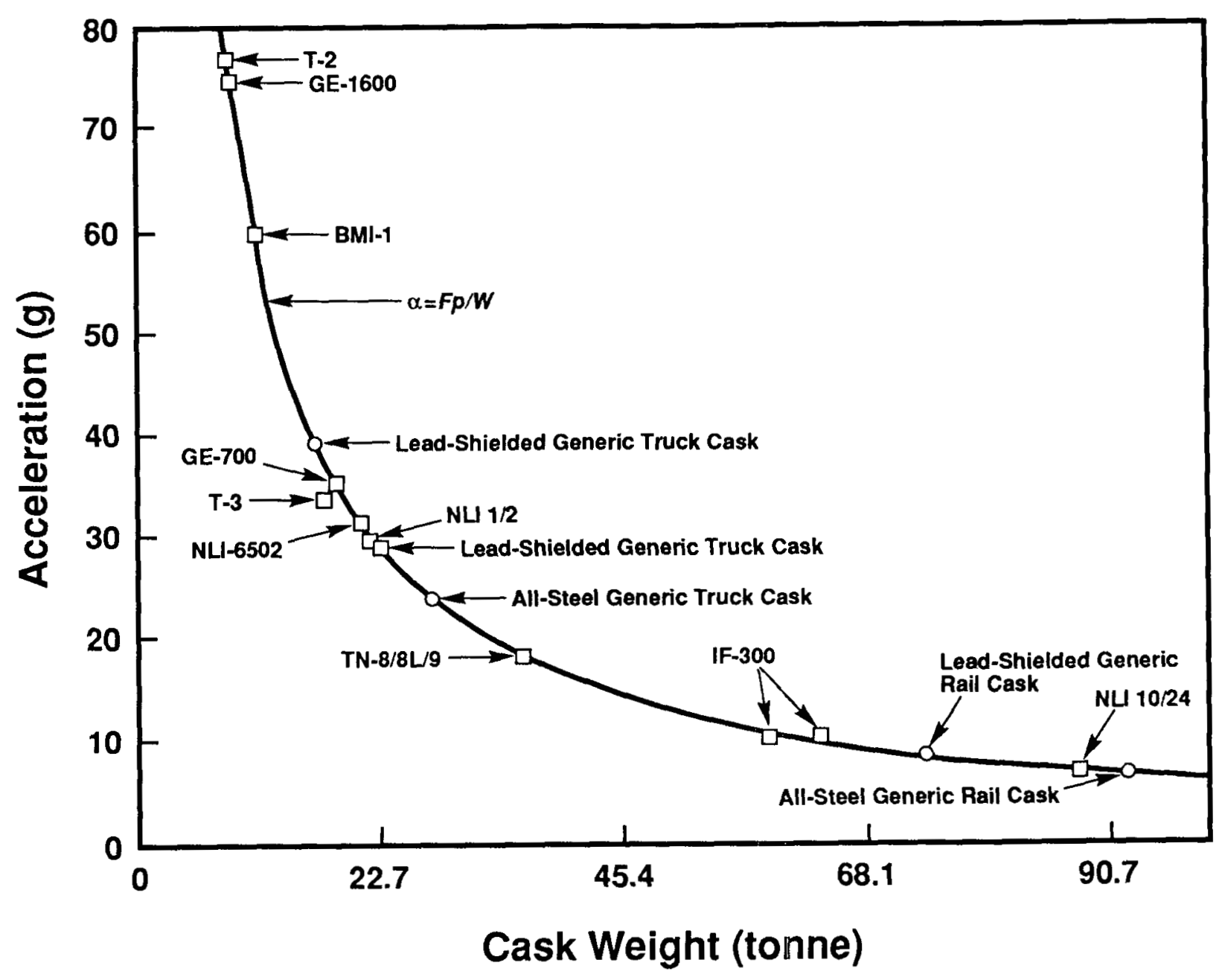

Figure 25. Maximum Punch-Drop Accelerations

\subsection{Summary}

The mechanical and thermal environments of fuel assemblies in spentfuel transport casks were characterized for casks subjected to the normal and hypothetical accident transport conditions defined by $10 \mathrm{CFR} 71$. Results for mechanical environments are presented in the form of peak $g$ accelerations or accelerations as a function of time. Results for thermal environments are presented in the form of peak temperatures or temperaturetime histories.

Normal transport conditions that were considered include shock and vibration during road and rail transport, a $0.3-\mathrm{m}$ drop onto a rigid target, and thermal response of spent fuel in casks under $38^{\circ} \mathrm{C}$ ambient temperature with full solar heat input. Hypothetical accident conditions that were quantified include cask impact on a rigid target following a 9.0-m drop, cask impact onto a mild steel bar following a $1.0-\mathrm{m}$ drop, and thermal response of spent fuel in casks subjected to a fully engulfing fire of half-hour duration at $800^{\circ} \mathrm{C}$. 


\subsection{References}

[EP86] EPRI, "The Castor/V21 PWR Spent Fuel Storage Cask: Testing and Analyses," Electric Power Research Institute, Palo Alto, CA, EPRI NP-4887, November 1986.

[FI87] Fischer, L., "Transport Container Response to Severe Highway and Railway Accident Conditions," Lawrence Livermore National Laboratory, Livermore, CA, NUREG/CR-4829, February 1987.

[MA77] Magnuson, C. F., "Shock and Vibration Environments for Large Transport Containers During Truck Transport (Part 1)," Sandia National Laboratories, Albuquerque, NM, SAND77-1110, September 1977.

[MA78] Magnuson, C. F., "Shock and Vibration Environments for Large Transport Containers During Truck Transport (Part 2)," Sandia National Laboratories, Albuquerque, NM, SAND78-0337, NUREG/CR-0128, May 1978.

[MA80] Magnuson, C. F., "Shock Environments for Large Transport Containers During Rail-Coupling Operations," Sandia National Laboratories, Albuquerque, NM, SAND79-2168, NUREG/CR-1277, June 1980.

[MA82] Magnuson, C. F., "Shock and Vibration Environments Encountered During Normal Rail Transportation of Highway Cargo," Sandia National Laboratories, Albuquerque, NM, SAND82-0819, August 1982.

[NU85] NUPAC, "Safety Analysis Report for the NUPAC 125-B Fuel Transport Cask," Nuclear Packaging Inc., Federal Way, WA, Rev. 1, October 1985 .

[US85] U.S. DOE, "Spent Fuel Storage Requirements," U.S. Department of Energy, Richland, WA, DOE/RL-85-82, 1985. 


\subsection{FUEL ROD RESPONSE}

In this section, the general methodology for the fuel rod mechanical response analysis is described and demonstrated by an analysis of a GE $7 \times 7$ BWR and a B\&W $15 \times 15$ PWR assembly. Fuel rod failure frequencies were derived from deterministic calculations of fuel rod and assembly response and probabilistic failure evaluation. The general approach is described in Section 2.0, and the analysis models are presented in detail in Appendix III.

Detailed structural models of the fuel rods, assemblies, and cask internal hardware were developed. Pretransport conditions and material properties of spent fuel were used to conduct analyses for single assembly and single rod geometries. These analyses were performed for several caskdrop orientations. The analyses include evaluations of structural interactions between the basket and assemblies, and between assembly hardware and fuel rods.

The deterministically calculated fuel rod response was compared to probabilistic failure criteria distributions for each feasible failure mode. These criteria included residual cladding ductility and fracture properties as functions of irradiation and thermal environments. A probabilistic characterization of preexisting flaws was incorporated in the failure evaluation because of the dependence of the calculated failure frequencies on the initial flaw state of the cladding.

The load transfer paths from the cask to the fuel assemblies depend strongly on the drop orientation. Figure 26 illustrates the three possible drop events: end drop, side drop, and corner drop (initial impact and slapdown). For the side drop, the load path to the fuel rods is primarily through the basket to the spacer grids and end plates. For end-drop loading conditions, however, the load path is axially through each fuel rod from end plate to end plate. Thus, the side-drop response requires a multiple rod assembly model to simulate rod-to-rod interaction, while the end-drop response is simulated with a single rod model.

Corner-drop loading is made up of two drop events: initial impact and slapdown. For cask impact angles near vertical, the initial impact dominates and the assembly response resembles the end-drop condition. For impact angles near horizontal, the slapdown phase dominates and the assembly response resembles the side-drop condition. Thus, in the cornerdrop analysis slightly modified versions of the side- and end-drop models were used. Appendix III describes in detail the modeling assumptions for each drop orientation.

\subsection{Side-Drop Response}

The assembly side-drop analytical model uses multiple fuel rods to represent rod-to-rod interaction. A two-dimensional longitudinal slice model of an assembly consisting of a single row of rods is sufficient because the amount of out-of-plane rod-to-rod interaction is negligible 


\section{END DROP CONDITION}

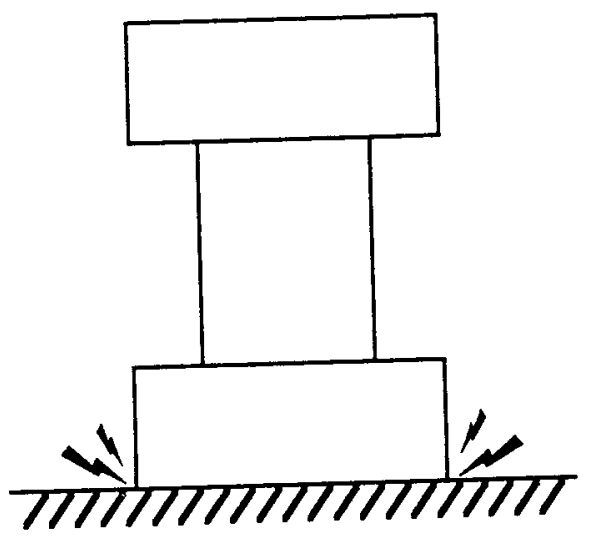

\section{SIDE DROP CONDITION}

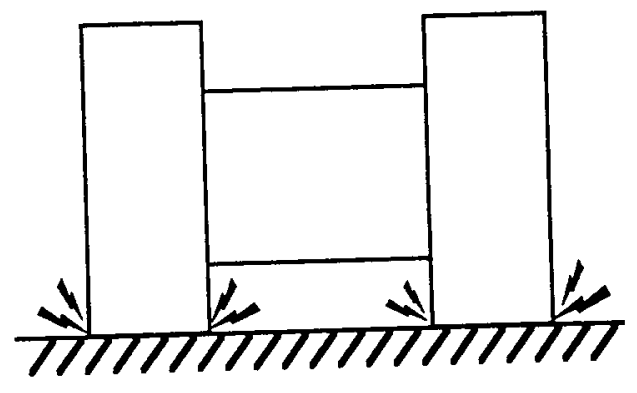

\section{CORNER DROP / SLAPDOWN}
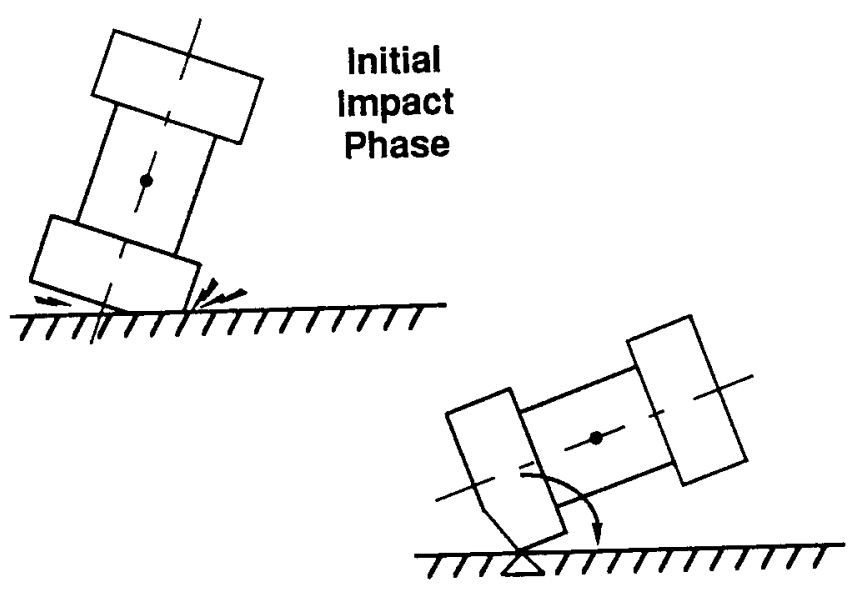

Pinned Phase

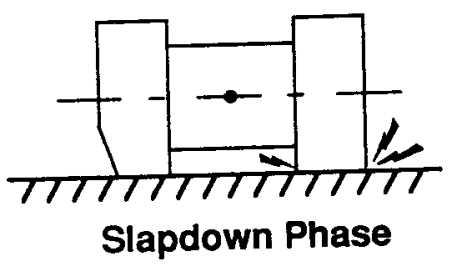

Figure 26. Cask Impact Orientations for the 9.0-m Drop 
relative to the in-plane response. Furthermore, neglecting the out-ofplane stiffness of the spacer grid is conservative because this stiffness brings the entire cross section into action, thereby increasing the inplane stiffness of the model.

As a result of the complexity of the spacer grid structure, a nonlinear spring element model was developed from separate analyses of the spacer grids and then incorporated into the assembly model. Detailed analyses of individual spacer grids were used to determine interaction between the spacer grid and fuel rods. The force transfer mechanism between the spacer grids and fuel rods is highly nonlinear as a result of flattening of the spacer contact springs and buckling of the spacer grid frame. (This sequence is illustrated in Figure 27.) Initially, the spacer grids support the full weight of the rods. As the load on the rods is increased, the spacer contact springs begin to flatten out and lose their resistance. Further load on the rods is transferred to the spacer grid frame members until they reach their critical buckling load. After buckling, the spacer grids no longer support the fuel rods independently and the fuel rods deflect until they come to rest on top of each other and begin to participate in carrying the load. After the buckling load of the spacer grid is reached, any increase in loading is supported by the rods themselves. The cladding ovalizes until it touches the fuel pellets as the rod loads increase. Further loading is transmitted through pellet-clad contact as the rods crush on top of each other. The force-deformation relationship for these four steps is illustrated in Figure 27 . The spacer grid spring elements (developed in Appendix III) model the load transfer stages through a piecewise linear force-displacement relationship, as illustrated in Figure 28. The spring elements are positioned between fue 1 rods at the spacer grid locations.

In addition to the spacer spring elements, contact spring elements are positioned along the length of the fuel rods between spacer grids to model rod-to-rod interaction. Two types of support conditions are used between rod elements and end plates in the side-drop assembly models. Tie rods and control-rod guide tubes are modeled with rigid attachments to the end plates of BWR and PWR assemblies. The rest of the assembly rods are free to move perpendicular to the end plates and were modeled assuming sliding resistance from the end plates is negligible. These rods are constrained to follow the transverse and rotational motion of the end plates but are free to slide in and out of the end plate. The tie rod and control-rod guide tube can carry axial loads.

Figure 29 illustrates a typical assembly side-drop computational model. This model consists of fuel rods as multiple beam elements, rigid end plates, and contact and spacer grid springs positioned between the rods. A typical PWR assembly model has over 480 elements. The contact springs allow rod-to-rod load transfer when the rods are stacked on top of each other. Rod stacking is further enhanced by inward rotation of the end plates, which allows the top rods to deflect more than the bottom rods. This rotation is driven by the tie rods. The top tie rods pull the end plate in, while the bottom rods restrain the inward translation of the end plate. 


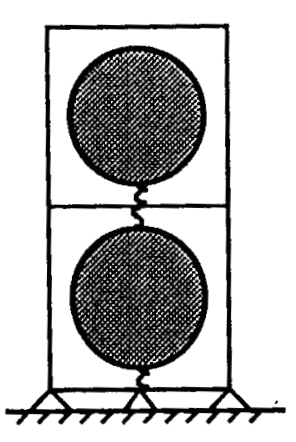

A

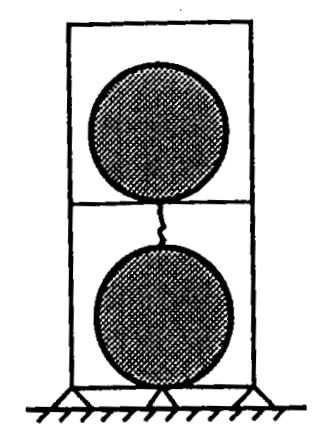

B

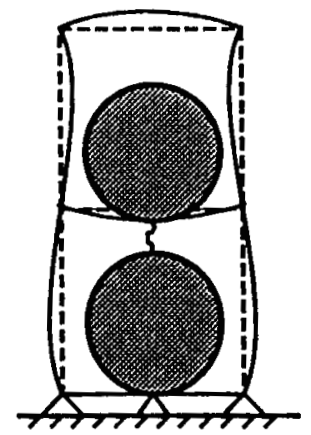

C

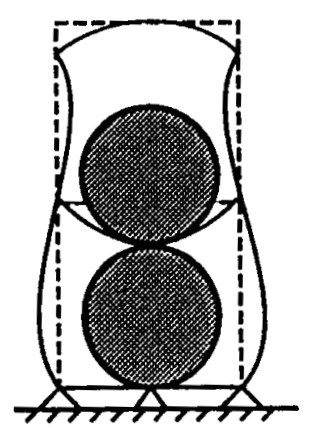

$\mathbf{D}$

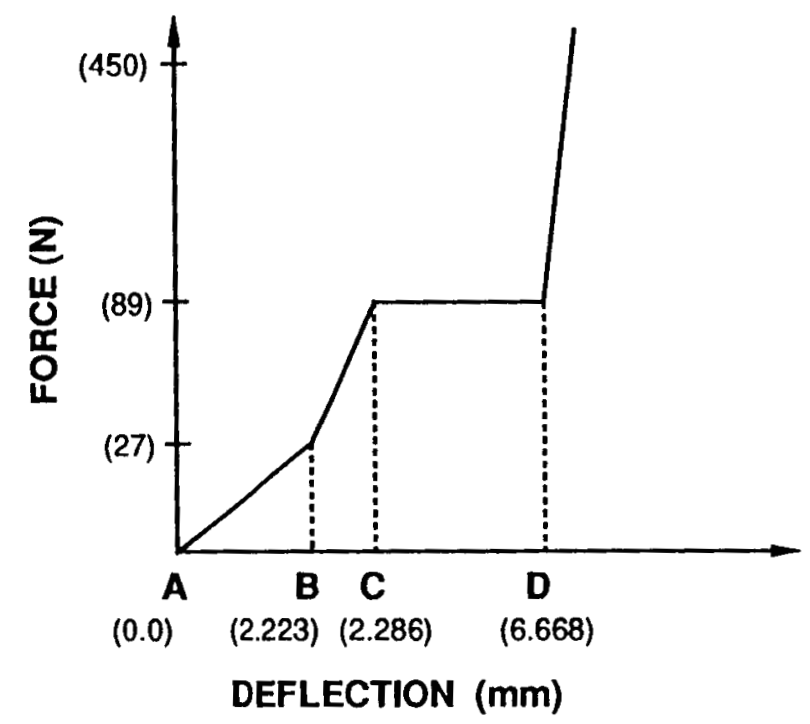




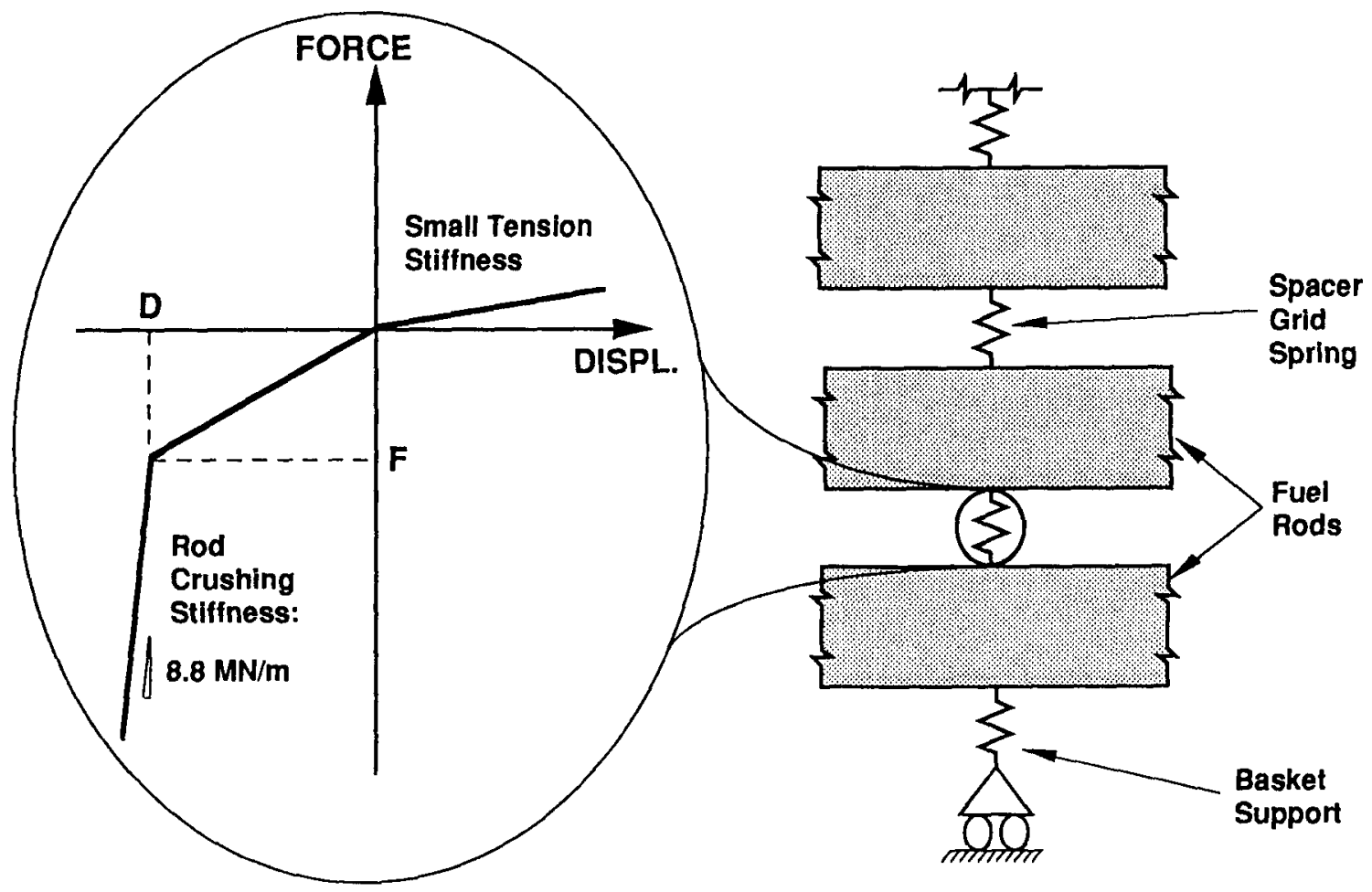

$D=$ Design gap between fuel rods
$F=$ Force required to buckle spacer grid frame

Figure 28. Two-Dimensional Assemb1y Model, the Spacer Grid Spring Elements and the Force-Displacement Relationship

The basket structure is included as spring supports in the side-drop model and is assumed to provide very stiff support for the fuel assemblies at either discrete points or continuously along the length of the basketassembly interface. The type of support (discrete versus continuous) and the relative stiffness between the assembly and basket can have significant effects on assembly response.

In the side-drop analysis sequence for free-drop loads a dynamic timemarching procedure was used. The free-drop condition was simulated with an initial velocity input to all components of the structural model. For the generic casks, initial velocities of $13.4 \mathrm{~m} / \mathrm{s}$ for the $9.0-\mathrm{m}$ drop and $2.4 \mathrm{~m} / \mathrm{s}$ for the $0.3-\mathrm{m}$ drop were typical. The momentum that resulted from these initial velocities was initiated by a retarding force that was equal to the product of the mass of the assemblies and the cask rigid-body deceleration time history. This retarding force was applied to each of the basket-assembly support points. Figure 30 illustrates the side-drop loading description. 


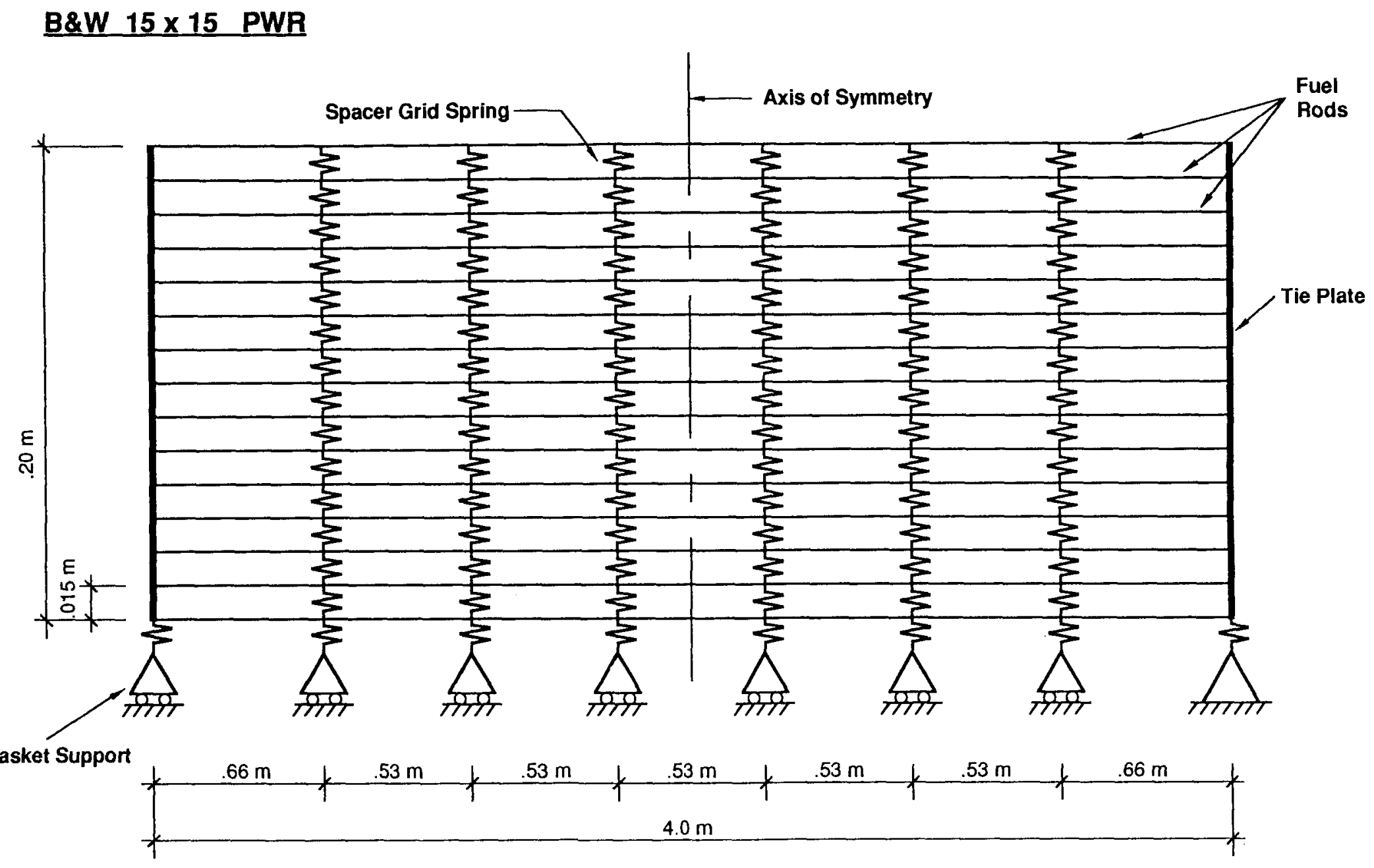

Figure 29. Example Computational Model for Side-Drop Analysis 


\section{CASK SIDE DROP}
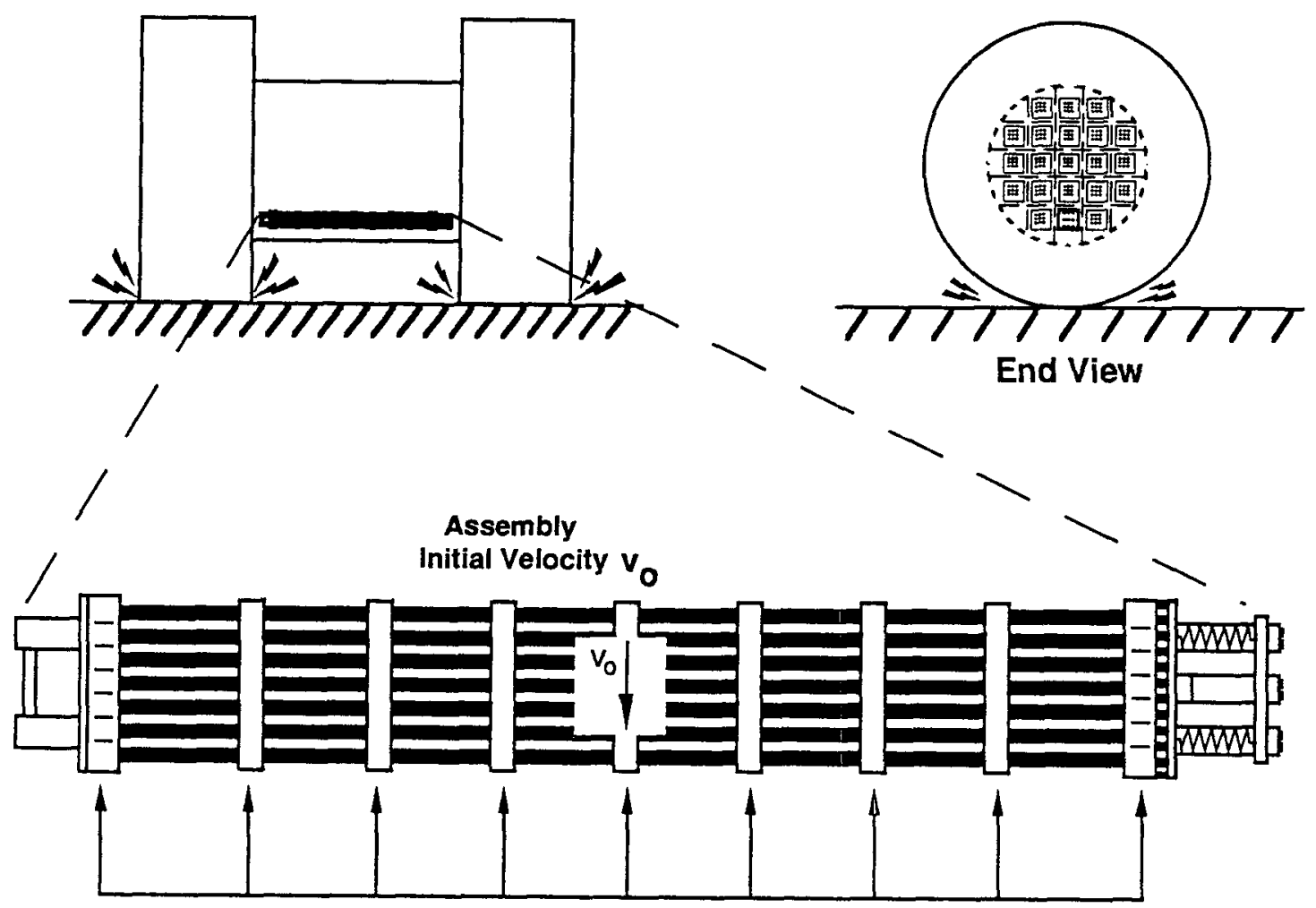

$F(t)=$ Acceleration $x$ Mass

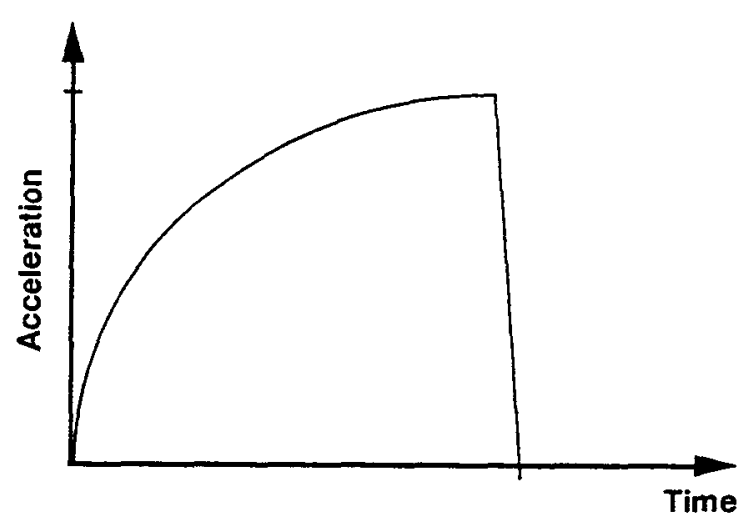

ACCELERATION TIME HISTORY

Figure 30. Assembly Side-Drop Loading Condition and Free-Body Diagram 


\subsection{End-Drop Response}

Under end-drop loading, the primary load path is axially along the length of the fuel rods. If all rods are assumed to have the same deformation pattern, a single rod model can be used to define the assembly enddrop response because the primary load path is essentially the same for all rods in the assembly. This assumption is conservative because for this loading condition rod-to-rod interaction caused by variations in deformation patterns among rods will reduce rather than increase lateral deformations that govern fuel rod failure.

As in the side-drop model, beam elements based on cladding geometric and material properties were used to model the fuel rods. However, only a portion of the fuel mass was assumed to adhere to the cladding and was included in the computational model. Those portions of the fuel mass not connected to the cladding were assumed to exert no load on the fuel rod cladding. The stiffness of the fuel mass adhering to the cladding was conservatively neglected.

Lateral constraints on the fuel assembly play an important role in determining fuel rod response. Unrestricted lateral displacements between spacer grids significantly increase the likelihood of fuel rod failure. These displacements could occur with an open-frame basket design (i.e., support provided only at discrete locations). In contrast, continuous basket designs provide a continuous lateral constraint along the length of the assembly and result in significantly less lateral displacement.

Figure 31 illustrates the process by which lateral deformation is limited to the width of gaps between the assembly and basket and between rods in the assembly.

The end-drop computational model (Figure 32) consists of a single fuel rod made up of segmented beam elements, spacer grid spring elements, and lateral constraint springs between the spacer grids. The interconnecting nonlinear spacer grid spring element models are similar to those in the side-drop analysis; however, the end-drop spacer grid spring element characteristics are based on the deformation pattern of a single rod in the assembly. The representative spacer grid model is illustrated in Figure 32. This shows the force-deformation relationship of the assembly's center rod from initial displacement until all rods are in contact with each other and with the basket support. Each step of the force-deformation curve represents the buckling of an individual spacer grid cell.

Initial rod bowing was included in the single rod model to permit the possibility of fuel rod buckling. The bowing profile was conservatively defined with the maximum plausible lateral offset in the lowest buckling mode shape of the rod. This lowest buckling mode produced maximum lateral displacements with the least amount of external energy.

Preliminary static buckling analyses were performed on individual assemblies to help quantify assembly response to the end-drop events. These analyses indicate that under static loading, buckling of freestanding assemblies (unsupported) occurs at loads significantly less than 


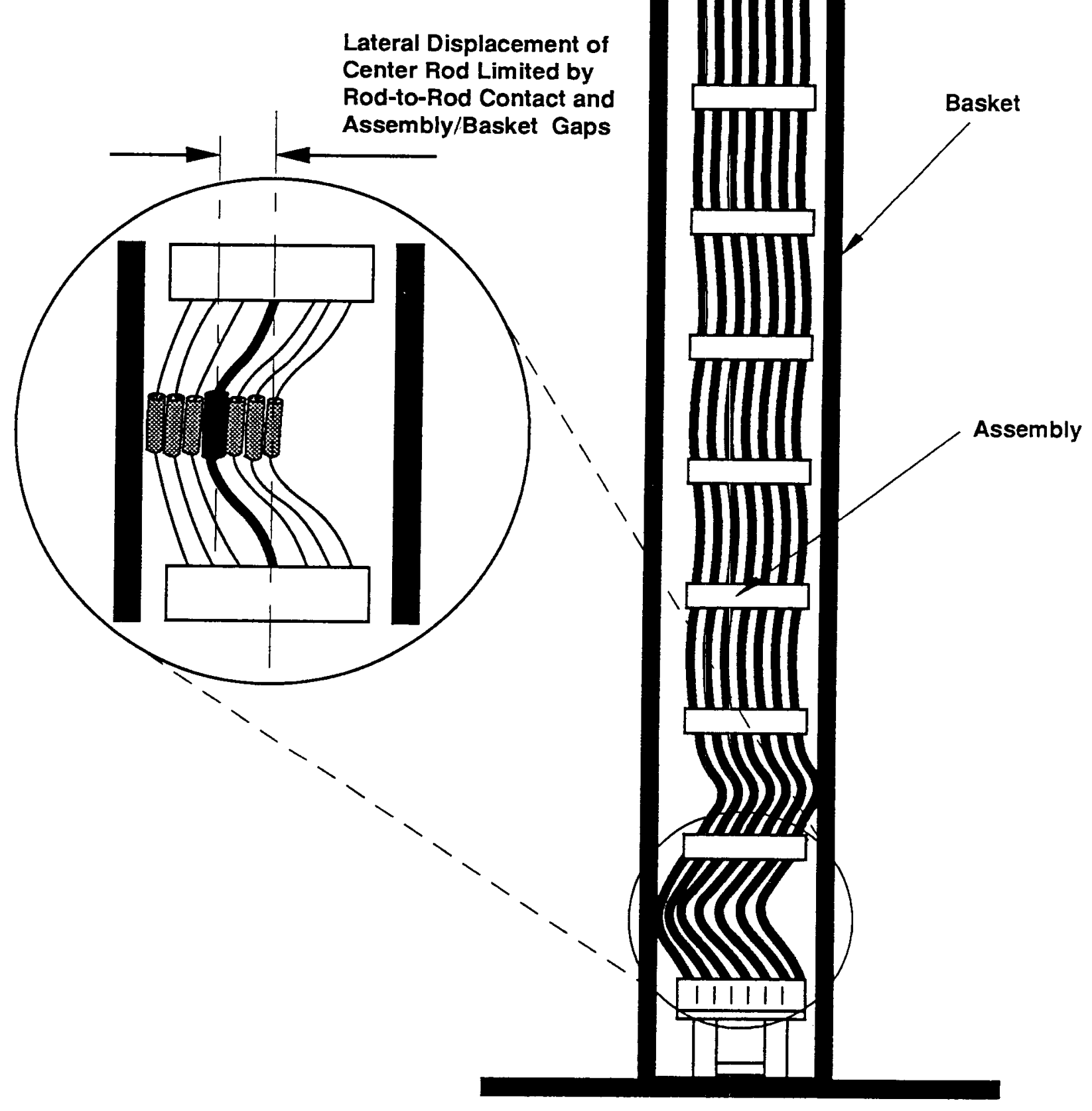

Figure 31. Lateral Displacement of Assembly Limited by Rod-to-Rod Contact and Basket-Assembly Gaps 
B = Maximum Bowing, Rod-To-Rod Design Gap

$C=$ Lateral Displacement Restrained By Assembly/Basket Geometry

metry

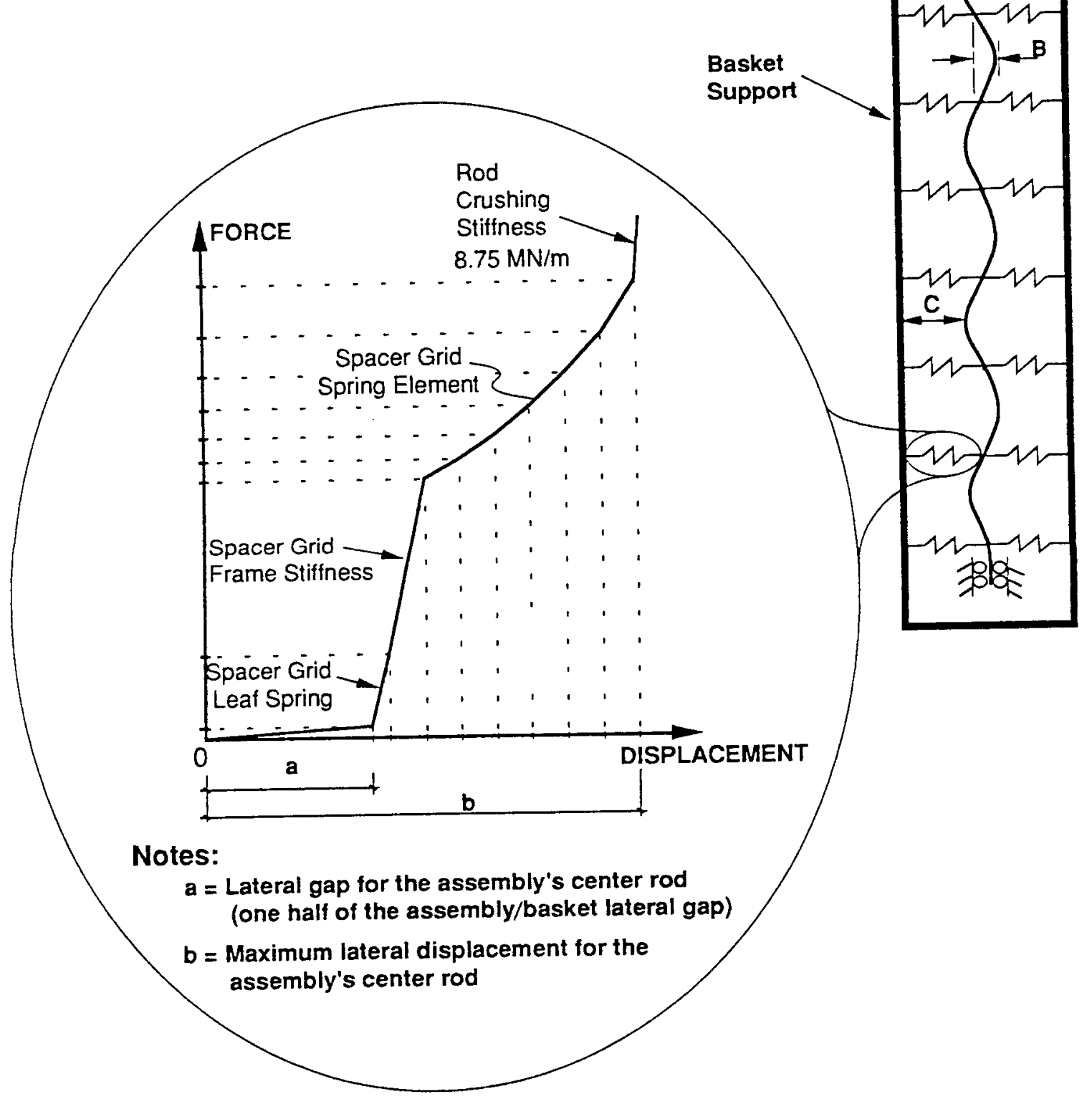

Figure 32. Example Single Rod End-Drop Assembly Mode1

regulatory impact loads. Thus, the features that constrain lateral deformation play an important role in the end-drop assembly response.

Similar to the side-drop case, the end-drop assembly model was analyzed by developing acceleration time histories (see Section 3.0). Figure 33 illustrates the loading on the end-drop model. 


\section{CASK END DROP}

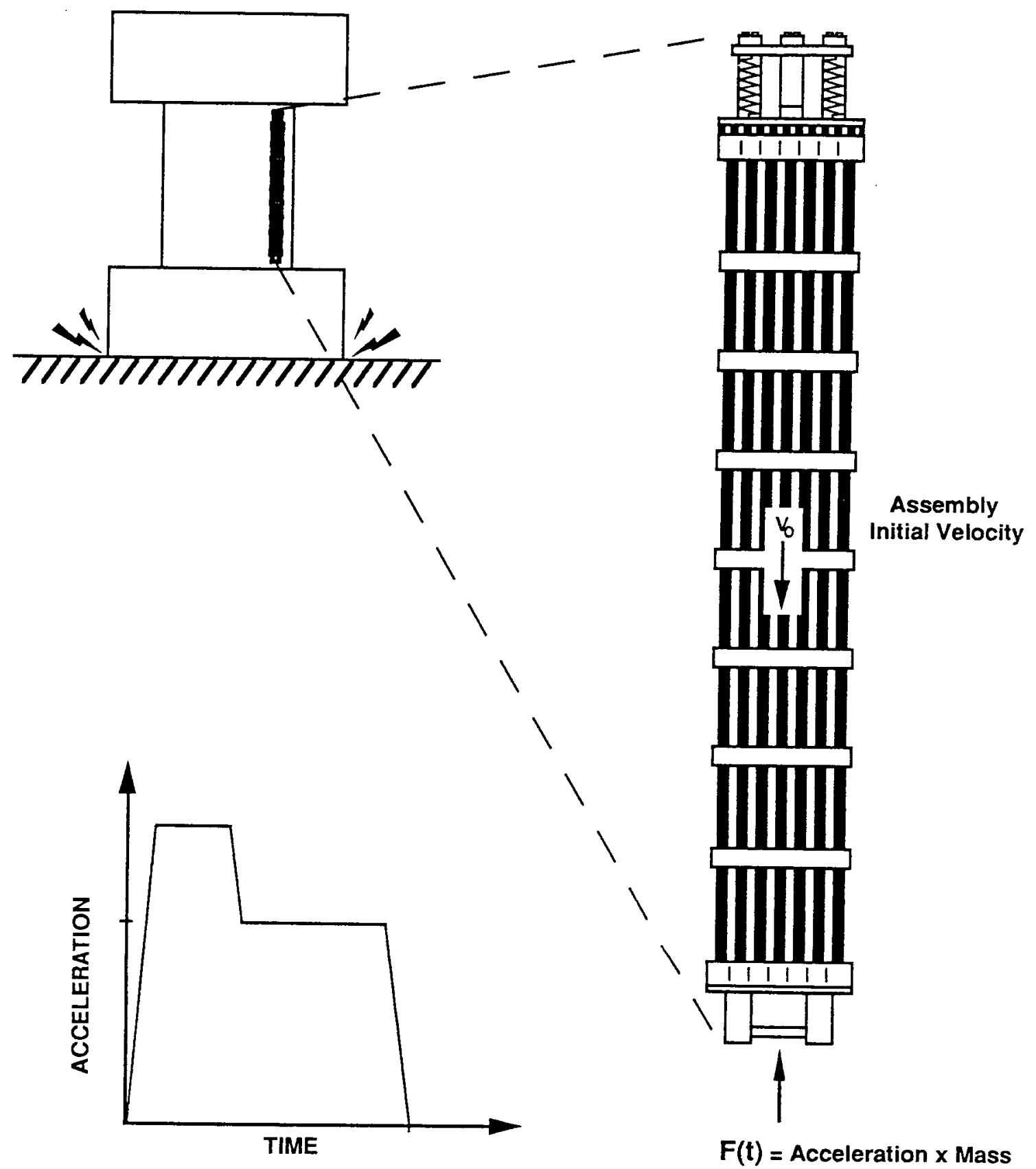

ACCELERATION TIME HISTORY

Figure 33. Assembly End-Drop Loading Condition and Free-Body Diagram 
Vertical gaps between the assembly and cask increase the deceleration of the assembly. This increase is caused by a relative velocity impact between the assembly and cask, which occurs after the cask begins to decelerate. A schematic diagram of the vertical gap load history is illustrated in Figure 34 .

\subsection{Corner-Drop and Slapdown Response}

The corner-drop response is divided into two separate events: initial impact and slapdown. Initial impact is defined as the deceleration loading corresponding to the crushing of the cask impact limiter at its initial impact location. The initial impact response dominates during cask drop at angles close to the end-drop condition.

After the initial impact phase brings the cask to rest in the upright position, a secondary impact or slapdown event will occur if the center of mass of the cask is positioned outside the impact footprint. This slapdown event is most critical when the drop angle is very small, that is, when the cask is nearly in its side-drop configuration.

These two impact events can be separately analyzed because one response dominates, depending on the impact angle. In addition, the initial end-on impact and slapdown event affect opposite ends of the cask. The effect of initial impact is higher for one end of the assembly, while the effect of slapdown is higher for the opposite end. Thus, although both events occur in sequence, only analysis of the controlling event is required.

The initial stress state in the fuel assembly caused by the initial impact loading was ignored in the slapdown analysis because the rotational interval between initial impact and slapdown allowed the assembly to return to its initial condition. Also, the critical slapdown loading conditions were at drop angles where negligible plastic response existed in the fuel assemblies. Thus, the fuel assembly returned to its original elastic state before the secondary impact.

Initial impact was modeled with the single rod model (discussed in Section 4.2) and with the addition of lateral and rotational loading caused by the angle of impact. Figure 35 illustrates the initial impact load history. The slapdown assembly response is similar to the side-drop response; thus, a modified version of the two-dimensional slice model was used to characterize the slapdown event (midplane symmetry was not assumed). Figure 36 illustrates the slapdown event.

\subsection{Thermal Response}

In addition to the normal and regulatory accident cask-drop analyses, fuel rod responses under thermally induced increases in pressure were evaluated. The expected fuel rod temperature distribution during a regulatory fire event is based on a thermal analysis presented in Appendix II. The initial conditions of the fuel rod, which include cladding inner surface crack distribution and gas pressure, are required as input data. With these initial conditions and forcing functions, individual fuel rods were analyzed to determine burst failure probability as a result of overpressurization from thermal environments. 


\section{Velocity (Cask) $=$ Velocity (Assembly) $=13.4 \mathrm{~m} / \mathrm{sec}$}

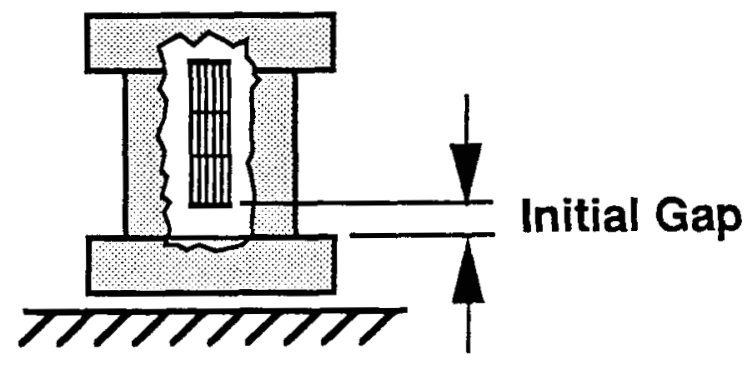

\section{Velocity (Assembly) $=13.4 \mathrm{~m} / \mathrm{sec} \quad$ Velocity (Cask) $<13.4 \mathrm{~m} / \mathrm{sec}$}

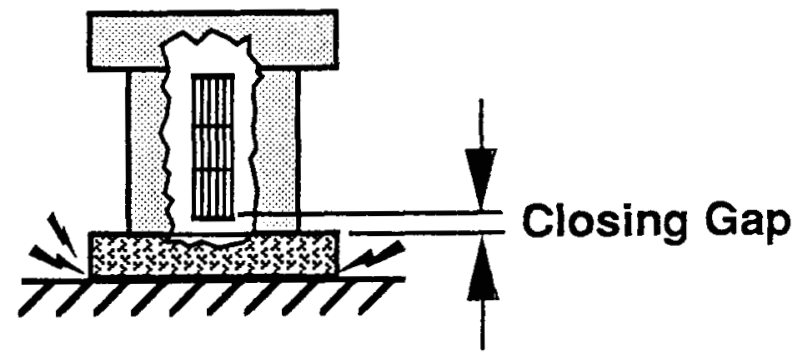

Velocity (Assembly) $=$ Velocity $($ Cask $)<13.4 \mathrm{~m} / \mathrm{sec}$

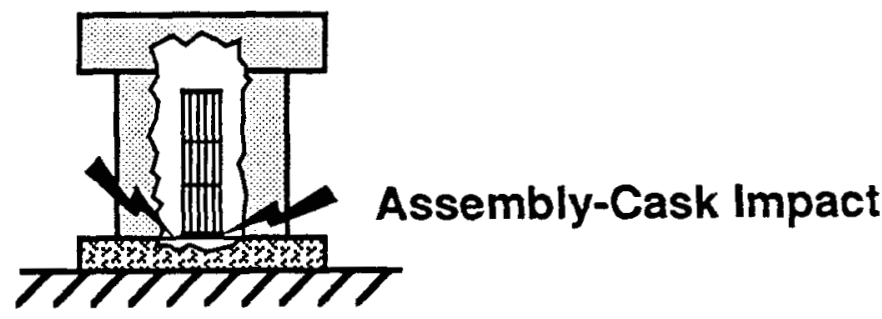

Figure 34. Illustration of End-Drop and Initial Velocity Loading

\subsection{Shock and Vibration Response}

A fuel assembly is subjected to cyclic loading conditions as a result of random shock and vibration loading during normal transport conditions. The sensitivity of fuel rod failure due to fatigue was investigated. Analyses (discussed in Appendix III) indicate that the magnitudes of the cyclic loads are such that the stresses induced in the cladding are below the endurance limit of the Zircaloy cladding. Even an infinite number of cyclic loads apparently would not propagate existing cracks into fuel rod failures. 


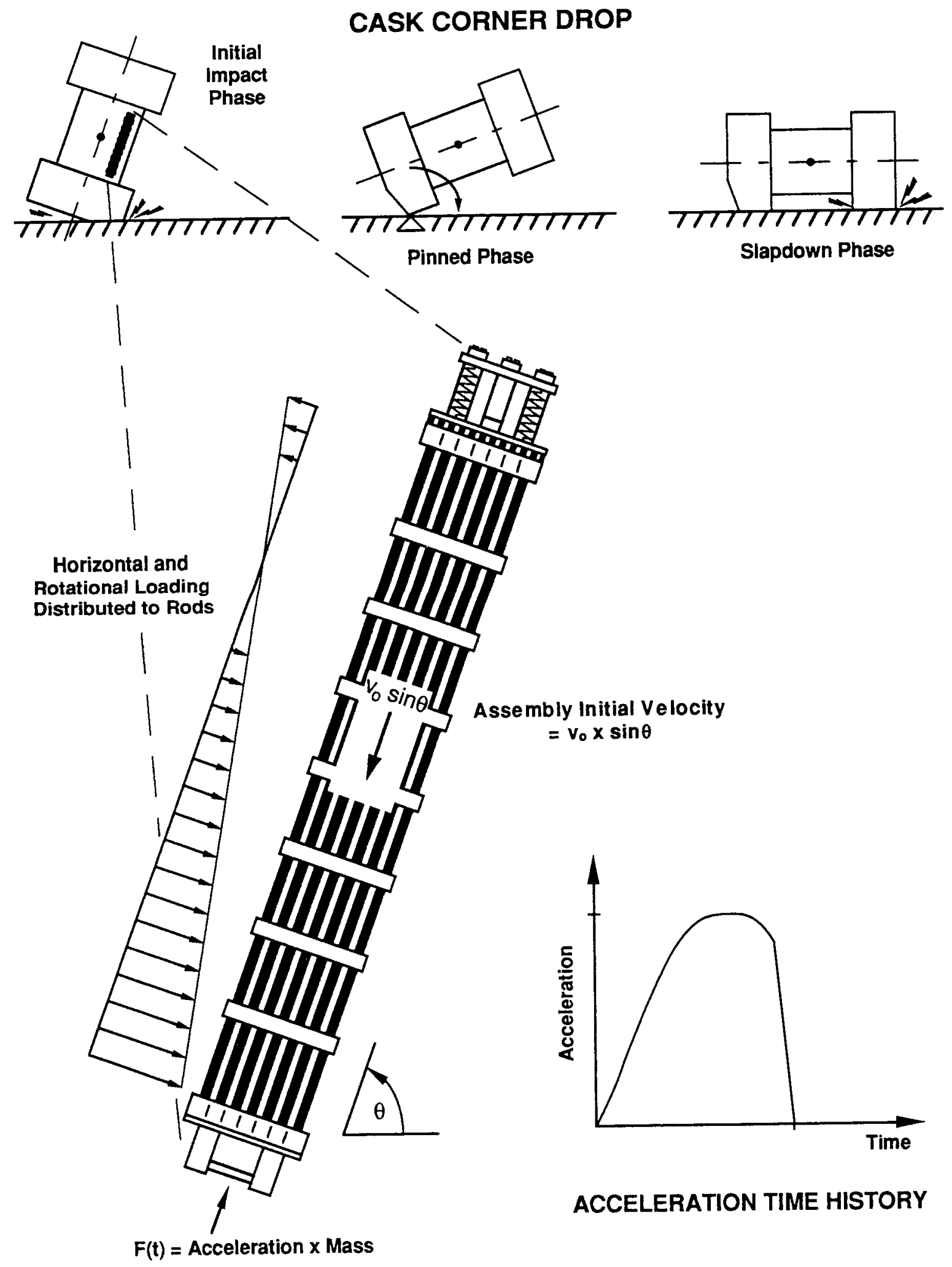

Figure 35. Assembly Corner Drop, Initial Impact Loading Condition, and Free-Body Diagram 


\section{CASK CORNER DROP}

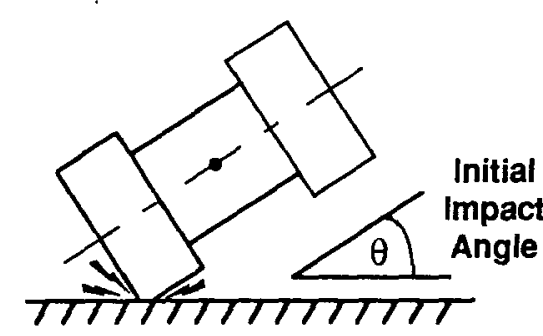

Initial Impact Phase

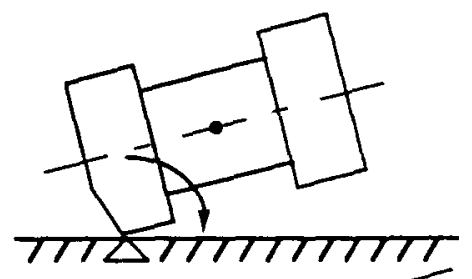

Pinned Phase -
Slapdown Phase

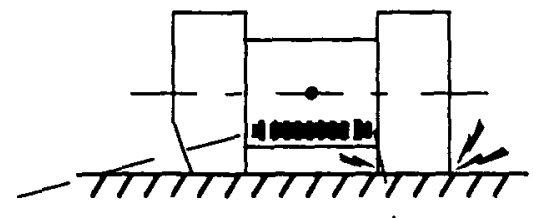

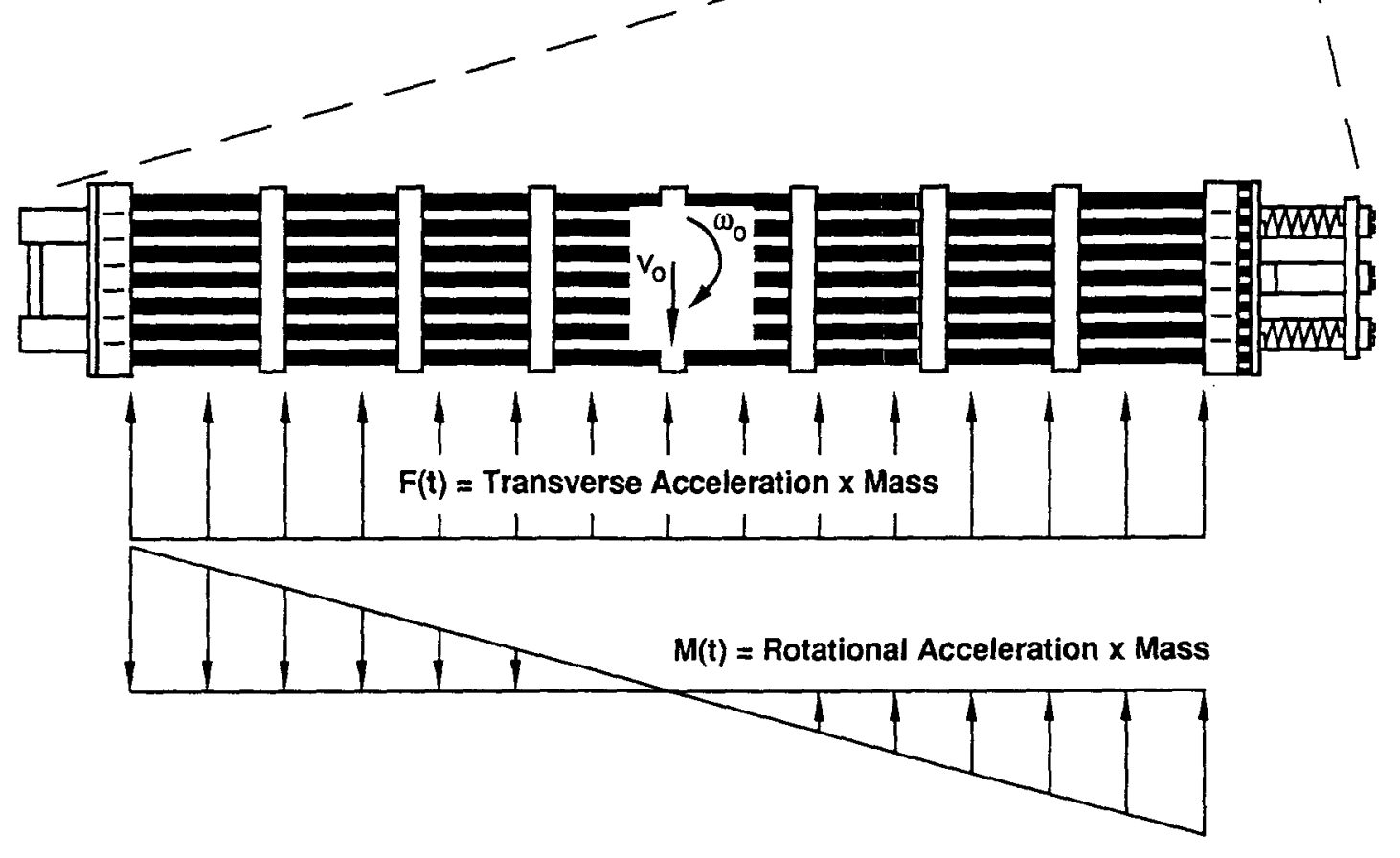
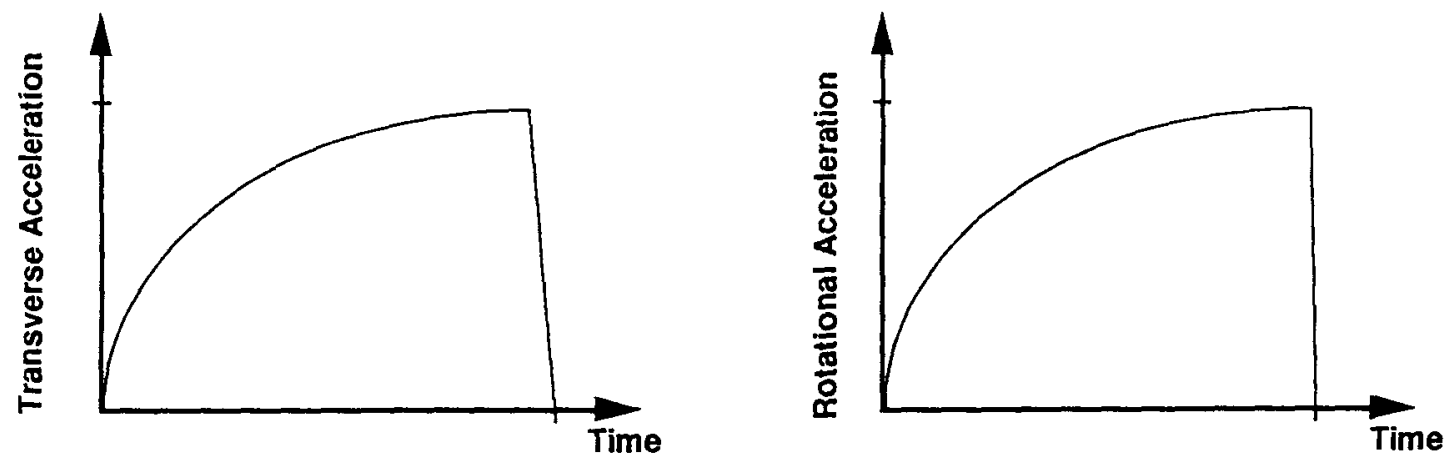

ACCELERATION TIME HISTORIES

Figure 36. Assembly Corner Drop, Slapdown Loading Condition, and Free-Body Diagram 


\subsection{Failure Criteria and Failure Modes}

A cladding breach can occur by one of two failure mechanisms: ductile tearing as a result of excessive strain or material fracture at a generated or preexisting crack. The phenomena are inherently stochastic; that is, they are naturally expressed in the terms of probability distributions.

Ductile tearing of irradiated Zircaloy occurs on the average at 48 strain in a pure biaxial tensile stress state when the hoop-to-axial stress ratio, b, is 0.9 or larger [MI76]. A median failure strain of 88 was defined for uniaxial stress states when $b$ is smaller than 0.1 . For intermediate stress states, the failure criterion was 1 inearly interpolated between the 4 and $8 \%$ values. Expressed in percent strain, the failure criterion was the following:

$$
\bar{\varepsilon}_{f}(b)=8.0-5.0(b-0.1) .
$$

These values for $\bar{\varepsilon}_{f}$ are the median values of a distribution that depends on $\mathrm{b}$. Random factors related to the micrometallurgical state and history of the material can result in rupture taking place in particular instances at strain values that are either above or below the median. The standard deviation of the distribution, $\sigma$, is similarly b-dependent. From limited data and an assumed normal form for the distribution, $\sigma$ is 0.758 when $b$ is 0.9 , and $\sigma$ is 1.258 when $b$ is 0.1 . For intermediate values, $\sigma$ can be interpolated as the following:

$$
\sigma(b)=1.25-0.625(b-0.1) .
$$

Given a strain $x$ at a particular location and the value of the biaxial stress ratio $b$, the probability of failure from ductile tearing can be determined from the integral of the normal probability density, which is shown in Figure 37:

$$
P(x)=\frac{1}{\sigma \sqrt{2 \pi}} \int_{-\infty}^{x} \exp \left(\frac{-(y-\bar{\varepsilon})^{2}}{2 \sigma^{2}}\right) d y
$$

where

$$
\begin{aligned}
& \mathrm{x}=\text { strain } \\
& \mathrm{y}=\text { integration variable (strain) } \\
& \bar{\varepsilon}=\text { mean failure strain } \\
& \sigma=\text { standard deviation of the distribution. }
\end{aligned}
$$

The second failure mechanism, called the material fracture, occurs at a generated or preexisting part-wall crack. Such cracks will propagate through the cladding wall when the local stress intensity factor $\left(\mathrm{K}_{\mathrm{IC}}\right)$ 


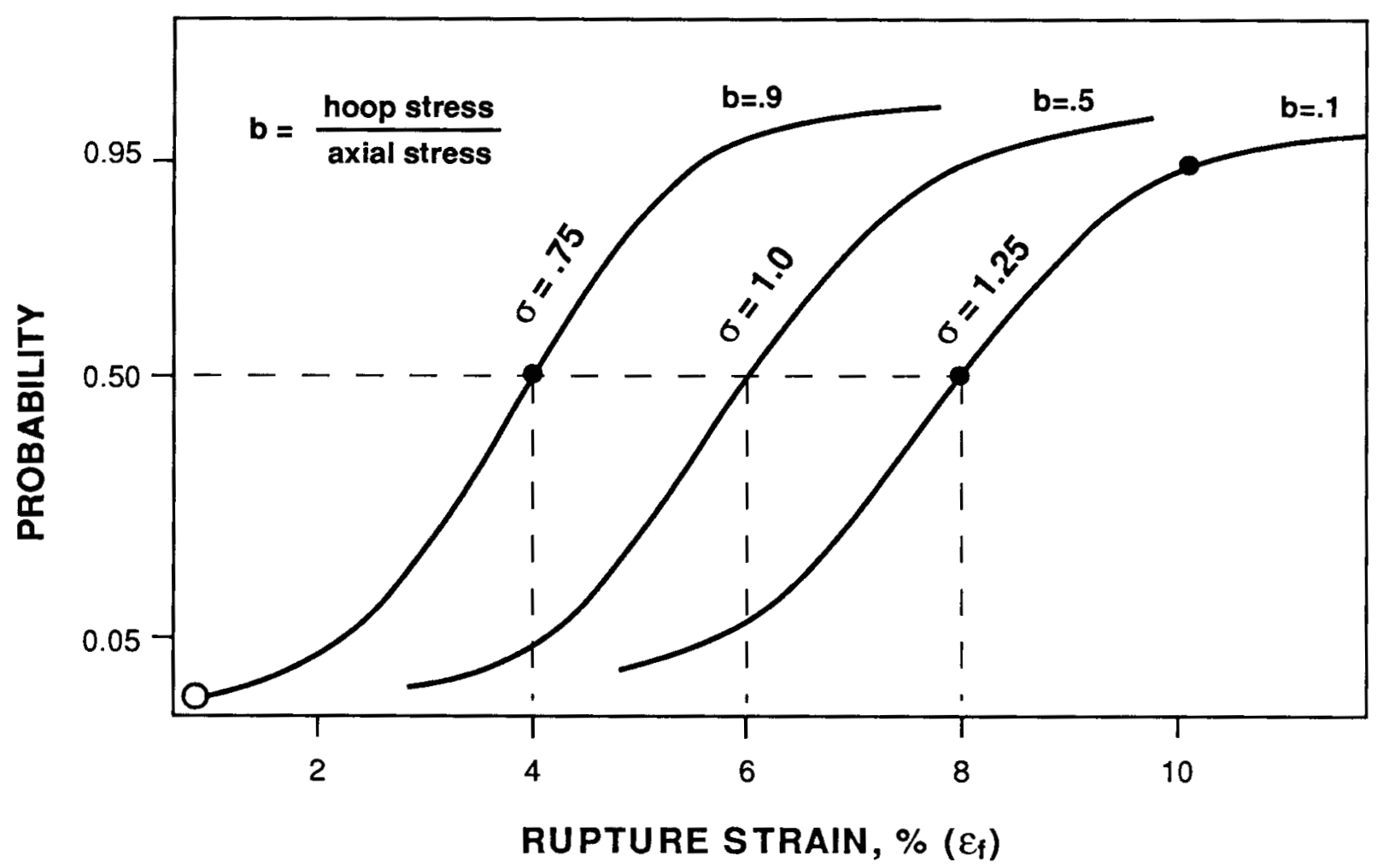

- Experimentally based data points used to develop rupture strain failure curves

$O$ - Failure probability defined as $1 \times 10^{-6}$ at elastic limit strain $=0.8 \%$

PROBABILITY OF FAILURE FOR STRAIN, $x$

$$
\begin{aligned}
& F(x)=\frac{1}{\sigma \sqrt{2 \pi}} \int_{-\infty}^{x} \exp \left(\frac{-(y-\mu)^{2}}{2 \sigma^{2}}\right) d y \\
& \mu=\text { Mean Value of Distribution } \\
& \sigma=\text { Standard De viation of Distribution }
\end{aligned}
$$

Figure 37. Probability Distribution for Cladding Rupture Strain as a Function of Biaxial Stress Ratio 
exceeds a normally distributed critical value $\left(\mathrm{K}_{\mathrm{IC}}\right)$, referred to as the material fracture toughness (see Appendix III, Section 4.2, for an explanation of stress intensity factors). Median values of $K_{I C}$ for Zircaloy cladding were correlated with neutron fluence, the cladding temperature, and the flaw orientation [BA87]:

$$
\ln \mathrm{K}_{\mathrm{IC}}=\mathrm{A}-0.134 \phi_{t}+4.14 \times 10^{-4} \mathrm{~T} \phi_{t},
$$

where

$$
\begin{aligned}
& \mathrm{K}_{\mathrm{IC}}=\text { units of } \mathrm{MPa} \sqrt{\mathrm{m}} \\
& \phi_{\mathrm{t}}=\text { neutron fluence in } 10^{21} \mathrm{n} / \mathrm{cm}^{2}(>1 \mathrm{MeV}) \\
& \mathrm{T}=\text { the temperature in }{ }^{\circ} \mathrm{C} \\
& \mathrm{A}=3.84+9 \times 10^{-4} \mathrm{~T} \text { for axial cracks } \\
& \mathrm{A}=3.91+3.6 \times 10^{-4} \mathrm{~T} \text { for circumferential cracks. }
\end{aligned}
$$

The standard deviation of the distribution is $5.5 \mathrm{MPa} \sqrt{\mathrm{m}}$; an example probability density curve is shown in Figure 38 for a transport temperature of $93^{\circ} \mathrm{C}$ and a fluence level of $7 \times 10^{21} \mathrm{n} / \mathrm{cm}^{2}$.

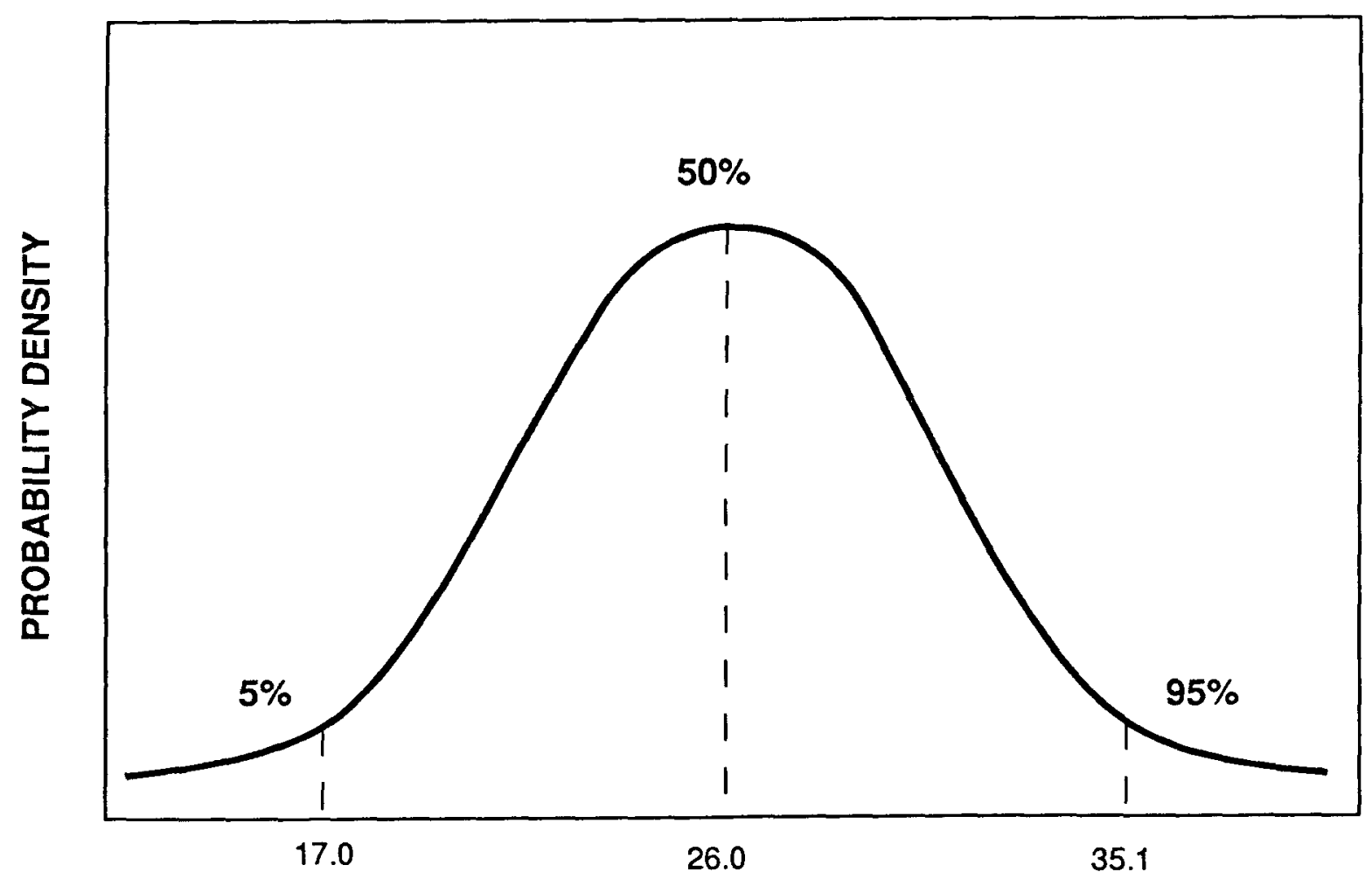

FRACTURE TOUGHNESS (MPa $\sqrt{\mathrm{m}})$

Figure 38. Probability Density Function for Cladding Fracture Toughness at $93^{\circ} \mathrm{C}$ and $7 \times 10^{21} \mathrm{n} / \mathrm{cm}^{2}$ 
Fuel rod cladding is expected to have part-wall cracks before transport, principally as a result of manufacturing flaws deepened by the mechanism of PCI during reactor operation. In this analysis, these preexisting cracks were assumed to have an exponential distribution. Distribution constants derived in Appendix III are based on the deepest preexisting flaw, referred to as critical flaw size, that would not fail while the fuel rod is in an operating reactor. Calculations performed with the fuel rod evaluation code FREY provide an estimate of 288 of the cladding thickness for the critical flaw size. In comparison, Tasooji, Einziger, and Miller have performed analyses for stress corrosion cracks from dry storage of spent fuel and predicted a critical crack depth of 208 of the cladding wall thickness [TA84]. In-reactor service records indicate approximately 1 in 10,000 PWR and 1 in 1000 BWR rods fail. An exponential distribution of part-wall cracks derived from these data is described in Appendix III. The resulting distribution of crack sizes is shown graphically in Figure 39. This distribution is based on limited experimental data and will ultimately require more extensive verification.

The probability of rod cladding failure was determined using the following calculation sequence:

- The stress intensity factor $K_{I}$ was evaluated for locations of peak stress, for cracks of an assumed depth $d$.

- The probability $P(d)$ for a crack of that size or larger was determined from the integral of the distribution function in Figure 39.

- The conditional probability $P(f \mid d)$ that the stress intensity results in rod failure was determined by integrating the probability density function for the fracture toughness (see Figure 38).

- The probability that a given part-wall crack results in cladding fracture is the product of the two probabilities: $P(f)=P(d) x$ $P(f \mid d)$.

This process was repeated for all crack sizes in the distribution at all candidate locations. The total probability of fracture failure for the rod was then determined by the sum of the individual results.

The two failure mechanisms discussed above are characteristic of the three cladding failure modes that could occur under impact loading conditions. The failure modes are defined below:

- Transverse Tearing--Rupture is initiated at the cladding outer surface because the local strain exceeds the ductility limit. This failure mode is governed by the $\bar{\varepsilon}_{f}$ criterion.

- Rod Breakage--A crack propagates from a transverse tear to full rod breakage under a bending load. This failure mode is governed by the $\mathrm{K}_{\mathrm{IC}}$ criterion. 


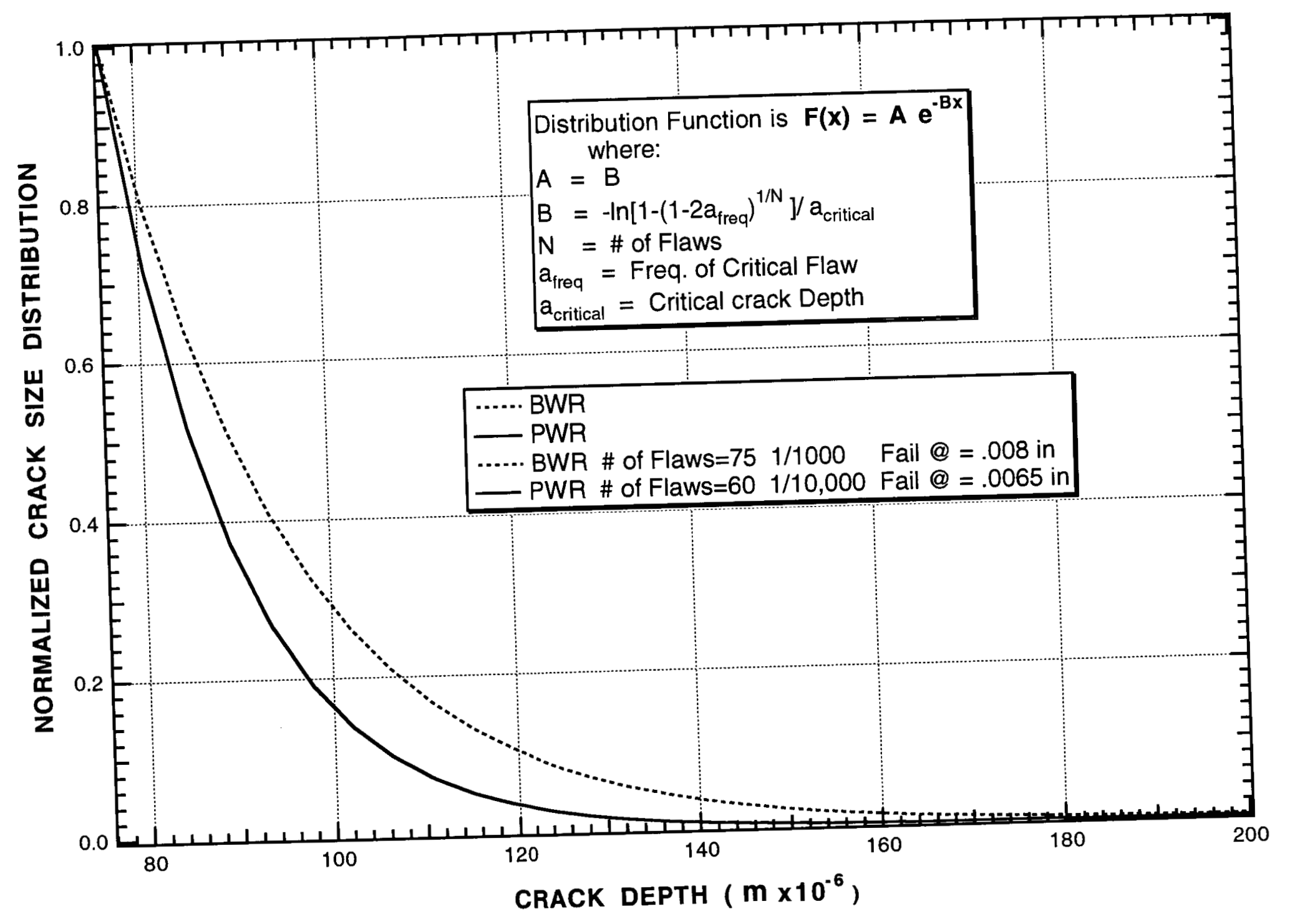

Figure 39. Crack Size Distribution 
- Longitudinal Tearing--A cladding fracture occurs as a result of a part-wall crack extending in the longitudinal direction under a compressive (pinch) load. This failure mode is governed by the $\mathrm{K}_{\mathrm{IC}}$ criterion.

Figure 40 again illustrates these three failure modes. A pinhole or narrow transverse crack results when the cladding material is stretched beyond its ductility limit. Large tensile strains occur in the fuel rods as they bend adjacent to and around the assembly spacer grids and end plates.

Cladding fracture also occurs when existing cracks propagate. Such a fracture can occur, although the bulk of the material is stressed well below the cladding yield strength. A comparative evaluation of manufacturing defect scratches due to handling and PCI part-wall cracks indicates that (1) PCI cracks are mainly found at pellet interfaces and initiated from shallow manufacturing defects and (2) these cracks are the primary source of potential material fracture. PCI cracks were oriented in the longitudinal direction; thus, propagation of these cracks requires high internal pressure or concentrated transverse loads that produce tensile hoop stress in the cladding.

Compressive pinch loads caused by rod-to-rod interaction under a sidedrop loading condition are the primary driving forces for longitudinal fracture. Rod breakage can occur if a transverse crack caused by ductile tearing propagates circumferentially and causes complete or partial cladding separation.

\subsection{Fuel Rod Failure Evaluation}

The deterministic analysis results of the fuel rod and assembly response models were used in conjunction with the probabilistic failure criteria discussed above to determine the conditional probabilities of fuel rod failure. The areas with the highest probability of failure resulting from side, end, and corner drops are defined as follows:

- Side Drop--Both ductile rupture and material fracture are plausible failure mechanisms for a side-drop event. Ductile rupture could occur and evolve into a transverse failure mode if the tensile bending strains of the fuel rod exceed the ductility limit of the cladding. These bending strains are highest at the end plates and spacer grids. Cladding material fracture could occur and evolve into a longitudinal tearing failure mode if the pinch loads are sufficiently high to cause propagation of inner surface cracks to through-wall cracks. The highest probability of failure occurs in rods in contact with the basket supports where the pinch loads are maximized. This material fracture failure mechanism is illustrated in Figure 41.

- End Drop--The most probable type of failure mechanism for the end-drop loading condition is ductile tearing of the cladding caused by sharp curvature bending of the fuel rod at its attachment to the bottom end plate, as illustrated in Figure 42. This could evolve into a transverse tearing failure mode. Large lateral 


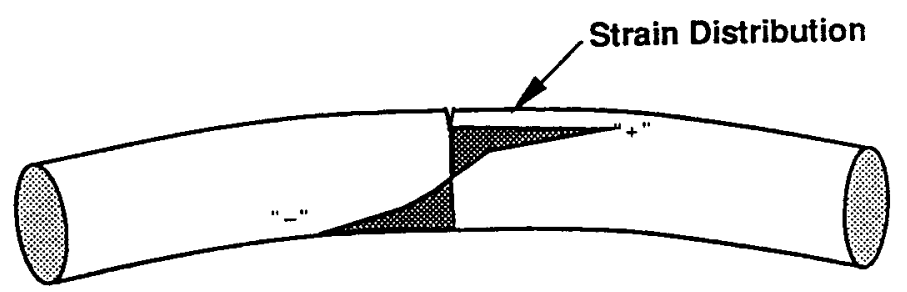

TRANSVERSE TEARING
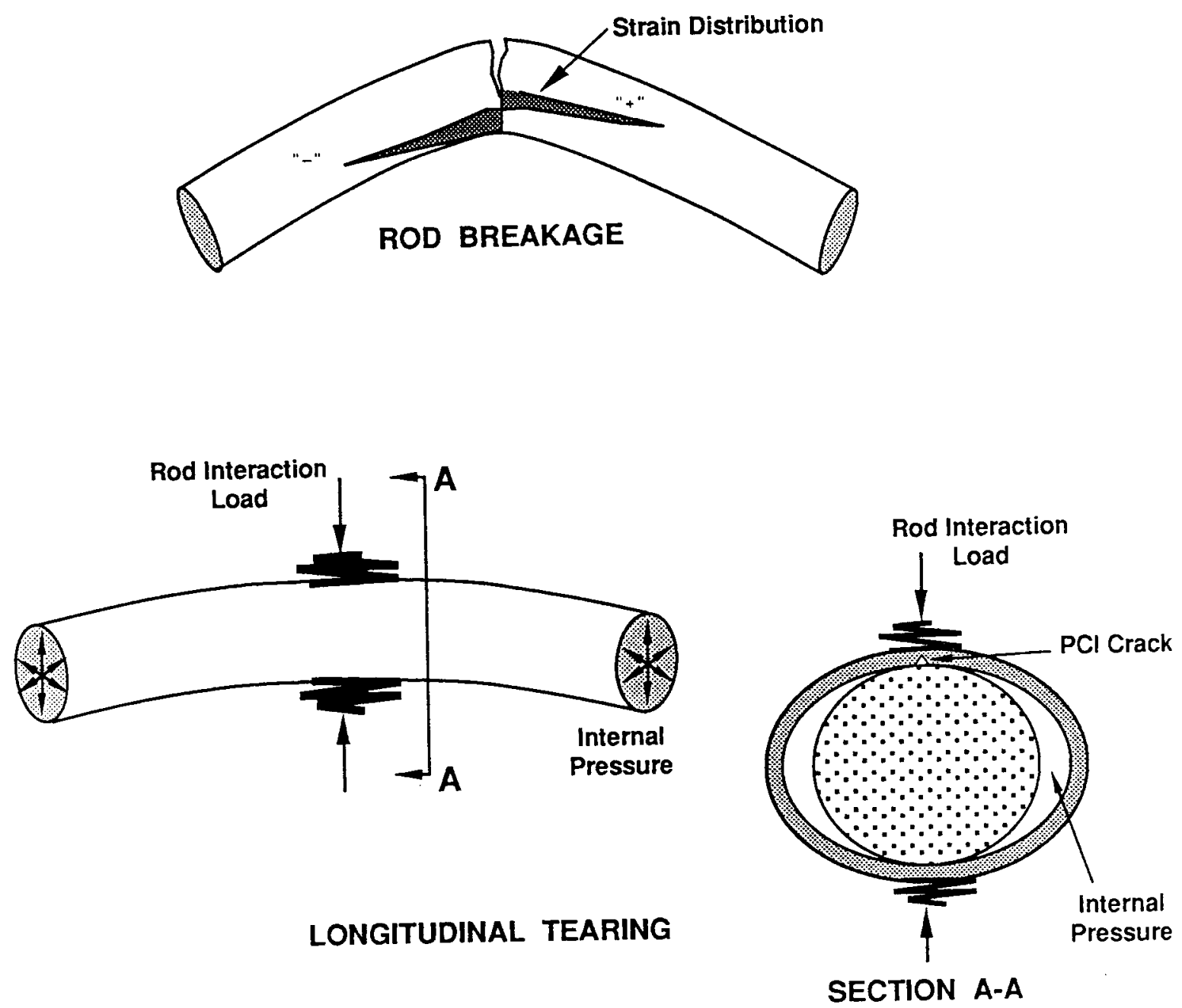

Figure 40. Fuel Rod Failure Modes 


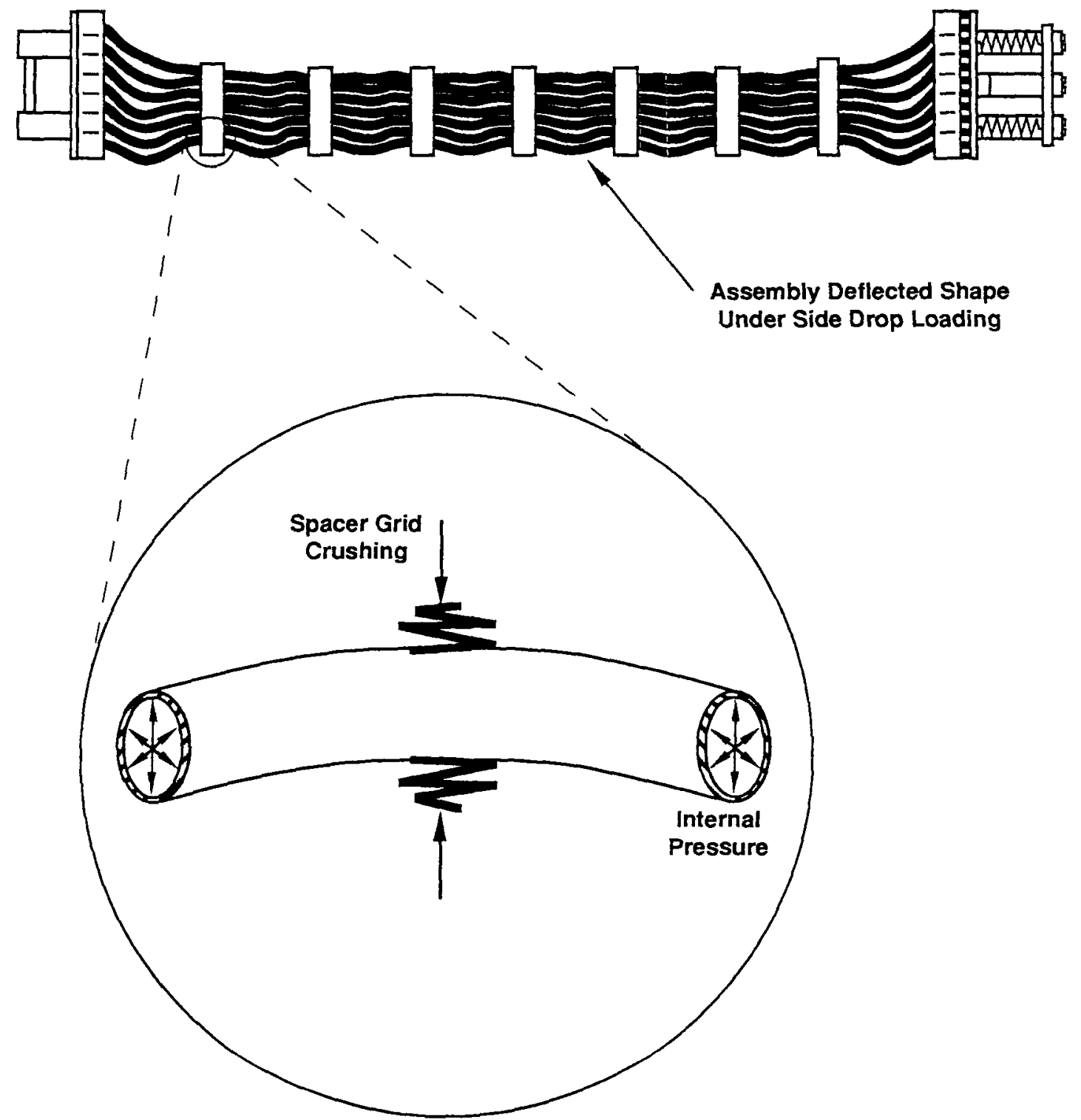

Figure 41. Cladding Material Fracture Failure Mode

deflections of the fuel rods may also lead to significant pinch loads between the fuel rods and the basket frame. This would provide a mechanism for a longitudinal tear to evolve from cladding inner surface part-wall cracks.

- Corner Drop--Fuel rod failures as a result of corner-drop initial impact and slapdown loads are similar to those from end-drop and side-drop loading. For the slapdown event, the probability of failure is highest where peak bending strains and pinch load are concentrated at the secondary impact (slapdown) end of the assembly. 


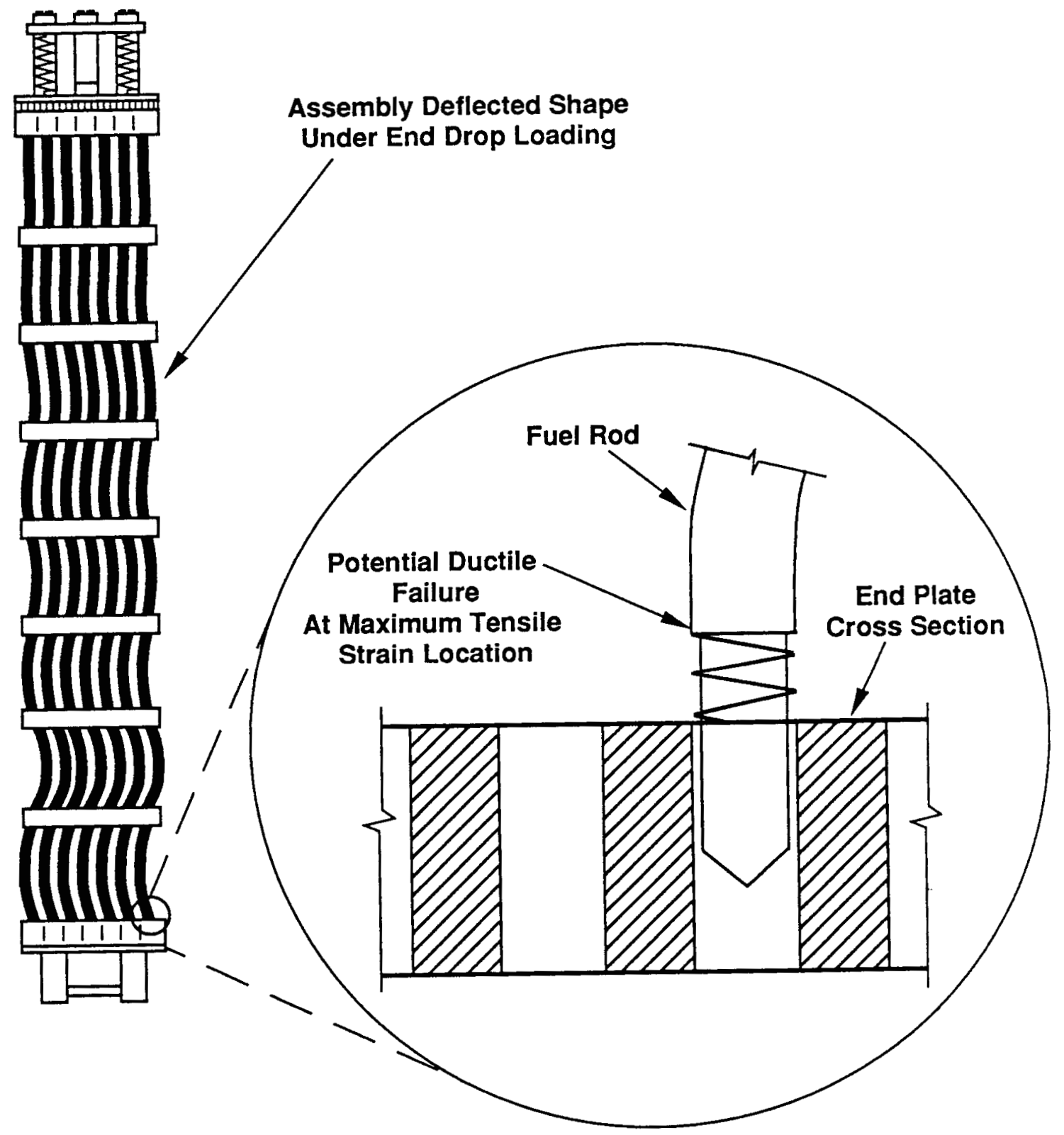

Figure 42. Cladding Material Rupture Failure Mode

\subsection{Example Analyses}

Example analyses of typical BWR and PWR assemblies were performed to illustrate the fuel rod response and failure methodology (Appendix III). For the example BWR and PWR analyses, the GE $7 \times 7$ and B\&W $15 \times 15$ 
assemblies were chosen, respectively. Each assembly was analyzed for impacts resulting from regulatory side-, end-, and corner-drop loading conditions. Failure probabilities were computed for both normal and accident transport conditions.

The failure probability assessment results for the example assemblies are given in Table 2. This table includes probabilities for the three failure modes, that is, longitudinal tearing, transverse tearing, and rod breakage, which are described in Section 4.6. Details of these analyses are given in Appendix III. Maximum peak tensile strains, rod-to-rod pinch forces, and conditional probabilities of failures for each failure mode were calculated for each of three axial fuel zones (end plate, spacer grid support, and midspan between spacer grids) for all applicable regulatory normal and hypothetical accident transport conditions. These probabilities were then combined to obtain the total failure probability per rod for each of the three failure modes, from which the cask fuel rod failure frequency was calculated by multiplying the highest probability of fuel rod failure by the number of rods in the transport cask.

The highest failure probability for the example GE $7 \times 7$ BWR assembly, approximately $8 \times 10^{-6}$ per rod per event, occurs during the initial impact phase of the $9.0-\mathrm{m}$ oblique corner-drop event. The methodology predicts a cask fuel rod failure frequency of approximately 0.02 for a rail cask containing fifty-two GE $7 \times 7$ BWR assemblies (48 fuel-bearing rods per assembly; 2496 fuel rods total). The methodology conservatively assumes that all the rods in an assembly are subjected to the peak stress states.

The highest failure probability for the example B\&W $15 \times 15$ PWR assembly, approximately $2 \times 10^{-4}$ per rod per event, occurs during both the slapdown phase of the $9.0-\mathrm{m}$ oblique corner-drop event and the $9.0-\mathrm{m}$ sidedrop event. The methodology predicts a cask fuel rod failure frequency of approximately 0.9 for a rail cask containing twenty-one B\&W $15 \times 15$ PWR assemblies (208 fuel-bearing rods per assembly; 4368 fuel rods total).

For normal transport conditions, a $0.3-\mathrm{m}$ side-drop event results in a failure probability of approximately $4 \times 10^{-8}$ per BWR rod and $3 \times 10^{-7}$ per PWR rod. This translates into cask fuel rod failure frequencies of $1 \mathrm{x}$ $10^{-4}$ for transport casks containing fifty-two BWR assemblies and $1.3 \times 10^{-3}$ for transport casks containing twenty-one PWR assemblies, respectively. 
Table 2

Sumary of Example Assembly Failure Probabilities Under Regulatory Transport Loading of Spent Fuel

\begin{tabular}{|c|c|c|c|c|c|c|c|c|c|c|c|c|c|}
\hline \multirow[b]{2}{*}{$\begin{array}{c}\text { Assembly } \\
\text { Loading Condition }\end{array}$} & \multirow[b]{2}{*}{$\begin{array}{r}\text { Drop } \\
\text { Angle }\end{array}$} & \multicolumn{3}{|c|}{ End Plate } & \multicolumn{3}{|c|}{$\begin{array}{c}\text { Spacer Grid } \\
\text { Support } \\
\end{array}$} & \multicolumn{3}{|c|}{$\begin{array}{l}\text { Midspan Between } \\
\text { Spacer Grids } \\
\end{array}$} & \multicolumn{3}{|c|}{$\begin{array}{c}\text { Failure Probability } \\
\text { Per Rod } \\
\end{array}$} \\
\hline & & $\begin{array}{l}\text { Tensile } \\
\text { Strain } \\
(z) \\
\end{array}$ & $\begin{array}{l}\text { Pinch } \\
\text { Load } \\
\text { (N) } \\
\end{array}$ & $\begin{array}{l}\text { No. } \\
\text { Per } \\
\text { Rod }\end{array}$ & $\begin{array}{l}\text { Tensile } \\
\text { Strain } \\
(x) \\
\end{array}$ & $\begin{array}{l}\text { Pinch } \\
\text { Load } \\
\text { (N) } \\
\end{array}$ & $\begin{array}{l}\text { No. } \\
\text { Per } \\
\text { Rod }\end{array}$ & $\begin{array}{l}\text { Tensile } \\
\text { Strain } \\
(z) \\
\end{array}$ & $\begin{array}{l}\text { Pinch } \\
\text { Load } \\
\text { (N) } \\
\end{array}$ & $\begin{array}{l}\text { No. } \\
\text { Per } \\
\text { Rod }\end{array}$ & $\begin{array}{c}\text { Longitudinal Slit } \\
\text { PCI } \\
\text { Part-Wall Crack } \\
\end{array}$ & $\begin{array}{l}\text { Transverse } \\
\text { Pinhole } \\
\text { Rupture } \\
\end{array}$ & $\begin{array}{c}\text { Rod } \\
\text { Breakage }\end{array}$ \\
\hline \multicolumn{14}{|l|}{ GE $7 \times>\mathrm{BWR}$} \\
\hline 9.0-m End Drop & 90 & 0.342 & 0 & 1 & 0.166 & 4.45 & 2 & 0.248 & 0 & 2 & $2 . E-10$ & $1 . E-09$ & $2 . E-13$ \\
\hline $\begin{array}{l}\text { 9.0-m Corner Drop } \\
\text { (Initial Impact) }\end{array}$ & 84 & 2.59 & 0 & 1 & 0.542 & 703 & 2 & 0.419 & 841 & 2 & $8 . E-10$ & 8.E-06 & 2.E-06 \\
\hline $\begin{array}{l}\text { 9.0-m Corner Drop } \\
\text { (Slapdown) }\end{array}$ & 2 & 0.99 & 0 & 1 & 1.16 & 7940 & 3 & 0.91 & 0 & 3 & $1 . E-06$ & 3.E- 07 & 2.E-07 \\
\hline 9.0-m Side Drop & 0 & 0.94 & 0 & 2 & 1.1 & 7562 & 5 & 0.84 & 0 & 6 & 2.E-06 & S.E-07 & 7.E-07 \\
\hline $\begin{array}{l}0.3-\mathrm{m} \text { Normal Drop } \\
\text { (Side Drop) }\end{array}$ & 0 & 0.63 & 0 & 2 & 0.56 & 2900 & 5 & 0.54 & 0 & 6 & $1 . E-08$ & 4.E-08 & 1.E-09 \\
\hline \multicolumn{14}{|l|}{ B\&W $15 \times 15$ PWR } \\
\hline 9.0-m End Drop & 90 & 2.5 & 0 & 1 & 1.04 & 84.5 & 2 & $\begin{array}{l}2.02 \\
0.844\end{array}$ & $\begin{array}{c}0 \\
2345\end{array}$ & $\begin{array}{l}1 \\
1\end{array}$ & $6 . E-10$ & 7.E-06 & 8.E-07 \\
\hline $\begin{array}{l}\text { 9.0-m Corner Drop } \\
\text { (Initial Impact) }\end{array}$ & 84 & 2.6 & 0 & 1 & 0.971 & 72.9 & 2 & $\begin{array}{l}2.08 \\
0.546\end{array}$ & $\begin{array}{c}0 \\
3006\end{array}$ & $\begin{array}{l}1 \\
1\end{array}$ & 2.E-09 & $9 . E-06$ & 1.E-06 \\
\hline $\begin{array}{l}\text { 9.0-m Corner Drop } \\
\text { (Slapdown) }\end{array}$ & 2 & 3.47 & 0 & 1 & 1.37 & 9728 & 2 & 1.02 & 0 & 3 & 2.E-05 & 2.E-04 & 2.E-05 \\
\hline 9.0-m Side Drop & 0 & 3.3 & 0 & 2 & 1.3 & 9265 & 6 & $\begin{array}{l}0.97 \\
1.2\end{array}$ & 0 & $\begin{array}{l}5 \\
2\end{array}$ & 2.E-05 & 2.E-04 & 5.E-05 \\
\hline $\begin{array}{l}0.3 \text {-m Normal Drop } \\
\text { (Side Drop) }\end{array}$ & 0 & 1.00 & 0 & 2 & 0.66 & 3560 & 6 & 0.72 & 0 & 7 & 3.E-08 & 3.E-07 & 2.E-08 \\
\hline Fire & $\mathrm{n} / \mathrm{a}$ & 0.8 & 0 & a & 0.8 & 0 & a & 0.8 & 0 & a & 1.E-11 & $0.0^{b}$ & $0.0^{\mathrm{b}}$ \\
\hline $\begin{array}{l}\text { Normal } \mathrm{c} \\
\text { Transport }\end{array}$ & $\mathrm{n} / \mathrm{a}$ & 0.252 & 0 & 1 & 0.1 & 79.2 & 2 & 0.203 & 0 & 2 & $0.0^{d}$ & 2.E-07 & 2.E-12 \\
\hline $\begin{array}{l}a_{\text {Fire analysis stre }} \\
\mathrm{b}_{\text {These failure mode }} \\
\mathrm{c}_{\text {Normal transport }} \\
\mathrm{d}_{\text {Stress intensity }}\end{array}$ & $\begin{array}{l}\text { ss is ba } \\
\text { s are no } \\
\text { s due to } \\
\text { actor is }\end{array}$ & $\begin{array}{l}\text { sed on part } \\
\text { applicabl } \\
\text { shock and } \\
\text { less than }\end{array}$ & $\begin{array}{l}\text {-wall cr } \\
\text { e to reg } \\
\text { vibratic } \\
\text { threshol }\end{array}$ & $\begin{array}{l}\text { sack i } \\
\text { zulato } \\
\text { on loa } \\
\text { ld val }\end{array}$ & $\begin{array}{l}\text { fuel wi } \\
\text { y fire } \\
\text { ing. } \\
\text { e. }\end{array}$ & $\begin{array}{l}\text { prob } \\
\text { ditior }\end{array}$ & & & & & & & \\
\hline
\end{tabular}




\subsection{References}

[BA87] Barsel1, A. W., "Nonlinear Statistical Analysis of Zircaloy-2 Fracture Toughness Data," Internal Correspondence, GA Technologies, San Diego, CA, February 1987.

[MI76] Miyamoto, Y., Y. Komatsu, T. Kakuma, and N. Nagai, "Mechanical Behavior of Zircaloy-2 Tubing Under Biaxial Stresses," Journal of Nuclear Materials, 61, p. 53 (1976).

[TA84] Tasooji, A., R. E. Einziger, and A. K. Miller, "Modeling of Zircaloy Stress-Corrosion Cracking: Texture Effects and Dry Storage Spent Fuel Behavior," in "Zirconium in the Nuclear Industry: Sixth International Symposium," American Society of Testing Materials, ASTM-STP-824, p. 595-626, 1984. 
This page is intentionally left blank. 


\subsection{EVALUATION OF POTENTIAL RADIONUCLIDE RELEASES}

Most radionuclides produced during irradiation of a fuel rod are trapped as impurities within the $\mathrm{UO}_{2}$ fuel crystal lattice. Some radionuclides migrate through the crystal lattice to the reactor surfaces because of the rather high temperatures involved during reactor operation and the relatively long durations during which the fuel remains at these temperatures. (A very small fraction is actually thrown out of the crystal by recoil or knockout processes.) However, once the fuel has been removed from the reactor, no additional migration occurs unless the fuel is again brought to high temperatures $\left(>1000^{\circ} \mathrm{C}\right)$. Such high temperatures cannot be attained during normal transport or under regulatory accident conditions when irradiated fuel is allowed to decay for $5 \mathrm{yr}$ before transport. Thus, only those radionuclides that migrated to the gap during reactor operation, a portion of the volatile species, and fuel fines (if any) would be available for release to the environment.

\subsection{Estimate of Tota1 Rod Activity}

The assessment of radioactive material release from fuel rods in transit began with an estimate of the total radionuclide inventory within the rods. This is typically performed using existing computational codes. The ORIGEN2 computer code [CR78, CR80, CR83] was chosen for this analysis. This code is widely used by the Office of Civilian Radioactive Waste Management (OCRWM) Program [R087] and the NRC in its severe accident analyses [KR85]. Sample results using ORIGEN2 are presented in Table 3 for some selected radionuclides contained in rods irradiated under both PWR and BWR conditions. Detailed analyses are presented in Appendix $I$.

\subsection{Determination of Gap Inventories}

Once the total radionuclide inventory was determined, the amount of radioactive material was estimated that would escape from the $\mathrm{UO}_{2}$ crystal lattice and be available for release from a fuel rod if the rod cladding lost its integrity. Three classes of radioactive material would exist in the gap: gases (tritium and $85 \mathrm{Kr}$ ), volatile species $\left(134 \mathrm{Cs}\right.$ and ${ }^{137} \mathrm{Cs}$ ), and nonvolatile species (fuel particles [fines] and their associated nuclides).

A methodology has been developed to estimate the gap inventories of $85 \mathrm{Kr}$ and the two cesium isotopes. This methodology is embodied in the standard ANSI/ANS-5.4-1982 [AN82] and is the recommended approach for estimating the gap fraction (i.e., the fraction of the total nuclide inventory contained within the the gap and plenum regions of a fuel rod) of $85 \mathrm{Kr}$. The calculations involved are most conveniently performed using a computer code because a detailed specification of the temperature and irradiation history of the fuel rod is required (see Appendix I). The standard, however, is at variance with experimental evidence for the cesium nuclides [L080, L081, J085, MA87, C087]. In this analysis, the same gap fraction used for ${ }^{85} \mathrm{Kr}$ was used for the $134 \mathrm{Cs}$ and $137 \mathrm{Cs}$ isotopes. While this approach is contrary to the standard, it is considered conservative because the volatile cesium isotopes were essentially treated as a gas. 
Table 3

Radionuclide Contents of Selected LWR Fuel Rods

\begin{tabular}{|c|c|c|c|c|}
\hline \multirow[b]{2}{*}{ Nuclide } & \multicolumn{2}{|c|}{ PWR Roda } & \multicolumn{2}{|c|}{ BWR Rodb } \\
\hline & $g$ & $\mathrm{Ci}$ & g & $\mathrm{Ci}$ \\
\hline${ }^{3} \mathrm{H}$ & $1.05 E-04$ & $1.02 E+00$ & $1.71 E-04$ & $1.66 \mathrm{E}+00$ \\
\hline $85 \mathrm{Kr}$ & $3.90 \mathrm{E}-02$ & $1.56 E+01$ & $4.54 E-02$ & $1.81 \mathrm{E}+01$ \\
\hline $90 \mathrm{Sr}$ & $1.03 E+00$ & $1.55 E+02$ & $1.18 E+00$ & $1.78 \mathrm{E}+02$ \\
\hline $90 \mathrm{Y}$ & $6.21 E-04$ & $1.55 E+02$ & $7.10 E-04$ & $1.78 \mathrm{E}+02$ \\
\hline${ }^{106} \mathrm{Ru}$ & $9.66 \mathrm{E}-03$ & $3.29 E+01$ & $1.42 \mathrm{E}-02$ & $4.84 E+01$ \\
\hline $106 \mathrm{Rh}$ & $9.23 \mathrm{E}-09$ & $3.29 E+01$ & 1. $36 E-08$ & $4.84 E+01$ \\
\hline $125 \mathrm{Sb}$ & $8.52 \mathrm{E}-03$ & $8.80 E+00$ & $1.28 \mathrm{E}-02$ & 1. $32 E+00$ \\
\hline $125 \mathrm{~m}_{\mathrm{Te}}$ & $1.19 E-04$ & $2.15 \mathrm{E}+00$ & $1.79 \mathrm{E}-04$ & $3.22 \mathrm{E}+00$ \\
\hline $134 \mathrm{Cs}$ & $5.80 E-02$ & $6.96 \mathrm{E}+01$ & $8.21 E-02$ & $9.85 E+01$ \\
\hline $137 \mathrm{Cs}$ & $2.36 E+00$ & $2.31 E+02$ & $3.59 \mathrm{E}+00$ & $3.52 E+02$ \\
\hline $137 \mathrm{~m}_{\mathrm{Ba}}$ & $4.07 \mathrm{E}-07$ & $2.19 E+02$ & $6.18 E-07$ & $3.33 E+02$ \\
\hline $144 \mathrm{Ce}$ & $6.00 E-03$ & $1.92 \mathrm{E}+01$ & $5.55 E-03$ & $1.78 \mathrm{E}+01$ \\
\hline $144 \mathrm{Pr}$ & $2.54 E-07$ & $1.92 E+01$ & $2.35 E-07$ & $1.78 \mathrm{E}+01$ \\
\hline $144 \mathrm{~m} \operatorname{Pr}$ & 1.27E-09 & 2. $30 E-01$ & $1.18 E-09$ & $2.13 \mathrm{E}-01$ \\
\hline${ }^{154} \mathrm{Eu}$ & $1.31 \mathrm{E}-01$ & $1.97 \mathrm{E}+01$ & $2.47 \mathrm{E}-01$ & $3.71 E+01$ \\
\hline $155 \mathrm{Eu}$ & $6.33 \mathrm{E}-03$ & $8.86 \mathrm{E}+00$ & 1. $20 \mathrm{E}-02$ & $1.68 \mathrm{E}+01$ \\
\hline $235 \mathrm{U}$ & 1. $39 E+01$ & $2.91 E-05$ & $5.60 \mathrm{E}+00$ & $1.18 \mathrm{E}-05$ \\
\hline $238 \mathrm{U}$ & $2.13 E+03$ & $7.02 E-04$ & $3.69 \mathrm{E}+03$ & $1.22 \mathrm{E}-03$ \\
\hline${ }^{238} \mathrm{Pu}$ & $4.97 \mathrm{E}-01$ & $8.45 \mathrm{E}+00$ & $8.91 E-01$ & $1.52 E+01$ \\
\hline $239 \mathrm{Pu}$ & 1. $14 \mathrm{E}+01$ & $7.04 \mathrm{E}-01$ & $1.95 \mathrm{E}+01$ & $1.21 \mathrm{E}+00$ \\
\hline $240 \mathrm{Pu}$ & $5.62 E+00$ & $1.29 \mathrm{E}+00$ & $1.02 E+01$ & 2. $33 E+00$ \\
\hline $241 \mathrm{Pu}$ & $2.27 \mathrm{E}+00$ & $2.49 \mathrm{E}+02$ & $4.42 E+00$ & $4.86 E+02$ \\
\hline $241_{\mathrm{Am}}$ & $8.48 \mathrm{E}-01$ & $2.71 E+00$ & $1.81 \mathrm{E}+00$ & $5.79 E+00$ \\
\hline $244 \mathrm{Cm}$ & $8.44 E-02$ & $6.92 \mathrm{E}+00$ & 4. $22 \mathrm{E}-01$ & $3.46 \mathrm{E}+01$ \\
\hline Balance & $\underline{5.55 \mathrm{E}+01}$ & $\underline{6.96 \mathrm{E}+01}$ & $1.41 \mathrm{E}+02$ & $8.46 \mathrm{E}+01$ \\
\hline Totals: & $2.22 E+03$ & 1. $33 E+03$ & $3.88 \mathrm{E}+03$ & $1.99 \mathrm{E}+03$ \\
\hline
\end{tabular}

aOconee-1 Rod 08639 irradiated to $38.2 \mathrm{MWD} / \mathrm{kg} U$ after 5 -yr decay [BA83]. bQuad Cities-1 Rod B5A-0139 irradiated to $33.7 \mathrm{MWD} / \mathrm{kg}$ U after 5-yr decay. 
No data have been derived describing the gap inventories of tritium in irradiated fuel rods. Tritium can be transported through the $\mathrm{UO}_{2}$ lattice considerably more rapidly than $85 \mathrm{Kr}$. This would tend to make the expected tritium gap fraction significantly larger than for $85 \mathrm{Kr}$. However, tritium is transported through Zircaloy much more rapidly than through $\mathrm{UO}_{2}$ and readily dissolves in the metal. These factors tend to decrease the expected gap inventory of tritium. Available cladding measurements indicate that approximately $40 \%$ of the tritium produced in PWR fuel rods is trapped in the Zircaloy cladding, and up to 608 has been observed in the cladding of a BWR fuel rod [G080]. Therefore, for the purposes of this analysis, the gap fraction of tritium was assumed to be 0.5 .

Similarly, data for the amount of fuel fines contained within a fuel rod are sparse. Consequently, a model for fuel fine release from rods with breached cladding was developed that is independent of a corresponding gap inventory. Meanwhile, a model is being developed to correlate fine production with severe impact pellet fracture; see Appendix IV for a discussion of preliminary work in that area.

Representative estimates of gap fractions for the two fuel rods identified in Table 3 are presented in Table 4. These estimates were generated using the FREY computer code [EP83] and available data. (See Appendix I for additional details.)

Table 4

Gap Concentrations of Selected Radionuclides

in PWR and BWR Fuel Rods

\begin{tabular}{|c|c|c|c|c|}
\hline \multirow[b]{2}{*}{ Nuclide } & \multicolumn{2}{|c|}{ PWR $^{a}$} & \multicolumn{2}{|c|}{ BWR $^{b}$} \\
\hline & $\begin{array}{c}\text { Gap } \\
\text { Fraction } \\
\text { Calculated } \\
\end{array}$ & $\begin{array}{c}\text { Calculated } \\
\text { Activity } \\
\text { (Ci) }\end{array}$ & $\begin{array}{c}\text { Gap } \\
\text { Fraction } \\
\text { Calculated } \\
\end{array}$ & $\begin{array}{c}\text { Calculated } \\
\text { Activity } \\
\text { (Ci) } \\
\end{array}$ \\
\hline $\begin{array}{l}85 \mathrm{Kr} \\
125 \mathrm{~m} \mathrm{Te} \\
134 \mathrm{Cs} \\
137 \mathrm{Cs}\end{array}$ & $\begin{array}{l}0.010 \\
0.010 \\
0.010 \\
0.010\end{array}$ & $\begin{array}{l}0.16 \\
0.02 \\
0.70 \\
2.31\end{array}$ & $\begin{array}{l}0.021 \\
0.021 \\
0.021 \\
0.021\end{array}$ & $\begin{array}{l}0.38 \\
0.07 \\
2.07 \\
7.37\end{array}$ \\
\hline $\begin{array}{l}\text { aOconee- } 1 \text { Rod } 0863 \\
\text { bQuad Cities-1 Fue } \\
\text { decay. } \\
\text { cExpressed as a fr }\end{array}$ & $\begin{array}{l}39 \text { irradiate } \\
1 \text { Rod B5A-C } \\
\text { Eaction of } t\end{array}$ & $\begin{array}{l}\text { to } 38.2 \mathrm{MWD} \\
9 \text { irradiate } \\
\text { total nuc1 }\end{array}$ & $\begin{array}{l}\text { 5-yr decay } \\
\text { MWD/kg U aft } \\
\text { ory. }\end{array}$ & er $5-y x$ \\
\hline
\end{tabular}




\subsection{Radionuclide Release from Fuel Rods}

Modern PWR fuel rods are pressurized with helium to approximately 3.04 MPa pressure; fission product gases added to this gap inventory generally increase the internal pressure of the rods by a few percent. (Krypton and xenon, which are primarily stable, constitute the fission gas inventory and were estimated in the same way the $85 \mathrm{Kr}$ gap fraction was determined.) Fuel rods irradiated in BWRs, however, contain helium at a pressure of approximately $0.30 \mathrm{MPa}$. This gas inventory can be significantly increased by the fission gas gap inventory. In the event of cladding breach, these fill and fission product gases will be released and act to purge some of the particulate material contained within the gap region.

If cladding failure occurs, virtually the entire gap inventories of tritium and $85 \mathrm{Kr}$ in the breached rods will be introduced into the free volume of the transport cask because these two nuclides are in gaseous form at all temperatures of concern. However, only the fraction of the cesium corresponding to the partial pressure of cesium in equilibrium with its condensed phase (probably cesium uranate compounds) will be in gaseous form. Hence, the amount of cesium purged from the gap depends on the temperature at which loss of cladding integrity occurs. A model developed to describe this release [LO79] is discussed in detail in Appendix IV. In addition to the cesium being purged from the rod at the time of cladding failure, additional diffusional release of cesium will occur after the internal and external pressures of the rod have reached equilibrium. This mode of release is unimportant because it requires temperatures and/or time periods in excess of those that would occur in normal or hypothetical accident conditions defined by the regulation.

Nonvolatile radioactive species in the form of fuel fines can also escape from rods through cladding breaches. The fines are ejected from the fuel rods at cladding failure by the purging action of fill and fission product gases. Thus, a sudden release of fuel fines occurs when the cladding integrity is initially lost, but no additional releases occur once equilibrium conditions are reached. Limited data are available for fines release at the time of cladding failure [LO80, L081, BU85]. Based on these data, roughly $0.003 \%$ of the fuel contained in rods appears to be released as fines during a rupture event.

For the purpose of this analysis, the composition of the fines was assumed to be identical to the average (i.e., as calculated by ORIGEN2) composition of the bulk fuel. But if the gap fraction for cesium exceeded 0.01 , the cesium inventories of the fuel fines were adjusted by a factor that is 100 times the gap fraction based on the observation [L081] that the fines act as local sorption (or condensation) sites for cesium migrating to the gap region (see Appendix IV).

For all three types of release (i.e., gaseous, volatile, and fuel fines), the amount released appeared to be independent of the location or size of the cladding failure within the scope of failures expected for regulatory transport conditions. In addition, only cesium releases were observed to be dependent on temperature. 


\subsection{Releases to a Cask Cavity at $530^{\circ} \mathrm{C}$}

Calculated results of radionuclide quantities that would be released from example PWR and BWR fuel rods for cladding failure at $530^{\circ} \mathrm{C}$ are presented in Table 5. The values in Table 5 are conservative because $530^{\circ} \mathrm{C}$ is higher than the allowable fuel temperature in transport casks and represents an upper limit on the expected fuel temperature during regulatory fire conditions (see Appendix II). Once the gases and vapors (volatile material) escape from the fuel rod, they should disperse homogeneously throughout the free volume of the cask. However, the dispersal of particulate material (i.e., fuel fines) is subject to attenuation processes, such as gravitational settling and deposition on surfaces, that tend to reduce the airborne concentration significantly. In principle, the attenuation can be estimated if the particle-size distribution of the ejected fuel fines is known; unfortunately, no adequate data exist. But only 108 of the fines distribute widely, while 90 s settle within a few inches of a breach location during burst rupture events [L080, L081, BU85]. Thus, a 108 aerosolized fuel fines fraction was used in this analysis.

With this methodology, example estimates were made for the concentrations of radionuclides released into an NLI-1/2 cask transporting PWR or BWR fuel assemblies. The results are presented in Table 6 . The estimates are based on release from a single fuel rod, release values listed in Table 5 , and free volume values of $0.155 \mathrm{~m}^{3}$ for the cask when transporting PWR assemblies and $0.129 \mathrm{~m}^{3}$ when transporting BWR spent fuel [SA91]. To arrange the radionuclides by relative toxicity, the concentrations are presented in terms of their corresponding $A_{2}$ values, as tabulated in $10 \mathrm{CFR}$ 71 [US90]. (Note that the default value $\mathrm{A}_{2}=0.4 \mathrm{Ci}$ has been employed to account for the balance of the radionuclides.)

An examination of the data presented in Table 6 indicates that the actinides contained in the fuel fines dominated radiotoxicity considerations. In terms of $\mathrm{A}_{2}$, the actinides contributed to approximately $90 \%$ of potentially releasable material into the environment. The results constitute the fuel product source term for the NLI-1/2 cask, with a single rod failure assumed. If all rods in an assembly and cask are identical, then the source concentrations scale linearly with the number of rods that have cladding failure.

\subsection{Example Containment Criteria}

The 10 CFR 71 criteria for containment integrity limit leak rates to no greater than $10^{-6}$ A2 per hr during normal transport conditions and no greater than $A_{2}$ in 1 wk during hypothetical accident conditions [US90]. These release rates, $R_{\mathbf{i}}$, were converted into maximum allowable leak rates, $L_{i}$, through the relation

$$
\mathrm{L}_{\mathrm{i}}=\mathrm{R}_{\mathrm{i}} / \mathrm{C}_{\mathbf{i}}
$$

for normal $(i=N)$ or accident $(i=A)$ conditions. The variable $C_{i}$ represents the appropriate source concentration. 
Table 5

Radionuclide Releases from Selected LWR

Fuel Rods Due to Cladding Failure at $530^{\circ} \mathrm{C}$

\begin{tabular}{|c|c|c|c|c|}
\hline \multirow[b]{2}{*}{ Nuclide } & \multicolumn{2}{|c|}{ PWR Roda } & \multicolumn{2}{|c|}{ BWR Rod $b$} \\
\hline & $\mathrm{g}$ & $\mathrm{Ci}$ & $\mathrm{g}$ & $\mathrm{Ci}$ \\
\hline $3_{\mathrm{H}^{c}}$ & $5.26 \mathrm{E}-05$ & $5.10 \mathrm{E}-01$ & $8.57 \mathrm{E}-05$ & $8.31 \mathrm{E}-01$ \\
\hline${ }^{85} \mathrm{Kr}^{\mathrm{d}}$ & $3.91 \mathrm{E}-04$ & $1.57 \mathrm{E}-01$ & $9.54 \mathrm{E}-04$ & $3.82 E-01$ \\
\hline $90 \mathrm{Sr}$ & $3.10 \mathrm{E}-05$ & $4.65 \mathrm{E}-03$ & $3.55 E-05$ & $5.33 E-03$ \\
\hline $90 \mathrm{Y}$ & $1.86 \mathrm{E}-08$ & $4.66 \mathrm{E}-03$ & $2.13 E-08$ & $5.33 E-03$ \\
\hline${ }^{106} \mathrm{Ru}$ & $2.90 \mathrm{E}-07$ & $9.86 \mathrm{E}-04$ & $4.27 E-07$ & $1.45 \mathrm{E}-03$ \\
\hline${ }^{106} \mathrm{Rh}$ & $2.77 E-13$ & $9.86 \mathrm{E}-04$ & $4.08 E-13$ & $1.45 E-03$ \\
\hline $125 \mathrm{Sb}$ & $2.56 \mathrm{E}-07$ & $2.64 \mathrm{E}-04$ & $3.83 \mathrm{E}-07$ & $3.96 E-04$ \\
\hline $125 \mathrm{~m}_{\mathrm{Te}}$ & $3.58 \mathrm{E}-09$ & $6.44 \mathrm{E}-05$ & $5.36 \mathrm{E}-09$ & $9.66 \mathrm{E}-05$ \\
\hline${ }^{134} \mathrm{Cs}^{\mathrm{e}}$ & $2.82 \mathrm{E}-06$ & $3.39 E-03$ & $5.39 E-06$ & $6.46 E-03$ \\
\hline $137_{\mathrm{Cs}}^{\mathrm{e}}$ & $1.15 \mathrm{E}-04$ & $1.13 \mathrm{E}-02$ & $2.35 \mathrm{E}-04$ & 2. $31 E-02$ \\
\hline $137 \mathrm{~m}_{\mathrm{Ba}}$ & 1. $22 \mathrm{E}-11$ & $6.56 \mathrm{E}-03$ & $1.86 \mathrm{E}-11$ & $9.98 \mathrm{E}-03$ \\
\hline $144 \mathrm{Ce}$ & $1.80 \mathrm{E}-07$ & $5.76 \mathrm{E}-04$ & $1.67 \mathrm{E}-07$ & $5.33 E-04$ \\
\hline $144 \mathrm{Pr}$ & $7.63 \mathrm{E}-12$ & $5.76 \mathrm{E}-04$ & $7.05 \mathrm{E}-12$ & $5.33 E-04$ \\
\hline $144 \mathrm{~m} P r$ & $3.81 \mathrm{E}-14$ & $6.91 \mathrm{E}-06$ & $3.53 E-14$ & $6.40 \mathrm{E}-06$ \\
\hline $154 \mathrm{Eu}$ & $3.94 \mathrm{E}-06$ & $5.91 \mathrm{E}-04$ & $7.42 E-06$ & $1.11 \mathrm{E}-03$ \\
\hline $155 \mathrm{Eu}$ & $1.90 \mathrm{E}-07$ & $2.66 \mathrm{E}-04$ & $3.60 \mathrm{E}-07$ & $5.04 \mathrm{E}-04$ \\
\hline $235 \mathrm{U}$ & $4.16 \mathrm{E}-04$ & $8.73 E-10$ & $1.68 \mathrm{E}-04$ & $3.53 E-10$ \\
\hline $238 \mathrm{U}$ & $6.38 \mathrm{E}-02$ & $2.10 \mathrm{E}-08$ & $1.11 \mathrm{E}-01$ & $3.65 \mathrm{E}-08$ \\
\hline${ }^{238} \mathrm{Pu}$ & $1.49 \mathrm{E}-05$ & $2.54 \mathrm{E}-04$ & $2.67 \mathrm{E}-05$ & $4.55 E-04$ \\
\hline${ }^{239} \mathrm{Pu}$ & $3.41 \mathrm{E}-04$ & $2.11 \mathrm{E}-05$ & $5.84 E-04$ & $3.62 E-05$ \\
\hline $240 \mathrm{Pu}$ & $1.69 \mathrm{E}-04$ & $3.88 \mathrm{E}-05$ & $3.05 E-04$ & $7.00 \mathrm{E}-05$ \\
\hline${ }^{241} \mathrm{Pu}$ & $6.80 E-05$ & $7.48 \mathrm{E}-03$ & 1. $32 \mathrm{E}-04$ & $1.46 \mathrm{E}-02$ \\
\hline $241_{A m}$ & $2.54 \mathrm{E}-05$ & $8.14 \mathrm{E}-05$ & $5.43 E-05$ & $1.74 \mathrm{E}-04$ \\
\hline $244 \mathrm{Cm}$ & $2.53 \mathrm{E}-06$ & $2.08 \mathrm{E}-04$ & $1.26 \mathrm{E}-05$ & $1.04 \mathrm{E}-03$ \\
\hline Balance & $1.66 \mathrm{E}-03$ & $2.09 \mathrm{E}-03$ & $4.24 \mathrm{E}-03$ & $2.54 \mathrm{E}-03$ \\
\hline Totals: & $6.71 E-02$ & 7. $12 \mathrm{E}-01$ & $1.18 \mathrm{E}-01$ & 1. $29 \mathrm{E}+00$ \\
\hline \multicolumn{5}{|c|}{ 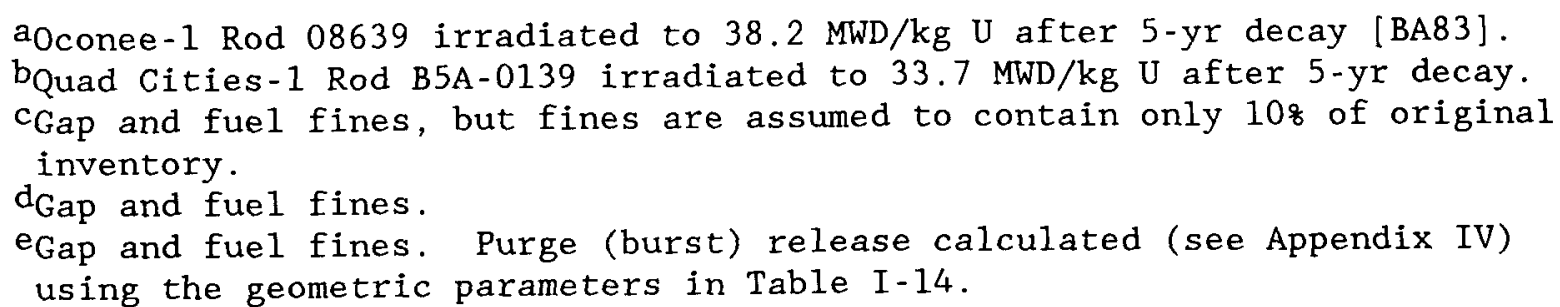 } \\
\hline
\end{tabular}


Table 6

Radionuclide Concentrations in an NLI-1/2 Transport Cask on Cladding Failure of a Single Fuel Rod

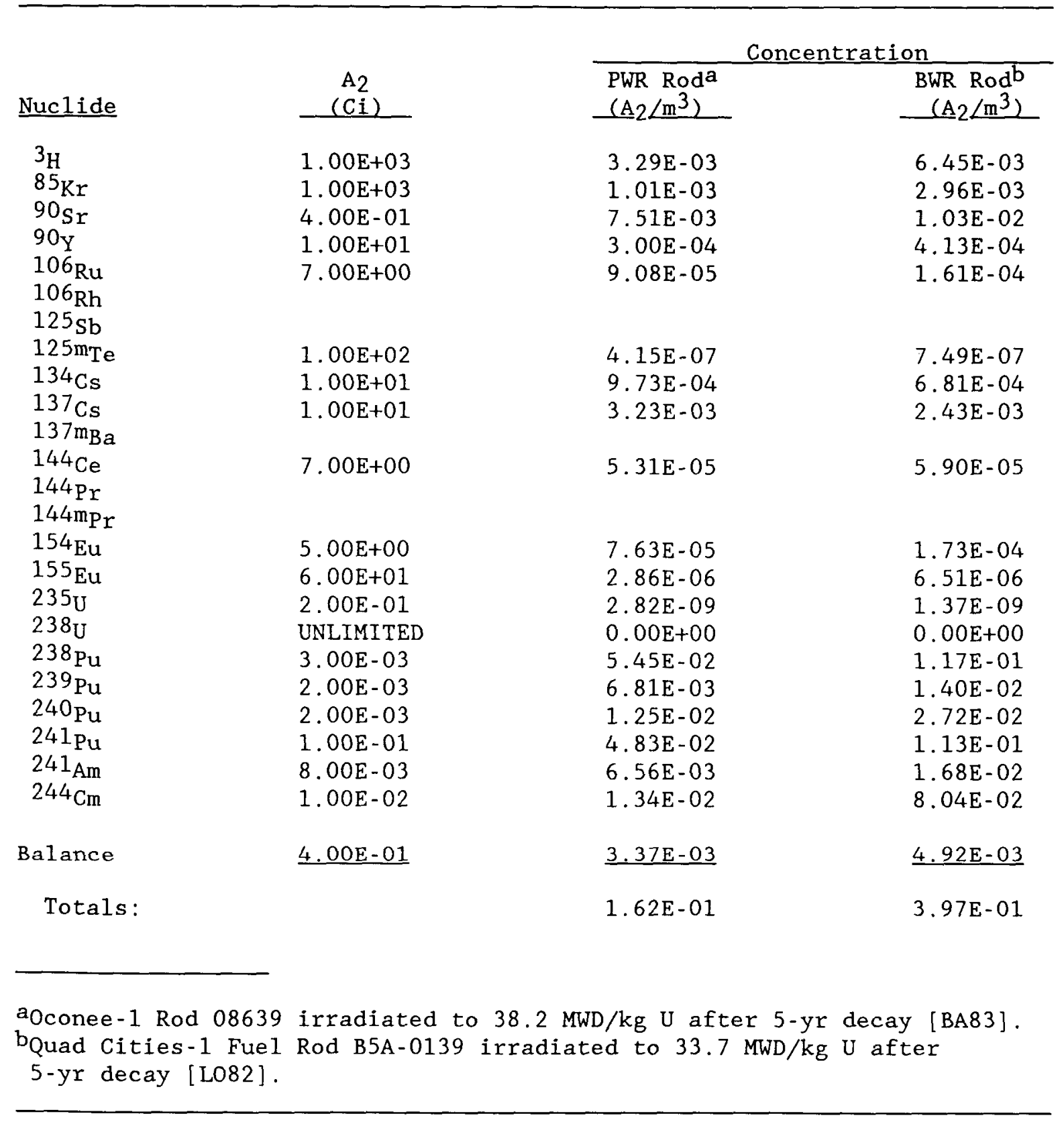


Containment criteria for the NLI-1/2 cask based on Equation (5-1) are presented in Table 7 in terms of leak rates.

Table 7

Containment Requirements for the NLI-1/2 Transport

Cask for Selected LWR Spent-Fuel Assemblies

with a Single Rod Failure

\begin{tabular}{|c|c|c|c|}
\hline $\begin{array}{l}\text { Transport } \\
\text { Condition }\end{array}$ & $\begin{array}{c}C_{\mathfrak{i}} \\
\left(\text { curie } / \mathrm{cm}^{3}\right) \\
\end{array}$ & $\begin{array}{c}\mathrm{R}_{\mathrm{i}} \\
\text { (curie/s) }\end{array}$ & $\begin{array}{r}\mathrm{L}_{i} \\
\left(\mathrm{~cm}^{3} / \mathrm{s}\right) \\
\end{array}$ \\
\hline $\begin{array}{l}\text { PWR Fuel } \\
\text { Normal } \\
\text { Accident }\end{array}$ & $\begin{array}{l}1.62 \times 10^{-7} \mathrm{~A}_{2} \\
1.62 \times 10^{-7} \mathrm{~A}_{2}\end{array}$ & $\begin{array}{l}2.78 \times 10^{-10} \mathrm{~A}_{2} \\
1.65 \times 10^{-6} \mathrm{~A}_{2}\end{array}$ & $\begin{array}{l}1.72 \times 10^{-3} \\
1.02 \times 10^{1}\end{array}$ \\
\hline $\begin{array}{l}\text { BWR Fuel } \\
\text { Normal } \\
\text { Accident }\end{array}$ & $\begin{array}{l}3.97 \times 10^{-7} \mathrm{~A}_{2} \\
3.97 \times 10^{-7} \mathrm{~A}_{2}\end{array}$ & $\begin{array}{l}2.78 \times 10^{-10} \mathrm{~A}_{2} \\
1.65 \times 10^{-6} \mathrm{~A}_{2}\end{array}$ & $\begin{array}{l}7.00 \times 10^{-4} \\
4.16\end{array}$ \\
\hline
\end{tabular}

During transport of a waterlogged rod in a spent-fuel assembly, a continuous but gentle purge of the gap region by water vapor occurs as the water evaporates and is driven from the rod. Because relatively small flow rates are involved, the purging action of the evaporating water does not result in fuel fine release but can cause additional releases of cesium. A bounding calculation was performed on the assumption that the entire rod is filled with water, and the cesium releases $\left(\right.$ at $100^{\circ} \mathrm{C}$ ) were estimated for the NLI - $1 / 2$ cask with the methodology described in Appendix IV. This mechanism resulted in additional cesium nuclide concentrations of $5.80 \mathrm{x}$ $10^{-14} \mathrm{~A} 2\left(\mathrm{Ci} / \mathrm{cm}^{3}\right)$ for a single rod in a PWR assembly and $2.66 \times 10^{-11} \mathrm{~A}_{2}$ $\left(\mathrm{Ci} / \mathrm{cm}^{3}\right)$ for a rod in a BWR assembly; both concentrations are negligible.

Appendix IV describes the effects of air ingress on radioactive material release during oxidation in high-temperature accident conditions. A convenient, rapid inflow cask leakage rate of $14 \mathrm{~cm}^{3} / \mathrm{s}$ was assumed. In Appendix IV, the accident is conservatively estimated to result in the oxidation of $0.582 \mathrm{~g}$ of fuel; in this section, this corresponds to 0.0268 of the fuel in a PWR rod and 0.0158 of the fuel in a BWR rod. If it is further conservatively assumed that the oxidized fuel behaves like fuel fines ejected when a fuel rod first undergoes cladding failure, then fuel oxidation would decrease the accident leakage criteria listed in Table 7 by approximately a factor of 10. However, the thermal conditions required for this amount of oxidation are in excess of those defined by the regulatory accident conditions. 


\subsection{References}

[AN82] American National Standards Institute, ANSI Standard N5.5-1982.

[BA83] Bain, G. M., and W. A. McInteer, "Data Package for High Burnup Fuel Samples," Babcock \& Wilcox, Lynchburg, VA, LRC4063, 1983.

[BU85] Burian, R. J., K. D. Kok, R. DiSalvo, M. E. Balmert, K. R. Freeman, and A. W. Pentiman, "Response of Spent LWR Fuel to Extreme Environments," Battelle Memorial Institute, Richland, WA, SAND857213, August 1985.

[C087] Collins, J. L., M. F. Osborne, and R. A. Lorenz, "Fission Product Tellurium Behavior Under Severe Light Water Reactor Accident Conditions, " Nuclear Technology, 77, p. 18 (1987).

[CR78] Croff, A. G., M. A. Bjerke, G. W. Morrison, and L. M. Patrie, "Revised Uranium-Plutonium Cycle PWR and BWR Models for the ORIGEN2 Computer Code," Oak Ridge National Laboratory, Oak Ridge, TN, ORNL/TM-6051, 1978.

[CR80] Croff, A. G., "ORIGEN2--A Revised and Updated Version of the Oak Ridge Isotope Generation and Depletion Code," Oak Ridge National Laboratory, Oak Ridge, TN, ORNL-5621, 1980.

[CR83] Croff, A. G., "ORIGEN2: A Versatile Computer Code for Calculating the Nuclide Compositions and Characteristics of Nuclear Materials," Nuclear Technology, 62, p. 335 (1983).

[EP83] Electric Power Research Institute, "FREY-01: Fue1 Rod Evaluation System," EPRI NP-3277-CCM, Palo Alto, CA, 1983.

[G080] Goode, J. H., R. G. Stacy, and V. C. A. Vaughen, "Comparison Studies of Head-End Reprocessing Using Three LWR Fuels," Oak Ridge National Laboratory, Oak Ridge, TN, ORNL/TM-7103, 1980.

[J085] Johnson, L. H., S. Stroes-Gascoyne, J. D. Chen, M. E. Attas, D. M. Sellinger, and H. G. Delaney, "The Relationship Between Fuel Element Power and the Leaching of $137 \mathrm{Cs}$ and $129 \mathrm{I}$ from Irradiated $\mathrm{UO}_{2}$ Fuel," in Proceedings: ANS Meeting on Fission Product Behavior and Source Term Research, Snowbird, UT, July 15-19, 1984, Electric Power Research Institute, EPRI NP-4113-SR, pp. 15-1 to 15-2, Ju1y 1985

[KR85] Kress, T. S., "Review of the Status of Validation of the Computer Codes Used in the Severe Accident Source Term Reassessment Study," Oak Ridge National Laboratory, Oak Ridge, TN, ORNL/TM-8842, BMI2104, April 1985.

[L079] Lorenz, R. A., J. L. Collins, and A. P. Malinauskas, "Fission Product Source Terms for the Light Water Reactor Loss-of-Coolant Accident," Nuclear Technology, 46, p. 404 (1979). 
[L080] Lorenz, R. A., J. L. Collins, and A. P. Malinauskas, "Fission Product Release from Highly Irradiated LWR Fuel," Oak Ridge National Laboratory, Oak Ridge, TN, NUREG/CR-0722, February 1980.

[L081] Lorenz, R. A., J. L. Collins, M. F. Osborne, R. L. Towns, and A. P. Malinauskas, "Fission Product Release from BWR Fuel Under LOCA Conditions," Oak Ridge National Laboratory, Oak Ridge, TN, NUREG/CR-1773, July 1981.

[L082] Lowry, L. M., and V. Pasupathi, "Postirradiation Examination of Fuel Rods from the Quad Cities-1 Reactor," Battelle Memorial Institute, Richland, WA, BCL-585-82-3, 1982.

[MA87] Malinauskas, A. P., and J. T. Be11, "The Chemistry of FissionProduct Iodine Under Nuclear Reactor Accident Conditions, " Nuclear Safety, 28, p. 505 (1987).

[R087] Roddy, J. W., and J. C. Mailen, "Radiological Characteristics of Light-Water Reactor Spent Fuel: A Literature Survey of Experimental Data," Oak Ridge National Laboratory, Oak Ridge, TN, ORNL/TM10105, December 1987.

[SA91] Sandoval, R. P., et a1., "Estimate of CRUD Contribution to Shipping Cask Containment Requirements," Sandia National Laboratories, Albuquerque, NM, SAND88-1358, January 1991.

[US90] "Packaging and Transportation of Radioactive Material," in U.S. Code of Federal Regulations, Title 10, Part 71, revised January 1, 1990. 


\subsection{SENSITIVITIES AND UNCERTAINTIES}

The dominant factors that can affect spent-fuel mechanical response, cladding failure frequencies, and radionuclide release estimates are related to the following sources: (1) cask and impact limiter design, (2) basket design, (3) assembly design, (4) fuel rod initial state, (5) in-reactor operational conditions, and (6) in-transport operational conditions. Sensitivities and uncertainties associated with each of these sources are discussed in this section.

\subsection{Cask and Limiter Design Parameters}

Figure 43 illustrates the basic geometric features of a typical spentfuel cask. The structural stiffness of a spent-fuel transport cask is larger by several orders of magnitude than the stiffness of a spent-fuel assembly. Therefore, cask shell stiffness is not a dominant sensitivity parameter. The cask impact limiter design does directly govern the magnitude and duration of loads transmitted to the assemblies. The total impulse momentum that is proportional to the area under the cask deceleration time history is independent of the limiter design for impact after a $9.0-\mathrm{m}$ free drop. However, the magnitude and duration of deceleration are functions of impact limiter stiffness and crush strength. Because the limiters are much softer than the cask, variations in limiter crush strength and crush area (footprint) directly affect the magnitude of deceleration transmitted to the assemblies.

The current method of modeling impact limiter response is based on the assumption that the impact limiter undergoes fully plastic crushing irrespective of drop height. For small drop heights such as $0.3-\mathrm{m}$ drop conditions, this may be overly conservative because the limiter may remain elastic. The analyses and results presented here are based on one value of crush strength (see Appendix II). Crush strength can be replaced by force deflection characteristics when these data are available.

Based on these cask and limiter design parameters, three sensitivity parameters were identified: limiter crush strength, limiter stiffness (force displacement relation), and footprint area (or deceleration history).

\subsection{Basket Design Parameters}

The basket design plays a major role in determining spent-fuel assembly response to impact events. The dominant parameters that significantly affect assembly response during impact conditions are the basket structural stiffness, extent of lateral constraints and gaps between the assembly and the basket frame, and gaps between the basket and cask (see Figure 43).

In the assembly response model used for this analysis (see Appendix III) it is assumed that the basket structural stiffness is very large relative to the structural stiffness of the assemblies. Furthermore, the 


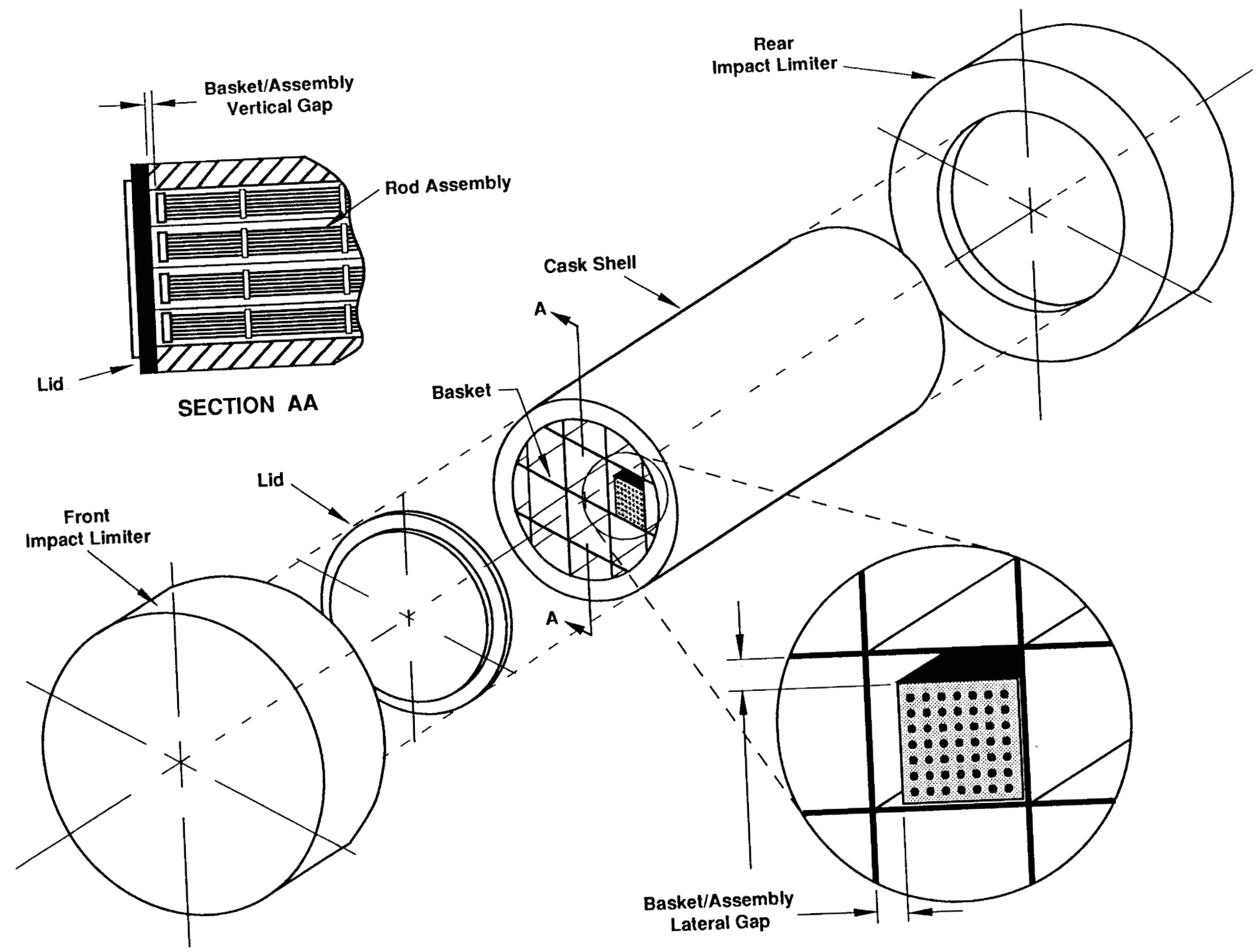

Figure 43. Typical Cask Design Components 
basket is assumed to retain its original shape so that each assembly response can be isolated from the rest of the assemblies in the cask. These assumptions imply that the basket frame supports each assembly independently. If the basket stiffness does not meet these requirements, assembly-to-assembly interaction could occur. For large rail casks with many assemblies, the probability of fuel rod failure may increase significantly if appreciable interassembly interaction occurs as a result of basket-frame collapse. For example, during side-drop loading, assembly-toassembly load transfer will greatly increase the total load on the bottom assemblies and thus increase the pinch loads on the lower fuel rods. As described in Appendix III, the pinch loads are responsible for cladding rupture emanating from a PCI-induced crack. Similarly, basket frame collapse could lead to larger deformations of top assemblies (and hence higher cladding strains) in the upper fuel rods.

Lateral assembly constraints provided by the basket limit the magnitude of the lateral displacements that can be sustained by fuel rods. Open-frame baskets similar to the IF- 300 basket shown in Figure 44 allow large lateral fuel rod displacements between basket supports, thereby increasing bending strains at the support points. Under end-drop loading, the buckling load of most fuel rods is less than the impact load in a $9.0-\mathrm{m}$ drop event. This lack of buckling strength causes large lateral deformations and, consequently, high tensile bending strains in the cladding. However, continuous baskets or baskets with more frequent restraints against lateral deflections restrict the fuel rod lateral deformations and thus result in lower failure probabilities, provided that the basket structure is designed to resist plastic deformations and collapse.

Both vertical and lateral basket-cask gaps and basket-assembly gaps are important sensitivity parameters for spent-fuel response analysis. Vertical gaps can potentially increase assembly response as a result of secondary impact between the assembly and the cask bottom. Some limited sensitivity analyses conducted for the example cases described in Appendix III show that the maximum cladding tensile strain (one of the critical parameters in determining fuel rod failure) increases from 1.2 to 2.48 when the vertical gap is increased from zero to $8 \mathrm{~cm}$.

Lateral gaps between the fuel assembly and basket can also have a major effect on assembly response. As mentioned above, during end-drop loading, large lateral deflections of the fuel rods can occur. These lateral deflections, however, are bounded and limited in magnitude by an amount equivalent to the gaps between the assembly and the basket, and by the spacing between individual fuel rods. Figure 45 illustrates typical lateral gaps between basket cells and assemblies. The example analyses of typical BWR and PWR assemblies described in Appendix III show variations of peak tensile strain with lateral deformations of the fuel rods.

Thus, the dominant sensitivity parameters in this category consist of basket-frame structural stiffness, open-frame versus continuous basket design, and the extent of lateral and vertical gaps between assemblies, basket structure, and cask shell. 


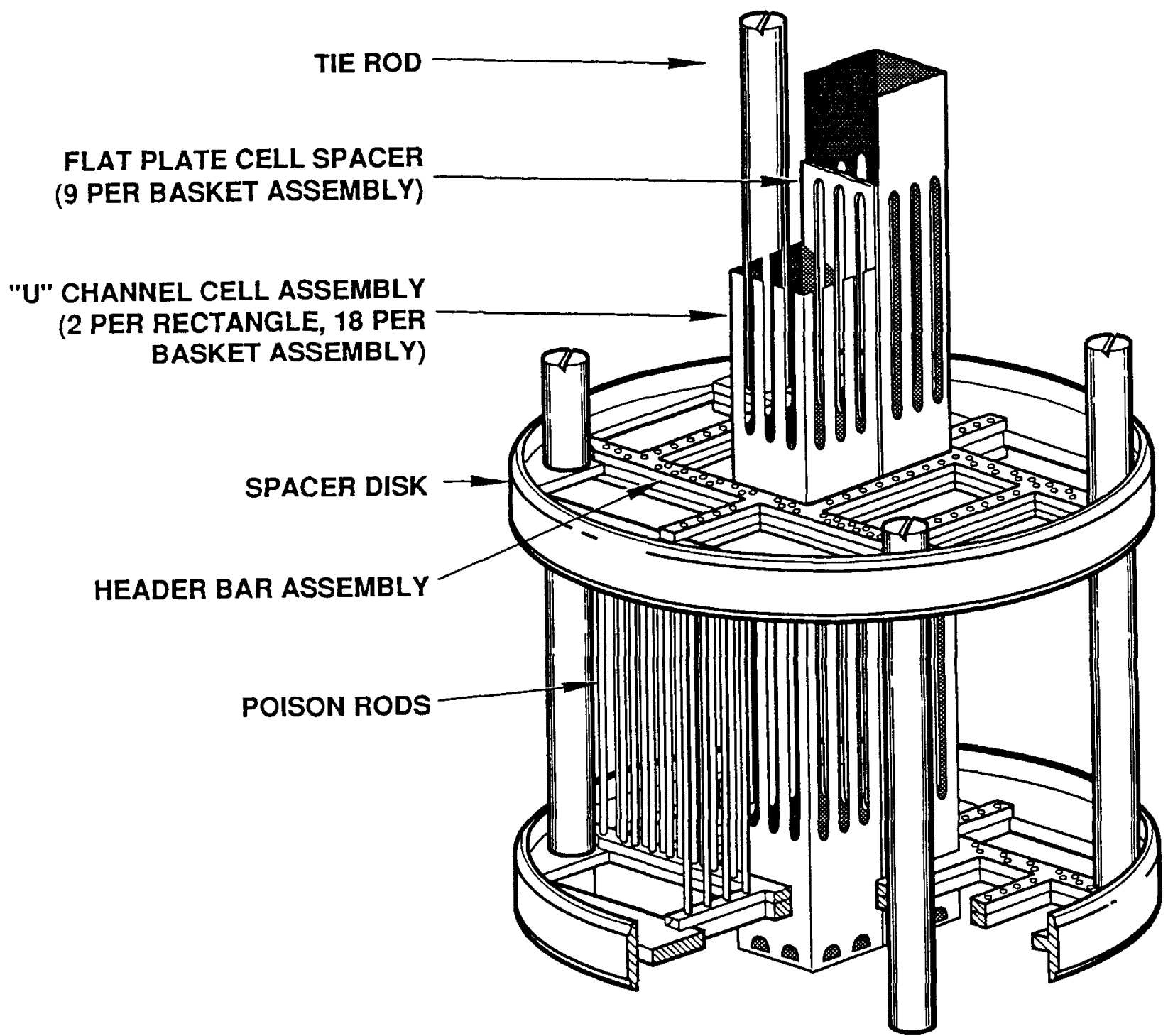

Figure 44. IF-300 BWR Fuel Basket Detail

\subsection{Assemb1y Design Parameters}

The most important assembly design parameters that influence fuel rod mechanical response are effectiveness of and distance between spacer grids, rod cladding diameter and thickness, and the number and spacing of rods in the assembly.

In addition to keeping the fuel rods in their proper positions, the spacer grids provide lateral constraints for the rods and flexural rigidity to the assembly. The longitudinal spacing of the spacer grids affects assembly response in any orientation. For end-drop loadings, the critical buckling load of the rods is a function of the square of the spacer grid spacing. The effect of damaged or missing grids is equivalent to increasing the spacing. For side drops, the bending strength of the rod is 


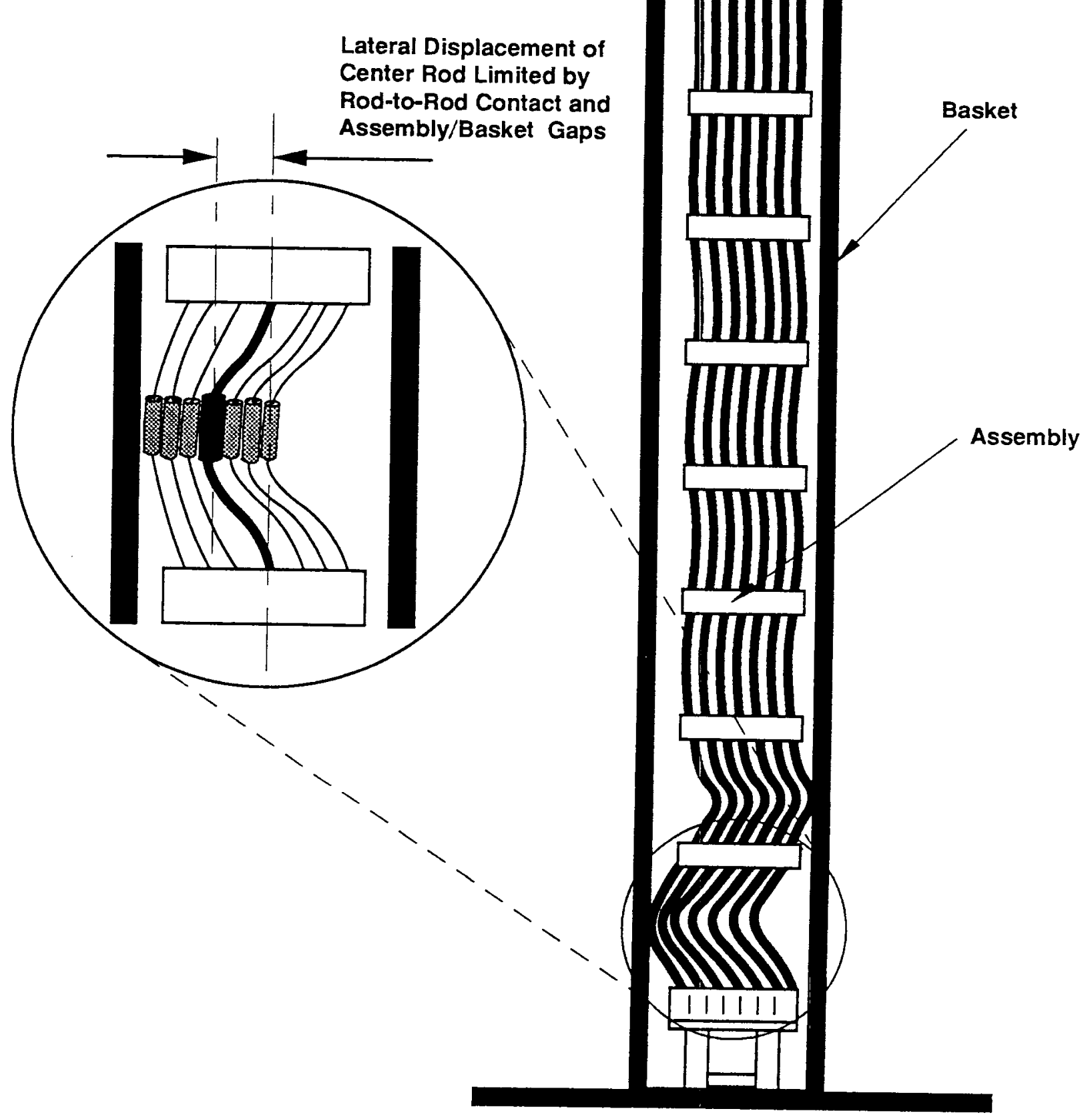

Figure 45. Lateral Displacement of Assembly Limited by Rod-to-Rod Contact and Basket-Assembly Gaps 
related to the square of a span of the rod between supports. Corner drops are affected in a similar manner because the corner-drop event is dominated by either an end-drop or a side-drop response.

Another critical dimension that affects fuel rod response is the rod diameter. Bending strains induced in rod cladding are a function of the square of the cladding outer diameter.

Other assembly design parameters that affect fuel rod mechanical response to a lesser degree include cladding thickness, number of rods in a row, and spacing between individual rods in the assembly. The influence of the latter parameter has already been discussed (see Section 6.2) in connection with the basket-assembly gaps. The number of rods per row affects rod-to-rod interaction. cladding thickness affects the moment of inertia, therefore affecting the bending stiffness of the cladding. This parameter, however, can be combined with the rod diameter as a diameter-tothickness ratio that governs both longitudinal and cross-sectional bending stiffness of the cladding.

Thus, within each of the BWR and PWR fuel types, the dominant sensitivity parameters in this category are the fuel rod outer diameter, the span between spacer grids, cladding thickness, the number of rods in the assembly, and rod-to-rod spacing.

\subsection{Initial State Parameters of Fuel Rods}

The dominant initial condition parameters affecting fuel rod response are relative frequency and size of the PCI-induced cracks, effective mass of the fuel pellets that adheres to the cladding, initial bowing of the rods, and, if applicable, the number and location of rods missing from the assembly.

Size and distribution of partial cracks in the fuel rod cladding play a major role in determining the probability of rod failure. One of the most probable types of cladding failure predicted is the extension of existing PCI-induced partial cracks (see Appendix III for details). The probabilistic cladding failure evaluation is based on a distribution for penetration depth of existing partial cracks in the fuel cladding.

Figure 46 illustrates the effect of changes in the number of cladding partial cracks on the 9 -m side-drop rod failure probability. As the number of cracks increases, the failure probability increases at a decreasing rate and becomes nearly constant at a large number of cracks. Best estimates for the number of cracks for PWR and BWR fuels used in the example analyses (described in Section 4.0) are based on the assumption that the probable number of PCI-induced cracks that exist in a fuel rod is roughly equal to the number of pellet interfaces that are strain and fission product concentration sites. Thus, these locations are more susceptible to stress corrosion cracking from the PCI mechanism (see Appendix I for details).

Figures 47 and 48 illustrate the effect of changes in the part-wall crack distribution on the $9-\mathrm{m}$ side-drop rod failure probability. Figure 47 shows the effects of varying the exponential part-wall crack distribution. 

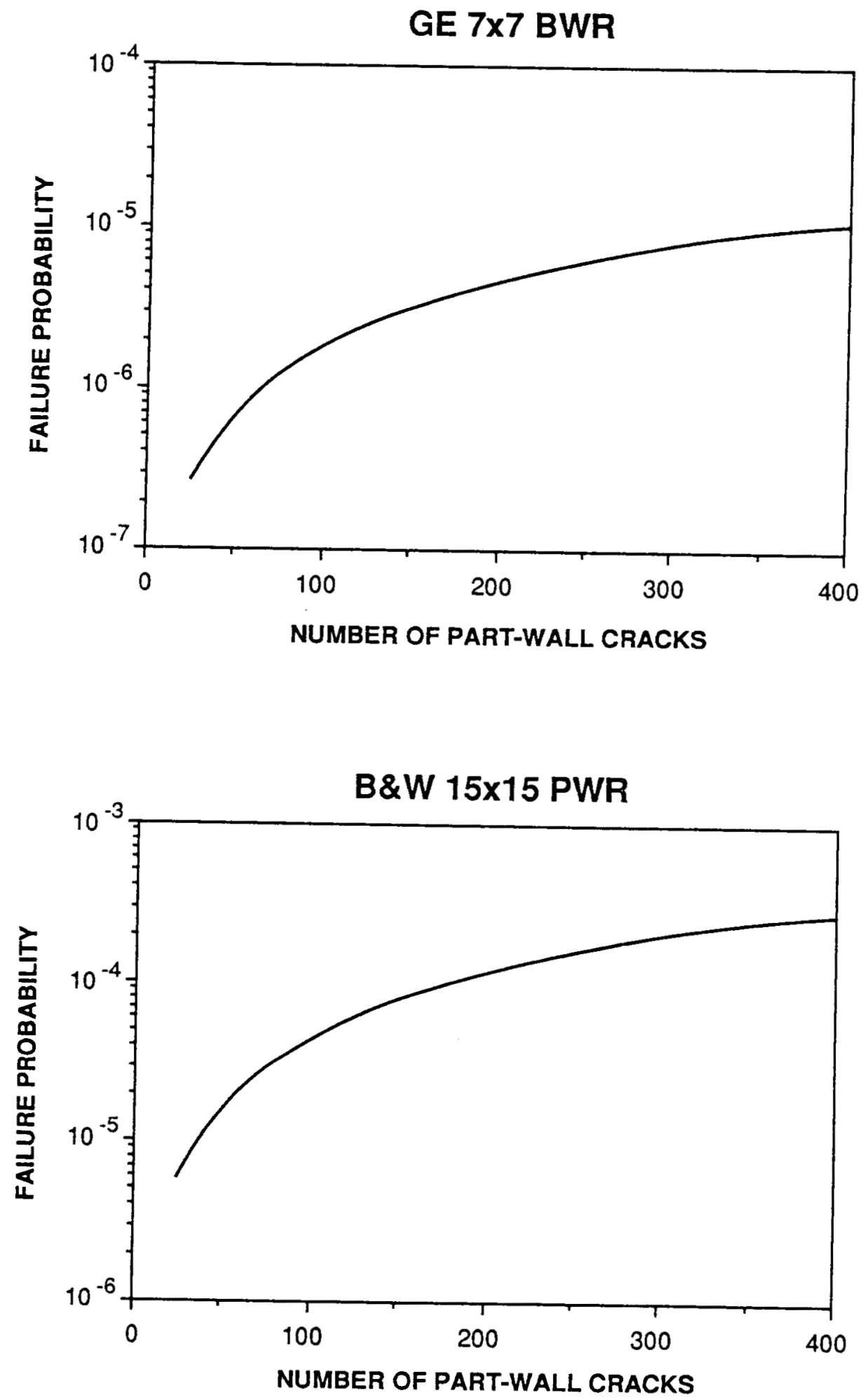

Figure 46. Material Fracture Failure Probability Versus the Number of Part-Wall Cracks for Example BWR and PWR Assemblies 
EXPONENTIAL CRACK DISTRIBUTIONS

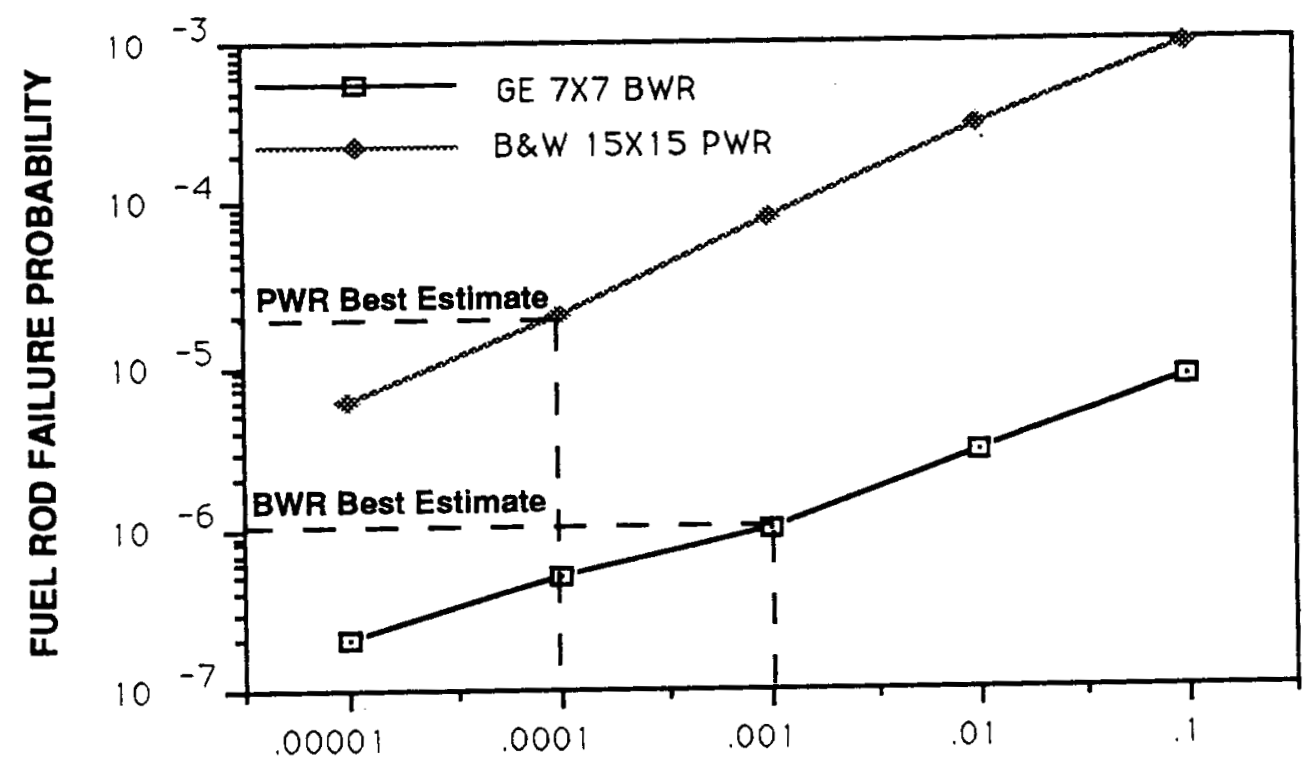

FREQUENCY OF CRITICAL CRACK SIZE

Figure 47. Exponential Distribution Material Fracture Failure Probability Versus the Frequency of Critical Crack Size for Example BWR and PWR Assemblies

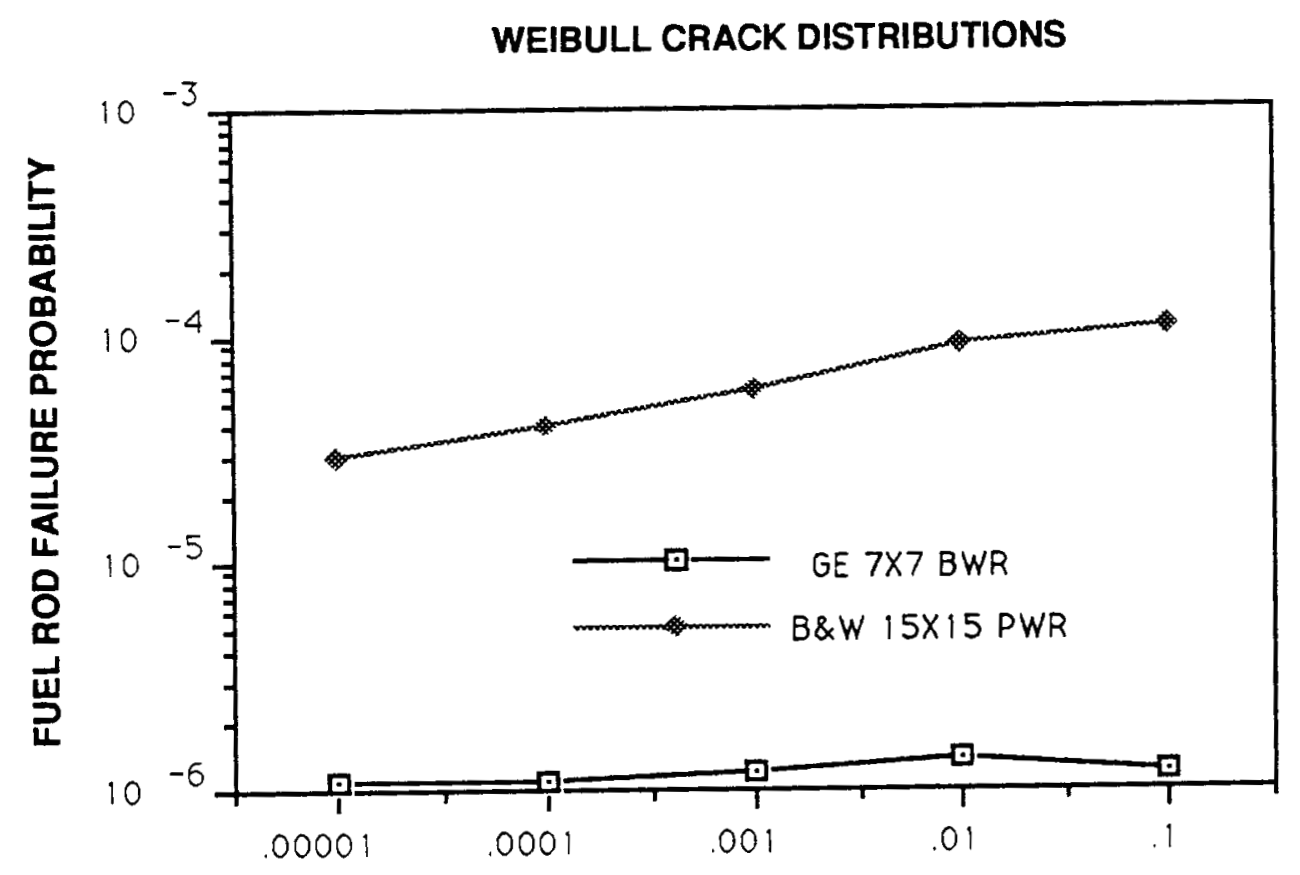

FREQUENCY OF CRITICAL CRACK SIZE

Figure 48. Weibull Distribution Material Fracture Failure Probability Versus the Frequency of Critical Crack Size for Example BWR and PWR Assemblies 
Different distributions were evaluated by increasing or decreasing the probability of occurrence of the critical crack depth. Best-estimate values of 1 in 1000 and 1 in 10,000 for BWR and PWR fuel, respectively, were obtained from fuel performance literature on the probability of rod failure within the reactor (see Appendix I for details). Because the exponential distribution was tied to this single data point, variations in the critical crack frequency caused shifts in the distribution. These curves indicate that extreme variations in the frequency of critical cracks result in a one-to two-order-of-magnitude change in the probability of fuel rod failure.

Figure 48 illustrates the effects of varying crack depth using a Weibull distribution. The Weibull distribution requires two parameters to evaluate the crack depth distribution: the critical crack size frequency and an assumption that 508 of the depth of the crack exceeds 108 of the wall thickness. Different distributions were evaluated by increasing or decreasing the probability of occurrence of the critical crack depth as performed in the exponential distributions described above. Evaluation of the resulting distributions shows that the failure probability is insensitive to the critical crack size frequency, although the assumption that 508 of the flaws were greater than 108 of the wall thickness has no experimental basis. Both figures indicate that for reasonable variations in crack size distributions, the fuel rod failure probability varies by approximately one order of magnitude. This translates to no greater than one PCI-related rod failure in a rail cask per regulatory accident.

The fuel rod response model developed for these analyses did not assess tensile and bending stiffness of the fuel pellets under all loading orientations. The amount of mass of the pellets included in the analysis model was based on loading orientation. For side-drop and lateral load components of corner-drop events, 100 of the fuel mass is considered active. However, for the end-drop event, only a portion of the fuel mass was included in the fuel response model. The other portion of the fuel mass was calculated not to adhere to the cladding and therefore is selfsupporting, that is, passive. The probability of fuel rod failure is sensitive to the percentage of fuel mass attached to the cladding.

For PWR fuel, the range of active mass was from 75 to 1008 , compared to approximately 108 for BWR fuel. The effects of in-reactor creep-down, ridging, and fuel-clad adhesion determined the amount of fuel mass that contributed to the loading on cladding under end-drop conditions. In PWR rods, these effects were prominent and a majority of the fuel pellets did adhere to the cladding. However, in BWRs where the cladding is thicker and reactor system pressure is approximately half of the reactor system pressure of PWRs, the cladding remains freestanding and thus its adhesion is greatly reduced.

A limited sensitivity analysis of fuel pellet mass effects is presented in Appendix III. The single rod-buckling analysis indicates that variations in effective fuel mass can cause large variations in the fuel rod-buckling load. For the BWR example assembly, the buckling load varied from $220 \mathrm{~g}$ (220 times gravity) for 108 effective fuel mass to $54 \mathrm{~g}$ for 1008 
effective fuel mass. For the PWR example assembly, the load varied from 40 to $32 \mathrm{~g}$ for 75 to $100 \%$ effective fuel mass.

The effect of initial pretransport bowing of the fuel rods on the fuel rod mechanical response was strongest under end-drop conditions because initial bowing promotes buckling. The fuel rod response model used in the example analyses conservatively assumes that the initial shape of the rod corresponds to the lowest buckling mode (see Figure 49), with a maximum amplitude equal to the design clearance between adjacent rods. This is a conservative value relative to experimental bowing measurements (see Appendix III). The maximum measured bowings of free-hanging fuel rods were 1.3 and $0.89 \mathrm{~cm}$ for PWR and BWR rods, respectively. The rods were hung vertically with unsupported lengths of approximately $4.1 \mathrm{~m}$. However, maximum unsupported rod lengths were typically approximately $51 \mathrm{~cm}$ in most assemblies. By linear interpolation, maximum expected bowings would have been approximately 0.18 and $0.10 \mathrm{~cm}$ for PWR and BWR rods. These values are smaller than the design clearances between rods $(0.351$ and $0.445 \mathrm{~cm}$ for the PWR and BWR examples in Appendix III) that were used in this analysis as the basis for defining the initially bowed geometry.

The effect of missing rods such as the corner rods in BWR $11 \times 11$ assemblies or the removal of damaged rods is equivalent to having larger lateral gaps (see Section 6.2).

\subsection{In-Reactor Operational Parameters}

In-reactor operational parameters that affect spent-fuel mechanical response and radionuclide releases if cladding ruptures are interdependent include power history, burnup, imposed fast neutron fluence, and reactor water chemistry. The imposed fast neutron fluence primarily affected the following cladding mechanical and failure properties: the elastic modulus, Poisson's ratio, yield strength, ultimate tensile strength, ductility, and fracture toughness. The deleterious effects of some of these properties, however, saturated at relatively low fluences--on the order of 1 x $10^{21}$ $\mathrm{n} / \mathrm{cm}^{2}(>1 \mathrm{MeV})$. Others such as fracture toughness continued to degrade with fast fluence in a linear dependence up to the limit of available experimental data, which was in the range of $7 \times 10^{21} \mathrm{n} / \mathrm{cm}^{2}(>1 \mathrm{MeV})$. This value is well below the design lifetime fluence for current LWR fuel designs. However, a linear extrapolation to higher fluence may not have been conservative, especially in the extended burnup range [ 9 to $10 \times 10^{21}$ $\left.\mathrm{n} / \mathrm{cm}^{2}(>1 \mathrm{MeV})\right]$. High burnup fuel was identified as a case for sensitivity analysis. Figure 50 shows fracture toughness probability distributions for various values of temperature and fast fluence.

Reactor water system chemistry affected the form of uniform and nodular cladding corrosion and CRUD buildup. Some BWR fuel rods were affected by CRUD-induced localized corrosion and deep craters (up to 758 of cladding thickness in some cases). Similarly, some PWR fuel rods were affected by debris erosion in the lower few inches of the fuel rods. The conditions were identified for sensitivity analysis of cladding thickness reduction and material ductility reductions as a result of local oxide buildup. 


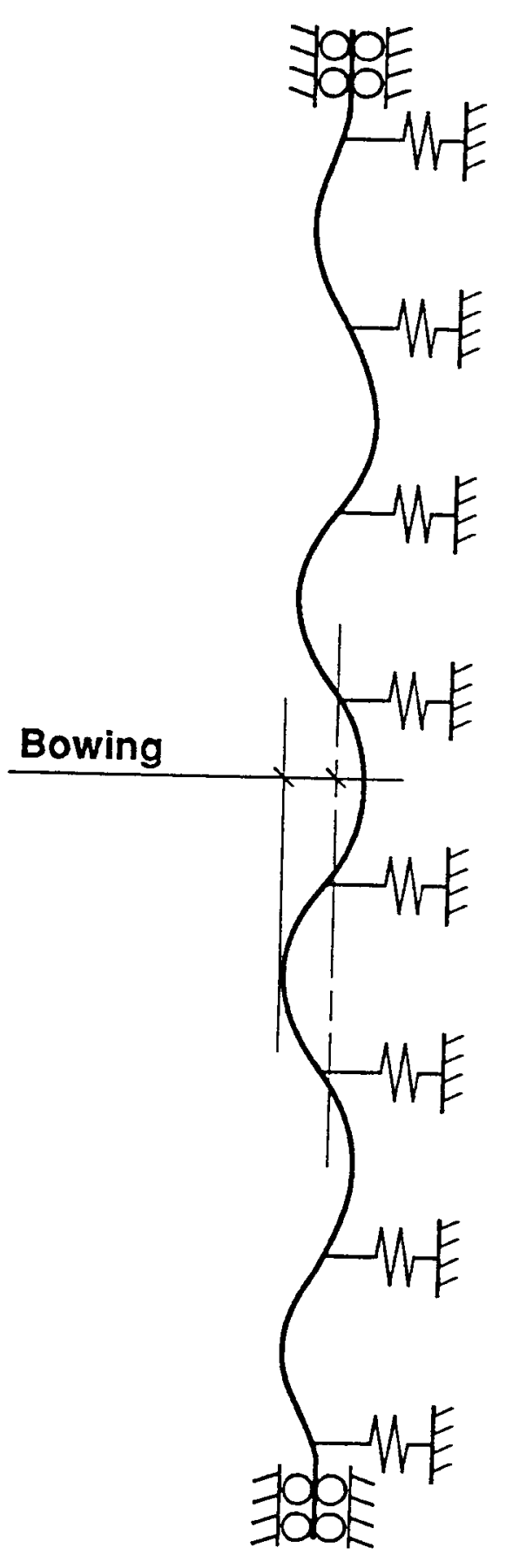

Figure 49. Lowest Buckling Mode Shape of a Fuel Rod 

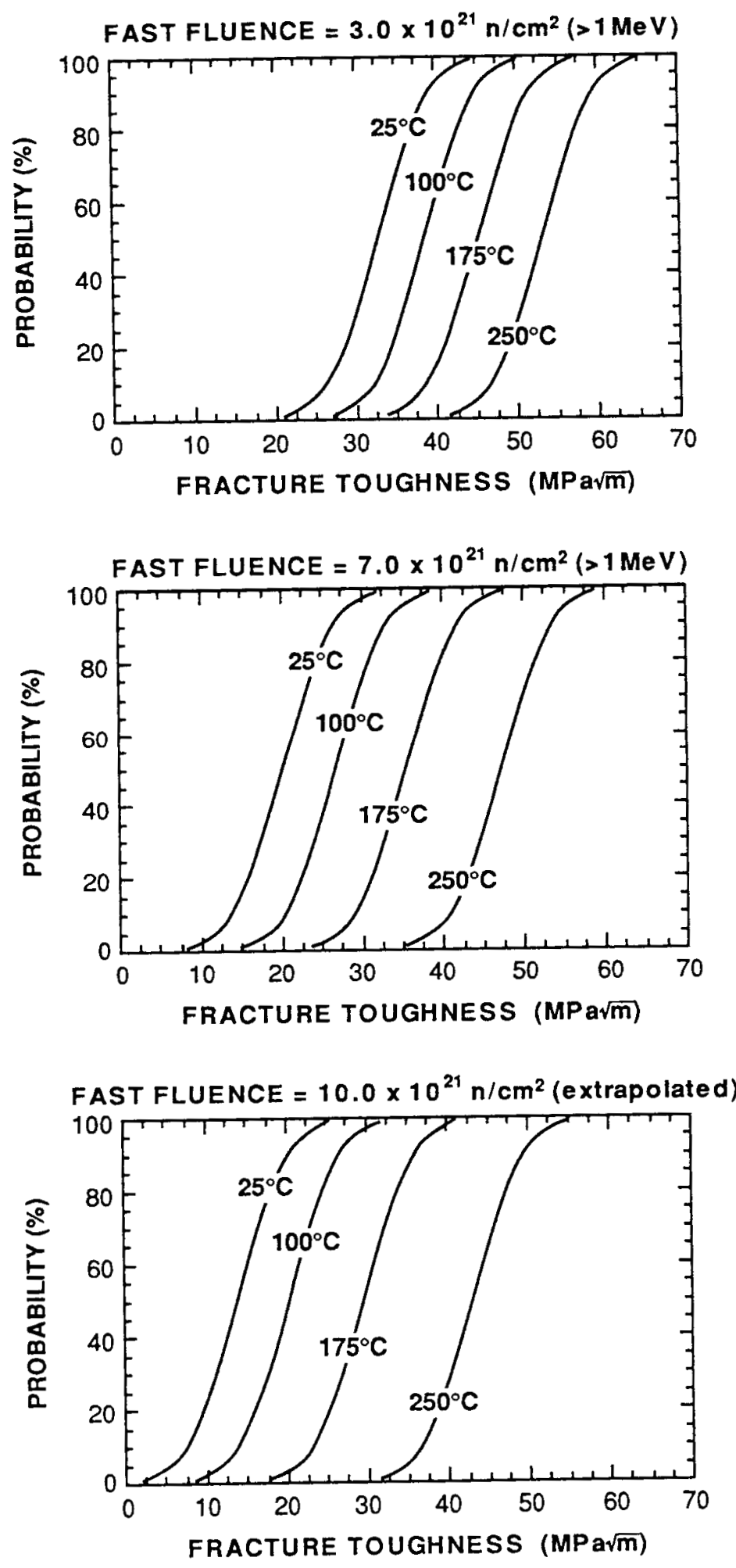

Figure 50. Cumulative Probability Distributions for Cladding Fracture Toughness at Various Temperatures and Fast Fluences 
Burnup power history also governs both fission and activation product inventory and in-core fission gas release to the fuel-cladding gap. The extent of fission gas release is a dominant sensitive parameter because of interdependence of diffusion and mobility characteristics of volatile species and noble gases. Sensitivity analyses provide a means of quantifying fission gas release with variations in average power history, axial power profiles, and burnup limits. Extended burnup influences fission gas release and product generation and, possibly, the extent of cladding hydriding and its effect on cladding material properties and failure criteria. Burnup also affects the quantity of fuel fines.

\subsection{In-Transport Operational Parameters}

Dominant in-transport operational parameters include the fuel temperature, possibility of excessive cask-handling events before transport, and consideration of a regulatory fire following a regulatory impact event.

Fuel temperature affects fracture toughness of the cladding and solubility of hydrides in Zircaloy material. The failure criterion used for flawed cladding, called fracture toughness, degrades significantly at low temperatures. Reprecipitation of hydrides in the form of platelets at lower temperatures further degrades fracture toughness. Therefore, the consequences of transporting old fuel, particularly in very cold weather, were identified for sensitivity analysis.

For the example analyses, the regulatory events were treated discretely. Thus, the effect of treating normal conditions as preceding accident conditions (impact followed by an engulfing fire) was not considered. This sequencing of events was identified for sensitivity analysis.

\subsection{Summary of Sensitive Parameters and Sources of Uncertainties}

A preliminary sensitivity evaluation into the effects of selective parameters on the probability of longitudinal slit fuel rod failure is illustrated in Figures 51 and 52 for the example assembly analyses described in Section 4.0. Each comparison was generated by varying a single parameter from the base case analysis described in detail in Appendix III. These figures indicate that fuel rod transport temperature appears to be the most influential sensitivity parameter, followed by fluence level and critical crack depth. The frequency, distribution, and number of part-wall cracks are all items that have less influence on the probability of fuel rod failure.

Table 8 provides a summary of dominant parameters discussed in this section and their effects. 


\section{GE 7X7 BWR Sensitivity Parameters}

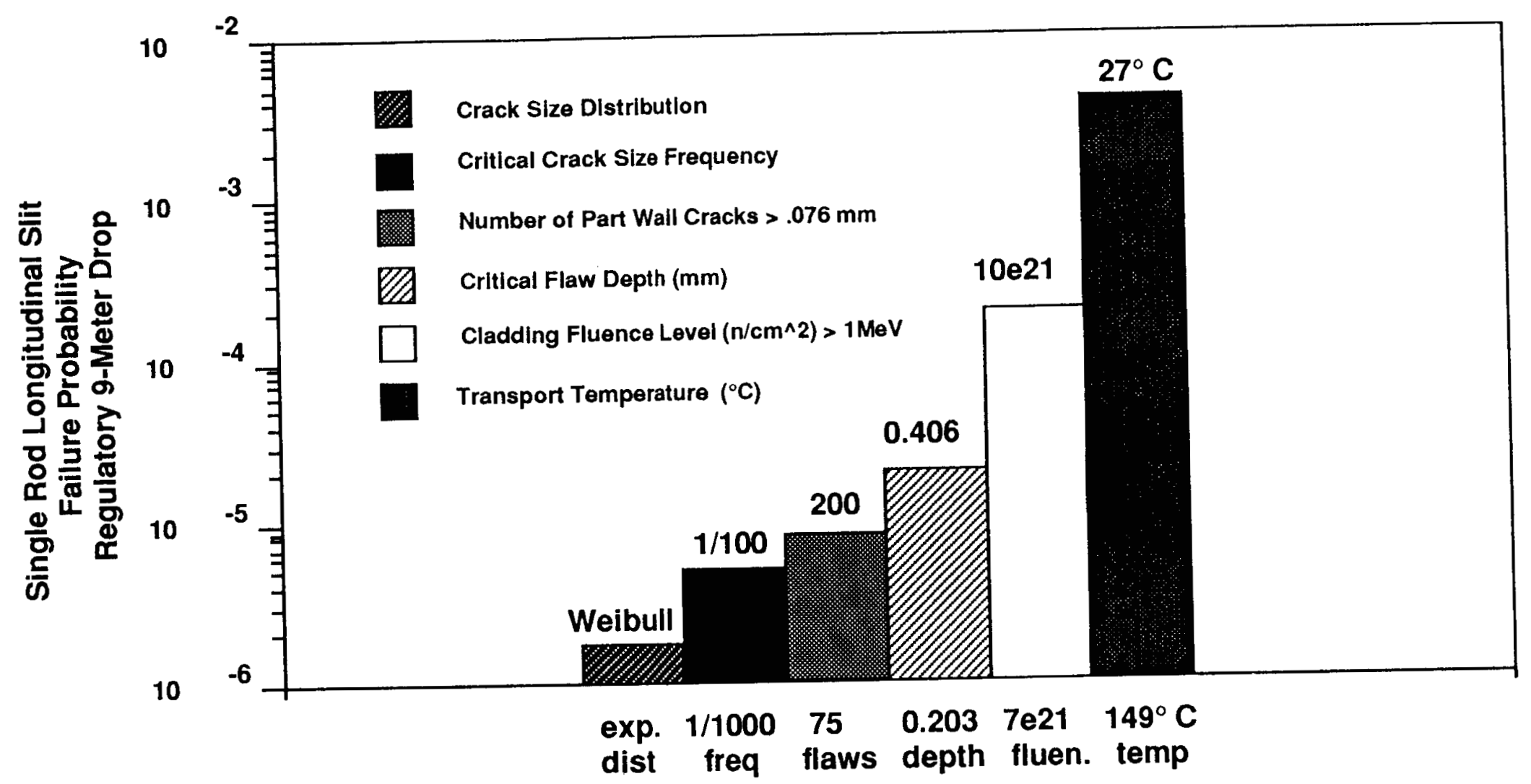




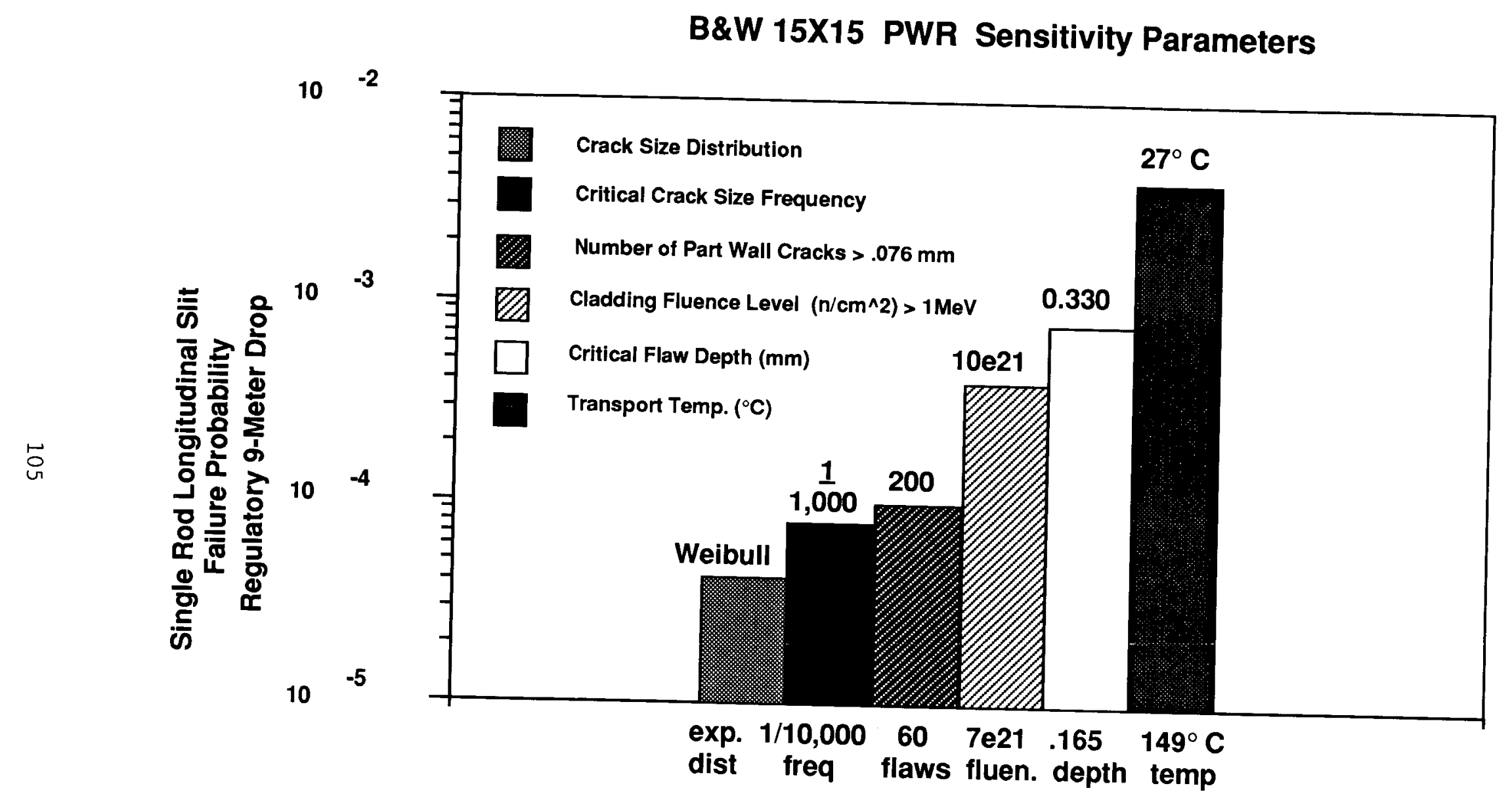

Figure 52. Example PWR Assembly and the Sensitivity of Material Fracture Failure Probability 
Table 8

Summary of Sensitivity Parameters

\begin{tabular}{|c|c|c|c|}
\hline \multicolumn{2}{|r|}{ Source } & $\begin{array}{l}\text { Sensitivity } \\
\text { Parameters }\end{array}$ & $\begin{array}{l}\text { Cladding Response } \\
\text { Variable(s) Affected }\end{array}$ \\
\hline \multirow[t]{3}{*}{1.} & $\begin{array}{l}\text { Cask and limiter } \\
\text { design }\end{array}$ & $\begin{array}{l}\text { Impact limiter } \\
\text { crush strength }\end{array}$ & $\begin{array}{l}\text { Amplitude and duration } \\
\text { of deceleration }\end{array}$ \\
\hline & & $\begin{array}{l}\text { Impact limiter } \\
\text { crush area }\end{array}$ & $\begin{array}{l}\text { Amplitude and duration } \\
\text { of decelaration }\end{array}$ \\
\hline & & $\begin{array}{l}\text { Impact limiter } \\
\text { elastic stiffness }\end{array}$ & $\begin{array}{l}\text { Amplitude and duration of } \\
\text { deceleration for small } \\
\text { drop heights (e.g., } 0.3-\mathrm{m} \\
\text { drop) }\end{array}$ \\
\hline \multirow{4}{*}{\multicolumn{2}{|c|}{ 2. Basket design }} & $\begin{array}{l}\text { Stiffness and } \\
\text { collapse strength }\end{array}$ & $\begin{array}{l}\text { Multi-assembly interac- } \\
\text { tion causing higher } \\
\text { strains, pinch loads } \\
\text { corresponding to stress } \\
\text { intensity factors }\end{array}$ \\
\hline & & $\begin{array}{l}\text { Open frame versus } \\
\text { continuous } \\
\text { support }\end{array}$ & $\begin{array}{l}\text { Tensile strains at } \\
\text { basket support points } \\
\text { and end-plate supports }\end{array}$ \\
\hline & & $\begin{array}{l}\text { Lateral gaps/ } \\
\text { constraints }\end{array}$ & $\begin{array}{l}\text { Tensile strains at } \\
\text { spacer grids, basket, } \\
\text { and end plate }\end{array}$ \\
\hline & & Vertical gaps & $\begin{array}{l}\text { Secondary impact leading } \\
\text { to larger amplitude } \\
\text { deceleration, causing } \\
\text { larger postbuckling } \\
\text { tensile strains }\end{array}$ \\
\hline \multirow{3}{*}{\multicolumn{2}{|c|}{ 3. Assembly design }} & Spacer grid span & $\begin{array}{l}\text { Fuel rod buckling, } \\
\text { tensile strains }\end{array}$ \\
\hline & & $\begin{array}{l}\text { Fuel rod cross- } \\
\text { sectional dimension } \\
\text { (diameter and } \\
\text { thickness) }\end{array}$ & $\begin{array}{l}\text { Tensile bending strains, } \\
\text { pinch-load induced stress } \\
\text { intensity factors }\end{array}$ \\
\hline & & $\begin{array}{l}\text { Number of rods per } \\
\text { row }\end{array}$ & $\begin{array}{l}\text { Rod-to-rod load } \\
\text { transfer (pinch loads) }\end{array}$ \\
\hline
\end{tabular}


Table 8

Summary of Sensitivity Parameters

(Concluded)

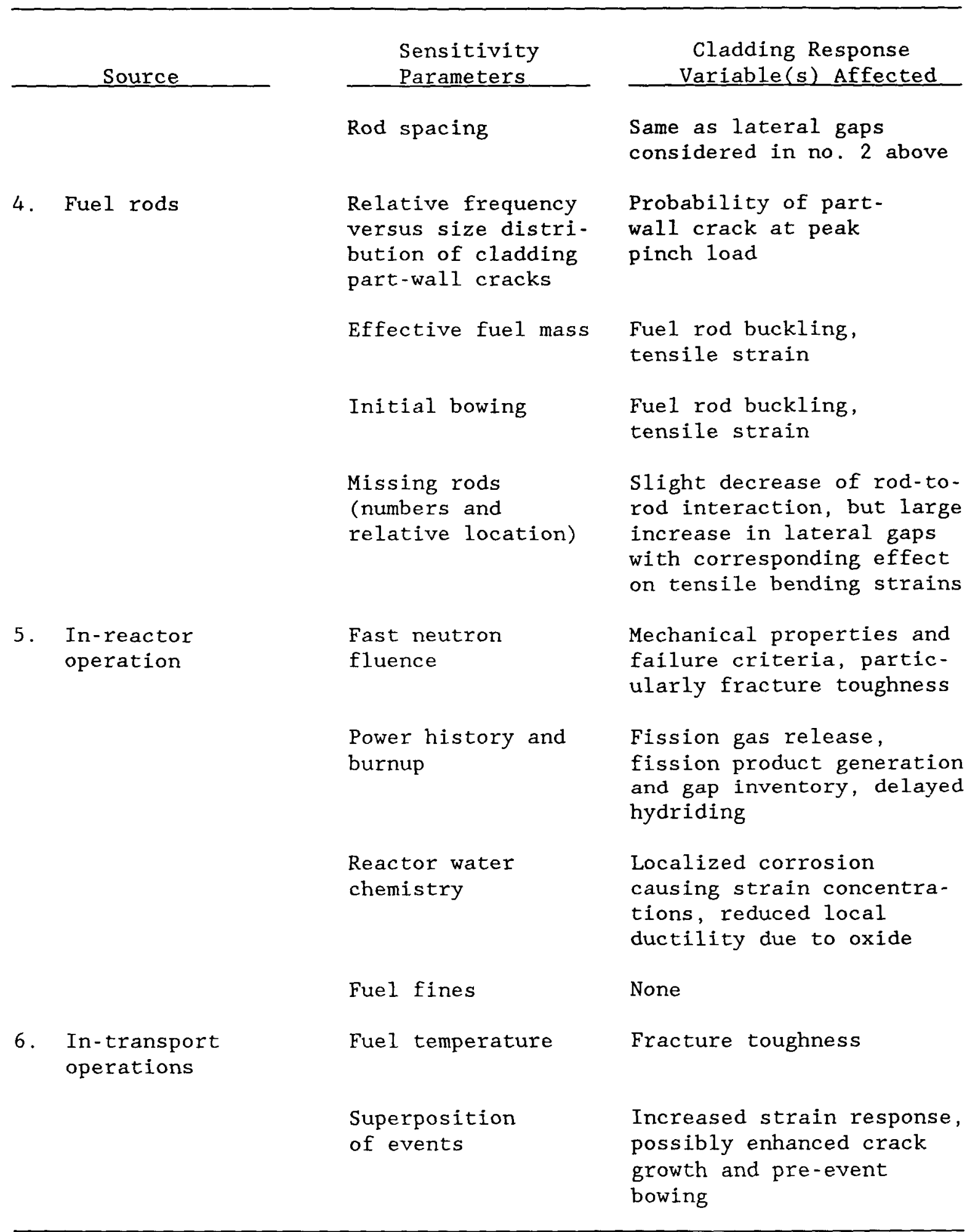


This page is intentionally left blank. 


\subsection{CONCLUSIONS AND RECOMMENDATIONS}

The source-term methodology described in this report was applied to two example cases: a $7 \times 7$ BWR assembly and a $15 \times 15$ PWR assembly. Thus, the following conclusions are based primarily on the results of these example analyses. However, the methodology was sufficiently developed so that the present general conclusions form a firm basis for making appropriate recommendations.

\subsection{Conclusions}

1. The phenomena involved in determining the fuel-related source term in spent-fuel transport casks can be modeled fairly accurately on the basis of existing analytical capabilities, material properties data, and operational history information. However, some important data are sparse or lacking. Therefore, major compensating assumptions were made that affected the results in crucial ways. These assumptions are associated with numerous sensitive parameters and data uncertainties.

2. For the example cases, the failure frequency is less than one rod per rail cask accident event. An assumption of massive fuel rod failure for the containment design of spent-fuel transport casks is unrealistically very conservative.

3. PCI incipient breaches (partial cracks) emerged as the most prominent initial fuel condition that affects the in-transport failure probability for both BWR and PWR fuels.

4. PWR spent fuel is more vulnerable to failure during transport than BWR fuel under the conditions of these analyses. The smaller PWR fuel rod diameters are mostly responsible for this condition. Rod failure as a result of initial PCI crack growth is more probable for PWR fuel than BWR fuel, although BWR fuel is more vulnerable to PCI failures while it is in the reactor.

5. The fuel fines, rather than the gaseous or volatile species, dominate the potentially releasable source term. However, the methodology for estimating the radionuclides contained in the fuel fines that could be purged with the gases during a cladding breach is based on a very limited amount of data. These data indicate that 0.0038 of the fuel contained in an intact fuel rod is released as fuel fines following a rupture.

6. The effect of a regulatory fire following the impact test sequence will be investigated in future sensitivity analyses.

7. Oxidation and leaching radionuclide release mechanisms are not plausible events during regulatory accident conditions. 
8. The fuel rod response, and consequently the failure probability, is affected by two types of uncertainties. The first is related to input parameters and can be addressed through sensitivity analyses and data development. The second, however, is related to the analytical models and was not easily evaluated. For example, a lumped parameter (spring and mass) model, which is the predominant working model for current licensing submittals, involves much greater uncertainty than a continuum finiteelement-based model. The nature of the fuel rod response is such that these lumped parameter-type models can be totally inadequate. The approach discussed here employed the continuum finite-element-based model throughout.

9. Obvious gaps exist in databases required to support this methodology at both input level and verification level. These gaps could not be totally closed through sensitivity analyses. Further experiments are required to obtain necessary data and to verify the method and results.

10. The design of cask impact limiters, fuel spacer grids, and cask basket structure significantly affects spent-fuel mechanical response.

11. For a given impulse momentum, the shape of the cask deceleration versus time history can be more important to fuel response than the amplitude of the deceleration (i.e., g-loading). This is because of a superimposed secondary impact due to internal gaps and dynamic amplification that depend on the assembly's natural frequencies relative to the frequency of the forcing function. Therefore, the stiffness and crush strength of impact limiters that govern the shape of the deceleration-time curve could significantly affect assembly response.

12. The higher the temperature while in transit, the lower the failure probability, provided that the burst temperature of the fuel is not exceeded. This is because of the beneficial effects of higher temperature on cladding fracture toughness. For the same reason, transport of very old fuel in sub- $0^{\circ} \mathrm{C}$ weather must be evaluated as a sensitivity case.

\subsection{Recommendations}

The results of the present analysis serve two important purposes. First, they demonstrate that a state-of-the-art technically defensible source-term methodology can be developed. Second, quantitative values for the potentially releasable fuel-bearing source term were obtained for the first time and will be used to support short-term needs of the overall program. A complete summary of this fuel contribution source-term methodology is presented in Section 2.0 of this report. Further development, refinement, and validation of this methodology should emphasize the following elements: 
1. Preliminary sensitivity analyses are discussed in detail in Section 6.0 of this report. A criterion should be established for selecting further analysis cases based on design variations, structural vulnerabilities, and source-term inventory.

2. The integrated computer model (STACE) can be the vehicle for applying the resulting complete source-term methodology to cask analysis and design.

3. The fuel-fines model should be enhanced to include a pellet fragmentation model that would estimate additional fines and their particle size distribution that may result during accident loading conditions.

4. Based on pretest sensitivity analyses, experiments should be designed to provide necessary missing input data and to verify individual models, as well as the overall integrated analysis sequence. These experiments should include the following elements: spacer grid stiffness and strength, assembly deflections, fuel rod resistance to pinch loads, fuel-fines particle size distribution and dispersion analyses, verifying experiments for PCI crack distribution and size, cladding ductility evaluations (bend tests), and integrated tests for single rod and assembly-drop conditions. 
This page is intentionally left blank. 
APPENDIX I

INITIAL CONDITIONS OF SPENT FUEL BEFORE TRANSPORT

I - I 
This page is intentionally left blank. 


\section{I.1 Introduction}

Spent-fuel rod susceptibility to damage and cladding breach during transport depends on the condition of the fuel rod as it is loaded into the transport cask. This condition is dependent on the history of the fuel rod. Fuel rods are manufactured in a variety of specifications and irradiated under differing reactor conditions. After irradiation, the fuel is moved to a water storage pool, where it generally is consolidated and then placed into dry storage before transport. The fuel is then transported directly to a repository for disposal or to a monitored retrievable storage (MRS) facility, where it is temporarily stored dry, repackaged, or consolidated before final transport to a repository. The pretransport condition of the spent fuel also depends on the handling path of the fuel rods.

The cladding on a small amount of fuel rods breaches while in the reactor. The breached rod may be identified or only the assembly containing the breached rod. Some breached rods remain internally dry or become waterlogged due to the ingress of reactor coolant water. The same mechanisms that cause breaches also cause a lesser degree of damage to intact fuel rod cladding.

After removal from the reactor, the fuel is moved to a water storage pool, where it generally is left as an intact assembly or consolidated. It may remain in the water pool at a modest temperature (usually $<85^{\circ} \mathrm{C}$ ) until transported from the reactor site. However, the fuel may be moved into dry storage, which usually involves higher temperatures. Four paths can be used in the handling of the fuel assembly from irradiation to initial transport (paths 1 to 4 in Figure I-1). If the spent fuel is transported to an MRS, the assembly is subjected to further handling scenarios. The fuel is left intact or is consolidated or reconsolidated. The fuel may then be put into dry lag storage for future transport or immediately repackaged for transport to the repository. A minimum of four handling paths through an MRS can be identified (paths 5 to 8 in Figure I-1).

Spent fuel has 20 handling paths from reactor to repository. Four paths involve one transport (paths 1 to 4, Figure I-1) and 16 paths (paths 1 to 4 combined with paths 5 to 8 in Figure I-1) involve two transports. Because the fuel behavior in each step along a path is dependent on the condition of the fuel as it exits a prior step, rigorous analysis would require a sequential analysis of each individual handling path. The analysis in this appendix does not include the effect of each handling path of fuel rod degradation; the potential fuel rod degradation during each step is evaluated independently.

The physical characteristics of the fuel rod, fuel assembly, and baskets affecting rod responses to subsequent transport environments are detailed in this appendix. Causes, characteristics, and inventory estimates of in-reactor cladding breaches are discussed, along with the behavior of waterlogged fuel. Rod behavior during storage in the reactor pool, transfers, consolidation, and dry storage are reviewed, along with analysis of potential cladding damage caused in the reactor by hydriding, oxidation, incipient cracks, embrittlement, and mechanical wear. 


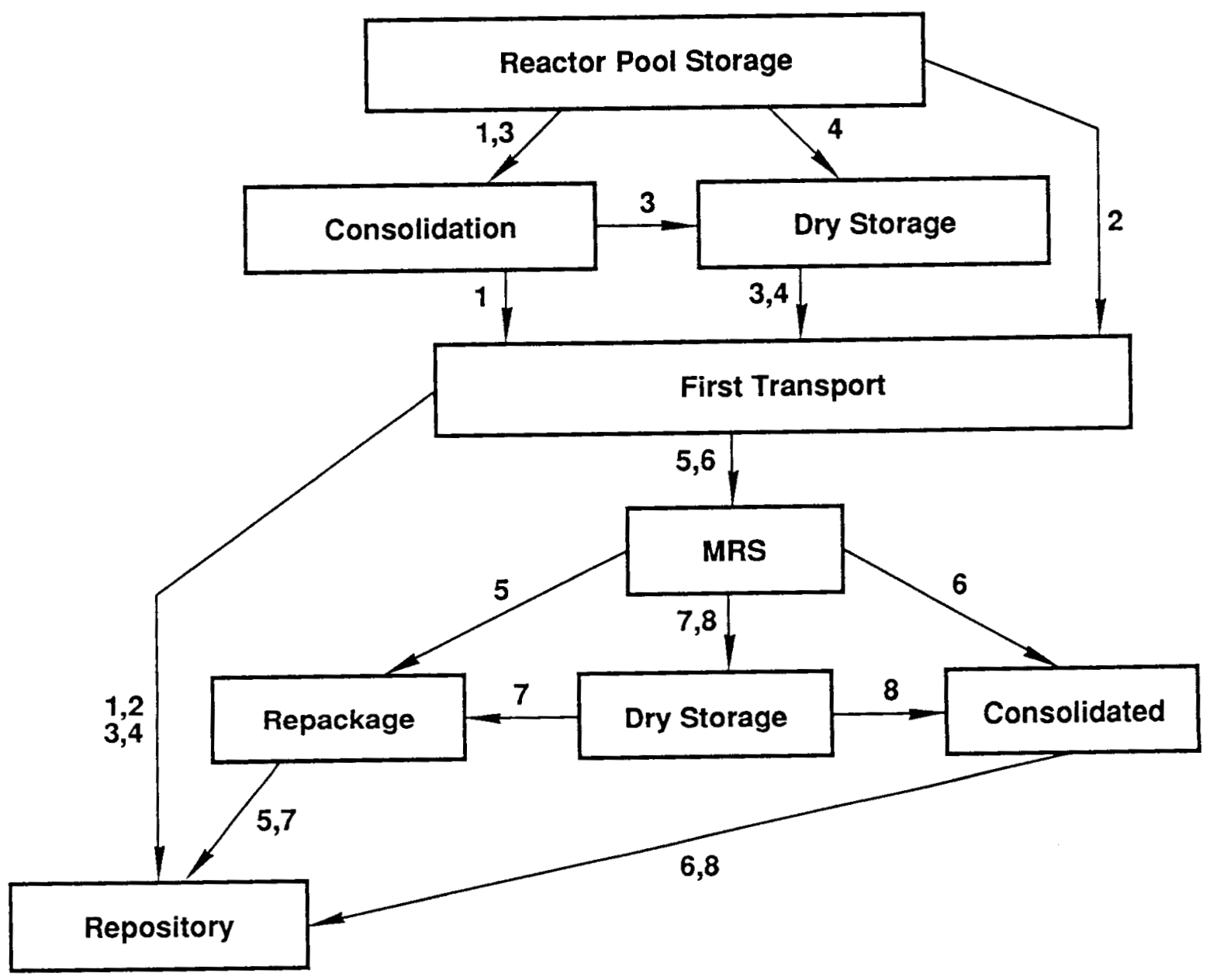

Figure I-1. Fuel Rod Handling Paths. (The numbers 1 through 8 represent the eight paths.)

\section{I.2 Physical Conditions of As-Manufactured Fuel Components}

The concentration of dispersible radioactive species in the cask is a function of fuel rod and assembly properties, and assembly and basket geometry. Rod characteristics affect rod strength, stiffness, stress in the cladding, and general rod response. Assembly geometry and material composition have a large impact on rod and assembly response because a critical dimension of the fuel assemblies is the distance between spacer grids. The spacer grid spacing affects rod response to impacts in all orientations. Also, the basket and cask design can have a major effect on fuel rod response in transport accident conditions. Differences in basket and cask design can produce a large variation in assembly response. The most significant parameters of the basket and casks are (1) basket and assembly axial and longitudinal gaps, (2) characteristics of interfaces 
between basket and assembly (i.e., continuous support versus discrete assembly support), and (3) characteristics of interfaces between the basket and the cask.

\section{Fuel Rods and Assemblies}

A light water reactor (LWR) fuel assembly consists of fuel rods and guide tubes held together in arrays (usually square) by Zircaloy or Inconel spacer grids, springs, and hardware. The arrays range from $6 \times 6$ to $11 \times 11$ for boiling water reactor (BWR) assemblies, and $14 \times 14$ to $18 \times 18$ for pressurized water reactor (PWR) assemblies. The five current fuel vendors are (1) Babcock and Wilcox ( $B \& W)$, (2) Combustion Engineering, Inc. (CE), (3) Advanced Nuclear Fuels Corporation (ANF) (previously known as Exxon Nuclear Company, Inc.), (4) Westinghouse Electric Corp. (WE), and (5) General Electric Company (GE). ANF and WE manufacture both BWR and PWR fuel assemblies. GE manufactures only BWR fuel, and both $B \& W$ and $C E$ make only PWR fuel assemblies. Normally, the assemblies are referred to by their manufacturer and array size (e.g., CE $14 \times 14$ ). There are, however, many subtypes within each of these categories that differ slightly in dimensions and materials. As shown in Tables I-1 and I-2, some of these subtypes have very limited usage.

A fuel rod consists of several sintered $\mathrm{UO}_{2}$ pellets stacked on end in a sealed cladding sheath comprised of Zircaloy-4, Zircaloy-2, or for a small percentage of the rods, stainless steel. Some GE rods have a metallurgically bonded zirconium layer inside the cladding. Diagrams of typical rods are shown in Figures I-2 and I-3. The pellets are held in place by a stainless steel or Inconel spring inside a plenum that is designed to accommodate fission gas released from the pellets during irradiation. This plenum is usually on top of the rod. The newer generation PWR and BWR rods are pressurized with argon or helium to improve heat conduction and reduce the contraction of the cladding onto the pellets due to external pressure in the reactor, known as cladding creep-down. The active length of the fuel column varies with the rod type. Common rod type dimensions are tabulated in Table I-3.

The pellets are 92 to $96 \%$ theoretically dense and have a grain size ranging from 5 to $20 \mu \mathrm{m}$. They are usually dished to provide room for irradiation swelling and are chambered to reduce the likelihood of pelletclad interaction (PCI). Pellet length ranges from 1.0 to $1.5 \mathrm{~cm}$. Current enrichment ranges from 0.8 to approximately $4.58235 \mathrm{U}$ with projections to 5.58. The enrichment can vary within assemblies of the same subtype. In some ANF rods, enrichment can vary within the rod itself; this trend may increase. The rods are designed to meet fuel management objectives for each individual reactor. In addition, some rods have pellets containing up to $108 \mathrm{Gd}_{2} \mathrm{O}_{3}$ (burnable poison). Some $\mathrm{B} \& W$ rods have $\mathrm{B}_{4} \mathrm{C}$ poison pellets at the top and bottom of the stack. Currently, rods tend to have larger arrays with smaller diameter rods to reduce in-core thermal gradients. Comprehensive information on fuel rod dimensions and materials is provided by Notz [N087]. 
Table I - 1

BWR Assembly Discharges as of December 1985 [NO87]

\begin{tabular}{|c|c|c|c|c|}
\hline Manufacturer & Array & Version & Number & $\begin{array}{c}\text { Average Burnup } \\
\text { (MWD/MTHM) }\end{array}$ \\
\hline $\mathrm{AC}^{\mathrm{a}}$ & $10 \times 10$ & & $153^{b}$ & 14,538 \\
\hline ANF & $6 \times 6$ & Dresden-1 & 66 & 4,906 \\
\hline ANF & $6 \times 6$ & Humboldt Bay & 126 & 9,037 \\
\hline ANF & $7 \times 7$ & $\mathrm{BWR} / 2$ & 260 & 23,552 \\
\hline ANF & $8 \times 8$ & JP-3 & 228 & 26,848 \\
\hline ANF & $9 \times 9$ & Big Rock Point & 4 & 20,981 \\
\hline ANF & $10 \times 10$ & LaCrosse & 78 & 17,307 \\
\hline ANF & $11 \times 11$ & Big Rock Point & 110 & 24,172 \\
\hline$G E$ & $6 \times 6$ & Dresden-1 & 366 & 20,283 \\
\hline GE & $6 \times 6$ & Humboldt Bay & 176 & 17,168 \\
\hline GE & $7 \times 7$ & $\mathrm{BWR} / 2,3: \mathrm{VI}$ & 1,542 & 16,782 \\
\hline GE & $7 \times 7$ & $\mathrm{BWR} / 2,3: \mathrm{V} 2$ & 5,469 & 17,050 \\
\hline GE & $7 \times 7$ & $\mathrm{BWR} / 4,5$ & 5,745 & 18,874 \\
\hline$G E$ & $7 \times 7$ & $\mathrm{BWR} / 1$ & 88 & 18,187 \\
\hline GE & $8 \times 8$ & $\mathrm{BWR} / 2,3$ & 3,489 & 26,008 \\
\hline GE & $8 \times 8$ & $\mathrm{BWR} / 4,5$ & 7,481 & 24,470 \\
\hline $\mathrm{GE}$ & $9 \times 9$ & $\mathrm{BRP}$ & 69 & 17,568 \\
\hline GE & Unknown & $\mathrm{BWR} / 2,3$ & 628 & 25,462 \\
\hline GE & Unknown & $B W R / 4,5$ & 324 & 7,908 \\
\hline UN NUCa & $6 \times 6$ & Dresden-1 & 457 & 17,634 \\
\hline NFSa & $9 \times 9$ & $\mathrm{BRP}$ & 5 & 16,857 \\
\hline Unknown & $8 \times 8$ & & 508 & 29,352 \\
\hline Unknown & $11 \times 11$ & & 4 & 20,923 \\
\hline \multicolumn{5}{|c|}{$\begin{array}{l}\mathrm{AC}=\mathrm{Allis} \text { Chalmers } \\
\mathrm{ANF}=\text { Advanced Nuclear Fuels } \\
\mathrm{GE}=\text { General Electric } \\
\text { UN NUC = United Nuclear } \\
\text { NFS = Nuclear Fuel Service }\end{array}$} \\
\hline \multicolumn{5}{|c|}{$\begin{array}{l}\text { aNot currently produced. } \\
\text { bonly two left; remainder were reprocessed. }\end{array}$} \\
\hline
\end{tabular}


Table I-2

PWR Assembly Discharges as of December 1985 [N087]

\begin{tabular}{|c|c|c|c|c|}
\hline Manufacturer & Array & Version & Number & $\begin{array}{c}\text { Average Burnup } \\
\text { (MWD/MTHM) }\end{array}$ \\
\hline$B \& W$ & $14 \times 14$ & Ginna & 2 & 24,397 \\
\hline$B \& W$ & $15 \times 15$ & Mark B & 2,767 & 26,604 \\
\hline $\mathrm{B} \& W$ & $15 \times 15$ & St. Steel & 627 & 30,809 \\
\hline $\mathrm{B} \& W$ & $17 \times 17$ & Mark C & 4 & 29,516 \\
\hline Generic & C14 & Design & 787 & 24,089 \\
\hline $\mathrm{CE}$ & $14 \times 14$ & Standard & 1,798 & 28,814 \\
\hline $\mathrm{CE}$ & $14 \times 14$ & Ft. Calhoun & 277 & 26,987 \\
\hline $\mathrm{CE}$ & $15 \times 15$ & Palisades & 273 & 15,956 \\
\hline $\mathrm{CE}$ & $16 \times 16$ & Onofre & 65 & 14,743 \\
\hline $\mathrm{CE}$ & $16 \times 16$ & St. Lucie/2 & 80 & 12,580 \\
\hline $\mathrm{CE}$ & $16 \times 16$ & ANO $/ 2$ & 226 & 26,559 \\
\hline ANF & $14 \times 14$ & West & 384 & 35,070 \\
\hline ANF & $14 \times 14$ & Top Rod & 66 & 36,835 \\
\hline ANF & $14 \times 14$ & CE (Ft. Calhoun) & 70 & 34,677 \\
\hline ANF & $15 \times 15$ & WE & 565 & 31,097 \\
\hline ANF & $15 \times 15$ & $\mathrm{CE}$ & 272 & 27,526 \\
\hline ANF & $15 \times 16$ & Yankee & 192 & 28,608 \\
\hline Unknown & $15 \times 16$ & & 93 & 26,890 \\
\hline WE & $13 \times 14$ & Indian Pt. 1 & 160 & 16,474 \\
\hline WE & $14 \times 14$ & $\mathrm{ZCA}$ & 561 & 28,565 \\
\hline WE & $14 \times 14$ & ZCB & 1,260 & 31,030 \\
\hline WE & $14 \times 14$ & $\mathrm{SC}$ & 363 & 28,089 \\
\hline WE & $14 \times 14$ & Mode1 C & 71 & 30,442 \\
\hline WE & $15 \times 15$ & $\mathrm{ZC}$ & 3,901 & 28,498 \\
\hline WE & $15 \times 15$ & OFA & 1 & 18,327 \\
\hline WE & $15 \times 16$ & Yankee & 56 & 25,832 \\
\hline WE & $17 \times 17$ & Standard & 2,794 & 26,138 \\
\hline \multicolumn{5}{|c|}{$\begin{array}{l}\mathrm{B} \& W=\text { Babcock and Wilcox } \\
C E=\text { Combustion Engineering } \\
\mathrm{ANF}=\text { Advanced Nuclear Fuels } \\
\mathrm{WE}=\text { Westinghouse Electric }\end{array}$} \\
\hline
\end{tabular}




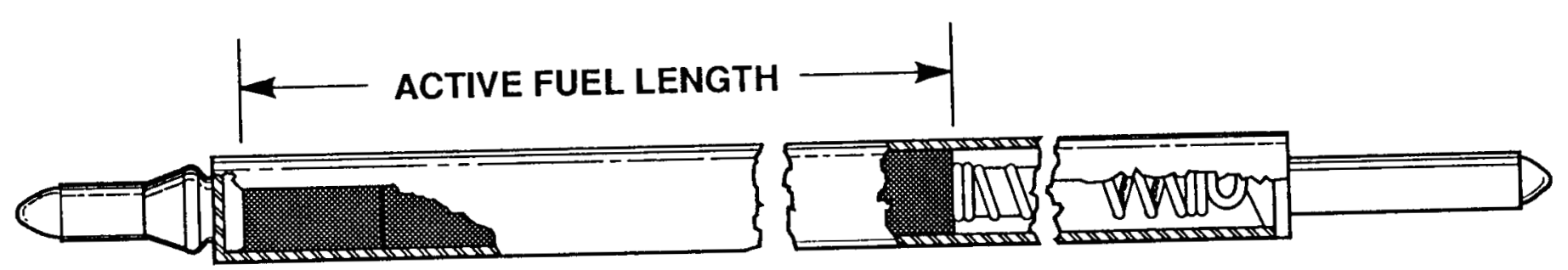




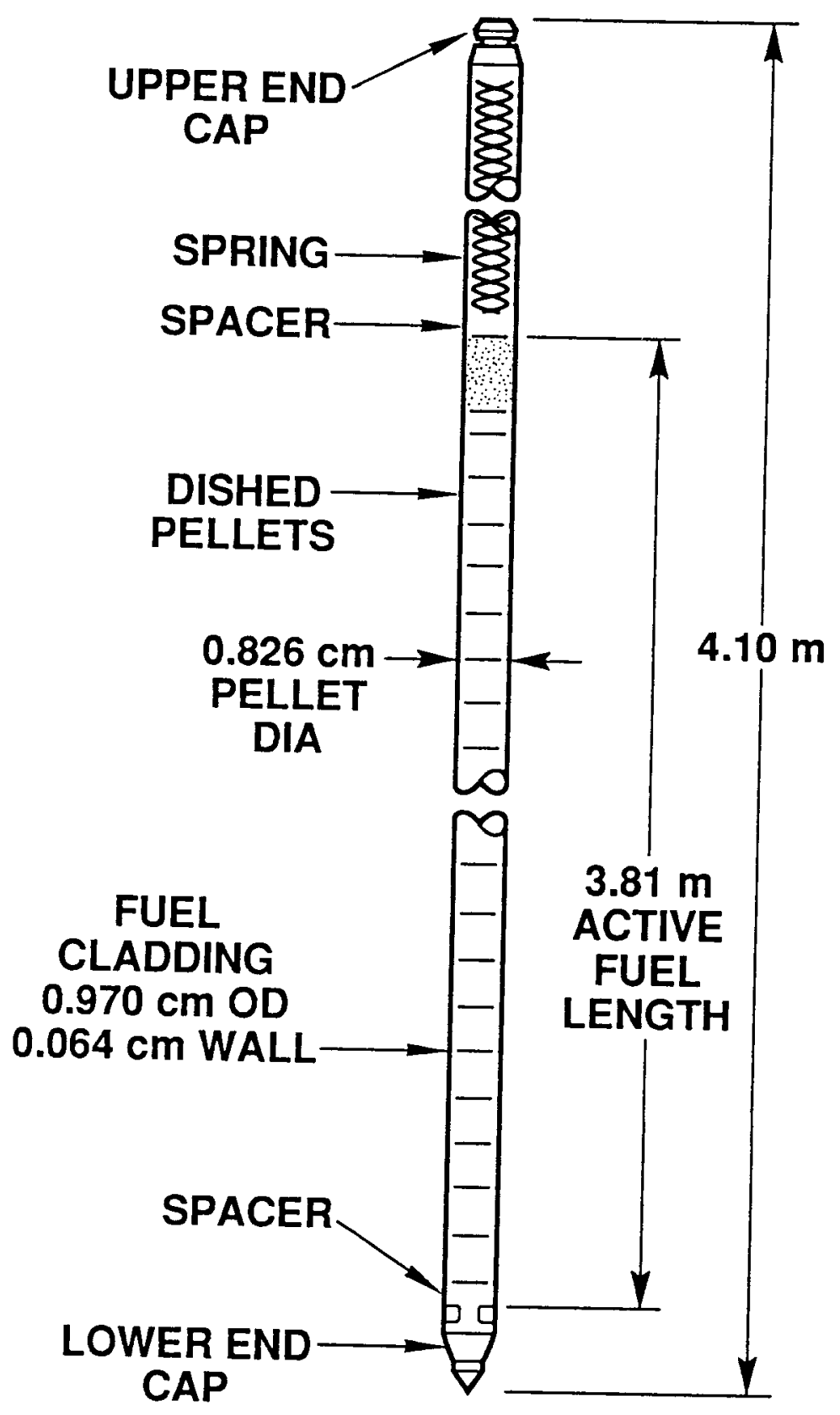

Figure I-3. CE $16 \times 16$ PWR Fuel Rod [WE79] 
Table I-3

As-Manufactured Physical Characteristics of LWR Fuel Rods [N087]

\begin{tabular}{|c|c|c|c|c|c|c|c|c|c|c|c|}
\hline Manufacturer & Array & Version & $\begin{array}{l}\text { Rod } \\
\text { Di am } \\
(\mathrm{cm}) \\
\end{array}$ & $\begin{array}{l}\text { Rod } \\
\text { Length } \\
\text { (m) }\end{array}$ & $\begin{array}{c}\text { Rod Fuel } \\
\text { Length } \\
\text { (m) } \\
\end{array}$ & $\begin{array}{l}\text { Rod } \\
\text { Weight } \\
\text { (kg) }\end{array}$ & $\begin{array}{c}\text { Clad } \\
\text { Thickness } \\
\quad(\mathrm{cm}) \\
\end{array}$ & $\begin{array}{c}\text { Clad } \\
\text { Material } \\
\end{array}$ & $\begin{array}{c}\text { Pressurized } \\
\text { Range } \\
\text { (MPa) } \\
\end{array}$ & $\begin{array}{c}\text { Fuel } \\
\text { Density } \\
(z \quad \text { Theo) } \\
\end{array}$ & $\begin{array}{c}\text { Grain } \\
\text { Size } \\
(\mu \mathrm{m}) \\
\end{array}$ \\
\hline$B \& W$ & $14 \times 14$ & & & & & & & & & & \\
\hline$B \& W$ & $15 \times 15$ & Mark B & 1.0922 & 3,9039 & 3.6017 & 3.17 & 0.0673 & Zircaloy -4 & 2.86 & 95 & 10 to 14 \\
\hline$B \notin W$ & $15 \times 15$ & St. Steel & 1.0718 & 3.2181 & 3.0607 & 2.68 & 0.0419 & SS 304 & 0.28 & 95 & 10 to 14 \\
\hline B\&W & $17 \times 17$ & Mark C & 0.9626 & 3.8785 & 3.6322 & 2.22 & & & 3.00 & 95 & 10 to 14 \\
\hline$B \& W$ & $15 \times 15$ & Mark BZ & 1.0922 & 3.9039 & 3.6017 & 3.18 & 0.0673 & Zircaloy -4 & 2.86 & 95 & 10 to 14 \\
\hline $\mathrm{CE}$ & $14 \times 14$ & Standard & 1.1176 & 3.7338 & 3.4798 & 3.13 & 0.0711 & Zircaloy -4 & 2.07 to 3.10 & 94 to 95 & $>5$ \\
\hline CE & $14 \times 14$ & Fort Calhoun & 1.1176 & 3.4798 & 3.2512 & 2.95 & 0.0711 & Zircaloy -4 & 2.07 to 3.10 & 94 to 95 & $>5$ \\
\hline $\mathrm{CE}$ & $15 \times 15$ & Pallsades & 1.0617 & 3.5560 & 3.3528 & 2.63 & 0.0660 & Zircaloy-4 & 2.07 to 3.10 & 94 to 95 & $>5$ \\
\hline $\mathrm{CE}$ & $16 \times 16$ & Onofre & 0.9702 & 4.0894 & 3.8100 & 2.59 & 0.0635 & Zircaloy -4 & 2.07 to 3.10 & 94 to 95 & $>5$ \\
\hline CE & $16 \times 16$ & St. Lucie/2 & 0.9702 & 3.7084 & 3.4721 & 2.36 & 0.0635 & Zircaloy-4 & 2.07 to 3.10 & 94 to 95 & $>5$ \\
\hline $\mathrm{CE}$ & $16 \times 16$ & ANO $/ 2$ & 0.9702 & 4.0894 & 3.8100 & 2.59 & 0.0635 & Zircaloy -4 & 2.07 to 3.10 & 94 to 95 & $>5$ \\
\hline $\mathrm{CE}$ & $16 \times 16$ & System 80 & 0.9702 & 4.0894 & 3.8100 & 2.59 & 0.0635 & Zircaloy -4 & 2.07 to 3.10 & 94 to 95 & $>5$ \\
\hline ANF & $14 \times 14$ & WE & 1.0769 & 3.7871 & 3.6068 & 3.03 & 0.0762 & Zircaloy -4 & 2.00 & 94 & Prop. \\
\hline ANF & $14 \times 14$ & $\mathrm{CE}$ & 1.1176 & 3.7211 & 3.4061 & 3.13 & 0.0787 & Zircaloy -4 & 2.59 & 94 & Prop. \\
\hline ANF & $14 \times 14$ & Top Rod & 1.0591 & 3.8608 & 3.6576 & 2.94 & 0.0749 & Zircaloy -4 & 2.10 & 94 & Prop. \\
\hline ANF & $15 \times 15$ & WE & 1.0769 & 3.8633 & 3.6576 & 3.05 & 0.0762 & Zircaloy -4 & 2.00 & 94 & Prop. \\
\hline ANF & $15 \times 15$ & $C E$ & 1.0519 & 3.5407 & 3.3477 & 2.67 & 0.0762 & Zircaloy -4 & 2.11 & 94 & Prop. \\
\hline ANF & $17 \times 17$ & WE & 0.9144 & 3.8608 & 3.6576 & 2.21 & 0.0635 & Zircaloy -4 & 2.00 & 94 & Prop. \\
\hline WE & $14 \times 14$ & Std/ZCA & 1.0718 & 3.8709 & 3.6880 & 3.03 & 0.0571 & Zircaloy-4 & 0 to 3.17 & 91 to 95 & 8 to 20 \\
\hline WE & $14 \times 14$ & OFA & 1.0160 & 3.8582 & 3.6576 & 2.78 & 0.0617 & Zircaloy -4 & 1.72 to 2.41 & 95 & 8 to 20 \\
\hline WE & $14 \times 14$ & $\mathrm{Std} / \mathrm{ZCB}$ & 1.0718 & 3.8709 & 3.6880 & 3.03 & 0.0571 & Zircaloy -4 & 0 to 3.17 & 91 to 95 & 8 to 20 \\
\hline WE & $14 \times 14$ & Std/SC & 1.0718 & 3.2181 & 3.0480 & 2.98 & 0.0419 & SS 034 & 0 to 2.07 & 93 to 95 & 8 to 20 \\
\hline WE & $14 \times 14$ & Model C & 1.1176 & 3.7185 & 3.4721 & 3.11 & 0.0660 & Zircaloy -4 & 1.99 to 2.76 & 95 & 8 to 20 \\
\hline WE & $15 \times 15$ & Std/ZC & 1.0718 & 3.8582 & 3.6576 & 3.11 & 0.0614 & Zircaloy -4 & 0 to 3.28 & 95 & 8 to 20 \\
\hline WE & $15 \times 15$ & OFA & 1.0718 & 3.8582 & 3.6576 & 3.08 & 0.0419 & Zircaloy -4 & 1.99 to 2.41 & 95 & 8 to 20 \\
\hline WE & $15 \times 15$ & Std/ZC & 1.0718 & 3.2181 & 3.0988 & 2.98 & 0.0571 & SS 034 & 0 & 95 & 8 to 20 \\
\hline WE & $17 \times 17$ & Standard & 0.9499 & 3.8506 & 3.6576 & 2.44 & 0.0571 & Zircaloy -4 & 1.99 to 3.45 & 95 & 8 to 20 \\
\hline WE & $17 \times 17$ & OFA & 0.9144 & 3.8506 & 3.6576 & 2.24 & 0.0571 & Zircaloy -4 & 1.99 to 2.41 & 95 & 8 to 20 \\
\hline WE & $17 \times 17$ & Vantage 5 & 0.9144 & 3.8684 & 3.6576 & 1.82 & 0.0571 & Zircaloy -4 & & 95 & \\
\hline WE & $17 \times 17$ & $\mathrm{XLR}$ & 0.9499 & 4.4856 & & & & & & & \\
\hline WE & $13 \times 13$ & & & & & & & & & & \\
\hline
\end{tabular}


Table I-3

As-Manufactured Physical Characteristics of LWR Fuel Rods [N087] (Concluded)

\begin{tabular}{|c|c|c|c|c|c|c|c|c|c|c|c|}
\hline \multicolumn{12}{|c|}{ BWR Fuel Rods } \\
\hline Manufacturer & Array & Version & $\begin{array}{r}\text { Rod } \\
\text { Di am } \\
(\mathrm{cm}) \\
\end{array}$ & $\begin{array}{c}\text { Rod } \\
\text { Length } \\
\text { (m) } \\
\end{array}$ & $\begin{array}{c}\text { Rod Fuel } \\
\text { Length } \\
\text { (m) } \\
\end{array}$ & $\begin{array}{l}\text { Rod } \\
\text { Weight } \\
\text { (kg) } \\
\end{array}$ & $\begin{array}{c}\text { Clad } \\
\text { Thickness } \\
(\mathrm{cm}) \\
\end{array}$ & $\begin{array}{c}\text { Clad } \\
\text { Material } \\
\end{array}$ & $\begin{array}{c}\text { Pressurized } \\
\text { Range } \\
\text { (MPa) } \\
\end{array}$ & $\begin{array}{c}\text { Fuel } \\
\text { Density } \\
(z \text { Theo) }\end{array}$ & $\begin{array}{c}\text { Grain } \\
\text { Size } \\
(\mu \mathrm{m}) \\
\end{array}$ \\
\hline ANF & $6 \times 6$ & GE & 1.4351 & 2.9641 & 2.7508 & 4.0188 & 0.1168 & Zircaloy -2 & 0 & 93.5 & Prop. \\
\hline ANF & $7 \times 7$ & GE & 1.4478 & 4.0182 & 3.6576 & 5.6290 & 0.1168 & Zircaloy-2 & 0 & 95 & Prop. \\
\hline ANF & $8 \times 8$ & $J P-3$ & 1.2293 & 4.0309 & 3.6880 & 4.0596 & 0.0914 & Zircaloy-2 & 0.3102 & 94.5 & Prop. \\
\hline ANF & $8 \times 8$ & $J P-4,5$ & 1.2293 & 4.1503 & 3.8100 & 4.1503 & 0.0914 & Zircaloy-2 & 0.2068 & 94.5 & Prop. \\
\hline ANF & $9 \times 9$ & $J P-4,5$ & 1.0769 & 4.1605 & 3.8100 & 3.1796 & 0.0762 & Zircaloy-2 & 0.4137 & 94.5 & Prop. \\
\hline ANF & $10 \times 10$ & $\mathrm{AC}$ & 1.0007 & 2.2860 & 2.1082 & 1.6011 & 0.0558 & SS 34811 & & & Prop. \\
\hline ANF & $11 \times 11$ & $\mathrm{GE}$ & 1.1404 & 1.9812 & 1.7780 & 1.6782 & 0.0863 & Zircaloy-2 & 0.2068 & 94 & Prop. \\
\hline ANF & $9 \times 9$ & $\mathrm{BRP}$ & 1.4300 & & 1.7272 & & & Zircaloy-2 & & & Prop. \\
\hline ANF & $6 \times 6$ & Humboldt Bay & & & & & & & & & \\
\hline WE & $8 \times 8$ & QUAD+ & 1.1633 & 4.0792 & 3.8100 & & 0.0736 & Zircaloy-2 & & 95 & \\
\hline GE & $6 \times 6$ & Dresden-1 & 1.4274 & 2.9718 & 2.6416 & & & Zircaloy-2 & & & \\
\hline $\mathrm{GE}$ & $6 \times 6$ & Humboldt Bay & 1.4300 & & 2.0066 & & & & & & \\
\hline GE & $7 \times 7$ & $/ 2,3: \mathrm{V}_{1}$ & 1.4478 & 4.0919 & 3.6576 & & 0.0812 & Zircaloy-2 & & & \\
\hline GE & $7 \times 7$ & 14,5 & 1.4300 & 4.0919 & 3.7084 & & 0.0812 & Zircaloy-2 & & & \\
\hline GE & $7 \times 7$ & Humboldt Bay & 1.2344 & & 2.0066 & & & & & & \\
\hline GE & $8 \times 8$ & 12,3 & 1.2192 & 4.0919 & 3.6576 & & 0.0812 & Zircaloy-2 & & & \\
\hline$G E$ & $8 \times 8$ & $14,5: V_{1}$ & 1.2446 & 4.0919 & 3.7084 & & 0.0812 & Zircaloy-2 & & & \\
\hline GE & $8 \times 8$ & $/ 4,5: v 2$ & 1.2446 & 4.0919 & 3.7084 & & 0.0812 & & & & \\
\hline GE & $9 \times 9$ & BRP & 1.4300 & & 1.7780 & & & Zircaloy-2 & & & \\
\hline GE & $11 \times 11$ & $\mathrm{BRP}$ & 1.1404 & & 1.7780 & & & & & & \\
\hline
\end{tabular}

Prop. = Proprietary

Note: Blank entries indicate no data available in the database. 
The fuel rods are held between nozzles or end fittings by spacer springs and guide tubes (see Figures I-4 and I-5 for typical assembly geometries). The arrays are given rigidity for handling by assembly hardware that consists of end plates, nozzles or end fittings, and connecting rods or guide tubes. The connecting rods of BWR assemblies consist of a limited number of rods (tie rods) bolted into the upper and lower end plates. The remaining rods are free to move in the spacer grids within the limitations of the hold-down springs. These assemblies can be dismantled. PWR assemblies, in contrast, are held together by the control rod guide tubes that are welded or bolted to the end fittings. Like BWR assemblies, the remaining rods are free to move between the end plates and are limited only by the hold-down spring. Except in special cases, only the newer $17 \times 17$ PWR arrays can be dismantled; the remainder of the PWR arrays must be cut apart. Assembly dimensions are shown in Table I-4.

Sixteen to twenty-four fuel rod slots in PWR assemblies have control rod guide tubes or instrument tubes inside that are slightly larger than a fuel rod. CE $14 \times 14$ and $16 \times 16$ assemblies use extra-large guide tubes that are $-2.5 \mathrm{~cm}$ in diameter; each guide tube replaces four rods. A few assembly versions use eight solid Zircaloy guide bars instead of control rod guide tubes. Generally, the guide tubes are comprised of Zircaloy-4. No control rod guide tubes are used in a BWR assembly, but one or two rod positions may be occupied by a water tube. (See Figure I-6 for typical locations of control rod guide tubes and tie rods.)

The fuel rods contained in an assembly are held in place by egg-crateshaped spacer grids that provide both lateral and axial support. Figure I-7 shows a photograph of a typical BWR grid. BWR fuel assemblies usually contain seven grids evenly spaced along the assembly length, while PWRs contain six to eight grids uniformly distributed in the middle of the assembly, with an additional grid at its ends. These grids are typically 3.8 to $6.4 \mathrm{~cm}$ wide (see Table I-5). A photograph of a PWR grid is shown in Figure I-8. Both BWR and PWR grids usually contain three contact springs inside each grid cell. Contact springs support the rods in the grid cells.

The spacer grids are welded directly to the guide tubes in $C E$ assemblies and brazed to a stainless steel grid sleeve that is then swaged to the guide tube in WE assemblies [WE79]. The two end grids are welded to the end fittings in the $\mathrm{B} \& \mathrm{~W}$ assembly, but the intermediate grids are held in place by spring contact with the guide tubes and are free to move. The GE BWR assemblies have tabs welded to one hollow Zircaloy-2 tube that has a square end plug. The grids are axially positioned with the tabs in the corner of a grid cell. The hollow Zircaloy 2 tube is rotated by $90^{\circ}$ so that the tubes maintain the grids in the proper axial location. Placement of the square end plug in the end plate locks the assembly [WE79].

Spacer grids are usually comprised of Zircaloy-4, a combination of Zircaloy-4 grids with Incone1-718 springs, or Incone1-718 alone (see Table I-6). Sometimes stainless steel or other Inconel alloys have been used. In a few assemblies, the end grids are of a different material than those in the active fuel zone. (See Table I-6 for a listing of the number of grids and materials for each assembly type.) Many Inconel grids are replaced with Zircaloy for neutron economy, but this requires modification to increase the grid strength [NE88]. 


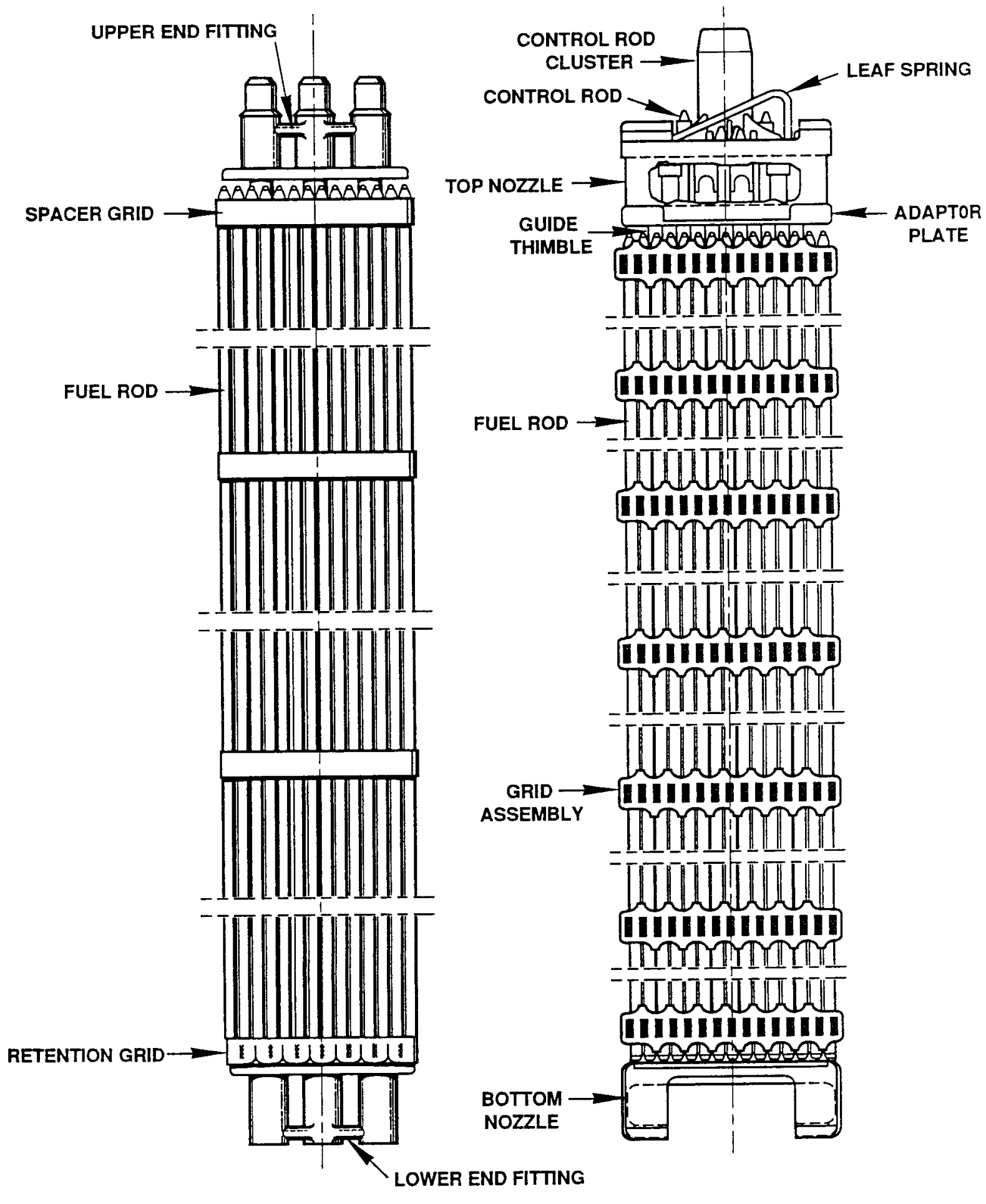

(a)

(b)

Figure I-4. Typical PWR Assemblies [IA76]: (a) CE $14 \times 14$; (b) WE $15 \times 15$ 


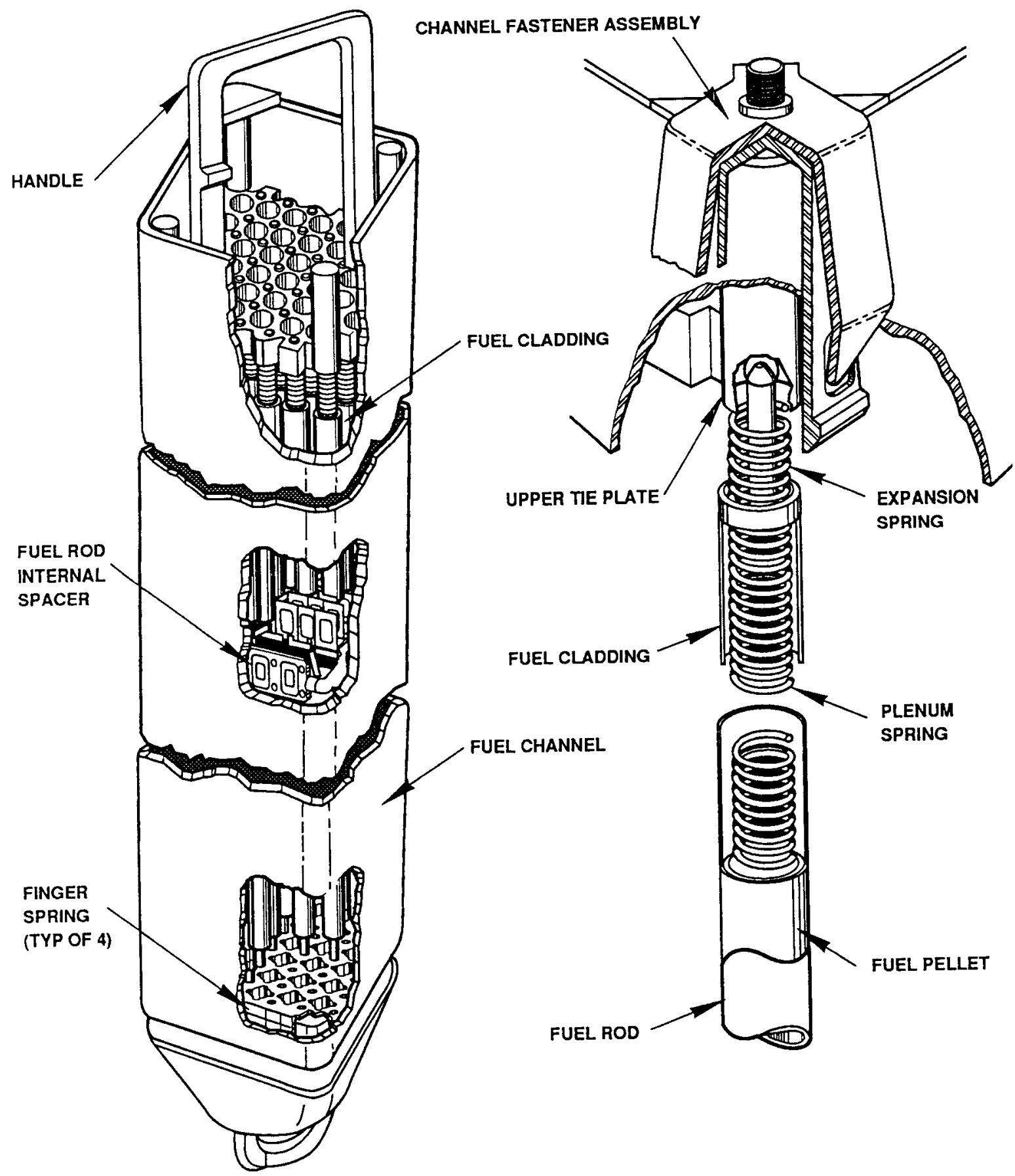

Figure I-5. Typical BWR Assembly [IA76] 
Table I-4

Fuel Assembly Dimensions [N087]

\begin{tabular}{|c|c|c|c|c|c|c|c|}
\hline Manufacturer & Array & Version & $\begin{array}{c}\text { Assembly } \\
\text { Width } \\
(\mathrm{cm}) \\
\end{array}$ & $\begin{array}{c}\text { Assembly } \\
\text { Length } \\
\text { (m) } \\
\end{array}$ & $\begin{array}{c}\text { Assembly } \\
\text { Weight } \\
(\mathrm{kg}) \\
\end{array}$ & $\begin{array}{l}\text { Typ. No. } \\
\text { of Rods }\end{array}$ & $\begin{array}{c}\text { Rod } \\
\text { Pitch } \\
(\mathrm{cm}) \\
\end{array}$ \\
\hline$B \& W$ & $14 \times 14$ & & & & & & \\
\hline$B \& W$ & $15 \times 15$ & Mark B & 21.6814 & 4.2062 & 687.9 & 203 & 1.4427 \\
\hline$B \& W$ & $15 \times 15$ & St. Steel & 21.5036 & 3.4950 & 569.7 & 204 & 1.4300 \\
\hline B\&WW & $15 \times 15$ & Mark C & 21.6814 & 4.2087 & 682.7 & 264 & 1.2750 \\
\hline$B \& W$ & $15 \times 15$ & Mark BZ & 21.6814 & 4.2062 & 687.9 & 208 & 1.4427 \\
\hline $\mathrm{CE}$ & $14 \times 14$ & Standard & 20.5740 & 4.2672 & 576.1 & 164 & 1.4732 \\
\hline $\mathrm{CE}$ & $14 \times 14$ & Fort Calhoun & 20.5740 & 3.9878 & 553.4 & 168 & 1.4732 \\
\hline $\mathrm{CE}$ & $15 \times 15$ & Palisades & 20.5740 & 3.7871 & 616.9 & 204 & 1.3970 \\
\hline $\mathrm{CE}$ & $16 \times 16$ & Onofre & 20.8280 & 4.7498 & 648.6 & 224 & 1.2852 \\
\hline $\mathrm{CE}$ & $16 \times 16$ & St. Lucie/2 & 20.5740 & 4.2926 & 589.7 & 224 & 1.2852 \\
\hline $\mathrm{CE}$ & $16 \times 16$ & ANO $/ 2$ & 20.5740 & 4.8006 & 648.6 & 232 & 1.2852 \\
\hline $\mathrm{CE}$ & $16 \times 16$ & System 80 & 20.5740 & 4.9022 & 648.6 & 220 & 1.2852 \\
\hline ANF & $14 \times 14$ & WE & 19.7180 & 4.0970 & 576.5 & 179 & 1.4122 \\
\hline ANF & $14 \times 14$ & $\mathrm{CE}$ & 20.5994 & 3.9928 & 586.0 & 176 & 1.4732 \\
\hline ANF & $14 \times 14$ & Top Rod & 19.7180 & 4.0970 & 551.1 & 179 & 1.4122 \\
\hline ANF & $15 \times 15$ & $\mathrm{WE}$ & 21.4020 & 4.0970 & 650.0 & 204 & 1.4300 \\
\hline ANF & $15 \times 15$ & $\mathrm{CE}$ & 20.9550 & 3.7820 & 607.4 & 216 & 1.3970 \\
\hline ANF & $17 \times 17$ & WE & 21.4020 & 4.0995 & 611.4 & 264 & 1.2598 \\
\hline WE & $14 \times 14$ & std/ZCA & 19.7104 & 4.1833 & 590.6 & 179 & 1.4122 \\
\hline WE & $14 \times 14$ & OFA & 19.7104 & 4.1833 & 533.9 & 179 & 1.4122 \\
\hline WE & $14 \times 14$ & std/ZCB & 19.7104 & 4.1833 & 590.6 & 179 & 1.4122 \\
\hline WE & $14 \times 14$ & Std/SC & 19.7104 & 3.5179 & 565.6 & 180 & 1.4122 \\
\hline WE & $14 \times 14$ & Model C & 20.3962 & 3.9928 & 582.0 & 176 & 1.4732 \\
\hline WE & $15 \times 15$ & $\mathrm{Std} / \mathrm{ZC}$ & 21.4223 & 4.1656 & 667.7 & 204 & 1.4300 \\
\hline WE & $15 \times 15$ & OFA & 21.3969 & 4.1046 & 661.8 & 204 & 1.4300 \\
\hline$W E$ & $15 \times 15$ & $\mathrm{Std} / \mathrm{SC}$ & 21.3868 & 3.5179 & 644.6 & 204 & 1.4300 \\
\hline WE & $17 \times 17$ & Standard & 21.4223 & 4.2468 & 672.2 & 264 & 1.2598 \\
\hline WE & $17 \times 17$ & OFA & 21.4223 & 4.2468 & 622.8 & 264 & 1.2598 \\
\hline WE & $17 \times 17$ & Vantage 5 & 21.4020 & 4.0665 & 619.2 & 264 & 1.2598 \\
\hline WE & $17 \times 17$ & XLR & 21.4122 & 5.0546 & & & 1.2598 \\
\hline WE & $13 \times 13$ & & & & & & \\
\hline
\end{tabular}


Table I-4

Fue1 Assembly Dimensions [NO87]

(Concluded)

BWR Fue1 Rods

\begin{tabular}{|c|c|c|c|c|c|c|c|c|c|}
\hline Manufacturer & & cra & & Version & $\begin{array}{c}\text { Assembly } \\
\text { Width } \\
(\mathrm{cm}) \\
\end{array}$ & $\begin{array}{l}\text { Assembly } \\
\text { Length } \\
\text { (m) }\end{array}$ & $\begin{array}{c}\text { Assembly } \\
\text { Weight } \\
(\mathrm{kg}) \\
\end{array}$ & $\begin{array}{l}\text { Typ. No. } \\
\text { of Rods } \\
\end{array}$ & $\begin{array}{c}\text { Rod } \\
\text { Pitch } \\
(\mathrm{cm}) \\
\end{array}$ \\
\hline $\mathrm{AC}$ & 10 & $\mathrm{x}$ & 10 & & & & & 100 & 1.4351 \\
\hline ANF & 6 & $\mathrm{x}$ & 6 & GE & 10.8585 & 3.4112 & 148.8 & 35 & 1.7627 \\
\hline ANF & 7 & $\mathrm{x}$ & 7 & $\mathrm{GE}$ & 13.3273 & 4.3510 & 280.8 & 48 & 1.8745 \\
\hline ANF & 8 & $\mathrm{x}$ & 8 & JP- 3 & 13.3375 & 4.3510 & 254.9 & 63 & 1.6281 \\
\hline ANF & 8 & $\mathrm{x}$ & 8 & $J P-4,5$ & 13.3375 & 4.4729 & 266.7 & 62 & 1.6281 \\
\hline $\mathrm{ANF}$ & 9 & $\mathrm{x}$ & 9 & $J P-3$ & 13.3375 & 4.3510 & 252.6 & 79 & 1.4528 \\
\hline ANF & 9 & $\mathrm{x}$ & 9 & $J P-4,5$ & 13.3375 & 4.4729 & 260.8 & 79 & 1.4528 \\
\hline ANF & 10 & $\mathrm{x}$ & 10 & $\mathrm{AC}$ & 14.2595 & 2.6035 & 171.0 & 96 & 1.4147 \\
\hline ANF & 11 & $\mathrm{x}$ & 11 & $\mathrm{GE}$ & 16.5481 & 2.1336 & 207.3 & 117 & 1.4655 \\
\hline ANF & 9 & $\mathrm{x}$ & 9 & $\mathrm{BRP}$ & 16.5100 & 2.0828 & & & 1.7957 \\
\hline ANF & 6 & $\mathrm{x}$ & 6 & Humboldt Bay & & 2.4130 & & 36 & \\
\hline WE & 8 & $\mathrm{x}$ & 8 & QUAD+ & 13.9700 & 4.4577 & 272.2 & 64 & 1.5468 \\
\hline $\mathrm{GE}$ & 6 & $\mathrm{x}$ & 6 & Dresden-1 & 10.1600 & 3.4290 & & 36 & 1.8034 \\
\hline GE & 6 & $\mathrm{x}$ & 6 & Humboldt Bay & 10.1600 & 2.4130 & & 36 & \\
\hline GE & 7 & $\mathrm{x}$ & 7 & $/ 2,3: \mathrm{V} 1$ & 14.0157 & 4.3434 & 272.2 & 49 & 1.8745 \\
\hline $\mathrm{GE}$ & 7 & $\mathrm{x}$ & 7 & $/ 2,3: \mathrm{V} 2$ & 14.0157 & 4.3434 & 272.2 & 49 & 1.8745 \\
\hline GE & 7 & $\mathrm{x}$ & 7 & 14,5 & 14.0157 & 4.4704 & 272.2 & 49 & 1.8745 \\
\hline GE & 7 & $\mathrm{x}$ & 7 & Humboldt Bay & 10.1600 & 2.4130 & & 49 & 1.6154 \\
\hline $\mathrm{GE}$ & 8 & $\mathrm{x}$ & 8 & $/ 2,3$ & 14.0157 & 4.3434 & 272.2 & & 1.6256 \\
\hline $\mathrm{GE}$ & 8 & $\mathrm{x}$ & 8 & $/ 4,5: V 1$ & 14.0157 & 4.4704 & 272.2 & 63 & 1.6256 \\
\hline GE & 8 & $\mathrm{x}$ & 8 & $14,5: v 2$ & 14.0157 & 4.4704 & 272.2 & 62 & 1. 6256 \\
\hline $\mathrm{GE}$ & 9 & $\mathrm{x}$ & 9 & BRP & 16.5100 & 2.0828 & & 81 & 1.7957 \\
\hline $\mathrm{GE}$ & 11 & $\mathrm{x}$ & 11 & $\mathrm{BRP}$ & 16.5100 & 2.0828 & & 111 & 1.4655 \\
\hline
\end{tabular}




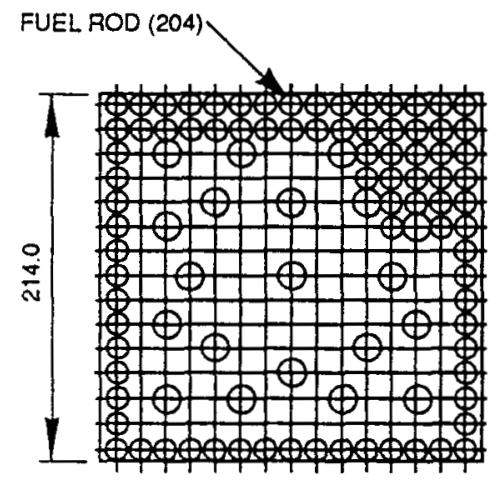

(a)

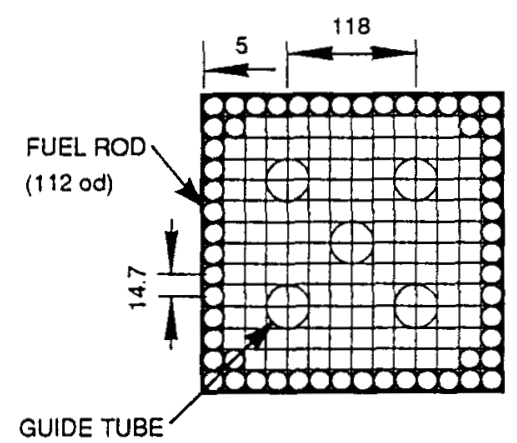

(b)

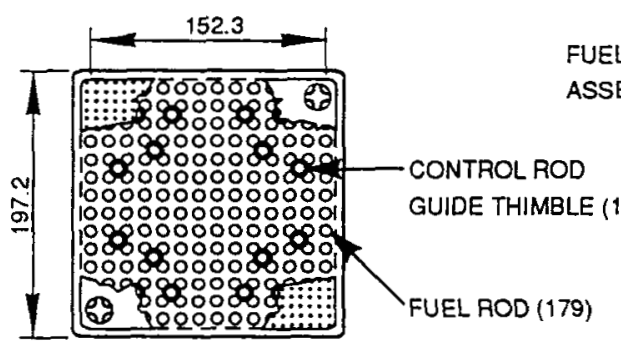

(c)

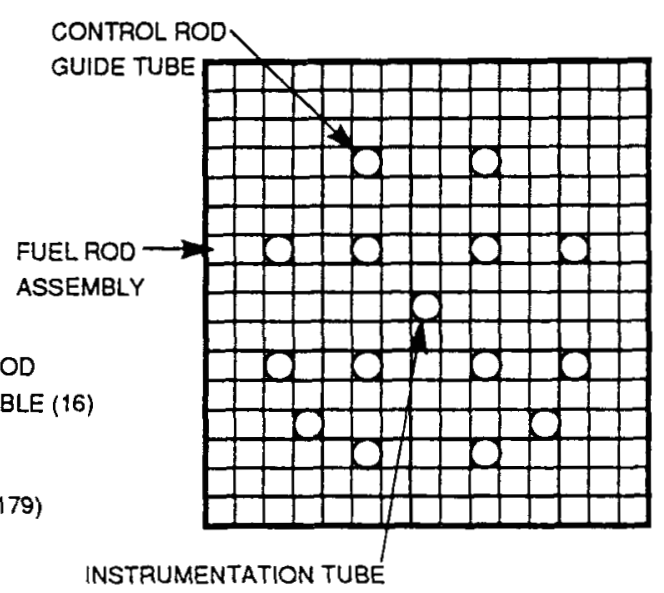

(d)

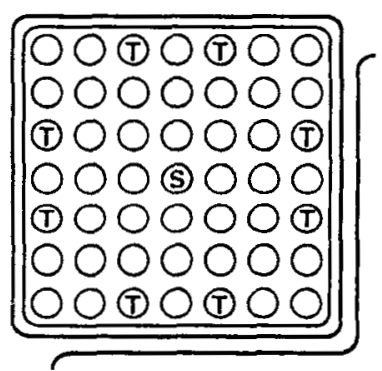

(e)

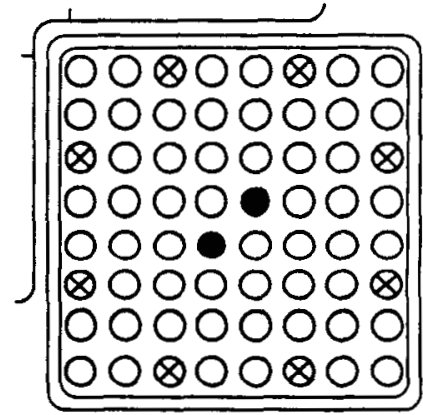

FUEL MODULE

- FUel RODS

O WATER RODS

$\otimes$ TIE ROOS

(f)

Figure I-6. Control Rod Guide Tube and Tie Rod Locations [IA76]:

(a) WE $15 \times 15$, (b) CE $14 \times 14$, (c) WE $14 \times 14 \mathrm{ZCA}$, (d) B\&W $15 \times 15$, (e) GE $7 \times 7$, and (f) GE $8 \times 8$. 

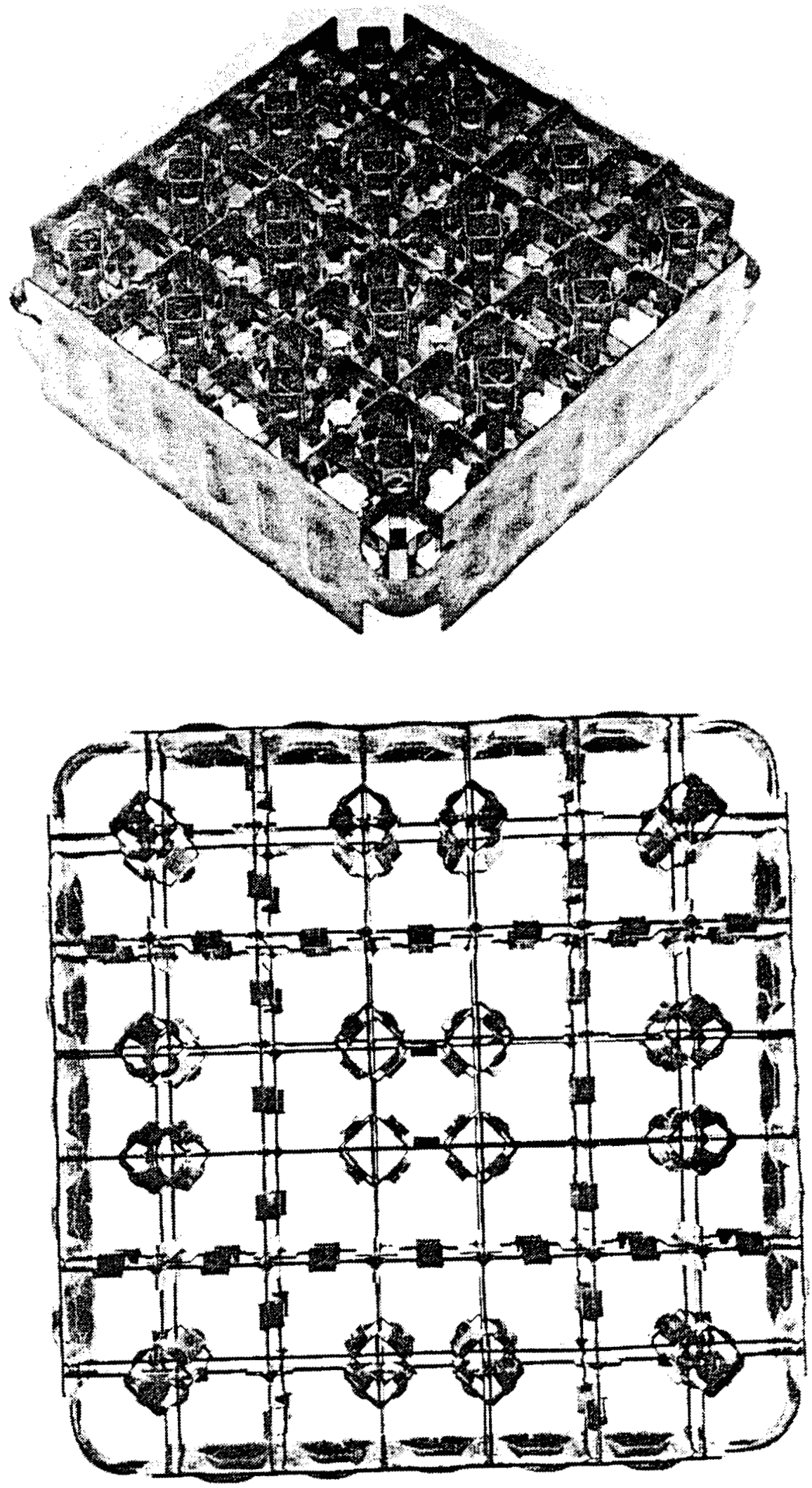

Figure I-7. Photograph of GE $7 \times 7$ BWR Spacer Grid 
Table I-5

Spacer Grid Widths a

\begin{tabular}{|c|c|c|}
\hline Manufacturer ${ }^{b}$ Fuel/Reactor & $\begin{array}{l}\text { Array } \\
\text { Size } \\
\end{array}$ & $\begin{array}{l}\text { Spacer Width } \\
\frac{(\mathrm{cm})}{}\end{array}$ \\
\hline \multicolumn{3}{|l|}{ BWR } \\
\hline $\begin{array}{ll}\text { ANF } /(G E) & \\
\text { ANF } /(G E) & (B W R-2) \\
\text { ANF } /(G E) & (\text { BWR-3, } 3,5 \& 6) \\
G E & \\
G E & \\
G E & \end{array}$ & $\begin{array}{l}7 \times 7 \\
8 \times 8 \\
8 \times 9 \\
7 \times 7 \\
8 \times 8 \\
8 \times 8\end{array}$ & $\begin{array}{l}6.35 \max \\
6.35 \max \\
6.35 \max \\
4.128 \\
4.128 \\
4.128\end{array}$ \\
\hline \multicolumn{3}{|l|}{ PWR } \\
\hline $\begin{array}{l}\mathrm{ANF} /(\mathrm{CE}) \\
\mathrm{ANF} /(\mathrm{WE}) \\
\mathrm{ANF} /(\mathrm{CE}) \\
\mathrm{ANE} /(\mathrm{WE}) \\
\mathrm{WE}\end{array}$ & 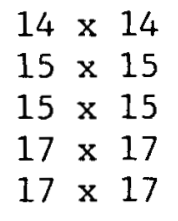 & $\begin{array}{l}6.99 \max \\
6.99 \max \\
6.99 \max \\
6.99 \max \\
5.441\end{array}$ \\
\hline \multicolumn{3}{|c|}{$\begin{array}{l}\text { aBWR and PWR Bundle Design, DWG No. SK-3-21911, U.S. DOE Hanford Engi- } \\
\text { neering Development Laboratory (HEDL), August } 1978 \text {. } \\
\text { bIf no reactor is indicated, then the assembly and reactor manufacturer } \\
\text { are the same. }\end{array}$} \\
\hline
\end{tabular}

The spring force of the spacer grid springs is lowered by irradiation. After exposures of up to $1 \times 10^{22} \mathrm{n} / \mathrm{cm}^{2}$ (E $\left.>0.8 \mathrm{MeV}\right)(\sim 20 \mathrm{GWD} / \mathrm{MTHM})$, the central grids have only 10 to 208 of their initial spring force. However, the upper and lower springs maintain $-40 \%$ of their initial fabricated spring force even at high burnups [ST82, KN85].

When a PWR assembly is fabricated, the end-plate-to-end-plate dimension is $-5 \mathrm{~cm}$ longer than the rods to accommodate differential irradiation growth. The rods are free to elongate and move within the spacer grids. At the end of irradiation, they may be seated on the bottom end plate. Because the assembly expands so much less than the rods, the degree of remaining clearance is closely monitored by surveillance of rod elongation. This elongation can differ significantly between adjacent rods. At $10 \mathrm{GWD} / \mathrm{MTU}$, elongation is between zero and 0.28 and increases to from 0.5 to 1.28 at -45 to $50 \mathrm{GWD} / \mathrm{MTU}$ [KN85, ST82, EX82, C082, MA85, BA85b, AN82b]. Based on a $3.9-\mathrm{m}$ rod, the approximate remaining gap between the fuel rods and end plates would be 5.1 to $4.3 \mathrm{~cm}$ at low burnup and 3.1 to $0.5 \mathrm{~cm}$ at high burnup. 

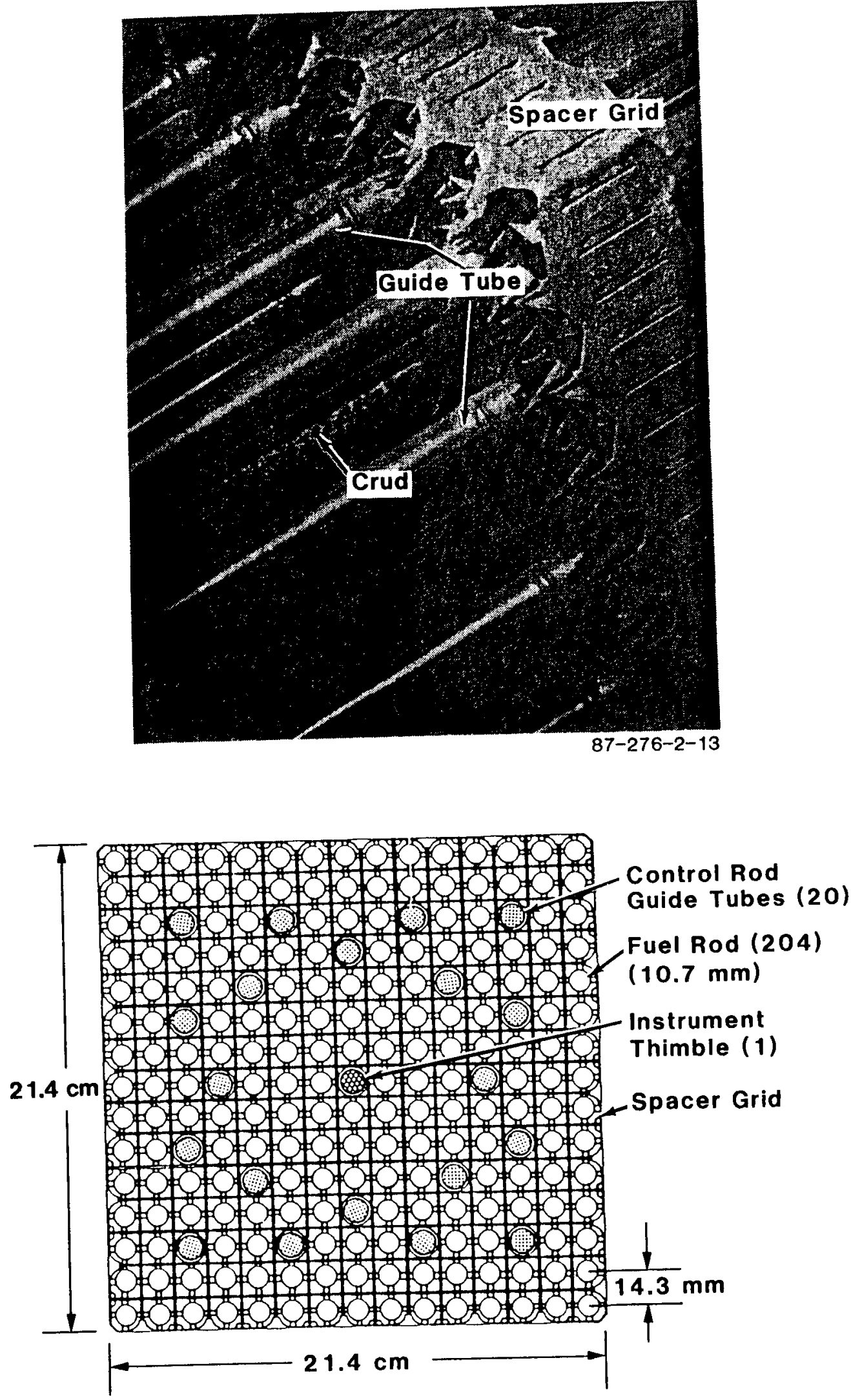

Figure I-8. Description of B\&W $15 \times 15$ PWR Spacer Grid 
Table I-6

Guide Tube and Spacer Grid Data for LWR Assemblies [NO87]

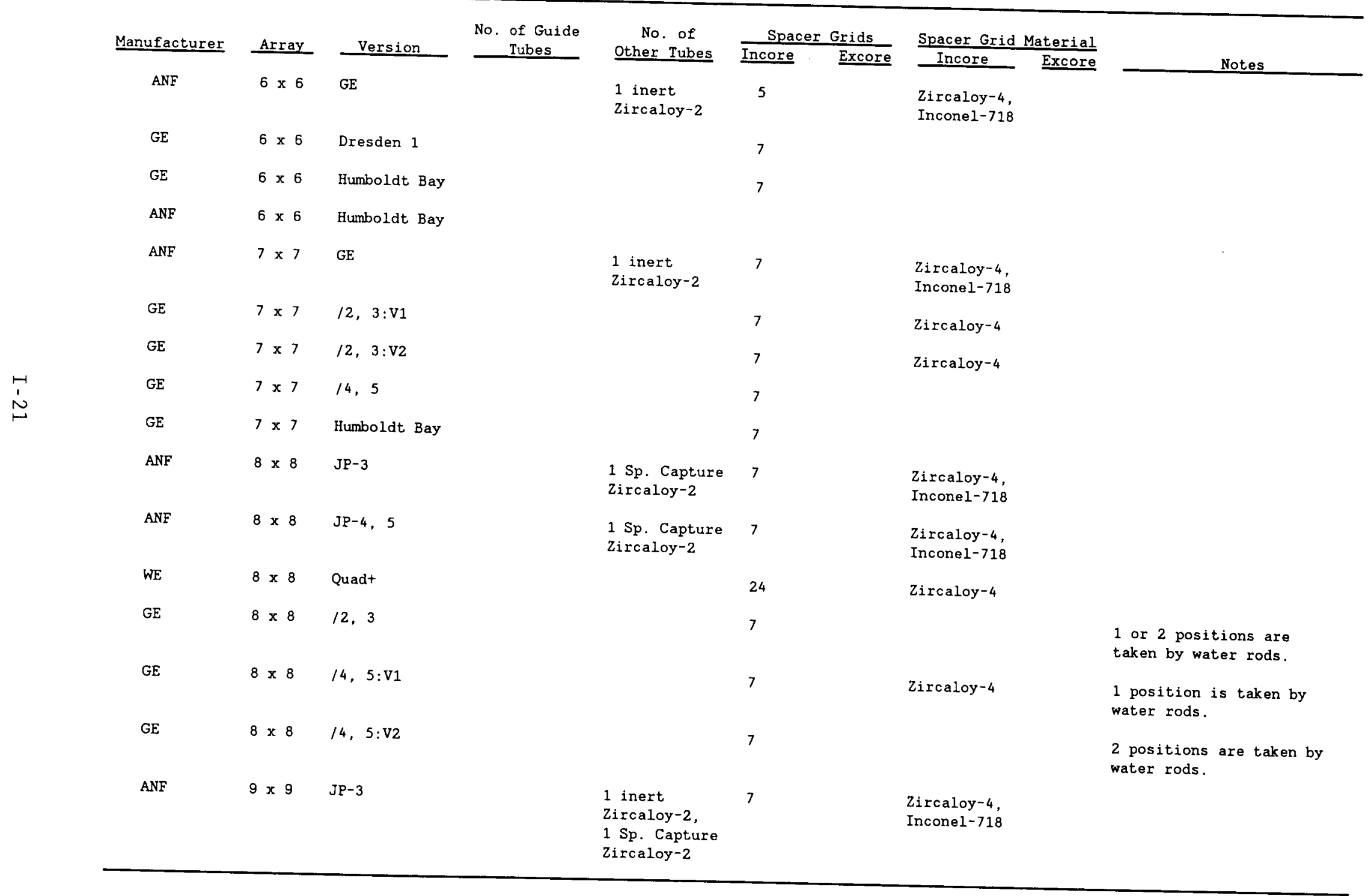


Table I- 6

Guide Tube and Spacer Grid Data for LWR Assemblies [N087] (Continued)

\begin{tabular}{|c|c|c|c|c|c|c|c|c|c|}
\hline \multirow[b]{2}{*}{ Manufacturer } & \multirow[b]{2}{*}{ Array } & \multirow[b]{2}{*}{ Version } & \multirow{2}{*}{$\begin{array}{l}\text { No. of Guide } \\
\text { Tubes } \\
\end{array}$} & \multirow{2}{*}{$\begin{array}{c}\text { No. of } \\
\text { Other Tubes } \\
\end{array}$} & \multicolumn{2}{|c|}{ Spacer Grids } & \multicolumn{2}{|c|}{ Spacer Grid Material } & \multirow[b]{2}{*}{ Notes } \\
\hline & & & & & Incore & Excore & Incore & Excore & \\
\hline ANF & $9 \times 9$ & $J P-4,5$ & & $\begin{array}{l}1 \text { inert } \\
\text { Zircaloy-2, } \\
1 \text { Sp. Capture } \\
\text { Zircaloy-2 }\end{array}$ & 7 & & $\begin{array}{l}\text { Zircaloy-4, } \\
\text { Inconel-718 }\end{array}$ & & \\
\hline GE & $9 \times 9$ & BRP & & & & & & & \\
\hline ANF & $9 \times 9$ & $\mathrm{BRP}$ & & & & & & & \\
\hline $\mathrm{AC}$ & $10 \times 10$ & & & & 3 & & $\begin{array}{l}\text { SS, } \\
\text { Zircaloy-4 }\end{array}$ & & $\begin{array}{l}3 \text { insert rods and a } \\
\text { spacer capture rod. }\end{array}$ \\
\hline ANF & $10 \times 10$ & $\mathrm{AC}$ & & $\begin{array}{l}3 \text { inert SS-ANF, } \\
\text { Inconel X-750, } \\
1 \text { Sp. Capture } \\
\text { SS-ANF, } \\
\text { Inconel X-750 }\end{array}$ & 3 & & $\begin{array}{l}\text { SS-ANF, } \\
\text { Inconel } X-750\end{array}$ & & \\
\hline ANF & $11 \times 11$ & GE & & $\begin{array}{l}3 \text { inert } \\
\text { nonfueled } \\
\text { Zircaloy-2, } \\
\text { Inconel X-750, } \\
1 \text { unfueled Sp. } \\
\text { Capture } \\
\text { Zircaloy-2 }\end{array}$ & 3 & & $\begin{array}{l}\text { Zircaloy }-4 \\
\text { Inconel-718 }\end{array}$ & & \\
\hline $\mathrm{GE}$ & $11 \times 11$ & BRP & & & & & & & \\
\hline WE & $13 \times 13$ & & & & & & & & \\
\hline$B \& W$ & $14 \times 14$ & & & & & & & & \\
\hline $\mathrm{CE}$ & $14 \times 14$ & Standard & 5 Zircaloy -4 & & $\begin{array}{l}7 \\
1 \text { lower }\end{array}$ & 1 plenum & $\begin{array}{l}\text { Zircaloy }-4 \\
\text { Inconel- } 625\end{array}$ & Zircaloy -4 & $\begin{array}{l}\text { Extra-large guide tubes. } \\
\text { Each tube is } \\
\text { approximately } 2.5 \mathrm{~cm} \text { in } \\
\text { diameter and displaces } 4 \\
\text { fuel rod positions. }\end{array}$ \\
\hline
\end{tabular}


Table I-6

Guide Tube and Spacer Grid Data for LWR Assemblies [NO87] (Continued)

\begin{tabular}{|c|c|c|c|c|c|c|c|c|c|}
\hline & & & No. of Guide & No. of & Space & Grids & Spacer Grid & Material & \\
\hline Manufacturer & Array & Version & Tubes & Other Tubes & Incore & Excore & Incore & Excore & Notes \\
\hline $\mathrm{CE}$ & $14 \times 14$ & Fort Calhoun & 5 Zircaloy -4 & & $\begin{array}{l}7 \\
1 \text { lower }\end{array}$ & 1 plenum & $\begin{array}{l}\text { Zircaloy }-4 \\
\text { Inconel- } 625\end{array}$ & Zircaloy -4 & $\begin{array}{l}\text { Extra-large guide tubes. } \\
\text { Each tube is } \\
\text { approximately } 2.5 \mathrm{~cm} \text { in } \\
\text { diameter and displaces } 4 \\
\text { fuel rod positions. }\end{array}$ \\
\hline ANF & $14 \times 14$ & WE & $\begin{array}{l}16 \\
\text { Zircaloy }-4\end{array}$ & $\begin{array}{l}1 \text { Instrument, } \\
\text { 2ircaloy }-4\end{array}$ & $\begin{array}{l}6 \text { vaned, } \\
2 \text { non- } \\
\text { vaned }\end{array}$ & 1 plenum & $\begin{array}{l}\text { Zircaloy-4, } \\
\text { Inconel-718 }\end{array}$ & $\begin{array}{l}\text { Zircaloy-4, } \\
\text { Inconel-718 }\end{array}$ & \\
\hline ANF & $14 \times 14$ & $\mathrm{CE}$ & $\begin{array}{l}5 \\
\text { Zircaloy }-4\end{array}$ & & 8 & & $\begin{array}{l}\text { Zircaloy-4, } \\
\text { Inconel-718 }\end{array}$ & & \\
\hline ANF & $14 \times 14$ & Top Rod & $\begin{array}{l}16 \\
\text { Zircaloy }-4\end{array}$ & $\begin{array}{l}1 \text { Instrument, } \\
\text { Zircaloy }-4\end{array}$ & $\begin{array}{l}3 \text { vaned, } \\
3 \text { non- } \\
\text { vaned }\end{array}$ & 1 plenum & $\begin{array}{l}\text { Zircaloy-4, } \\
\text { Inconel-718 }\end{array}$ & $\begin{array}{l}\text { Zircaloy }-4, \\
\text { Inconel-718 }\end{array}$ & \\
\hline ANF & $14 \times 14$ & Std/ZCA & 17 SS-304 & & 6 & 1 plenum & Inconel-718 & $\begin{array}{l}\text { Inconel-718, } \\
\text { West. Braze }\end{array}$ & \\
\hline WE & $14 \times 14$ & OFA & $\begin{array}{l}17 \\
Z_{\text {ircaloy }-4}\end{array}$ & & $\begin{array}{l}5 \\
1 \text { lower }\end{array}$ & & $\begin{array}{l}\text { Zircaloy-4, } \\
\text { Inconel-718 }\end{array}$ & & \\
\hline WE & $14 \times 14$ & Std/ZCB & $\begin{array}{l}17 \\
\text { Zircaloy }-4\end{array}$ & & 6 & 1 plenum & Incone1-718 & $\begin{array}{l}\text { Inconel-718, } \\
\text { West. Braze }\end{array}$ & \\
\hline WE & $14 \times 14$ & Std/SC & $16 \mathrm{SS}-304$ & & 6 & 1 plenum & Inconel-718 & Inconel-718 & \\
\hline WE & $14 \times 14$ & Model C & $\begin{array}{l}5 \\
\text { Zircaloy-4 }\end{array}$ & & 8 & 1 plenum & Incone1-718 & Incone1-718 & $\begin{array}{l}8 \text { grid sleeves SS-304. } \\
\text { The spacer grids are } \\
\text { brazed to SS grid } \\
\text { sleeves to fix their } \\
\text { position along the guide } \\
\text { tubes. }\end{array}$ \\
\hline ANF & $14 \times 14$ & Fort Calhoun & & & & & $\begin{array}{l}\text { Zircaloy-4, } \\
\text { Inconel- } 7188\end{array}$ & & \\
\hline
\end{tabular}


Table I-6

Guide Tube and Spacer Grid Data for LWR Assemblies [N087]

(Continued)

\begin{tabular}{|c|c|c|c|c|c|c|c|c|c|}
\hline & & & No. of Guide & No. of & Spacer & $\underline{r}$ Grids & Spacer Grid & Material & \\
\hline Manufacturer & Array & Version & Tubes & Other Tubes & Incore & Excore & Incore & Excore & Notes \\
\hline$B \& W$ & $15 \times 15$ & Mark B & $\begin{array}{l}16 \\
\text { Zircaloy } 4\end{array}$ & $\begin{array}{l}1 \text { Instrument } \\
\text { Zircaloy }-4\end{array}$ & 6 & $\begin{array}{l}1 \text { plenum, } \\
1 \text { bottom }\end{array}$ & Incone $1-718$ & $\begin{array}{l}\text { Incone1-718, } \\
\text { Incone1-718 }\end{array}$ & $\begin{array}{l}7 \text { grid supports in core } \\
\text { Zircaloy } 4 .\end{array}$ \\
\hline$B £ W$ & $15 \times 15$ & St. Steel & $20 S S-304$ & $\begin{array}{l}1 \text { Instrument } \\
\text { SS-304 }\end{array}$ & 6 & 1 plenum & Inconel-718 & Inconel-718 & \\
\hline $\mathrm{CE}$ & $15 \times 15$ & Palisades & $\begin{array}{l}8 \text { guide bars } \\
\text { solid } \\
\text { Zircaloy- } 4\end{array}$ & $\begin{array}{l}1 \text { Instrument } \\
z_{\text {ifcaloy }-4}\end{array}$ & $\begin{array}{l}8 \\
1 \text { lower }\end{array}$ & 1 plenum & $\begin{array}{l}\text { Zircaloy }-4, \\
\text { Inconel-625 }\end{array}$ & Zircaloy -4 & $\begin{array}{l}\text { No guide tubes. Single, } \\
\text { centrally located } \\
\text { instrument tube. Struc- } \\
\text { tural support provided } \\
\text { by guide bars, two on } \\
\text { each side of the } \\
\text { assembly. }\end{array}$ \\
\hline ANF & $15 \times 15$ & WE & $\begin{array}{l}20 \\
\text { Zircaloy }-4\end{array}$ & $\begin{array}{l}1 \text { Instrument } \\
\mathrm{Z} \text { ircaloy }-4\end{array}$ & $\begin{array}{l}5 \text { vaned, } \\
1 \text { non- } \\
\text { vaned }\end{array}$ & 1 plenum, & $\begin{array}{l}\text { Zircaloy }-4 \\
\text { Inconel-718 }\end{array}$ & $\begin{array}{l}\text { Zircaloy }-4 \\
\text { Inconel }-718\end{array}$ & \\
\hline ANF & $15 \times 15$ & $\mathrm{CE}$ & $\begin{array}{l}8 \text { guide bars } \\
\text { Zircaloy }-4\end{array}$ & $\begin{array}{l}1 \text { Instrument } \\
\mathrm{Z}_{\text {ircaloy }-4}\end{array}$ & 9 & 1 plenum & $\begin{array}{l}\text { Zircaloy } 4 \\
\text { Inconel- } 718\end{array}$ & $\begin{array}{l}\text { Zircaloy }-4 \\
\text { Inconel-718 }\end{array}$ & \\
\hline WE & $15 \times 15$ & Std/ZC & $\begin{array}{l}21 \\
\text { Zircaloy }-4\end{array}$ & & 6 & 1 plenum & Inconel-718 & $\begin{array}{l}\text { Incone1-718, } \\
\text { West. Braze }\end{array}$ & $\begin{array}{l}7 \text { grid sleeves SS-304. } \\
1 \text { guide tube may be a } \\
\text { centrally located } \\
\text { instrument tube. }\end{array}$ \\
\hline WE & $15 \times 15$ & OFA & $\begin{array}{l}21 \\
\text { Zircaloy }-4\end{array}$ & & $\begin{array}{l}5 \\
1 \text { lower }\end{array}$ & 1 plenum & $\begin{array}{l}\text { Zircaloy-4, } \\
\text { Incone1-718 }\end{array}$ & Incone1-718 & $\begin{array}{l}1 \text { guide tube may be a } \\
\text { centrally located } \\
\text { instrument tube. } 5 \\
\text { intermediate grid } \\
\text { assemblies. }\end{array}$ \\
\hline WE & $15 \times 15$ & $\mathrm{Std} / \mathrm{SC}$ & $21 \mathrm{SS}-304$ & & 6 & 1 plenum & Incone1-718 & $\begin{array}{l}\text { Inconel-718, } \\
\text { West. Braze }\end{array}$ & $\begin{array}{l}1 \text { guide tube may be a } \\
\text { centrally located } \\
\text { instrument tube. }\end{array}$ \\
\hline
\end{tabular}


Table I-6

Guide Tube and Spacer Grid Data for LWR Assemblies [NO87] (Continued)

\begin{tabular}{|c|c|c|c|c|c|c|c|c|c|}
\hline \multirow[b]{2}{*}{ Manufacturer } & \multirow[b]{2}{*}{ Array } & \multirow[b]{2}{*}{ Version } & \multirow{2}{*}{$\begin{array}{l}\text { No. of Guide } \\
\text { Tubes } \\
\end{array}$} & \multirow{2}{*}{$\begin{array}{c}\text { No. of } \\
\text { Other Tubes } \\
\end{array}$} & \multicolumn{2}{|c|}{ Spacer Grids } & \multicolumn{2}{|c|}{ Spacer Grid Material } & \multirow[b]{2}{*}{ Notes } \\
\hline & & & & & Incore & Excore & Incore & Excore & \\
\hline B\&W & $15 \times 15$ & Mark BZ & $\begin{array}{l}16 \\
\text { Zircaloy-4 }\end{array}$ & 1 Instrument & 6 & $\begin{array}{l}1 \text { bottom } \\
1 \text { plenum }\end{array}$ & Zircaloy -4 & $\begin{array}{l}\text { Incone1-718 } \\
\text { Incone1-718 }\end{array}$ & $\begin{array}{l}\text { Replaces the inter- } \\
\text { mediate six Inconel } \\
\text { spacer grids with } \\
\text { Zircaloy-4 spacers. } \\
7 \text { grid supports in } \\
\text { core Zircaloy } 4 .\end{array}$ \\
\hline ANF & $15 \times 16$ & WE & $\begin{array}{l}8 \text { guide bars } \\
\text { Zircaloy }-4\end{array}$ & $\begin{array}{l}1 \text { Instrument } \\
\text { Zircaloy }-4\end{array}$ & 5 & 1 plenum & $\begin{array}{l}\text { Zircaloy } 4, \\
\text { Inconel-718 }\end{array}$ & $\begin{array}{l}\text { Zircaloy }-4 \\
\text { Inconel- } 718\end{array}$ & \\
\hline WE & $15 \times 16$ & & $\begin{array}{l}8 \text { guide bars } \\
Z \text { ircaloy }-4\end{array}$ & $\begin{array}{l}1 \text { Instrument } \\
\text { Zircaloy- } 4\end{array}$ & & & & & $\begin{array}{l}8 \text { Zircaloy }-4 \text { solid guide } \\
\text { bars and a single, } \\
\text { centrally located } \\
\text { instrument tube. }\end{array}$ \\
\hline $\mathrm{CE}$ & $15 \times 16$ & Yankee Rowe & $\begin{array}{l}8 \text { guide bars } \\
\text { Zircaloy-4 }\end{array}$ & $\begin{array}{l}1 \text { Instrument } \\
\text { 2ircaloy }-4\end{array}$ & 1 lower & 1 plenun & $\begin{array}{l}\text { Zircaloy }-4, \\
\text { Inconel-625 }\end{array}$ & Zircaloy -4 , & \\
\hline ANE & $15 \times 16$ & Yankee Rowe & & & 5 & 1 plenum & $\begin{array}{l}\text { Zircaloy-4, } \\
\text { Inconel-718 }\end{array}$ & $\begin{array}{l}\text { Zircaloy }-4, \\
\text { Inconel-718 }\end{array}$ & \\
\hline CE & $16 \times 16$ & Onofre & $\begin{array}{l}5 \\
2 \text { ircaloy }-4\end{array}$ & & 9 & $\begin{array}{l}1 \text { plenum } \\
1 \text { lower } \\
\text { end } \\
\text { region }\end{array}$ & Zircaloy -4 & $\begin{array}{l}\text { Zircaloy }-4 \\
\text { Inconel-625 }\end{array}$ & $\begin{array}{l}5 \text { extra-large guide } \\
\text { tubes in a square array. } \\
\text { Displaces } 4 \text { fuel rod } \\
\text { positions. }\end{array}$ \\
\hline $\mathrm{CE}$ & $16 \times 16$ & St. Lucie/2 & $\begin{array}{l}5 \\
\text { Zircaloy }-4\end{array}$ & & 8 & $\begin{array}{l}1 \text { plenum } \\
1 \text { lower } \\
\text { end } \\
\text { region } \\
\text { core }\end{array}$ & $Z_{\text {ircaloy }-4}$ & $\begin{array}{l}\text { Zircaloy-4 } \\
\text { Inconel-625 }\end{array}$ & $\begin{array}{l}5 \text { extra-large guide } \\
\text { tubes in a square array. } \\
\text { Displaces } 4 \text { fuel rod } \\
\text { positions. }\end{array}$ \\
\hline $\mathrm{CE}$ & $16 \times 16$ & $\mathrm{ANO} / 2$ & $\begin{array}{l}5 \\
\text { Zircaloy }-4\end{array}$ & & 10 & $\begin{array}{l}1 \text { plenum } \\
1 \text { lower } \\
\text { end } \\
\text { region }\end{array}$ & Zircaloy -4 & $\begin{array}{l}\text { Zircaloy-4 } \\
\text { Incone1-625 }\end{array}$ & $\begin{array}{l}5 \text { extra-large guide } \\
\text { tubes in a square array. } \\
\text { Displaces } 4 \text { fuel rod } \\
\text { positions. }\end{array}$ \\
\hline
\end{tabular}


Table I- 6

Guide Tube and Spacer Grid Data for LWR Assemblies [N087] (Concluded)

\begin{tabular}{|c|c|c|c|c|c|c|c|c|c|}
\hline \multirow[b]{2}{*}{ Manufacturer } & \multirow[b]{2}{*}{ Array } & \multirow[b]{2}{*}{ Version } & \multirow{2}{*}{$\begin{array}{l}\text { No. of Guide } \\
\text { Tubes }\end{array}$} & \multirow{2}{*}{$\begin{array}{c}\text { No. of } \\
\text { Other Tubes } \\
\end{array}$} & \multicolumn{2}{|c|}{ Spacer Grids } & \multicolumn{2}{|c|}{ Spacer Grid Material } & \multirow[b]{2}{*}{ Notes } \\
\hline & & & & & Incore & Excore & Incore & Excore & \\
\hline $\mathrm{CE}$ & $16 \times 16$ & System 80 & $\begin{array}{l}5 \\
\text { Zircaloy-4 }\end{array}$ & & 9 & $\begin{array}{l}1 \text { plenum } \\
1 \text { lower } \\
\text { end } \\
\text { region }\end{array}$ & Zircaloy -4 & $\begin{array}{l}\text { Zircaloy }-4 \\
\text { Inconel- } 625\end{array}$ & $\begin{array}{l}5 \text { extra-large guide } \\
\text { tubes in a square array. } \\
\text { Displaces } 4 \text { fuel rod } \\
\text { positions. }\end{array}$ \\
\hline B\&W & $17 \times 17$ & Mark C & $\begin{array}{l}24 \\
\text { Zircaloy }-4\end{array}$ & $\begin{array}{l}1 \text { Instrument } \\
\text { Zircaloy }-4\end{array}$ & 6 & $\begin{array}{l}1 \text { bottom, } \\
1 \text { plenum }\end{array}$ & Inconel-718 & Incone1-718 & $\begin{array}{l}\text { Centrally located } \\
\text { instrument tube. Grids } \\
\text { are not welded into } \\
\text { place on the instrument } \\
\text { tube but are supported } \\
\text { by a series of grid } \\
\text { sleeves that surround } \\
\text { the instrument tube. }\end{array}$ \\
\hline ANF & $17 \times 17$ & WE & $\begin{array}{l}24 \\
\text { Zircaloy }-4\end{array}$ & $\begin{array}{l}1 \text { Instrument } \\
\text { Zircaloy }-4\end{array}$ & $\begin{array}{l}6 \text { vaned, } \\
1 \text { non- } \\
\text { vaned }\end{array}$ & 1 plenum & $\begin{array}{l}\text { Zircaloy }-4, \\
\text { Incone1-718 }\end{array}$ & $\begin{array}{l}\text { Zircaloy }-4, \\
\text { Inconel- } 718\end{array}$ & $\begin{array}{l}\text { May have } 10 \text { spacer } \\
\text { grids. }\end{array}$ \\
\hline WE & $17 \times 17$ & Standard & $\begin{array}{l}25 \\
\text { Zircaloy }-4\end{array}$ & & 6 & 1 plenum & Inconel-718 & $\begin{array}{l}\text { Inconel-718, } \\
\text { West. Braze }\end{array}$ & $\begin{array}{l}6 \text { grid sleeves } \mathrm{SS}-304 . \\
\text { May have centrally } \\
\text { located instrument tube. }\end{array}$ \\
\hline WE & $17 \times 17$ & OFA & $\begin{array}{l}24 \\
\text { Zircaloy-4 }\end{array}$ & & $\begin{array}{l}5 \\
1 \text { lower }\end{array}$ & 1 plenum & $\begin{array}{l}\text { Zircaloy-4 } \\
\text { Inconel- } 718\end{array}$ & $\begin{array}{l}\text { Incone } 1-718 \\
\text { West. Braze }\end{array}$ & $\begin{array}{l}1 \text { grid sleeve in gas } \\
\text { plenum SS-304. I B. } \\
\text { grid sleeve in core SS- } \\
304 \text {. May have centrally } \\
\text { located instrument tube. }\end{array}$ \\
\hline WE & $17 \times 17$ & Vantage 5 & $\begin{array}{l}24 \\
\text { Zircaloy-4 }\end{array}$ & $\begin{array}{l}1 \text { Instrument } \\
\text { Zircaloy }-4\end{array}$ & 6 lower & 1 plenum & $\begin{array}{l}\text { Zircaloy }-4 \\
\text { Inconel-718 }\end{array}$ & Inconel-718 & \\
\hline WE & $17 \times 17$ & XLR & & & & & & & \\
\hline
\end{tabular}




\section{I.3 Pretransport Spent-Fuel Characteristics}

\section{I.3.1 Fue1 Rod Breach Behavior}

Some fuel rods begin transport with breached cladding. The radionuclide release from these rods during transport differs from intact rods and depends on characteristics of the failure. Section I.3.1.1 discusses in-core cladding breaches due to hydriding, fretting, baffle jetting, PCI, and corrosion. This includes a description of the mechanism, breach characteristics, and statistics. The behavior of waterlogged fuel is also discussed. Section 1.3.1.2 discusses fuel rod breaches and cladding damage that occurs during handling and storage. Section I.3.1.3 discusses the amount of defective fuel in storage.

\section{I.3.1.1 In-Reactor Fuel Rod Cladding Breaches}

The overall materials performance of LWR fuel rods is excellent despite sporadic events involving cladding breaching or fuel rod damage. A cladding breach is defined as a loss of fission gas containment. In-core damage is defined as any structural change in a fuel rod or assembly that results in abnormal maintenance, early discharge of the assembly, or replacement of its component parts. In the 1970s, fuel breaches were associated with cladding hydriding and PCI. In the 1980s, in-reactor breaches were also caused by baffle jetting (associated with changes in assembly designs), flow and debris-induced fretting, and cladding corrosion [including CRUD-induced localized corrosion (CILC)]. In-core reliability increased in the $1980 \mathrm{~s}$ as a result of improved fuel design and plant operating procedures, and better control of the coolant water chemistry.

\section{Hydriding Breaches}

Fuel rod breaching caused by hydriding has been discussed extensively by Garzarolli et a1. [GA79], Locke [L074], and Bailey and Wu [BA88a]. Primary hydriding results from residual moisture in the fuel pellet. Hydrogeneous impurities attack the inside cladding surface and form local hydride blisters up to $0.64 \mathrm{~cm}$ in diameter [LO74]. These blisters, which resemble sunbursts, are weak spots in the cladding that may eventually burst. Improvements in fuel fabrication techniques have reduced the moisture content in pellets and subsequently reduced the occurrence of rod breach due to internal hydriding.

A second source of hydrogen results from corrosion of the Zircaloy cladding. Approximately 108 of the hydrogen produced by corrosion diffuses into the cladding [PY85]. At current burnup levels, the hydrogen level in cladding of $\leq 150 \mathrm{ppm}$ does not significantly degrade Zircaloy mechanical properties. Secondary hydriding occurs in rods that have been ruptured by other means. Primary coolant water enters the fuel cladding gap, where radiolysis and corrosion produce hydrogen that diffuses into the cladding. Massive localized hydriding leads to hydride blisters and deterioration of cladding mechanical properties; additional cladding perforations can occur at the blisters [GA79]. 
Hydriding was the dominant breach mechanism in BWRs in the early 1970s. Twenty-five BWR plants had hydriding breaches during this period [GA79]. The amount of BWR rods affected by hydriding peaked at $\sim 0.58$ in 1969. By 1977, the rod breach level due to hydriding had dropped to less than 0.018 . Changes in manufacturing procedures to reduce the as manufactured moisture content of fuel have significantly reduced the amount of primary hydriding breaches. Breach levels for PWRs during the same period dropped from 0.35 to 0.018 ; only five plants were affected. Six PWR fuel rods breached by hydriding in 1982 [BA84a], and less than five fuel assembly breaches by hydriding were observed in 1985 [BA88a]. No hydriding-caused fuel breaches were reported in 1986.

\section{Fretting Breaches}

Debris-induced fretting is the mechanical erosion of the exterior fuel rod cladding wall caused by the flow-induced impact of metallic debris from the coring of plugged steam generators. Several debris-induced fretting breaches in PWRs have occurred since 1980 (see McDonald and Kaiser [MC85] or Sofer et al. [S088] for a picture of debris fretting). These incidents are summarized in Table I-7 [BA88a]. The estimated fractions of fuel rod breaches range from 0.038 in 1983 to $7 \times 10^{-48}$ in 1986.

Table I - 7

Breaches Due to Debris-Induced Fretting in PWRs

\begin{tabular}{lcc}
\hline Year & $\begin{array}{c}\text { Breached } \\
\text { Rods }\end{array}$ & $\begin{array}{c}\text { Breached } \\
\text { Assemblies }\end{array}$ \\
\cline { 2 - 2 } 1983 & 122 & 77 \\
1984 & a & 21 \\
1986 & 3 & 3
\end{tabular}

a Number unknown.

Flow-induced fretting [BA88a] is caused by flow-induced vibration of fuel rods against spacer grids. Grid-to-rod fretting typically occurs along the bottom grid in a fuel assembly because of the increased vibration caused by inlet flow turbulence. Five PWR fretting incidents were reported from 1971 to 1977, and two BWR incidents occurred from 1969 to 1971 [GA79]. The associated rod breach fractions are approximately 0.05 to 0.018 for BWRs and 0.01 to $0.04 \%$ for PWRs. Flow-induced fretting breaches occurred in two PWR power plants [two fuel rods in Yankee Rowe ( $C-E$ ) and one rod in Salem-2 (WE)] during 1985 and 1986. The estimated rod breach fraction is negligibly small (1.0 x 10-5 


\section{Baffle Jetting Breaches}

Fuel rod breaches are caused by excessive rod vibration from coolant jetting through baffle joints. These breaches were first noticed in WE PWRs in 1973 [BA88a]. Baffle jetting is caused by a coolant pressure differential across the baffle plate joints. Baffle jetting incidents since 1973 are listed in Table I-8 [BA88a]. In 1973, one rod had a 28-cm section of cladding missing between spacer grids. Two rods breached in 1978. One rod had a severed bottom and a $2.4-\mathrm{m}$-long axial split, and some fuel pellets were missing. In 1985, the fuel cladding on one rod was breached to the extent that pellets could be seen through a hole in the cladding. In 1986, video inspections revealed that several rods in a McGuire-1 fuel assembly were damaged by baffle jetting. Four intact pellets and pieces of other pellets were found in the core.

Table I-8

PWR Fuel Rods Breached Due to Baffle Jetting [BA88a]

\begin{tabular}{lcc}
\hline Year & $\begin{array}{c}\text { Number of } \\
\text { Fuel Rods }\end{array}$ & $\begin{array}{c}\text { Number of } \\
\text { Fuel Assemblies }\end{array}$ \\
1973 & 3 & 1 \\
1976 & - & 1 \\
1978 & 2 & 1 \\
1980 & 2 & 2 \\
1981 & - & 2 \\
1982 & - & 17 \\
1983 & - & 11 \\
1984 & - & 1 \\
1985 & 8 & 10 \\
1986 & 18 & 3 \\
& & \\
\hline
\end{tabular}

Thirty-three breached rods were identified in 8 of the 49 assemblies suspected of baffle jetting damage, which was equal to approximately four rods per assembly. If it is assumed that each of the remaining 41 damaged assemblies also has four breached rods, 197 rods have breached due to baffle jetting. When the total existing PWR fuel inventory is considered (see Table I-9), -0.138 of the assemblies and $0.0025 \%$ of the rods have been damaged by baffle jetting.

Although the baffle jetting breach level is low, such damage is severe and may involve secondary hydriding breaches in damaged fuel assemblies. Special clips to bridge several fuel rods have been designed for ANF assemblies to reduce baffle jetting vibration [S085]. These clips are attached to peripheral rods of assemblies in core periphery locations known to be susceptible to baffle jetting. 
Table I-9

PWR Baffle Jetting Breach Statistics

\begin{tabular}{lcc}
\hline & Rods & Assemblies \\
$\begin{array}{l}\text { Number of units in operation } \\
\text { or discharged through 1986 }\end{array}$ & $8 \times 10^{6}$ & $3.7 \times 10^{4}$ \\
Number of breached units & 197 & 49 \\
Failure 8 & 0.0025 & 0.13 \\
\hline
\end{tabular}

\section{PCI Breaches}

LWR breaches can also occur as a result of PCI; that is, when the cladding is stressed by the mechanical interaction between the pellet and the cladding. The brittle nature of a PCI breach has been attributed to stress corrosion cracking that is caused by fission products such as iodine, cesium, or cadmium [CU80, DA84, SH83, SH84, CU78]. The potential for PCI breaching is a function of reactor operating conditions. PCI is associated with severe power ramping at moderate and high burnup levels and off-normal reactor operating conditions, such as anticipated transients without scram. Postirradiation examination of PCI defects from power reactors and power ramp experiments shows fine cracks in the interior cladding surface that are often associated with X-shaped markings on the cladding outer surface.

The first observation of PCI breach was in 1964, but after 1970, PCI was recognized as a performance-limiting effect. Through 1977, the estimated PCI fuel rod breach levels have been 0.01 to $0.06 \%$ in BWRs and 0.007 to $0.04 \%$ in PWRs [GA79]. Procedures involving modest power increases during startup were implemented at BWRs and PWRs to eliminate the PCI problem. Only one case of PCI rod breach has been identified since 1977: a sipping test in 1986 at the Oyster Creek BWR Plant revealed 47 fuel assemblies with cladding breaches probably caused by PCI [BA88a]. If it is assumed that at least two rods per assembly breached, the general breach level during 1986 in BWR fuel was -0.068 .

Fuel design changes have been proposed and developed to allow removal of administrative controls on fuel reactivity ramps while continuing to control PCI. The GE zirconium barrier fuel provides a materials solution to the PCI problem in BWRs [BA88a]. A thin layer of pure zirconium is metallurgically bonded to the inside of the Zircaloy-2 cladding. This soft layer absorbs most of the stress at the pellet-pellet interface, thus eliminating one condition (excessive stress) necessary for PCI. Other designs, such as the ANF $9 \times 9$ fuel and the WE Asea-Atom water-cross BWR fuel, also provide solutions to PCI by reducing the thermal gradients across the pellet [NY85]. PCI breach rates are not expected to increase in 
the future even with higher burnup fuel [BA89]. Partial through-the-wall cracks caused by PCI are discussed later in this appendix.

\section{Cladding Corrosion Breaches}

Fuel rod breaches due to CRUD deposition were reported in BWR plants from 1973 through 1976 at an average yearly level of 0.0028 [GA79]. In 1979 , approximately 75 rods breached in 31 fuel assemblies ( $8 \times 8$ design) in the Vermont Yankee BWR P1ant [BA81] as a likely result of excessive CRUD deposition. Also in 1979, approximately 25 rods in nine fuel assemblies in the Maine Yankee PWR (C-E) Plant breached, probably due to CRUD deposition [BA81]. The estimated rod breach level in 1979 was $0.05 \%$ for BWRs and $0.007 \%$ for PWRs.

Thirty-five fuel rod breaches as a result of CILC in 11 fuel assemblies were reported by the Hatch-1 BWR Power Plant in 1981 [BA82]. Full core sipping in 1982 revealed the presence of 31 leaking fuel assemblies in the Browns Ferry-2 BWR Plant [BA84a]. Nineteen leaking fuel assemblies were detected in the Hatch-2 BWR Plant in 1983 [BA84a]. CILC cladding breaches (see Bailey et al. [BA85a] for a picture of a CILC breach) occurred at burnup levels of 15 to 20 GWD/MTU and were highly concentrated in ( $U, G d$ ) $\mathrm{O}_{2}$ rods in high-power-density BWR plants with Cubearing condenser tubing and filter demineralizer cleanup systems [BA88a]. CILC CRUD is $2508 \mathrm{CuO}$ with $208 \mathrm{Fe}_{2} \mathrm{O}_{3}$, while standard CRUD is primarily $\mathrm{Fe}_{2} \mathrm{O}_{3}$ with minor amounts of $\mathrm{Cu}$ [BA85a]. If it is assumed that each breached assembly in Hatch-2 and Browns Ferry-2 has approximately three rod breaches per assembly (the same as Hatch-1 in 1981), the breach level for GE $8 \times 8$ rod arrays from 1981 through 1983 due to CILC ranged from 0.02 to 0.078 . Improvements in plant water chemistry and cleanup systems have reduced high concentrations of $\mathrm{Cu}$ in the reactor coolant water and subsequent CILC breaches.

\section{I.3.1.2 Damage During Handling and Storage}

Before transport, spent fuel is transferred out of the reactor core and placed in a storage pool. The fuel remains in this pool or is placed in dry storage. In either the reactor spent-fuel storage-water pool or in an MRS facility, the fuel may be consolidated to save space. The handling and residence of the assembly in storage can have an effect on the pretransport rod cladding condition.

\section{Reactor Pool Storage and Transfer Experience}

Pacific Northwest Laboratory (PNL) evaluated the condition of LWR spent fuel on arrival at interim storage, fuel reprocessing, and disposal facilities [BA83a]. Known damage to dropped or bumped assemblies was documented by Bailey and Wu [BA88a]. Observed incidents included spacer grid damage, nozzle damage, bent and bowed rods, deformed bails, scratches, nut and bolt damage, and other events. During handling operations, 34 fuel assemblies were dropped, 2 PWR fuel assemblies and 1 unidentified fuel assembly fell from a vertical to a horizontal position, and three PWR assemblies tilted from the vertical position and rested on another object. Fuel assemblies have been dropped 0.13 to $9.1 \mathrm{~m}$ in water. One assembly was 
dropped in water, and a momentary release of radioactivity occurred that became airborne.

Some fuel rods broke into two pieces during incidents involving BWR assemblies in 1982 [BA88a]. These are the only known incidents of handling-induced fuel breach. Approximately 27,446 assemblies have been discharged from BWRs as of 1986 [N087]. If the assemblies before 1979 are considered to be $7 \times 7$ and those after 1979 to be $8 \times 8$, and if it is assumed that the two damaged assemblies each contained at least two broken rods, then the handling-induced breach rate for BWR rods is $-0.0003 \%$. No rod breaches resulting from handling of irradiated PWR assemblies have been noted.

As of 1986, no cases of fuel breach resulting from wet storage have been observed. CRUD loosening and flaking from Oconee rods have been observed during extended pool storage.

\section{Spent-Fuel Consolidation}

Only one fuel rod breach has occurred as a result of spent-fuel consolidation. Wet consolidation of six fuel assemblies was performed at the West Valley Demonstration project in 1986 [W086]. Each assembly contained fuel rods from 1979 from the $R$. E. Ginna reactor. The cladding of one fuel rod was breached during rod pulling. Apparently, a flat portion of the rod was caught on a spacer grid, and the rod underwent clad collapse. Four PWR assemblies at Oconee, one PWR assembly at Maine Yankee, five PWR assemblies at Battelle Columbus Laboratories (BCL), six PWR assemblies at Millstone-2, and 36 PWR assemblies at Prairie Island have all been successfully consolidated with no resulting rod breach [GI88]. Dry rod consolidation of WE $15 \times 15$ spent-fuel assemblies was successfully completed at Idaho National Engineering Laboratory (INEL) in 1987 [VI88]. Rods from 48 Surry and Turkey Point PWR fuel assemblies were pulled without any resulting rod breaches; each contained 204 rods in the 25 GWD/MTU burnup range. The estimated rod breach level from these consolidation projects is $<0.005 \%$. Prediction of the breach rates that would occur in production line rod-consolidation activities is difficult based on the breach rates given above.

\section{Dry Storage}

Some fuel is stored in dry storage either at reactor sites or at an MRS facility before transport to a repository. The dry storage duration could range from a few years to up to $40 \mathrm{yr}$. The condition of fuel rod cladding when the fuel is loaded into transportation casks depends on changes in material properties or the physical condition of the cladding. Such changes could occur as a result of hydriding and crack propagation during the dry storage cycle. Extensive experimental analyses [EI84b, EI82, EI84a, OL85, J087, J086] and analytical evaluations [SA88, J083] have been conducted in recent years to identify cladding degradation mechanisms and characteristics, and to determine storage atmospheres and temperatures that will permit dry storage with minimum degradation. Mechanisms evaluated include creep rupture, stress-corrosion cracking, delayed hydrogen cracking and hydride reorientation, Zircaloy cladding oxidation, and the effects of CRUD and fuel oxidation. The extent of material 
degradation depends on storage temperature and duration, atmosphere, and rod stress that, in turn, depend on fuel design, time out of reactor, and fuel configuration (intact assemblies or consolidated fuel).

Based on these evaluations, a maximum dry storage temperature in the $380^{\circ} \mathrm{C}$ range in an inert atmosphere is recommended. The inert atmosphere prevents cladding oxidation, and if cladding breaches, fuel oxidation. The recommended temperature is based on data for fracture mechanisms as a function of temperature and stress. All dry storage degradation mechanisms except oxidation produce breaching by a slow crack growth mechanism. Any such defects are in the form of pinholes or cracks varying in size from 1 to $30 \mu \mathrm{m}$ [TA84]. These defects should not grow into a size that threatens gross rod integrity because the initial breach vents the internal pressure [YA80]. Under recommended dry storage conditions, delayed hydrogen cracking caused by hydrogen migration in the cladding to the crack tip would be secondary to stress corrosion cracking (SCC). However, the possibility of severely weakening the cladding by hydride reorientation has not been eliminated. A complete discussion of dry storage degradation mechanisms is provided by Sanders et al. [SA88].

As of 1983, only 1 in 5600 PWR rods has breached in short-term ( $<3$ yr) dry storage tests [J083], which is equivalent to a breach rate of 0.028 . These evaluations indicate that the breach probability for fuel rods in dry storage is low. An additional $9800 \mathrm{PWR}$ rods have been consolidated and are currently stored in dry storage casks [VI88]. These rods have not been evaluated for cladding breach. To date, no dry storage demonstrations have been conducted on BWR rods. The BWR rod breach rate is estimated to be less than or equal to the PWR rod breach rate because BWR rods have much lower internal pressures.

\section{Waterlogged Fuel Rods}

If a fuel rod loses its cladding integrity while in the core, the internal gas diffuses to the primary coolant. Depending on the size of the breach and subsequent reactor operating conditions, water could be forced into the cladding gap. Some of these rods would undergo secondary hydriding defects and some would be removed from the reactor core with water remaining in the gap. The amount of assemblies containing waterlogged rods is not known. Using ultrasonic inspection, Boehm and Foerch [B085] examined more than 160,000 German and Belgian fuel rods and found water in more than 300. Therefore, at least 0.198 of the rods had a defect large enough to admit water. No information concerning the inventory history was given; therefore, it is not possible to determine if the breach rate represents the inventory in general or a select group of assemblies.

The expected performance of waterlogged fuel rods under dry storage conditions was analyzed by Woodley [W084]. Woodley examined the possibilities of repressurizing the waterlogged fuel rod by resealing cladding defects, as well as cladding and fuel oxidation. Although many radiological reactions can take place, Woodley's analysis indicates that waterlogged rods would not degrade further if they are placed in dry storage. The probability of resealing was not determined. 
Several investigations have been conducted to determine if the water in waterlogged rods will vent during cask-drying operations. Two waterlogged BWR rods containing reactor-induced breaches and two intentionally defected rods (one PWR and one BWR rod) were tested in a hot cell at a heating rate simulating the temperature rise in a storage cask during pump-down and dry-out operations [K086]. In the intentionally defected rods, some fission gas $(0.5$ and 218 from the PWR and BWR rods, respectively) was released from the fuel when heated with water present. Approximately $50 \%$ of the water was removed at $100^{\circ} \mathrm{C}$ [GI85, K086], and the remainder was slowly released during storage or remained bound in the fuel. Essentially, all water was removed from the rod on heating to $400^{\circ} \mathrm{C}$.

The behavior of waterlogged PWR rods when heated to $400^{\circ} \mathrm{C}$ in a hot ce11 was studied by Peehs and Fleisch [PE86]. Approximately 3 to 6 grams of water were released during the simulated cask-drying operation. The presence of ${ }^{85} \mathrm{Kr}$ and ${ }^{3} \mathrm{H}$ was detected in the released moisture. Further tests using a vacuum drying procedure of $31 \mathrm{hr}$ duration removed all water from the rods [PE86]. The limited analytical and experimental work to date suggests that moisture is released from waterlogged rods during dry storage conditions without causing additional rod damage. The released water may be accompanied by some fission product release.

\section{I.3.1.3 Defective Fue1 in Storage}

Because evaluating rod breach fractions without considering yearly trends is misleading, an analysis of the rod breach rate by year was conducted. A defective fuel assembly contains one or more breached rods and/or damaged hardware. As of December 1985, a total of 27,446 BWR assemblies and 18,123 PWR assemblies have been discharged from licensed U.S. reactors. As shown in Figures $I-9$ and $I-10$, the rod breach level for various breach mechanisms decreased considerably with time as a result of improvements in fuel designs and reactor operations.

The breach level for each mechanism and its period of relevance are shown in Table I-10. A breach level (i.e., breach fraction/year) was determined using the trends for each mechanism in the period of relevance (see Figure I-11). Since 1978, the breach fraction for PWR rods has leveled off at $\sim 0.028$. Earlier fractions were much higher. BWR rates are similar, with some sporadically higher years. These breach levels are approximately an order of magnitude lower than those measured by Boehm and Foerch on foreign fuel [B085] using an ultrasonic probe. The cause of this discrepancy has not been investigated.

\section{I.3.2 Postirradiation Fue1 Pellet Condition}

During irradiation, the fuel pellets and cladding undergo physical and dimensional changes. The pellets crack during the startup ramp and swell or become more dense depending on the manufacturing technique used to fabricate them. Cladding on PWR rods creeps down on the pellets because of higher external coolant pressure. The fuel and cladding can interact chemically, bonding some fuel fragments to the cladding. Figure I-12 shows a typical cross section of an irradiated fuel rod ( 28 GWD/MTU). 


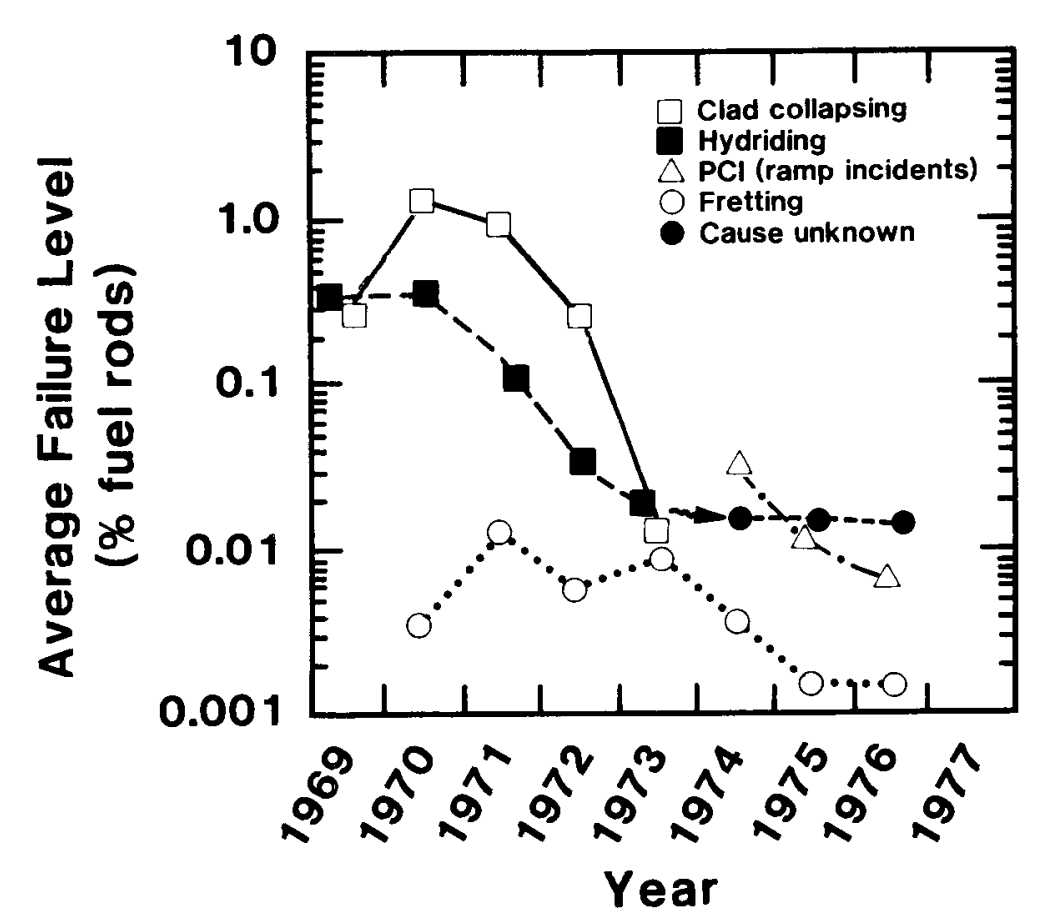

Figure I-9. Overall Significance of Fuel Breaches in PWR Plants [GA79]

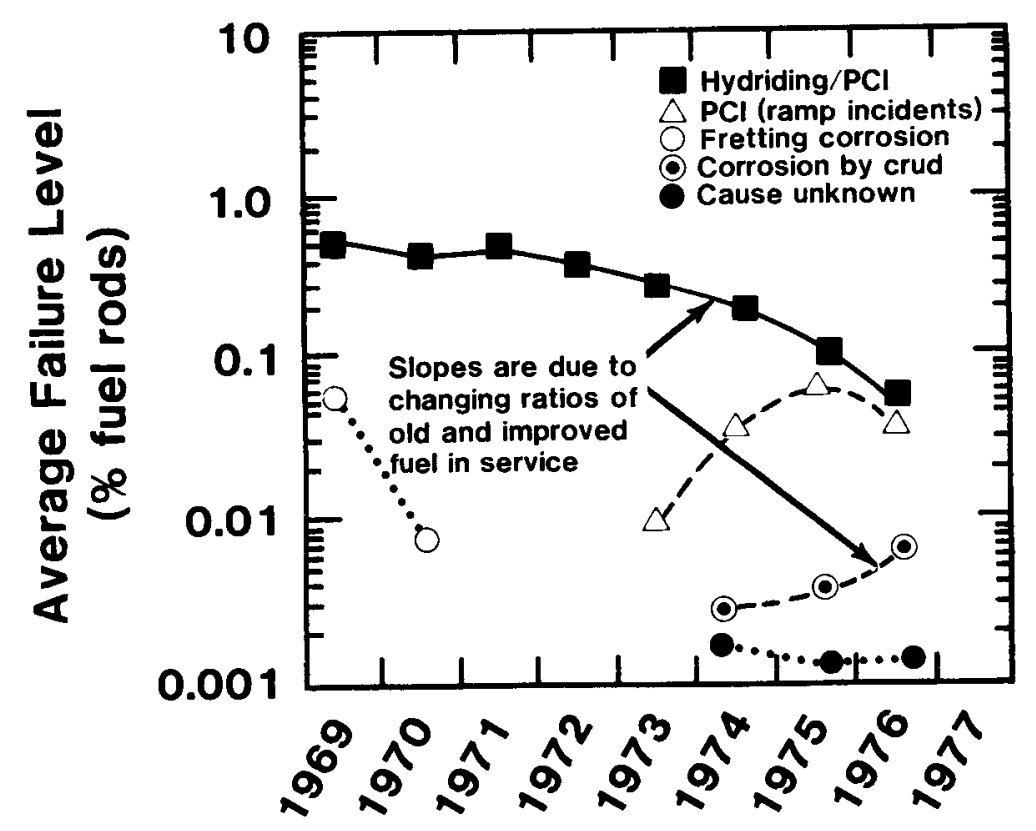

Year

Figure I-10. Overall Significance of Fuel Breaches in BWR Plants [GA79] 
Table I-10

Fuel Rod Breach Levels in BWRs and PWRs

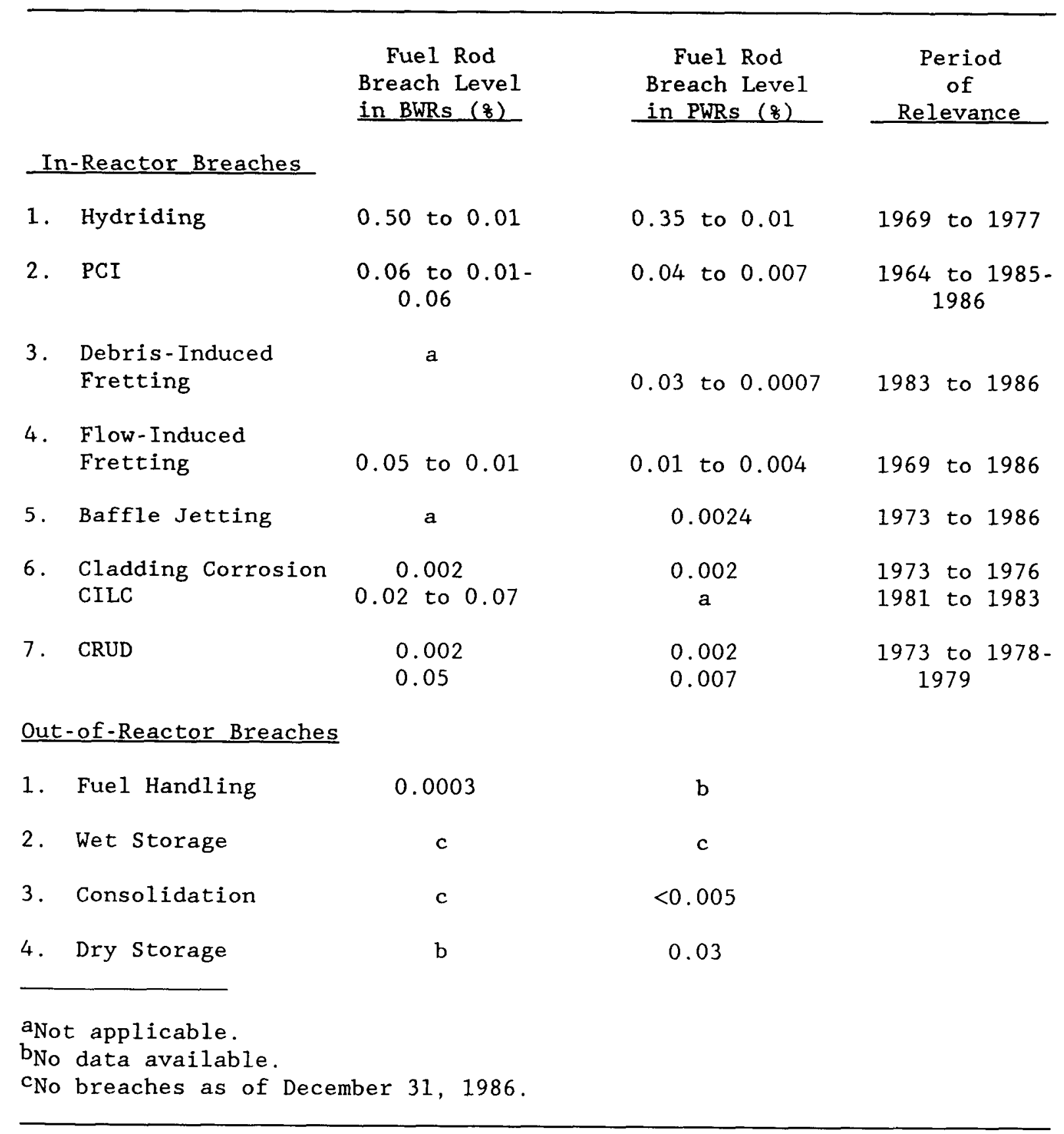




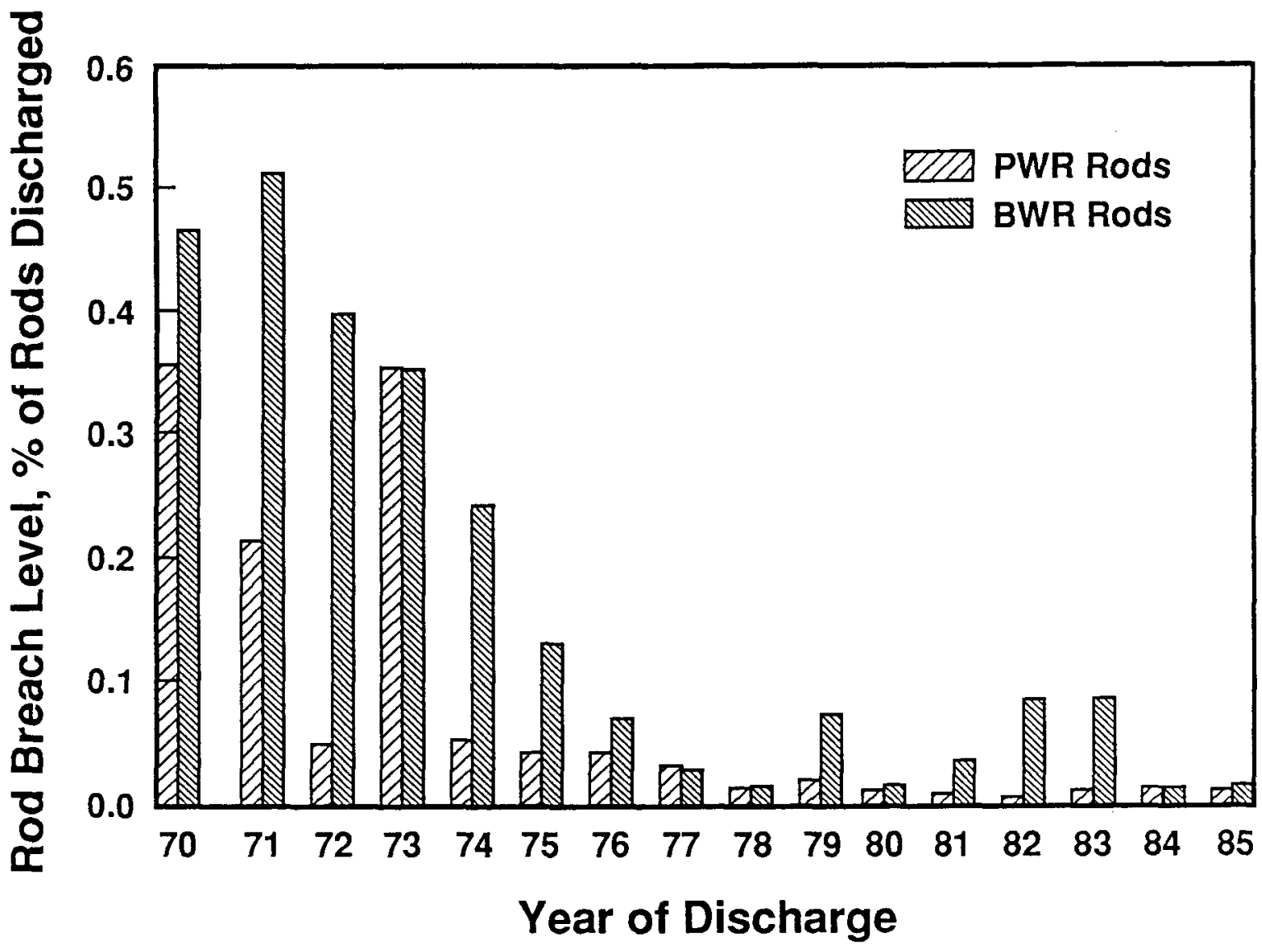

Figure I-11. Rod Breach Levels

Approximately eight to twelve cracks are in the general radial direction. Cracks also form in the longitudinal direction (see Figure I-13) so that a previously whole pellet consists of -20 to 40 interlocking pieces after irradiation. The pellet cracks during the first irradiation cycle and apparently does not crack further during subsequent cycles. Frequently, the sharp corners of the dished pellets are crushed into many small fragments (see Figure I-14).

Because of high external coolant pressure, the PWR cladding creeps down onto the pellets, closing the fuel-cladding gap, ovalizing the cladding, and forming ridges at the pellet-to-pellet interfaces where there is high stress from pellet contact. These effects are accentuated with each irradiation cycle. A typical profilometer trace (Figure I-15) of a PWR rod at 28 GWD/MTU shows both ovality and ridging. In this example, the ridge dimension is from 13 to $51 \mu \mathrm{m}$.

The combination of interlocked pellet fragments and ridging tends to hold the fragments in place. This is confirmed by the severe difficulty with which fuel from irradiated PWR cladding can be removed. Shaking and hammering will not remove it, and cladding splitting is necessary. 


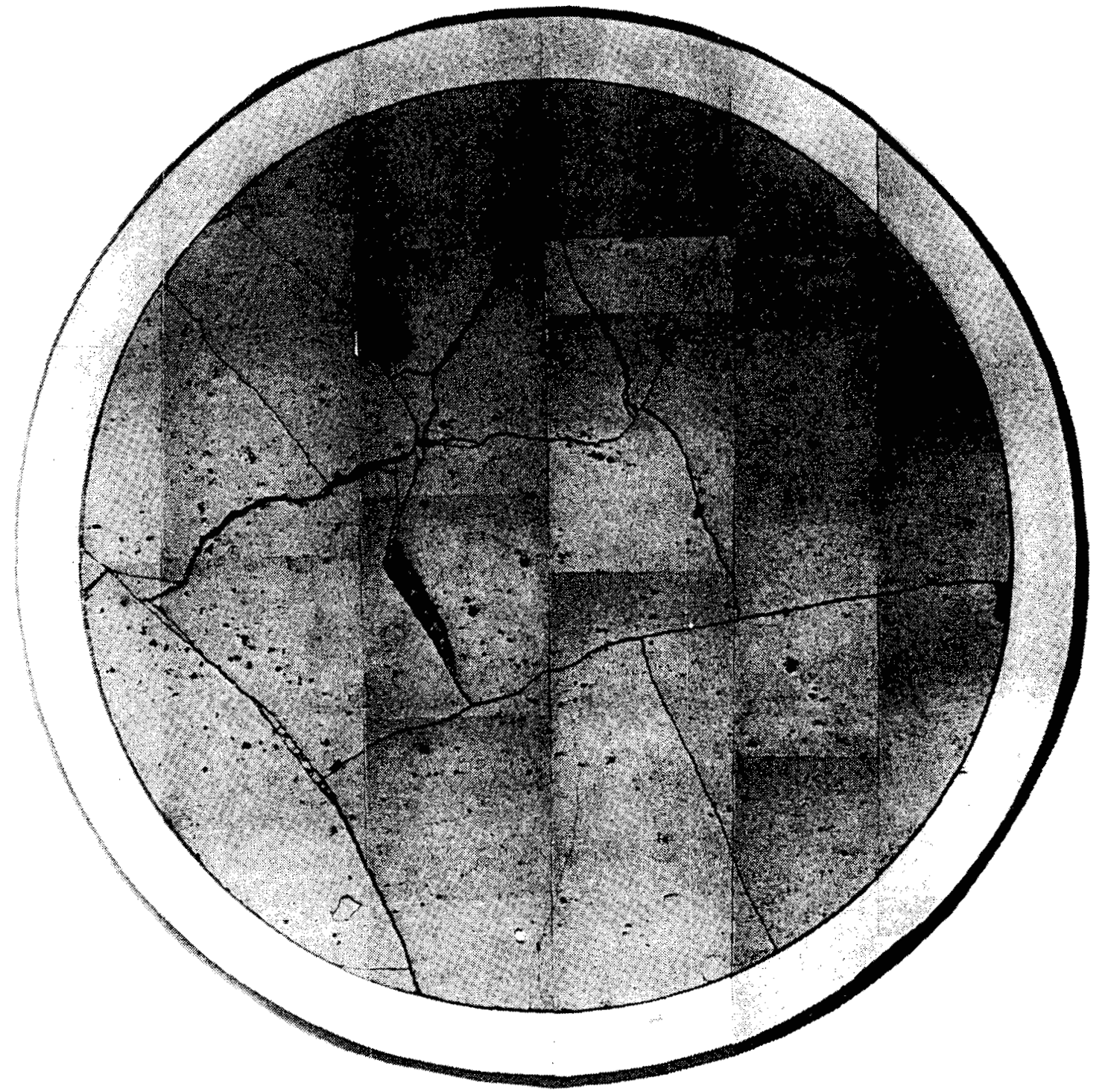

Figure I-12. Cross Section of As-Irradiated Turkey Point PWR Fuel Rod

Essentially 758 (for $14 \times 14$ assemblies) to 1008 (for $17 \times 17$ assemblies) of PWR fuel mechanically adheres to the cladding after a few cycles of irradiation.

BWR pellets also crack into 20 to 40 fragments during irradiation; radial and longitudinal cracks are formed (see Figures I-16 and I-17). The chambered corners of the pellets reduce small fragment formation. The cladding on BWR rods does not creep down and ridges are not formed. The gap does close during irradiation as a result of differential thermal expansion, so some fuel-cladding chemical bonding occurs. Because of the lack of ridging, BWR spent fuel is easily removed from the cladding 


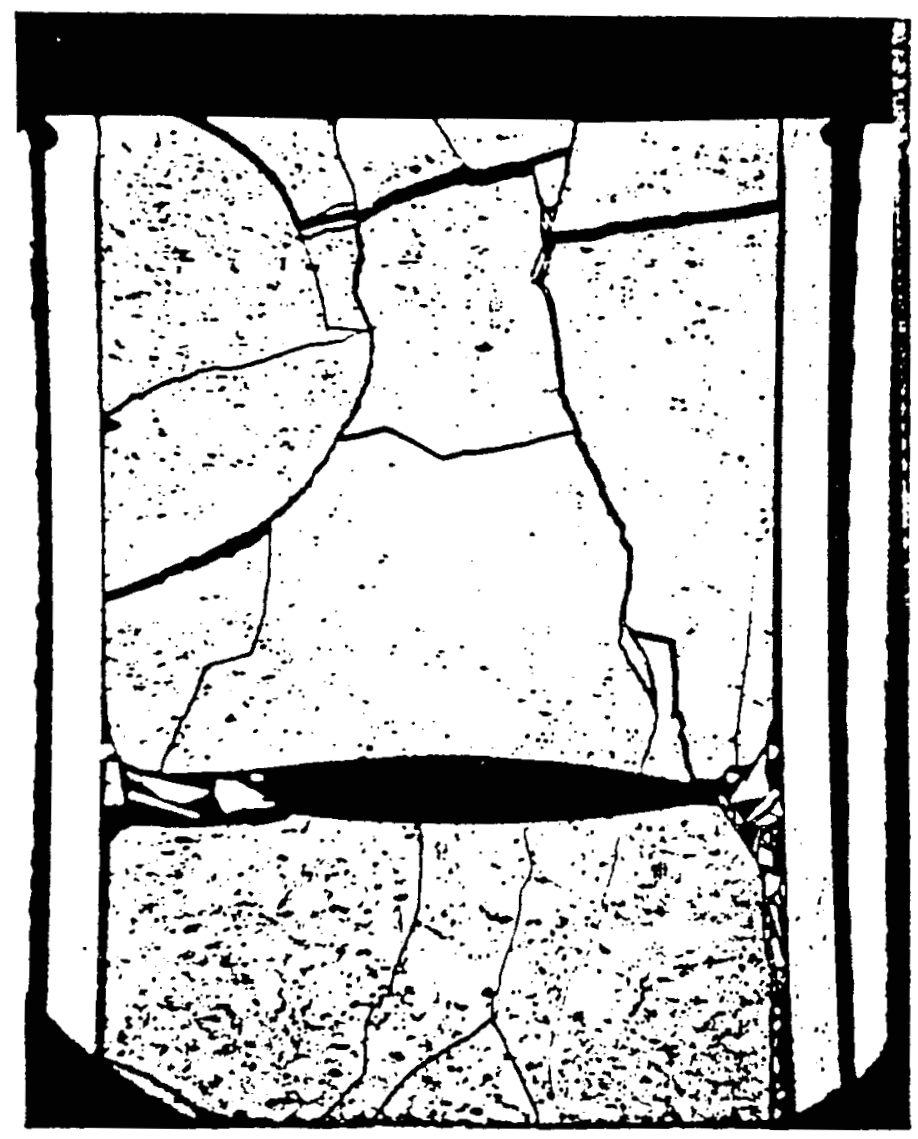

Figure I-13. Longitudinal Cross Section of a Turkey Point Fuel Rod After Irradiation. (Note: This rod had been intentionally pressurized after irradiation to expand the cladding; therefore, the fuel cladding gap is larger than normal.)

by shaking or tapping. Essentially, the pellet stack is freestanding inside the cladding, except for the small amount of fuel (approximately 108) that may be chemically bonded.

\section{I.3.3 Intact Rod Cladding Damage}

As-manufactured cladding has a distribution of small mechanical defects and chemical nonhomogeneities, but in general has a homogenous, uniform wall thickness. During irradiation, mechanical properties of cladding change and defects occur as part of localized wall thinning. Inreactor mechanisms, such as fretting, baffle jetting, or CILC, can cause cladding damage. However, there are presently no measurements of incipient damage caused by these mechanisms, nor have models been developed to predict it. Defects in fuel rods with intact cladding can be caused by a hydride redistribution in storage at temperatures reaching $380^{\circ} \mathrm{C}$, or uniform and nodular corrosion of the external Zircaloy surface in the reactor, or incipient PCI crack growth. These effects change the 


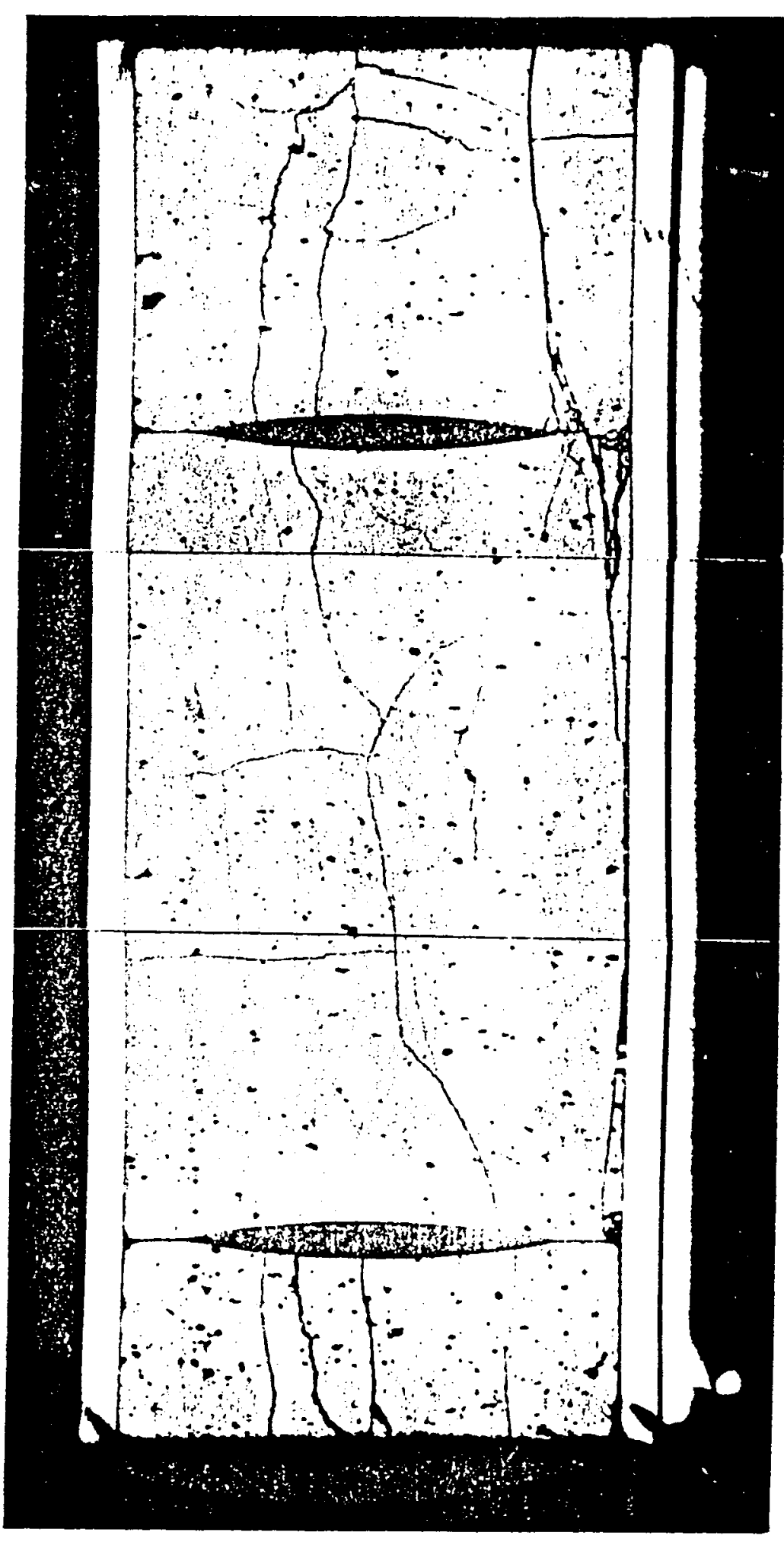

Figure I-14. Mosaic of a Longitudinal Cross Section of a Turkey Point Fuel Rod at the Spacer Grid Location of Sample G9-18 (2.0 m) at $7 \mathrm{x}$ [DA81] 


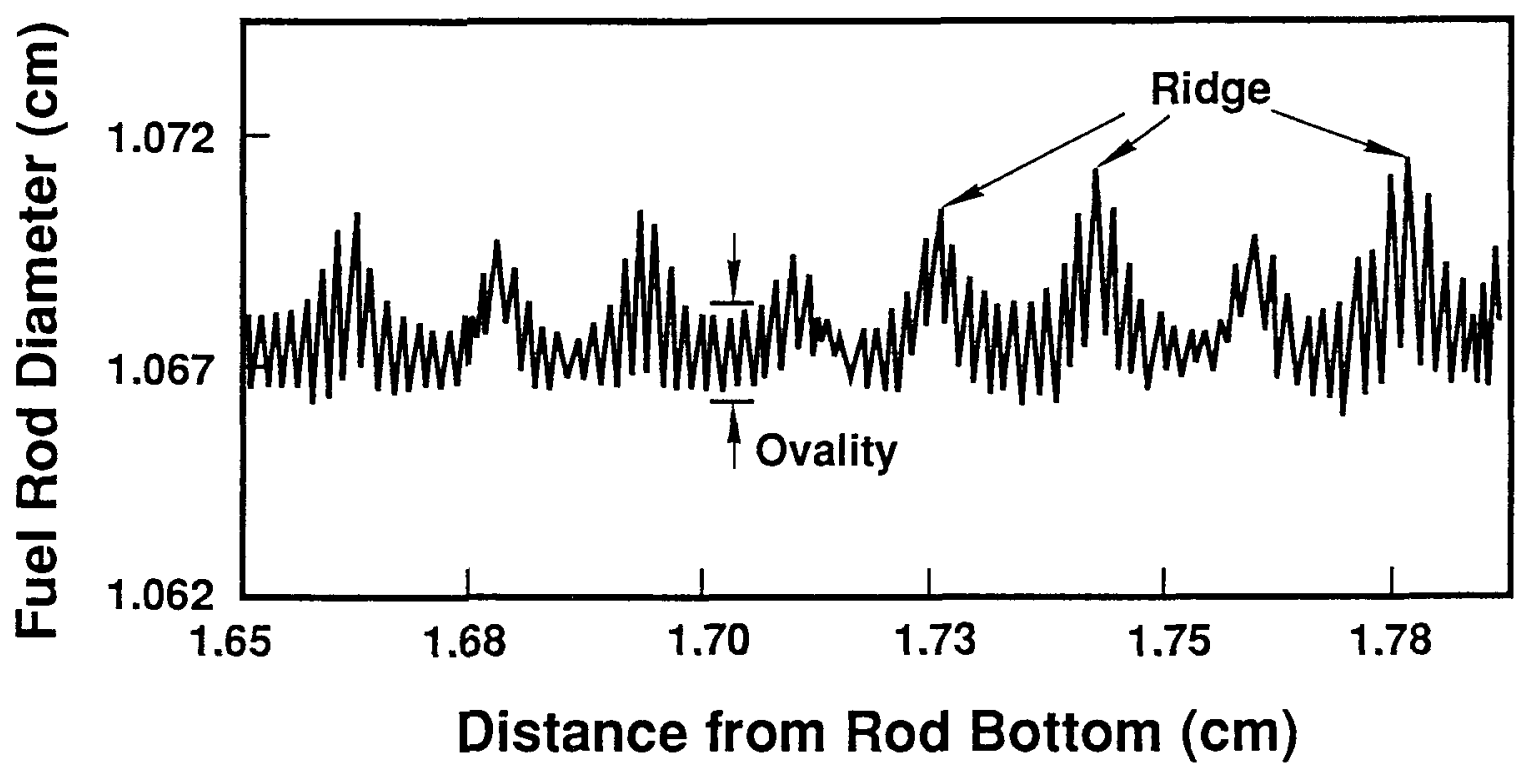

Figure I-15. Profilometry Scan of Rod DO1-19 Showing Continuous Localized Ridging at Pellet Interfaces Between 1.65 and $1.78 \mathrm{~m}$ from Rod Bottom (Neg. 8007580-6) [DA81]

mechanical properties of the cladding and hence the cladding propensity to breach during transport.

\section{I.3.3.1 Hydride Distribution and Degradation}

\section{In the Reactor}

As-fabricated Zircaloys contain approximately $10 \mathrm{ppm}$ of hydrogen. During reactor service, approximately $10 \%$ of hydrogen generated by aqueous corrosion of the reactor cladding can diffuse into the cladding. During reactor operations, the hydrogen levels are usually low enough (less than $100 \mathrm{ppm}$ ) and temperatures are high enough to prevent the hydrogen from exceeding its terminal solubility in the cladding, thereby remaining in solution in the Zircaloy (see Figure I-18). The hydrogen content of the cladding in extended burnup fuel apparently can be as high as $450 \mathrm{ppm}$ [GR88]. In PWR fuel, the hydrogen concentration is not uniform throughout the cladding, but peaks at approximately two-thirds of the length of the fuel column [EI82] (see Figure I-19). A variety of radial hydride distributions have also been observed, but in general, the radial distribution is uniform. No data have been obtained for BWR fuel rods.

\section{Storage and Transportation}

When cladding temperature is reduced (as it is during cool down in dry storage, pool storage, or possibly transportation), the solubility limit of the hydrogen can be exceeded, precipitating brittle hydride platelets 


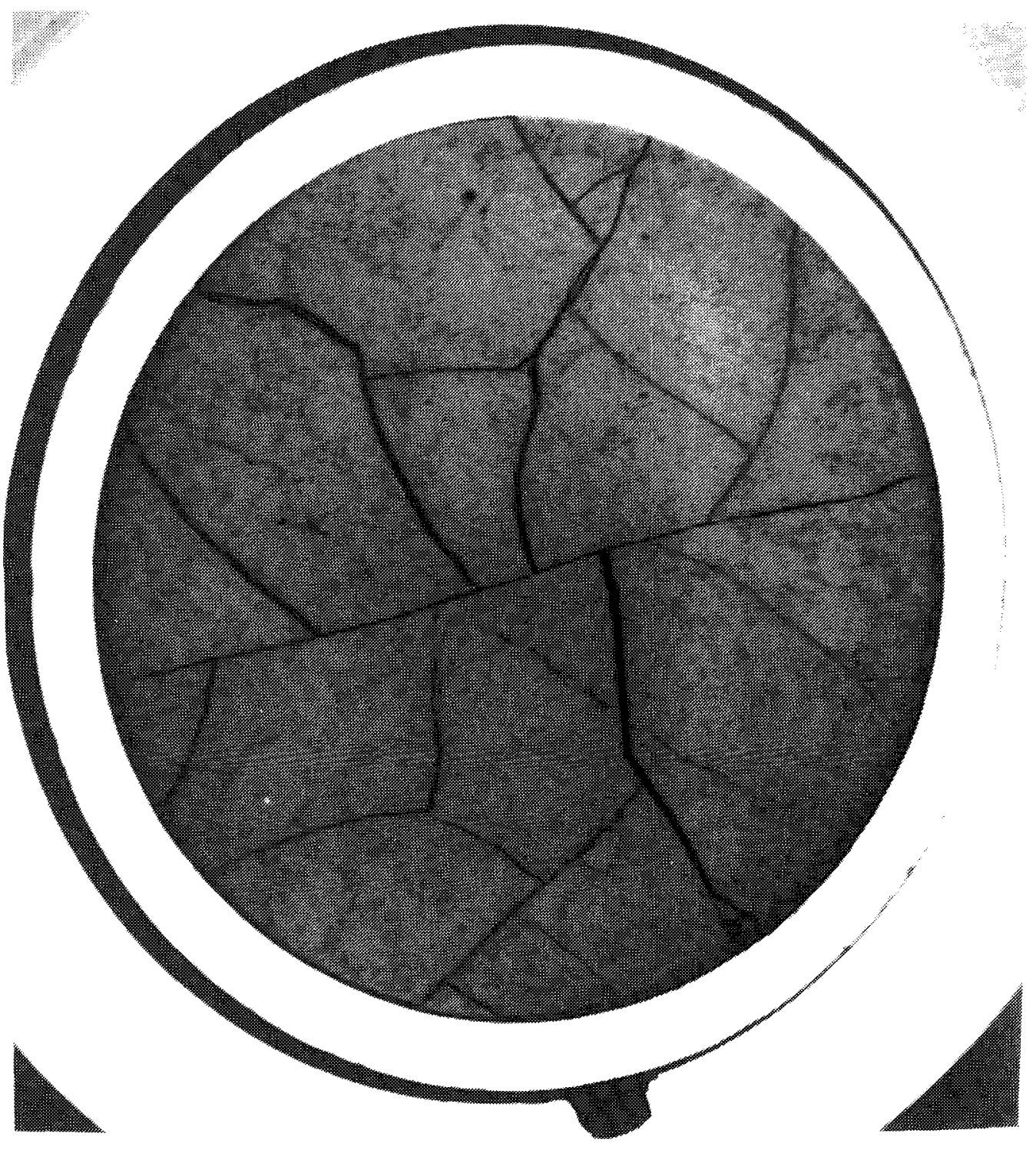

Figure I-16. Photomacrograph of an As-Polished Transverse Sample of Cooper BWR Fuel from the Peak Power Region ( $10 \mathrm{x})$ Burnup of 28 GWD/MTU [GU88c]. (Note: Photo taken at an oblique angle to fuel surface.)

(see Figure I-20). In general, these hydrides reduce the cladding fracture toughness, impact strength, and ductility at a low strain rate. The degree of embrittlement critically depends on the hydride orientation relative to the applied stress. Factors such as cladding composition, fabrication history, temperature, chemical environment, cooling rate, and excessive residual stress levels influence hydride orientation [N083]. The effect of hydriding on the mechanical properties of zirconium and its alloys are described in detail by Northwood and Kosasih [N083] and are summarized below. 


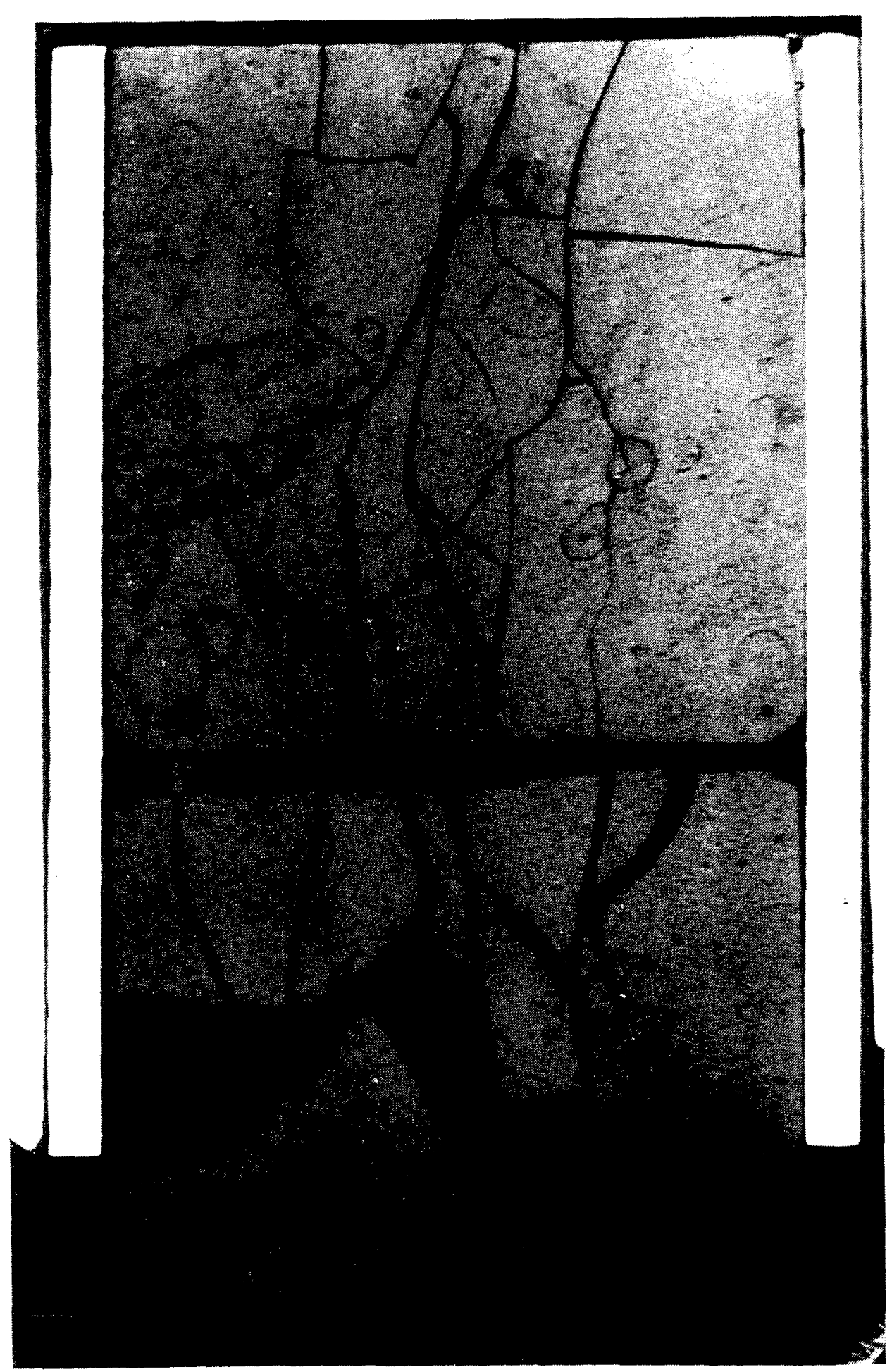

Figure I-17. Photomacrograph of an As-Polished Longitudinal Sample of Cooper BWR Fuel from the Peak Power Region ( $10 \mathrm{x}$ ) Burnup of 28 GWD/MTU (Neg. No. 87074D-5) [GU88c]. (Note: Photo taken at an oblique angle to fuel surface.) 
$\left({ }^{\circ} \mathrm{C}\right)$

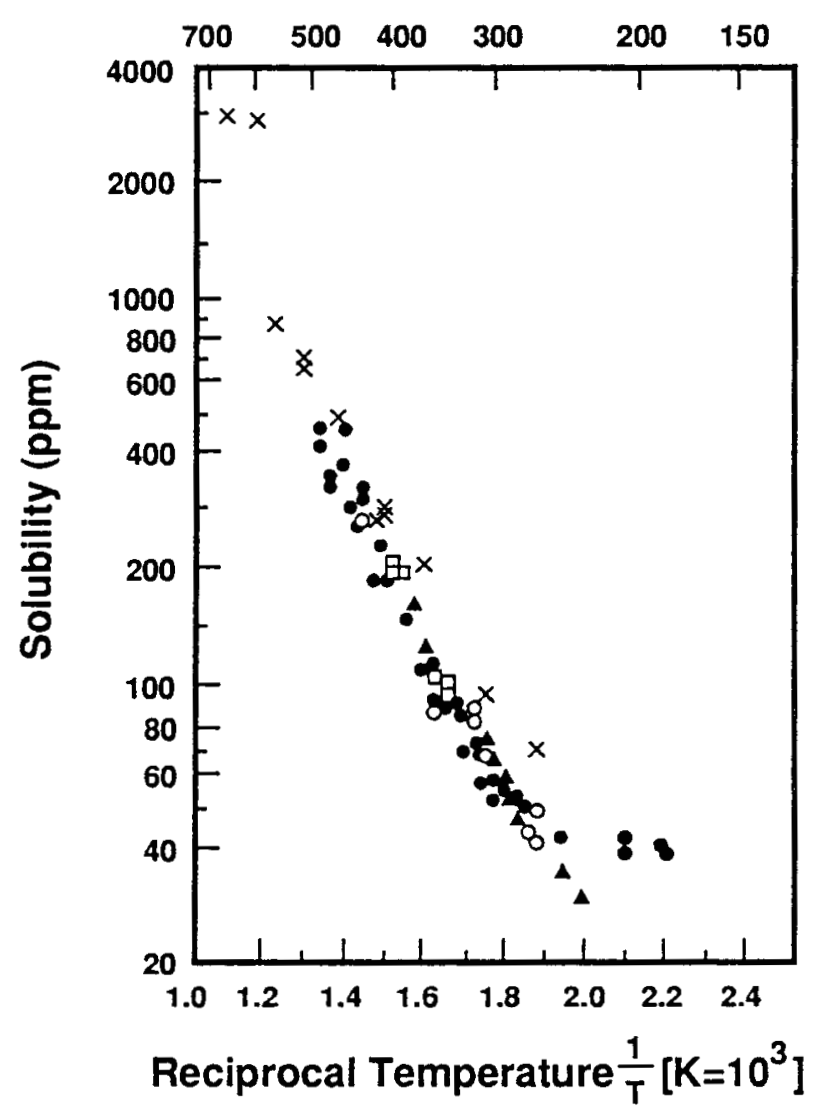

Figure I-18. Hydrogen Solubility in Zircaloy [GA79]

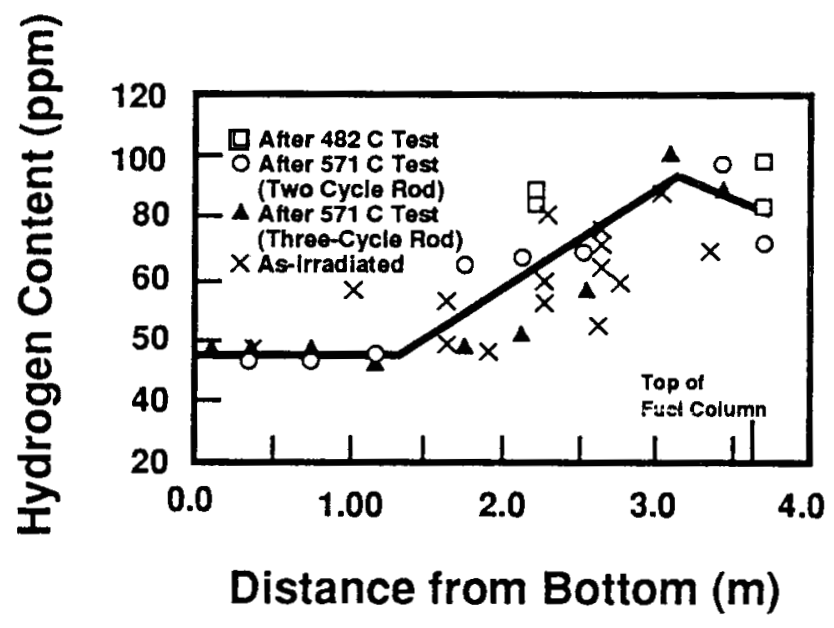

Figure I-19. Hydrogen Content of Cladding at Various Locations from the Bottom of the Rod [EI82] 


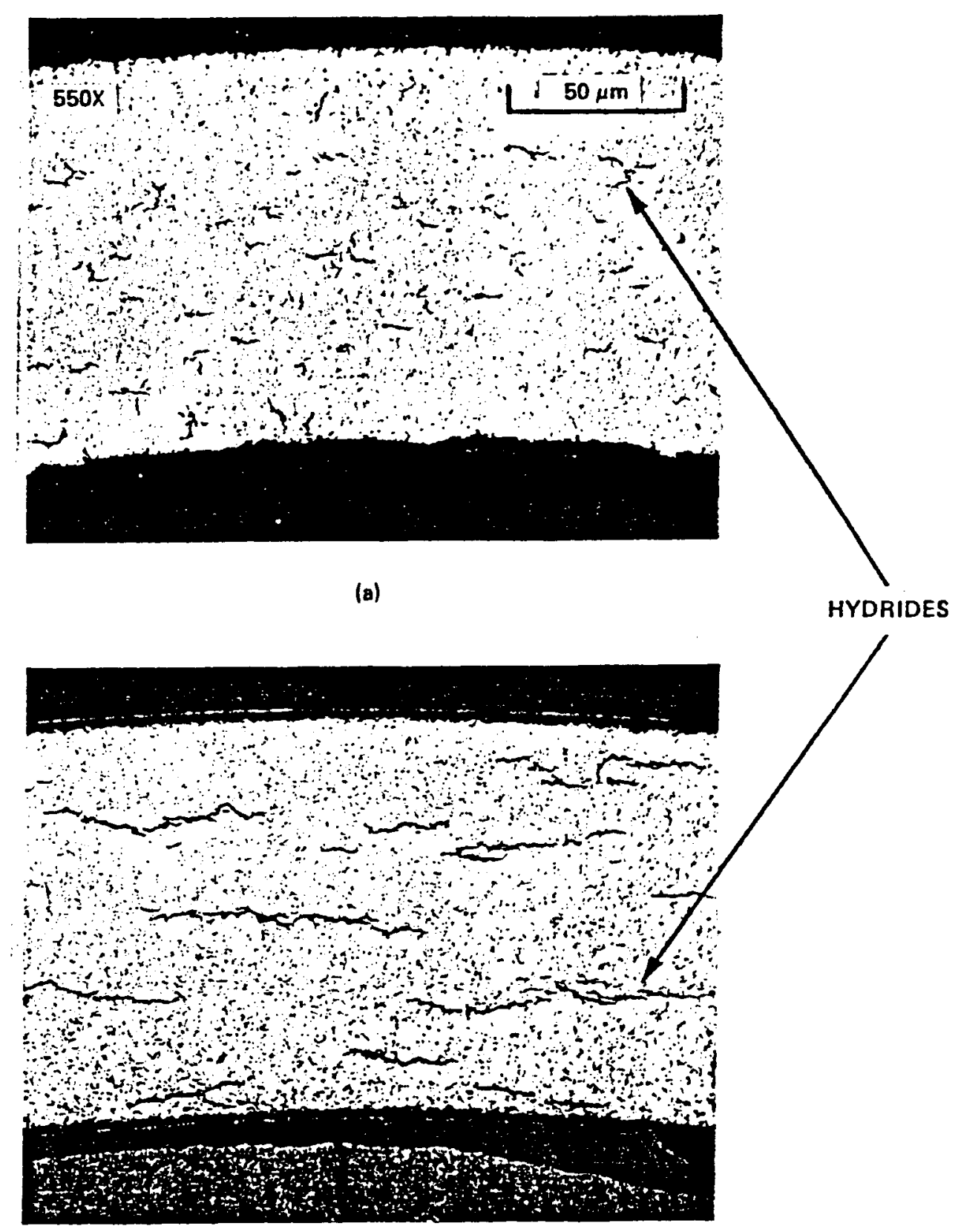

(b)

Figure I-20. (a) Pre- and (b) Post-test Hydride Structure [EI82] 
Northwood and Kosasih [N083] evaluated the deleterious effects of hydrogen on the increase of Zircaloy ductility when

1. The temperature drops below $200^{\circ} \mathrm{C}$;

2. The hydrogen content increases;

3. For equal concentrations, the hydrides become larger;

4. Hydride platelets become oriented perpendicular to the tensile axis.

As previously discussed, the hydrogen solubility decreases with temperature, thus causing precipitation of brittle hydrides. The transition temperature from ductile to brittle behavior increases as hydrogen content increases (see Figures I-21 and I-22). The cladding has a maximum of 150 to $400 \mathrm{ppm}$ of hydride and parts of the cladding can become brittle in the range of 75 to $150^{\circ} \mathrm{C}$. Consequently, there is potential for cladding failure or damage during low-temperature cask operations.

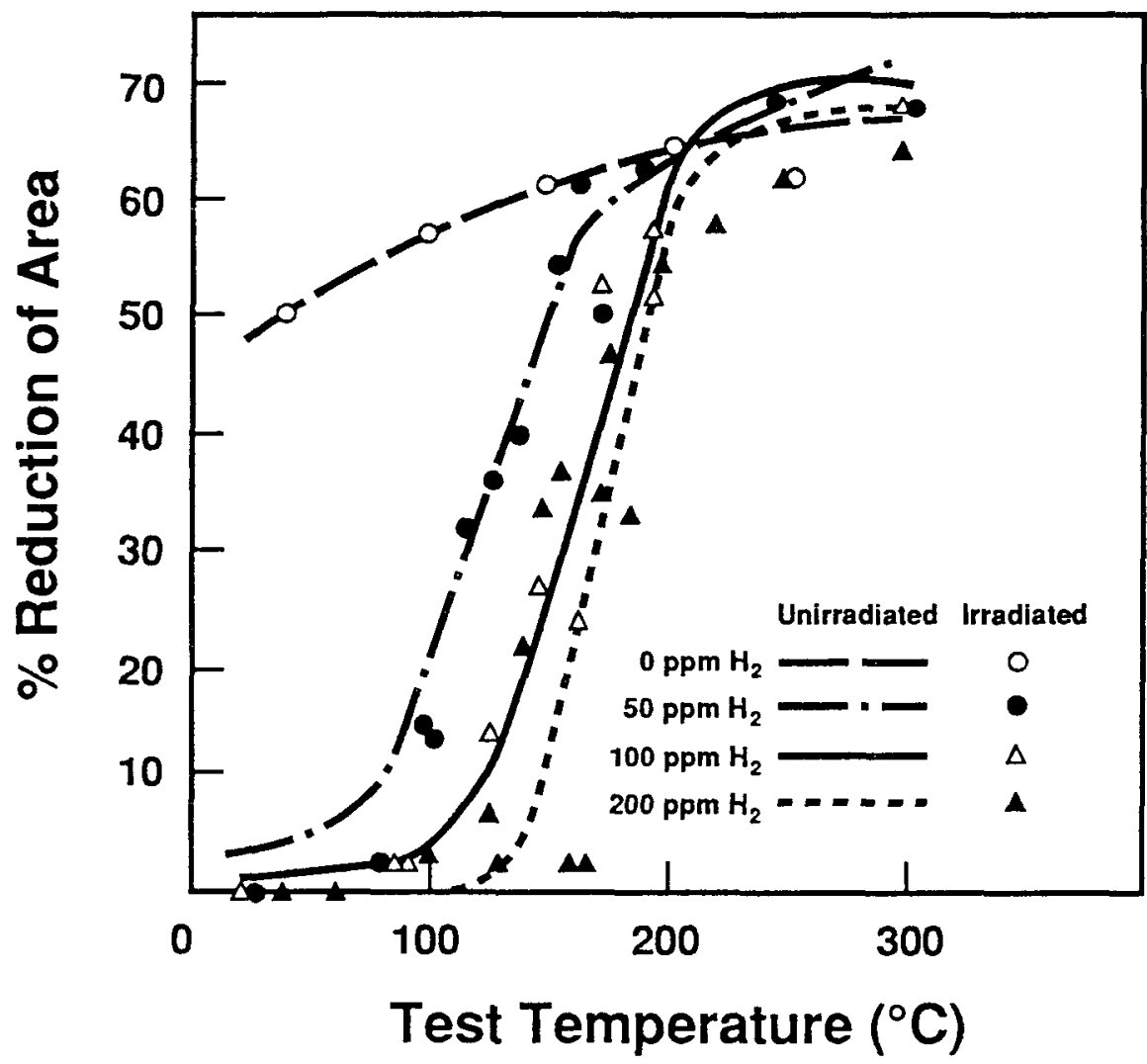

Figure I-21. Reduction of the Area of a Short Transverse Tensile Specimen from a Rolled Zircaloy-2 Slab as a Function of Temperature and Hydrogen Content. Basal poles are in the stress direction. Specimens were irradiated $50^{\circ} \mathrm{C}$ to $4.3 \times 10^{19}$ $\mathrm{n} / \mathrm{cm}^{2}(>1 \mathrm{MeV})$ [PI72]. 


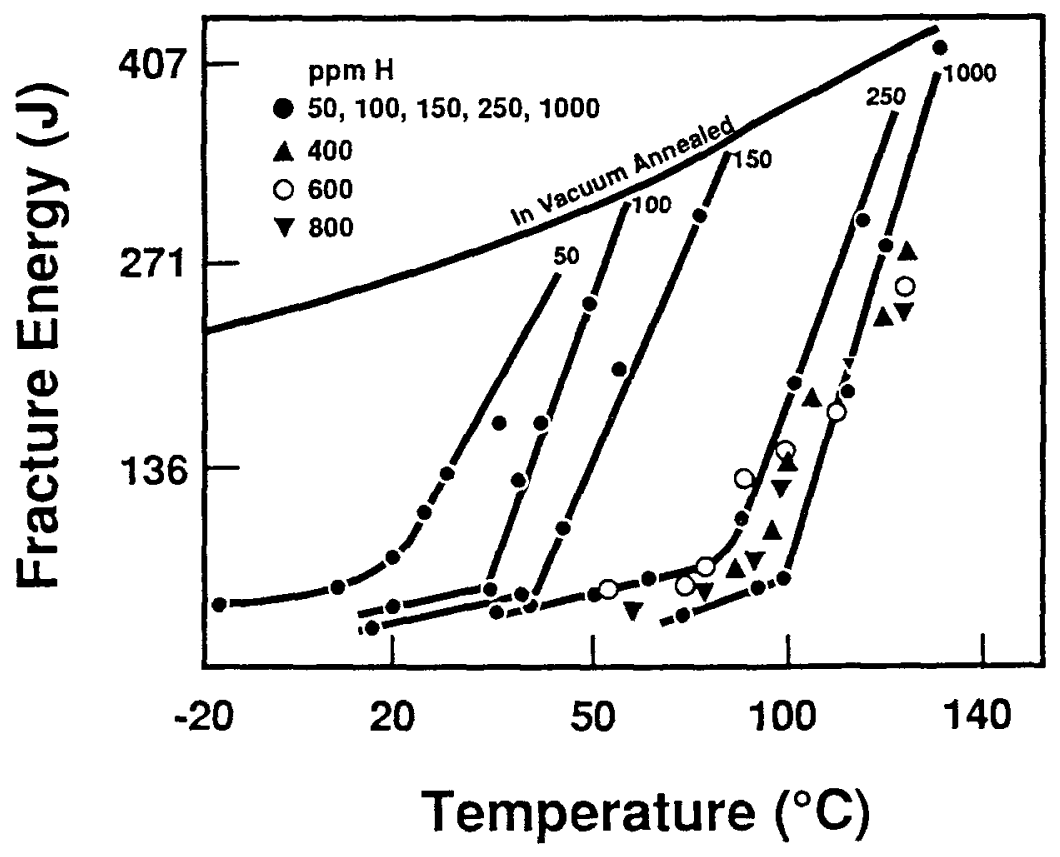

Figure I-22. Influence of Hydrogen on the Fracture Energy Transition Temperature [GA79]

Hydrides oriented parallel to the tensile stress have little effect on cladding mechanical properties at room temperature, but hydrides normal to the tensile stress reduce the ultimate tensile strength and ductility of the cladding [N083] (see Figure I-23). Radially oriented hydrides can provide a ready crack path under hoop stress. Cold-drawing fabrication techniques produce a texture in the Zircaloy that fosters circumferentially oriented hydrides. However, if internal stress in the rod is sufficient to overcome the residual stress due to texture, the hydrides precipitate radially. Rarely, if ever, are radial hydrides observed in as-irradiated cladding. Based on the internal stress threshold for reorientation and maximum internal pressure in the rods, any operations that involve fast cladding cool down should not cause hydride reorientation. However, extensive hydride reorientation has been observed in moderately pressurized PWR rods when cooled at a moderate rate of $5^{\circ} \mathrm{C}$ per hr [EI84c] (see Figure $(-24)$.

There is no evidence to indicate that reorientation will not occur during operations such as dry storage. No data are available at this time to indicate the fraction of hydrides that will reorient as a function of cooling rate and time. As shown in Figure $I-23$, reorientation significantly reduces ductility. Hence, the effects of mechanical properties of Zircaloy with circumferential hydrides must be considered in predicting cladding transport behavior after extended dry storage and when the fuel undergoes a slow cool down. 


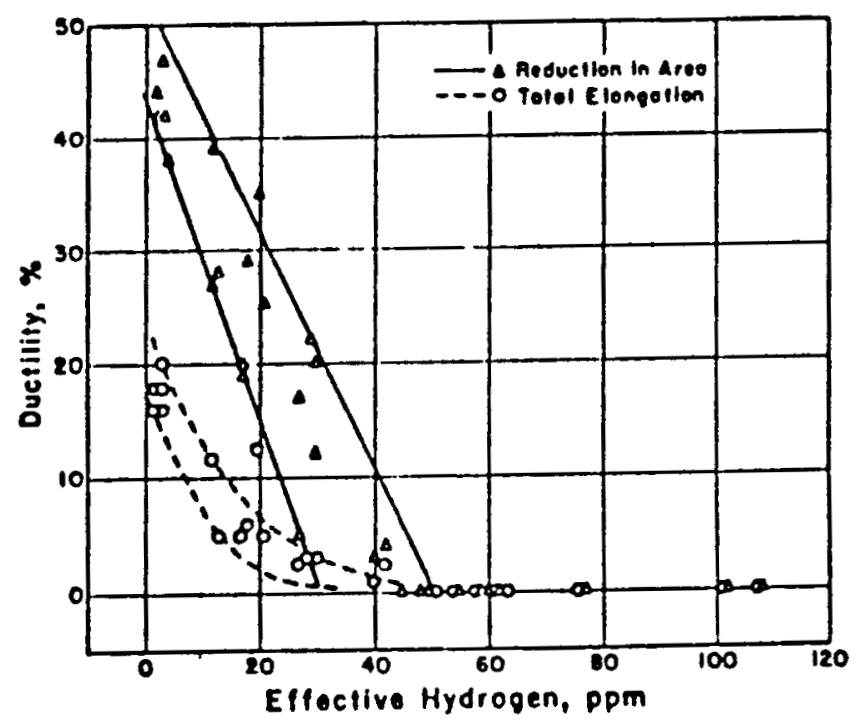

Figure I-23. Variations in Tensile Properties of Zircaloy with the Effective Hydrogen Content. Effective hydrides are those oriented in the radial direction [MA63].

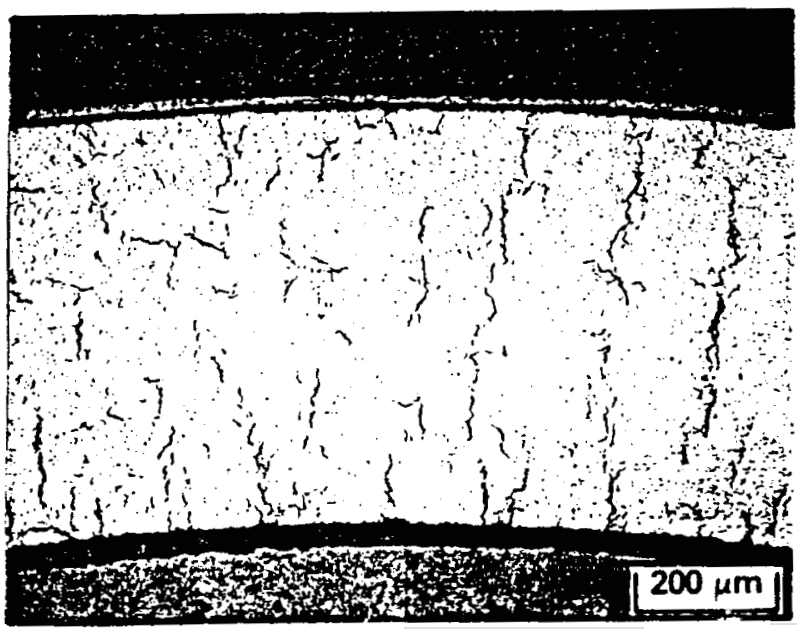

Figure I-24. Reoriented Hydrides After a Slow Cooling of PWR Cladding Under an Internal Pressure Hoop Stress [EI84c] 


\section{I.3.3.2 In-Reactor External Cladding Oxidation}

Zircaloy can oxidize when exposed to steam or water. This process is extensively analyzed and reviewed by Garzarolli et al. [GA80]. The oxidation process proceeds in two phases. A cubic rate law is followed initially until a critical oxide thickness is reached (i.e., transition). Then the oxidation process proceeds by a linear rate law. The oxide layer may be black, mottled, gray, or even tan. When a heat flux is applied, as in the case of reactor operation, the weight gain at transition remains the same while the time to transition shortens.

The amount of cladding oxidation can be affected by in-reactor fast neutron flux, oxygen level, fuel rod power, coolant chemistry, pH, CRUD presence, and prior oxidation history [GA80]. A detailed analytical model of the oxidation process is not necessary because extensive oxide measurements and characterizations over a wide range of variables are available and sufficient to provide correlations.

A uniform oxide layer forms on BWR irradiated cladding. This oxide layer has greater thickness than on unirradiated material [VI75]. In addition, nodules form in some BWR cladding [GA78a]. This nodule oxidation can be as great as 25 times the amount of uniform corrosion, eventually coalescing into a uniform oxide layer [N078]. The nodules tend to form more readily near dissimilar metal contacts, such as fuel rod and spacer grid interfaces. The growth rate of the uniform oxide layer is always found to be low on Zircaloy-2 cladding in a BWR environment [HO88]. When the rod has undergone a burnup of $-30 \mathrm{GWD} / \mathrm{MTU}$, the uniform oxide layer has reached a maximum of $-20 \mu \mathrm{m}$ (a $13-\mu \mathrm{m}$ metal loss out of an $\sim 800-\mu \mathrm{m}$ cladding thickness), but nodular corrosion at the same burnup could result in layer thickness as high as $-120 \mu \mathrm{m}$ ( $80-\mu \mathrm{m}$ metal 1oss). New processes for forming Zircaloy-2 BWR cladding that reduce the size of alloying element precipitates result in a lower oxide thickness [H088].

Most irradiated PWR cladding ( $-600 \mu \mathrm{m}$ thick) has a typical posttransition uniform oxide layer and its thickness is greater than expected, based on studies of unirradiated Zircaloy-4. Rarely is nodular corrosion observed under normal operating conditions. The oxide thickness peaks at approximately two-thirds of the rod length (which, as expected, corresponds with the axial location of the maximum hydrogen content). Dips in the oxide thickness occur at the spacer grids. Extensive measurements of the peak oxide thickness ( $-35 \mu \mathrm{m}$ oxide $=23 \mu \mathrm{m}$ metal) irrespective of the axial location have been made for many fuels at burnups up to -40 GWD/MTU burnup (see Figure I-25). Measurements of oxide thickness on rods from Calvert Cliffs, Ft. Calhoun, and ANO-2 reactors that have been exposed to burnups as high as 55 GWD/MTU fall within the trends of the bounds established at the lower burnups [H088, AN88]. However, the oxide layer on some highburnup, high-power test rods has exceeded a circumferential average thickness of $140 \mu \mathrm{m}$ (93- $\mu \mathrm{m}$ metal loss out of $\sim 600-\mu \mathrm{m}$ original metal) [H088]. To combat possible excessive oxidation at higher burnups, new cladding heat treatments, such as beta quenching [GR88] and changes in the Zircaloy alloy, are being examined. 


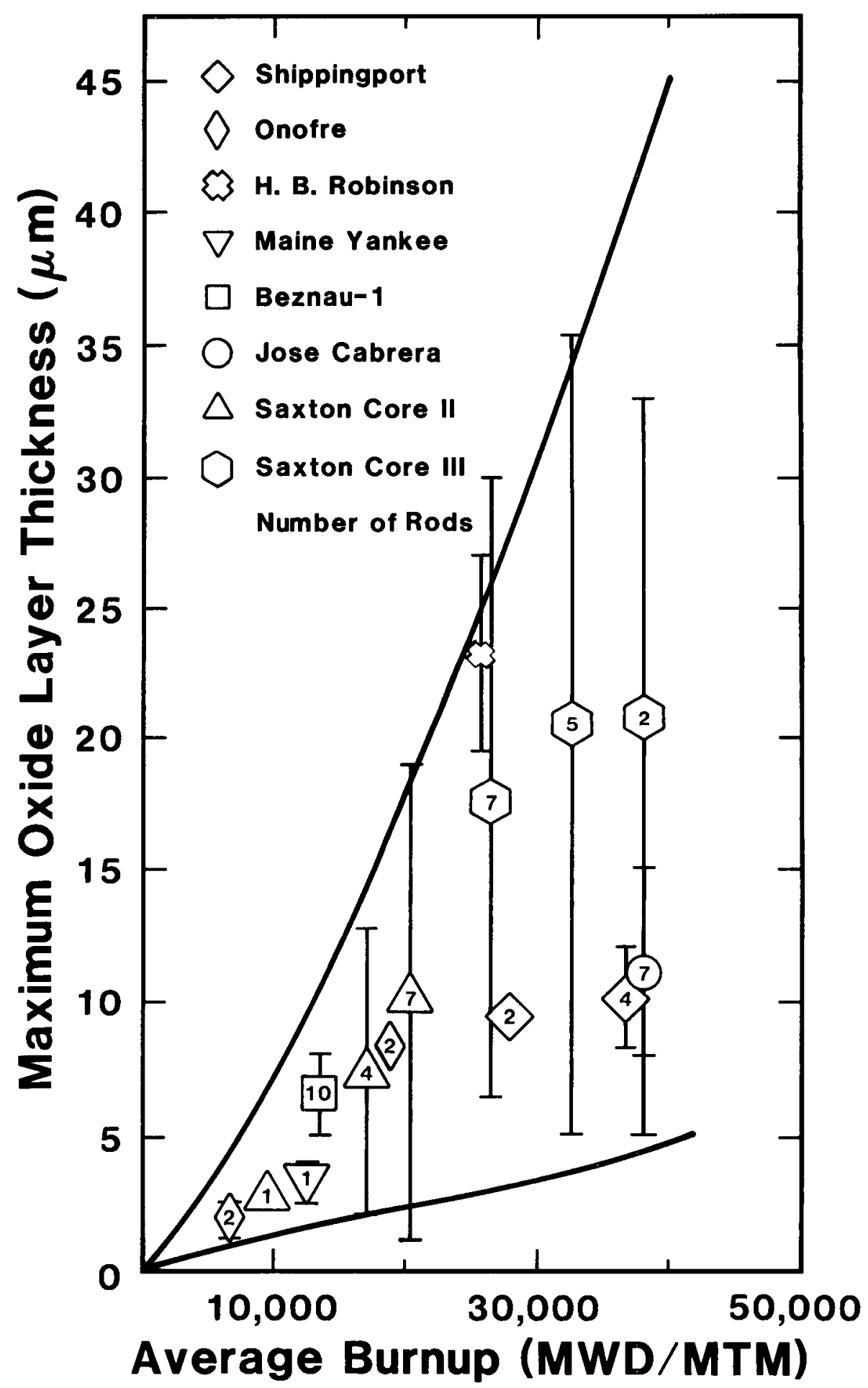

Figure I-25. Maximum Oxide Layer Thickness of Non-KWU Fuel Rods Versus Burnup [GA80] 


\section{I.3.3.3 Determination of Incipient Breaches}

The most prominent source of partial cracks (partially through the wall) in irradiated spent-fuel rods is PCI-induced stress corrosion cracking [R077]. The PCI breach mechanism is governed by a fuel-clad mechanical interaction (FCMI) force in a complex time-dependent process that depends on operational parameters such as fuel location, burnup, power ramp rates, and material parameters such as fuel and cladding mechanical properties.

\section{Incipient Breaches in the Reactor}

FCMI causes highly localized hoop stresses in the cladding at pelletto-pellet interfaces and at pellet cracks adjacent to the inner surface of the cladding. These interfacial localized stresses can lead to crack initiation. The process is likely assisted by the presence of the fission product iodine [YA80, ST83] or other aggressive species, notably Cs and Cd [SY77, DA84] in the fuel rod gap. Once a crack forms, it generally propagates into a through-the-wall crack if the FCMI driving force persists. Test data in iodine atmospheres [YA80, ST83] used in the laboratory to simulate the aggressive environment in the fuel rod gap indicate that crack propagation time is very short in comparison with crack initiation time. Thus, the PCI breach criterion is characterized by breach time (the sum of both initiation and propagation times) as a function of temperature and yield strength. Such a failure criterion has been developed based on extensive laboratory data and was incorporated into the FREY [RA87] fuel behavior code. This code has been validated by power and test reactor experiments.

Depending on the rod breach statistics chosen, rod breach frequency is between $10^{-4}$ and $2 \times 10^{-3}$ breaches per rod. One to 20 out of 10,000 rods have at least one through-the-wall crack. Assemblies containing rods with cracks that propagate through the wall during service are usually discharged in the next scheduled outage. Postirradiation examination (PIE) data indicate that the remaining rods, while unbreached, may contain partial cracks. Seven cladding cracks of varying depth were found in three unbreached high fission gas release rods from the Maine Yankee reactor after operating at $36 \mathrm{~kW} / \mathrm{m}$. The maximum crack depth observed was 658 of the wall thickness. No incipient defects were found in the low gas release rods [R077], and none in unbreached rods from Dresden 3, Oskarshamn-1, or Point Beach-1 [PA78].

An unbreached rod could contain a partial crack with a depth equal to a critical size that, if subjected to a subsequent operational ramp, would extend in an unstable manner and breach the cladding wall. The critical crack size has only a 18 probability of breach if the rod is left in the reactor. For a range of stresses below $517 \mathrm{MPa}$, the critical crack size is 208 of the wall thickness. This was calculated by Tasooji et al. [TA84] using an SCC model developed by Miller et al. [MI81].

A critical crack size was determined for this analysis by PCI analyses of a $7 \times 7$ BWR fuel assembly using the FREY fuel behavior code that was validated for PCI breach predictions. Operating powers and power ramps 
that were representative of normal duty were considered. The analysis follows a trial-and-error procedure in which an ID crack of a given depth was introduced. Starting from a cold gap determined by the fuel relocation model in FREY for the discharge burnup, a power ramp to the operating level was applied and the stress intensity factor $\mathrm{K}_{I}$ at the crack tip element was calculated and compared to the fracture toughness $\mathrm{K}_{\mathrm{IC}}$. The mean average power levels specified for the operating histories were typically $28 \mathrm{~kW} / \mathrm{m}$ for a BWR. When a typical peaking factor of 1.3 that allows for small overpower excursions was used, the top-of-the-ramp power level was $36 \mathrm{~kW} / \mathrm{m}$.

Because the cladding breach rate due to PCI ranges from 0.01 to 0.068 [BA88b, S088], probability of occurrence of the critical part-wall crack is $6 \times 10^{-4}$ to $1 \times 10^{-4}$ at a breach probability of 508. The appropriate failure criterion is the mean $K_{I C}$ value given by (also see Appendix III)

$$
\ln \mathrm{K}_{\mathrm{IC}}=\mathrm{A}-0.134 \phi_{t}+4.14 \times 10^{-4} \mathrm{~T} \phi_{t} \text {, }
$$

where

$$
\begin{aligned}
\mathrm{K}_{\mathrm{IC}} & =\text { units of } \mathrm{MPa} \sqrt{\mathrm{m}} \\
\phi_{\mathrm{t}} & =\text { neutron fluence in } 10^{21} \mathrm{n} / \mathrm{cm}^{2}(>1 \mathrm{MeV}) \\
\mathrm{T} & =\text { temperature in }{ }^{\circ} \mathrm{C} \\
\mathrm{A} & =3.84+9 \times 10^{-4} \mathrm{~T} \text { for axial cracks, and } \\
\mathrm{A} & =3.91+3.6 \times 10^{-4} \mathrm{~T} \text { for circumferential cracks. }
\end{aligned}
$$

Results of FREY code analyses using initial crack sizes ranging from 76 to $610 \mu \mathrm{m}$ (see Figure I-26) show the power corresponding to a 508 breach probability plotted as a function of normalized crack depth. As shown in Figure I-26, the critical crack depth corresponding to a power of $36 \mathrm{~kW} / \mathrm{m}$ is $28 \%$ of the clad thickness. The curves for 2 and 988 breach probabilities are also shown for comparison.

The agreement between the FREY and Tasooji analyses is good considering the differences in approach. A critical crack depth equivalent to $28 \%$ of the wall thickness is incorporated into the fuel rod breach models derived in Appendix III.

\section{Dry Storage Incipient Breaches}

Unfortunately, little is known of the partial crack propagation that occurs in the fuel rod cladding during dry storage because the evaluations were made based on a time-to-breach analysis. Because experimental programs were not conducted for the purpose of characterizing distributions of incipient defects after dry storage, no experimental data exist concerning defect distributions developed during dry storage.

\section{Manufacturing Flaws}

Flaws due to manufacturing include pits, grooves, and scratches in the Zircaloy tubing. The scanning electron microscopy (SEM) examination by Tucker [TU76] quantifies the size and distribution of these flaws. This flaw size distribution for unirradiated as-fabricated material was verified 


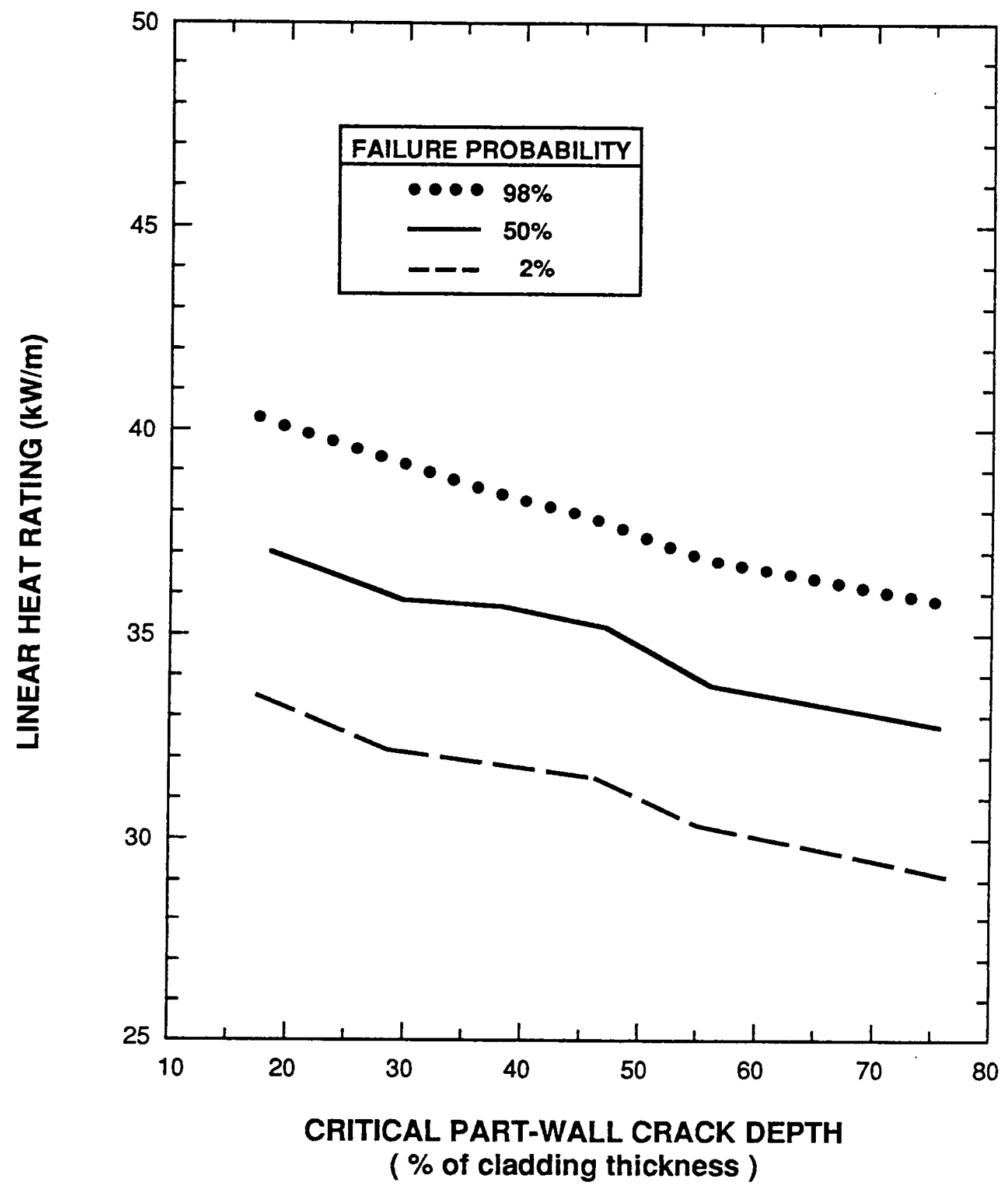

Figure I-26. Linear Heat Rating (kW/m) Versus Critical Incipient Crack Depth (Percent of Cladding Thickness) 
by Miller [MI81] based on the strength of cladding materials, and is given by

$$
\phi\left(C_{0}\right)=1-\exp \left[-k A_{S} C_{0}^{-p / 2}\right],
$$

where

$$
\begin{aligned}
& \phi\left(C_{0}\right)= \text { is the probability of the existence of a flaw at least of } \\
& \text { size } C_{0} \\
& A_{S}= \text { the surface area of interest } \\
& k \text { and } p=9.84 \times 10^{-10} \text { and } 5.175 \text { for unirradiated Zircaloy. }
\end{aligned}
$$

This distribution translates into small probabilities of substantial cracks in the cladding. Tucker indicates that less than 58 of the flaws are greater than 28 of the wall thickness [TU76]. Because the acceptance criteria for Zircaloy tubing is generally $10 \%$ of clad thickness, manufacturing flaws greater than 108 of clad thickness have negligibly small probabilities.

\section{Operationally Induced (PCI) Cracks}

The complex time-dependent behavior leading to operationally induced (PCI) cracks makes quantifying their number and distribution without costly hot-cell data extremely difficult. Limited data are described in the following subsection; however, these data are not adequate to construct valid distributions for PCI partial cracks in BWR and PWR fuels at end-oflife burnup levels. The following subsections and Appendix III describe a method to estimate the number of cracks and construct appropriate distributions for PCI cracks.

\section{Available Data}

Limited information from in-service inspections can be used to develop a flaw size distribution. The available data are subject to uncertainty because of the inadequate inspection process (i.e., the difficulty in detecting the small flaw sizes). One reason that 1ittle data are available on in-service flaws is that part-wall cracks appear prevalent only in failed rods. As described previously, the extensive investigation of Point Beach-1 (PWR), Dresden 3 (BWR), and Oskarshamn-1 (BWR) revealed no partwall defects in any of the inspected unfailed fuel rods [PA78]. These studies include detailed inspections of over 50 rods that were specially selected because they were believed to have part-wall cracks. Although one may infer that presently available techniques are not capable of detecting PCI incipient defects, investigations in Maine Yankee Core 1 did reveal the seven part-wall cracks mentioned previously and described in detail below.

The examinations of Maine Yankee Core 1 were from the detailed inspection of 656 rods [FU76]. These data were developed from the inspection of four assemblies that had known leakers, and thus contained significantly more flaws than one would expect from other assemblies in the Maine Yankee Core and most other reactors. The distribution of part-wall cracks that were found is tabulated in Table I-11. These limited data demonstrate the rarity of significant part-wall cracks. 
Table I-11

PCI Incipient Defects from Maine Yankee Core 1

\begin{tabular}{|c|c|c|c|c|c|c|}
\hline $\begin{array}{c}\text { Number of } \\
\text { Flaws }\end{array}$ & 2 & 1 & 1 & 1 & 1 & 1 \\
\hline $\begin{array}{l}\text { Depth of } \\
\text { Crack }\end{array}$ & $0.040 \mathrm{~mm}$ & $0.114 \mathrm{~mm}$ & $0.203 \mathrm{~mm}$ & $0.236 \mathrm{~mm}$ & $0.305 \mathrm{~mm}$ & $0.457 \mathrm{~mm}$ \\
\hline \multicolumn{7}{|l|}{$\begin{array}{l}\text { Percent of } \\
\text { Clad }\end{array}$} \\
\hline Thickness & $7 \frac{8}{8}$ & 178 & 308 & 358 & 448 & 658 \\
\hline
\end{tabular}

Estimating the Amount of Cracks

Partial cladding cracks in irradiated fuel rods are almost always found opposite radial pellet cracks at pellet-to-pellet interfaces [GA78b, R0771. Considering that ridging does not take place near the ends of the fuel column (where the linear power is lower) and that a pellet is $-1.5 \mathrm{~cm}$ long, then the amount of active pellet-to-pellet interfaces is $(\mathrm{L}-76) / 1.5$, where $\mathrm{L}$ is the fuel column length in centimeters. If this is multiplied by $\pi \mathrm{DW}$, where $\mathrm{D}$ is the cladding inside diameter and $\mathrm{W}$ is the stressed interface width (in this case $0.1 \mathrm{~cm}$ ), then -50 to $60 \mathrm{~cm}^{2}$ of PWR cladding and 50 to $75 \mathrm{~cm}^{2}$ of BWR cladding per rod is susceptible to partial cracking. In tests of unirradiated Zircaloy in an iodine atmosphere, Cubicciotti [CU80] found only one partial crack per $\mathrm{cm}^{2}$ in the unbreached segments. This amounts to 50 to 60 partial cracks per PWR rod and 50 to 75 partial cracks per BWR rod.

Another approach is to consider that incipient cladding cracks form only in $a \mathrm{~mm}$ band around radial fuel cracks. The area available for cracking becomes

$$
A_{c}=\left[\frac{(L-76)}{1.5}\right] \text { W N } 0.2 \mathrm{~cm}^{2}
$$

where

$$
\begin{aligned}
& N=\text { the number of radial cracks } \\
& W=\text { the pellet-to-pellet interface width }(0.1 \mathrm{~cm})
\end{aligned}
$$

Eight to twelve radial cracks are estimated in a cross-sectional plane if transverse ceramography is used. Therefore, $A_{c}$ is between 30 and $45 \mathrm{~cm}^{2}$ and there should be -30 to 45 partial cracks per rod. 
Comparison of these estimates with the available data discussed above reveals that the estimated crack amounts appear very conservative. If

these crack amounts are accurate, then most of the cracks are probably very small and thus are not measurable under current inspection techniques.

\section{Crack Depth Distribution}

For these analyses, the frequency of a physical phenomenon such as a crack size, which tends to have a low probability of existence, is represented below a critical state with a negative exponential function. For this application, the distribution is estimated as

$$
p(x)=A e^{-B x},
$$

where $p(x)$ is the relative frequency of occurrence of a defect of a given size and $A$ and $B$ are constants. It can be shown that $A=B$ from the requirement that the area under the curve of Equation (I-4) equals unity. To determine a value for $B, P C I$ failure data reported in the fuel performance literature reveal that approximately one rod in 1000 and one rod in 10,000 fails in BWRs and PWRs, respectively. From these data, the implication can be drawn that any cracks found in the remainder of the rods must be subcritical; otherwise, the reactor operations are such that failure would be inevitable once the crack reaches or exceeds a critical size. The constant $B$ is evaluated on this basis by assuming that the frequency of exceedence of the critical crack size is equal to the frequency of rod breaches in the reactor. This process is further discussed in Appendix III as it applies to the probabilistic evaluation of fuel rod failure of example BWR and PWR assemblies.

Distributions other than the exponential distribution discussed above may be used, such as the following Weibull distribution [BE70]:

$$
\mathrm{p}(\mathrm{x})=\frac{\beta}{\alpha^{\beta}} \mathrm{x}^{\beta-1} \mathrm{e}^{-\left(\frac{\mathrm{x}}{\alpha}\right)^{\beta}}
$$

This is an extreme-value distribution that emphasizes the lower bound of the data. Similar to the exponential distribution, $p(x)$ is the relative frequency of occurrence of a defect of a given size and $\alpha$ and $\beta$ are constants. However, unlike the exponential distribution, the Weibull curve requires two unique datums to define the distribution. The Weibull distribution can be constructed assuming that $50 \%$ of the flaws are deeper than 108 of the wall thickness, in addition to the critical flaw frequency assumptions presented above.

Figure I-27 provides a comparison between the exponential and Weibull distributions, and a distribution for manufacturing flaws constructed from actual data. Figure I-27 shows that the exponential and Weibull distributions provide similar representations for in-service cracks. The exponential distribution is used in the example analyses in Appendix III 


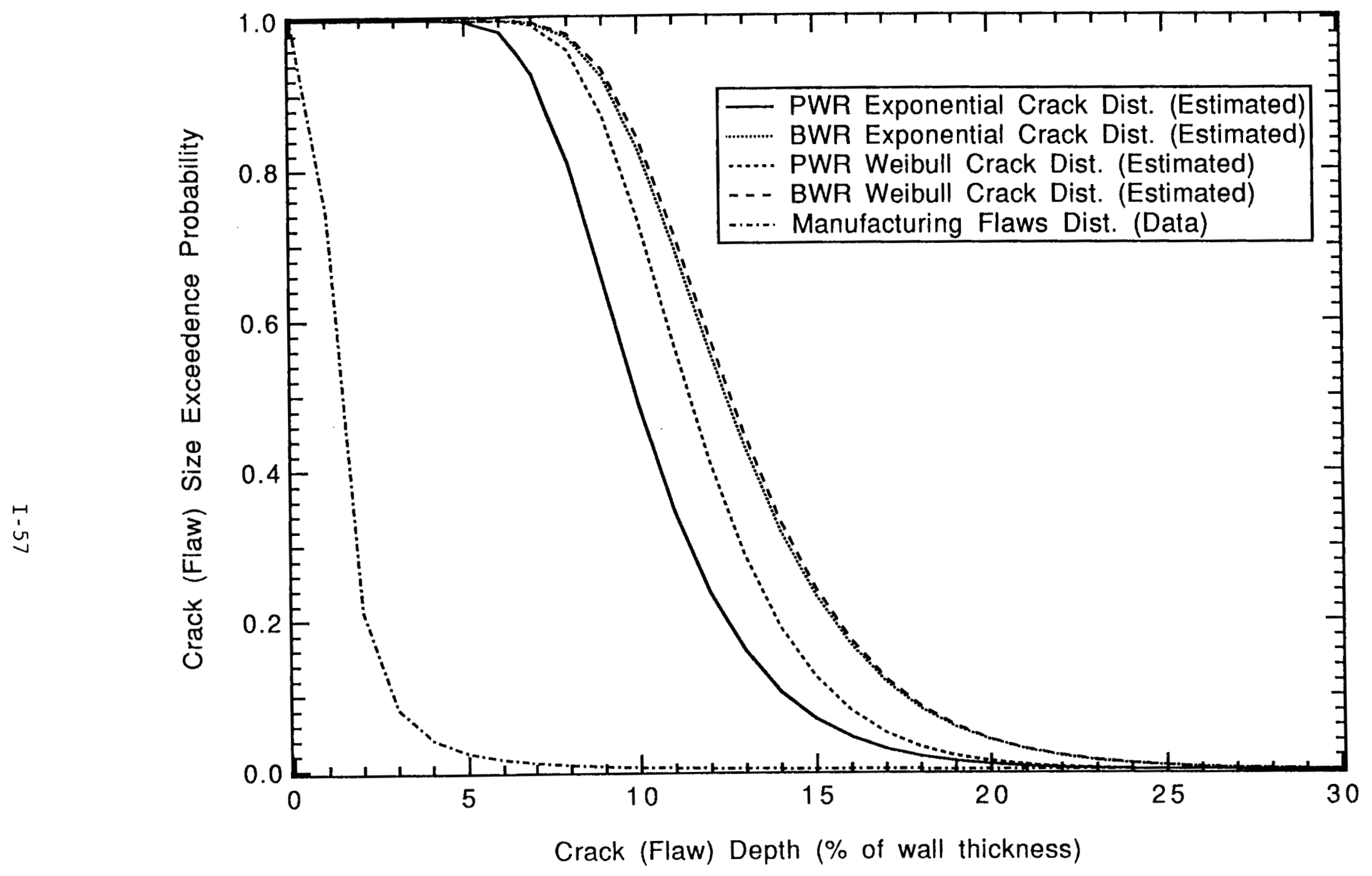

Figure I-27. Comparison of Exponential, Weibull, and Manufacturing Crack (Flaw) Size Exceedence Probability Distributions 
because it requires only the single data point to define the distribution. If sensitivity analyses of the resulting fuel rod cladding breaches indicate that this distribution is an important variable, then further benchmark evaluations are necessary.

The Maine Yankee data discussed previously can be used to verify the assumptions above that were used to construct the exponential distribution. The amount of possible flaws is estimated by multiplying 656 rods by 60 flaws per rod (as computed above), which equals 39,360 flaws. The amount of flaws exceeding the critical depth of 288 of the wall thickness is four. Thus, the critical flaw size frequency is $4 / 39,360=1 \times 10^{-4}$. While this result may be coincidentally close to the value assumed for the frequency of exceedence of PWR PCI cracks, this result verifies the reasonableness of these assumptions until further data are available.

\section{I.4 Radionuc1ide Inventory}

Large amounts of radionuclides are produced within fuel rods during reactor operation. Reasonably accurate estimates of the nuclide composition of irradiated fuel can be obtained if the irradiation history can be specified. Furthermore, reliable estimates of the nuclide fuel composition can be calculated for any time after reactor shutdown if the nuclide fuel composition is known at shutdown time because the decay characteristics of virtually all pertinent radionuclides can be established to a high level of accuracy. Many computer programs have been created and are routinely used to estimate radionuclide compositions of irradiated fue1; the most widely used is the computer code ORIGEN2 [CR80, CR83].

ORIGEN2 is used to support these analyses because ORIGEN2 has already been proposed for use in the programs of the Office of Civilian Radioactive Waste Management (OCRWM) of the U.S. Department of Energy's (DOE) programs [R087] and is extensively employed by the U.S. Nuclear Regulatory Commission (NRC) in its severe accident assessment programs [KR85]. A1though ORIGEN2 is a state-of-the-art computer code, the results of ORIGEN2 do not exactly agree with radiochemical assays. A recent comparison [R087] of ORIGEN2 calculations with radiochemical assays of irradiated fuel specimens noted that whereas agreement between calculation and experimental determination is good (within 10\%) for many radionuclides such as $137 \mathrm{Cs}$, it is only fair (within 50\%) for others, such as $134 \mathrm{Cs}$. For a few nuclides, the agreement is poor. Surprisingly, one example is $154 \mathrm{Eu}$, which is often used as a fuel marker because it is a gamma emitter that tends to remain associated with $\mathrm{UO}_{2}$. However, the isotopes that are radiologically significant from a health risk perspective, e.g., ${ }^{137} \mathrm{Cs}$, are well determined by ORIGEN2.

Similar comparisons were conducted by the Materials Characterization Center (MCC) for two average burnup PWR fuels [BA84a] ( -32 GWD/MTU), one BWR fuel ( 32 GWD/MTU), and one high burnup PWR fuel (47 GWD/MTU). The results are summarized in Table I-12. ORIGEN2 predicts $126 \mathrm{Sn}$ to be four to five times the measured value and ${ }^{79} \mathrm{Se}$ to be six to seven times the measured value irrespective of fuel type. For BWR fuel, ORIGEN2 tends to overpredict the inventory for many isotopes. For the high burnup PWR fuel, ORIGEN2 both over- and underpredicts select isotopes. However, given the 
Table I-12

Ratio of Measured to Predicted Values

of Selected Nuclides [GU88a, BA84b, GU88b] ${ }^{a}$

\begin{tabular}{|c|c|c|c|}
\hline Radioisotope & PWR & BWR & $\begin{array}{l}\text { High Burnup } \\
\text { PWR }\end{array}$ \\
\hline $234 \mathrm{U}$ & - & - & 0.77 \\
\hline $235 \mathrm{U}$ & - & - & 0.73 \\
\hline${ }^{235} \mathrm{U}$ & - & - & - \\
\hline $238 \mathrm{U}$ & - & - & - \\
\hline $238 \mathrm{Pu}$ & - & - & - \\
\hline${ }^{239} \mathrm{Pu}$ & - & 0.81 & 0.83 \\
\hline${ }^{240} \mathrm{Pu}$ & - & - & - \\
\hline${ }^{241} \mathrm{Pu}$ & - & 0.77 & 0.82 \\
\hline $242 \mathrm{Pu}$ & - & - & 1.20 \\
\hline $237_{\mathrm{Np}}$ & - & - & 0.71 \\
\hline $241_{\mathrm{Am}}$ & - & 0.71 & 1.32 \\
\hline $243 \mathrm{Cm}+244 \mathrm{Cm}$ & - & 0.79 & - \\
\hline${ }^{14} \mathrm{C}$ & 1.23 & - & - \\
\hline${ }^{79} \mathrm{Se}$ & 0.15 & 0.14 & 0.12 \\
\hline${ }^{90} \mathrm{Sr}$ & - & - & - \\
\hline${ }^{99} \mathrm{Tc}$ & - & - & 0.73 \\
\hline $126 \mathrm{Sn}$ & 0.23 & 0.24 & 0.19 \\
\hline${ }^{129} \mathrm{I}$ & - & - & - \\
\hline $135 \mathrm{Cs}$ & - & - & - \\
\hline $137_{\mathrm{Cs}}$ & - & - & - \\
\hline
\end{tabular}

uncertainties associated with other aspects of the methodology, ORIGEN2 results can be considered sufficiently accurate for consequence assessment purposes. Once the operational parameters (i.e., enrichment, power history, and burnup) and material composition of the fuel and hardware have been specified, ORIGEN2 can be employed to estimate the nuclide composition of the fuel assemblies prepared for shipment. These results represent the primary source term for radioactivity release.

For example, two assembly-specific sample calculations using ORIGEN2 were performed. One of these represents Rod 08639 that was contained in a B\&W $15 \times 15$ PWR fuel assembly (Assembly 1D13) and irradiated to an average burnup of $38.2 \mathrm{MWD} / \mathrm{kg} \mathrm{U}$ through four cycles of the Oconee-1 reactor [BA83b]. The second calculation was for Fuel Rod BSA-0139, which was part of a GE $7 \times 7$ BWR assembly irradiated to an average burnup of $33.7 \mathrm{MWD} / \mathrm{kg} \mathrm{U}$ through five cycles of the Quad Cities-1 reactor [L082]. Those radionuclides that contributed more than 0.018 to the fue1 assembly's total radioactivity are listed in Table I-13. Based on the ratio of inventory to $A_{2}$ value, the remaining nuclides have little effect on results of transport 
Table I-13

Radionuclide Contents of Selected LWR Fuel Rods

\begin{tabular}{|c|c|c|c|c|}
\hline \multirow[b]{2}{*}{ Nuclide } & \multicolumn{2}{|c|}{ PWR Rod ${ }^{a}$} & \multicolumn{2}{|c|}{ BWR Rodb } \\
\hline & g & $\mathrm{Ci}$ & $\mathrm{g}$ & $\mathrm{Ci}$ \\
\hline${ }^{3} \mathrm{H}$ & $1.05 E-04$ & $1.02 E+00$ & $1.71 \mathrm{E}-04$ & $1.66 \mathrm{E}+00$ \\
\hline $85 \mathrm{Kr}$ & $3.90 \mathrm{E}-02$ & $1.56 \mathrm{E}+01$ & $4.54 \mathrm{E}-02$ & $1.81 \mathrm{E}+01$ \\
\hline $90 \mathrm{Sr}$ & $1.03 E+00$ & $1.55 \mathrm{E}+02$ & $1.18 \mathrm{E}+00$ & $1.78 E+02$ \\
\hline $90 Y$ & $6.21 E-04$ & $1.55 \mathrm{E}+02$ & $71.0 \mathrm{E}-04$ & $1.78 \mathrm{E}+02$ \\
\hline $106_{\mathrm{Ru}}$ & $9.66 \mathrm{E}-03$ & $3.29 \mathrm{E}+01$ & $1.42 E-02$ & $4.84 E+01$ \\
\hline $106 \mathrm{Rh}$ & $9.23 \mathrm{E}-09$ & $3.29 E+01$ & 1. $36 \mathrm{E}-08$ & $4.84 E+01$ \\
\hline $125 \mathrm{Sb}$ & $8.52 E-03$ & $8.80 \mathrm{E}+00$ & $1.28 \mathrm{E}-02$ & 1. $32 \mathrm{E}+01$ \\
\hline $125 \mathrm{~m}_{\mathrm{Te}}$ & $1.19 \mathrm{E}-04$ & $2.15 \mathrm{E}+00$ & $1.79 \mathrm{E}-04$ & $3.22 \mathrm{E}+00$ \\
\hline $134 \mathrm{Cs}^{\mathrm{c}}$ & $5.80 \mathrm{E}-02$ & $6.96 \mathrm{E}+01$ & $8.21 \mathrm{E}-02$ & $9.85 \mathrm{E}+01$ \\
\hline $137 \mathrm{Cs}^{\mathrm{c}}$ & $2.36 \mathrm{E}+00$ & $2.31 \mathrm{E}+02$ & $3.59 E+00$ & $3.52 E+02$ \\
\hline $137 \mathrm{~m}_{\mathrm{Ba}}$ & $4.07 \mathrm{E}-07$ & $2.19 \mathrm{E}+02$ & $6.18 \mathrm{E}-07$ & $3.33 E+02$ \\
\hline $144 \mathrm{Ce}$ & $6.00 \mathrm{E}-03$ & $1.92 \mathrm{E}+01$ & $5.55 \mathrm{E}-03$ & $1.78 \mathrm{E}+01$ \\
\hline $144 \mathrm{Pr}$ & $2.54 \mathrm{E}-07$ & $1.92 \mathrm{E}+01$ & $2.35 \mathrm{E}-07$ & $1.78 \mathrm{E}+01$ \\
\hline $144 \mathrm{mPr}$ & $1.27 \mathrm{E}-09$ & $2.30 \mathrm{E}-01$ & $1.18 E-09$ & $2.13 \mathrm{E}-01$ \\
\hline $154 \mathrm{Eu}$ & 1. $31 \mathrm{E}-01$ & $1.97 \mathrm{E}+01$ & $2.47 \mathrm{E}-01$ & 3. $71 \mathrm{E}+01$ \\
\hline $155 \mathrm{Eu}$ & $6.33 E-03$ & $8.86 E+00$ & 1. $20 \mathrm{E}-02$ & $1.68 \mathrm{E}+01$ \\
\hline $235 \mathrm{U}$ & $1.39 \mathrm{E}+01$ & $2.91 \mathrm{E}-05$ & $5.60 \mathrm{E}+00$ & 1. $18 \mathrm{E}-05$ \\
\hline $238 \mathrm{U}$ & $2.13 E+03$ & $7.02 E-04$ & $3.69 E+03$ & $1.22 \mathrm{E}-03$ \\
\hline $238 \mathrm{Pu}$ & $4.97 \mathrm{E}-01$ & $8.45 E+00$ & $8.91 \mathrm{E}-01$ & 1. $52 \mathrm{E}+01$ \\
\hline${ }^{239} \mathrm{Pu}$ & 1. $14 \mathrm{E}+01$ & $7.04 \mathrm{E}-01$ & $1.95 \mathrm{E}+01$ & $1.21 \mathrm{E}+00$ \\
\hline${ }^{240} \mathrm{Pu}$ & $5.62 \mathrm{E}+00$ & $1.29 \mathrm{E}+00$ & $1.02 \mathrm{E}+01$ & $2.33 \mathrm{E}+00$ \\
\hline${ }^{241} \mathrm{Pu}$ & $2.27 \mathrm{E}+00$ & $2.49 \mathrm{E}+02$ & $4.42 E+00$ & $4.86 E+02$ \\
\hline $241_{\mathrm{Am}}$ & $8.48 \mathrm{E}-01$ & $2.71 \mathrm{E}+00$ & $1.81 \mathrm{E}+00$ & $5.79 E+00$ \\
\hline $244 \mathrm{Cm}$ & $8.44 \mathrm{E}-02$ & $6.92 E+00$ & $4.22 \mathrm{E}-01$ & $3.46 \mathrm{E}+01$ \\
\hline Balance & $\underline{5.55 E+01}$ & $\underline{6.96 E+01}$ & $1.41 \mathrm{E}+02$ & $8.46 \mathrm{E}+01$ \\
\hline Totals: & $2.22 \mathrm{E}+03$ & $1.33 \mathrm{E}+03$ & $3.88+03$ & $1.99 \mathrm{E}+03$ \\
\hline \multicolumn{5}{|c|}{$\begin{array}{l}\text { a Oconee-1 Rod } 08639 \text { irradiated to } 38.2 \mathrm{MWD} / \mathrm{kg} \mathrm{U} \text { after } 5-\mathrm{yr} \text { dec } \\
\text { bQuad Cities-1 Fuel Rod B5A-0139 irradiated to } 33.7 \mathrm{MWD} / \mathrm{kg} \mathrm{U} \text { a } \\
\text { decay. } \\
\text { cTotal mass of all Cs isotopes: } 6.42 \mathrm{~g} \text { (PWR) and } 9.96 \mathrm{~g} \text { (BWR). }\end{array}$} \\
\hline
\end{tabular}

cask safety assessments. Note also that these example radionuclide inventories may not be upper-bound values. 


\section{I.5 Gap Inventory of an Irradiated Fuel Rod}

Most fission and activation products formed within fuel rods are contained as impurity atoms and ions within the $\mathrm{UO}_{2}$ fuel matrix. However, because of thermal motion, however, some of these impurity species diffuse to crystal surfaces and are released from the $\mathrm{UO}_{2}$ lattice. (A very small fraction is actually ejected from the $\mathrm{UO}_{2}$ structure directly, as a result of knockout or recoil mechanisms.) Once released from the $\mathrm{UO}_{2}$ lattice, these species migrate to the voids within the fuel pellets and within the spaces between the pellets and cladding (the fuel-cladding gap). Hence, during irradiation while the fuel is at an elevated temperature, a gap inventory of radionuclides is developed. Because the gas phase component of this inventory affects heat transfer characteristics of the fuel rods, identifying the expected gap inventory has been the subject of considerable study [PA83, MA87]. Most of these analyses have focused on noble gas fission products.

A detailed analysis of fission-gas gap-inventory assays of irradiated fuel rods resulted in the publication of the American National Standards Institute (ANSI) standard ANSI/ANS-5.4-1982 [AN82a]. This standard measures gap inventory as a fraction of total inventory and relates the gap inventory of a particular nuclide to fuel burnup, temperature, and the amount of time that fuel is at a given temperature. Because these parameters are not constant in space and time, this standard requires that the fuel pellet column within a rod be divided into a minimum of six radial nodes of equal volume or radial increment, and a minimum of ten axial nodes of equal length. In addition, the irradiation period is to be apportioned into a series of burnup time increments corresponding to burnup periods of a maximum of $2000 \mathrm{MWD} / \mathrm{MTU}$ so that the temperature and power in each increment can be assumed constant. Thus, the calculational process can be accomplished easily if a suitable computer program is used.

ANSI/ANS-5.4-1982 was primarily developed to account for the buildup of $\mathrm{Xe}$ and $\mathrm{Kr}$ isotopes in the connected void space of the fuel rod. Extension to moderately volatile species (iodine, $\mathrm{Cs}$, and $\mathrm{Te}$ ) is accomplished by prescribing the appropriate diffusion parameters in terms of the corresponding parameter for the noble gases. Thus, the effective diffusion coefficient for the $C_{s}$ isotopes ( $D^{\prime} C_{s}$, for example) is related to the noble gas diffusion coefficient, $D^{\prime}$ noble, through the equation

$$
D^{\prime} \mathrm{Cs} / \mathrm{D}^{\prime} \text { noble }=2 \text {. }
$$

These relations were largely based on diffusion measurements involving small samples of unclad, lightly irradiated $\mathrm{UO}_{2}$. More recently, direct measurements of gap inventories of noble gases and moderately volatile fission products have been conducted. These measurements suggest instead that the gap inventories of noble gases and moderately volatile species are equal when expressed as fractions of total inventory [MA87, L080, L081, J085, C087]. This implies that, contrary to the ANSI/ANS standard,

$$
D^{\prime} \text { Cs } / D^{\prime} \text { noble }=1 \text {. }
$$


For safety assessments involving spent fuel, it is therefore recommended that the gap inventories of noble gases be calculated from the measured fission gas release, or as prescribed by ANSI/ANS-5.4-1982.

However, the Cs nuclide gap inventories expressed as fractions of the total Cs inventory should be made equal to the value for the noble gases. Thus, if the inventory of $85 \mathrm{Kr}$ is $10 \%$ of the total amount of $85 \mathrm{Kr}$ in the fuel rod, then the gap inventories of $134 \mathrm{Cs}$ and $137 \mathrm{Cs}$ should also be 108 of their corresponding total inventories in the rod.

Numerous measurements of fission gas release have been performed by puncturing irradiated LWR rods. The majority of BWR rods in the database were unpressurized and showed gas release as high as 258 (see Figure I-28). However, the release tended to be low $(<28)$ for PWR rods that were pressurized, although it increased with burnup. Higher gas release can occur when the rod is not pressurized, such as the Maine Yankee or Connecticut Yankee rods, or if the fuel is operated at temperatures that encourage grain growth, such as the two high Calvert Cliff rods (43 GWD/MTU, $25 \mathrm{~kW} / \mathrm{m}$ ) [GU88d]. Newer pressurized BWR rods are expected to release less fission gas on the average, although pressurization less than in PWR rods and power peaking in some BWR rods make predicting BWR gap releases difficult.

Sample calculations using the ANSI/ANS-5.4-1982 standard were also performed for the same two cases for which the ORIGEN2 calculations were made (see Section 1.4). The calculations were performed using the FREY fuel rod analysis routine [RA87] that in most cases predicts the fission gas release within a factor of two. When the code is not accurate within a factor of two, the measured value can be significantly higher than the calculated value when peaks in the reactor power profile are underestimated.

Geometric and operational characteristics input to the ORIGEN2 code are given in Table I-14. Selected results of the calculations are given in Table I-15. These data indicate that the gap inventory of fission gases in the PWR rod is 1.028 of total fission gas inventory and the gap inventory in the BWR rod is $2.05 \%$ of the total inventory. Direct measurements of the gap inventory of the Oconee-1 rod yielded 3.358 of the total fission gas inventory [BA83b], whereas measurements of the Quad Cities-1 rod yielded a gap inventory value of 13.5\% [L082]. The calculated fission gas release for both rods and measured fission gas release for the BWR rods are in line with the bounds shown in Figures I-28 and I-29. The measured release for the PWR rods is slightly higher than expected. (The discrepancy for the BWR fuel rod is indicative of the difficulty in modeling the axial power profile accurately.)

Because of their mobility, the noble gas fission products are uniformly distributed throughout the gap region of the fuel rod. The gap inventories of fission products in condensible form are distributed on the surfaces defining the gap region in a manner determined by the temperature distribution of the fuel surfaces. (The partial pressure of the gas phase is uniform but very low, however.) Regardless of physiochemical form, all long-lived isotopes of a given element are distributed identically (as opposed to uniformly) throughout a fuel rod. Thus, the gap inventory of 
Table I-14

Input Data for Gap Inventory Calculations

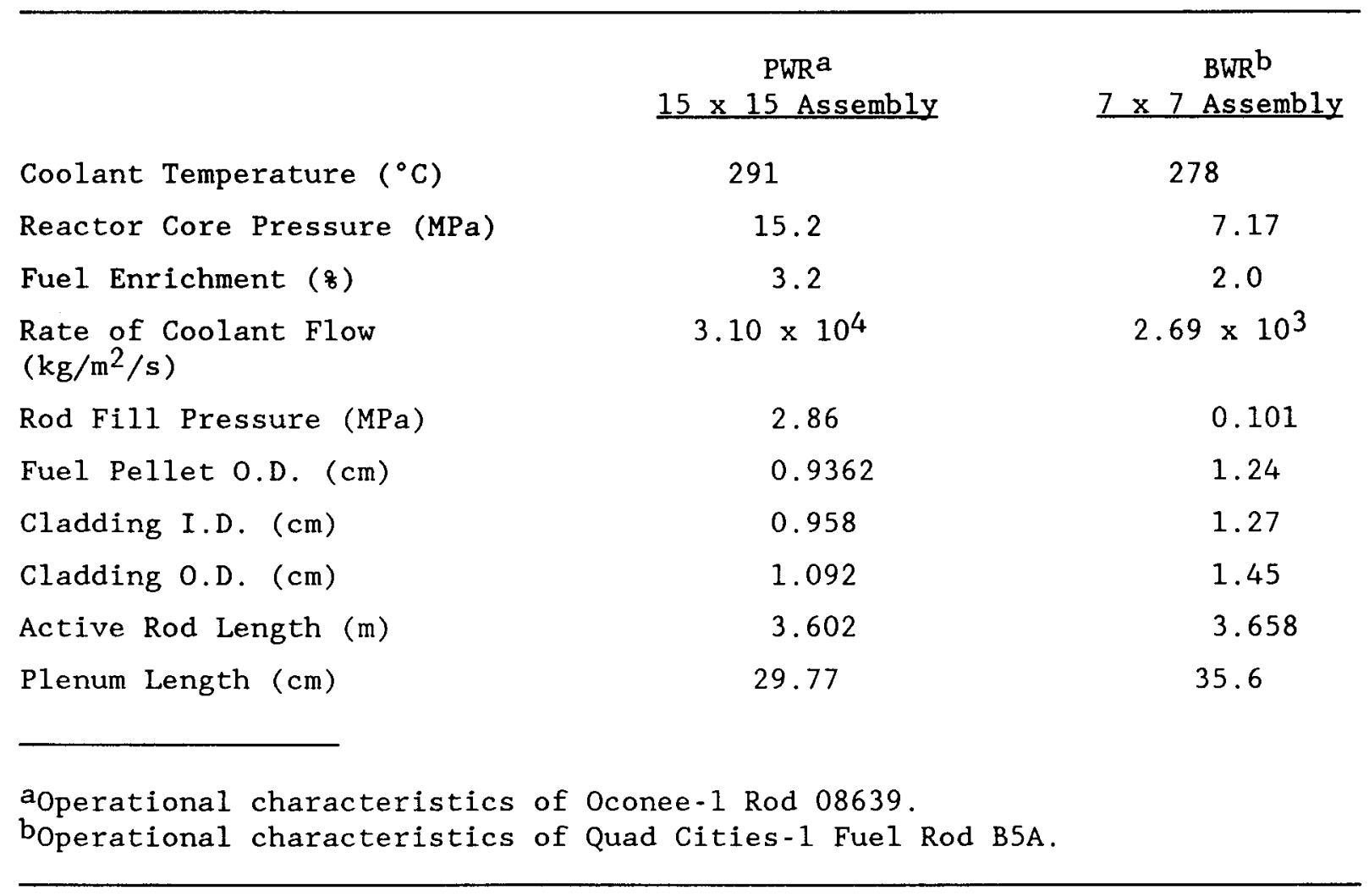

Table I-15

Calculated Fission Gas Production and Gap Inventory

in Irradiated PWR and BWR Fuel Rods

\begin{tabular}{|c|c|c|c|c|c|}
\hline \multirow[b]{2}{*}{$\begin{array}{l}\text { Fuel Rod } \\
\text { Type } \\
\end{array}$} & \multicolumn{3}{|c|}{ Fission Gas } & \multicolumn{2}{|c|}{$\begin{array}{c}\text { Fission } \\
\text { Gas Composition } \\
\text { in Gap }\end{array}$} \\
\hline & $\begin{array}{c}\text { Burnup } \\
(\mathrm{MWD} / \mathrm{kg} \mathrm{U})\end{array}$ & $\begin{array}{c}\text { Produced } \\
\text { (mo1) }\end{array}$ & $\begin{array}{l}\begin{array}{c}\text { Released to Gap } \\
(\mathrm{mol})\end{array} \\
\end{array}$ & $\begin{array}{c}\begin{array}{c}\text { Krypton } \\
(\text { mol) }\end{array} \\
\end{array}$ & $\begin{array}{r}\text { Xenon } \\
(\mathrm{mo} 1) \\
\end{array}$ \\
\hline PWR $^{a}$ & 38.3 & $5.55 \times 10^{-2}$ & $5.64 \times 10^{-4}$ & $8.64 \times 10^{-5}$ & $4.78 \times 10^{-4}$ \\
\hline $\mathrm{BWR}^{\mathrm{b}}$ & 33.7 & $9.08 \times 10^{-2}$ & $1.86 \times 10^{-3}$ & $2.90 \times 10^{-4}$ & $1.6 \times 10^{-3}$ \\
\hline $\begin{array}{l}\text { a Oconee-1 } \\
\text { bQuad Cit } \\
\text { decay. }\end{array}$ & $\begin{array}{l}\text { Rod } 08639 \\
\text { ies-1 Fue } 1\end{array}$ & radiated to & $\begin{array}{l}38.2 \mathrm{MWD} / \mathrm{kg} \mathrm{U} \text { aft } \\
\text { cradiated to } 33.7\end{array}$ & $\begin{array}{l}\text { er } 5-y r \text { decay } \\
\text { MWD } / \mathrm{kg} \text { U aft }\end{array}$ & er 5-yr \\
\hline
\end{tabular}




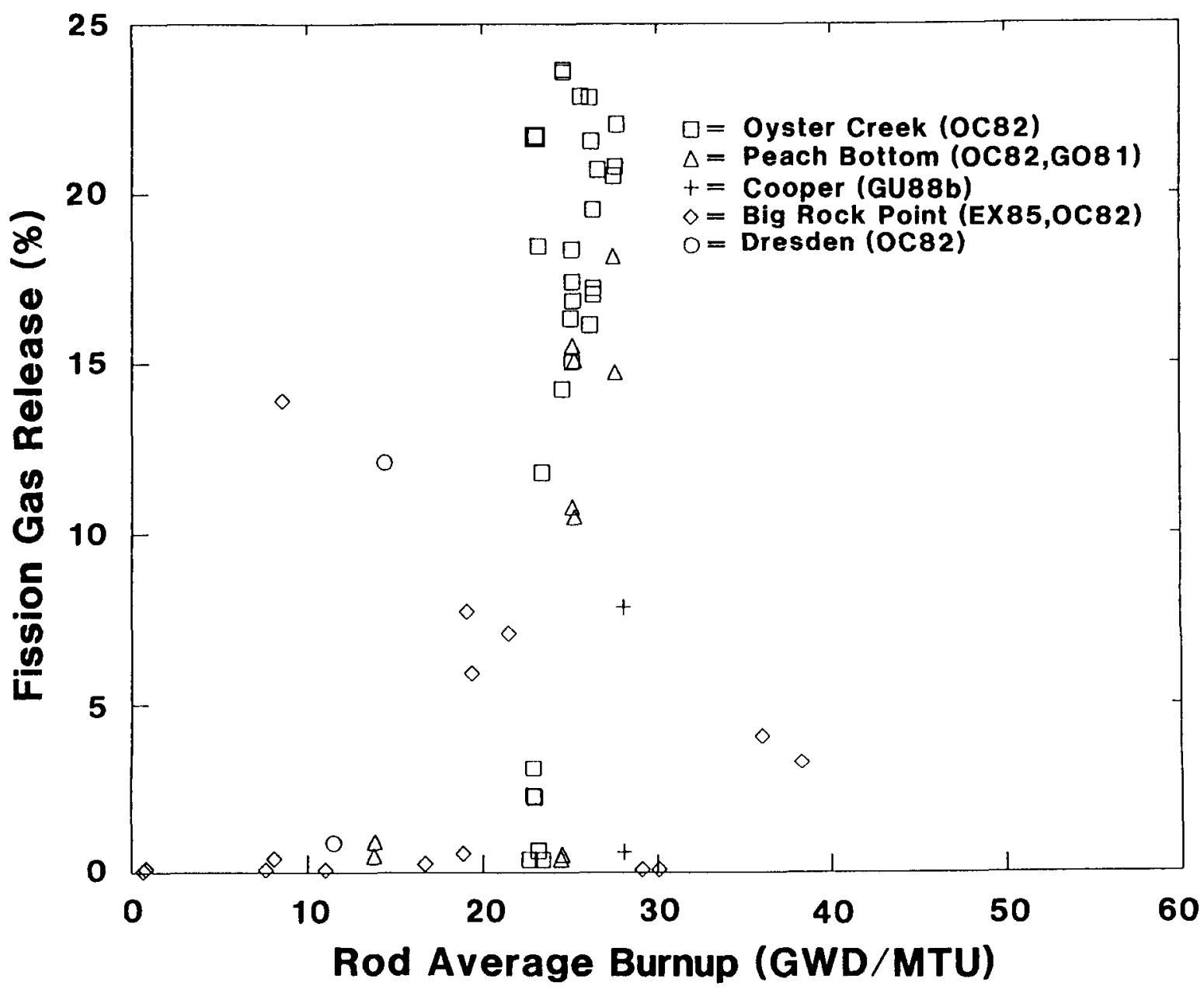

Figure I-28. Fission Gas Release for BWR Rods

the $85 \mathrm{Kr}$ expressed as a fraction of the total inventory of $85 \mathrm{Kr}$ is equal to the gap inventory calculated for total $\mathrm{Kr}$. Similarly, the gap inventories of the long-lived Cs isotopes are considered identical to those of the fission gases, but are not distributed within the gap region in the same way.

Detailed investigations of the interim inventories in BWR and PWR rods of up to $36 \mathrm{GWD} / \mathrm{MTU}$ were performed by Bleier [BL84]. In this analysis, the fraction of $\mathrm{H}^{3}$ released to the gap was always very small. The $\mathrm{H}^{3}$ distributes itself between the fuel and the cladding as shown in Table I-16. For the purpose of this analysis, it is conservatively assumed that 508 of the $\mathrm{H}^{3}$ inventory is present in the gap region. (See also Appendix IV for a discussion of $\mathrm{H}^{3}$ behavior.) 


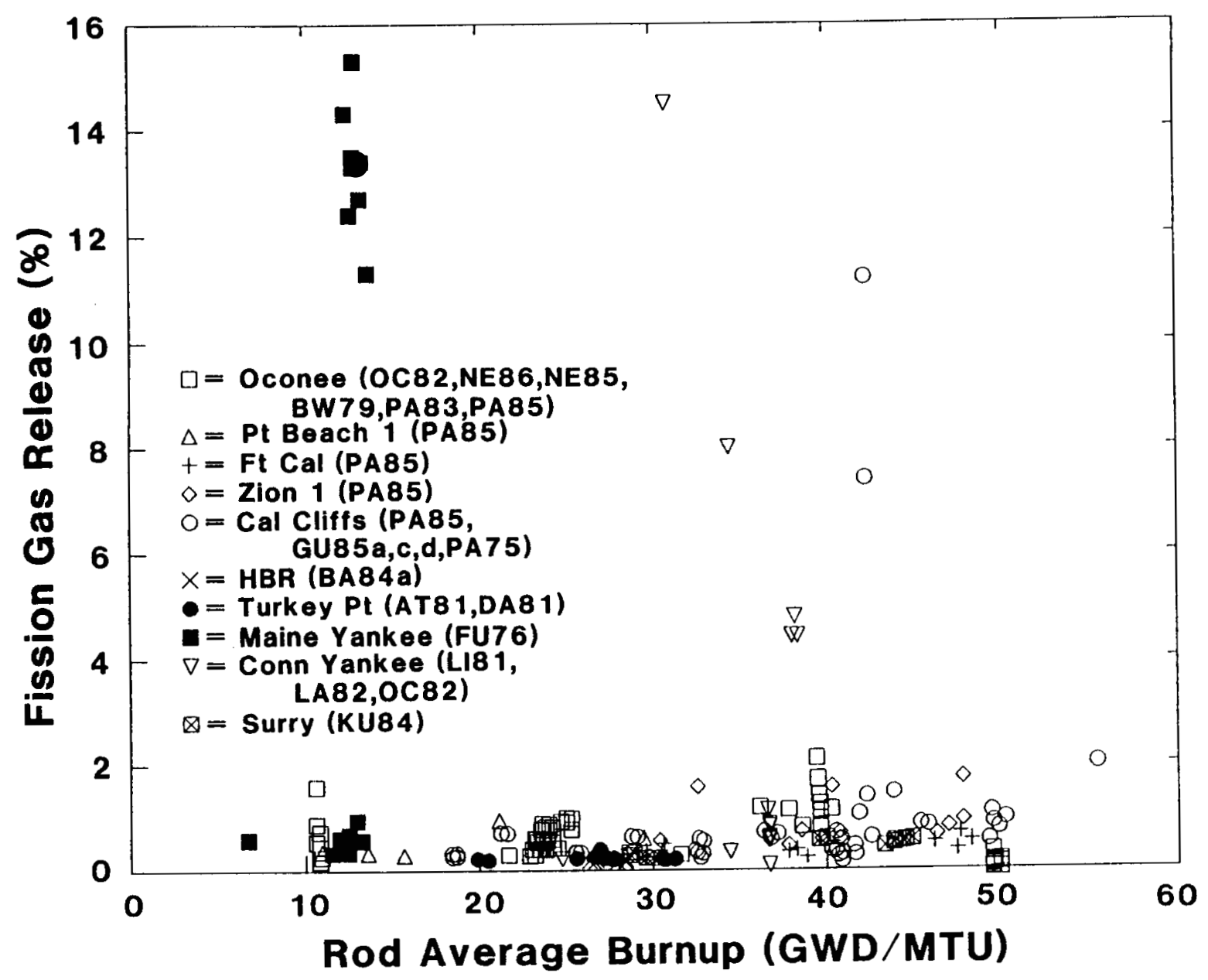

Figure I-29. Fission Gas Release for PWR Rods

Table I-16

Tritium Distribution as a Percentage of the Inventory

in LWR Spent Fue1 [BL84]

\begin{tabular}{lll}
\hline & $\underline{\text { PWR }}$ & $\underline{\text { BWR }}$ \\
Fuel & 37 & 50 \\
Cladding & 63 & 50 \\
\hline
\end{tabular}

There are no data concerning gap inventories of Te nuclides. However, Te releases from the fuel rod-cladding combination, even at elevated temperatures, are delayed until a significant fraction of the Zircaloy 
cladding is oxidized [C087]. Otherwise, the Te gap inventory is conservatively estimated to be identical to that of Cs. For all other nuclides of interest to spent-fuel safety assessments, the gap inventories may be considered negligible.

\section{I.6 Conclusions and Recommendations}

There are 23 variations of BWR fuel assemblies and 32 variations of PWR fuel assemblies made by five different manufacturers. Many variations have small differences in rod length or rod diameter. Most BWR assemblies have either $7 \times 7$ or $8 \times 8$ arrays, while most PWR assemblies have $14 \times 14$ and $15 \times 15$ arrays. While some fuel rods are clad with stainless steel, most BWR and PWR rods are clad with Zircaloy-2 and Zircaloy-4, respectively. Recent improvements in fuel rod design and manufacture have decreased fuel breach rates considerably. A small amount of rods come out of the reactor breached, and an additional small fraction are breached by handling. Most of these breaches are small pinholes. The actual damage incurred depends on the particular handling scenario. When estimating cladding damage, the following should be considered:

1. There is no count of the intact rods that have been damaged in the reactor by such mechanisms as fretting and baffle jetting, or by previous core handling and consolidation. However, the fraction of such rods is expected to be small.

2. Storage in the reactor water pool apparently does not cause fuel rod deterioration.

3. There appears to be enough conservatism built into the temperature storage limit determinations so that partial crack growth in dry storage by SCC, creep rupture, or delayed hydrogen cracking should be very slow.

4. The possibility of hydride reorientation during dry storage could degrade the mechanical properties of Zircaloy cladding. This is of special concern with newer, high burnup fuel when high cladding hydrogen contents have been observed.

5. The oxide layer that forms on the cladding surface during irradiation should be considered wastage. For PWRs, metal losses as high as $93 \mu \mathrm{m}$ with a mean metal loss of $\sim 17 \mu \mathrm{m}$ have been observed. For BWRs, uniform metal losses of $-13 \mu \mathrm{m}$ and nodular metal losses of $80 \mu \mathrm{m}$ have been observed.

6. Partial cracks occur on the inner surface of the cladding. Calculations indicate that for a high percentage of the rods, the maximum partial crack does not exceed 25 to $30 \%$ of the cladding wall thickness. Before transport, a maximum of 60 incipient cracks are estimated to exist in a PWR fuel rod and 75 incipient cracks are estimated to exist in a BWR fuel rod. 
7. Approximately 71 to 1008 of the fuel can be considered mechanically bonded to the PWR cladding after a few irradiation cycles, but other than some chemically bonded (10\%) fuel, most BWR pellets are free of the cladding.

8. ORIGEN2 was used in these example analyses for determining the radionuclide inventory of spent fuel. Most radionuclides remain with the fuel, but some, such as $C s$, iodine, and $T e$, are released to the pellet-cladding gap in the same ratio as the fission gas release. The fission gas release can be calculated using the ANSI-N5.4 standard or an upper and lower estimate can be determined from fission gas surveillance data.

\section{I.7 References}

[AN82a] American Nuclear Society, "Method for Calculating the Fractional Release of Volatile Fission Products from Oxide Fuel," ANSI/ANS5.4-1982.

[AN82b] Andrews, M. G., S. C. Hatfield, and E. J. Ruzauskas, "Operating Experience with Combustion Engineering Fuel at High Burnup," in "Proceedings of ANS Topical Meeting on LWR Extended Burnup Fuel Performance and Utilization," Williamsburg, VA, p. 1-5, April 1982.

[AN88] Andrews, M. G., G. P. Smith, and M. A. Shubert, "Experience and Developments with Combustion Engineering Fuel," in "Proceedings of ANS Topical Meeting on LWR Fuel Performance," Williamsburg, VA, p. 90, April 1988.

[BA81] Bailey, W. J., et al., "Fuel Performance Annual Report for 1980," Pacific Northwest Laboratories, Richland, WA, NUREG/CR-2410, December 1981.

[BA82] Bailey, W. J., and M. Tokar, "Fuel Performance Annual Report for 1981," Pacific Northwest Laboratories, Richland, WA, NUREG/CR-3001, December 1982 .

[BA83a] Bailey, W. J., "Experience with Fue1 Damage Caused by Abnormal Conditions in Handling and Transporting Operations," in "Proceedings of 7 th International Symposium on Packaging and Transportation of Radioactive Materials (PATRAM 83)," Conference No. $830528,1: 802-807,1983$.

[BA83b] Bain, G. M., and W. A. McInteer, "Data Package for High Burnup Fuel Samples," Babcock and Wilcox, Lynchburg, VA, LRC4063, 1983.

[BA84a] Bailey, W. J., and M. Tokar, "Fuel Performance Annual Report for 1982," Pacific Northwest Laboratories, Richland, WA, NUREG/CR-3602, March 1984.

[BA84b] Barner, J. O., "Characteristics of LWR Spent Fue1 MCC-Approved Testing Material--ATM-101," Pacific Northwest Laboratories, Richland, WA, PNL-5109, January 1984. 
[BA85a] Bailey, W. E., M. O. Marlowe, and R. A. Proebstle, "Trends in BWR Fuel Performance," in "Proceedings of ANS Topical Meeting on LWR Fuel Performance," Pacific Northwest Laboratories, Richland, WA, DOE/NE/34130, p. 1-3, April 1985.

[BA85b] Bairiot, D. H., and M. Lippens, "High Burnup Performance of BN Fuels in LWRs," in "Proceedings of ANS Topical Meeting on LWR Extended Burnup Fuel Performance and Utilization," Williamsburg, VA, p. 2-65, April 1982.

[BA88a] Bailey, W. J., and S. Wu, "Fuel Performance Annual Report for 1986," Pacific Northwest Laboratories, Richland, WA, NUREG/CR-3950, Vol. 4, March 1988.

[BA88b] Baily, W. E., L. D. Noble, R. G. Serenka, C. S. Kennedy, and R. A. Proebstle, "Performance Experience of GE Boiling Water Reactor Fuel," (GE), American Nuclear Society Topical Meeting on LWR Fuel Performance, April 17-20, Williamsburg, VA, 1988.

[BA89] Bailey, W. J., "Extended-Burnup LWR Fue1: The Amounts, Characteristics, and Potential Effects of Interim Storage," Pacific Northwest Laboratories, Richland, WA, PNL-6717, March 1989.

[BE70] Benjamin, J. R., and C. C. Allin, Probability, Statistics, and Decision of Civil Engineers, New York: McGraw-Hill Publishing Company, 1970 .

[BL84] Bleier, A., R. Kroebel, K. H. Neeb, and E. Schneider, "The Inventories and Behavior in Dry-Cladding of Spent LWR-Fuel Rods," in "Proceedings of ANS Conference Fuel Reprocessing and Waste Management," Jackson, WY, August 1984.

[B085] Boehm, H. H., and H. Foerch, "Operational Experience Gained with the Failed Rod Detection System in Nuclear Power Plants," in "Proceedings of Waste Management '85," R. Post, ed., p. 259, 1985.

[C082] Coleman, T. A., G. M. Bain, T. D. Pyecha, J. S. Tulenko, R. G. Snipes, and J. D. Kortheuer, "Extended Burnup Fuel Performance and Utilization," in "Proceedings of ANS Topical Meeting on LWR Fuel Performance," Williamsburg, VA, p. 1-39, April 1982.

[C087] Collins, J. L., M. F. Osborne, and R. A. Lorenz, "Fission Product Tellurium Behavior Under Severe Light Water Reactor Accident Conditions," Nuclear Technology, 그, p. 18 (1987).

[CR80] Croff, A. G., "ORIGEN2--A Revised and Updated Version of the Oak Ridge Isotope Generation and Depletion Code," Oak Ridge National Laboratory, Oak Ridge, TN, ORNL-5621, 1980.

[CR83] Croff, A. G., "ORIGEN2: A Versatile Computer Code for Calculating the Nuclide Compositions and Characteristics of Nuclear Materials," Nuclear Technology, 62, p. 335 (1983). 
[CU78] Cubicciotti, D., and R. L. Jones, "EPRI NASA Cooperative Project on Stress Corrosion Cracking of Zircaloys," Electric Power Research Institute, Palo Alto, CA, EPRI NP-717, 1978.

[CU80] Cubicciotti, D., R. L. Jones, and B. C. Syrett, "Stress Corrosion Cracking of Zircaloy," Electric Power Research Institute, Palo Alto, CA, PRI NP-1329, March 1980.

[DA81] Davis, R. B., "Pre-Test Non-Destructive Examination Data Summary Report on Turkey Point Spent Fuel Assemblies D01, D04, and D06 for the Climax Spent Fuel Tests," Electric Power Research Institute, Palo Alto, CA, HEDL-TME 80-83, January 1981.

[DA84] Dannhauser, G., A. Atrens, and G. Baro, "Stress Corrosion Cracking of Zircaloy 4 Cladding Tubes, Part 2. Cs/Cd Mixtures and Fractography, " Journal Nuclear Material, 126, p. 103 (1984).

[EI82] Einziger, R. E., S. A. Atkin, V. Pasupathi, and D. Stellrecht, "High Temperature Post Irradiation Material Performance of Spent Pressurized Water Reactor Fuel Rods Under Dry Storage Conditions," Nuclear Technology, 57, p. 65-80 (1982).

[EI84a] Einziger, R. E., and J. A. Cook, "Pre-Test Visual Examination of LWR Rods Used in the Long-Term Low-Temperature Whole Rod Test," Electric Power Research Institute, Palo Alto, CA, NUREG/CR-3285, 1984.

[EI84b] Einziger, R. E., and J. A. Cook, "LWR Spent Fuel Dry Storage Behavior at $229^{\circ} \mathrm{C}, "$ Electric Power Research Institute, Palo Alto, CA, NUREG/CR-3708, 1984.

[EI84c] Einziger, R. E., and R. Kohli, "Low-Temperature Rupture Behavior of Zircaloy-Clad Pressurized Water Reactor Spent Fuel Rods Under Dry Storage Conditions," Nuclear Technology, 67, p. 107 (October 1984).

[EX82] Exarhos, C. A., D. E. Bentley, T. R. Blair, L. F. Van Swam, and F. P. Walquist, "High Burnup Performance of ENC Fuel in the H. B. Robinson PWR," in "Proceedings of ANS Topical Meeting on LWR Extended Burnup Fuel Performance and Utilization," Williamsburg, VA, pp. 1-59, April 1982.

[FU76] Fuhrman, N., V. Pasupathi, D. B. Scott, S. M. Temple, S. R. Pati, and T. E. Hollowe11, "Evaluation of Fuel Rod Performance in Maine Yankee Core I," Electric Power Research Institute, Palo Alto, CA, EPRI NP-218, November 1976.

[GA78a] Garzarolli, F., R. Manzel, S. Reschke, and E. Tenckhoff, "KWU Review on Corrosion and Dimensional Behavior of Zircaloy Under Water Reactor Conditions," in "Proceedings of the Fourth International Conference in Zirconium in the Nuclear Industry," Stratford-on-Avon, England, ASTM-STP-681, June 1978. 
[GA78b] Garzarolli, F., R. Manzel, M. Peeks, and H. Stehle, "Observations and Hypotheses on Pellet-Clad Interaction Failure, " Kerntechnik 20, Jahrgang, No. 1 (1978).

[GA79] Garzarolli, F., J. Von Jan, and H. Stehle, "The Main Causes of Fuel Element Failure in Water-Cooled Power Reactors," Atomic Energy Review, 17, No. 1 (1979).

[GA80] Garzarolli, F., D. Jorde, R. Manzel, G. W. Parry, and P. G. Smerd, "Review of PWR Fuel Rod Waterside Corrosion Behavior," Electric Power Research Institute, Palo Alto, CA, EPRI NP-1472, August 1980.

[GI85] Gilbert, E. R., et al., "Assessment of Nitrogen as an Atmosphere for Dry Storage of Spent LWR Fuel," Pacific Northwest Laboratories, Richland, WA, PNL-5569, September 1985.

[GI88] Gilbert, E. R., W. J. Bailey, and A. B. Johnson, "Results of Studies in the Behavior of Spent Fuel in Storage," Journal of Nuclear Material Management, p. 27 (April 1988).

[GR88] Grapengresser, B., A. R. Massih, O. Nylund, L. Hyden, S. O. Anderson, E. Patrakka, and G. Ronnberg, "High Burnup Fuel Evaluation Activities in Sweden and Finland," in "Proceedings of the ANS Topical Meeting on LWR Fuel Performance," Williamsburg, VA, April 1988.

[GU88a] Guenther, R. J., D. E. Blahnik, T. K. Campbe11, U. P. Jenquin, J. E. Mendel, and C. K. Thornhill, "Detailed Characterization of LWR Fuel Rods for the U.S. Civilian Radioactive Waste Management Program," presented at the Berlin Meeting of the Materials Research Society, Berlin, Germany, November 1988.

[GU88b] Guenther, R. J., D. E. Blahnik, T. K. Campbe11, U. P. Jenquin, J. E. Mende 1, L. E. Thomas, and C. L. Thornhil1, "Characterization of Spent Fuel Approved Testing Material--ATM-103," Pacific

Northwest Laboratories, Richland, WA, PNL-5109-103, April 1988.

[GU88c] Guenther, R. J., D. E. Blahnik, T. K. Campbe11, U. P. Jenquin, J. E. Mendel, L. E. Thomas, and C. K. Thornhill, "Characterization of Spent Fuel Approved Testing Material--ATM-105," Pacific Northwest Laboratories, Richland, WA, PNL-5109-105, 1988.

[GU88d] Guenther, R. J., D. E. Blahnik, T. K. Campbell, U. P. Jenquin, J. E. Mende1, and C. K. Thornhil1, "Characterization of Spent Fuel Approved Testing Material-ATM-106," Pacific Northwest Laboratories, Richland, WA, PNL-5109-106 (UC-70), pp. 4, 21, October 1988 .

[H088] Holzer, R., and H. Knaab, "Recent Fuel Performance Experience and Implementation," in "Proceedings of the ANS Topical Meeting on LWR Fue1 Performance," Williamsburg, VA, p. 69, April 1988. 
[IA76] IAEA (Internationa1 Atomic Energy Association), "Vol. X: Power and Research Reactors," "Directory of Nuclear Reactors," International Atomic Energy Association, Vienna, Austria, 1976.

[J083] Johnson, A. B., and E. R. Gilbert, "Technical Basis for Storage of Zircaloy-Clad Spent Fuel in Inert Gases," Pacific Northwest Laboratories, Richland, WA, PNT.-4835, September 1983.

[J085] Johnson, L. H., S. Stroes-Gascoyne, J. D. Chen, M. E. Attas, D. M. Sellinger, and H. G. Delaney, "The Relationship Between Fuel Element Power and the Leaching of $137 \mathrm{Cs}$ and ${ }^{129} \mathrm{I}$ from Irradiated $\mathrm{UO}_{2}$ Fue1," in "Proceedings: ANS Meeting on Fission Product Behavior and Source Term Research," Snowbird, UT, July 15-19, 1984, Electric Power Research Institute, EPRI NP-4113-SR, p. 15-1 to 15-2, July 1985 .

[J086] Johnson, A. B., Jr., E. R. Gilbert, and J. C. Dobbins, "Evaluation of a PWR Fuel Assembly Subject to Air Storage," in "Proceedings of the Third International Spent Fuel Storage Technology Symposium/Workshop, " Conference-860417, Pacific Northwest Laboratories, Richland, WA, PNL-SA-13878, p. W55-W62.

[J087] Johnson, A. B., Jr., J. C. Boddins, and F. R. Zaloudek, "Assessment of Integrity of Spent Fuel Assemblies Used in Dry Storage Demonstration at the Nevada Test Site," Pacific Northwest Laboratories, Richland, WA, PNL-6207, July 1987.

[KN85] Knaab, H., and R. Von Jan, "Fuel Performance Evaluation and Improved Fuel Utilization by Pool Site Fuel Services," in "Proceedings of the ANS Topical Meeting on LWR Fuel Performance," Orlando, FL, DOE/NE/34130, p. 1-35, April 1985.

[K086] Kohli, R., and V. Pasupathi, "Investigation of Water-Logged Spent Fuel Rods Under Dry Storage Conditions," Pacific Northwest Laboratories, Richland, WA, PNL-5987, September 1986.

[KR85] Kress, T. S., "Review of the Status of Validation of the Computer Codes Used in the Severe Accident Source Term Reassessment Study," Oak Ridge National Laboratory, Oak Ridge, TN, ORNL/TM-8842, BMI2104, April 1985.

[L074] Locke, D. H., "Review of Experience With Water Reactor Fuels 1968 1973, " Nuclear Engineering and Design, 33, p. 94 (1974).

[L080] Lorenz, R. A., J. L. Collins, and A. P. Malinauskas, "Fission Product Release from Highly Irradiated LWR Fuel," Oak Ridge National Laboratory, Oak Ridge, TN, NUREG/CR-0722, February 1980.

[L081] Lorenz, R. A., J. L. Collins, M. F. Osborne, R. L. Towns, and A. P. Malinauskas, "Fission Product Release From BWR Fuel Under LOCA Conditions," Oak Ridge National Laboratory, Oak Ridge, TN, NUREG/CR-1773, July 1981. 
[L082] Lowry, L. M., and V. Pasupathi, "Postirradiation Examination of Fuel Rods from the Quad Cities-1 Reactor," Battelle-Columbus Laboratories, Columbus, OH, BCL-585-82-3, 1982 .

[MA63] Marshal1, R. P., and M. R. Louthan, Jr., Tensile Properties of Zircaloy with Oriented Hydrides, Transactions of ASM, Vol. 56, p. 693,1963 .

[MA85] Mathison, J. E., L. W. Newman, W. A. McInteer, and S. M. Bain, "Recent Operating Experience with B\&W Fuel with Emphasis on Extended Burnup," in "Proceedings of the ANS Topical Meeting on LWR Fuel Performance," Orlando, FL, DOE/NE/34130, p. 2-47, April 1985.

[MA87] Malinauskas, A. P., and J. T. Bell, "The Chemistry of Fission Product Iodine Under Nuclear Reactor Accident Conditions, "Nuclear Safety, 28, p. 505 (1987).

[MC85] McDona1d, S. G., and R. S. Kaiser, "The Impact of Metallic Debris on Fuel Performance--A Case History," in "Proceedings of the ANS Topical Meeting on LWR Fuel Performance," Orlando, FL, DOE/NE/34130, p. 2-3, April 1985.

[MI81] Miller, A. K., H. Ocken, and A. Tasooji, "Iodine Stress Corrosion Cracking of Zircaloy: Laboratory Data, A Phenomenological Mode1, and Predictions of In-Reactor Behavior," Journal of Nuclear Material, 99, p. 254 (1981).

[NE88] Newman, L. W., and H. A. Hassan, "Fuel Performance Overview," in "Proceedings of the ANS Topical Meeting on LWR Fuel Performance," Williamsburg, VA, p. 81, April 1988.

[N078] Norfolk, D. J., "The Development of a Mechanism for Zircaloy-2 Oxidation Below $800 \mathrm{~K}$," Central Electricity Generating Board, Berkeley Nuclear Laboratories, London, United Kingdom, RD/B/N4369, November 1978.

[N083] Northwood, D. O., and U. Kosasih, "Hydrides and Delayed Hydrogen Cracking in Zirconium and Its Alloys," International Metals Reviews, 28, p. 2 (1983).

[N087] Notz, K., Characteristics of Spent Fuel. High-Level Waste, and Other Radioactive Wastes Which May Require Long-Term Isolation, Vo1. 1 of 6, DOE/RW-0184, December 1987.

[NY85] Nylund, 0., "Design and Operational Behavior of SVEA Water Cross BWR Fuel, Light Water Reactor Fuel Performance," in "Proceedings of the ANS Topical Meeting on Light Water Reactor Fuel Performance," Orlando, FL, p. 7-45, April 22-24, 1985.

[OL85] Olsen, C. S., "The Performance of Defected Spent LWR Fuel Rods in Inert Gas and Dry Air Storage Atmospheres," Pacific Northwest Laboratories, Richland, WA, NUREG/CR-4074, January 1985. 
[PA78] Pasupathi, V., J. S. Perrin, E. Roberts, E. H. Pilger, N. R. Metcalf, G. R. Schmidt, H. S. Rosenbaum, U. E. Wolff, W. L. Bell, R. F. Mattas, J. E. Sanecki, and L. A. Neimark, "Determination and Microscopic Study of Incipient Defects in Irradiated Power Reactor Fuel Rods," Electric Power Research Institute, Palo Alto, CA, EPRI-NP-812, July 1978.

[PA83] Papazoglou, T. P., and H. H. Davis, "EPRI/B\&W Cooperative Program on PWR Fuel Rod Performance," Electric Power Research Institute, Palo Alto, CA, EPRI NP-2848, p. 4-34, March 1983.

[PE86] Peehs, M., and J. Fleisch, "LWR Spent Fuel Behavior," Journal Nuclear Materials, 137, p. 190 (1986).

[PI72] Pickman, D. O., "Properties of Zircaloy Cladding," Nuclear Engineering and Design, 21, p. 212-236 (1972).

[PY85] Pyecha, T. D., G. M. Bain, W. A. McInteer, and C. H. Phan, "Waterside Corrosion of PWR Fuel Rods Through Burnups of 50 GWD/MTU," in "Proceedings of the ANS Topical Meeting on LWR Fuel Performance," DOE/NE/34130, p. 3-17, April 1985.

[RA87] Rashid, Y. R., A. J. Zangari, and R. S. Dunham, "FREY-01; Fuel Rod Evaluation System, 1-4," Electric Power Research Institute, Palo Alto, CA, EPRI NP03277-CCM, August 1987.

[R077] Roberts, J. T. A., E. Smith, N. Fuhrman, and D. Cubicciotti, "On the Pellet-Cladding Interaction Phenomenon, "Nuclear Technology, 35, p. 136 (August 1977).

[R087] Roddy, J. W., and J. C. Mailen, "Radiological Characteristics of Light-Water Reactor Spent Fuel: A Literature Survey of Experimental Data," Oak Ridge National Laboratory, Oak Ridge, TN, ORNL/TM-10105, December 1987.

[SA88] Sanders, T. L., C. A. Ottinger, L. A. Brentlinger, D. G. Dippold, J. L. Brimhall, E. R. Gilbert, and R. H. Jones, "The Feasibility of and Incentives for Implementing a Transportable Storage Cask (TSC) Concept for Interim Dry Storage of Spent Fue1," Sandia National Laboratories, Albuquerque, NM, SAND88-2481, (draft) 1988.

[SH83] Shannon, S. H., and D. R. Olander, "Stress Corrosion Cracking of Zircaloy by Cd, I, and Metal Iodides," Journal of Nuclear Material, 113 , p. 234 (1983).

[SH84] Sharkawy, S. W., A. S. Abou-Zahra, and F. H. Hammad, "Liquid Metal Embrittlement of Zircaloy-2 Tubes by Cs and/or Cd," Journal of Nuclear Material, 126, p. 184 (1984).

[S085] Sofer, G. A., L. F. P. Van Swam, and C. A. Exarhos, "Performance of Exxon Nuclear Company Fuel in Light Water Reactors, Light Water Reactor Fuel Performance," in "Proceedings of the ANS Topical Meeting on Light Water Reactor Fuel Performance," Orlando, FL, p. 53, 1985 . 
[S088] Sofer, G. A., L. E. Hansen, L. F. P. Van Swam, and J. F. Patterson, "Performance of ANF Corporation Fuel in LWRs," in "Proceedings of the ANS Topical Meeting on LWR Fuel Performance," Williamsburg, VA, p. 41, April 1988.

[ST82] Stehle, H., P. Fuchs, H. Knaab, and R. Manzel, "KWU Experience and Analyses of LWR Fuel with Respect to High Burnup," in "Proceedings of the ANS Topical Meeting in LWR Extended Burnup Fuel Performance and Utilization," Williamsburg, VA, p. 1-83, April 1982.

[ST83] Steinberg, E., M. Peehs, and H. Stehle, "Development of the Crack Pattern During Stress Corrosion in Zircaloy-Tubes," Journal of Nuclear Material, 118, p. 286 (1983).

[SY77] Syrett, B. C., D. Cubicciotti, and R. L. Jones, "Embrittlement of Zircaloy -4 by Liquid $\mathrm{Cs}$ at $300^{\circ} \mathrm{C}$, " in Zirconium in the Nuclear Industry, A. L. Lowe and G. W. Parry, eds., American Society for Testing Materials, ASTM STP-633, p. 281, 1977.

[TA84] Tasooji, A., R. E. Einziger, and A. K. Miller, "Modeling of Zircaloy Stress Corrosion Cracking: Texture Effects and Dry Storage Spent Behavior," in Zirconium in the Nuclear Industry, D. G. Franklin and R. B. Adamson, Eds., American Society for Testing Materials, ASTM STP-824, p. 595, 1984.

[TU76] Tucker, R. P., P. H. Kreyns, and J. J. Kearns, "The Effects of Internal Surface Flaws, Iodine Concentration, and Temperature on the Stress Corrosion Cracking Behavior of Zircaloy-4 Tubing," Westinghouse Bettis Atomic Power Laboratory, Report No. WAPD-TM1248, February 1976.

[VI75] Videm, K., "Uniform Corrosion and Hydrogen Pickup of Zirconium-Base Cladding Materials in Boiling Water Reactors, "Nuclear Engineering and Design, 33, p. 170 (1975).

[VI88] Vinjamuri, K., E. M. Feldman, C. K. Mullen, B. L. Griebenow, A. E. Arave, and R. C. Hill, "Dry Rod Consolidation Technology Project at the Idaho National Engineering Laboratory," EG\&G, Idaho Falls, ID, EG\&G-WM-8059, Apri1 1988.

[WE79] Weihermiller, W. B., and G. S. Allison, "LWR Nuclear Fuel Bundle Data for Use in Fuel Bundle Handling," Pacific Northwest Laboratories, Richland, WA, PNL-2575, September 1979.

[W084] Woodley, R. E., "Considerations to the Dry Storage of LWR Fuel Rods Containing Water," NUREG/CR-3658, June 1984.

[W086] Woodal1, C. B., and C. R. Johnson, "Rod Consolidation of Ginna Fue1," in "Proceedings of the 3rd International Spent Fuel Technology Symposium/Workshop," Conference Number 860417, Vo1. 1, Seattle, WA, p. s-368, April 8-10, 1986. 
[YA80] Yaggee, F. L., R. R. Mattas, and L. A. Neimark, "Characterization of Irradiated Zircaloys: Susceptibility to Stress Corrosion Cracking," Electric Power Research Institute, Palo Alto, CA, EPRI NP $-1557,1980$. 
This page is intentionally left blank. 


\section{APPENDIX II}

CHARACTERISTICS OF SPENT-FUEL TRANSPORTATION ENVIRONMENTS 
This page is intentionally left blank.

I I - 2 


\section{II.1 Introduction}

The objective of the spent-fuel environments characterization task is to quantify the mechanical and thermal environments of spent fuel in transport casks when subjected to the regulatory normal and hypothetical accident transport conditions. The characterized environments and the methodologies from which the characterizations were obtained are described in this appendix. The quantified environments are presented in formats that provide the basic inputs needed for an analytical model for spent-fuel response.

Formal specification of the transport conditions is provided in the U.S. Code of Federal Regulations (10 CFR 71). Normal and hypothetical accident transport conditions are summarized below; the accident conditions are listed sequentially.

Normal Transport Conditions (10 CFR 71.71)

1. Heat--An ambient temperature of $38^{\circ} \mathrm{C}$ in still air.

2. Cold--An ambient temperature of $-40^{\circ} \mathrm{C}$ in still air and shade.

3. Reduced external pressure--An external pressure of $24.5 \mathrm{kPa}$ absolute.

4. Increased external pressure--An external pressure of $140 \mathrm{kPa}$ absolute.

5. Vibration--Vibration normally incident to transportation.

6. Water spray--A water spray that simulates exposure to rainfall of approximately $5 \mathrm{~cm}$ per hr for at least $1 \mathrm{hr}$.

7. Free drop--Between 1.5 and $2.5 \mathrm{hr}$ after conclusion of the water spray test, a free drop must be performed from a distance of $0.3 \mathrm{~m}$. The cask weight is greater than 15 tonnes and strikes a flat, essentially unyielding horizontal surface that is in a position for maximum expected damage.

8. Penetration--Impact of the hemispherical end of a vertical steel cylinder of $3.2 \mathrm{-cm}$ diameter and $6-\mathrm{kg}$ mass that is dropped from a height of $1.0 \mathrm{~m}$ onto the exposed cask surface that is expected to be most vulnerable to puncture. The long axis of the cylinder must be perpendicular to the cask surface.

Hypothetical Accident Conditions (10 CFR 71.73)

1. Free drop--A free drop of the cask from a 9-m distance striking a flat, essentially unyelding horizontal surface in a position for maximum expected damage. 
2. Puncture-A free drop of the cask from a $1-m$ distance in a position for maximum expected damage onto the upper end of a mild steel bar. The bar is solid, vertical, and cylindrical and is mounted on an essentially unyielding horizontal surface. The bar must be $15 \mathrm{~cm}$ in diameter with the top horizontal and its edge rounded to a radius of $0.6 \mathrm{~cm}$ maximum, and of such a length as to cause maximum damage to the cask, but not less than $20 \mathrm{~cm} 1 \mathrm{ong}$. The long axis of the bar must be vertical.

3. Thermal--Exposure of the whole cask for 30 min minimum to a heat flux not less than that of a radiation environment of $800^{\circ} \mathrm{C}$ with an emissivity coefficient of at least 0.9. For calculation purposes, the surface absorptivity must be either the value that the cask may be expected to possess if exposed to a fire, or 0.8 ; the greater of these two values is used. In addition, convective heat input must be included (when significant) on the basis of still ambient air at $800^{\circ} \mathrm{C}$. Artificial cooling must not be applied after cessation of external heat input. The effects of solar radiation may be neglected before, during, and following the test.

4. Immersion (fissile material)--When water in-leakage has not been assumed for criticality analysis, the cask must be immersed under a head of water of at least $0.9 \mathrm{~m}$ for a minimum period of $8 \mathrm{hr}$ in a position for maximum expected leakage.

5. Immersion (a11 casks)--An undamaged cask subjected to water pressure equivalent to immersion under a minimum 15-m head of water for a period of at least $8 \mathrm{hr}$.

The most severe normal and hypothetical accident transport conditions defined in $10 \mathrm{CFR} 71$ are considered in this characterization of the spentfuel environment. The normal transport conditions considered in this report are heat, vibration (with associated shock), and free drop. The hypothetical accident transport conditions that are addressed are free drop, puncture, and thermal. Other transport conditions are less severe in terms of the potential for causing significant spent-fuel response.

In this analysis, generic or representative cask designs rather than specific casks are used to characterize spent-fuel transportation environments. This methodology was developed to provide general environmental characteristics rather than specify environmental characteristics that are applicable only to very specific cask designs. An analysis tool was designed that can be used to conservatively estimate the potential mechanical environments of spent fuel in a cask during normal and accident transport conditions. The thermal response analyses of the generic casks that were analyzed suggest that unacceptable thermal response of spent fuel in any certifiable cask is improbable for both normal and accident transport conditions.

The general environmental conditions that spent fuel may encounter are summarized as follows: 
1. Shock and vibration of a cask in either over-the-road or over-therail transport modes are passed essentially unaltered to the spent fuel in the cask. The accelerations associated with most shocks typically are $10 \mathrm{~g}$ or less in any of the three principal cask directions. Shocks associated with rail-coupling events can be more severe. Accelerations due to vibration are random in character, tend to be high frequency, and have peak amplitudes of less than $1 \mathrm{~g}$.

2. The peak rigid-body accelerations of a cask in impacts are usually limited to $100 \mathrm{~g}$ or less, regardless of cask orientation at impact because of impact limiter design and materials. The rigid-body accelerations are passed to the spent fuel inside the cask, including possible amplification of the loading caused by gaps between the assembly and cask internals. (See Appendix III [Figures III-12 through III-14] for calculations of assembly acceleration load histories.)

3. If the thermal response of the spent fuel in the cask is acceptable under normal transport conditions (e.g., the peak fuel temperatures do not exceed the acceptable limit of $380^{\circ} \mathrm{C}$ for longterm storage of spent fuel [US85]), then the thermal response of the fuel will almost certainly be acceptable for the regulatory hypothetical fire accident.

Representative cask concepts are described in Section II.2. In Section II.3, several useful methods for estimating accelerations of casks subjected to the regulatory impact and puncture events are described and applied to the analysis of representative cask concepts. Characterization of spent-fuel shock and vibration environments are discussed in section II.4. Section II.5 describes the thermal response analyses of representative cask concepts. Section II.6 summarizes the results of this analysis.

\section{II.2 Cask Concepts and Spent-Fue1 Descriptions}

There are presently about 20 different designs for certified spentfuel casks. Many of these casks are comprised of primarily stainless steel for the principal load-carrying structural members and containment boundary, and lead or depleted uranium for gamma shield material. The gamma shields are sandwiched between thick stainless-steel plates at each end of the cask or between stainless-steel shells on the cylindrical sides of the cask. A low-density, low-Z neutron shield material, such as borated water or resin, is used on the external cylindrical surface of the cask.

To decrease the accelerations and, hence, decrease the structural load of a cask during an impact, energy absorbers, referred to as impact limiters, are used at each end of the transport container. Several existing casks use impact limiters constructed from grain-oriented balsa wood that is encased in a thin metal shell. Metal inserts may be used in portions of the limiters to reduce deformation for some impact orientations. A few existing and proposed casks use metal fins or toroidal rings at the cask ends as impact limiters. 
Four generic cask concepts that are representative of existing systems have been developed. These concepts are based on the six generic models initially considered by Fischer [FI87]. Each of the four representative casks is assumed to use a balsa wood impact limiter or an equivalent crushable foam or aluminum honeycomb impact limiter.

The four casks considered in this appendix include an all-steel truck cask, a lead-shielded truck cask, an all-steel rail cask, and a leadshielded rail cask. The truck casks are sized to a capacity of 1 PWR and 3 BWR spent-fuel assemblies based on geometric constraints. The rail casks are assumed to have a capacity of 21 PWR assemblies; the BWR capacity of the rail casks has not been determined. These fuel capacities represent truck casks that are roughly the same size as current legal-weight truck casks. However, the rail cask concepts are more representative of casks that have been designed for transporting long-cooled spent fuel.

The dimensions of the lead-shielded truck and rail cask concepts are provided in Figure II-1. These dimensions also apply to the all-steel truck and rail casks except that the total thickness of the cylindrical wall for each all-steel cask is $D=31.12 \mathrm{~cm}$, and the dimensions for $E$ and $F$ (indicated in the figure) are suitably changed while keeping the cask cavity radius constant. A neutron shield is not shown in Figure II-1, and none was assumed in the analyses of representative cask concepts.

In the impact analyses, each cask is assumed to have balsa wood impact limiters for mitigating impact energies and forces. The balsa wood grain is in the direction of principal crush strength and energy absorption. This grain is assumed to be aligned in the axial direction of the cylindrical portion of the impact limiter, which has a radius $F$ in Figure II-1 and a height of $101.6 \mathrm{~cm}$. Grain orientation is assumably radial in the annular portion of the limiter, which is situated along the sides of the cask. The height (i.e., lengthwise along the cask side) of this annular portion is not specified by Fischer [FI87]. Based on Figure II-1, this height was initially estimated to be $17.8 \mathrm{~cm}$. Analyses of the casks for the $9.0-\mathrm{m}$ drop impact suggest higher values for this dimension are required for an acceptable impact limiter design. The balsa wood thickness at the ends of these cask models (as provided by Fischer [FI87]) is much more than is required to provide impact protection to the casks.

The impact limiter design shown in Figure II-1 was varied to provide a more balanced design. A hole was added in the end of each impact limiter that reduced the end-drop peak acceleration levels without strongly affecting accelerations in other impact orientations. All generic cask rigid-body analyses discussed in these appendices were based on this design variant.

Nominal physical properties of the materials used in the representative casks are summarized in Table II-1. The properties listed are the same as those used by Fischer [FI87]. Characteristics of typical PWR and BWR fuel assemblies that could be transported in the representative casks are presented in Table II- 2 . 


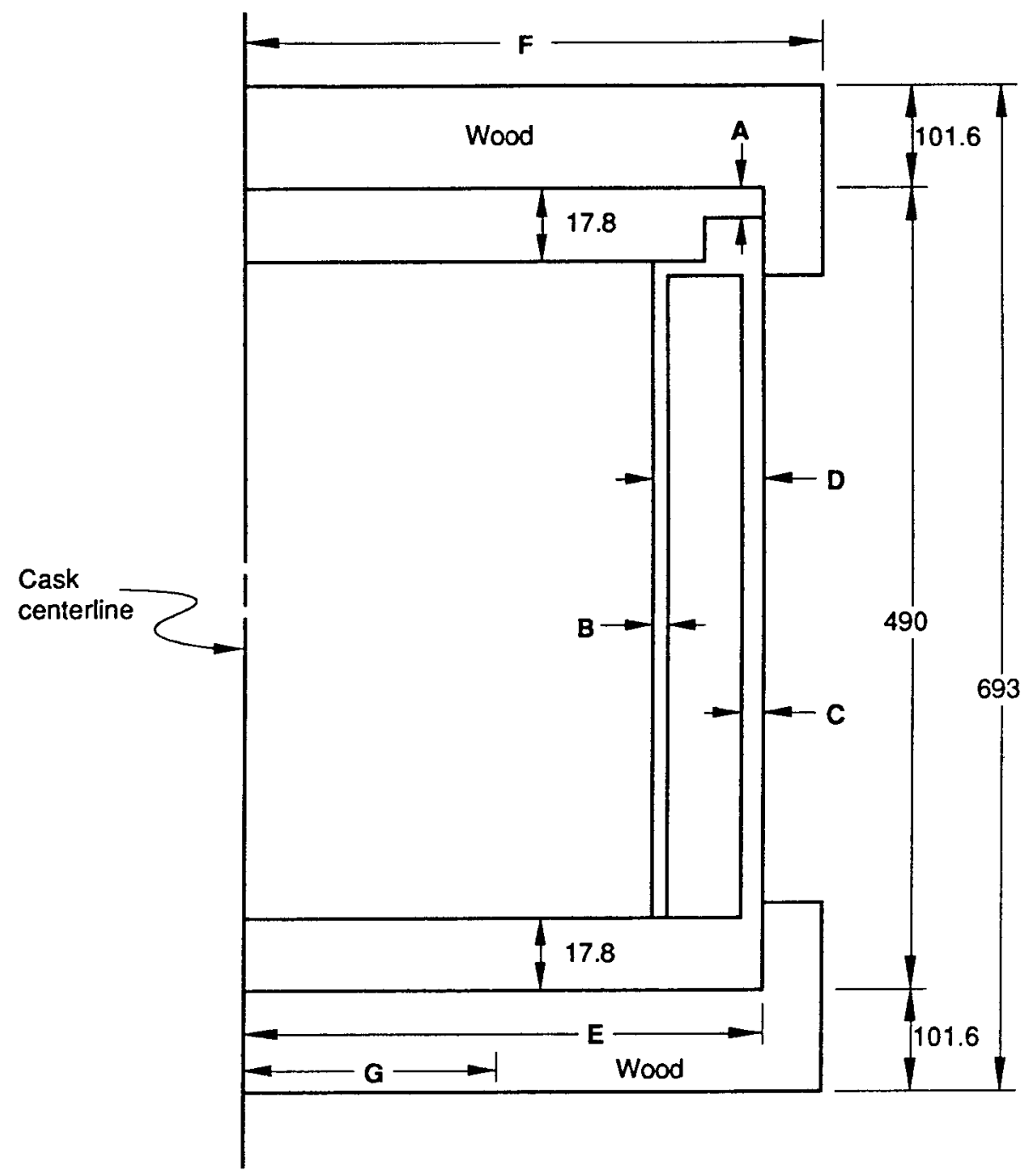

$\begin{array}{ccc}\text { Dim. } & \begin{array}{c}\text { Truck } \\ (\mathrm{cm} .)\end{array} & \begin{array}{c}\text { Rail } \\ (\mathrm{cm} .)\end{array} \\ \text { A } & 3.8 & 7.6 \\ \text { B } & 1.3 & 3.8 \\ \text { C } & 3.18 & 6.35 \\ \text { D } & 17.8 & 20.3 \\ \text { E } & 34.93 & 96.5 \\ \text { F } & 97.15 & 147.3 \\ \text { G } & 24.4 & 76.2\end{array}$

* Note: A hole of radius $G$ was included in the generic rigid body cask analyses discussed in Section II.3.4. The hole was not included in the analytic and finite element calculations in Sections II.3.1 and II. 3. 3 .

Figure II-1. Representative Lead-Shielded Cask Dimensions (cm) [FI87] 
Table II - 1

Physical and Mechanical Properties of Cask Materials

\begin{tabular}{|c|c|c|c|c|c|}
\hline Material & $\begin{array}{l}\text { Density } \\
\left(\mathrm{g} / \mathrm{cm}^{3}\right)\end{array}$ & $\begin{array}{l}\text { Young's } \\
\text { Modulus } \\
\text { (GPa) }\end{array}$ & $\begin{array}{l}\text { Hardening } \\
\text { Modulus } \\
\text { (GPa) }\end{array}$ & $\begin{array}{c}\text { Poisson's } \\
\text { Ratio } \\
\end{array}$ & $\begin{array}{c}\text { Yield or } \\
\text { Crush Stress } \\
(\mathrm{MPa}) \\
\end{array}$ \\
\hline $\begin{array}{l}304 \text { Stain- } \\
\text { less Steel }\end{array}$ & & & & & \\
\hline $\begin{array}{l}\text { Less Steel } \\
\text { Lead }\end{array}$ & $\begin{array}{r}7.96 \\
11.34\end{array}$ & $\begin{array}{r}190 \\
15.3\end{array}$ & $\begin{array}{l}1.38 \\
0.31\end{array}$ & $\begin{array}{l}0.29 \\
0.43\end{array}$ & $\begin{array}{r}172 \\
29.6\end{array}$ \\
\hline Balsa Wood & 0.144 & 4.07 & --- & 0.01 & 11.7 \\
\hline
\end{tabular}

Table II-2

Characteristics of Representative BWR and PWR Fuel Assemblies

\begin{tabular}{|c|c|c|}
\hline Characteristic & BWR & PWR \\
\hline Manufacturer, Array & GE $7 \times 7$ & B\&W $15 \times 15$ \\
\hline Rod Diameter $(\mathrm{cm})$ & 1.439 & 1.09 \\
\hline Cladding Thickness (cm) & 0.0876 & 0.0673 \\
\hline Pitch $(\mathrm{cm})$ & 1.875 & 1.443 \\
\hline Assembly Length (m) & 4.06 & 3.96 \\
\hline Number of Spacers & 7 & $6+1$ at each end \\
\hline Midspan Spacer Separation $(\mathrm{cm})$ & 51 & 53 \\
\hline Zircaloy Modulus (MPa) & $1.02 \times 10^{5}$ & $1.02 \times 10^{5}$ \\
\hline Weight (kg) & 279 & 687.2 \\
\hline
\end{tabular}

The approximate mass of the various components of the representative casks may be determined based on the dimensions in Figure II-1, material densities in Table II-1, and fuel assembly weights in Table II-2. These masses are summarized for each of the four cask concepts in Table II- 3 .

\section{II.3 Generic Cask Impact Analyses}

Several analysis techniques are available for estimating the accelerations of spent-fuel casks and contents when subjected to the normal transport condition of the $0.3-\mathrm{m}$ free drop and the hypothetical accident conditions of the $9.0-\mathrm{m}$ free drop and the $1.0-\mathrm{m}$ free drop-puncture. The analysis techniques include analytical solutions, one-dimensional lumped parameter modeling, finite element modeling and analysis, and multidimensional dynamic response analysis using rigid-body kinematics. 
Table II - 3

Representative Casks Mass Summaries ( $\mathrm{kg}$ )

\begin{tabular}{|c|c|c|c|c|c|}
\hline Type & Stee1 & Lead & Limiters & Fue 1-PWR & Total \\
\hline All-Steel Truck & 25,200 & - - - & 1,280 & 687 & 27,170 \\
\hline Lead-Shielded Truck & 3,970 & 10,900 & 1,000 & 687 & 15,560 \\
\hline Al1-Steel Rail & 75,200 & - - - & 2,520 & 14,430 & 92,150 \\
\hline Lead-Shielded Rail & 28,500 & 28,000 & 2,200 & 14,430 & 73,130 \\
\hline
\end{tabular}

Analytical solutions make possible rapid estimation of the peak accelerations of a cask during an impact situation for specific impact orientations. The lumped parameter method is used to evaluate the dynamic response of the system modeled for simple (e.g., end-on) impact orientations. The finite element modeling method provides the most detailed data concerning the dynamic response of the modeled cask to the impact. Multidimensimal dynamic response analysis using rigid-body kinematics is the principal impact analysis technique used in this report. This methodology gives estimates for the transient acceleration components of a cask during impact.

\section{II.3.1 Analytical Modeling Methods}

Simple, approximate analytical expressions are available for predicting the decelerations of a spent-fuel cask and the amount of deformation sustained by its impact limiter in the regulatory $0.3-\mathrm{m}$ and 9.0-m drop impacts for end-on, side, and corner (CMC) impact orientations. With minor reinterpretation, the approximate analytical expressions for the cask accelerations for end-on and side impact orientations also provide estimates for accelerations in the hypothetical accident puncture condition.

The approximate analytical expressions for accelerations of casks in impacts and for impact limiter deformations are based solely on application of Newton's second law of motion and the principle of the conservation of energy. In this subsection, the simple analytical expressions are described. Specific results have been obtained by applying these analytical expressions to analysis of the four representative cask concepts. The analysis approaches described here are based on the simplified analysis methods discussed by Adams et a1. [AD81].

\section{End-On Impact Analysis}

In the end-on impact orientation for the $0.3-\mathrm{m}$ or $9.0-\mathrm{m}$ free drop condition, the limiter at the impacting end of the cask is assumed to crush uniaxially at a constant crush stress, $\sigma_{c}$ (assuming the energy absorbed in crushing the thin metal shell that encases the crushable material can be ignored). Designating the backed cross-sectional area of the impact 
limiter's end as A (which is constant during the end-on impact), the constant decelerative force $F$ exerted on the cask as the limiter crushes is given by $F=\sigma_{\mathrm{c}} \mathrm{A}$. Using Newton's Second Law with $\mathrm{m}$ as cask mass and $\mathrm{g}$ as acceleration due to gravity, the constant deceleration, $a$, in $g$ of the cask during the end-on free drop impact is given by

$$
\mathrm{a}=\frac{\sigma_{\mathrm{c}}^{\mathrm{A}}}{\mathrm{mg}}
$$

The acceleration estimate in Equation (II-1) also applies to the $1.0-\mathrm{m}$ end-on free drop onto a puncture bar (without the cask impact limiter in place) if $\sigma_{c}$ is identified as the flow stress of the mild steel in the bar, and $A$ is the cross-sectional area of the bar. The product $\sigma_{\mathrm{C}} \mathrm{A}$ is then the largest force that the bar can exert on the end (or the side) of the cask.

Returning to the 0.3 - or $9.0-\mathrm{m}$ free-drop condition, the energy absorbed by (or work performed on) the crushable impact limiter material as it deforms through a distance $t$ during the end-on impact is the crush force $\sigma_{\mathrm{c}} \mathrm{A}$ multiplied by the distance $t$ through which it acts, i.e., $\sigma_{\mathrm{c}} \mathrm{At}$. The kinetic energy of the spent-fuel cask system at the moment of impact is equivalent to the initial gravitational potential energy associated with the drop height, $h$, of 0.3 or $9.0 \mathrm{~m}$. This cask impact energy is given by mgh. If the additional (and sma11) change in the gravitational potential energy of the cask as its impact limiter deforms through the distance $t$ is ignored, then the approximate crush depth of the limiter can be found by equating the cask impact energy to the impact limiter material calculations. Thus, the expression for the approximate crush depth is given by

$$
t=\frac{\mathrm{mgh}}{\sigma_{c} \mathrm{~A}}
$$

It is evident from the above expressions for a and $t$ that crush depth, drop height, and deceleration (in g) of a cask are approximately related by the expression $t=h / a$. This result is independent of the crush strength and cross-sectional area of the impact limiter.

Results of applying these formulas to the analysis of the representative cask concepts are presented in Figure II-2. Note that the nominal crush strength of the balsa wood used in the representative casks is $11.7 \mathrm{MPa}$. Figure II-2 shows that the deceleration predicted for the casks in end-on $9.0-\mathrm{m}$ drop impact ranges from approximately $100 \mathrm{~g}$ for the a11-steel rail cask to $215 \mathrm{~g}$ for the lead-shielded truck cask. For these two extremes, the respective balsa wood crush thicknesses using the relation $t=h / a$ are 9.1 and $4.3 \mathrm{~cm}$. Both thicknesses are only a small fraction of the total thickness $(101.6 \mathrm{~cm})$ assumed for the impact limiter at the ends of the representative casks. The end-on $9.0-\mathrm{m}$ drop impact decelerations could be decreased by reducing the crush strength of the impact limiter material, decreasing the contact area $A$ by making the limiter in the shape of an annulus, or combining these two approaches. 


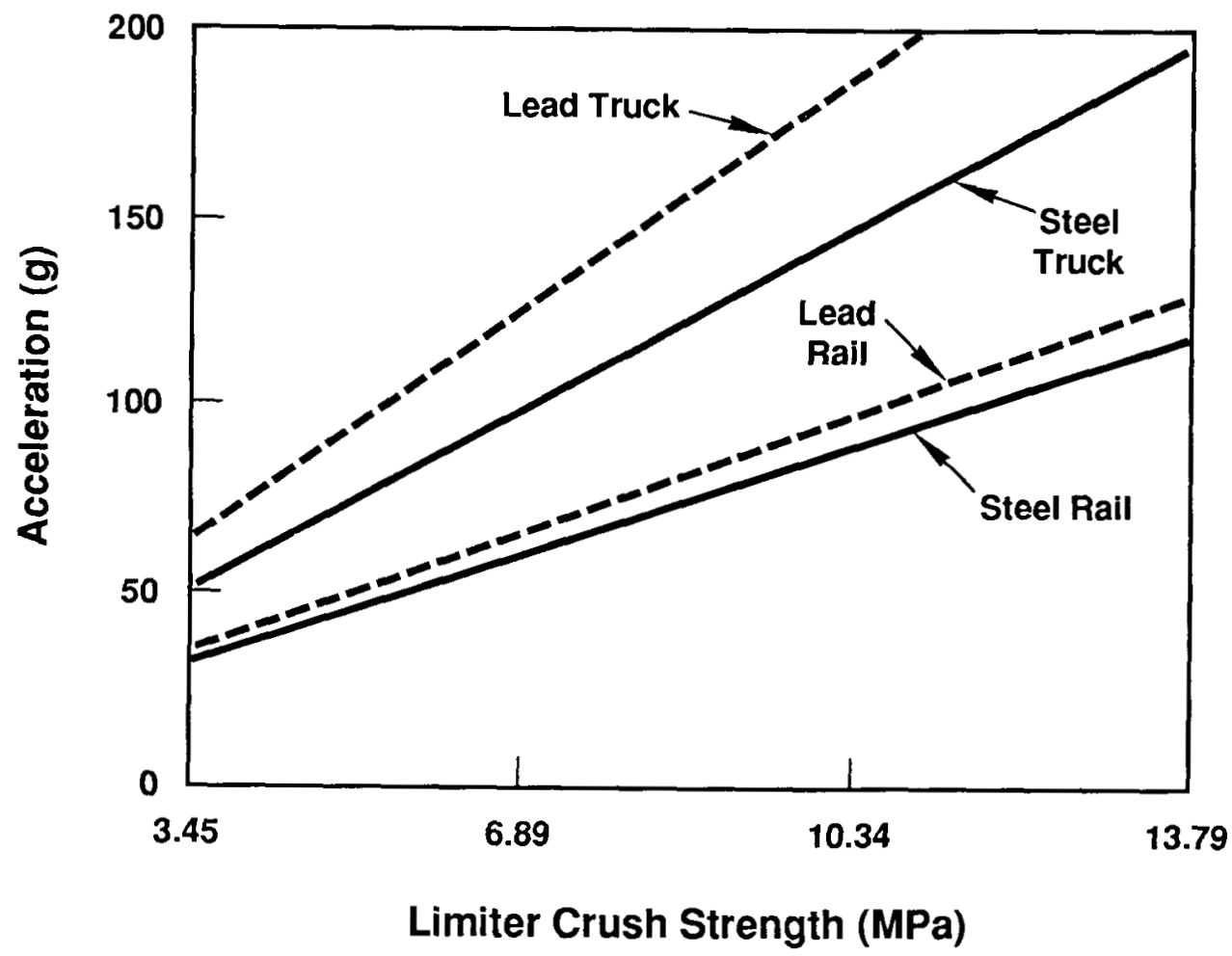

Figure II-2. Representative Cask Acceleration Predictions as a Function of Crush Strength for End-On Impact

\section{Side-Impact Analysis}

Only the portion of the impact limiters that extends along the cylindrical sides of the representative cask concepts is assumed to be effective in decelerating the containers in the simple analysis of the side-impact orientation for 0.3 - and $9.0-\mathrm{m}$ free drops. For side impact, the limiter is assumed to crush as shown in Figure II-3 [AD81]. This figure shows an end view of one of the annular impact limiter sections and the cask body. This limiter has an outer radius, $r_{0}$, and an inner radius, $r_{i}$, that is the same as the outer radius of the cask. Only the portion of the limiter labeled Area 1 in Figure II- 3 is assumed to be involved in the initial phases of the side impact. During this phase, the decelerative force on the cask gradually increases until the limiter surface (including contributions from each end of the cask) in contact with the impact surface has an area of $4 \mathrm{r}_{i} \mathrm{~L}$ in which $\mathrm{L}$ is the axial length of one impact limiter along the side of the cask. At this point, it is assumed that the cask crushes the limiter along the inner surface of the limiter (Area 2 in Figure II-3) without further increasing the contact area between the limiter and the rigid target. The deceleration of the cask is essentially constant during the crush of Area 2.

By ignoring the insignificant deceleration achieved during the first phase of the response and considering only the balance of forces present during the second phase of the side impact, an analytical expression for cask deceleration as a function of impact limiter crush strength can be 


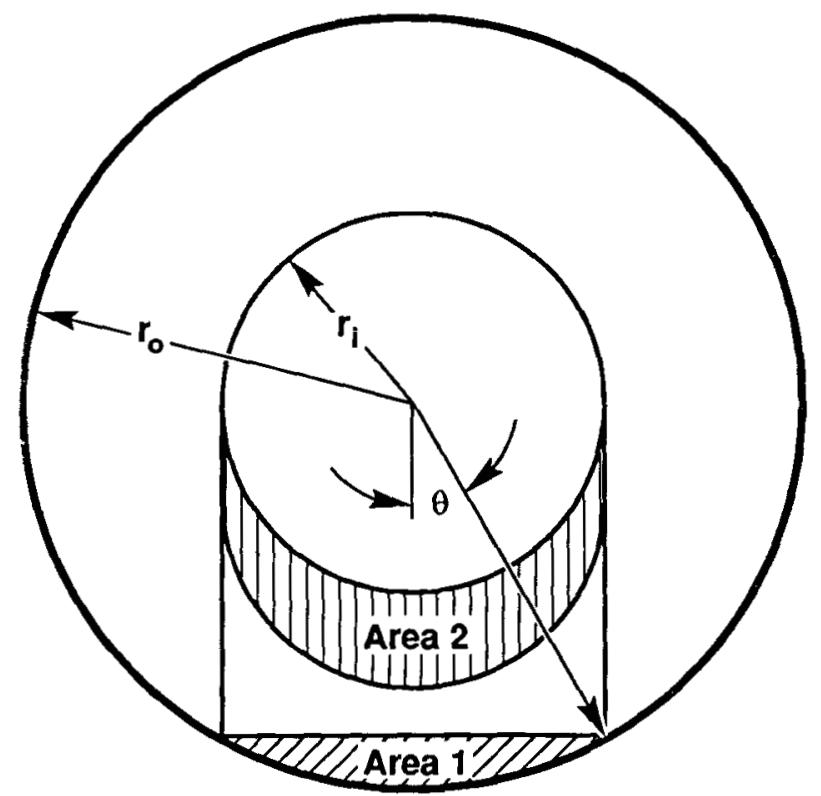

Figure II-3. Assumed Impact Limiter Deformation During Side Impact [AD81]

obtained. During the second phase of the side impact, the total decelerative force acting on the cask is $F=4 \mathrm{r}_{i} L \sigma_{c}$. When Newton's second law of motion is applied, the cask deceleration, $a$, in $g$ is given by

$$
a=\frac{4 r_{i} L \sigma_{c}}{m g} .
$$

A convenient expression for the depth, $t$, of the impact limiter material crushed during side impacts is also readily derived if deformation and energy removal during the first phase of the impact are ignored. As in the end-on impact analysis, the kinetic energy of the cask system at the moment of impact in the side orientation is equivalent to the gravitational potential energy, mgh, associated with the drop of the cask system from height $h$. This kinetic energy is expended in crushing the impact limiter material and is given by the product of the crush force $4 \mathrm{r}_{i} L \sigma_{c}$ and the distance, $t$, through which it acts. Equating the expression for kinetic energy to limiter material calculations provides the following approximate expression for the limiter crush depth in side impacts:

$$
t=\frac{m g h}{4 r_{i} \sigma_{c}}
$$

As with end-on impacts, cask deceleration and impact limiter crush depth are related by the expression $t=h / a$ for the side-impact situation and are at least as accurate as the equation approximations for $a$ and $t$.

Equations (II-3) and (II-4) were applied to the analysis of the 9.0-m free-drop side impact for the four representative cask concepts. Analyses 
results for the lead-shielded concepts cask are shown in Figure II-4. From the typical results plotted in Figure II-4, representative casks are predicted with the simple analyses to considerably lower decelerations in the side impact ( 20 to $50 \mathrm{~g}$ ) than in the end-on impact ( 100 to $215 \mathrm{~g}$ ), provided that sufficient limiter material is available so that lock-up or bottoming does not occur during the impacts. The principal reason is that the impact limiter area that is effective in decelerating these casks is considerably greater for the end-on impact orientation than for the side impact. The limiter crush depth achieved in the end-on impact, however, is much less than that predicted for the side-impact orientation.

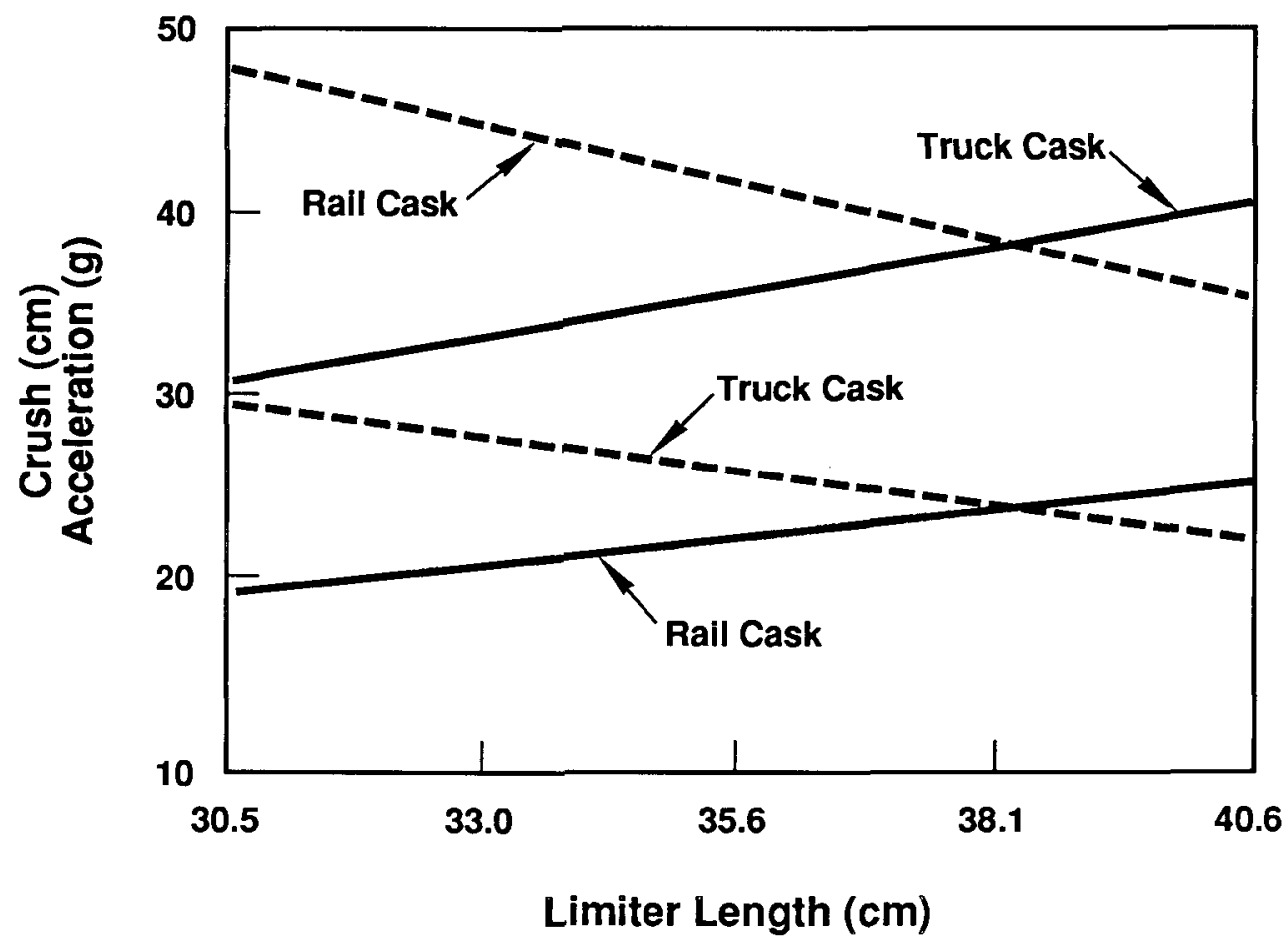

Figure II-4. Lead-Shielded Cask Decelerations Versus Limiter Length for Side Impact. The solid line indicates cask deceleration and the dashed line indicates the limiter crush depth.

If metal inserts are used in the impact limiters of the representative casks to control the deformation extent that is achieved in side impacts, then cask accelerations for side impacts could exceed those predicted for end-on impacts. The predicted decelerations for existing casks presented in Section II.3.5 show that cask decelerations for various impact orientations are design-dependent and either the side or the end-on impact situation may produce the largest cask accelerations.

\section{Center-of-Mass-Over-Corner (CMC) Impact Analysis}

Simple analysis of the 0.3 - and $9.0-\mathrm{m} \mathrm{CMC} \mathrm{impacts} \mathrm{for} \mathrm{spent-fuel} \mathrm{casks}$ is accomplished using the same methodology as the end-on and side-impact analyses. Complications of rigid-body rotation and secondary impact are 
not included in the analysis of the CMC impact because the center-of-mass system of the cask is assumed to be directly above the point of contact between the impact limiter and the rigid target, and the total decelerative force exerted by the limiter on the cask is assumed to act only through the center-of-mass. In practice, a regulatory drop test involving a perfect CMC impact would be difficult to achieve.

A schematic illustrating the deformation of the limiter at the impacting end of a cask in CMC impact is shown in Figure II-5 [AD81]. The angle $\alpha$ in Figure II-5 is the impact angle. For a CMC impact, the impact angle is given by

$$
\tan \alpha=\frac{2 r_{0}}{1}
$$

if it is assumed that the center-of-mass of the cask system is located at the geometric center of the cask body.

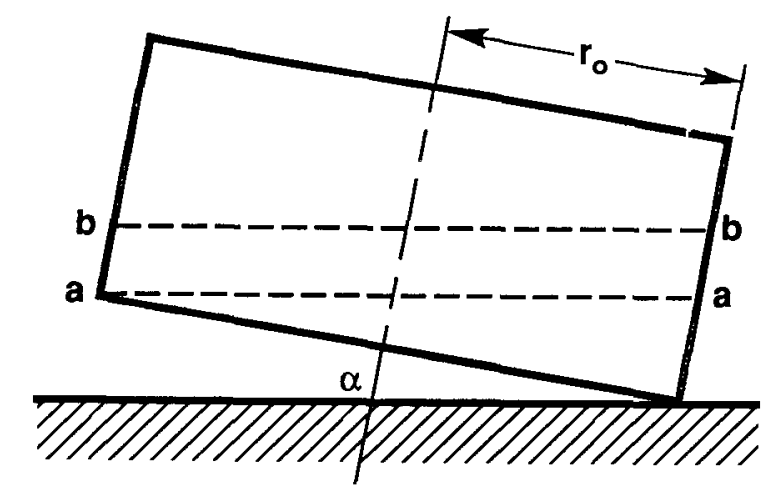

Figure II-5. Schematic of CMC Impact Showing the Impact Limiter Only [AD81]

In this equation, $r_{0}$ is the outer radius of the impact limiter and 1 is the overall length of the cask-impact limiter system. For the representative cask models, the angle $\alpha$ ranges from $15.6^{\circ}$ for the leadshielded truck cask to $24.5^{\circ}$ for the all-steel rail cask.

During the early phases of a CMC impact, the contact area between the impact limiter and the impact surface increases nonlinearly. The shape of this contact area is a portion of an ellipse. The contact area monotonically increases with increasing limiter deformation until the line marked a-a in Figure II-5 is reached. Cask deceleration is nonlinear and time-dependent during the first phase of a CMC impact. The second phase involves further limiter deformation from line a-a to the final deformation indicated by line $b-b$. During the second phase of the impact, the impact limiter contact area is constant and takes the shape of an ellipse and the 
decelerative force exerted by the limiter on the cask body is essentially constant. Cask deceleration is greatest during the second phase because the contact area is greater during the second phase of the impact than during the first phase.

If it is assumed that the initial kinetic energy of the cask system is sufficient to cause the impact limiter to crush beyond line a-a in Figure II-5, it is possible to develop a simple expression for the constant deceleration of the cask during the second phase of impact that is analogous to the side impact equations. During the second phase of a CMC impact, the contact area between the impact limiter and the impact surface is $\pi r_{0}{ }^{2} / \cos \alpha$ and the decelerative force on the cask system is the limiter material crush strength $\sigma_{c}$ multiplied by the contact area. Therefore, Newton's second law, the deceleration (in g) of the cask during this response phase, is given by

$$
a=\frac{\pi r_{o}^{2} \sigma_{c}}{m g \cos \alpha} .
$$

The cask deceleration is predicted to be only 4 to 108 greater for the CMC impact orientation than for the end-on impact dropped from the same height. This is shown by comparing Equation (II-6) to the expression for the cask deceleration obtained in the end-on impact analysis and considering the impact angles for the representative casks ( 15.6 to $24.5^{\circ}$ ) that lead to a CMC impact. Also, it is assumed that bottoming of the limiter is not achieved in either impact orientation and deformation will continue beyond line a-a in Figure II-5 for the CMC impact. Because of this observation, numerical analysis results using Equation (II-6) are not presented for CMC impacts to the four representative cask models.

Average Decelerations and Impact Durations

It is possible to develop simple relations between the average accelerations (in $\mathrm{g}$ ) of cask systems as a result of impact following the 0.3 - and $9.0-\mathrm{m}$ drops, time duration of the impact, and impact limiter crush depth. The following analysis is suggested by results presented by Huerta and Yoshimura [HU88].

Consider a rigid cask of mass $m$ impacting a rigid target as shown in Figure II-6. The effective impact area of the impact limiter on this cask is $A$, and the total crush depth is $t$. The limiter material has a rigidperfectly plastic crush behavior as indicated in Figure II- 6 .

With the same assumptions for the impact process as for end-on impacts, the motion equation for this cask is given by Newton's second law,

$$
\frac{\mathrm{d}^{2} \mathrm{y}}{\mathrm{d} \tau^{2}}=\frac{\sigma_{\mathrm{c}} \mathrm{A}}{\mathrm{m}},
$$




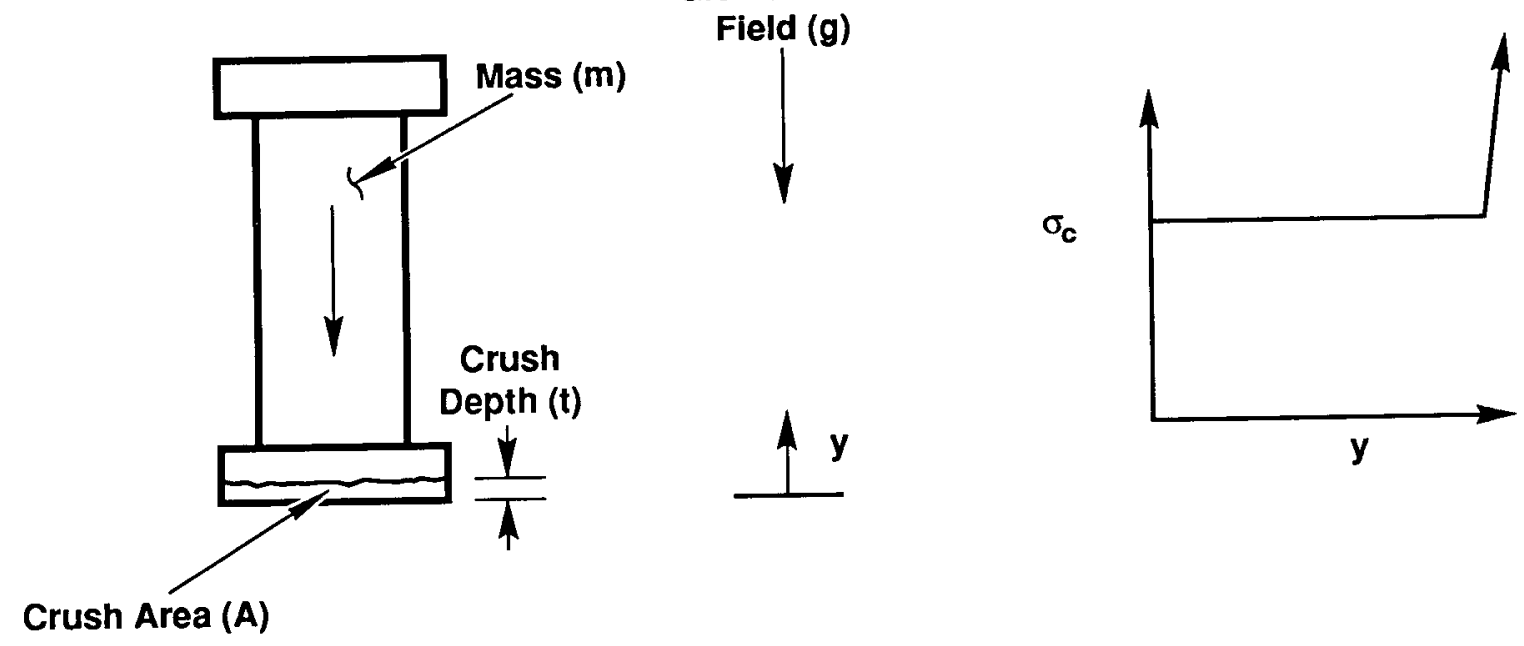

Gravitational

Field (g)

Figure II-6. Simplified End-On Impact Mode1 (left) and Crush Strength Versus Deformation Characteristics of Impact Limiter Material (right)

where

$$
\begin{aligned}
y & =\text { the vertical displacement taken upward (Figure II-6) } \\
\tau & =\text { time } \\
\sigma_{c} & =\text { impact limiter material constant crush strength. }
\end{aligned}
$$

Cask initial conditions at the instant of impact $(\tau=0)$ are $y(0)=0$ and $\mathrm{dy}(0) / \mathrm{d} \tau=-\mathrm{v}_{0}$. The cask velocity $\mathrm{v}_{0}$ at impact is related to the cask drop height $h$ by $v_{0}=\sqrt{2 g h}$, in which $g$ is the acceleration due to gravity.

Integrating the cask motion equation twice with respect to time and introducing the initial conditions gives the following results for the cask velocity, $v$, and displacement, $y$, as a function of time:

$$
\begin{gathered}
\mathrm{v}=\frac{\sigma_{c}^{\mathrm{A}}}{\mathrm{m}} \tau-\mathrm{v}_{\mathrm{o}}, \\
\mathrm{y}=\frac{\sigma_{c}^{\mathrm{A}}}{2 \mathrm{~m}} \tau^{2}-\mathrm{v}_{\mathrm{o}} \tau .
\end{gathered}
$$

From the cask velocity equation, the time, $\tau_{0}$, at which the cask first comes to rest is given by $r_{0}=\mathrm{mv}_{\mathrm{O}} / \sigma_{\mathrm{C}} \mathrm{A}$. From the cask motion equation, the deceleration, $a$, in $g$ of the cask is given by $a=\sigma_{c} A / m g$. The cask 
deceleration combined with the expression for $\tau_{0}$ yields $\tau_{0}=v_{0} / g a$. Substituting for $v_{0}$ then yields

$$
\tau_{0}=\frac{\sqrt{2 h / g}}{a}
$$

If this last expression for $\tau_{0}$ is substituted in the equation for cask displacement as a function of time, the crush depth, $t$, of the impact limiter at time $\tau_{0}$ that is the time at which the crush depth is maximum is found to satisfy the following relation:

$$
t=\frac{h}{a}
$$

The above equations for $\tau_{0}$ and $t$ describe the relationships of between maximum impact limiter crush depth, average cask deceleration (in g), and impact duration. All are a function of drop height.

A plot of the equation $t=h / a$ (actually $a=h / t$ ) for a $9.0-m$ drop height is shown in Figure II-7; the corresponding impact duration $\tau_{0}$ is indicated at selected points in the figure. This curve is based upon a similar curve developed by Huerta and Yoshimura [HU88]. Figure II-7 shows that when using highly simplifying assumptions, the average cask deceleration simply depends on the amount of crush up. The more crush distance consumed, the lower the $g$ loading and the longer the time duration of the impact event. It is assumed that there is no bottoming of the impact limiter material. Interestingly, such parameters as crush area A, limiter material crush strength $\sigma_{\mathrm{c}}$, and cask mass $m$ do not appear explicitly in the final relations provided above.

\section{II.3.2 Lumped Parameter Modeling}

The lumped parameter modeling method is possibly the simplest analysis method available for predicting the dynamic response of spent-fuel cask systems subjected to regulatory free-drop and puncture impact conditions in the end-on orientation. In this modeling approach, the spent-fuel cask system is discretized into several masses coupled by springs. By appropriately defining initial and boundary conditions for the mass points in the lumped parameter model and solving the system of coupled second-order spring-mass equations for the dynamic response of the coupled masses, one can estimate the lower response frequencies of the cask system and predict the temporal history of the displacement, velocity, and acceleration of each mass point in the model. The lumped parameter models of representative casks described below were analyzed using the SHELL SHOCK code [GR84].

The lumped parameter modeling method can be used to analyze spent-fuel casks subjected to the regulatory free-drop and puncture conditions for the end-on impact orientation. Its one-dimensional nature, however, limits the use of this method for investigating cask response for other impact orientations. Only the $9.0-\mathrm{m}$ free-drop, end-on impact situation is considered in this report. 


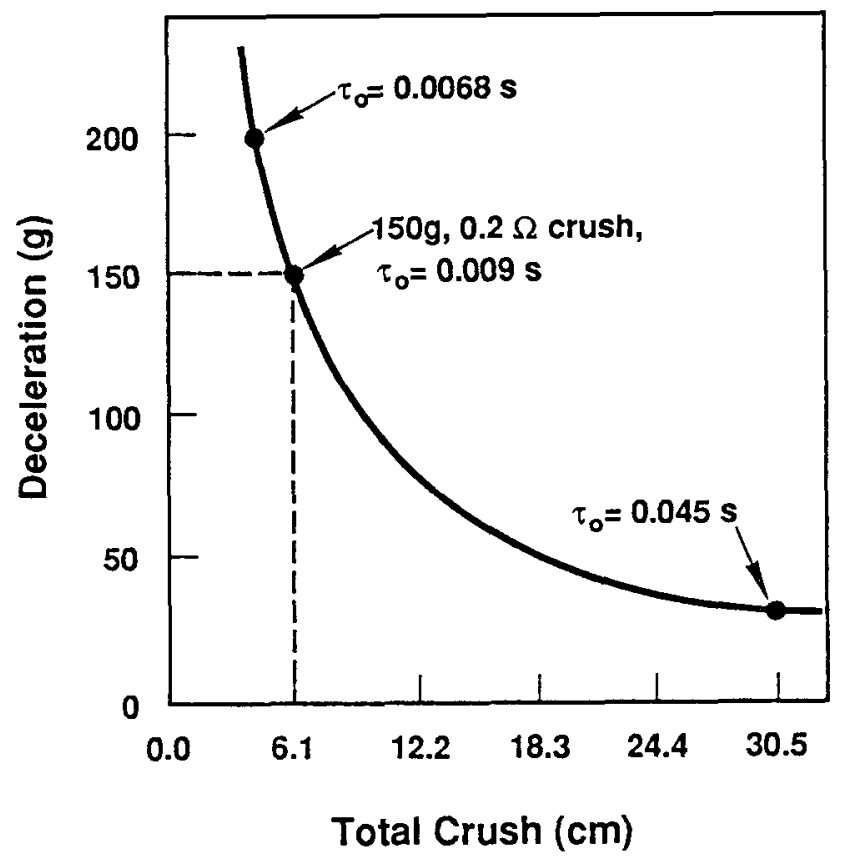

Figure II-7. Average Deceleration Versus Limiter Crush Depth for the 9.0-m Drop [HU88]

The method used in discretizing a typical cask system into a lumped parameter model is illustrated by Figure II-8. The top drawing is an outline of the representative all-steel truck cask that indicates the monolithic steel body of the cask, impact limiter at each end of the system, and single PWR fuel assembly contained in the cask cavity. The point labeled 1 at the left end of the drawing is assumed to be the impact point and is held fixed.

Also labeled on the lower drawing in Figure II- 8 are seven points (2 through 8 ) at which mass is concentrated for the lumped parameter mode1 of this cask. The mass point labeled 9 in the lower drawing in Figure II- 8 is the simplest explicit representation of the PWR spent-fuel assembly in the cask. In the lumped parameter model of one of the representative rail casks, there could be 21 such fuel assembly masses (numbered 9 through 29), one for each PWR assembly. BWR fuel payloads could be handled in a similar fashion.

The lower drawing in Figure II- 8 shows the specific coupling between the mass points in this spring-mass model of the representative all-steel truck cask with a payload of one PWR spent-fuel assembly. A similar coupling scheme can be used in models of the lead-shielded truck cask and representative rail casks, except that 21 spring-masses must be used to represent the spent fuel in these containers.

The representative cask lumped parameter models are based on the approach by Adams et a1. [AD81]. Details of the development process of these models are briefly described in the following paragraphs. Some results of sensitivity analyses performed with several lumped parameter models are also presented. 

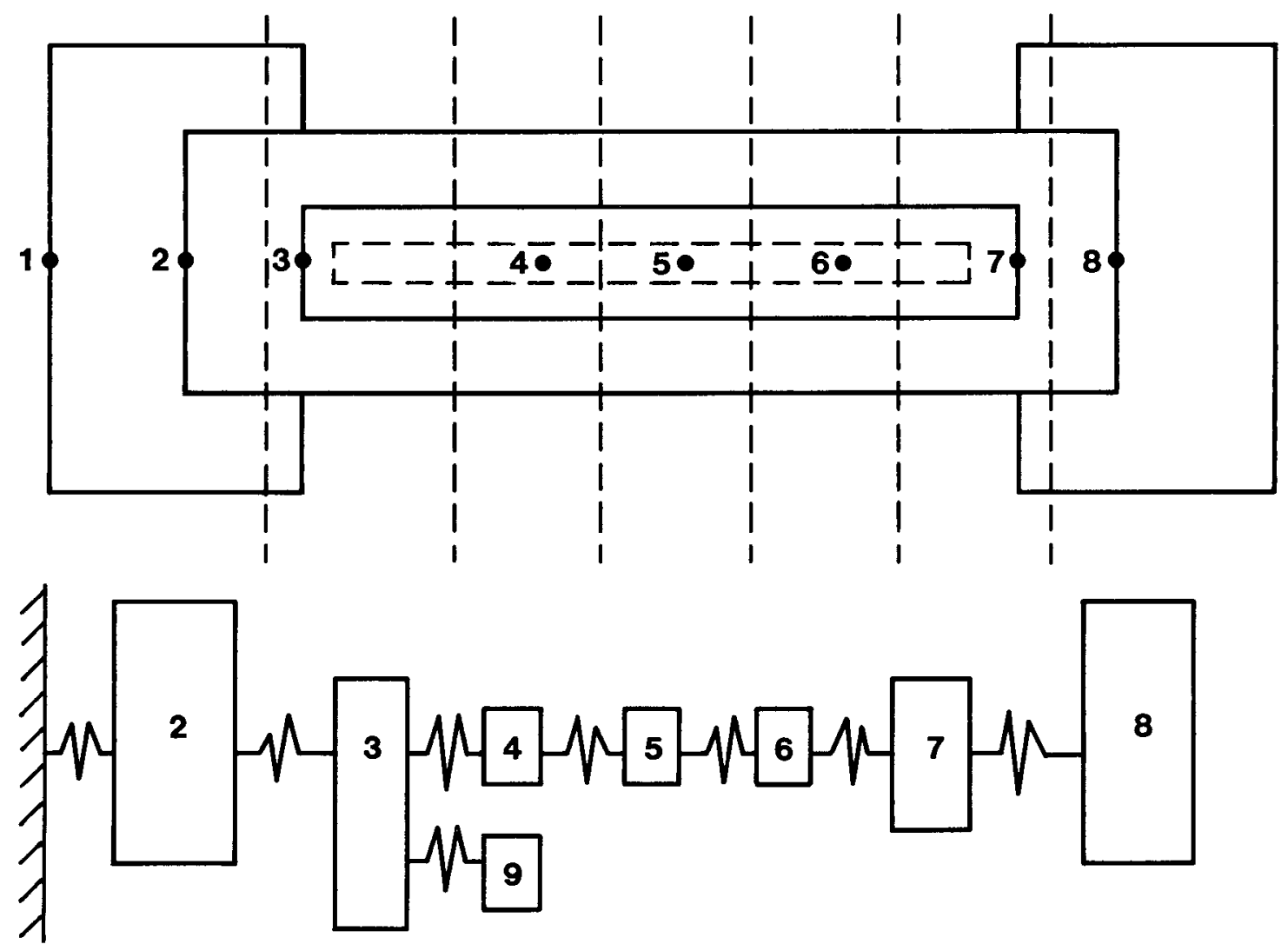

Figure II-8. Outline of Steel Truck Cask (top drawing) and Its Lumped Parameter Mode1 (bottom drawing)

The cask drawing in Figure II-8 indicates with the six vertical, dashed lines the manner in which mass would be allocated to each cask mass point for the lumped parameter model. The dashed vertical lines are located midway between each labeled mass point. The mass of the cask body and/or impact limiter between pairs of dashed lines is assumed to be concentrated at the mass point between the lines. At the cask ends, the impact limiter mass and the mass of the small portion of the cask end beyond the left-and right-most dashed lines is assumed to be concentrated at mass points 2 and 8 . No mass is assigned to mass point 1 . Finally, in its simplest explicit representation, the entire fuel assembly mass is concentrated at its mass point. The concentrated masses for all of the representative cask concepts are summarized in Table II-4, assuming the mass lumping scheme illustrated in Figure II-8.

The couplings between the mass points in the example cask lumped parameter models are calculated several ways depending on the anticipated interaction between the mass point pairs. Between mass points 1 and 2 in the models, the coupling is defined in terms of the force-deflection characteristics of the balsa wood impact limiter. The crush strength of 
Table II -4

Lumped Parameter Model Masses ( $\mathrm{kg}$ )

\begin{tabular}{|c|c|c|c|c|}
\hline Mass ID & Truck--Steel & Truck--Lead & Rail--Steel & Rail--Lead \\
\hline$M_{1}$ & 0 & 0 & 0 & 0 \\
\hline $\mathrm{M}_{2}$ & 1,119 & 738 & 3,764 & 3,118 \\
\hline $\mathrm{M}_{3}$ & 3,450 & 1,993 & 10,730 & 8,152 \\
\hline$M_{4}$ & 5,785 & 3,443 & 16,230 & 12,060 \\
\hline$M_{5}$ & 5,785 & 3,443 & 16,230 & 12,060 \\
\hline$M_{6}$ & 5,785 & 3,443 & 16,230 & 12,060 \\
\hline $\mathrm{M}_{7}$ & 3,450 & 1,993 & 10,730 & 8,152 \\
\hline M8 & 1,119 & 738 & 3,764 & 3,118 \\
\hline Mg etc. (PWR) & 687 & 687 & 687 & 687 \\
\hline Mg etc. (BWR) & 279 & 279 & -. & - - \\
\hline
\end{tabular}

the balsa wood in these cask models is assumed to be $11.7 \mathrm{MPa}$. The cask and impact limiter diameter average is assumed to define the diameter of the limiter crush area that decelerates the cask. The force-deflection curve of the limiter is bilinear, consisting of elastic and flat portions. The elastic portion continues up to a strain of 0.0029 , which is based on balsa wood properties given in Table II- 1 . The flat portion involves continual deflection at a constant force that is given by the balsa wood crush stress multiplied by the limiter area which stops the cask.

The couplings in the example cask models between mass points 2 through 8 are modeled as linear springs. The constant $k$ is given by $k=$ $\mathrm{AE} / \mathrm{L}$, in which $\mathrm{A}$ is the cross-sectional area, $\mathrm{E}$ is Young's modulus, and $\mathrm{L}$ is the distance between the coupled mass points. These spring constants are calculated accounting for changes in the cross-sectional area between some mass points in the cask length and material property differences. Also, these calculations account for whether springs associated with portions of the cask models for different materials act in parallel or series.

In their simplest explicit representation, the fuel assembly mass points are assumed to be coupled to the cask mass point labeled 3 , which is the inner surface of the case at the impacting end. The fuel assembly springs are assumed to be linear and to act only under compressive forces, and are calculated from the equation $\mathrm{k}=\mathrm{AE} / \mathrm{L}$. Only the cross-sectional area of Zircaloy in the individual fuel rods of the assembly is used for calculating the assembly spring stiffness. Half the length of the fuel assembly is also used for $L$ in the stiffness computations. For simplicity, additional stiffness from fuel oxide was not considered in evaluating the spring stiffness for the fuel assemblies nor was possible reduction in the assembly stiffness due to its potential for undergoing axial buckling.

The couplings calculated for lumped parameter models for the four representative casks are summarized in Table II-5. 
Table II - 5

Lumped Parameter Model Stiffnesses $\left(10^{9} \mathrm{~N} / \mathrm{m}\right)$

\section{Stiffness ID}

$k_{1-2}$ elastic
$k_{2}-3$
$k_{3}-4$
$k_{4}-5$
$k_{5}-6$
$k_{6}-7$
$k_{7}-8$
$k_{3}-9$, etc. (PWR)
$k_{3}-9$, etc. (BWR)

In the SHELL SHOCK analyses of the cask lumped parameter models for end-on impact, each concentrated mass point is assigned an initial axial velocity of $13.40 \mathrm{~m} / \mathrm{s}$ that corresponds to the impact speed associated with the $9.0-\mathrm{m}$ drop impact test. The SHELL SHOCK analyses of the models are then performed, and predictions for the response of the various model mass points are then examined.

The model illustrated in Figure II-8 represents only one possible lumped parameter modeling approach for the representative cask concepts. The sensitivity of the predicted cask response to the amount of springmasses used in a lumped parameter model was investigated by performing two series of SHELL SHOCK analyses. Each involved several different lumped parameter models. The sensitivity of the predicted response of the empty all-steel truck cask to the amount of masses representing this system was evaluated in the first series. In the second series, a single spring-mass representation of the all-steel truck cask was considered that contained one PWR spent-fuel assembly. The assembly was modeled with a varying amount of lumped mass points. The effect of assumed damping on the predicted cask and fuel assembly responses was also evaluated in the second analyses series.

Seven different lumped parameter models of the empty all-steel truck cask with impact limiters were analyzed in the end-on impact orientation using SHELL SHOCK. The seven models involved discretizations of the cask into $6,7,8,9,11,12$, and 16 concentrated masses. The values for the concentrated masses and couplings were calculated using the procedure previously described. Response sensitivity to the modeling scheme was investigated by examining predicted results for the cask mass point located at the impacting end of the cask, shown as mass point 3 in Figure II- 8 . As examples, the predicted displacement, velocity, and acceleration (in $g$ ) of this cask mass point are presented in Figures II-9, II-10, and II-11. These characteristics were obtained in the analyses of cask models involving seven, eight, and nine mass points. The predicted responses for 


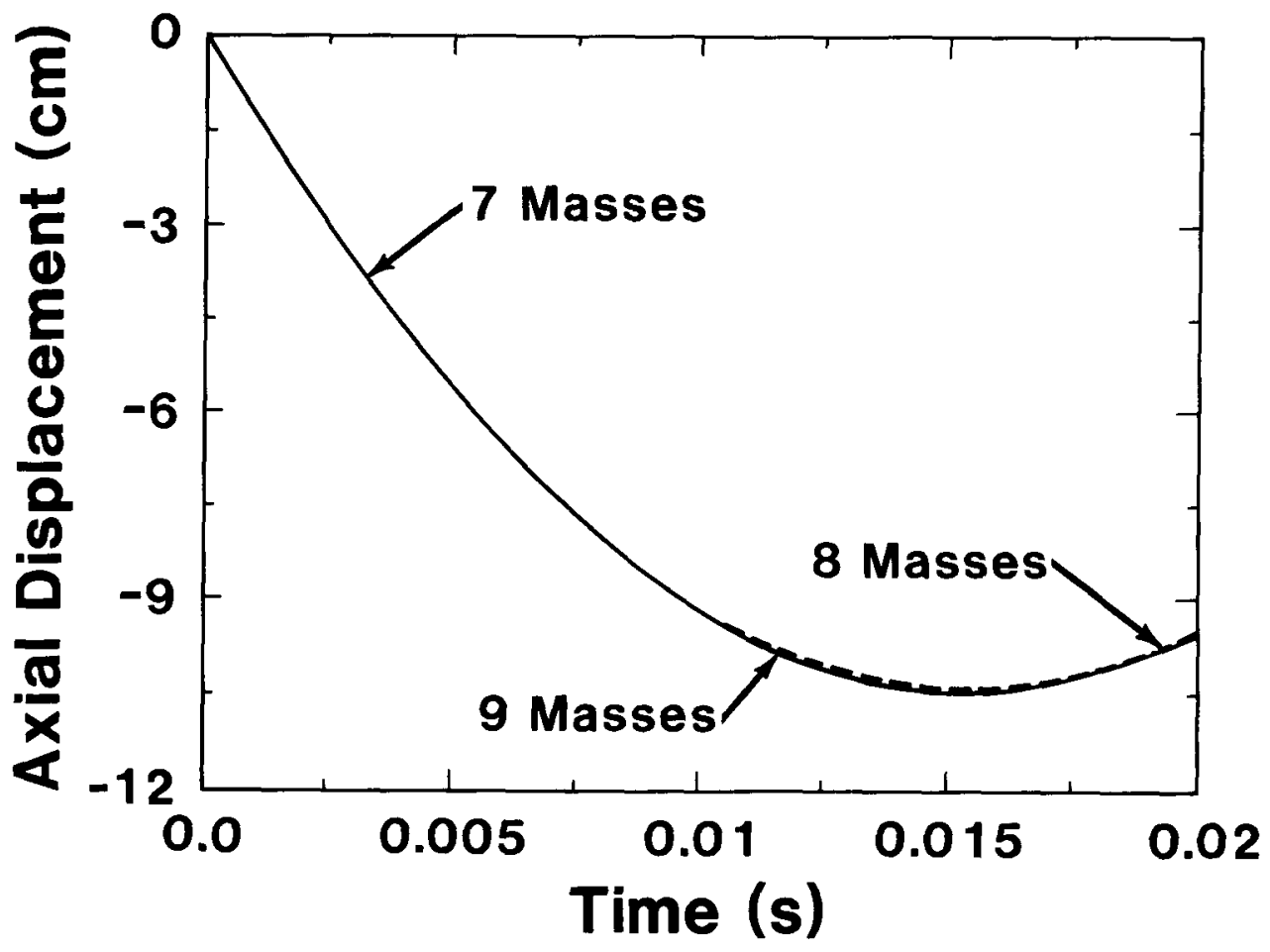

Figure II-9. Displacement History of the All-Steel Truck Cask End for Three Discretizations for End-On Impact

the other four models of this cask were bracketed by the results plotted in these figures.

The predicted displacement of the cask end is essentially the crush depth of the impact limiter. Figure II-9 illustrates that the predicted displacement of the cask end and the impact duration are not very sensitive to the amount of masses used to model the cask. The axial velocity predictions for the cask end mass manifest only modest dependence on the amount of model masses, as seen in Figure II-10. Finally, the predicted deceleration histories shown in Figure II-11 present evidence of the sensitivity of the predicted response to the amount of mass points used in the model, especially in terms of frequency content. However, the sensitivity is not particularly severe, and using an eight-mass-point model of the cask system, as depicted in Figure II-8, is adequate for present purposes.

The SHELL SHOCK analyses addressed the predicted response sensitivity to the model representing the single PWR fuel assembly transported in the all-steel truck cask. The eight-mass-point discretization of the all-steel truck cask concept was used in these analyses. In these analyses, five different lumped parameter models of the assembly were considered. These models involved one, three, five, seven, and nine equally-spaced mass point discretizations of the assembly. An odd number of mass points was considered in each case so that one was located at the axial midlength of 


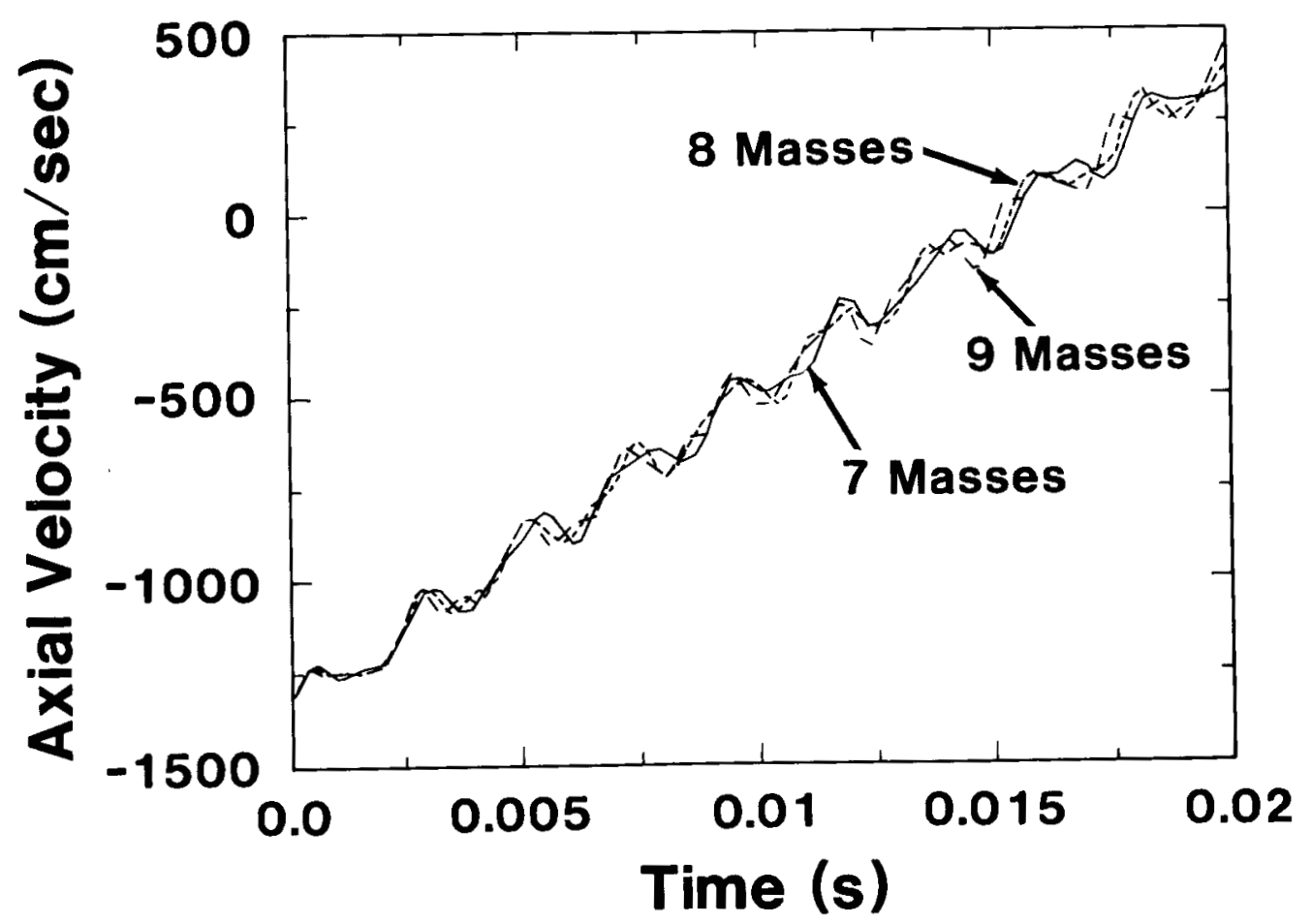

Figure II-10. Velocity History of the All-Steel Truck Cask End for Three Discretizations for End-On Impact

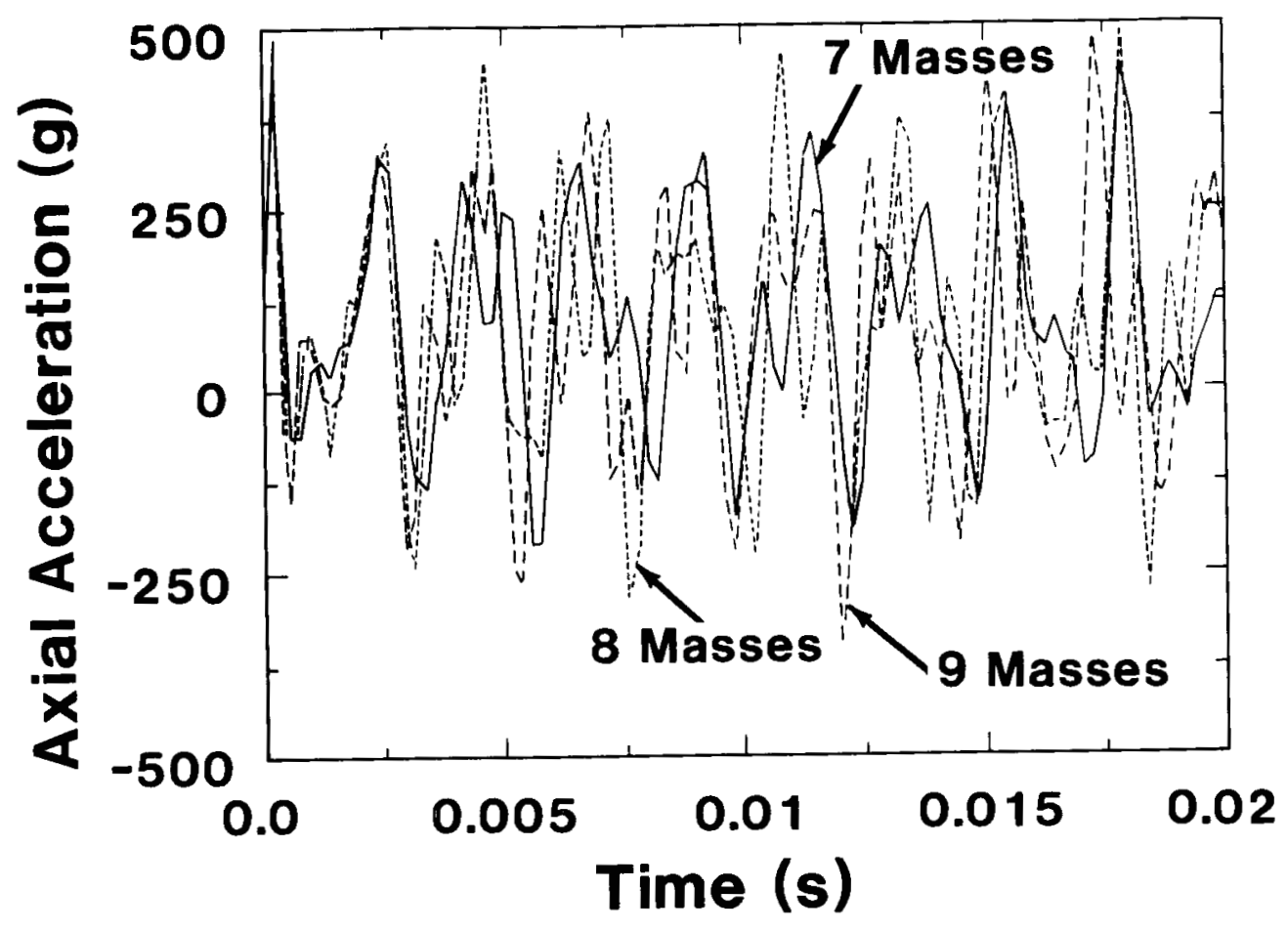

Figure II-11. Acceleration History of the Al1-Stee1 Truck Cask End for Three Discretizations for End-On Impact 
the assembly. The responses of this mass point and the cask mass point to which the fuel assembly model was attached were monitored. A compressiononly spring was used between the impacting end and the interior cask end of the assembly. The couplings between other mass points in the fuel assembly models were assumed to be linear. The spring constants for the fuel assembly couplings were calculated as described earlier in this section. These fuel model sensitivity analyses indicate that the predicted cask response was relatively insensitive to the amount of mass points used in the fuel assembly model.

These analyses were performed with and without stiffness proportional damping specified in SHELL SHOCK. Damping levels of 2.5 and 5.08 at $1000 \mathrm{~Hz}$ were considered. As an example of the results, Figure II-12 shows a comparison of the predicted cask acceleration histories for the all-steel truck cask using a seven-mass-point model for its single fuel assembly, assuming no damping and the two different levels of damping. Not surprisingly, damping strongly influenced the late-time acceleration response predictions. Unfortunately, the correct level of damping to use in the analyses is not known.

Based on the sensitivity analyses reported above, the cask acceleration response of the four representative cask concepts were analyzed with SHELL SHOCK. An eight-mass-point cask model and a single mass point for each fuel assembly in the casks were used. Figure II-13 is an example of the typical results that were obtained.

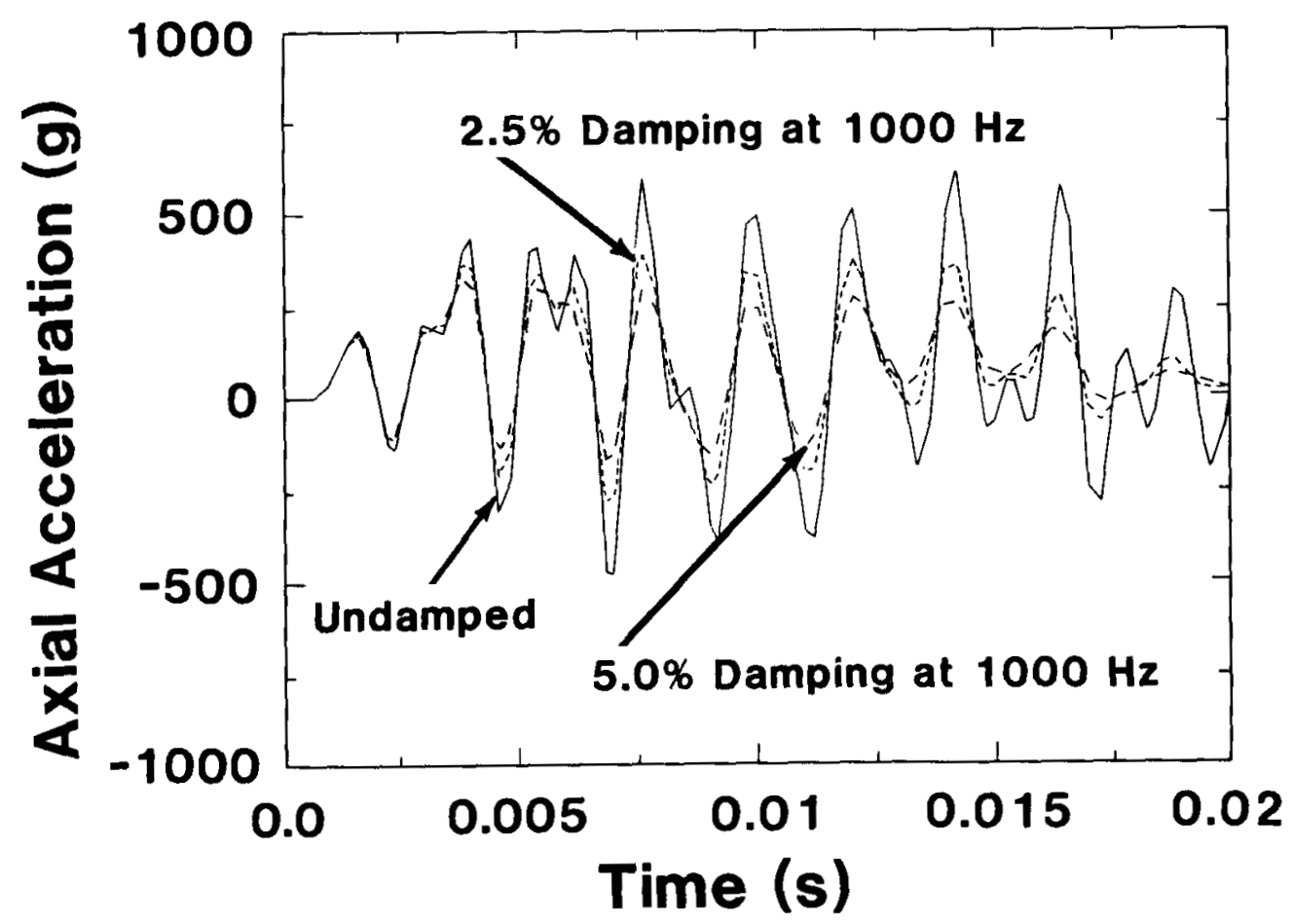

Figure II-12. Acceleration History of the All-Steel Cask End for Assumed Levels of Damping Using Seven-Mass Points for the Fue 1 Assembly 


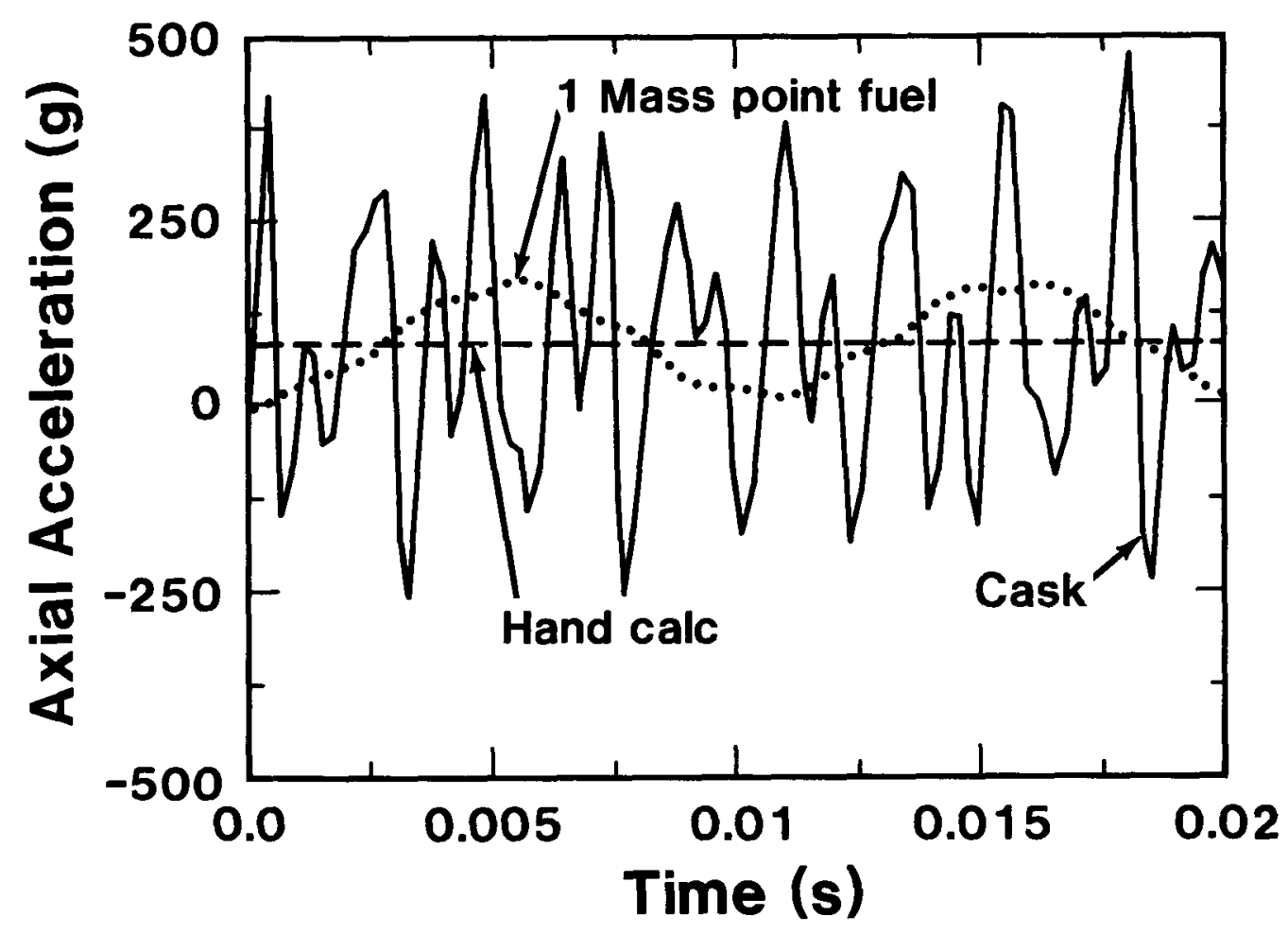

Figure II-13. Acceleration History of the All-Steel Truck Cask End and PWR Fuel Assembly for End-On Impact

Figure II-13 presents the predicted $9.0-\mathrm{m}$ free-drop acceleration histories for the end mass of the all-steel truck cask as well as the center-of-mass acceleration of the PWR assembly in the cask. Also shown in Figure II-13 is the predicted constant cask acceleration using the simple end-on analysis results. Considerable high frequency content is evident in the cask deceleration prediction in Figure II-13. The magnitude of the dynamic deceleration of the cask was generally predicted in the -250 to $-375-\mathrm{g}$ range. The large swings in the cask accelerations seen in Figure II-13 were due primarily to elastic ringing. The spent fuel in the cask did not undergo these accelerations; the fuel assemblies responded principally to cask rigid-body accelerations.

\section{II.3.3 Finite Element Modeling}

The finite element method is the analysis technique capable of predicting in greatest detail the multidimensional dynamic response of spent-fuel casks to the regulatory free-drop and puncture conditions for any impact orientation. The DYNA2D code [HA84] was used to perform a limited number of finite element analyses.

The finite element analyses were performed for the all-steel truck and rail cask concepts under 9.0 -m free drop and end-on impact conditions. For brevity, only the results of the finite element analyses of the all-steel 
truck cask are discussed below. Figure II-14 presents the finite element mesh used to model the all-steel truck cask. The outline of the cask is shown in the left side of the figure and the element zoning in the right. Four node, axisymmetric quadrilateral finite elements were used in the cask model. The lower impact limiter in the cask model was the impacting end in the analyses.

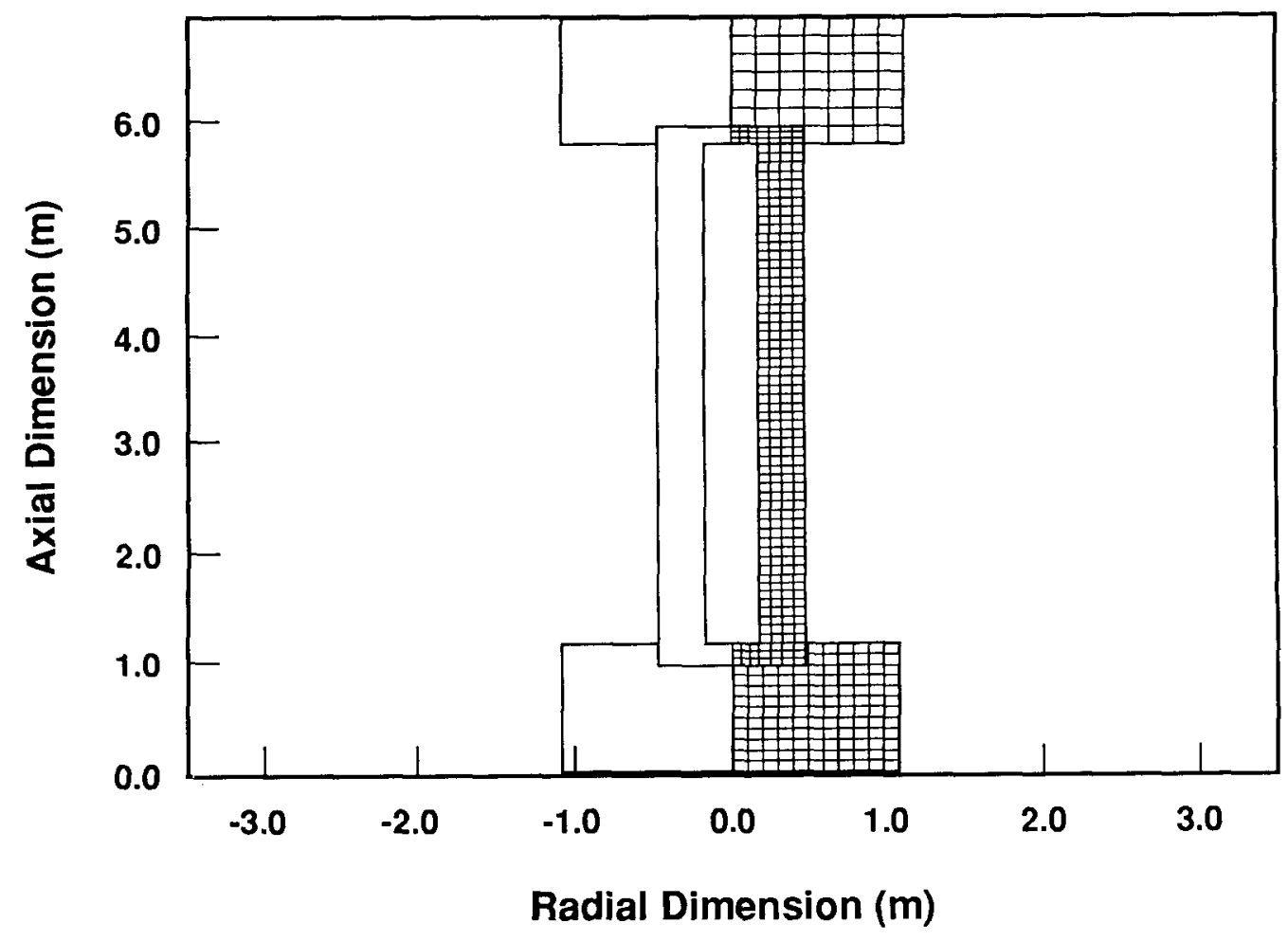

Figure II-14. Finite Element Model for the All-Steel Truck Cask

The truck cask model consisted of the all-steel cask body and balsa wood impact limiter at each end of the container. Spent fuel was not modeled either explicitly or as additional lumped mass in this cask model. Assuming the cask contains PWR fuel and using the mass estimates in Table II-3, the mass of the single fuel assembly in the truck cask represented only approximately 38 of the total loaded cask mass. Because the fuel mass is a small fraction of the total system mass, the effect of the former on the cask response in the end-on impact is not significant.

The mechanical properties of the steel and balsa wood used in the finite element analysis of this cask concept are given in Table II-1. The material response of the steel was modeled as elastic-plastic with hardening. The balsa wood material response was modeled as elasticperfectly plastic.

In the analyses of the regulatory accident end-on impact, each node in the truck cask model was given an initial axial velocity of $-13.40 \mathrm{~m} / \mathrm{s}$ that 
is the speed attained after a free fall of $9.0 \mathrm{~m}$. The DYNA2D Stonewall option [HA84] was used to define a perfectly rigid target surface at the impacting end of the model. A slide line without friction was also defined between the lower end of the cask body and the lower impact limiter. Finally, a tied interface was used between the upper limiter and upper end of the cask body.

Analysis of the cask finite element model was performed interactively using the re-zoning option in DYNA2D. This was necessary to control node penetration along the sliding interface between the cask and the impact limiter at the impacting end. Re-zoning was performed in the analysis every $0.005 \mathrm{~s}$ (model time) during the response until the cask body began to rebound.

The displacement and acceleration histories for several nodes located along the inner surface of the truck cask model at the impact end were monitored during the analysis. The displacement histories predicted for three of these nodes presented in Figure II-15 may be compared to the SHELL SHOCK displacement predictions for this cask shown in Figure II-9. Because more impact limiter deformation was predicted in the finite element analysis, a smaller portion of the limiter than was assumed in the lumped parameter analyses may be effective in decelerating the cask.

Figure II-16 presents the acceleration history predicted for one node at the impacting end of the truck cask. For frequency content and amplitude, this history compares fairly well with the acceleration predictions given in Figure II-13 as determined with SHELI SHOCK.

In general, the predictions obtained in the few finite element analyses of the representative all-steel truck and rail casks tend to corroborate the models used and results obtained by the SHELL SHOCK analyses of these casks. Both analysis approaches predict that the accelerations of the casks in end-on impact situations are temporarily complex, with amplitudes several times larger (typically a factor of two or three) than the constant acceleration predictions obtained by the simple analysis method described in Section II.3.1. The large amplitude acceleration spikes are typically very short in duration and have little effect on the structural response of the cask. Also, because the acceleration spikes are of such short duration, they do not couple well into the low-frequency mechanical system presented by the spent-fuel assemblies inside the casks. The introduction of damping in both dynamic analysis methods would help decrease the amplitudes of the swings predicted in the acceleration histories. Unfortunately, the proper amount of damping to be used in the dynamic analyses is not known.

\section{II.3.4 Rigid-Body Kinematics Analysis of Impact}

The rigid-body kinematics analysis of impact described in this section treats a cask as a two-dimensional rigid body with three degrees of freedom: two translational and one rotational, as shown in Figure II-17. This method provides acceleration histories for any impact orientation and excludes the high-frequency response associated with deformable casks. The SLAM computer code (Spent fueL cask impact Analysis Method) was developed 


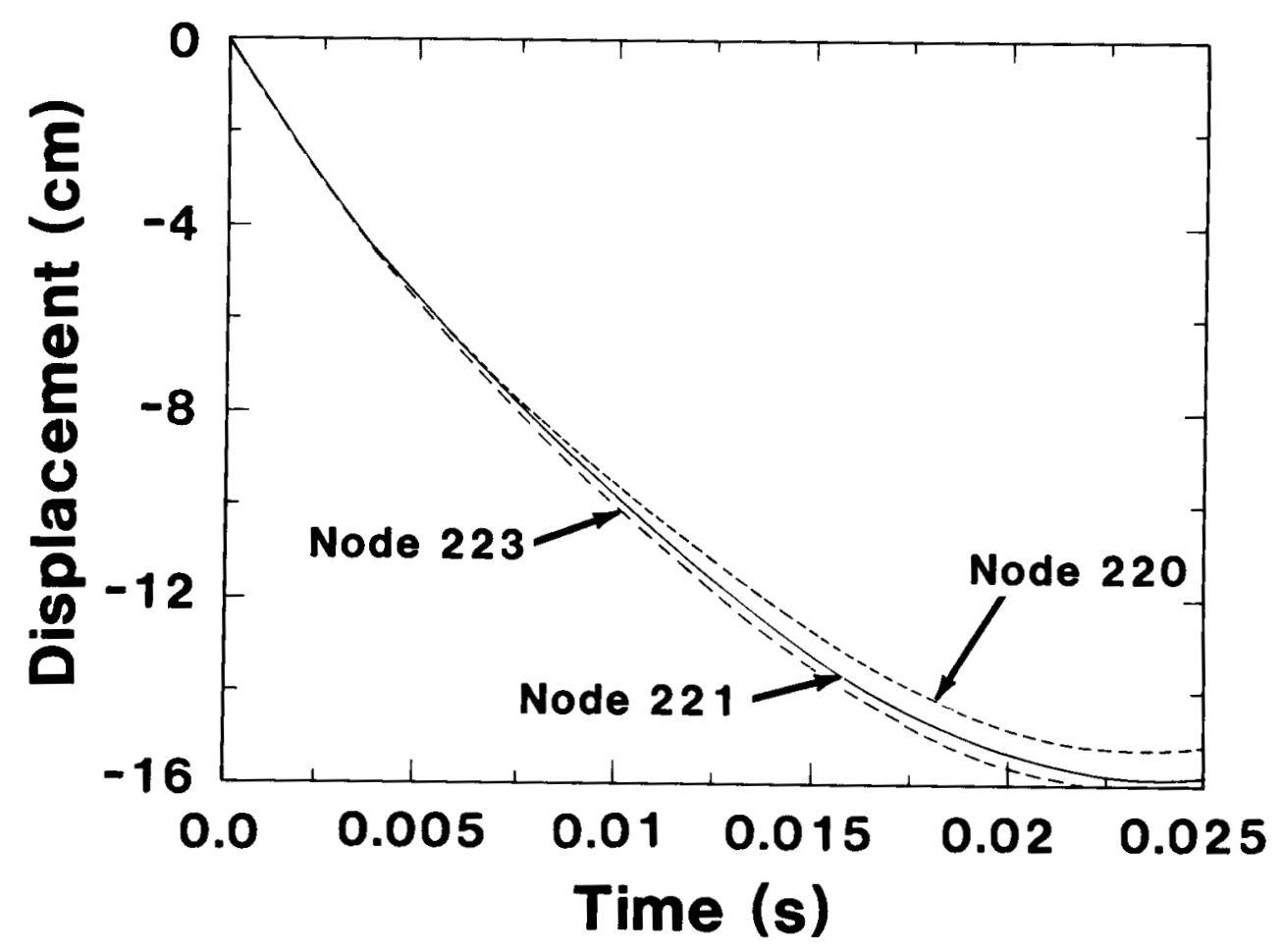

Figure II-15. Displacement Histories for Three All-Steel Truck Cask Nodes Predicted by the Finite Element Analysis

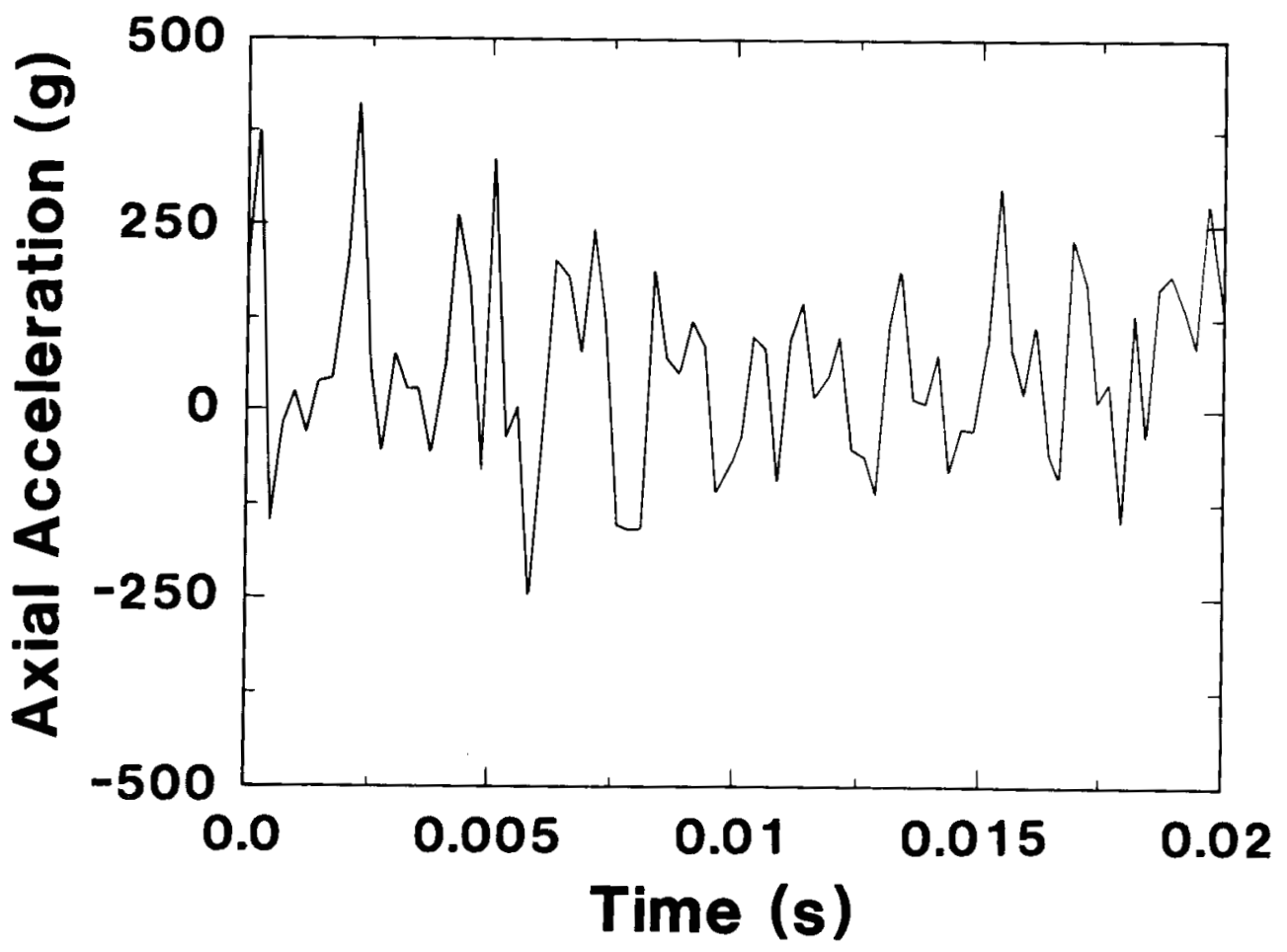

Figure II-16. Acceleration History Predicted by Finite Element Analysis of the All-Steel Truck Cask During End Impact 


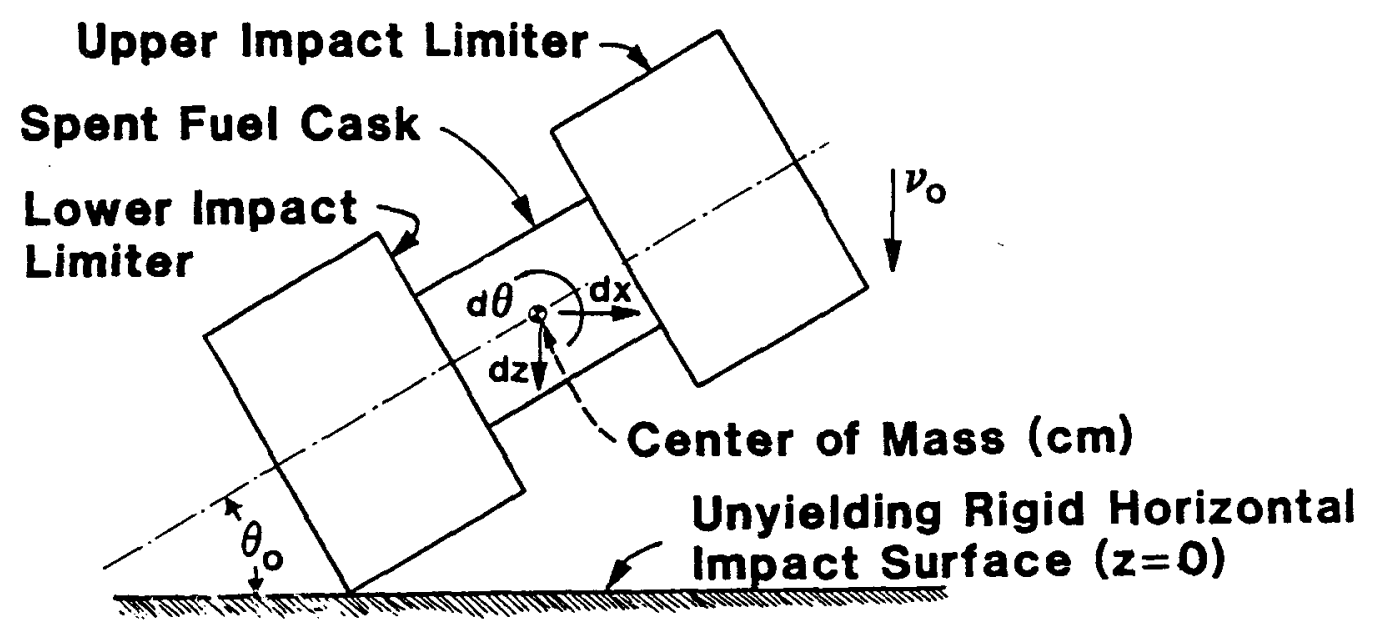

Figure II-17. Rigid-Body Cask Degrees of Freedom

to analyze the rigid-body response of spent-fuel casks to impact environments. (Other codes are also now available.) The following subsections describe the development, validation, and application of the SLAM code.

\section{Rigid-Body Kinematics Cask Mode1}

As shown in Figure II-18, impact is assumed to occur in three phases: an initial impact phase, a pinned phase, and a slapdown phase. In general, the lower (lower-left) limiter impacts first during the initial impact phase, followed by the upper (upper-right) impact limiter during the slapdown phase. The side-on impact is an exception, in which both limiters impact simultaneously.

A summary of the capabilities and limitations of a rigid-body model is listed in Table II-6. The major assumptions in constructing the spent-fuel cask model are (1) the cask behaves as a rigid body and (2) the deformation response of the impact limiters can be simplified as illustrated in Table II -6 .

The assumption that the cask-fuel system behaves as a rigid body results in the high-frequency response being neglected in the analysis. This assumption is reasonable because the additional high-frequency acceleration components that would occur with a flexible cask model do not significantly affect the response of the spent-fuel assemblies that have much lower fundamental frequency characteristics.

Many assumptions (e.g., axisymmetry, shape, contact area geometry, and stress distribution) relating to impact limiter behavior during crush up 

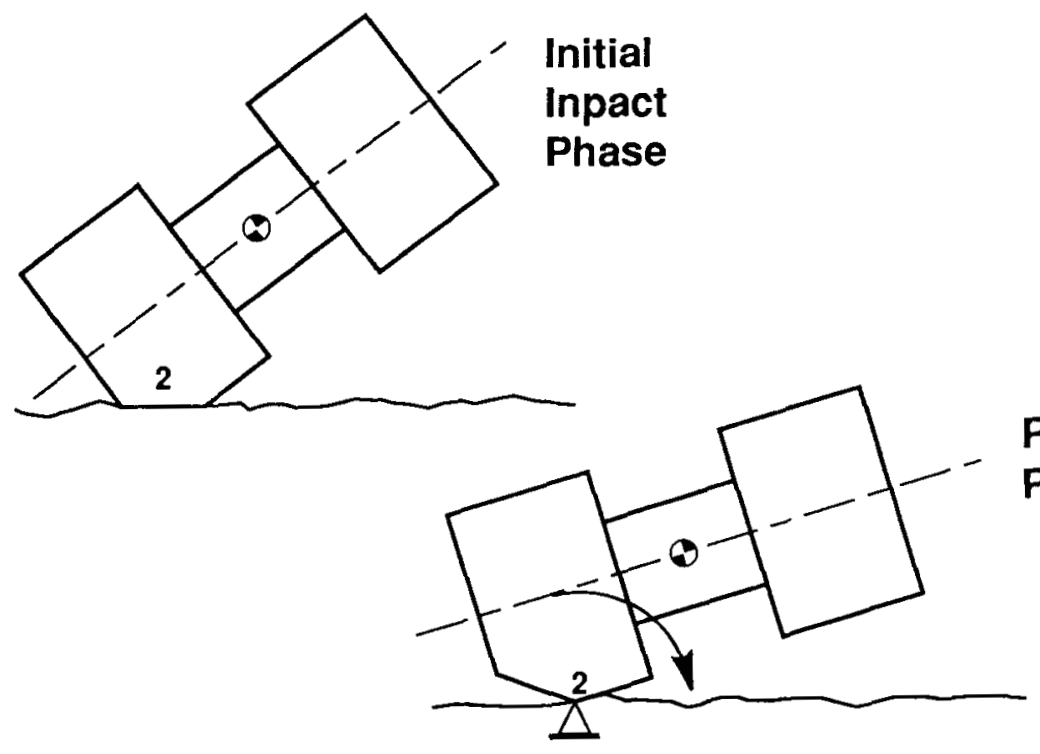

Pinned Phase

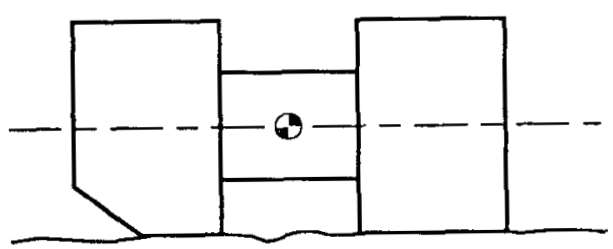

Slapdown Phase

Figure II-18. Rigid-Body Impact Phases

result in an overestimation of accelerations. For example, the entire contact area is assumed to be effective in decelerating the cask. However, those regions not backed by the cask may not be fully effective, causing actual acceleration levels to be less than the predicted levels. By using the total contact area, a realistic upper bound on the accelerations is obtained.

Stress distributions that are normal to the contact area are assumed to be uniform and equal to the crush strength of the impact limiter material; i.e., impact limiter contact stresses are independent of impact limiter strains. Figure II-19 indicates some strain dependence of the impact limiter stresses. However, for most of the working range of soft impact limiters, the stresses are equal to the crush strength $\left(\sigma_{\mathrm{C}}\right)$, as shown in Figure II-19. Idealizing the stresses as being independent of strain results in an overprediction of stresses in the elastic regions of the contact area. The extra stress assumed in the elastic regions of the impact limiter contact areas causes a slight overprediction of accelerations and provides an upper bound on the spent-fuel assembly accelerations.

The assumptions regarding deformable impact limiter behavior could be eliminated by incorporating user-definable force-deformation curves into 
Table II-6

Rigid-Body Model Capabilities and Limitations

Al1 Phases of Impact

- Each cask is a rigid body with deformable impact limiters.

- Each cask-impact limiter combination is axisymmetric around its centerline, symmetric around a plane through the center of mass, and normal to the cask centerline.

- All motion is two-dimensional (in the $x-z$ plane).

- Impact limiters are cylindrical.

- The contact area $\left(A_{C}\right)$ equals the intersection of a finite length cylinder and an infinite plane (impact surface).

- The crush strength $\left(\sigma_{\mathrm{c}}\right)$ is constant over the contact area.

- Each impact limiter normal force equals the crush strength multiplied by the contact area $\left(\mathrm{N}=\sigma_{\mathrm{C}} \mathrm{A}_{\mathrm{C}}\right)$.

- Each impact limiter normal force acts at the centroid of the contact area (center of pressure).

- Relative motion (slip) between each impact limiter and the impact surface may occur during all phases with frictional forces.

Initial Impact Phase

- Crush up of initial impacting end occurs.

- A small amount of rotation may occur.

- The initial impact phase terminates when the velocity of the initial end is zero.

Pinned Phase

- No impact limiter crushing occurs.

- Pin/roller is inserted at the edge of the initial crush up.

- The pinned phase does not occur for $\theta>\theta_{\mathrm{CMC}}$ and small $\theta$.

- The pinned phase terminates when the upper impact limiter contacts the impact surface. 
Table II-6

Rigid-Body Model Capabilities and Limitations

(Concluded)

\section{Slapdown Phase}

- Crush up of upper impact limiter occurs.

- A small amount of rotation may occur.

- The slapdown phase does not occur for $\theta>\theta \mathrm{CMC}$.

- The slapdown phase (and problem) terminates when the velocity of the upper impact end is zero.

the cask model. This option is not currently available in SLAM but is planned as a future task. Two methods are proposed for the forcedeformation capability: (1) an analytical formula with user-supplied stiffness and strength parameters and (2) interpolation of tabular data that could be experimentally or analytically obtained, e.g., finite element analyses. The impact limiter force-deformation behavior (tabular or formula) would be supplied as a cask angle function.

In addition to the calculation of impact limiter contact areas discussed above, axisymmetric assumptions are used for computing mass properties. Mass properties (weight and moment of inertia) can be computed in SLAM from geometry and material densities; however, these mass properties may also be supplied directly as input data. If the impact limiter behavior and mass properties are determined external to the code, then the assumption of axisymmetry is not required in the analysis. Other assumptions regarding the location of the impact limiter normal forces and the insertion of a pin or roller are required, but they have negligible effects on the initial impact phase accelerations and relatively small effects on accelerations occurring during the pinned and slapdown phases.

The parameters that describe each problem are shown in Figure II-20. The mass properties $W$ and $I_{\mathrm{cm}}$ may be defined by the code user or they may be computed from other input parameters. If $\mathrm{W}$ and $\mathrm{I}_{\mathrm{cm}}$ are defined by the user, then $t_{\text {end, }} t_{\text {inner, }} t_{\text {shield, }} t_{\text {outer, }} m_{\text {fuel }}, \rho_{\text {cask}}, \rho_{\text {shield, }}$ and $\rho$ limiter need not be specified.

\section{Newton's Second Law}

Figure II-21 shows the forces acting on a cask during impact. Applying Newton's second law of motion for each degree of freedom gives the following three equations representing dynamic equilibrium: 


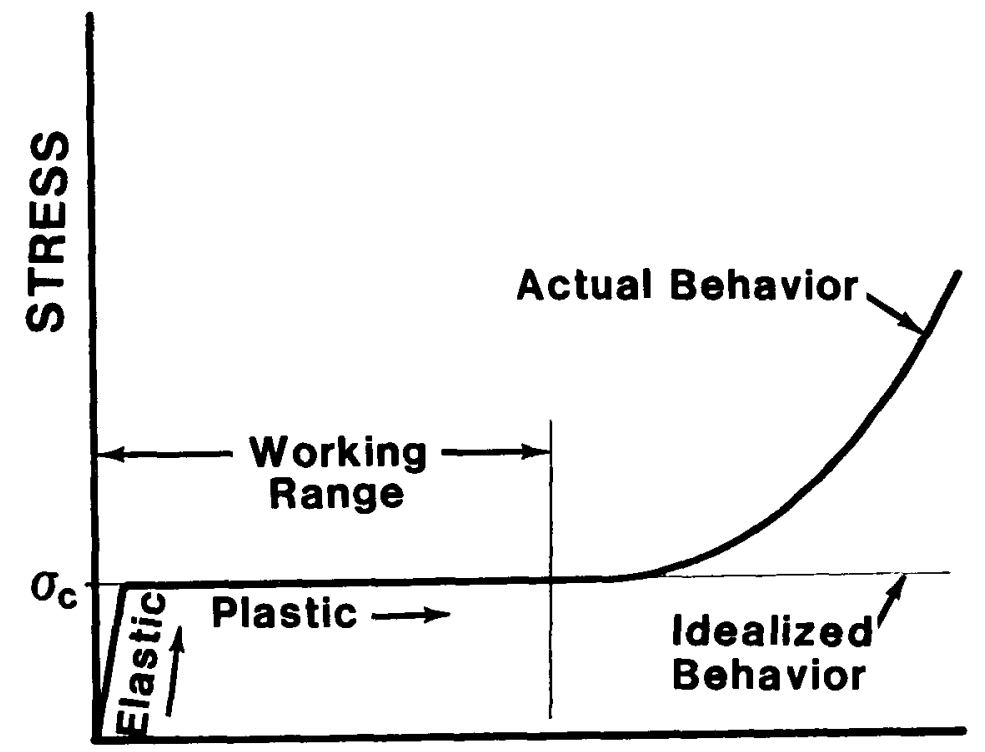

STRAIN

Figure II-19. Stress and Strain Behavior of the Impact Limiter

$$
\begin{aligned}
& \Sigma \mathrm{F}_{\mathrm{x}}=\mathrm{m}_{\mathrm{cm}}=\mathrm{F}_{2}+\mathrm{F}_{4} \\
& \Sigma \mathrm{F}_{\mathrm{z}}=\mathrm{m} \ddot{z}_{\mathrm{cm}}=\mathrm{W}-\mathrm{N}_{2}-\mathrm{N}_{4} \\
& \Sigma M_{\mathrm{y}}=\mathrm{I}_{\mathrm{cm}} \ddot{\theta}=\hat{z}_{2} \mathrm{~F}_{2}+\hat{\mathrm{z}}_{4} \mathrm{~F}_{4}+\hat{\mathrm{x}}_{2} \mathrm{~N}_{2}+\hat{\mathrm{x}}_{4} \mathrm{~N}_{4},
\end{aligned}
$$

where

$$
\begin{aligned}
& \mathrm{m}=\text { the mass of the cask and impact limiters } \\
& \mathrm{W}=\mathrm{mg}=\text { the weight of the cask and impact limiters } \\
& F_{i}=\text { the horizontal friction force at point } i \\
& \mathrm{~N}_{i}=\text { the vertical force associated with impact limiter crush up } \\
& \text { acting normal to the impact surface at point } i \\
& x_{i}, z_{i}=\text { coordinates of point } i \text { with respect to the center of mass } \\
& \mathrm{cm}=\text { a value at the center of mass } \\
& 2,4=\text { values at the contact area centroids of the lower and } \\
& \text { upper impact limiters. }
\end{aligned}
$$

\section{Center-of-Mass Coordinate System}

Though using the center of mass as a reference point is often convenient, this point is moving. Therefore, the following equations are used to relate center-of-mass coordinates to a fixed coordinate system $(\mathrm{x}, \mathrm{z})$ : 


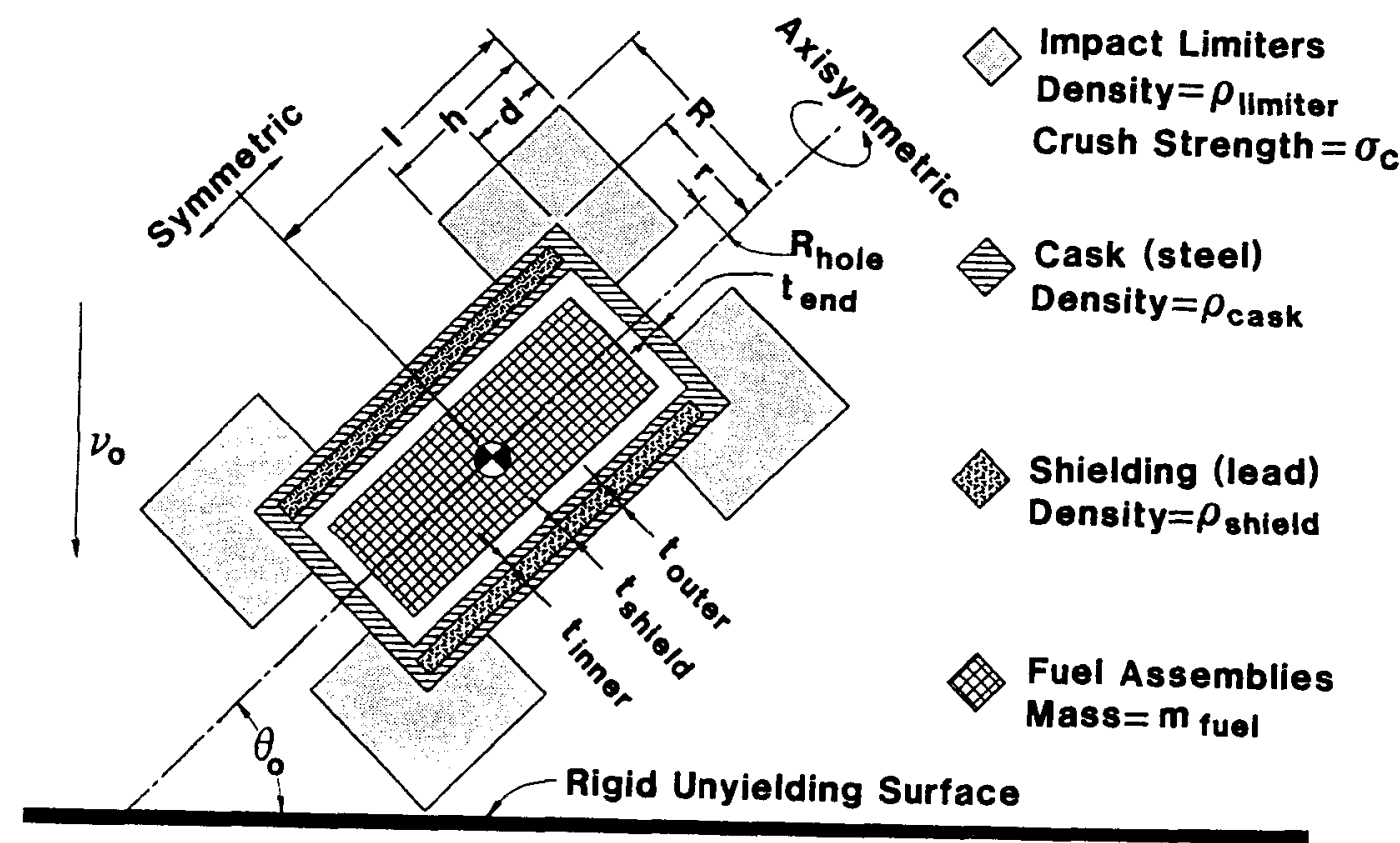

Parameter

Description

$\begin{aligned} \mathrm{R} & \text { Impact Limiter Radius } \\ \tau & \text { Cask Radius } \\ \mathrm{R}_{\mathrm{hole}} & \text { Impact Limiter Hole Radius } \\ 1 & \text { Center-of-Mass Height } \\ \mathrm{h} & \text { Impact Limiter Height } \\ \mathrm{d} & \text { Impact Limiter Depth } \\ \sigma_{\mathrm{c}} & \text { Impact Limiter Crush Strength } \\ \mathrm{v}_{\mathrm{o}} & \text { Impact Velocity } \\ \Delta \mathrm{t} & \text { Time Step } \\ \mu_{\mathrm{S}} & \text { Static Coefficient of Friction } \\ \mu_{\mathrm{k}} & \text { Kinetic Coefficient of Friction } \\ \mathrm{W} & \text { Total Cask Weight } \\ \mathrm{I}_{\mathrm{cm}} & \text { Mass Moment of Inertia } \\ t_{\text {end }} & \text { Cask End Thickness } \\ t_{\text {inner }} & \text { Inner Cask Wall Thickness } \\ t_{\text {shield }} & \text { Shielding Thickness } \\ \mathrm{T}_{\text {outer }} & \text { Outer Cask Wall Thickness } \\ \mathrm{m}_{\mathrm{fuel}} & \text { Mass of Fuel } \\ \rho_{\text {cask }} & \text { Cask Density (typically steel) } \\ \rho_{\text {shield }} & \text { Shielding Density (typically lead) } \\ \rho_{1 \text { imiter }} & \text { Density of Impact Limiters }\end{aligned}$

Figure II-20. Rigid-Body Model Parameters 


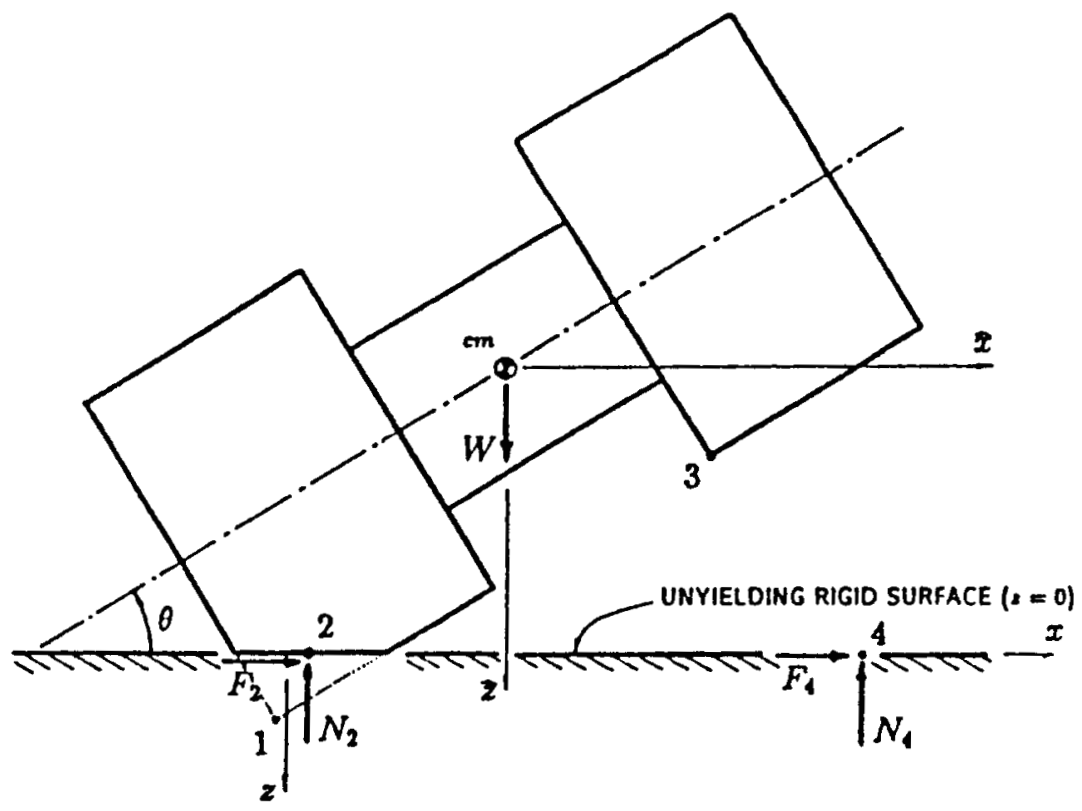

Figure II-21. Cask and Impact Limiter Forces

$$
\begin{aligned}
& x=x_{c m}+\hat{x} \\
& z=z_{c m}+\hat{z} \\
& \dot{x}=\dot{x}_{c m}+\hat{z} \dot{\theta} \\
& \dot{z}=\dot{z}_{c m}-\hat{x} \dot{\theta} \\
& \ddot{x}=\ddot{x}_{c m}+\hat{z} \ddot{\theta}-\hat{x} \dot{\theta}^{2} \\
& \ddot{z}=\ddot{z} c m-\hat{x} \ddot{\theta}-\hat{z}^{2} .
\end{aligned}
$$

Given a description of motion of the center of mass and the rotation of the cask body, the motion of any other point in the system is known. In particular, four points in the cask/impact limiter system are of interest and are shown in Figure II-21.

$$
\begin{aligned}
& \hat{x}_{1}=x_{1}-x_{c m}=R \sin \theta-1 \cos \theta \\
& \hat{z}_{1}=z_{1}-z_{c m}=R \cos \theta+1 \sin \theta
\end{aligned}
$$




$$
\begin{gathered}
\hat{x}_{2}=x_{2}-x_{c m}=x_{2}-x_{1}+x_{1}-x_{c m}=x_{c p 2}+\hat{x}_{1} \\
\hat{z}_{2}=z_{2}-z_{c m}=-z_{c m} \\
\hat{x}_{3}=x_{3}-x_{c m}=R \sin \theta+(1-h) \cos \theta \\
\hat{z}_{3}=z_{3}-z_{c m}=R \cos \theta-(1-h) \sin \theta \\
\hat{x}_{4}=x_{4}-x_{c m}=x_{4}-x_{1}+x_{1}-x_{c m}=x_{c p 4}+\hat{x}_{3} \\
\hat{z}_{4}=z_{4}-z_{c m}=-z_{c m} .
\end{gathered}
$$

In Equations (II-23) and (II-27), $x_{\operatorname{cp} 2}$ and $x_{c p 4}$ are the $x$ coordinates of the center of pressures for the lower and upper impact limiter contact surface relative to points 1 and 3 . Expressions for the center of pressures are developed later with the limiter contact area equations.

\section{Friction and Slip Conditions}

The slip condition is based on the sum of the horizontal friction forces ( $F$ ) and the sum of the vertical limiter normal forces (N):

$$
F=F_{2}+F_{4}=m \ddot{x}_{c m}
$$

When $\mathrm{F}$ becomes greater than $\mathrm{N} \mu_{\mathrm{S}}$, slippage will occur at the limiter contact surfaces for the remainder of the solution, where $\mu_{\mathrm{S}}$ is the static coefficient of friction. The value of $\mu \mathrm{s}$ is a code input parameter. If $\mathrm{F}$ is always less than $\mathrm{N} \mu_{\mathrm{s}}$, then slip will not occur.

Once slip occurs, then the kinetic friction forces are computed by

$$
\begin{aligned}
& F_{2}=-\operatorname{sgn}\left(\dot{x}_{2}\right) N_{2} \mu_{k} \\
& F_{4}=-\operatorname{sgn}\left(\dot{x}_{4}\right) N_{4} \mu_{k},
\end{aligned}
$$

where

$$
\begin{aligned}
\operatorname{sgn}(\dot{x})= & +1 \text { for } \dot{x}>0 \\
\operatorname{sgn}(\dot{x})= & 0 \text { for } \dot{x}=0 \\
\operatorname{sgn}(\dot{x})= & -1 \text { for } \dot{x}<0 \\
\mu_{k}= & \text { kinetic coefficient of friction. This is a code input } \\
& \text { parameter. }
\end{aligned}
$$




\section{Boundary Conditions}

As outlined in Table II-7, four sets of boundary conditions are used to describe the various phases of impact with and without slippage at the contact surfaces. The acceleration and velocity boundary conditions (e.g., $\dot{\mathrm{x}}_{2}=\dot{\mathrm{z}}_{2}=\mathrm{x}_{2}=\mathrm{z}_{2}=0$ ) can be restated in terms of center-of-mass quantities using Equations (II-17) through (II-20):

$$
\begin{aligned}
& \dot{\mathrm{x}}_{2}=0 \rightarrow \dot{\mathrm{x}}_{\mathrm{cm}}=-\hat{\mathrm{z}}_{2} \dot{\theta} \\
& \dot{\mathrm{z}}_{2}=0 \rightarrow \dot{\mathrm{z}}_{\mathrm{cm}}=+\hat{\mathrm{x}}_{2} \dot{\theta} \\
& \ddot{\mathrm{x}}_{2}=0 \rightarrow \ddot{\mathrm{x}}_{\mathrm{cm}}=-\hat{\mathrm{z}}_{2} \ddot{\theta}+\hat{\mathrm{x}}_{2} \dot{\theta}^{2} \\
& \ddot{z}_{2}=0 \rightarrow \ddot{z}_{\mathrm{cm}}=+\hat{\mathrm{x}}_{2} \ddot{\theta}+\hat{\mathrm{z}}_{2} \dot{\theta}^{2} .
\end{aligned}
$$

Table II -7

Rigid-Body Cask Boundary Conditions

\begin{tabular}{cccc} 
Case & Slip? & Pinned? & Conditions \\
\cline { 3 - 4 } 1 & No & No & $\ddot{\mathrm{x}}_{2}=\dot{\mathrm{x}}_{2}=0$ if $\mathrm{N}_{2}>\mathrm{N}_{4}$ \\
& & & $\ddot{\mathrm{x}}_{4}=\dot{\mathrm{x}}_{4}=0$ if $\mathrm{N}_{4}>\mathrm{N}_{2}$ \\
2 & No & Yes & $\ddot{\mathrm{x}}_{2}=\dot{\mathrm{x}}_{2}=0$, and \\
3 & Yes & No & $\ddot{\mathrm{z}}_{2}=\dot{\mathrm{z}}_{2}=0$ \\
4 & Yes & Yes & $\ddot{z}_{2}=\dot{\mathrm{z}}_{2}=0$ \\
\hline
\end{tabular}

\section{Equations of Motion}

Substitution of each set of boundary conditions into Equations (II-12) through (II-14) yields four sets of differential equations that may be expressed as

$$
\begin{aligned}
& \frac{\mathrm{d}}{\mathrm{d} t} \dot{\theta}=\ddot{\theta}(t) \\
& \frac{\mathrm{d}}{\mathrm{dt}} \dot{\mathrm{x}}_{\mathrm{cm}}=\ddot{x}_{\mathrm{cm}}(t)
\end{aligned}
$$




$$
\frac{\mathrm{d}}{\mathrm{dt}} \dot{z}_{\mathrm{cm}}=\ddot{z}_{\mathrm{cm}}(t)
$$

The specific equations $\left(\theta(t), \mathrm{x}_{\mathrm{cm}}(t), z_{\mathrm{cm}}(t)\right)$ for each set of boundary conditions are given in Table II-8. Equations (II-27) through (II-38)

along with the following three equations comprise six ordinary simultaneous differential equations to be integrated in time.

$$
\begin{aligned}
& \frac{d}{d t}(\theta)=\dot{\theta} \\
& \frac{d}{d t}\left(z_{c m}\right)=\dot{z}_{c m} \\
& \frac{d}{d t}\left(x_{c m}\right)=\dot{x}_{c m} .
\end{aligned}
$$

Equations (II-42) through (II-47) give the initial conditions representing cask position and velocity just before impact:

$$
\begin{aligned}
\theta(0) & =\theta_{0} \\
\dot{\theta}(0) & =0 \\
\mathrm{z}_{\mathrm{cm}}(0) & =-R \cos \theta_{0}-1 \sin \theta_{0} \\
\dot{\mathrm{z}}_{\mathrm{cm}}(0) & =\mathrm{v}_{\mathrm{o}} \\
\mathrm{x}_{\mathrm{cm}}(0) & =+1 \cos \theta_{0}-R \sin \theta_{0} \\
\dot{\mathrm{x}}_{\mathrm{cm}}(0) & =0 .
\end{aligned}
$$

A first-order Euler scheme and a fourth-order Runge-Kutta scheme are available in the SLAM code to numerically integrate the six differential equations of motion. The results presented in this report all used the Runge-Kutta scheme.

\section{Impact Limiter Contact Area}

Each impact limiter crushes as it comes into contact with the rigid impact surface. The motion equations developed above require the values for each limiter normal force $\left(\mathrm{N}_{2}\right.$ and $\left.\mathrm{N}_{4}\right)$ and the location where the force acts relative to the initial impacting corner (center of pressures, $x_{c p 2}$ and $\left.x_{\mathrm{cp}}\right)$. Limiter crush equations are developed here for one limiter but are applicable to both. The crushing force (N) acting on the limiter that is normal to the target surface is assumed to be a product of a constant crush stress $\left(\sigma_{\mathrm{C}}\right)$ and the contact area $\left(\mathrm{A}_{\mathrm{C}}\right)$ : 
Table II -8

Equations of Motion for the Rigid-Body Cask Model

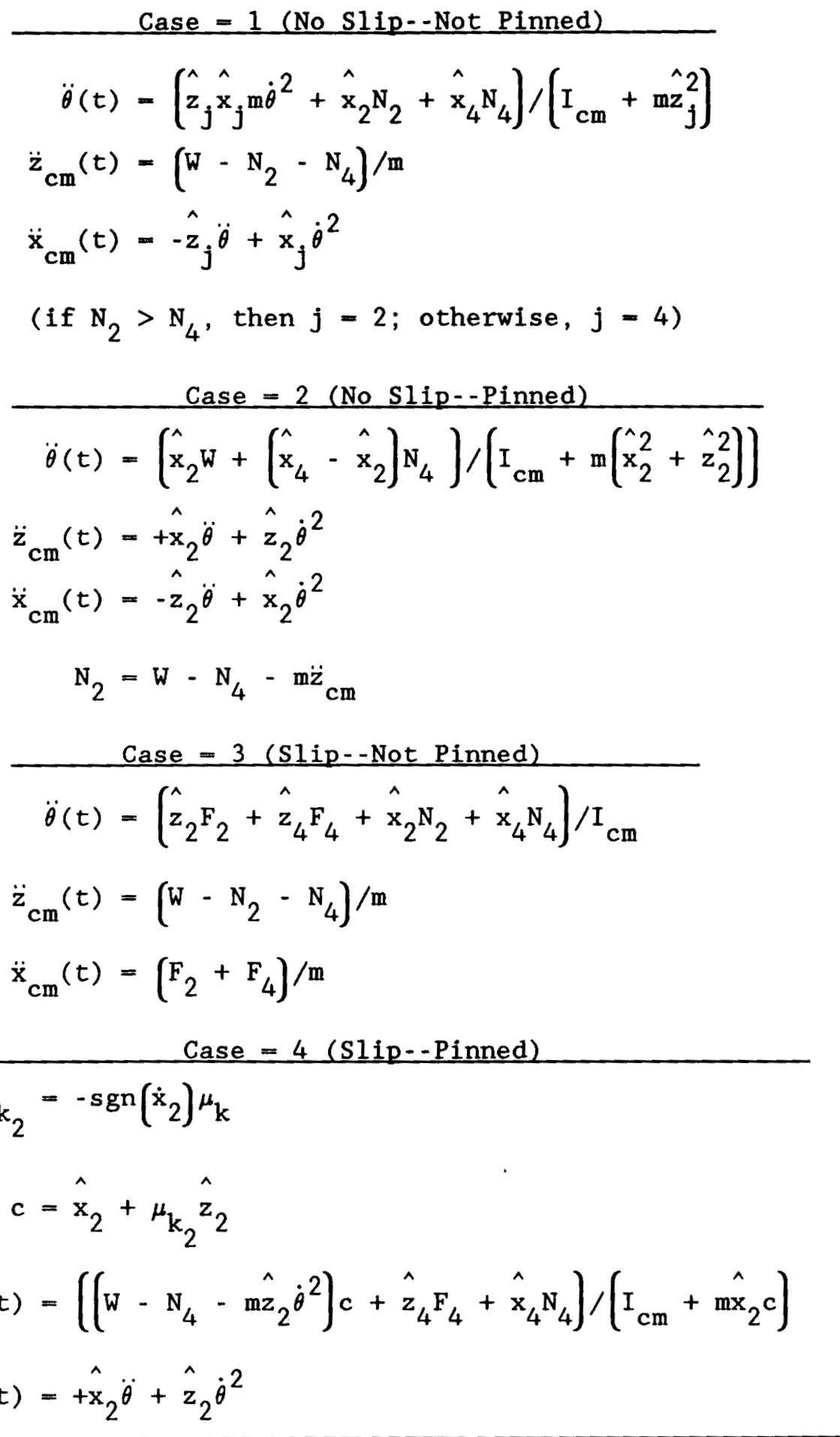


Table II -8

Equations of Motion for the Rigid-Body Cask Model

(Concluded)

$$
\begin{aligned}
N_{2} & =W-N_{4}-m \ddot{z}_{c m} \\
F_{2} & =\mu_{k_{2} N_{2}} \\
\ddot{x}_{c m}(t) & =\left(F_{2}+F_{4}\right)^{/ m}
\end{aligned}
$$

$$
N=\sigma_{c} A_{c}
$$

As the limiter crushes and the cask rotates, the contact area changes. Figure II-22 shows the types of contact areas (footprints) that may occur for cylindrical limiters. In general, the contact area is a function of limiter deformation $(\delta)$ and the angle $(\theta)$ between the cask axis and the impact surface.

As shown in Figure II-23, the contact area for an end-on impact orientation $(\theta=\pi / 2)$ is

$$
A_{c}=\pi R^{2}
$$

and the center of pressure is

$$
x_{c p}=-R
$$

For the side-on impact orientation $(\theta=0)$, the contact area is given by

$$
A_{c}=2 h \sqrt{2 \delta R-\delta^{2}}
$$

and the center of pressure is

$$
x_{c p}=\frac{h}{2}
$$

where

$$
\begin{aligned}
& \mathrm{R}=\text { the outer radius of the impact limiter and } \\
& \mathrm{h}=\text { limiter height. }
\end{aligned}
$$



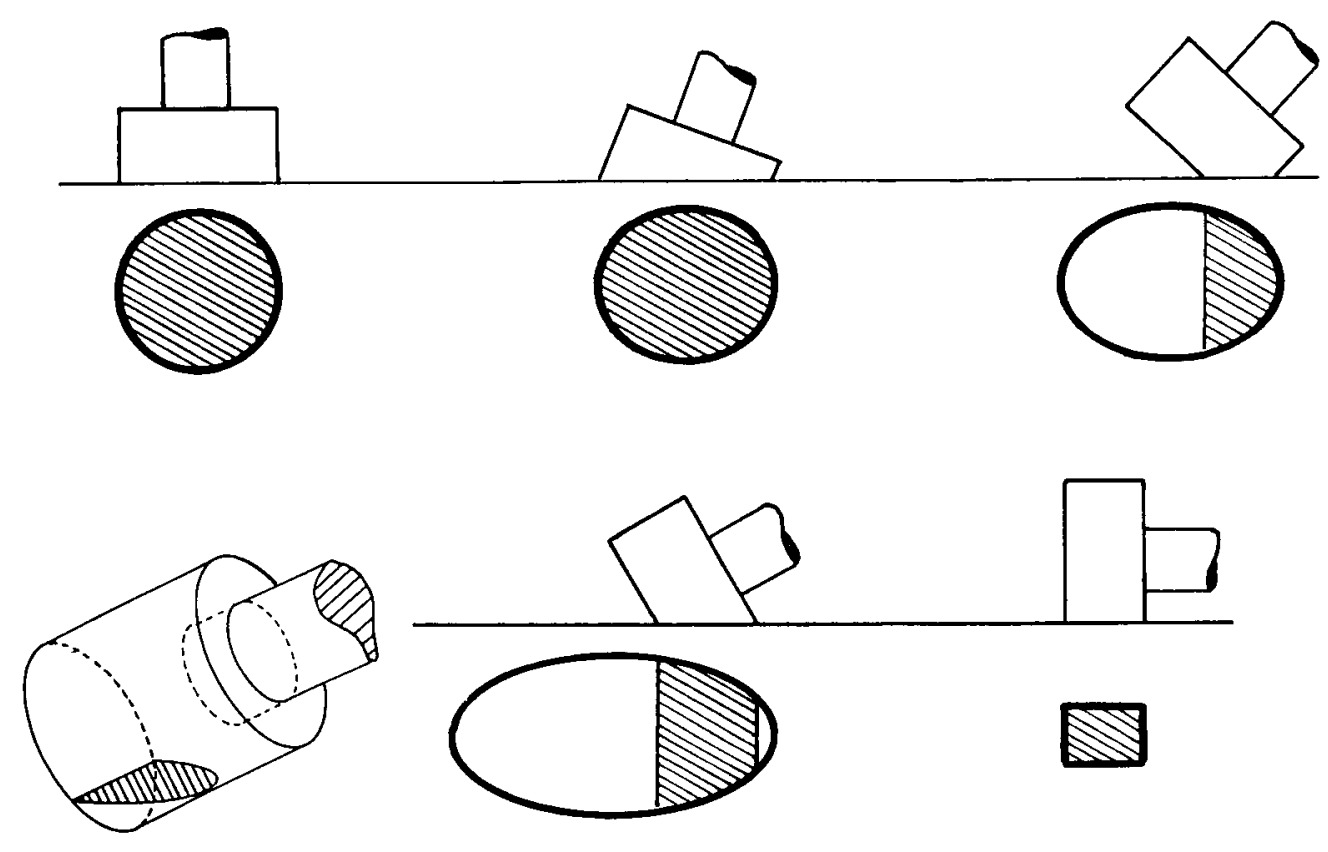

Figure II-22. Impact Limiter Contact Areas
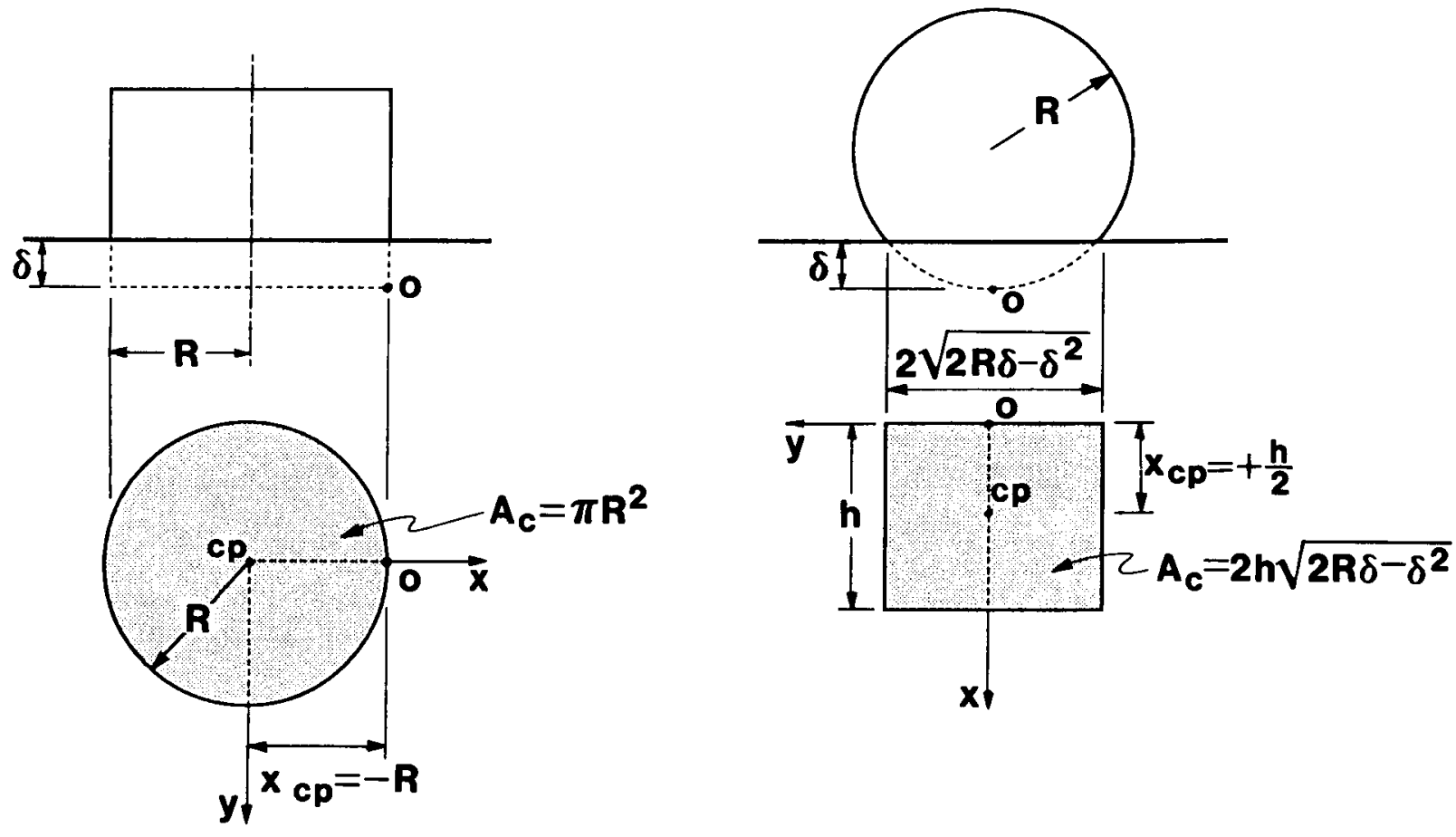

Figure II-23. Impact Limiter Contact Areas for End-On and Side-On Impact 
For oblique cask angles, the contact area is some portion of an ellipse as shown in Figure II-24. The major (a) and minor (b) ellipse dimensions are given by

$$
\begin{aligned}
& \mathrm{a}=\frac{\mathrm{R}}{\sin |\theta|} \\
& \mathrm{b}=\mathrm{R} .
\end{aligned}
$$

The equation describing the ellipse outline is given by

$$
\frac{x^{2}}{a^{2}}+\frac{y^{2}}{b^{2}}=1 \text {, }
$$

or, solving for $y$,

$$
y= \pm \frac{b}{a} \sqrt{a^{2}-x^{2}}
$$

The contact area can be determined by integrating Equation (II-56) from $\mathrm{x}_{\mathrm{L}}$ to $\mathrm{x}_{\mathrm{R}}$ :

$$
\begin{gathered}
A_{c}=2 \int_{x_{L}}^{x_{R}} y d x \\
A_{c}=2 \int_{x_{L}}^{x_{R}} \frac{b}{a} \sqrt{a^{2}-x^{2}} d x \\
A_{c}=\frac{b}{a}\left[x_{R} \sqrt{a^{2}-x_{R}^{2}}+a^{2} \sin ^{-1}\left(\frac{x_{R}}{a}\right)-x_{L} \sqrt{a^{2}-x_{L}^{2}}-a^{2} \sin ^{-1}\left(\frac{x_{L}}{a}\right)\right] .
\end{gathered}
$$

The limits of integration $\left(x_{L}\right.$ and $x_{R}$ ) are given by

$$
\begin{aligned}
& \mathrm{x}_{\mathrm{L}}=\frac{\mathrm{R}}{\sin \theta}-\frac{\delta}{\sin \theta \cos \theta} \geq-\mathrm{a} \\
& \mathrm{x}_{\mathrm{R}}=\frac{\mathrm{R}}{\sin \theta}-\frac{\delta}{\sin \theta \cos \theta}+\frac{\mathrm{h}}{\cos \theta} \leq \mathrm{a}
\end{aligned}
$$

As shown in Figure II-24, limiter deformation $(\delta)$ is equal to vertical (z) coordinate at the impact limiter corner:

$$
\begin{aligned}
& \delta=z_{1} \text { (lower impact limiter) } \\
& \delta=z_{3} \text { (upper impact limiter) . }
\end{aligned}
$$




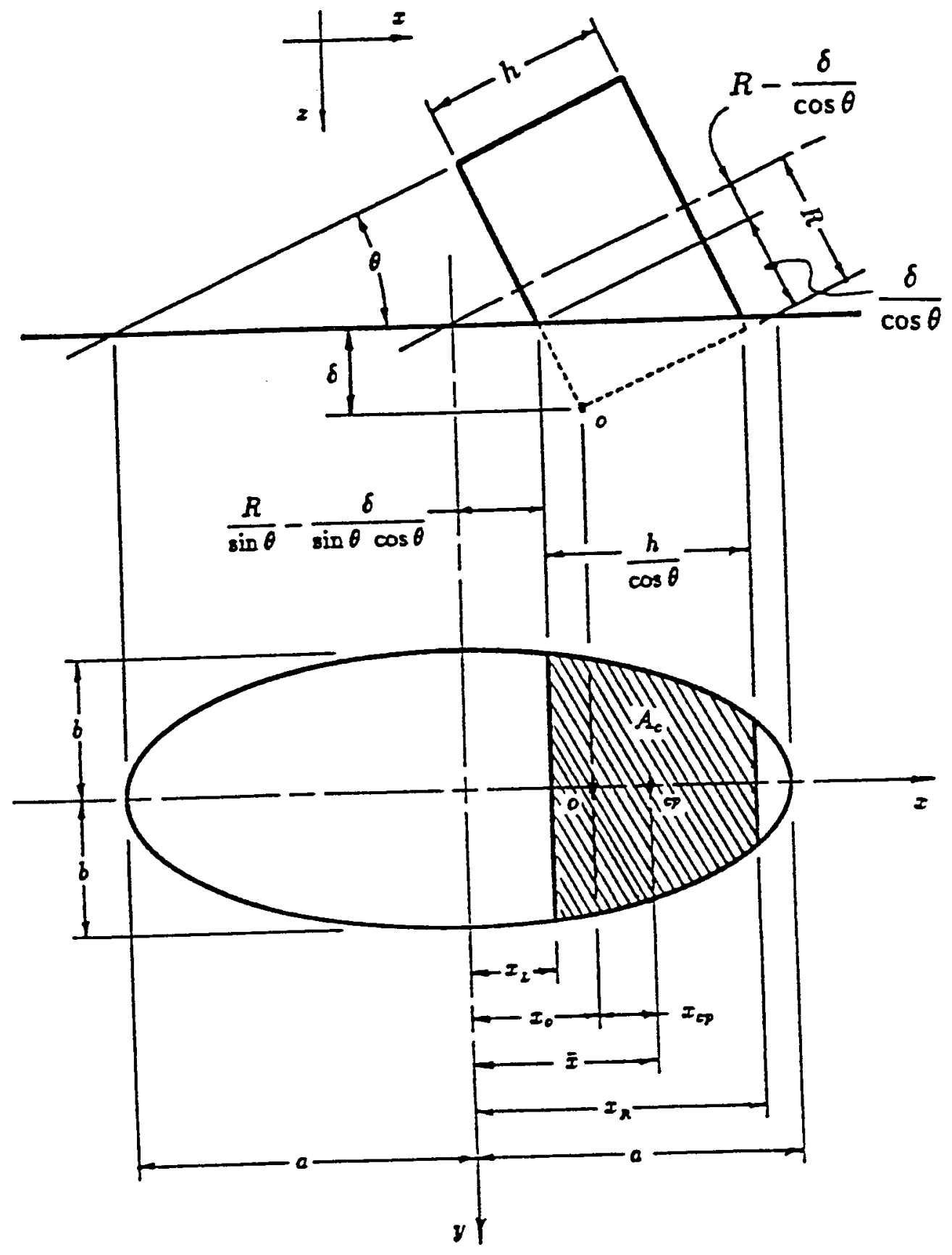

Figure II-24. Impact Limiter Contact Area for Oblique Impact 
The resulting crush force is assumed to act at the crush area centroid (center of pressure), $\bar{x}$, which is determined using the first moment of area theorem:

$$
\begin{aligned}
& \bar{x}=\frac{2}{A_{c}} \int_{x_{L}}^{x_{R}} x y d x \\
& \bar{x}=\frac{2}{A_{c}} \int_{x_{L}}^{x_{R}} x \frac{b}{a} \sqrt{a^{2}-x^{2}} d x \\
& \bar{x}=-\frac{2 b}{3 a A_{c}}\left[\sqrt{\left(a^{2}-x_{R}^{2}\right)^{3}}-\sqrt{\left(a^{2}-x_{L}^{2}\right)^{3}}\right] .
\end{aligned}
$$

Equation (II-66) provides the position of the contact area centroid relative to the center of the ellipse. The centroid position can also be referenced to the corner of the impact limiter where initial impact occurs $\left(x_{0}\right)$. Point o corresponds to point 1 on the lower limiter and point 3 on the upper limiter. The location of point o relative to the ellipse center is given by

$$
\mathrm{x}_{0}=\frac{\mathrm{R}}{\sin \theta}-\frac{\delta}{\sin \theta \cos \theta}+\delta \tan \theta .
$$

Thus, the position of the contact area center of pressure (centroid) relative to point $o$ is given by

$$
x_{c p}=\bar{x}-x_{o}
$$

A cylindrical hole in the end of an impact limiter is treated as follows. The equations governing the hole geometry are the same as those developed above for a solid cylindrical limiter, except that $R$ is replaced by $\mathrm{R}_{\text {hole }}$, and $\delta$ is replaced by $\delta_{\text {hole }}$ :

$$
\delta_{\text {hole }}=\delta \cdot\left(\mathrm{R}-\mathrm{R}_{\text {hole }}\right) \cos \theta .
$$

The effect of the hole is ignored until $\delta$ hole becomes nonnegative. Once the contact areas ( $A_{\text {solid }}$ and $A_{h o l e}$ ) and center of pressures ( $x_{\text {solid }}$ and $x_{h o l e}$ ) are computed for the solid impact limiter and limiter hole, the effect of the hole may be subtracted from the solid impact limiter to give the actual values:

$$
\begin{aligned}
A_{c} & =A_{\text {solid }}-A_{\text {hole }} \\
x_{c p} & =\left(x_{\text {solid }} A_{\text {solid }}-x_{\text {hole }} A_{\text {hole }}\right)^{/ A_{c}} .
\end{aligned}
$$




\section{Model Validation}

The SLAM code was validated by comparing results with hand calculations and experimental data. All validation calculations were performed on a quarter-scale model of the NUPAC 125-B cask that was tested at Sandia National Laboratories (SNL) [NU85]. Note that accelerations presented here for the quarter-scale cask model are four times greater than corresponding accelerations would be for the full-size cask. The quarter-scale cask parameters are given in Table II -9 .

Table II.9

Input Parameters for the Quarter-Scale NUPAC 125 -B Cask

\begin{tabular}{cc}
\hline & \\
Parameter & Value \\
\hline $1(\mathrm{~cm})$ & 88.75 \\
$\mathrm{R}(\mathrm{cm})$ & 38.10 \\
$\mathrm{r}(\mathrm{cm})$ & 20.8 \\
$\mathrm{~h}(\mathrm{~cm})$ & 47.65 \\
$\mathrm{~d}(\mathrm{~cm})$ & 22.9 \\
$\mathrm{~W}(\mathrm{~kg})$ & 1286 \\
$\mathrm{I}_{\mathrm{Cm}}\left(\mathrm{kg}-\mathrm{m}^{2}\right)$ & 267.7 \\
$\sigma_{\mathrm{C}}(\mathrm{MPa})$ & 4.83 \\
$\mathrm{v}_{\mathrm{O}}(\mathrm{m} / \mathrm{s})$ & 13.4 \\
$\Delta \mathrm{t}(\mathrm{s})$ & 0.0005 \\
\hline
\end{tabular}

\section{Comparisons to Experiment}

The NUPAC 125-B scale model was instrumented with accelerometers. Accelerometer data were collected for end-drop, side-drop, and CMC obliquedrop $\left(62.5^{\circ}\right)$ impact orientations. The data were then filtered to remove elastic body vibratory responses, leaving only the rigid-body accelerations. Acceleration histories were computed using SLAM for the quarterscale model at each impact orientation. Figures II-25 through II-27 show the computed accelerations for the end-drop, side-drop, and oblique-drop $\left(62.5^{\circ}\right)$ impact orientations. Figures II-28 through II-30 show the computed accelerations superimposed on the measured accelerations. Good agreement in the acceleration histories was observed for each angle of impact.

Some differences exist in the shape of each curve in Figures II-28 through II-30. These differences are probably a result of the elastic response that occurs during initial loading and unloading of the impact limiters. A rigid-perfectly-plastic material response (constant crush strength) is used in SLAM for each limiter; however, in reality some elastic response will occur. Additionally, a constant nominal crush strength of $4.83 \mathrm{MPa}$ was used in SLAM, when actually the crush strength of 


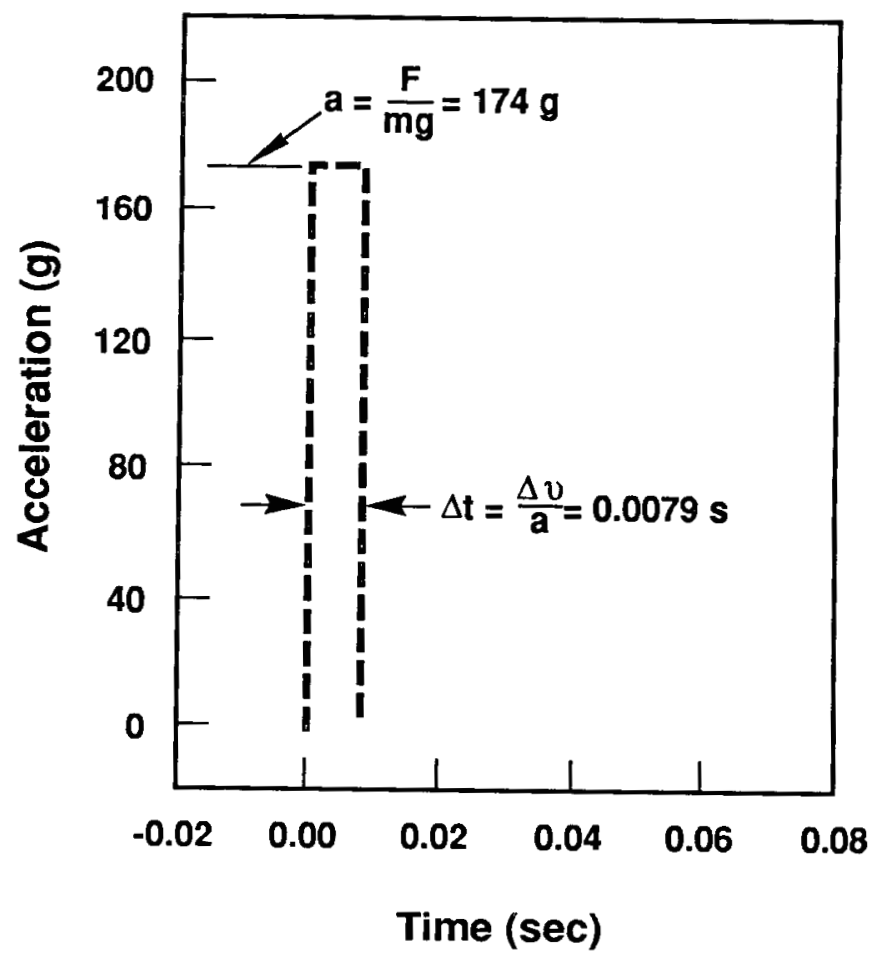

Figure II-25. End-Drop Analytical Accelerations for the NUPAC 125-B Mode1

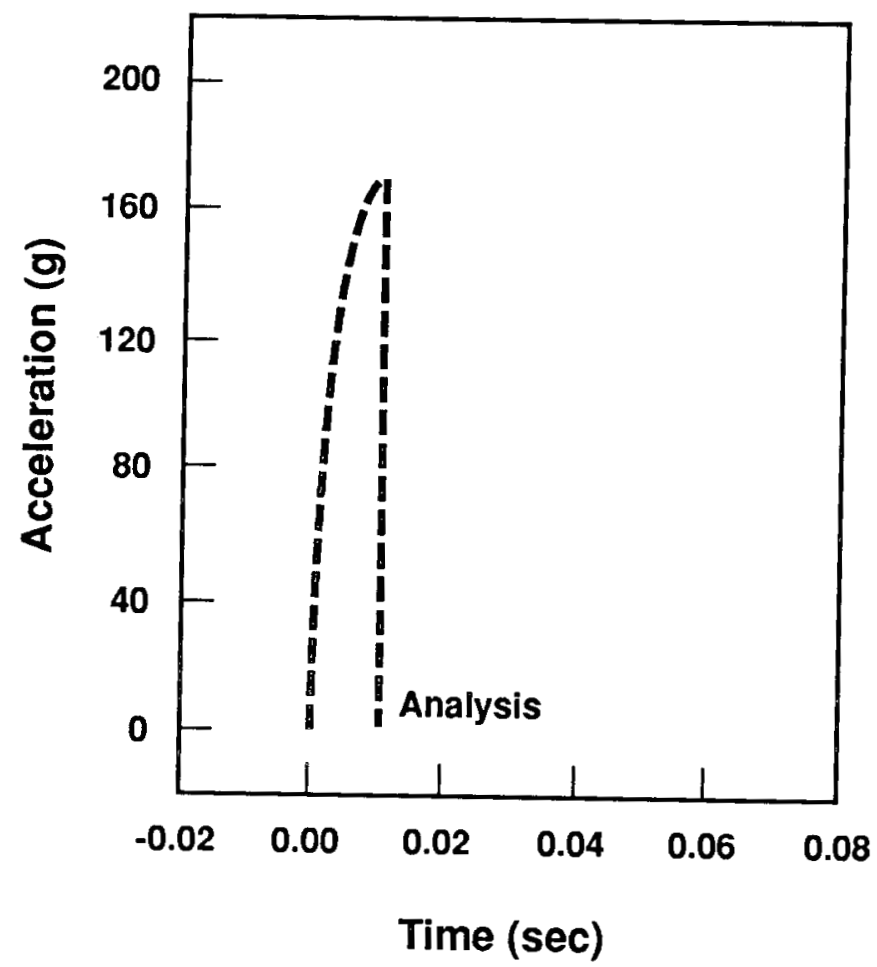

Figure II-26. Side-Drop Analytical Accelerations for the NUPAC 125-B Model 


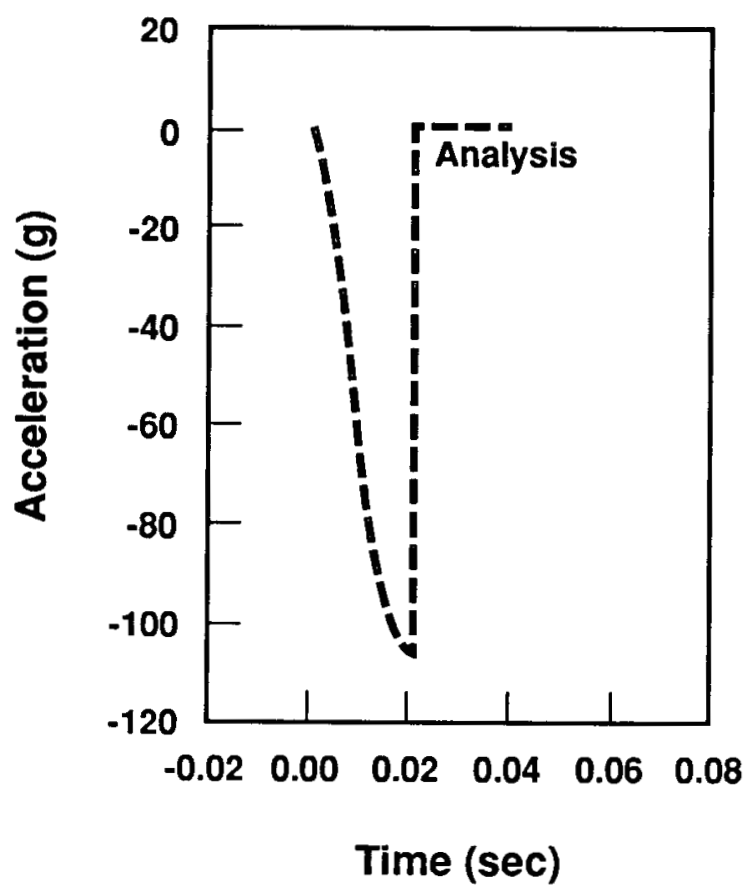

Figure II-27. Oblique-Drop Analytical Accelerations for the NUPAC 125-B Mode 1

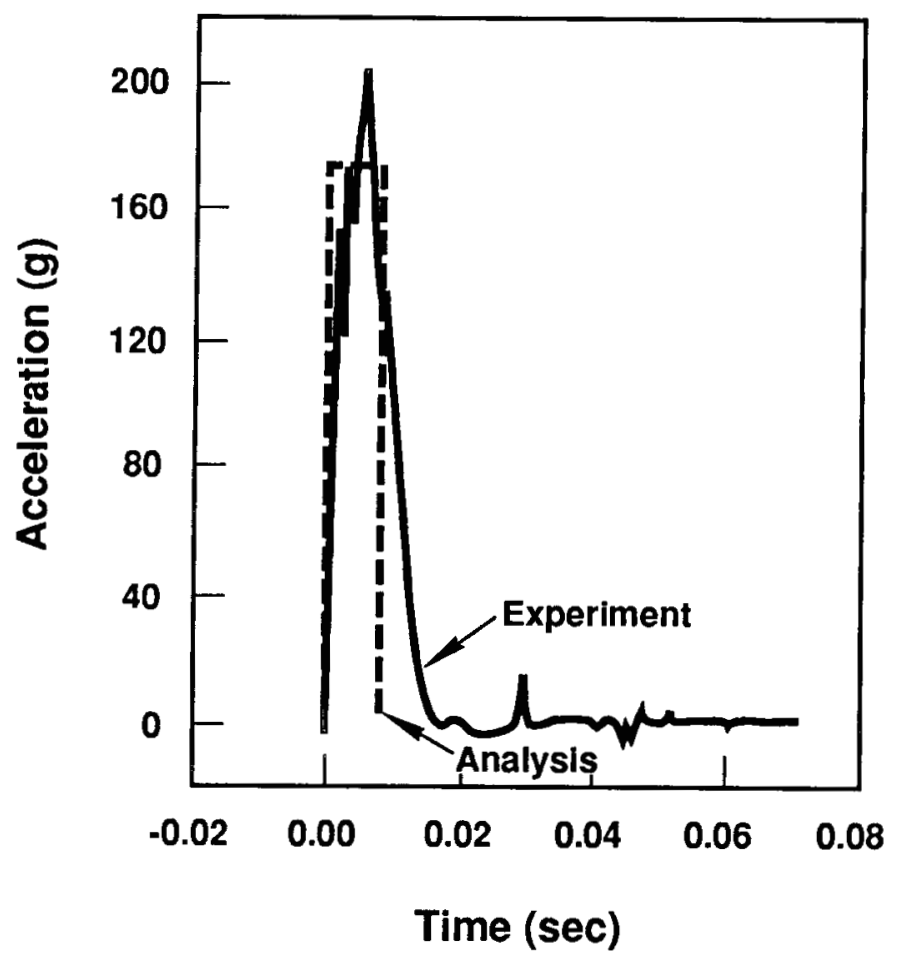

Figure II-28. End-Drop Analytical and Experimental Accelerations for the NUPAC 125-B Mode 1 


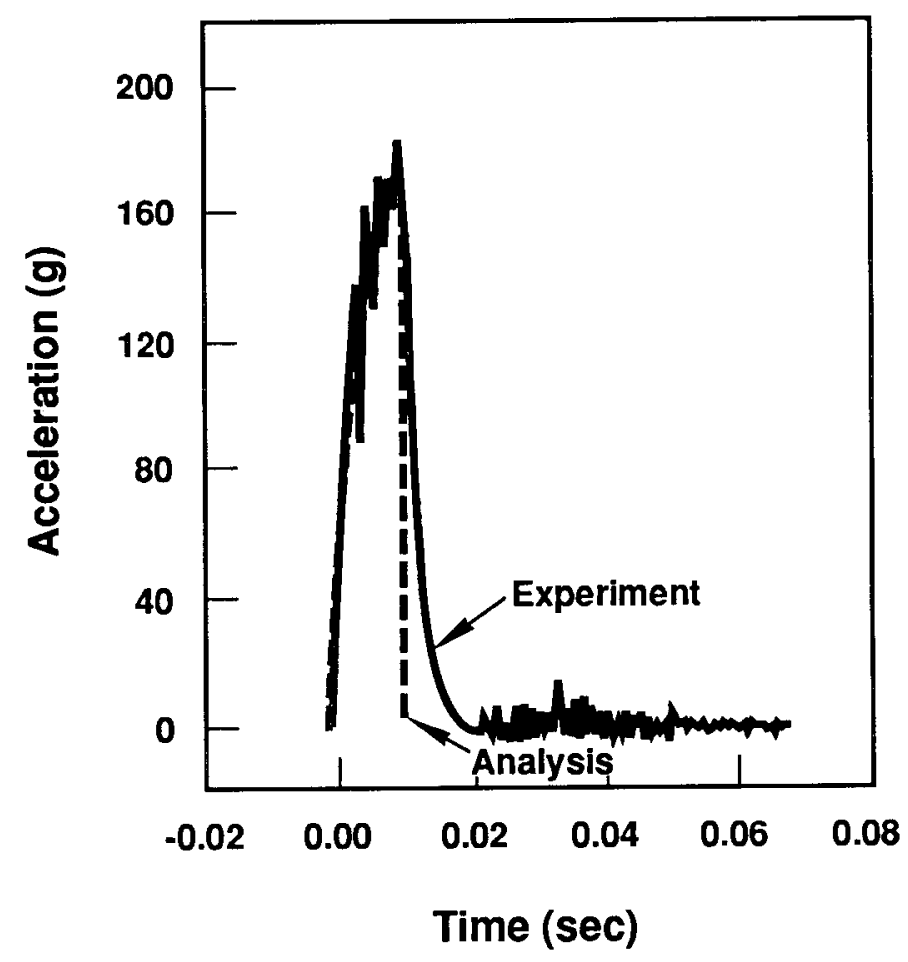

Figure II-29. Side-Drop Analytical and Experimental Accelerations for the NUPAC 125-B Model

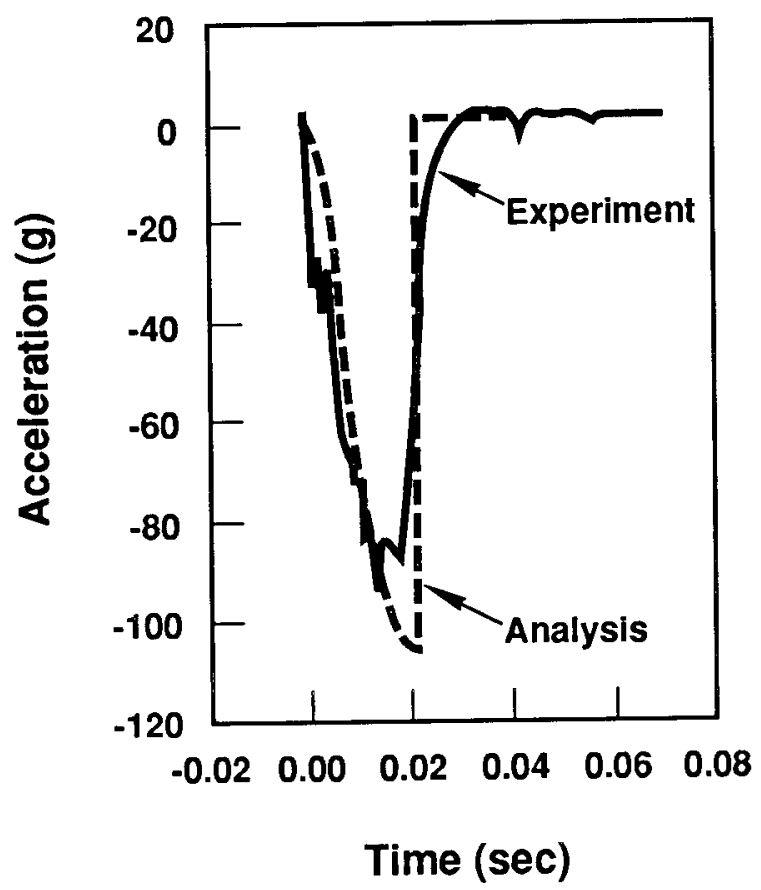

Figure II-30. Oblique-Drop Analytical and Experimental Accelerations for the NUPAC 125-B Model 
the limiter material is a function of strain in the material. Even with the rigid-perfectly-plastic assumption, SLAM can reliably predict acceleration levels and durations.

\section{Comparisons to Closed-Form Solutions}

Comparisons to hand calculations were also performed for the end- and side-drop impact orientations. Figure II-25 shows the predicted acceleration history for the end-drop impact of the quarter-scale NUPAC 125 - B cask. SLAM predicts a constant acceleration of $174 \mathrm{~g}$ for a $0.0078-\mathrm{s}$ duration. This agrees exactly with the acceleration ( $g$ ) calculated by hand using Newton's second law (Equation II-1):

$$
\mathrm{a}=\frac{\mathrm{F}}{\mathrm{m}}=\frac{\sigma_{\mathrm{c}} \pi \mathrm{R}^{2}}{\mathrm{~m}}=\frac{(4.83) \pi(0.381)^{2}}{1286}=174 \mathrm{~g} \text {. }
$$

For a constant acceleration, the duration is given by

$$
\Delta t=\frac{\Delta v}{a}=\frac{13.4}{174 \times 9.81}=0.0079 \mathrm{~s} .
$$

For the side-drop impact, force on the impact limiters is the contact area for both limiters $\left(2 A_{c}\right.$ ) given by Equation (II-51), multiplied by the crush strength $\left(\sigma_{\mathrm{c}}\right)$ :

$$
F=2 \sigma_{c} A_{c}=4 h \sigma_{c} \sqrt{2 R \delta-\delta^{2}} .
$$

Integrating this force deformation relationship yields the effect on the cask during impact:

$$
\mathrm{U}=\int_{0}^{\delta} \mathrm{Fd} \delta=2 \mathrm{~h} \sigma{ }_{c}\left[(\delta-\mathrm{R}) \sqrt{2 \mathrm{R} \delta-\delta^{2}}+\mathrm{R}^{2} \sin ^{-1}\left(\frac{\delta-\mathrm{R}}{\mathrm{R}}\right)+\frac{\pi}{2} \mathrm{R}^{2}\right] .
$$

Conservation of energy requires that the effect on the cask during impact must be equal to the change in kinetic and potential energy of the cask:

$$
\mathrm{U}=\frac{1}{2} \mathrm{mv} \mathrm{o}_{\mathrm{o}}^{2}-\frac{1}{2} \mathrm{mv} \mathrm{v}^{2}-\mathrm{mg} \delta
$$

Solving Equation (II-76) for cask velocity as a function of limiter deformation gives

$$
v=\sqrt{v_{0}^{2}-2 g \delta-\frac{4 h R^{2} \sigma_{c}}{m}\left[\frac{\pi}{2}+\sin ^{-1}\left[\frac{\delta-R}{R}\right]+\frac{\delta-R}{R} \sqrt{\frac{2 \delta}{R}-\left(\frac{\delta}{R}\right)^{2}}\right]}
$$


Equation (II-77) is plotted in Figure II-31 along with the corresponding velocity versus the deformation curve computed by SLAM for the quarter-scale NUPAC 125-B cask. The SLAM acceleration agrees well with the closed-form solution.

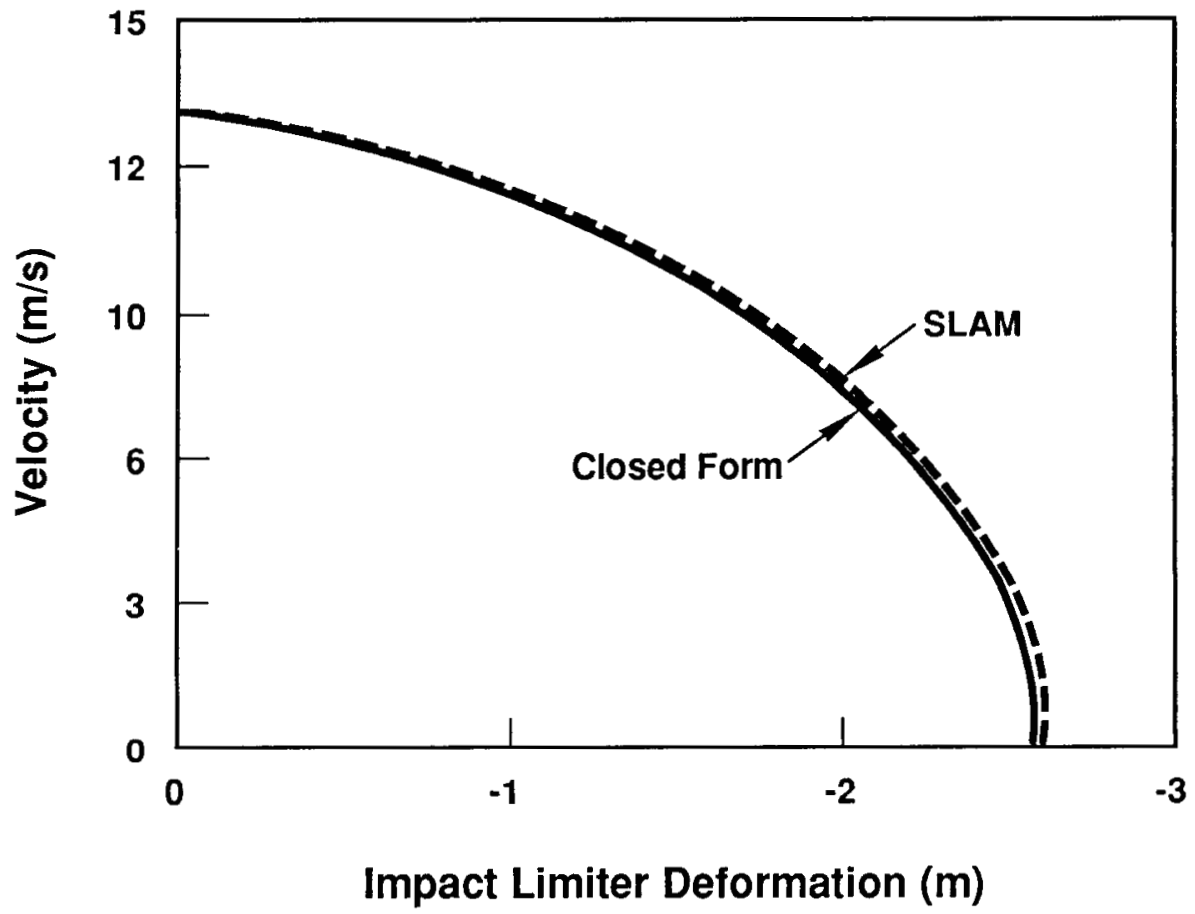

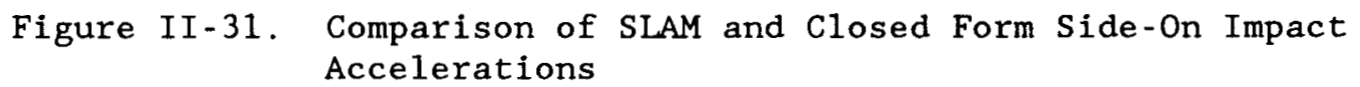

Energy conservation is tracked within SLAM during each impact solution. Energy distribution during the impact process for the end-and side-drop cases is shown in Figures II-32 and II-33. As work is done during the crush up of the impact limiters, the kinetic energy

$\left(\mathrm{KE}=1 / 2 \mathrm{~m}\left(\dot{\mathrm{x}}_{\mathrm{cm}}{ }^{2}+\dot{\mathrm{z}}_{\mathrm{cm}}{ }^{2}\right)+1 / 2 \mathrm{I}_{\mathrm{cm}} \dot{\theta}^{2}\right)$ decreases. In addition, a small decrease in potential energy $\left(P E=-m g z_{c m}\right)$ occurs, while the total energy $(\mathrm{TE}=$ work $+\mathrm{KE}+\mathrm{PE})$ remains constant.

An additional check can be made for an oblique drop on a frictionless $(\mu=0)$ surface. As shown in Figure II-34, the horizontal position of the cask center of mass remains constant because there are no horizontal forces for a frictionless case.

\section{Results for Representative Casks}

The representative cask designs described in Section II.2 were analyzed using SLAM to determine representative acceleration loadings on the spent-fuel assemblies. The inner and outer cask dimensions and fuel masses were the same as those specified in Section II.2. However, 1imiters for each cask were redesigned to yield a more balanced design in which peak 


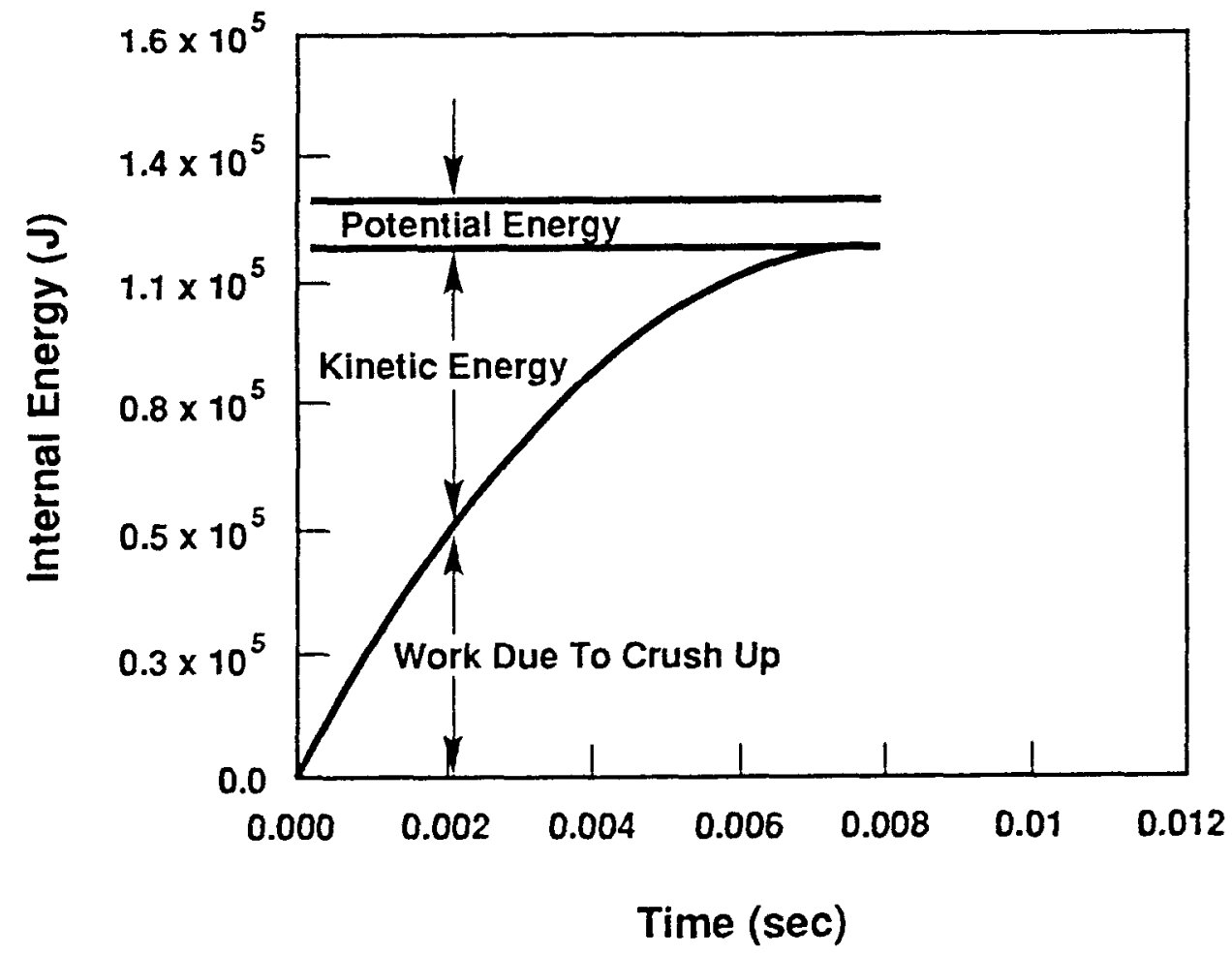

Figure II-32. SLAM End-Drop Conservation of Energy Check

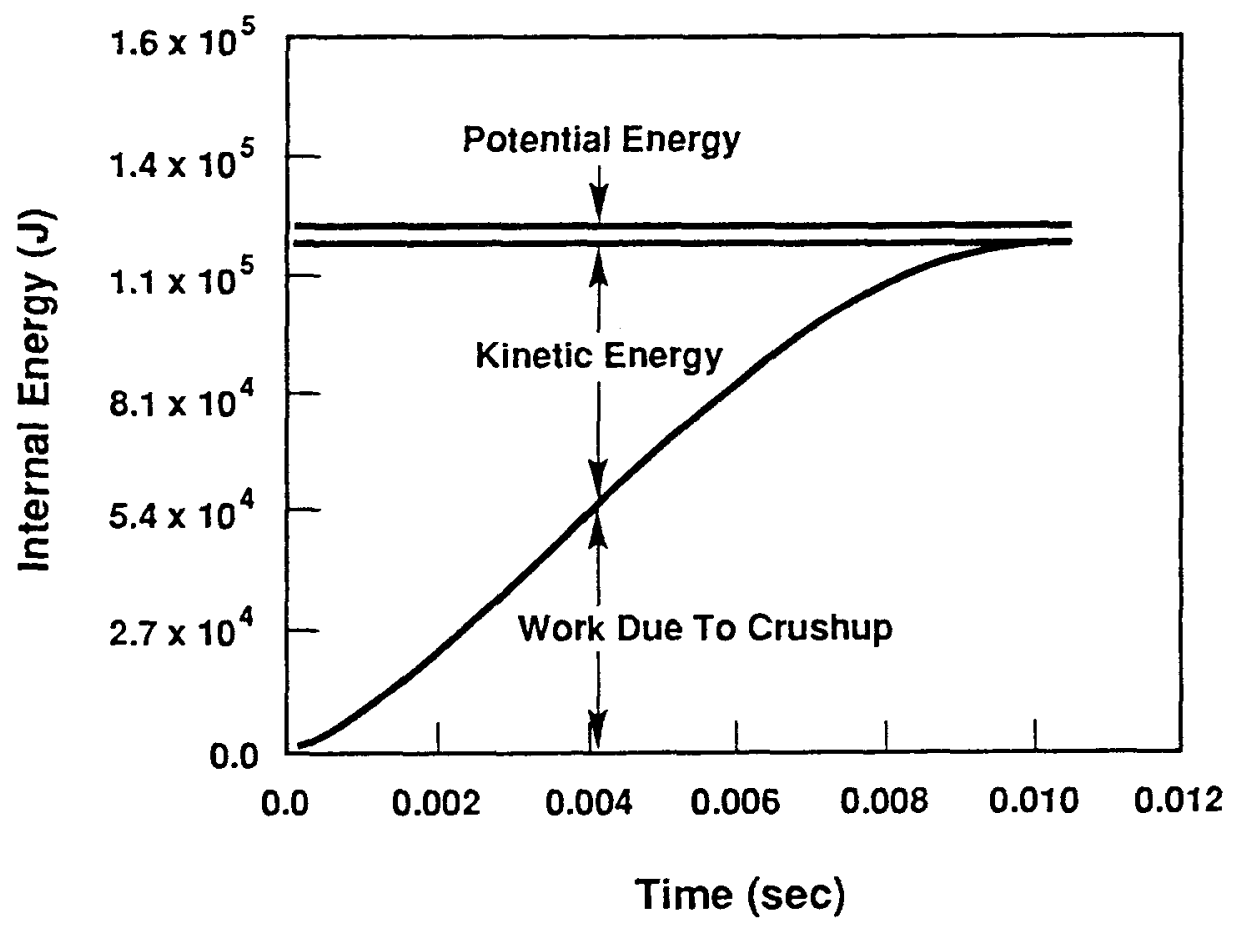

Figure II-33. SLAM Side-Drop Conservation of Energy Check 


\section{Quarter-Scale NUPAC 125-B Cask Impact Angle $=62.5^{\circ}$}

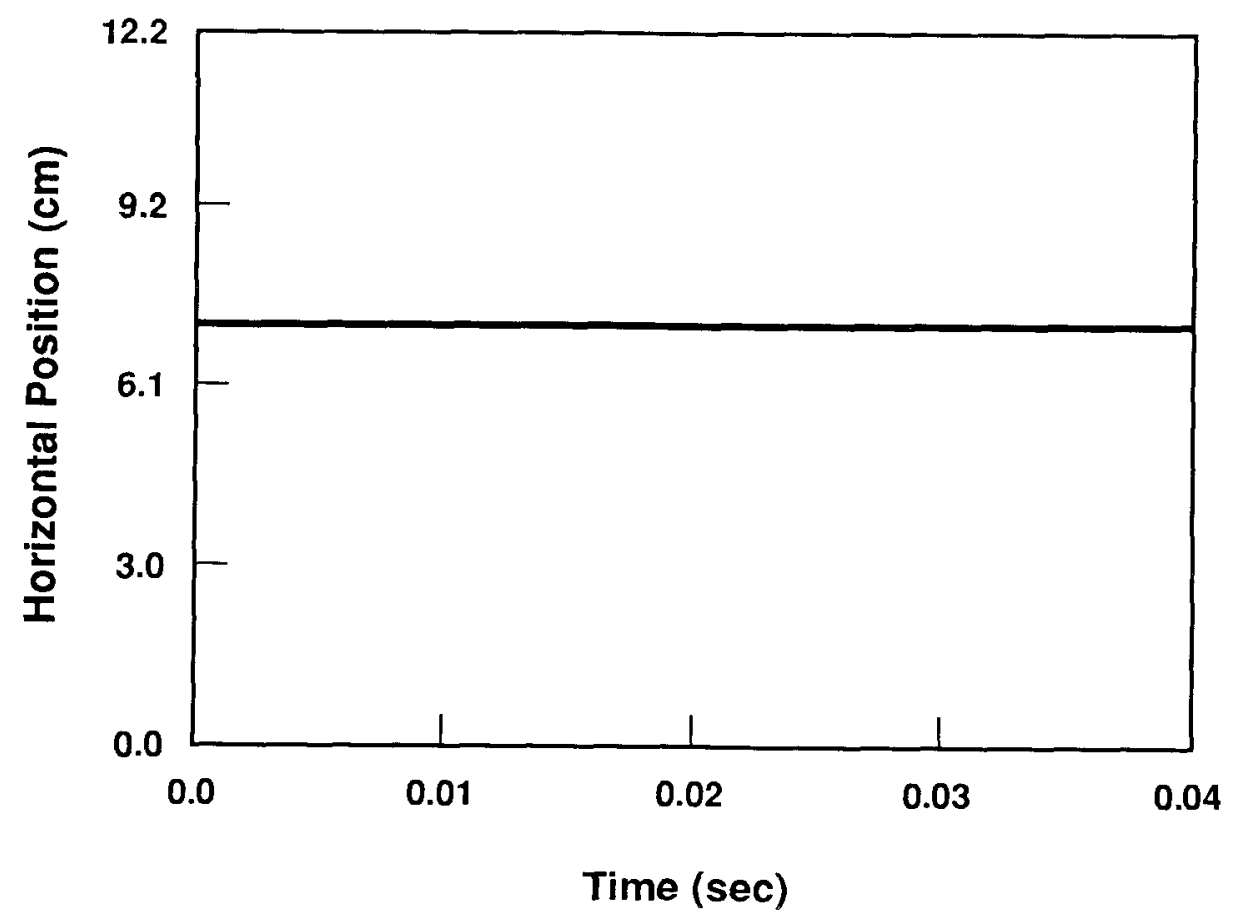

Figure II-34. Horizontal Center-of-Mass Position for Oblique Impact on a Frictionless Surface

vertical accelerations have less variance with the impact angle. This was done by adding a hole to the base of the impact 1 imiter to reduce the contact in the end-impact case.

Limiter redesign results in a lower crush strength $(6.9 \mathrm{MPa})$ and modified dimensions, including the addition of a hole in the top and bottom of each limiter that reduces the end-drop acceleration levels without affecting accelerations at other impact orientations. In addition, the limiter height is adjusted to achieve the same accelerations for side-on $(\theta=0)$ impact as for end-on $(\theta=\pi / 2)$ impact. Sufficient thicknesses of material are provided so that total strains are less than 508 for all impact angles. The resulting code input parameters for the four generic cask designs are shown in Table II-10. See Figure II-20 for the definition of dimensions.

SLAM was used to analyze each of the four example casks subjected to the $9.0-\mathrm{m}$ drop regulatory accident condition, assuming both a no-slip condition $(\mu=\infty)$ and a frictionless condition $(\mu=0)$. An additional normal transport condition requires that a spent-fuel cask withstand a $0.3-\mathrm{m}$ drop impact. The lead-shielded truck cask was subjected to the $0.3-\mathrm{m}$ drop regulatory normal condition for a frictionless target. Both 0.3 - and 9.0-m drop analyses were performed over a 0 (side-on) to $90^{\circ}$ (end-on) range of impact angles. The 0.005-s time step $(\Delta t)$ used in the analyses was obtained from convergence studies in which further refinement of $\Delta t$ 
Table II -10

Representative Cask Input Parameters

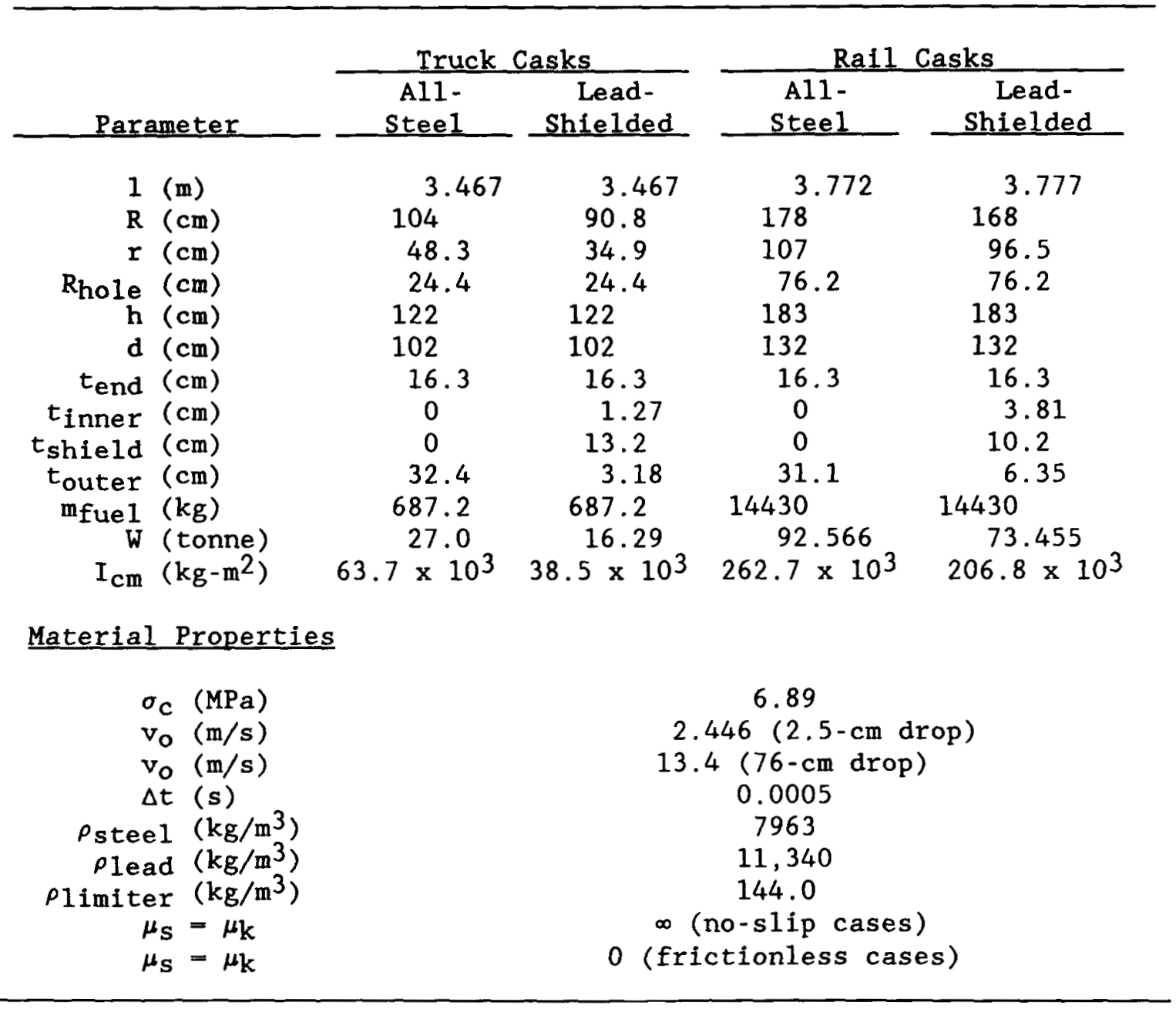

produced no additional accuracy. Over 150 runs were conducted. Execution times were minimal, averaging $3 \mathrm{CPU} s$ per run on a MicroVax computer.

The lead-shielded truck cask impacting a frictionless surface gives larger accelerations when compared to accelerations for the other cask designs and impact surface friction. Vertical acceleration histories for the lead-shielded truck cask subjected to the $0.3-\mathrm{m}$ drop are plotted simultaneously with the 9.0 -m drop results in Figures II-35, II-36, II-37, and II-38 for impact angles of $0,30,60$, and $90^{\circ}$. The $0.3-\mathrm{m}$ drop slapdown events, which are not a regulatory condition, are shown for comparison only. Angular acceleration histories are shown in Figures II-39 and II-40 for impact angles of 30 and $60^{\circ}$. Impact angles of 0 and $90^{\circ}$ do not result in any angular accelerations, and due to the assumption of the frictionless impact surface, horizontal accelerations are zero. 
Lead-Shielded Truck Cask (frictionless)

Impact Angle $=\mathbf{0} .0$ degrees

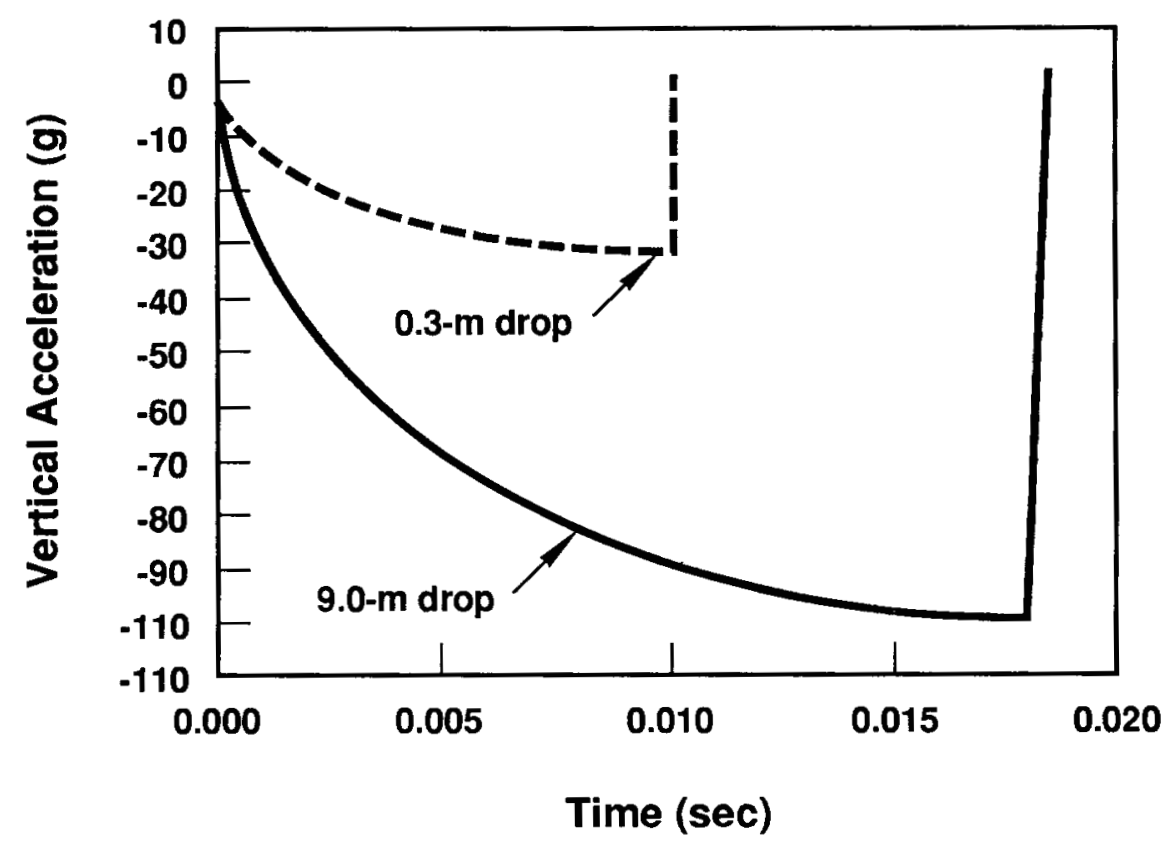

Figure II-35. Vertical Acceleration Histories for the 0.3- and 9.0-m Drops $\left(\theta_{0}=0^{\circ}\right)$

Lead-Shielded Truck Cask (frictionless)

Impact Angle $\mathbf{=} \mathbf{3 0} .0$ degrees

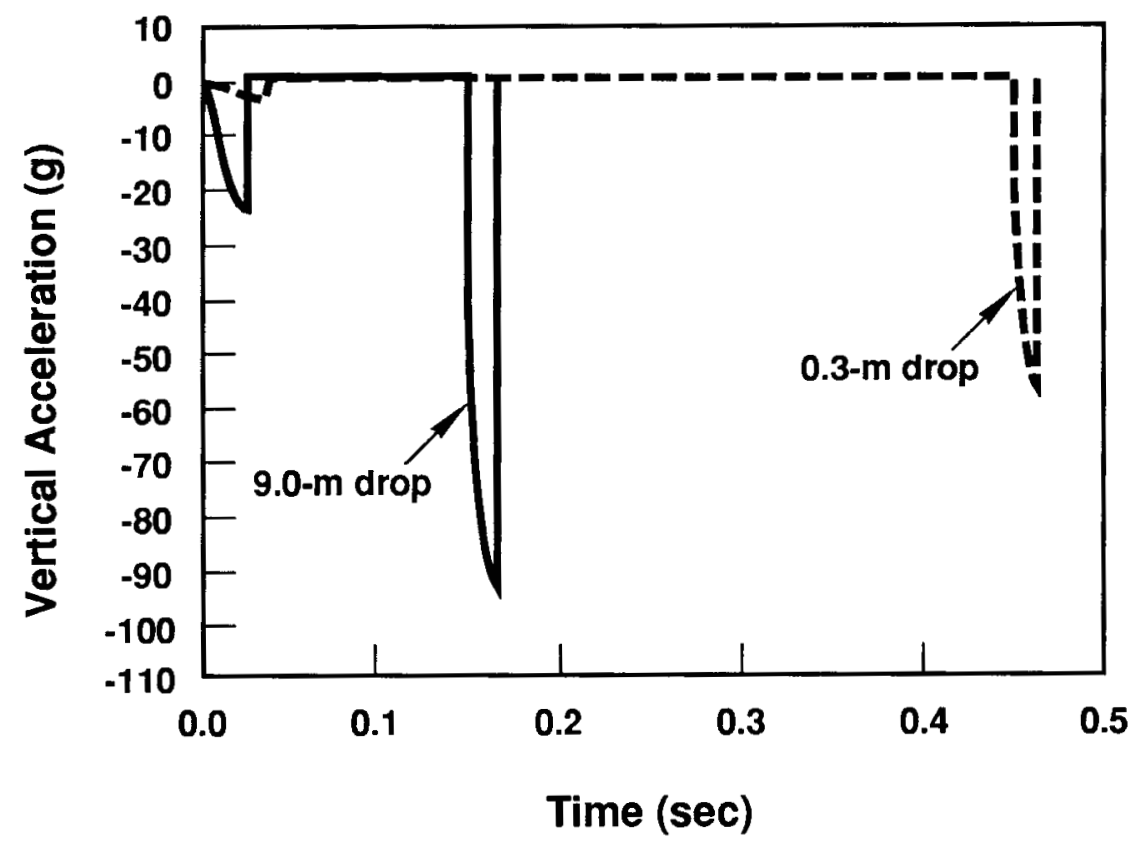

Figure II-36. Vertical Acceleration Histories for the 0.3- and 9.0-m Drops $\left(\theta_{0}=30^{\circ}\right)$ 


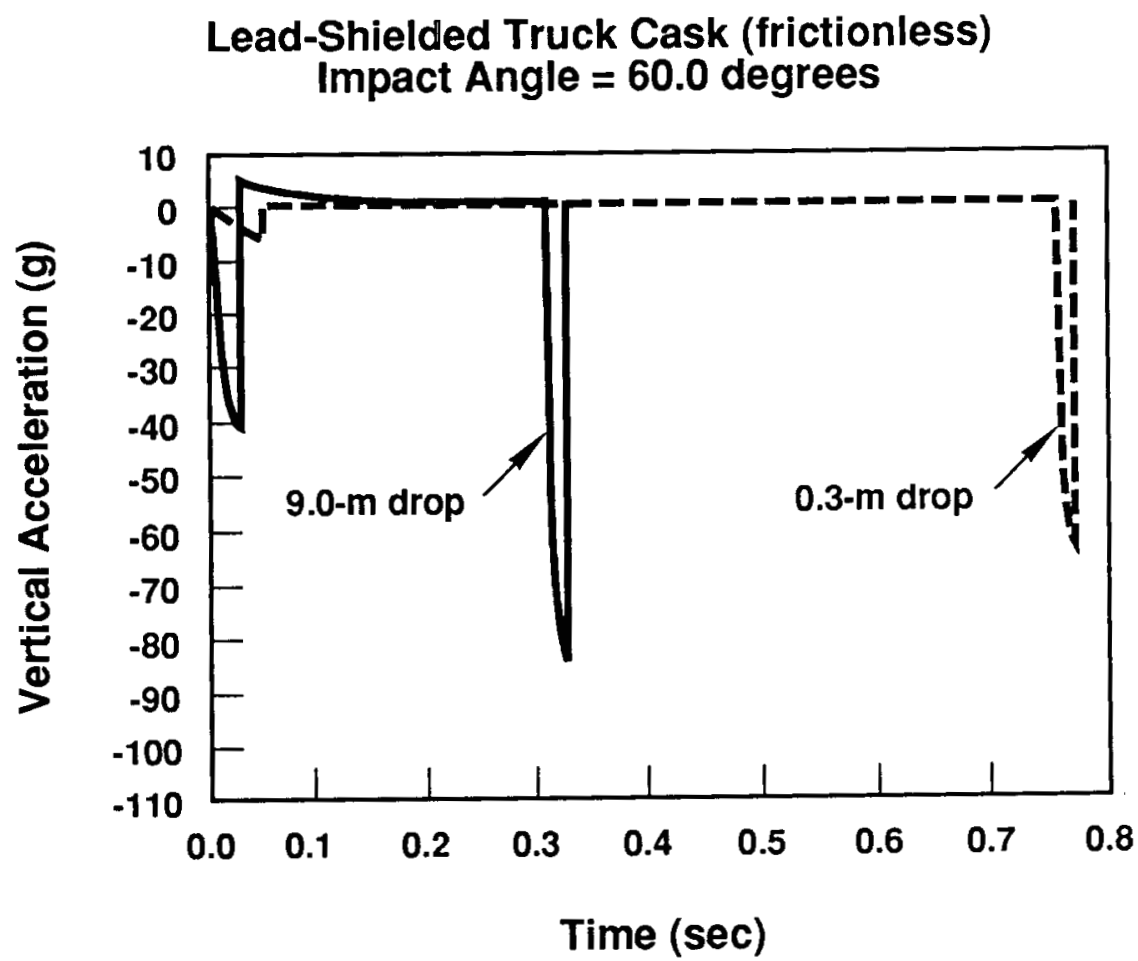

Figure II-37. Vertical Acceleration Histories for the 0.3- and 9.0-m Drops $\left(\theta_{0}=60^{\circ}\right)$

\section{Lead-Shielded Truck Cask (frictionless)}

Impact Angle $=90.0$ degrees

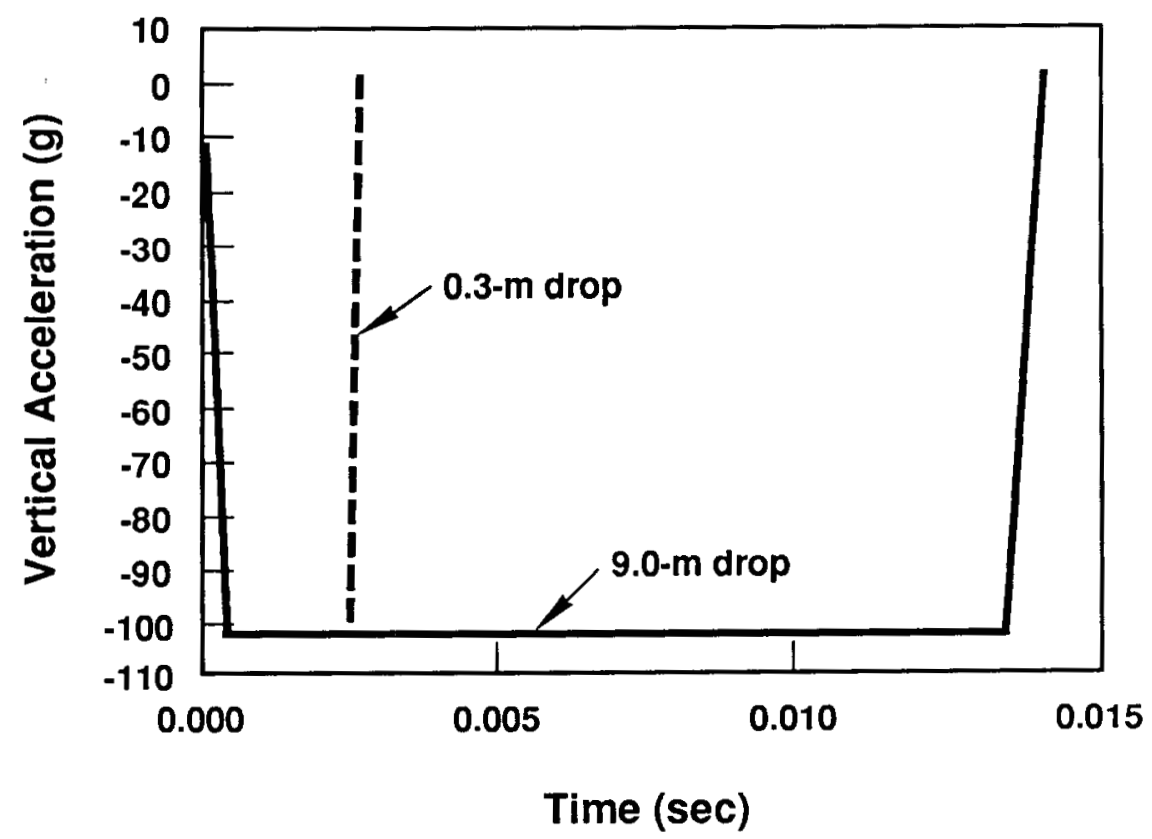

Figure II-38. Vertical Acceleration Histories for the 0.3- and 9.0-m Drops $\left(\theta_{0}=90^{\circ}\right)$ 


\section{Lead-Shielded Truck Cask (frictionless) Impact Angle $\mathbf{=} \mathbf{3 0} .0$ degrees}

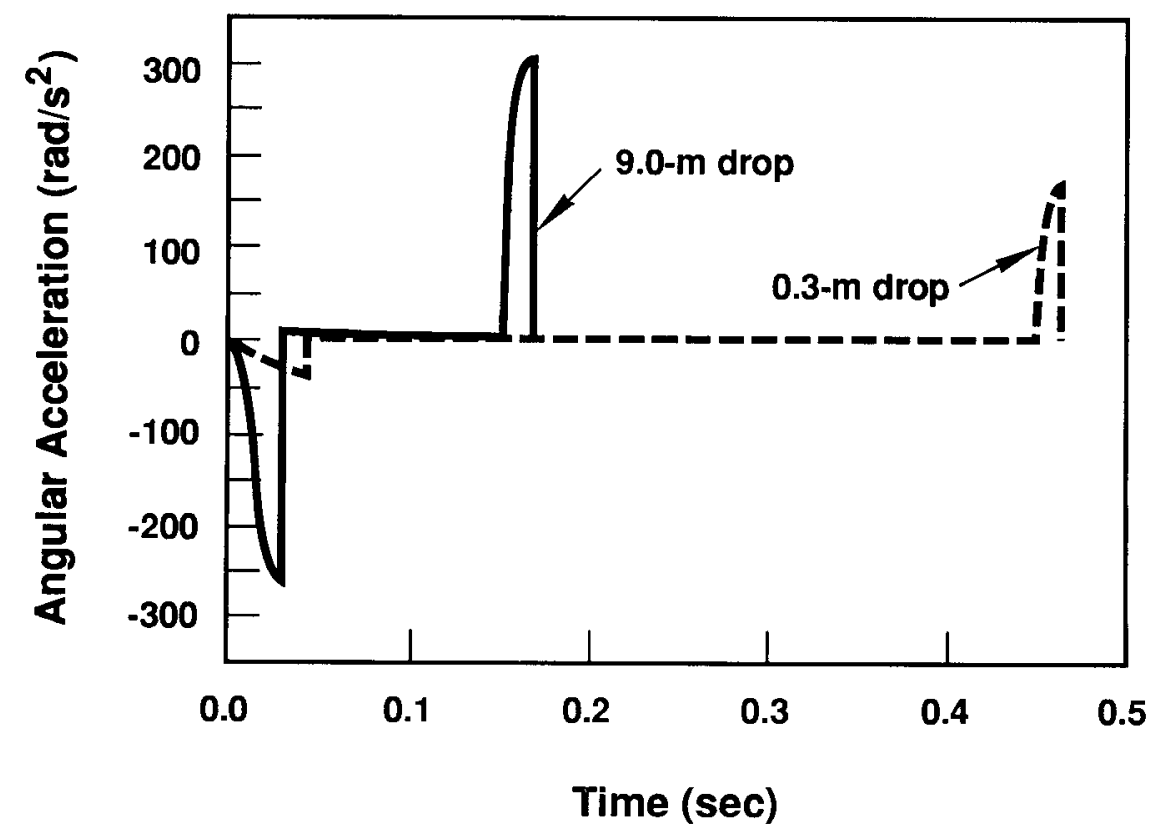

Figure II-39. Angular Acceleration Histories for the 0.3- and 9.0-m Drops $\left(\theta_{0}=30^{\circ}\right)$

\section{Lead-Shielded Truck Cask (frictionless) Impact Angle $\mathbf{= 6 0 . 0}$ degrees}

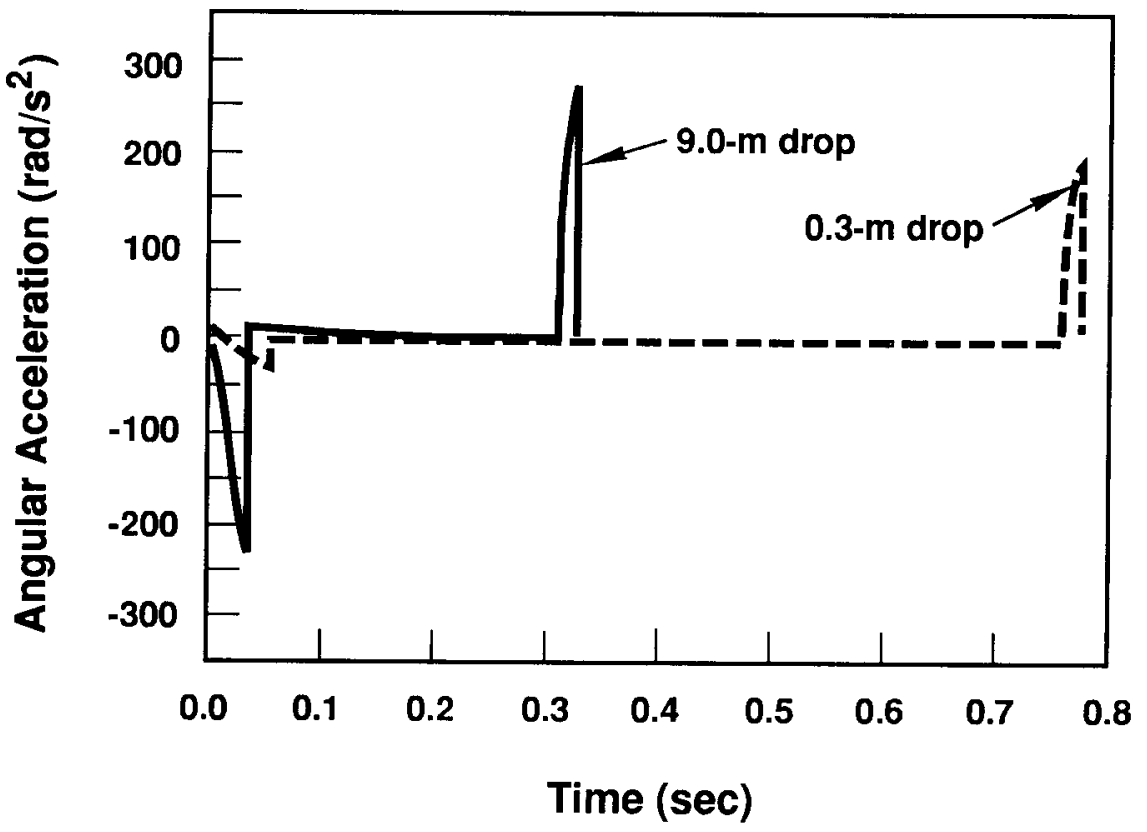

Figure II-40. Angular Acceleration Histories for the 0.3- and 9.0-m Drops $\left(\theta_{0}=60^{\circ}\right)$ 
Typical generic cask accelerations determined for the end and side drops agree excellently with accelerations computed using closed-form methods presented in Section II.3.1. Typical histories for oblique-angle impact orientations consist of two acceleration humps. The first hump corresponds to the cask acceleration as the limiter at the initial impact end deforms. The second hump in the plotted results is the predicted accelerations as the second limiter deforms during the slapdown phase. The flat portion between the two humps corresponds to the pinned phase of the calculations during which the accelerations are small.

Acceleration histories can be characterized by the accelerations' peak magnitude and time duration during initial and slapdown impact phases. Figure II-41 shows the peak vertical accelerations as a function of impact angle for the lead-shielded truck cask impacting a frictionless surface. Maximum initial and slapdown accelerations are plotted for the 0.3-m normal and $9.0-\mathrm{m}$ accident conditions. Envelopes of the combined initial impact and slapdown acceleration levels are also shown.

\section{Lead-Shielded Truck Cask (frictionless)}

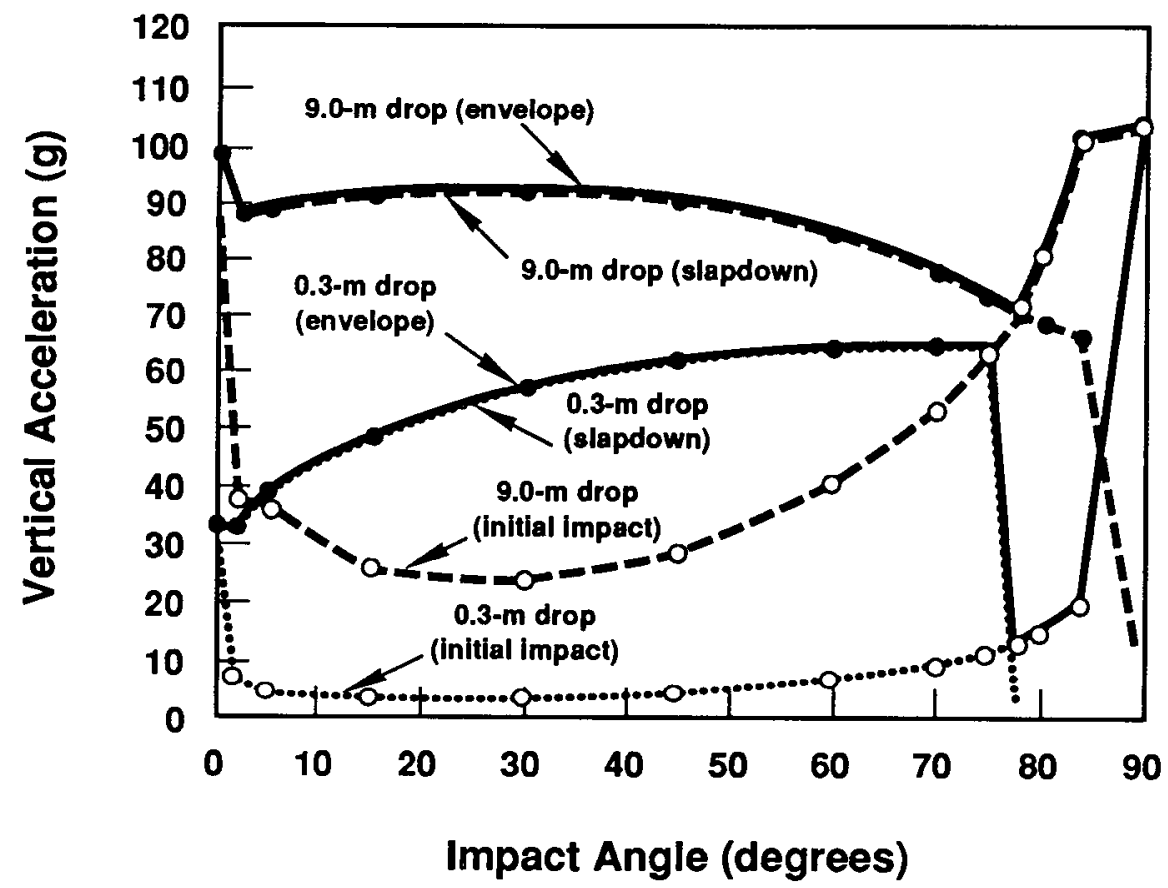

Figure II-41. Peak Accelerations for the 0.3- and 9.0-m Drops

Note that for the $90^{\circ}$ end-impact orientations, cask accelerations are independent of drop height. This observation is consistent with hand calculation Equation (II-1). However, hand calculations and SLAM both assume that stress on the impact limiter contact surface is always equal to limiter crush strength, resulting in overestimation of accelerations. This is particularly true for the $0.3-\mathrm{m}$ drop event during which the limiters have lower plastic deformations than for a $9.0-\mathrm{m}$ drop. 
For impact angles other than $90^{\circ}$, the $0.3-\mathrm{m}$ drop accelerations are less than the corresponding accelerations for the $9.0-\mathrm{m}$ drop, especially during the initial impact phase. As a result of the assumptions regarding limiter stresses and contact area geometries, predicted $0.3-\mathrm{m}$ drop accelerations are more conservative than for the 9.0-m drop. However, for both drop heights, an upper bound on accelerations is provided.

Figure II-42 shows the acceleration time durations as a function of impact angle for the lead-shielded truck cask impacting a frictionless surface. Initial and slapdown impact durations are plotted for the $0.3-\mathrm{m}$ normal and 9.0-m accident drop conditions. For all angles except those close to end-on impact, the time duration of the initial impact is greater for the $0.3-\mathrm{m}$ drop when compared to the $9.0-\mathrm{m}$ drop. However, slapdown impact durations are similar for both drop heights.

Figures II-43 through II-54 show typical acceleration histories for the $9.0-\mathrm{m}$ end-drop, side-drop, and oblique impact angles of 30 and $60^{\circ}$ for all four cask concepts. Only the vertical component of acceleration is nonzero for the end- and side-on impacts, and no pinned and slapdown phases occur. All three components of acceleration are plotted for 30 and $60^{\circ}$ impact angles for each of the representative casks using both the frictionless $(\mu=0)$ and no $\operatorname{slip}(\mu=\infty)$ conditions. The horizontal $\left(\mathrm{x}_{\mathrm{cm}}\right)$ accelerations are not shown for the frictionless cases because they are zero.

\section{Lead-Shielded Truck Cask (frictionless) Time Duration of Initial Impact Phase}

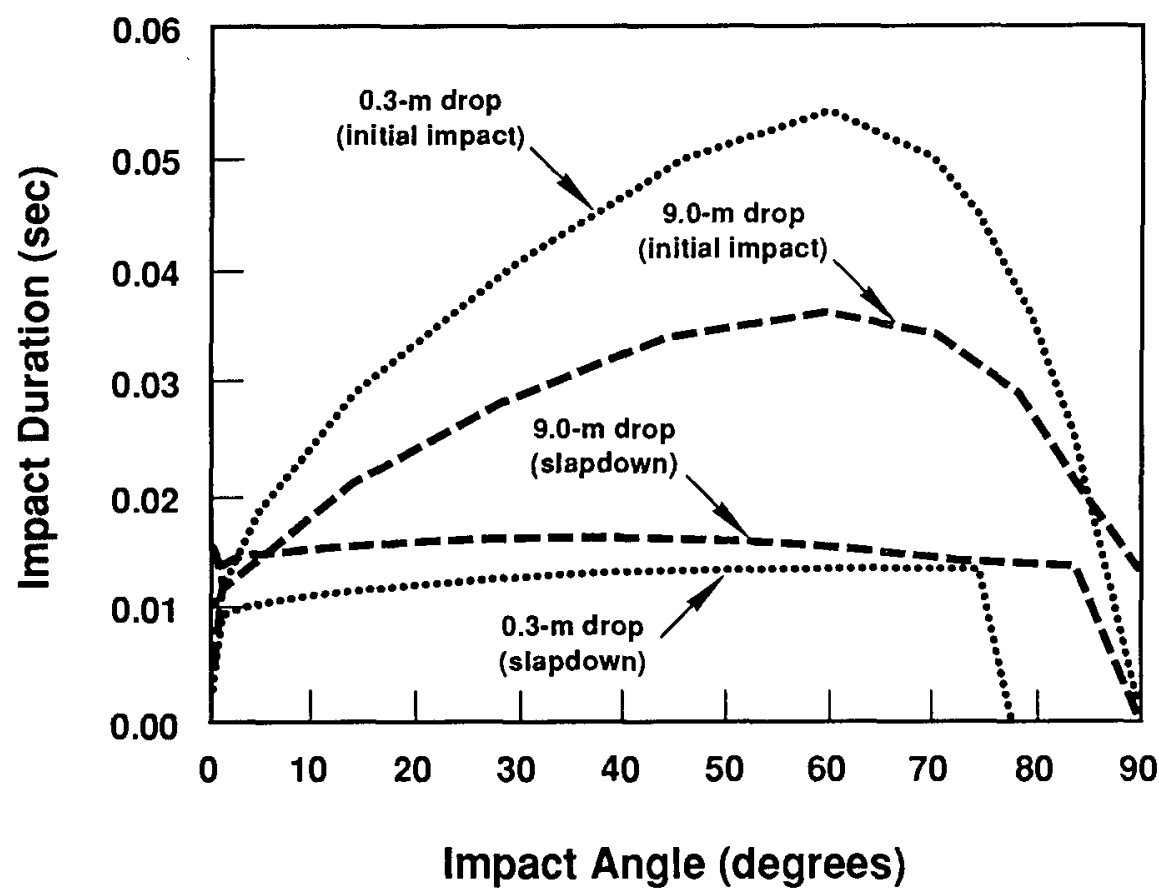

Figure II-42. Impact Durations for the 0.3- and 9.0-m Drops 


\section{Vertical Accelerations \\ Impact Angle $=90$ degrees (end drop)}

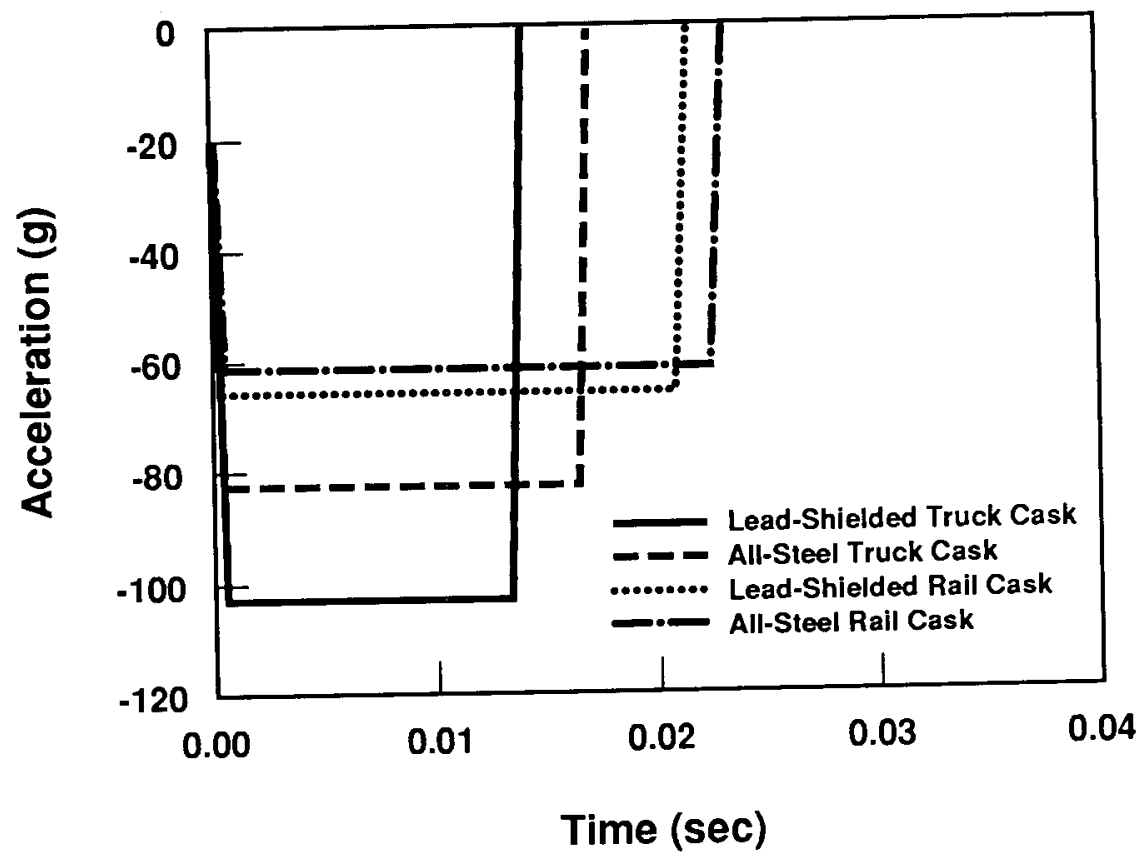

Figure II -43. Vertical Acceleration Histories for the End Drop $\left(\theta=90^{\circ}\right)$

Vertical Accelerations

Impact Angle $=0$ degrees (side drop)

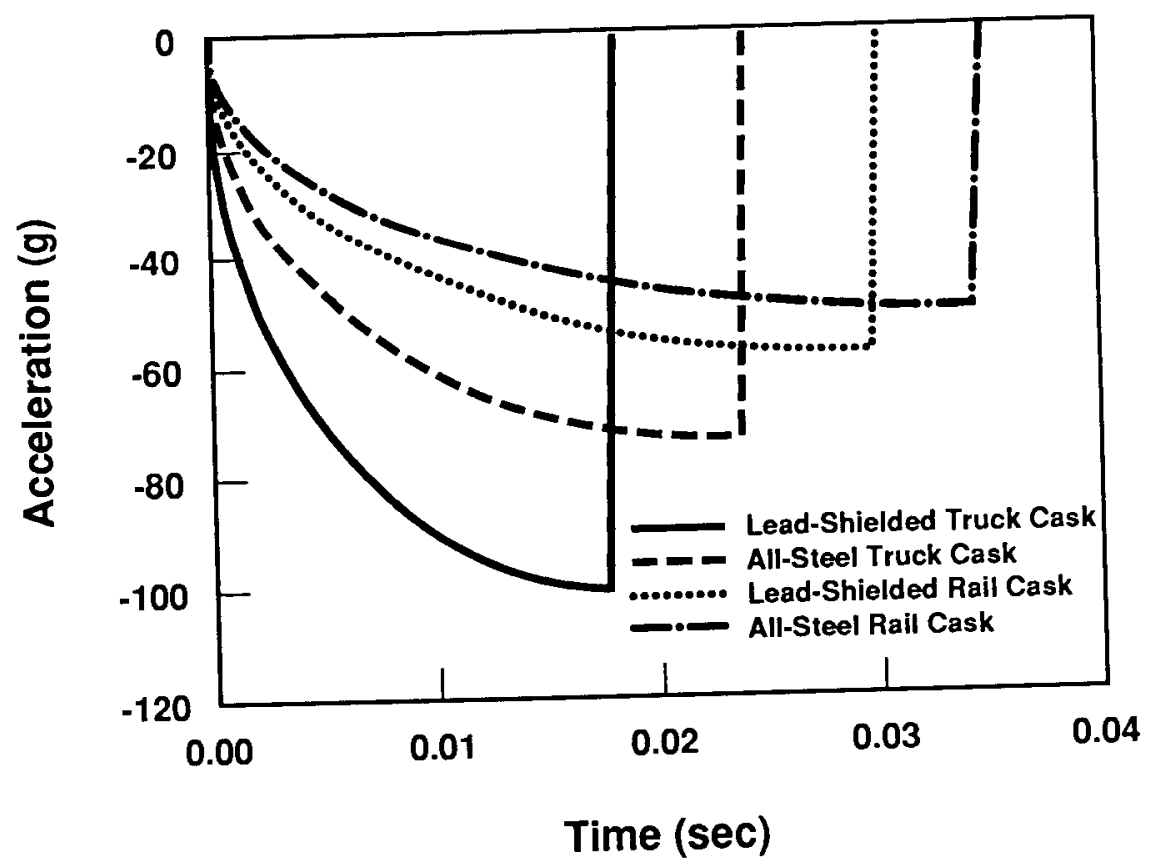

Figure II-44. Vertical Acceleration Histories for the Side Drop $\left(\theta=0^{\circ}\right)$ 


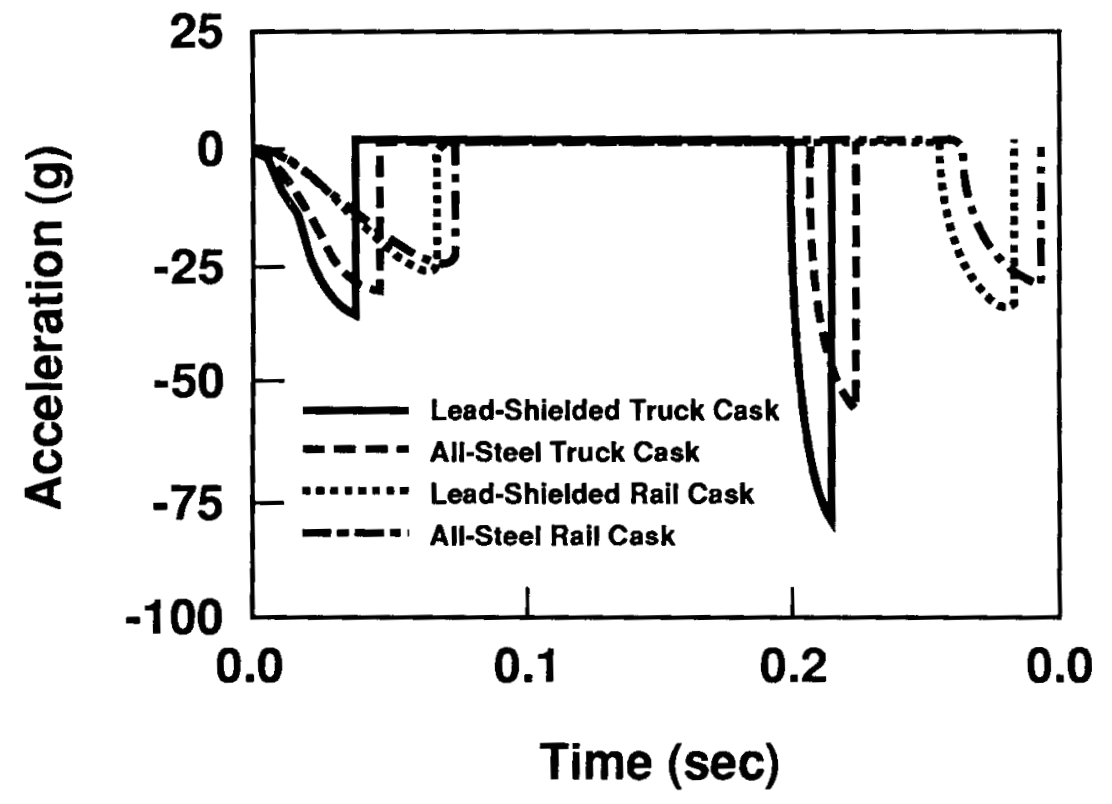

Figure II-45. Vertical Acceleration Histories Where $\theta=30^{\circ}$ (no slip)

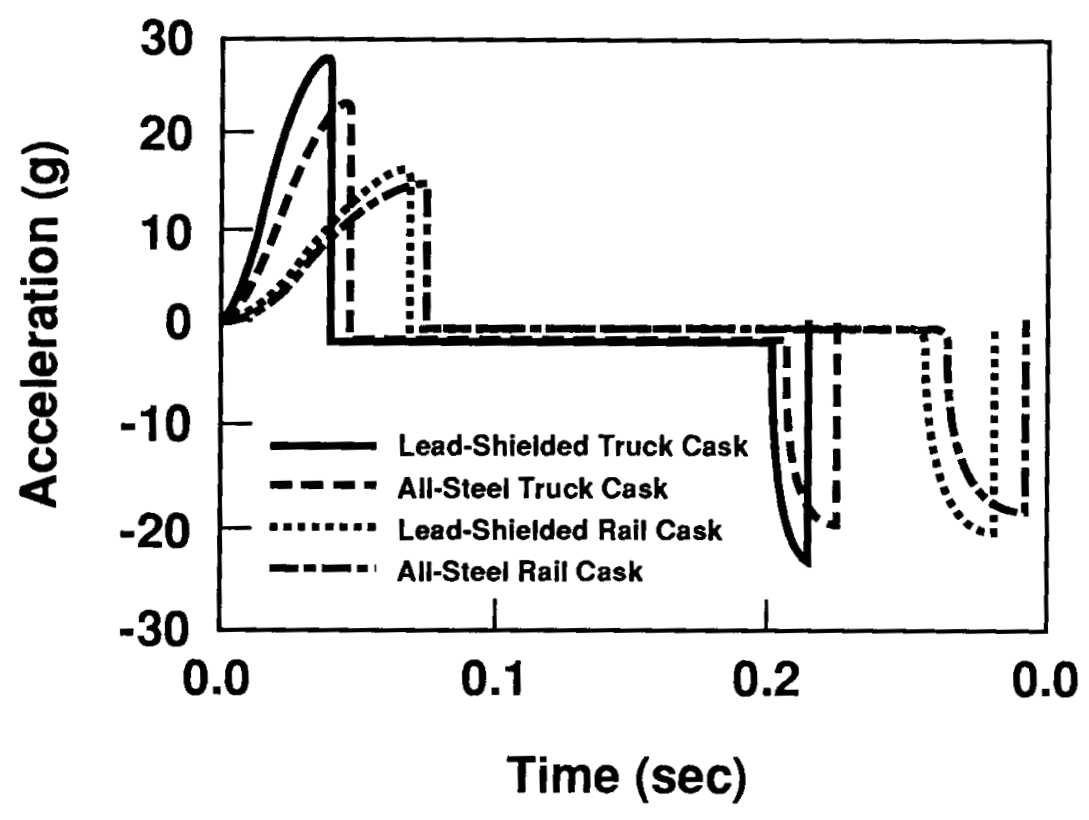

Figure II-46. Horizontal Acceleration Histories Where $\theta=30^{\circ}$ (no slip) 


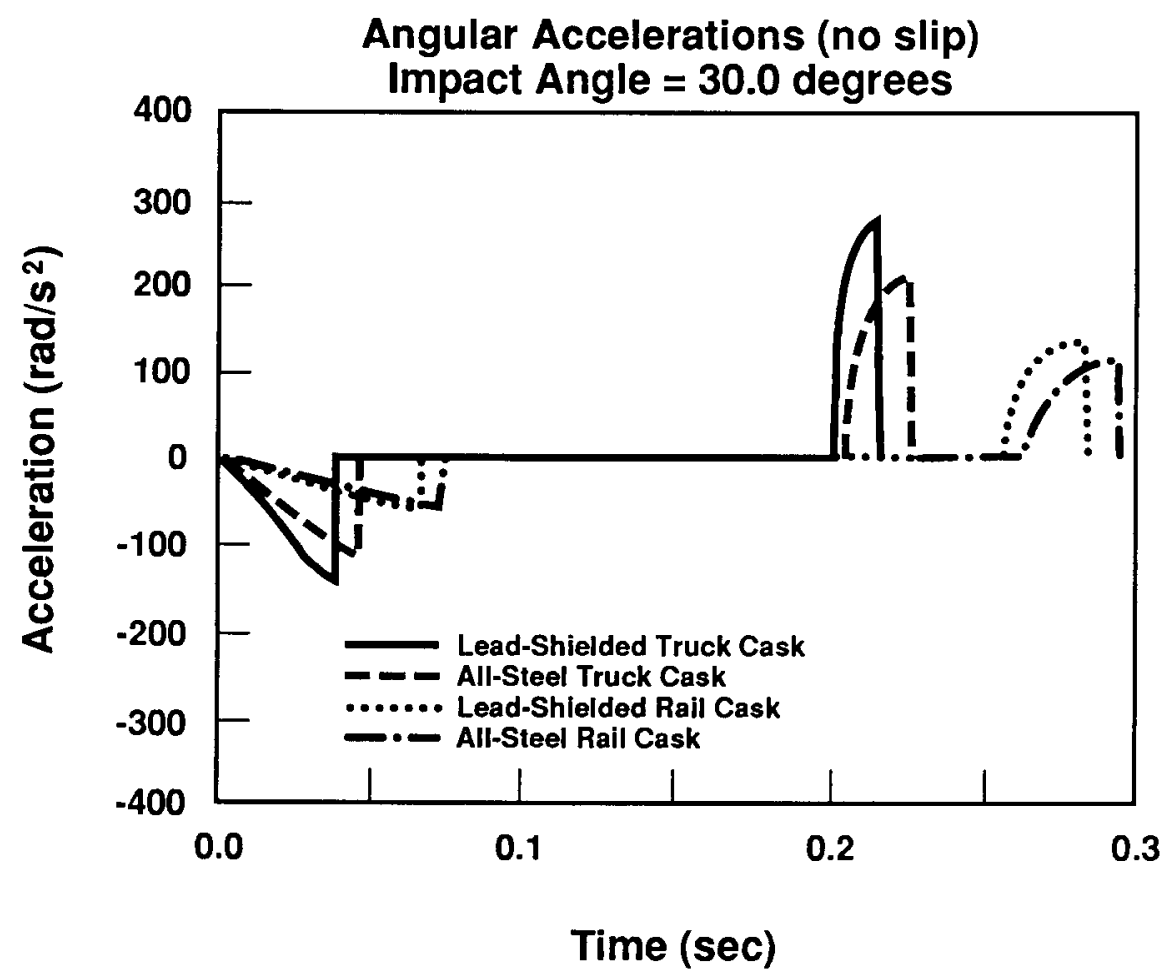

Figure II-47. Angular Acceleration Histories Where $\theta=30^{\circ}$ (no slip)

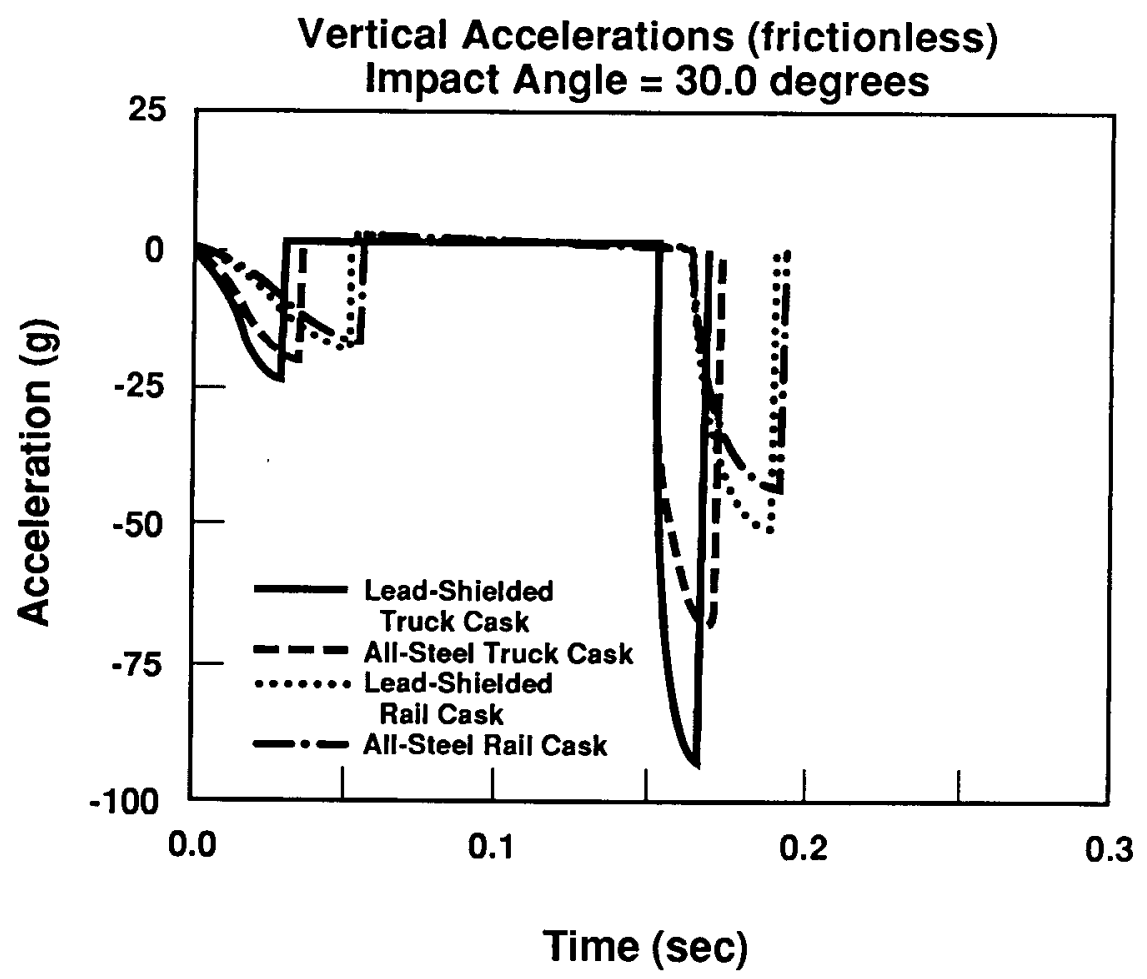

Figure II-48. Vertical Acceleration Histories Where $\theta=30^{\circ}$ (frictionless) 


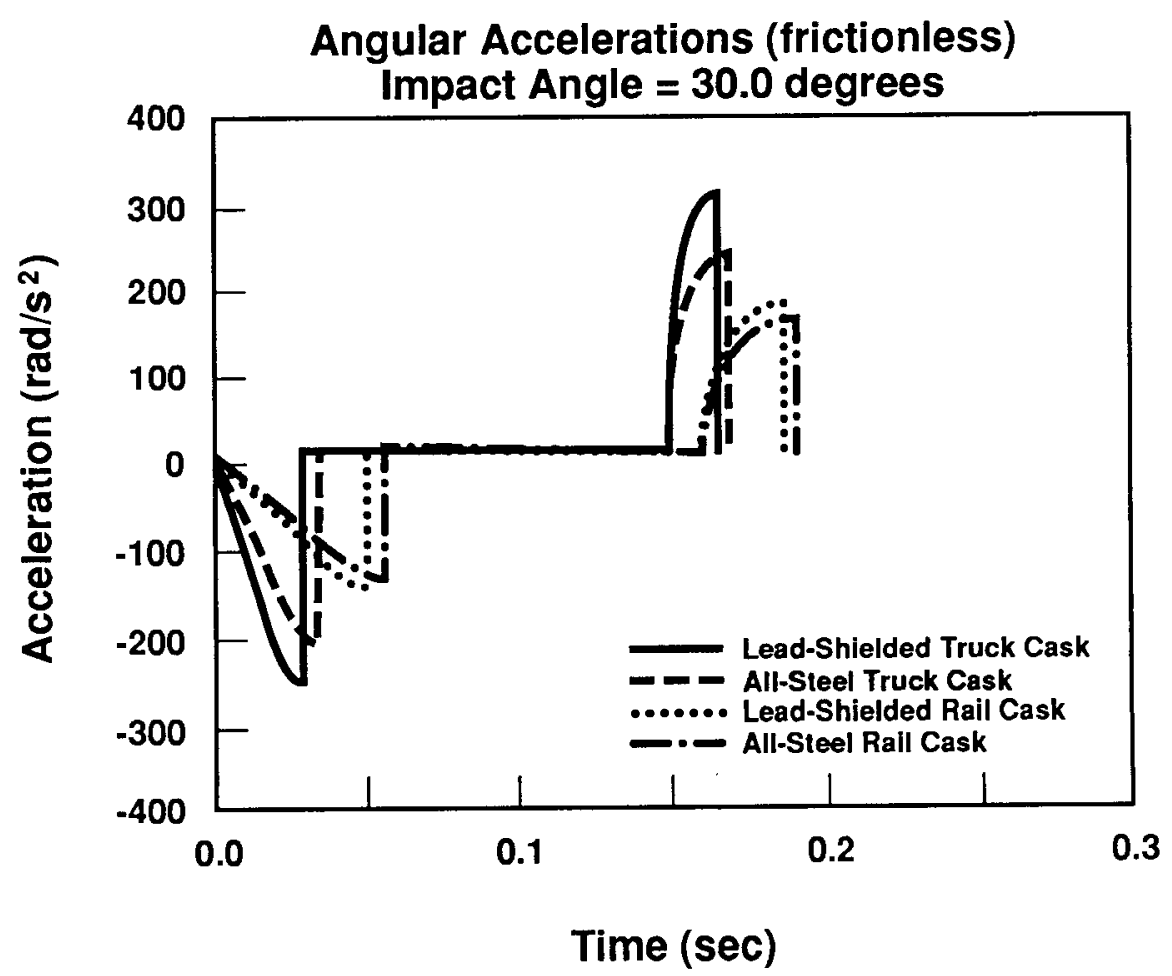

Figure II-49. Angular Acceleration Histories Where $\theta=30^{\circ}$ (frictionless)

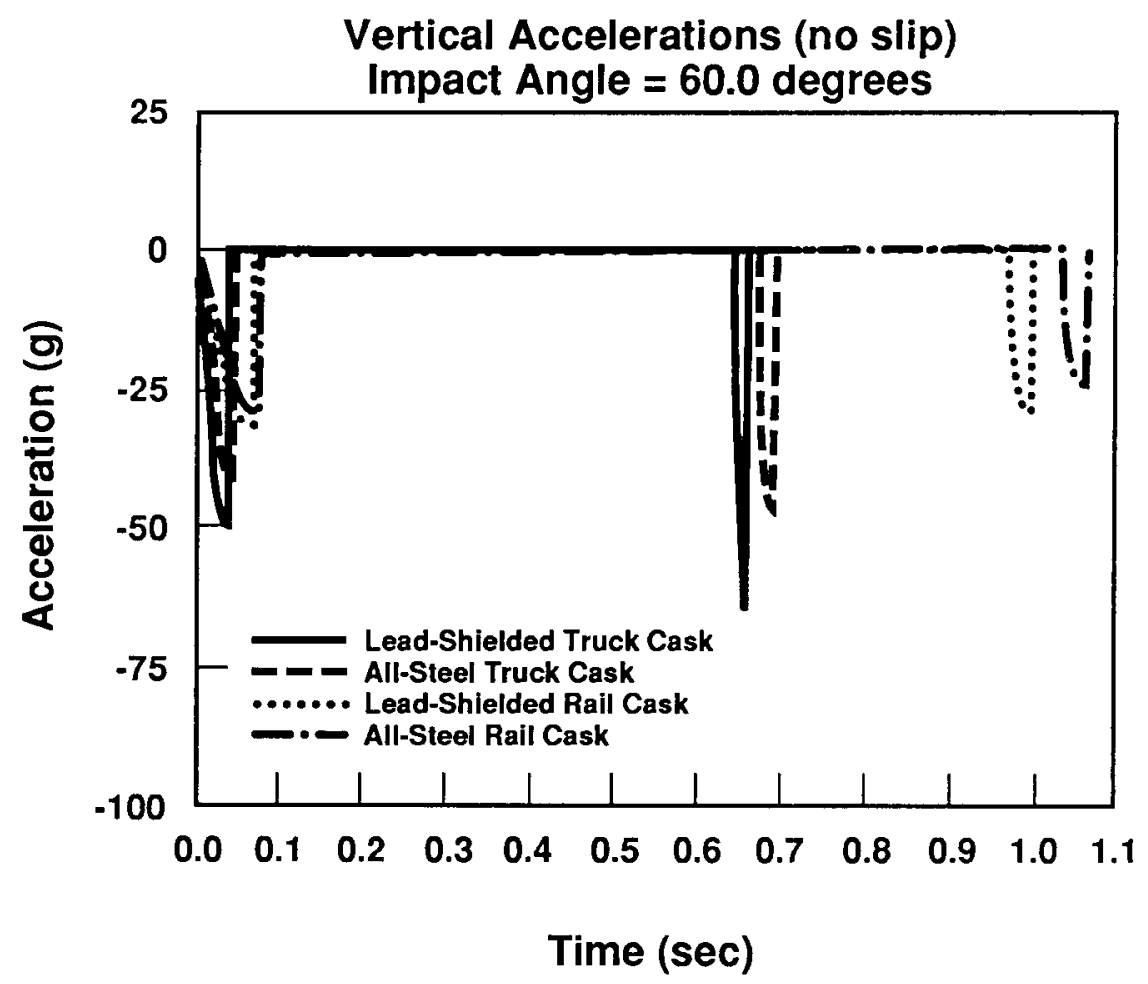

Figure II-50. Vertical Acceleration Histories Where $\theta=60^{\circ}$ (no slip) 


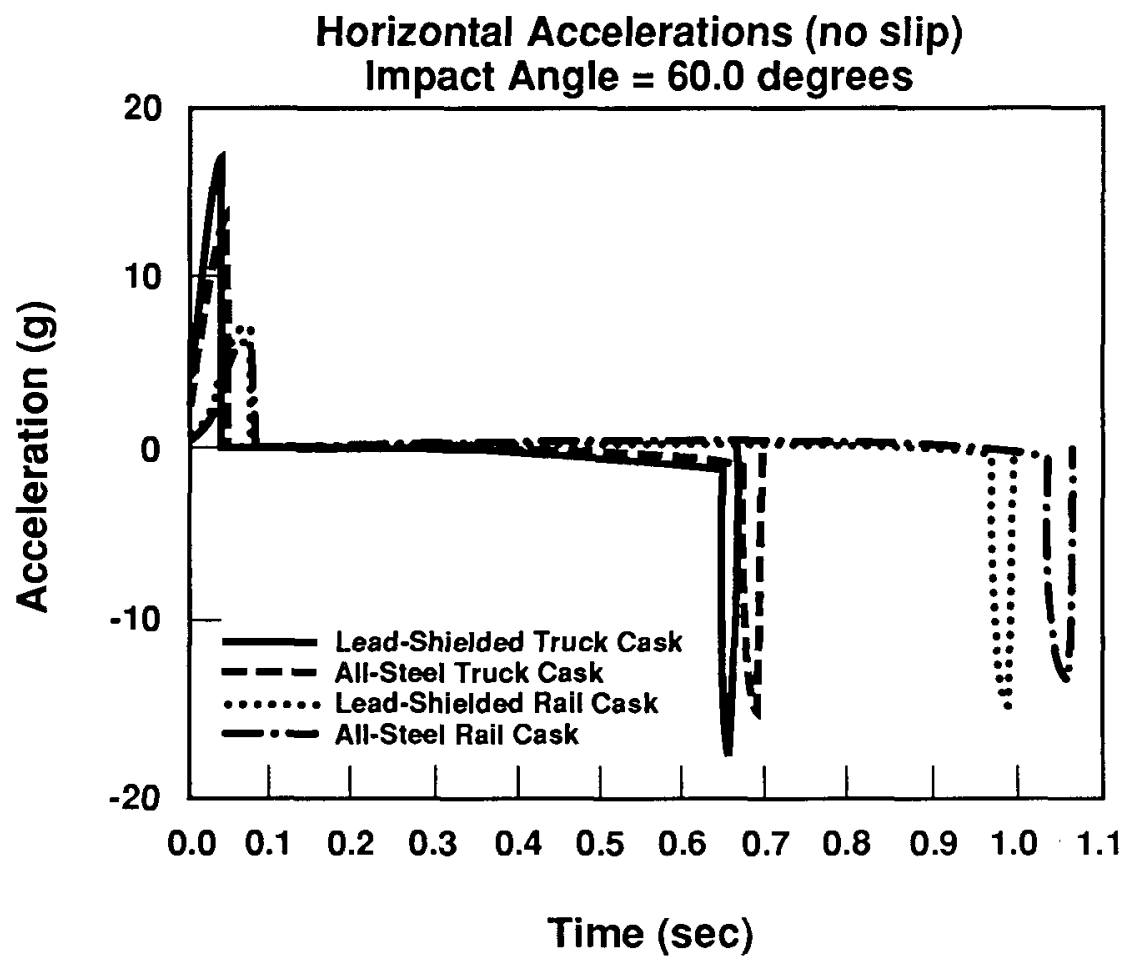

Figure II-51. Horizontal Acceleration Histories Where $\theta=60^{\circ}$ (no slip)

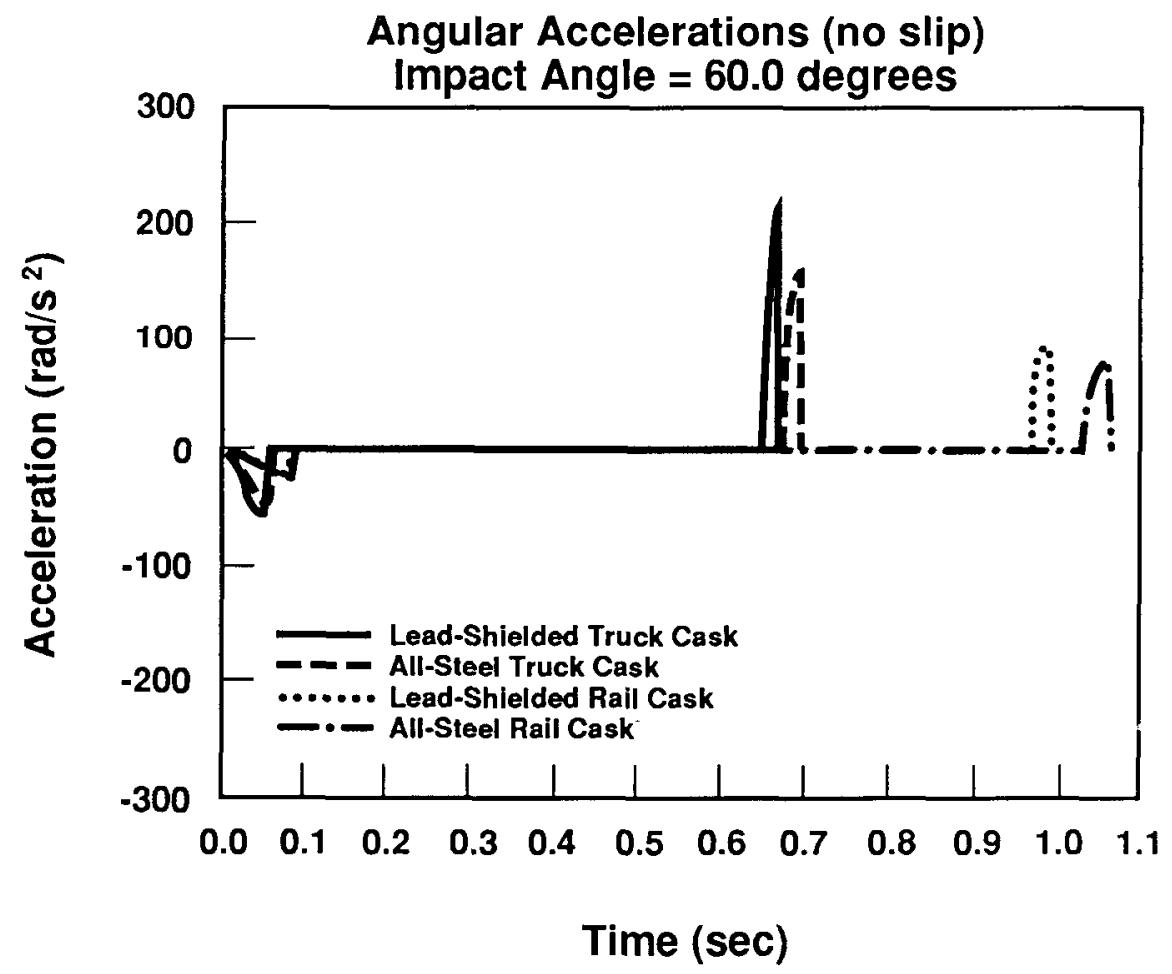

Figure II-52. Angular Acceleration Histories Where $\theta=60^{\circ}$ (no slip) 


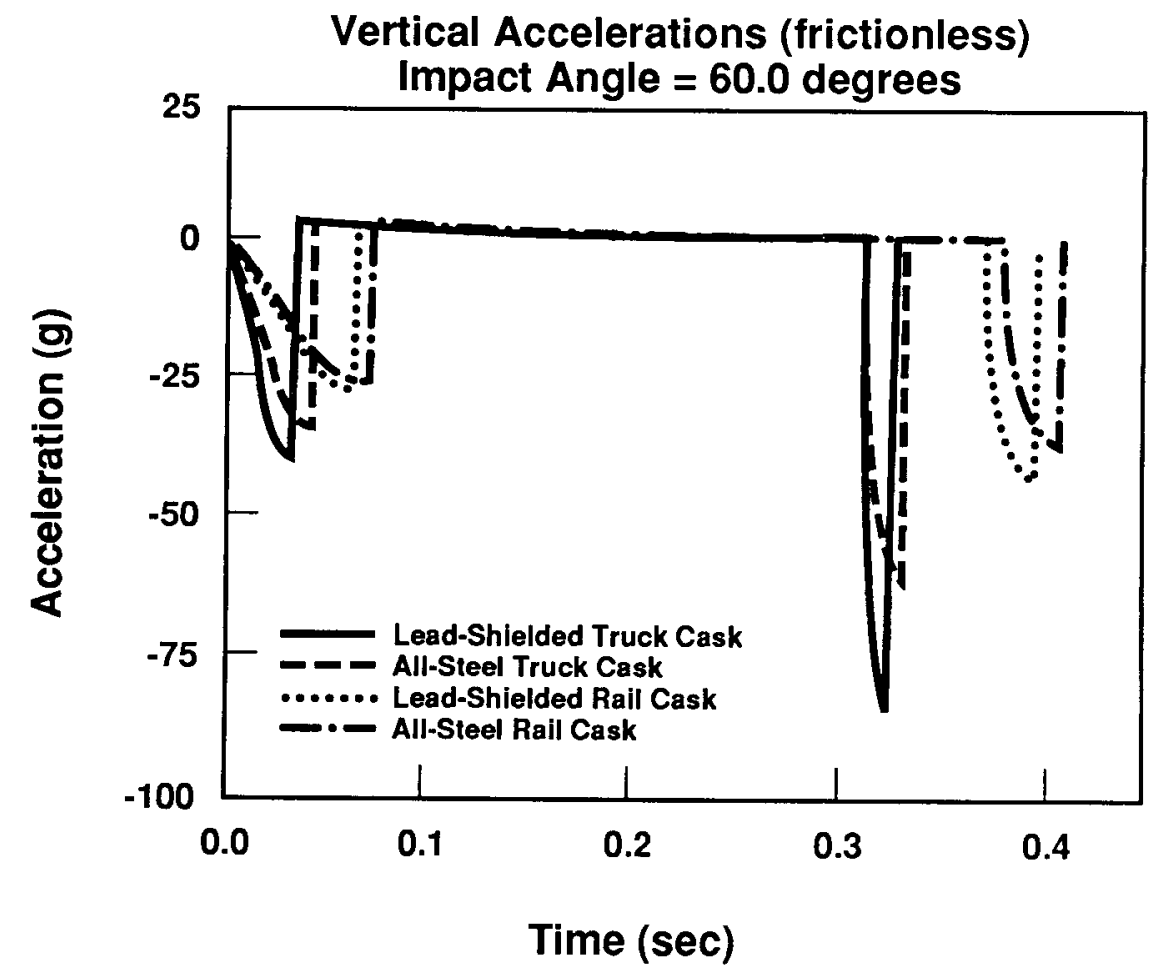

Figure II-53. Vertical Acceleration Histories Where $\theta=60^{\circ}$ (frictionless)

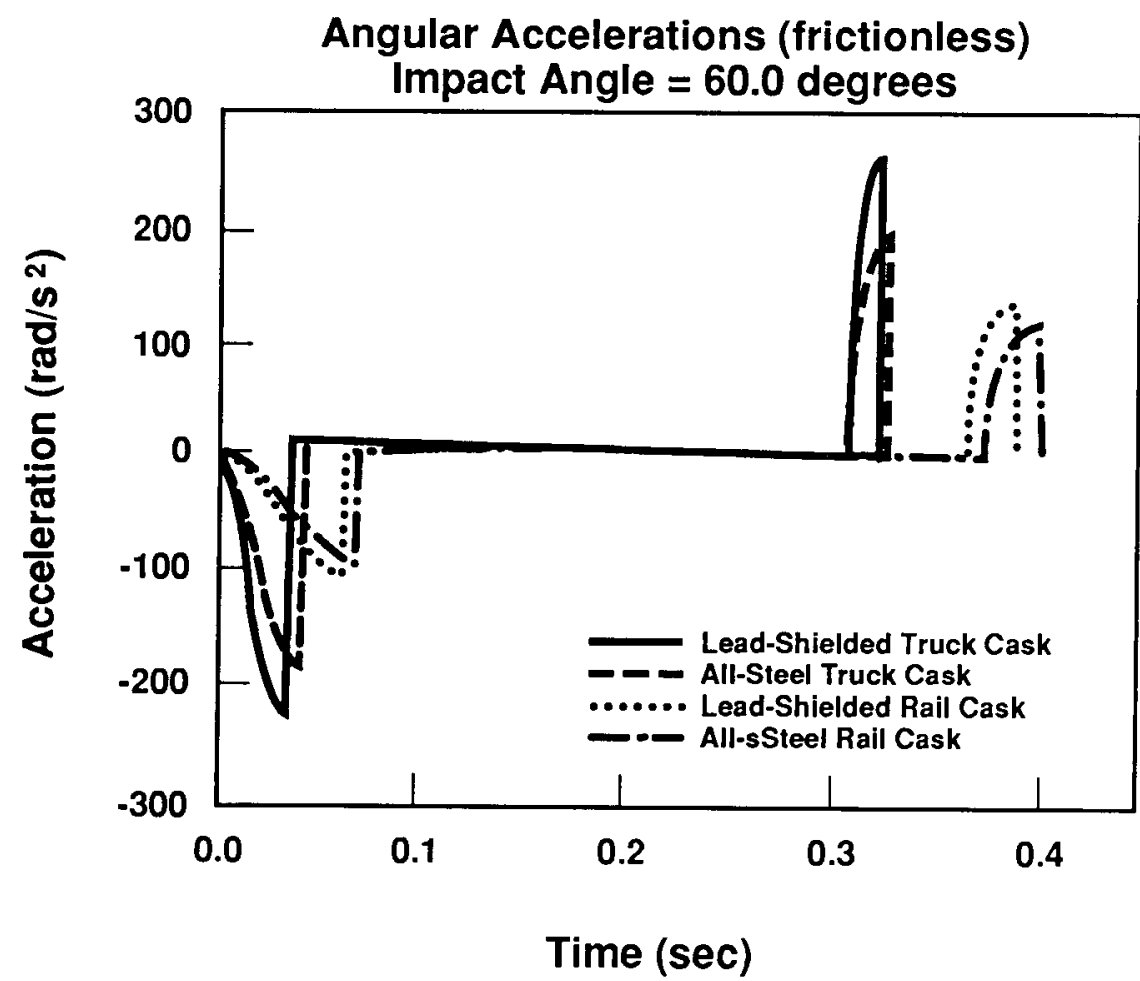

Figure II-54. Angular Acceleration Histories Where $\theta=60^{\circ}$ (frictionless) 
Peak acceleration levels for initial impact, slapdown, and all phases are plotted in Figures II-55 through II-63 as a function of the impact angle. These figures give an overall view of the effect of cask design, friction (or absence of), and slapdown versus initial impact on peak accelerations.

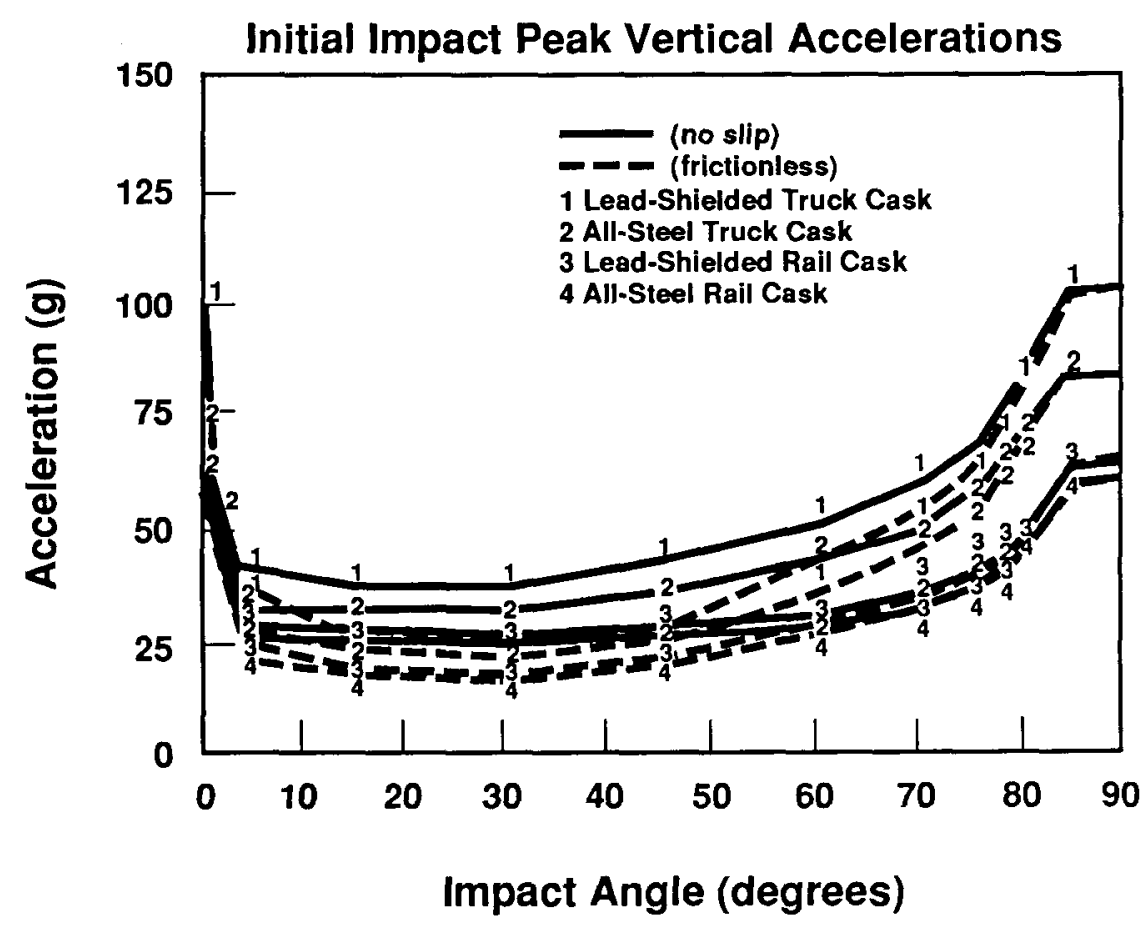

Figure II-55. Peak Vertical Accelerations for Initial Impact

Some general conclusions may be drawn from the figures presented here. Acceleration results clearly indicate that cask peak accelerations may occur during the slapdown phase rather than during initial impact. Lack of impact surface friction increases accelerations for oblique impacts, particularly during slapdown. Peak vertical center-of-mass acceleration levels are $100 \mathrm{~g}$ or less and are representative of actual cask designs that will be reviewed in Section II.3.5. The above observations are made for the generic cask concepts described earlier; note that other viable impact limiter designs could produce accelerations significantly above or below $100 \mathrm{~g}$.

In summary, rigid-body assumptions eliminate undesired high-frequency cask response modes and provide accurate acceleration time histories for spent-fuel response models. The model has been validated against closed form and experimental results, and has been applied to four generic cask designs for various impact angles and impact surface friction characteristics. 


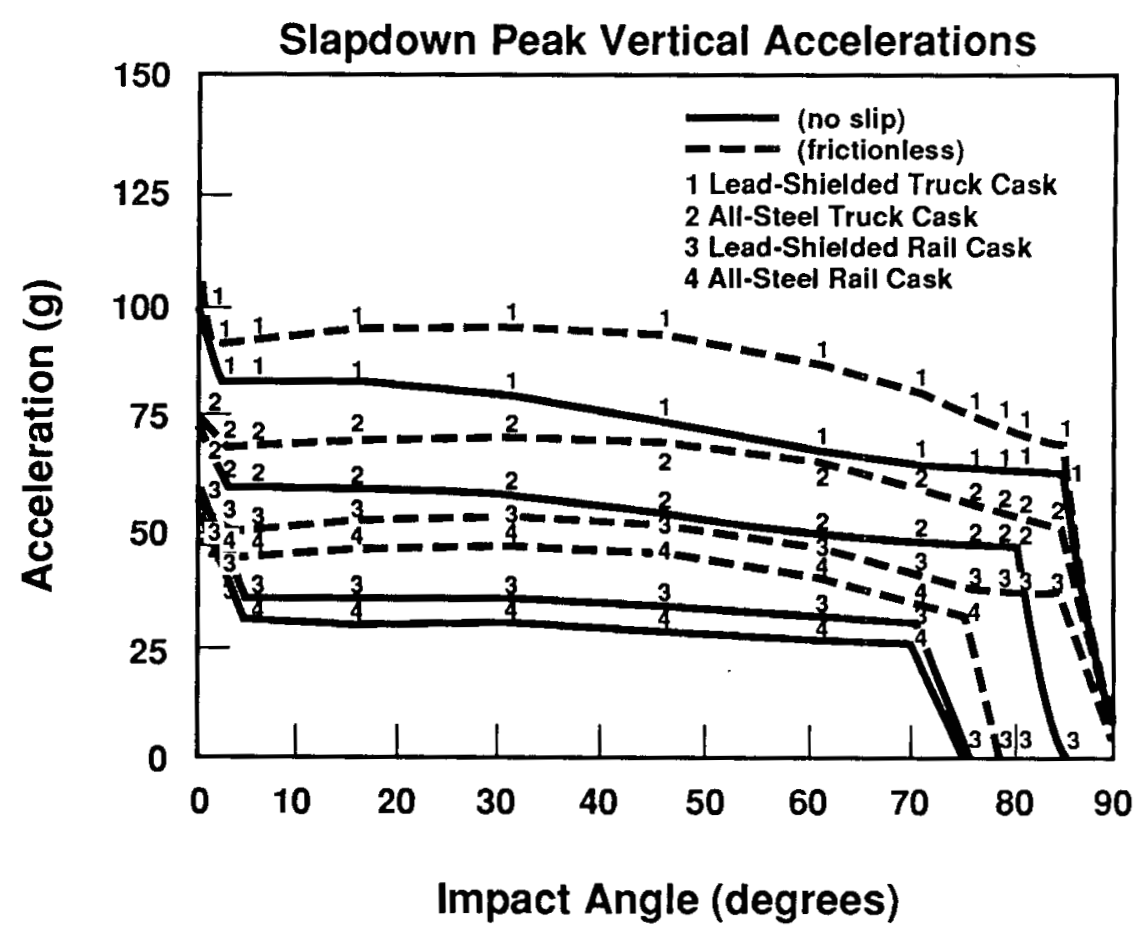

Figure II-56. Peak Vertical Accelerations for Slapdown

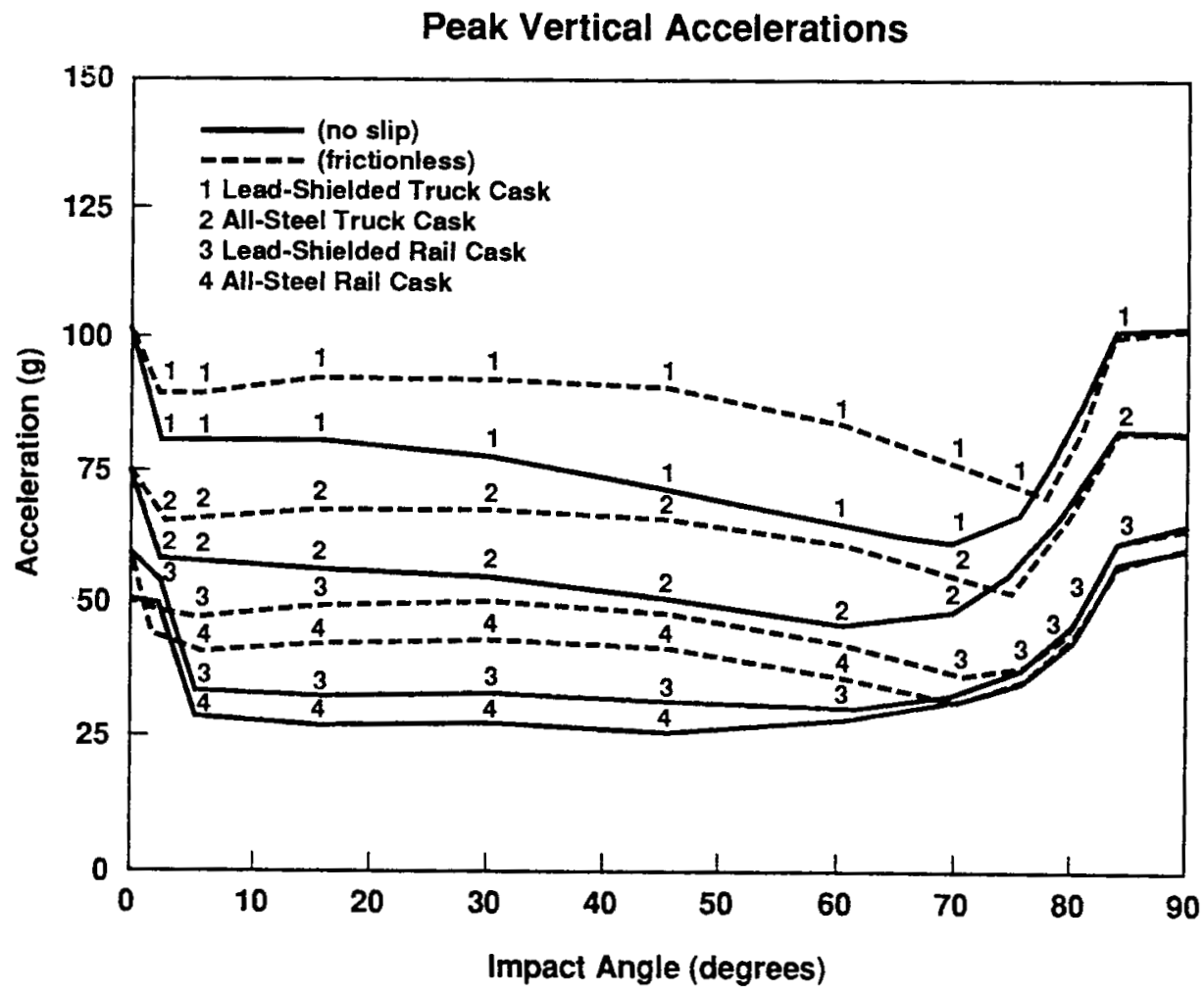

Figure II-57. Peak Vertical Accelerations for A11 Phases 


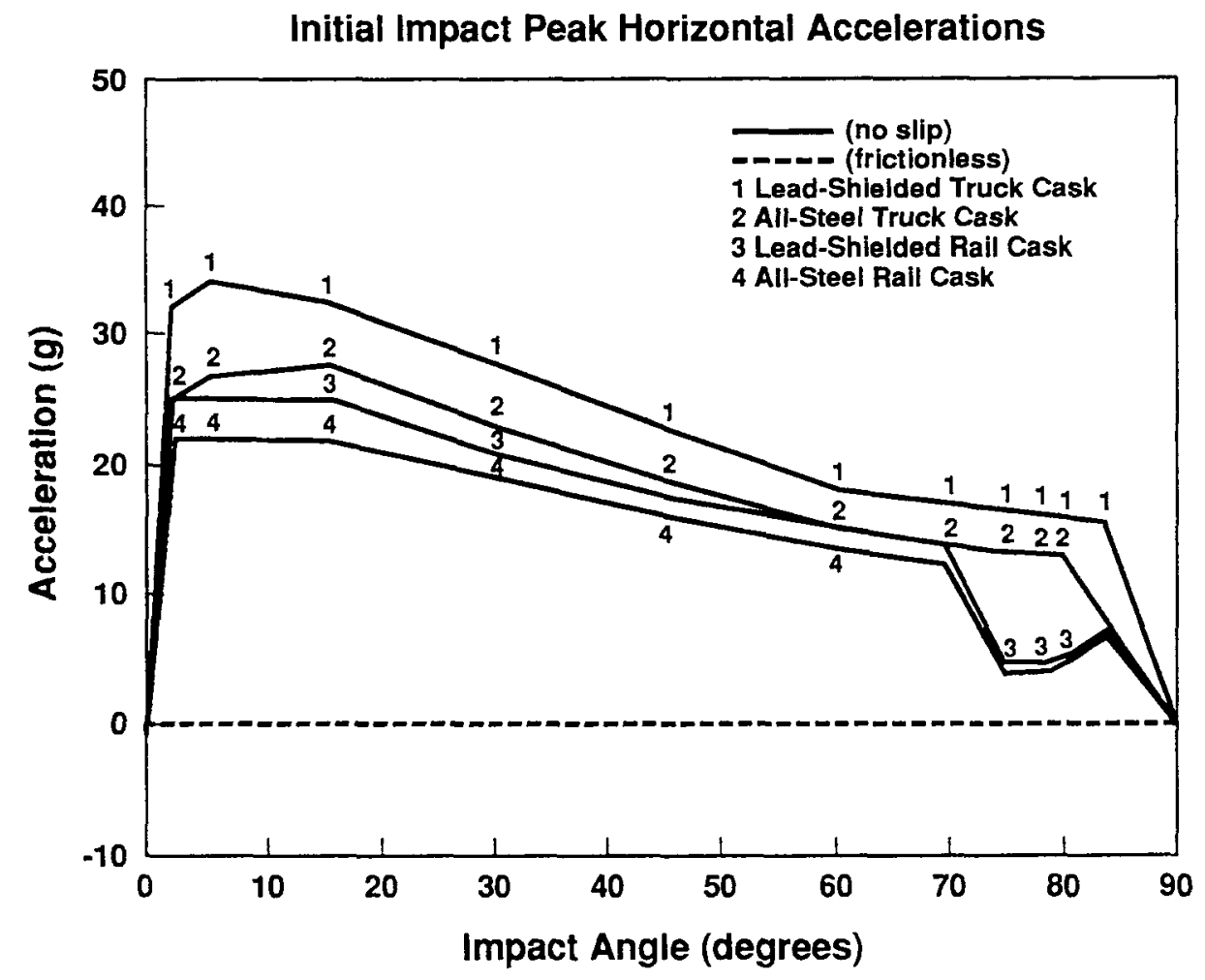

Figure II-58. Peak Horizontal Accelerations for Initial Impact

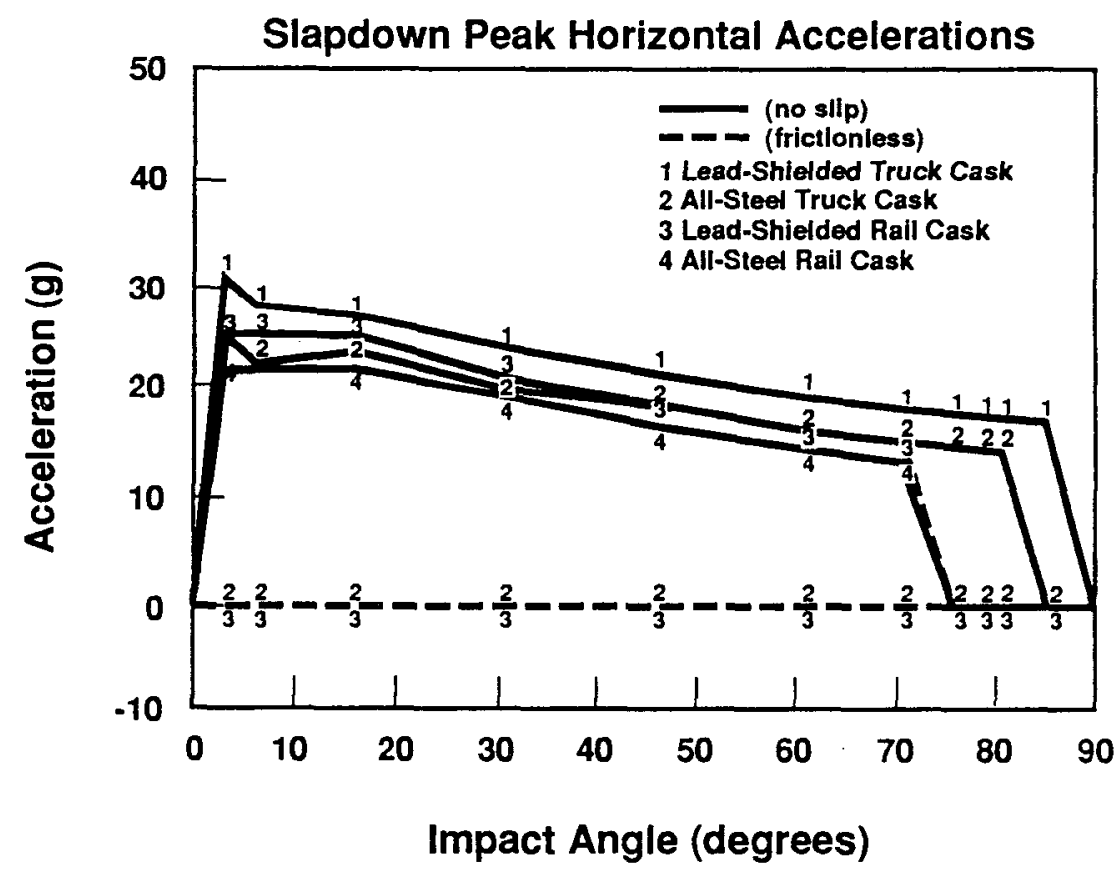

Figure II-59. Peak Horizontal Accelerations for Slapdown 


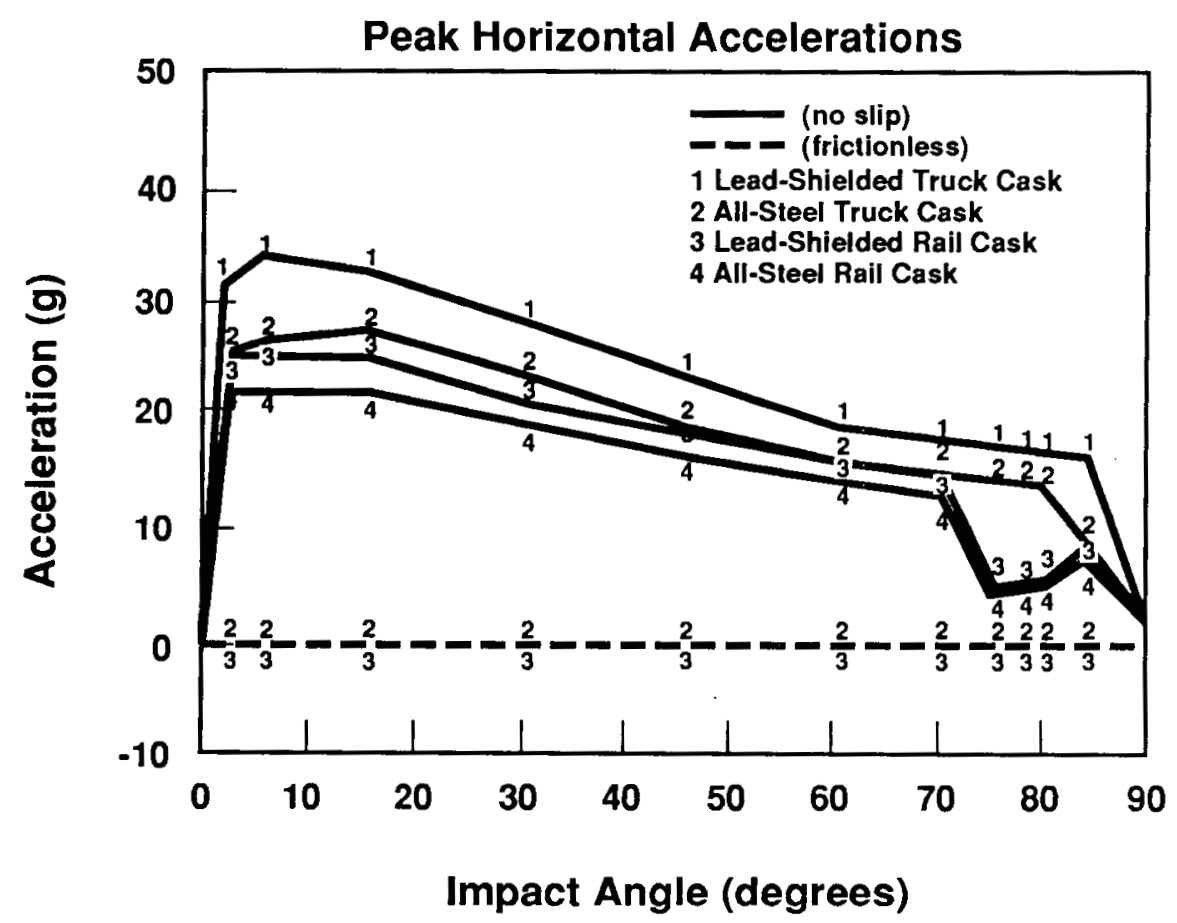

Figure II-60. Peak Horizontal Accelerations for All Phases

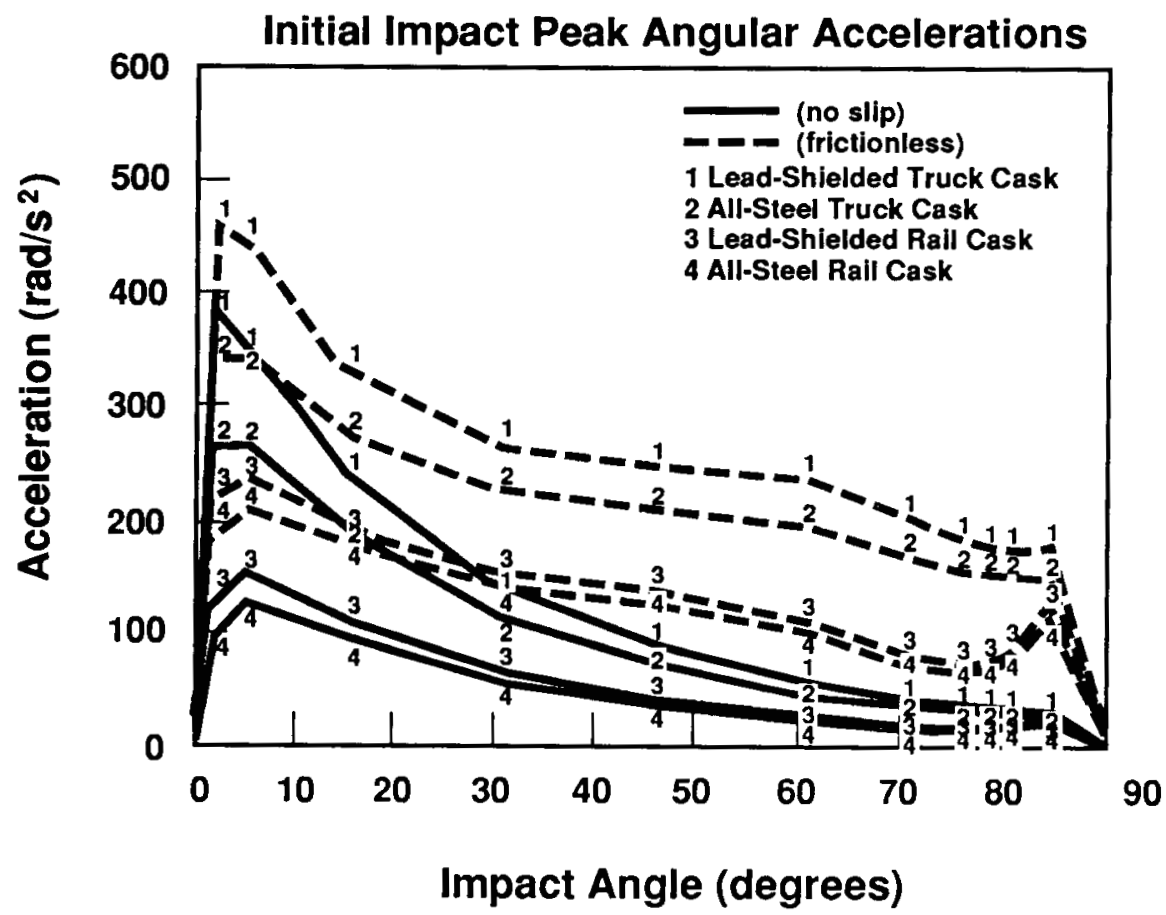

Figure II-61. Peak Angular Accelerations for Initial Impact 


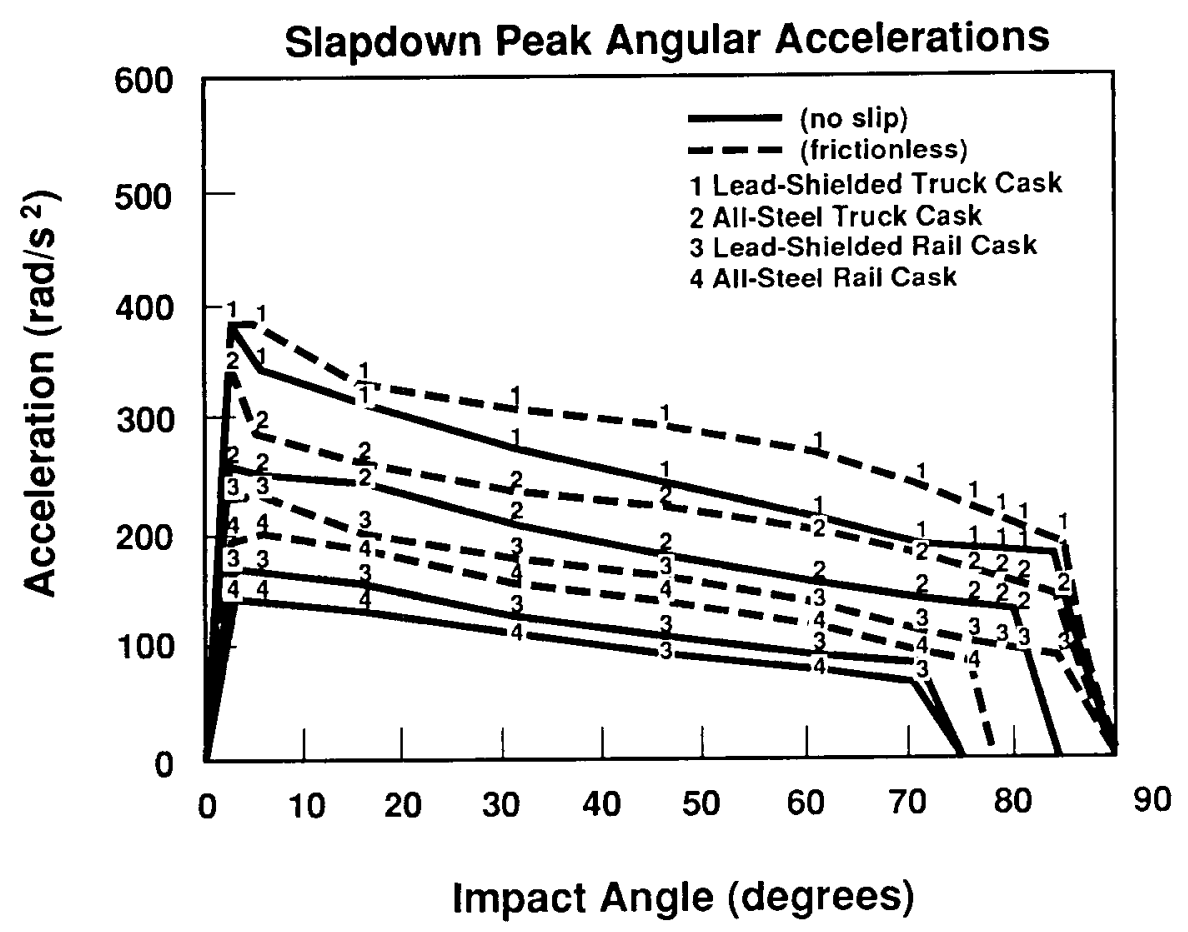

Figure II-62. Peak Angular Accelerations for Slapdown

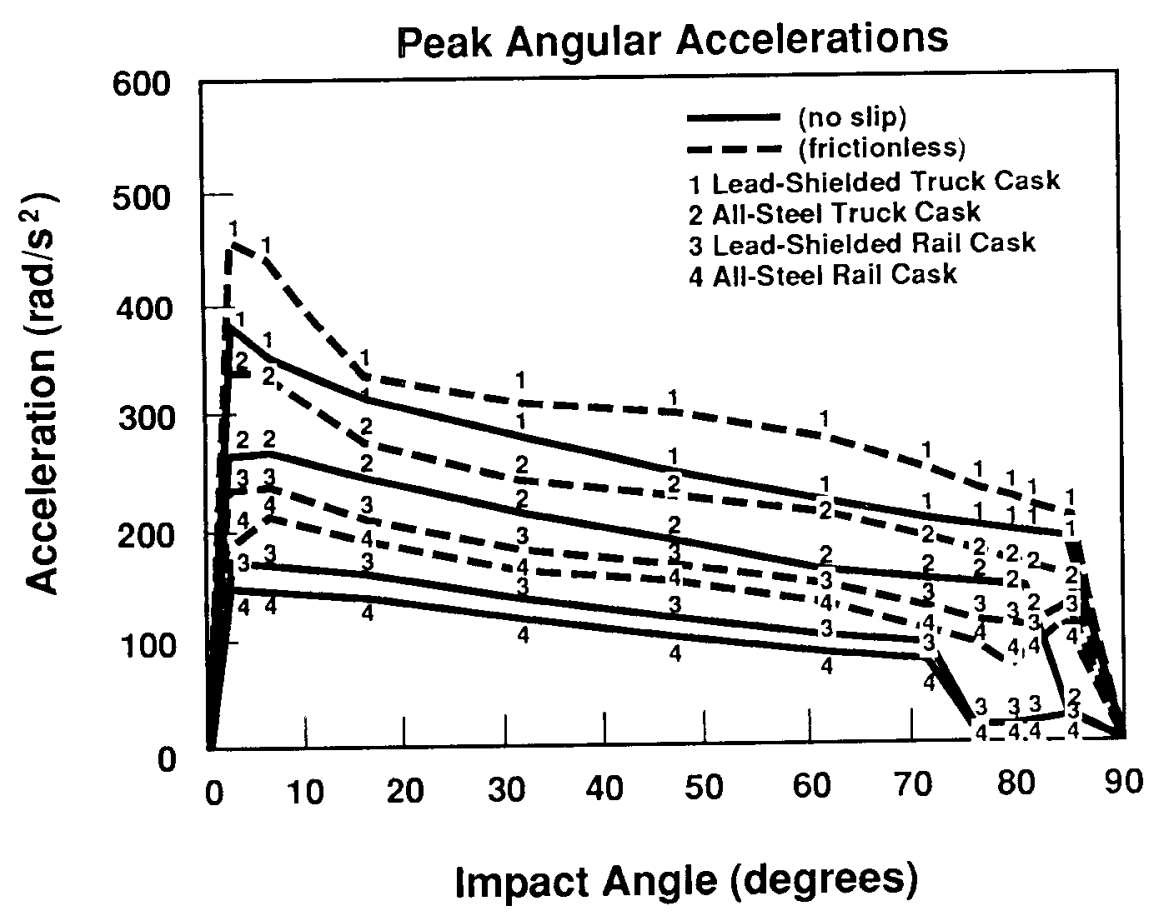

Figure II-63. Peak Angular Accelerations for A11 Phases 


\section{II.3.5 Predicted Decelerations of Currently Certified U.S. Casks}

A survey of currently certified spent-fuel casks as of October 1986 using cask SARPs was conducted. Table II-11 shows acceleration loading predicted for 14 of the casks for the regulatory 9.0 -m drop test. Unless otherwise noted, all values in Table II-11 are peak acceleration values.

Table II-11 shows that there is a wide range in predicted or measured acceleration values that are largely dependent on the basic impact limiter type. End-drop peak acceleration values, for instance, range from 29.4 to $375 \mathrm{~g}$, and side drop values vary from 32.1 to $500 \mathrm{~g}$. The very high accelerations ( 375 and $500 \mathrm{~g}$ ) were predicted for a cask (BMI-1) that does not have impact 1 imiters.

The review of the SARPs show that in no case were actual accelerometer measurements reported for the 9.0-m drop of a full-size cask. Results were either calculated using one-dimensional energy-balance-type analyses that measured on scale models, or deduced from static load-deformation tests on limiters. Also, acceleration measurements reported on scale models (TN-8, $8 \mathrm{~L}$, and 9) are peak values for the direction of impact only. No transient deceleration data are available.

Several cask SARPs also present estimates for cask accelerations expected as a result of the 1.0-m free-drop puncture condition. These estimates are summarized in Table II-12. The cask accelerations for the puncture condition were less than those predicted for the $9.0-\mathrm{m}$ free drop.

\section{II.3.6 Summary Remarks}

In summary, the following remarks can be made regarding the analytical methods and results pertaining to spent-fuel acceleration loadings resulting from the regulatory 9.0-m drop accident condition. Some particular characteristics of the four analysis methods presented herein are summarized in Table II-13.

Three analysis methods were described in the previous sections. The simple analytical hand calculations described in Section II.3.1 give quick estimates for accelerations in end-on, side-on, and CMC impacts; however, this method is limited to these three orientations only. The lumped parameter modeling technique described in Section II.3.2 provides detailed acceleration time histories; however, this method is also limited to those impact orientations in which no rotation occurs (end-on, side-on, and CMC). The finite element modeling technique described in Section II. 3.3 can be used to analyze casks at any arbitrary impact angle but is rather expensive. The lumped parameter and finite element results give detailed acceleration histories, including the high-frequency content associated with vibrations in deformable casks. Most of the high-frequency content in cask response will be damped out and will not transfer to the fuel assemblies because of the large impedance differences between the cask and fuel rods. Therefore, because the cask vibrational modes do not contribute significantly to fuel rod acceleration loadings, a rigid-body analysis is most appropriate. 
Table II -11

Peak Acceleration Loadings for Certified Casks for the Regulatory 9.0-m-Drop Impact Test (from SARPs)

\begin{tabular}{|c|c|c|c|c|c|c|c|}
\hline \multirow[b]{2}{*}{ Cask } & \multirow{2}{*}{$\begin{array}{c}\text { Primary } \\
\text { Transport } \\
\text { Mode }\end{array}$} & \multirow{2}{*}{$\begin{array}{l}\text { Impact } \\
\text { Limiter } \\
\text { Type } \\
\end{array}$} & \multicolumn{4}{|c|}{$\begin{array}{l}\text { Peak Acceleration } \\
\text { for } 9.0-\mathrm{m} \text { Drop (g) }\end{array}$} & \multirow{2}{*}{$\begin{array}{l}\text { Basis for } \\
\text { Acceleration Levels }\end{array}$} \\
\hline & & & Top & Bottom & Side & Corner & \\
\hline $\begin{array}{l}\text { NAC - } 1 \\
(\text { NFS - 4) }\end{array}$ & $\begin{array}{l}\text { Legal weight } \\
\text { truck }\end{array}$ & $\begin{array}{l}\text { Balsa } \\
\text { wood }\end{array}$ & 44.7 & 76.6 & 96 & 45.5 & $\begin{array}{l}\text { One-dimensional } \\
\text { analysis. }\end{array}$ \\
\hline NLI - 1/2 & $\begin{array}{l}\text { Legal weight } \\
\text { truck }\end{array}$ & $\begin{array}{l}\text { Balsa } \\
\text { wood }\end{array}$ & 39.4 & 39.4 & $32.1-38.6$ & 37.5 & $\begin{array}{l}\text { One-dimensional } \\
\text { analysis. }\end{array}$ \\
\hline $\begin{array}{l}\text { TN-8, TN-8L, } \\
\text { TN-9 }\end{array}$ & $\begin{array}{l}\text { Overweight } \\
\text { truck }\end{array}$ & $\begin{array}{l}\text { Balsa } \\
\text { wood }\end{array}$ & $\begin{array}{l}90 \\
155^{c}\end{array}$ & -- & $\begin{array}{c}155^{a} \\
75-90^{d}\end{array}$ & $80^{b}$ & $\begin{array}{l}\text { Extrapolated from } \\
\text { scale model test. }\end{array}$ \\
\hline NLI - $10 / 24$ & Rail & & 29.4 & 29.4 & 80.75 & $30.95^{\mathrm{e}}$ & $\begin{array}{l}\text { Static load test } \\
\text { of } 1 / 8 \text { limiter models. }\end{array}$ \\
\hline IF -300 & Rail & $\begin{array}{l}\text { Steel } \\
\text { fins }\end{array}$ & 178 & 234 & $70.6-109$ & $\begin{array}{l}56.8^{f} \\
68.7 g\end{array}$ & $\begin{array}{l}\text { Drop height divided } \\
\text { by total crush of fin. }\end{array}$ \\
\hline BMI - 1 & $\begin{array}{l}\text { Not } \\
\text { specified }\end{array}$ & $\begin{array}{l}\text { None } \\
\text { (lead) }\end{array}$ & $375^{h}$ & $375^{h}$ & 500 & 128 & $\begin{array}{l}\text { One-dimensional } \\
\text { energy balance. }\end{array}$ \\
\hline $\mathrm{T}-3$ & Truck & $\begin{array}{l}\text { Poly - } \\
\text { urethane } \\
\text { foam }\end{array}$ & $\begin{array}{l}81.1- \\
87.8\end{array}$ & $\begin{array}{l}81.1- \\
87.8\end{array}$ & 94.5 & 50.2 & $\begin{array}{l}\text { One-dimensional energy } \\
\text { balance. }\end{array}$ \\
\hline NLI - 6502 & Truck & $\begin{array}{l}\text { Steel } \\
\text { end } \\
\text { skirts }\end{array}$ & (i) & (i) & (i) & (i) & $\begin{array}{l}\text { Not measured during } \\
\text { testing. }\end{array}$ \\
\hline
\end{tabular}


Table II-11

Peak Acceleration Loadings for Certified Casks for the Regulatory

9.0-m-Drop Impact Test (from SARPs)

(Concluded)

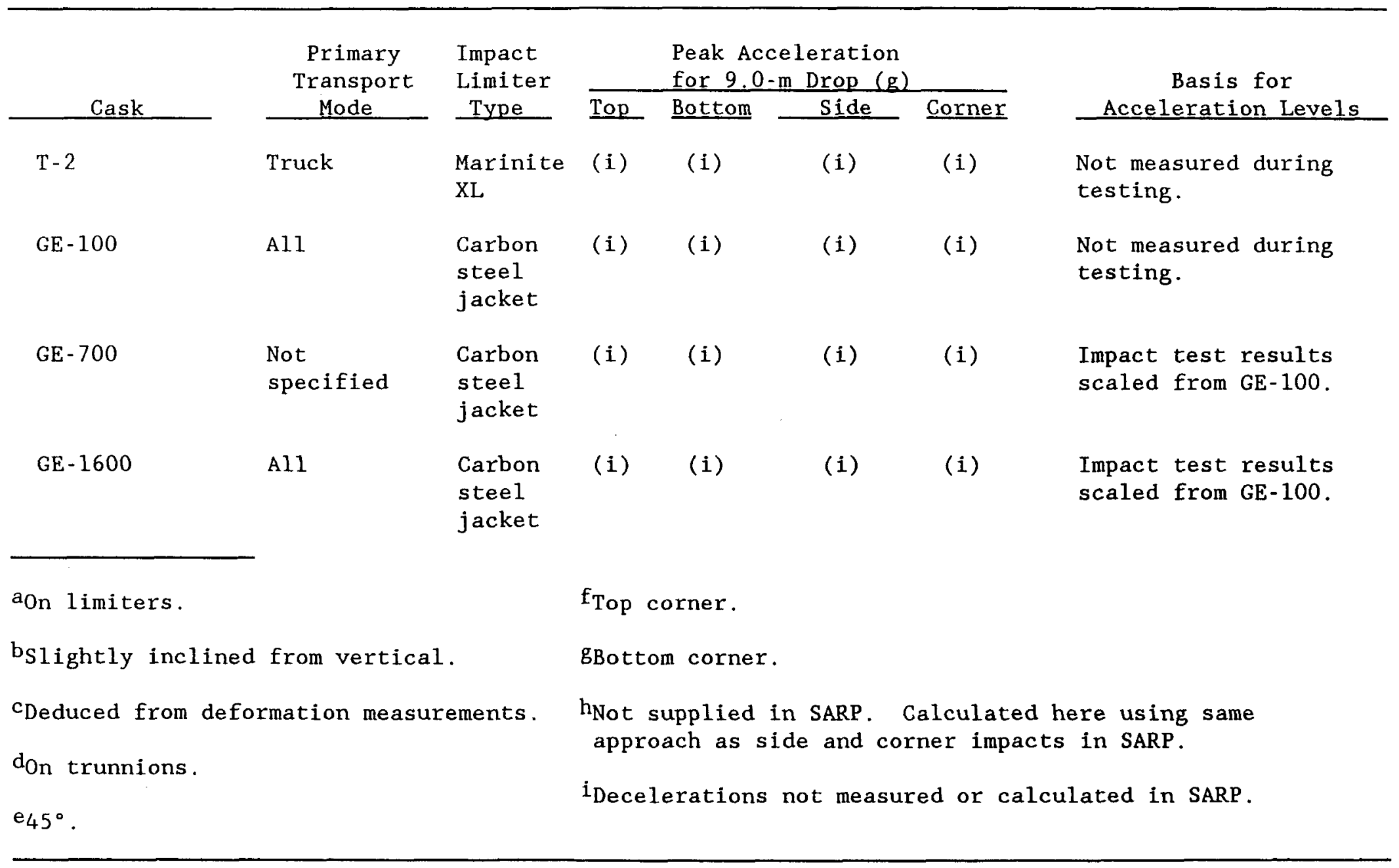


Table II -12

Accelerations for Certified Casks for the Regulatory

1.0-m Free-Drop Puncture Condition

(from SARPs)

\begin{tabular}{|c|c|c|}
\hline Cask & $\begin{array}{l}\text { Acceleration } \\
(\mathrm{g})\end{array}$ & $\begin{array}{c}\text { Method of } \\
\text { Determination }\end{array}$ \\
\hline $\begin{array}{l}\text { NLI }-1 / 2 \\
\mathrm{TN}-8\end{array}$ & $\begin{array}{l}27.6 \\
10-30 \text { end } \\
10 \text { side }\end{array}$ & $\begin{array}{l}\text { Simple beam analysis from } 1 / 2 \\
\text { model data. }\end{array}$ \\
\hline $\begin{array}{l}\text { NLI }-10 / 24 \\
\text { IF }-300\end{array}$ & $\begin{array}{r}7.1 \\
7.2\end{array}$ & Puncture bar failure assumption. \\
\hline T-3 & 33.5 & Puncture bar failure assumption. \\
\hline
\end{tabular}

Table II -13

Qualitative Comparison of Analytical Methods

for Cask Drop Events

\begin{tabular}{|c|c|c|c|}
\hline Method & $\begin{array}{c}\text { Relative } \\
\text { Complexity }\end{array}$ & $\begin{array}{c}\text { Impact } \\
\text { Orientations } \\
\end{array}$ & $\begin{array}{c}\text { Predicts High- } \\
\text { Frequency Cask } \\
\text { Vibration } \\
\text { Response? } \\
\end{array}$ \\
\hline $\begin{array}{l}\text { Hand } \\
\text { calculations }\end{array}$ & Simple & $\begin{array}{l}\text { Side, end, } \\
\text { and CMC drop }\end{array}$ & No \\
\hline $\begin{array}{l}\text { Lumped } \\
\text { parameter } \\
\text { modeling }\end{array}$ & Moderate & End drop only & Yes \\
\hline $\begin{array}{l}\text { Finite } \\
\text { element } \\
\text { analysis }\end{array}$ & Complex & Arbitrary & Yes \\
\hline $\begin{array}{l}\text { Rigid-body } \\
\text { kinematic } \\
\text { model (SLAM) }\end{array}$ & Moderate & Arbitrary & No \\
\hline
\end{tabular}


The rigid-body kinematic model most efficiently determines spent-fuel accelerations contained in transport casks when impacting a rigid target at an arbitrary angle. Several conclusions regarding the rigid-body cask results can be made:

1. The rigid-body kinematic model results are in excellent agreement with the NUPAC 125-B quarter-scale model experimental accelerations and closed-form solutions.

2. Center-of-mass vertical accelerations for the four generic cask concepts are less than $100 \mathrm{~g}$ for all angles of impact, and are within the range reported for existing casks.

3. Accelerations for other cask designs could vary significantly from the $100 \mathrm{~g}$ reported here, as observed for some currently certified casks.

4. The modeling assumptions that were used regarding impact limiter behavior are conservative and provide a realistic upper bound on spent-fuel accelerations. Rigid-body assumptions eliminate undesirable high-frequency response modes and provide accurate acceleration history information used in the spent-fuel response models (Appendix III).

5. Results of generic cask analyses indicate the following:

a. Lighter casks (e.g., generic lead-shielded truck casks) have the highest accelerations.

b. When slapdown occurs, the resulting slapdown accelerations are as large as or greater than the initial impact accelerations. (The $0.3-\mathrm{m}$ normal slapdown events described in the text are not regulatory loads.)

c. End- and side-drop impacts are insensitive to friction effects.

d. Frictionless impact surfaces enhance accelerations for oblique impact angles, particularly during slapdown.

\section{II.4 Shock and Vibration Characterization}

\section{II.4.1 Introduction}

During normal transport of a spent-fuel shipping cask by truck or rail, vehicle shock and vibration occurs. This transient loading condition is transmitted through the vehicle and ultimately is transferred to the truck or rail shipping cask. The rigid-body response resulting from this transient input is needed for fuel response analyses. 
Typical truck- and rail-car-mounted transport configurations are shown in Figure II-64. Input of transient loading during normal transport primarily occurs at the tires for the truck configuration, and at both the wheels and couplers in the rail configuration. The loading is normally partitioned into the two categories mentioned above: shock or vibration. Vibration is continuous excitation caused by, for example, the inevitable surface roughness of varying quality roads for truck transport. Shock is a higher-intensity loading occurring relatively infrequently (for trucks, approximately once per mile) that is due, for instance, to potholes in roads.

As described below, a wealth of testing has been performed over the past several decades to quantify the shock and vibration environment of conventional cargo transport modes such as truck and rail. In much of this work spent-fuel casks have been analyzed.

Figure II-65 shows a spring-mass model for input excitation to a truck trailer body from a prescribed road surface roughness, $X(t)$ [GL86]. The initial shock and vibration input function, $X(t)$, passes into a one-degreeof-freedom damped oscillator representing the tires and an additional onedegree-of-freedom oscillator representing axle spring response. The truck bed, tie-downs, and cask could be represented using spring-mass systems.

The input $X(t)$ may then be significantly modified by these coupled spring-mass systems before reaching the cask. Clearly, the location on the transportation system where response signals (typically accelerometer) are measured is important if proper interpretation of the shock and vibration environment is to be made.

A detailed chronological review of available shock and vibration analyses is presented in this section with emphasis on recent rail and truck experiments using spent-fuel transport casks. This review is briefly summarized and analysis concepts are defined in the following section. Simplified bounding input loading curves are supplied based on the upper bounds of data provided, and shock and vibration results are summarized.

\section{II.4.2 Summary and Discussion of Available Shock/Vibration Data}

In most of the references reviewed, the following quantitative definitions of shock and vibration apply.

1. Vibration is assumed to encompass the lower $99 \%$ of all peaks of the recorded acceleration in each frequency band selected, whereas the upper 18 of the peaks are assumed to be associated with shock.

2. Foley et al. state that the upper distribution may occupy as much as $60 \%$ of the total range of amplitude found in the frequency band [F072a]. Further, this upper distribution appears to be produced by events that are recurrent and discrete in nature, whereas the lower 998 of peaks represent continuous excitation (see Figure II-66). 


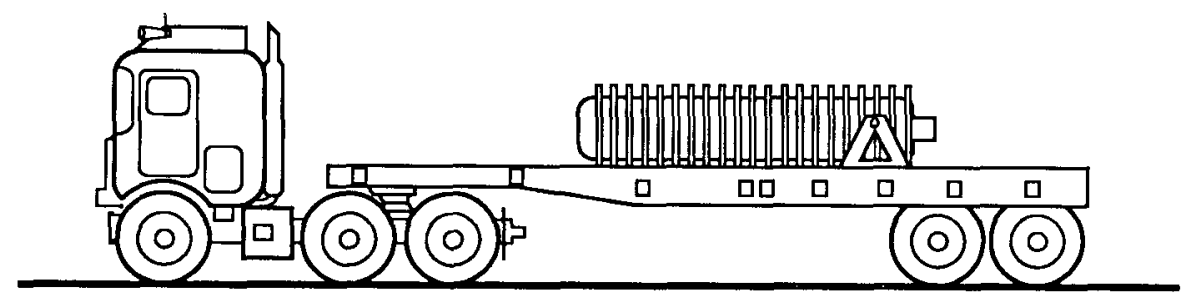

\section{a. Truck Configuration}

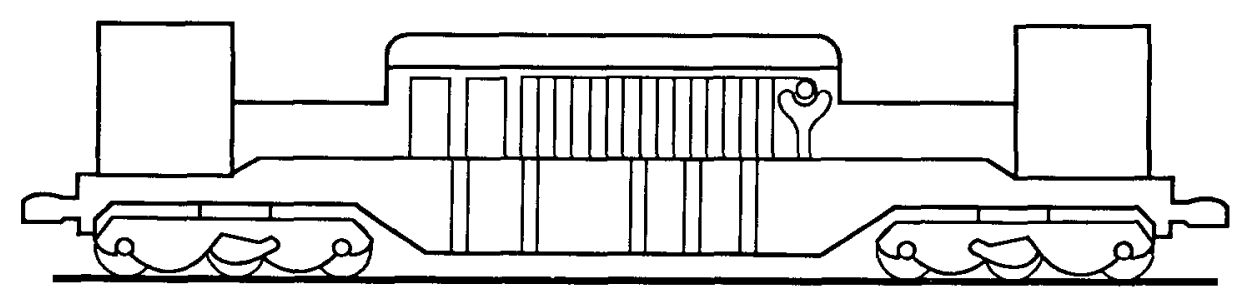

\section{b. Rail Configuration}

Figure II-64. Typical Truck and Rail Transport Configurations for SpentFue1 Casks

3. In the references surveyed, vibration data are generally presented in the form of plots of acceleration amplitude as a function of frequency. Shock data, however, are generally presented in the form of shock response spectra. A shock response spectrum is a plot of the peak response (in this case, acceleration) of a set of single-degree-of-freedom spring-mass systems of varying natural frequency subjected to the same input signal. (Details for response spectra use are presented by Biggs [BI64] and Thomson [TH72].)

4. In the references surveyed, accelerations are usually recorded at the cask-to-carrier (trailer or rail car) interface. For a rigid cask-carrier connection, recorded accelerations are therefore those of the cask. For a cask not rigidly mounted, accelerations recorded at the cask-to-carrier interface will be conservative relative to those of the cask, based on results reported by Glass and Gwinn [GL86, 87]. 

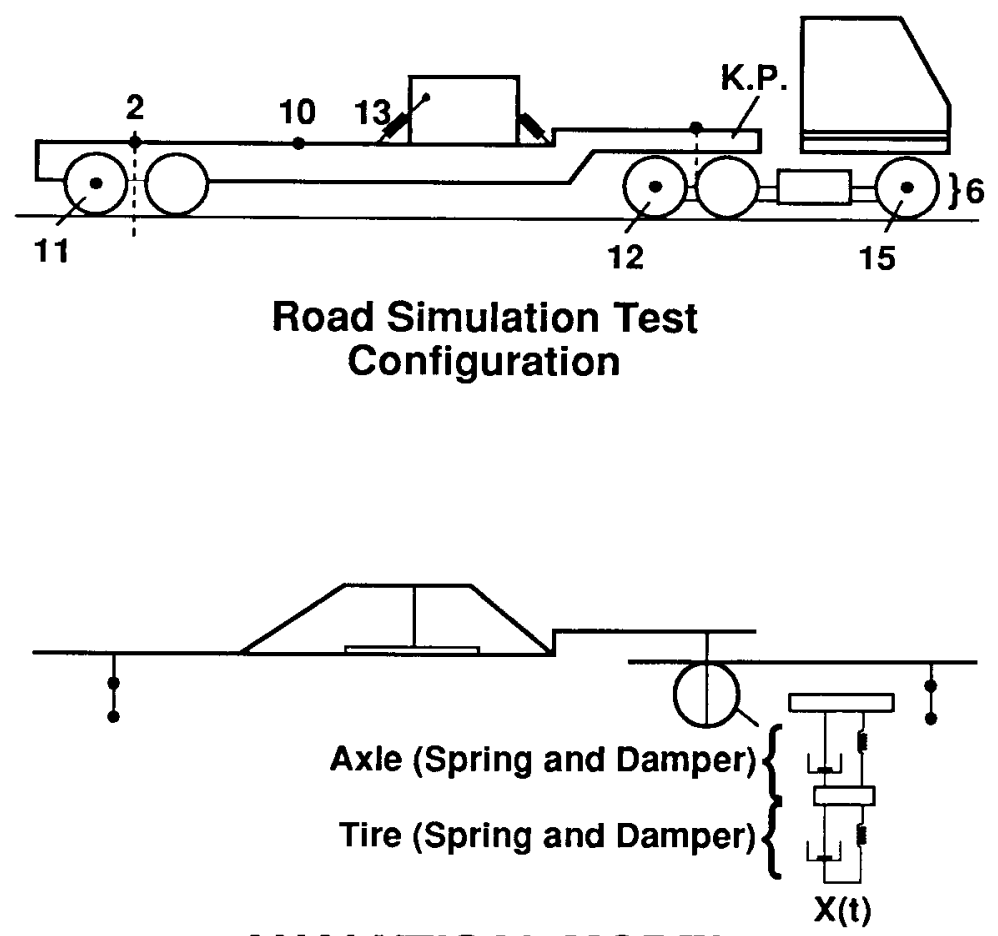

ANALYTICAL MODEL

Figure II-65. Spring-Mass Model for Input Excitation to the Truck Trailer Body from Prescribed Road Surface Roughness, X(t). [GL86]

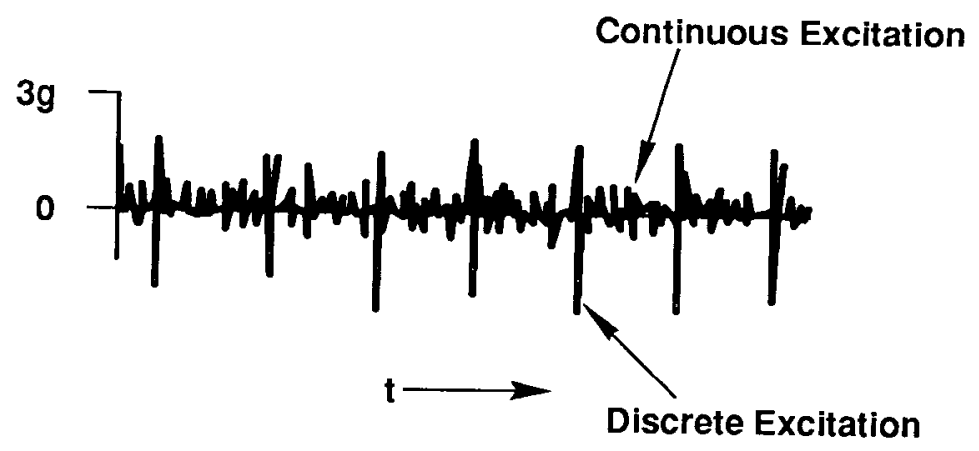

Figure II-66. Acceleration-Time History Illustrating the Presence of Discrete Shock Signals Superimposed on Continuous Vibration [GE72] 
Work up to approximately 1960 is summarized for both rail and road vehicles by Harris and Crede [HA61]. Possible average and peak acceleration values of truck and trailer cargo areas are presented.

A large quantity of work on truck and rail shock and vibration has been reported by SNL for weapons shipments as well as transport casks. SNL has also established a Transportation Technical Environmental Information Center [DA82] for the collection and dissemination of transportation environment information. More recent shock reported and vibration analyses include the following (in chronological order):

\section{Foley and Gens}

Foley and Gens [F071] reported results on the transportation of a radioactive materials cask from Paducah, KY, to Oak Ridge, TN, by railroad, returning by truck to Paducah. The cargo was a relatively light (13.61-tonne) laminated uranium Fuel Element Transport Cask and associated tiedown system that had been developed by Union Carbide Corporation. The bimodal testing provided an opportunity to determine rail and truck transport environments under identical loading conditions with identical instrumentation. However, at least for the rail data, the cask represented an unrealistically light load.

During the rail shipment, the cask was positioned at the center of the rail car and tied down by tie rods connected through the floor to the car frame and to plates that had been welded to the frame. Key locations for both rail and truck at which data were recorded were those described as the "input to cargo" interface of the vehicle and cargo systems, although other vehicle and cargo locations were also instrumented. Comparisons of truck versus rail transport indicate that while the dynamic environments of rail and truck transport in this test program differ in precise detail, the overall environmental input to the cargo differs little.

The measured (vibration-superimposed) shock data are presented in the form of acceleration shock response spectra for rail and truck transport, as shown in Figures II-67 and II-68. Apparently, both Figures II-67 and II-68 represent the maximum measured shock response for al1 three axes and all shock events.

The measured vibration data are summarized in Figures II-69 and II-70 for the rail and truck systems, respectively. Additional similar rail and truck shock and vibration data from a variety of other sources are presented in related analyses by Foley [FO72b] and Gens [GE72].

\section{Magnuson and Wilson}

Magnuson and Wilson [MA77a] present a thorough review and discussion of previous tests on truck and rail spent-fuel cask shipments. Using the same definitions for shock and vibration given in Section II.4.1, they summarize vibration results for seven different truck and tractor-trailer configurations. Included in the compilation are trucks equipped with both conventional spring and air-cushioned suspension systems. Cargo weights varied from no-load to 13.61 tonnes. The reported results are presumably the highest values recorded and are shown in Table II-14. 


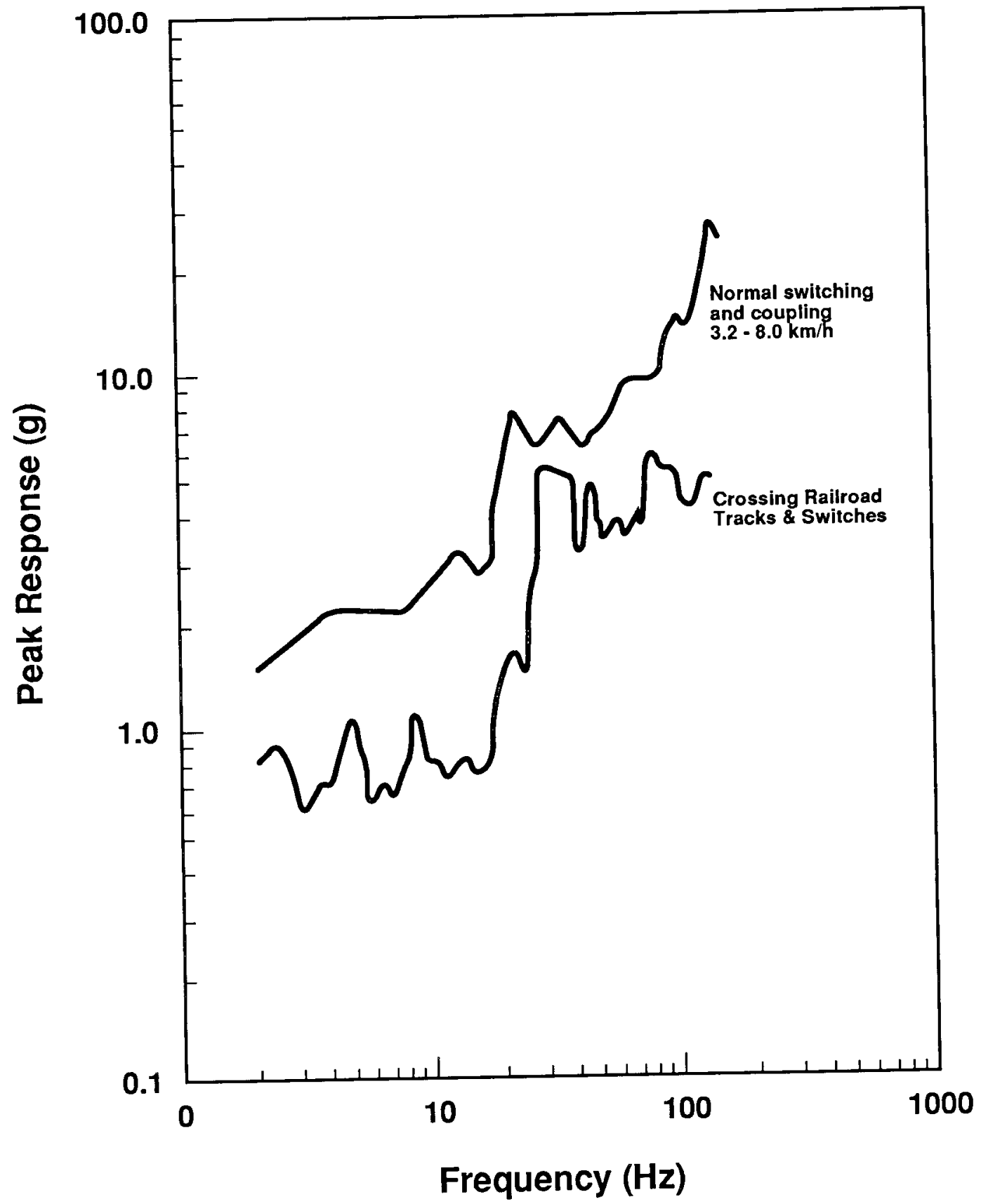

Figure II-67. Comparison of Two Rail Shock Spectrum Envelopes [F071] 


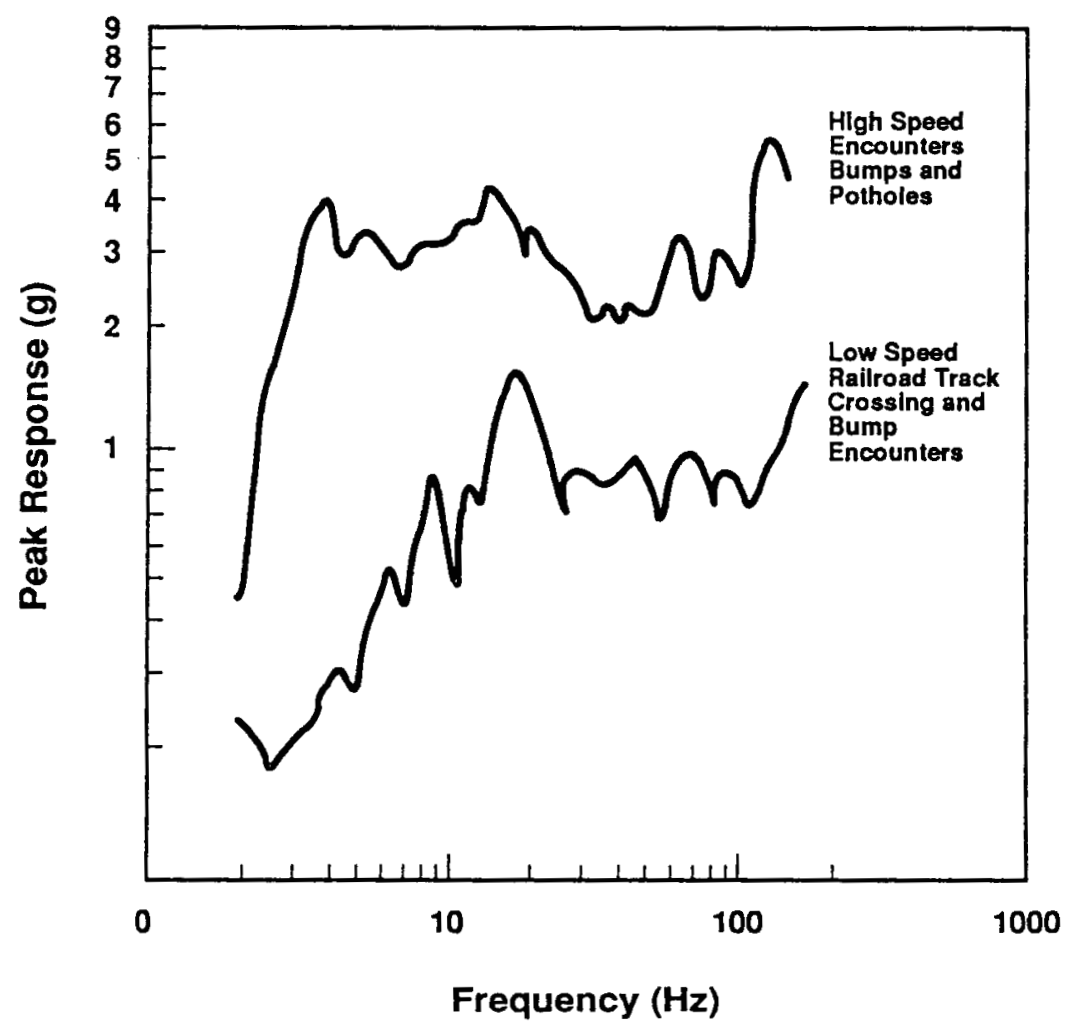

Figure II-68. Shock Response Spectrum Envelopes at All Three Axes for Truck Transport Events at High-and Low-Encounter Speeds [F071]

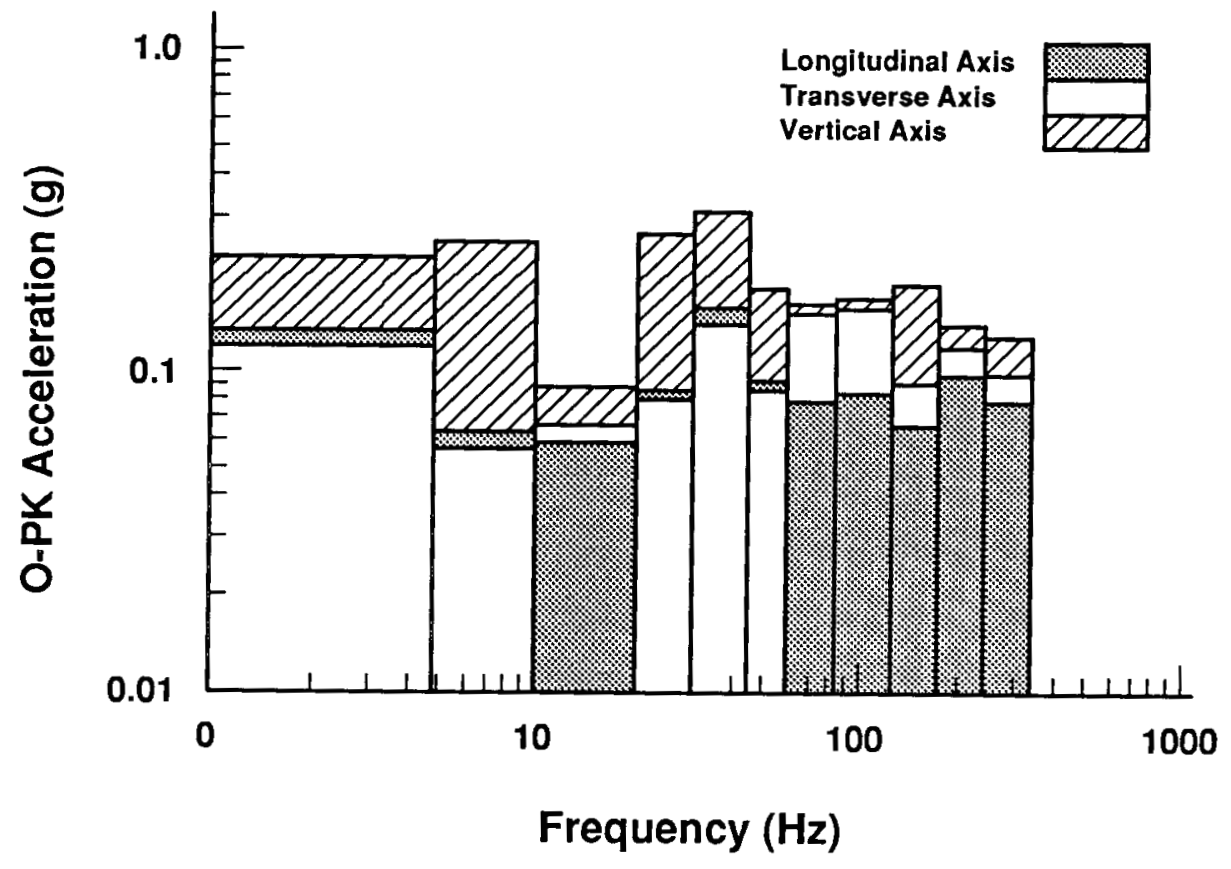

Figure II-69. Rail Vibration Summary at 998 Level [F071] 


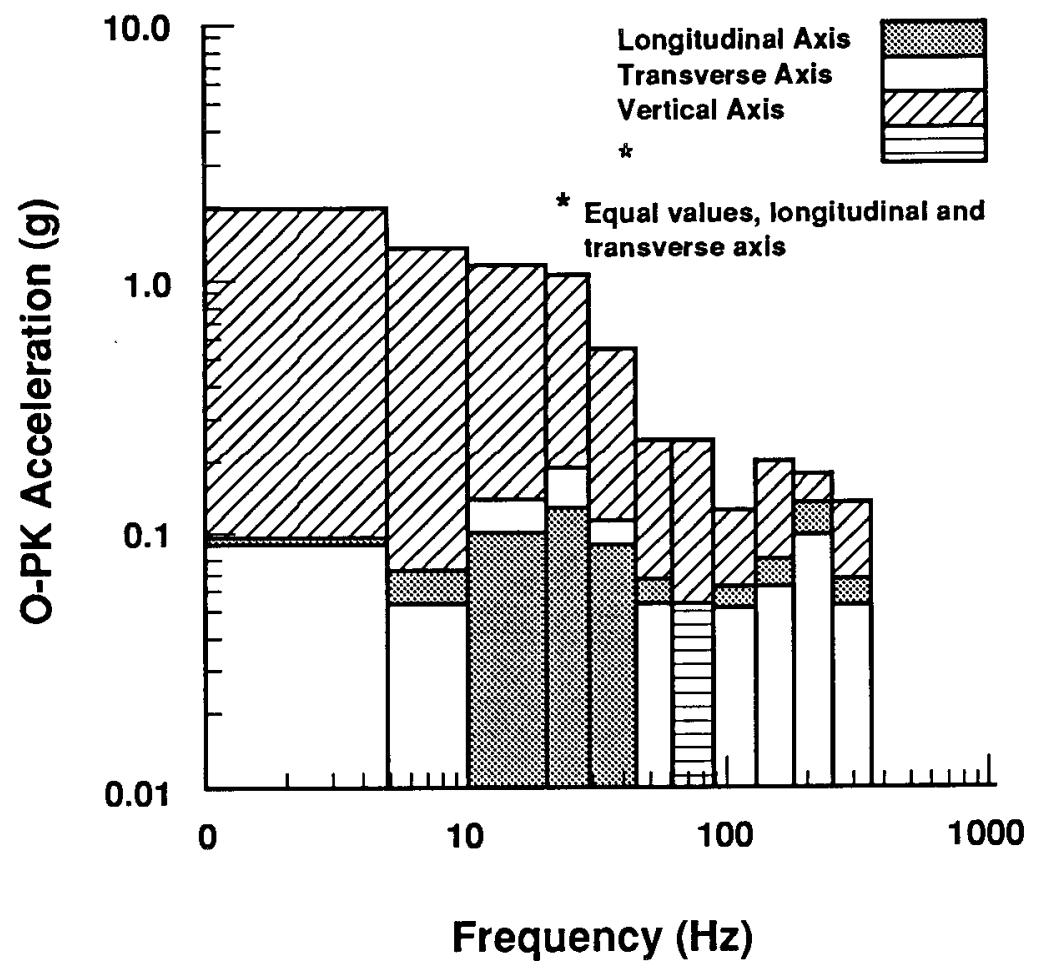

Figure II-70. Truck Vibration Summary at 998 Level [F071]

Table II - 14

Truck Vibration Data [MA77a]

\begin{tabular}{|c|c|c|c|}
\hline \multirow[b]{2}{*}{$\begin{array}{l}\text { Frequency } \\
\text { Band }(\mathrm{Hz})\end{array}$} & \multicolumn{3}{|c|}{$\begin{array}{l}\text { Measurements on Cargo Floor }(\mathrm{g}) \\
998 \text { Level of Zero to Peak Amplitude }\end{array}$} \\
\hline & $\begin{array}{l}\text { Longitudinal } \\
\text { Axis }\end{array}$ & $\begin{array}{l}\text { Transverse } \\
\text { Axis } \\
\end{array}$ & $\begin{array}{l}\text { Vertical } \\
\text { Axis } \\
\end{array}$ \\
\hline $0-5$ & 0.10 & 0.10 & 2.00 \\
\hline $5-10$ & 0.08 & 0.06 & 1.04 \\
\hline $10-20$ & 0.84 & 0.15 & 1.68 \\
\hline $20-40$ & 0.51 & 0.24 & 1.20 \\
\hline $40-80$ & 0.36 & 0.42 & 0.50 \\
\hline $80-120$ & 0.24 & 0.27 & 0.87 \\
\hline $120-180$ & 1.23 & 0.21 & 0.63 \\
\hline $180-240$ & 0.87 & 0.12 & 0.87 \\
\hline $240-350$ & 0.24 & 0.15 & 0.63 \\
\hline $350-500$ & 0.24 & 0.15 & 0.42 \\
\hline $500-700$ & 0.87 & 0.15 & 0.87 \\
\hline $700-1000$ & 1.50 & 0.87 & 1.17 \\
\hline $1000-1400$ & 0.87 & 1.17 & 1.17 \\
\hline $1400-1900$ & 0.39 & 0.24 & 0.87 \\
\hline
\end{tabular}


Shock data were obtained from the same tests as the truck vibration data for the following events: potholes, railroad crossings, and bridge approaches. The responses at the interface of the cargo and its floor are given in the form of shock response envelopes for 38 damping, as shown in Figure II-71. These shock-producing road irregularities occur approximately once per mile of travel.

Rail data presented by Magnuson and Wilson [MA77a] are based on the 13.61-tonne rail cask shipment detailed by Foley and Gens [FO71]. The resulting vibration was cast in a somewhat different form and is presented in Table II-15.

Superimposed shock data were obtained during the same test rail series for different events (crossing rail joints, traveling through switches, and run-in and run-out); the results are shown in Figure II-72. These data are similar to those presented in the lower curve of Figure II-67 except directional information is also given. Again, the rail results are limited because they are based on an unrealistically low cask mass.

For the rail-coupling events isolated shock data that are not superimposed with normal vibrations are also presented by Magnuson and Wilson [MA77a]. These data are based on rail-coupling tests conducted in the early 1960 s using ATMX cars with very light 4.5 -tonne cargoes. The applicability of these rather severe rail-coupling data to spent-fuel response analyses is questionable, though conservative, due to the extremely light cargo. This analysis by Magnuson and Wilson shows that for rail cars equipped with standard draft gear, shock magnitude at the cargo decreases as the cargo weight increases until the coupler springs bottom out.

Magnuson and Wilson also include a simplification of the shock definition: a single pulse representation of shock. Magnuson and Wilson list specific pulses that produce response spectra that envelope those obtained in the shock tests discussed above. Half-sine pulses specified with magnitude and duration are also presented. For example, the rail shock response data in Figure II-72 are conservatively bounded from above by a half-sine pulse response spectrum, as shown in Figure II-73. Here, only a single half-sine pulse is used to bound vertical, longitudinal, and transverse spectra because these spectra are similar. Table II-16 gives the half-sine pulses for which response spectra conservatively bound the shock response spectra for truck and rail transport modes. These spectra only apply to the relatively light fuel assemblies contained within the spent-fuel cask.

\section{Magnuson}

For shock and vibration data for heavier cargoes, Magnuson [MA77b] performed acceleration measurements during transport of a 20-tonne spentfue 1 cask by truck from Mercury, NV, to Albuquerque, NM. The cask was supported on each end by structures fastened to structural members of the trailer. Accelerometers were mounted in longitudinal, transverse, and vertical directions at the structure supporting the cask. 


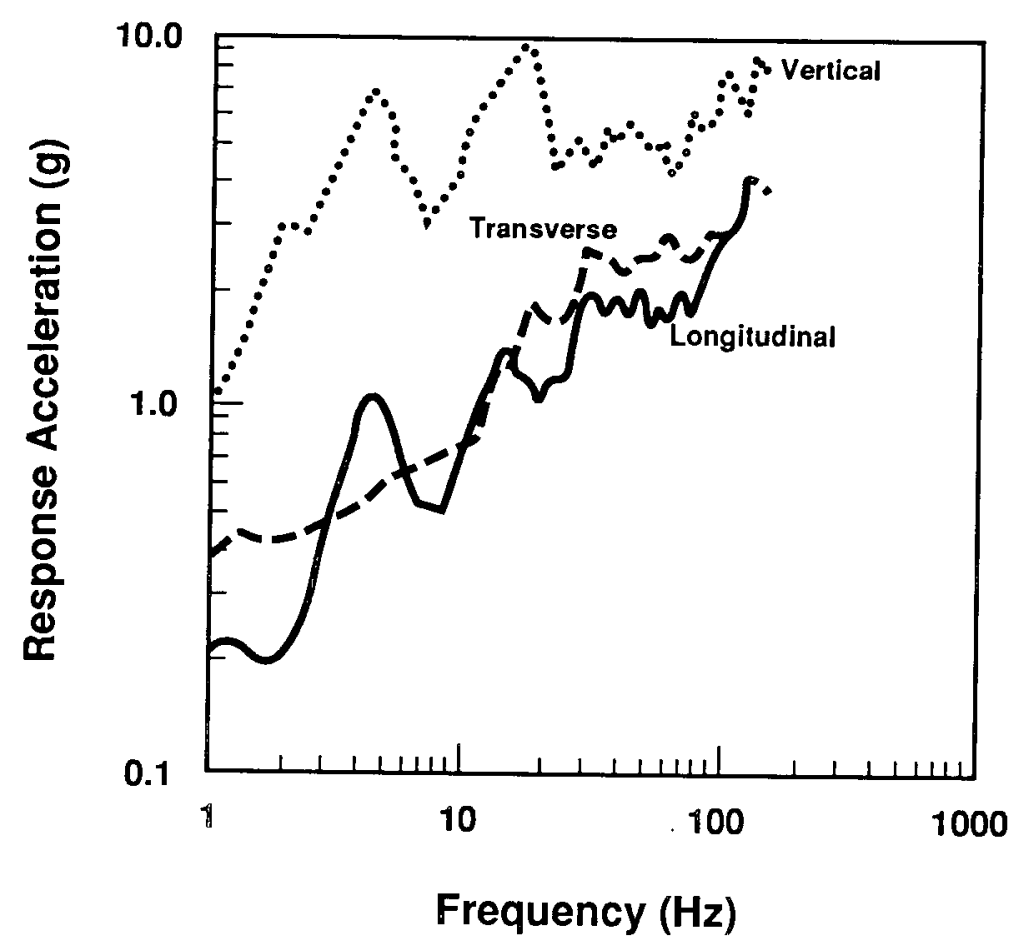

Figure II-71. Truck Superimposed Shock Response Envelopes with 38 Damping [MA77a]

Table II-15

Train Vibration Data [MA77a]

\begin{tabular}{|c|c|c|c|}
\hline \multirow[b]{2}{*}{$\begin{array}{l}\text { Frequency } \\
\text { Band }(\mathrm{Hz})\end{array}$} & \multicolumn{3}{|c|}{$\begin{array}{l}\text { Measurements on Cargo Floor (g) } \\
998 \text { Level of } 0 \text { to Peak Amplitude }\end{array}$} \\
\hline & $\begin{array}{l}\text { Longitudinal } \\
\text { Axis } \\
\end{array}$ & $\begin{array}{l}\text { Transverse } \\
\text { Axis } \\
\end{array}$ & $\begin{array}{l}\text { Vertical } \\
\text { Axis } \\
\end{array}$ \\
\hline 0.5 & 0.14 & 0.14 & 0.37 \\
\hline $5-10$ & 0.072 & 0.072 & 0.14 \\
\hline $10-20$ & 0.072 & 0.072 & 0.10 \\
\hline $20-30$ & 0.10 & 0.10 & 0.27 \\
\hline $30-45$ & 0.19 & 0.14 & 0.37 \\
\hline $45-60$ & 0.10 & 0.10 & 0.27 \\
\hline $60-87$ & 0.10 & 0.19 & 0.19 \\
\hline $87-125$ & 0.10 & 0.19 & 0.19 \\
\hline $125-175$ & 0.10 & 0.10 & 0.19 \\
\hline $175-250$ & 0.10 & 0.14 & 0.14 \\
\hline $250-350$ & 0.10 & 0.10 & 0.14 \\
\hline
\end{tabular}




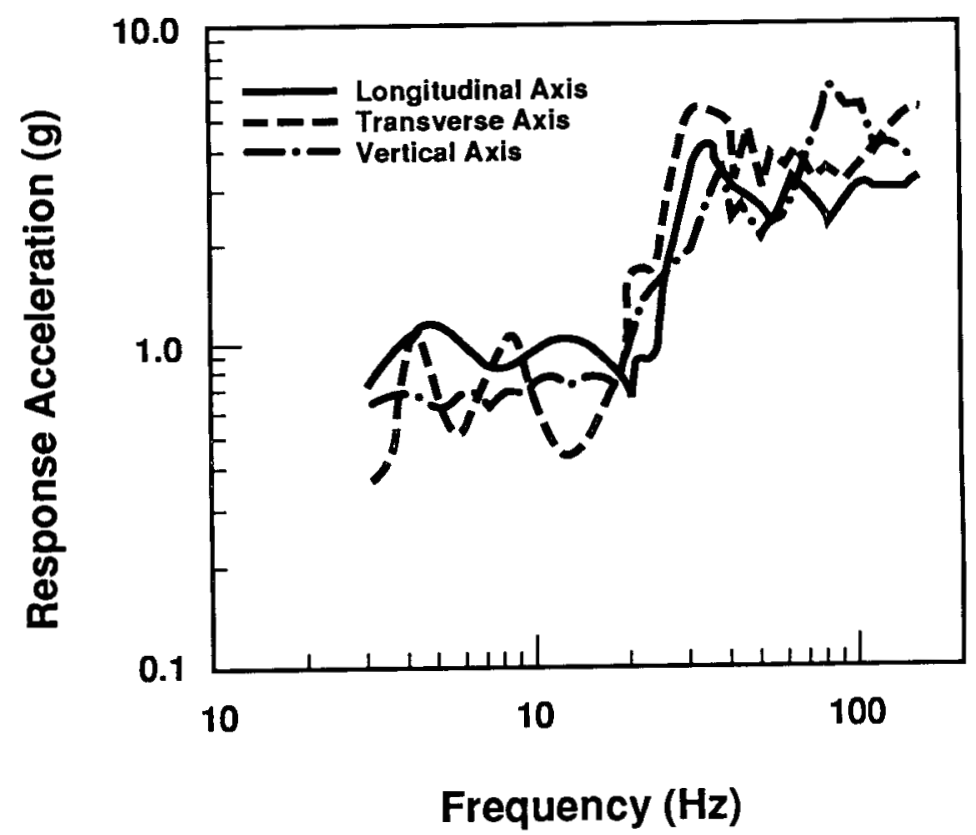

Figure II-72. Superimposed Shock Response Envelopes for Rail Transport with 38 Damping [MA77a]

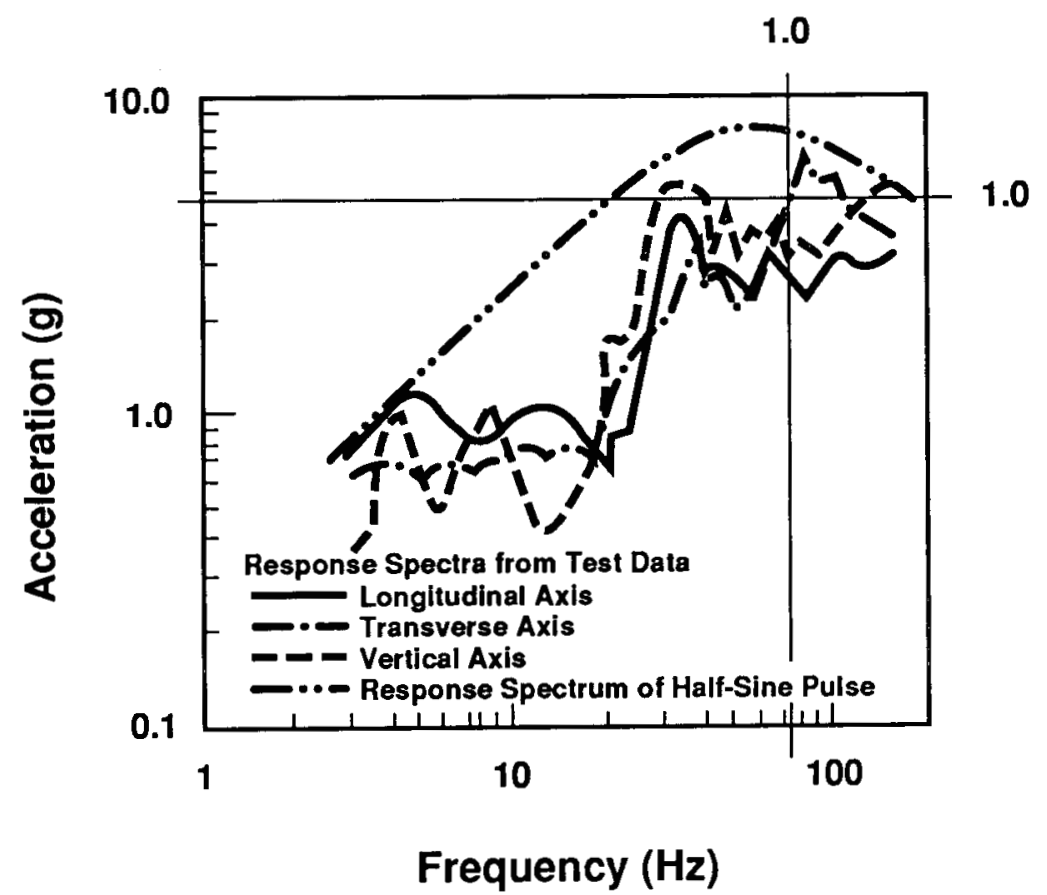

Figure II-73. Test Data Response Spectra Enveloped by Simple Pulse Response Spectrum [MA77a] 
Table II - 16

Bounding Half-Sine Pulses [MA77a]

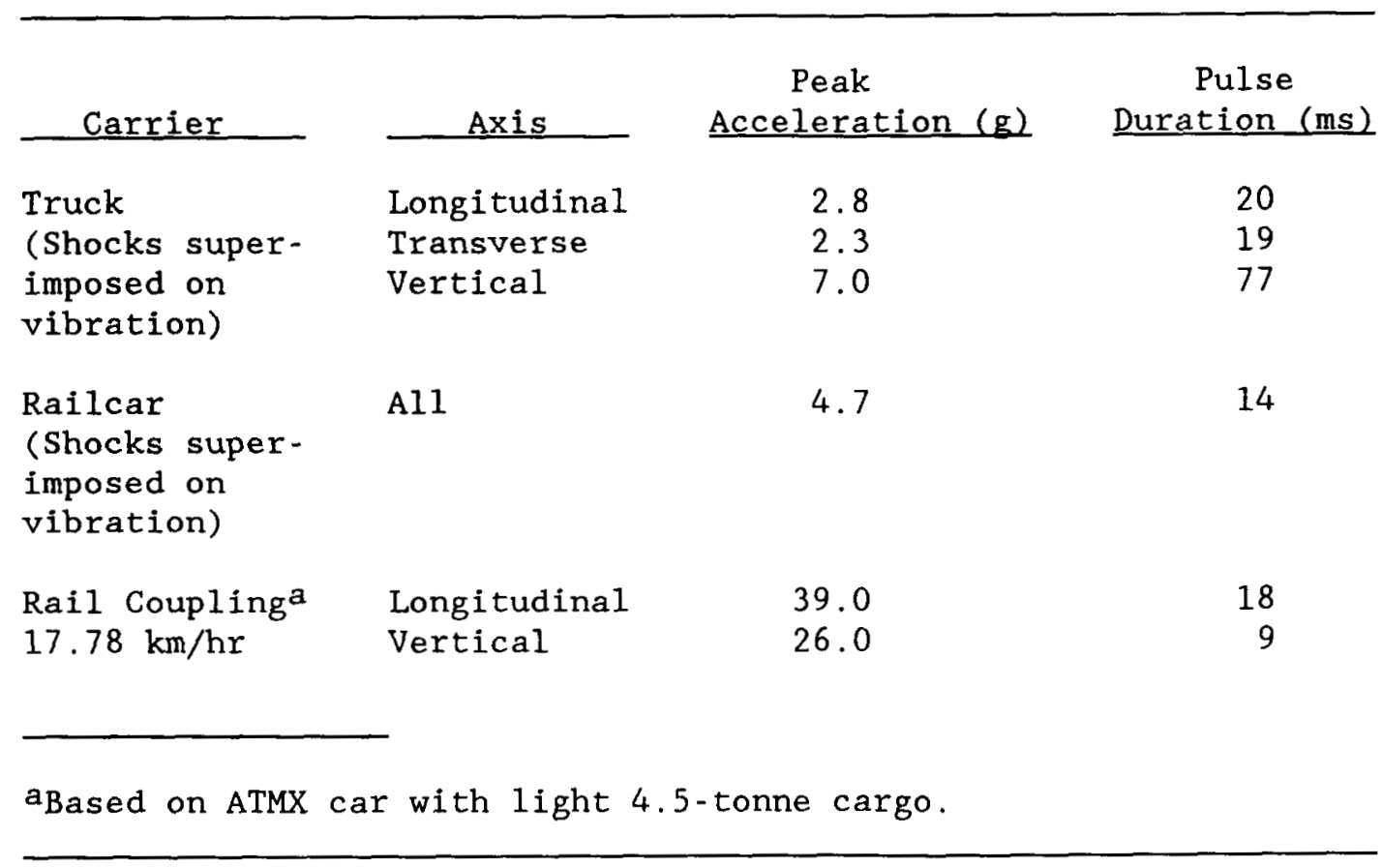

Again, vibration data presented by Magnuson are zero-to-peak acceleration amplitude levels that envelope 998 of all amplitudes measured in each frequency band. The remaining 18 of the data representing superimposed shock was treated separately. Also, acceleration amplitudes were measured at the interface between the cargo and its floor.

The shock data are again presented in the form of single-degree-offreedom response spectra. Half-sine pulses that bound the corresponding spectra are given. Vibration results, that is, the input to the cargo, are shown in Table II-17. Shock spectra results with various superimposed shock events are shown for longitudinal, transverse, and vertical axes in Figures II-74 through II-76.

Again, half-sine pulses were developed in which the shock response spectra bound from above the shock spectra that is constructed from accelerometer data. Results of bounding the response spectra of absolute peak responses are listed in Table II-18. A comparison of shock vibration results with those of Magnuson and Wilson [MA77a] is also presented in Magnuson. Note that Magnuson investigates transport of a 20-tonne cargo, whereas truck data compiled by Magnuson and Wilson [MA77a] involve shipments varying from no-load to 13.6 tonnes. One notable difference is that the shock response produced in the vertical direction during the heavier cargo shipment is less than the response reported for the lighter cargo across the entire frequency spectrum. The peak acceleration of the equivalent half-sine pulse decreased from 7.0 to $2.6 \mathrm{~g}$. 
Table II -17

Truck Vibration Data for a 20-Tonne Cargo [MA77b]

\begin{tabular}{|c|c|c|c|}
\hline \multirow[b]{2}{*}{$\begin{array}{l}\text { Frequency } \\
\text { Band (Hz) }\end{array}$} & \multicolumn{3}{|c|}{$\begin{array}{l}\text { Input to Cargo ( } \mathrm{g}) \\
998 \text { Leve } 1 \text { of } 0 \text { to Peak Amplitude }\end{array}$} \\
\hline & $\begin{array}{l}\text { Longitudinal } \\
\text { Axis }\end{array}$ & $\begin{array}{l}\text { Transverse } \\
\text { Axis } \\
\end{array}$ & $\begin{array}{l}\text { Vertical } \\
\text { Axis } \\
\end{array}$ \\
\hline $0-5$ & 0.14 & 0.14 & 0.27 \\
\hline $5-10$ & 0.19 & 0.19 & 0.19 \\
\hline $10-20$ & 0.27 & 0.27 & 0.27 \\
\hline $20-40$ & 0.10 & 0.27 & 0.27 \\
\hline $40-80$ & 0.14 & 0.14 & 0.52 \\
\hline $80-120$ & 0.07 & 0.10 & 0.52 \\
\hline $120-180$ & 0.07 & 0.10 & 0.52 \\
\hline $180-240$ & 0.05 & 0.10 & 0.52 \\
\hline $240-350$ & 0.05 & 0.10 & 0.52 \\
\hline $350-500$ & 0.05 & 0.05 & 0.14 \\
\hline $500-700$ & 0.04 & 0.04 & 0.07 \\
\hline $700-1000$ & 0.03 & 0.07 & 0.07 \\
\hline $1000-1400$ & 0.01 & 0.04 & 0.05 \\
\hline $1400-1900$ & 0.01 & 0.05 & 0.05 \\
\hline
\end{tabular}

Magnuson

Magnuson then reported additional shock and vibration data during the truck shipment of heavy cargo [MA78]. The truck cargo consisted of a spent-fuel cask and its supporting structure with a total weight of 25 tonnes, which was heavier than previously reported truck shipments. The truck was driven from Mercury, NV, to Albuquerque, NM.

Accelerometers in three directions were mounted not directly on the cask, but rather near each end of the cask on the cask tie-down structure. Magnuson interpreted the accelerations [MA78] as being measured at the interface between the cargo and the cargo floor.

Again, the data were partitioned into vibration and superimposed shock on vibration. The shock data were again obtained from measurements coinciding with the truck encountering railroad crossings, cattle-guard crossings, and bridge approaches. For cargo weighing more than 13.6 tonnes, there is little difference in the vibration amplitudes regardless of the suspension system type of the trailer. A comparison of vibration levels for three different cargo weights is shown in Figures II-77 through II-79 for longitudinal, transverse, and vertical directions .

Shock response spectra generated by Magnuson [MA78] are compared to earlier results in Figures II-80 through II-82 for the longitudinal, 


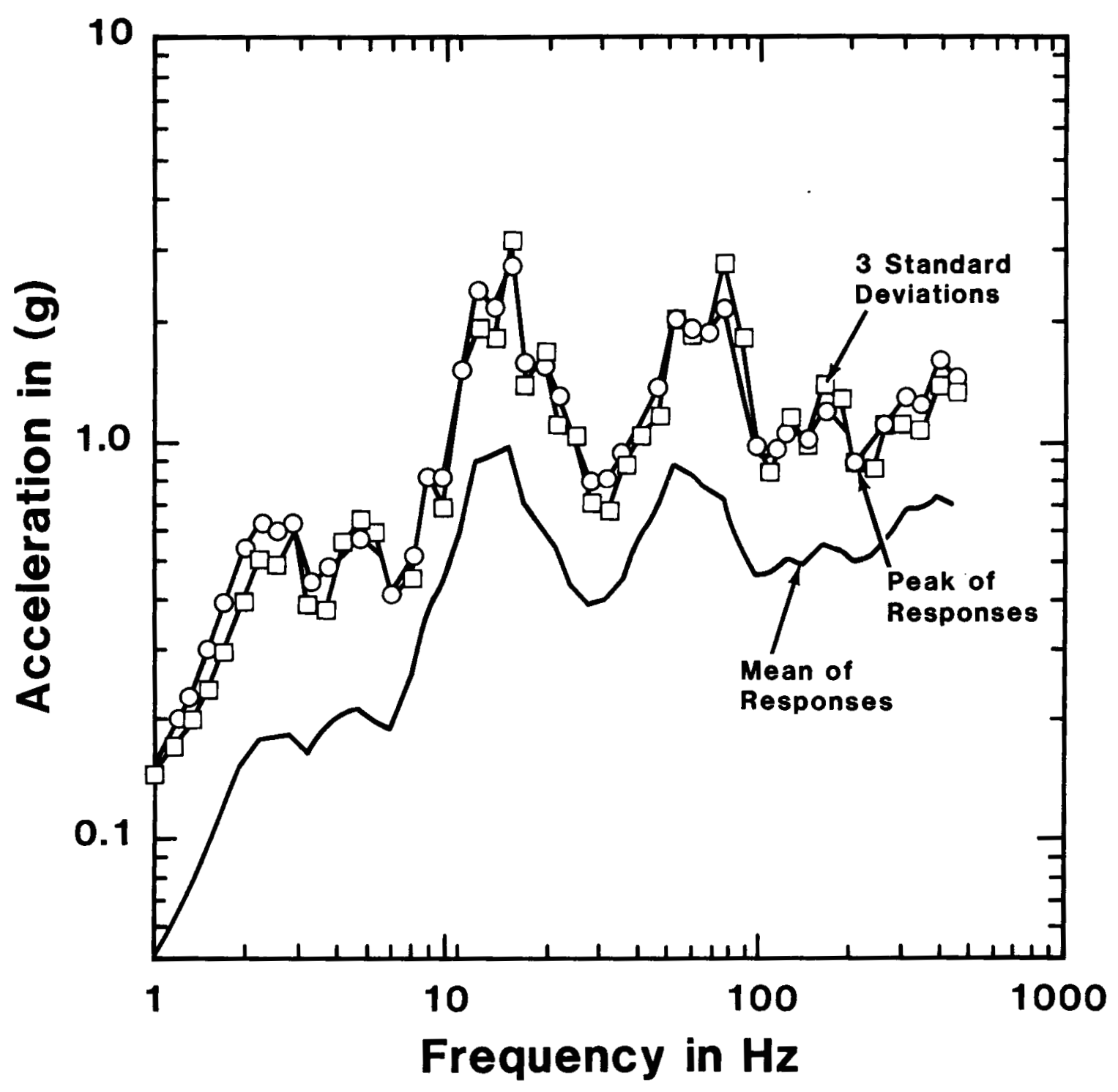

Figure II-74. Superimposed Shock Response Spectra with 38 Damping for the Longitudinal Axis [MA77b]

transverse, and vertical axes. No general statement can be made regarding the influence of cask mass on the resulting shock spectra. The simple half-sine pulses given in Table II-19 conservatively represent the maximum expected severities of superimposed shock for a cargo weighing 25 tonnes (based on absolute peak response).

\section{Fields}

Fields [FI78] developed a spring-mass-dashpot dynamic model of a rail car system for spent-fuel transport casks to estimate the vibrational response of the spent-fuel assemblies to time-varying track roughness. However, the results are limited to excitation and response in the vertical 


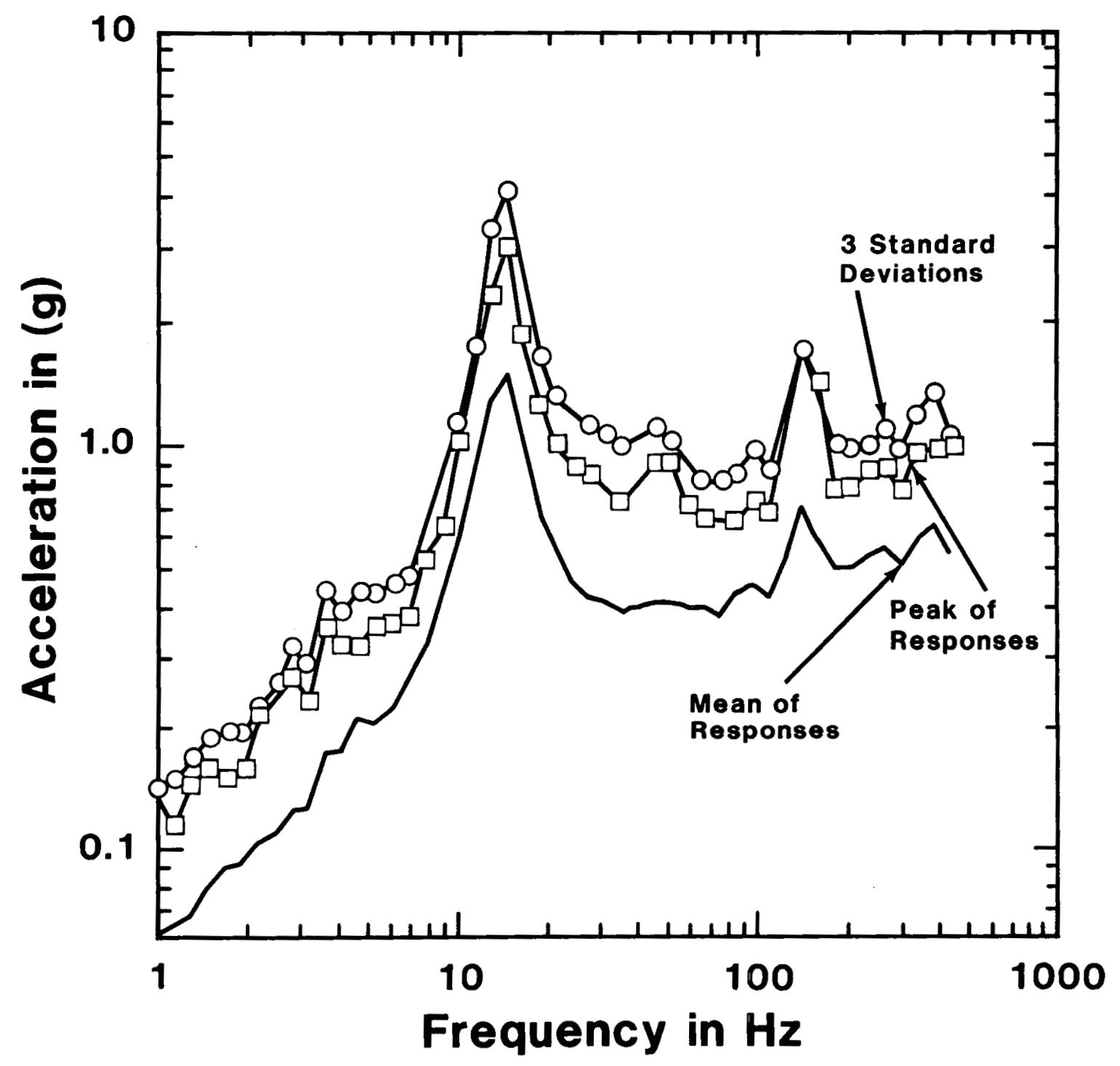

Figure II-75. Superimposed Shock Response Spectra with 38 Damping for the Transverse Axis [MA77b]

direction resulting from the vertical displacement of the rail car trucks only. Unfortunately, the analysis was terminated before realistic input properties and track conditions could be evaluated.

\section{Forest}

Forest [FO79] reported the results of a series of tests conducted to determine shock and vibration environments of simulated, full-scale spentfuel casks during rail and truck transport. The casks were simulated using an assembly of large concrete blocks that contained a prototype transport module with dumny fuel. 


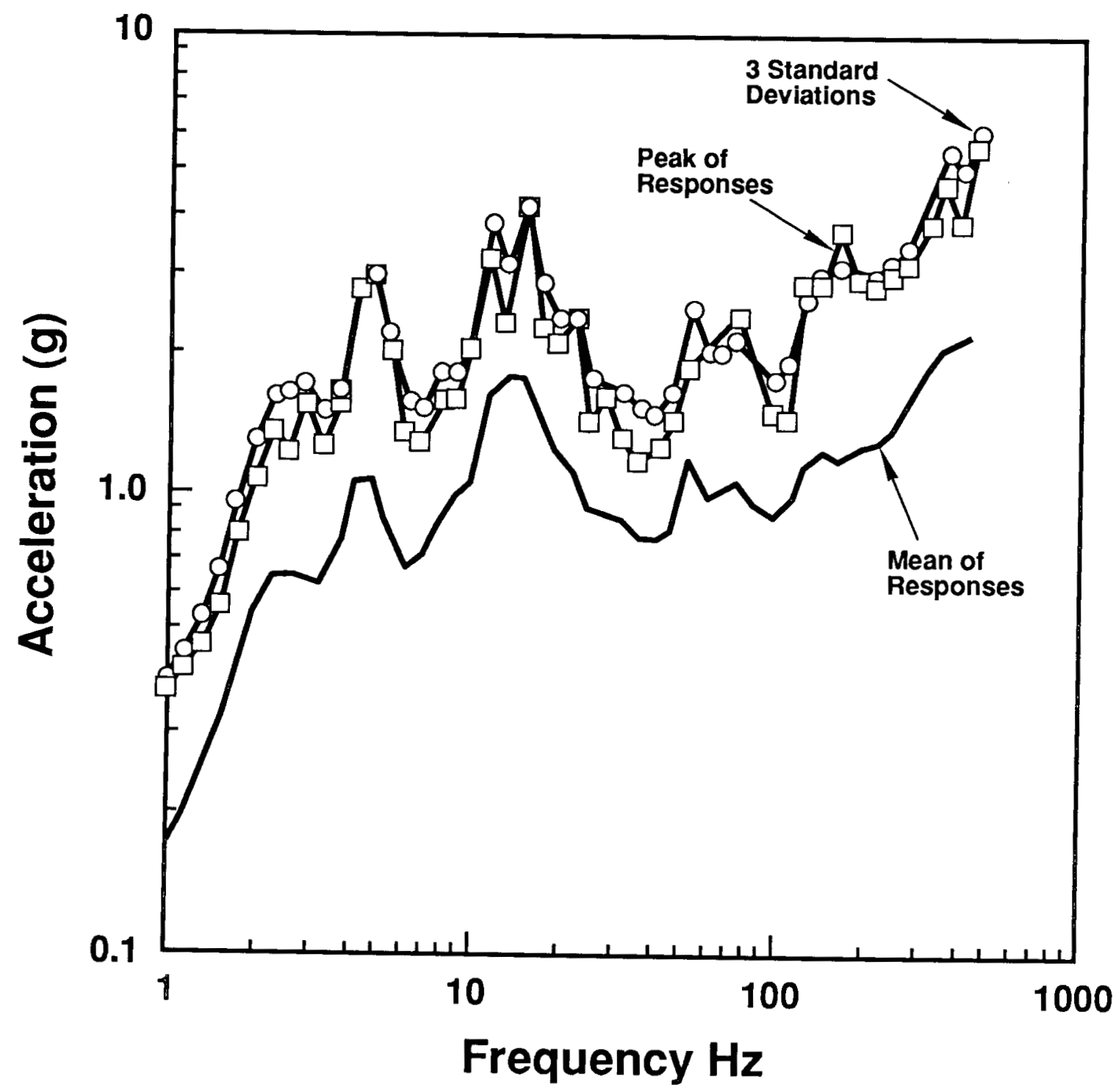

Figure II-76. Superimposed Shock Response Spectra with 3\% Damping for the Vertical Axis [MA77b]

\section{Table II-18}

Bounding Half-Sine Pulses

for Truck Vibration Data [MA77b]

\begin{tabular}{lcc}
\hline \multicolumn{1}{c}{ Axis } & $\begin{array}{c}\text { Peak } \\
\text { Acceleration (g) }\end{array}$ & $\begin{array}{c}\text { Peak } \\
\text { Duration (ms) }\end{array}$ \\
Longitudina1 & 1.9 & 32 \\
Transverse & 1.7 & 50 \\
Vertical & 2.6 & 67 \\
\hline
\end{tabular}




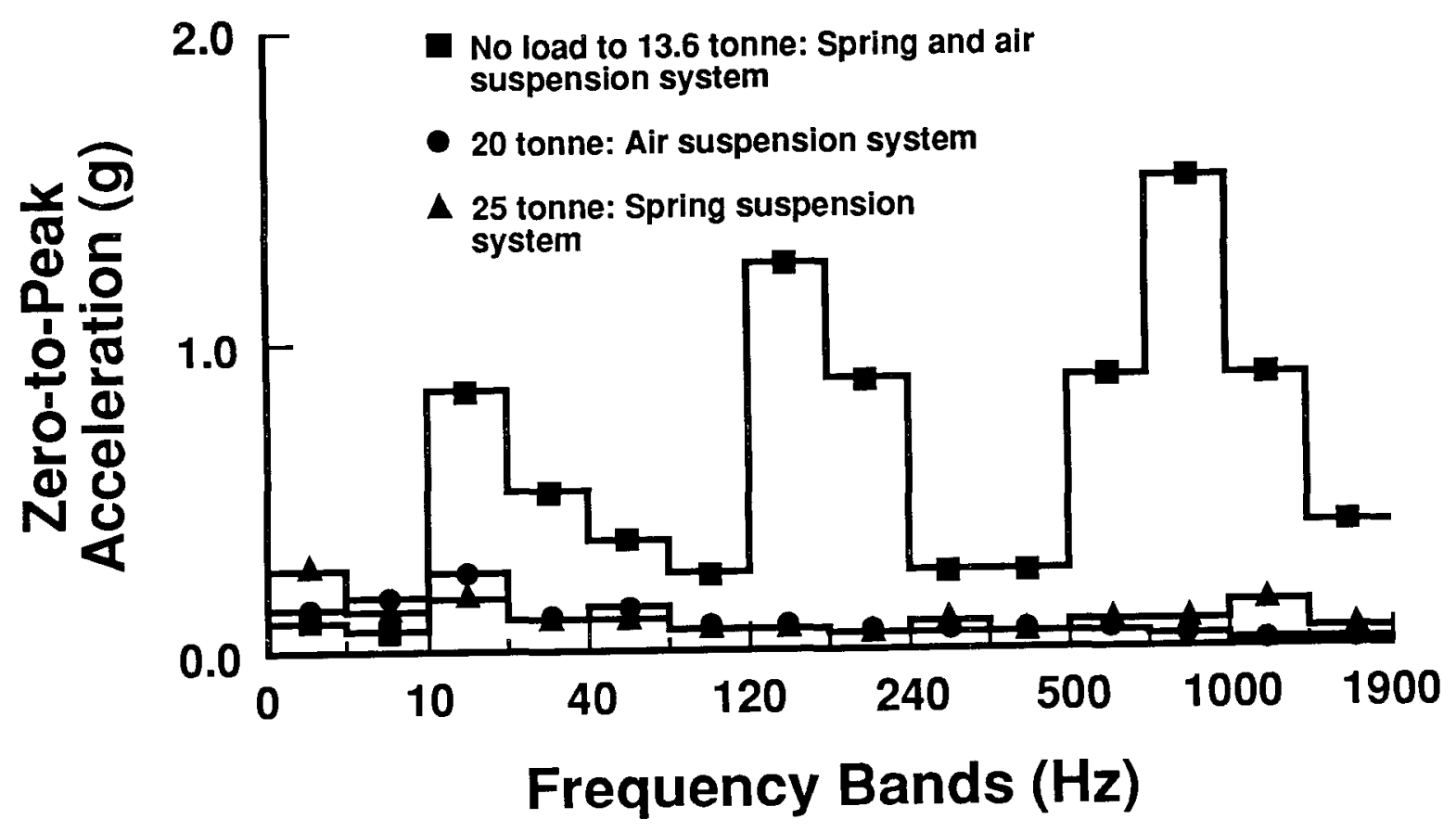

Figure II-77. Comparison of Truck Vibration Data for the Longitudinal Axis [MA78 ]

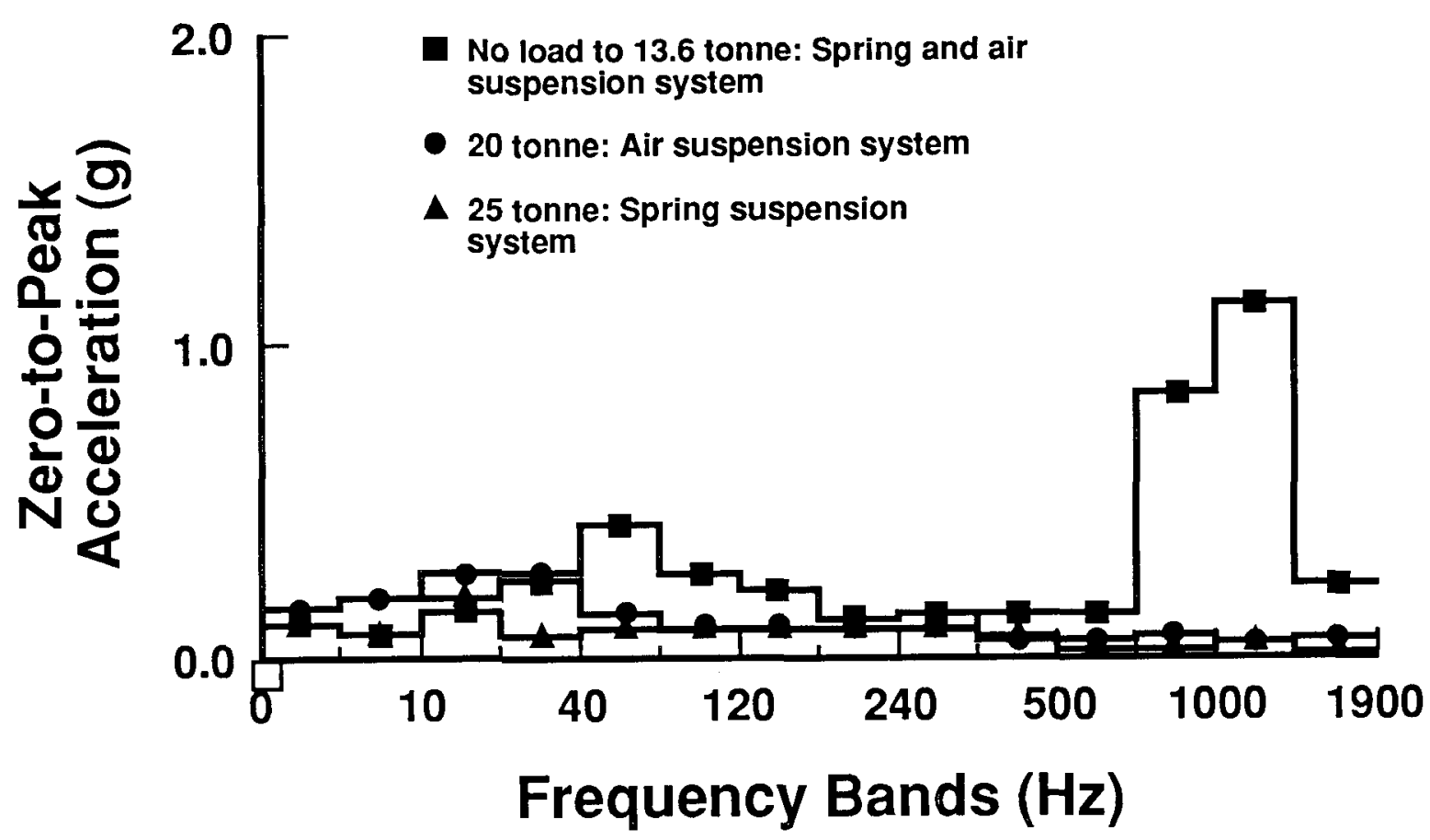

Figure II-78. Comparison of Truck Vibration Data for the Transverse Axis [MA78] 


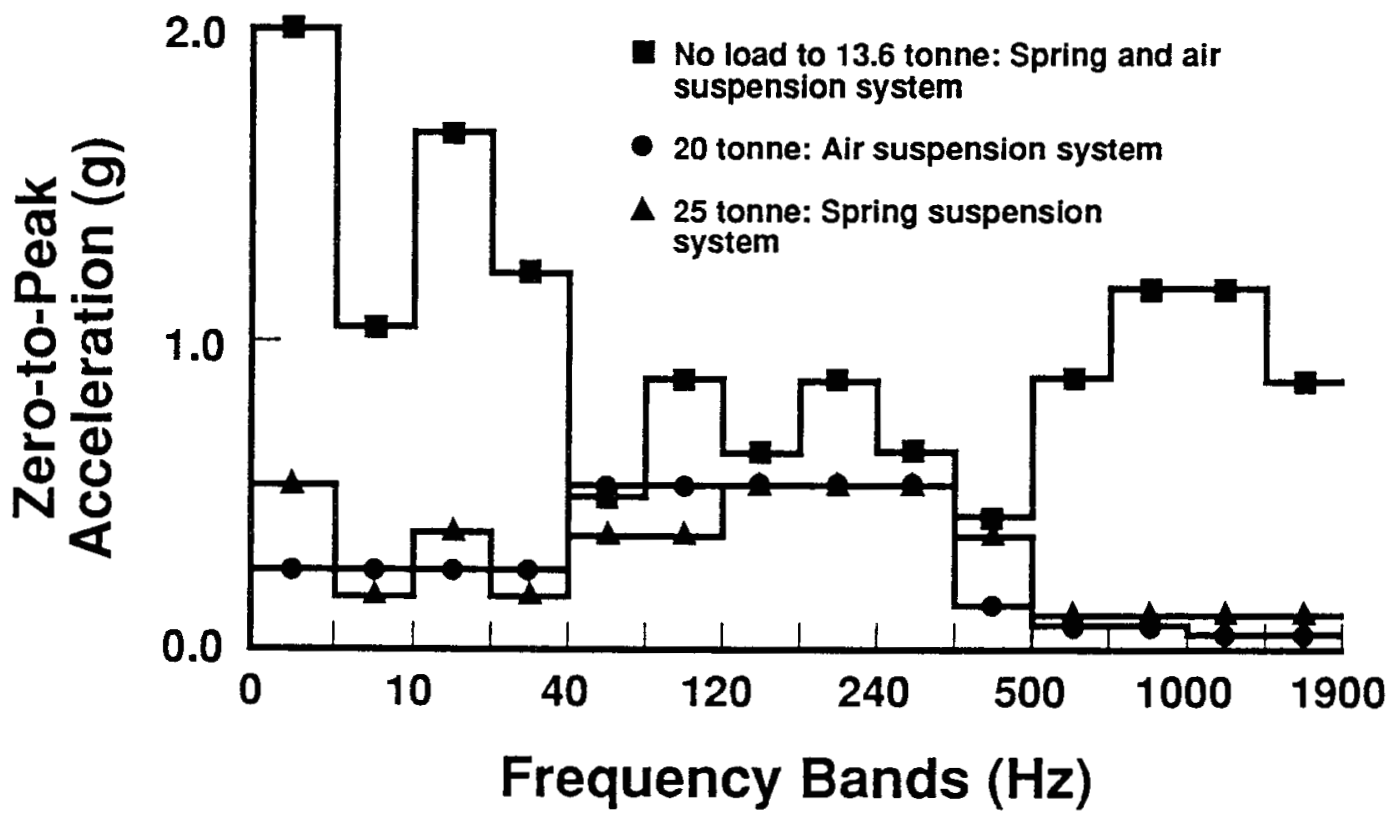

Figure II-79. Comparison of Truck Vibration Data for the Vertical Axis [MA78]

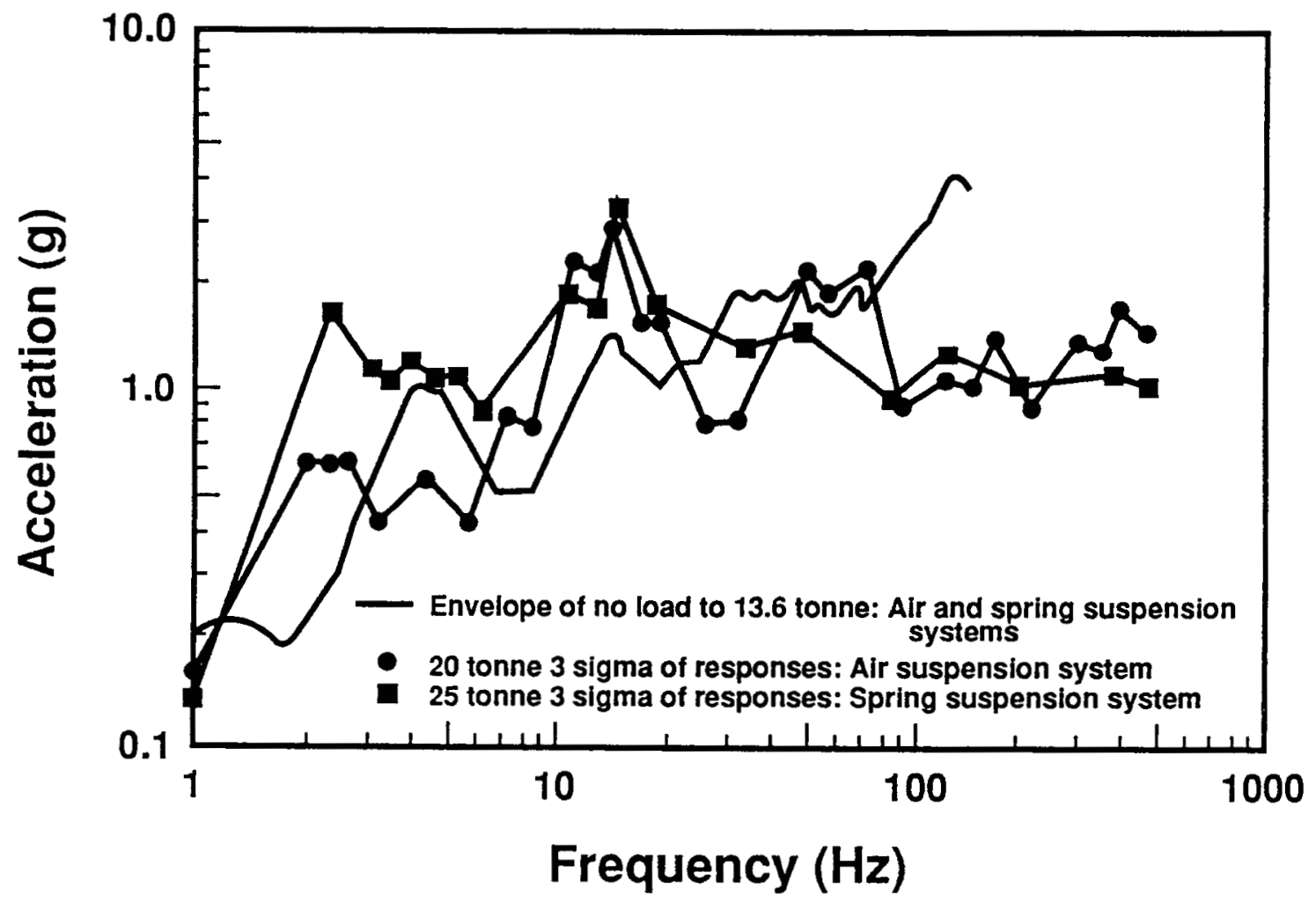

Figure II-80. Comparison of Response Spectra from Truck Data with 38 Damping for the Longitudinal Axis [MA78] 


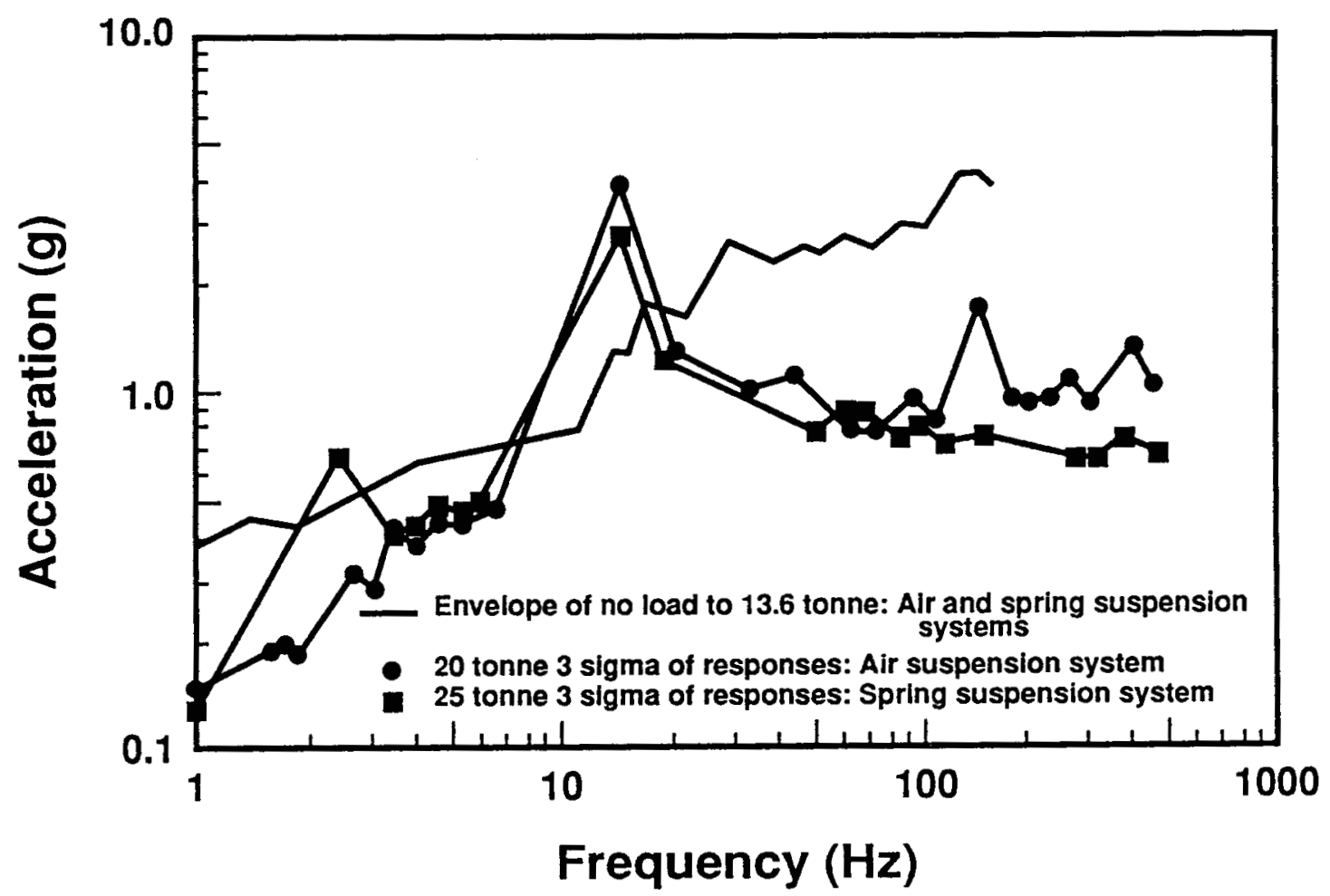

Figure II-81. Comparison of Response Spectra from Truck Data with 38 Damping for the Transverse Axis [MA78]

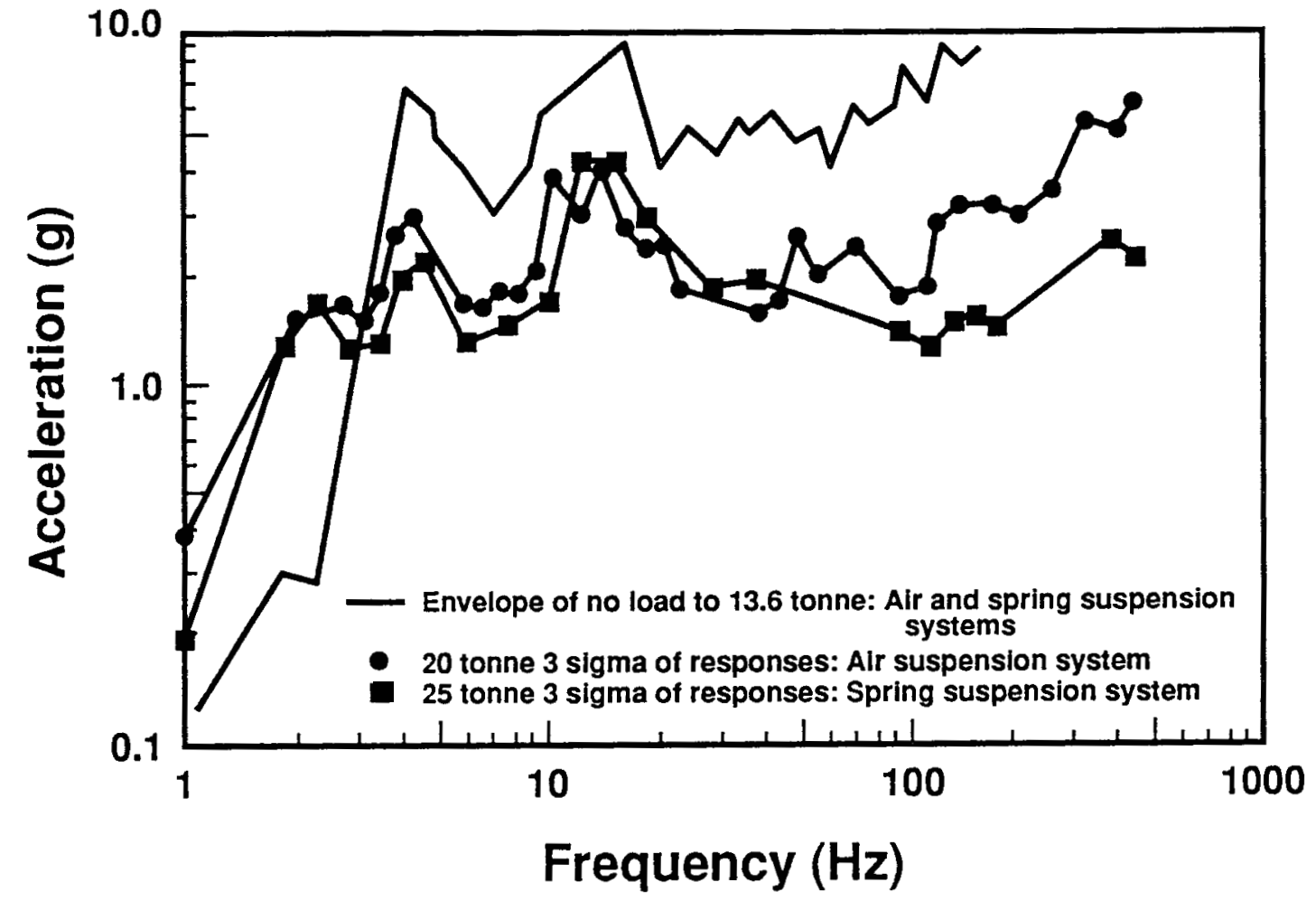

Figure II-82, Comparison of Response Spectra from Truck Data with 38 Damping for the Vertical Axis [MA78] 
Table II - 19

Bounding Truck Half-Sine Pulses [MA78]

\begin{tabular}{lcc}
\hline \multicolumn{1}{c}{ Axis } & $\begin{array}{c}\text { Peak } \\
\text { Acceleration (g) }\end{array}$ & $\begin{array}{c}\text { Peak } \\
\text { Duration (ms) }\end{array}$ \\
\cline { 3 - 3 } Longitudinal & 1.8 & 91 \\
Transverse & 1.3 & 59 \\
Vertical & 2.9 & 59 \\
\hline
\end{tabular}

Attempts were made to place accelerometers on the base plate of the concrete mass assembly as well as on the transport modules so that an interface transfer function could be obtained. Apparently this was the first attempt to obtain shock and vibration measurements directly at the fuel assemblies.

Generally, while the data reduction by Forest [F079] is incomplete, trends that are similar to the test data cited in previous references can be observed. For example, rail car impacts during coupling were found to impose the greatest shock loads on the fuel.

\section{Magnuson}

Magnuson [MA80] developed additional shock and vibration data for rail-coupling operations involving large transport casks. (Previous railcoupling data covered cargo weights of up to only -4.5 tonnes.

Superimposed shock and vibration of rail cars previously covered the transport of a 13.6-tonne spent-fuel cask, which is still extremely light for rail shipment.) The cargo on the rail cars consisted of either 36(including skid) or 64-tonne casks. Different rail car designs were also evaluated.

During coupling operations, rail cars were permitted to roll freely into other cars and tests revealed impact velocities of approximately $11 \mathrm{mph}$. This impact velocity bounds 99.88 of observed coupling operations. Accelerometers were mounted on both the rail car structure and the casks. However, specific results for cask-mounted accelerometers were not reported. Again, the results are provided in a single-degree-of-freedom response format. Composite spectra from various test events performed by Magnuson [MA80] are presented for longitudinal, transverse, and vertical axes in Figures II-83 through II-85. The half-sine input pulses in Table II- 20 conservatively bound the maximum shock intensities reported by Magnuson [MA80] for different cargo weights and rail car coupling devices.

\section{Magnuson}

The purpose of more recent work by Magnuson [MA82] was to obtain additional vibration and superimposed shock data for rail shipments of 


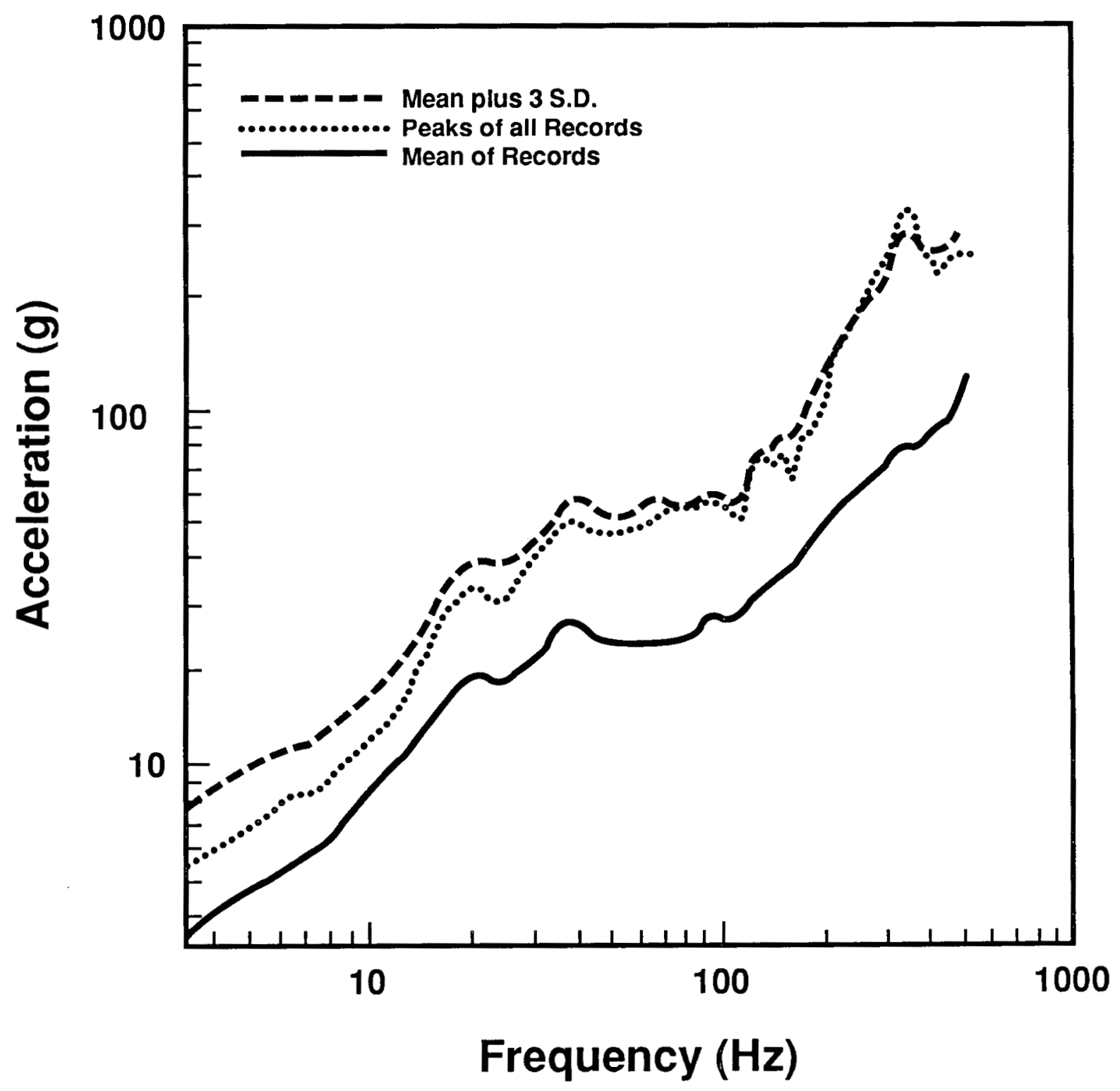

Figure II-83. Composite Response Spectra of Rail Car Structure for the Longitudinal Axis with Standard Draft Gear, 36- and 64-Tonne Cargo, 12.91 to $17.98 \mathrm{~km} / \mathrm{hr}$ Impact Velocity, and 38 Damping [MA80] .

heavy cargo. Data were obtained during rail shipment of a 45-tonne cargo consisting of an empty spent-fuel cask and associated equipment. The shipment was from Denver, CO, to Albuquerque, NM. Again, the data were presented as follows: for vibration, the 99-percentile levels of acceleration amplitude, and for shock, the shock response spectra. The spent-fuel transport cask was tied to the instrumented rail car by two cables. In addition, wood blocking was used to prevent longitudinal and transverse motion of the cask relative to the rail car. Accelerometers were mounted on the rail car structure to measure the input from the rail 


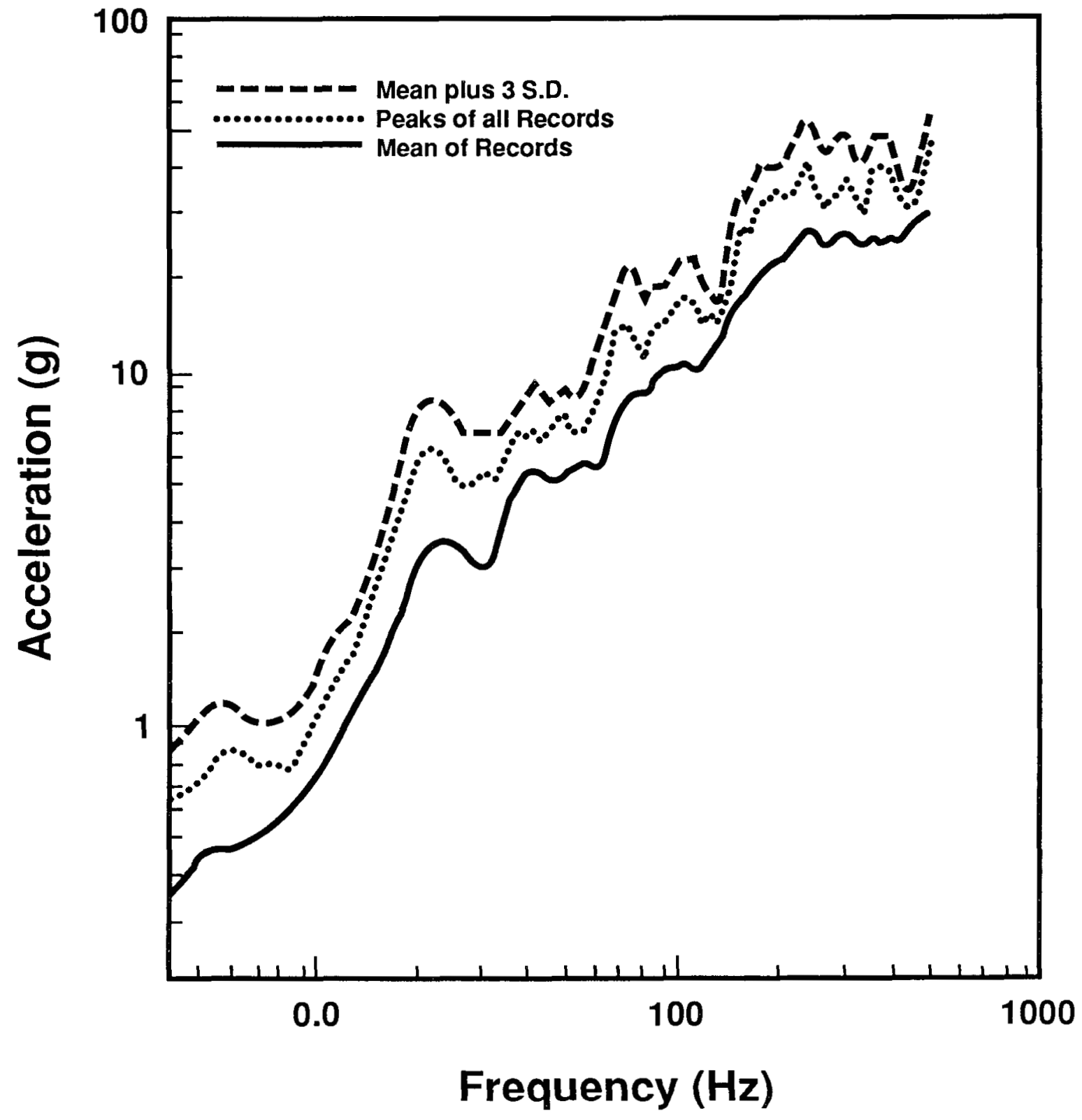

Figure II-84. Composite Response Spectra of Rail Car Structure for the Transverse Axis with Standard Draft Gear, 36- and 64-Tonne Cargo, 12.91 to $17.98 \mathrm{~km} / \mathrm{hr}$ Impact Velocity, and 38 Damping [MA80].

car to the cargo. No accelerometers were placed directly on the transport cask.

The reported vibration data are shown in Figure II-86 and appear to represent a generic definition of input to the cargo. Also shown again are the zero-to-peak acceleration amplitude levels that include 998 of all amplitudes measured in each frequency band. The shock spectra generated are shown in Figure II-87. The shock data from 16 specific shockgenerating events (e.g., crossing bridges and switches) were first reduced 


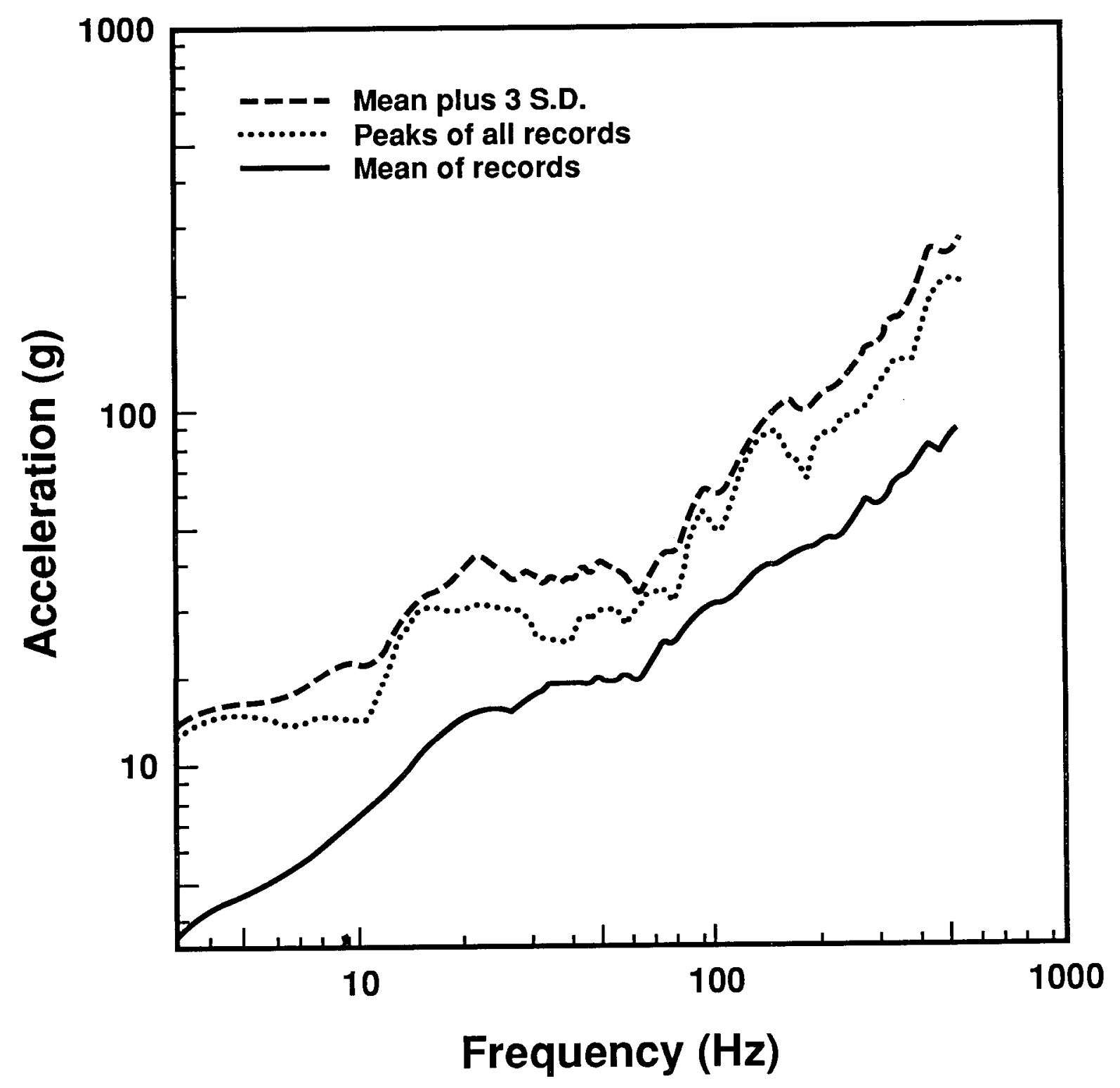

Figure II-85. Composite Response Spectra of Rail Car Structure for the Vertical Axis with Standard Draft Gear, 36- and 64-Tonne Cargo, 12.91 to $17.98 \mathrm{~km} / \mathrm{hr}$ Impact Velocity, and 38 Damping [MA80].

in response spectra format. Then the individual response spectra were combined and the results are the spectra shown. Note that the plotted results correspond to mean-plus-three standard deviation amplitude envelopes of the shock response spectra, a slightly different format than most of the results previously discussed. Unfortunately, no equivalent half-sine pulse was developed. 
Table II -20

Bounding Half-Sine Pulses for Rail-Coupling Shocks [MA80]

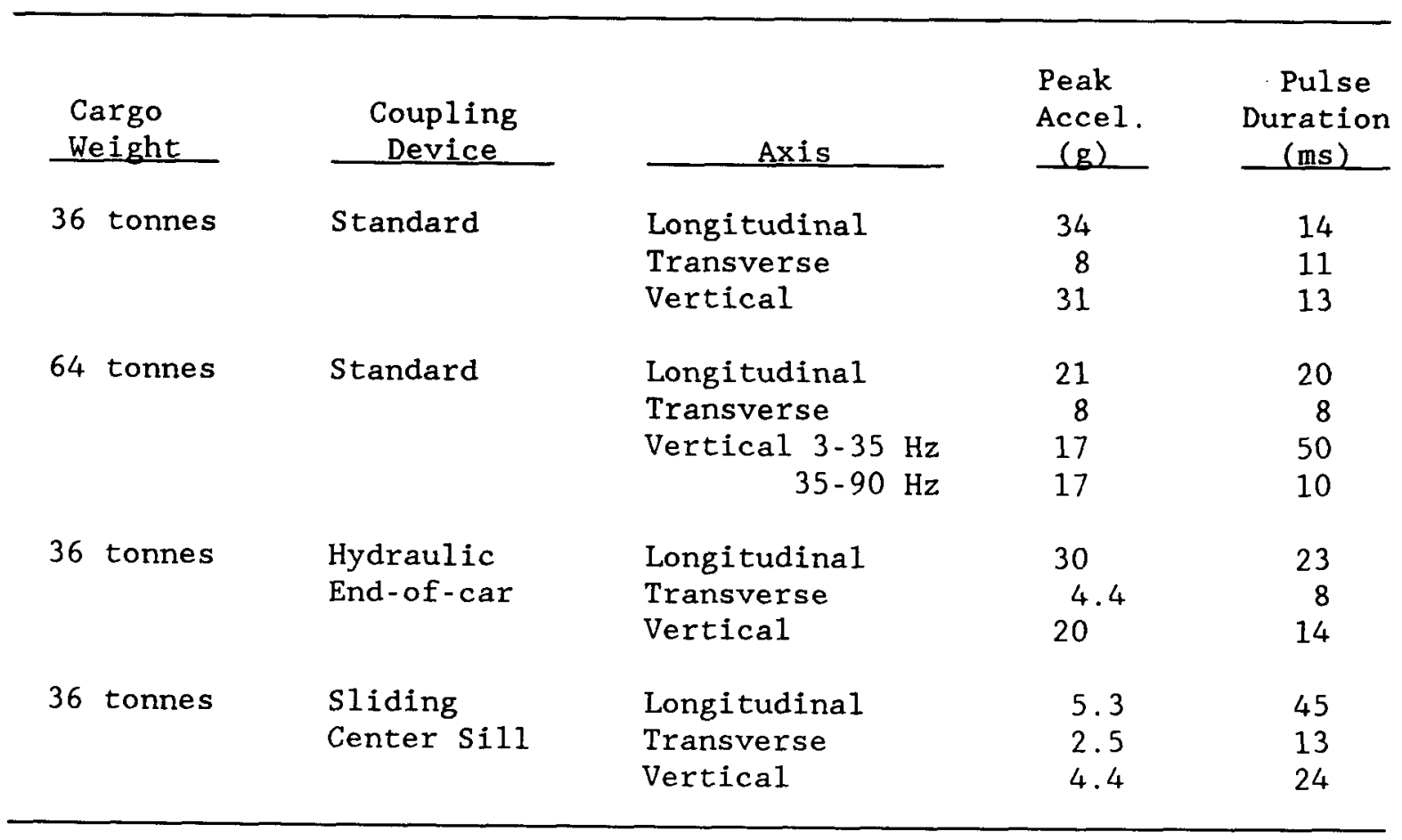

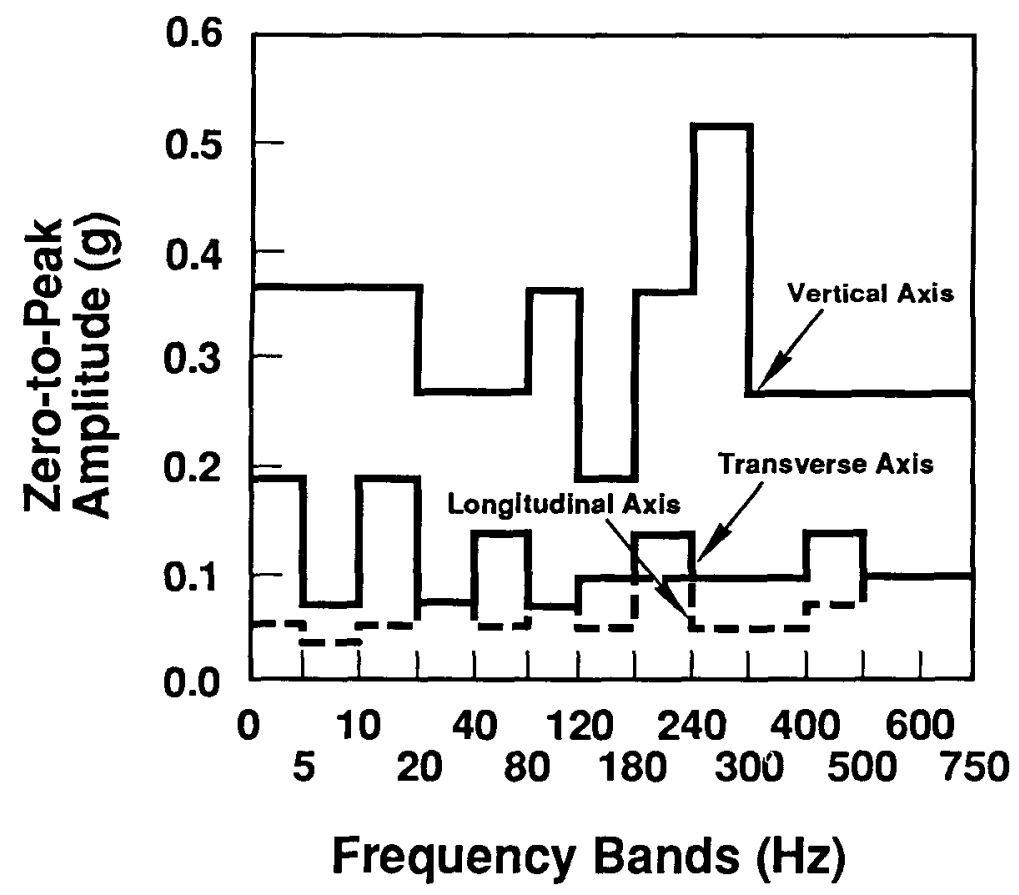

Figure II-86. Input to Cargo of Rail Vibration in (g) Including the 99 Percentile Level of Zero-to-Peak Amplitudes [MA82] 


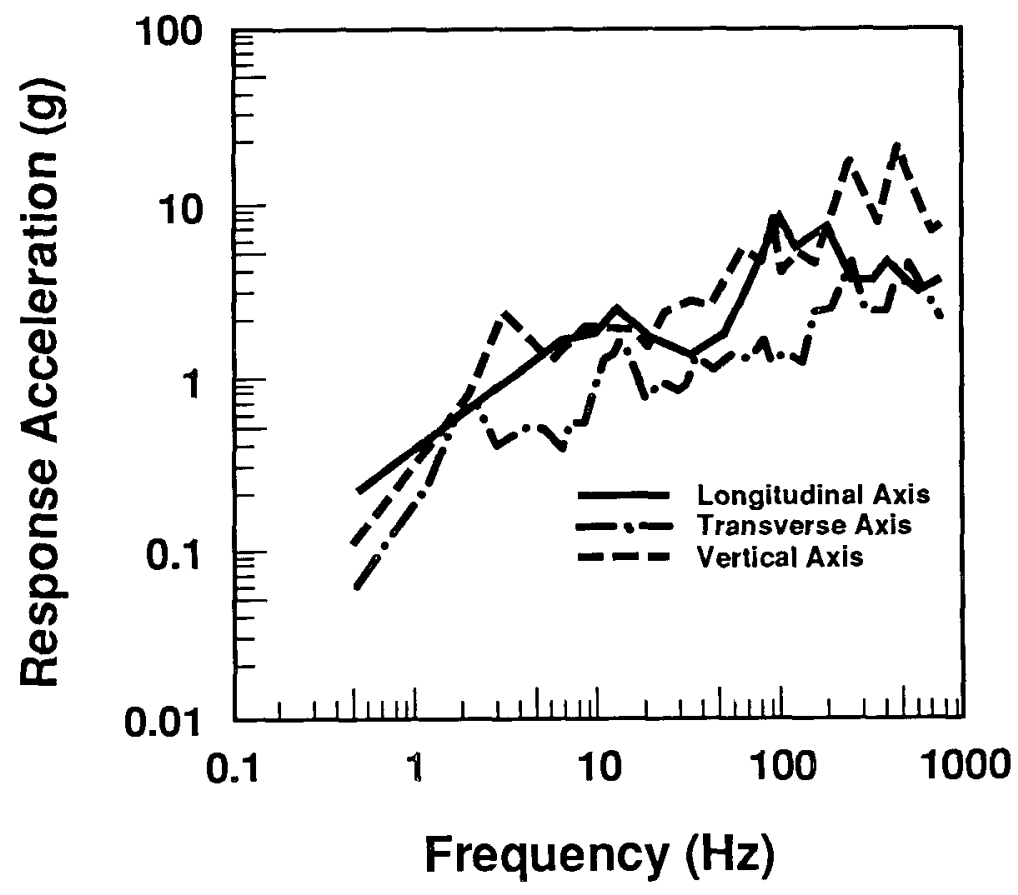

Figure II-87. Mean-Plus-Three Standard Deviation Amplitude Envelopes of Shock Response Spectra with 38 Damping [MA82]

\section{Fields}

Fields [FI83] has developed a model to simulate the dynamic behavior of transport casks and their rail car transporters during normal transport conditions. The computer model CARDS was validated on the basis of comparison with a previously conducted series of rail car coupling tests. The CARDS program is a complex, two-dimensional, multi-degree-of-freedom model that determines the horizontal, vertical, and rotational motion of both the cask and rail transporter during coupling operations. The goal was to provide a method for determining the forces transmitted to radioactive material packages so that input data for a broad range of package-tiedown structural assessments are available. The program is directed toward the rail car coupling environment because of its loading severity.

\section{Glass and Gwinn}

Glass and Gwinn [GL86] analyzed normal shock and vibration environments encountered during truck transportation of nuclear materials. They concluded that previous analyses (as discussed in the Draft ANSI N14.23 Standard [AN80]) have resulted in an overly conservative shock spectra description of the environments anticipated during normal transport conditions.

Glass and Gwinn used combined road simulation experiments, over-theroad tests, and the development of an analytical model. The system 
response to a known input was used to define frequency response functions for the system.

With the exception of Fields' analysis [FI78,83], efforts up to 1986 have been empirical. Some road and rail systems represent actual caskshipment configurations while others do not. These roads and rail systems are instrumented and tested in representative rail or road environments. Thus, a new predictive analytical method was developed and verified whereby the response of a specific system can be calculated for varying trailer damping, stiffness, geometry, and cargo mass parameters.

With the exception of Forest's analysis [F079], this appears to be the first over-the-road test program in which accelerometers were mounted directly on the cask as well as at the usual trailer system locations. (Some accelerometers were cask-mounted by Magnuson [MA80], but no data were reported.) Note that the least severe responses were measured on the cask lid. At that location, all modes except the first trailer bounce mode were damped out.

Glass and Gwinn [GL87] also recently reported shock and vibration data for the TRUPACT transport package. This Type B package was subjected to a series of normal transport conditions, including various road types and shock events. Accelerometers were located at the four mounting corners. Acceleration-time histories were reduced to power spectral densities that provide vibrational energy as a function of frequency.

The results indicate that the railroad grade crossing produced the maximum average vertical accelerations. Maximum accelerations for all observed events were less than $1 \mathrm{~g}$. Only vertical accelerations were reported. As opposed to earlier analyses, no clear distinction is made between shock and vibration.

A summary of the key experimental shock and vibration studies reviewed above is presented in Table II-21. Shock and vibration data presented and bounded in the following section are drawn from several of these studies.

\section{II.4.3 Simplified Bounding Input Loading Curves}

As shown in Section II.4.2 and Table II-20, much shock response data are available for truck and rail transport modes of spent-fuel casks. From this data, representative, simplified shock response curves and equivalent pulses have been selected for this analysis that are conservative, as well as meaningful, for generic spent-fuel casks transported by truck and rail car. These curves should be the most realistically bounding (on the basis of cask mass) of the available response spectra.

\section{Bounding Truck Shock Spectrum}

The bounding acceleration shock response spectrum for truck cask contents is shown in Figure II-88. The curve includes longitudinal, transverse, and vertical axes and is based on the 20- and 25-tonne cargo data by Magnuson [MA77a, MA77b, MA78]. These data are shown in Figures II-80 through II-82 for the three axes of motion. Note that the suggested 
Table II-21

Summary of Recent Shock and Vibration Studies

\begin{tabular}{|c|c|c|c|c|}
\hline Author & Year & Rail/Truck & Cargo & Comments \\
\hline Foley \& Gens & 1971 & Both & $\begin{array}{l}\text { 13.6-tonne shipping } \\
\text { cask }\end{array}$ & $\begin{array}{l}\text { Evaluates light cargo, particularly for } \\
\text { the rail-shock response spectrum. Dynamic } \\
\text { environments were similar for truck and } \\
\text { rail. }\end{array}$ \\
\hline Magnuson \& Wilson & 1977 & Both & $\begin{array}{l}\text { No-load to } 13.6 \text { tonnes; } \\
4.5 \text { tonne for rail- } \\
\text { coupling tests }\end{array}$ & $\begin{array}{l}\text { Evaluates 1ight cargo. Provides a summary } \\
\text { of earlier test results, including seven } \\
\text { different truck and tractor-trailer } \\
\text { configurations. Observed that shock } \\
\text { decreases as cargo weight increases. } \\
\text { Provides shock response spectra, bounding } \\
\text { single pulse representation, and rail- } \\
\text { coupling data. }\end{array}$ \\
\hline Magnuson & 1977 & Truck & $\begin{array}{l}20 \text {-tonne spent-fuel } \\
\text { shipping cask }\end{array}$ & $\begin{array}{l}\text { Provides shock response spectra and single } \\
\text { pulse representation. Evaluates heavy } \\
\text { cargo. }\end{array}$ \\
\hline Magnuson & 1978 & Truck & $\begin{array}{l}25 \text {-tonne spent-fuel } \\
\text { shipping cask }\end{array}$ & $\begin{array}{l}\text { Provides shock response spectra and single } \\
\text { pulse representation. Evaluates heavy } \\
\text { cargo. }\end{array}$ \\
\hline Forest & 1979 & Both & $\begin{array}{l}\text { Large concrete blocks } \\
\text { containing shipping } \\
\text { modules }\end{array}$ & $\begin{array}{l}\text { Accelerometers were placed on both } \\
\text { concrete and shipping modules. Data } \\
\text { reduction was incomplete. Rail-coupling } \\
\text { tests were performed. }\end{array}$ \\
\hline
\end{tabular}


Table II-2I

Summary of Recent Shock and Vibration Studies

(Concluded)

\begin{tabular}{|c|c|c|c|c|}
\hline Author & Year & Rail/Truck & Cargo & Comments \\
\hline Magnuson & 1980 & $\begin{array}{l}\text { Rail-coupling } \\
\text { only }\end{array}$ & 36- to 64-tonne casks & $\begin{array}{l}\text { Evaluates different rail car designs. } \\
\text { Provides shock response spectra and } \\
\text { single pulse representation. Evaluates } \\
\text { heavy cargo. }\end{array}$ \\
\hline Magnuson & 1982 & Rail & 45-tonne cargo & $\begin{array}{l}\text { Provides shock response spectra. } \\
\text { Includes no equivalent pulse. Evaluates } \\
\text { heavy cargo. }\end{array}$ \\
\hline Glass \& Gwinn & 1986 & Truck & $\begin{array}{l}14.1 \text {-tonne to } 16.8 \text {-tonne } \\
\text { casks }\end{array}$ & $\begin{array}{l}\text { Road Simulator was used. Predictive } \\
\text { analytical method was developed. } \\
\text { Includes no shock response spectra or } \\
\text { equivalent pulse. Work recognizes the } \\
\text { questionable interpretation of earlier } \\
\text { transportation data, particularly for } \\
\text { tiedown loads. }\end{array}$ \\
\hline Glass \& Gwinn & 1987 & Truck & $\begin{array}{l}\text { TRUPACT (Type B } \\
\text { package) } 22.7 \text {-tonne } \\
\text { gross weight }\end{array}$ & $\begin{array}{l}\text { Acceleration-time signals were reduced to } \\
\text { power spectral densities. These give the } \\
\text { vibrational energy as a function of shock } \\
\text { response spectra. Includes no equivalent } \\
\text { pulse. The Type B package was evaluated. }\end{array}$ \\
\hline
\end{tabular}




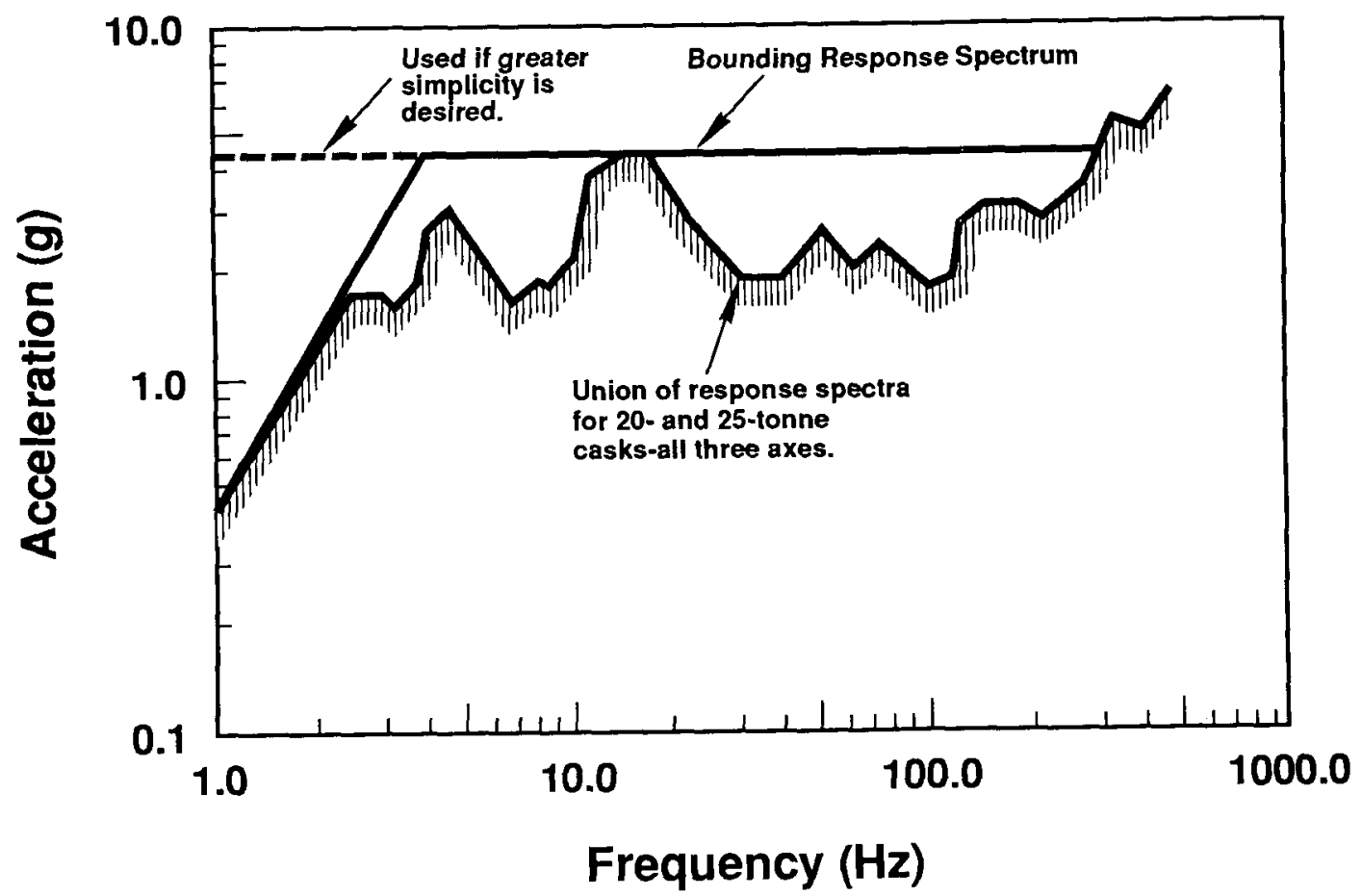

Figure II-88. Bounding Acceleration Shock Response Spectrum for 38 Damping on all Three Axes for Truck

bounding response spectrum in Figure II-88 does not always bound truck data in the 0- to 13.6-tonne range. This range in truck cask cargo weights is unrealistically low. Also shown in Figure II-88 is the union of data for longitudinal, transverse, and vertical axes; comparison with the bounding curve indicates that the bounding curve is generally quite conservative.

The bounding spectrum appears to be a bilinear curve (in the log-log plane) consisting of an initial linear portion followed by a line of constant $4.4-\mathrm{g}$ acceleration to a maximum frequency of $300 \mathrm{~Hz}$. For even greater simplicity, the dashed line may be used, but this may result in overly conservative results. However, if greater precision is needed, more complicated bounding curves can be constructed from Figure II-88. Also, individual bounding spectra for each of the three axes can be drawn in a similar manner using Figures II-80 through II-82.

\section{Bounding Rail Shock Spectrum}

The bounding rail shock spectrum shown in Figure II-89 consists of a straight line. Figure II-89 also shows the union of all rail data (see Figure II-87) presented by Magnuson [MA82]. The data are based on the realistic cargo mass for rail shipment of 45 tonnes and include longitudinal, transverse, and vertical axes. Note that a variety of shockgenerating events, such as crossing bridges and switches, are included in the bounded rail data. Coupling data, however, are not included. 


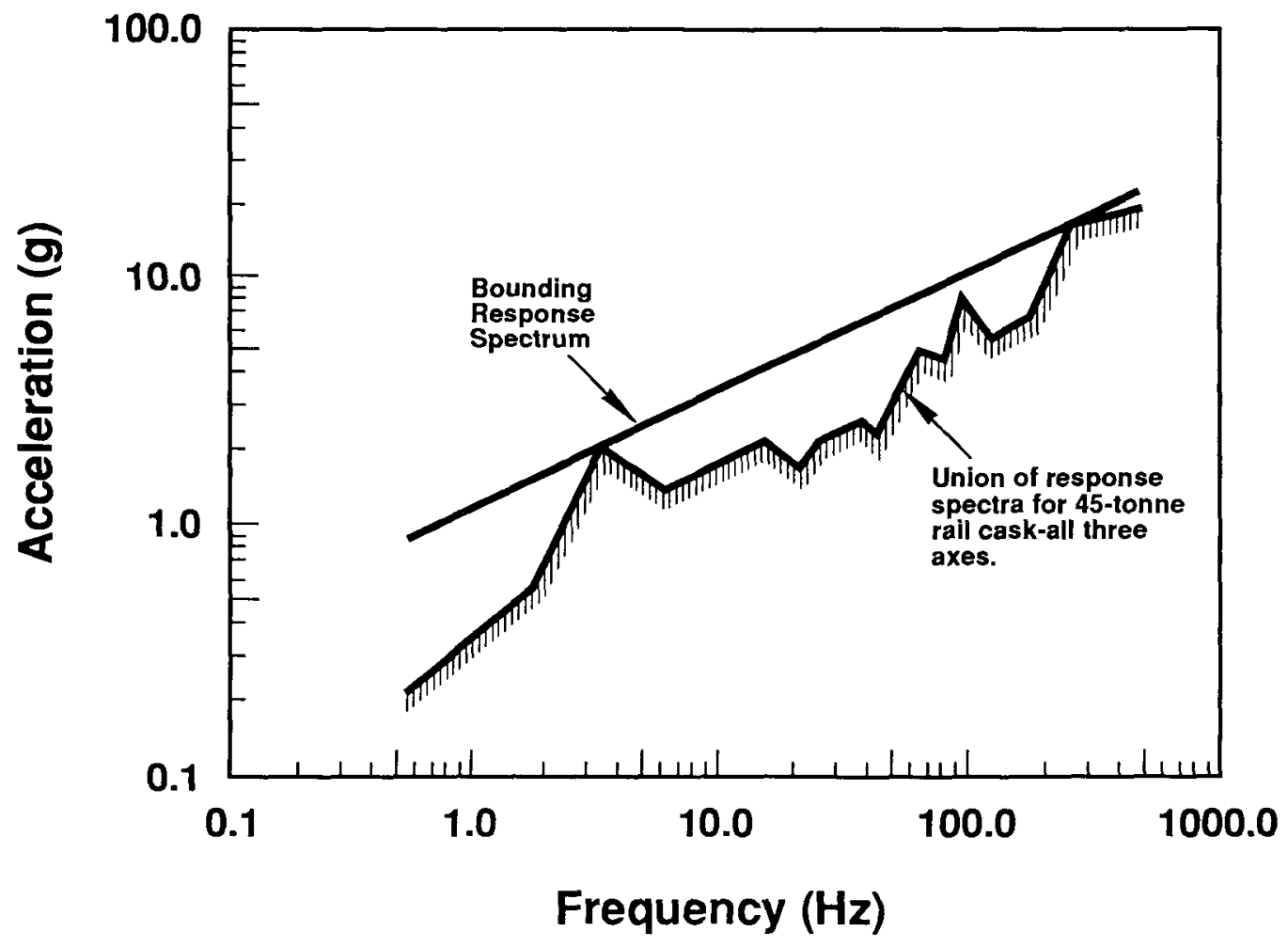

Figure II-89. Bounding Acceleration Shock Response Spectrum for 38 Damping on All Three Axes for Rail

\section{Bounding Rail-Coupling Shock Spectrum}

The bounding rail shock spectrum during coupling operations is considerably more severe than that for over-the-rail normal transport. The union of response spectra during coupling for all three axes (Figures II-83 through II-85) for the 36- to 64-tonne casks described by Magnuson [MA80] is shown in Figure II-90, along with a straight-line bounding response spectrum. Note that the coupling event represented by these curves corresponds to impacts in the range of -13 to $17.7 \mathrm{~km} / \mathrm{hr}$. These high accelerations may be overly conservative for spent-fuel transport events because normal coupling provides peak accelerations of approximately $10 \mathrm{~g}$, as shown in Figure II-90. Also, applying these data to linear-dynamic and quasi-static analyses would be overly conservative because these peak accelerations are of very short duration.

\section{Equivalent Pulse-Truck Shock}

With a graphical superposition procedure described by Magnuson [MA77a], a bounding response spectrum is plotted in Figure II-91 over the union of all response spectra from the 20-and 25-tonne truck cask cases. This spectrum is valid to slightly over $100 \mathrm{~Hz}$ and corresponds to a halfsine pulse of the form

$$
a(t)= \begin{cases}a_{0} \sin \left(\frac{\pi t}{\tau}\right) & 0<t<\tau \\ 0 & t<0 \text { and } t>\tau\end{cases}
$$




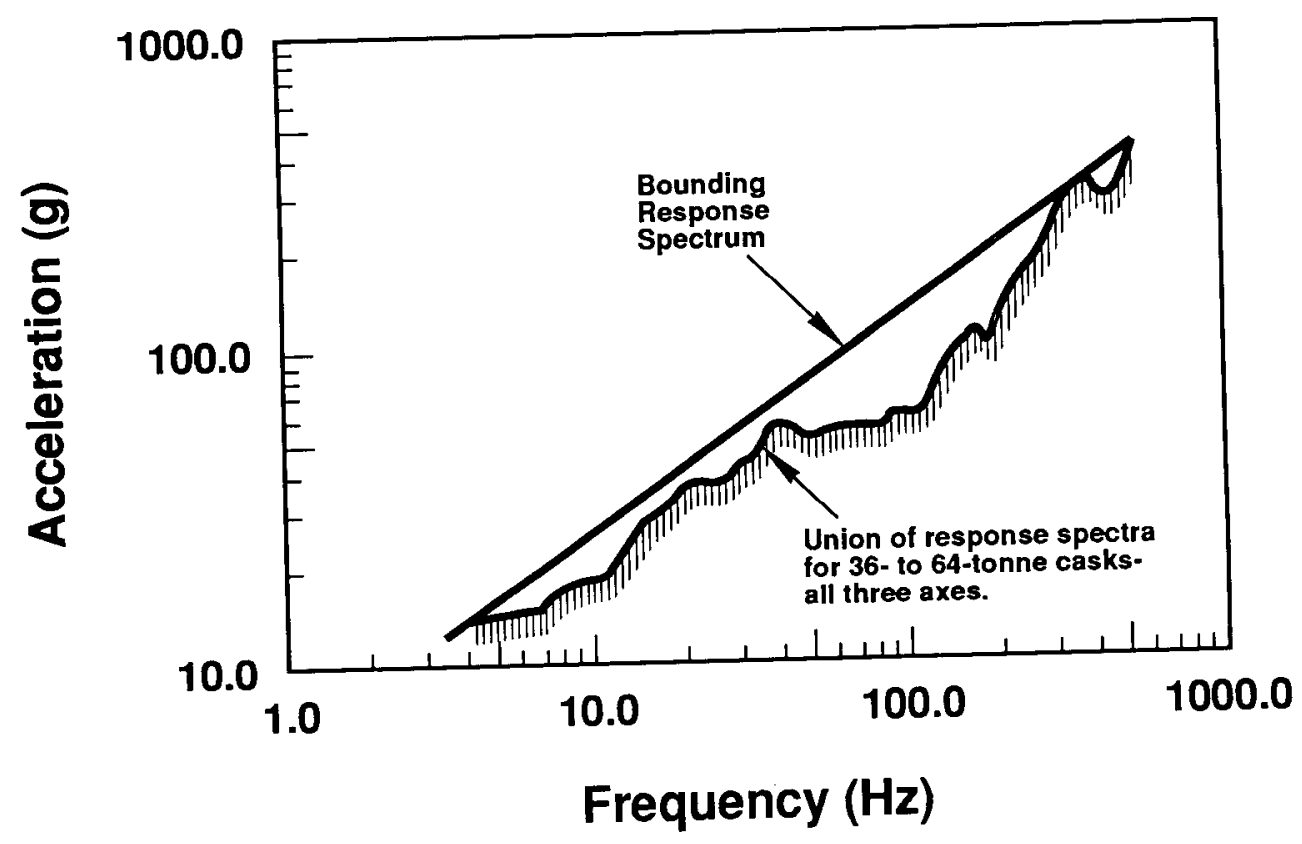

Figure II-90. Bounding Acceleration Shock Response Spectrum for 38 Damping on All Three Axes for Rail Coupling

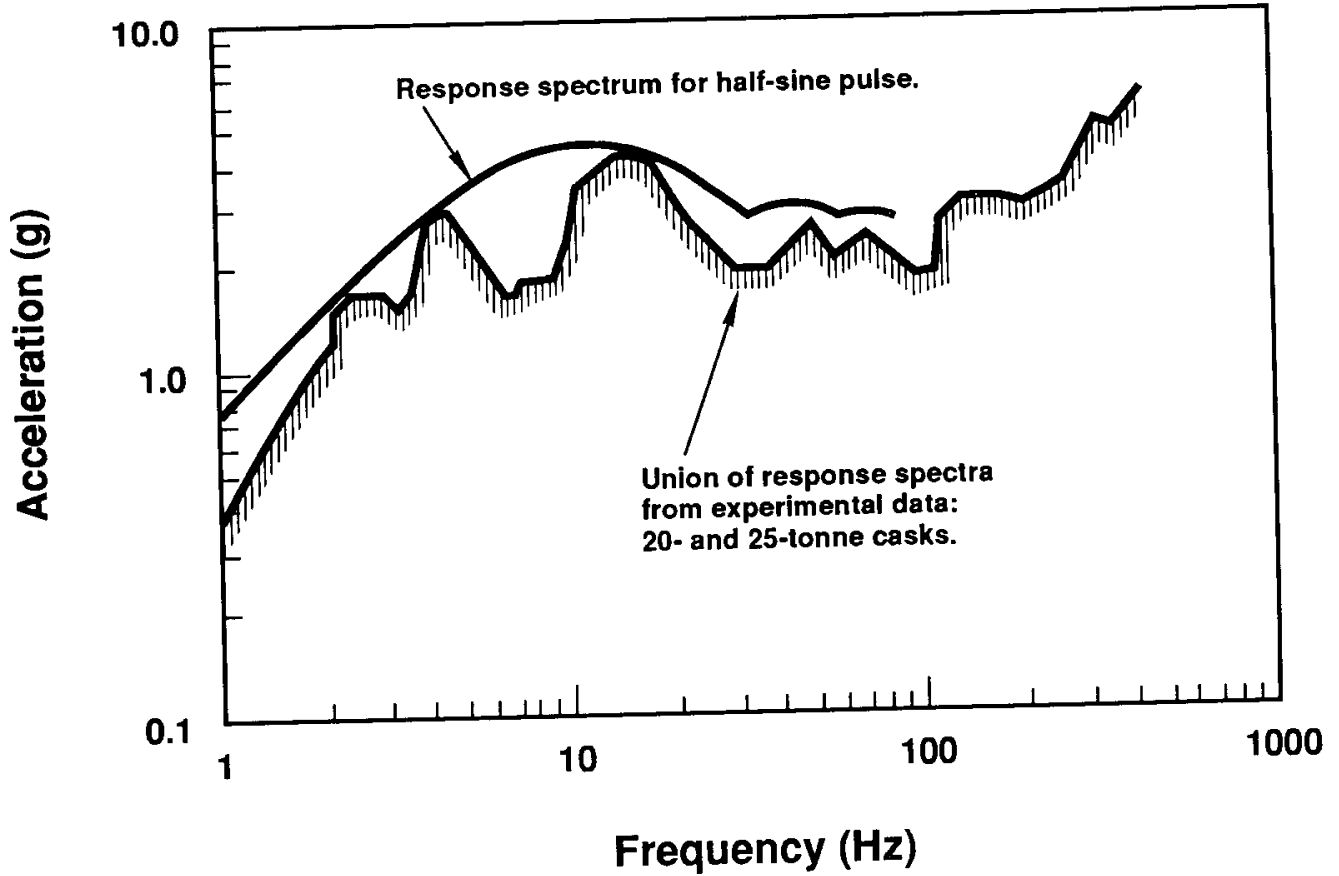

Figure II-91. Bounding Half-Sine Pulse Response Spectrum for Truck Shock 
where

$$
\begin{aligned}
t & =\text { time } \\
\tau & =\text { pulse duration } \\
a_{0} & =\text { peak magnitude }
\end{aligned}
$$

The equivalent pulse magnitude and duration for the half-sine pulse are

$$
\begin{aligned}
a_{0} & =2.7 \mathrm{~g} \\
\tau & =80.0 \mathrm{~ms} .
\end{aligned}
$$

\section{Equivalent Pulse Rail Shock}

When a procedure identical to the procedure for truck shock is used, constants for an equivalent half-sine pulse for rail shock loading conditions are

$$
\begin{aligned}
& a_{o}=2.4 \mathrm{~g} \\
& \tau=83.0 \mathrm{~ms} .
\end{aligned}
$$

The response spectrum corresponding to this half-sine pulse is shown in Figure II-92. It bounds the composite rail data presented above up to a frequency of $43 \mathrm{~Hz}$.

Equivalent Pulse/Rail-Coupling Shock

Here, an equivalent half-sine pulse has the constants

$$
\begin{aligned}
& a_{0}=33.2 \mathrm{~g} \\
& \tau=30.1 \mathrm{~ms} .
\end{aligned}
$$

Figure II-93 shows the bounding shock spectrum corresponding to this half-sine pulse, along with the composite spectra for rail-coupling experiments. The selected half-sine pulse is valid up to a frequency of $53 \mathrm{~Hz}$. Again, these rail-shock data will provide unreasonably large fuel loads if peak accelerations are analyzed independently of time.

\section{Bounding Truck Vibrations}

Bounding truck vibration data recommended for generic cask systems are shown in Figure II-94. This curve bounds data (see Figures II-77 through II-79) reported by Magnuson [MA77b, MA78] for longitudinal, transverse, and vertical axes. Also, the data correspond to realistic masses for truck casks in the range of 20 to 25 tonnes (vibration levels tend to be inversely related to cask mass). As discussed in Section II.4.2, the vibration data correspond to the maximum acceleration within the frequency bands shown occurring in the lower 998 of the peaks. The upper 18 of the peaks within a given frequency band correspond to shock. 


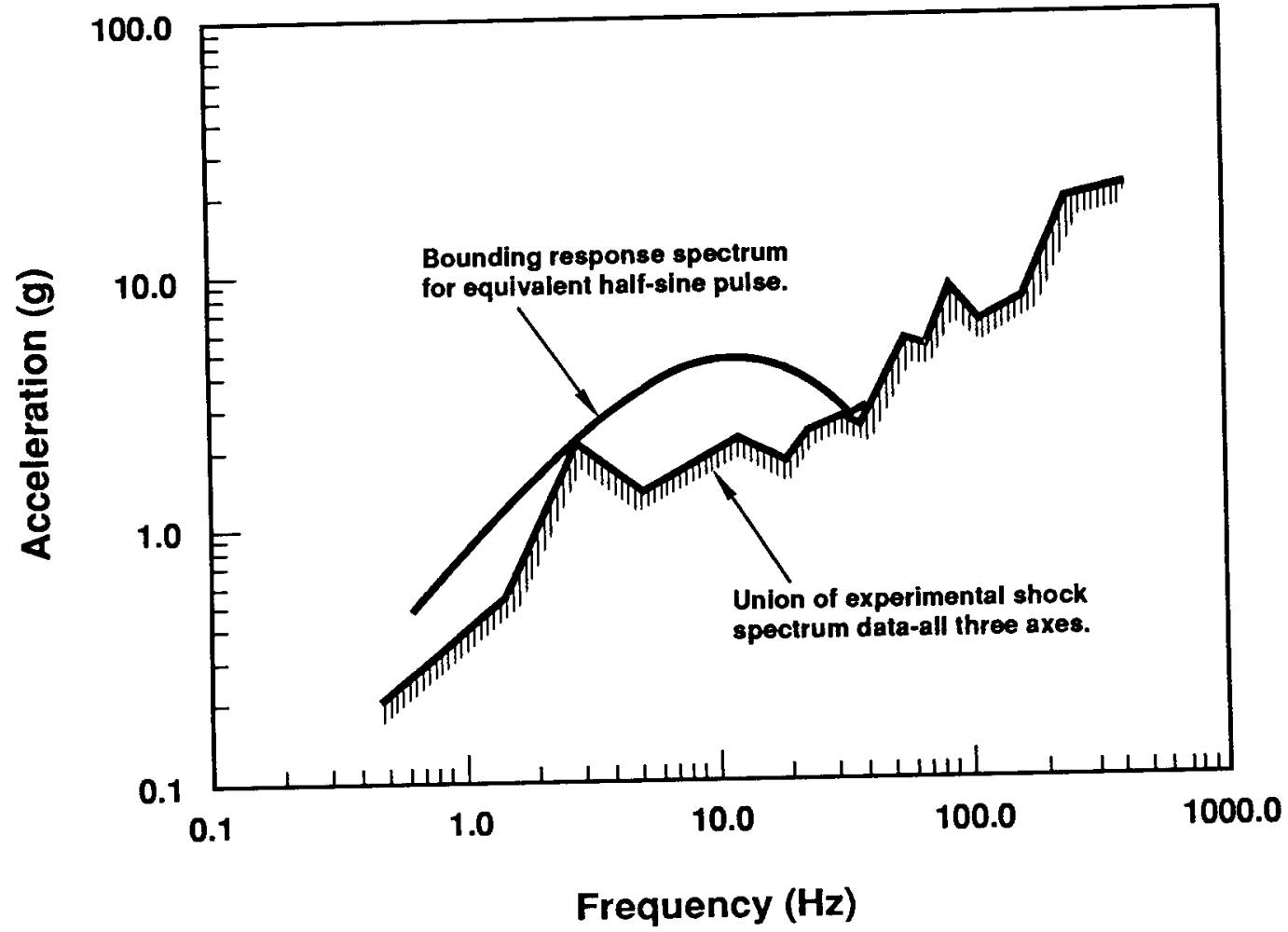

Figure II-92. Bounding Half-Sine Pulse Response Spectrum for Rail Shock

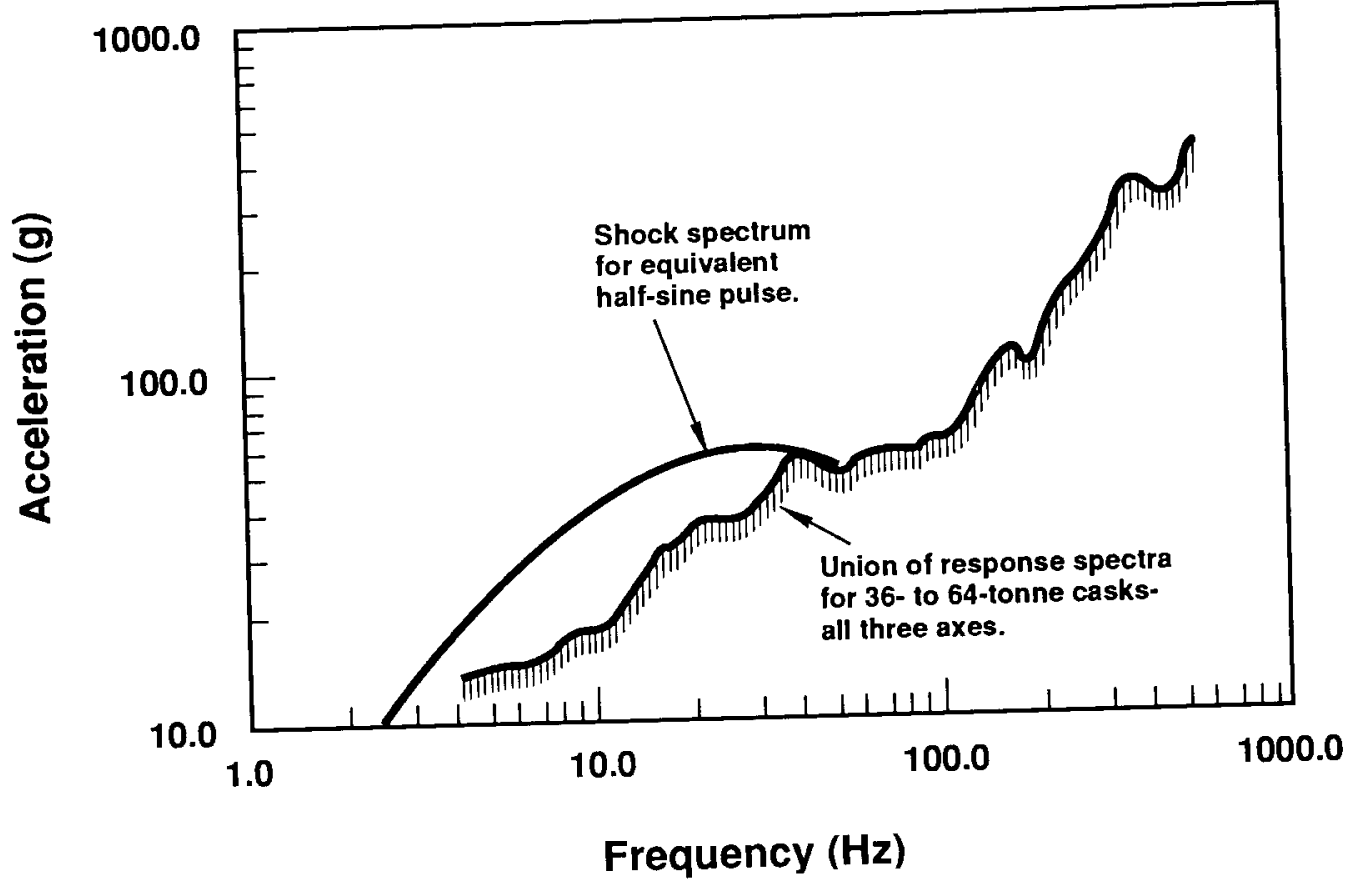

Figure II-93. Bounding Half-Sine Pulse Response Spectrum for Rail-Coupling Shock 


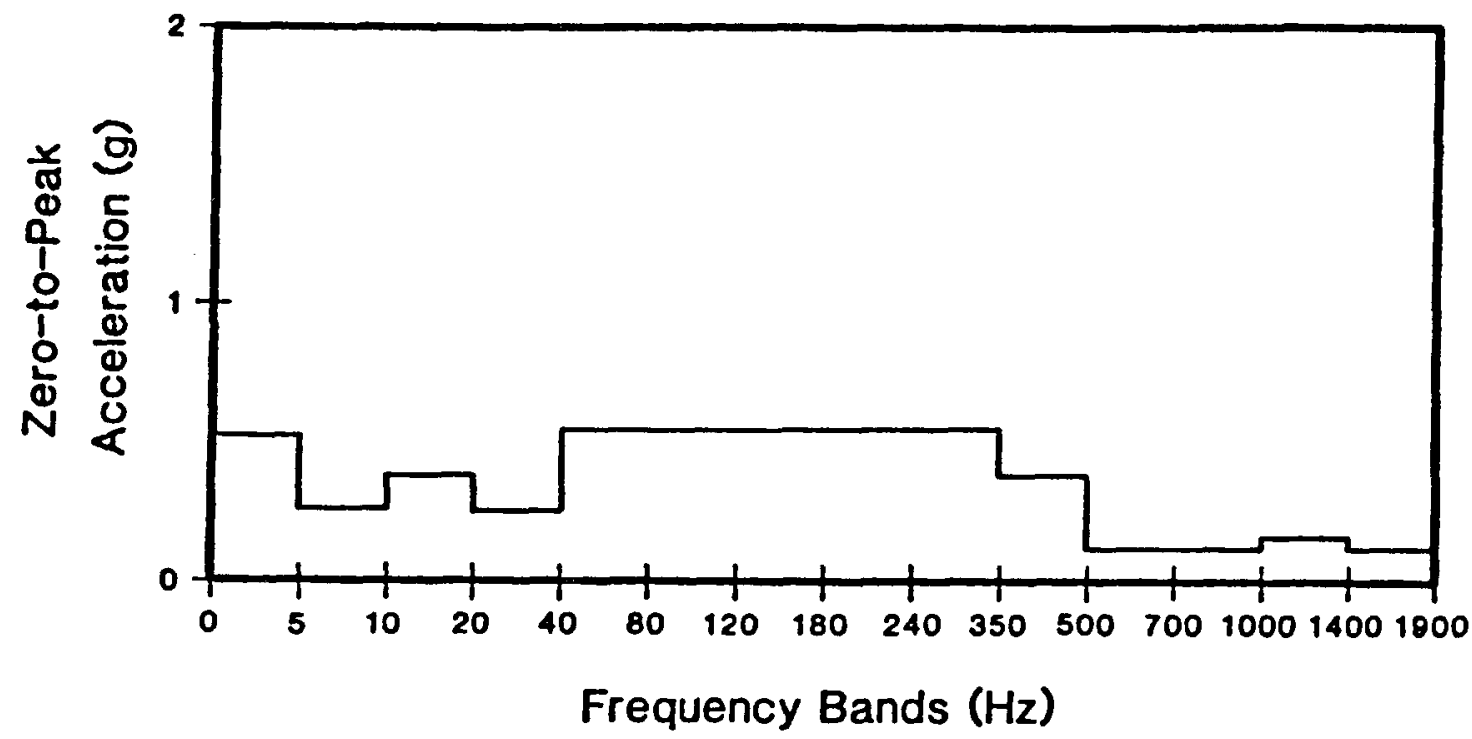

Figure II-94. Bounding Truck Vibration Data for All Three Axes

\section{Bounding Rail Vibrations}

Bounding rail vibration data recommended for generic cask systems are shown in Figure II-95 [MA82]. The data (see Figure II-86) correspond to vertical axis 99 -percentile peak accelerations in each frequency band. The bound shown in Figure II-95 is valid for all three axes because vertical accelerations bound those along longitudinal and transverse directions. These vibration levels are based on a realistic rail cask mass of 45 tonnes.

\section{5 Cask Therma1 Analyses}

\section{II.5.1 Introduction}

Thermal analyses of two of the four representative cask concepts were performed to characterize expected thermal environments of spent fuel in these generic casks. The primary purposes of these analyses were to quantify temperatures of spent fuel in the cask under both normal transport conditions and hypothetical accident transport conditions, and to contrast predicted temperatures with temperatures that are estimated to be necessary to cause thermally induced fuel failure mechanisms such as clad rupture.

Thermal analyses were performed on two representative casks: the lead-shielded truck cask with one PWR spent-fuel assembly and the leadshielded rail cask with an assumed payload of 21 PWR spent-fuel assemblies. Both cask concepts provide essentially the same thermal barrier as the corresponding all-steel casks. 


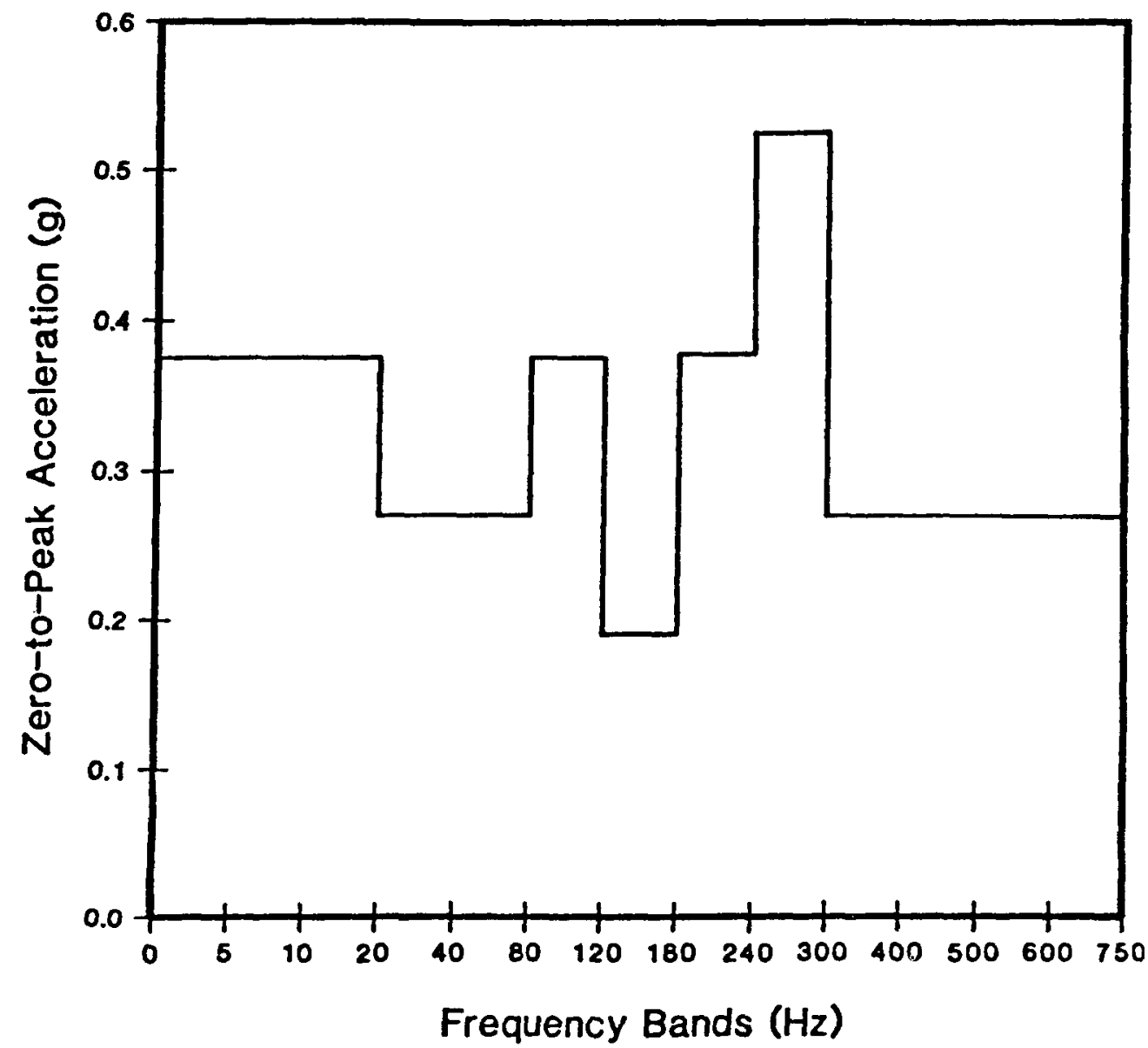

Figure II-95. Bounding Rail Vibration Data for All Three Axes

Two thermal environments were considered in the cask thermal response analyses:

1. Normal transport conditions in which the casks are assumed to be subjected to full solar input and an ambient temperature of $38^{\circ} \mathrm{C}$;

2. Regulatory fire accident conditions in which the casks are subjected to a totally engulfing, $800^{\circ} \mathrm{C}$, half-hour duration fire. The flame emissivity for this fire and the absorptivity of the outer surface of the cask are assumed to be 0.9 and 0.8 , respectively. The thermal responses of the cask during the fire and subsequent cool-down period are considered.

The thermal analyses of the lead-shielded cask concepts were performed using a finite element thermal analysis code that is two dimensional. A pie section representing one-eighth of each cask was modeled and assumed to be located at the axial midlength of the cask. The casks were also assumed to be oriented horizontally. Because the analyses 
were two dimensional, heat transfer in the cask axial direction was not modeled. Convection of the assumed helium-cavity gas was not modeled in the truck cask, but only (approximately) in the rail cask analyses. By neglecting axial heat transfer and only approximating (or ignoring) helium convection, a conservative estimate of the interior temperatures of the casks was obtained.

Even with a conservative modeling approach, acceptable thermal response is predicted for the lead-shielded truck cask with a spent-fuel decay heat generation rate of up to approximately $3.0 \mathrm{~kW}$. A lower decay rate of $1.0 \mathrm{~kW}$ per assembly was predicted to lead to acceptable thermal response for the representative lead-shielded rail cask. Higher estimates of the thermal performance of the casks would probably be achieved with a more detailed three-dimensional thermal analysis of each cask and modification of the cask designs to include fins and perhaps neutron shielding. Scoping results were the only goal of these thermal response analyses.

\section{II.5.2 Cask Thermal Analysis Models}

The thermal analyses of the representative lead-shielded casks were performed using the finite element method rather than the more common finite difference method. The finite element method was used because of the analyst's considerable experience with the technique and the immediate availability of an appropriate finite element thermal analysis code.

The finite element method is a flexible analysis technique that enables rapid modeling of complex $1=1$ problem geometries. Heat transfers by conduction and radiation are readily implemented. The finite element thermal analysis program that was available for the present analyses can only approximately model convection in the cask interior. As discussed below, however, convection is not expected to be a significant effect in either of the analyzed casks as long as they are assumed to be oriented horizontally.

The thermal response analyses of the two representative casks were performed with the TOPAZ2D finite element thermal analysis code [SH86]. TOPAZ2D is capable of solving thermal response problems in two-dimensional planar and axisymmetric geometries. Problems that are solvable with TOPAZ2D may involve steady-state or time-dependent response and temperature-dependent material response (with phase change). The code models heat transfer by conduction, convection, and radiation. It outputs a user-readable summary of the problem results and data sets that may be plotted with the finite element data post-processing program, ORION [HA83].

Before the representative cask thermal analyses were performed, several simple thermal analysis problems for which theoretical solutions are available were modeled and analyzed with TOPAZ2D to verify code functioning and its implementation into the source-term methodology. These simple test problems included features such as internal heat generation, temperature-dependent material properties, heat transfer by conduction and radiation, and time-dependent response. Three of these test problems and the results predicted by TOPAZ2D are briefly described below. 


\section{Transient Response of a Nonlinear Solid}

The first problem considered is discussed in the TOPAZ2D User's Manual. TOPAZ2D was used to calculate the transient response of a onedimensional slab of thickness 1 that is heated on the front surface by a constant and uniform heat $\mathrm{flux} q$, and insulated on the rear surface. This problem is illustrated in Figure II-96. The slab material is assumed to be homogeneous and isotropic, with nonlinear conductivity $K(T)$ and heat capacity $\mathrm{C}(\mathrm{T})$ given by the following equations (with temperature in ${ }^{\circ} \mathrm{C}$ ):

$$
\begin{aligned}
& \mathrm{K}(\mathrm{T})=\mathrm{K}_{\mathrm{o}}\left[1+\beta\left(\mathrm{T}-\mathrm{T}_{\mathrm{o}}\right)\right] \\
& \mathrm{C}(\mathrm{T})=\mathrm{C}_{\mathrm{o}}\left[1+\beta\left(\mathrm{T}-\mathrm{T}_{\mathrm{o}}\right)\right],
\end{aligned}
$$

where

$$
\begin{aligned}
\mathrm{q} & =7.94 \times 10^{6} \mathrm{~J} / \mathrm{m}^{2} \mathrm{~s} \\
\mathrm{l} & =0.00955 \mathrm{~m} \\
\beta & =0.00278^{\circ} \mathrm{C}^{-1} \\
\mathrm{~K}_{\mathrm{O}} & =1.728 \mathrm{~J} / \mathrm{m}-{ }^{\circ} \mathrm{C}-\mathrm{s} \\
\mathrm{C}_{\mathrm{O}} & =209.2 \mathrm{~J} / \mathrm{kg}^{\circ}{ }^{\circ} \mathrm{C} .
\end{aligned}
$$

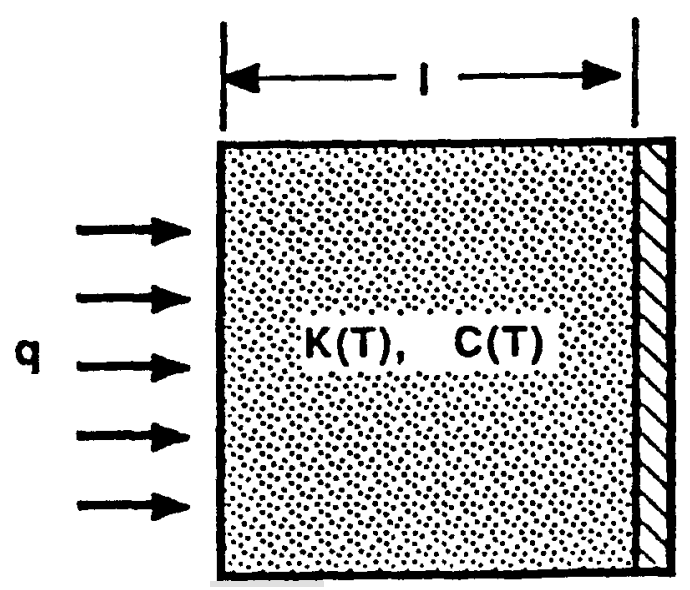

Figure II-96. Nonlinear Slab Thermal Analysis Problem

The slab material density was $8009 \mathrm{~kg} / \mathrm{m}^{3}$, and its initial temperature was $21.3^{\circ} \mathrm{C}$.

The transient response for this slab can be analytically solved for the special case in which the ratio $\alpha=\mathrm{K} / \rho \mathrm{C}$ is a constant [ST70]. Here the solution is

$$
\frac{\beta \mathrm{T}_{\mathrm{o}} \theta^{2}}{2}+\theta=\frac{\mathrm{ql}}{\mathrm{K}_{\mathrm{o}} \mathrm{T}_{0}}\left[\frac{\mathrm{X}^{2}}{2}-\mathrm{X}+\frac{1}{3}+\mathrm{F}-\frac{2}{\pi^{2}} \mathrm{n}_{=1}^{\infty} \frac{1}{\mathrm{n}^{2}} \cos \mathrm{n} \pi \mathrm{Xe}^{-\mathrm{Fn}^{2} \pi^{2}}\right]
$$


where

$$
\begin{aligned}
& \theta=\left(\mathrm{T}-\mathrm{T}_{\mathrm{o}}\right) / \mathrm{T}_{\mathrm{O}} \\
& \mathrm{X}=\mathrm{x} / 1 \\
& \mathrm{~F}=\alpha \mathrm{t} / 1^{2} .
\end{aligned}
$$

The finite element model of this problem involved 40 quadrilateral elements through the slab's thickness. The thickness of the first 10 elements from the heated slab surface was $0.0305 \mathrm{~mm}$. Larger zoning was used for the remainder of the problem mesh.

The transient solution of this problem was continued to a response time of $10^{-4} \mathrm{hr}$. Table II-22 compares the TOPAZ2D slab surface temperature predictions with those estimated with the analytical solution to this problem. The TOPAZ2D predictions agree with the analytical results to within better than 18 .

\begin{tabular}{|c|c|c|}
\hline $\begin{array}{l}\text { Time } \\
(\mathrm{hr})\end{array}$ & $\begin{array}{l}\text { Analytical } \\
\text { Result }\left({ }^{\circ} \mathrm{C}\right)\end{array}$ & $\begin{array}{c}\text { TOPAZ2D } \\
\text { Prediction }\left({ }^{\circ} \mathrm{C}\right)\end{array}$ \\
\hline $1.0 \times 10^{-6}$ & 197.49 & 196.49 \\
\hline $1.0 \times 10^{-5}$ & 394.28 & 390.87 \\
\hline $1.0 \times 10^{-4}$ & 755.42 & 748.58 \\
\hline
\end{tabular}

Table II -22

Nonlinear Slab Temperature Predictions

\section{Steady Response Example with a Heat Source}

The second test problem involves a slab of material of thickness $2 \mathrm{~L}_{1}$ that generates heat volumetrically at a constant rate $q$. The slab is encased on its two faces by another material of thickness ( $L_{2}-L_{1}$ ) on each face. The constant conductivities of the two materials are designated $\mathrm{K}_{1}$ and $\mathrm{K}_{2}$. The external surface of the outer material in the problem geometry is assumed to transfer heat to its environment, which has a temperature of $T_{\infty}$, at a rate characterized by a constant heat transfer coefficient, $h$. Figure II-97 illustrates this analysis problem.

The analytical solution for the steady-state temperature distributions in each slab is given by the following [AR66]:

In the central slab at location $\mathrm{x}$,

$$
\mathrm{T}_{1}(\mathrm{x})=\mathrm{T}_{\infty}+\frac{\mathrm{qL}_{1}^{2}}{2 \mathrm{~K}_{1}}\left\{1-\left(\frac{\mathrm{x}^{2}}{\mathrm{~L}_{1}}\right)-2\left(\frac{\mathrm{K}_{1}}{\mathrm{~K}_{2}}\right)+2 \frac{\mathrm{L}_{2} \mathrm{~K}_{1}}{\mathrm{~L}_{1} \mathrm{~K}_{2}}\left(1+\frac{\mathrm{K}_{2}}{\mathrm{hL}_{2}}\right)\right\}
$$




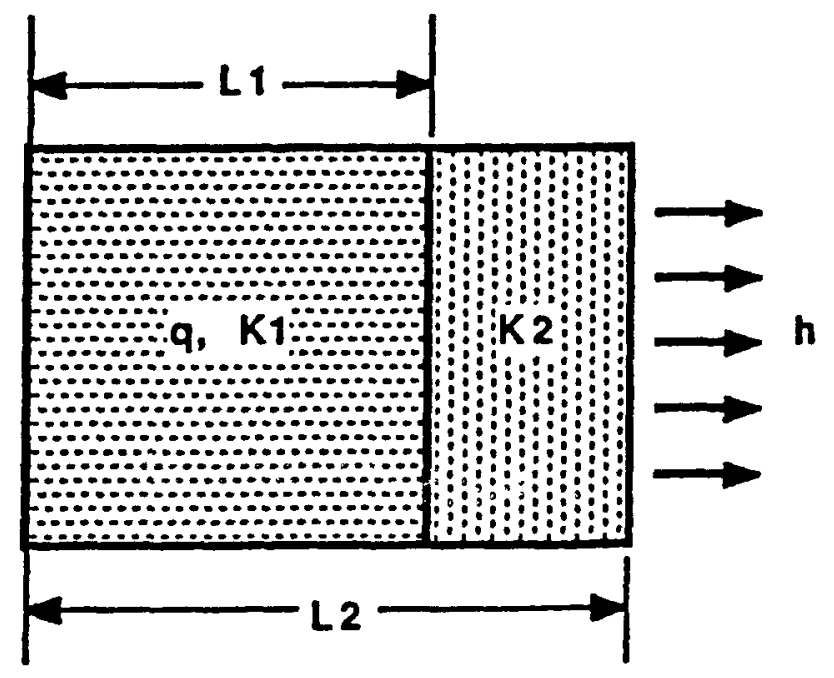

Figure II-97. Layered Slabs with Internal Heat Generation

In the outer slab region,

$$
\mathrm{T}_{2}(\mathrm{x})=\mathrm{T}_{\infty}+\frac{\mathrm{qL}_{1}^{2}}{\mathrm{~K}_{2}}\left\{-\left(\frac{\mathrm{x}}{\mathrm{L}_{1}}\right)+\frac{\mathrm{L}_{2}}{\mathrm{~L}_{1}}\left(1+\frac{\mathrm{K}_{2}}{\mathrm{hL}_{2}}\right)\right\},
$$

where

$$
\begin{aligned}
\mathrm{L}_{1} & =0.305 \mathrm{~m} \\
\mathrm{~L}_{2} & =0.457 \mathrm{~m} \\
\mathrm{~T}_{\infty} & =37.8{ }^{\circ} \mathrm{C} \\
\mathrm{K}_{1} & =69.1 \mathrm{~J} / \mathrm{m}-{ }^{\circ} \mathrm{C}-\mathrm{s} \\
\mathrm{K}_{2} & =276 \mathrm{~J} / \mathrm{m}^{\circ}{ }^{\circ} \mathrm{C}-\mathrm{s} \\
\mathrm{h} & =568 \mathrm{~J} / \mathrm{m}^{2}-{ }^{\circ} \mathrm{C}-\mathrm{s} \\
\mathrm{q} & =20.7 \times 10^{3} \mathrm{~J} / \mathrm{m}^{3}-\mathrm{s} .
\end{aligned}
$$

With these parameter values, $\mathrm{T}_{1}(\mathrm{x})=66.25-45.7 \mathrm{x}^{2}$, and $\mathrm{T}_{2}(\mathrm{x})=59.31$ $22.9 \mathrm{x}$. In both equations, $\mathrm{x}$ is restricted to values applicable for the particular slab in question.

This problem was modeled with TOPAZ2D using 10 equally sized quadrilateral elements through the central slab's half thickness and 5 elements through the outer slab. Symmetry was invoked to enable modeling of only half the problem. A comparison of the predicted and analytically derived temperatures through thickness of the two slabs is given in Table II-23. The TOPAZ2D results agree with the analytical solution to five digits. 
Table II -23

Slab with Heat Source-Temperature Predictions

\begin{tabular}{lcc}
\hline $\begin{array}{c}\text { Location } \\
(\mathrm{m})\end{array}$ & $\begin{array}{c}\text { Analytical } \\
\left.\text { Result }{ }^{\circ} \mathrm{C}\right)\end{array}$ & $\left.\begin{array}{c}\text { Topaz2D } \\
\text { Prediction }\end{array}{ }^{\circ} \mathrm{C}\right)$ \\
\cline { 3 - 3 } 0.00 & 66.25 & 66.25 \\
0.061 & 65.69 & 65.69 \\
0.152 & 62.78 & 62.78 \\
0.213 & 59.44 & 59.44 \\
0.305 & 52.36 & 52.36 \\
0.396 & 50.28 & 50.28 \\
0.457 & 48.89 & 48.89 \\
\hline
\end{tabular}

Radiative Exchange Between Conducting Fins

This test problem is suggested in the TOPAZ2D User's Manual. It involves estimation of the temperatures along the surfaces of two 1 -m-long, $0.05-\mathrm{m}$-thick conducting fins joined at a common base and oriented at $45^{\circ}$ with respect to each other. The base of the fins is assumed to be held at a constant temperature of $1 \mathrm{~K}$. The fin surface emissivity is taken as 0.5 and fin material conductivity is assumed to be $2.835 \times 10^{-7} \mathrm{~W} / \mathrm{m}-\mathrm{K}$; the problem geometry is shown schematically in Figure II-98.

The analytical solution to this problem is provided by Sparrow et al. [SP61]. For brevity, only the analysis results are cited here.

In the TOPAZ2D mode1 of this problem, each fin was assembled from 10 quadrilateral finite elements. View factors for the element surfaces that couple between the two fins were calculated with the FACET code. Using these factors, the TOPAZ2D model was analyzed for the surface temperatures along the length of the fin starting at the fin base (location 0.0) up to the fin tip (location 1.0). A comparison of the analytical and TOPAZ2D temperature predictions along the fins is given in Table II-24. The TOPAZ2D predictions agree with the analytical results to within approximately 18 .

\section{Cask Models}

The finite element models of the two generic lead-shielded casks were assembled from four-node quadrilateral elements using the PATRAN code [PD84]. The finite element mesh for the lead-shielded truck cask is shown in Figure II-99; the mesh for the lead-shielded rail cask is shown in Figure II-100. Global material zones of each model are also shown in these figures. 


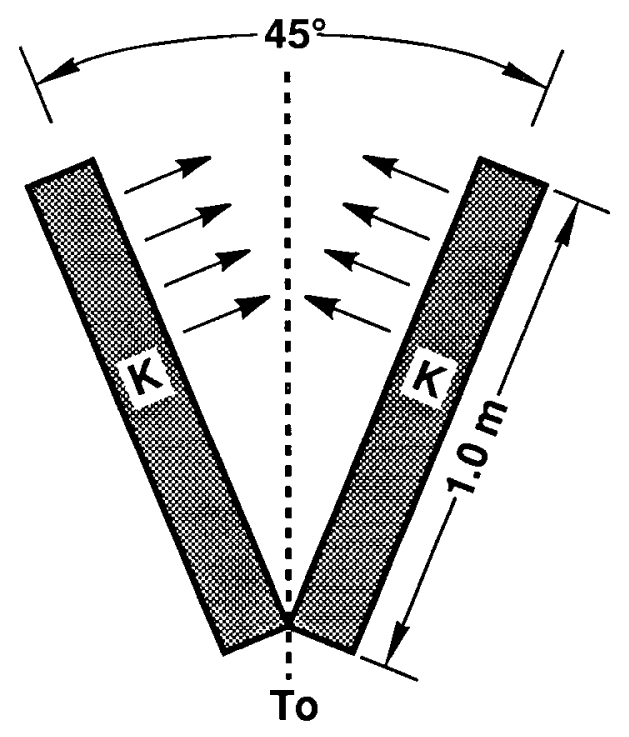

Figure II-98. Radiative Exchange Between Conducting Fins

Table II -24

Temperature Predictions of Radiative Heat Exchange Between Fins

\begin{tabular}{ccc}
\hline $\begin{array}{c}\text { Location } \\
(\mathrm{m})\end{array}$ & $\begin{array}{c}\text { Analytical } \\
\text { Result }(\mathrm{K})\end{array}$ & $\begin{array}{c}\text { Topaz2D } \\
\text { Prediction }(\mathrm{K})\end{array}$ \\
0.00 & 1.0000 & 1.0000 \\
0.2 & 0.9136 & 0.9080 \\
0.4 & 0.8483 & 0.8428 \\
0.6 & 0.8027 & 0.7999 \\
0.8 & 0.7752 & 0.7733 \\
1.0 & 0.7659 & 0.7640 \\
\hline
\end{tabular}

The one-eighth pie section finite element models are representative of the casks at the axial midlengths. With the casks horizontally oriented, their interior temperatures are anticipated to be highest at axial midlength because the decay heat profile along the length of the spent-fuel assemblies is maximum in this vicinity [EP86]. According to EPRI [EP86], the decay heat generation rate is estimated to be a factor of 1.2 greater near the axial midlength of a typical PWR spent-fuel assembly than it would be if the decay heat generation rate were uniform along the entire active length of the assembly. Experimental evidence from measured decay heat profiles [EP86] confirms the power-peaking factor estimate of 1.2. 

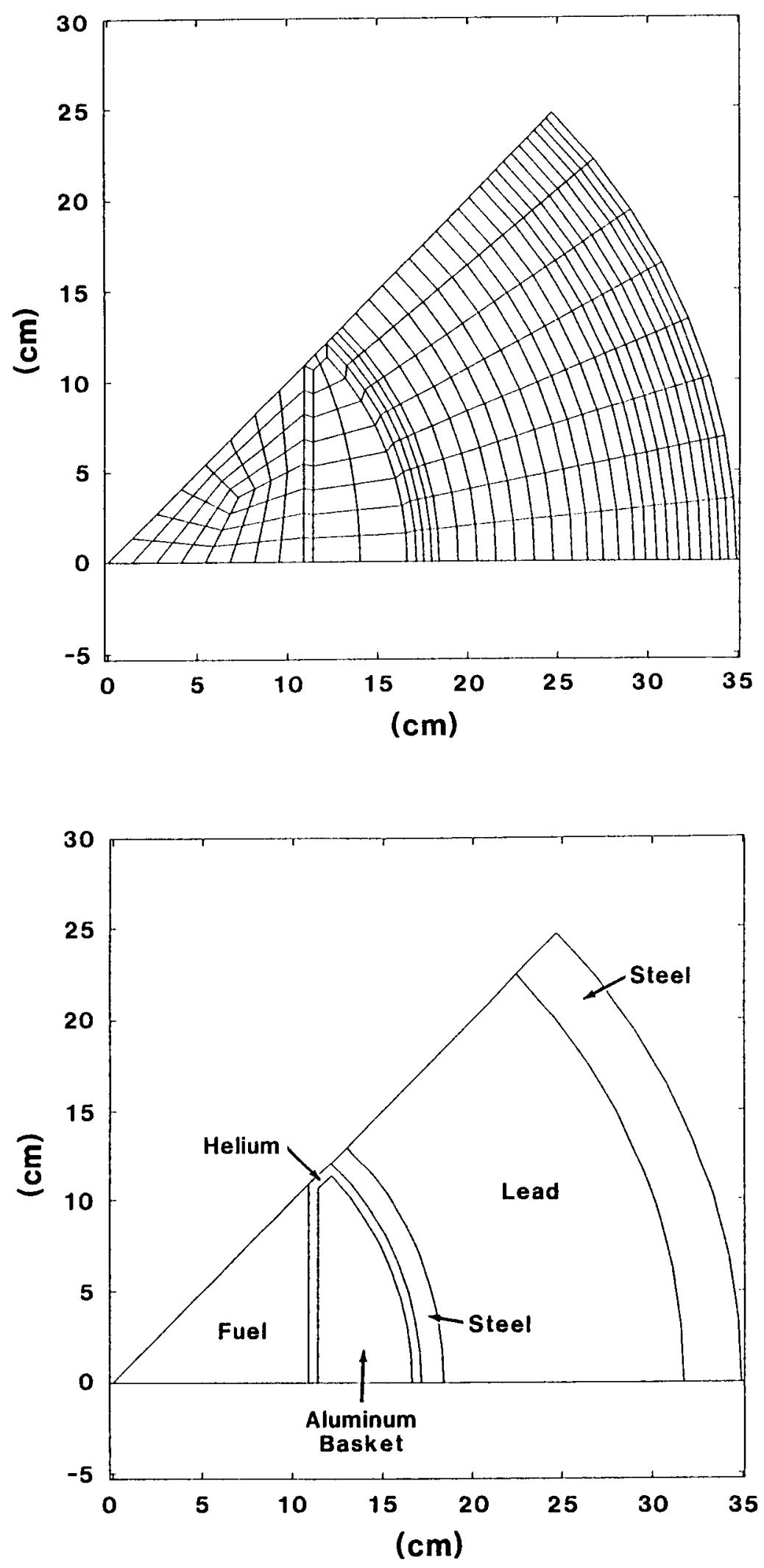

Figure II-99. Finite Element Mesh (top) and Material Zones (bottom) for the Lead-Shielded Truck Cask 

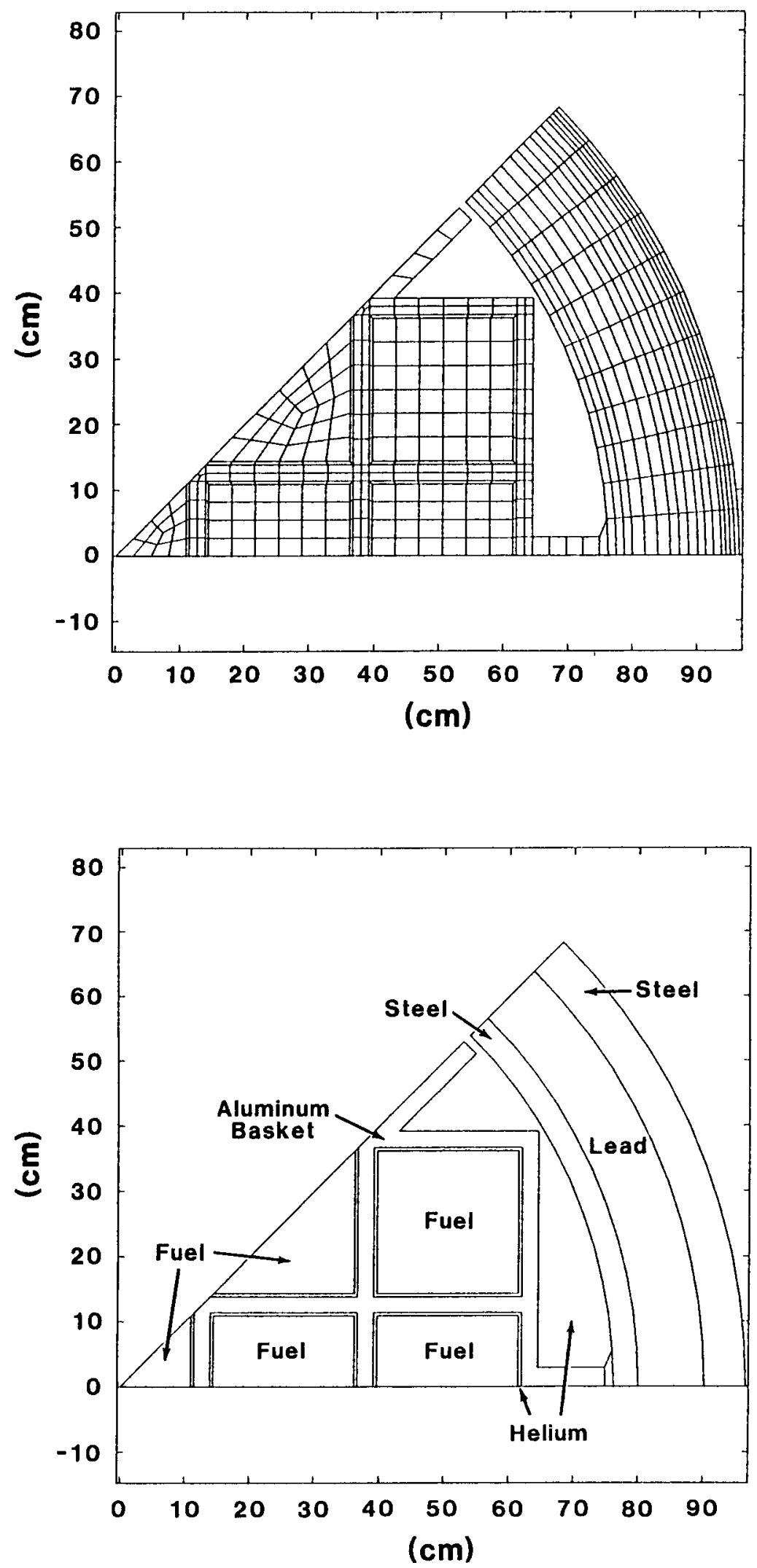

Figure II-100. Finite Element Mesh (top) and Material Zones (bottom) for the Lead-Shielded Rail Cask 
A decay-heat-peaking factor of 1.2 was used in analyses of the two representative casks. If the total decay heat generation rate of a PWR spent-fuel assembly in the lead-shielded truck cask is assumed to be $1.0 \mathrm{~kW}$, then the volumetric rate of heat generation used for the finite element model fuel region of the cask is specified for such a magnitude that when multiplied by the total active volume of the fuel assembly model, a decay heat generation rate of $1.2 \mathrm{~kW}$ is obtained.

The finite element model for the lead-shielded truck cask consists of 314 nodes and 274 quadrilateral elements. The triangular region in this model, which represents one-eighth of a PWR spent-fuel assembly, has an edge length of $10.89 \mathrm{~cm}$. The inner radius of the cask cavity is $17.145 \mathrm{~cm}$ and the inner steel shell thickness is $1.27 \mathrm{~cm}$. The outer radius is 34.925 $\mathrm{cm}$, and the outer steel shell thickness is $3.175 \mathrm{~cm}$. The helium-filled gap between the fuel zone and the aluminum basket wedge, and between this wedge and the cask inner wall is $0.5 \mathrm{~cm}$ wide. This solid aluminum basket wedge is similar to the wedge used in the NLI-1/2 cask for transporting PWR spent fuel [NL80].

The finite element model of the lead-shielded rail cask consists of 547 nodes and 478 quadrilateral elements. The edge length of the entire fuelassembly zone in this model is $21.78 \mathrm{~cm}$. The equivalent of $2.625 \mathrm{PWR}$ spent-fuel assemblies is included in this one-eighth section of the cask, which corresponds to a full fuel load of 21 PWR spent-fuel assemblies. The fuel assemblies are contained in individual cells of a solid aluminum basket. The basket was modeled as solid aluminum to simplify the thermal analyses. In an actual application, a neutron poison might be incorporated in at least some of the basket cell walls for criticality control. The thickness of the cell walls is $2.5 \mathrm{~cm}$.

The $0.5-\mathrm{cm}$-wide gap between the outer surface of the fuel zones and the adjacent basket wall in each fuel cell is assumed to be filled with helium. The helium also fills the space between the outer surface of the basket and the inner wall surface of the cask. The two finlike extensions on the outer side of the basket that point out toward the cask wall are included to improve heat dissipation, to assist in positioning the basket inside the cask cavity, and to aid in computation of view factors for estimating radiative heat transfer between the basket and cask wall.

The inner radius of the wall in the finite element model of the rail cask is $76.2 \mathrm{~cm}$ and the outer radius is $96.52 \mathrm{~cm}$. The inner steel shell thickness is $3.81 \mathrm{~cm}$, and outer steel shell thickness is $6.35 \mathrm{~cm}$. The helium-filled space between the basket outer surface and inner cask surface in the rail cask model is not paved with finite elements. Instead, the bulk node feature available in TOPAZ2D was used to model the helium in this portion of the model. Modeling the helium in this space with finite elements would overestimate conductive heat transfer from the basket to the cask wal1. Heat transfer in this large open space most likely occurs by convection rather than by direct conduction through the helium. With the bulk node approach to modeling convection, a node is specified in the helium-filled space between the cask wall and basket. The total helium mass located in this space is associated with this node, which is also assumed to have helium thermal material properties. The bulk node is 
specified to couple thermally with all element surfaces bounding the helium-filled space via a heat transfer law of the form

$$
q=f(T)\left(T_{s i}^{a}-T_{B}^{a}\right)^{b}
$$

where

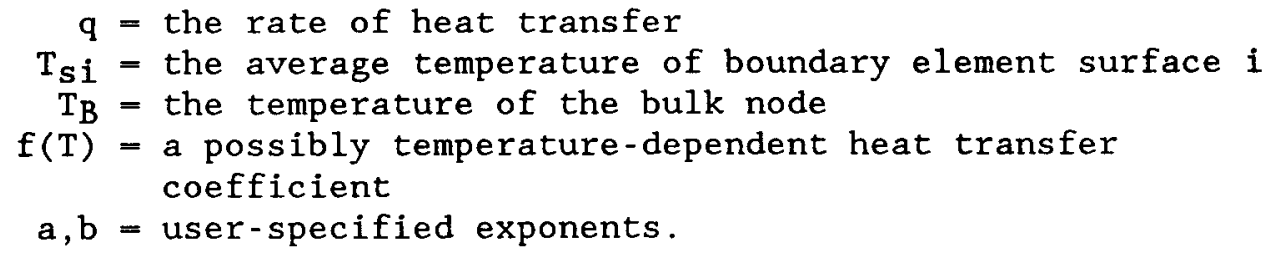

The values of the exponents were $a=1.0$ and $b=1.0$ for the thermal response analyses of the lead-shielded rail cask. The value (assumed constant) of $f(T)$ in the rail cask analyses was $1.7 \times 10^{-3} \mathrm{~J} / \mathrm{s}-\mathrm{cm}^{2}-{ }^{\circ} \mathrm{C}$, which is the average of the typical range $\left(5.7\right.$ to $\left.28.4 \mathrm{~J} / \mathrm{m}^{2}{ }^{\circ} \mathrm{C}-\mathrm{s}\right)$ of heat transfer coefficients for gases (free convection), as given by Arpaci [AR66].

Heat transfer by radiation across the helium-filled gaps and voids in the analyses for both representative casks was modeled using view factors calculated for the external element surfaces of the fuel, basket and cask inner shell material zones, and a heat transfer law of the form

$$
q=F(T)\left(T_{i}^{4}-T_{j}^{4}\right),
$$

where

$$
\begin{aligned}
\mathrm{q}= & \text { the rate of heat transfer } \\
\mathrm{T}_{\mathbf{i}}, \mathrm{T}_{\mathbf{j}}= & \text { the temperatures of surfaces } i \text { and } \mathrm{j} \text { that are } \\
& \text { interchanging heat radiatively } \\
\mathrm{F}(\mathrm{T})= & \text { a factor assumed to be temperature-independent } \\
& \text { in the analyses that accounts for the view factors, } \\
& \text { surface emissivities, and Stefan-Boltzmann constant } \\
& \left(5.672 \times 10^{-12} \mathrm{~J} / \mathrm{s}-\mathrm{cm}^{2}-^{\circ} \mathrm{C}\right) .
\end{aligned}
$$

The spent-fuel emissivity surface was assumed to be 0.8 , which is the emissivity measured for typical PWR spent fuel, as reported by EPRI [EP86]). The spent-fuel emissivity of aluminum surfaces and the cask stainless steel inner wall was assumed to be 0.2 and 0.5 , respectively; these two emissivities are the same values used in the NLI-1/2 SARP [NL80].

The view factors used in the analyses of the representative casks were calculated with the FACET code [SH83]. The input of this code is the geometry of the system at hand, specification of the surfaces for which view factors are desired, and, if applicable, identification of surfaces that cannot intercouple. This last feature rapidly calculates the view factors for systems such as the rail cask model in which many surfaces are involved throughout the cask interior, but are not in view of each other. 
FACET is capable of evaluating view factors for three-dimensional models and uses the Hottel cross string method [HO67] to calculate the view

factors for two-dimensional planar models. The code outputs a data file of view factors that is readable by TOPAZ2D.

A symmetry plane approximation is not available in FACET. Consequently, consistent estimates of the factors are obtained with the code only if each surface for which view factors are to be calculated is entirely (or nearly so) surrounded by other surfaces for which factors are also desired. For the two representative cask thermal analysis models, some of the surfaces for which view factors are desired are not completely surrounded by other surfaces. This applies particularly to those surfaces near the zero and $45^{\circ}$ planes of symmetry. However, because the gaps are small in the cask models, the error in factors calculated when there is a lack of total enclosure is not substantial. For large gaps, factor errors would be manifested in the thermal analysis results as a nonphysical energy loss mechanism. The procedure used to develop a suitable material model for the fuel zones of the two cask models was the most affected by the requirement of nearly total surface enclosure for calculating view factors.

Heat transfer by conduction was only assumed across the threematerial zones (steel-lead-steel) of the cask walls. The possibility of voids in the lead zone and the presence of thermal contact resistance across the interfaces between dissimilar materials were not considered. These would tend to slightly increase the cask interior temperatures under normal transport conditions, but would also help maintain lower temperatures in the fire environment. The possibility of phase change, for example, lead melt, particularly during the fire response analyses, was also not included. Modeling phase changes would considerably slow timestepping in the analyses. Also, not modeling the phenomenon is believed to be conservative because thermal energy that would be absorbed as latent heat in the phase change process (solid to liquid) is otherwise available during the heating phase to penetrate more deeply into the cask interior. Latent heat associated with a phase change would also be a heat source during the post-fire cooldown phase of the analyses. But such released heat energy would then be transferred out from, as well as in toward, the center of the cask. Hence it is anticipated that higher temperatures are predicted by ignoring phase changes.

Three modes of thermal energy transfer were modeled at the exterior surface of the cask models. The first of these consisted of thermal energy input as a result of solar radiation. The solar energy flux modeled as flowing into the cask at its outer surface was $0.1354 \mathrm{~J} / \mathrm{cm}^{2}-\mathrm{s}$ [CR74]. This energy flux was assumed to act uniformly on the cask exterior surface under normal transport conditions and throughout the cask response to the fire environment.

The second mode of thermal energy transfer modeled at the outer surface of the cask was heat transfer by free convection. The mathematical form assumed for heat transfer by this process is based on a correlation given by Kreith [KR73] for free convection from a horizontal cylindrical surface: 


$$
\mathrm{q}=1.02 \times 10^{-4}\left(\mathrm{~T}_{\mathrm{s}}-\mathrm{T}_{\mathrm{A}}\right)^{1 / 3}\left(\mathrm{~T}_{\mathrm{s}}-\mathrm{T}_{\mathrm{A}}\right)
$$

where

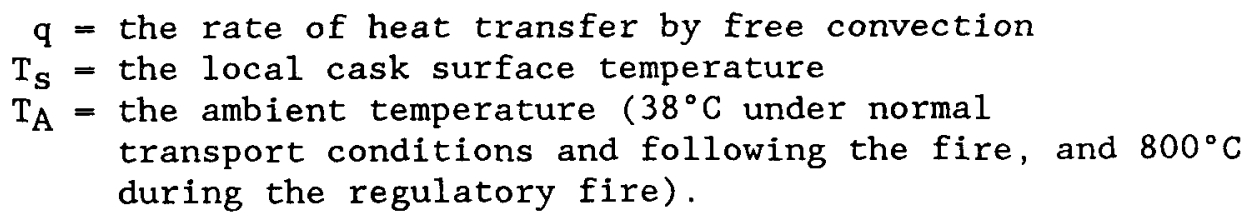

The coefficient in the equation was chosen so that the units for $q$ are $\mathrm{J} / \mathrm{cm}^{2}-\mathrm{s}$.

The third mechanism modeled for heat transfer from the outer surface of the cask was by radiation. Heat transfer in this case is governed by the expression [AR66]

$$
\mathrm{q}=\sigma \mathrm{F}\left(\mathrm{T}_{\mathrm{S}}^{4}-\mathrm{T}_{\mathrm{A}}^{4}\right)
$$

where

$$
\begin{aligned}
\sigma= & \text { the Stefan-Boltzmann constant } \\
\mathrm{T}_{\mathrm{S}}= & \text { the local cask surface temperature } \\
\mathrm{T}_{\mathrm{A}}= & \text { the ambient temperature (with the values given in } \\
& \text { the previous paragraph). }
\end{aligned}
$$

$F$ is defined in terms of view factor $F_{i j}$ of the cask surface with respect to its surroundings, cask surface emissivity $\varepsilon_{1}$, and ambient emissivity $\varepsilon_{2}$. For the above, $F_{i j}=1.0, \varepsilon_{1}=0.5$ under normal transport conditions and postfire cooldown and 0.8 during the fire, and $\varepsilon_{2}=0.9$ during the fire and 1.0 otherwise. The explicit expression for F [NL80] is

$$
F=\left(\frac{1}{F_{i j}}+\frac{1}{\varepsilon_{1}}+\frac{1}{\varepsilon_{2}}-2\right)^{-1} .
$$

When the above emissivity and view factor values are used, $F=0.5$ under normal transport conditions and during the postfire cooldown period, and $F=0.7347$ during the regulatory fire.

\section{II.5.3 Cask Material Models}

The thermal analyses of the representative lead-shielded casks were performed using temperature-dependent material properties for the cask and spent-fuel assembly materials. Basic material properties used in the analyses are summarized in Table II-25. The thermal properties listed in Table II-25 for $\mathrm{UO}_{2}$ and Zircaloy-2 were taken from Glasstone and Sesonske [GL67]. Properties for aluminum and lead are those used in the NLI-1/2 SARP [NL80]. The aluminum properties were modeled as temperature-

independent. The density and temperature-dependent specific heat for steel 
Temperature-Dependent Properties of the Basic Cask Model Materials. (See text for the various sources of these data.)

\begin{tabular}{|c|c|c|c|c|}
\hline Material & $\begin{array}{l}\text { Temperature } \\
\left({ }^{\circ} \mathrm{C}\right)\end{array}$ & $\begin{array}{l}\text { Density } \\
\left(\mathrm{g} / \mathrm{cm}^{3}\right) \\
\end{array}$ & $\begin{array}{c}\text { Specific } \\
\text { Heat } \\
\left(\mathrm{J} / \mathrm{gm}-{ }^{\circ} \mathrm{C}\right)\end{array}$ & $\begin{array}{l}\text { Thermal } \\
\text { Conductivity } \\
\left(\mathrm{J} / \mathrm{cm}-\mathrm{s}-{ }^{\circ} \mathrm{C}\right)\end{array}$ \\
\hline Uranium Oxide & $\begin{array}{r}24 \\
93 \\
260 \\
538 \\
815 \\
1093\end{array}$ & 10.96 & $\begin{array}{l}0.251 \\
0.264 \\
0.293 \\
0.307 \\
0.321 \\
0.335\end{array}$ & $\begin{array}{l}0.092 \\
0.092 \\
0.074 \\
0.050 \\
0.038 \\
0.031\end{array}$ \\
\hline Zircaloy-2 & $\begin{array}{r}24 \\
93 \\
260 \\
538 \\
815 \\
1093\end{array}$ & 6.55 & 0.297 & $\begin{array}{l}0.116 \\
0.119 \\
0.124 \\
0.125 \\
0.125 \\
0.125\end{array}$ \\
\hline Helium & $\begin{array}{r}27 \\
327 \\
627 \\
927 \\
1227\end{array}$ & $1.6 \times 10^{-4}$ & 5.234 & $\begin{array}{l}1.48 \times 10^{-3} \\
2.44 \times 10^{-3} \\
3.40 \times 10^{-3} \\
4.36 \times 10^{-3} \\
5.32 \times 10^{-3}\end{array}$ \\
\hline Aluminum & - & 2.71 & 0.926 & 1.675 \\
\hline Steel & $\begin{array}{l}100 \\
400 \\
495 \\
700 \\
816\end{array}$ & 7.91 & $\begin{array}{l}0.518 \\
0.565 \\
0.580 \\
0.612 \\
0.630\end{array}$ & $\begin{array}{l}0.147 \\
0.191 \\
0.205 \\
0.235 \\
0.252\end{array}$ \\
\hline Lead & $\begin{array}{l}106 \\
204 \\
327 \\
427 \\
527 \\
691\end{array}$ & 11.35 & $\begin{array}{l}0.132 \\
0.136 \\
0.142 \\
0.141 \\
0.141 \\
0.139\end{array}$ & $\begin{array}{l}0.339 \\
0.317 \\
0.209 \\
0.156 \\
0.151 \\
0.150\end{array}$ \\
\hline
\end{tabular}


were also obtained from NLI [NL80]. Thermal conductivity of steel was evaluated using the expression $\mathrm{K}(\mathrm{T})=0.13217+0.1465 \times 10^{-3} \mathrm{~T}\left(\mathrm{~T}\right.$ in ${ }^{\circ} \mathrm{C}$ and $\mathrm{K}(\mathrm{T})$ in $\left.\mathrm{J} / \mathrm{cm}-\mathrm{s}-{ }^{\circ} \mathrm{C}\right)$, given by EPRI [EP86]. Helium conductivity was calculated from a similar expression $\mathrm{K}(\mathrm{T})=1.394 \times 10^{-3}+0.32 \times 10^{-5} \mathrm{~T}$ [EP86]. The specific heat and density of helium were also taken from EPRI [EP86].

The material properties for aluminum, helium, stainless steel, and lead, as given in Table II-25, were used directly in the finite element thermal response analyses of the representative cask concepts. Additional effort was required to obtain useful temperature-dependent material properties for the spent-fuel zones in the finite element models. This effort was required because the spent-fuel assemblies in the analyses were not modeled explicitly with fuel pin arrays with each pin consisting of a pure fuel core surrounded by a layer of Zircaloy cladding. Rather, they were modeled as homogenized material zones occupying the same crosssectional area as the actual spent-fuel pin arrays. The density and temperature-dependent heat capacity of the homogenized spent-fuel material were estimated in a procedure that gives the same total mass and energy absorption capability for the fuel model as for the actual fuel assembly. Deriving the required density and heat capacity values was possible using only the dimensions of the fuel pins and homogenized fuel zones, and the $\mathrm{UO}_{2}$ and Zircaloy-2 material properties given in Table II-25.

\section{II.5.4 Spent-Fuel Effective Conductivity}

This section describes the complex procedure that was used to estimate temperature-dependent effective conductivities of the homogenized fuel

material. In heat transfer, the effective conductivities derived for spent fuel are approximately equivalent to the effective conductivity that results from the interplay of conduction, convection, and radiation in the fuel pin array in a spent-fuel assembly. Briefly, the steps involved in determining effective conductivities for spent fuel include the following:

1. TOPAZ2D modeling and analysis of a fuel pin array,

2. Comparison of analysis results with the theoretical solution of a similar heat conduction problem,

3. Derivation of effective conductivities based on the comparison, and

4. Verification of the effective conductivities by analysis of a spent-fuel assembly for which measured temperatures are available.

Details of the procedure estimating equivalent spent-fuel conductivities are presented in the remainder of this section.

PWR Spent Fuel

It was assumed that PWR spent fuel in the representative cask thermal analyses was similar to Surry Nuclear Power Plant spent fuel involved in the tests described by EPRI [EP86]. The Surry fuel assembly is a standard 
Westinghouse (WE) $15 \times 15$ PWR fuel design. A cross section showing some details of this fuel assembly type is presented in Figure II-101 [EP86].

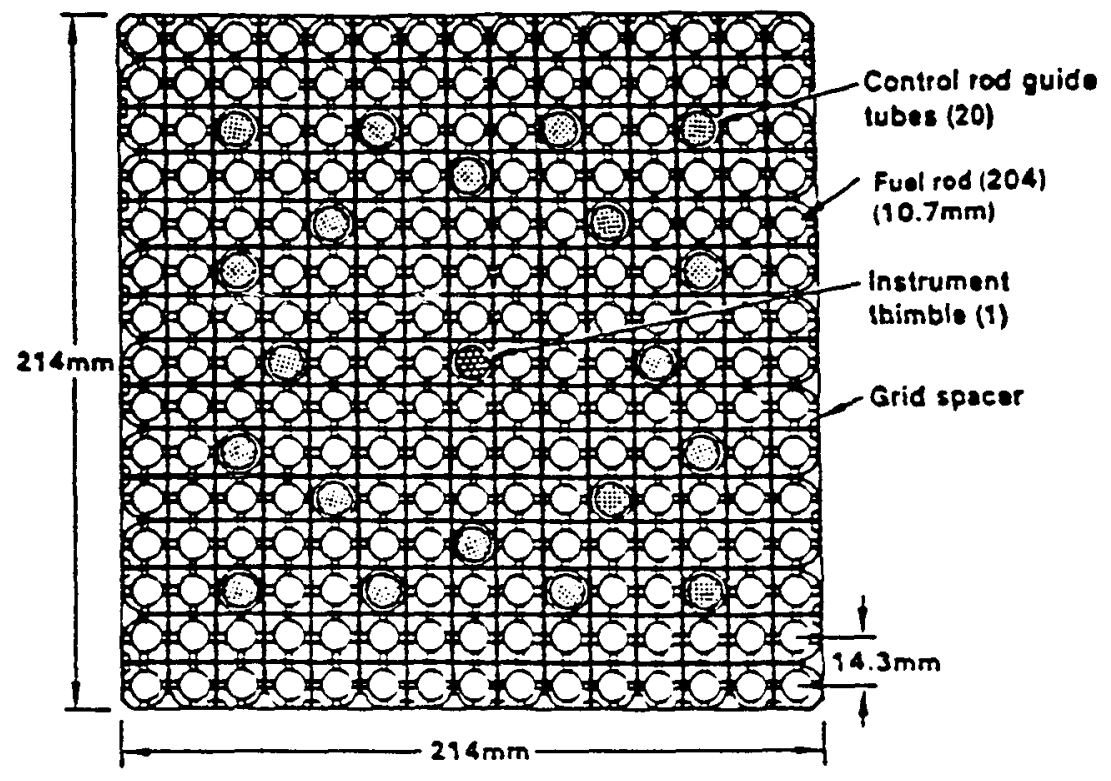

Figure II-101. Cross Section of the Surry $15 \times 15$ PWR Fue1 Assembly [EP86]

The standard WE $15 \times 15$ fuel assembly design consists of a $15 \times 15$ array of equally spaced tubes and pins, which includes 204 fuel pins, 20 control rod guide tubes, and 1 in-core instrumentation thimble. The array locations of the control rod guide tubes and the instrumentation thimble are shown in Figure II-102. The fuel pin array is held together by 7 -grid spacers (approximately equally spaced along the length of the pins) and hardware at each end. The overall fuel assembly length is $405.8 \mathrm{~cm}$ and the cross-sectional dimensions are $21.4 \mathrm{~cm} \times 21.4 \mathrm{~cm}$. The active length of the fuel region in the pins is $365.8 \mathrm{~cm}$.

The fuel in the pins consists of a stack of $\mathrm{UO}_{2}$ pellets. Each pellet has a nominal diameter of $0.9294 \mathrm{~cm}$. Surrounding the pellet stack is a Zircaloy- 4 tube. In the thermal analyses, the material properties of Zircaloy- 2 were used in place of Zircaloy-4 properties. Thermal properties of the two materials are not significantly different. The outer diameter of the Zircaloy tube is $1.071 \mathrm{~cm}$ and the thickness is $0.0617 \mathrm{~cm}$. There is a small gap between the fuel pellet outer surface and the surrounding Zircaloy tube.

\section{Simplified Fue1 Pin Model}

Thermal analyses of the casks that calculate peak spent-fuel temperatures should include an explicit representation in the analysis models of individual fuel pins in each cask spent-fuel assembly. Clearly, such an approach would lead to large analysis models. Because only a 

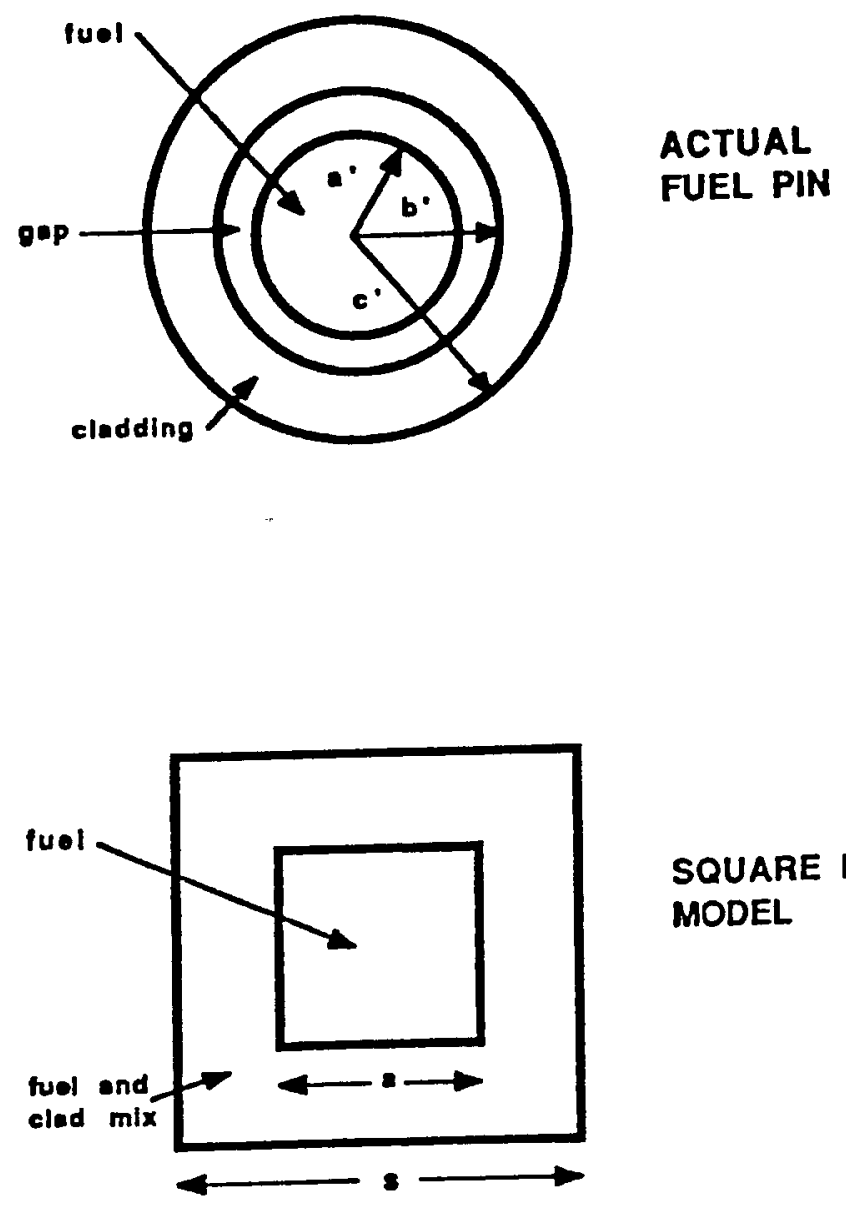

SQUARE PIN

MODEL

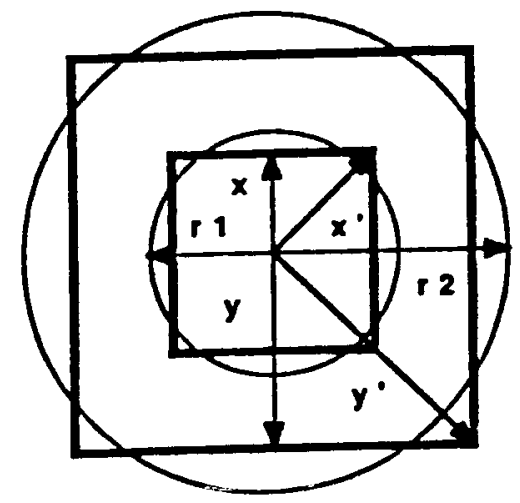

AVERAGE RADII OF SQUARE PIN MODEL

Figure II-102. Simplified Model of an Actual Fuel Pin 
first-order thermal analysis of each cask is required for the present analysis, a much simpler model of the assemblies is adequate. However, the effective conductivity of a spent-fuel assembly in a cask is dependent on the construction of the assembly from many individual pins, each of which is radiatively coupled to many of its neighboring pins (i.e., any other pin in the array by which it can be affected). Furthermore, thermal response of individual pins is conductively and convectively coupled to other pins because of the presence of a fluid (in this case, helium) between the pin spaces. The thermal response of the spent-fuel assembly is also dependent on the immediate surroundings of the assembly, such as the walls of a cask basket.

The first step in determining effective conductivity of a fuel assembly array involves developing a simplified model of a single fuel pin that is more convenient to assemble in larger models than a circular pin. This simplified model, in cross section, consists of a square pure fuel core surrounded by a square annulus that is comprised of a fuel-clad mixture. The outer perimeter of the fuel-clad mixture zone was taken to be equal to the outer cladding surface perimeter of an actual fuel pin. The first two drawings from the top in Figure II-102 illustrate the cross section of a real fuel pin and its simplified square model. The values for the dimensions labeled in the figure are $a^{\prime}=0.4647 \mathrm{~cm}, b^{\prime}=0.4738 \mathrm{~cm}$, $c^{\prime}=0.5355 \mathrm{~cm}, a=0.5047 \mathrm{~cm}$, and $\mathrm{s}=0.84116 \mathrm{~cm}$. A six- or eight-sided model of the fuel pin could also have been selected, but this would have substantially complicated the analysis models described in the following subsection.

Thermal material properties of the solid fuel core in the center of the simplified fuel pin model are those listed for $\mathrm{UO}_{2}$ in Table II-25. The approximate thermal material properties of the fuel-clad mixture can be calculated from the given dimensions using $\mathrm{UO}_{2}$ and Zircaloy basic material properties.

The equivalent density and heat capacity of the fuel-clad mixture were calculated using the rule of mixtures. Designating $\rho_{f}$ as the density of $\mathrm{UO}_{2}, \mathrm{C}_{\mathrm{f}}$ as its specific heat, $\rho_{\mathrm{c}}$ as the density of Zircaloy-2, and $\mathrm{C}_{\mathrm{C}}$ as the cladding's specific heat, then the equivalent density $\rho_{f c}$ of the fuelclad mixture is given by

$$
\rho_{f c}=\frac{\rho f\left[\pi a^{, 2}-a^{2}\right]+\pi \rho_{c}\left[c^{, 2}-b^{, 2}\right]}{s^{2}-a^{2}},
$$

and the equivalent specific heat $C_{f c}$ is given by

$$
C_{f c}=\frac{\rho_{f} C_{f}\left[\pi a^{, 2}-a^{2}\right]+\rho_{c} C_{c} \pi\left[c^{\prime 2}-b^{\prime 2}\right]}{\rho_{f c}\left(s^{2}-a^{2}\right)} .
$$


To estimate the effective conductivity of the fuel-clad mixture, the bottom drawing in Figure II-102 is first used to define the average radii $r_{1}$ and $r_{2}$ of the inner and outer squares of the simplified fuel pin model

$$
\begin{aligned}
& r_{1}=\frac{1}{2}\left(x+x^{\prime}\right)=0.30462 \mathrm{~cm} \\
& r_{2}=\frac{1}{2}\left(y+y^{\prime}\right)=0.50769 \mathrm{~cm} .
\end{aligned}
$$

Next, the effective conductivity $K_{f c}$ of the fuel-clad mixture (which gives the same temperature drop between $r_{1}$ and $r_{2}$ in the simple fuel pin model as between $r_{1}$ and $c^{\prime}$ in the actual pin) is determined. This is done by using Fourier's law for heat transfer expressed in cylindrical coordinates:

$$
q=-2 \pi r k \frac{d T}{d r}
$$

where

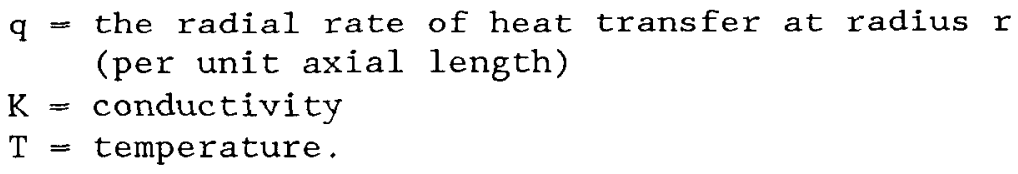

If $\mathrm{T}_{1}$ is the temperature at $r=r_{1}, T_{3}$ is the temperature at $c^{\prime}, K_{f}$ is the thermal conductivity of $\mathrm{UO}_{2}$, and $\mathrm{K}_{\mathrm{C}}$ is the conductivity of Zircaloy, solution of the above differential equation for the actual fuel pin gives

$$
\mathrm{T}_{3}-\mathrm{T}_{1}=-\frac{\mathrm{q}}{2 \pi}\left[\frac{1}{\mathrm{~K}_{\mathrm{f}}} \ln \left(\mathrm{a}^{\prime} / \mathrm{r}_{1}\right)+\frac{1}{\mathrm{~K}_{\mathrm{c}}} \ln \left(\mathrm{c}^{\prime} / \mathrm{a}^{\prime}\right)\right] .
$$

If $\mathrm{T}_{1}$ is the temperature at $\mathrm{r}=\mathrm{r}_{1}$ and $\mathrm{T}_{3}$ the temperature at $\mathrm{r}=\mathrm{r}_{2}$, the temperature drop across the fuel-clad mixture region (approximated as a circular annulus) of the simplified fuel pin model is given by

$$
\mathrm{T}_{3}-\mathrm{T}_{1}=-\frac{\mathrm{q}}{2 \pi \mathrm{K}_{\mathrm{fc}}} \ln \left(\mathrm{r}_{2} / \mathrm{r}_{1}\right)
$$

Equating the two expressions for the temperature difference $T_{3}-T_{1}$ results in

$$
\mathrm{K}_{\mathrm{fc}}=\frac{\ln \left(\mathrm{r}_{2} / \mathrm{r}_{1}\right)}{\frac{1}{\mathrm{~K}_{\mathrm{f}}} \ln \left(\mathrm{a}^{\prime} / \mathrm{r}_{1}\right)+\frac{1}{\mathrm{~K}_{\mathrm{c}}} \ln \left(\mathrm{c}^{\prime} / \mathrm{a}^{\prime}\right)} .
$$


The material properties in Table II-25 and the fuel pin and its simplified model dimensions are finally used to evaluate the above expressions for $\mathrm{K}_{\mathrm{fc}}$, $\rho_{\mathrm{fc}}$, and $\mathrm{C}_{\mathrm{fc}}$. The results of these evaluations are summarized in Table II-26.

Table II -26

Equivalent Thermal Material Properties for the Simplified Fuel Pin Model

\begin{tabular}{cccc}
\hline $\begin{array}{c}\text { Temperature } \\
\left({ }^{\circ} \mathrm{C}\right)\end{array}$ & $\begin{array}{c}\text { Density, } \rho \text { fc } \\
\left(\mathrm{g} / \mathrm{cm}^{3}\right)\end{array}$ & $\begin{array}{c}\text { Specific } \\
\text { Heat }, C_{f c} \\
\left(\mathrm{~J} / \mathrm{gm}-{ }^{\circ} \mathrm{C}\right)\end{array}$ & $\begin{array}{c}\text { Thermal } \\
\begin{array}{c}\text { Conductivity, } \\
\left(\mathrm{J} / \mathrm{cm}-\mathrm{S}-{ }^{\circ} \mathrm{C}\right)\end{array}\end{array}$ \\
\cline { 1 - 1 } 24 & 13.08 & 0.261 & 0.0879 \\
93 & & 0.271 & 0.0883 \\
260 & & 0.294 & 0.0746 \\
538 & & 0.305 & 0.0533 \\
815 & & 0.316 & 0.0417 \\
1093 & & 0.327 & 0.0346 \\
\hline
\end{tabular}

\section{Simplified Pin Array Analyses}

Availability of a simple model of an individual fuel pin makes possible approximate modeling of a fuel pin array. The fuel pin array is modeled with the spacing used in typical PWR fuel assemblies, with helium filling the space between the individual pin models, and with the whole array surrounded by a square steel tube. The outer surface of the square steel tube is assumed to be maintained at a constant temperature. The fuel core of each fuel pin array is assumed to generate heat internally at a specified constant rate. If the temperature difference between the central portion of the array and the isothermal surface is not too great, such a fuel pin array and surrounding isothermal surface are thermally quite similar to the two-dimensional problem of a square region of dimensions $2 a \times 2 a$ and a material with an effective constant thermal conductivity $\mathrm{K}_{\mathrm{eff}}$ that also generates heat at a constant uniform rate $Q$ with its boundary temperature remaining at a constant value $\mathrm{T}_{0}$. Figure II-103 illustrates this simple problem in heat conduction. Its solution is readily derived, and given in series form by Arpaci [AR66]. The rapidly converging series solution can be used to show that the temperature $\mathrm{T}_{c}$ at the center of the square region is given by the following equation, keeping three terms in the series to evaluate the numerical coefficient:

$$
\mathrm{T}_{\mathrm{c}}=\mathrm{T}_{\mathrm{o}}+0.29468 \frac{\mathrm{Qa}^{2}}{\mathrm{~K}_{\mathrm{eff}}}
$$




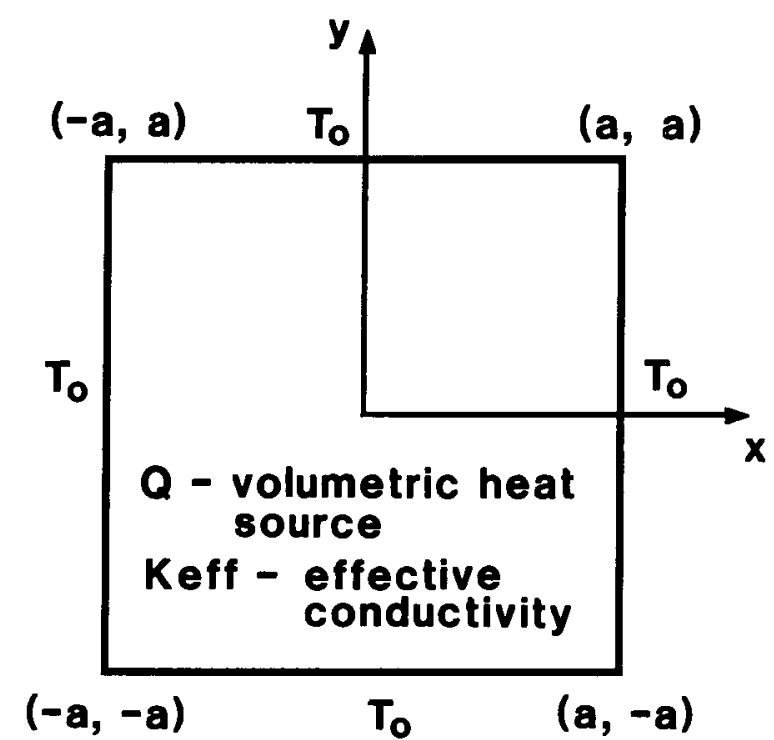

Figure II-103. A Problem in Heat Conduction Similar to an Enclosed Array of Fuel Pins

The previous analytical solution was used to estimate an effective conductivity for a fuel pin array as follows:

1. The fuel pin array was assembled using the simplified fuel pin model. This array was surrounded by a $0.635-\mathrm{cm}$-thick square steel tube. View factors were calculated with FACET for radiative heat transfer among individual fuel pins and between the pins and the inside surface of the steel tube. The pure fuel region of each pin was assigned the same constant rate of heat generation. The volumetric rate of heat generation was selected to correspond to a total spent-fuel assembly decay heat generation rate of $1.02 \mathrm{~kW}$. This heat generation rate corresponds to the heat generation rate of a spent-fuel assembly for which measured temperatures are available (see the following section). A finite element model of the array was developed.

2. TOPAZ2D was then used to predict temperatures throughout the fuel pin array for four specified boundary temperatures of the steel tube outer surface: $127,227,327$, and $427^{\circ} \mathrm{C}$. The temperature predicted at the center of the array as a function of boundary temperature was recorded.

3. The expression for $T_{C}$ given above was next used in conjunction with the predicted center temperatures of the finite element model to obtain values for $K_{e f f}$ that would yield the same central temperatures in the analytical problem. The constant rate of heat generation used in the finite element model of the fuel pin array 
was homogenized to obtain a value for $Q$ that would give the same total rate of heat generation in the analytical problem as would be involved in the finite element model.

4. Finally, the derived values for $K_{\text {eff }}$ were used to model a fuel assembly inside a cask basket. Temperatures predicted for this fuel assembly were compared to measured temperatures reported by EPRI [EP86] to verify adequacy of the effective conductivity values.

Originally, the intent was to model a full $15 \times 15$ array with the simplified fuel pin model. However, it was estimated that an excessive amount of computer time would be required to calculate the view factors for the 960 surfaces $(921,600$ matrix elements) involved in such a problem. Hence a smaller $9 \times 9$ array entailing 360 surfaces $(129,600$ matrix elements) was modeled instead. The finite element mesh for this array is shown in Figure II-104.

The edge length of the fuel pin cell (i.e., the simple fuel pin model plus associated helium) used in the $9 \times 9$ array model was $1.452 \mathrm{~cm}$. The distance between the outer row surface of the simple pin models in the array and the inner surface of the surrounding steel tube was $0.635 \mathrm{~cm}$. The thermal material properties for the pure fuel pin core, helium, and steel used in this array model are those given in Table II-25. The equivalent thermal material properties from Table II-26 were used for the fuel-clad mixture zones.

Temperature contours predicted with TOPAZ2D for the pin array subjected to a $327^{\circ} \mathrm{C}$ boundary temperature condition are shown in Figure II-105. The temperature difference between the adjacent contours in the figure is $1.5^{\circ} \mathrm{C}$. The total temperature difference between the boundary and the array center appears to be quite small. This increases the likelihood that a constant value for the effective conductivity will be sufficient to analytically reproduce the calculated temperature profile.

The peak temperatures predicted in the four analyses of the $9 \times 9$ fuel pin array and the corresponding calculated effective conductivity values are summarized in Table II-27. Note that the temperature difference between the array center and the boundary is predicted to decrease as the boundary is increased. This indicates that radiative heat transfer plays an increasing role in the total heat transfer inside the array as the temperatures increase; increasing heat transfer by radiation serves to smooth out temperature gradients in the array.

A linear fit to the effective conductivity estimates in Table II-27 using the boundary temperature as the independent variable $\mathrm{T}\left({ }^{\circ} \mathrm{C}\right)$ is given by $K_{\text {eff }}(\mathrm{T})=1.633 \times 10^{-5} \mathrm{~T}+3.53 \times 10^{-3}$ with units $\mathrm{J} / \mathrm{cm}-\mathrm{s}-{ }^{\circ} \mathrm{C}$. This equation produces all calculated effective conductivities in Table II-27 to within an accuracy of 38 . The estimated conductivities could also have been fit to the average temperature of the pin array evaluated in each analysis to give a more precise correlation for the temperature dependence of the effective conductivity. Because the difference between the boundary temperature and average array temperature is small in each of the four 


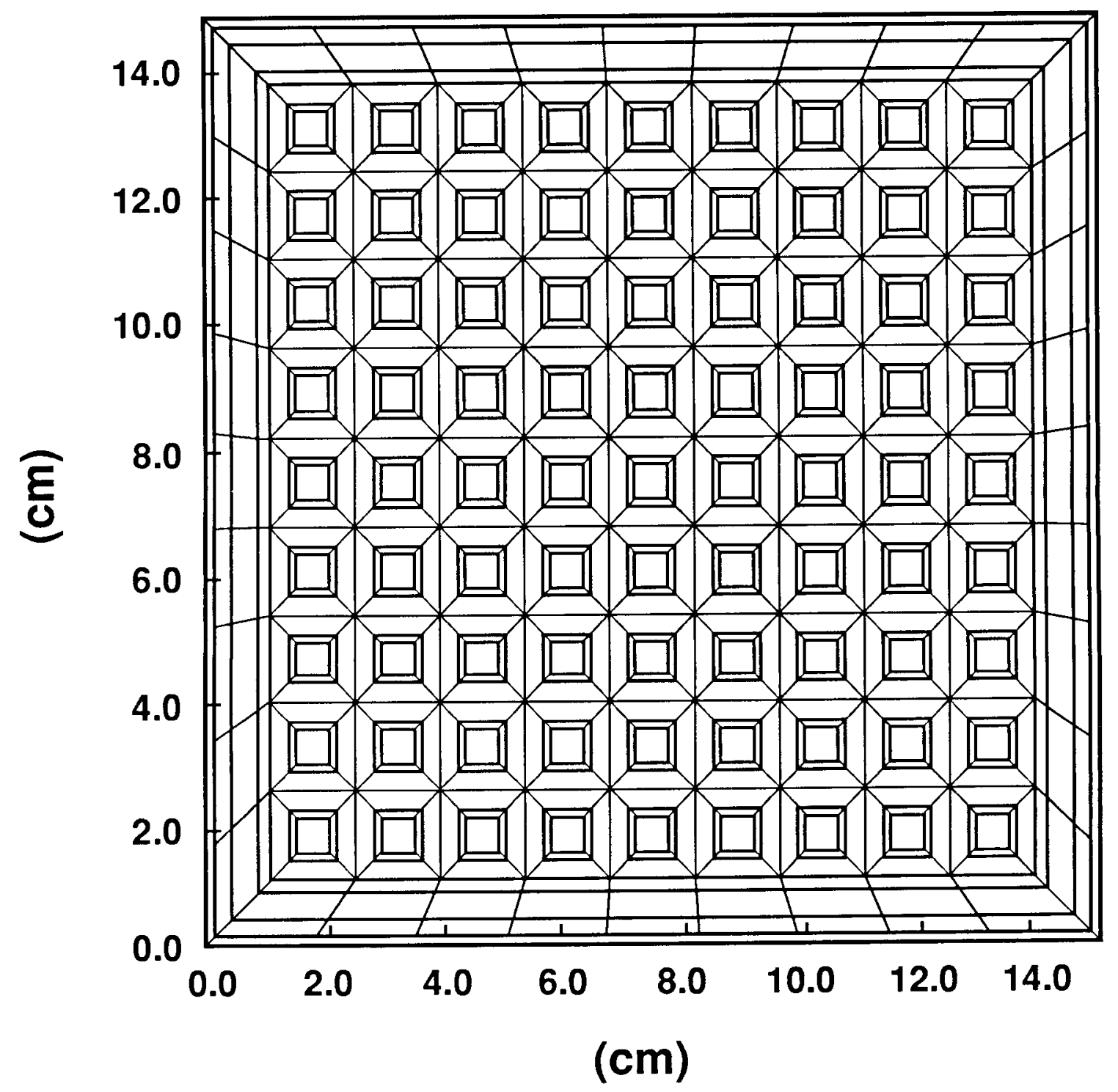

Figure II-104. Finite Element Mesh of the Steel-Tube-Enclosed $9 \times 9$ Simplified Fuel Pin Model Array

analyzed cases, the results of using average effective conductivity fit temperatures would also be small.

The $9 \times 9$ fuel pin array problem was next reanalyzed using homogenized, or smeared, thermal material properties for the fuel plus its cladding and associated helium. The density and temperature-dependent specific heats of the smeared fuel were evaluated using the rule of mixtures and the fuel pin cells shown in Figure II-106. A11 familiar dimensions in the figure have the same values previously given. 


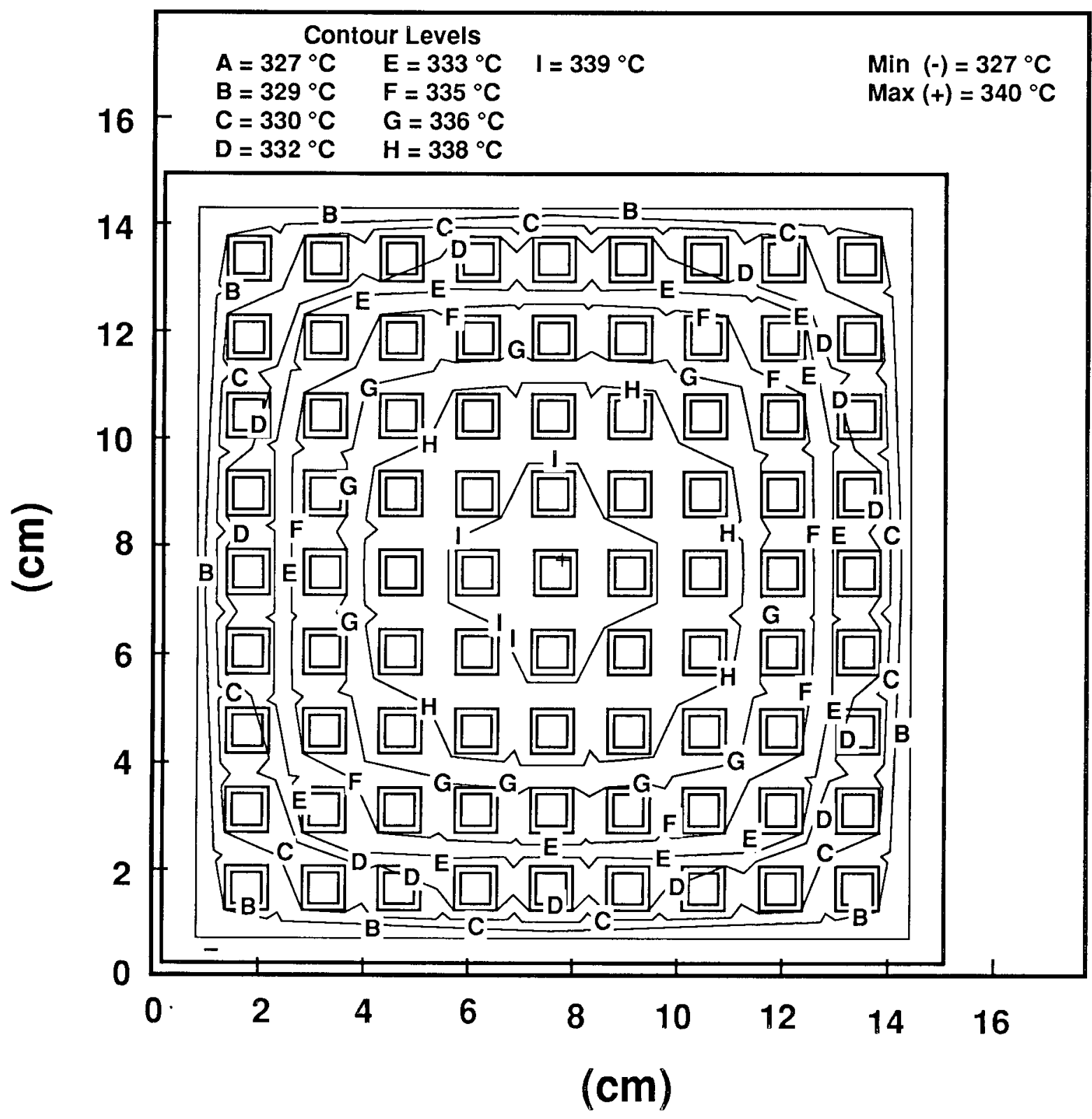

Figure II-105. Predicted Temperature Contours in the $9 \times 9$ Pin Model for $327^{\circ} \mathrm{C}$ Boundary Temperatures

The new dimension $\mathbf{s}^{\prime}$ designating the edge length of the fuel pin cell is equal to $1.452 \mathrm{~cm}$. Based on the geometries indicated in Figure II-106, the rule of mixtures expression for the effective density $\rho_{\text {eff }}$ of the smeared fuel is given by

$$
\rho_{\text {eff }}=\frac{1}{s^{\prime}}\left[\rho_{\mathrm{f}} \pi \mathrm{a}^{\prime 2}+\rho_{\mathrm{c}} \pi\left(\mathrm{c}^{\prime 2}-\mathrm{b}^{\prime 2}\right)+\rho_{\mathrm{He}}\left(\mathrm{s}^{\prime 2}-\pi \mathrm{c}^{\prime 2}\right)\right],
$$


Table II -27

Effective Conductivity Estimates for the $9 \times 9$

Simplified Fuel Pin Array

\begin{tabular}{ccc}
\hline $\begin{array}{c}\text { Boundary } \\
\begin{array}{c}\text { Temperature } \\
\left({ }^{\circ} \mathrm{C}\right)\end{array}\end{array}$ & $\begin{array}{c}\text { Array Center } \\
\text { Temperature } \\
\left({ }^{\circ} \mathrm{C}\right)\end{array}$ & $\begin{array}{c}\text { Effective } \\
\text { Conductivity } \\
\left(\mathrm{J} / \mathrm{cm}-\mathrm{S}-{ }^{\circ} \mathrm{C}\right)\end{array}$ \\
\cline { 1 - 1 } 127 & 147.1 & \\
227 & 242.8 & $5.76 \times 10^{-3}$ \\
327 & 339.8 & $9.36 \times 10^{-3}$ \\
427 & 437.9 & $1.06 \times 10^{-3}$ \\
\hline
\end{tabular}

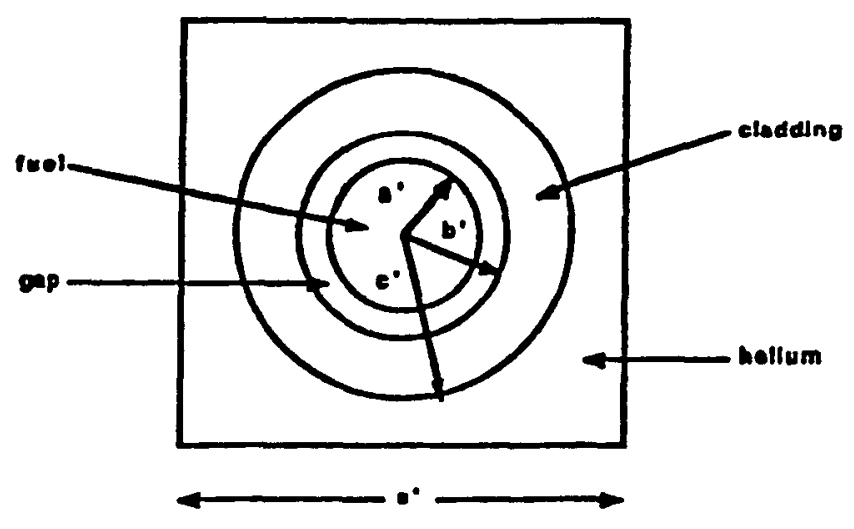

ACTUAL FUEL PIN

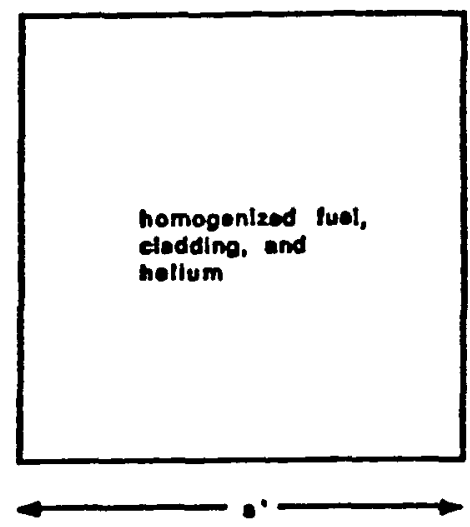

HOMOGENIZED FUEL PIN MODEL

Figure II-106. Actual and Homogenized Fuel Pin Cells

in which $\rho_{\mathrm{f}}, \rho_{\mathrm{C}}$, and $\rho_{\mathrm{He}}$ are the densities of $\mathrm{UO}_{2}$, Zircaloy, and helium. Similarly, the effective specific heat $C_{e f f}$ of the smeared fuel is given by

$C_{\text {eff }}=\frac{1}{\rho_{\text {eff }} s^{\prime 2}}\left[\rho_{f} C_{f} \pi a^{\prime 2}+\rho_{c} C_{c} \pi\left(c^{\prime 2}-b^{\prime 2}\right)+\rho_{H e} C_{H e}\left(s^{\prime 2}-\pi c^{\prime 2}\right)\right],($ II - 102)

where $C_{f}, C_{C}$, and $C_{H e}$ are the specific heats of $\mathrm{UO}_{2}$, cladding, and helium.

Using the specified dimensions and basic material properties from Table II-25, the effective thermal properties for homogenized spent fuel 
are determined and given in Table II-28. The effective thermal conductivities of the smeared fuel given in the table were evaluated using the linear fit to the estimated effective conductivities given above.

Table II -28

Effective Thermal Material Properties of Homogenized Smeared Spent Fuel

\begin{tabular}{cccc}
\hline $\begin{array}{c}\text { Temperature } \\
\left({ }^{\circ} \mathrm{C}\right)\end{array}$ & $\begin{array}{c}\text { Specific } \\
\text { Density } \\
\left(\mathrm{g} / \mathrm{cm}^{3}\right)\end{array}$ & $\begin{array}{c}\text { Effective } \\
\text { Heat } \\
\text { Capacity } \\
\left(\mathrm{J} / \mathrm{gm}-{ }^{\circ} \mathrm{C}\right)\end{array}$ & $\begin{array}{c}\text { Effective } \\
\text { Thermal } \\
\text { Conductivity } \\
\left(\mathrm{J} / \mathrm{cm}-{ }^{\circ}{ }^{\circ} \mathrm{C}\right)\end{array}$ \\
24 & 4.135 & 0.258 & 0.0039 \\
93 & & 0.269 & 0.0050 \\
260 & & 0.294 & 0.0078 \\
538 & & 0.306 & 0.0123 \\
815 & & 0.318 & 0.0168 \\
1093 & & 0.329 & 0.0214 \\
\hline
\end{tabular}

The finite element model of the smeared $9 \times 9$ fuel pin array inside the helium-filled square steel tube is shown in Figure II-107. The outer surface temperature of the steel tube was maintained at $327^{\circ} \mathrm{C}$ in this model analysis. The temperature contours predicted with TOPAZ2D for this model are shown in Figure II-108. These contours agree quite well with those predicted for the explicit model of the $9 \times 9$ pin array (see Figure II105). The peak temperature, which was located at the center of the array, was predicted to be $339.8^{\circ} \mathrm{C}$ in both analyses.

\section{Effective Conductivities Validation}

Measured temperatures for Surry Nuclear Power Plant spent fuel contained in the 21 PWR capacity Castor-V/21 spent-fuel storage cask [EP86] were used to investigate the quality of the estimated effective conductivities derived for smeared spent fuel. These temperature data were obtained for the storage cask oriented in the horizontal or vertical direction and backfilled with helium or nitrogen. Temperatures were measured at six locations along the length of the spent-fuel assemblies in the cask at 10 radial locations. Figure II-109 indicates the locations of thermocouple lances relative to the various spent-fuel assemblies in the cask interior. Some of the thermocouple lances were placed inside guide tubes of a few assemblies. Figure II-109 indicates which guide tubes were used. Two lances measured temperatures in the cask basket near the fuel assemblies.

The temperatures measured for assembly Al, control rod guide tubes numbers 1 and 3 , and location $E$ near the outer surface of $A 1$ were used to evaluate the quality of the effective smeared fuel thermal conductivities. 


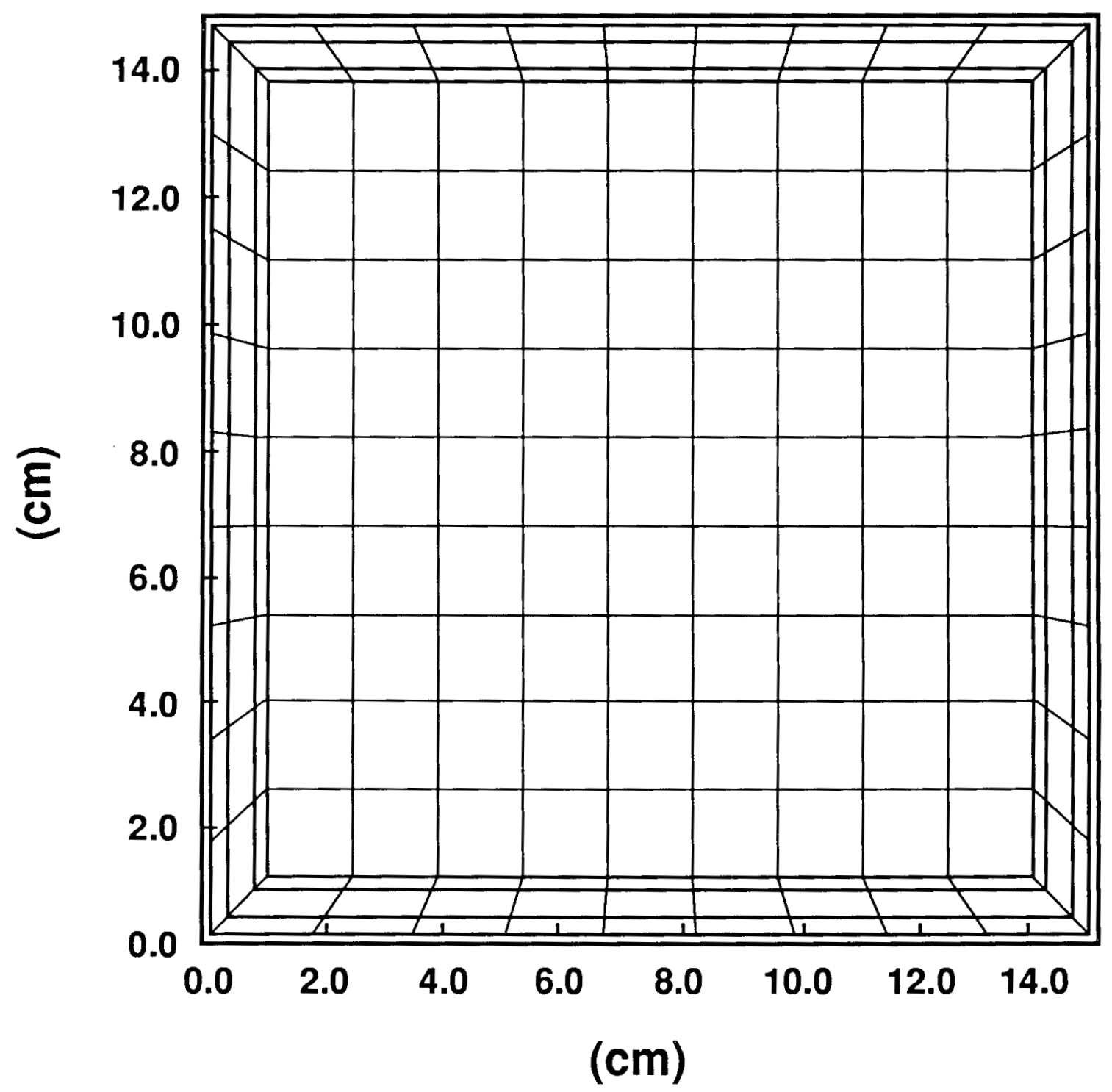

Figure II-107. Finite Element Mesh of the Steel-Tube-Enclosed Homogenized Smeared $9 \times 9$ Fuel Pin Array

For the cask in the horizontal orientation that was backfilled with helium, peak temperatures measured at these locations occurred at an axial position approximately $242 \mathrm{~cm}$ from the bottom end of the cask. The temperatures reported in the EPRI study are $356.4^{\circ} \mathrm{C}$ at $\mathrm{Al}-1,360.5^{\circ} \mathrm{C}$ at $\mathrm{Al}-3$, and $347.6^{\circ} \mathrm{C}$ at $\mathrm{E}$. The decay heat generation rate of assembly $\mathrm{Al}$ was specified as being $1.02 \mathrm{~kW}$ with a 1.2 -power peaking factor in the vicinity where the previously mentioned temperatures were measured. 


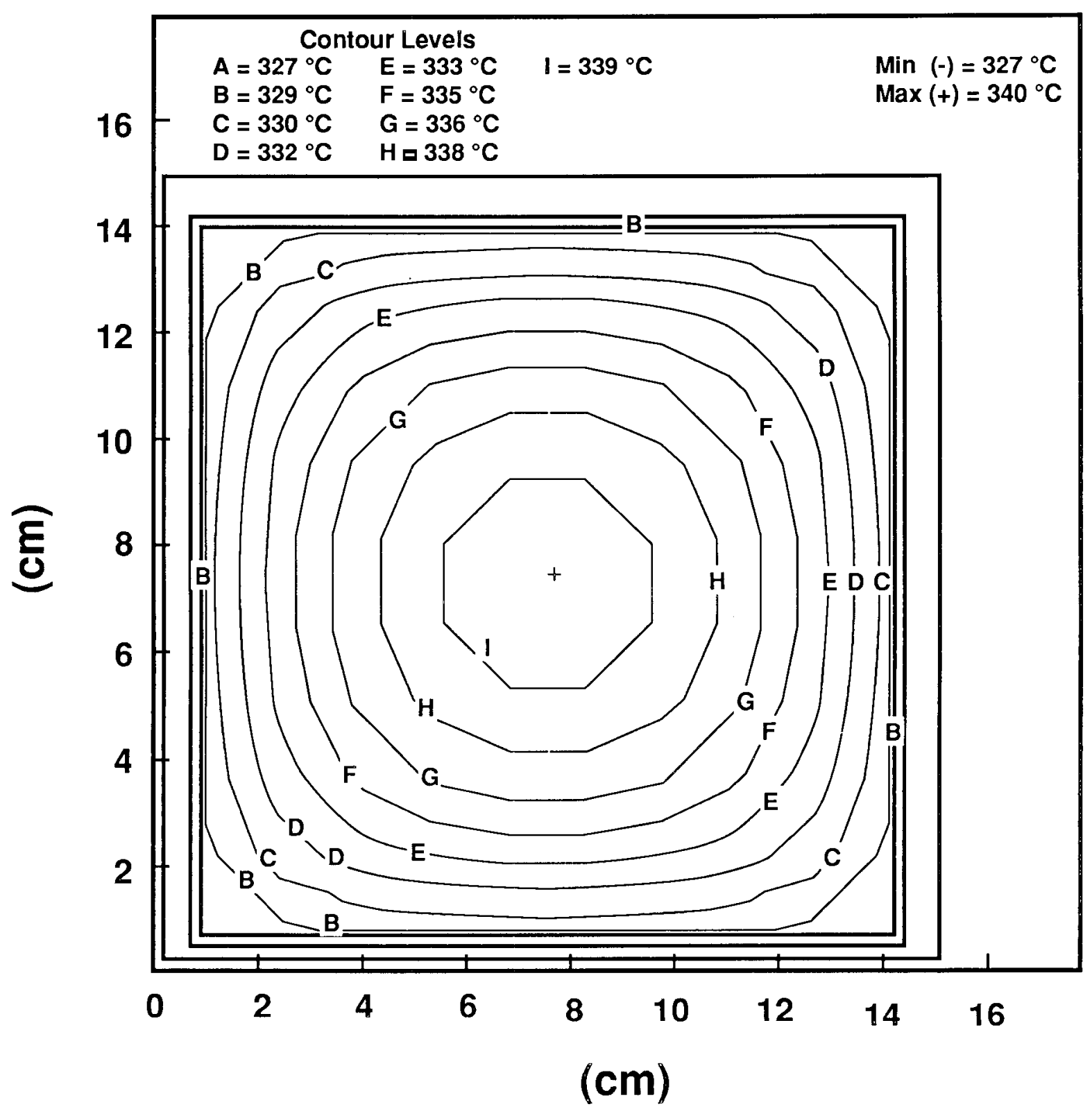

Figure II-108. Predicted Temperature Contours for the Homogenized $9 \times 9$ Fuel Pin Array $\left(327^{\circ} \mathrm{C}\right.$ boundary temperature)

The measured radial temperature profiles for the horizontal cask backfilled with helium (see Figure II-110) indicate that the temperature gradients were rather small in the central portion of the cask. EPRI also stated that convection seemed to be relatively unimportant when the cask was horizontally oriented. This suggests that the temperature measured at location $E$ was probably fairly close to the outer surface temperature of the steel basket tube surrounding spent-fuel assembly Al in the Castor cask. Furthermore, this surface was probably approximately isothermal. 


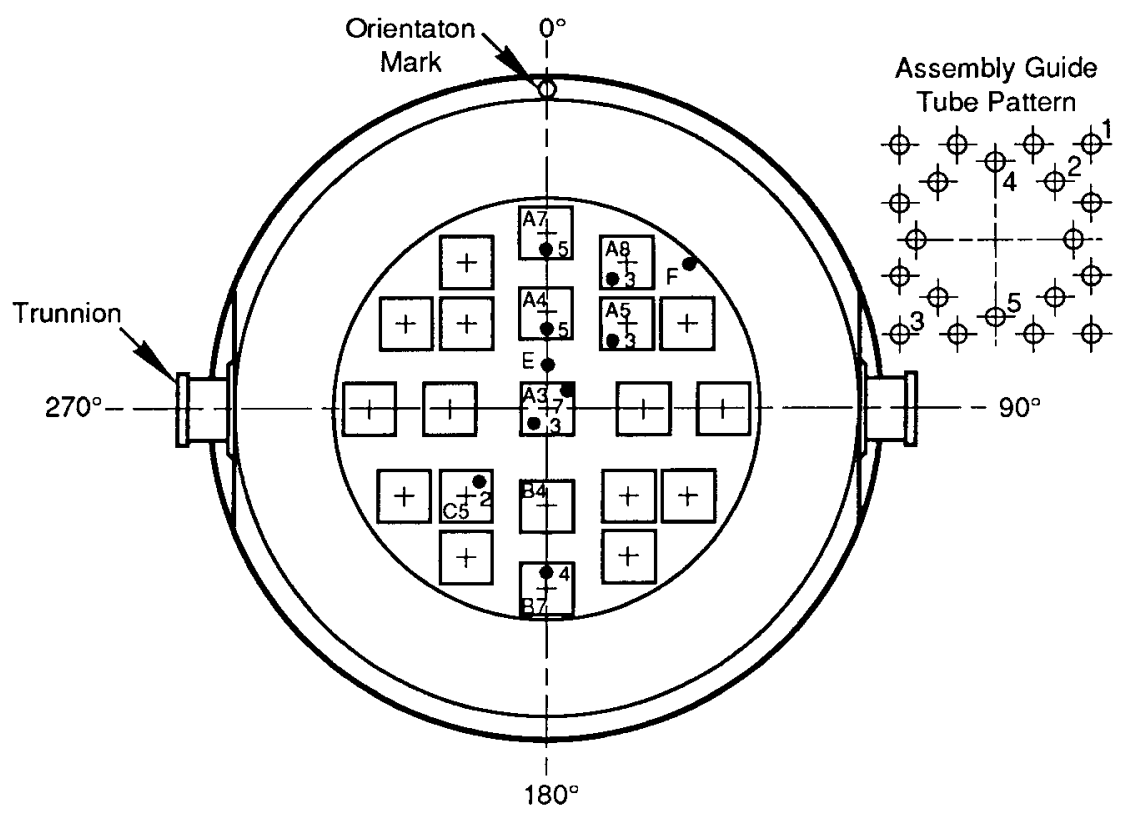

Figure II-109. Thermocouple Lance Locations in Castor-V/21 Spent-Fuel Temperature Measurement Tests [EP86]

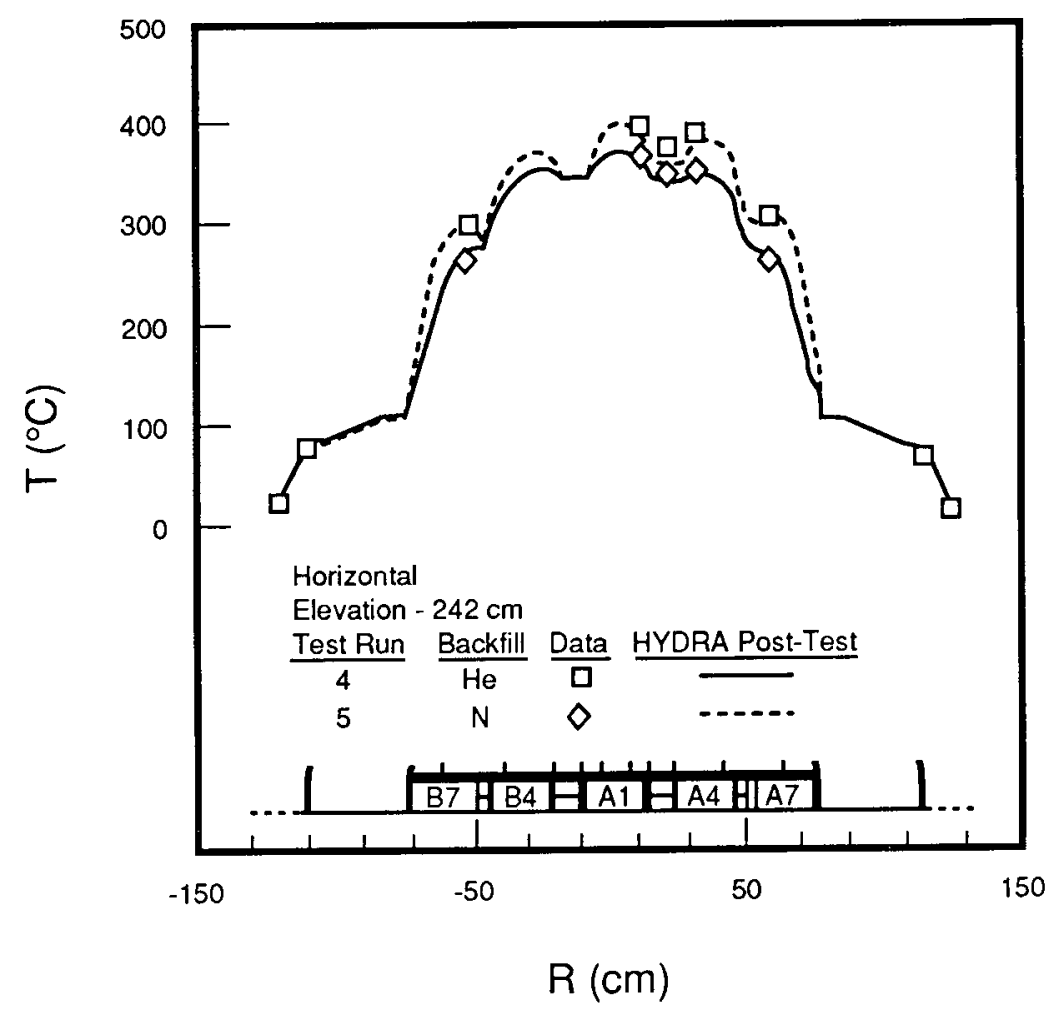

Figure II-110. Measured Radial Temperature Profiles of Horizontal Helium-Backfilled Castor-V/21 Casks [EP86] 
To confirm the usefulness of the effective thermal conductivities of smeared spent fuel, a homogenized spent-fuel finite element model of assembly $A l$ and its surrounding steel basket tube was developed and analyzed with TOPAZ2D. This model consists of a $15 \times 15$ array of quadrilateral elements with smeared fuel thermal properties surrounded by a $0.6353-\mathrm{cm}-$ thick layer of helium and a $0.635-\mathrm{cm}$-thick square steel tube. View factors for radiative heat transfer between the outer surface of the smeared fuel array and inner surface of the steel tube were calculated using FACET. The volumetric heat generation rate of the smeared fuel was specified as a magnitude corresponding to a total assembly decay heat generation rate of $1.02 \mathrm{~kW}$ with a 1.2 -power peaking factor. The finite elements in the array corresponding to the 21-guide tube and instrumentation thimble locations in the assembly were not assigned volumetric heat generation. The outer surface temperature of the steel tube was specified as $347.6^{\circ} \mathrm{C}$. Figure II-111 shows the finite element model used in this analysis.

The temperature contours predicted for this $15 \times 15$ smeared fuel pin array problem are shown in Figure II-112. The predicted temperature at location $\mathrm{Al}-1$ is $371.4^{\circ} \mathrm{C}$ (compared to a measured temperature of $360.5^{\circ} \mathrm{C}$ ) and $362.3^{\circ} \mathrm{C}$ for location $\mathrm{Al}-3$ (compared to the measured value of $356.4^{\circ} \mathrm{C}$ ). Here, using the effective thermal conductivities for smeared fuel resulted in conservative (i.e., high) temperature predictions in the spent-fuel assembly interior.

One reason for the higher predicted fuel pin temperatures may be that a fairly large helium-filled gap $(0.635 \mathrm{~cm}$ wide) was assumed between the outer surface of the smeared fuel and the inner steel tube surface. Insufficient information is given by EPRI [EP86] to determine the true width of the gap. A smaller gap would lead to lower predicted temperatures in the smeared fuel interior. However, the converse is true if the actual gap is larger than the modeled gap.

Another source for the discrepancy is that the effective conductivities were evaluated from analyses of a $9 \times 9$ fuel pin array and applied to a $15 \times 15$ array. This is a large extrapolation for a physical situation involving a highly nonlinear process such as radiative heat transfer. Also, the individual pins in the $9 \times 9$ array were modeled as squares in cross section rather than as circles. Thus, the actual radiative heat transfer was only approximately modeled in the analyses.

Finally, the measured temperatures may also have been less than actual temperatures in assembly $\mathrm{Al}$. This is because it is not known (according to EPRI [EP86]) if the thermocouples were in contact with the sides of the guide tubes. If they were not, the guide tube wall temperatures could have been higher than the measured temperatures.

In any event, the temperature predictions obtained with the smeared fuel properties were shown to be in reasonable (and conservative) agreement with experimental data. Thus, the smeared spent-fuel material model is deemed adequate for the task at hand, namely, first-order thermal response analysis of the representative lead-shielded cask concepts. 


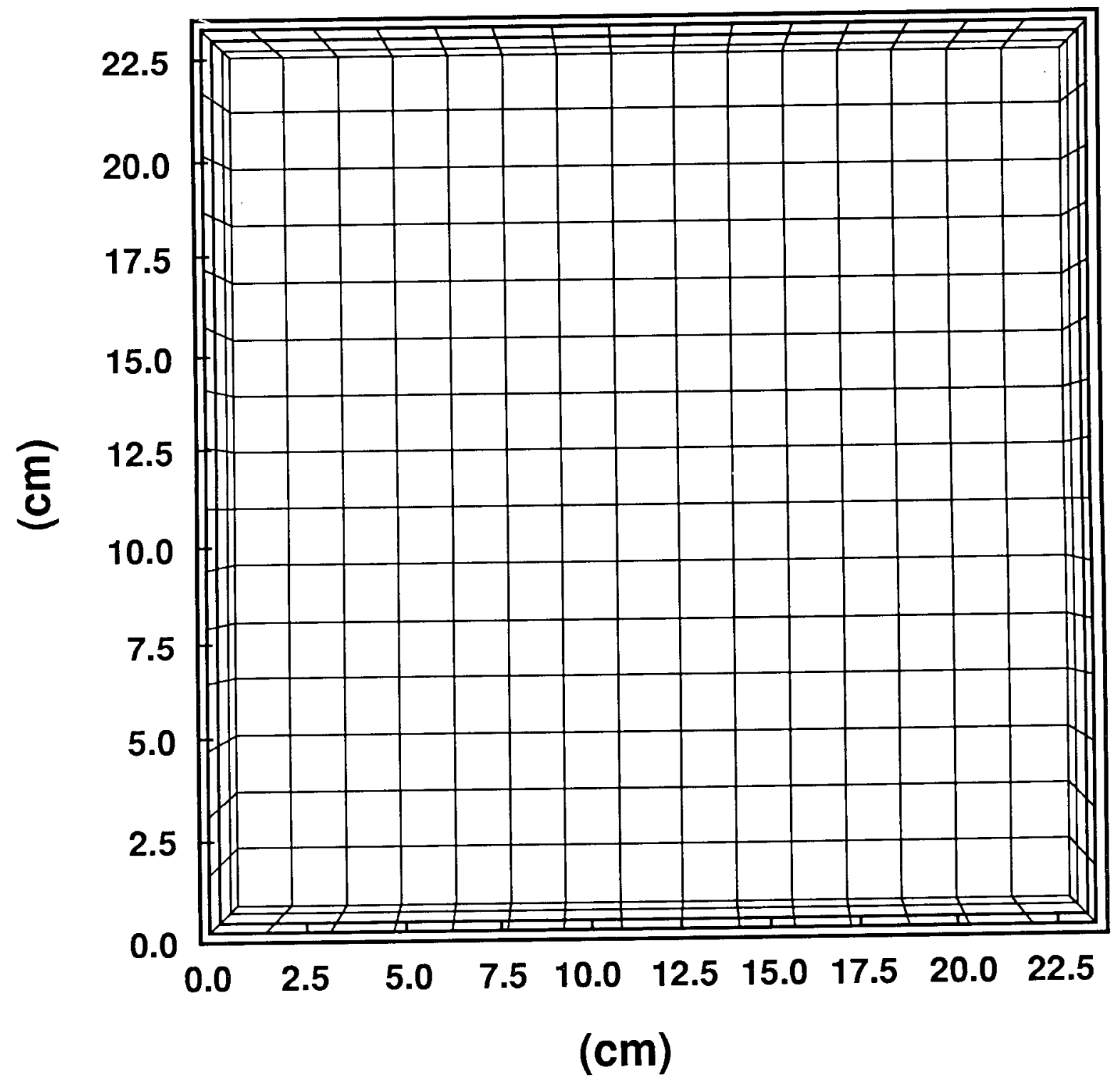

Figure II-111. Finite Element Mesh of Assembly A1 and Its Basket Tube in the Castor $-\mathrm{V} / 21$ Cask

\section{II.5.5 Cask Thermal Response Results}

The thermal analysis models of the representative lead-shielded truck and rail cask concepts were presented in Section II.5.2. The thermal material properties for helium, lead, aluminum, and steel used in these cask analyses are those given in Table II-25. The effective material properties from Table II-28 were used for the smeared fuel zones in the cask finite element models. 


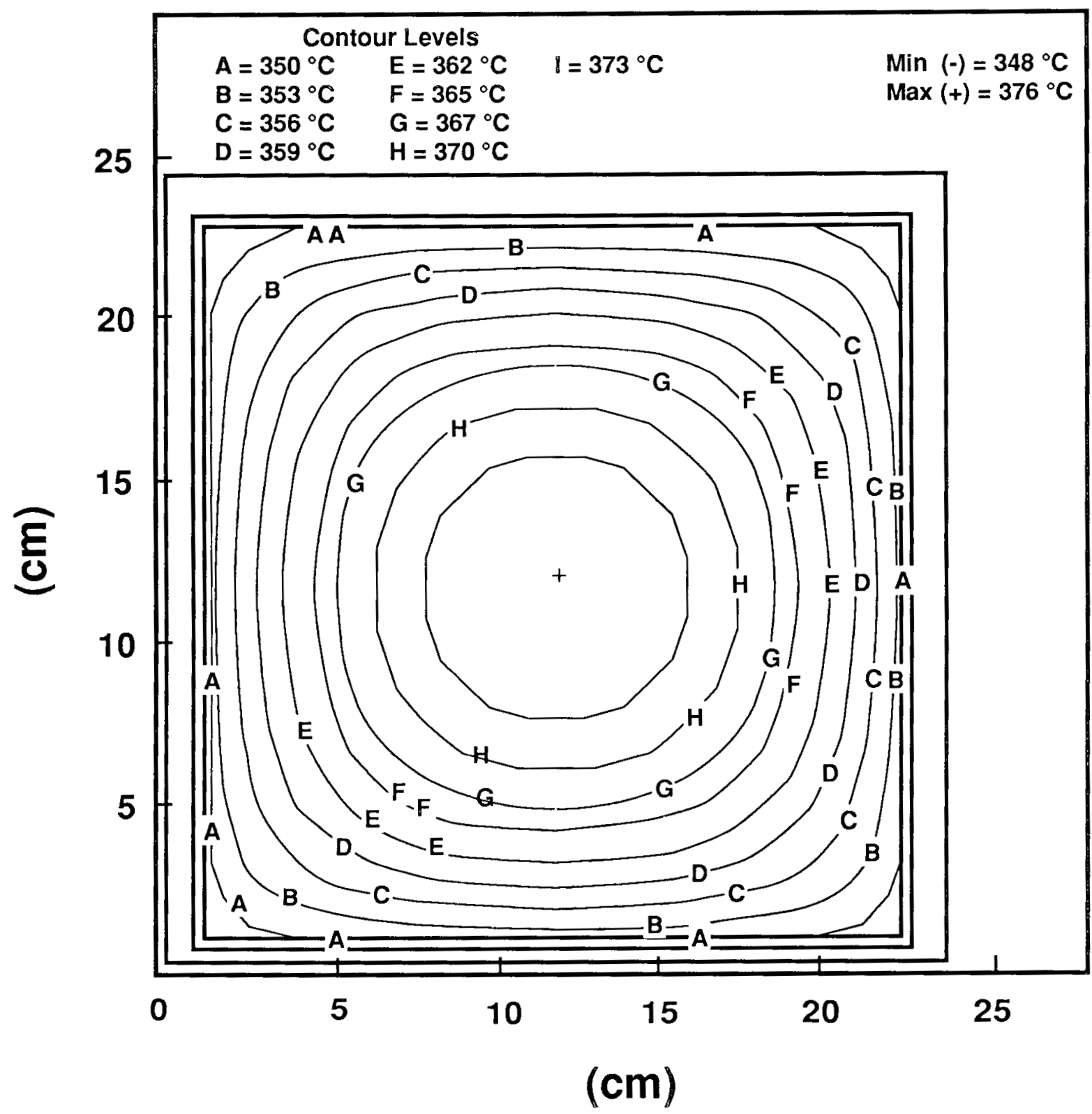

Figure II-112. Predicted Temperature Contours for Assembly AI in the Castor-V/21 Cask

The first analyses of the cask models involved determination of the total decay heat loads that could be transported in the casks under the normal transport conditions without exceeding the allowable temperature guidelines for spent-fuel storage. According to the DOE [US85], a reasonable allowable temperature for spent-fuel storage is a peak cladding temperature of $380^{\circ} \mathrm{C}$. 
The lead-shielded truck cask model was analyzed for five different decay heat generation rates $(1.0,1.5,2.0,2.5$, and $3.0 \mathrm{~kW})$ of the single PWR spent-fuel assembly contained in the cask. A 1.2 -power peaking factor was used in each of these analyses. Figure II-113 shows the predicted peak fuel temperature of the cask as a function of the assembly decay heat. From this figure, a $3.0-\mathrm{kW}$ assembly in the truck cask is estimated to have a peak fuel temperature $\left(315^{\circ} \mathrm{C}\right)$ that meets the conservative allowable temperature limit. Figure II-114 presents temperature contours in the fuel zone (top) and cask wall (bottom) predicted for a $3.0-\mathrm{kW}$ decay heat load in the cask under steady-state (normal) conditions.

\section{Lead-Shielded Truck Cask-1 PWR-Max Fuel Temperature}

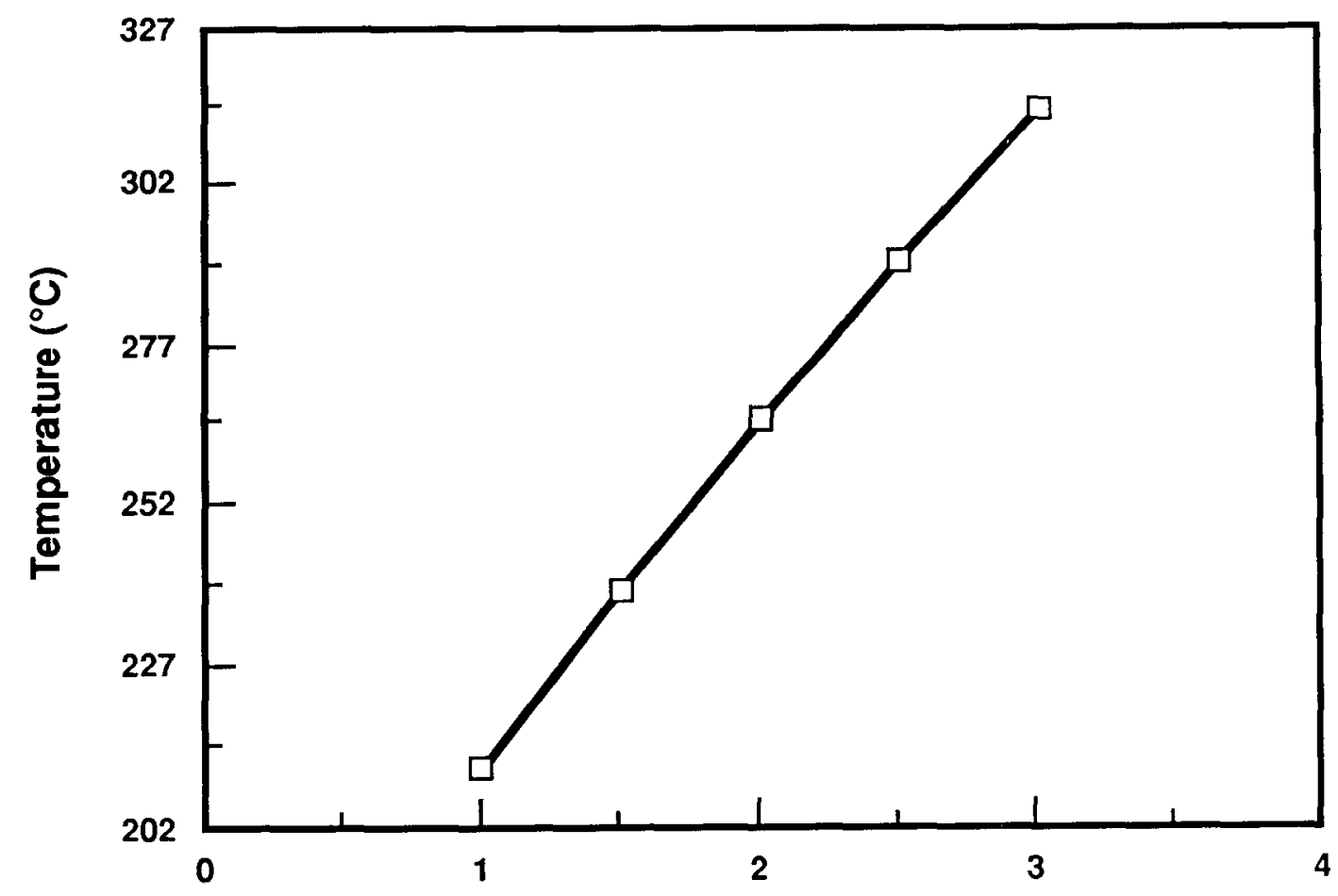

Assembly Source (kW)

Figure II-113. Predicted Peak Fuel Temperature in Truck Casks as a Function of Assembly Decay Heat Generation Rate

With a 1.2-power peaking factor used again, four decay heat rates $(0.75,1.0,1.25$, and $1.5 \mathrm{~kW})$ per assembly were considered in the normal transport condition analyses of the lead-shielded rail cask. The predicted peak fuel temperatures in this cask are shown as a function of assembly heat generation rate in Figure II-115. A decay heat rate of $1.0 \mathrm{~kW}$ per spent-fuel assembly results in a predicted peak fuel temperature of $307^{\circ} \mathrm{C}$. The temperature contours for the normal transport conditions of this cask when loaded with 21 1.0-kW PWR assemblies are shown in Figure II-116. 

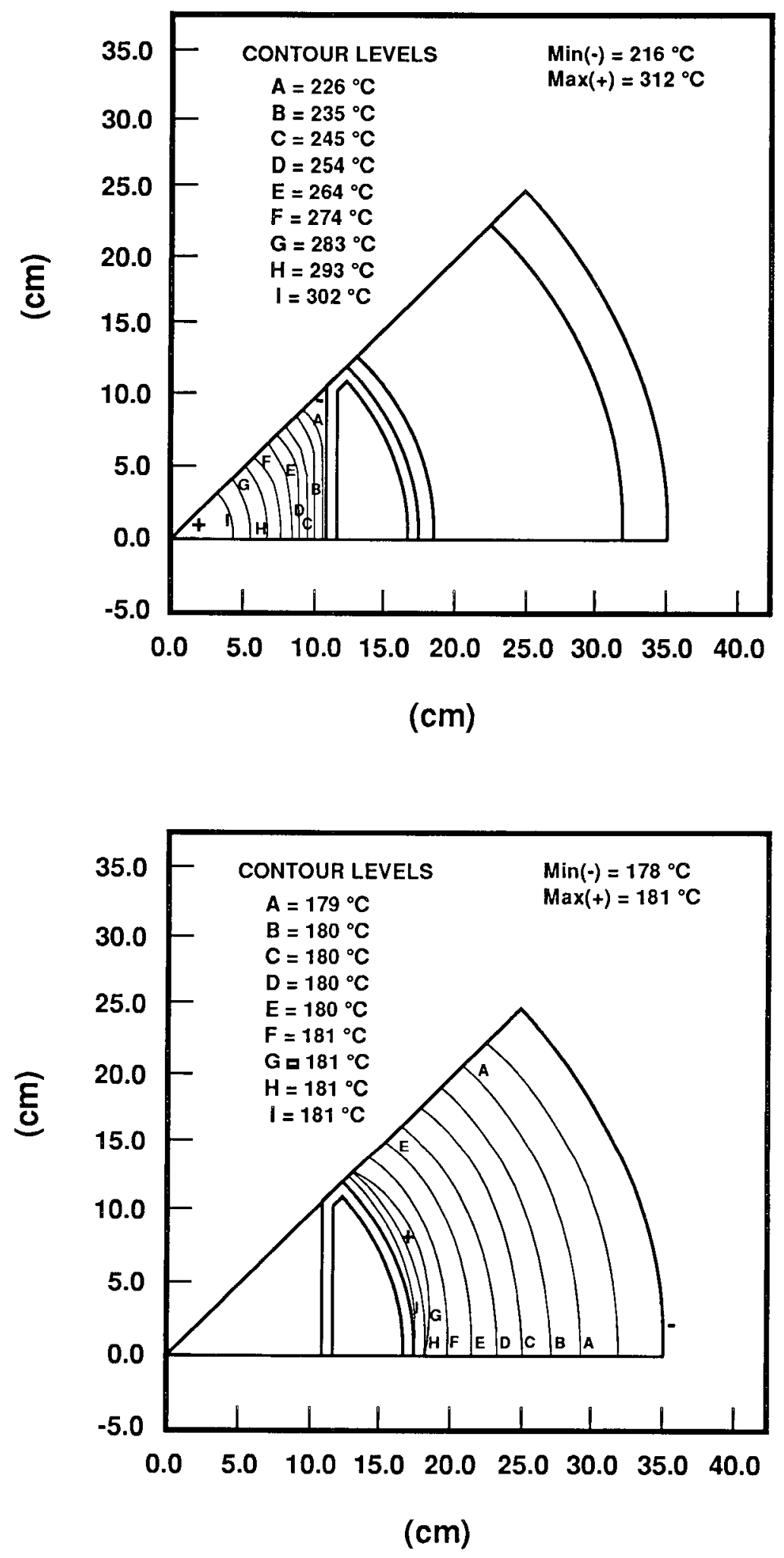

Figure II-114. Predicted Temperature Contours in Fuel (top) and Wall (bottom) of the Truck Cask for $3.0-\mathrm{kW}$ Fuel Under Normal Transport Conditions 


\section{Lead-Shielded Rail Cask-21 PWR-Max Fuel Temperature}

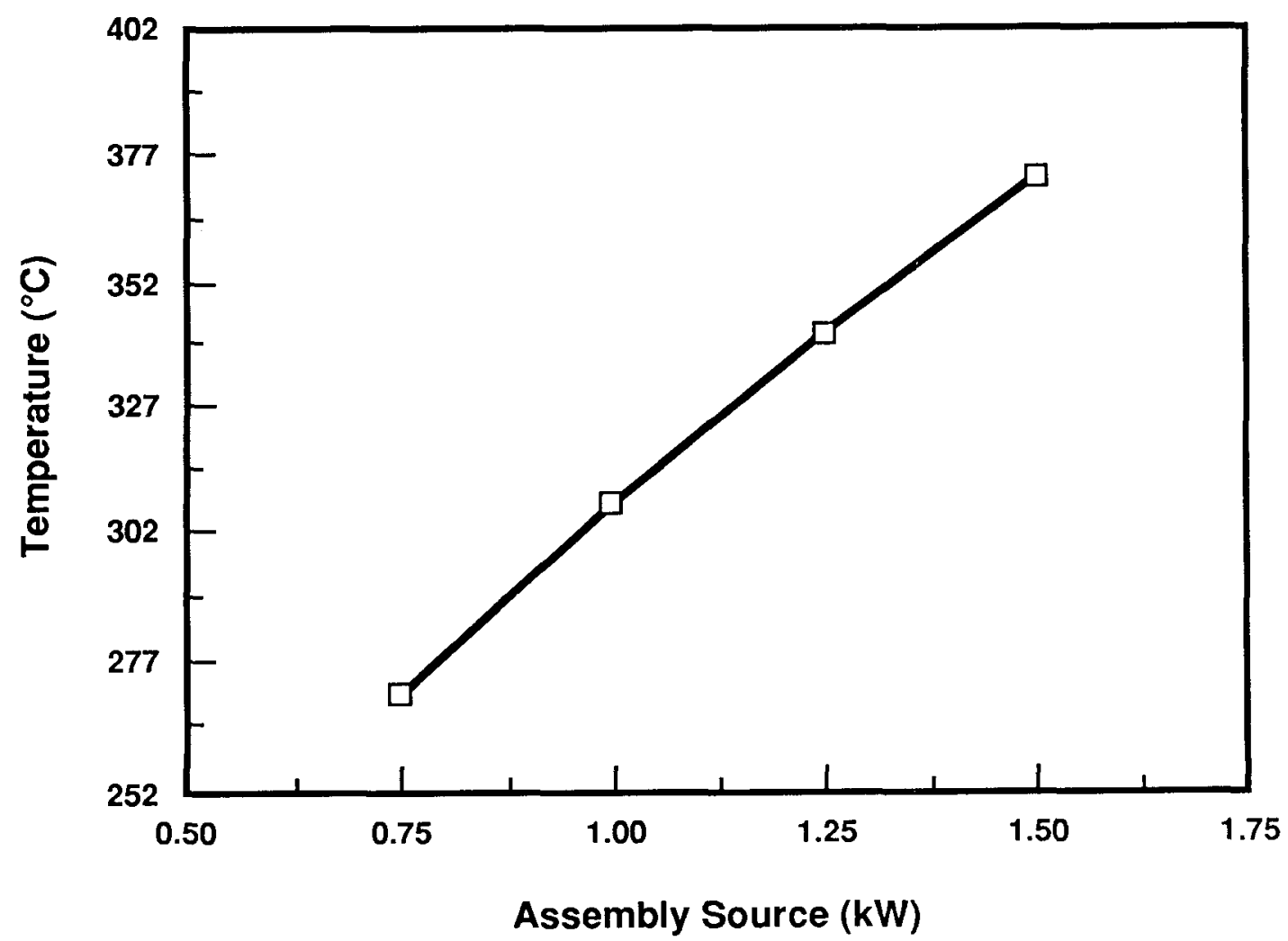

Figure II-115. Predicted Peak Fuel Temperature in the Rail Cask as a Function of Assembly Decay Heat Generation Rate

The thermal response to the regulatory fire accident of the leadshielded truck cask was evaluated assuming the decay heat generation rate of the cask's single PWR assembly was $3.0 \mathrm{~kW}$. The thermal response of the cask was followed for a total model time of $8 \mathrm{hr}(0.5 \mathrm{hr}$ during the fire followed by a 7.5-hr cooldown phase). This was enough time for the temperature at each cask location to reach maximum and then gradually begin to cool.

Temperature-time histories at five cask locations are presented to illustrate the analyses results. The five cask locations include node 1 at the center of the fuel assembly, node 94 at the center of the aluminum basket wedge, node 82 on the inner surface of the cask wall, node 159 near the middle of the lead-shielding, and node 302 on the cask outer surface. Figure II-117 indicates the approximate locations of these nodes in the cask model. 

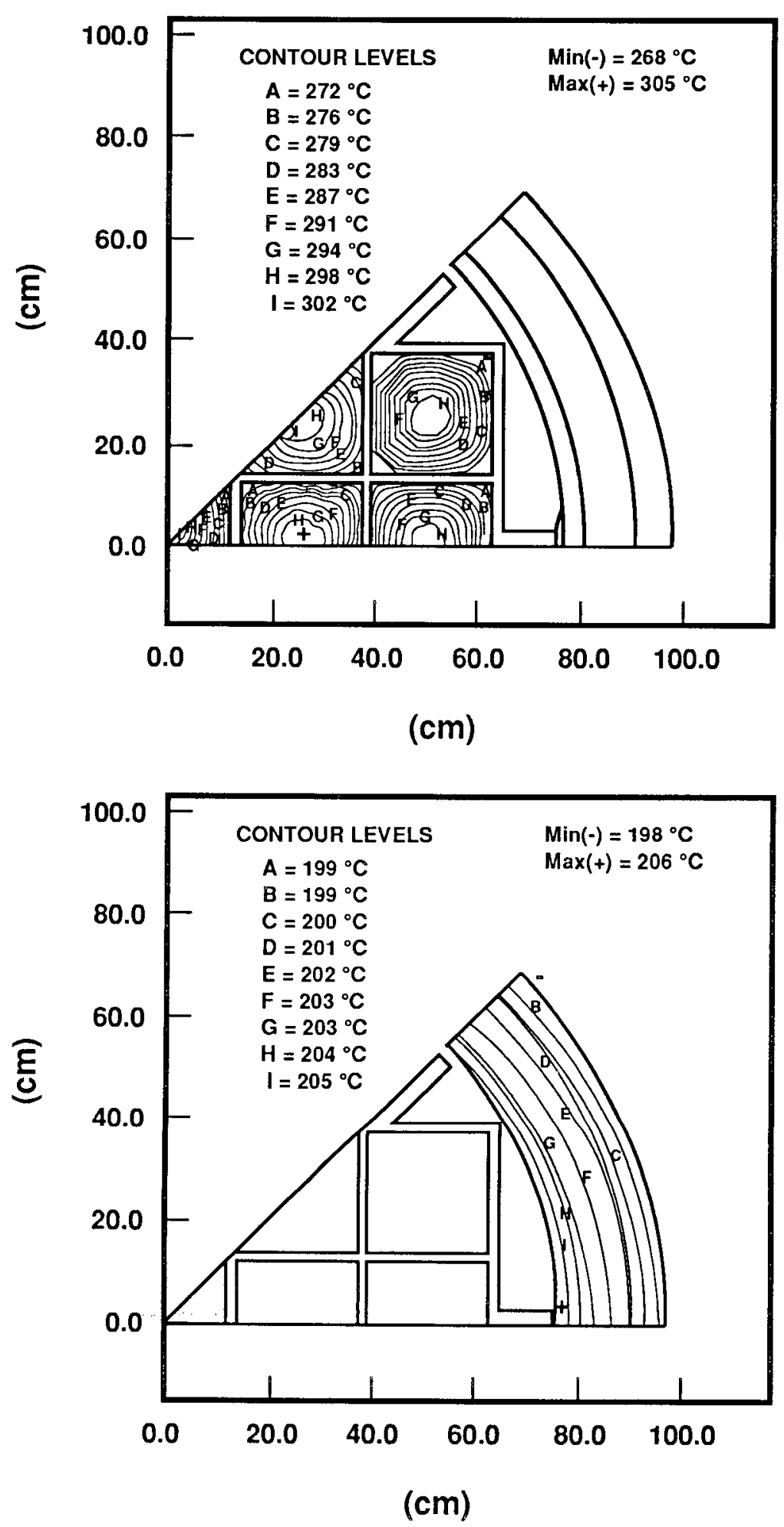

Figure II-116. Predicted Temperature Contours in Fuel (top) and Wall (bottom) of the Rail Cask for $1.0-\mathrm{kW}$ Fuel Under Normal Transport Conditions 


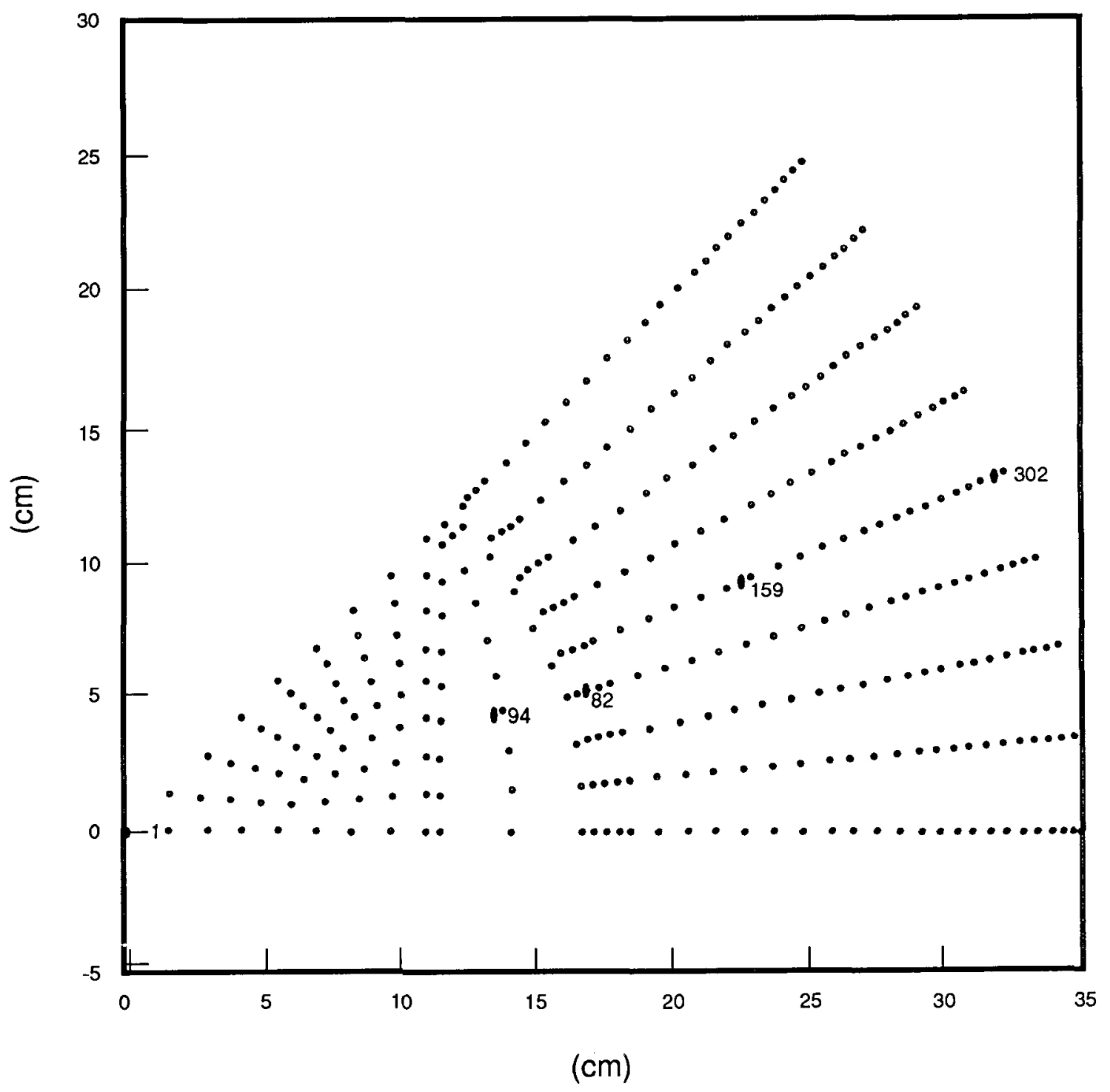

Figure II-117. Truck Cask Node Locations for Which Fire Accident Temperature Histories Are Presented

The predicted temperature histories of the above cask model nodes are shown in Figure II-118. Figure II-118 shows that the fuel in the cask is predicted to undergo a temperature increase of approximately $100^{\circ} \mathrm{C}$ up to a temperature of approximately $402^{\circ} \mathrm{C}$. This peak temperature is estimated to occur almost $3 \mathrm{hr}$ after the end of the regulatory fire.

Softening of the aluminum basket segment in this cask is predicted to be a potential problem. This problem can be minimized by using aluminum that is capable of withstanding high temperatures or by using other basket materials such as stainless steel. Changing to steel would only moderately increase cask internal temperatures. Note, however, that the basket wedge 


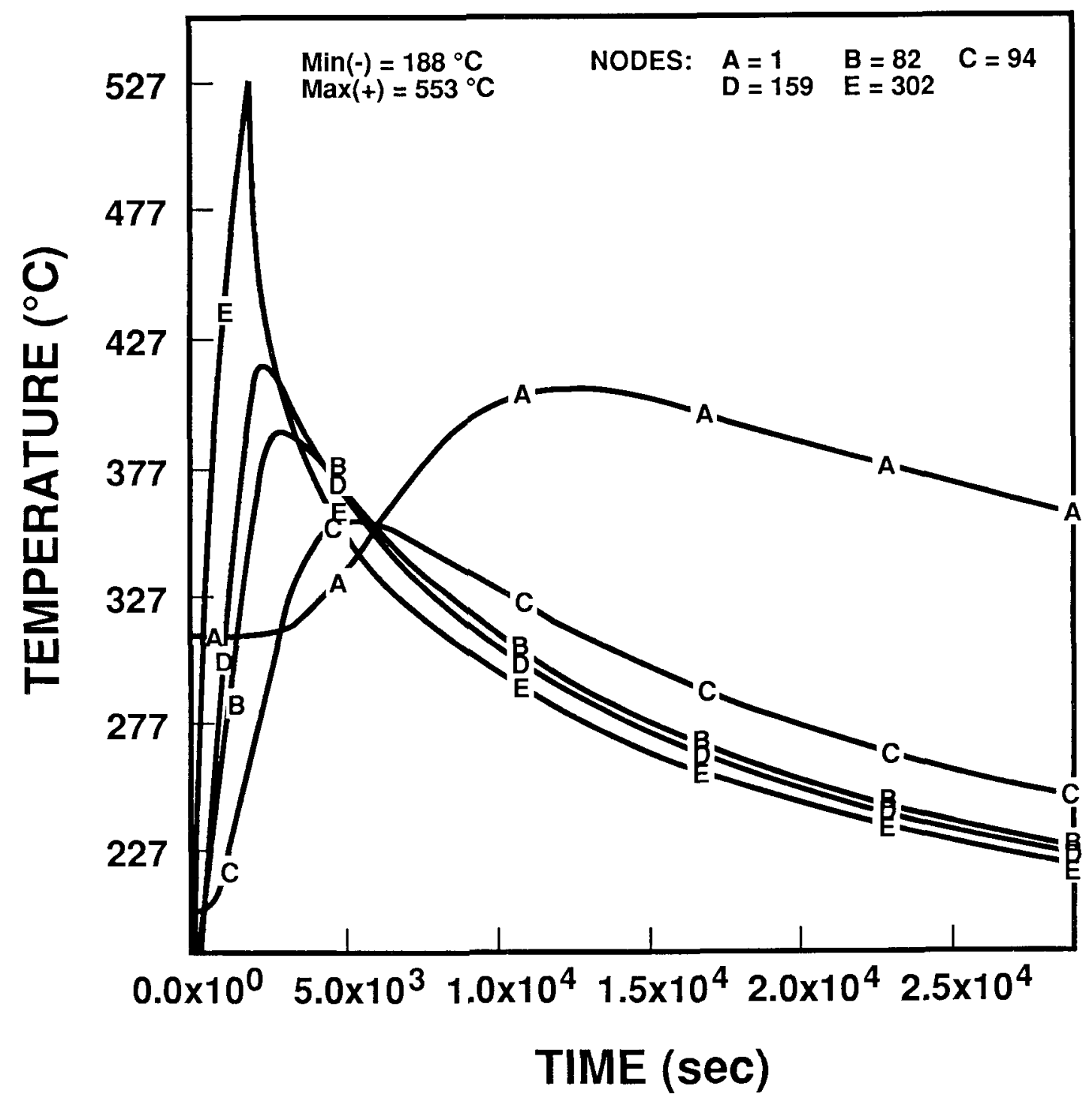

Figure II-118. Predicted Regulatory Fire Accident Temperature $\left({ }^{\circ} \mathrm{C}\right)$ Histories of Several Truck Cask Nodes

in this cask design concept is not required to carry any significant structural loads. Thus, softening of the basket material may not present a significant problem.

The results plotted in Figure II-118 also suggest that lead melt that begins at a temperature of approximately $327^{\circ} \mathrm{C}$ is predicted to occur during cask response to the regulatory fire. Provisions would be necessary in the cask design to accommodate the lead expansion and melt, or the cask could be provided with a thermal shield to help prevent lead melt. A simple thermal shield concept could involve a steel jacket around the cask. A hydrogenous material could be inside the jacket that would provide 
additional neutron shielding under the normal transport conditions without seriously impairing heat rejection from the cask interior. Under accident conditions, the hydrogenous material could be vented or burned away, leaving the steel jacket in place to protect the outer cask steel shell from the direct effects of the fire. Such a shield is used in the NLI-1/2 cask [NL80].

The regulatory fire accident analysis of the lead-shielded rail cask was performed assuming the decay heat generation rate of each spent-fuel assembly in the cask was $1.0 \mathrm{~kW}$. This analysis was continued for a model response time of $30,000 \mathrm{~s}$ (i.e., for nearly $8 \mathrm{hr}$ after termination of the $0.5-h r$ duration regulatory fire). At this point in time, all cask locations were predicted to have begun to cool.

Temperature history predictions at five locations in the rail cask model are presented to indicate the general results. The cask locations involved include node 6 at the cask outer surface, node 168 at the cask inner surface, node 213 at the outer surface of the fuel basket, node 427 in an inner portion of the basket, and node 482 at a fuel assembly center. Node 482 was the hottest location by $0.2^{\circ} \mathrm{C}$ under the normal transport conditions. Figure II-119 shows the locations of these five nodes in the finite element model of the rail cask.

The predicted temperature-time histories of the above rail cask nodes for the regulatory fire conditions are shown in Figure II-120. Because the thermal mass in comparison to its external surface area is much greater for the lead-shielded rail cask than for the lead-shielded truck cask, it is not surprising that the regulatory fire is predicted to have less effect on the thermal mass than it does on the external surface area. The figure indicates that the spent-fuel temperature at node 482 is predicted to increase only about 40 to $-347^{\circ} \mathrm{C}$ as a result of the fire. This node reaches a peak temperature at the end of the analysis (nearly $8 \mathrm{hr}$ after the end of the fire).

As with the lead-shielded truck cask, lead melt is also predicted to occur in the rail cask. Once again, a thermal shield could easily alleviate this problem without substantial effect on the temperature distribution in the cask interior in the normal transport conditions.

The time histories in Figure II-120 also suggest that the aluminum basket in the rail cask is predicted to be fairly isothermal. The temperature histories of the two basket nodes (nodes 213 and 427) are predicted to be quite similar throughout the response to the regulatory fire. Thermal softening of the aluminum could also be a concern in this cask.

\section{II.5.6 Thermal Analyses Conclusions}

The purposes of the thermal analyses of the representative cask concepts are twofold: 


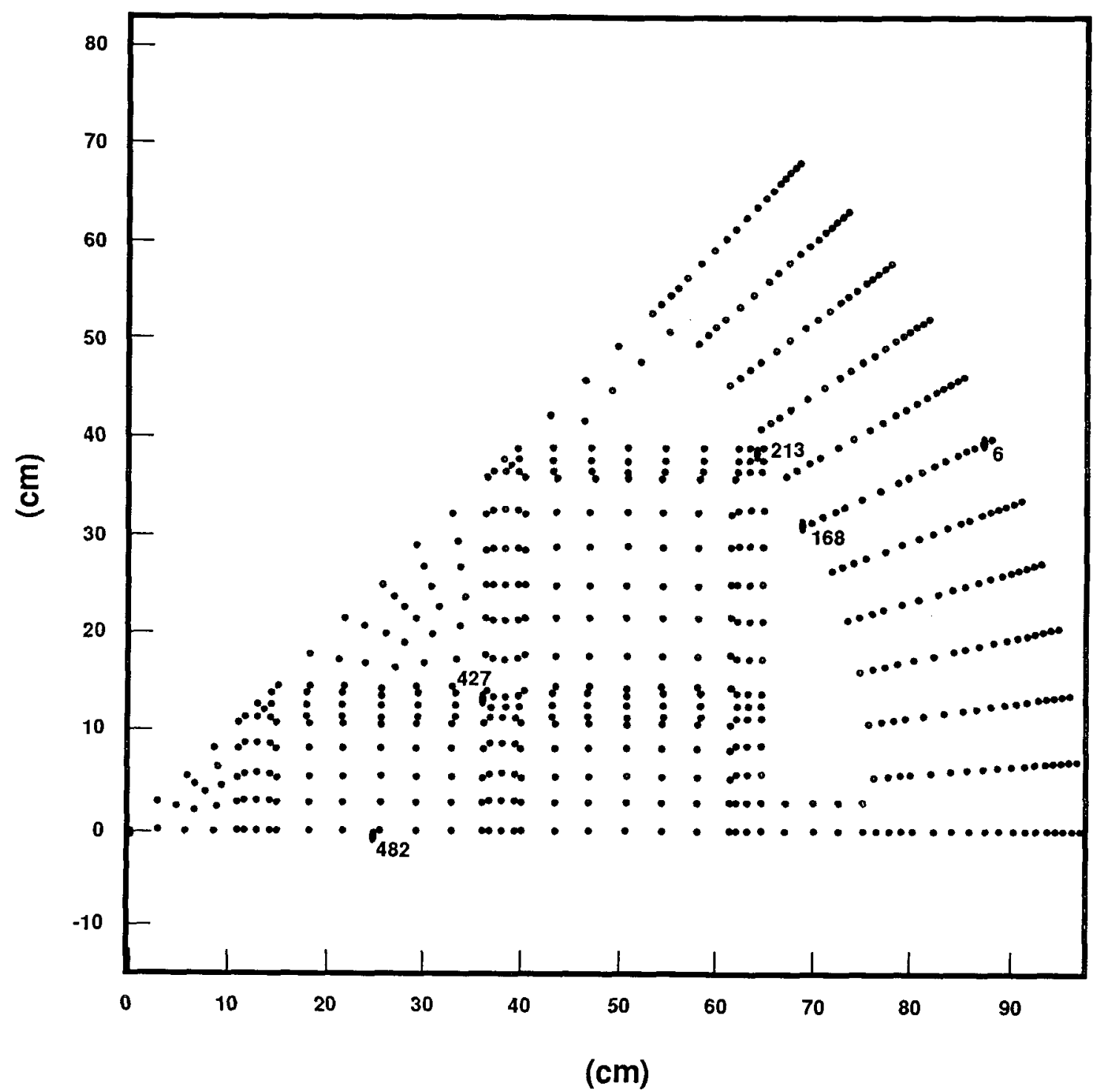

Figure II-119. Rail Cask Node Locations for Which Fire Accident Temperature Histories Are Presented

1. To quantify the temperatures of spent fuel in the casks under normal transport conditions and hypothetical accident conditions, and

2. To contrast the predicted temperatures to those estimated as necessary to cause thermally induced fuel failure mechanisms.

The thermal response analyses were performed using two-dimensional planar models of one-eighth pie sections of the casks taken from their axial midlength. The cask models were analyzed using the finite element method. 


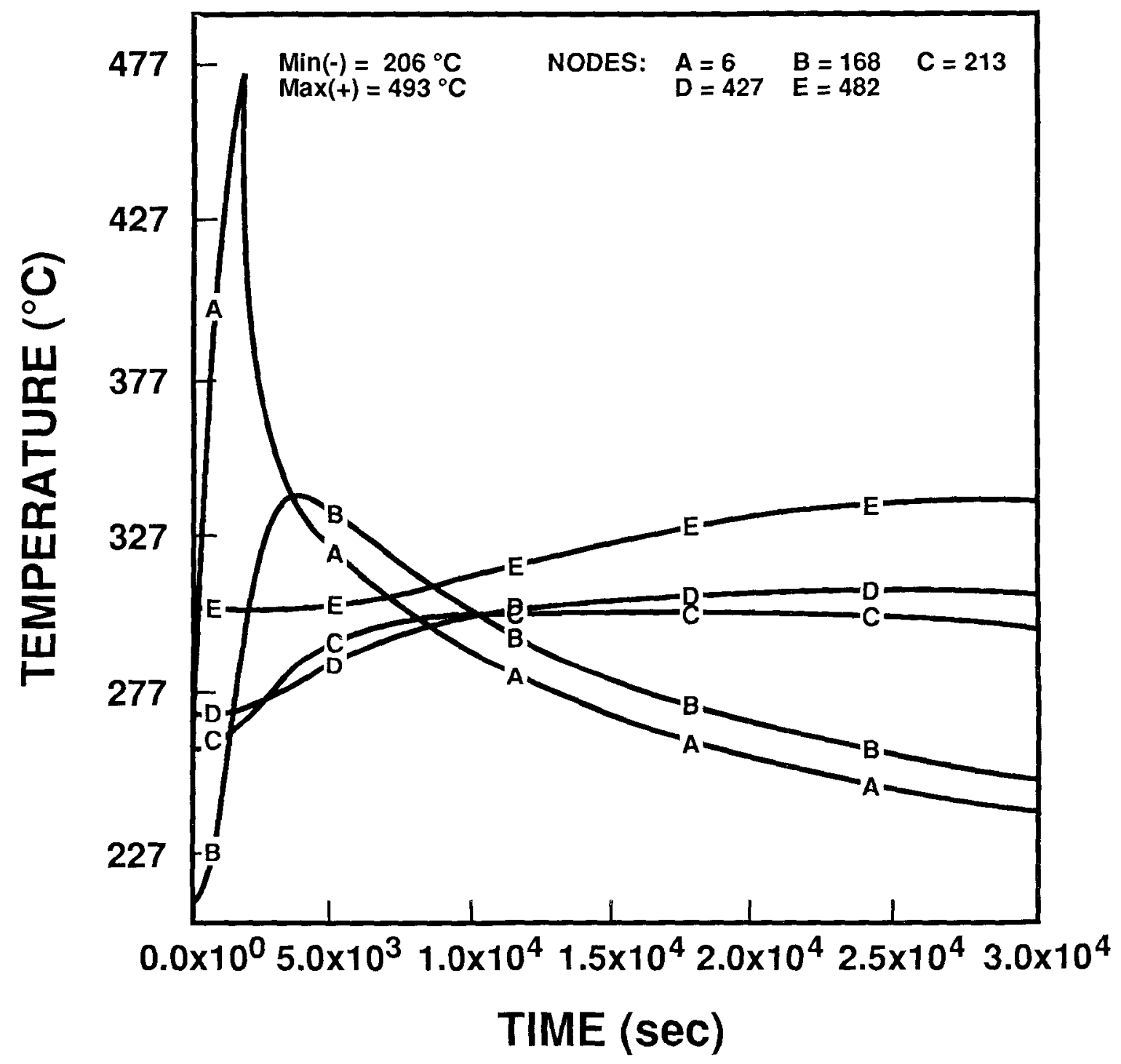

Figure II-120. Predicted Regulatory Fire Accident Temperature $\left({ }^{\circ} \mathrm{C}\right)$ Histories of Several Rail Cask Nodes

The analyses of the lead-shielded truck cask for the normal transport conditions resulted in a predicted peak fuel temperature of $315^{\circ} \mathrm{C}$ for a PWR spent-fuel assembly decay heat generation rate of $3.0 \mathrm{~kW}$. For the leadshielded rail cask, the predicted peak fuel temperature under normal transport conditions was $307^{\circ} \mathrm{C}$ for a decay heat rate of $1.0 \mathrm{~kW}$ per assembly in the cask. Both peak temperatures are less than the allowable spent-fuel storage temperature of $378^{\circ} \mathrm{C}$.

Burian identifies five thermally induced failure modes of LWR fuel by surveying available data on the thermal response of LWR fue1 [BU85]. These include cladding melting, eutectic formation involving cladding and other 
elements, cladding oxidation and embrittlement, cladding burst, and cladding failure as a result of fuel ratcheting. According to Burian, only cladding burst of the five failure modes is possible as a result of an extreme thermal environment during spent-fuel transport in a shipping cask.

Cladding burst is shown by Burian [BU85] to depend on such factors as differential pressure across the cladding wall, cladding wall temperature, and heating rate. For spent fuel in a cask subjected to a fire, the heating rate is low (less than $1^{\circ} \mathrm{C} / \mathrm{s}$ ). From tests involving tubes with typical initial PWR internal pressures, Burian indicates that clad rupture of sound irradiated fuel rods may occur at a cladding temperature of $725^{\circ}$ to $750^{\circ} \mathrm{C}$ or above.

The transient analysis of the lead-shielded truck cask for response to the regulatory fire environment indicates that the peak fuel temperature of the cask would be $-402^{\circ} \mathrm{C}$. For the lead-shielded rail cask, the peak fuel temperature is predicted to be $347^{\circ} \mathrm{C}$ for the regulatory fire. Thus, the peak fuel temperatures in these casks for the thermal conditions analyzed are less than the $-727^{\circ} \mathrm{C}$ temperature estimated by Burian as being required to induce fuel failure by burst rupture. See Appendix III for more detailed evaluation of the effects of regulatory fire on spent fuel.

\section{II.6 Summary Remarks}

The most severe normal and hypothetical accident transport conditions defined in $10 \mathrm{CFR} 71$ are considered in this characterization of the spentfuel environment. The conditions for which the spent-fuel environments are investigated and quantified include the following:

1. Cask acceleration response to the $0.3-\mathrm{m}$ and $9.0-\mathrm{m}$ drop impact events,

2. Shock and vibration normally occurring in over-the-road and overthe-rail transport, and

3. Cask and contents thermal response to the normal thermal environment and to the regulatory hypothetical fire scenario.

Four analysis methods are considered for quantifying cask accelerations resulting from the 0.3- and 9.0-m drop impacts. Based on the nature of the analytical model for spent-fuel response, the rigid-body kinematics analysis method is considered most suitable for generating the necessary quantified environments. This method provides acceleration time history estimates for two translational and one rotational degree of freedom of the impacting cask, which is modeled as a rigid-body with attached impact limiters. A computer code is described that implements the rigid-body kinematics analysis method. This code is used to estimate the impact accelerations for four representative cask concepts. The code can be used to estimate the impact-induced rigid-body accelerations for any cask with impact limiters for which the force-deflection characteristics are known. 
An extensive survey of relevant measured shock and vibration data for both truck and rail transport modes is discussed. The most appropriate data from this survey are used to construct simplified conservative envelopes that bound the shock and vibration data from above. Two procedures for using the data in the analytical model for spent-fuel response are proposed: modal analysis coupled with acceleration shock spectra and equivalent half-sine pulse coupled with transient time integration.

Finally, the thermal response analyses of two of the representative cask concepts are described. First, the maximum PWR decay heat loads are determined that could be carried in the two casks under normal transport conditions without exceeding acceptable peak fuel temperatures (based on the recommendations by the DOE [US85]). Then the transient responses of the casks to the regulatory fire are investigated. Thermally induced failure (i.e., cladding rupture) of the spent fuel in the casks was assessed to be unlikely.

The quantified impact, shock, and vibration environments presented in this report are believed to be realistic. Shock and vibration environments are considered to be clearly conservative. The quality of the characterizations and degree of conservatism could be assessed and improved, however, by performing a few additional tests. Interesting and rather simple tests that would specifically address the conservatism of the shock and vibration environments could be performed with accelerometer-instrumented fresh fuel inside instrumented casks shipped by truck and rail. A considerably more complicated test verifying whether casks respond as rigid bodies during impacts could be performed with accelerometer-instrumented fresh fuel or fuel models inside a drop-tested obsolete cask or a cask model (possibly even subscale). This test would not only verify the rigid-body response of the cask, but also would provide direct information concerning the actual accelerations of spent fuel during cask impact.

\section{II.7 References}

[AD81] Adams, C., A Comparison of Analytical Techniques for Analyzing a Nuclear-Spent-Fuel Shipping Cask Subjected to an End-On Impact, Sandia National Laboratories, Albuquerque, NM, NUREG/CR-2018, SAND80-1870, June 1981.

[AN80] American National Standards Institute, Draft American National Standard Design Basis for Resistance to Shock and Vibration of Radioactive Material Packages Greater Than One Ton in Truck Transport, ANSI Standard N14.23, May 1980.

[AR66] Arpaci, V. S., Conduction Heat Transfer (Reading, MA: AddisonWesley Publishing Company, 1966).

[BI64] Biggs, J. M., Introduction to Structural Dynamics (New York: McGraw-Hill, Inc., 1964). 
[BU85] Burian, R. J., Response of Spent LWR Fuel to Extreme Environments, Sandia National Laboratories, Albuquerque, NM, SAND85-7213, August 1985 .

[CR74] CRC, CRC Handbook of Chemistry and Physics (Cleveland, OH: CRC Press, 1974).

[DA82] Davidson, C. A., and J. T. Foley, Transportation Technical Environmental Information Center Index, Sandia National Laboratories, Albuquerque, NM, SAND82-1200, June 1982.

[EP86] EPRI, The Castor $-V / 21$ PWR Spent Fue1 Storage Cask: Testing and Analyses, Electric Power Research Institute, Palo Alto, CA, EPRI NP-4887, November 1986.

[FI78] Fields, S. R., SAVIT--A Dynamic Model to Predict Vibratory Motion Within a Spent Fuel Shipping Cask--Rail Car System, Hanford Engineering Development Laboratory, Rich1and, WA, March 1978.

[FI83] Fields, S. R., Dynamic Analysis to Establish Normal Shock and Vibration of Radioactive Material Shipping Packages, Vol. 3, Hanford Engineering Development Laboratory, Richland, WA, NUREG/CR2146, HEDL-TME 83-18, October 1983.

[FI87] Fischer, L., Shipping Container Response to Serve Highway and Railway Accident Conditions, Lawrence Livermore National Laboratory, Livermore, CA, NUREG/CR-4829, February 1987.

[F071] Foley, J. T., and M. B. Gens, "Shock and Vibration Measurements During Normal Rail and Truck Transport," International Journal of Radiation Engineering, 1, 5, pp. 453-474 (1971).

[F072a] Foley, J. T., M. B. Gens, and C. F. Magnuson, "Current Predictive Models of the Dynamic Environment of Transportation," in Proceedings of the Institute of Environmental Sciences, May 5, 1972.

[F072b] Foley, J. T., Transportation Shock and Vibration Descriptions for Package Designers, Sandia National Laboratories, Albuquerque, NM, SC-M-72 0076, July 1972.

[F079] Forest, J. W., Irradiated Fuel Transportation Shock and Vibration Program Rail and Truck Field Tests, Ontario Hydro Research Division Report, 79-149-K, March 29, 1979.

[GE72] Gens, M. B., The Transportation and Handling Environment, Sandia National Laboratories, Albuquerque, NM, SC-DC-72-1386, September 1972 .

[GL67] Glasstone, S., and A. Sesonske, Nuclear Reactor Engineering (New York: Van Nostrand Reinhold Company, 1967). 
[GL86] Glass, R. E., and K. W. Gwinn, "Shock and Vibration Environments for Truck-Transported Nuclear Waste: Test and Analysis," Journal of the Institute of Nuclear Materials Management, XV, pp. 319-323 (June $22-25,1986$ ).

[GL87] Glass, R. E., and K. W. Gwinn, TRUPACT-I Over-the-Road Test, Sandia National Laboratories, Albuquerque, NM, SAND87-0518, October 1987.

[GR84] Grant, J., and V. Gabrielson, Shell Shock Structural Code, Sandia National Laboratories, Albuquerque, NM, SAND83-8011, March 1984.

[HA61] Harris, C. M., and C. E. Crede, "Shock and Vibration in Road and Rail Vehicles," in Shock and Vibration Handbook (New York: McGrawHil1, 1961), Chap. 45, pp. 45-1 to 45-39.

[HA83] Hallquist, J. O., ORION: An Interactive Post-Processor for the Analysis Codes NIKE2D, DYNA2D and TACO2D, Rev. 1, Lawrence Livermore National Laboratory, Livermore, CA, UCID-19310, July 1983.

[HA84] Hallquist, $J$. O., User's Manual for DYNA2D--An Explicit TwoDimensional Hydrodynamic Finite Element Code with Interactive Rezoning, Rev. 2, Lawrence Livermore National Laboratory, Livermore, CA, UCID-18756, January 1984.

[H067] Hottel, H. C., and A. F. Sarafim, Radiative Transfer (New York: McGraw-Hill, 1967).

[HU88] Huerta, M., and H. Yoshimura, A Discussion of Impact Limiters for Radioactive Material Packagings, Sandia National Laboratories, Albuquerque, NM, to be published.

[KR73] Kreith, F., Principles of Heat Transfer--Third Edition (New York: Harper \& Row, Publishers, 1973).

[MA77a] Magnuson, C. F., and L. T. Wilson, Shock and Vibration Environments for Large Shipping Containers on Rail Cars and Trucks, Sandia National Laboratories, Albuquerque, NM, SAND76-0427, NUREG 766510, June 1977.

[MA77b] Magnuson, C. F., Shock and Vibration Environments for Large Shipping Containers During Truck Transport (Part I), Sandia National Laboratories, Albuquerque, NM, SAND77-1110, September 1977.

[MA78] Magnuson, C. F., Shock and Vibration Environments for a Large Shipping Container During Truck Transport (Part II), Sandia National Laboratories, Albuquerque, NM, SAND78-0337, NUREG/CR-0128, May 1978. 
[MA80] Magnuson, C. F., Shock Environments for Large Shipping Containers During Rail Coupling Operations, Sandia National Laboratories, Albuquerque, NM, SAND79-2168, NUREG/CR-1277, June 1980.

[MA82] Magnuson, C. F., Shock and Vibration Environments Encountered During Normal Rail Transportation of Heavy Cargo, Sandia National Laboratories, Albuquerque, NM, SAND82-0819, August 1982.

[NL80] NLI, Safety Analysis Report--NLI $1 / 2$ Spent Fuel Shipping Cask, NL Industries, Inc., Wilmington, DE, 1980.

[NU85] NUPAC, Safety Analysis Report for the NUPAC 125-B Fuel Shipping Cask, Rev. 1, Nuclear Packaging Inc., Federal Way, WA, October 1985.

[PD84] PDA, PATRAN User's Guide, PDA Engineering, Santa Ana, CA, July 1984.

[SH83] Shapiro, A. B., FACET--A Radiation View Factor Computer Code for Axisymmetric, 2D Planar, and 3D Geometries with Shadowing, Lawrence Livermore National Laboratory, Livermore, CA, UCID-1987, August 1983.

[SH86] Shapiro, A. B., TOPAZ2D--A Two-Dimensional Finite Element Code for Heat Transfer Analysis, Electrostatic, and Magnetostatic Problems, Lawrence Livermore National Laboratory, Livermore, CA, UCID-20824, July 1986.

[SP61] Sparrow, E. M., E. R. Eckert, and T. F. Irvin, "The Effectiveness of Radiating Fins with Mutual Irradiation," Journal of the Aerospace Sciences (October 1961).

[ST70] Stewart, R. E. D., and F. C. Wessling, On Heat Conduction in Solids, Sandia National Laboratories, Albuquerque, NM, SC-RR-70334,1970 .

[TH72] Thomson, W. T., Theory of Vibration with Applications (Englewood Cliffs, NJ: Prentice-Ha11, Inc., 1972).

[US85] U.S. DOE, Spent Fuel Storage Requirements, U.S. Department of Energy, Richland Operations Office, Richland, WA, DOE/RL-85-2, 1985 . 
This page is intentionally left blank. 
APPENDIX III

SPENT-FUEL RESPONSE TO TRANSPORT ENVIRONMENTS 
This page is intentionally left blank.

I I I - 2 


\section{III.1 Introduction}

The spent-fuel response model provides a basis for determining the probability, type, and location of fuel rod failure during normal and regulatory accident transport conditions. This appendix describes the detailed structural and material models of the fuel assemblies and cask internal hardware that were developed. With the as-shipped conditions and material properties of spent fuel, analytical methods were developed for whole-assembly and single-rod geometries. These analyses were performed for all potentially critical cask drop orientations. The analysis models allow interaction between a cask basket and assemblies within and between assembly hardware and constituent fuel rods.

Spacer grids are the major elements of impact-force transfer mechanisms. The stiffness of assembly spacer grids significantly influences impact deformation of the fuel assemblies and the response level of the individual rods. Thus, emphasis was placed on spacer grid modeling and characterization of the highly nonlinear force-displacement relationships between the spacer grid springs and fuel rods.

The calculated fuel rod response to a specific environment was evaluated against criteria developed for all feasible fuel failure modes. These failure criteria account for the residual cladding ductility and fracture properties as functions of irradiation and thermal environments. Because force transfer is characteristically different for side and end drops, two types of models were developed to determine fuel rod cladding response to loads characteristic of normal and accident transport conditions.

The first model deals with side-drop and slapdown loading conditions. A set of two-dimensional longitudinal slice models of the fuel assembly is used to characterize these loading conditions. These models are capable of treating the significant rod-to-rod interaction that occurs during sidedrop loading conditions. The side-drop models are analyzed dynamically using a typical cask deceleration time history (see Appendix II). The slapdown component of a corner-drop loading is analyzed with a modified version of the side-drop model because the assembly response under slapdown loading is similar to the response under side-drop loading.

The second type of model deals with the end-drop loading condition for which the fuel rod response is assumed to be characterized by a single rod. This single-rod approximation of the assembly is believed to be conservative because there is little rod-to-rod load transfer during enddrop loading conditions. The single-rod model includes the constraints of the spacer grids and basket frame; however, it conservatively neglects rodto-rod contact between the grids. The end-drop models were analyzed similarly to the side-drop model, with deceleration time histories modified to model any increase in load caused by gaps between the assemblies and cask.

All plausible burst and breaking modes of failure are discussed. Burst failure is postulated for overpressurization of the fuel rods as a result of external heat input. The onset of failure is determined on the 
basis of the stresses, strains, and rod-to-rod crush forces, which are deterministically calculated. A probabilistic approach is used for determining the ductile and brittle failure limits of the cladding. A fracture-mechanics-based failure criterion is used to analyze rods with part-wall cracks. Two types of failure are considered: circumferential tearing and longitudinal tearing. Circumferential tearing is driven primarily by longitudinal forces caused by excessive fuel rod bending. Longitudinal tearing is caused by large hoop stresses in the rods. These stresses are caused by a combination of internal rod pressurization and rod ovalization at the spacer grids as a result of rod-to-rod pinch forces.

The following sections provide a general description of the methodology used in determining fuel rod failure. Example analyses of a $7 \times 7$ boiling water reactor (BWR) assembly of General Electric (GE) design and a $15 \times 15$ pressurized water reactor (PWR) assembly of Babcock \& Wilcox (B\&W) design are provided to illustrate the procedure. Figure III-1 illustrates the terms herein that describe the fuel rod, assembly, and cask components.

Section III.2 describes all feasible fuel rod failure modes, including burst and breakage. Section III.3 describes the structural modeling and analysis methodology of the fuel assemblies. The finite element method used to determine the magnitude, location, and distribution of fuel rod stresses and strains is also described. Section III.4 establishes the fuel rod failure criteria. The number and locations of predicted fuel rod failures are based on deterministic response analyses and probabilistic material failure criteria. A fracture mechanics approach is used to determine failure of part-wall cracks. Section III.5 describes the structural analysis of two example assemblies, GE $7 \times 7$ BWR and B\&W $15 \times$ 15 PWR, that are used to demonstrate the analysis methodology. Detailed descriptions of the spacer grid analyses of these assemblies are included. The analyses characterize the fuel assembly response under side-, end-, and corner-drop loading conditions. Section III.6 discusses the methodology for fuel failure evaluation. This section combines the end-, side-, and corner-drop deterministic analyses results with a probabilistic assessment of part-wall crack number, size, distribution, and tensile strain failure limits to determine the frequency of fuel rod failure. The resulting failure probabilities are based on the example analyses of Section III.5 and do not necessarily represent bounding calculations.

\section{III.2 Failure Modes}

Fuel rod cladding failure mechanisms can be divided into two categories: burst failure and breaking failure. Burst failure is defined as a local rupture of the fuel rod cladding and is characterized by a pinhole or longitudinal slit in the cladding caused by fuel rod overpressurization (most likely during fire) in conjunction with an imperfection of the fuel rod cladding. Breaking failure is the rupture of the fuel rod cladding caused by either longitudinal or transverse tearing of the cladding material as a result of normal and accident transport conditions. Fuel rod breaking is caused by an external mechanical load on 


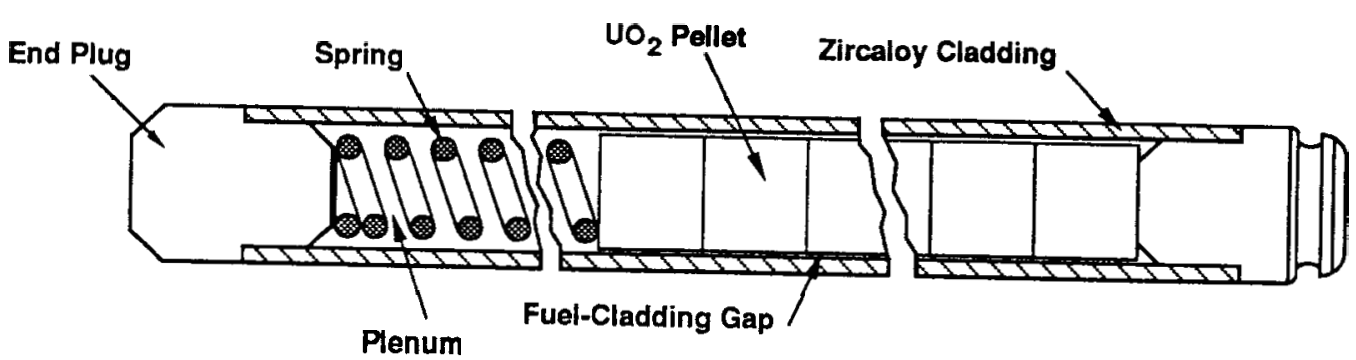

FUEL ROD DEFINTIONS
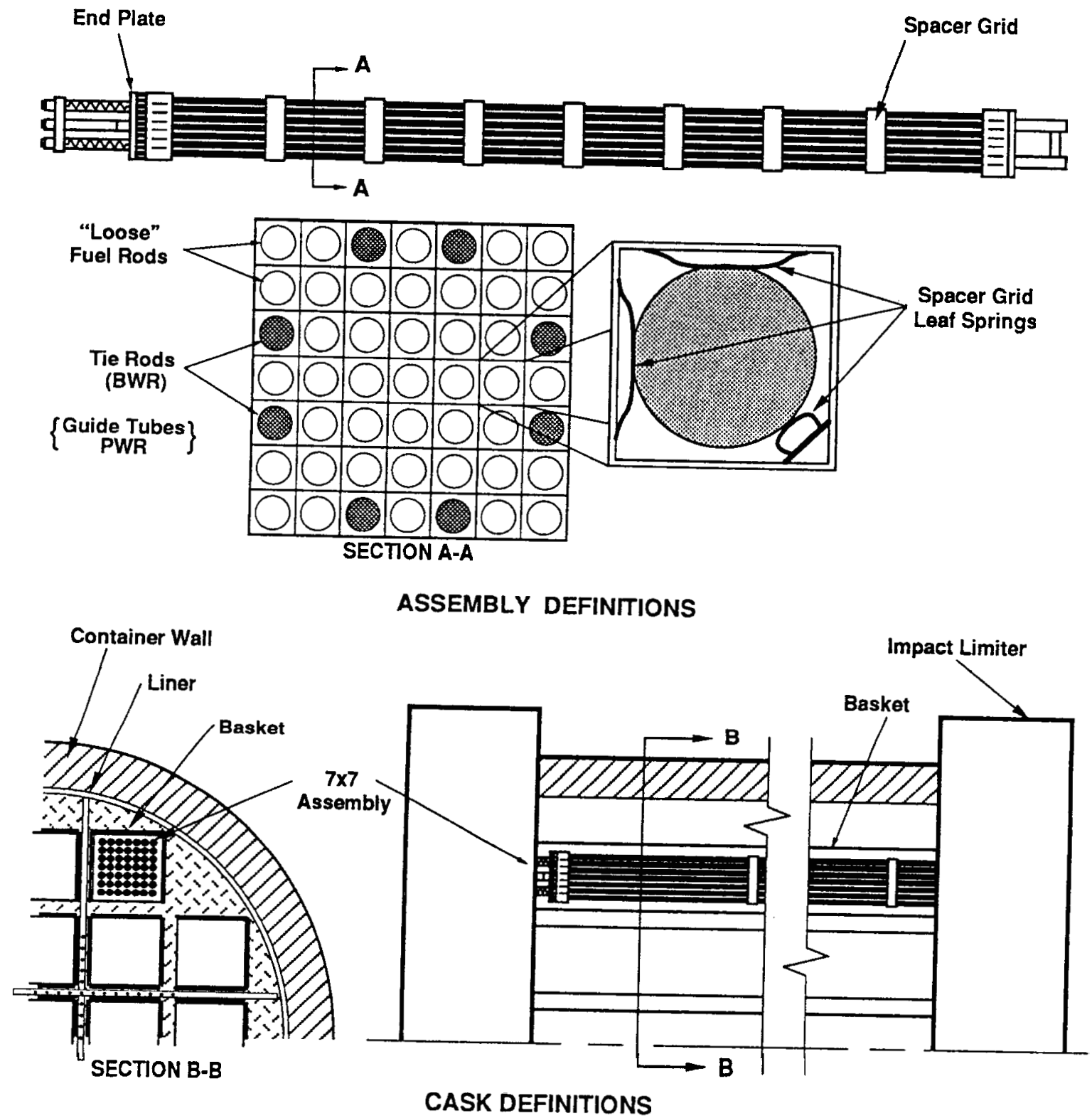

Figure III-1. Fue1 Rod, Assembly, and Cask Component Definitions 
the fuel rods. However, the breaking failure modes also take into account the stresses in the fuel rods resulting from internal pressurization.

\section{III.2.1 Burst Fallure}

The following sections discuss the plausibility of burst failure as a result of fire, melting, oxidation, eutectic formation, and zirconium-water reaction.

Fire

Fuel rod failure as a result of thermally induced overpressurization is the most probable mode of burst-type failure. Burian [BU85] identifies cladding burst as the only plausible mode of fuel rod failure as a result of extreme thermal environment during spent-fuel transport in shipping casks. Experimental analyses [BU85] indicate that failures in irradiated fuel rods occur at cladding temperatures in the range of 725 to $750^{\circ} \mathrm{C}$. For rod failure to occur, a 4-hr duration of this peak temperature is required. The peak regulatory fire temperature (outside the cask) is $800^{\circ} \mathrm{C}$, as defined by 10 CFR 71 . Thermal analyses performed to determine the corresponding maximum fuel rod temperature history are described in Appendix II. An upper bound maximum fuel temperature based on a peak initial fuel temperature of $380^{\circ} \mathrm{C}$ and a regulatory fire is $530^{\circ} \mathrm{C}$ (see Appendix II). The fuel rod temperature history for this event, shown in Figure III-2, was extrapolated from the truck cask regulatory fire analysis of Appendix II.

The only mechanism for thermally induced failure is the extension of existing part-wall cracks in the cladding as a result of fuel rod overpressurization because the peak fuel temperature of $530^{\circ} \mathrm{C}$ is well below the required temperature of $725^{\circ} \mathrm{C}$ for burst failure of sound fuel rods. Other factors, such as oxidation wastage, can further reduce cladding strength by reducing its thickness. Experimental measurements of inreactor oxidation indicate maximum oxide thicknesses in the range of 4 to 108 of the cladding wall thickness for PWRs and up to 128 of the wall thickness for nodules in BWRs [AN79, AN85, IA87]. These data are from spent fuel with burnup levels to $50,000 \mathrm{MWD} / \mathrm{MTU}$. The corresponding reduction in cladding thickness is less because of its higher density. Computations in the following section show that further reduction in thickness during transport is negligible. Thus, it is reasonable to assume that oxidation wastage results in minimal reduction of cladding strength because the maximum change in cladding thickness is roughly $10 \%$. To investigate these effects, detailed thermal stress analyses were performed using the FREY [RA87] analysis code. The stress results of these analyses were used in conjunction with the failure criteria described in Section III. 4 to determine the probability of this fuel rod failure mode. The analysis of an example PWR assembly is described in Section III.5; the corresponding failure probability of the example PWR assembly is outlined in Section III. 6. 


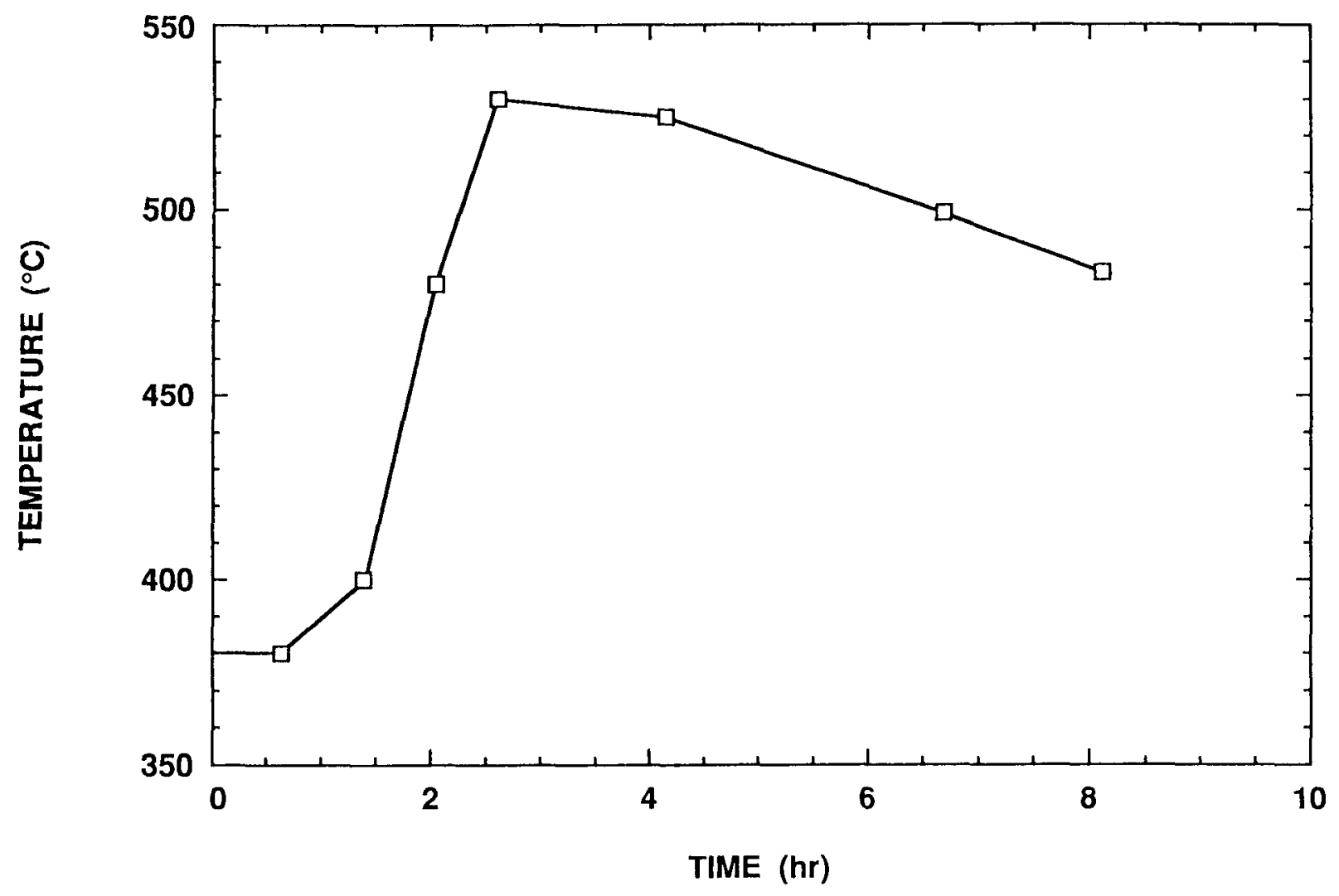

Figure III-2. Example Analysis of a Truck Cask Regulatory Fire-Assembly Temperature History

\section{Me1ting}

Fuel rod melting will occur for temperatures in the range of $1650^{\circ} \mathrm{C}$ [BE80]. Because the maximum temperature likely to occur during a hypothetical accident fire is $530^{\circ} \mathrm{C}$ (Appendix II), melting is not a plausible failure mode for transport conditions.

\section{Zircaloy Oxidation Below $500^{\circ} \mathrm{C}$}

If there is a leak in a transport cask, oxygen may ingress and the Zircaloy cladding may oxidize. The amount of oxidation will depend on oxygen availability and oxidation kinetics. An evaluation of the oxidelayer thickness is illustrated below.

For the purpose of this evaluation, it is assumed that the leak is large enough to permit an ample supply of oxygen, and that the oxidation amount is controlled by the kinetics. Clean Zircaloy surfaces oxidize according to a $t^{1 / 3}$ power 1 aw until some transition oxide thickness is reached that is dependent on temperature. After transition, the oxide film continues to grow according to a " $t$ " (i.e., linear) rate law. In general, the rods attain the transition thickness while in the reactor [HI76]. 
Therefore, for these calculations, posttransition kinetics can be used. Lower temperature data have been collected, evaluated, and fit to existing models by Garzarolli [GA80]. The data are best fit to a model (called the EPRI model) by the equation

$$
\Delta \mathrm{W}=2.21 \times 10^{9} \times \exp (-28,200 / \mathrm{RT}) \times \mathrm{t},
$$

where

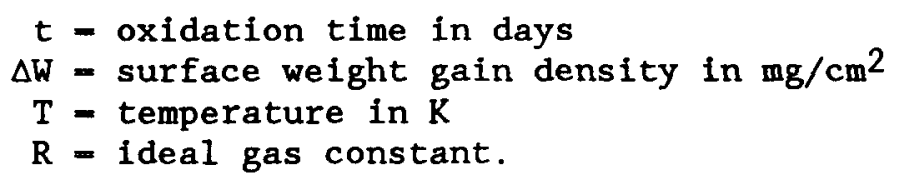

Below $350^{\circ} \mathrm{C}$, a model by Dalgaard [GA80] yields more conservative results (see Figure III-3). This model is

$$
\Delta \mathrm{W}=1.84 \times 10^{7} \times \exp (-22,200 / \mathrm{RT}) \times \mathrm{t} .
$$

Three cases are considered in this analysis:

1. The fuel rod surface temperature maintained at $300^{\circ} \mathrm{C}$ for $30 \mathrm{~min}$;

2. The fuel rod surface temperature maintained at $300^{\circ} \mathrm{C}$ for $30 \mathrm{~min}$ and followed by $1 \mathrm{hr}$ at $400^{\circ} \mathrm{C}$, then $2 \mathrm{hr}$ at $450^{\circ} \mathrm{C}$;

3. The fuel rod surface temperature held between 100 and $300^{\circ} \mathrm{C}$ for 1 to 2 weeks to simulate normal transport conditions.

The surface weight gain density, $\Delta \mathrm{W}$, can be converted to a Zircaloy cladding thickness that oxidizes. The oxidized material volume is $\mathrm{V}=\mathrm{Ah}$, where $A$ is the oxidized area and $h$ is the thickness of the oxidized material. The mass of oxidized Zircaloy is $\rho \mathrm{Ah}$, where $\rho$ is the Zircaloy density. Therefore,

$$
\Delta \mathrm{W} \times \mathrm{A}=\rho \mathrm{Ah} \quad \text { or } \quad \mathrm{h}=\Delta \mathrm{W} / \rho \text {. }
$$

If a Zircaloy density equal to the density of zirconium is used (i.e., $\left.6.45 \mathrm{~g} / \mathrm{cm}^{3}\right)$, the thickness in $\mu \mathrm{m}$ is given by

$$
\mathrm{h}(\mu \mathrm{m})=0.0457 \Delta \mathrm{W} .
$$

Calculations of the expected thickness of oxidized Zircaloy for the three cases and the different models are given in Table III-1. The conservative column in each case refers to the highest amount of oxidation that is predicted independent of the model.

The largest amount of oxidation is predicted for the case of normal transport for 2 weeks at $300^{\circ} \mathrm{C}$. One may expect $0.038 \mu \mathrm{m}$ of the Zircaloy cladding to oxidize. Considering that cladding thicknesses range from 0.76 to $0.89 \mathrm{~mm}$, even for the worst-case cladding, thinning as a result of Zircaloy oxidation is negligible. 


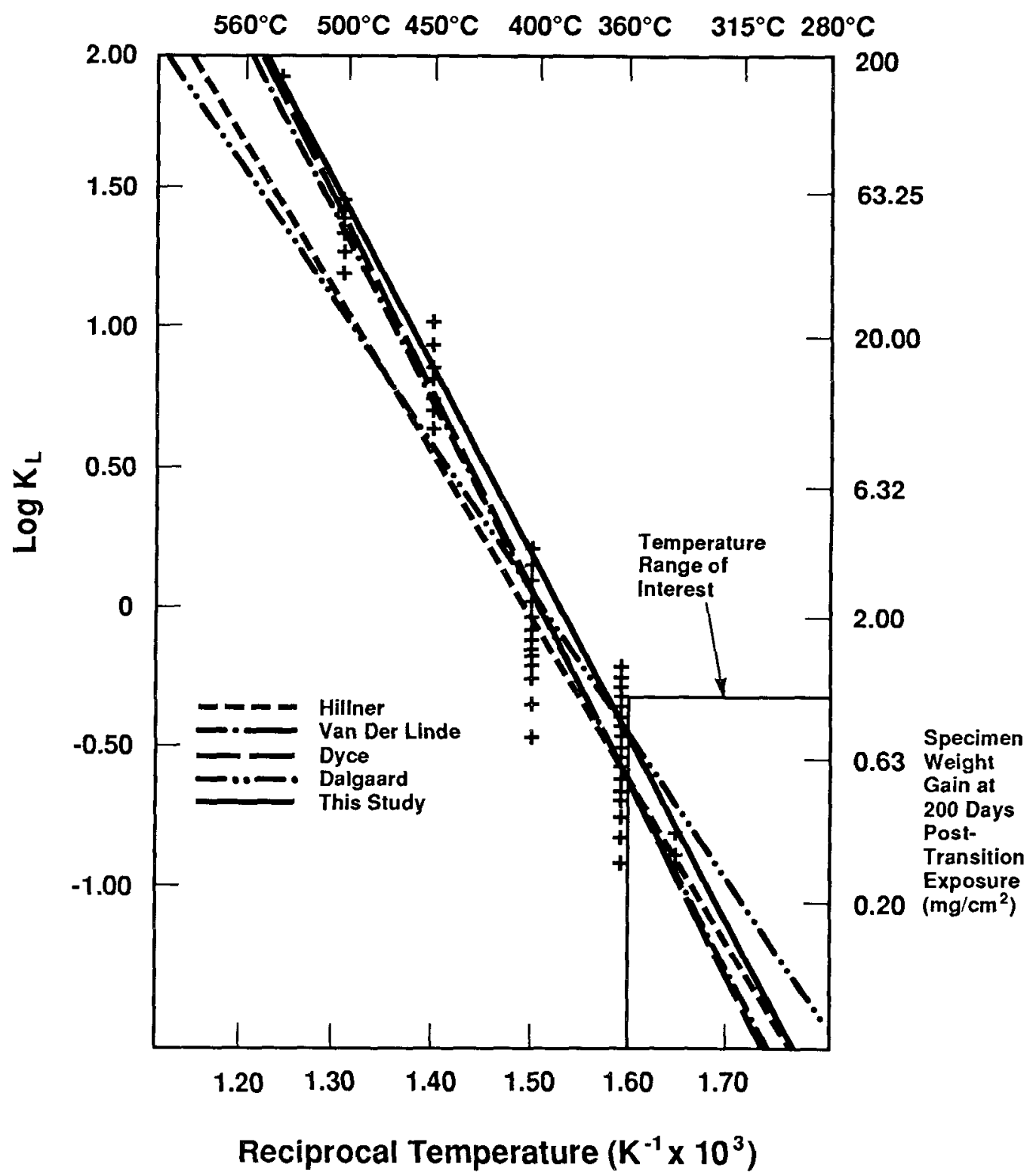

Figure III-3. Comparison of Posttransition Corrosion Models with Data [GA80]

\section{Eutectic Formation}

A eutectic reaction between the Inconel in the spacers and Zircaloy cladding requires a temperature of $930^{\circ} \mathrm{C}$ [BU85]. Because the maximum fuel rod temperature calculated for a regulatory accident is $530^{\circ} \mathrm{C}$, eutectic failure will not occur during transport. Eutectic reactions between stainless steel and Zircaloy or Inconel also require temperatures $>1000^{\circ} \mathrm{C}$ and therefore are not plausible failure modes. 
Table III-1

Zircaloy Oxidation During Transport Conditions

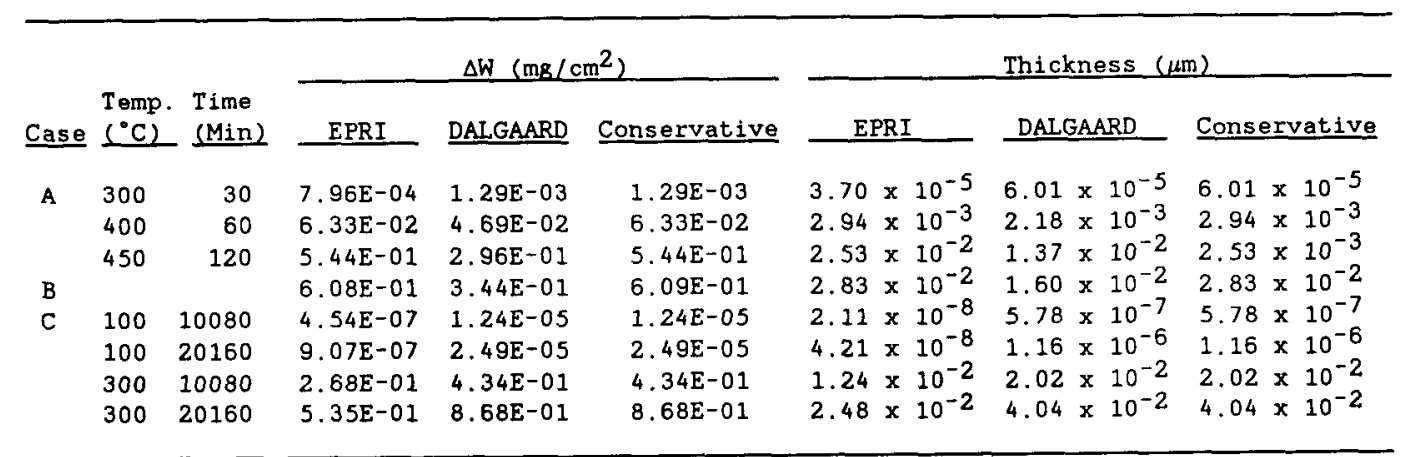

\section{Zirconium-Water Reaction}

The zirconium-water reaction, if credible, would have three potentially hazardous results: (1) hydrogen gas would be liberated, (2) heat would be given off as the reaction proceeds, and (3) the cladding would embrittle. However, this exothermic reaction is not significant until temperatures over $980^{\circ} \mathrm{C}$ are reached [LE77]. Again, this temperature requirement is higher than the maximum temperature to which the fuel rods may be exposed under accident conditions. Thus, the zirconium-water reaction is not a plausible failure mode.

\section{III.2.2 Breaking Failure}

Fuel rod breaking may occur if external loading exceeds fuel rod strength. Rod breaking is postulated for both circumferential and longitudinal directions.

\section{Circumferential Tearing}

Circumferential tearing is driven by longitudinal forces in the fuel rods. Two levels of circumferential failure can be distinguished. The onset of circumferential tearing creates a pinhole-size failure in the cladding. If loading increases, the circumferential crack can propagate around the circumference of the rod and result in large openings in the cladding and possible separation of the rod into multiple pieces.

\section{Longitudinal Tearing}

Longitudinal tearing is caused by large hoop stresses in the fuel rod. Longitudinal breakage is characterized by pinholes or longitudinal slits in the cladding. Hoop stresses in the cladding are primarily driven by rod internal pressure and by ovalization as a result of external pinch loads. 
The probability of breaking failure depends on the fuel response. A methodology for determining if breaking failure will occur is discussed in detail in Section III.4.

\section{III.3 Structura1 Modeling}

The spent-fuel response model provides a methodology for determining if fuel rod breakage occurs in the fuel assemblies under normal transport and regulatory accident loading conditions. Fuel rod failure is determined through a combination of deterministic stress analyses and probabilistic failure assessment based on a part-wall crack distribution and a probabilistic fracture mechanics criterion. This section describes the modeling assumptions, load definition, and spacer grid and assembly models developed for determining the analytical response of the spent fuel. Section III.5 illustrates the analytical modeling and sensitivity evaluations of example PWR and BWR assemblies.

\section{III.3.1 Mode1ing Assumptions}

Many assumptions are required to develop a methodology for modeling fuel assemblies under transport loading conditions. The majority of the assumptions 1 isted below are conservative and were chosen to reduce the modeling effort required for the analyses. The effects of these assumptions on the fuel rod response are minimal and generally result in a slight overestimation of the fuel rod response. Other assumptions described below are best estimates of basket and cask design parameters. The effects of variations in these assumptions have been evaluated through parametric sensitivity analyses. The modeling assumptions for fuel rods, spacer grids, assemblies, baskets, casks, and analysis procedures are provided below. A basis for each is also provided to better quantify each assumption.

\section{Fuel Rod Assumptions}

Assumption. Tensile and bending stiffness from the spent-fuel pellets is neglected.

Basis. The fuel rod analysis models conservatively assume that the cladding provides all the tensile and bending structural stiffness of the fuel rods. This assumption is based on the very low tensile strength (100 $\mathrm{MPa}$ versus $690 \mathrm{MPa}$ for the cladding) of the spent-fuel pellets [HA80] and on their initially cracked state. The compressive stiffness of the pellets is accounted for as a restraint against cladding ovalization and compressive loads under end-drop loading conditions.

Assumption. Fuel rod ovalization is a secondary mode of deformation that is considered only locally, at locations of peak pinch loads.

Basis. The main deformation modes of the fuel rods are axial (extension or shortening) and bending. Fuel rod ovalization is a secondary mode of deformation and is constrained by the fuel pellets. Local crushing between the cladding and fuel pellets may occur at the spacer grid or basket supports; however, this deformation pattern is very localized and can be 
neglected in the assembly models. Local crushing effects on fuel rod cladding are treated in the fuel rod failure models.

Assumption. The effective fuel mass varies significantly between PWR and BWR fuel rods. Under side-drop loading, the full fuel pellet mass (100\%) of both PWR and BWR rods is structurally active. However, for end-drop loading conditions, only the pellet portion directly attached to the cladding is active. For the example analyses in Section III.5 (PWR and BWR fuel rods under end-drop loading conditions), 75 and $10 \%$ of the fuel mass are assumed to be effective in loading the cladding of PWR and BWR rods, respectively.

Basis. The mass model used in the example PWR analysis assumes that the fuel pellets are sufficiently attached to the cladding so that 758 of the fuel mass under end-drop loading is supported by the cladding, whereas the other $25 \%$ is supported by the pellets stacking on top of other pellets. For the BWR model for which pellet-clad interaction (PCI) is minimal, only $10 \%$ of the fuel is assumed to load the cladding under end-drop loading conditions. The effects of in-reactor creepdown, ridging, and fuel-clad adhesion determine the effective weight of the pellets. These effects are discussed in detail in Appendix I.

Assumption. Fuel rod bowing is conservatively assumed to occur in all rods in the assembly and to have the shape of the lowest buckling mode of the fuel rod, with a maximum lateral deflection equal to the design clearance between adjacent fuel rods.

Basis. This magnitude-of-bowing assumption is conservative in that most rods show a very small amount of bowing, and only a few rods, if any, will show maximum bowing. These maximum values are conservative relative to the experimental bowing measurements of free-hanging spent-fuel rods [GE71]. The maximum measured bowings were 1.27 and $0.89 \mathrm{~cm}$ for PWR and BWR rods, respectively. The rods were hung from one end only, and the unsupported lengths measured $\sim 4.06 \mathrm{~m}$. However, the maximum unsupported rod lengths are typically 53.3 and $50.8 \mathrm{~cm}$ for the PWR and BWR assemblies, respectively. If a linear relation between the unsupported lengths is assumed, the maximum bow would be $\sim 0.18$ and $0.10 \mathrm{~cm}$ for the PWR and BWR assemblies, respectively. These values are much smaller than the design clearance between the rods used in the initially bowed rod analyses. The lowest buckling mode shape (Figure III-4) is the most conservative shape of rod bowing in that deformation of these bowed rods results in maximum lateral displacements with the least amount of energy input. The fuel rod bending strains, which are a basis of the fuel rod failure criteria, are directly related to these lateral displacements.

\section{Spacer Grid Assumptions}

Assumption. Spacer grid sliding in the longitudinal direction (Figure III-5) relative to the fuel rods is neglected. The distance between grid frames is assumed to remain constant throughout the loading event. 


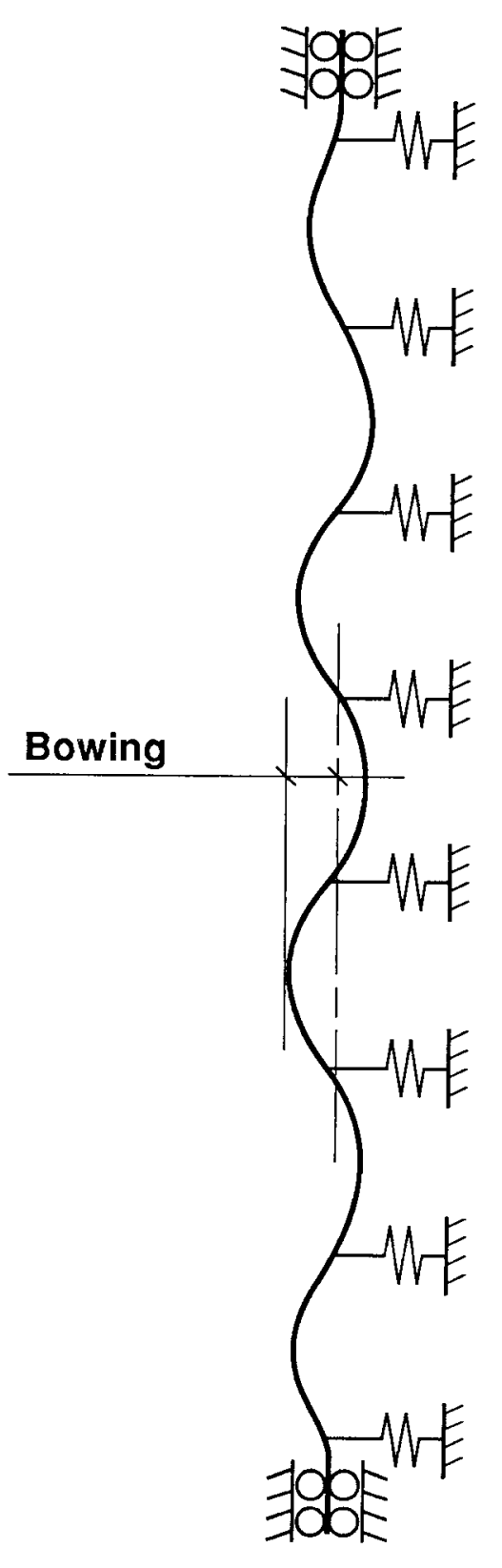

Figure III-4. Lowest Buckling Mode Shape of the Fuel Rod

Basis. Spacer grid movement in the longitudinal direction is restricted by the grid leaf springs and crushing of the grids; thus, significant deformation is very unlikely. 


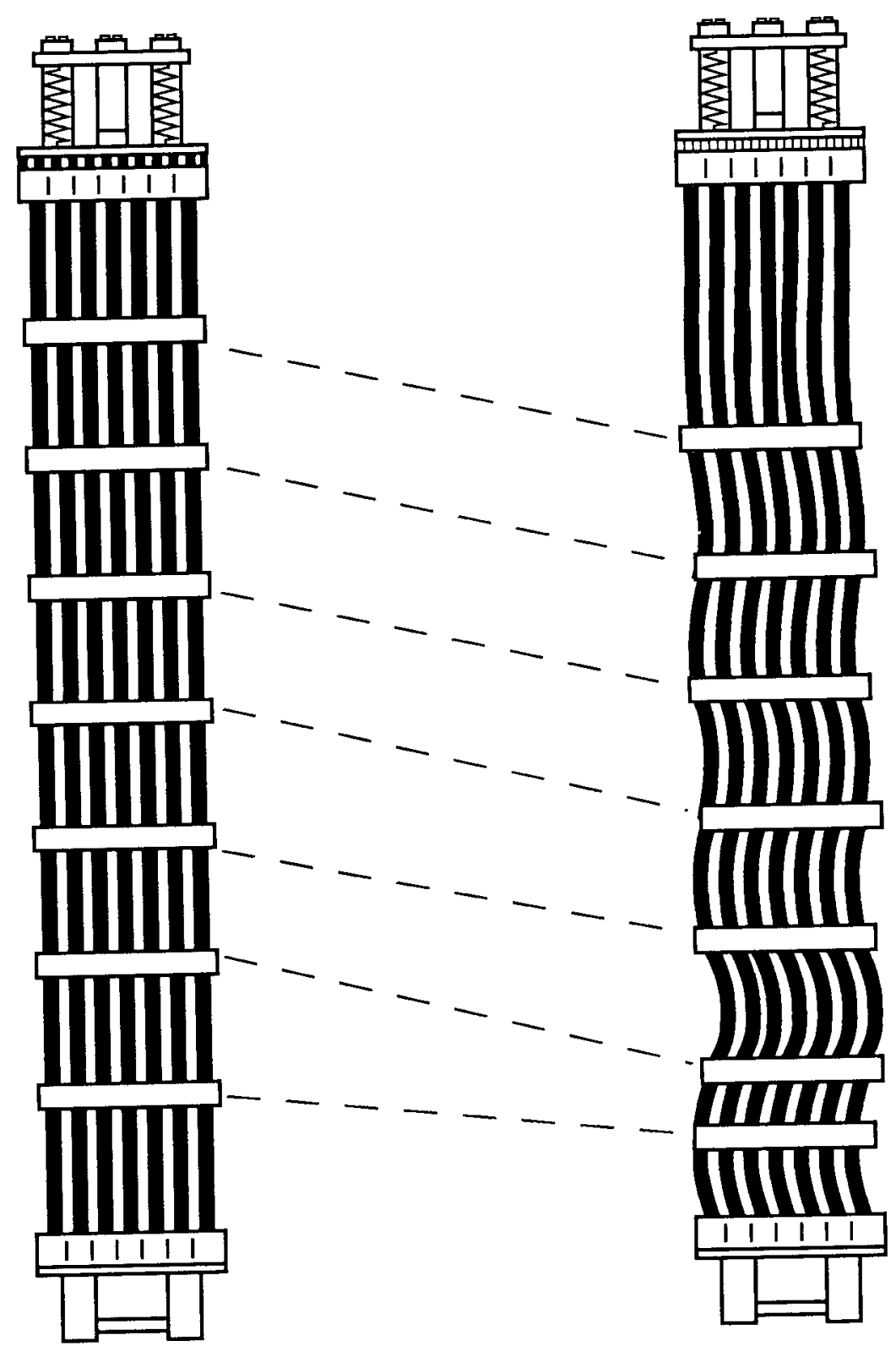

UNDEFORMED

ASSEMBLY

DEFORMED ASSEMBLY

Figure III-5. Longitudinal sliding of Spacer Grids 


\section{Assemb1y Assumptions}

Assumption. The assembly models include only fuel rods. No properties of nonfuel rods are modeled. (Future analyses could easily incorporate the properties of nonfuel rods.)

Basis. The analysis models assume that the assemblies are completely filled with loose fuel rods and tie rods or guide tubes. The guide tubes and other assembly components are assumed to be structurally equivalent to fuel rods. This assumption is conservative in that it adds more mass to the analysis model without increasing the assembly stiffness.

Assumption. The end-drop model assumes no rod-to-rod load transfer except at the spacer grids. Lateral displacement constraints are based on all assembly rods having the same deformation pattern.

Basis. This assumption is conservative in that all rods will not deform in the same pattern. Rod-to-rod interaction will limit the lateral deflection of the fuel rods. The fuel rod maximum bending strains (the main basis of the end-drop failure criteria) are directly related to these fuel rod lateral deflections. The end-drop model includes lateral restraints along the fuel rod length between spacer grids to prevent unlimited lateral displacement. These lateral restraints, shown in Figure III-6, are based on all fuel rods having the same deformation pattern, with their lateral deformation constrained by the assembly and basket.

Assumption. Under end-drop loading, the responses of the assembly tie rods and loose rods are assumed to be identical.

Basis. During the peak response times of end-drop loading, all rods are compressed between the end plates. Thus, both loose rods and tie rods can be assumed to be rigidly attached to the end plates and to have the same stiffness characteristics. Gaps between the loose rods and the end plates are included as part of the assembly basket gap.

Assumption. The tie plates and nozzles of the assemblies are very stiff relative to the fuel rods and spacer grid stiffnesses.

Basis. This assumption is conservative in that flexibility in the tie plate and nozzle would reduce the magnitude of the deceleration response transmitted between the cask and the fuel rods. Plastic deformation of these components, conservatively neglected by the assembly model, will absorb energy and dampen the assembly response.

Assumption. The stiffness of the assemblies that are out of plane with the load direction is neglected.

Basis. This assumption is conservative in that out-of-plane rod-to-rod interaction will only increase the assembly stiffness. Increases in assembly stiffness will reduce the peak bending strains in the fuel rods and the probability of their failure. 


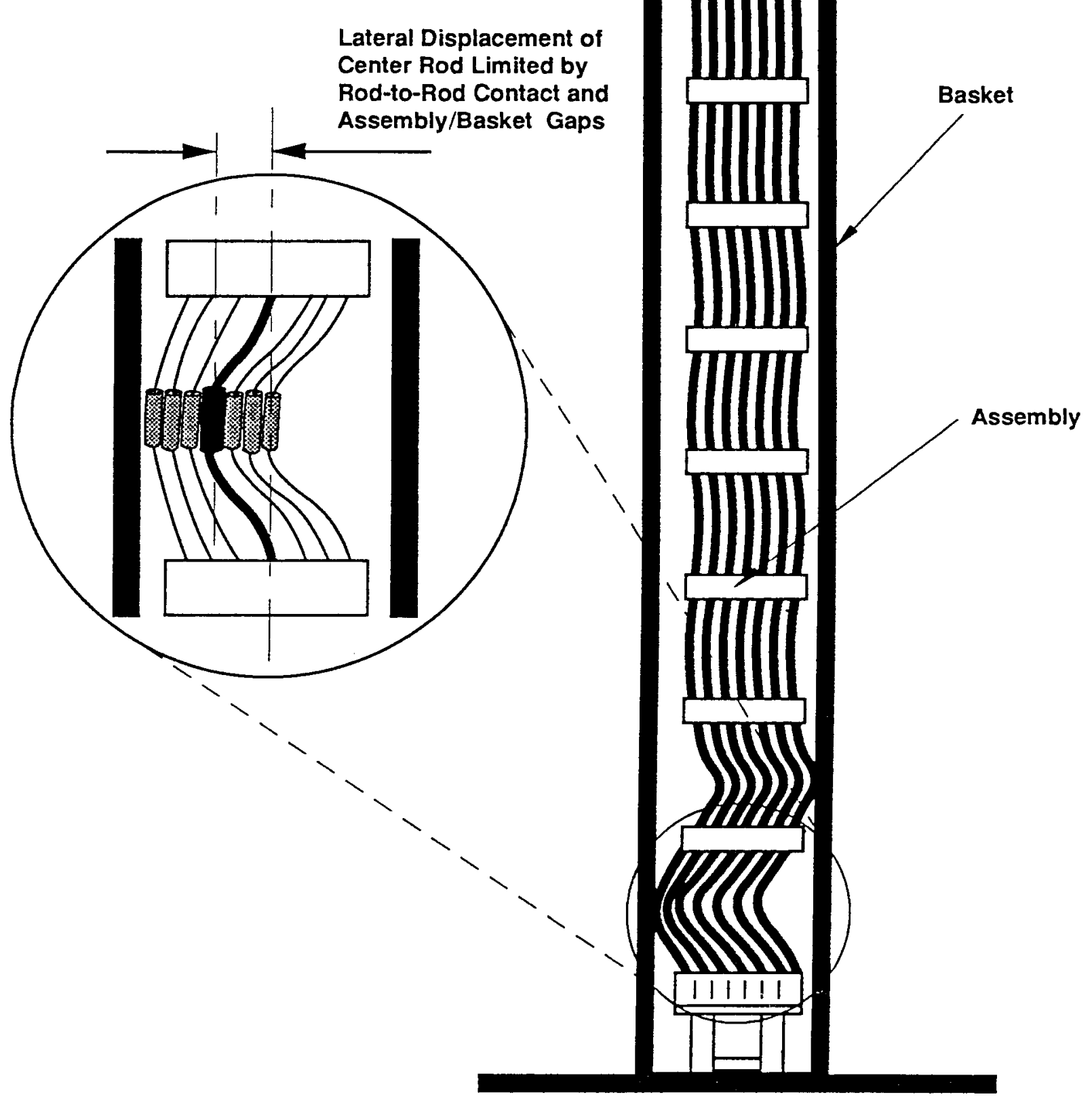

Figure III-6. Lateral Displacement of Assembly Limited by Rod-to-Rod Contact and Assembly-Basket Gaps 


\section{Basket Assumptions}

Assumption. The structural stiffness of the basket is very great in relation to the fuel assemblies; thus, assembly-to-assembly load transfer is negligible.

Basis. This assumption must be evaluated on a basket-by-basket design basis because assembly-to-assembly load transfer would significantly increase the assembly response.

Assumption. Basket-cask gaps are negligible.

Basis. This assumption is consistent with current basket and cask designs in which basket-cask gaps are small enough that they can be neglected.

However, large gaps will increase the fuel rod response; these gaps must be evaluated on a design-by-design basis.

Assumption. For the example analysis for side-drop loading, the basket supports are assumed to be distinct supports that coincide with the spacer grid supports.

Basis. This assumption is made to simplify the example analyses performed (see Section III.5). For baskets with continuous supports, this assumption is conservative in that these supports will spread out the rod-to-rod pinch loads (a major basis of the side-drop failure criteria) and limit the bending deformation of the assembly between support points. However, modifications to the basket boundary conditions may be required on a design-by-design basis.

\section{Cask Assumptions}

Assumption. The cask body is not modeled and is assumed to be very stiff relative to the stiffness of assemblies. This assumption conservatively neglects any energy absorption by the cask body.

Basis. Because the cask stiffness is orders of magnitude greater than the fuel assembly stiffness, flexibility of the cask is a second-order effect and can be neglected.

\section{Analysis Assumptions}

Assumption. The corner-drop initial impact and slapdown loading conditions can be analyzed independently.

Basis. The corner-drop initial impact and slapdown loading conditions are treated as independent events in that the peak stresses and strains for each of these loading conditions occur at opposite ends of the assembly. For the critical initial impact loading conditions, the corresponding slapdown loading magnitude at the bottom end of the assembly is an order of magnitude less than the corresponding initial impact. Conversely, the initial impact response at the top end of the assembly is negligible for all drop angles and thus does not need to be added to the slapdown analyses that concentrate loading to the top of the assembly. 
Assumption. Damping of the analytical model is limited to numerical damping, which varies as a function of time step. The numerical damping never exceeded $1.6 \%$ of the critical damping of the structure.

Basis. Numerical damping is provided to make the solution well behaved. This damping value is conservative in that mechanisms such as crushing of the assembly appurtenances, friction, and so on will produce significantly more assembly damping than the numerical value.

\section{III.3.2 Assembly Mechanical Loading Conditions}

This section defines the process of applying the normal transport and regulatory accident free-drop loading conditions to fuel assemblies. Appendix II describes the regulatory loading conditions in detail. Typical cask deceleration time histories for all angles of regulatory accident and normal transport free drops are developed using the SLAM program (see Appendix II). The magnitude and durations of the load histories are functions of the cask mass, impact limiters, drop height, and drop angle. Two drop heights are considered: $9.0 \mathrm{~m}$ for the regulatory accident condition and $0.3 \mathrm{~m}$ for the normal transport condition. Time histories for the upper-bound-normal and accident drops from 0.3 and $9.0 \mathrm{~m}$ are used in the example analyses described in Section III.5. The peak center-of-mass decelerations of the example assemblies for all drop angles are less than $100 \mathrm{~g}$ for the particular cask models evaluated.

The fuel assembly response to the regulatory free-drop event is related to the drop angle. Regulatory drops can be divided into three distinct categories: side drop, end drop, and corner drop. Figure III-7 illustrates these drop angles.

The rigorous methodology developed and described herein for defining the response of fuel assemblies under these regulatory drop conditions is characterized as time-history dynamic analysis. The cask deceleration load histories are applied directly to the fuel assembly structural analysis models. The dynamic analyses capabilities provide for variations in loading duration, as well as for the effects of gaps between the assembly and cask.

A free-body diagram of the side-drop assembly loading condition is illustrated in Figure III-8. An initial velocity $(13.4 \mathrm{~m} / \mathrm{s}$ for the $9.0-\mathrm{m}$ free drop) is input to all assembly components. The momentum as a result of this initial velocity loading is reacted by an equivalent momentum applied in the form of a retarding force at the basket support points. The retarding force is equal to the mass of the assembly multiplied by the cask rigid-body deceleration history. An example deceleration time history is shown in Figure III-8 and corresponds to the upper bound response of a lead-shielded truck cask, as described in Appendix II. The increase in force as a function of time is caused by the increase in crush area over the duration of the event, which causes an increase in cask deceleration.

The end-drop assembly loading is illustrated in Figure III-9. Similar to the side drop, an initial velocity is input to all components of the assembly and reacted by a retarding-force time history; the impact-limiter 


\section{END DROP CONDITION}

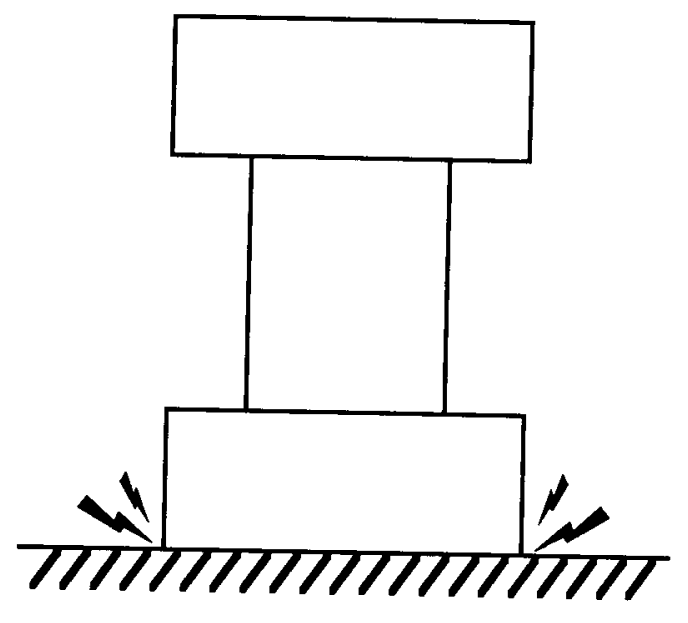

\section{SIDE DROP CONDITION}

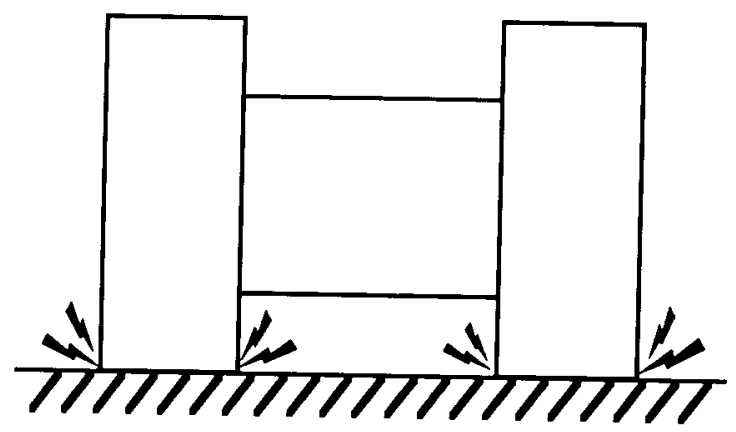

CORNER DROP / SLAPDOWN
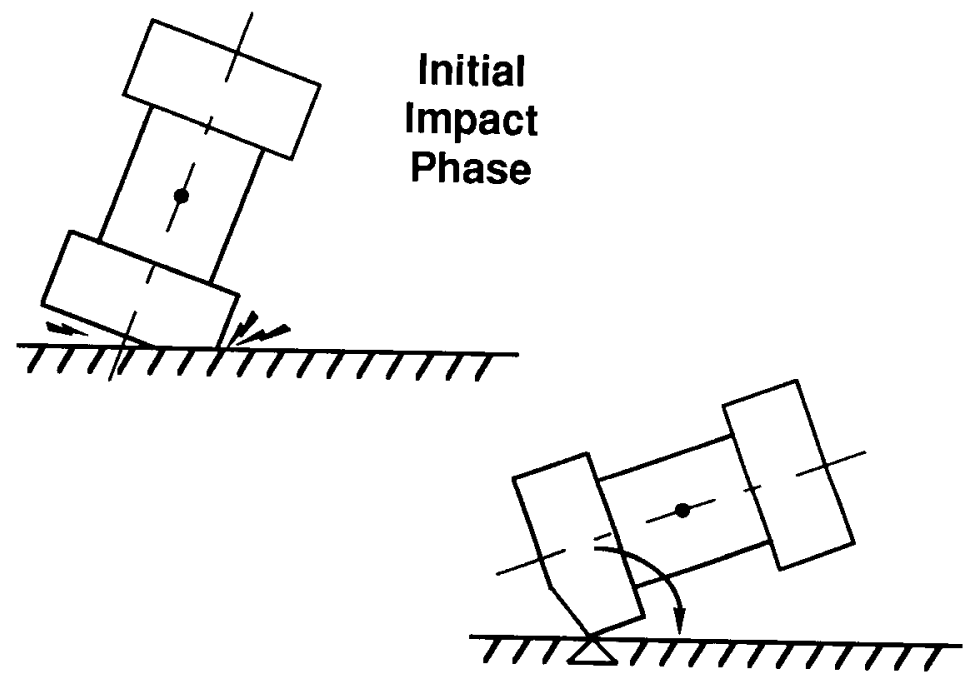

Pinned Phase

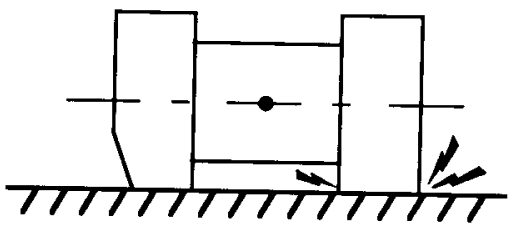

Slapdown Phase

Figure III-7. Cask Drop Impact Orientations 


\section{CASK SIDE DROP}
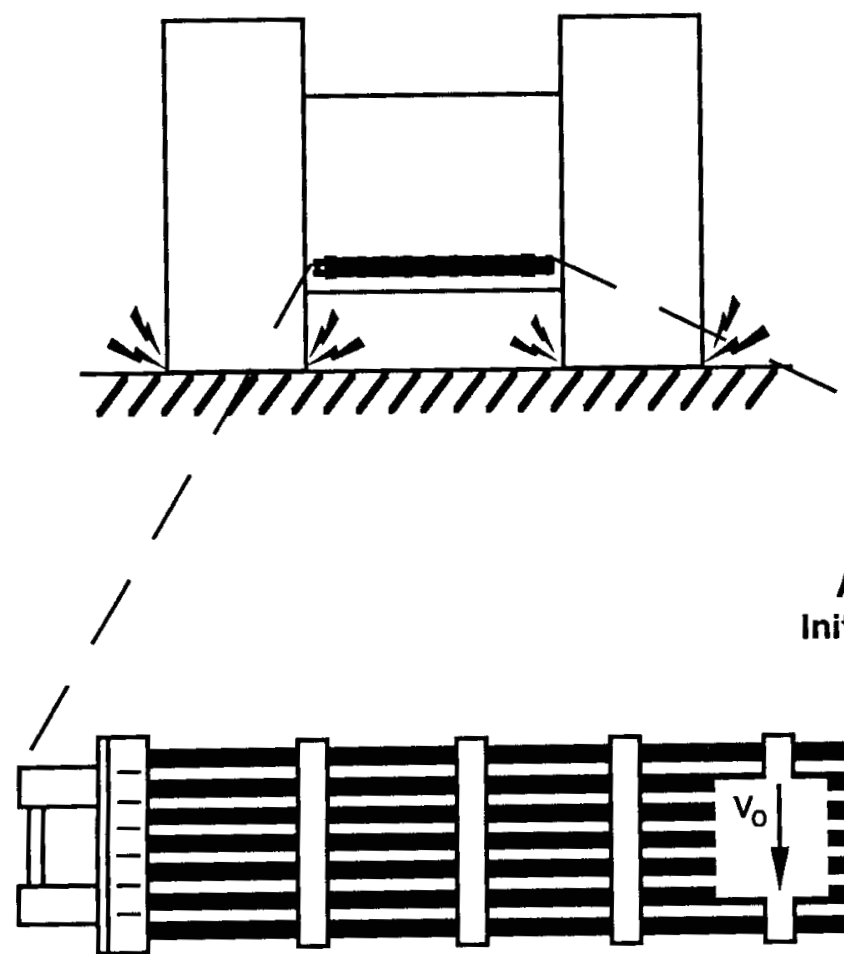
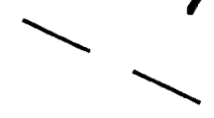

Assembly Initial Velocity, $v_{0}$

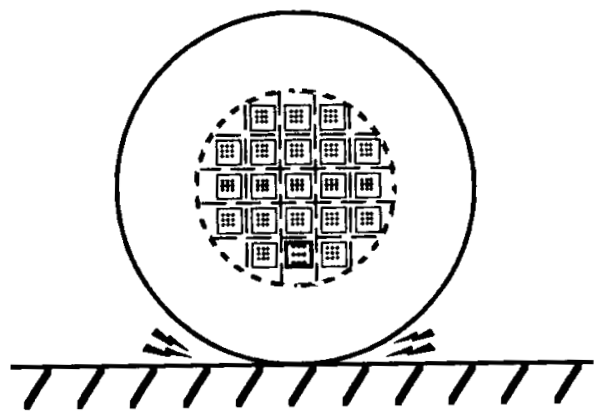

End View

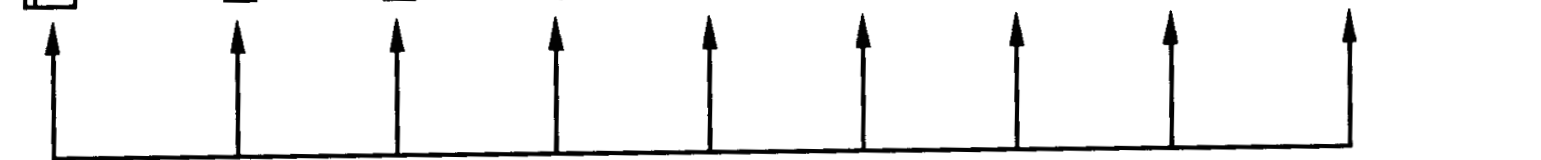

$F(t)=$ Acceleration $\times$ Mass

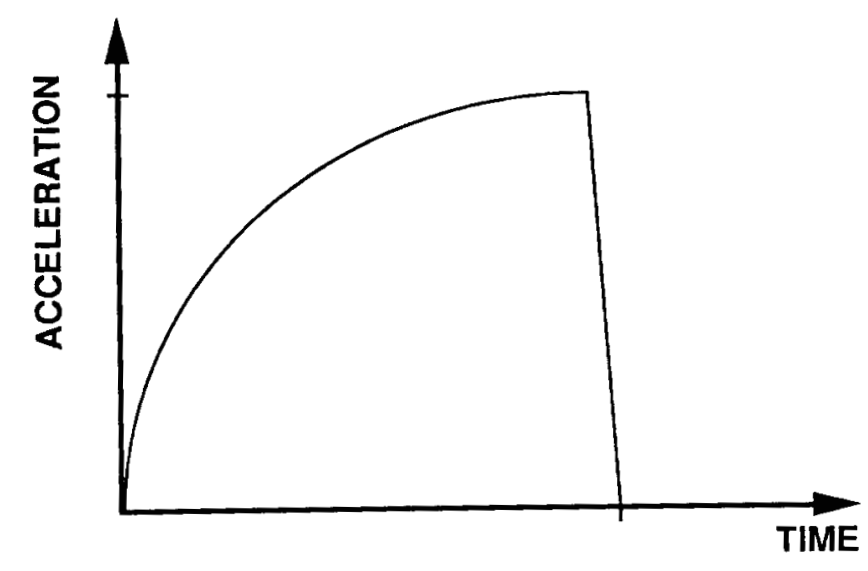

ACCELERATION TIME HISTORY

Figure III-8. Free-Body Diagram: Assembly Side-Drop Loading Condition 


\section{CASK END DROP}

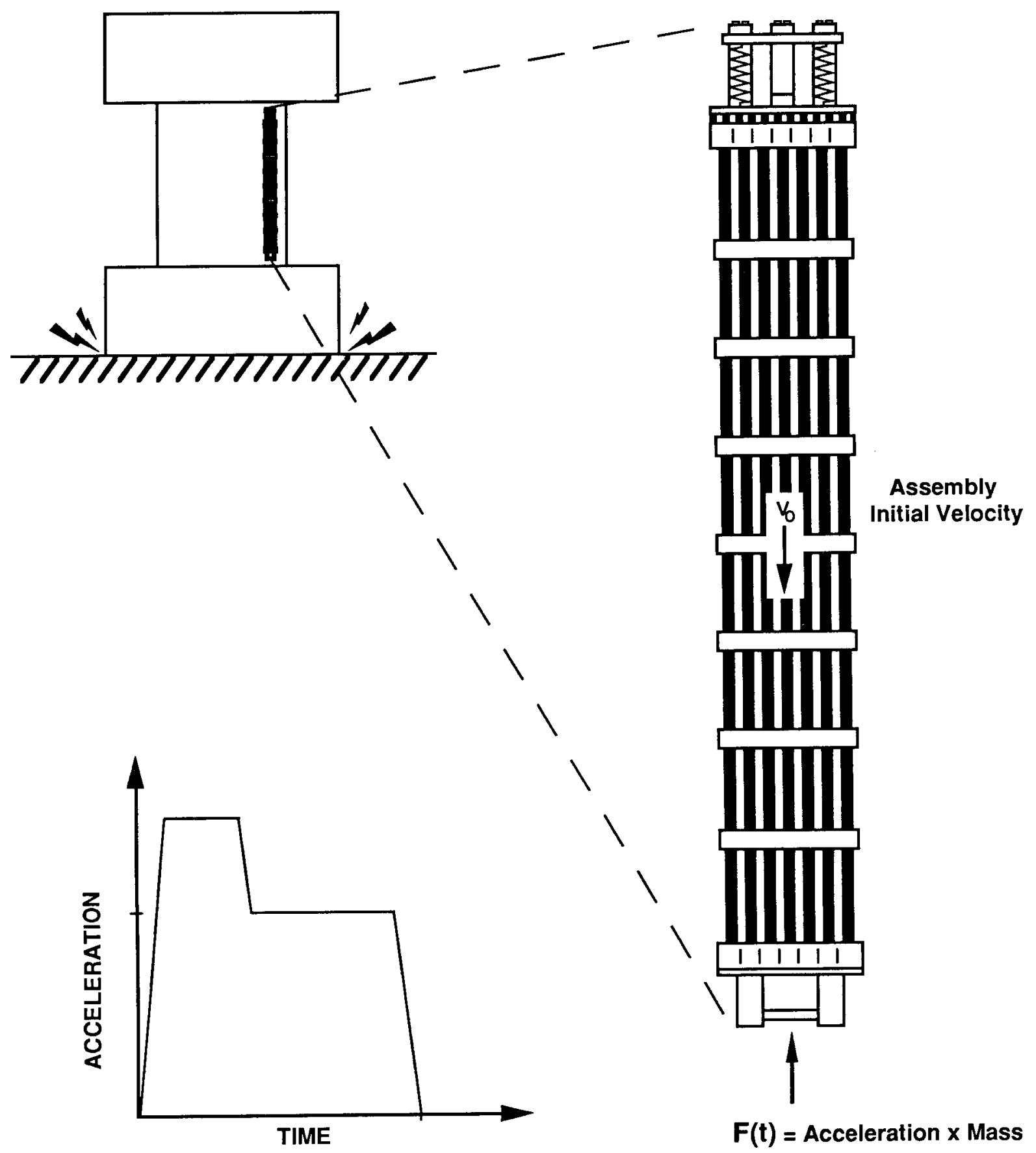

\section{ACCELERATION TIME HISTORY}

Figure III-9. Free-Body Diagram: Assembly End-Drop Loading Condition 
retarding force is applied to the bottom of the assembly. The flat-shaped end-drop deceleration curve is the result of a constant impact-limiter crush area during the entire loading event.

The corner-drop loading consists of two separate loading events: initial impact and slapdown. For cask drop angles near vertical, the initial impact dominates and the assembly response resembles the end-drop response. For corner-drop impact angles near horizontal, slapdown dominates and the assembly response resembles the side-drop response. Assembly free-body diagrams for both the initial impact and slapdown loading conditions are shown in Figures III-10 and III-11.

For modeling purposes, the initial impact loading can be divided into longitudinal and transverse components. The impulse load on the assembly in the longitudinal direction is input similar to the end drop. An initial velocity is imposed on the entire assembly and reacted with the impactlimiter retarding force at the assembly end plate. The lateral and rotational loading components, which are small compared to the axial load, are input as distributed loads on the assembly components and reacted at the basket supports on the downward side of the assembly.

The slapdown loading (Figure III-11) is applied to all assembly components in the form of translational and rotational initial velocities, which are based on the cask deceleration time history. The initial velocities of the fuel assembly are reacted by a retarding-force history equal to the assembly mass multiplied by the cask deceleration time history. Similar to the side drop, the increase in the magnitude of the slapdown force over time is a result of the increase in the impact-limiter crush area. A simplified dynamic analysis of a slapdown loading condition is described in the example analyses of Section III.5.

Gaps between the assembly and the cask and/or basket supports can increase the response of the assembly for all angles of drop orientation. This response is amplified by a relative velocity impact between the assembly (accelerating at $1 \mathrm{~g}$ to close the gap) and the cask already decelerating after impact. The cask/assembly vertical gap loading is modeled by combining the effects of the relative velocity impact of the assembly and the cask with the cask deceleration defined by the impact limiters. Figure III-12 illustrates the three-step process for the initial gap loading condition. When the cask impacts the rigid surface, the fuel assembly continues to accelerate at $1 \mathrm{~g}$ downward until the assembly-cask gap closes. On impact, the relative velocity impact between the cask and assembly is superimposed on the steady deceleration response of the cask impact limiters. After impact, the cask and assembly continue to decelerate together in that the assembly is assumed then to be fixed to the bottom of the cask.

To model this gap effect, an impact-force pulse is developed using the relative velocity between the assembly and cask, assuming an unyielding target condition. The infinitely large stiffness assumption for the assembly nozzle and cask support is conservative. The maximum peak force and pulse area (or rod momentum range) depend directly on the relative velocity in the vertical gap. This cask-assembly pulse is added to the 


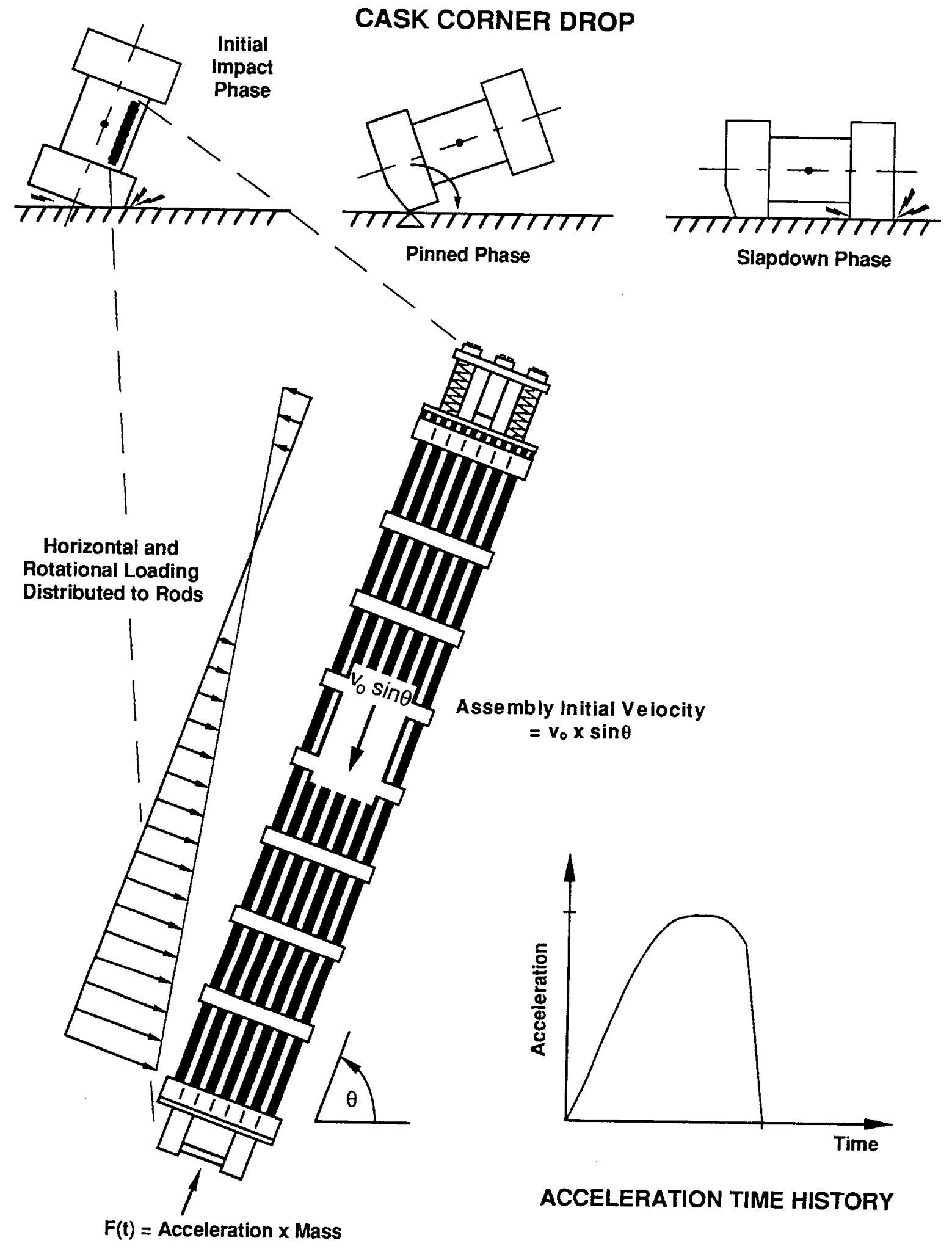

Figure III-10. Free-Body Diagram: Assembly Corner Drop, Initial Impact
Loading Condition 


\section{CASK CORNER DROP}
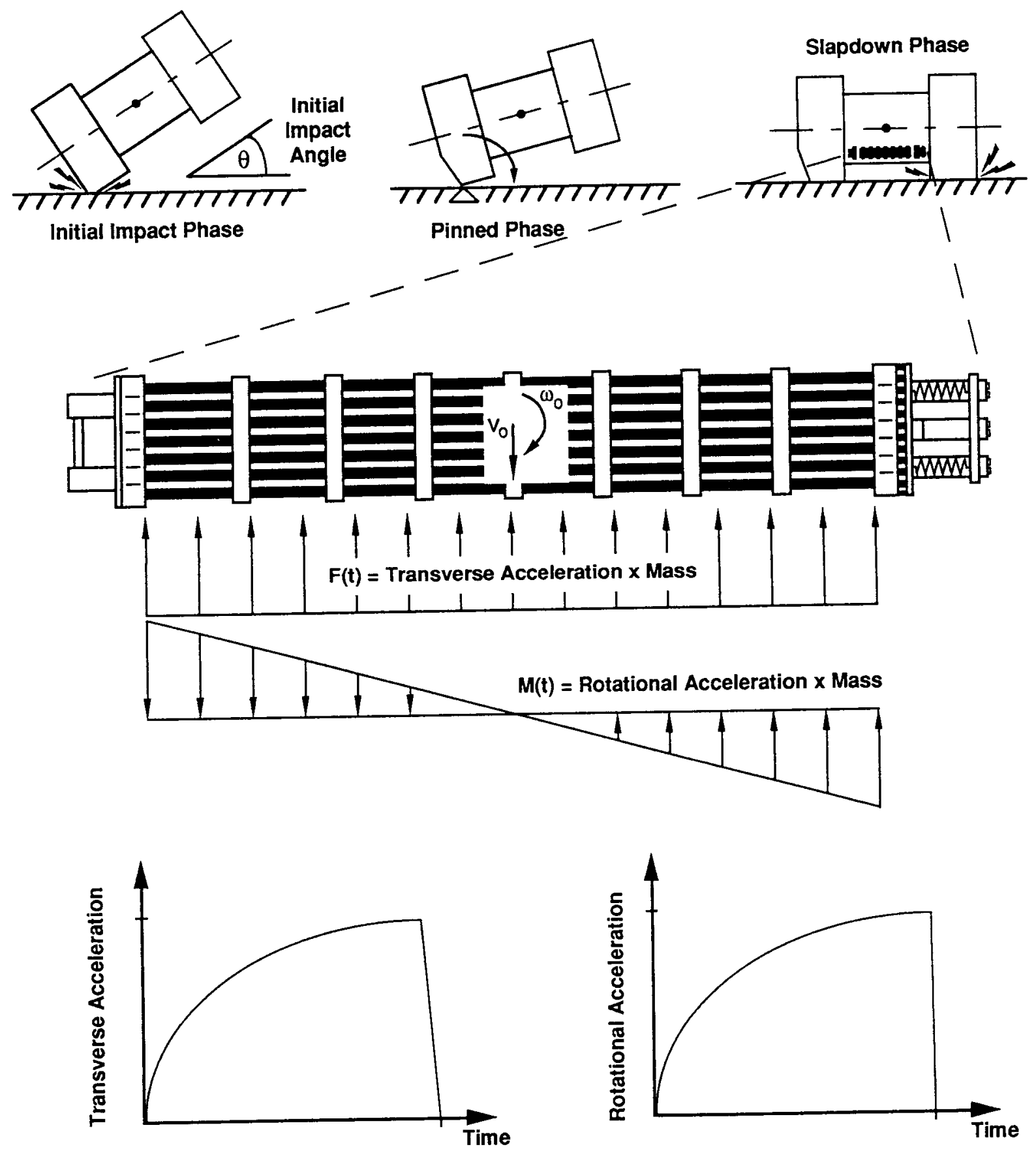

ACCELERATION TIME HISTORIES

Figure III-11. Free-Body Diagram: Assembly Corner Drop, Slapdown Loading Condition 


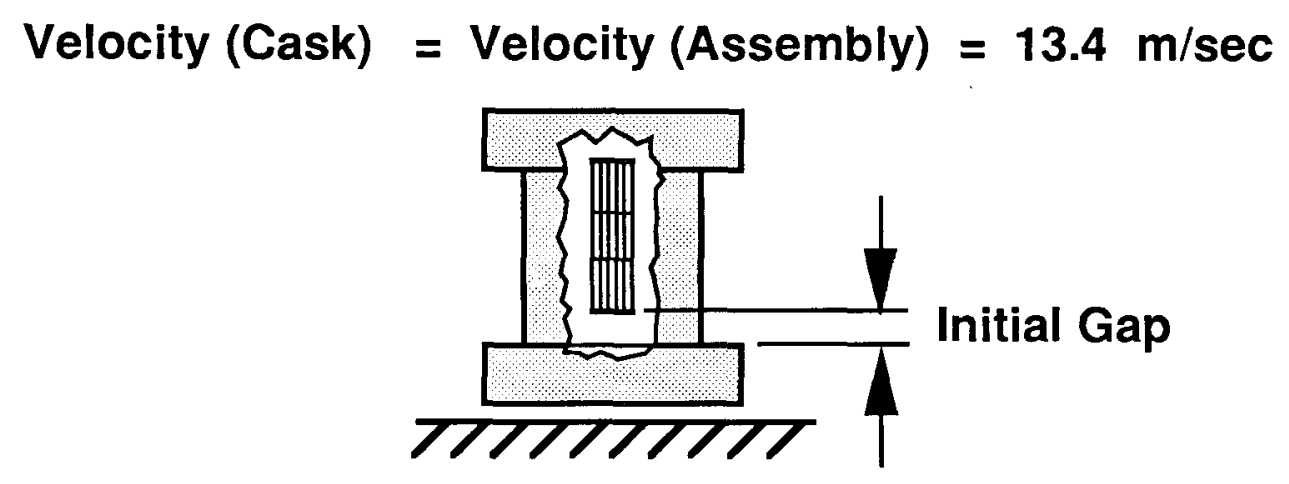

Velocity (Assembly) $=13.4 \mathrm{~m} / \mathrm{sec}$ Velocity (Cask) $<13.4 \mathrm{~m} / \mathrm{sec}$

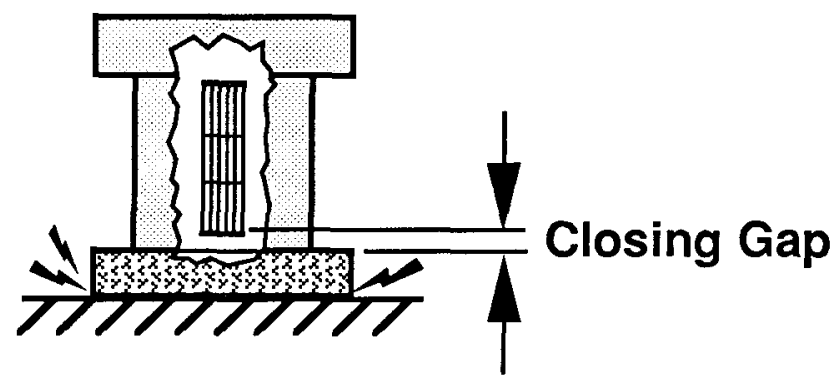

Velocity (Assembly) $=$ Velocity $($ Cask $)<13.4 \mathrm{~m} / \mathrm{sec}$

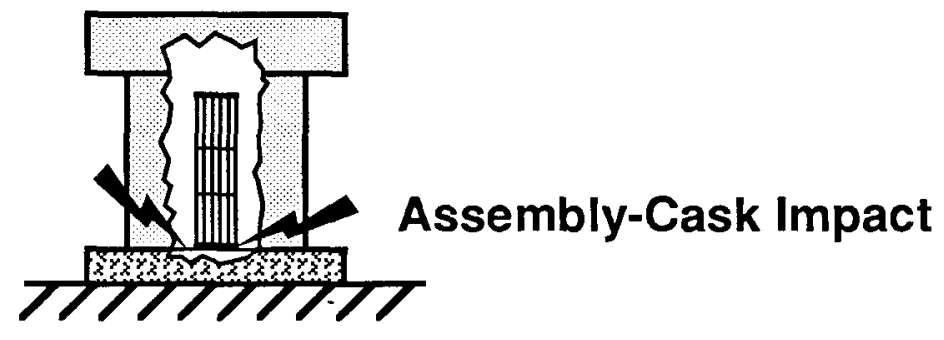

Figure III-12. Illustration of End Drop: Initial Velocity Loading 
cask-limiter deceleration pulse. However, the initial phase of the casklimiter pulse (which corresponds to the time before the assembly-cask impact) is eliminated because the assembly will not undergo this deceleration. Thus, the total area of the combined cask-assembly and casklimiter pulses is approximately equivalent to the assembly initial momentum from the initial drop. The pulse areas of the no-gap and gap conditions are equivalent; however, as the gap increases, pulse duration decreases and peak deceleration magnitude increases. Figures III-13 and III-14 illustrate the assembly deceleration pulse.

A small amount of damping is included numerically for all dynamic analyses to produce a well-behaved solution. For the example analyses of Section III.5, this damping never exceeds the equivalent of 1.68 of critical damping. This value is conservative in that damping as a result of fuel PCI, rod-to-rod interaction, and plasticity (excluding the cladding, which is modeled) will introduce significantly more damping into the assembly response.

\section{III.3.3 Spacer Grid Model}

Each fuel assembly contains spacer grids that provide both lateral and axial support to the fuel rods between the end plates (see Appendix I). Because the response of these spacer grids is very complex, it is necessary to analyze the grids apart from the fuel assemblies to determine their response. Results of these analyses are included in the side-, end-, and corner-drop assembly computational models by incorporating nonlinear spring elements between the rods.

The spacer grids provide a highly nonlinear load transfer mechanism between the fuel rods and the cask and basket. For the side-drop loading condition, loads from the rods onto the grids cause the grids to buckle. Thus, the pre- and postbuckling response of the grids must be computed to quantify their response. This section provides a detailed description of the load deflection mechanism of the grids. It also describes the analytical models used for the grids and the corresponding nonlinear spring elements that were developed for input into the assembly models.

\section{Structural Response}

Detailed models of the BWR and PWR spacer grids are required to determine their force/deflection relationship. The force deflection characteristics of the spacer grids can be described by the following fourstep process: (1) initial loading on the fuel rod deflects the spacer leaf springs until they bottom out on the spacer frame; (2) additional loading causes deflection of the frame itself until it buckles; (3) on buckling of the frame, each affected rod deflects without additional resistance until it contacts the top of the rod underneath; and (4) additional loading causes rod-to-rod compression, and further deflection is limited by the crushing strength of the rods. This four-step process, shown in Figure III-15, has the following characteristics. 


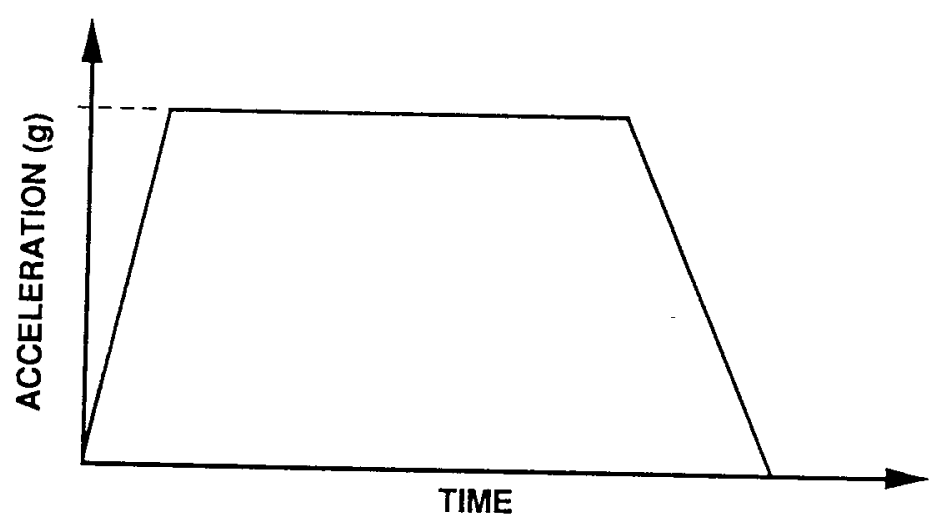

CASK ACCELERATION HISTORY

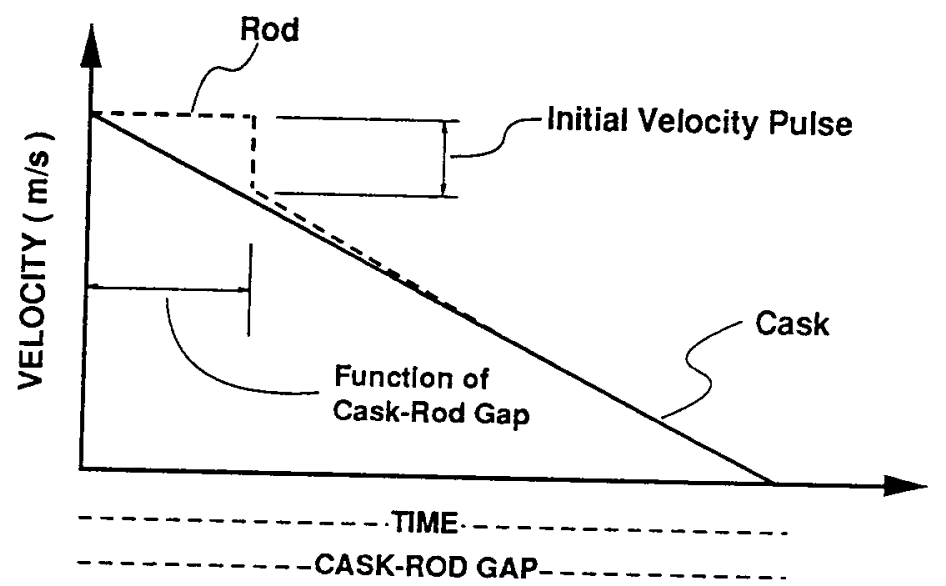

CASK - ROD RELATIVE VELOCITY

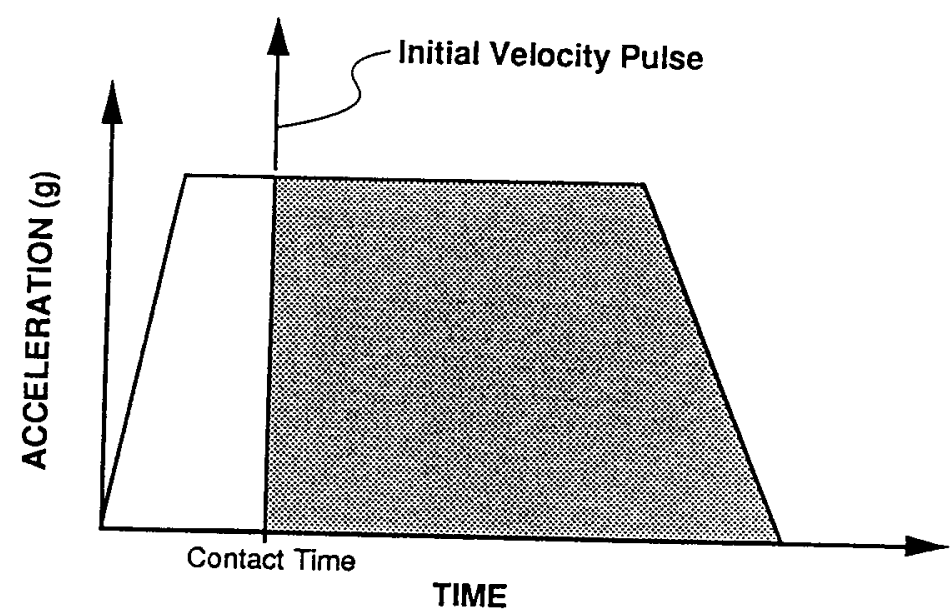

ROD LOADING HISTORY

Figure III-13. Cask-Assembly End Drop: Initial Velocity Loading Schematic
Diagram 

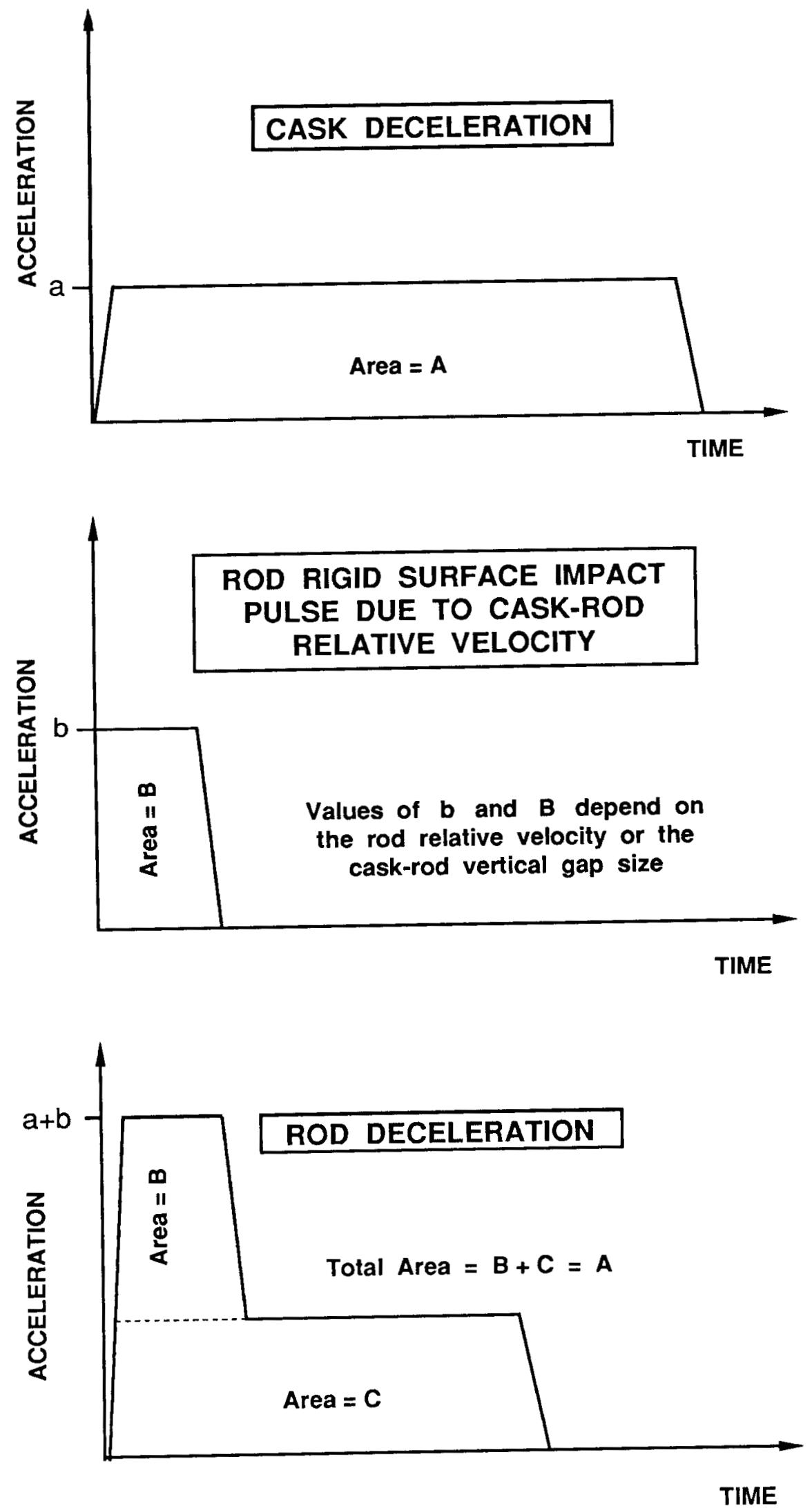

Figure III-14. Development of Cask-Rod Vertical Gap Deceleration Loading 

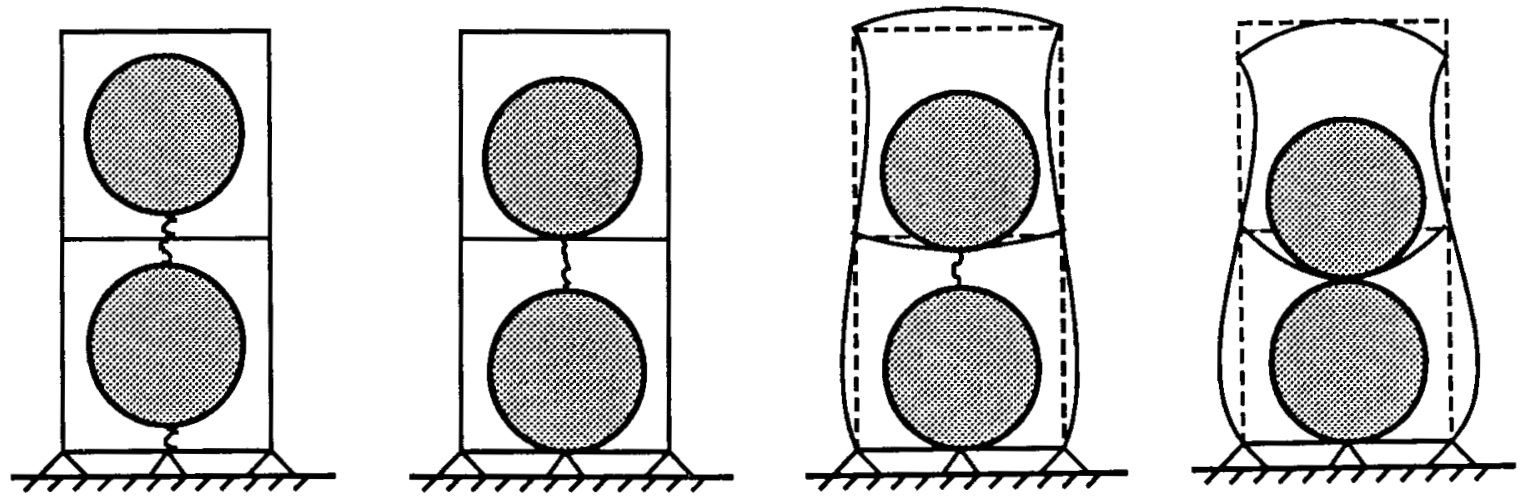

A

B

C

D

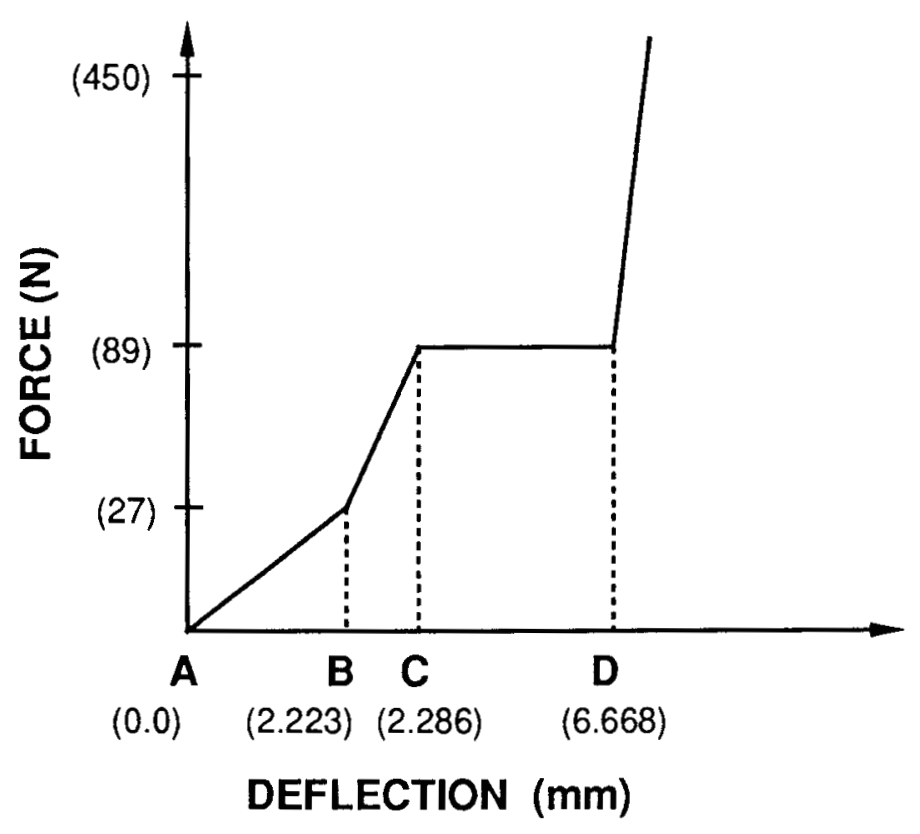

Figure III-15. Schematic Diagram of Spacer Grid Response

1. Initial loading from the fuel rods crushes the leaf springs onto the spacer grid frame. If, for example, the spacer leaf springs have a stiffness of $12.3 \times 10^{3} \mathrm{~N} / \mathrm{m}$ and they bottom out after a deflection of $0.222 \mathrm{~cm}$, they will carry a load of only $26.7 \mathrm{~N}$ before they bottom out on the grid frame.

2. Further loading after the spacer contact springs bottom out results in deflection of the spacer frame. The example spacer grid frames (see Section III.5) provide resistance to approximately 66.7 to $75.6 \mathrm{~N}$ per rod, whereupon the frame begins to buckle.

3. After buckling, the frame offers minimal resistance to further load; therefore, the load must be transmitted directly from rod to rod in the buckled cells. The amount of rod deflection occurring 
after the frame buckles is directly related to the location of the rod in the spacer grid. The higher the rod is in the grid, the larger its deflection before it contacts the rod directly below it.

4. Further grid deflection is limited by the fuel rods. As the load on the rods increases, more frames buckle until all rods are stacked directly on top of one another. Further deflection is limited by the crushing stiffness of the rods.

\section{Computational Model}

The assembly spacer-grid nonlinear spring element described here is based on analysis of a single spacer grid cell. The basis of this cell model has been verified through extensive modeling of entire spacer grid frames, as described in the example analyses presented in Section III.5.

A typical spacer grid cell model is shown in Figure III-16. This model is analyzed with geometric and material nonlinearities to capture the minimum buckling response of the spacer grid frame. The force-displacement spring element developed for the assembly models is based on the failure load of this frame and the individual spacing between spacer grids and fuel rods.

\section{Assembly Spacer Grid Element}

The spacer grid nonlinear spring elements developed for the side- and end-drop assembly models are illustrated in Figures III-17 and III-18. The side-drop spacer grid element (Figure III-17) is applicable to all grids in the assembly model because the entire response of the grid frame includes the combined responses of individual spacer-grid fuel rod element interactions. The magnitude of force on each grid is a result of the combined force from its corresponding fuel rod and all rods above it. Thus, because the loading on the grids increases from top to bottom, the bottom grid cells will buckle first. Subsequent loading will buckle consecutive grid bays upward through the cross section. The forces developed among the fuel rods of the assembly model provide a means of quantifying the pinch loads among the rods. The rod-to-rod pinch loads are computed from the spring element forces minus the buckling strength of the frame because the frame structure is not explicitly included in the assembly model. These pinch loads are input into the failure criteria developed in Section III.4.

The end-drop spacer grid spring element simulates the interaction between a center rod and the entire assembly and is based on a typical rod in the center of an assembly. Lateral forces on the elements correspond to lateral forces on each rod in the assembly. The lateral deformations of the center rod of the assembly are based on the assumption that an identical force is being applied to all other rods in the assembly. The assumption that forces on all of the rods are equal is consistent with the basic conservative assumptions of the single-rod end-drop model that assumes all rods deform together. Figure III-18 illustrates the interaction of the assembly spacer grid spring element. 


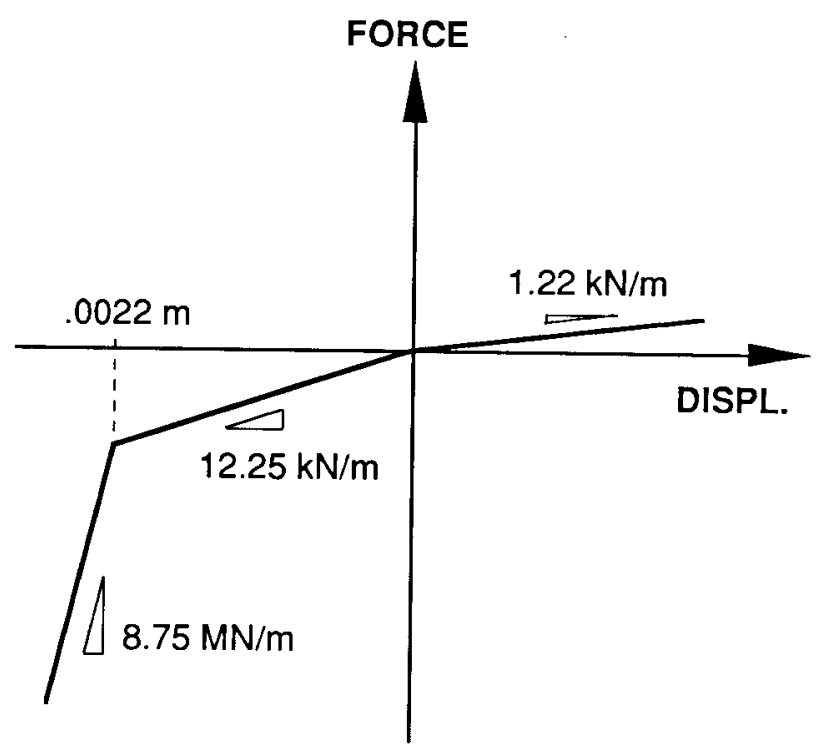

\section{LEAF SPRING FORCE-DEFLECTION}

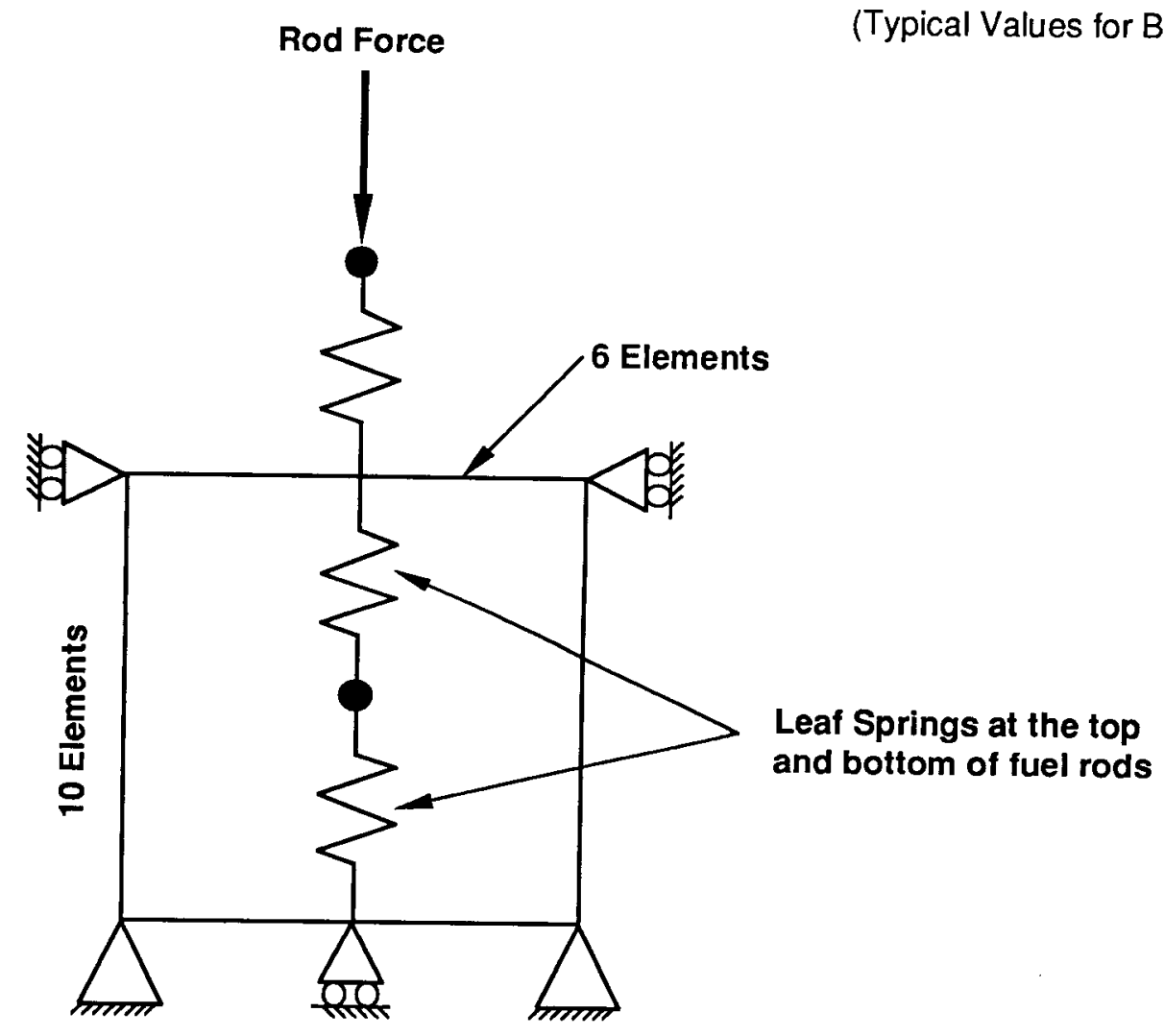

Figure III-16. Single-Cel1 Spacer Grid Analysis Model 


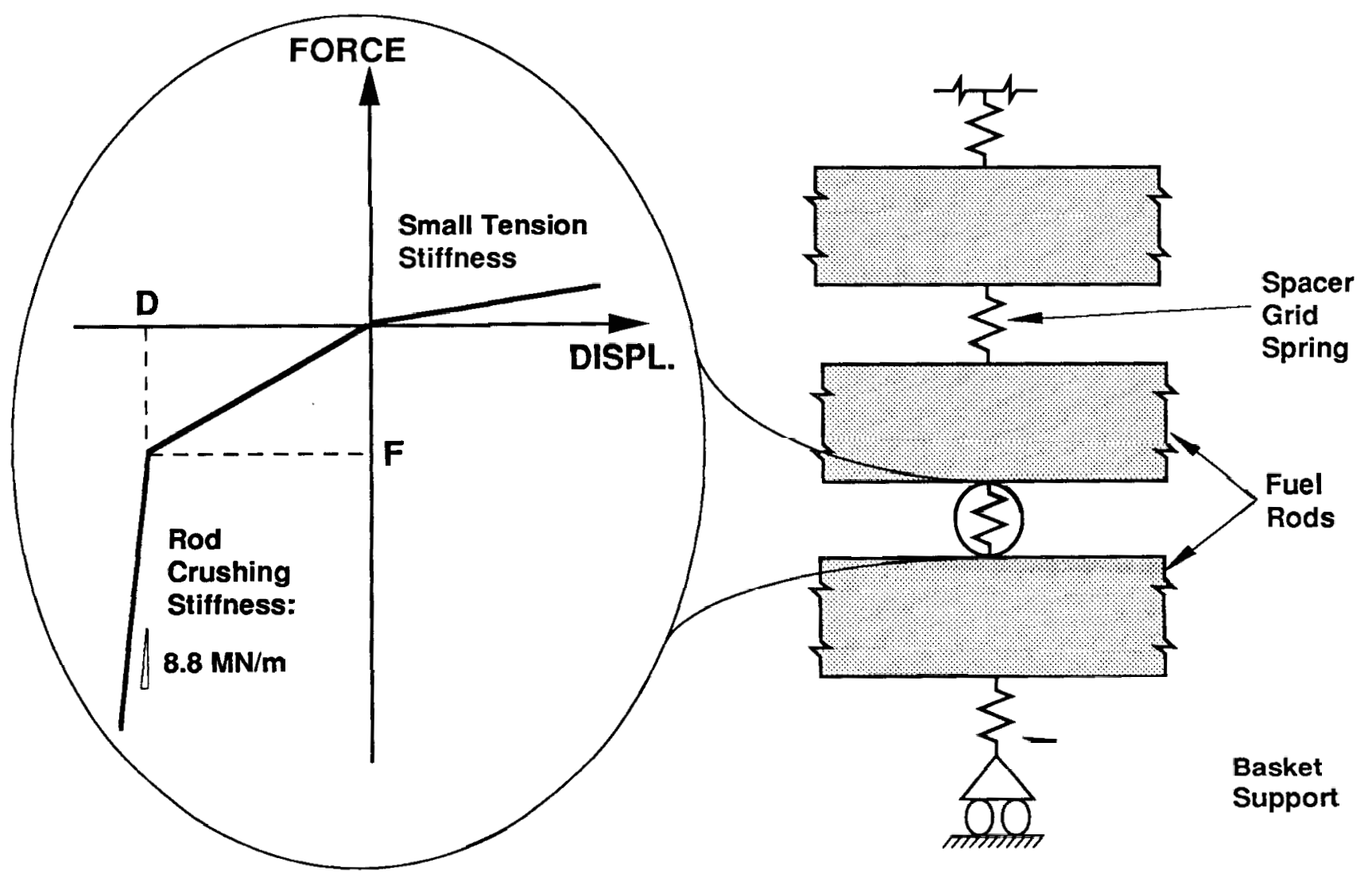

$D=$ Design gap between fuel rods

$F=$ Force which is required to buckle spacer grid frame

Figure III-17. Two-Dimensional Assembly Mode1: Spacer Grid Spring Element, Force-Displacement Relationship

The initial flat portion of the curve models the assembly-basket gap. Each step of the stepped portion of the force-displacement curve (Figure III-18) corresponds to the buckling of an individual grid cell. Thus, the assembly center rod model requires the number of steps on the forcedisplacement curve to be equal to one-half the total number of cells in the spacer grid.

\section{III.3.4 Assemb1y Mode1}

The spacer grid elements developed, as described in the previous section, are a single part of the complex assembly models. Different assembly models are used to analyze the various cask drop orientations, such as side drops and end drops, because the load paths for predominantly side-drop and end-drop loadings are characteristically different. The corner drops are analyzed using a combination of slightly modified end- and side-drop models. 
$B=$ Maximum Bowing , Rod-To-Rod Design Gap

C = Lateral Displacement Restrained By Assembly-Basket Geometry

C. Laterat Displacement Restraned By Assembly-Basket Geomety

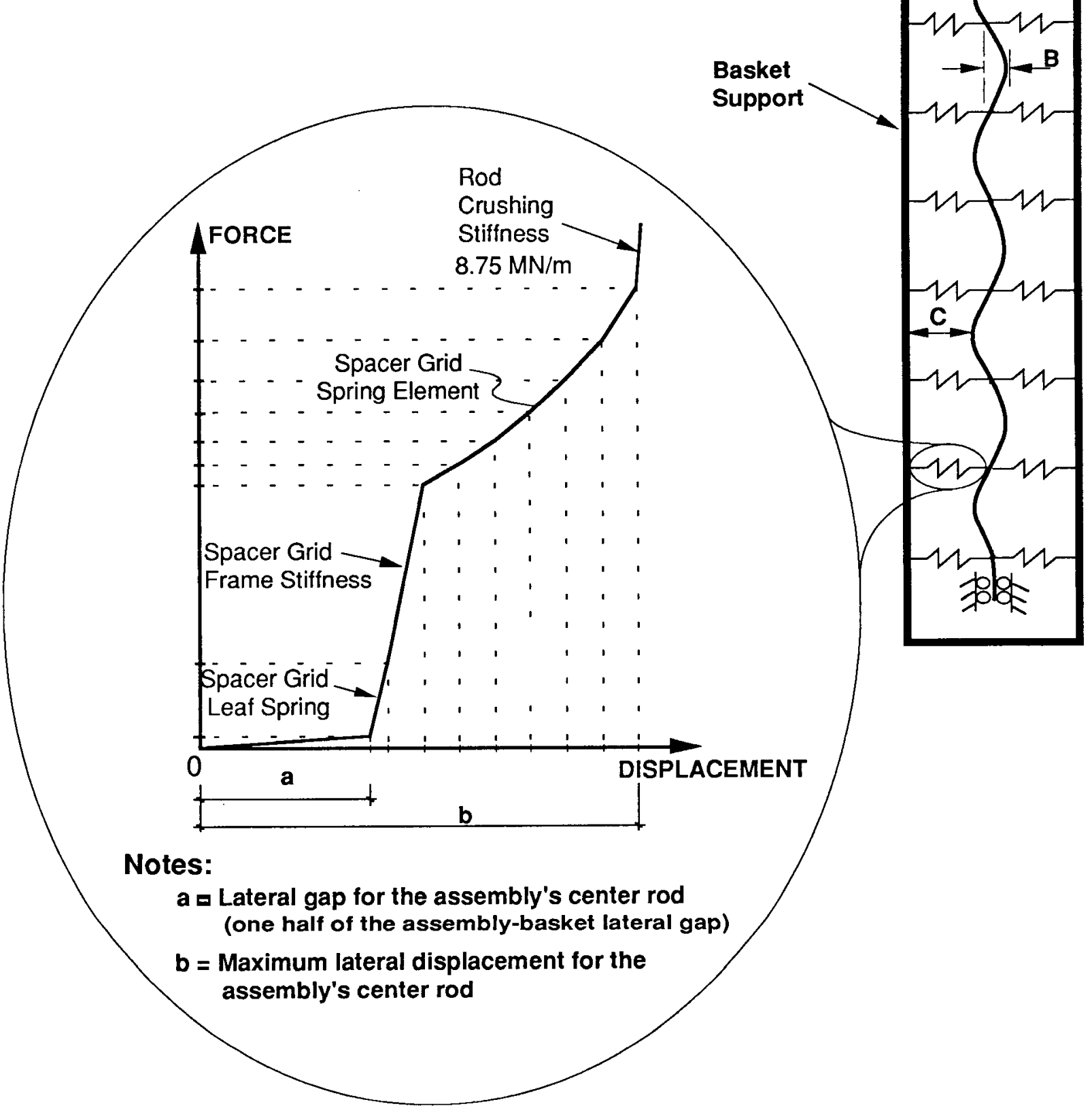

Figure III-18. Spacer Grid Model: Typical Single-Rod End-Drop Assembly

\section{Side-Drop Model}

Significant rod-to-rod interaction occurs in the vertical load plane during a side drop. Little interaction occurs out of the load plane. Only two-dimensional slice models (single rows of rods) of the fuel assemblies are analyzed because neglecting out-of-plane stiffness 
is conservative. By providing an analysis of all the geometrically different slices, two-dimensional assembly models detail the response of all assembly rods.

Two-dimensional slice models of typical BWR and PWR assemblies are shown in Figures III-19 through III-23. These models consist of multiplebeam elements used to model the fuel rods, end plates modeled as rigid beam members, spacer grids modeled with nonlinear force-deflection spring elements (as described in the previous section), and contact spring models of rod-to-rod contact between spacer grids.

Three or more two-dimensional longitudinal slice models are used in the analyses of both BWR and PWR assemblies to model the various assembly cross sections. For each assembly, a two-dimensional slice model is developed for each geometrically different vertical cross section. Figure III-21 shows a cross section of a typical BWR fuel assembly and the corresponding slice analysis models. Loading orientations that are not perpendicular to an assembly face were conservatively neglected because the number of rods stacked on top of each other is always less than or equal to the perpendicular slices and the lateral (relative to the load direction) restraints from the basket and adjacent rods are much greater.

With the exception of their connection to the tie plate, BWR assembly tie rods and loose rods are modeled identically. The tie rods are completely fixed to the end plate. Internal loose rods are fixed to the end plate in all directions except axial translation perpendicular to the tie plate. This boundary condition allows the loose rods to slide axially in and out of the tie plate, provided the fuel rod does not slide completely out of the end plate. (A maximum allowable relative axial displacement of $1.78 \mathrm{~cm}$ is based on current end plate and rod end nozzle designs.) These loose-rod boundary conditions are not valid if the fuel axial deformations of the rod allow the loose fuel rods to completely separate from the end plate. Because the loose rods are allowed to deflect axially (in and out of the end plate), they do not allow an axial load to be transmitted through them. Thus, the response of the loose rods is pure bending. The tie rods, however, are fixed to the tie plate and develop axial stresses in addition to bending stresses. Figure III-20 describes the fuel rod/tie plate modeling in detail. For PWR assemblies, the guide tubes are modeled similarly to the BWR tie rods.

Contact spring elements are used to model rod-to-rod load transfer in the spans between spacer grids and between end plates and adjacent spacer grid springs. As the load increases on the fuel rods, the end plates rotate as a result of axial loads in the tie rods. The rotation of the end plate (inward at the top and outward at the bottom) allows the fuel rods to stack on top of one another. This phenomenon is modeled through contact springs that provide support between fuel rods once the gaps between rods close. A description of the contact springs is shown in Figure III-20. On contact, a stiffness equivalent to the crushing strength of the fuel rods is used for the springs to simulate rod-to-rod interaction. 


\section{BWR: GE $7 \times 7$ BUNDLE}

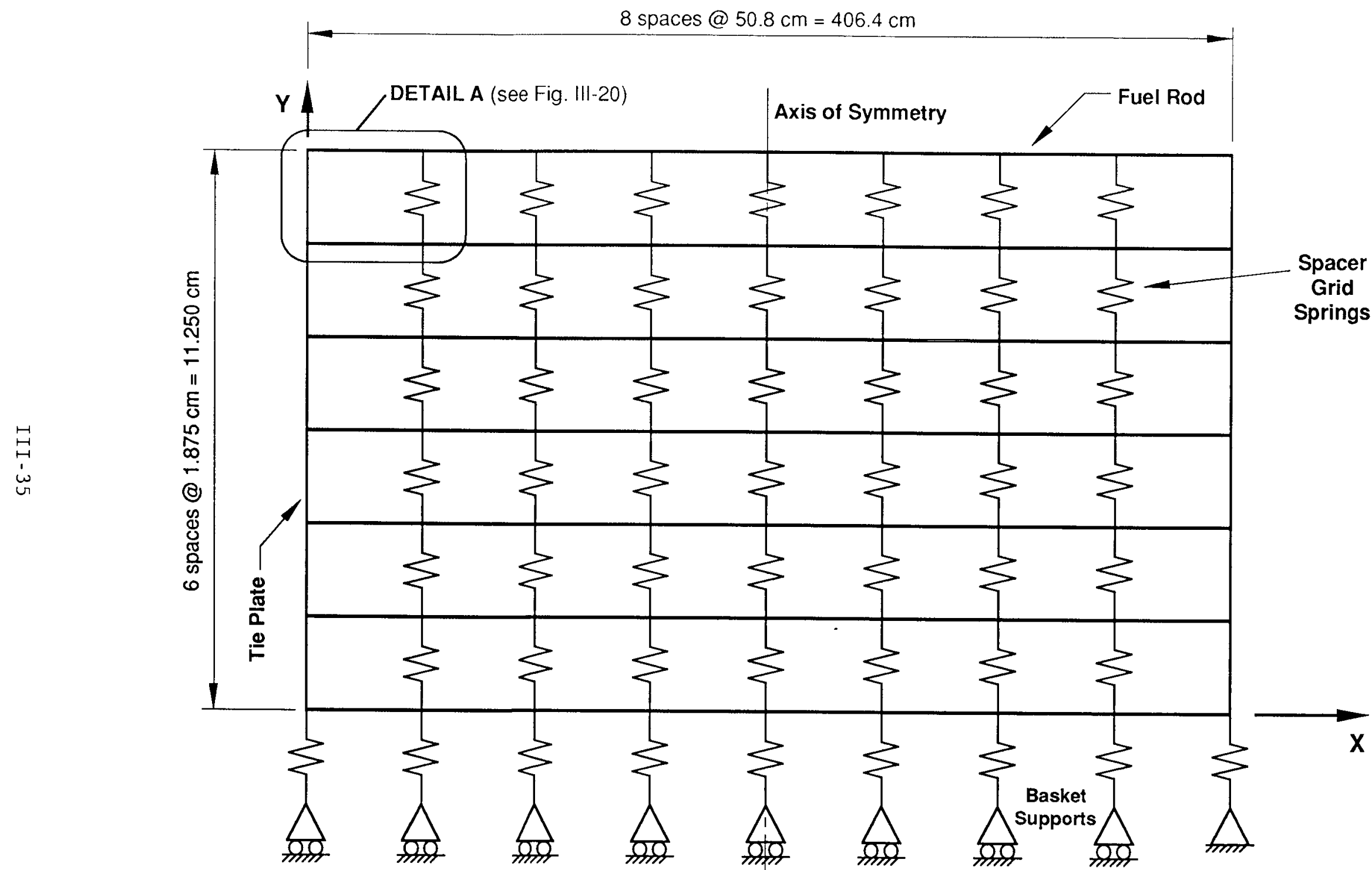

Figure III-19. Example BWR GE 7 × 7 Bundle Side Drop: Two-Dimensional Assembly Model Description 


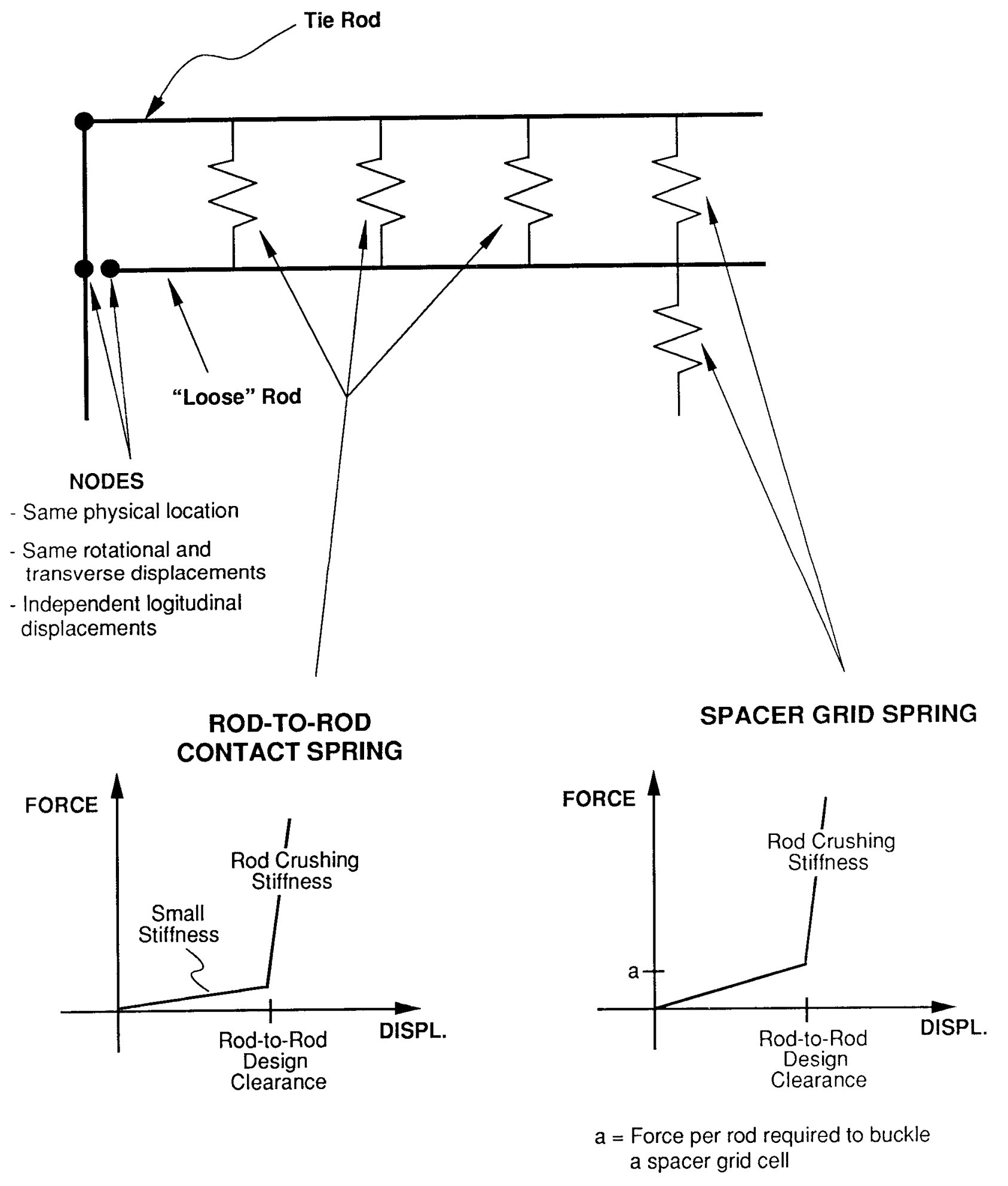

Figure III-20. Example BWR Side Drop: Two-Dimensional Assembly Model, Detail A 


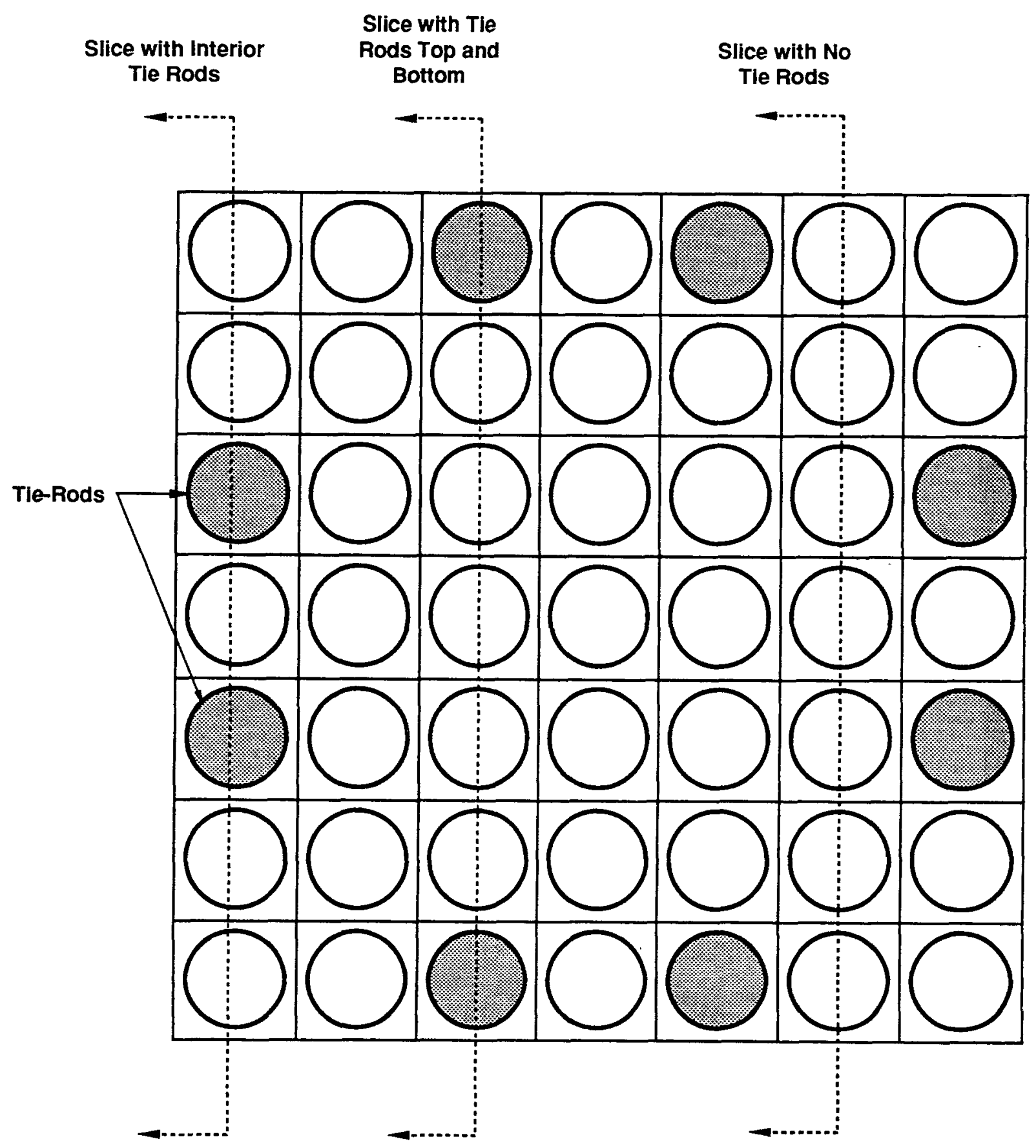

Typical BWR Cross Section at Spacer Grid ( GE 7×7 Assembly)

Figure III-21. BWR Side Drop: Two-Dimensional Assembly Model Cross Sections 


\section{B\&W $15 \times 15$ PWR}

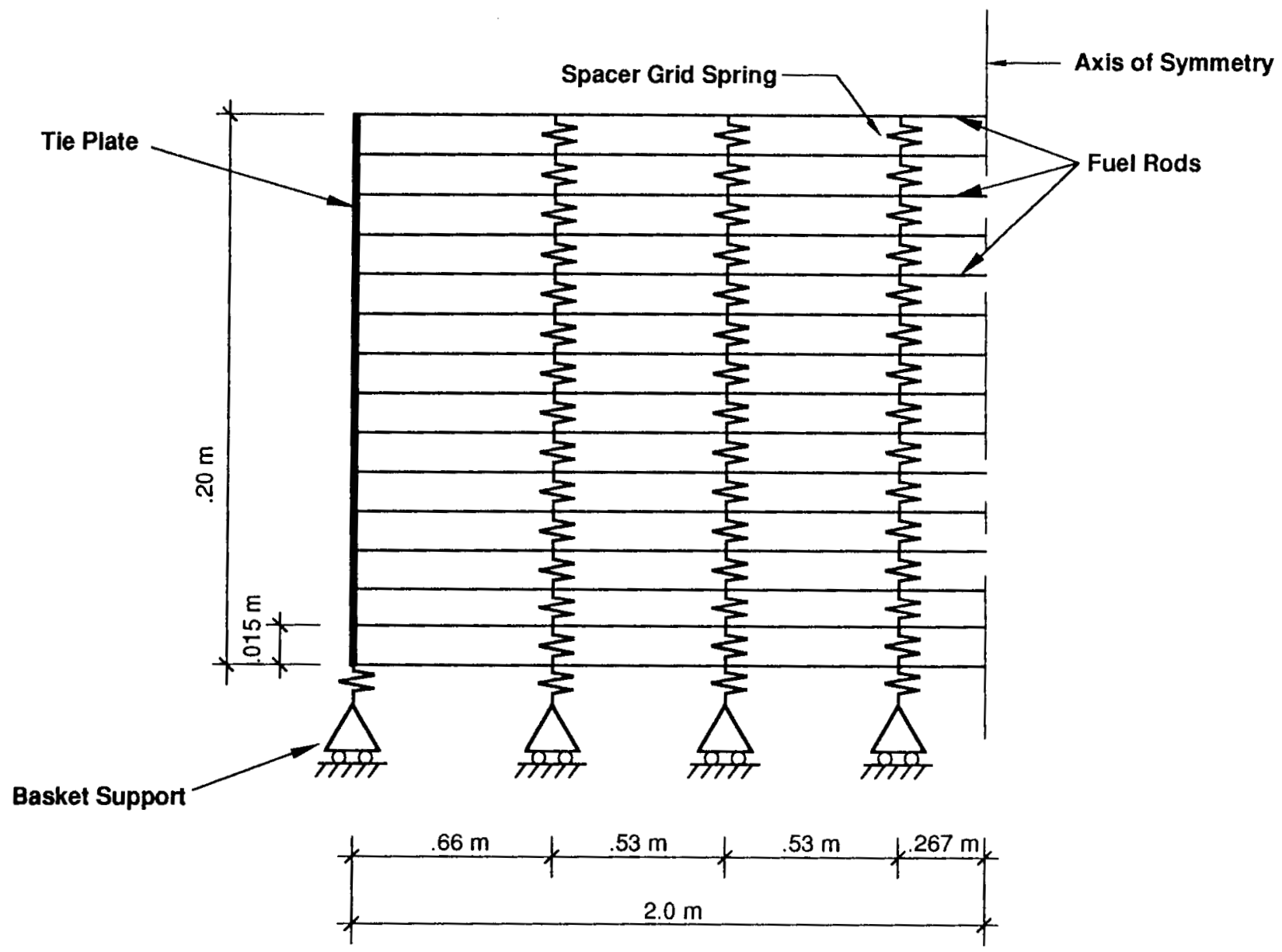

Figure III-22. Example PWR Side Drop: Two-Dimensional Assembly Model Description

The two-dimensional finite element model used to analyze the assembly response uses symmetry boundary conditions on a half model to simulate the entire assembly. For the side-drop analyses, the symmetry boundary condition provides an exact simulation of the full assembly response. The base of the assembly model is restrained vertically at the basket supports for the spacer grid and end plates. The vertical assembly restraints, as shown in Figure III-19, are modeled by coupling the vertical displacements of the spacer grid and end plate supports together at their interface points to the cask basket. The impact-limiter forcing function (described in Section III.3.2) is then applied to these support points simultaneously to simulate a very stiff connection between the support points and the impact limiter. This assumes that the basket supports coincide with the spacer grid supports and are rigid relative to the assembly. This boundary 


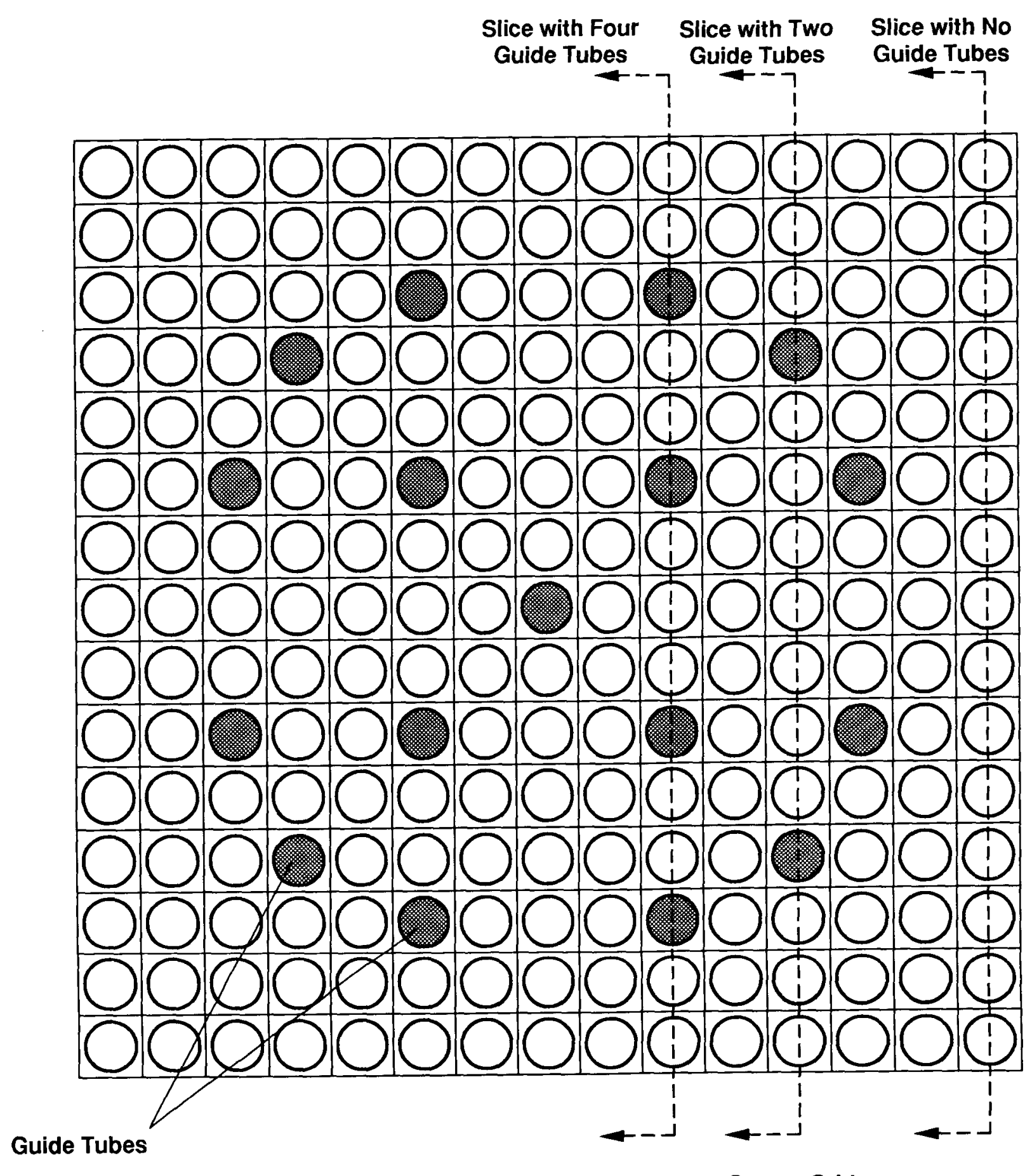

Typical PWR Cross Section at Spacer Grid (B\&W 15x15 Assembly)

Figure III-23. PWR Side Drop: Two-Dimensional Assembly Slice Mode1 Cross Sections 
condition is conservative compared to a continuous basket, in that a continuous basket would provide a continuous support along the length of the assembly and thus restrict the fuel rods' deflections between spacer grids that are allowed by discrete supports. Different support conditions in the assembly model may be required for each specific basket being analyzed. The end plate is assumed to be fixed at its base in the vertical (relative to side-drop orientation) direction only, thus allowing any translation or rotations of the end plate to occur naturally.

The highly nonlinear analysis of the side-drop model requires an incremental solution with small time steps and multiple iterations. Analyses are performed on both BWR and PWR models using the deceleration load histories of the cask under normal and accident conditions as described in Section III.3.2 and in Appendix II. Example BWR and PWR assembly side-drop analyses are described in Section III.5.

\section{End-Drop Mode1}

The end-drop condition is defined as an impact on the top or bottom end of a cask so that the fuel rod assemblies are loaded axially. In this condition, all assembly rods are assumed to be loaded simultaneously and equally as they impact with the tie plate (differences caused by unequal rod lengths are incorporated into the assembly-basket gap).

The end-drop model developed herein conservatively assumes that rodto-rod interaction is limited to all rods deforming in the same pattern (see Figure III-6). This assumption is based on the rods not being in contact with each other before the impact loading event, and all rods having the same deformed shape and deflecting in the same direction at the same time during impact loading. This assumption is conservative in that intermittent rod-to-rod interaction caused by random lateral deformations of a true end drop will restrict lateral deflections of the rods and thus reduce their maximum bending strains (the basis of the end-drop failure criteria).

Under end-drop loading, all assembly rods are in compression. Because both tie rods and loose rods behave the same way under compressive loading, there is no need to differentiate between the two in the model. Neglecting intermittent rod-to-rod interaction and differences between tie and loose rods allows the end-drop loading condition to be modeled with a single rod. Figure III-24 shows example single-rod models for PWR and BWR assemblies. Each model consists of beam elements, spacer grid spring elements, and contact spring elements.

The beam elements have a hollow circular cross section that models the cladding stiffness and material properties. The tensile and bending stiffness of the fuel is neglected. The beam element cross section remains circular during deformations. Fuel rod ovalization is considered locally when necessary to determine if material fracture will occur.

Smaller beam element lengths are used at the bottom of the rod to capture more accurately the strain profile near the impact end of the assembly. The number of elements between the spacer springs is shown in Figure III-24. Nonlinear spacer spring elements are used to model the 


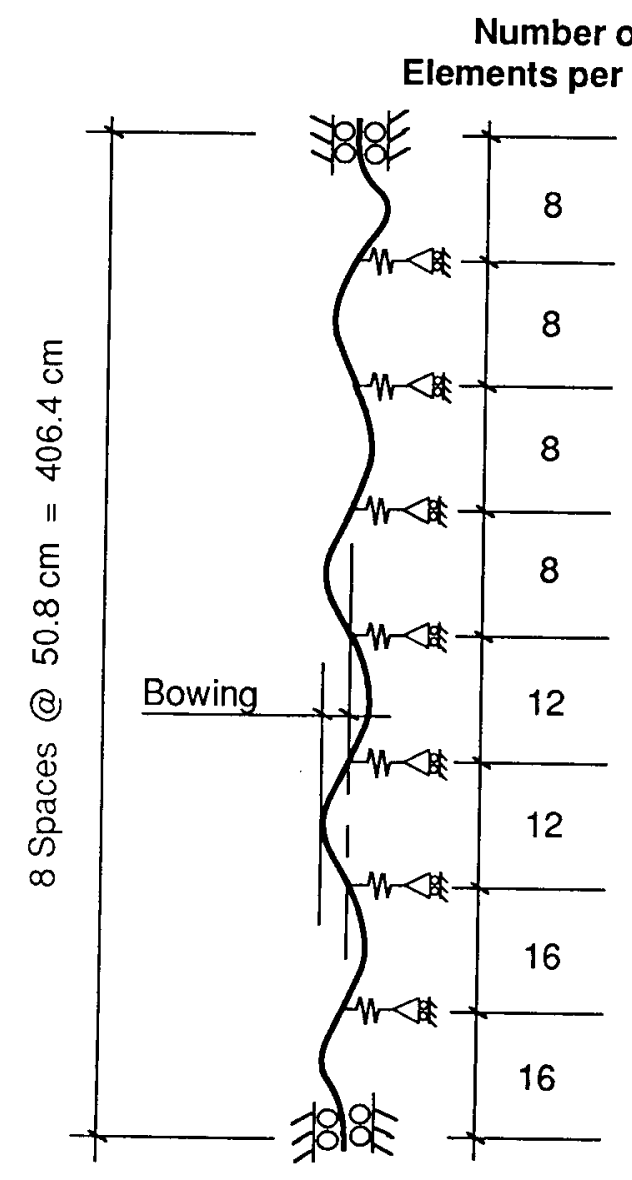

Outside Diameter $=1.430 \mathrm{~cm}$

Wall Thickness $=0.081$

Bowing $=0,0.222,0.445$

Active Fuel Mass $=10 \%$

Active Rod Weight $=1.405 \mathrm{~kg}$

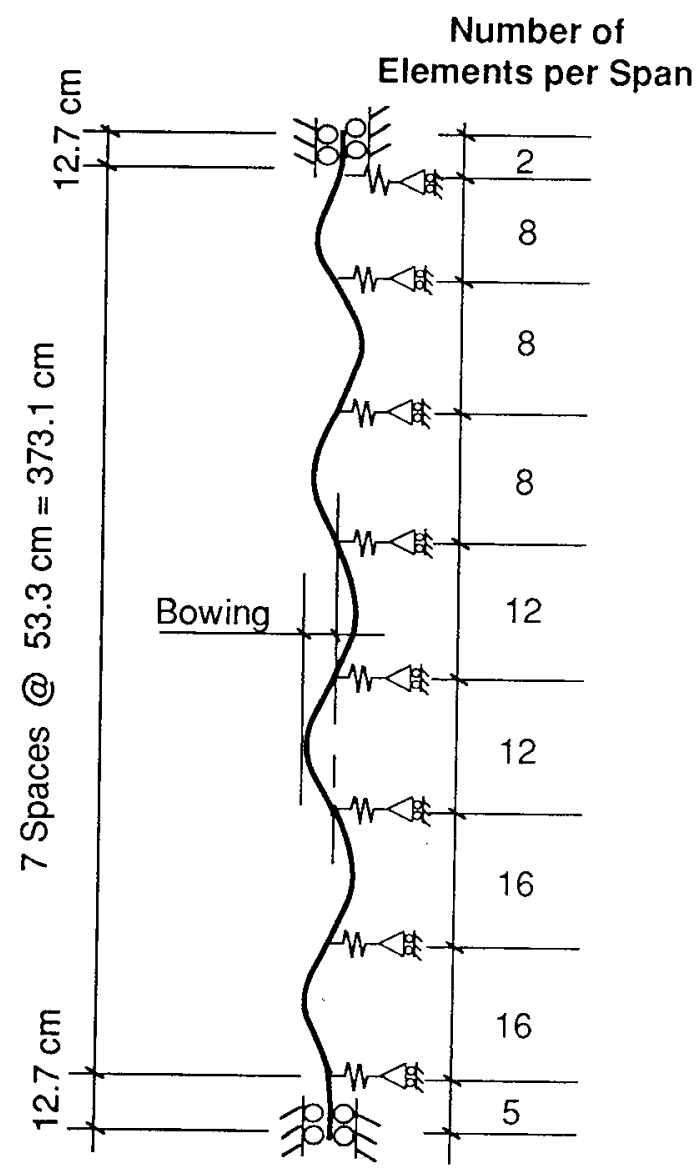

Outside Diameter $=1.092 \mathrm{~cm}$

Wall Thickness $=0.0673$

Bowing $=0,0.175,0.350$

Active Fuel Mass $=75 \%$

Active Rod Weight $=2.575 \mathrm{~kg}$

Figure III-24. Example BWR and PWR End-Drop Single-Rod Models 
lateral supports provided by the spacer grids in each assembly. The spring element force-displacement properties are based on the grid analyses described in Section III.3.3. These properties (illustrated in Figure III-18) are for the center rods of each assembly, if it is assumed that all of the fuel rods are loaded simultaneously and exhibit the same deformation characteristics.

Maximum bowing equal to the design clearance among rods in the assemblies is assumed for all rods. As previously described, this assumption is conservative. Rod weights are based on the amount of active fuel mass or portion of the fuel mass that is attached to the cladding. The nonactive fuel mass is the portion that is free to move in the rod and therefore does not load the cladding during an end-drop impact event.

The rod mass includes the mass of the cladding and active fuel, as well as some additional mass for the top tie plates, and is distributed uniformly along the assembly length. The top and bottom of the rod are fixed in the lateral and rotational directions to simulate the end-plate constraint. The end-drop model is analyzed using time-marching dynamic analysis techniques described in Section III.3.2.

\section{Corner Drop}

There are two distinct sequential events for corner-drop impacts when the cask center of gravity is outside the center of gravity of the impact footprint: initial impact and slapdown. When the center of gravity of the cask falls inside the impact footprint, the cask responds only in the initial impact mode in a manner similar to the end drop. At initial impact, the cask decelerates vertically as the impact limiter crushes; however, at the same time, the cask generates rotational acceleration around its contact point. The initial impact phase ends when the translational velocity of the cask is zero. Depending on the instability of the cask, a secondary impact occurs after the initial impact when the top or free end of the cask impacts the rigid surface. This secondary impact is referred to as the slapdown event. This section describes the models used to establish the assembly responses to the initial impact and slapdown regulatory corner-drop events.

Under regulatory accident 9-m corner drops for which the cask is nearly vertical, the initial impact dominates the typical cask response (see Appendix II). The initial impact response for these large angles is similar to the end-drop response. A modified version of the end-drop single-rod model is used to model the initial impact corner drop. The secondary slapdown impact for angles greater than 45 degrees loads the opposite end of the assembly with a significantly smaller magnitude of loading, and so it can be neglected.

For angles less than 45 degrees, the slapdown response begins to dominate the cask response and an analysis of the slapdown impact is used to characterize the cask response. The slapdown response is very similar to that of the side-drop response; therefore, a modified version of the two-dimensional assembly side-drop model is used to determine the fuel assembly response under slapdown loading conditions. 


\section{Initial Impact Model}

For large angles, the initial impact response of the fuel assembly is very similar to the end-drop loading condition; thus, a modified version of the single-rod models previously described is used for the initial impact analyses. Dynamic analyses are performed similarly to the end-drop response, with the addition of a nonuniform load distribution along the assembly length, which is used to simulate the horizontal and rotational accelerations of the cask. Example analyses performed for drop angles of 84, 70, and 45 degrees for the PWR assembly, and 84 and 45 degrees for the BWR assembly are described in Section III.5. Figure III-25 illustrates BWR and PWR typical initial impact models. The only difference between the initial impact model and the end-drop model is the inclusion of lateral load.

\section{Slapdown Mode1}

For angles less than 45 degrees from the side-drop condition, the slapdown response dominates the loading on the assembly. Slapdown occurs when the orientation of the cask is in nearly a horizontal position, and so cask deceleration loading is perpendicular to the fuel rods. This orientation of the fuel rod to loading direction is the same as the sidedrop orientation previously analyzed. Hence a modified version of the twodimensional assembly model used for the side-drop analyses is used to analyze the corner-drop loading conditions. Because a nonuniform loading distribution is required to account for the rotational acceleration of the cask, the model must be extended from a symmetry half-model to a full model.

The maximum slapdown loading occurs for the representative casks in Appendix II at an initial impact angle offset 2 degrees from the side-drop position. This maximum is obtained from the superposition of the maximum vertical acceleration and maximum angular acceleration, as indicated in Figures II-57 and II-61. Figure III-26 illustrates a typical slapdown model. The time-history dynamic analysis of the slapdown loading condition is described in Section III.3.2. Half-assembly models are used for the slapdown example analyses presented in section III.5. These analyses conservatively assume that the assemblies are symmetrical around their more highly stressed side.

\section{III.3.5 Method of Analysis}

A procedure is presented in this section for the analysis of a fuel assembly impact response that is idealized as a finite element system using the computational models described in the preceding section. Because existing concepts and techniques have been adapted for these analyses, the procedure is presented briefly and references to other sources are provided for details.

\section{Finite Element Discretization}

The basic concept of a finite element method of discretization is idealization of a fuel rod assembly as an assemblage of discrete elements 

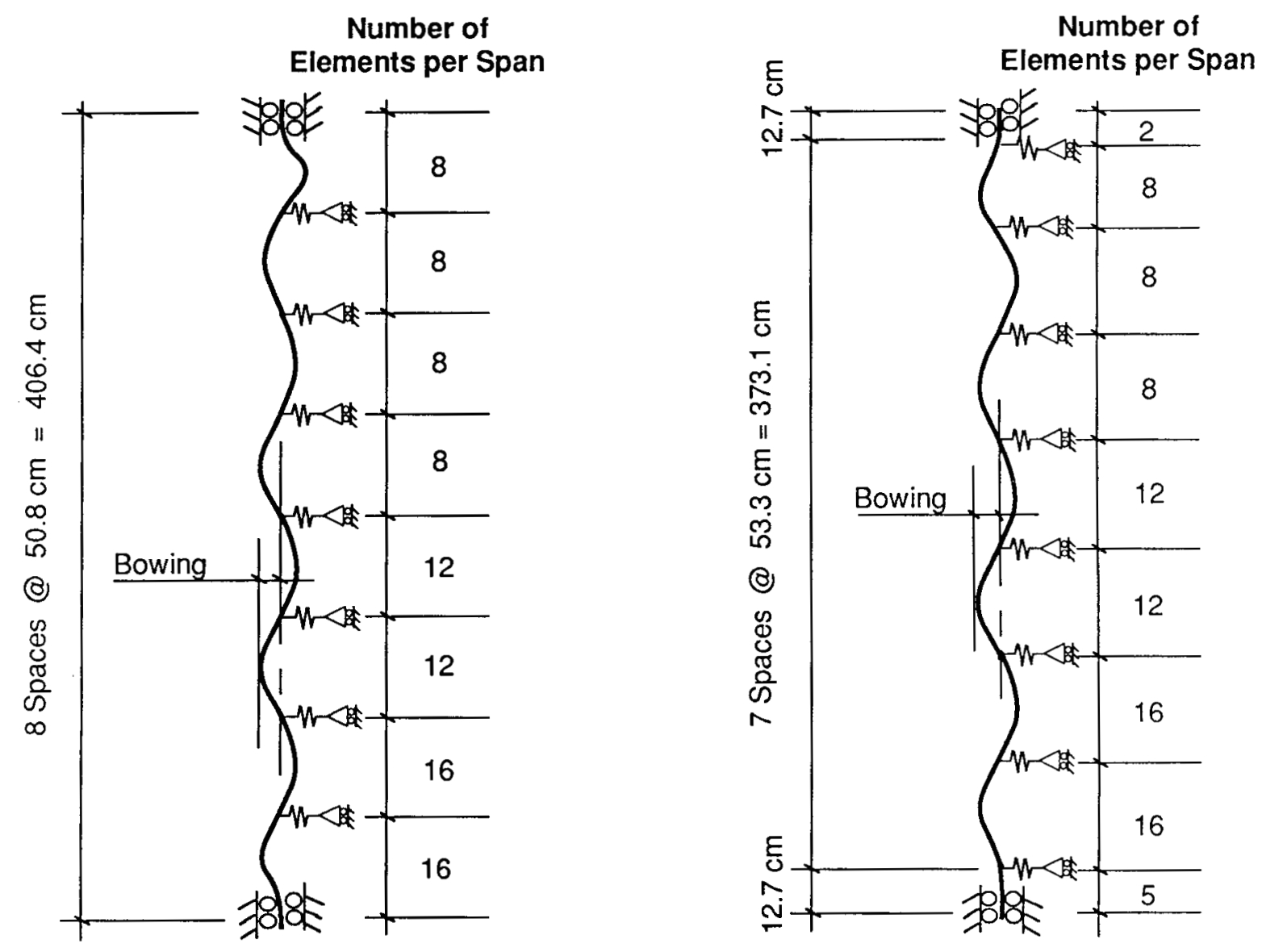

Outside Diameter $=1.430 \mathrm{~cm}$

Outside Diameter $=1.092 \mathrm{~cm}$

Wall Thickness $=0.081$

Wall Thickness $=0.0673$

Bowing $=0.445$

Bowing $=0.350$

Vertical Active Fuel Mass $=10 \%$

Vertical Active Fuel Mass $=75 \%$

Lateral Active Fuel Mass $=100 \%$

Lateral Active Fuel Mass $=100 \%$

B\&W $15 \times 15$ PWR

Figure III-25. Example BWR and PWR Corner Drop: Initial Impact Single-Rod 


\section{B\&W $15 \times 15 \quad$ PWR}

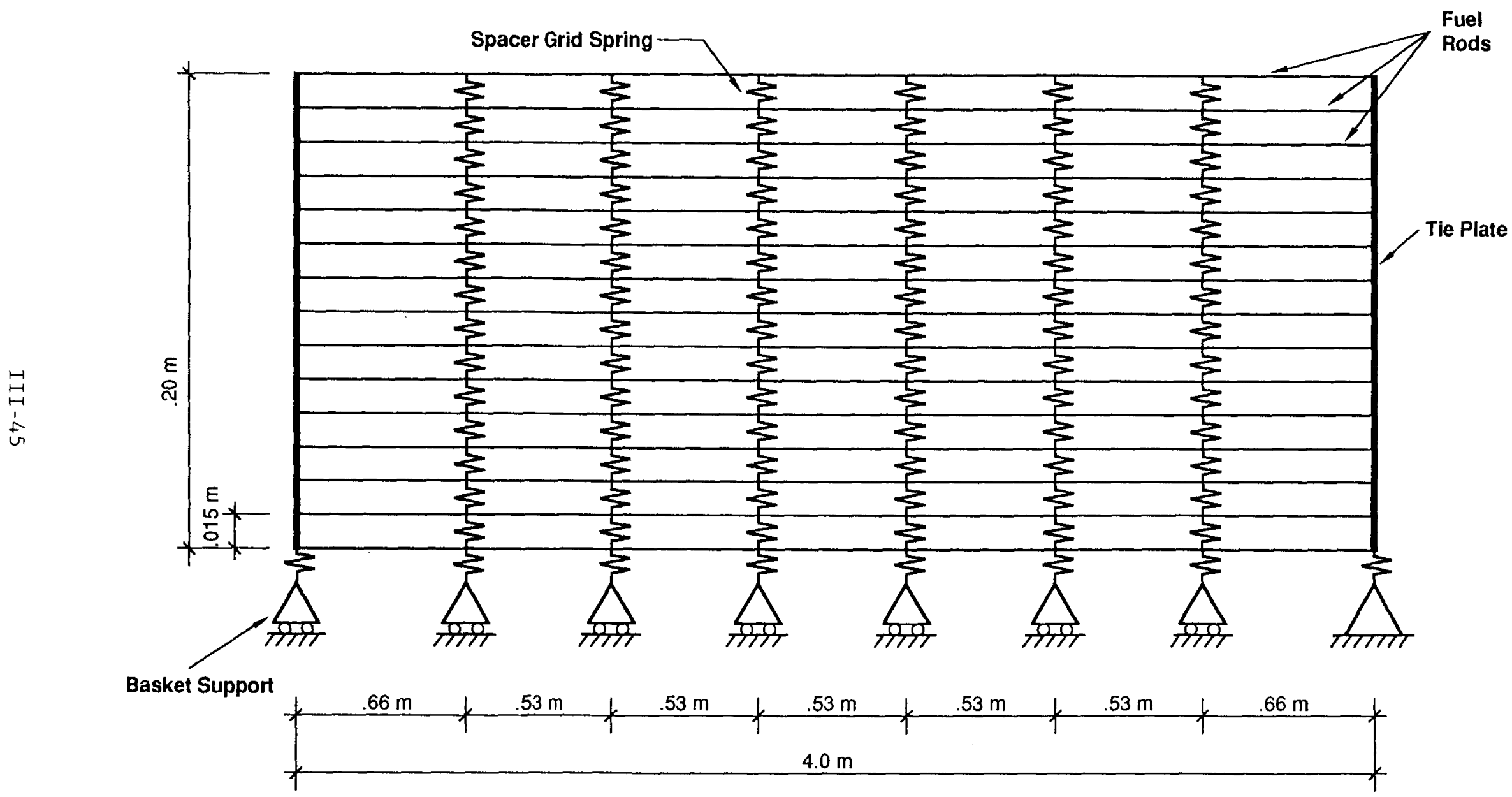

Figure III-26. Computational Model for Slapdown Analysis 
interconnected at their nodal points. The assembly is represented by twodimensional beam and spring elements of arbitrary lengths with suitable displacement variations. Consequently, assemblies with arbitrary shapes can be represented as a collection of finite elements. Each rod in the assembly is represented by a minimum of 30 beam elements. Furthermore, because the constitutive properties for each element are defined individually, arbitrary nonhomogeneities in the assembly can be conveniently handled in the finite element method.

The nonlinear response analysis is carried out as a step-by-step sequence of linear analyses. The duration of interest is discretized into a large number of small time steps; each $\delta t$ may be of different duration. Over any time step $\delta t$, the structure is assumed to have constant properties as determined by the history of stresses and deformations up to the beginning of the time step. A linear analysis determines the state of the system at the end of the time step.

The element stiffness matrix for any time step $\delta t$ can be calculated by standard procedures [ZI71] once the displacement field has been assumed. The displacement field is a function of the element's geometry and constitutive properties determined at the beginning of the $\delta t$. The basic steps for evaluating the element stiffness matrix are described by Clough [CL69]. The time-dependent stiffness matrix, $K(t)$, of the complete structural assemblage is obtained from current individual element stiffness matrices. This matrix is obtained most conveniently by direct stiffness assembly procedures. The stiffness matrix is very sparse and can easily be arranged into a banded form.

A consistent mass matrix for the beam elements can be derived using the same displacement interpolation functions; it possesses the same coupling properties between adjacent elements as the stiffness matrix. Energy dissipation in yielding of the cladding at large deformations is accounted for by the hysteretic property included in the stress/strain relationship that forms the basis of the stiffness matrix formulation. A small amount of numerical damping is introduced to improve the automatic time-stepping solver performance by reducing the numerical noise of the solution caused by changes in time-step size. The Hilber-Hughes-Taylor operator [HI78] is used to define this numerical damping. A generalized viscous damping matrix, $C$, proportional to the mass and stiffness matrices $M$ and $K$ (i.e., $C=\alpha_{0} M+\alpha_{1} K$ ), is conservatively neglected for the shortduration impact analyses.

\section{Equations of Motion}

The equation of motion for the fuel rod assembly that is idealized as a finite element system and is subjected to impact loading at time $t$ is

$$
f_{I}(t)+f_{S}(t)=P(t),
$$

where $f_{I}, f_{S}$, and $P(t)$ are the vectors of inertial forces, elastic restoring forces, and applied forces. Similarly, the equation of motion at time $t+\delta t$ is 


$$
f_{I}(t+\delta t)+f_{S}(t+\delta t)=P(t+\delta t) .
$$

The incremental equation for a time increment $\delta t$ is obtained by subtracting Equation (III-4) from Equation (III-5):

$$
\Delta f_{I}(t)+\Delta f_{S}(t)=\Delta P(t)
$$

where

$$
\begin{aligned}
\Delta f_{I}(t) & =f_{I}(t+\delta t)-f_{I}(t) \\
\Delta f_{S}(t) & =f_{S}(t+\delta t)-f_{S}(t) \\
\Delta P(t) & =P(t+\delta t)-P(t)
\end{aligned}
$$

Denoting the vector of nodal point displacements relative to the impact surface as $r(t)$, the accelerations as $r(t)$, and the corresponding increments over the time interval $t$ to $t+\delta t$ as $\delta \mathrm{r}$ and $\delta \mathrm{r}$, the incremental forces of Equation (III-7) can be expressed as follows:

$$
\begin{aligned}
\Delta \mathrm{f}_{\mathrm{I}}(t) & =\mathrm{M} \delta \dot{r} \\
\Delta \mathrm{f}_{\mathrm{S}}(t) & =\mathrm{K}(\mathrm{t}) \delta \mathrm{r} \\
\Delta \mathrm{P}(\mathrm{t}) & =\mathrm{M}_{\mathrm{a}} \mathrm{A}(\mathrm{t}) \mathrm{a} .
\end{aligned}
$$

The mass matrix, $M$, remains constant, whereas the stiffness matrix, $K$, varies according to the state of the system at the beginning of the time increment $\delta t$ and on the prior deformation and stress history. $M_{a}$ and $A(t)$ are scalar quantities equal to the assembly mass and acceleration time history; a is a matrix relating the scalar quantities to the support points of the assembly. Substituting Equations (III-8), (III-9), and (III-10) into Equation (III-6) gives

$$
M \delta \ddot{r}+K(t) \delta r=M_{a} A(t) a .
$$

\section{Step-by-Step Integration}

The impact response of the fuel assembly is obtained by solving the equation of motion, Equation (III-11), using a step-by-step method of integration. The total relevant duration is divided into short time steps, and the response is calculated during each step by treating the system as linear, i.e., having the properties determined at the time-step beginning. At the end of the step, the properties are modified to conform to the deformation and stress state at, and its history up to, that time; thus, the nonlinear analysis is approximated as a sequence of analyses of successively changing linear systems. Several methods are presently available for integrating Equation (III-11).

The ABAQUS [HI88] general-purpose finite element program was used for the analysis described in Section III.5. In this implicit-integration dynamic analysis, automatic time stepping is based on the concept of halfstep residuals [HK79]. The time-stepping operator defines the velocities and accelerations in terms of displacement at the end of the step and conditions at the beginning of the step. Equilibrium is then established at $(t+\delta t)$; this ensures an equilibrium solution at the beginning and end of any individual time step. However, these solutions do not guarantee 
equilibrium throughout the step. The time-step control is based on measuring the equilibrium error (the force residuals) at some point during the step by using the integration operator with the solution obtained at $(t+t)$ to interpolate within the step. The evaluation is performed at the half step $(t+\delta t / 2)$. If the maximum entry in this residual vector (the maximum "half-step residual") is greater than a user-specified tolerance, the time step is considered too large and is reduced by a factor of four. If the maximum half-step residual is less than half the user-specified tolerance, the time step is increased by a quarter for the next increment-otherwise, it is deemed adequate.

ABAQUS uses Newton's method as a numerical technique for solving the nonlinear equilibrium equations. The motivation for this choice is primarily the convergence rate obtained by using Newton's method compared to the convergence rates exhibited by alternate methods (usually modified Newton or quasi-Newton methods).

The displacements generated from the solution of Equation (III-11) are back-substituted into the beam stiffness equation to determine the fuel member forces and strains of the rods. Only axial strains are associated with the beam elements used to model the fuel rods. Ovalization effects are treated by local finite element models of two-dimensional cross sections of the cladding and fuel pellet using the FREY code. The results from the ABAQUS analysis are used as input into these detailed analyses.

Material plasticity of the cladding is based on an isotropic elastic, von Mises yield criterion, and an isotropic hardening material. Details of this material model are provided by Hibbett [HI88]. The strain and force data from the analysis results are incorporated into the failure criterion discussed in the following section to predict the fuel rod failure probability.

\section{4 Failure Criteria}

The fuel rod failure criteria presented herein are based on the fuel deterministic structural response of the fuel rod (see Section III.3) and the probabilistic evaluation of the material properties of the cladding. The results of the assembly response analyses are examined against failure criteria to determine the type, extent, and probability of fuel rod cladding failure for a given loading condition. The methodology and criteria used to quantify fuel rod failure from the calculated response are described here. Failure evaluations (e.g., analyses of PWR and BWR assemblies) are presented in Section III.6.

The release of rod contents under normal transport or regulatory accident conditions is postulated for two types of failure: material rupture as a result of strains exceeding the ductility limit, and fracture as a result of excessive stress on an existing crack in the material. The resulting failure may produce a small tear in the hoop direction, a longitudinal slit, or complete fuel rod severance. Figures III-27 and III-28 illustrate these two failure mechanisms. Three modes of failure are described by these two failure mechanisms: (1) transverse tearing initiated by material rupture, (2) rod breakage caused by crack 


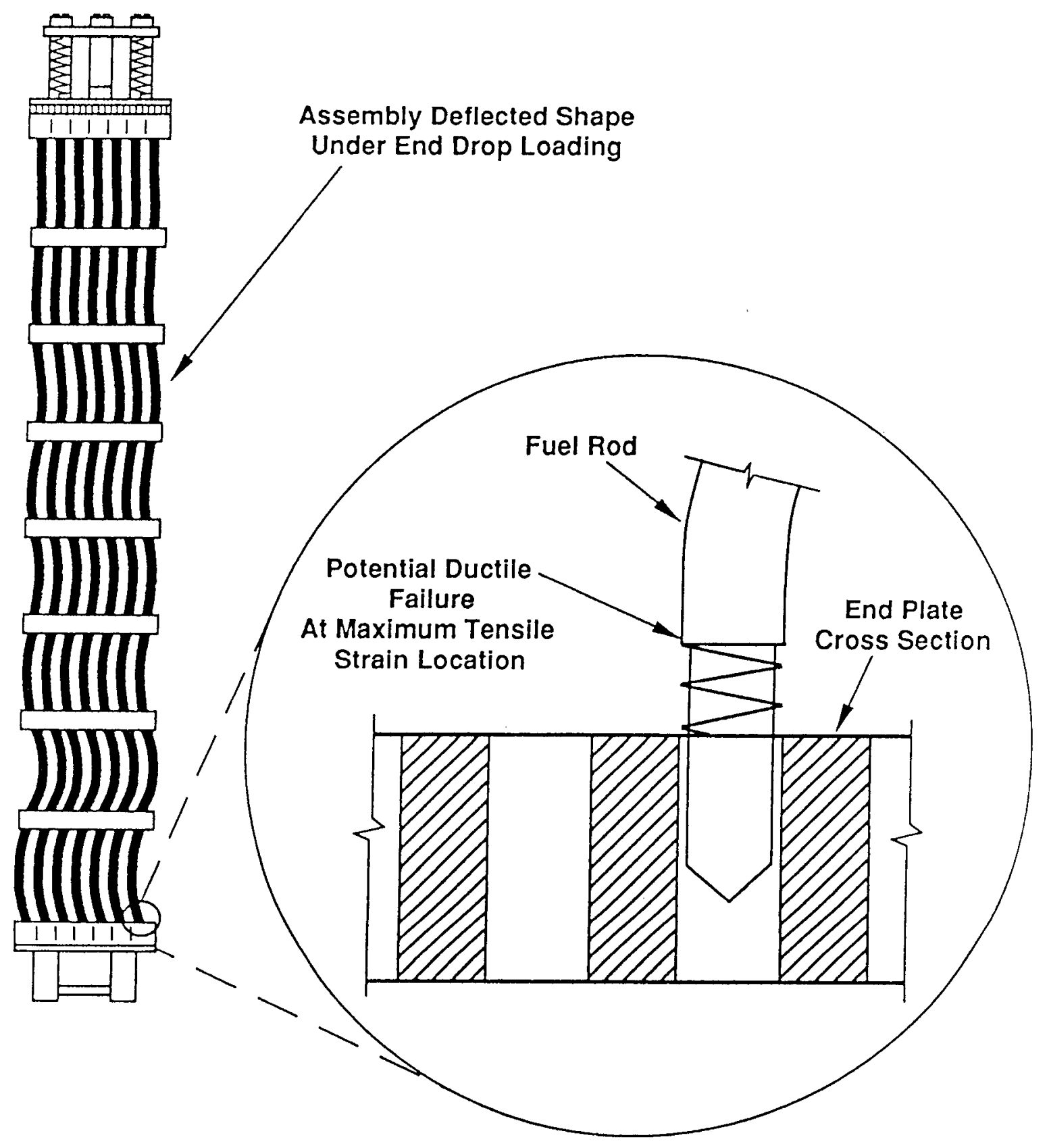

Figure III-27. Cladding Material Rupture Failure Mode 


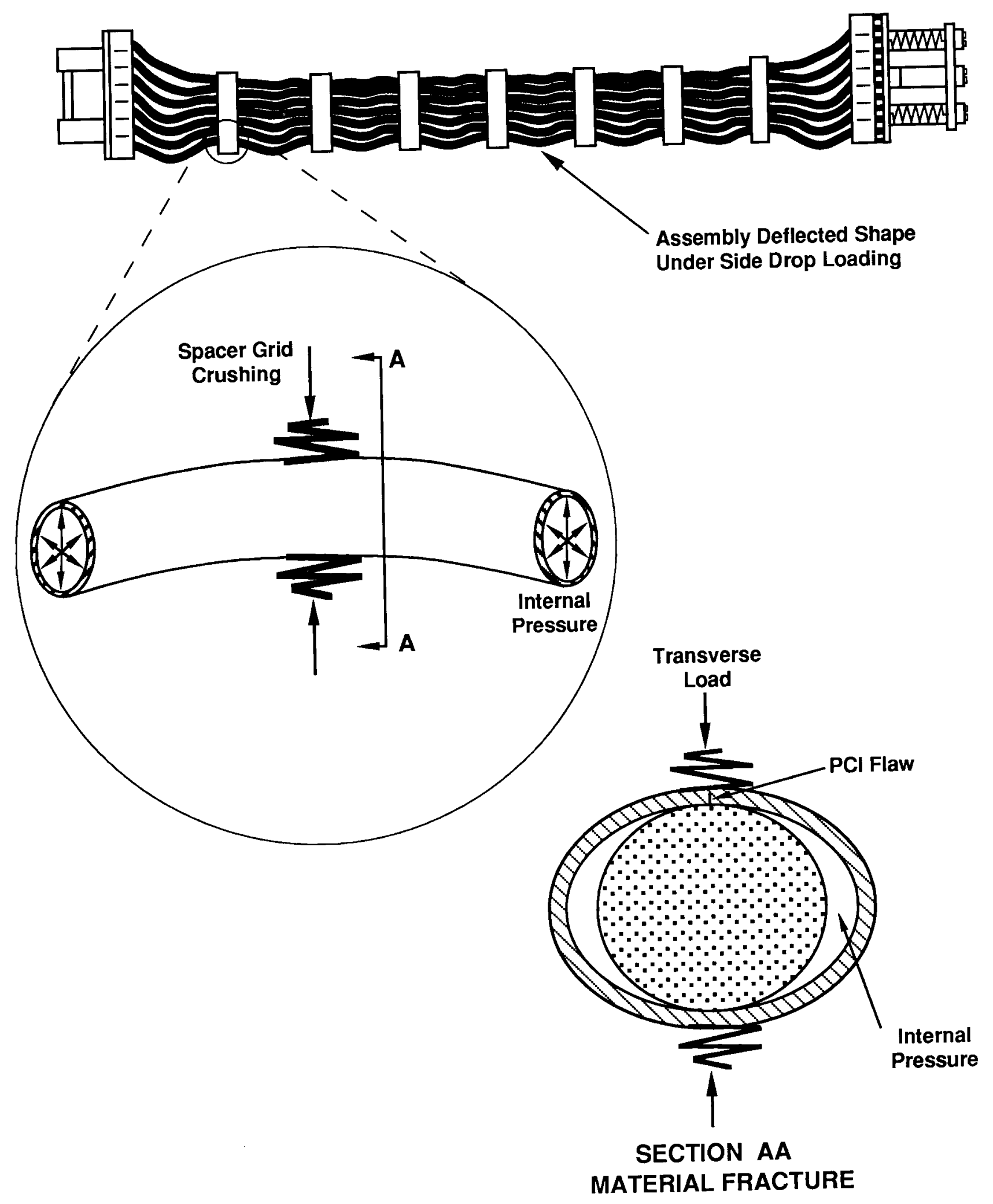

Figure III-28. Cladding Material Fracture Failure Mode 
propagation, and (3) longitudinal tearing initiated by material fracture. Figure III-29 illustrates these three mechanical failure modes. The failure criteria used to determine these failure modes are the material rupture and material fracture failure mechanisms described in this section.

\section{III.4.1 Material Rupture}

This failure mechanism is associated with stretching the cladding material past its ductility limit. As a fuel rod bends around a spacer restraint or tie plate connection, large axial strains may develop in the outer fibers of the rod. Test data [GE80] indicate that rupture can occur for irradiated Zircaloy at nominal bending strains of $8 \%$. Tests were performed at room temperature for high-exposure fuel $>12,000 \mathrm{MWD} / \mathrm{MTU}$ (fast neutron fluence $>1.7 \times 10^{21} \mathrm{n} / \mathrm{cm}^{2}$ ). These data were obtained from bending irradiated fuel rods around smooth mandrels of large diameter, with the bending strain computed from the curvature and strength of the material. However, this nominal bending strain of $\sim 88$ may be considerably higher than the rupture strain under biaxial loading. For example, data from combined axial and circumferential loading of irradiated Zircaloy cladding [BA77] show a considerable reduction in ductility, with some failure strains as low as 28 and with a median of -48 . These failure strain test data are applicable to high-exposure fuel from room temperature to $600^{\circ} \mathrm{C}$. The reduction in ductility is attributed to the state of biaxial tension. Large-radius bending tends to create a uniaxial stress state in the cladding, which is relatively benign from a ductile tearing perspective. Cladding ovalization during bending, however, tends to create a multiaxial state of stress, introducing circumferential bending as well.

Some areas of a rod are subjected to transverse loads from spacer interaction, causing ovalization of the cross section and subsequent areas of high transverse stress. Cladding locations subjected to such biaxial tension could fail at substantially lower effective strains. Thus, for the material rupture failure criterion, a failure strain as a function of biaxial stress state is employed. Figure III-30 defines this material rupture strain as a function of biaxial stress ratio. Experimental data used to define these curves are noted in Figure III-30. For a hoop tensile stress to axial tensile stress ratio of 0.9 or larger, an effective strain of 48 is assumed to be the median value required to produce tearing. For a hoop-to-axial tensile stress ratio of 0.1 or less, a median effective cladding strain of 88 is assumed to produce tearing. A linear interpolation between these values is used for intermediate biaxial stress ratios.

The curves shown in Figure III-30 are constructed from available data using a Gaussian distribution. The standard deviation was determined as follows: for the biaxial stress at a ratio of 0.1 or smaller (uniaxial bending), the $10 \%$ positive failure strain was taken as roughly the 958 value, that is, 958 of failures occur for a 108 strain. From symmetry, this defines 68 strain as the criterion for fuel rod cladding failure for 58 of the rods. With these values, the standard deviation is calculated as 1.258 strain for the $b=0.1$ curve. For the biaxial stress ratio of 0.9 or larger (pressurized rods under tension), the median value of the failure data is -48 positive strain. Because the lower end of this curve 
FAILURE MODE
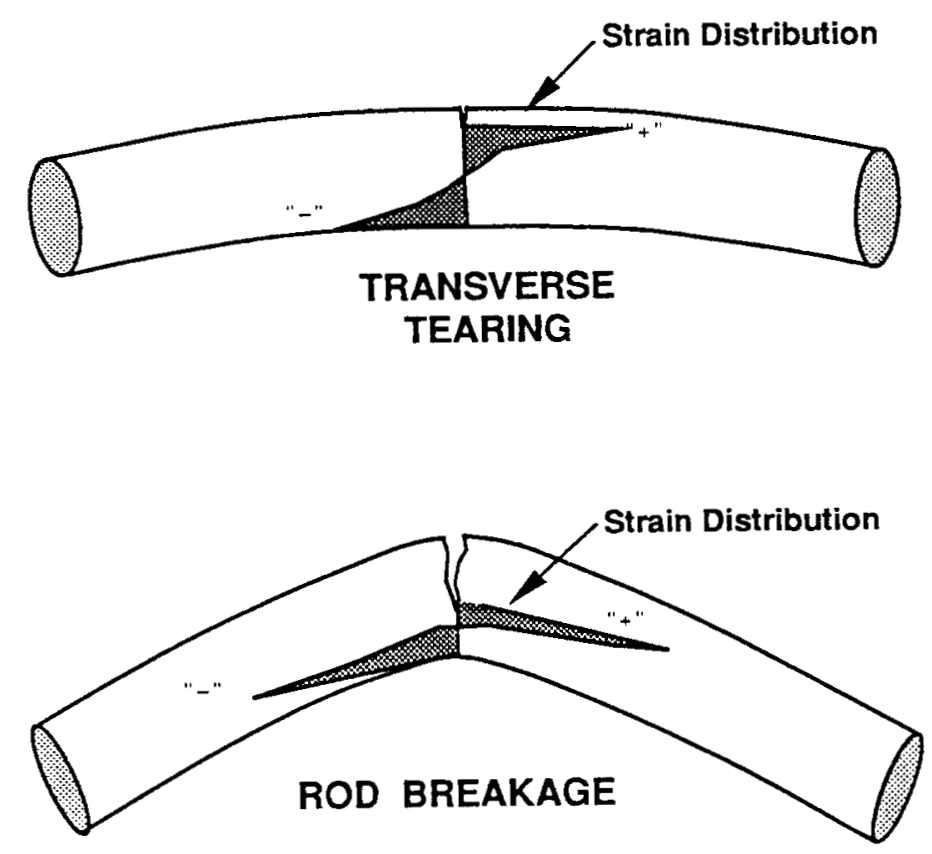

FAILURE MEASURE

$\varepsilon \geq \varepsilon_{\mathrm{f}}$

\section{$\mathrm{K}_{\mathrm{I}} \geq \mathrm{K}_{\mathrm{IC}}$}
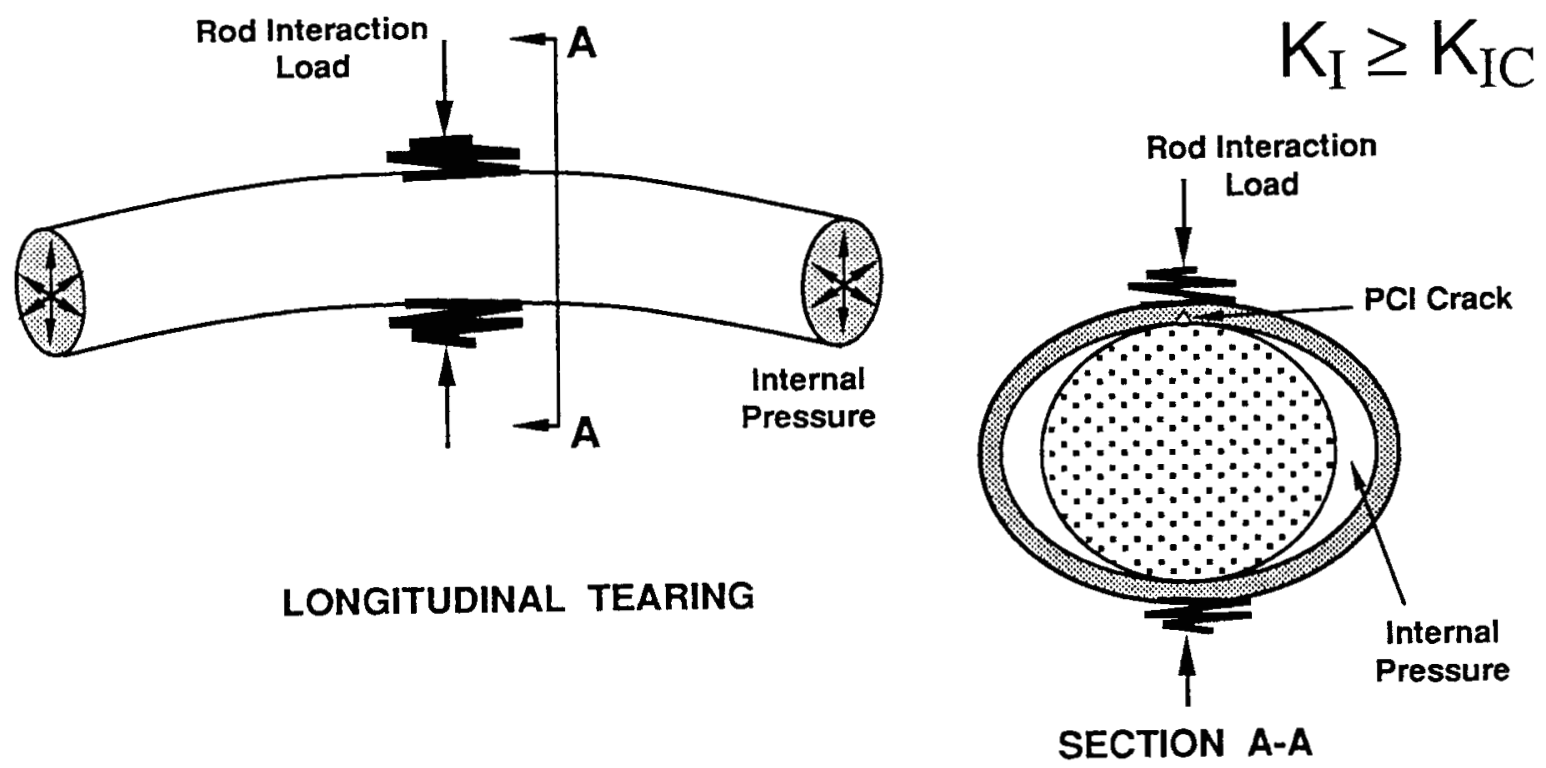

Figure III-29. Fuel Rod Failure Modes 


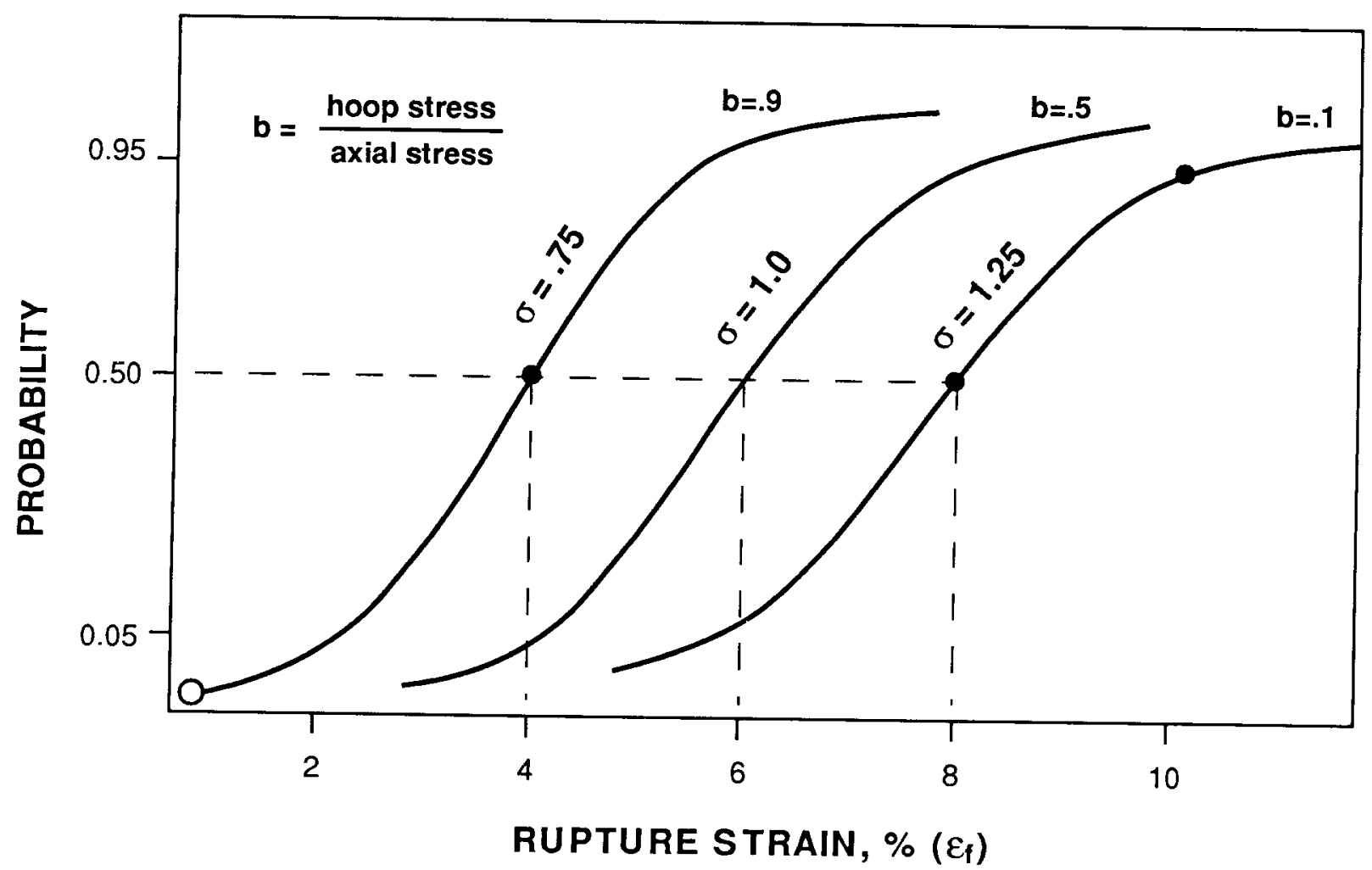

- Experimentally based data points used to develop rupture strain failure curves

$O$ - Failure probability defined as $1 \times 10^{-6}$ at elastic limit strain $=0.8 \%$

PROBABILITY OF FAILURE FOR STRAIN, $x$

$$
\begin{aligned}
& F(x)=\frac{1}{\sigma \sqrt{2 \pi}} \int_{-\infty}^{x} \exp \left(\frac{-(y-\mu)^{2}}{2 \sigma^{2}}\right) d y \\
& \mu=\text { Mean Value of Distribution } \\
& \sigma=\text { Standard Deviation of Distribution }
\end{aligned}
$$

Figure III-30. Probability Distribution for Cladding Rupture Strain as a Function of Biaxial Stress Ratios 
approaches the elastic limit of the material, the standard deviation was defined so that a strain near the elastic limit of $0.8 \%$ would produce a failure probability of $1 \times 10^{-6}$. These two data points define the standard deviation for this curve: 0.758 strain. The standard deviation for an intermediate biaxial stress ratio is based on a linear interpolation between uniaxial $(b=0.1)$ and biaxial $(b=0.9)$ values; $\sigma=1.08$ strain for $b=0.5$.

The biaxiality of the assembly response models is computed as follows: the transverse pinch load on rods as a result of rod-to-rod crushing (at spacer grids or adjacent to basket supports) is used to determine the rod hoop stress. This hoop stress is combined with that of internal pressure and the result is used to determine the ratio of hoop stress to axial stress. The axial stress in the outer fiber of the rod is caused by longitudinal bending as a result of the fuel rod mechanical response. Based on the ratio of hoop axial stress (the biaxial stress ratio), a failure strain criterion for the rod stress state is defined. The peak strain in the rod based on the bending stress state is evaluated against the failure strain distribution to determine the failure probability. This is accomplished by normalizing the standard probability density function to the strain level for rupture and integrating to find the probability of failure, $P(x)$, for a given strain value, $x$, as

$$
P(x)=\frac{1}{\sigma \sqrt{2 \pi}} \int_{-\infty}^{x} \exp \left(\frac{-(y-\mu)^{2}}{2 \sigma^{2}}\right) d y,
$$

where

$$
\begin{aligned}
& \mu=\text { the mean value and } \\
& \sigma=\text { the standard deviation. }
\end{aligned}
$$

As an example illustrating the failure distributions above, if the axial strain from bending is $\sim 18$ and the cladding material has a hoop-toaxial stress ratio of 0.9 from transverse loading as a result of spacer crushing, the mean rupture strain is 48 . The failure probability, $P$, for 18 strain is then

$$
P(18)=\frac{1}{0.75 \sqrt{2 \pi}} \int_{-\infty}^{1} \exp \left(\frac{-(y-4.0)^{2}}{2(0.75)^{2}}\right) d y \simeq 3.2 \times 10^{-5} \text { failures per rod }
$$

This postulated material rupture failure defines a circumferential pinhole opening in the cladding. Potential propagation of this initial breach is discussed in the following section on material fracture. 


\section{III.4.2 Material Fracture}

Both initiation of a longitudinal cladding pinhole failure and extension of the circumferential breach involve a material fracture analysis approach. The longitudinal failure mode must be considered because it can occur under loads producing stresses well below the ductility limits of the material. Part-wall cracks resulting from fabrication defects introduced before reactor service (possibly from cold working, welding, or service-induced effects such as those caused by PCI or mechanical interference) are required as an initial condition for material fracture. PCI occurs during reactor service when the fuel pellets relocate and swell beyond the gap clearance and impinge on the inner bore of the cladding. This triggers stress corrosion cracking and produces small cracks in the cladding material that are oriented in the longitudinal direction [MI84] (see Appendix I for a detailed description of the process involved in the formation of PCI part-wall cracks).

These longitudinal part-wall cracks may propagate under internal pressure or transverse loads that produce hoop stress. If unstable propagation occurs, a longitudinal slit in the rod will be produced. No evaluation is required for crack arrest in order to estimate the tear length for this failure scenario because the cladding openings will remain as longitudinal slits.

Circumferential cracks such as manufacturing defects or pinholes created from the material rupture failure mode may propagate under bendinginduced axial loads. Unstable propagation in this case causes large openings in the cladding and ultimately fuel rod breakage into separate pieces.

The following information is needed to evaluate the potential for cladding fracture as a result of preexisting flaws: (1) the stress distribution in the cladding, (2) an estimate of the flawed state of the cladding, and ( 3 ) an estimate of the material fracture toughness (appropriately degraded by radiation embrittlement and other environmental effects). From the first two, the applied stress intensity of linear elastic fracture mechanics (LEFM) is calculated; comparison to the fracture toughness data then determines the likelihood of crack propagation. The fracture evaluation is based on (1) probabilistic size and distribution of part-wall cracks, (2) probabilistic material fracture toughness properties, and (3) deterministic stress distributions based on a given cask/fuel design and the regulatory normal or accident loading condition.

Dynamic fracture toughness properties were not considered because no relevant data were found for irradiated Zircaloy cladding and because the strain rates under impact loading $\left(\sim 1\right.$ to $\left.10 \mathrm{~s}^{-1}\right)$ are well below the projectile-type impact loading rates where dynamic properties are crucial. This reduction in strain rate is caused by the cask impact limiters, which dramatically reduce the rate of loading applied to the fuel rods. Therefore, despite the fact that fracture toughness does decrease with increasing strain rate, the error as a result of overestimating the dynamic fracture toughness is small relative to the other uncertainties. 
The fracture toughness of a material is the critical value of the mode stress intensity factor that causes the material to fracture from stress at a sharp crack. Figure III-31 illustrates the three modes (I, II, III) of crack extension. All of the fracture failures discussed here are based on Mode I crack extension. Considerable toughness measurements on irradiated Zircaloy- 2 material have been obtained, including data from Hanford $\mathrm{N}$ reactor pressure tubes [MI85]. A statistical analysis of these data [BA87] gives a correlation for fracture toughness, $\mathrm{K}_{I C}$, as a function of neutron fluence, temperature, and flaw orientation as

$$
\ln K_{I C}=A-0.134 \sigma_{t}+4.14 \times 10^{-4} \mathrm{~T} \sigma_{t},
$$

where

$$
\begin{aligned}
\mathrm{K}_{I C} & =\text { fracture toughness in } \mathrm{MPa} \sqrt{\mathrm{m}} \\
\Phi_{\mathrm{t}} & =\text { the neutron fluence in } 10^{21} \mathrm{n} / \mathrm{cm}^{2}(>1 \mathrm{MeV}) \\
\mathrm{T} & =\text { the temperature in }{ }^{\circ} \mathrm{C} \\
\mathrm{A} & =3.84+9 \times 10^{-4}(\mathrm{~T}) \text { for axial cracks } \\
\mathrm{A} & =3.91+3.6 \times 10^{-4}(\mathrm{~T}) \text { for circumferential cracks. }
\end{aligned}
$$

This correlation has a standard deviation of $5.5 \mathrm{MPa} \sqrt{\mathrm{m}}$ for the data used. A conservative approach is to assume fuel with high burnup and long cooling times out of the reactor (i.e., lowest fracture toughness). Thus, if a temperature of $93.3^{\circ} \mathrm{C}$ and a fast fluence of $7 \times 10^{21} \mathrm{n} / \mathrm{cm}^{2}$ are assumed, Equation (III-13) gives a mean fracture toughness of $26.0 \mathrm{MPa} \sqrt{\mathrm{m}}$ for axial cracks and $26.56 \mathrm{MPa} \sqrt{\mathrm{m}}$ for circumferential cracks. If the standard deviation of $5.5 \mathrm{MPa} \sqrt{\mathrm{m}}$ for axial cracks is applied, a probability density function for this example fracture toughness can be developed as described in Figure III-32. This function is integrated to determine the probability of crack propagation for a calculated stress intensity factor. For example, for a calculated stress intensity value of $17.0 \mathrm{MPa} \sqrt{\mathrm{m}}$, the probability of of material having this or a lower value of fracture toughness is 58. Figure III-33 illustrates the effect of variations of temperature and fast fluence on the cladding fracture toughness.

The most uncertain parameter in the material fracture evaluation is the cracked condition of the cladding. Little information from either preservice or inservice inspection is available to reduce the uncertainty, and even the few data available are subject to uncertainty related to the inspection process (i.e., the probability of not detecting an existing crack). Appendix I provides details for two different part-wall crack distributions. To illustrate the development of a failure criterion, consider a distribution of the form

$$
\mathrm{p}(\mathrm{x})=\mathrm{Ae}-\mathrm{Bx},
$$

which represents the relative frequency of occurrence of a defect of a given size, $x$. Such a distribution is often used to correlate phenomena that have terminal characteristics such as crack depths and particle survival probabilities. The distribution is applied here because data on variations in part-wall crack size of fuel rod cladding are sparse. 

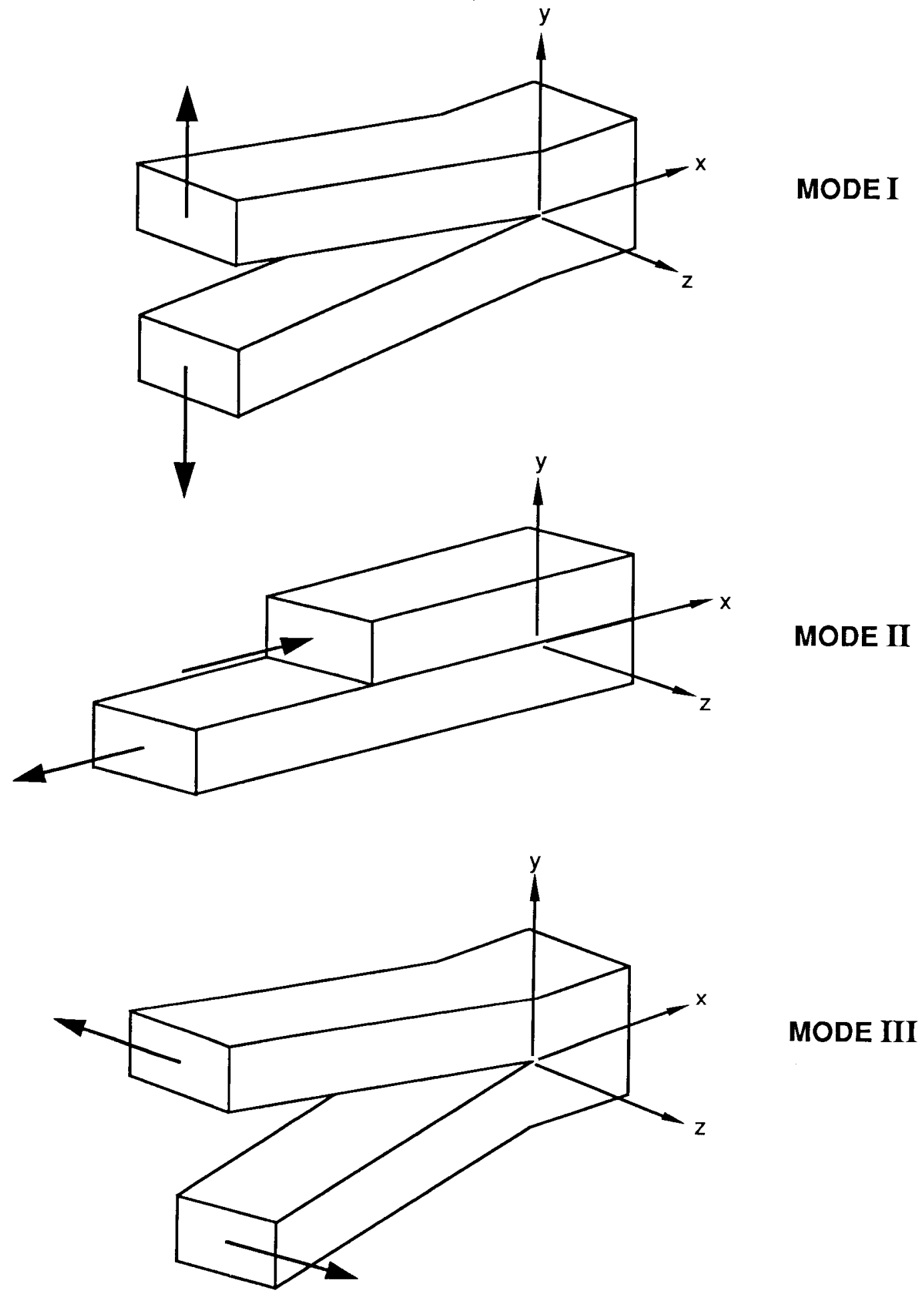

Figure III-31. The Three Basic Fracture Mechanics Modes of Crack Surface Displacements 


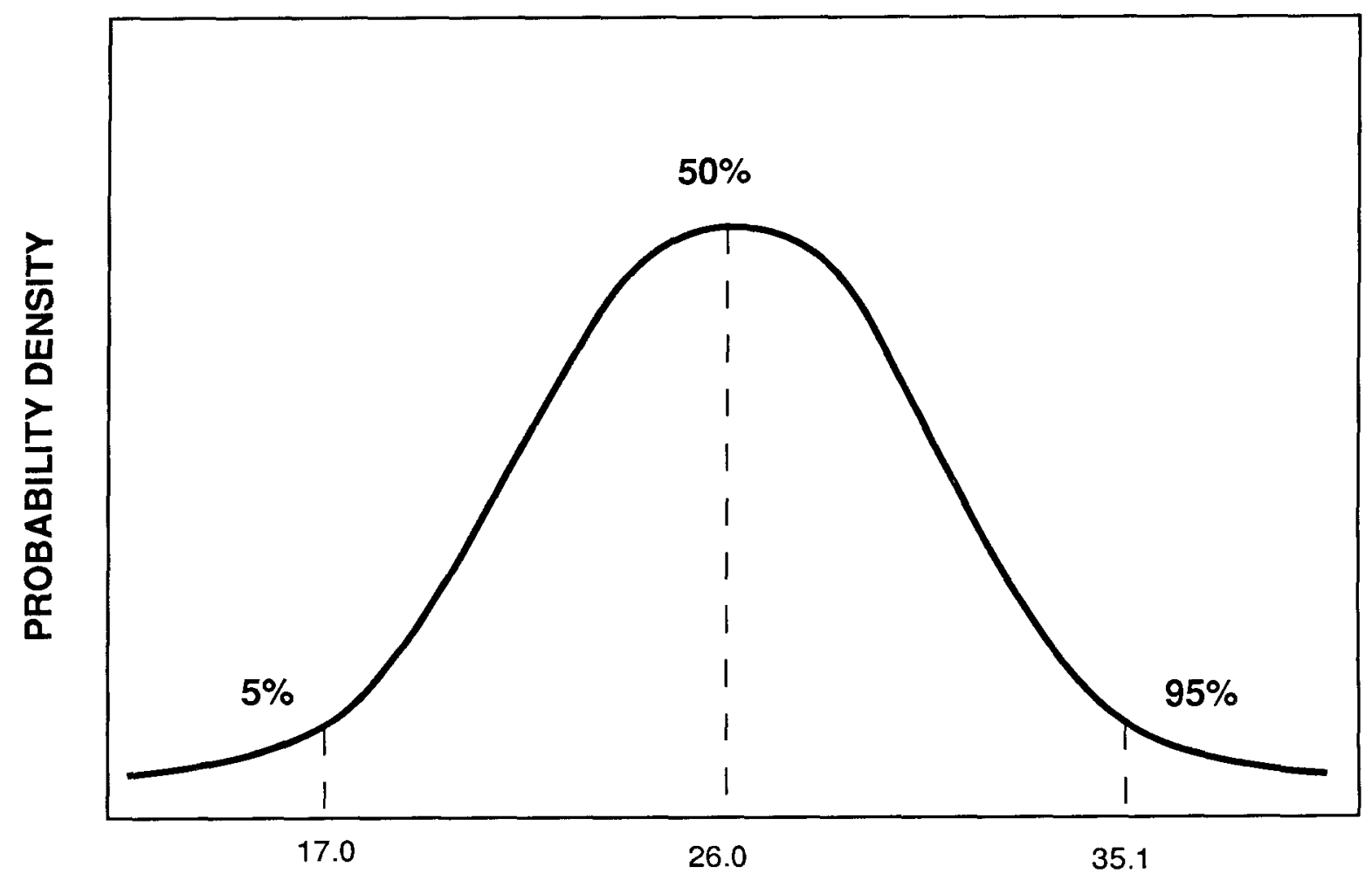

FRACTURE TOUGHNESS (MPa $\sqrt{\mathrm{m}}$ )

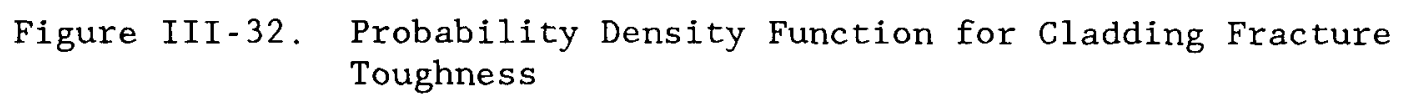

Given the type of distribution of crack sizes in the cladding of spent-fuel rods, parameters of crack sizes can be determined. The integral of Equation (III-14) must be unity (the possibility of having a crack of any size 1.0):

$$
\int_{0}^{\infty} A e^{-B x} d x=1.0, \text { therefore, } A=B
$$

To evaluate the single parameter $B$, one point on the distribution curve (i.e., a crack size and the frequency of its occurrence) can be determined.

In-reactor service records [AN79] indicate that approximately 1 in 10,000 rods fails as a result of PCI for $15 \times 15$ PWR assemblies and -1 in 1000 rods fails for $7 \times 7$ BWR assemblies. If the critical size for these PCI-induced cracks to cause propagation to rupture can be determined, then a calibration point for the crack distribution function can be deduced. To this end, supplemental analyses were carried out with the FREY computer 

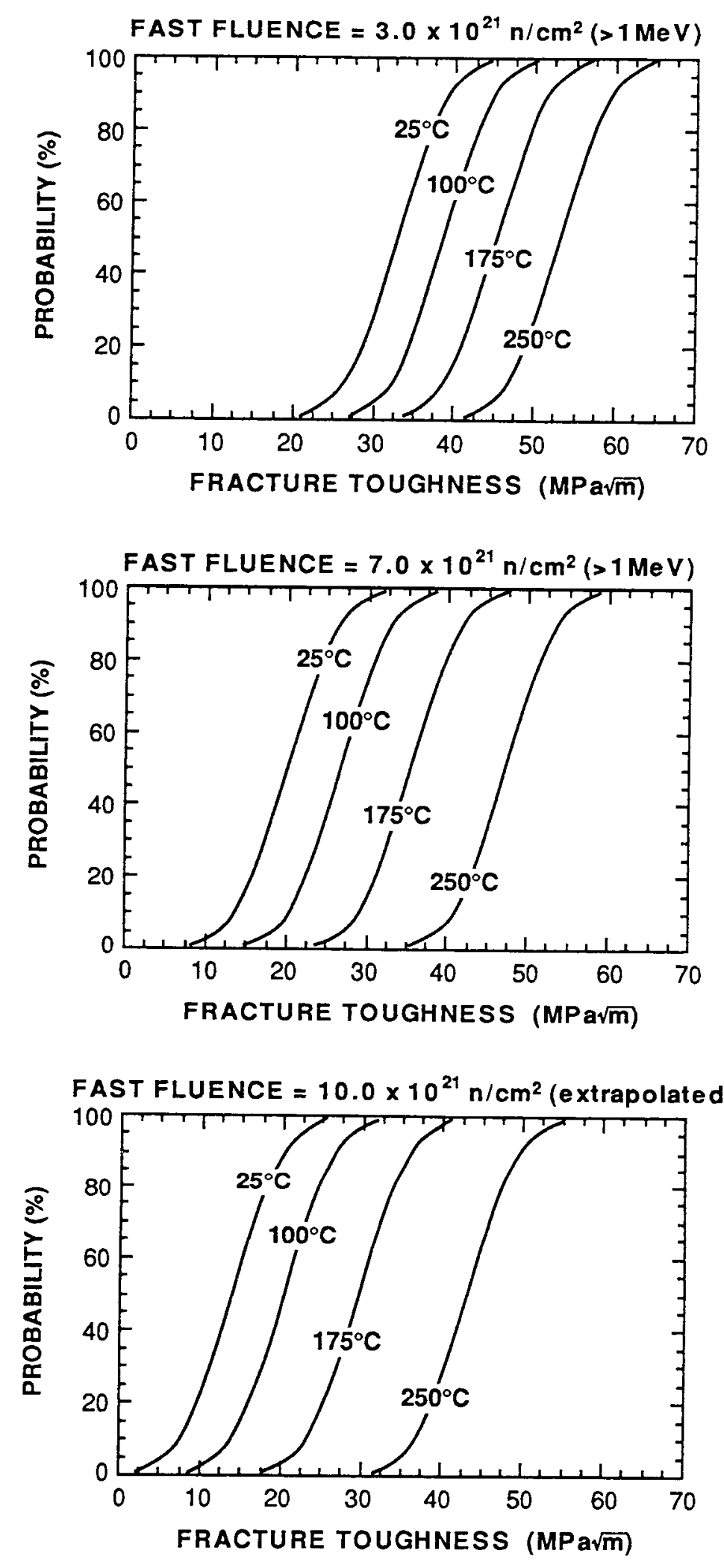

Figure III-33. Cumulative Probability Distributions for Cladding Fracture Toughness at Various Temperatures and Fast Fluences 
program [RA87] to find the critical crack depth of the cladding that would cause fuel fracture under operating conditions. These supplemental analyses and other corroborating independent analyses [MI84] are described in Appendix I. From these analyses, the critical crack depth for both BWR and PWR rods is 288 of the cladding thickness. Figure III-34 illustrates the distribution function for the example PWR and BWR assemblies.

A lower bound depth of $76 \mu \mathrm{m}$ for part-wall cracks is included in the failure criterion presented here because it is the smallest measurable crack depth and parametric analyses show that smaller cracks produce significantly smaller potential failure probabilities and therefore can be neglected. Parametric analyses using the FREY [RA87] computer program were used to quantify variations in stress intensity (based on rod-to-rod pinch load) versus depth of the part-wall cracks.

Part-wall cracks induced by PCI under reactor service conditions may propagate through the wall of the cladding under pinch loads caused by rodto-rod crushing during a free-drop horizontal impact event. These partwall cracks on the inside diameter of the cladding are propagated by tensile hoop stresses at the crack tip. Figure III-35 illustrates the loading mechanism causing propagation of a PCI part-wall crack. The cladding ovalizes under these pinch loads, resulting in tensile stresses on the inside surface and compressive stresses on the outside surface of the rod directly under the load. When the initial gap between the cladding and fuel pellet closes, a portion of the load is transferred directly across the pellet. The FREY [RA87] computer program was employed to model the part-wall crack and PCI and to calculate the stress intensity factor explicitly from the singular displacement field at the crack tip.

The results of these parametric analyses performed for example BWR and PWR assemblies are illustrated in Figure III-36. The minimum crack depth of $76 \mu \mathrm{m}$ is illustrated in Figure III-36, along with the critical crack depths for the two example assemblies. For part-wall cracks less than $76 \mu \mathrm{m}$, the magnitude of stress intensity drops dramatically; thus, it is not necessary to include these cracks in the crack size probability distribution because the probability of material fracture is small. Figure III-36 also illustrates that small fluctuations in the critical crack depth do not significantly increase the calculated stress intensity.

The integral of Equation (III-14) from size $x$ to infinity gives the probability that the crack depth will be $\mathrm{x}$ or larger:

$$
P(x)=\int_{x}^{\infty} B e^{-B x} d x
$$

If there are $\mathrm{N}$ cracks ( $76 \mu \mathrm{m}$ and greater) in a rod, the probability that at least one crack of size $s$ or larger exists is

$$
P_{S}=1-[1-P(s)]^{N},
$$




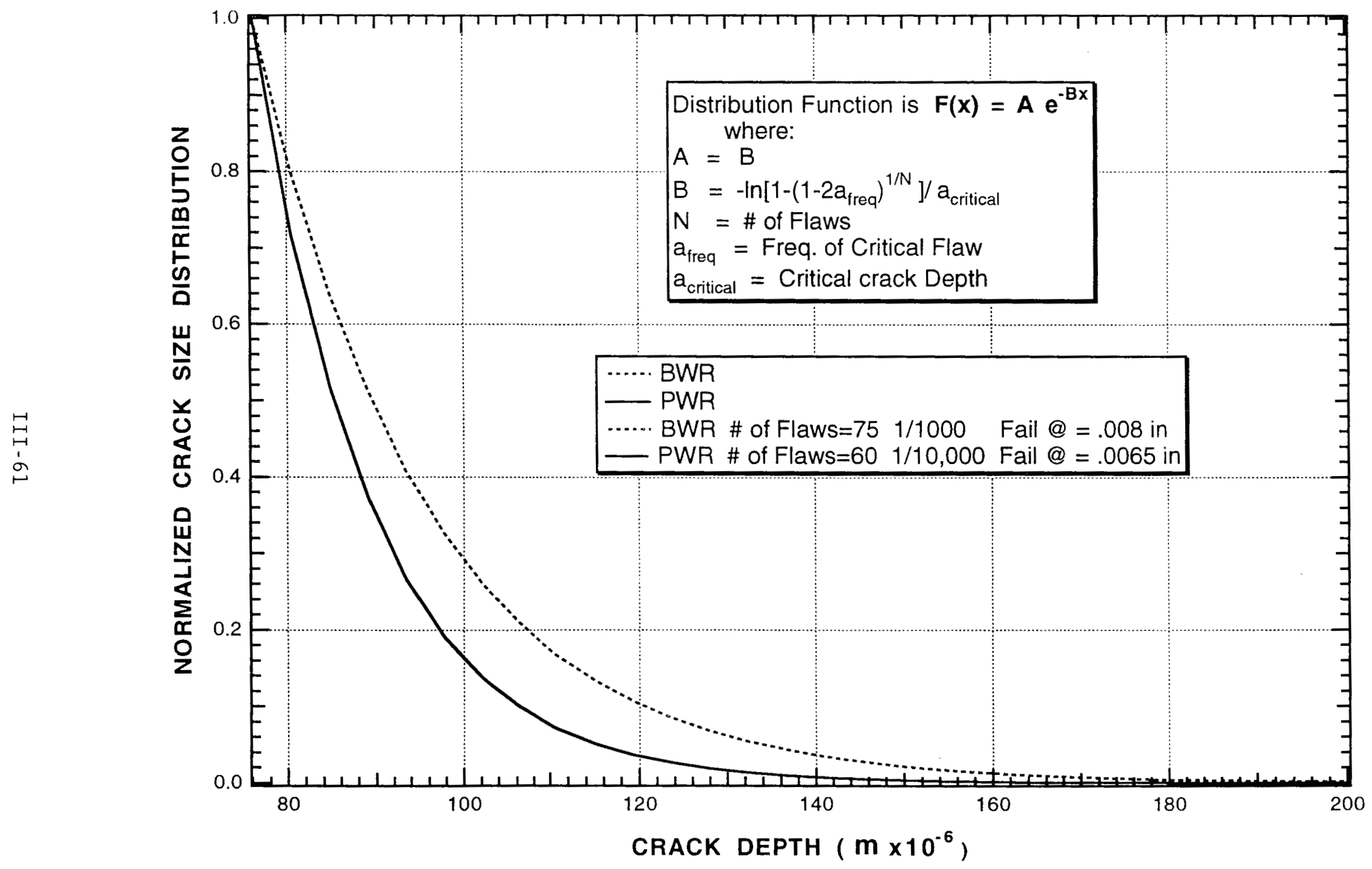

Figure III-34. Crack Size Distribution 


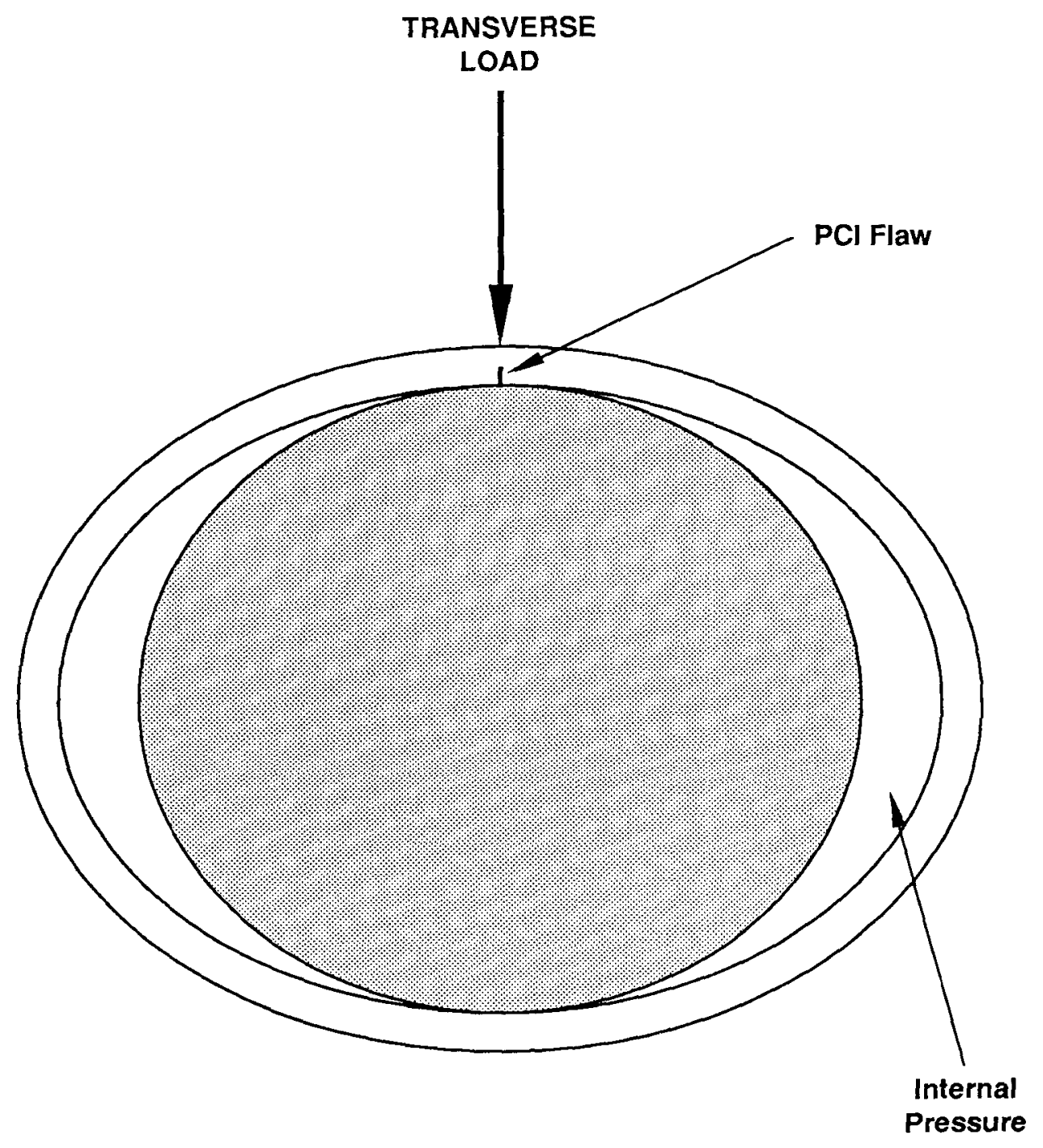

Figure III-35. PCI Flaw Under Pinch Load

where $P(s)$ is defined by Equation (III-16). The probability of a rod failure is the product of $P_{S}$ and $P_{k}$, where $P_{k}$ is the probability that the crack of size s will cause failure. The crack size distribution and number of cracks per rod can be related because the probability of rod failure and the critical crack size that causes failure is known. Also, as described Appendix I, the critical size that is determined corresponds to the $50 \%$ failure probability. For example, for BWRs,

$$
\mathrm{P}_{\mathrm{k}}\left(1-[1-\mathrm{P}(200 \mu \mathrm{m})]^{\mathrm{N}}\right\}=1 / 1000,
$$




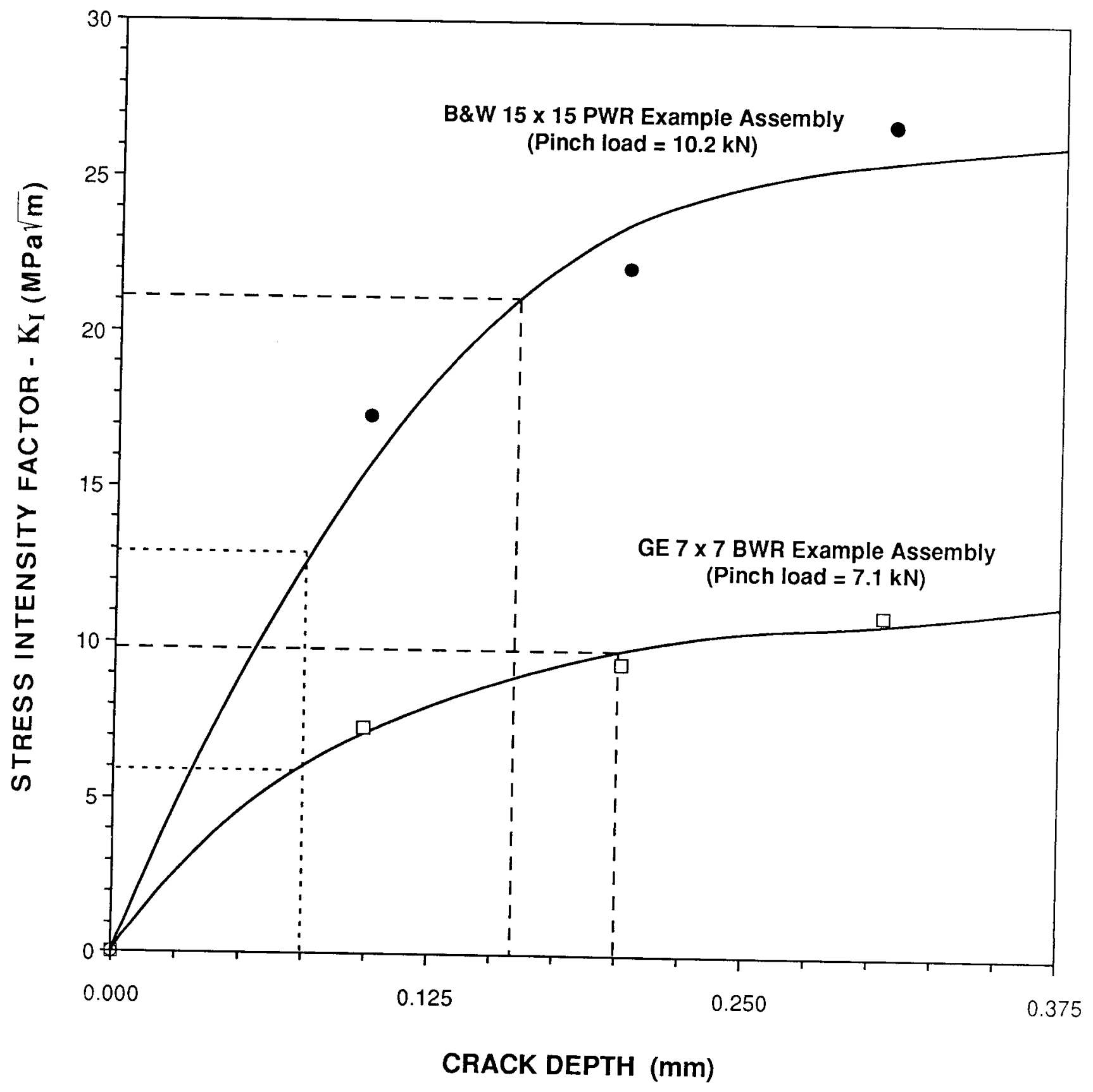

Figure III-36. Example Stress Intensity Values Versus Part-WalI Cracks Under Pinch Loads 
where

$$
\begin{aligned}
\mathrm{P}_{\mathrm{k}} & =0.5 \\
\mathrm{P}(200 \mu \mathrm{m}) & =\text { probability that the crack will be the critical size } \\
& \text { or larger } \\
\mathrm{N} & =\text { the number of } \mathrm{PCI} \text { - induced part-wall cracks per rod. }
\end{aligned}
$$

By establishing a value for $N$, the distribution function can be calibrated to known rod failure data and can be subsequently used to compute the probability of a PCI crack of any size in a rod. The crack distribution function is thus defined from the parameter $B$ as follows:

$$
\int_{A_{\text {c rit }}}^{\infty} B e^{-B x} d x=1-\left[1-2 A_{\text {freq }}\right]^{1 / N}
$$

or

$$
B=-\ln \left[1-\left(1-2 A_{\text {freq }}\right)^{1 / N}\right] / A_{c r i t},
$$

where

$$
\begin{aligned}
A_{\text {crit }}= & \text { the critical crack size ( } 28 \% \text { of the cladding } \\
& \text { thickness for BWR and PWR rods) } \\
A_{\text {freq }}= & \text { observed rod failure rate } \\
\mathrm{N}= & \text { total number of PCI part-wall cracks per rod. }
\end{aligned}
$$

The total number of PCI cracks per rod can be estimated by assuming a crack exists at each fuel pellet interface in the high-power section of the rod. This is estimated from fuel performance data showing axial

distributions of diametral changes. The current best estimate is 75 PCI cracks per rod for BWRs and 60 for PWRs (see Appendix I). Equations (III-16) and (III-17) are thus used to calculate the probability that a given crack size exists in the rod.

The stress intensity factor expressions that relate stress distribution, crack size flaws, and fracture toughness for all potential part-wall crack orientations can then be quantified. Cladding flaws can be either of the part-wall surface crack type or embedded flaws. Because most of the credible crack generation mechanisms are associated with surface phenomena and because surface cracks represent a more conservative condition, it is appropriate to consider only LEFM stress intensity expressions that apply to surface cracks in cylindrical tubes. In particular, axial and circumferential surface cracks on either the inside or outside of the cladding must be considered for Mode I crack extension. Figure III-37 illustrates the locations of postulated cracks in fuel rod cladding and the associated terminology. Mode I crack extension is illustrated in Figure III-31.

The following terms illustrated in Figure III-37 are used to describe the geometry of part-wall cracks: 


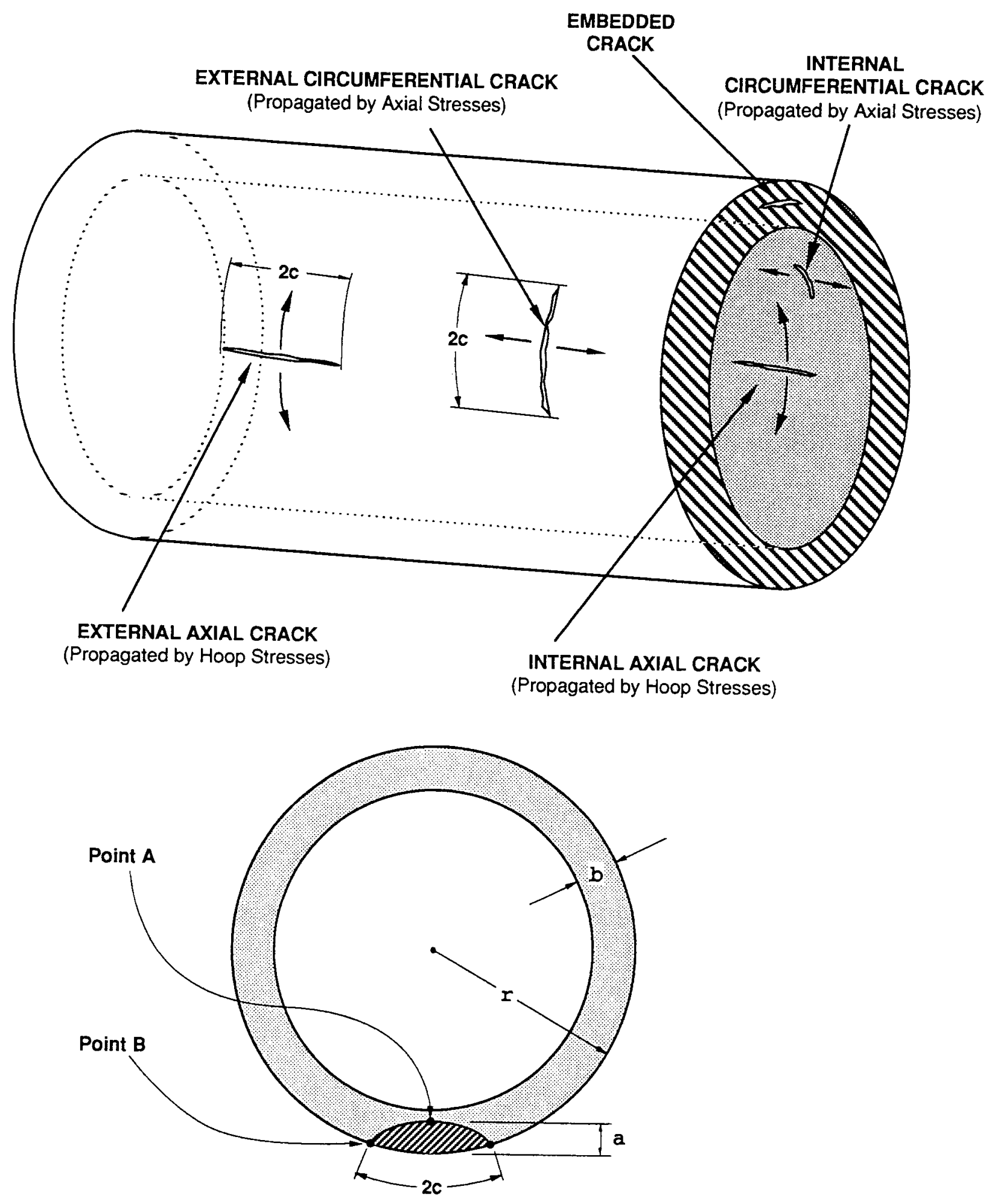

Figure III-37. Locations of Potential Part-Wall Cracks 


$$
\begin{aligned}
& a=\text { crack depth } \\
& b=\text { cladding thickness } \\
& c=\text { half crack length } \\
& r=\text { cladding external radius }
\end{aligned}
$$

Most of the LEFM surface flaw stress intensity expressions for tubes have been derived as the result of concerns about brittle fracture of pressurized vessels or piping, with a corresponding emphasis on internal surface cracks. The most widely used of these expressions are general expressions for both axial and circumferential internal surface cracks in cylinders developed by Labbens [LA76] and Buchalet and Bamford [BU76]. The stresses in an uncracked cylinder wall are identified and fitted to a polynomial of the form

$$
\sigma(\mathrm{x})=\mathrm{A}_{0}+\mathrm{A}_{1} \mathrm{x}+\mathrm{A}_{2} \mathrm{x}^{2}+\mathrm{A}_{3} \mathrm{x}^{3}, \quad 0 \leq \mathrm{x} \leq \mathrm{b}
$$

where

$$
\begin{aligned}
\sigma(\mathrm{x})= & \text { circumferential stress (for axial cracks) or axial } \\
& \text { stress (for circumferential cracks) } \\
\mathrm{b}= & \text { wall thickness of the cylinder } \\
\mathrm{A}_{0}, \mathrm{~A}_{1}, \mathrm{~A}_{2}, \mathrm{~A}_{3}= & \text { coefficients of membrane, bending (etc.) stress. }
\end{aligned}
$$

The stress intensity expression is given by

$$
\mathrm{K}_{\mathrm{I}}=\sqrt{\pi \mathrm{a}}\left[\mathrm{A}_{0} \mathrm{~F}_{1}+\frac{2 \mathrm{a}}{\pi} \mathrm{A}_{1} \mathrm{~F}_{2}+\frac{\mathrm{a}^{2}}{2} \mathrm{~A}_{2} \mathrm{~F}_{3}+\frac{4 \mathrm{a}^{3}}{3 \pi} \mathrm{A}_{3} \mathrm{~F}_{4}\right]
$$

where

$$
\begin{aligned}
F_{1}, F_{2}, F_{3}, F_{4}= & \text { referred to as magnification factors that are } \\
& \text { tabulated in graphical form from extensive } \\
& \text { finite element calculations (see Figure III-38). }
\end{aligned}
$$

The magnification factors are functions of the dimensionless crack depth $a / b$ and are presented as a family of curves for different ratios of cylinder thickness-to-radius $(b / r)$. For example, $F_{1}$ for a circumferential crack depth $a / b=0.3$ is -1.3 for $b / r=0.1$, whereas $F_{1}$ is -1.5 for an axial crack under the same conditions.

These stress intensity solutions were derived for infinitely long cracks; that is, the flaw aspect ratio (a/c) tends to zero. More recently, expressions for semielliptical surface flaws have been developed, with $a / c=1 / 3$. Heliot et al. [HE79] and McGowan and Raymund [MG79] concentrated on axial semielliptical flaws on the internal surface of a cylinder. Raju and Newman [RA82] developed expressions for semielliptical flaws on the interior or exterior surface of a cylinder. Again, the circumferential stress field for the uncracked geometry is fitted with expression

$$
\sigma(x)=A_{0}+A_{1} x+A_{2} x^{2}+A_{3} x^{3}, 0 \leq x \leq b,
$$




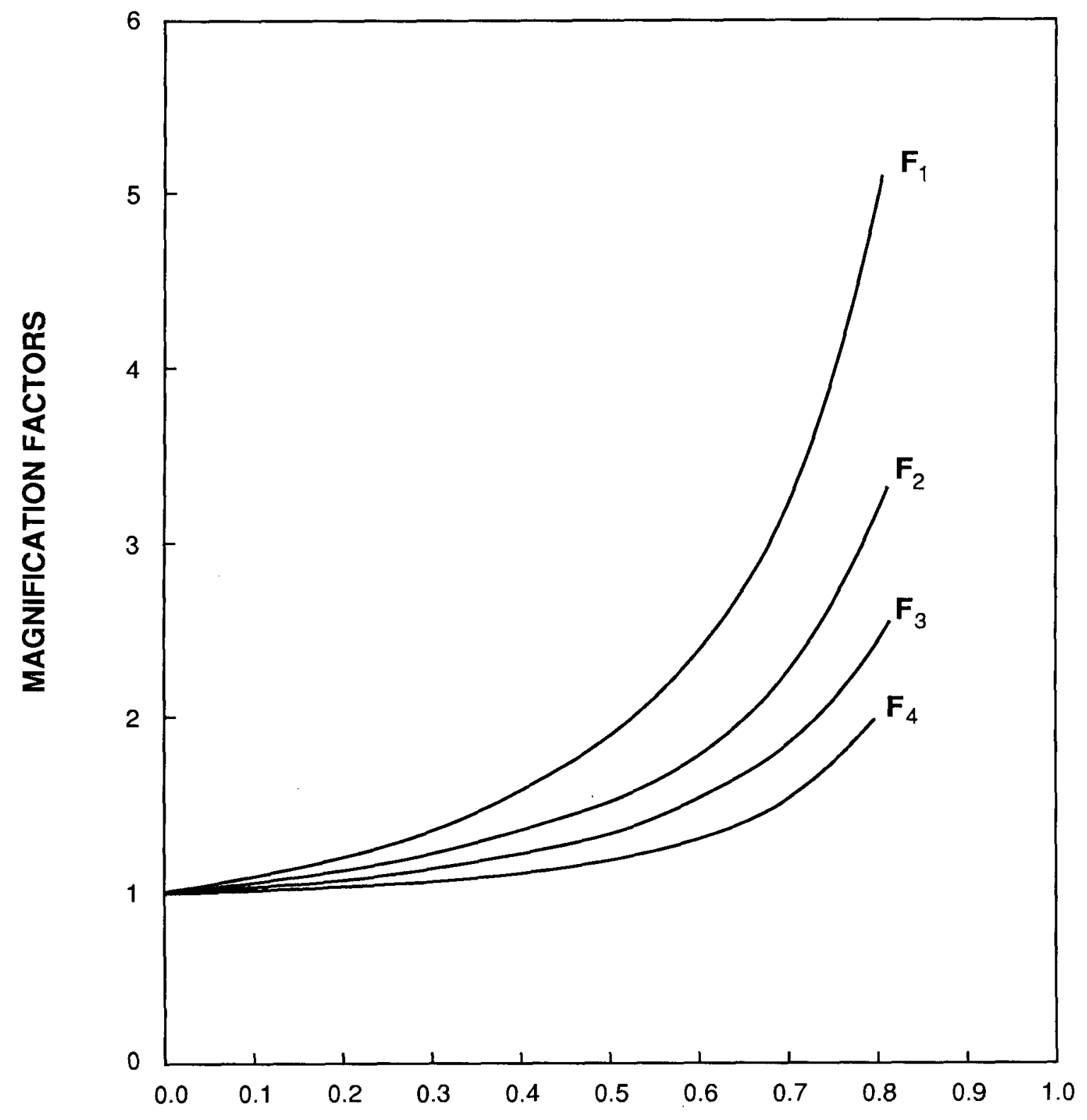

FRACTIONAL DISTANCE THROUGH WALL $(a / b)$

Figure III-38. Stress Intensity Magnification Factors for Axial Crack in a Cylinder $(b / r=0.1)$ 
and the applied stress intensity is described by

$$
K_{I}=\sqrt{\frac{\pi a}{Q}}\left[G_{0} A_{0}+G_{1} A_{1} a+G_{2} A_{2} a^{2}+G_{3} A_{3} a^{3}\right] .
$$

The parameter $Q$ is the square of the complete elliptic integral of the second type and is approximately equal to

$$
\mathrm{Q}=1+1.464(\mathrm{a} / \mathrm{c})^{1.65}
$$

For a crack with $a / c=1 / 3, Q$ becomes 1.20 . The magnification factors $G_{0}$, $G_{1}, G_{2}$, and $G_{3}$ are obtained from tabular values (Table III-2) [RA82].

No such complete treatment is available for internal circumferential cracks in that the stress intensities for such flaws are not governing for most stress states of interest. External circumferential surface flaws are of interest, however, especially for bending loads. Raju and Newman [RA86] have recently developed a general expression for the applied stress intensity

$$
\mathrm{K}_{\mathrm{I}}=\mathrm{S}_{\mathrm{i}} \sqrt{\frac{\pi \mathrm{a}}{\mathrm{Q}}} \mathrm{F}
$$

where the subscript $i$ is either $t$ for tension loads or $b$ for bending loads. $Q$ is given by Equation (III-23), and the shape factor $F$ is tabulated as a function of $\mathrm{r} / \mathrm{b}, \mathrm{a} / \mathrm{b}$, and $\mathrm{a} / \mathrm{c}$, with different tables for tension and bending loads (Table III-3). Values are given for the intersection of the crack with the free surface (point $B$ ) and for the point of maximum crack penetration (point A) (Figure III-37). For example, for $a / b=0.2$, $\mathrm{r} / \mathrm{b}=10$, and $\mathrm{a} / \mathrm{c}=0.6, \mathrm{~F}=1.071$ at point $\mathrm{A}$ and $\mathrm{F}=0.913$ at point $\mathrm{B}$.

In summary, for a given stress state in a rod, the appropriate stress intensity expression can be evaluated for a distribution of part-wall crack sizes. The probability of this crack size existing can be determined from Equations (III-16) and (III-17). Fracture probability for the crack can be calculated from the stress intensity factor and fracture toughness distribution. Probability of the crack existing at the location of the peak stress can be determined from geometric considerations. The product of these three conditional probabilities is then the probability that failure occurs. The probability or frequency of failure for all cask rods can be determined by considering the various rod zones (tie plate connection, spacer grid location, and the span between spacer grids) and the rod locations (the bottom rod of an assembly experiences more load than the top rod in a side drop).

For normal transport conditions in which random shock and vibration loads are induced as well as the regulatory $0.3-\mathrm{m}$ free-drop handling condition, the material fracture criterion is based on the fatigue crack propagation equations of linear elastic fracture mechanics. The fatigue crack propagation equation for steady crack growth is

$$
\mathrm{da} / \mathrm{dN}=\mathrm{A}(\Delta \mathrm{K})^{\mathrm{n}},
$$


Table III -2

Influence Coefficients, Gj, for Semielliptical Surface Cracks

Inside a Cylinder $(b / R=0.1)$

\begin{tabular}{|c|c|c|c|c|c|c|c|c|c|c|}
\hline \multirow[b]{2}{*}{$\begin{array}{l}\text { Type of } \\
\text { Loading }\end{array}$} & \multirow[b]{2}{*}{$\frac{2 \phi}{\pi}$} & \multicolumn{3}{|c|}{$a / c=0.2$} & \multicolumn{3}{|c|}{$\mathrm{a} / \mathrm{c}=0.4$} & \multicolumn{3}{|c|}{$\mathrm{a} / \mathrm{c}=1.0$} \\
\hline & & $\frac{\mathrm{a}}{\mathrm{b}}=0.2$ & $\frac{a}{b}=0.5$ & $\frac{a}{b}=0.8$ & $\frac{a}{b}=0.2$ & $\frac{a}{b}=0.5$ & $\frac{\mathrm{a}}{\mathrm{b}}=0.8$ & $\frac{\mathrm{a}}{\mathrm{b}}=0.2$ & $\frac{a}{b}=0.5$ & $\underline{a}=0.8$ \\
\hline \multirow{5}{*}{$\begin{array}{l}\text { Uniform } \\
\left(\mathrm{G}_{0}\right)\end{array}$} & 0 & 0.607 & 0.791 & 1.179 & 0.777 & 0.936 & 1.219 & 1.140 & 1.219 & 1.348 \\
\hline & 0.25 & 0.740 & 0.932 & 1.284 & 0.810 & 0.948 & 1.164 & 1.068 & 1.126 & 1.200 \\
\hline & 0.5 & 0.945 & 1.188 & 1.568 & 0.940 & 1.076 & 1.243 & 1.033 & 1.074 & 1.091 \\
\hline & 0.75 & 1.073 & 1.366 & 1.798 & 1.038 & 1.180 & 1.357 & 1.019 & 1.055 & 1.090 \\
\hline & 1.0 & 1.115 & 1.427 & 1.872 & 1.072 & 1.217 & 1.393 & 1.015 & 1.050 & 1.090 \\
\hline \multirow{5}{*}{$\begin{array}{l}\text { Linear } \\
\left(\mathrm{G}_{1}\right)\end{array}$} & 0 & 0.079 & 0.138 & 0.253 & 0.125 & 0.176 & 0.259 & 0.197 & 0.221 & 0.255 \\
\hline & 0.25 & 0.206 & 0.268 & 0.374 & 0.246 & 0.291 & 0.356 & 0.359 & 0.377 & 0.397 \\
\hline & 0.5 & 0.422 & 0.503 & 0.619 & 0.442 & 0.487 & 0.538 & 0.541 & 0.554 & 0.555 \\
\hline & 0.75 & 0.603 & 0.705 & 0.859 & 0.608 & 0.657 & 0.727 & 0.669 & 0.683 & 0.703 \\
\hline & 1.0 & 0.673 & 0.783 & 0.960 & 0.672 & 0.723 & 0.806 & 0.715 & 0.729 & 0.760 \\
\hline \multirow{5}{*}{$\begin{array}{l}\text { Quadratic } \\
\left(G_{2}\right)\end{array}$} & 0 & 0.023 & 0.052 & 0.104 & 0.043 & 0.069 & 0.106 & 0.074 & 0.085 & 0.099 \\
\hline & 0.25 & 0.075 & 0.105 & 0.154 & 0.097 & 0.119 & 0.149 & 0.153 & 0.162 & 0.170 \\
\hline & 0.5 & 0.237 & 0.277 & 0.331 & 0.256 & 0.279 & 0.302 & 0.333 & 0.339 & 0.337 \\
\hline & 0.75 & 0.429 & 0.480 & 0.560 & 0.441 & 0.466 & 0.505 & 0.514 & 0.520 & 0.533 \\
\hline & 1.0 & 0.514 & 0.571 & 0.671 & 0.523 & 0.549 & 0.601 & 0.588 & 0.596 & 0.618 \\
\hline \multirow{5}{*}{$\begin{array}{l}\text { Cubic } \\
\left(G_{3}\right)\end{array}$} & 0 & 0.010 & 0.027 & 0.056 & 0.021 & 0.036 & 0.056 & 0.038 & 0.044 & 0.051 \\
\hline & 0.25 & 0.032 & 0.049 & 0.077 & 0.044 & 0.058 & 0.074 & 0.075 & 0.080 & 0.085 \\
\hline & 0.5 & 0.146 & 0.169 & 0.199 & 0.161 & 0.174 & 0.187 & 0.218 & 0.222 & 0.219 \\
\hline & 0.75 & 0.332 & 0.363 & 0.412 & 0.346 & 0.360 & 0.385 & 0.417 & 0.420 & 0.424 \\
\hline & 1.0 & 0.438 & 0.462 & 0.529 & 0.441 & 0.456 & 0.493 & 0.512 & 0.515 & 0.532 \\
\hline
\end{tabular}


Table III-2

Influence Coefficients, $\mathrm{Gj}$, for Semielliptical Surface Cracks (Continued) Inside a Cylinder $(b / R=0.25)$

\begin{tabular}{|c|c|c|c|c|c|c|c|c|c|c|}
\hline \multirow[b]{2}{*}{$\begin{array}{l}\text { Type of } \\
\text { Loading }\end{array}$} & \multirow[b]{2}{*}{$\frac{2 \phi}{\pi}$} & \multicolumn{3}{|c|}{$\mathrm{a} / \mathrm{c}=0.2$} & \multicolumn{3}{|c|}{$a / c=0.4$} & \multicolumn{3}{|c|}{$a / c=1.0$} \\
\hline & & $\underline{\mathrm{a}}=0.2$ & $\frac{\mathrm{a}}{\mathrm{b}}=0.5$ & $\frac{\mathrm{a}}{\mathrm{b}}=0.8$ & $\frac{\mathrm{a}}{\mathrm{b}}=0.2$ & $\frac{a}{b}=0.5$ & $\frac{\mathrm{a}}{\mathrm{b}}=0.8$ & $\frac{\mathrm{a}}{\mathrm{b}}=0.2$ & $\frac{a}{\mathrm{~b}}=0.5$ & $\frac{\mathrm{a}}{\mathrm{b}}=0.8$ \\
\hline $\begin{array}{l}\text { Uniform } \\
\left(\mathrm{G}_{0}\right)\end{array}$ & $\begin{array}{l}0 \\
0.25 \\
0.5 \\
0.75 \\
1.0\end{array}$ & $\begin{array}{l}0.606 \\
0.736 \\
0.935 \\
1.057 \\
1.097\end{array}$ & $\begin{array}{l}0.797 \\
0.925 \\
1.170 \\
1.343 \\
1.405\end{array}$ & $\begin{array}{l}1.201 \\
1.270 \\
1.549 \\
1.838 \\
1.959\end{array}$ & $\begin{array}{l}0.770 \\
0.801 \\
0.928 \\
1.024 \\
1.057\end{array}$ & $\begin{array}{l}0.924 \\
0.932 \\
1.056 \\
1.157 \\
1.193\end{array}$ & $\begin{array}{l}1.219 \\
1.154 \\
1.241 \\
1.385 \\
1.443\end{array}$ & $\begin{array}{l}1.128 \\
1.058 \\
1.025 \\
1.013 \\
1.009\end{array}$ & $\begin{array}{l}1.191 \\
1.105 \\
1.060 \\
1.045 \\
1.041\end{array}$ & $\begin{array}{l}1.316 \\
1.180 \\
1.088 \\
1.099 \\
1.105\end{array}$ \\
\hline $\begin{array}{l}\text { Linear } \\
\left(\mathrm{G}_{1}\right)\end{array}$ & $\begin{array}{l}0 \\
0.25 \\
0.5 \\
0.75 \\
1.0\end{array}$ & $\begin{array}{l}0.079 \\
0.205 \\
0.419 \\
0.598 \\
0.666\end{array}$ & $\begin{array}{l}0.141 \\
0.268 \\
0.498 \\
0.698 \\
0.776\end{array}$ & $\begin{array}{l}0.262 \\
0.372 \\
0.615 \\
0.876 \\
0.996\end{array}$ & $\begin{array}{l}0.123 \\
0.243 \\
0.438 \\
0.603 \\
0.666\end{array}$ & $\begin{array}{l}0.174 \\
0.287 \\
0.481 \\
0.650 \\
0.715\end{array}$ & $\begin{array}{l}0.263 \\
0.356 \\
0.540 \\
0.740 \\
0.828\end{array}$ & $\begin{array}{l}0.194 \\
0.356 \\
0.538 \\
0.667 \\
0.713\end{array}$ & $\begin{array}{l}0.214 \\
0.371 \\
0.550 \\
0.680 \\
0.726\end{array}$ & $\begin{array}{l}0.248 \\
0.393 \\
0.556 \\
0.708 \\
0.768\end{array}$ \\
\hline $\begin{array}{l}\text { Quadratic } \\
\left(\mathrm{G}_{2}\right)\end{array}$ & $\begin{array}{l}0 \\
0.25 \\
0.5 \\
0.75 \\
1.0\end{array}$ & $\begin{array}{l}0.023 \\
0.075 \\
0.236 \\
0.426 \\
0.511\end{array}$ & $\begin{array}{l}0.054 \\
0.106 \\
0.275 \\
0.477 \\
0.567\end{array}$ & $\begin{array}{l}0.108 \\
0.154 \\
0.330 \\
0.571 \\
0.692\end{array}$ & $\begin{array}{l}0.042 \\
0.096 \\
0.254 \\
0.439 \\
0.520\end{array}$ & $\begin{array}{l}0.068 \\
0.118 \\
0.276 \\
0.462 \\
0.545\end{array}$ & $\begin{array}{l}0.109 \\
0.150 \\
0.304 \\
0.513 \\
0.614\end{array}$ & $\begin{array}{l}0.072 \\
0.152 \\
0.332 \\
0.512 \\
0.588\end{array}$ & $\begin{array}{l}0.082 \\
0.159 \\
0.338 \\
0.519 \\
0.594\end{array}$ & $\begin{array}{l}0.097 \\
0.169 \\
0.339 \\
0.537 \\
0.623\end{array}$ \\
\hline $\begin{array}{l}\text { Cubic } \\
\left(G_{3}\right)\end{array}$ & $\begin{array}{l}\quad 0 \\
0.25 \\
0.5 \\
0.75 \\
1.0\end{array}$ & $\begin{array}{l}0.010 \\
0.032 \\
0.145 \\
0.330 \\
0.426\end{array}$ & $\begin{array}{l}0.028 \\
0.050 \\
0.168 \\
0.361 \\
0.460\end{array}$ & $\begin{array}{l}0.059 \\
0.077 \\
0.199 \\
0.419 \\
0.542\end{array}$ & $\begin{array}{l}0.021 \\
0.044 \\
0.160 \\
0.345 \\
0.439\end{array}$ & $\begin{array}{l}0.036 \\
0.057 \\
0.173 \\
0.358 \\
0.454\end{array}$ & $\begin{array}{l}0.059 \\
0.075 \\
0.188 \\
0.391 \\
0.509\end{array}$ & $\begin{array}{l}0.037 \\
0.075 \\
0.217 \\
0.416 \\
0.511\end{array}$ & $\begin{array}{l}0.043 \\
0.079 \\
0.221 \\
0.419 \\
0.515\end{array}$ & $\begin{array}{l}0.050 \\
0.085 \\
0.220 \\
0.431 \\
0.536\end{array}$ \\
\hline
\end{tabular}


Table III-2

Influence Coefficients, Gj, for Semielliptical Surface Cracks (Continued) Outside a Cylinder $(b / R=0.1)$

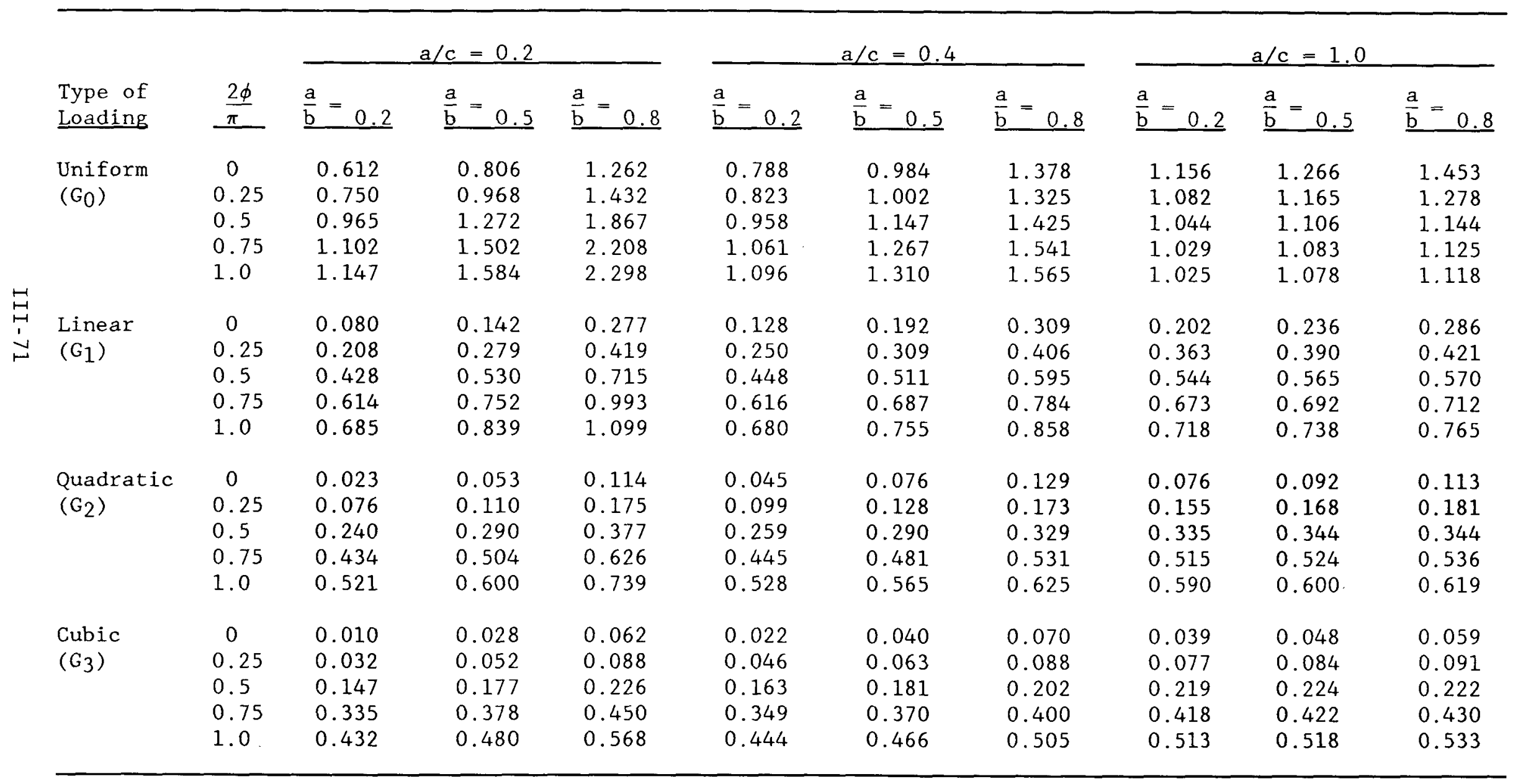


Table III-2

Influence Coefficients, Gj, for Semielliptical Surface Cracks (Concluded) Outside a Cylinder $(b / R=0.25)$

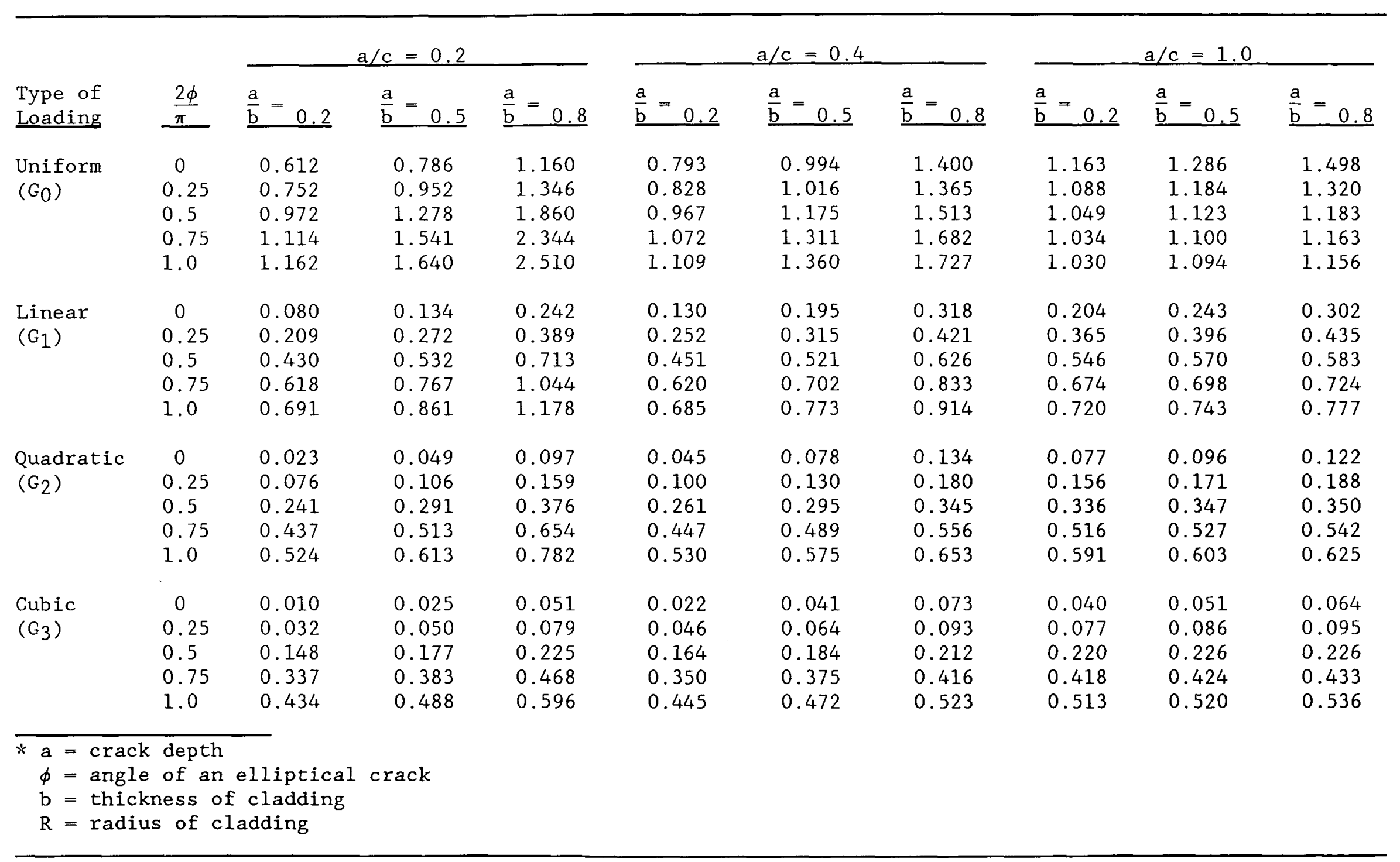


Normalized Stress-Intensity Factors for Surface Cracks in Pipes Under Tension and Bending Loads

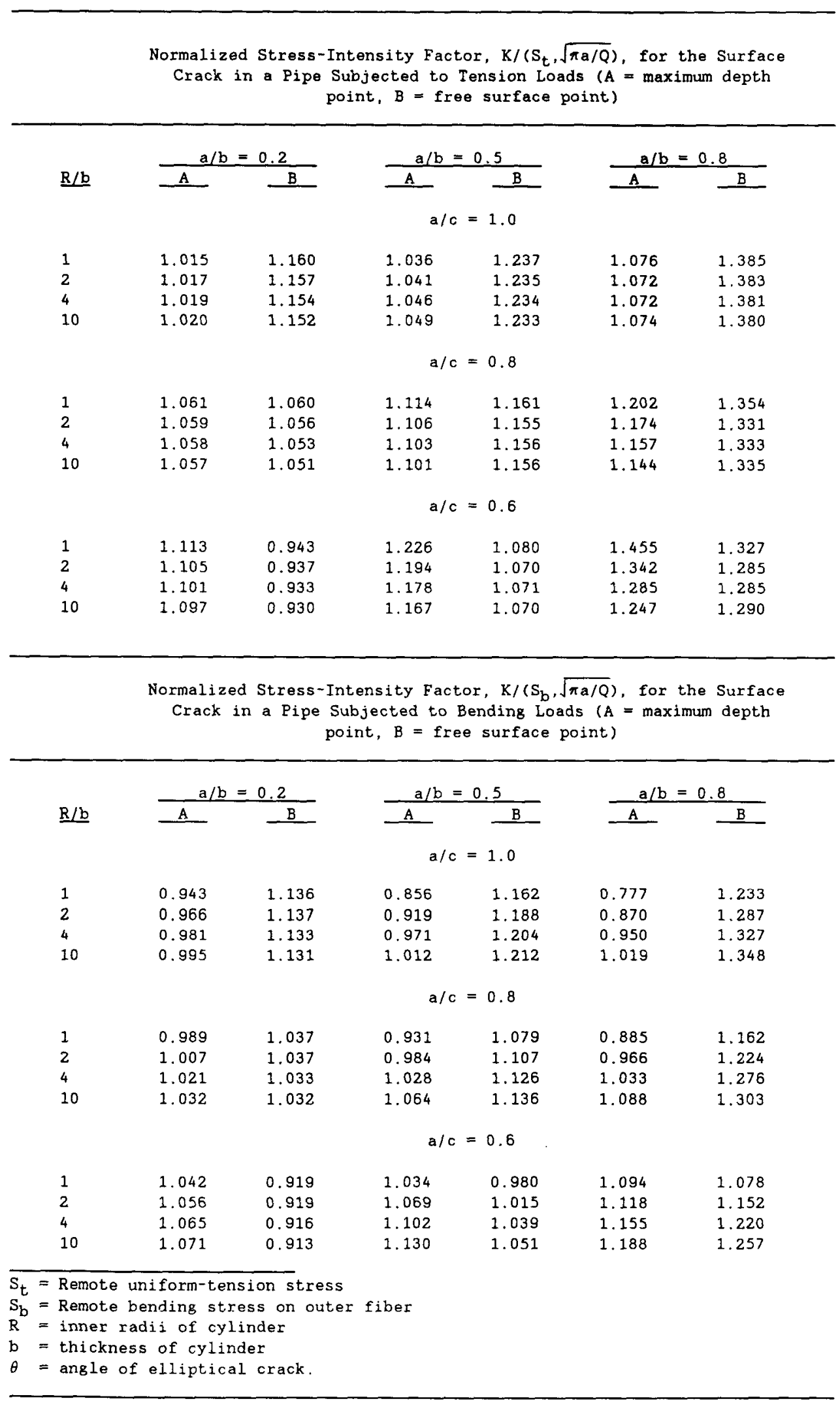


where

$$
\begin{aligned}
a & =\text { crack length } \\
\mathrm{N} & =\text { number of cycles } \\
\Delta \mathrm{K} & =\text { the stress intensity factor fluctuation } \\
\mathrm{A}, \mathrm{n} & =\text { material constants. }
\end{aligned}
$$

Metals such as Zircaloy exhibit a region of behavior in which cracks will not propagate under even an infinite number of cycles at some level of cyclic load [HA80]. This behavior is characterized by a material constant, $\Delta K_{t h}$, called the fatigue threshold for the cyclic stress-intensity factor fluctuation. For Zircaloy, the value of $\Delta \mathrm{K}_{\mathrm{th}}$ is $9.51 \mathrm{MPa} \sqrt{\mathrm{m}}$ [HA80]. Thus, for cyclic stresses that produce a $\Delta K$ on a given crack size and orientation less than this value, no crack growth occurs even after infinite cycles.

\section{III.4.3 Scoping Calculations}

Material fracture scoping calculations were performed to evaluate the relative importance of the fracture modes identified in the previous sections. Potential fracture scenarios involve (1) external circumferential cracks under bending, (2) external longitudinal cracks under pinch loads, and (3) internal longitudinal cracks under pinch loads. Internal circumferential cracks are not considered because no mechanism is available to create these cracks. External circumferential cracks can result from manufacturing defects or a through-wall tear initiated by material rupture. Figure III-37 illustrates the location of these cracks. The following equation governs this scenario:

$$
\mathrm{K}=\sigma_{\mathrm{b}} \mathrm{F} \sqrt{\frac{\pi \mathrm{a}}{\mathrm{Q}}}
$$

For manufacturing defects, the crack depth is small compared to the wall thickness; in this case, $Q \simeq 2.464$ and $F \simeq 1.113$. If it is assumed that $\sigma_{b}$ is equal to the yield stress of $483 \mathrm{MPa}$ and $\mathrm{K}$ is the 5 th percentile fracture toughness at $17.0 \mathrm{MPa} \sqrt{\mathrm{m}}$, the critical crack size for fracture is $0.076 \mathrm{~cm}$. Because this is equal to typical cladding wall thicknesses, the probability of failure as a result of stress at manufacturing defects is so small that it can be neglected.

For circumferential fracture created by material rupture, the crack depth is equal to the wall thickness and $F \simeq 1.38$. If $a=0.076 \mathrm{~cm}$ and $\sigma_{\mathrm{b}}=$ yield stress is assumed,

$$
\mathrm{K}=1.38 \sigma_{\mathrm{b}} \sqrt{\frac{\pi \mathrm{a}}{\mathrm{Q}}}=20.8 \mathrm{MPa} \sqrt{\mathrm{m}} .
$$

This implies that if crack initiation occurs as a result of excessive strain, the conditional failure probability for the breakage failure mode must be evaluated. 
External longitudinal cracks under tension are considered because of possible handling scratches at spacer grid locations. The pinch load from spacer crushing can put the clad outer surface in tension 90 degrees from the transverse load. If a linear stress state with the outer fiber near yield is assumed, then

$$
\sigma(\mathrm{x})=483-965 \mathrm{x} / \mathrm{b},
$$

and Equation (III-22) becomes

$$
\mathrm{K}=\sqrt{\frac{\pi \mathrm{a}}{\mathrm{Q}}}\left(483 \mathrm{G}_{0}-965 \mathrm{G}_{1} \mathrm{a} / \mathrm{b}\right) .
$$

For a scratch, $a / b$ and $a / c$ are small so that $Q \simeq 1.1, G_{0} \simeq 1.15$, and $G_{1} \simeq$ 0.70 . Substituting for the 5 th percentile fracture toughness, the critical crack size is approximately the wall thickness. A handling accident that imposes damage of this size cannot be considered a scratch, and this fracture scenario need not be considered further.

A PCI-induced crack is a longitudinal incipient crack on the inside surface of the cladding. When this crack occurs directly under a pinch load, the inside surface of the cladding is in tension. Assuming a linear distribution of stress and applying Equation (III-10) yields

$$
\mathrm{K}_{\mathrm{I}}=\sqrt{\pi \mathrm{a}}\left[483 \mathrm{~F}_{1}-965 \mathrm{~F}_{2} 2 \mathrm{a} / \pi\right] \text {, }
$$

where $F_{1} \simeq 1.5$ and $F_{2} \simeq 1.2$ for $a / b \simeq 0.25$. If a $0.020-\mathrm{cm}$ deep partial crack is assumed, $K_{I} \simeq 18.2 \mathrm{MPa} \sqrt{\mathrm{m}}$. PCI-induced cracks are known to be present, and the above scoping calculations indicate relatively high-stress intensity factors. Because the fuel pellets will carry some portion of the pinch load, this evaluation is best done with the FREY program [RA87], which models fuel-clad interaction and can directly calculate the stress intensity factor. The relationship between crack depth and stress intensity has been quantified for two example assemblies, as illustrated in Figure III-36.

For normal transport shock and vibration loading conditions, fuel rod failure as a result of fatigue must be evaluated. Calculations in Section III.5.8 for an example PWR rod show a maximum tensile stress of $155 \mathrm{MPa}$ and pinch loads of $80.1 \mathrm{~N}$ under the most critical shock and vibration loading condition, which is described in Appendix II. The axial stress is the driving force for circumferential external longitudinal part-wall cracks; therefore, the following equation applies:

$$
\mathrm{K}=\sigma_{\mathrm{b}} \mathrm{F} \sqrt{\frac{\pi \mathrm{a}}{\mathrm{Q}}} .
$$

Again, $F \approx 1.12, Q=2.464$; here $\sigma_{\mathrm{b}}=155 \mathrm{MPa}$. If the threshold value, $\Delta K_{t h}$, is substituted for $K$, the critical part-wall crack size for fatigue growth is calculated as $0.20 \mathrm{~cm}$. This is obviously impossible to achieve. If Equation (III-20) is considered for fatigue growth of PCI incipient cracks as a result of pinch load conditions, 


$$
\mathrm{K} \simeq 1.5 \sigma \sqrt{\pi \mathrm{a}}
$$

The hoop stress associated with an 80.1 pinch load (that cycled above the internal pressure) is approximated at $6.20 \mathrm{MPa}$. The crack size PCI critical to causing fatigue propagation (above $K=9.51$ ) is much larger than the wall thickness and can never occur. Thus, crack propagation under cyclic normal transport shock and vibration loading conditions appears to have a negligible probability of occurrence.

\section{III.5 Example Structural Analys is Results}

This section describes example analyses performed on typical BWR and PWR assemblies to illustrate the methodology for calculating spent-fuel assembly response under normal transport and regulatory accident drop conditions. Preliminary scoping analysis results and sensitivity evaluations of various design parameters are included with the detailed fuel rod response calculations. The development of the example assembly finite element models analyzed here is described in detail in Section III.3. The ABAQUS general-purpose finite element program [HI88] was used to perform the detailed spacer grid and assembly analyses. These example analyses resulted in representative data on the peak fuel rod stresses, strains, and pinch loads used to compute failure probabilities (see Section III.6). The results of these analyses are for illustration purposes only and do not represent an upper bound.

\section{III.5.1 Summary}

Table III-4 summarizes the structural analysis results of the example fuel assemblies. Peak strain and pinch load values and their locations are listed for each critical normal transport and regulatory accident loading condition.

A summary of the $B \& W 15 \times 15$ PWR assembly analysis results is as follows. The maximum tensile strain of 3.58 occurs under a $9.0-\mathrm{m}$ drop regulatory accident loading for a 2 -degrees-from-horizontal corner drop at slapdown. This 3.58 strain is located in the top fuel rods of the assembly adjacent to the end plate. The maximum initial impact corner drop (84-degree drop angle) strain is 2.68 and is located at the base of the assembly. The maximum end-drop tensile strain is 2.58 and it also occurs at the bottom end plate. The maximum side-drop tensile strain of 3.38 occurs in the top rods of the assembly for the cross sections without control rods.

The pinch load is the diametrically opposed force pair transmitted between adjacent rods. The pinch load for the B\&W PWR assembly is maximized during the slapdown loading condition. The maximum rod pinch load of $9728 \mathrm{~N}$ is distributed over the width of one spacer grid (approximately $2.5 \mathrm{~cm}$ ) and occurs in the bottom rods of the assembly.

The GE $7 \times 7$ BWR assembly analysis results can be summarized as follows. Under most loading conditions, the assembly remains nearly elastic. The maximum strain of 2.598 for the BWR fuel rods occurs in the 84-degree corner-drop initial impact orientation. 
Table III-4

Example Structural Analysis Results: Regulatory Transport Loading of Spent Fuel

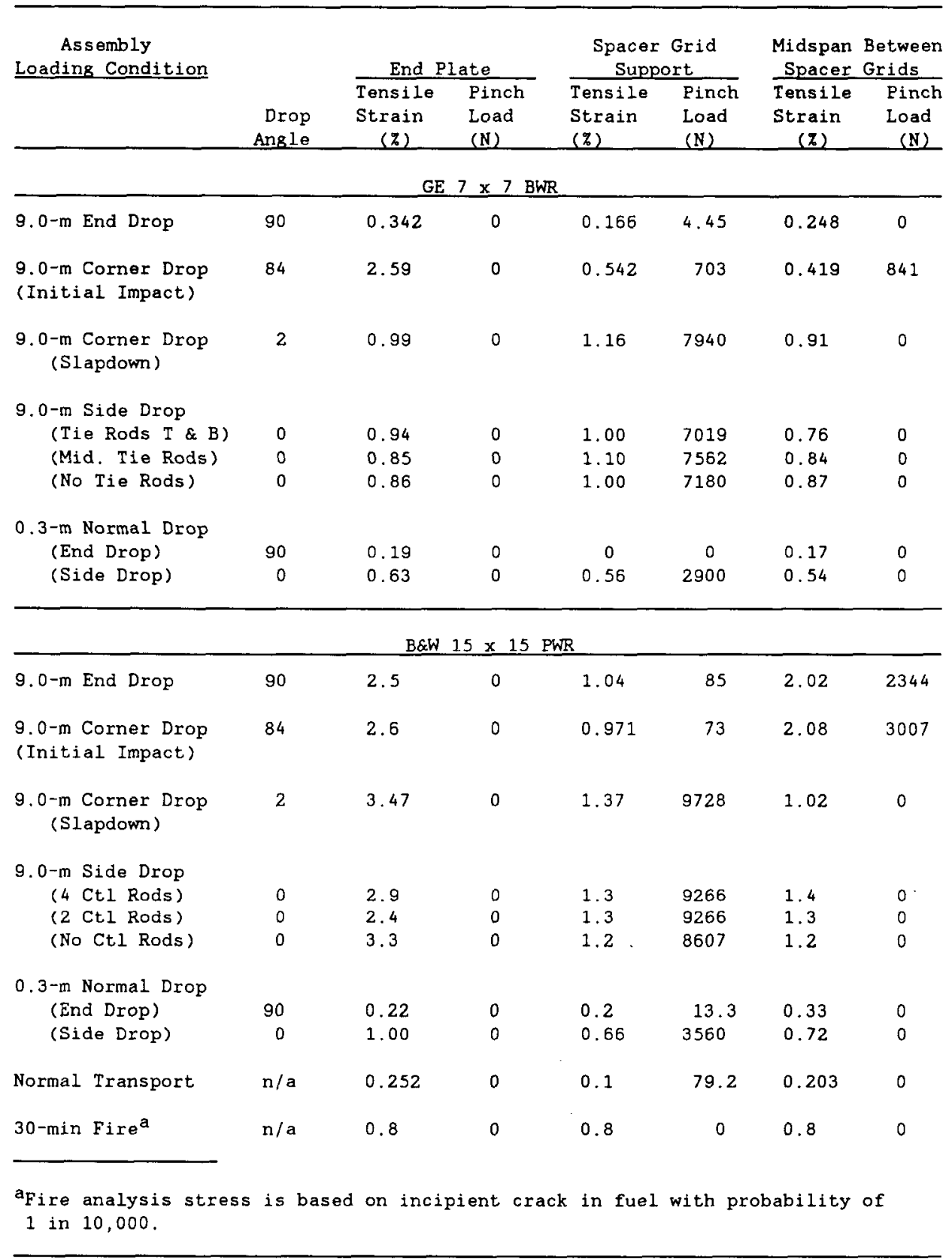


A description of the example assemblies and more detailed results of the hypothetical accident side-, end-, and corner-drop normal transport and regulatory fire analyses are presented in the corresponding sections.

\section{III.5.2 Example Assemblies}

Two example assemblies are chosen to illustrate the methodology developed in Section III.3 and to characterize the response of typical BWR and PWR fuel. Assemblies with unique material properties or geometric characteristics that vary significantly from typical spent fuel are not included in the present analysis. The example assemblies selected are based on the following criteria:

- The response of the example assemblies is not bounding.

- The fuel assemblies have a design common with a large number of fuel assemblies requiring future transport.

- The geometric and material properties must be typical of BWR/PWR fuel assemblies.

- Geometric, material, and burnup data must be readily available for the fuel.

For BWRs, the GE $7 \times 7$ is chosen because it is the most common BWR spent fuel, with five different types previously or currently produced. It has geometric and material characteristics typical of BWR fuel. There is a larger variety of PWR fuel designs than BWR. The B\&W $15 \times 15$ was selected because it is a common fuel assembly with typical fuel rod properties and an array of readily available data associated with it.

\section{GE $7 \times 7$ BWR Assemb1y Description}

The BWR example assembly is the GE $7 \times 7$. A typical GE $7 \times 7$ assembly is shown in Figure III-39 [N087], and a description of its geometric characteristics and material properties is provided in Table III-5 [N087]. This assembly is typically $14 \mathrm{~cm}$ wide and $4.34 \mathrm{~m}$ long. The fue 1 rod length is $4.017 \mathrm{~m}$. Assembly weight is $272 \mathrm{~kg}$, with each rod weighing $5.44 \mathrm{~kg}$. Typical GE $7 \times 7$ fuel rods have a $1.45-\mathrm{cm}$ outside diameter, with a cladding thickness of $0.081 \mathrm{~cm}$. The assembly contains seven spacer grids along its length, with an average span of $50.8 \mathrm{~cm}$ between grids.

\section{B\&W $15 \times 15$ PWR Assembly Description}

The PWR example assembly is the B\&W $15 \times 15$. A drawing of this assembly is shown in Figure III-40 [N087], and a description of its geometric characteristics and material properties is provided in Table III-6 [N087]. This assembly is $21.68 \mathrm{~cm}$ wide and $4.207 \mathrm{~m} \mathrm{long.}$

Individual rod lengths are $3.904 \mathrm{~m}$. The assembly weight is $687.2 \mathrm{~kg}$, with each rod weighing $3.18 \mathrm{~kg}$. Typical B\&W $15 \times 15$ fuel rods have a 1.09 $\mathrm{cm}$ outside diameter, with a cladding thickness of $0.0673 \mathrm{~cm}$. The assembly typically contains two spacer grids adjacent to each of its ends and six more along its length. The end spans measure $12.7 \mathrm{~cm}$, while the average midspan is approximately $53.3 \mathrm{~cm}$ between grids. 


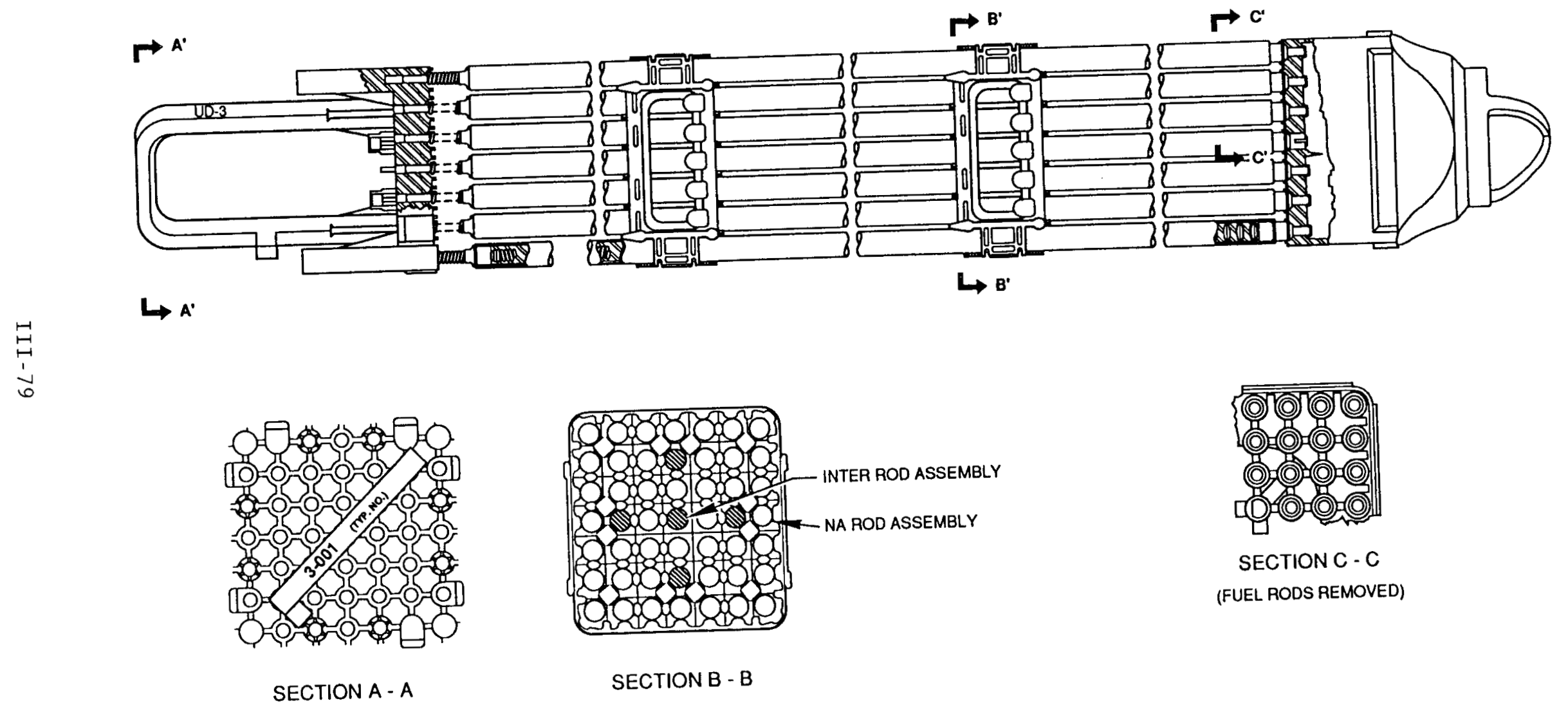

Figure III-39. GE $7 \times 7$ BWR Assembly Geometry 
Tab1e III - 5

Geometric and Material Properties of the GE $7 \times 7$ BWR Fuel Rod

Type of rod

Fuel rod

Fue 1 rod positions per assembly................ 49

Typical number of fuel rods per assembly............ 48

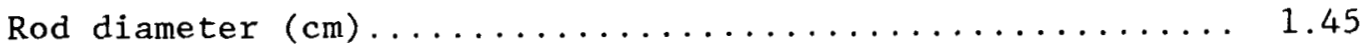

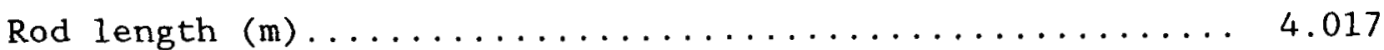

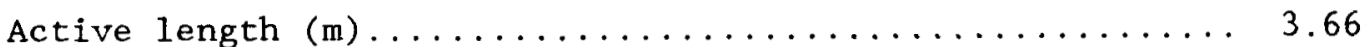

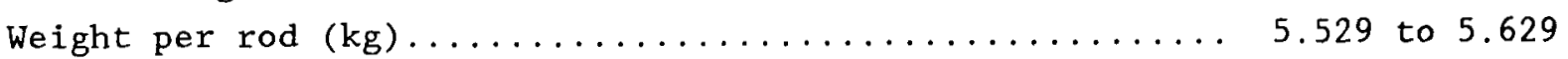

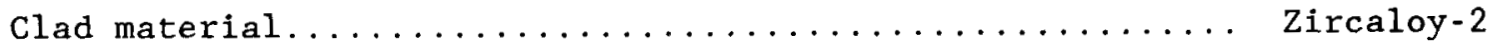

Clad thickness $(\mathrm{cm}) \ldots \ldots \ldots \ldots \ldots \ldots \ldots \ldots \ldots \ldots \ldots . \ldots \ldots 1$ to 0.117

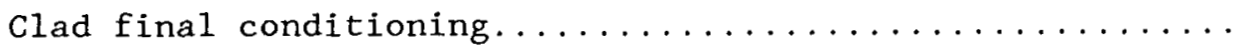

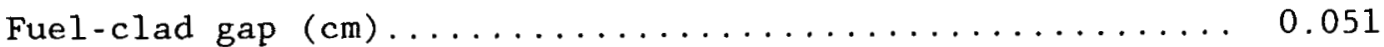

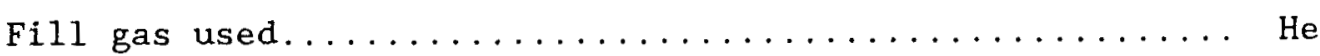

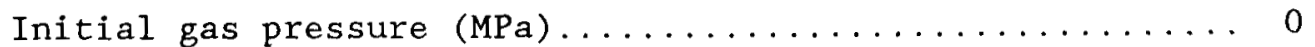

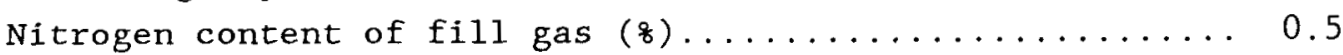

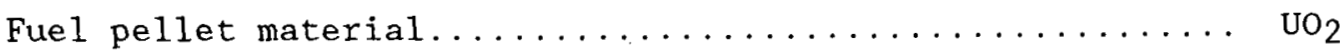

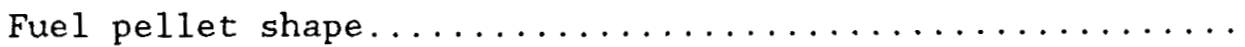

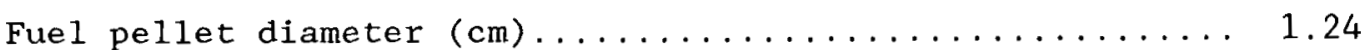

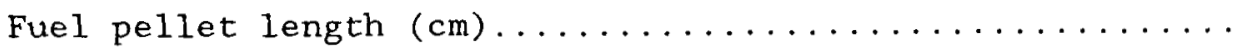

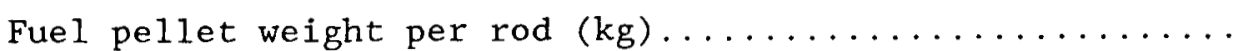

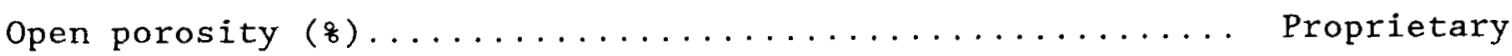

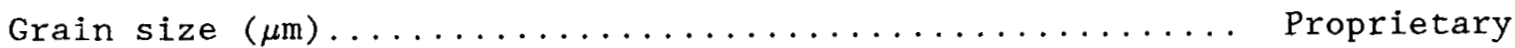

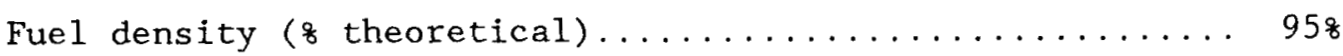

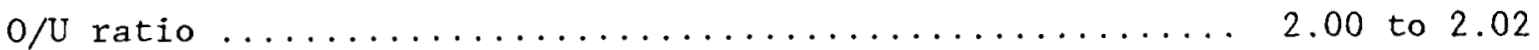

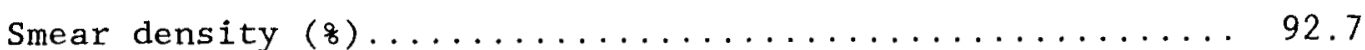

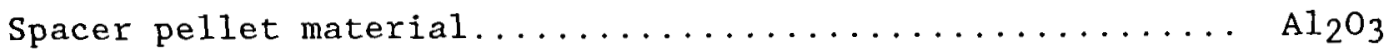

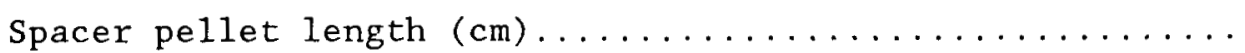

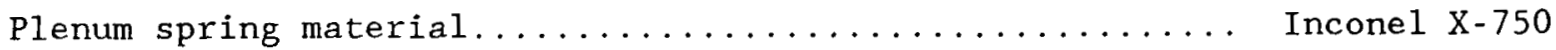

Plenum spring weight per assembly $(\mathrm{kg}) \ldots \ldots \ldots \ldots \ldots \ldots . \ldots . \ldots 059$

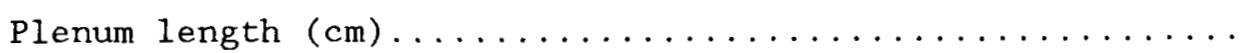

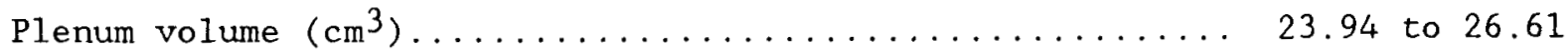

Note: A GE $7 \times 7$ BWR assembly contains four burnable poison fueled rods with typically 44.75 grams of gadolinia/rod. 

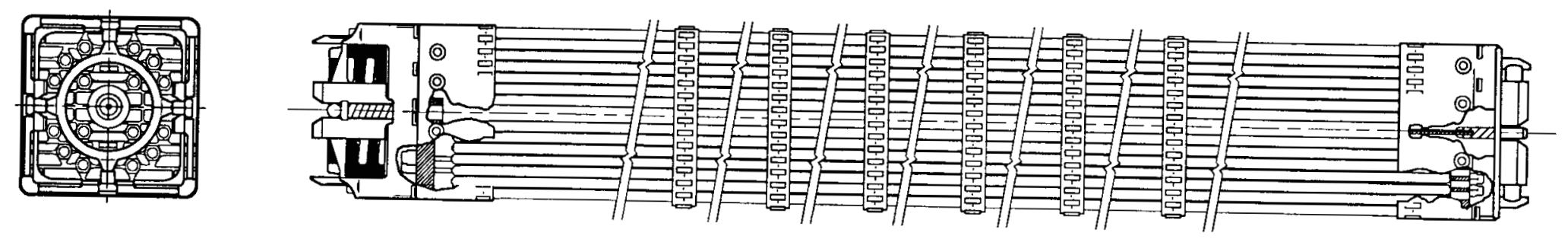

Fuel Assembly

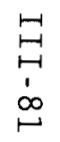
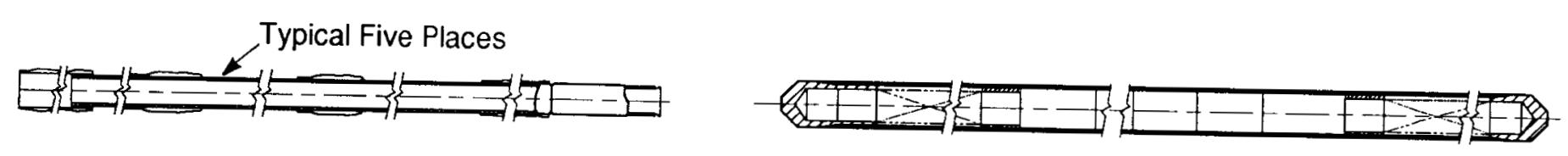

Instrumentation TLM Assembly Details

Fuel Rod Assembly
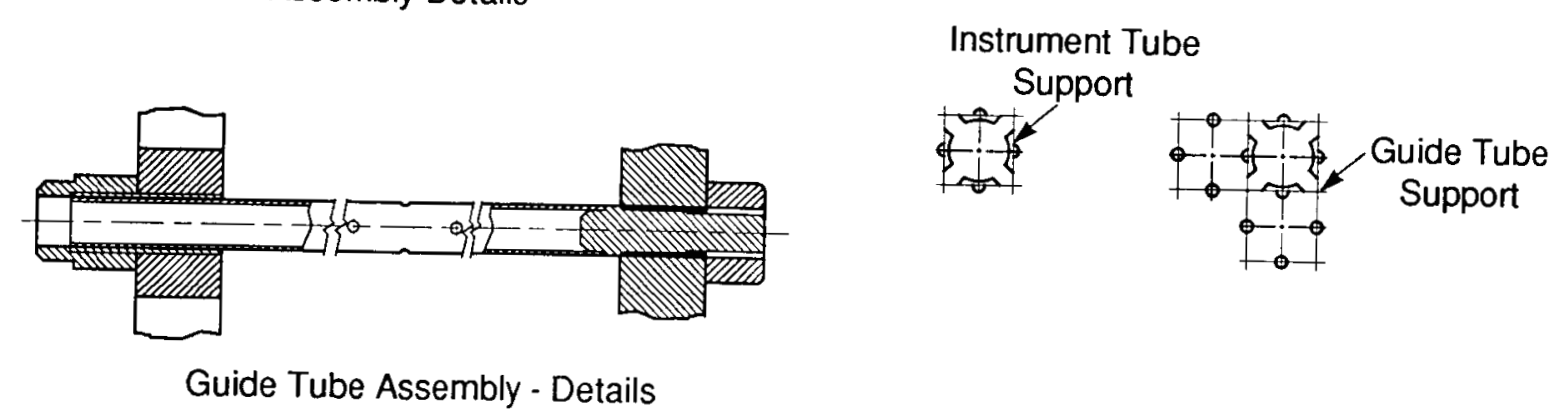

Guide Tube Assembly - Details

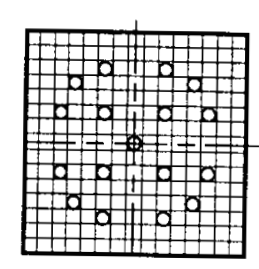

Section Z-Z

Figure III-40. B\&W $15 \times 15$ PWR Assembly Geometry 
Table III-6

Geometric and Material Properties of the B\&W $15 \times 15$ Mark B PWR Fuel Rod

Type of rod

Fuel rod positions per assembly.

Typical number of fuel rods per assembly

Rod diameter $(\mathrm{cm})$.

Rod length (m).

Active length (m)

Weight per rod $(\mathrm{kg})$

Clad material.

Clad thickness (cm)

Clad final conditioning.

Fuel-clad gap (cm)

Fil1 gas used.

Initial gas pressure (MPa)

Nitrogen content of fill gas ( 8 )

Fuel pellet material

Fuel pellet shape.

Fuel pellet diameter $(\mathrm{cm})$

Fuel pellet length (cm).

Fuel pellet weight per rod $(\mathrm{kg})$

Open porosity ( 8 )

Grain size $(\mu \mathrm{m})$

Fuel density ( 8 theoretical).

$0 / U$ ratio.

Smear density $\left(\mathrm{g} / \mathrm{cm}^{3}\right)$.

Spacer pellet material.

Spacer pellet length $(\mathrm{cm})$

Plenum spring material.

Plenum spring weight per assembly $(\mathrm{kg})$.

Plenum length (cm)

Plenum volume $\left(\mathrm{cm}^{3}\right)$
Fue1 rod

225

208

1.09

3.904

3.60

3.18

Zircaloy -4

0.0673

SRA

0.0107

$\mathrm{He}$

2.86

3.0

$\mathrm{UO}_{2}$

Dished, chamfered

0.9362

1.105

2.53

$<18$

10 to 14

95

2 to $2.02: 1$

9.75

Zircaloy-4

Stls. Steel 302

0.019

29.769

21.43 


\section{III.5.3 Spacer Grid}

Separately detailed analyses are required to quantify the highly nonlinear response of the spacer grid frames. These analyses are used to develop simplified nonlinear spring-element spacer grid models for incorporation into the assembly models.

Analyses of complete spacer grid frames and single-bay spacer grid models are presented in this section as verification of the single-cell spacer grid analysis procedure described in Section III.3.3. Analyses of both BWR and PWR example spacer grid frames and development of the assembly spacer grid spring elements are presented.

\section{BWR Spacer Grid Analyses}

The geometry of the GE $7 \times 7$ spacer grid is shown in Figure III-41; two analytical models are developed to verify its response. A slice model (row of cells) is analyzed to determine the complete force deflection time history of a typical row of rods, and a half-grid model is analyzed to the point of initial buckling to verify the assumptions made with the slice model. Both models are analyzed using material and geometric nonlinearities.

\section{Ful1 Spacer Grid Computationa1 Mode1}

A one-half spacer grid computational model including symmetry boundary conditions is used to generate the fuel rod/spacer grid interaction of a complete spacer grid frame. The model uses multiple beam-type finite elements to model the frame, which is 7 cells high by $3-1 / 2$ cells wide. Inside each cell, nonlinear springs are used in both the horizontal and vertical directions to simulate the interaction between the fuel rods and frame. These springs also constrain the frame's deflections consistent with the fuel rod geometry. Vertical loads are applied to the bottom spring of each cell to simulate loading from the fuel rods. Loads from each rod are ramped with equal magnitudes until the onset of buckling.

The full grid analysis predicts the onset of buckling of the BWR spacer grid at $-75.7 \mathrm{~N}$ of load per fuel rod. Figure III-42 shows a deflected shape of the full grid model. Notice that the response of the frame is nearly symmetric in all bays; thus, a single-bay model approximation seems reasonable. The single-bay slice model is presented in the next section and verifies this assumption.

\section{Single-Bay Spacer Grid Model}

The single-bay spacer grid verification model (Figure III-43) is analyzed similar to the full spacer grid model by ramping fuel rod loads uniformly in each spacer grid cell until the onset of buckling. On buckling, very small load increments are used to determine the postbuckling behavior of the spacer grid frame. 


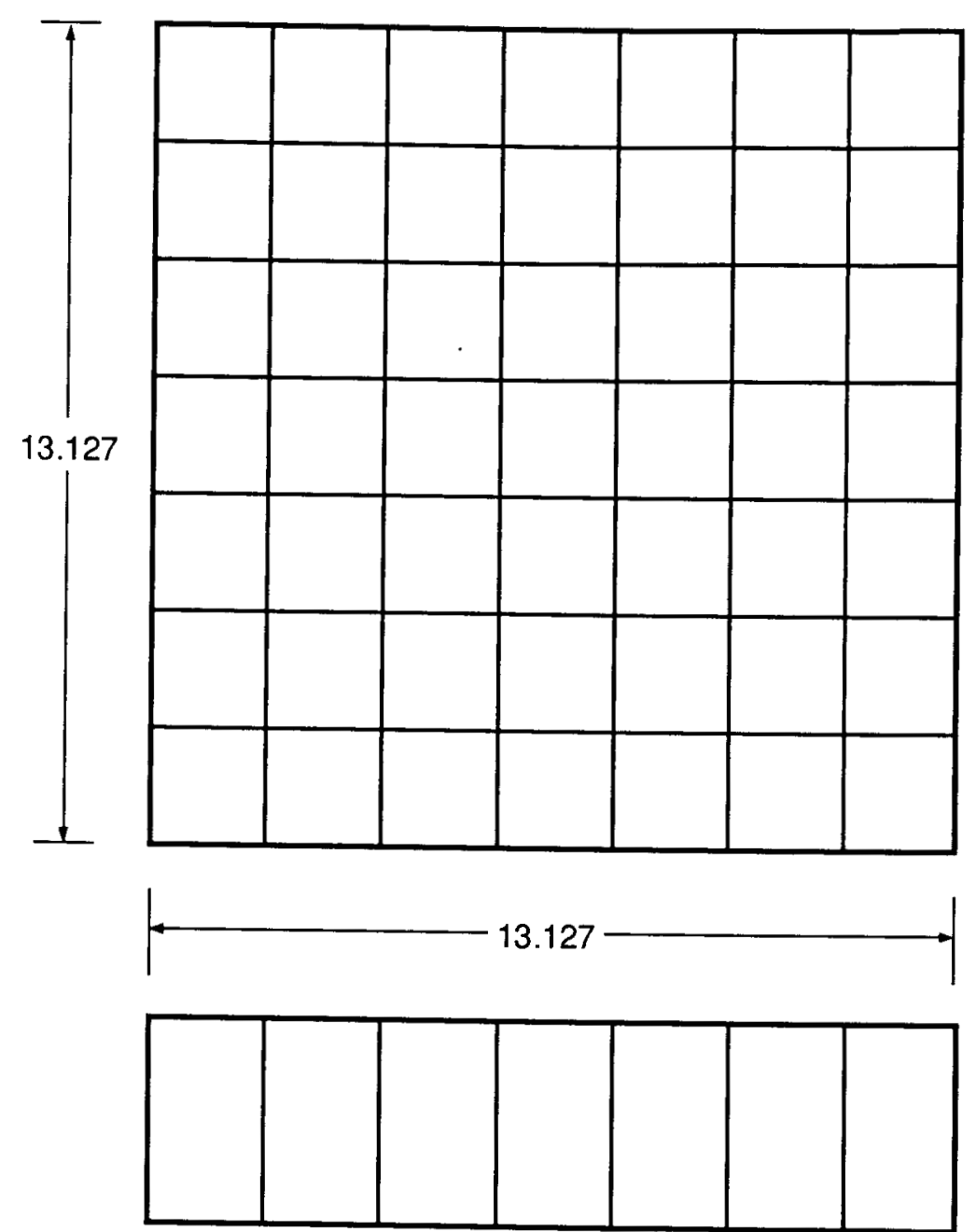

EXTERIOR FRAME
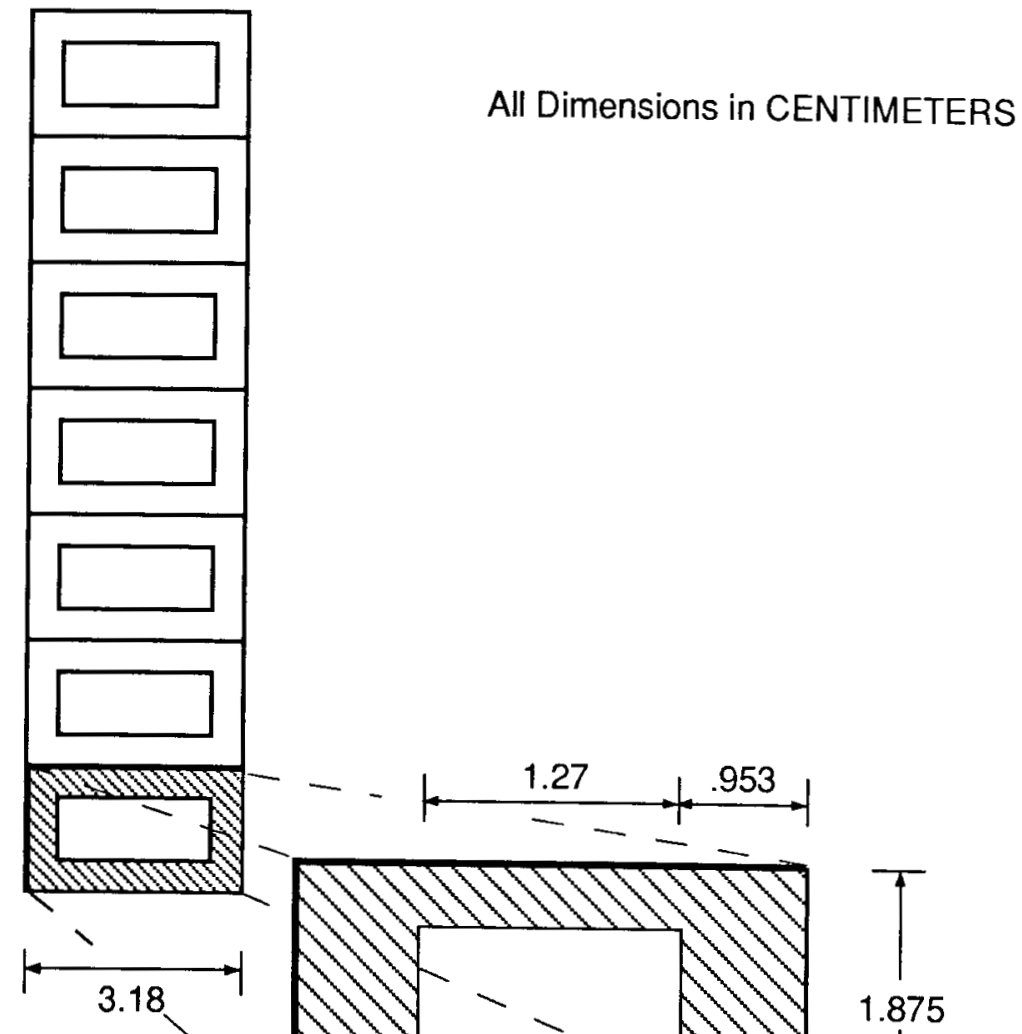

1.27

.953

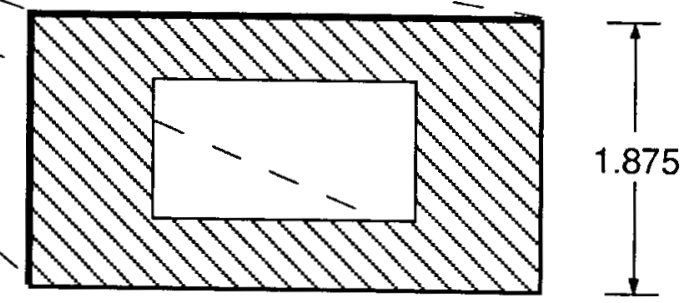

3.18 


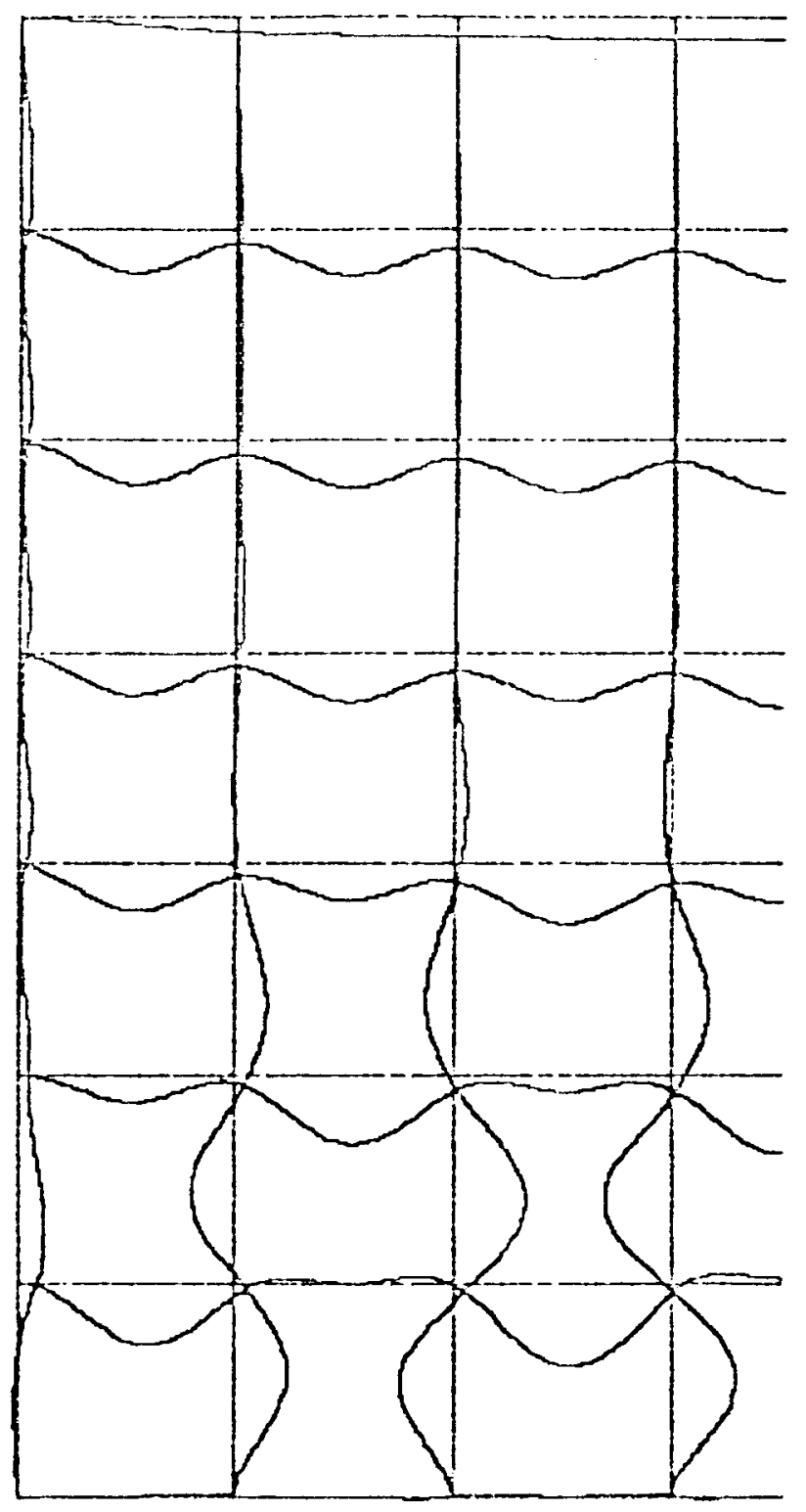

BUCKLING OCCURS AT

75.65 N PER ROD

Figure III-42. BWR One-Half Spacer Grid Mode1: Deflected Shape Plot 


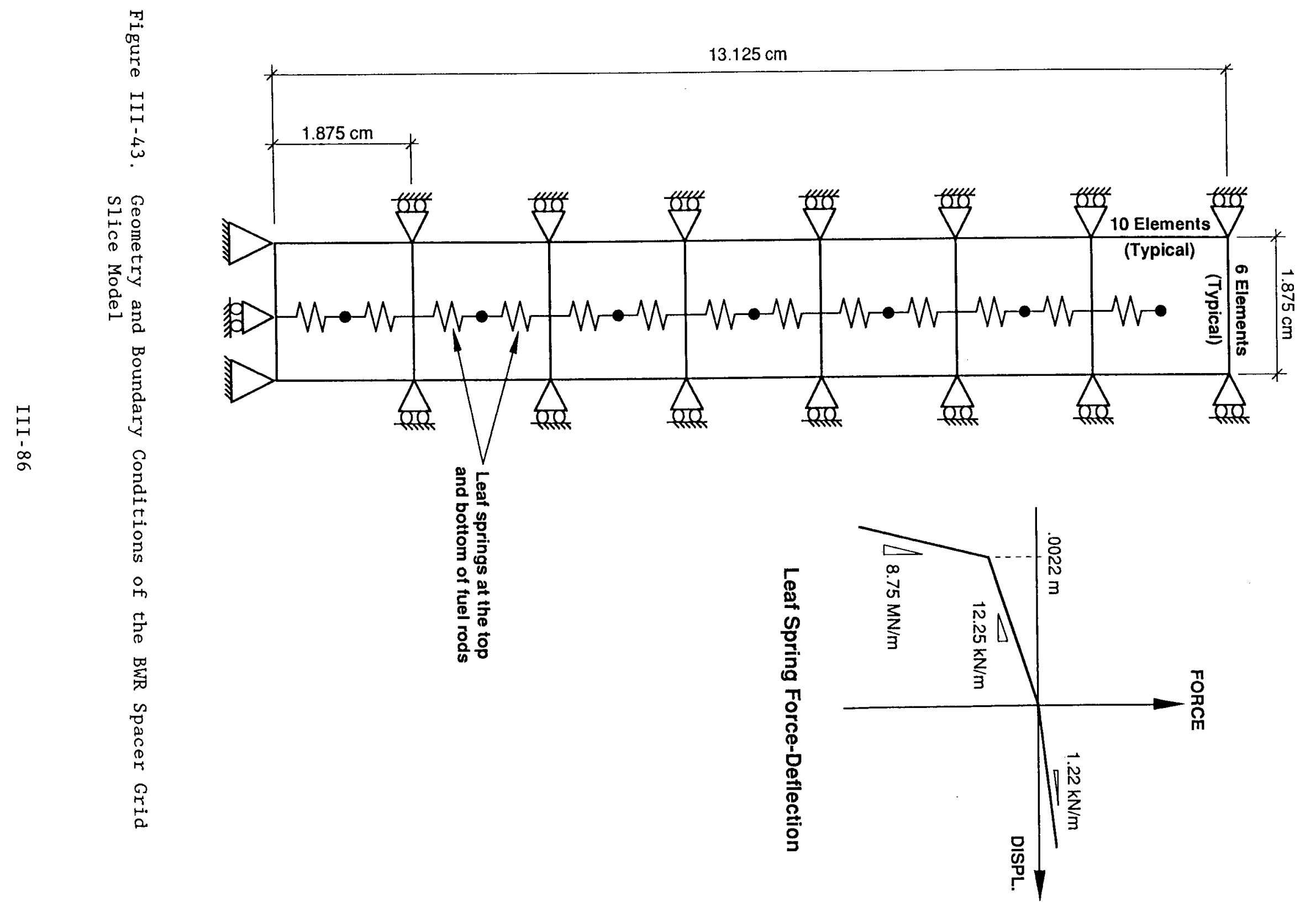


The single-bay slice model was verified by comparing its initial buckling loads with the previously mentioned full grid models. It buckled initially at $\sim 75.7 \mathrm{~N}$; thus, this single-bay model provides excellent agreement with the full grid model, which also buckled at $75.7 \mathrm{~N}$. A single-cel1 model (see Figure III-16) also provided a buckling load of $75.7 \mathrm{~N}$ and thus is the preferred method for future analyses.

Figure III-44 shows the deflected shape from the GE $7 \times 7$ single-bay slice model analysis. The force deflection spring elements developed for the example BWR assembly models are shown in Figure III-45. The springs used to simulate the spacer grids are modeled in series for two-dimensional side drop assembly analyses (discussed in the following section). Although all of the springs have similar force deformation curves, all rods will see the net deformation of the spacer springs below them as the assembly is loaded. Thus, the response of the complete spacer grid frame is simulated by the single-bay analyses. Similarly, each spring element will accrue the force from all rods above the spring of interest, and so the buckling force of $454 \mathrm{~N}$ for an individual cell, which is indicated in Figure III-45, is equivalent to a force of $75.7 \mathrm{~N}$ in each rod that buckles the bottom cell (454 $\mathrm{N}$ is equal to six rods multiplied by $75.7 \mathrm{~N}$ per rod).

Figure III-45 also shows the assembly spacer grid spring element force/displacement relationship relative to a single rod at the center of the assembly. The five incremental slopes of the force/deflection curve model the following events: (1) the gap between the assembly and basket, (2) the spacer grid leaf spring, (3) the spacer grid stiffness for a complete intact spacer grid, (4) the frame stiffness after one and two cells have buckled, and (5) buckling of all cells of the spacer grid and stacking of the fuel rods on top of one another.

\section{PWR Spacer Grid Analysis}

The geometry of the example B\&W $15 \times 15$ PWR spacer grid is shown in Figure III-46. Based on the correlation between the single-bay and the full grid models observed in the GE $7 \times 7$ spacer grid analyses, the B\&W $15 \times 15$ PWR force/deflection relationship was developed with a single-bay model similar to that of the BWR model. The deformed and undeformed shape plots of the single-bay B\&W $15 \times 15$ spacer grid are shown in Figure III-47.

The $B \& W 15 \times 15$ spacer grids respond similarly to those of the BWR spacer grid model. However, with the larger number of fuel rods, more load is transferred to the bottom cell of the frame and these grids buckle at a smaller load per rod than the BWR spacer grid. This occurs despite the fact that the PWR grid is stronger than the BWR grid. For example, the analyses indicate that the bottom cell of the PWR spacer model buckles at $66.8 \mathrm{~N}$.

The force/displacement spring elements for the B\&W $15 \times 15$ assembly models are shown in Figures III-48 and III-49. These models are similar to the BWR GE $7 \times 7$ models described above. The spacer grid spring element (Figure III-49) represents the spacer grids at the ends of most PWR assemblies. These spacer grids are unique in that they are attached to the end plate directly. Thus, the additional stiffness from this attachment has been modeled into these spring elements. 


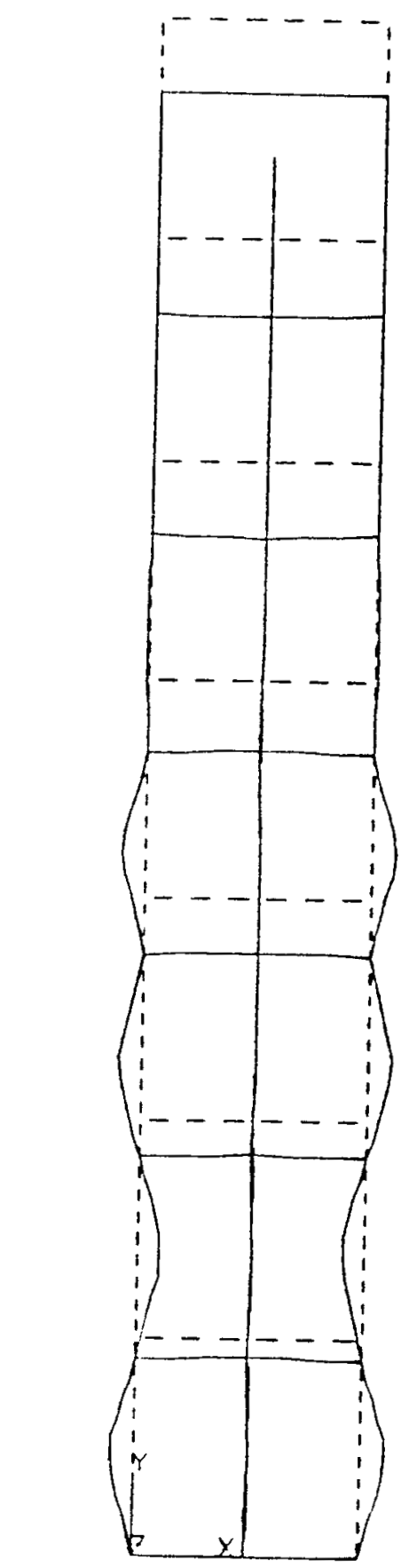

\section{GE BWR $7 \times 7$ SPACER GRID BUCKLING} 75.65 N PER ROD

Figure III-44. BWR Spacer Grid Slice Mode1: Deflected Shape Plot 

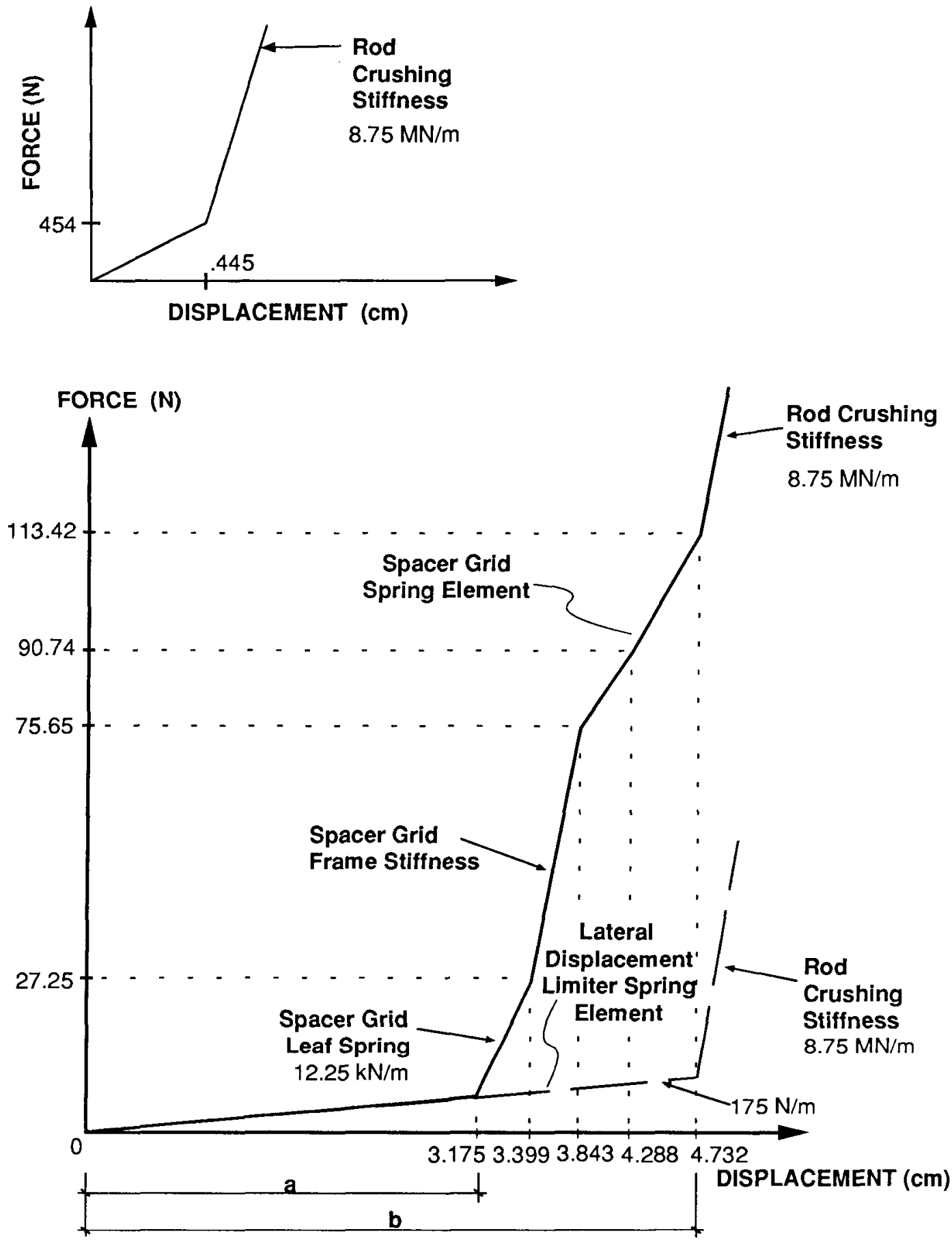

Notes:

The numerical values are typical of a BWR assembly center rod

$a=$ Lateral gap for the assembly's center rod (one half of the assembly-basket lateral gap)

$b=$ Maximum lateral displacement for the assembly's center rod

Figure III-45. GE $7 \times 7$ BWR Assembly Spacer and Grid Elements 

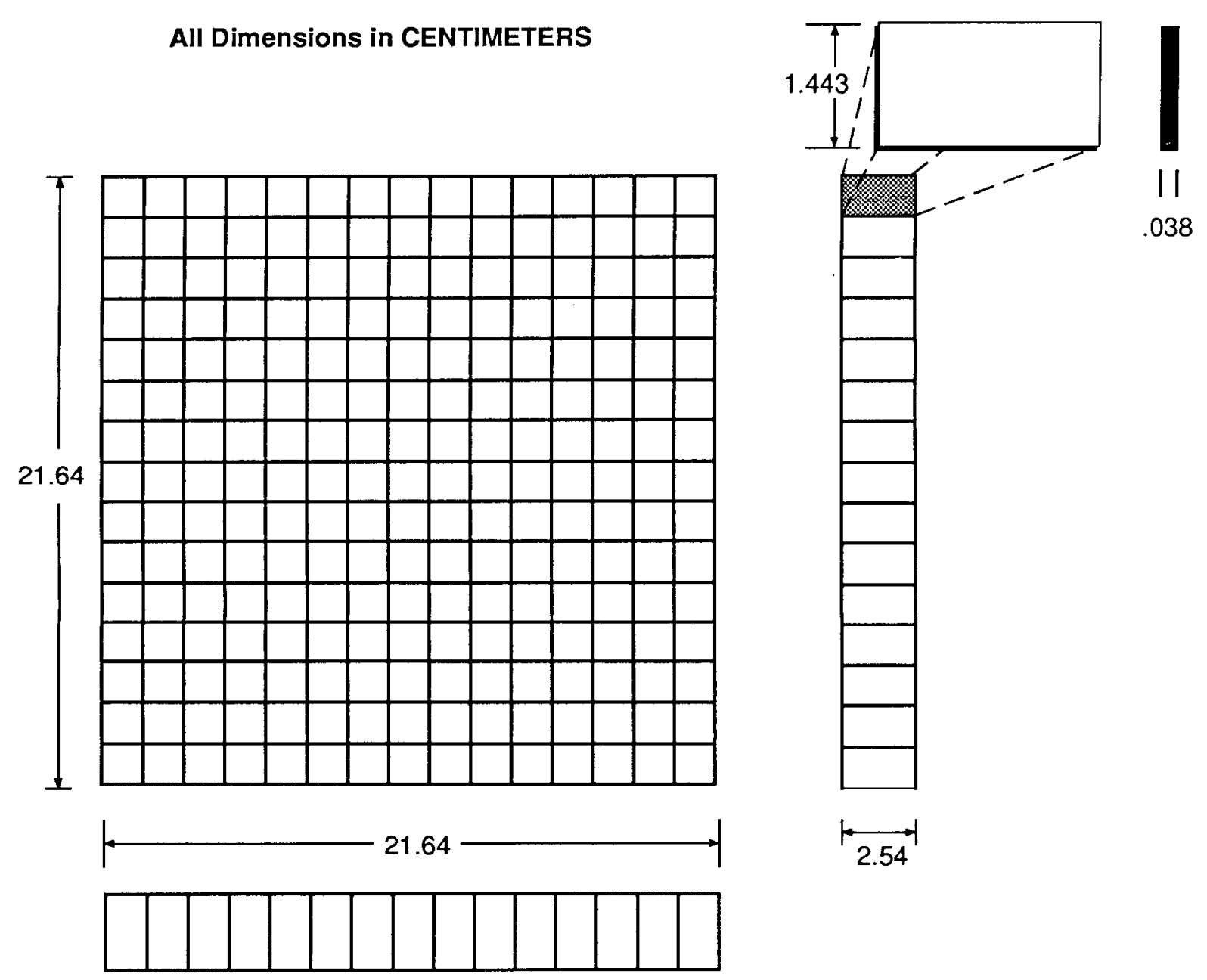

Figure III-46. Example PWR Spacer Grid Geometry

\section{III.5.4 Hypothetical Accident Side Drop}

Two types of analyses are performed to quantify the response of the example assembly under hypothetical accident side-drop loading conditions: quasistatic and time-history dynamic analyses. The static analyses provide a means of developing the detailed response model, as well as reasonable estimates of the fuel rod response. Using the results of the static analyses, dynamic analyses are performed to develop more accurate solutions. These analyses also provide a means of differentiating the fuel assembly response as a function of loading duration. Results of both analyses are presented here because the static analysis results aid in understanding the total response of the assemblies. 


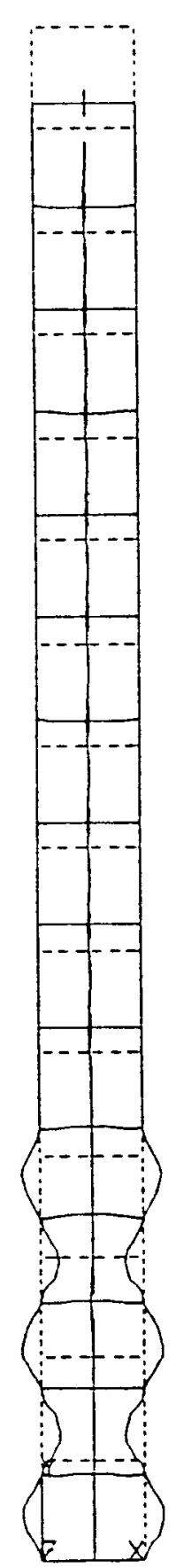

\section{B\&W PWR 15×15 \\ SPACER GRID BUCKLING}

66.7 N PER ROD

Figure III-47. PWR Spacer Grid Slice Model: Deflected Shape Plot 

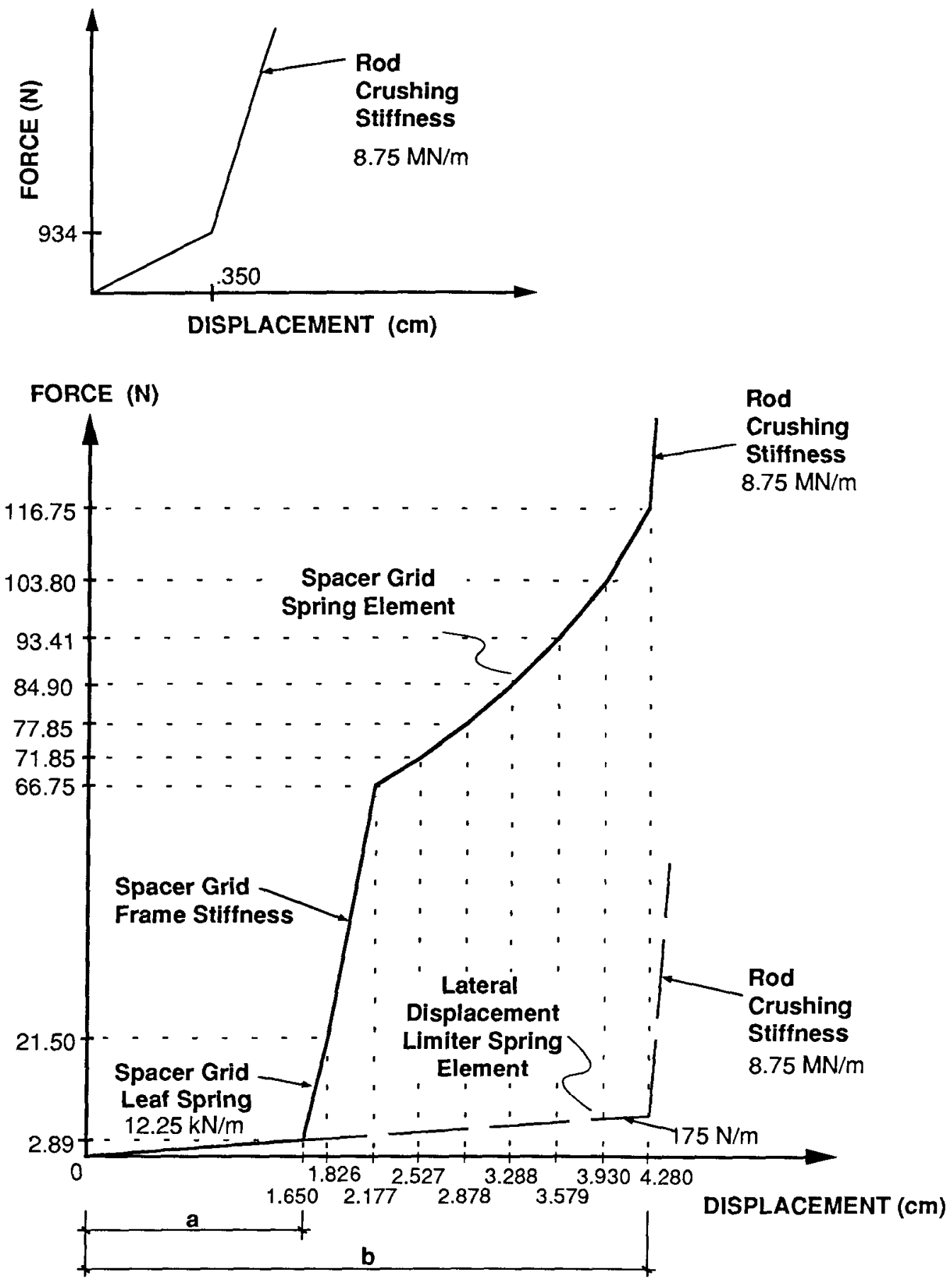

Notes:

The numerical values are typical of a PWR assembly center rod

$$
\begin{aligned}
& a=\text { Lateral gap for the assembly's center rod } \\
& \text { (one half of the assembly-basket lateral gap) } \\
& b=\text { Maximum lateral displacement for the } \\
& \text { assembly's center rod }
\end{aligned}
$$

Figure III-48. B\&W $15 \times 15$ PWR Assembly Spacer Grid Elements 


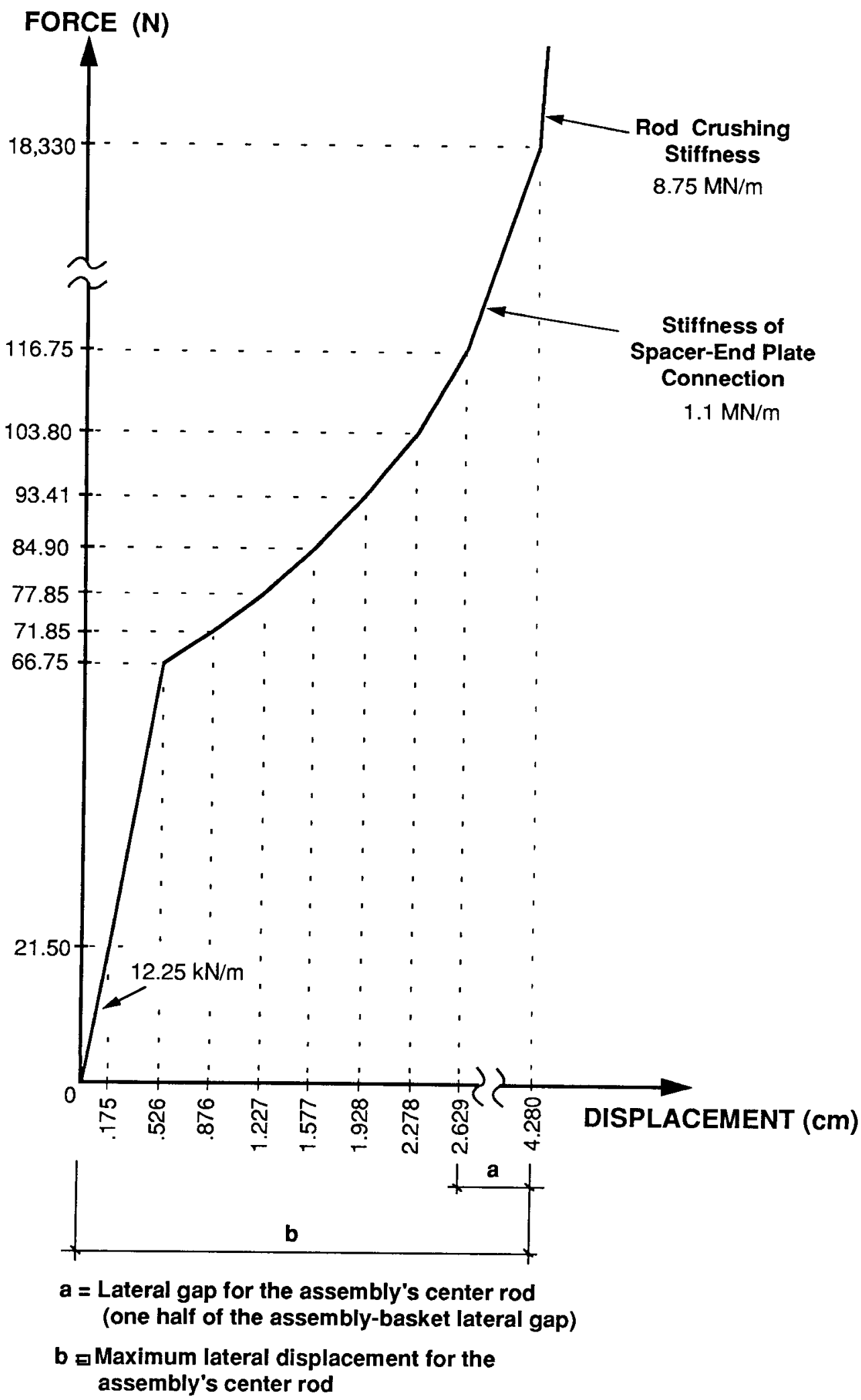

Figure III-49. PWR End Drop Mode1: Top and Bottom Spacer Grid Spring Element 
Similar analytical models are used in both the quasi-static and dynamic analyses. Details for the development of these models are provided in Section III.3.4. The main difference between the two models lies in their support conditions. For the quasi-static analysis, the basket support points are treated as fixed supports. For the dynamic analyses, the basket supports are coupled together and a retarding force equal to the assembly mass multiplied by the cask impact limiter deceleration history is applied.

Loadings for both the quasi-static and dynamic example analyses are based on the response of an example lead-shielded truck cask, as described in Appendix II. The quasi-static analysis is performed by ramping the loads uniformly up to a maximum of $100 \mathrm{~g}$. The dynamic analyses are performed using a deceleration history as shown in Figure III-8, with a peak load of $100 \mathrm{~g}$ and a total load duration of $18 \mathrm{~ms}$.

\section{GE $7 \times 7$ BWR Side Drop Quasi-static Analysis Results}

This section describes the results of the example regulatory accident quasi-static analyses of the GE $7 \times 7$ two-dimensional assembly side drop models. These models are illustrated in Figures III-19 and III-20. The response of the GE $7 \times 7$ BWR fuel assembly under quasi-static loading of $100 \mathrm{~g}$ can best be described by the deflected shape plots shown in Figure III-50. The three plots show the deflected shapes of the three assembly slice models: (1) slice with interior tie rods, (2) slice with top and bottom tie rods, and (3) slice without any tie rods. All three models show similar deformation patterns. Each spacer grid spring is crushed so that the fuel rods stack on top of one another away from the end plate. The magnitude of deflections at midspan between the end plate and first spacer grid-spring support varies slightly depending on tie rod positions. For the slice without any tie rods, all rods slide axially within the tie plate in a similar pattern. Thus, each rod follows a similar deformation pattern. For the slices with tie rods, the axial deformation of the tie rods themselves is limited to the deformation caused by tie plate rotation. Vertical deformations of the loose rods are also restricted when they stack on top of the axially stiffened tie rods. In all three slice models, the maximum positive strains of the fuel rods occur at their interface with the end plate. Figures III-51 through III-53 provide longitudinal outer-fiber strain plots along the length of the selected rods for the three slice locations. The maximum tensile outer fiber strain occurs in the assembly's top fuel rod slice without any tie rods. This 0.78 strain occurs at the interface between the end plate and the top rod.

Fuel rod pinch forces are developed when the spacer grids are buckled after loads from the top rods of the assembly are transferred from rod to rod to the basket supports. The pinch loads increase from the top to the bottom of the assembly as additional load is transformed from each new rod encountered between the top of the assembly and the basket support. For the quasi-static side drop analyses, rigid basket supports are modeled at each spacer grid. Thus, the maximum pinch loads occur in the bottom rods at the spacer grid support locations. Figure III-54 shows the distribution of pinch loads for the assembly slice with top and bottom tie rods. For the slice with interior tie rods, the maximum $(5680 \mathrm{~N})$ pinch load occurs at the spacer grid location adjacent to the end plate. 


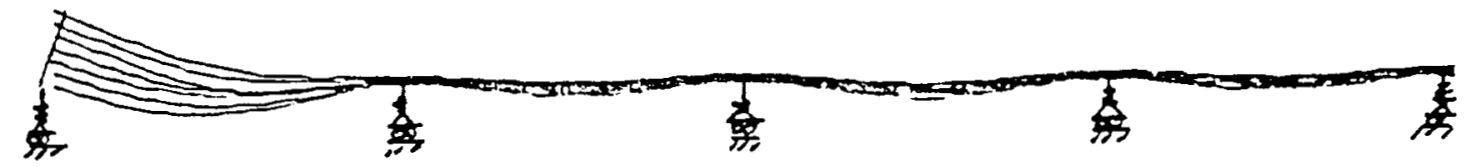

GE $7 \times 7$ BWR - SIDE SLICE WITH TIE-RODS - SIDE DROP

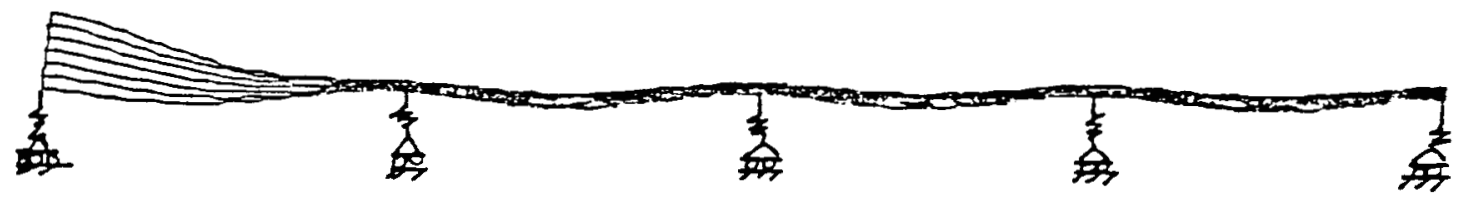

GE 7X7 BWR - MID SLICE WITH TIE-RODS - SIDE DROP

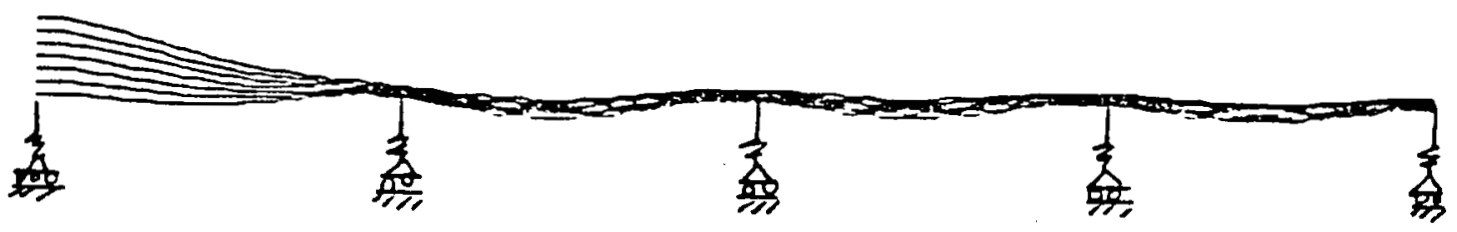

GE $7 \times 7$ BWR - MID SLICE WITHOUT TIE-RODS - SIDE DROP

Figure III-50. GE $7 \times 7$ BWR Side Drop Two-Dimensional Assembly, 100-g Quasi-static Analyses: Deflected Shape Plots 


\section{LONGITUDINAL STRAIN AT BOTTOM SURFACE OF ROD}

\section{$(\mathrm{m} / \mathrm{m})$}

$-4.00 \times 10^{-3}$

$-3.30 \times 10^{-3}$

$-2.60 \times 10^{-3}$

$-1.90 \times 10^{-3}$

$-1.20 \times 10^{-3}$

$-4.99 \times 10^{-4}$

$2.00 \times 10^{-4}$

$9.00 \times 10^{4}$

$1.60 \times 10^{-3}$

$2.30 \times 10^{-3}$

$3.00 \times 10^{-3}$

$-6.00 \times 10^{-3}$

$-5.10 \times 10^{-3}$

$-4.20 \times 10^{-3}$

$-3.30 \times 10^{-3}$

$-2.40 \times 10^{-3}$

$-1.50 \times 10^{-3}$

$-5.99 \times 10^{-4}$

$3.00 \times 10^{-4}$

$1.20 \times 10^{-3}$

$2.10 \times 10^{-3}$

$3.00 \times 10^{-3}$

$-4.00 \times 10^{-3}$

$-3.00 \times 10^{-3}$

$-2.00 \times 10^{-3}$

$-1.00 \times 10^{-3}$

$1.00 \times 10^{-9}$

$1.00 \times 10^{-3}$

$2.00 \times 10^{-3}$

$3.00 \times 10^{-3}$

$4.00 \times 10^{-3}$

$5.00 \times 10^{-3}$

$6.00 \times 10^{-3}$

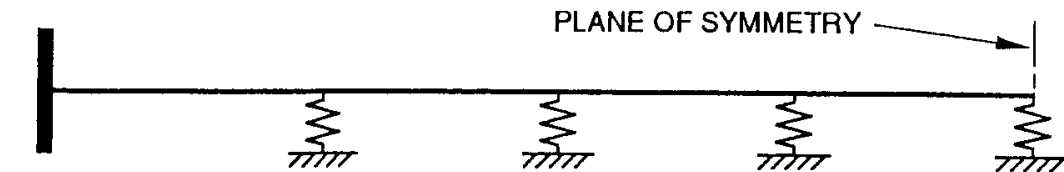

ROD LOCATION
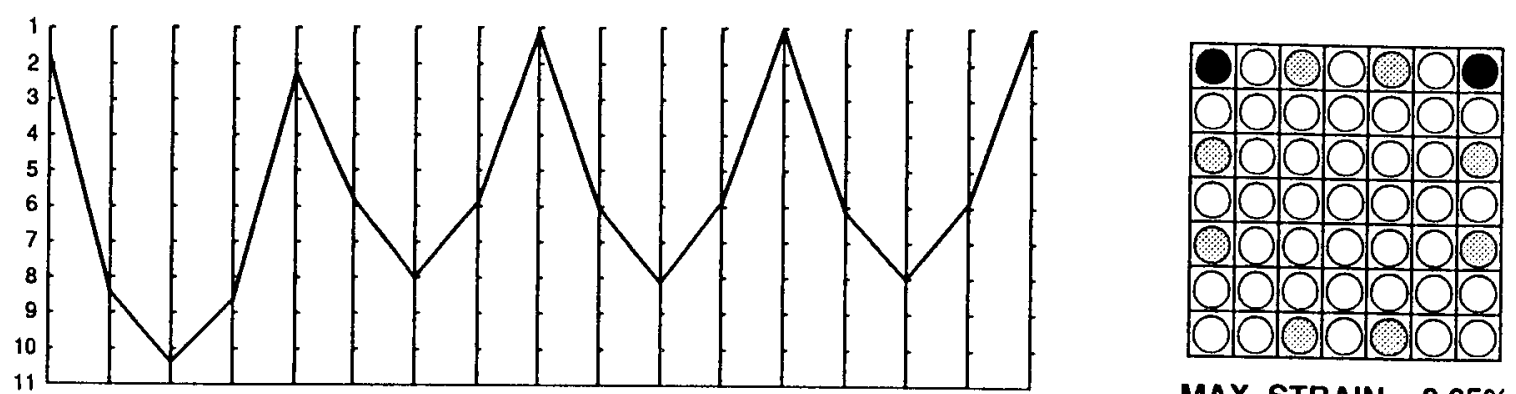

MAX. STRAIN $=0.25 \%$
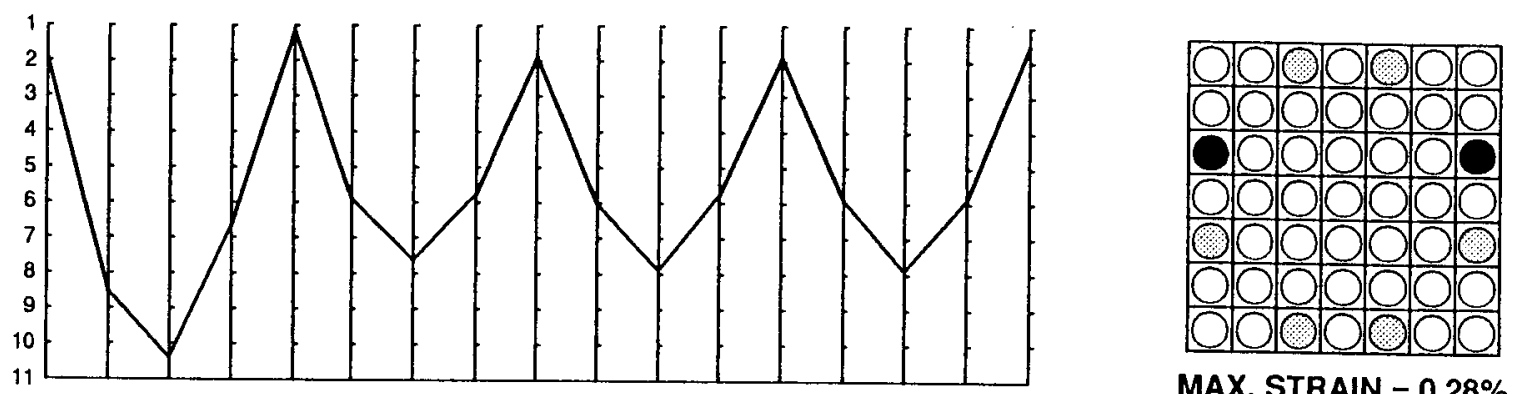

MAX. STRAIN $=0.28 \%$

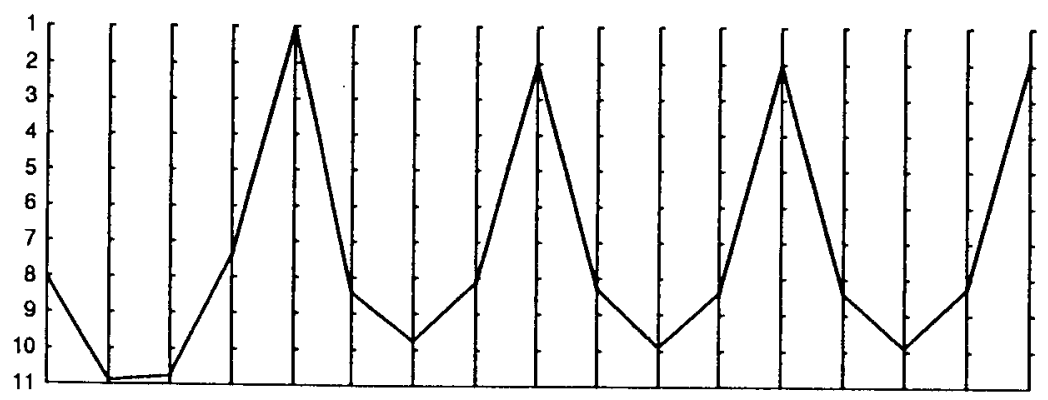

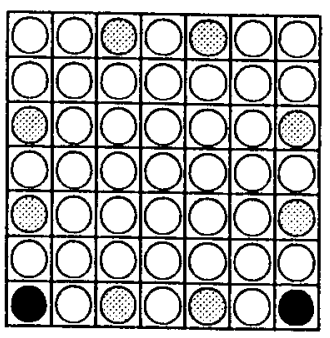

MAX. STRAIN $=0.6 \%$

Figure III-51. GE 7 × 7 BWR Side-Drop Two-Dimensional Assembly, 100-g Quasi-static Analysis: Longitudinal

\footnotetext{
Figure III-51. GE $7 \times 7$ BWR Side-Drop Two-Dimensional Assembly, 100-g Quasi-static
Outer Fiber Strain Profile Plots, Slice Model with Interior Tie Rods
} 


\begin{tabular}{|c|c|c|}
\hline & $\begin{array}{l}\text { LON } \\
\text { BOTI }\end{array}$ & $\begin{array}{l}\text { UDINAL STR } \\
\text { LSURFACE }\end{array}$ \\
\hline & & $(\mathrm{m} / \mathrm{m})$ \\
\hline & 1 & $-4.00 \times 10^{-3}$ \\
\hline & 2 & $-3.00 \times 10^{-3}$ \\
\hline & 3 & $-2.00 \times 10^{-3}$ \\
\hline & 4 & $-1.00 \times 10^{-3}$ \\
\hline & 5 & $1.00 \times 10^{-9}$ \\
\hline & 6 & $1.00 \times 10^{-3}$ \\
\hline & 7 & $2.00 \times 10^{-3}$ \\
\hline & 8 & $3.00 \times 10^{-3}$ \\
\hline & 9 & $4.00 \times 10^{-3}$ \\
\hline & 10 & $5.00 \times 10^{-3}$ \\
\hline & 11 & $6.00 \times 10^{-3}$ \\
\hline & 1 & $-3.00 \times 10^{-3}$ \\
\hline & 2 & $-2.20 \times 10^{-3}$ \\
\hline & 3 & $-1.40 \times 10^{-3}$ \\
\hline & 4 & $-5.99 \times 10^{-4}$ \\
\hline & 5 & $2.00 \times 10^{-4}$ \\
\hline 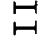 & 6 & $1.00 \times 10^{-3}$ \\
\hline$\dot{s}$ & 7 & $1.80 \times 10^{-3}$ \\
\hline & 8 & $2.60 \times 10^{-3}$ \\
\hline & 9 & $3.40 \times 10^{-3}$ \\
\hline & 10 & $4.20 \times 10^{-3}$ \\
\hline & 11 & $5.00 \times 10^{-3}$ \\
\hline & 1 & $-2.00 \times 10^{-3}$ \\
\hline & 2 & $-1.20 \times 10^{-3}$ \\
\hline & 3 & $-3.99 \times 10^{-4}$ \\
\hline & 4 & $4.00 \times 10^{-4}$ \\
\hline & 5 & $1.20 \times 10^{-3}$ \\
\hline & 6 & $2.00 \times 10^{-3}$ \\
\hline & 7 & $2.80 \times 10^{-3}$ \\
\hline & 8 & $3.60 \times 10^{-3}$ \\
\hline & 9 & $4.40 \times 10^{-3}$ \\
\hline & 10 & $5.20 \times 10^{-3}$ \\
\hline & 11 & $6.00 \times 10^{-3}$ \\
\hline
\end{tabular}

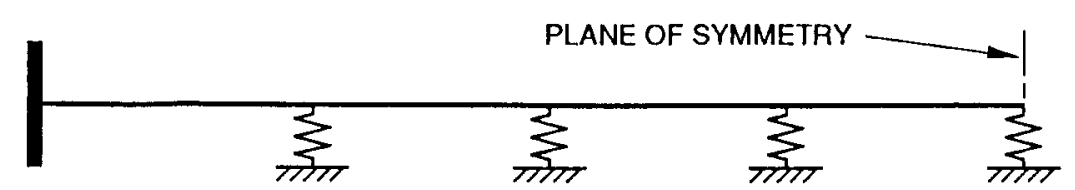

ROD LOCATION
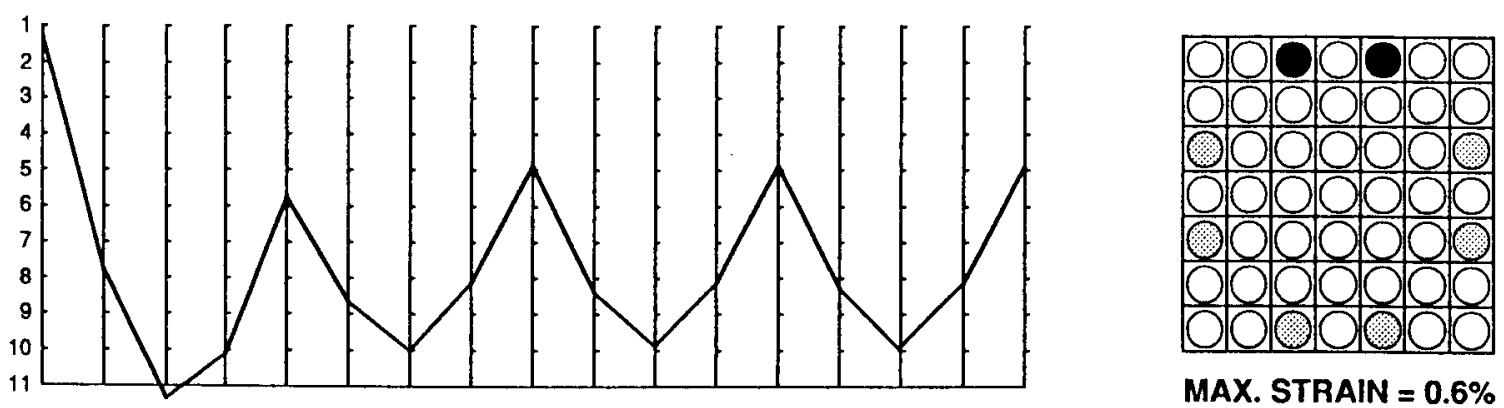

MAX. STRAIN $=0.6 \%$
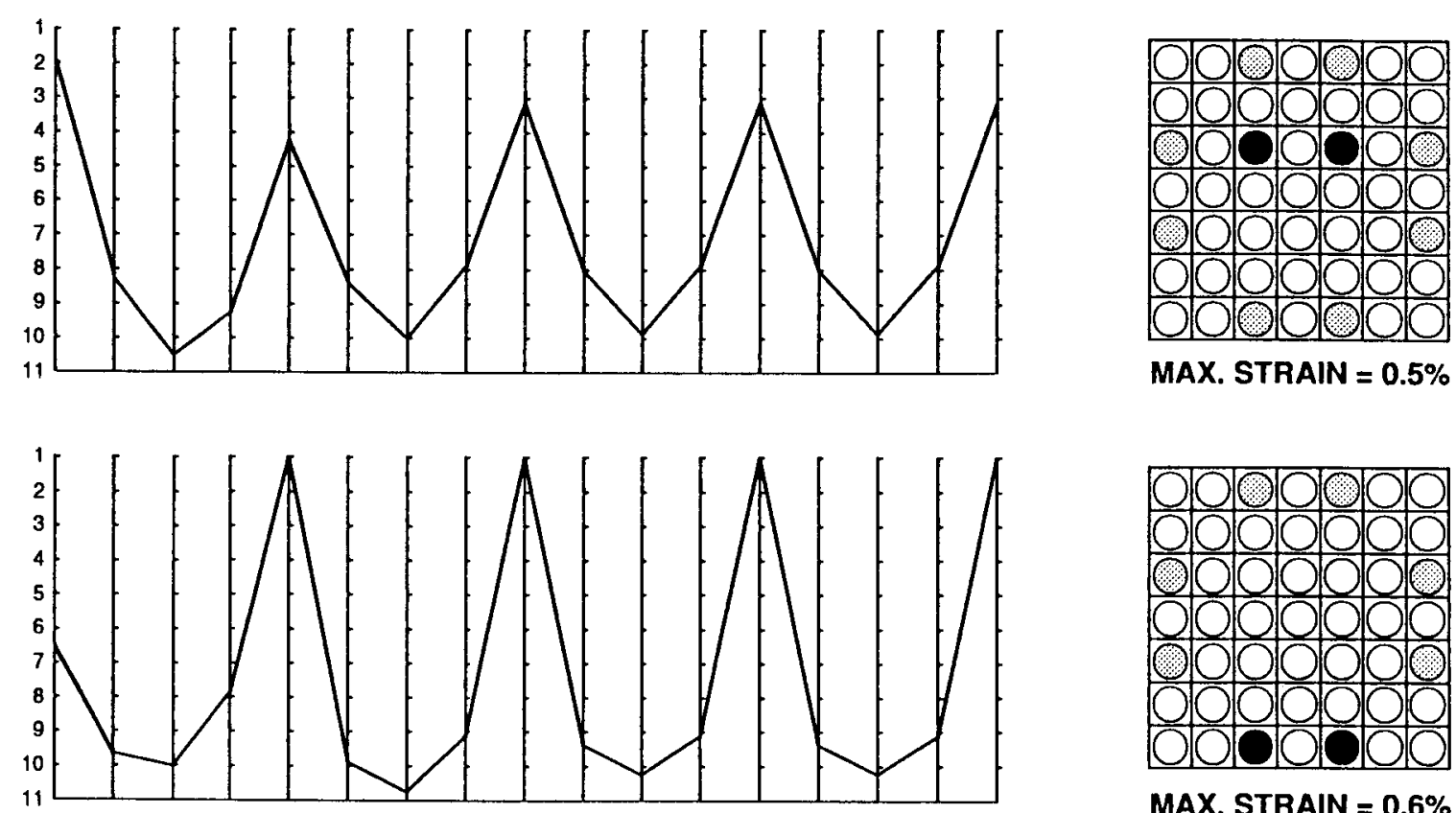

MAX. STRAIN $=0.5 \%$

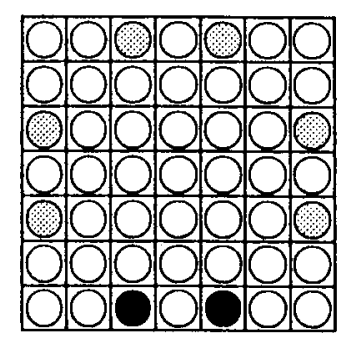

MAX. STRAIN $=0.6 \%$

Figure III-52. GE $7 \times 7$ BWR Side-Drop Two-Dimensional Assembly, 100-g Quasi-static Analysis: Longitudinal Outer Fiber Strain Profile Plots, Slice Model with Top and Bottom Tie Rods 


\section{LONGITUDINAL STRAIN AT BOTTOM SURFACE OF ROD}

$(\mathrm{m} / \mathrm{m})$

$-2.00 \times 10^{-3}$

$-1.10 \times 10^{-3}$

$-1.99 \times 10^{-4}$

$7.00 \times 10^{-4}$

$1.60 \times 10^{-3}$

$2.60 \times 10^{-3}$

$3.40 \times 10^{-3}$

$4.30 \times 10^{-3}$

$5.20 \times 10^{-3}$

$6.10 \times 10^{-3}$

$7.00 \times 10^{-3}$

$-3.00 \times 10^{-3}$

$-2.10 \times 10^{-3}$

$-1.20 \times 10^{-3}$

$-2.99 \times 10^{-4}$

$6.00 \times 10^{-4}$

$1.50 \times 10^{-3}$

$2.40 \times 10^{-3}$

$3.30 \times 10^{-3}$

$4.20 \times 10^{-3}$

$5.10 \times 10^{-3}$

$6.00 \times 10^{-3}$

$-3.00 \times 10^{-3}$

$-2.10 \times 10^{-3}$

$-1.20 \times 10^{-3}$

$-2.99 \times 10^{-4}$

$6.00 \times 10^{-4}$

$1.50 \times 10^{-3}$

$2.40 \times 10^{-3}$

$3.30 \times 10^{-3}$

$4.20 \times 10^{-3}$

$5.10 \times 10^{-3}$

$6.00 \times 10^{-3}$

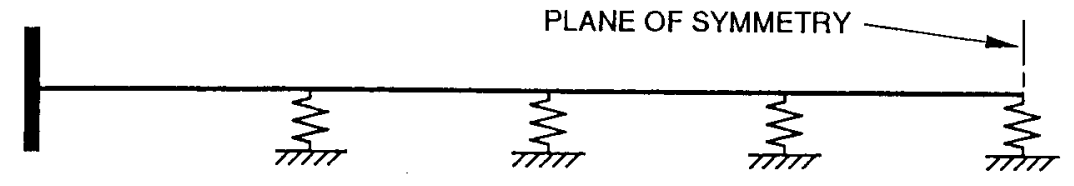

ROD LOCATION
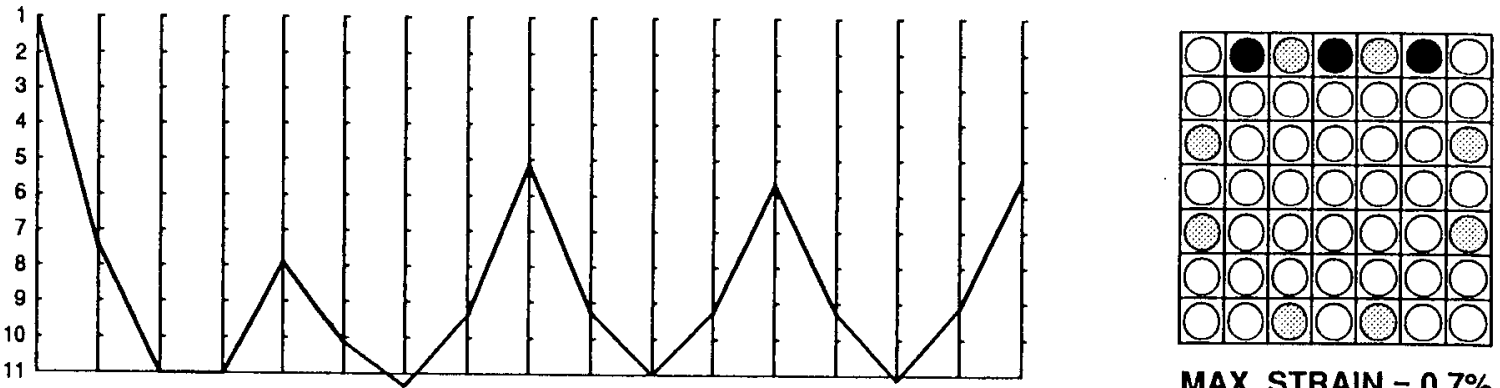

MAX. STRAIN $=0.7 \%$
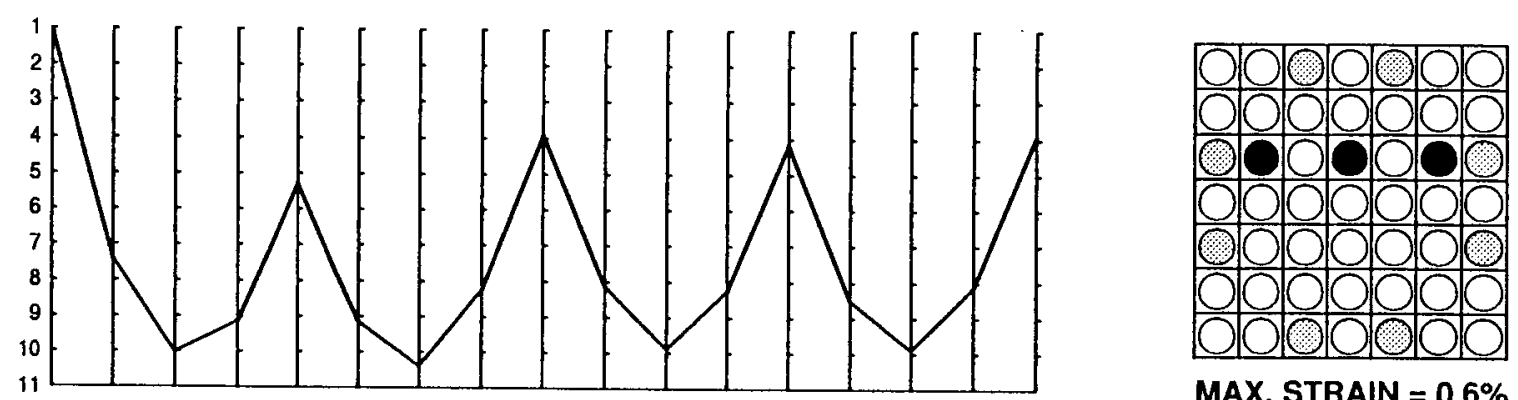

MAX. STRAIN $=0.6 \%$

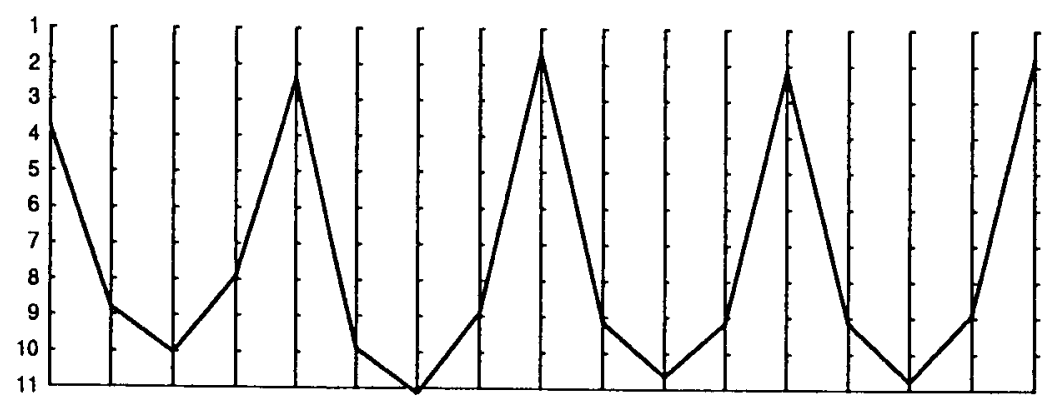

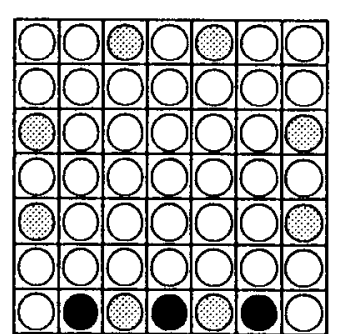

MAX. STRAIN $=0.55 \%$

Figure III-53. GE 7 × 7 BWR Side-Drop Two-Dimensional Assembly, 100-g Quasi-static Analysis: Longitudinal Outer Fiber Strain Profile Plots, Slice Model with No Tie Rods 


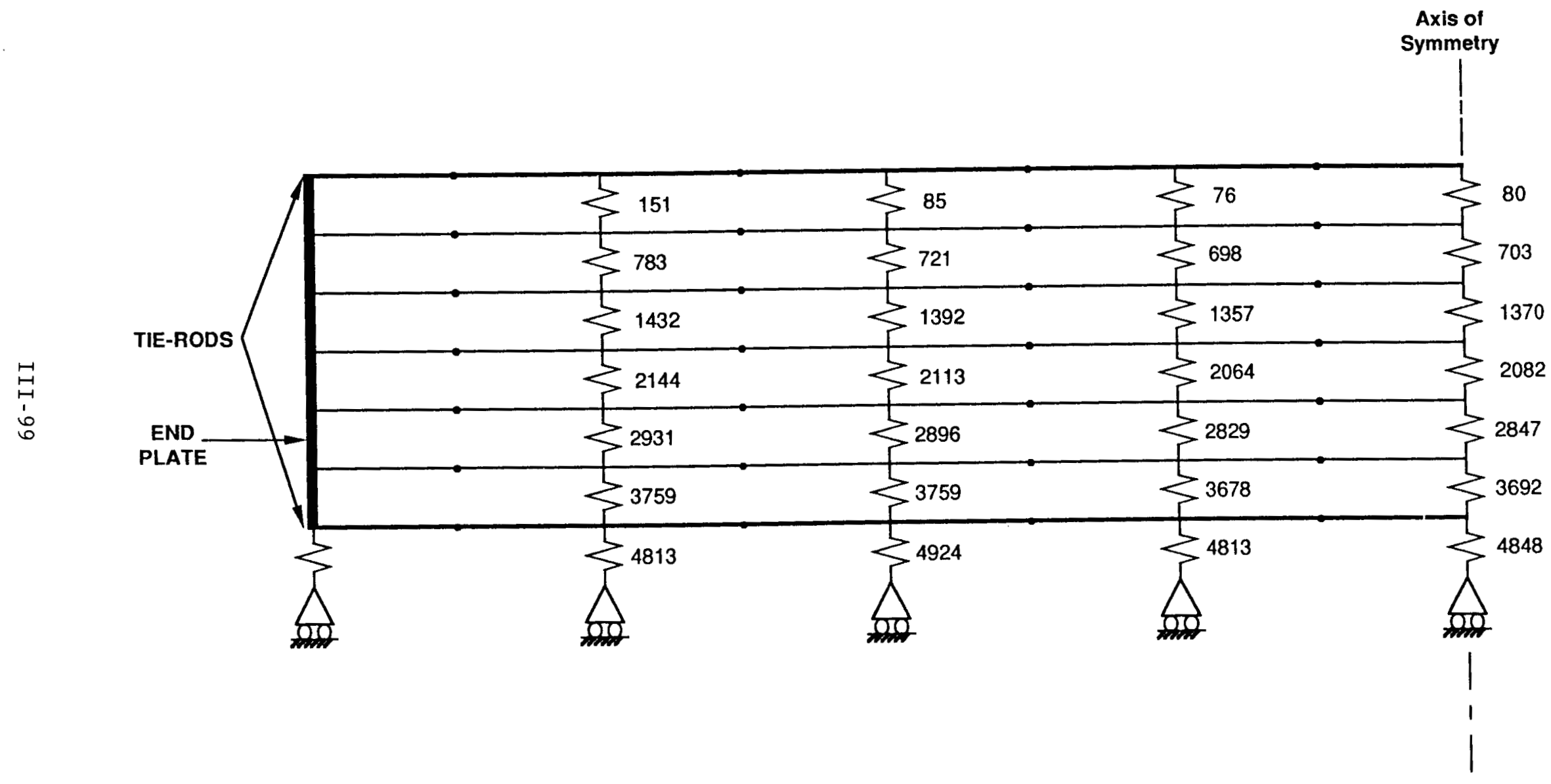

Figure III-54. GE $7 \times 7$ BWR Side-Drop Two-Dimensional Assembly, 100-g Analysis: Distribution of Spacer Grid Pinch Loads, Slice Model with Tie Rods Top and Bottom 
B\&W $15 \times 15$ PWR Side-Drop Quasi-static Analysis Results

In the $B \& W 15 \times 15$ quasi-static analysis, the two-dimensional slice models described in Figures III-22 and III-23 were used. The response of the PWR assembly under the regulatory accident loading of a $100-\mathrm{g}$ example for a lead-shielded truck cask is similar to the BWR response, as shown in Figure III-55. The three plots show the deflected shapes of the three assembly slice models: (1) slice with four control rods, (2) slice with two control rods, and (3) slice without any control rods. The control rods provide support similar to the support provided by BWR fuel assembly tie rods. The distribution of longitudinal outer fiber strains for the three different slice locations is similar to the distribution predicted by the BWR analysis. The maximum outer fiber tensile strain of 1.18 occurs in the top rod of the assembly slice without any control rods at the interface between the end plate and the rod. The magnitude of strains is slightly higher in the PWR assembly than in the BWR assembly because the PWR rods have a smaller bending stiffness.

The pinch force distribution for the PWR assembly is similar to the BWR analysis. Figure III-56 shows the pinch load distribution for the assembly slice with two control rods. For the assembly slice with two control rods, the maximum pinch load of $7188 \mathrm{~N}$ occurred in the spacer grid adjacent to the end plate.

\section{GE $7 \times 7$ BWR Side-Drop Dynamic Analysis Results}

Slightly modified versions of the GE $7 \times 7$ BWR assembly twodimensional slice models that were used for the static analyses are used in the side-drop dynamic analyses. The basket support points are replaced with springs that have zero tension stiffness and are very stiff in compression. These supports are linked to provide a mechanism for applying the cask impact limiter deceleration loading.

Because maximum response is reached in a short time (before damping forces can absorb much energy from the structure), only a small amount of numerical damping was introduced to improve the solution time. Numerical damping causes a maximum of 58 energy loss in each analysis. Damping forces neglected by the solution include the effects of rod-to-rod friction and the plastic crushing of the spacer grid frames.

The dynamic analysis is modeled with an initial velocity applied to all model components and a retarding deceleration-force time history applied to the basket support points. The initial velocity $(13.4 \mathrm{~m} / \mathrm{s})$ corresponds to the $9.0 \mathrm{-m}$ free drop of the regulatory accident condition. The retarding force is equal to the assembly mass (the modeled portion) multiplied by the deceleration history for the $9.0-\mathrm{m}$ side drop. Figure III- 8 shows the deceleration pulse shape of the cask. The example cask limiters reach a maximum deceleration of $100 \mathrm{~g}$ in $0.018 \mathrm{~s}$. For these example analyses, the lateral gap between the assembly and the basket is neglected. However, large lateral gaps between the assembly and basket may increase the assembly response. A free-body diagram of the assembly sidedrop dynamic analysis is also shown in Figure III-8. 


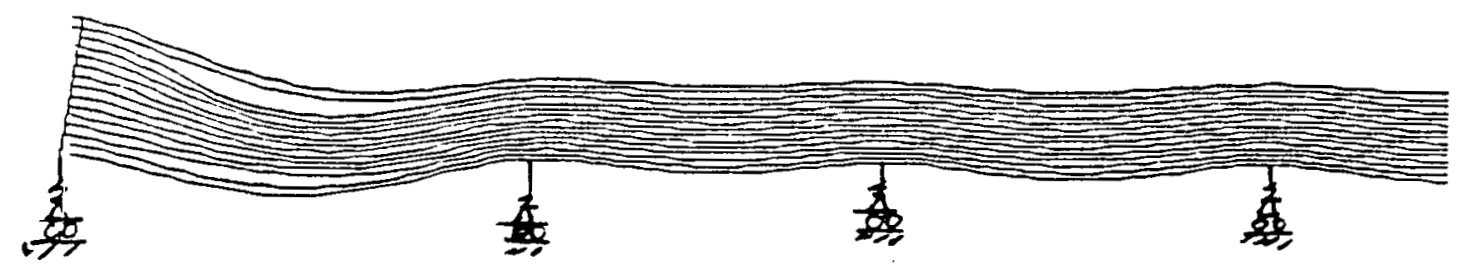

\section{B\&W 15X15 PWR - SLICE WITH FOUR TIE-RODS - SIDE DROP}

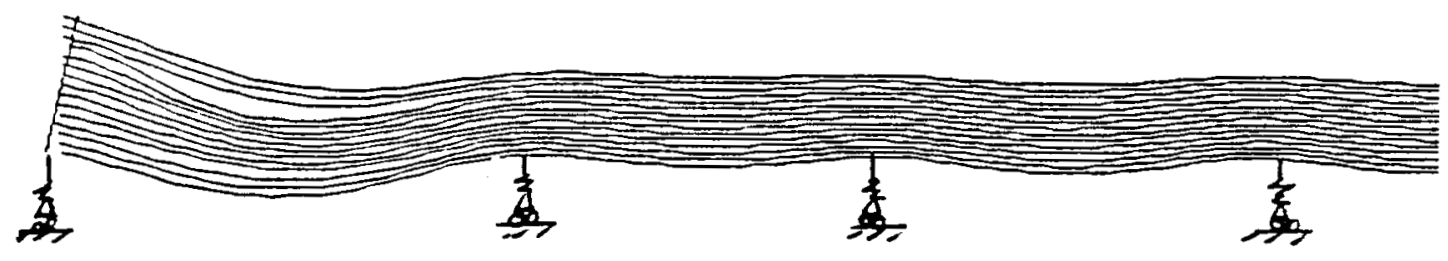

B\&W 15X15 PWR - SLICE WITH TWO TIE-RODS - SIDE DROP

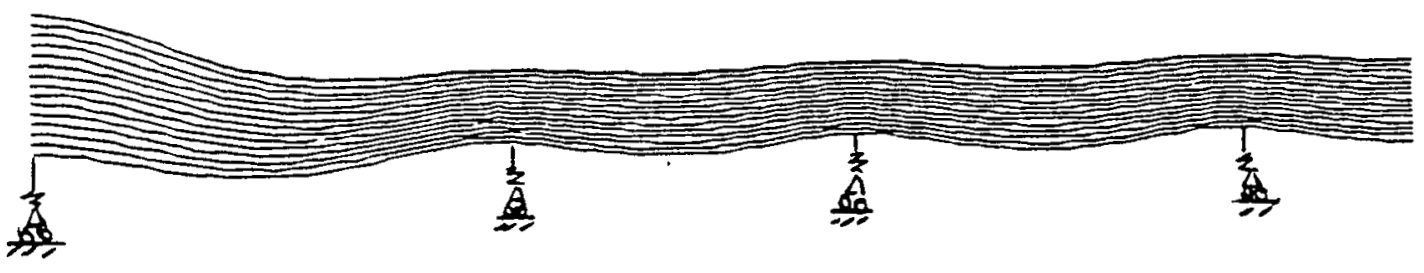

B\&W 15X15 PWR - MID SLICE WITHOUT CONTROL RODS - SIDE DROP

Figure III-55. B\&W $15 \times 15$ PWR Side-Drop, Two-Dimensional Assembly, 100-g Quasi-static Analysis: Deflected Shape Plots 


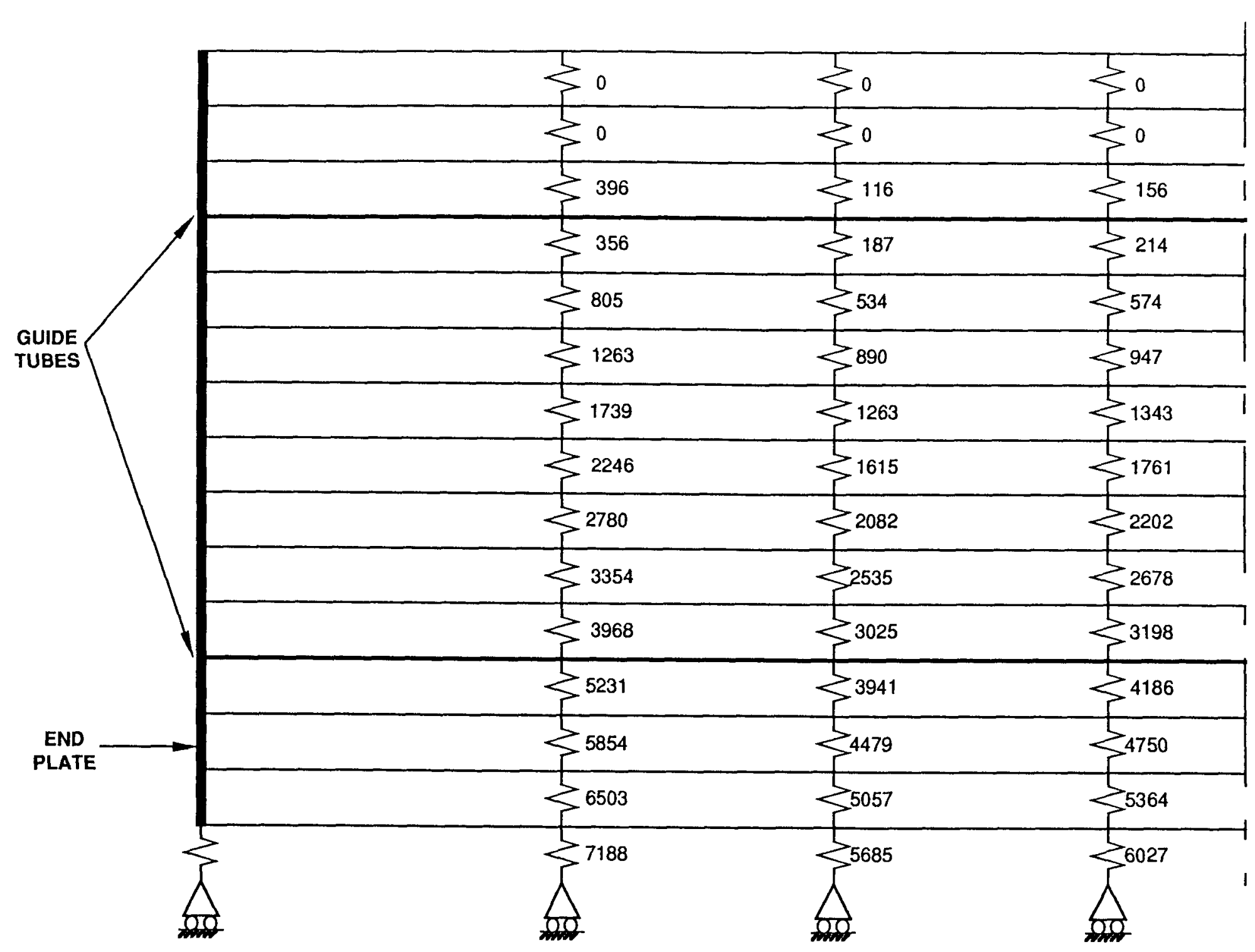

Axis of Symmetry

Figure III-56. B\&W 15 × 15 PWR Side-Drop, Two-Dimensional Assembly, 100-g Static Analysis: Distribution of Spacer Grid Pinch Loads, Slice Model with Tie Rods Top and Bottom 
Figure III-57 illustrates the deflected shapes of the three GE $7 \times 7$ side-drop slice models when the downward displacement of each assembly is at its maximum value. This occurs at approximately the end of the cask and assembly deceleration. This also corresponds to the time when the retarding force is maximized. In each of the three assembly slice models, the maximum deflection occurs in the top fuel rod midspan between the second and third spacer grids (measured from either end plate). The maximum deflection of the assembly is $4.06 \mathrm{~cm}$ in the slice with top and bottom tie rods.

The maximum tensile strain for all three BWR slice models is 1.18 . This peak strain occurs in the assembly bottom rod at the first two spacer grid locations in the slice with two interior tie rods. The maximum pinch force is $7562 \mathrm{~N}$, which also occurs at the first spacer grid location of the bottom rod in this slice. Figures III-58 and III-59 show, respectively, the maximum tensile strain and the maximum pinch force time histories for the example assembly. The jaggedness of the time history curves is a result of the coarseness of the finite element model.

Figure III-60 shows the maximum tensile strain distribution in the fuel rods at the spacer grids and midspan between them for the assembly slice with two interior tie rods. Figure III-60 also shows the corresponding maximum rod pinch forces at the spacer grids. Note that the maximum values in Figure III-60 do not occur at the same time; therefore, combining these maxima to produce failure probabilities (see Section III.6) is a conservative approach.

\section{B\&W $15 \times 15$ PWR Side-Drop Dynamic Analysis Results}

Similar to the BWR analysis, the two-dimensional assembly dynamic slice models for the $B \& W 15 \times 15$ PWR are slightly modified versions of the static models. Compression-only springs are used to model the basket support points. The dynamic models also include the additional spacer grid springs $12.7 \mathrm{~cm}$ away from the end plates. These springs were conservatively neglected in the static models. The effects of assembly and basket gaps are neglected, and the structural damping is applied to the PWR models in the same manner as described for the example BWR assembly analysis.

The deflected shapes of all three PWR slice models at the time of maximum downward displacements are shown in Figure III-61. In the slice with four control rods, the third fuel rod from the top has a maximum deflection of $6.10 \mathrm{~cm}$ midspan between the first and second spacer springs. In the other two slice models, the top fuel rod midspan between the first and second springs has maximum deflection. The controlling deflection is $6.60 \mathrm{~cm}$ in the slice with two control rods.

The assembly slice without any control rods produces the highest tensile strain: 3.38. This maximum strain occurs adjacent to the end plate in both the top fuel rod and third fuel rod from the top. The maximum rod pinch force of $9265 \mathrm{~N}$ occurs at the second spacer grid in the bottom fuel rod for the slice models with two and four control rods. The 

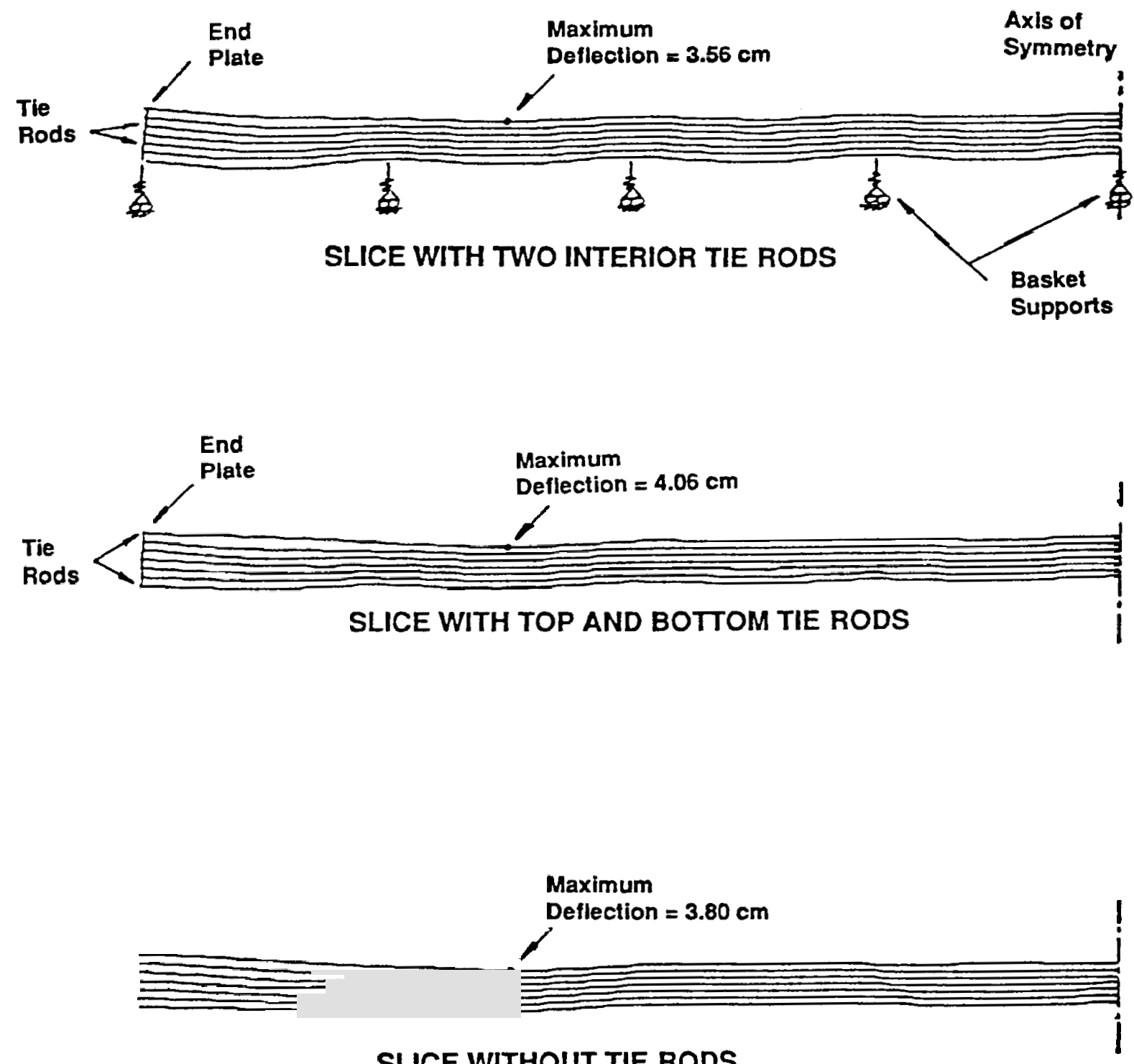

SLICE WITHOUT TIE RODS

Figure III-57. GE $7 \times 7$ BWR 9.0-m Side-Drop Dynamic Analysis: TwoDimensional Slice Model Deflected Shapes 


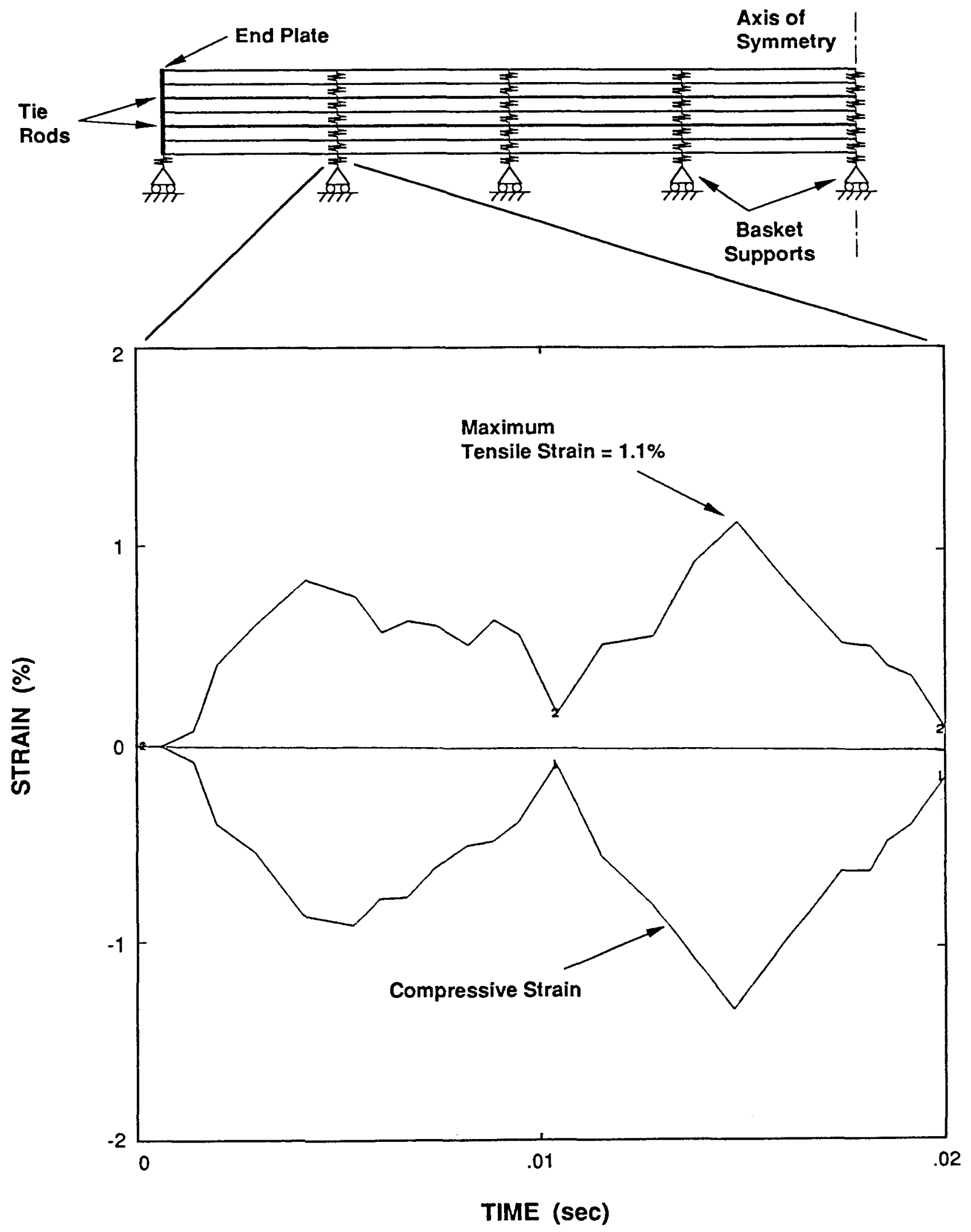

Figure III-58. GE $7 \times 7$ BWR 9.0-m Side-Drop Dynamic Analysis: Maximum Tensile Strain Time History 


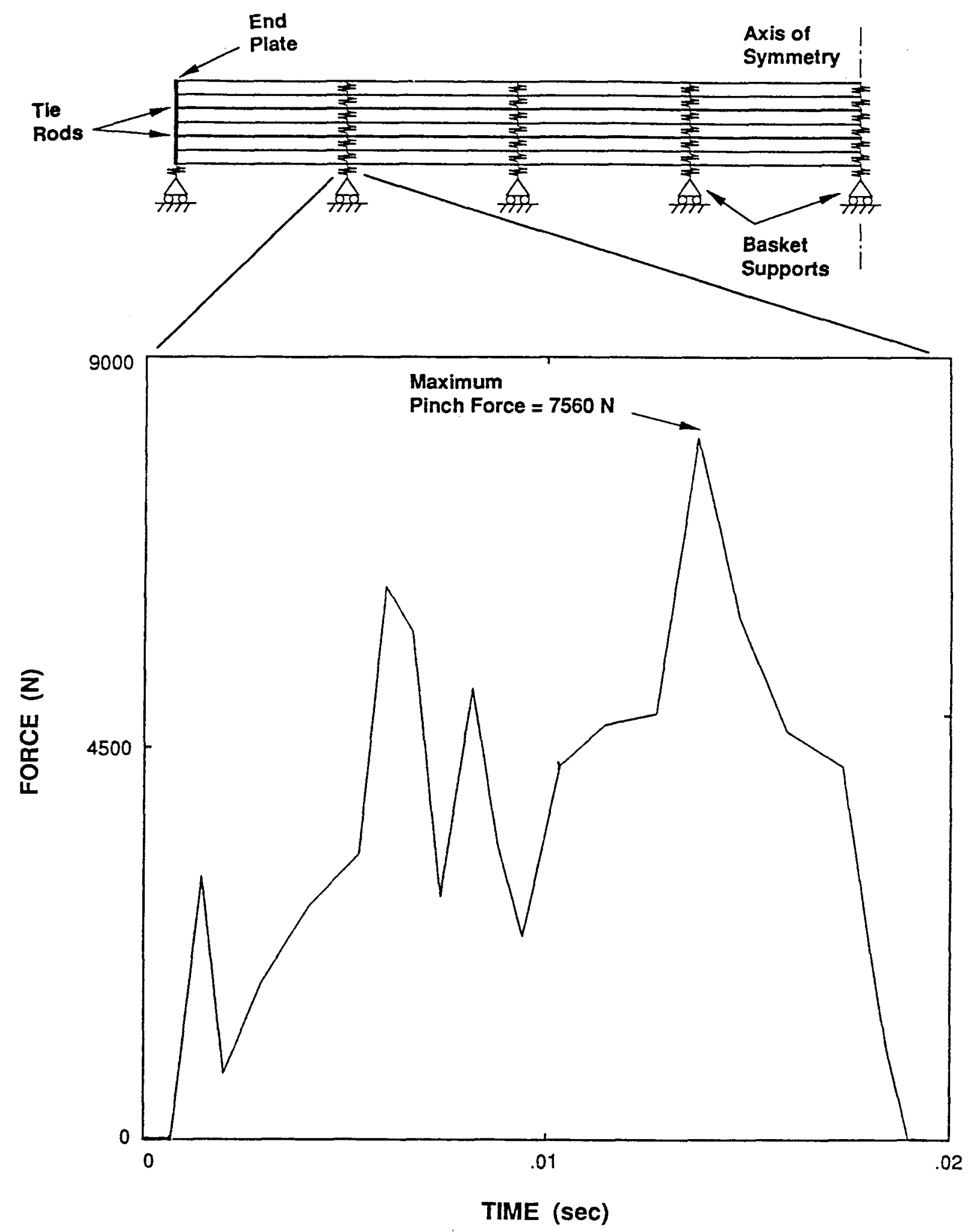

Figure III-59. GE $7 \times 7$ BWR 9.0-m Side-Drop Dynamic Analysis: Maximum Pinch Force Time History 


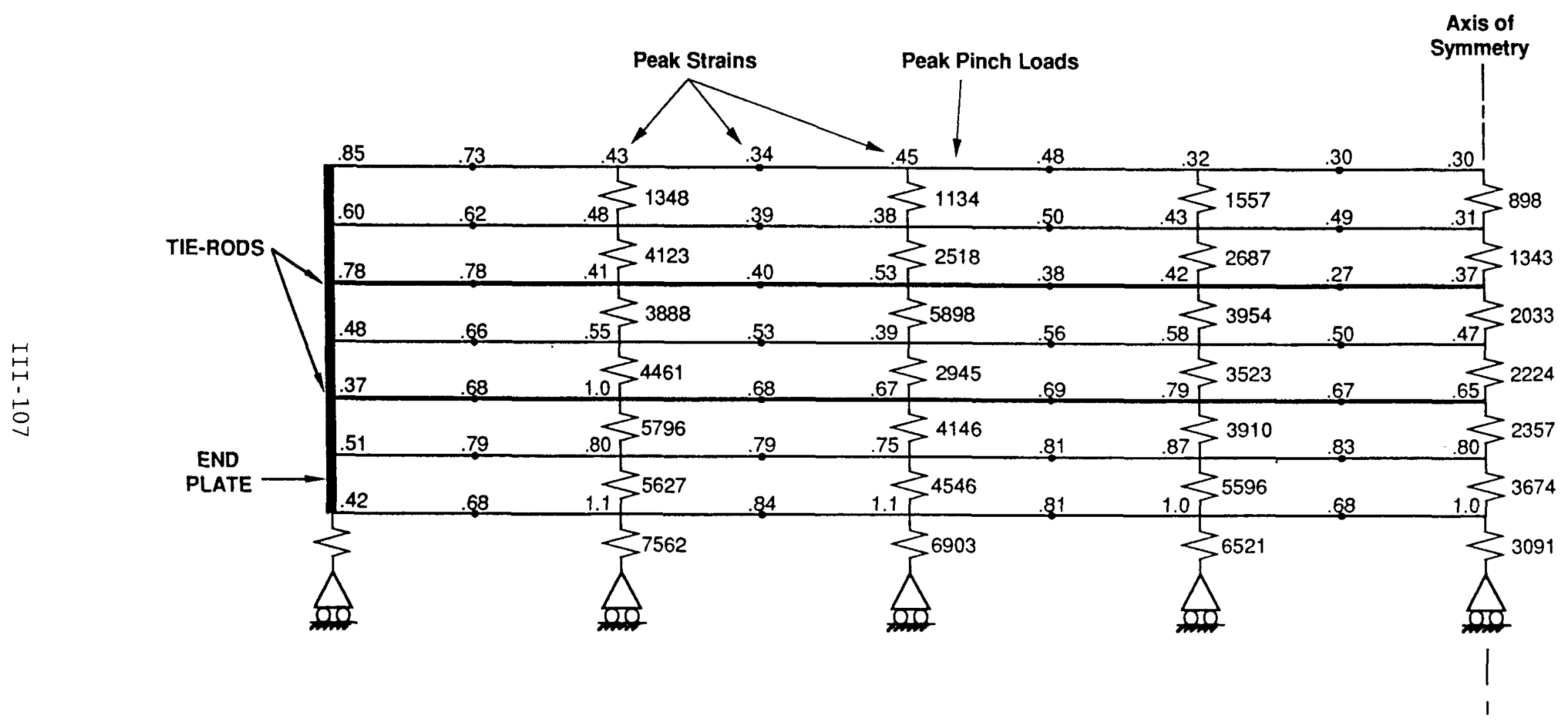

Figure III-60. BWR Example Assembly GE $7 \times 7$ S1ice Model with Two Interior Tie Rods: Summary of Peak Tensile Strains and Rod-to-Rod Pinch Forces (Note: Strains listed in Percentage and pinch forces listed in $\mathrm{N}$ do not occur at the same time.) 

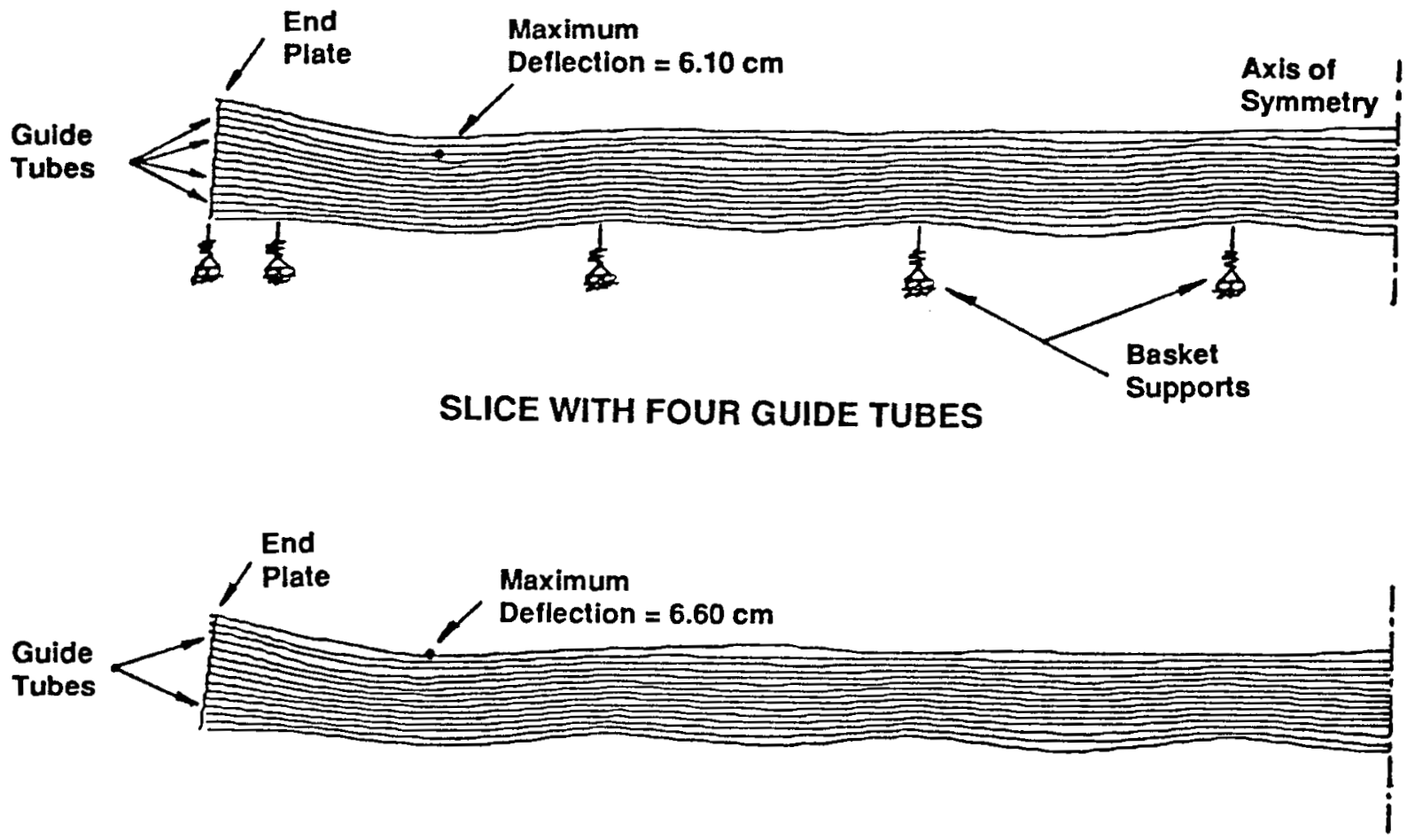

SLICE WITH TWO GUIDE TUBES

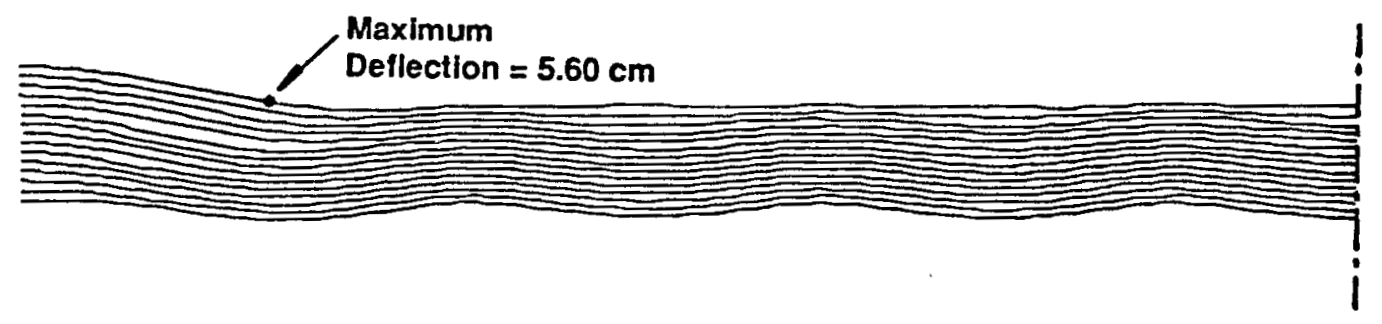

SLICE WITHOUT GUIDE TUBES

Figure III-61. B\&W $15 \times 15$ PWR 9.0-m Side-Drop Dynamic Analysis: TwoDimensional Slice Model Deflected Shapes 
corresponding maximum tensile strain is 1.38 in both slice models. Figures III-62 and III-63 show the maximum pinch force and its corresponding tensile strain time history for the assembly slice model with four control rods.

Figure III-64 shows the maximum tensile strain distribution at selected locations and the maximum pinch forces at the spacer grid locations for the slice with four control rods. These maxima do not occur at the same time; thus, any combination produces conservative results. Probabilistic evaluations of these example dynamic analyses are presented in Section III. 6.

\section{III.5.5 Hypothetical Accident End Drop}

In addition to rigorous time history dynamic analyses, the end-drop impact is characterized by buckling analyses, quasi-static collapse analyses, and sensitivity analyses of various design parameters. The response of fuel assemblies under end-drop loading conditions was analyzed both statically and dynamically. Static analyses are initially performed to estimate the end-drop response; however, collapse occurs at loads significantly less than the example regulatory accident load. Thus, buckling analyses were used to better characterize the assembly response under end-drop loading. These analyses provide a means of determining sensitivity of the buckling response with respect to active fuel mass.

Quasi-static analyses were then performed to further quantify the stress state of the fuel at the static collapse load limit. Finally, dynamic analyses were used to quantify the effects of fuel rod bowing and vertical gaps between fuel assemblies and the cask.

\section{Buckling Analysis}

Elastic buckling analyses of the example GE $7 \times 7$ BWR and B\&W $15 \times 15$ PWR assemblies are described here. Single-rod models with maximum initial bowing (see Figure III-24) were used to perform these analyses.

The models conservatively neglect the tensile and bending stiffness of the fuel rod pellets. For the buckling analysis, only the percentage of fuel pellet mass attached to the cladding is included in the fuel response model. Mass not connected to the cladding is assumed to be supported independently of the fuel rod cladding; $75 \%$ of the PWR fuel mass and 10\% of the BWR fuel mass are used in the analyses. The basis of these estimates is discussed in Appendix I.

Figure III-65 shows the buckling mode shapes of the example BWR and PWR assemblies. The small buckling strength of the PWR assembly ( $40 \mathrm{~g}$ for the $B \& W 15 \times 15$ assembly) indicates that under end-on impact loading conditions, large lateral displacements of PWR fuel assemblies will occur.

In addition to an evaluation of the buckling loads of the example assemblies, sensitivity analyses of the effects of fuel pellet mass are presented here. These analyses indicate that variations in effective fuel pellet mass can cause large variations in the fuel rod critical buckling load. For the BWR example assembly, the buckling load varies from 220 to $54 \mathrm{~g}$ based on 10 to $100 \%$ of the fuel pellet mass being active, and for the 


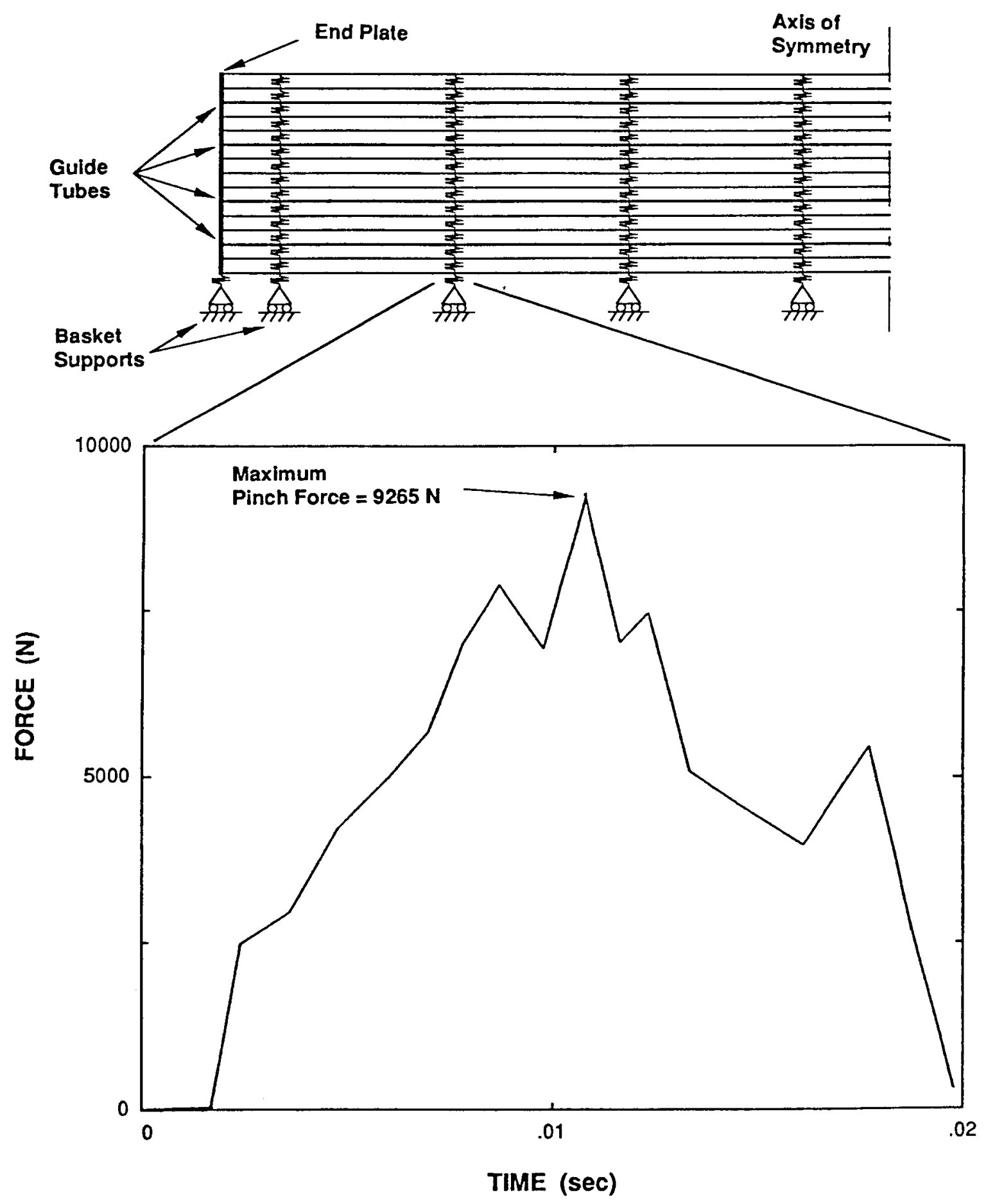

Figure III-62. B\&W $15 \times 15$ PWR 9.0-m Side-Drop Dynamic Analysis: Maximum Pinch Force Time History 


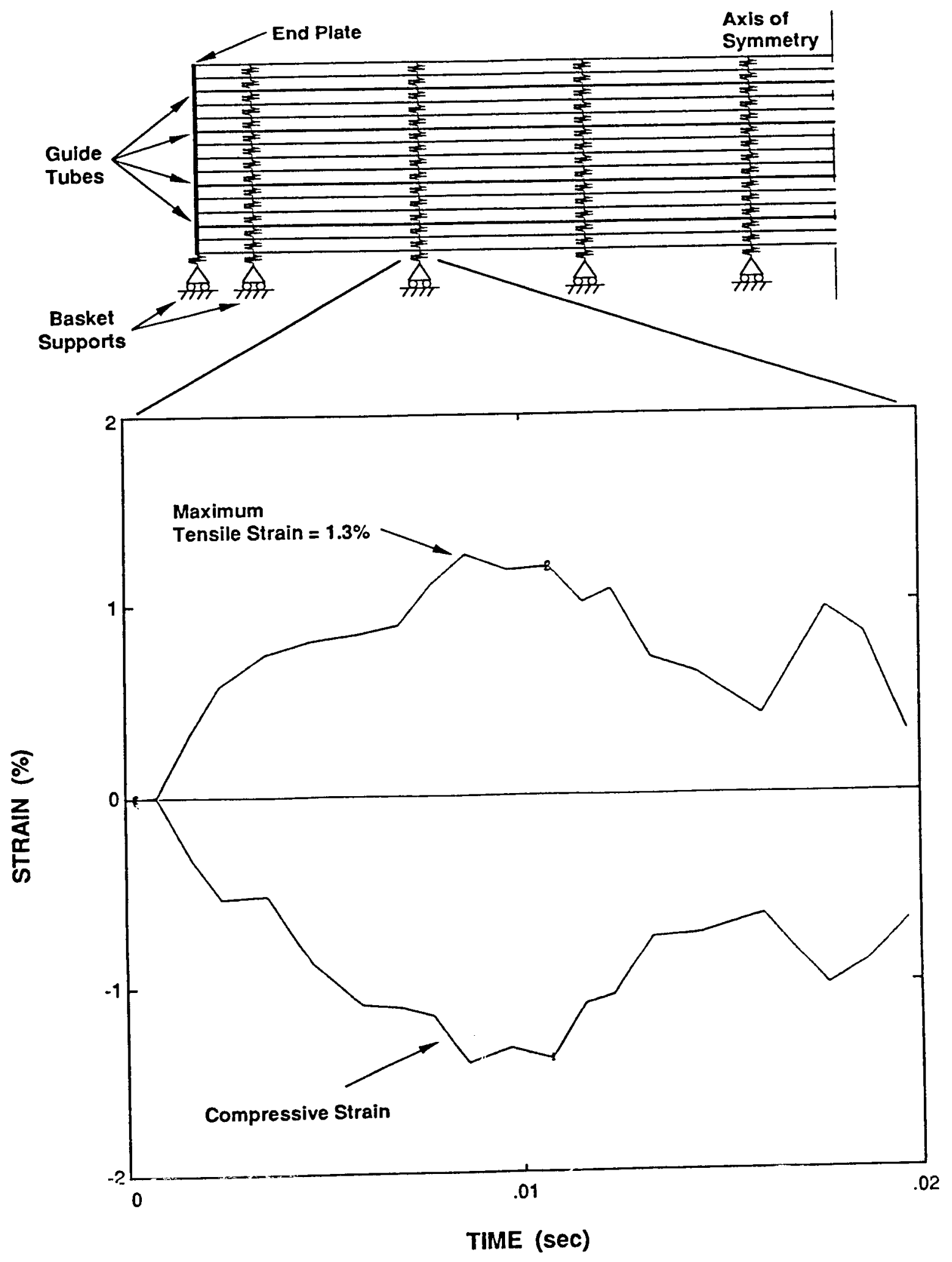

Figure III-63. B\&W $15 \times 15$ PWR 9.0-m Side-Drop Dynamic Analysis: Maximum Tensile Strain Time History 


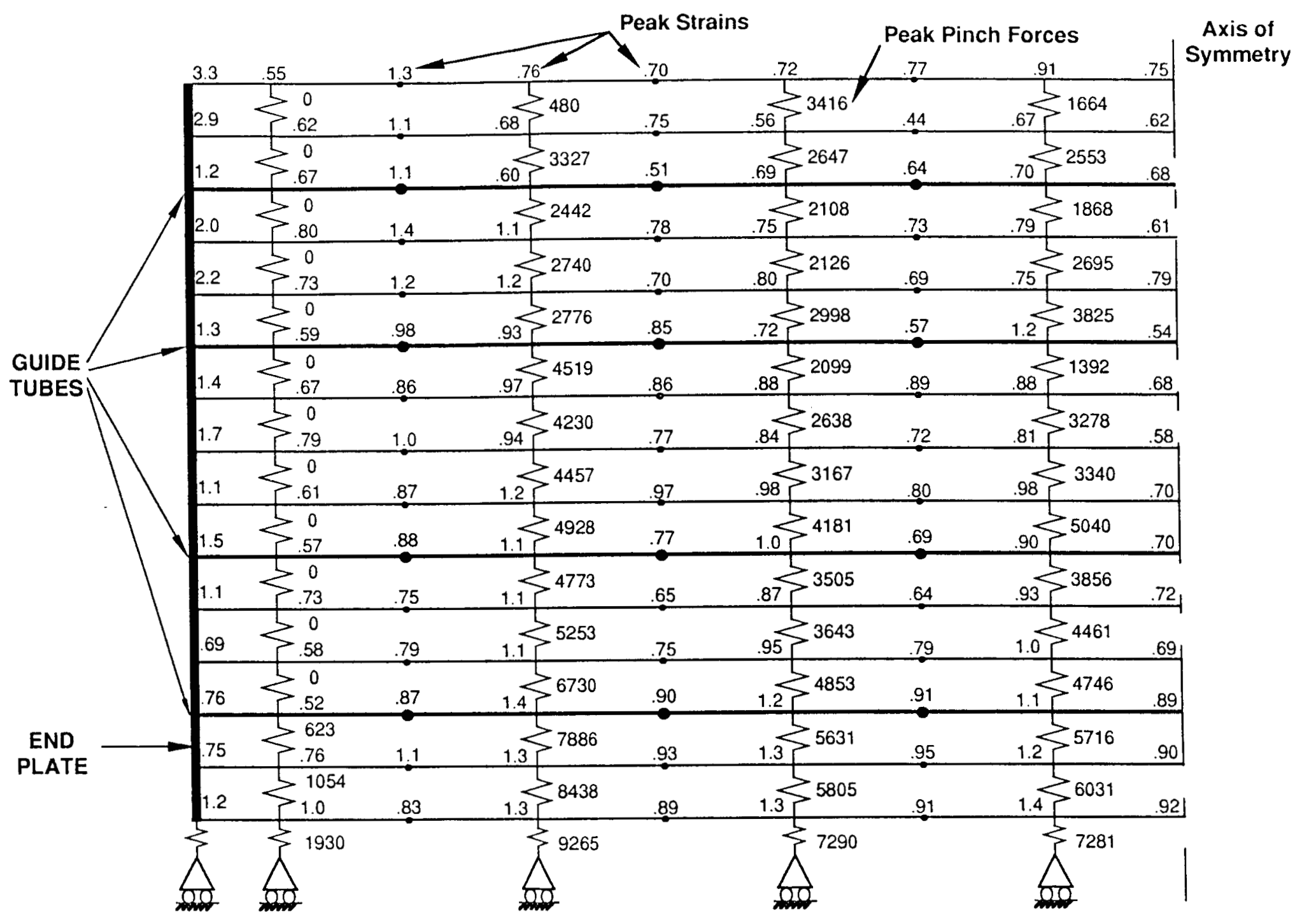

Figure III-64. PWR Example Assembly B\&W $15 \times 15$ Slice Model with Four Interior Control Rods: Summary of Peak Tensile Strains and Rod-to-Rod Pinch Forces (Note: Strains listed in 8 and pinch forces in $\mathrm{N}$ do not occur at the same time.) 

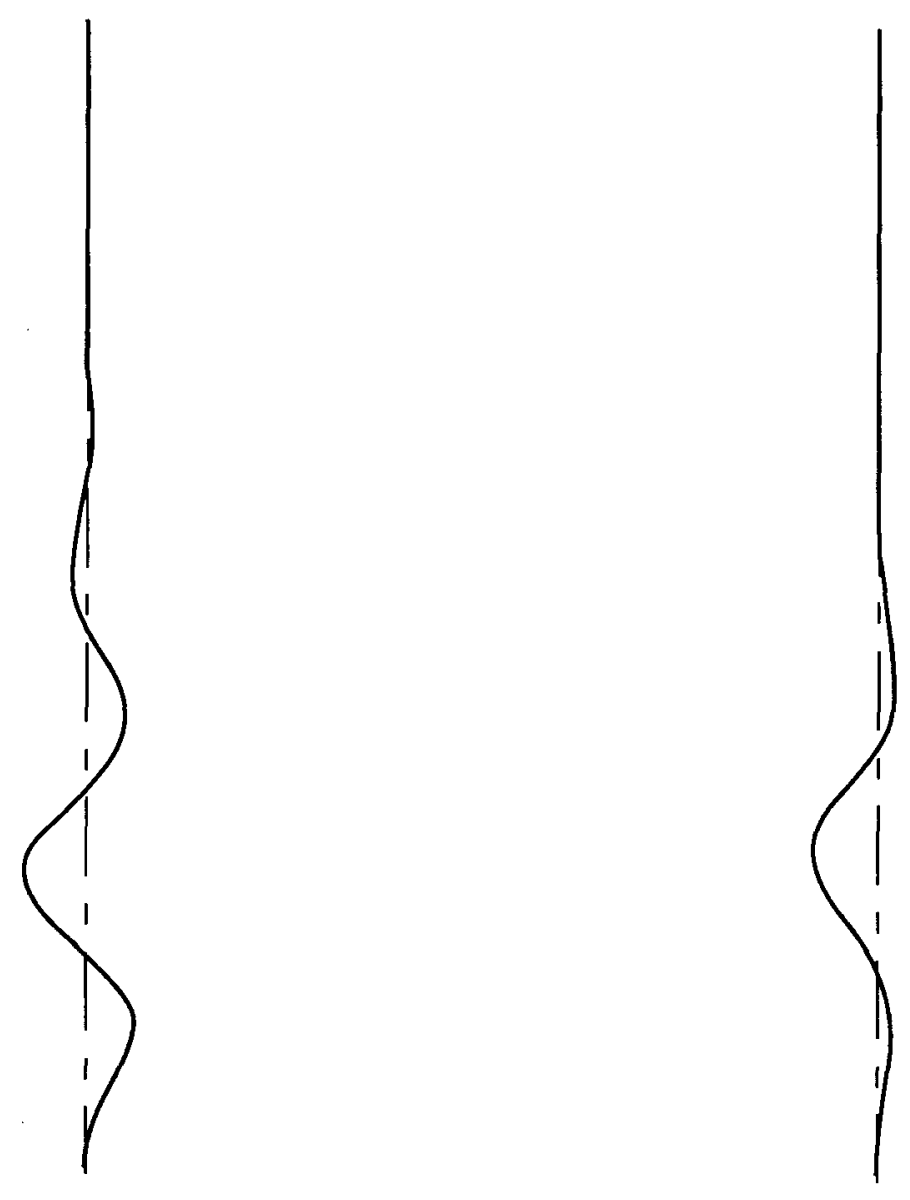

\title{
B\&W 15X15 PWR BUCKLING MODE SHAPE
}

\section{GE 7X7 BWR BUCKLING MODE SHAPE}

\author{
Figure III-65. B\&W $15 \times 15$ PWR and GE $7 \times 7$ BWR End-Drop Buckling Mode \\ Shapes
}

PWR example assembly, the buckling load varies from 40 to $32 \mathrm{~g}$ for 75 to 1008 active fuel mass. Although exceeding the static buckling load does not indicate that fuel rod failure will occur, it does have a significant effect on the probability of such failure. Figure III-66 illustrates the variation in static buckling load versus percentage of active fuel mass. Again, active fuel mass is assumed to add weight without increasing rod stiffness . 


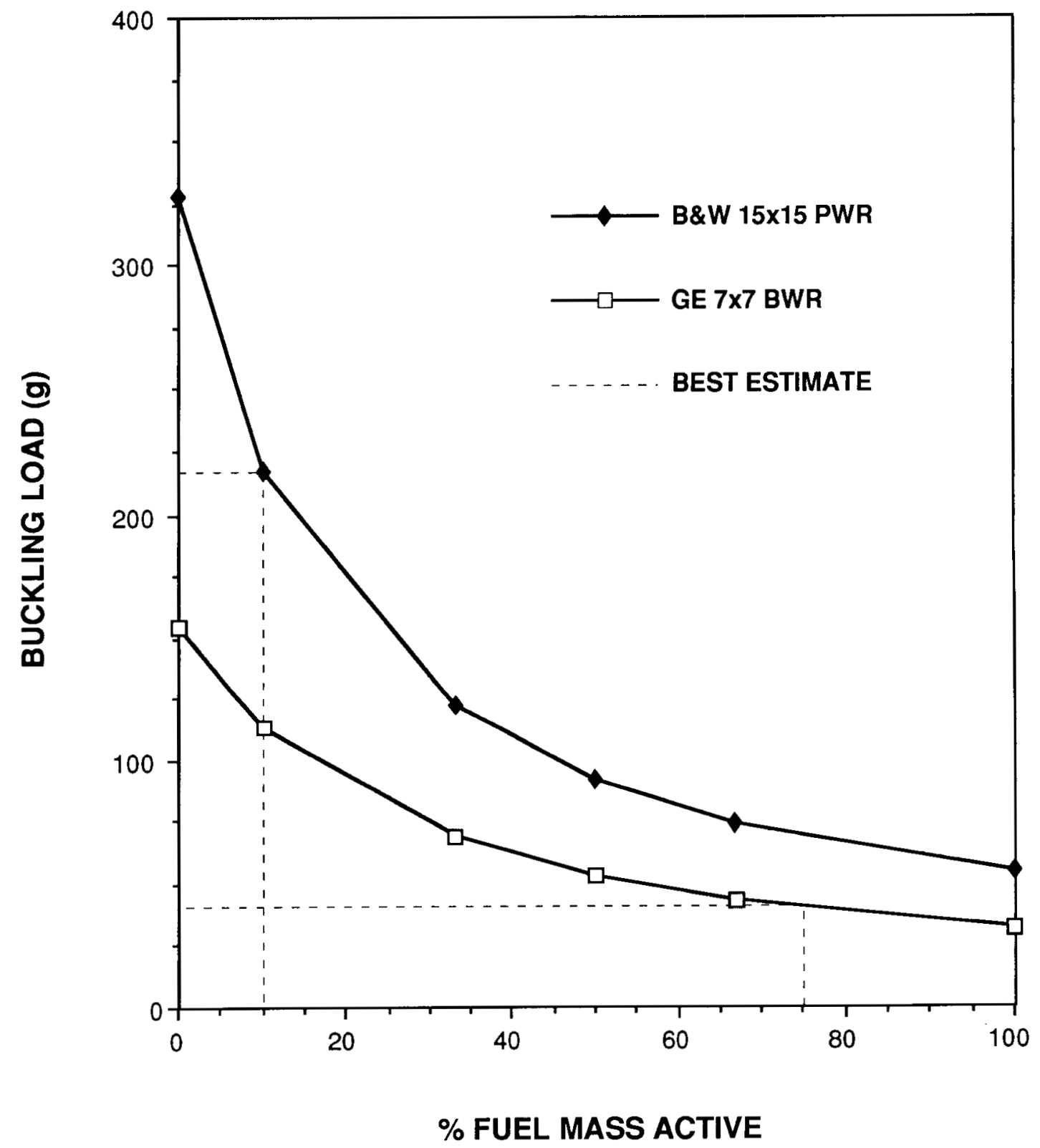

Figure III-66. Example Assemblies: Sensitivity of Buckling Load Versus Percentage of Fue1 Mass Active

\section{Quasi-static Analysis}

To further quantify the axial load capacity of the fuel assemblies, quasi-static collapse analyses were performed with single-rod models similar to those used in the buckling analysis. An incremental solution procedure is used to determine a relationship between the lateral deflections of fuel rods and the corresponding bending strains in the cladding. The magnitude of the tensile bending strain is used as a basis for the failure criterion (discussed in Section III.4). 
The rods are statically analyzed for a linearly ramped acceleration loading from zero to the static collapse load, or $100 \mathrm{~g}$ (end-drop deceleration of the example cask as described in Section III.3.2). Again, the models with the maximum rod bowings are analyzed. The rod weights include only the active fuel mass portions--75\% and 108 of the total fuel mass, respectively, for the example PWR and BWR rods.

As expected from the buckling analysis, the BWR rod does not collapse and remains fully elastic up to a maximum loading of $100 \mathrm{~g}$. However, the PWR rod inelastically collapsed at a loading of $37 \mathrm{~g}$. The deflected shapes at these loadings are shown in Figure III-67. The lateral deflections are greatly distorted for each assembly for clarity; however, both the BWR and PWR assemblies are distorted by the same magnitude. Using the same distortion scale allows a visual comparison between the two analyses.

A summary of the analysis results is presented in Figure III-68 for the PWR and BWR rods. Figure III-68 shows the relationship between the maximum lateral displacement (either positive or negative) and the maximum positive strain of the example assemblies plotted against the applied load in $g$. The location of maximum positive strain coincides with the location of maximum lateral displacement that occurs midway between the first and second spacer grids from the rod bottom, as indicated in Figure III-67. For the PWR model, the positive strain of 88 occurs when the maximum lateral displacement is $-12.2 \mathrm{~cm}$. However, it is not possible for a PWR rod in an assembly with continuous basket supports to deflect $12.2 \mathrm{~cm}$. A conservative estimate of $5.08 \mathrm{~cm}$ maximum lateral displacement corresponds to $0.9 \%$ tensile strain at $37 \mathrm{~g}$, which is less than the collapse load. The $5.08 \mathrm{~cm}$ limit on the lateral displacement will greatly increase the collapse load from $37 \mathrm{~g}$. Dynamic analyses are used to investigate these lateral displacements under the full $100-\mathrm{g}$ loading to obtain a more accurate solution for both assemblies.

\section{Dynamic Analysis}

In addition to providing detailed response of the example assemblies, dynamic analyses are used to investigate the sensitivity of the response to variations in vertical gaps between the assembly and basket, fuel rod bowing, and lateral displacement constraints. All of the analyses used are variations of the single-rod model discussed in Section III.3.4.

An automatic time-step solver is used for the dynamic analyses. A small amount of time-step-dependent numerical damping is introduced to improve the stability of the solution. During the analysis, the amount of damping varies as the time step changes: the larger the time step, the higher the damping. However, the amount of damping never exceeds $1.6 \%$ of the structure critical damping. This is very small in comparison with the natural damping caused by events such as crushing of assembly appurtenances, friction, and so on. No other damping is input into the analyses; therefore, the results are conservative.

The sensitivity of two conditions of assembly-cask vertical gap interaction was evaluated: (1) a no-gap condition in which the bottom of the rods remains in contact with the cask during the impact event and 


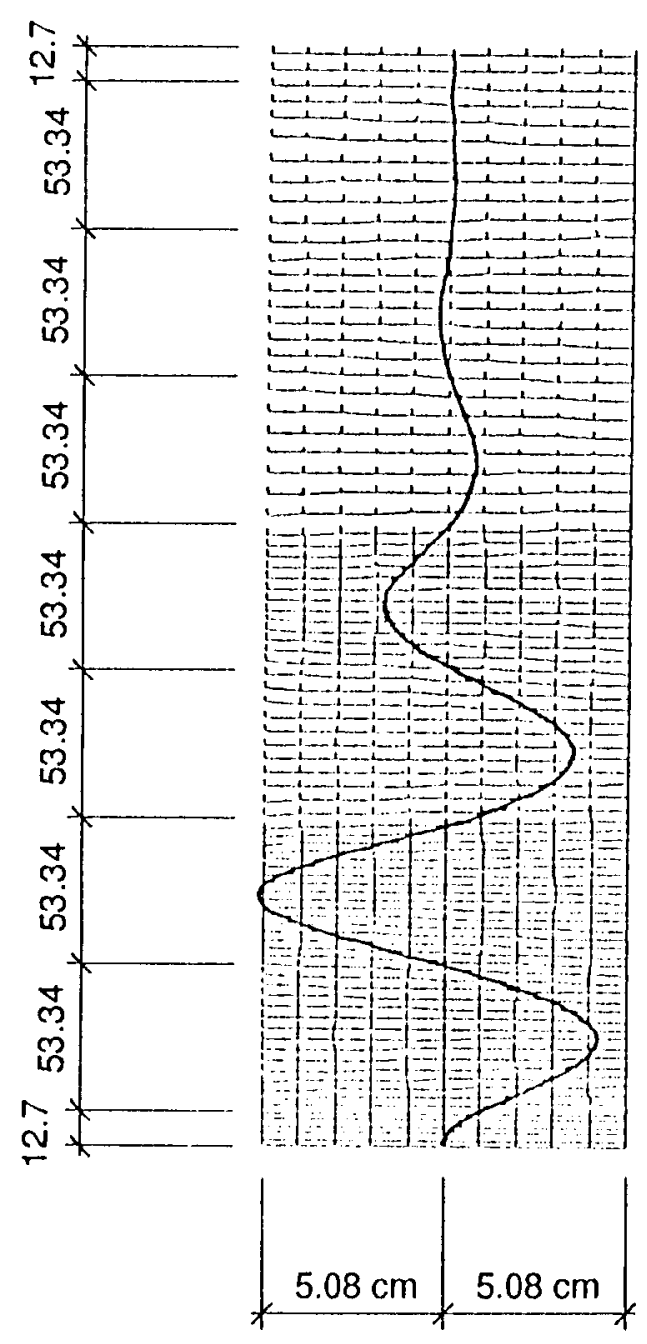

\section{B\&W 15x15 PWR Loading $=37 \mathrm{~g}$}
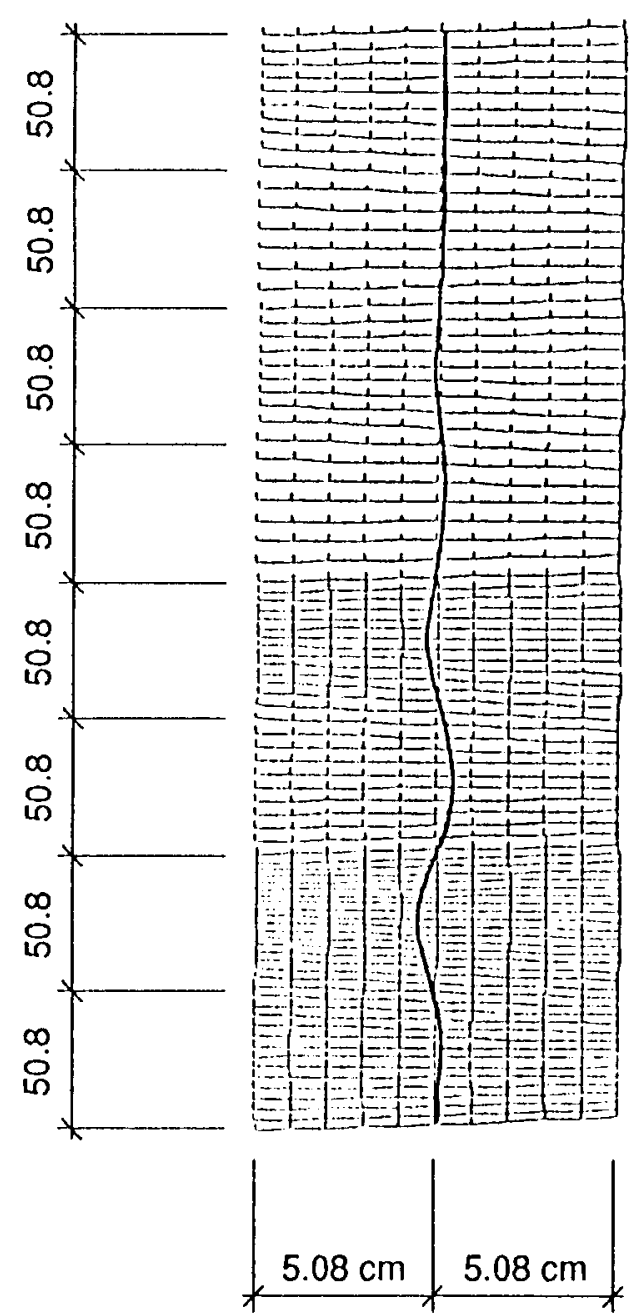

GE 7×7 BWR Loading $=\mathbf{1 0 0} \mathrm{g}$

Figure III-67. End-Drop Quasi-static Collapse Analyses: Deflected Shape Plots

(2) a gap condition in which there is a vertical gap between the rods and the cask, but in which once the gap is closed, the rods remain in contact with the cask.

In the no-gap condition, the rods decelerate with the cask and are therefore subjected to an initial velocity of $13.4 \mathrm{~m} / \mathrm{s}$ (for a 9.0 -m drop) and a retarding force pulse at their bottom equal to the deceleration history of the impact limiter multiplied by the assembly mass. This force pulse has an area equal to the rod momentum as a result of the $13.4 \mathrm{~m} / \mathrm{s}$ velocity and a peak constant acceleration loading of $100 \mathrm{~g}$ (the $9.0-\mathrm{m}$ end 

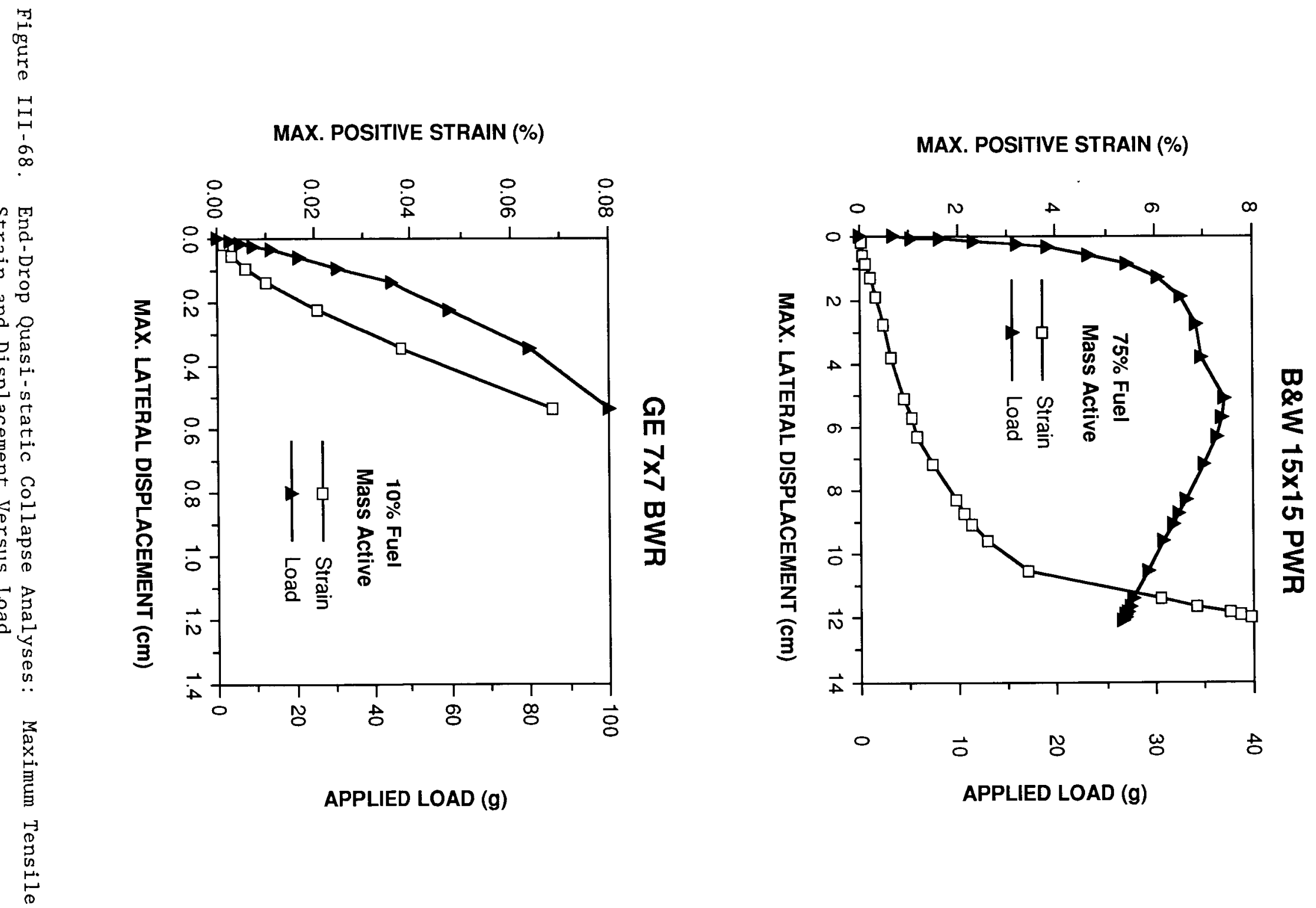
drop constant deceleration of the example cask). The acceleration loading pulse is shown in Figure III-9.

In the gap condition, the rods impact the cask after the cask begins to decelerate; after impact they are assumed to continue to decelerate similar to the cask. The rod/cask impact force pulse depends on the relative velocity of the rods with respect to the velocity of the cask, which is a function of the vertical gap size.

Three vertical gap sizes, $2.54,5.08$, and $7.62 \mathrm{~cm}$, are analyzed to determine the sensitivity of gap size. (A $2.54-\mathrm{cm}$ gap is most representative of current industry practice.) The corresponding rod/cask impact relative velocities of $1.83,3.66$, and $5.49 \mathrm{~m} / \mathrm{s}$ are analyzed to determine the rod/cask impact pulse. The relative velocity rigid-surface impact analyses show that there is a linear relationship among the pulse shapes of each PWR and BWR rod type. This linear relationship occurs because these responses remain elastic, except for a PWR rod with a $7.62-\mathrm{cm}$ gap. This case results in a very small amount of inelastic strain. The retarding force distribution for all analyzed relative velocity impacts is in the shape of a square pulse with constant duration that is proportional to the wave speed through the rod, and a peak force that changes linearly as a function of the gap size. Rod bowing has negligible effect on the pulse shapes for the vertical gap sizes that were considered. Table III-7 summarizes the rod-cask gap rigid-surface impact loadings.

Table III-7

Summary of Rod-Cask Gap Rigid-Surface Impact Loadings

Active Fuel Mass ( $(z)$

Rod Mass (kg)

Pulse Duration (ms)

2.54-cm Vertical Gap Peak Force (N)

$5.08-\mathrm{cm}$ Vertical Gap Peak Force (N)

$7.62-\mathrm{cm}$ Vertical Gap Peak Force (N)

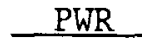

75

2.573

2.68

1757

3514

5271
BWR

10

1.406

1.6

1601

3203

These relative velocity retarding force histories are combined with the 9.0-m drop deceleration histories described in Section III.3.2. Each single-rod model is analyzed for the initial velocity of $13.4 \mathrm{~m} / \mathrm{s}(\mathrm{a} 9.0-\mathrm{m}$ drop) and a retarding force pulse corresponding to each vertical gap size. The results indicate that the different BWR rod bowing models remain elastic for 0 to $7.62-\mathrm{cm}$ gap sizes. However, only the PWR rod model without bowing remains elastic for all of the gap sizes. The elastic response for the unbowed rod is in good agreement with previous GE IF300 impact analyses [GE80]. Table III-8 shows the results for the PWR rod models with lateral gaps and both maximum and one-half maximum bowing (see Figure III-25). 
Table III - 8

Results for the PWR Rod Models with Lateral Gaps and Both Maximum and One-Half Maximum Bowing

\begin{tabular}{|c|c|c|c|c|c|c|c|c|}
\hline \multirow[b]{2}{*}{$\begin{array}{l}\text { Vertical Gap Size }(\mathrm{cm}) \\
\text { Maximum Lateral }\end{array}$} & \multicolumn{3}{|c|}{ Bowing $=0.351 \mathrm{~cm}$} & \multicolumn{5}{|c|}{ Bowing $=0.175 \mathrm{~cm}$} \\
\hline & 7.62 & 5.08 & 2.54 & 0 & 7.62 & 5.08 & 2.54 & 0 \\
\hline $\begin{array}{l}\text { Disposal }<5.08(\mathrm{~cm}) \\
\text { Maximum Tensile }\end{array}$ & 4.06 & 5.08 & 5.08 & 5.08 & 4.32 & 4.06 & 4.57 & 5.08 \\
\hline Strain $(8)$ & 2.4 & 1.9 & 1.4 & 1.2 & 1.7 & 1.5 & 1.1 & 1.4 \\
\hline
\end{tabular}

The maximum tensile strains listed in Table III-8 are those occurring at the time that the maximum lateral displacement reaches $5.08 \mathrm{~cm}$. This limitation is imposed assuming cask baskets will constrain rods from deflecting indefinitely. If this deflection limitation is not imposed, lateral deflections and peak tensile strains increase significantly. Figure III-69 characterizes this strain versus maximum lateral displacement relationship. As can be seen from Figure III-69, casks without lateral displacement constraints have significantly higher probabilities of fuel rod failures than casks with continuous baskets.

The foregoing parametric analyses illustrate the potential effects of vertical gaps and rod bowing on the maximum positive strain. However, to better quantify the PWR end-drop dynamic analysis, a more detailed model of the PWR rod is analyzed. More detailed spacer spring models are introduced as shown in Figures III-45 and III-49 that include the lateral gap between the assembly and basket. Based on the design drawing [NL76], a typical PWR basket cell opening has a width equal to $22.6 \mathrm{~cm}$. If the minimum PWR assembly width is $19.3 \mathrm{~cm}$, the maximum lateral assembly-basket gap is $3.30 \mathrm{~cm}$. Note that the spring property is for a rod at the center of the assembly, which itself is located at the center of the basket cell. Travel of the center rod is limited to a maximum of $2.629 \mathrm{~cm}$ as a result of spacer grid buckling; therefore, the total allowable lateral displacement is onehalf 3.30 plus 2.629 or $4.280 \mathrm{~cm}$. The spacer grid spring elements next to the end plates include the added stiffness of the box-plate connections with the end plates. The lateral bending stiffness of these connections is estimated to be $1.103 \times 10^{6} \mathrm{~N} / \mathrm{m}$. This stiffness is included in the force/displacement relation of the springs, as illustrated in Figure III -49 .

Additional lateral constraints among spacer grids are introduced to prevent lateral displacements larger than $1.685 \mathrm{in}$. This is achieved by including a lateral nonlinear spring at each finite element node of the rod model. The force/displacement property of this spring is shown in Figure III-18. The maximum bowed model is used for conservatism. However, only the no-gap and $2.54-\mathrm{cm}$ vertical gaps are considered here because these are best estimates of the expected assembly vertical gap. Figures III-70 through III-72 show the deflected shape and time history plots of the 


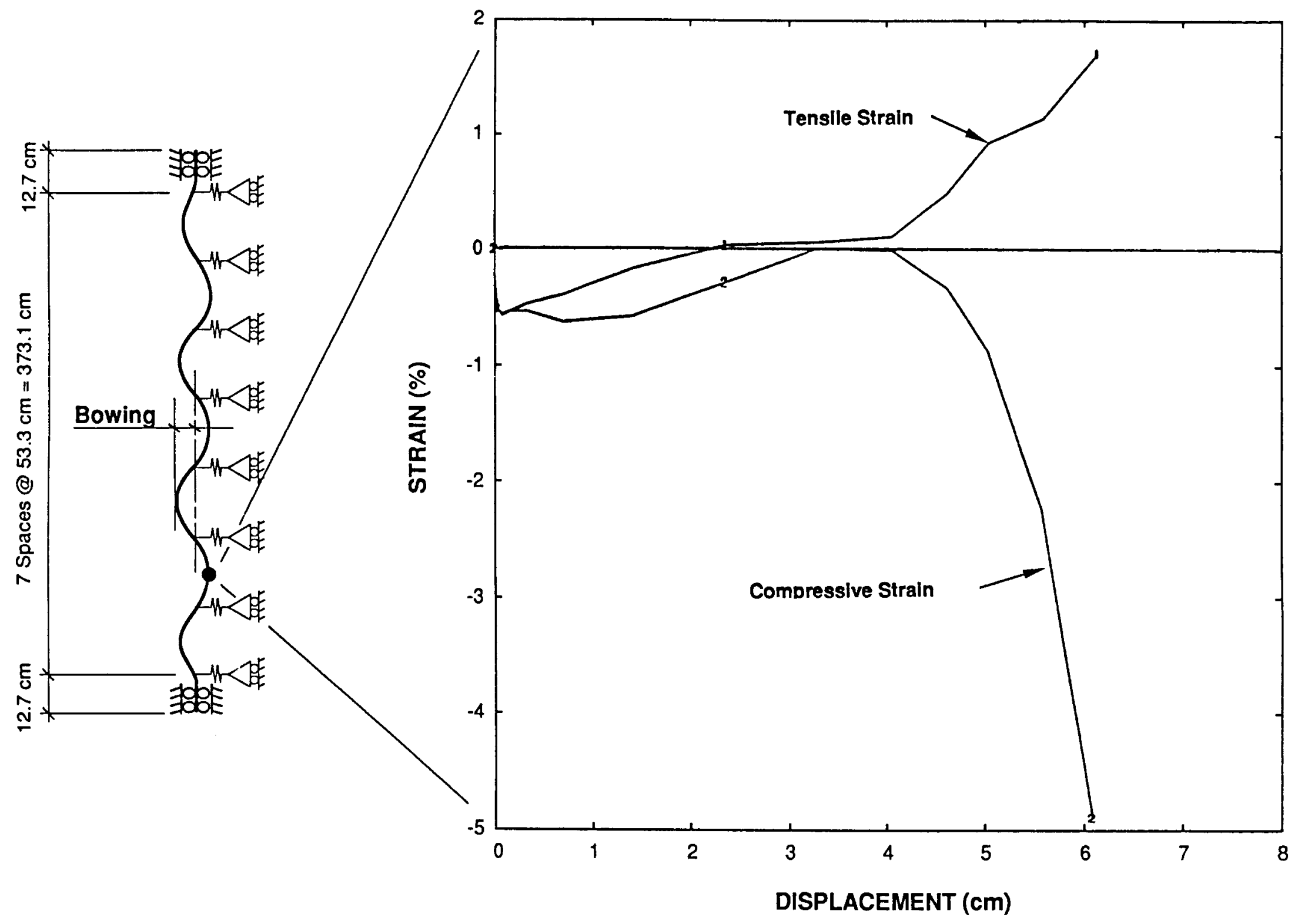

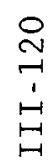

Figure III-69. B\&W $15 \times 15$ PWR End-Drop Analysis: Maximum Tensile and Compressive Rod Surface Strain Versus Maximum Lateral Displacement 


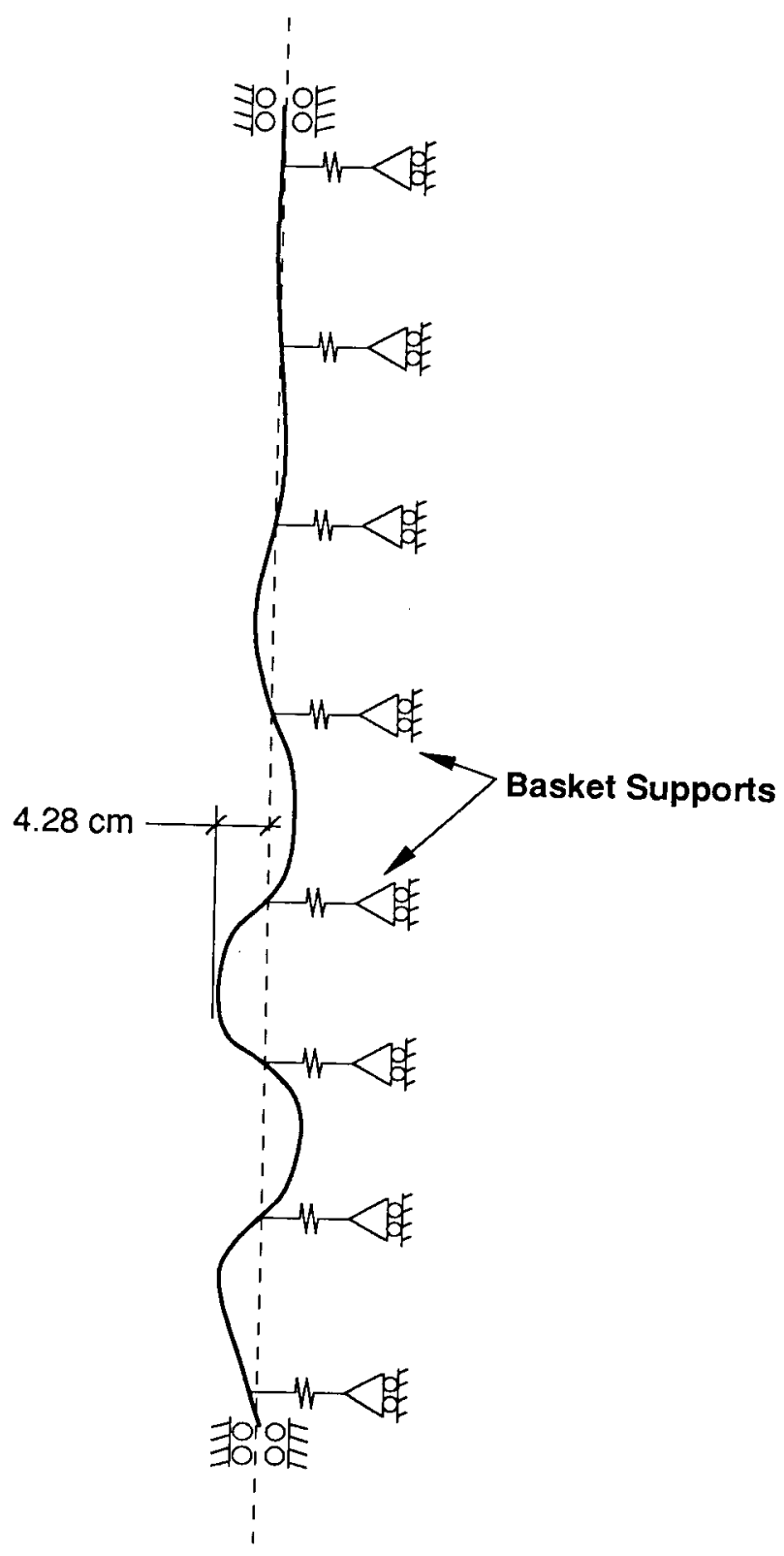

\section{DEFLECTED SHAPE \\ MAGNIFICATION FACTOR $=2.6$}

\footnotetext{
Figure III-70. B\&W $15 \times 15$ PWR 9.0-m End-Drop Analysis: Deflected Shape Plot
} 


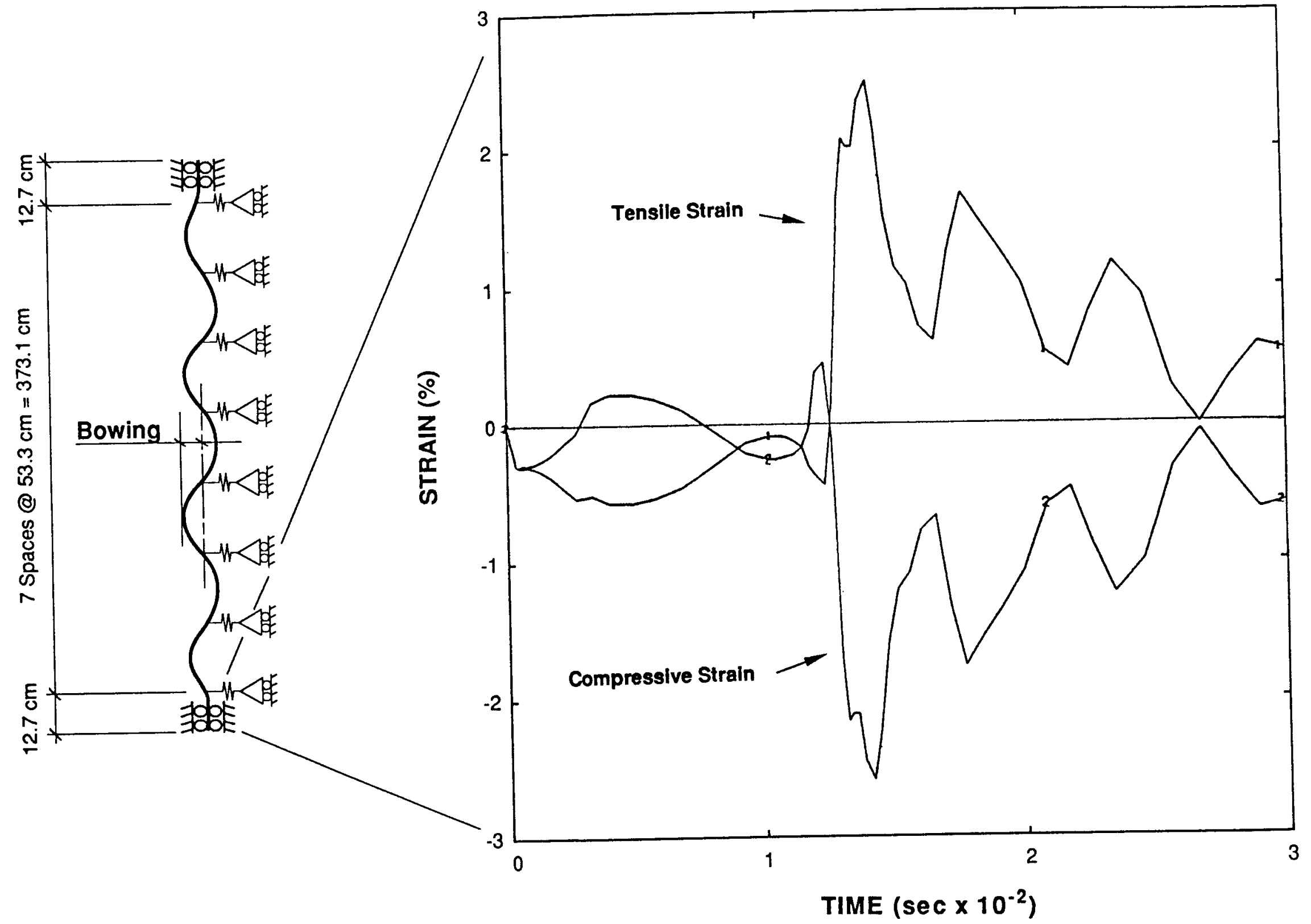



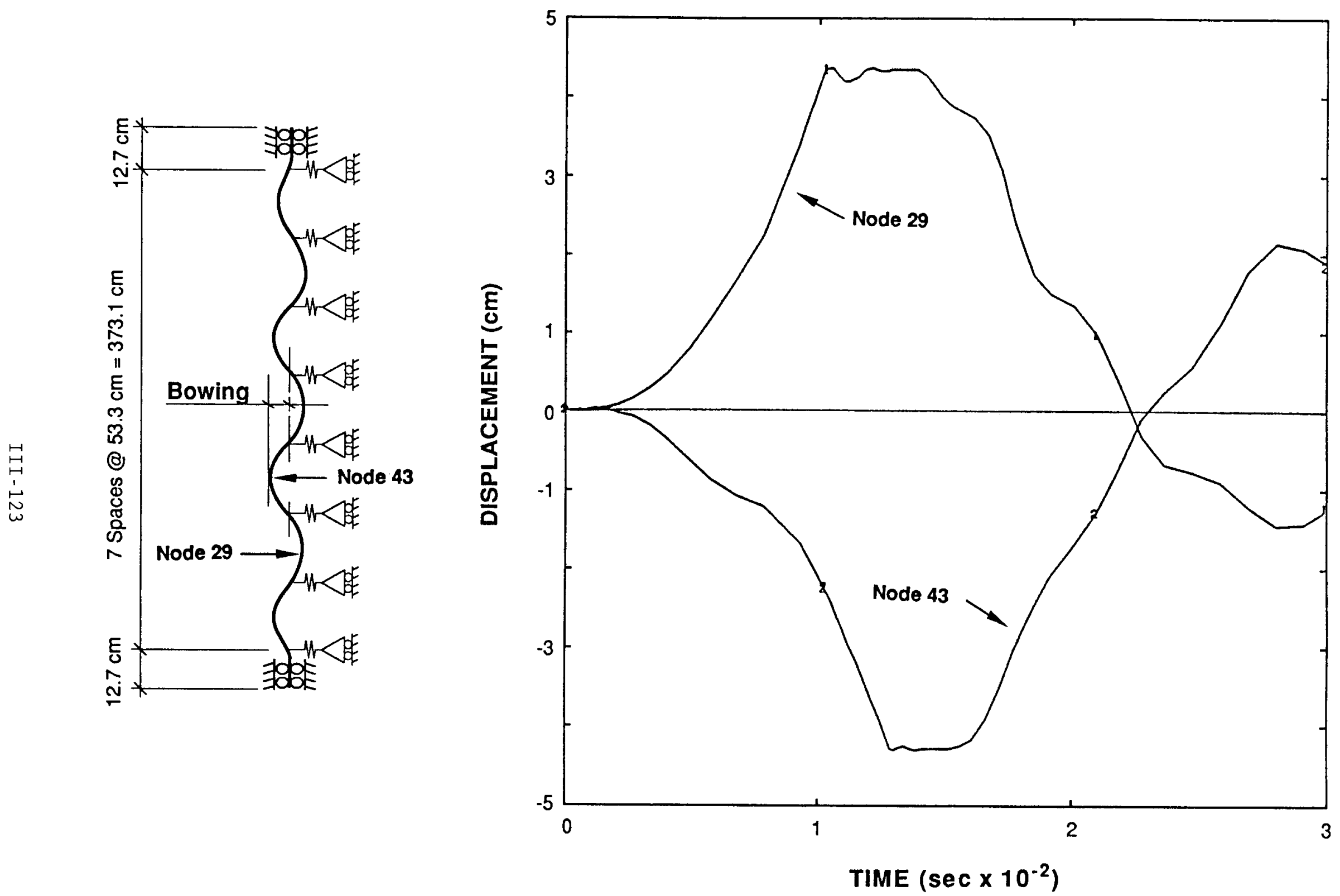

Figure III-72. B\&W $15 \times 15$ PWR 9.0-m End-Drop Analysis: Maximum Lateral Displacement Time History 
maximum lateral displacement as well as the maximum tensile strain for the $2.54-\mathrm{cm}$ vertical gap case. Below is a summary of the results. When the gap size is $0 \mathrm{~cm}$, the strain is 2.18 ; when it is $2.54 \mathrm{~cm}$, the strain is 2.58. Both cases are for the same amount of bowing, $0.351 \mathrm{~cm}$ at midspan, of a fuel rod in a B\&W $15 \times 15$ PWR fuel assembly.

Because the displacement is limited by the basket constraints for this analysis, pinch loading occurs between the rods and the basket. The maximum load is $2767 \mathrm{~N}$ and occurs between the second and third spacer grid supports from the rod bottom. The maximum tensile strains and the pinch load distributions are compared to probabilistic failure criteria to determine the probability of fuel rod failure. Probabilistic failure evaluations of the example assemblies are presented in Section III. 6.

\section{III.5.6 Hypothetical Accident Corner Drop}

As described in Section III.3, the corner-drop response is composed of two drop conditions: (1) initial impact and (2) slapdown. The initial impact response controls the corner-drop event for angles from 45 to 90 degrees from the side-drop orientation. For angles less than 45 degrees, the slapdown event controls the response. Because the initial impact response resembles the end-drop response, its analyses are performed similar to the end-drop analyses. The slapdown analysis is modeled similar to the side drop in that both analyses have loading exclusively normal to the fuel rods.

\section{Initial Impact Analyses}

This section summarizes the initial impact analyses of the example assemblies. As with the end-drop model, the initial impact is modeled with a single-rod assembly model. For scoping analyses, the assembly is limited to a maximum lateral displacement of $\pm 5.08 \mathrm{~cm}$. Analyses were performed for impact angles of 84,70 , and 45 degrees to determine the effect of angle on the maximum strains of the fuel rods. The results of the analyses are given in Table III-9.

Table III-9

Results of the Initial Impact Analyses Using a Single-Rod Assembly Model to Measure the Effect of Angle on the Maximum Strains of Fuel Rods

$\begin{array}{crr}\text { Impact Angle } & \text { Max. Tensile Strain 8 } & \text { Max. Tensile } \\ 84^{\circ} & 0.9 & 2.5 \\ 70^{\circ} & 0.6 & 1.0 \\ 45^{\circ} & 0.5 & 0.9\end{array}$


The 84-degree initial impact analysis provided the maximum response of the PWR assembly. The peak tensile strain (assuming a maximum lateral deflection of $5.08 \mathrm{~cm}$ ) is 2.58 and occurs in the center of the bottom bay of the rod adjacent to the tie plate. The 84-degree initial impact analysis also provided the BWR assembly maximum response. Its peak tensile strain of 0.98 occurs in the center of the bottom bay of the rod adjacent to the tie plate.

Results from a more detailed analysis of the 84-degree initial impact analysis for the PWR assembly using a $4.280-\mathrm{cm}$ lateral displacement limitation indicate that the maximum tensile strain is 2.668 and occurs at the assembly bottom end plate. The maximum pinch load for this analysis is $2527 \mathrm{~N}$.

\section{Slapdown Analyses}

The response of the example assemblies under slapdown loading conditions is patterned after the side-drop calculations because the slapdown response resembles the response of the side drop. Quasi-static analyses of the side-drop response are performed using a modified version of the side-drop one-half assembly model and a nonuniform loading distribution corresponding to the more highly loaded half of the assembly. At slapdown, the center-of-mass maximum deceleration of the example leadshielded cask is $90 \mathrm{~g}$. The corresponding maximum rotational deceleration is $395 \mathrm{rad} / \mathrm{s}$. Combining these translational and rotational accelerations produces a linearly varying deceleration distribution from $10 \mathrm{~g}$ at one tip to $170.33 \mathrm{~g}$ at the opposite tip. Figure III-73 illustrates the slapdown loading distribution.

The methodology for performing dynamic time history analyses of the slapdown loading event is described in section III.3.2. The dynamic analyses require a full model of an assembly slice because they cause significantly more redistribution of loading compared to the quasi-static loading condition. To avoid doubling the size of the two-dimensional assembly slice model, a uniform loading distribution is used as an approximation to maintain the one-half model symmetric boundary. Figure III-74 shows the uniform approximation of the nonuniform slapdown loading condition. The uniform load distribution is equivalent to the average initial velocity of the more highly loaded half of the assembly. This resulting uniform velocity profile approximation is nearly equivalent to the side-drop load distribution. Because the increase in load between this slapdown event and the side-drop event is less than 5\%, the response of the example assemblies under dynamic slapdown loading conditions is determined by linearly extrapolating the dynamic side-drop analyses results. These factored slapdown dynamic analysis results are presented in Table III-4. The dynamic results envelop the quasi-static slapdown analyses previously performed. A summary of the quasi-static analyses is as follows.

The quasi-static response of the assembly under slapdown loading is similar to the response under side-drop loading. For the PWR assembly, the peak tensile strain for the maximum slapdown load case is $1.87 \%$ and occurs in the top rod at its connection to the tie plate. Figure III-75 shows deflected shapes of each assembly under slapdown loading. For the BWR assembly, the peak positive strain for the maximum slapdown load case is 


\section{CORNER DROP SLAPDOWN \\ LOADING CONDITION}

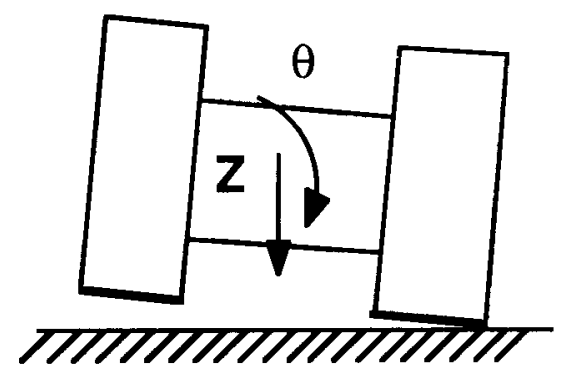

Maximum Slapdown Acceleration

$$
Z=90 \mathrm{~g} \quad \theta=395 \mathrm{rad} . / \mathrm{sec}
$$

\section{EQUIVALENT g LOAD DISTRIBUTION}

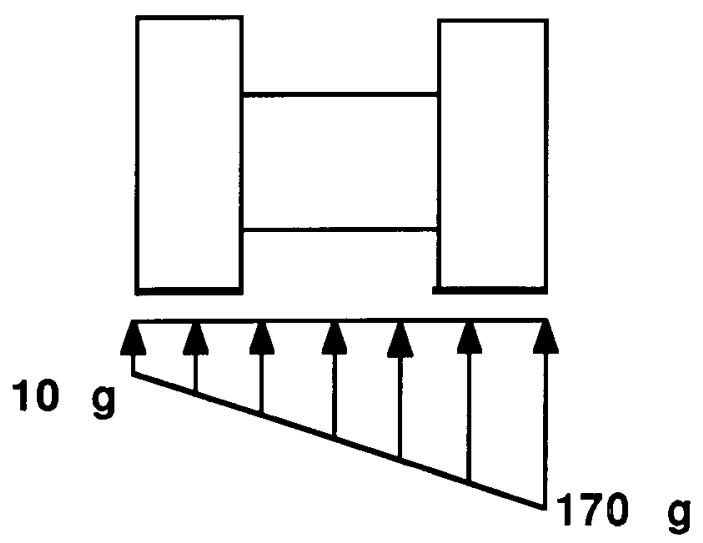

Figure III-73. Example Corner-Drop Slapdown Load Distribution: 9.0-m Drop of the Lead-Shielded Truck Cask 


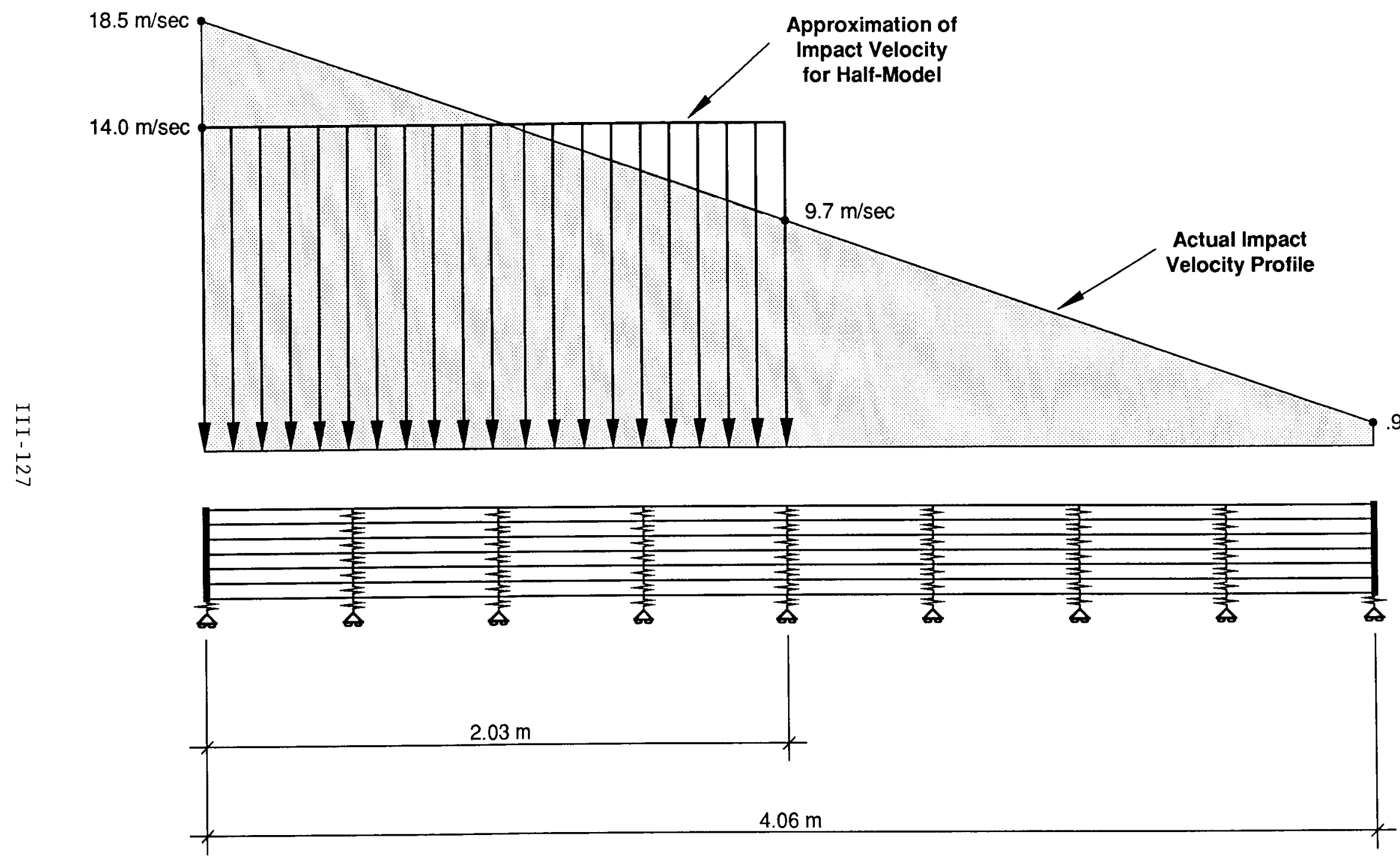

Figure III-74. Regulatory 9.0-m Corner Drop at 2-Degree Initial Impact Angle: Slapdown Impact Velocity Profile 


\section{GE 7X7 BWR - MID SLICE WITHOUT TIE-RODS - $2^{\circ}$ SLAPDOWN \\ $(\mathrm{T}=1 \mathrm{sec})$}

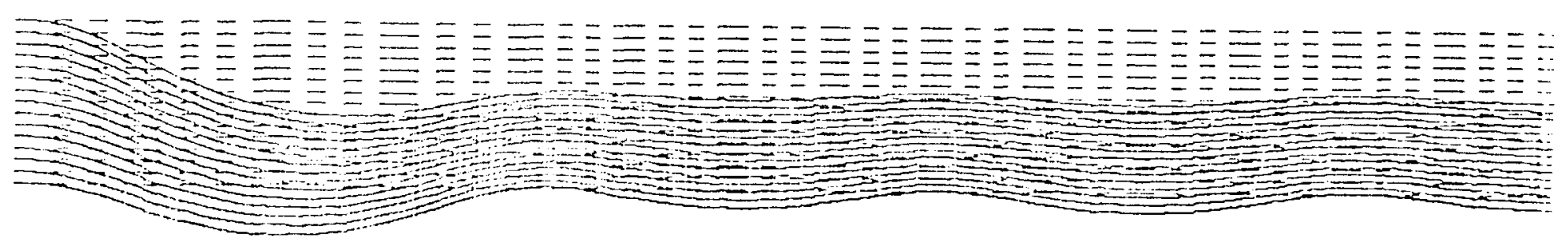

B\&W 15X15 PWR - MID SLICE WITHOUT TIE-RODS - $2^{\circ}$ SLAPDOWN

$(T=1$ sec $)$ 
0.928 , and it also occurs in the top rod of the assembly at its connection to the tie plate.

Pinch loads (i.e., diametrically opposed forces between adjacent rods) occur under slapdown loading similar to the previously described side-drop condition. The maximum crush force for the PWR assembly is $9208 \mathrm{~N}$ and occurs in the bottom rod assembly under the first spacer grid support adjacent to the assembly top end plate. The maximum slapdown force of the BWR assembly is $845 \mathrm{~N}$ and occurs at this same location.

\section{III.5.7 Regulatory Fire}

Failure of sound fuel rods as a result of thermally induced overpressurization is not a plausible failure mode, as discussed in section III. 2 . However, if partial cracks exist or cladding oxidation occurs, thermally induced fuel rod failure could occur at stresses below the ultimate strength of sound rods. To quantify the probability of this failure mode, a detailed analysis of the B\&W $15 \times 15$ PWR example assembly is performed to determine the peak strain history in the fuel rods for failure evaluation.

A two-dimensional slice model (Figure III-76) of an individual fuel rod is analyzed using the FREY fuel rod analysis code [RA87] to determine the effects of a regulatory fire on a preexisting partial crack in the fuel rod cladding. The geometry of a $0.0191-\mathrm{cm}$-deep partial crack is included in the model. This crack depth is the critical partial crack depth of PWR fuel for in-service PCI (Appendix I). This model is analyzed for the regulatory thermal loading history shown in Figure III-2 and is based on a peak initial condition fuel temperature of $380^{\circ} \mathrm{C}$ and a regulatory environment fire.

Analysis of the PWR fuel rod shows that the pressure inside the rods can rise from $8.584 \mathrm{MPa}$ at $380^{\circ} \mathrm{C}$ (computed from the internal gap pressure calculations in Appendix IV) before the fire to a maximum of $11.81 \mathrm{MPa}$ at the maximum expected postfire fuel temperature of $530^{\circ} \mathrm{C}$. The strain history at the highest stressed point in the cladding is depicted in Figure III-77, which shows a peak value of 0.88 . This strain that is below the elastic limit of the material is used in the probabilistic failure evaluation in Section III. 6.

\section{III.5.8 Normal Transport}

Normal transport loads can be divided into two categories:

- Shock and vibration loading caused by normal over-the-road operations and

- The 0.3-m normal regulatory drop event, which is intended to be an initial condition before entering the accident environments.

This section discusses the response of the example assemblies to both of these loading conditions. Probabilities of fuel rod failure for the more severe $0.3-\mathrm{m}$ drop condition are presented in Section III.6. The normal transport peak shock and vibration axial impact load (rail car coupling) is $34 \mathrm{~g}$. 


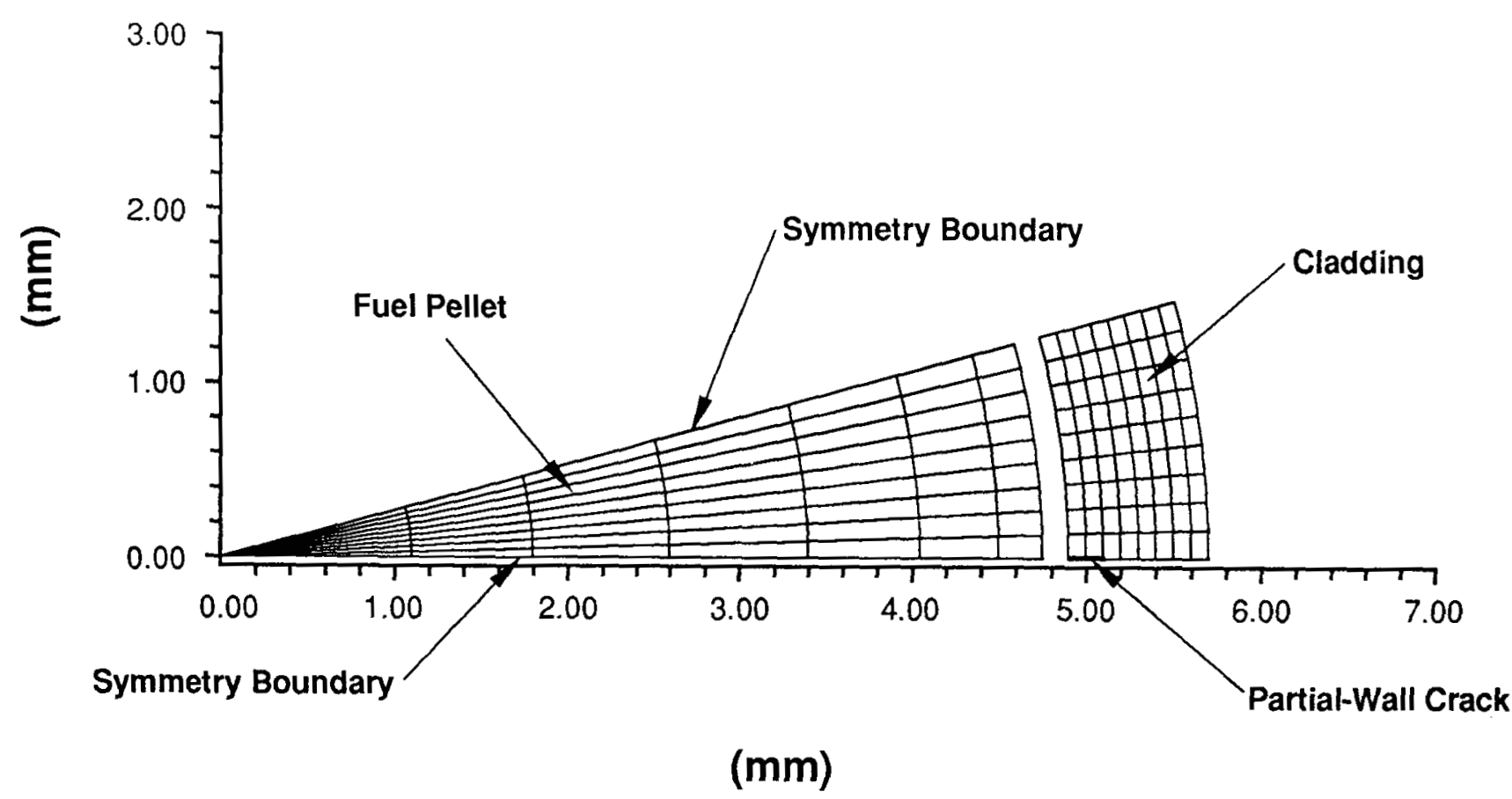

0
$\stackrel{-}{-1}$
$\stackrel{-1}{E}$

Figure III-76. Fire Analysis: Two-Dimensional Fuel Rod Slice Computational Model with Part-Wall Crack 
Regulatory Fire, $1.905 \times 10^{-4} \mathrm{~m}$ Incipient Crack in PWR Cladding

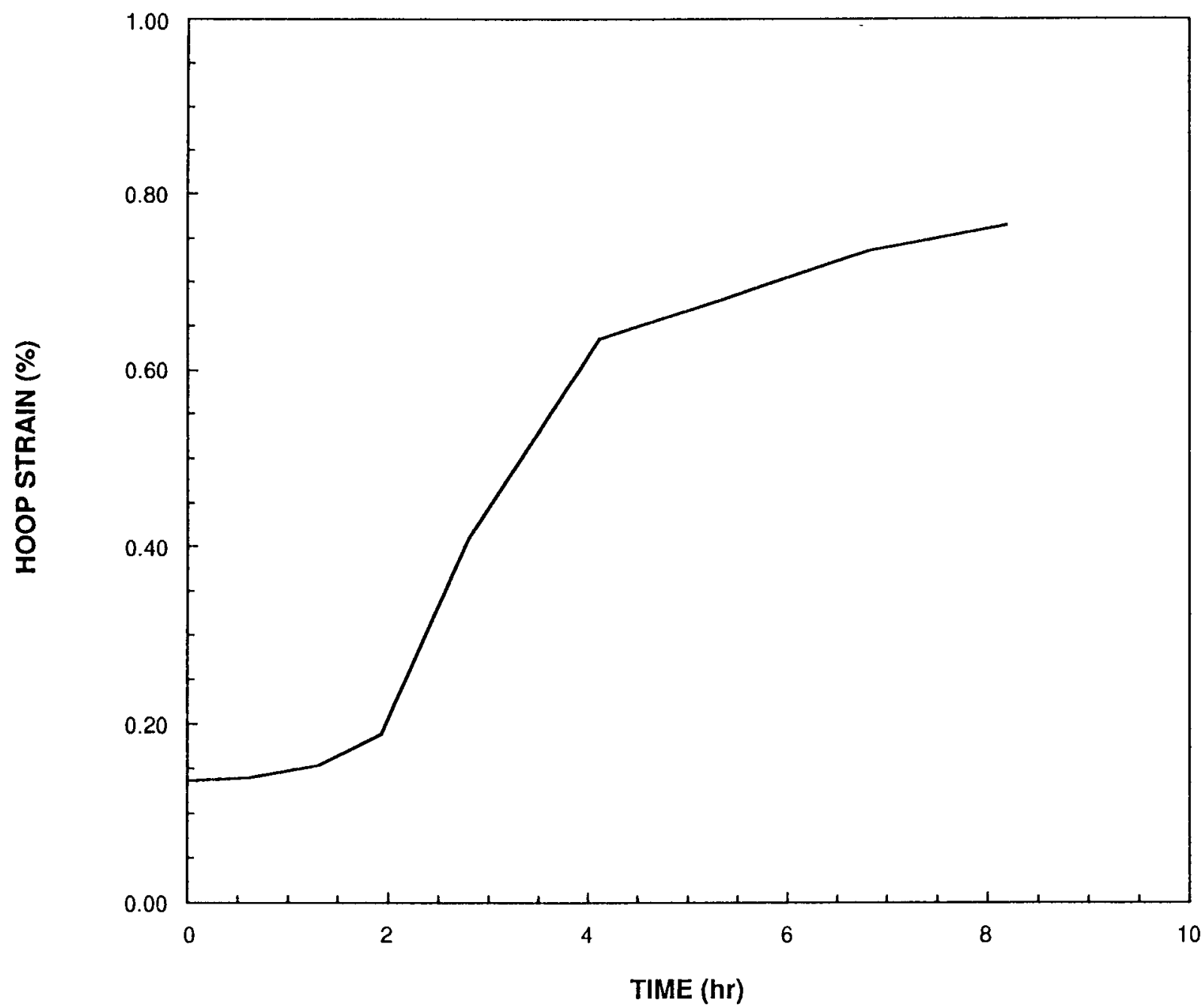

Figure III-77. Regulatory Fire: Maximum Hoop Strain History at a PartWall Crack in PWR Cladding ( $1.905 \times 10^{-4} \mathrm{~m}$ depth)

The corresponding maximum transverse loading is $10 \mathrm{~g}$. This load case is analyzed to quantify the probability of fuel rod failure under normal transport shock and vibration conditions. A modified version of the enddrop single-rod model is used to perform the analysis. This analysis is performed initially for the more critical B\&W 15 x 15 PWR assembly.

Because the resulting response is very low, no normal transport shock and vibration analyses of the BWR assembly were performed. 
Figure III-78 shows the deflected shape of the rod under these normal peak shock loading conditions. The analysis results show that the assembly will remain elastic under normal transportation shock and vibration loading conditions. The maximum tensile stress is $155 \mathrm{MPa}$ and occurs at the bottom of the rod adjacent to the end plate. The corresponding maximum spacer grid pinch force is $80.1 \mathrm{~N}$.

The normal transport regulatory drop event is defined as a 0.3-m cask drop in the most critical orientation. Slapdown is not considered a normal event. Cask deceleration time histories for the 0.3-m drop event of a typical lead-shielded truck cask are described in Appendix II. Each example assembly is analyzed for the $0.3-\mathrm{m}$ end- and side-drop orientations. For the end drop, a vertical $2.54-\mathrm{cm}$ gap between the assembly and the cask is assumed similar to the accident loading condition. However, the cask will come to rest before the assembly closes a gap of $0.635 \mathrm{~cm}$ or larger; therefore, the assembly will experience a rigid impact with the cask for the $0.3-\mathrm{m}$ end drop. Lateral gap between the assembly and the basket/cask is neglected for the side-drop condition. Thus, the maximum example cask deceleration developed in Appendix II is used for the side-drop loading condition.

The impact, velocity for the end drop is $2.54 \mathrm{~m} / \mathrm{s}$, which corresponds to $33.0 \mathrm{~cm}$ of free fall ( 30.5 plus $2.5 \mathrm{~cm}$ of assumed gap). The same example assembly single-rod models used in the accident drop analyses are used for the $0.3-\mathrm{m}$ drop events. The responses of the assemblies under this loading condition are negligible. The GE $7 \times 7$ BWR model has a maximum tensile strain of 0.198 at the end plate, with almost zero rod-to-rod pinch force. The B\&W $15 \times 15$ PWR model has a maximum tensile strain of $0.33 \%$ midspan between the first and second spacer grids from the bottom. It also has a maximum rod pinch force of $13.3 \mathrm{~N}$, with a corresponding tensile strain of 0.208 at the first spacer grid from the bottom.

Based on the $9.0-\mathrm{m}$ drop analyses, the slice models with maximum response from each example assembly are used for the side-drop loading condition. The models are analyzed with an initial velocity of $2.44 \mathrm{~m} / \mathrm{s}$ and a retarding force corresponding to the maximum example cask deceleration time history for the $0.3-\mathrm{m}$ side drop (see Appendix II). The analysis of the GE $7 \times 7$ BWR slice model with interior tie rods produces a maximum tensile strain of 0.638 at the end plate, and a maximum rod-to-rod pinch force of $2891 \mathrm{~N}$ at the first spacer grid from the end plate. The analysis of the B\&W $15 \times 15$ PWR slice model with four control rods produces a maximum tensile strain of 1.08 at the end plate, and a maximum rod-to-rod pinch force of $3559 \mathrm{~N}$ at the second spacer grid from the end plate. Figure III-79 presents the deflected shapes of the models showing the corresponding maximum values of tensile strains and rod pinch forces. Failure probability evaluations for the $0.3-\mathrm{m}$ drop conditions and the example assemblies are presented in Section III. 6.

\section{III.6 Example Failure Evaluation}

The methodology developed for analyzing the structural response of spent fuel during normal and accident conditions of transport has been presented in Section III.3. The methodology for evaluating this structural 


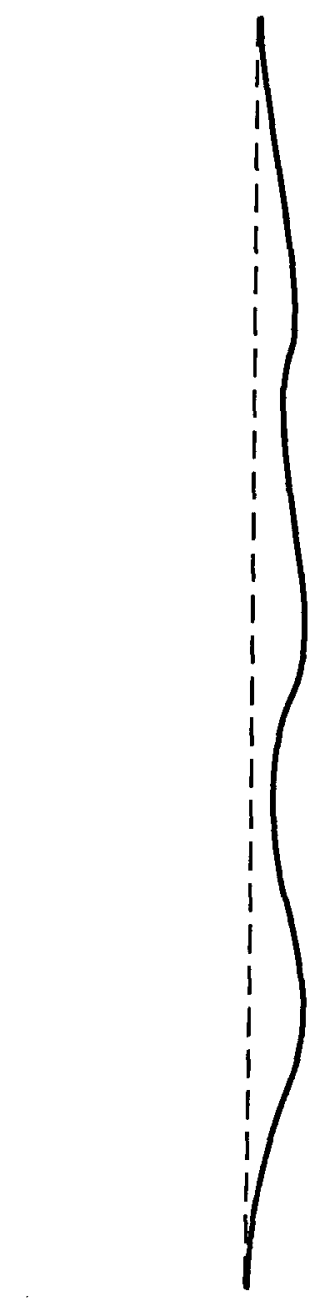

\section{MAG. FACTOR $=4.9$}

\section{SOLID LINES: Displaced Mesh DASHED LINES: Original Mesh}

Figure III-78. B\&W $15 \times 15$ PWR Normal Transport Analysis: Deflected
Shape

response against failure criteria to predict cladding breach is discussed in detail in Section III.4. Section III.5 illustrates the application of the structural response methodology to example BWR and PWR fuel assemblies. This section presents both a procedure for applying the methodology to actual failure evaluations and an assessment of clad failure probabilities for the example assemblies. 


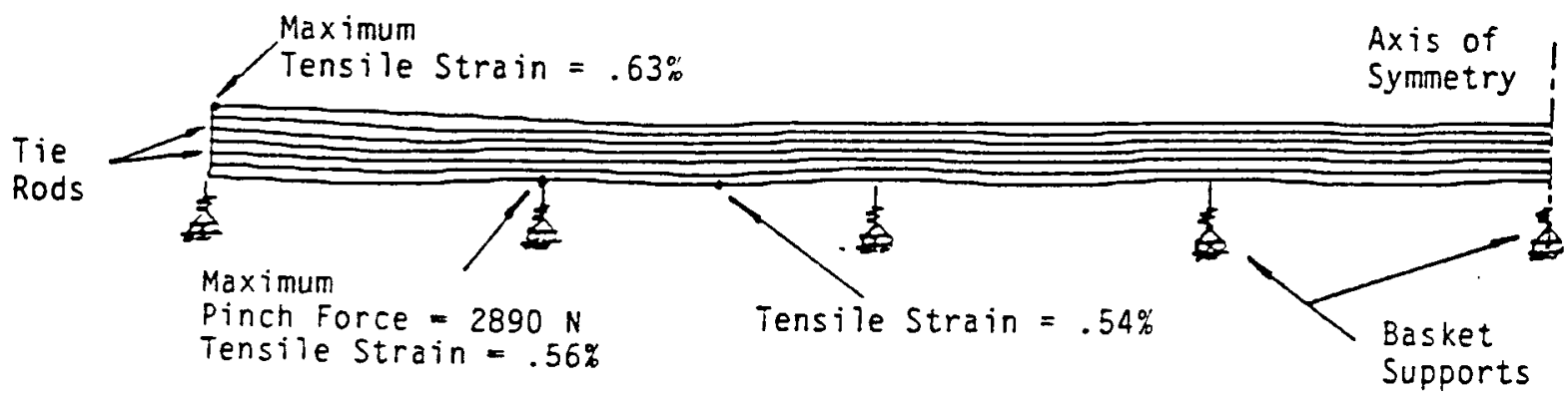

GE 7X7 BWR - SLICE WITH TWO INTERIOR TIE-RODS

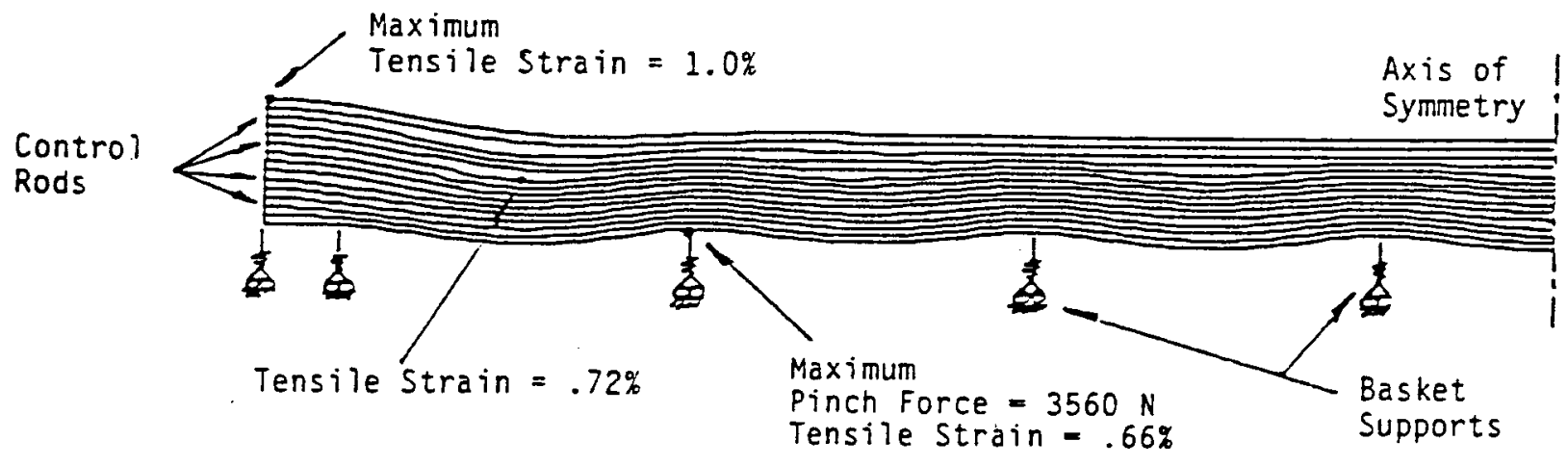

B\&W 15X15 PWR - SLICE WITH FOUR CONTROL RODS

Figure III-79. GE $7 \times 7$ BWR and B\&W $15 \times 15$ PWR Summary of 0.3-m Side-Drop Dynamic Analysis 


\section{III.6.1 Evaluation Procedure}

As discussed in Section III.4, a probabilistically based failure evaluation considers the following variables to establish the likelihood of clad failure for a given assembly under a given loading condition:

1. Statistical variation in the ductile rupture strain of the material;

2. Statistical variation in the brittle fracture toughness of the material;

3. Size, location, and frequency of part-wall cracks in the material;

4. Part-wall crack orientation; and

5. Distribution of stress/strain pinch forces along the rod.

For end drops, each rod behaves independently from others in the assembly until certain maximum deflection limits are reached. However, as the drop angle approaches horizontal, individual rods have substantially different loads, depending on their locations in the assembly. The bottom rods will experience higher transverse loading because of the weight of the rods above. This causes ovalization and hoop bending, and thus affects the likelihood of clad failure. (In a given model, each rod is representative of several rods in the full assembly. For example, in a side drop the resulting stress and load distribution on a bottom rod is based on the size and number of assemblies in the cask.) In addition, the stress distribution varies along the length of the rod because of interactions between various components, such as interactions with the tie plate and spacer grid. Each rod must therefore be divided into zones to evaluate the failure probability due to variation in stress. Finally, in the zones of each representative rod, the failure probability as a result of each potential failure mode must be evaluated.

The probability of material rupture is determined by evaluating the biaxial stress ratio at the point of interest and assigning an appropriate probability density function for the material rupture strain. Integration of the area under the density function (shown in Figure III-30) up to the calculated strain value will give the probability that the material has a rupture strain at or below the calculated strain. If an isolated event, $\mathrm{P}_{i}$, can happen $\mathrm{n}$ independent times, the probability of that event occurring is

$$
P=1-\left(1-P_{i}\right)^{n},
$$

where $P_{i}$ is the probability of tear for zone $i$.

Thus, the probability of rupture or tear at a zone because of a given stress/strain state is magnified according to Equation (III-28) by the number of occurrences of similar stress/strain states along the rod. The failure probability for each zone is then combined with all other zones as follows: 


$$
\mathrm{P}=1-\left[\left(1-\mathrm{P}_{1}\right) *\left(1-\mathrm{P}_{2}\right) *\left(1-\mathrm{P}_{3}\right) \times \ldots \times\left(1-\mathrm{P}_{\mathrm{N}}\right)\right] .
$$

The probability of subsequent breakage is determined by calculating the fracture probability at a through-wall crack under bending loads and multiplying this by tear probability because tearing must occur to develop a through-wall crack. This fracture probability is determined by evaluating the stress intensity expression defined in Equation (III-24) and the probability that the material has a lower fracture toughness. This procedure should be examined in some detail because linear elastic fracture mechanics is applied to a section well into the plastic regime of the material if excessive strain has caused tearing.

Material fracture probability from part-wall surface cracks is determined by summing failure probabilities from all cracks in the range of $76 \mu \mathrm{m}$ to the maximum probable size (see Section III.4). The characteristic crack size 1/B ( $B$ is defined in Equation [III-16]) is used to increment over the range of possible flaws. For each crack size, the probability of the crack existence, $P_{S}$, is found from Equation (III-17). The probability of this size crack causing fracture, $P_{k}$, is calculated by evaluating the stress intensity value due to the pinch load and integrating the probability density function defined in Figure III-32. Note that the stress intensity calculation can be made from either the stress intensity expressions defined in Section III.4 or from an explicit computer simulation of the crack. The probability of the part-wall crack occurring at the applied pinch load, $\mathrm{P}_{1}$,

where

$$
P_{1}=1-\left[1-\left(\frac{5}{180}\right)\left(\frac{5.08}{365.8}\right)^{\mathrm{N}}\right]
$$

is calculated by assuming that the crack must be within 5 degrees of the pinch load (top or bottom) and within $5.08 \mathrm{~cm}$ of the load along the length of the fuel column. $N$ is the number of part-wall cracks in the rod.

The probability of material fracture for each characteristic flaw size is the product of $\mathrm{P}_{S}, \mathrm{P}_{k}$, and $\mathrm{P}_{1}$. These are summed (as in Equation [III29]) to find the fracture probability in the rod at any zone, which is then magnified using Equation (III-28) for the number of occurrences and combined with probabilities from other zones using Equation (III-29).

\section{III.6.2 Probabilistic Assessment}

The likelihood of cladding failure for the example analyses can now be established. In an effort to provide conservatism while reducing the amount of data, the probabilistic assessment for these assemblies will be applied to the rod locations experiencing the maximum response rather than examining samples of rods experiencing different loads within the assembly. Thus, variables such as variation in rod bowing, vertical and horizontal gap differences, and rod location are considered to be maximized in all assembly rods for the present analysis. All rod zones are assumed to have the worst-case conditions for any particular drop angle. Table III-10 summarizes the probabilistic failure analyses calculations of the example assemblies under this worst-case scenario. The rows of Table III-10 identify drop angles considered for each of the BWR and PWR assembly types. 
Table III-10

Summary of Example Assemblies Failure Probabilities Under Regulatory Transport Loading of Spent Fuel

\begin{tabular}{|c|c|c|c|c|c|c|c|c|c|c|c|c|c|}
\hline \multirow[b]{2}{*}{$\begin{array}{c}\text { Assembly } \\
\text { Loading Condition }\end{array}$} & \multirow[b]{2}{*}{$\begin{array}{r}\text { Drop } \\
\text { Angle } \\
\end{array}$} & \multicolumn{3}{|c|}{ End Plate } & \multicolumn{3}{|c|}{$\begin{array}{c}\text { Spacer Grid } \\
\text { Support }\end{array}$} & \multicolumn{3}{|c|}{$\begin{array}{l}\text { Midspan Between } \\
\text { Spacer Grids }\end{array}$} & \multicolumn{3}{|c|}{$\begin{array}{c}\text { Failure Probability } \\
\text { Per Rod }\end{array}$} \\
\hline & & $\begin{array}{l}\text { Tensile } \\
\text { Strain } \\
(\pi) \\
\end{array}$ & $\begin{array}{l}\text { Pinch } \\
\text { Load } \\
\text { (N) }\end{array}$ & $\begin{array}{l}\text { No. } \\
\text { Per } \\
\text { Rod } \\
\end{array}$ & $\begin{array}{l}\text { Tensile } \\
\text { Strain } \\
(\%) \\
\end{array}$ & $\begin{array}{l}\text { Pinch } \\
\text { Load } \\
\text { (N) } \\
\end{array}$ & $\begin{array}{l}\text { No. } \\
\text { Per } \\
\text { Rod } \\
\end{array}$ & $\begin{array}{l}\text { Tensile } \\
\text { Strain } \\
(\bar{z}) \\
\end{array}$ & $\begin{array}{l}\text { Pinch } \\
\text { Load } \\
\text { (N) } \\
\end{array}$ & $\begin{array}{l}\text { No. } \\
\text { Per } \\
\text { Rod }\end{array}$ & $\begin{array}{c}\text { Longitudinal Slit } \\
\text { PCI } \\
\text { Part-Wall Crack } \\
\end{array}$ & $\begin{array}{l}\text { Pinhole } \\
\text { Rupture }\end{array}$ & $\begin{array}{c}\text { Rod } \\
\text { Breakage }\end{array}$ \\
\hline \multicolumn{14}{|l|}{$\mathrm{GE} 7 \times 7 \mathrm{BWR}$} \\
\hline 9.0-m End Drop & 90 & 0.342 & 0 & 1 & 0.166 & 4.45 & 2 & 0.248 & 0 & 2 & 2. E-10 & 1.E-09 & $2 . E-13$ \\
\hline $\begin{array}{l}\text { 9.0-m Corner Drop } \\
\text { (Initial Impact) }\end{array}$ & 84 & 2.59 & 0 & 1 & 0.542 & 703 & 2 & 0.419 & 841 & 2 & 8.E-10 & 8.E-06 & 2.E-06 \\
\hline $\begin{array}{l}\text { 9.0-m Corner Drop } \\
\text { (Slapdown) }\end{array}$ & 2 & 0.99 & 0 & 1 & 1.16 & 7940 & 3 & 0.91 & 0 & 3 & 1.E-05 & $3 . E-07$ & 2.E-07 \\
\hline 9.0-m Side Drop & 0 & 0.94 & 0 & 2 & 1.1 & 7562 & 5 & 0.84 & 0 & 6 & 2.E-05 & 5.E-07 & 7.E-07 \\
\hline $\begin{array}{l}\text { 0.3-m Normal Drop } \\
\text { (Side Drop) }\end{array}$ & 0 & 0.63 & 0 & 2 & 0.56 & 2900 & 5 & 0.54 & 0 & 6 & 1.E-08 & 4.E-08 & 1.E-09 \\
\hline \multicolumn{14}{|l|}{$\underline{B \& W} 15 \times 15 \mathrm{PWR}$} \\
\hline 9.0-m End Drop & 90 & 2.5 & 0 & 1 & 1.04 & 84.5 & 2 & $\begin{array}{l}2.02 \\
0.844\end{array}$ & $\begin{array}{c}0 \\
2345\end{array}$ & $\begin{array}{l}1 \\
1\end{array}$ & $6 . E-10$ & 7.E-06 & 8.E-07 \\
\hline $\begin{array}{l}\text { 9.0-m Corner Drop } \\
\text { (Initial Impact) }\end{array}$ & 84 & 2.6 & 0 & 1 & 0.971 & 72.9 & 2 & $\begin{array}{l}2.08 \\
0.546\end{array}$ & $\begin{array}{c}0 \\
3006\end{array}$ & $\begin{array}{l}1 \\
1\end{array}$ & 2.E-09 & 9.E-06 & 1.E-06 \\
\hline $\begin{array}{c}\text { 9.0-m Corner Drop } \\
\text { (Slapdown) }\end{array}$ & 2 & 3.47 & 0 & 1 & 1.37 & 9728 & 2 & 1.02 & 0 & 3 & 2.E-05 & $2 . E-04$ & 2.E-05 \\
\hline 9.0-m Side Drop & 0 & 3.3 & 0 & 2 & 1.3 & 9265 & 6 & $\begin{array}{l}0.97 \\
1.2\end{array}$ & 0 & $\begin{array}{l}5 \\
2\end{array}$ & $2 . E-05$ & 2.E-04 & 5.E-05 \\
\hline $\begin{array}{c}\text { 0.3-m Normal Drop } \\
\text { (Side Drop) }\end{array}$ & 0 & 1.00 & 0 & 2 & 0.66 & 3560 & 6 & 0.72 & 0 & 7 & 3.E-08 & 3.E-07 & 2. $E-08$ \\
\hline Fire & $\mathrm{n} / \mathrm{a}$ & 0.8 & 0 & a & 0.8 & 0 & a & 0.8 & 0 & a & 1.E-11 & $0.0^{\mathrm{d}}$ & $0.0^{\mathrm{d}}$ \\
\hline $\begin{array}{l}\text { Norma } 1^{c} \\
\text { Transport }\end{array}$ & $\mathrm{n} / \mathrm{a}$ & 0.252 & 0 & 1 & 0.1 & 79.2 & 2 & 0.203 & 0 & 2 & $0.0^{\mathrm{b}}$ & 2.E-07 & 2.E-12 \\
\hline $\begin{array}{l}a_{\text {Fire analysis stre }} \\
\mathrm{b}_{\text {Stress intensity }} \\
\mathrm{c}_{\text {Normal }} \text { transport } \\
\mathrm{d}_{\text {These failure mode }}\end{array}$ & $\begin{array}{l}\text { is is ba } \\
\text { ictor is } \\
\text { due to } \\
\text { are no }\end{array}$ & $\begin{array}{l}\text { ess than } \\
\text { hock and } \\
\text { applicabl }\end{array}$ & $\begin{array}{l}\text { wall } \\
\text { aresho } \\
\text { ibrati } \\
\text { to re }\end{array}$ & $\begin{array}{ll}d & \text { va } \\
\text { n } & 10 \\
& \end{array}$ & $\begin{array}{l}\text { fuel w. } \\
\text { e. } \\
\text { ing. } \\
y \text { fire }\end{array}$ & ition & ilit & of $1 \mathrm{ir}$ & 000 . & & & & \\
\hline
\end{tabular}


The major columns identify the three zones for clad failure evaluation, along with the calculated probability of failure. Within each zone along the rod, maximum values found in response of the zone are tabulated.

Once the conditional probability of failure either by material rupture or fracture is calculated, it is multiplied by the frequency of occurrence. The conditional probabilities for each zone are then summed. The frequency of occurrence is the number of times the indicated response occurs along the rod. For this assessment, the maximum response is used along with a best estimate of the number of occurrences along the length of the rod. The probability of pinhole failure is the sum of the probabilities of material rupture and PCI crack propagation. Note that the probability of material fracture of a PCI part-wall crack will only be feasible at a zone that has significant transverse load to produce hoop stress. The probability of fuel rod breakage is calculated by requiring that a throughwall crack has been already formed according to the material rupture criterion, and therefore has this conditional probability as a multiplier.

\section{III.6.3 Summary}

Based on the maximum response assumptions used in Table III-10, the highest failure probability for the PWR example assembly occurs from a 9.0-m side drop that results in a pinhole or longitudinal slit failure probability of $2.0 \times 10^{-4}$ per rod per event. The next highest likelihood of failure is for an oblique corner drop with slapdown, with either a pinhole or longitudinal slit failure probability of $1.7 \times 10^{-4}$ per rod per event. Thus, for a PWR rail cask containing 33 assemblies ( 7425 rods) and conservatively assuming all rods in the assembly are subjected to the worst-case loading, one rod will have a pinhole or longitudinal slit failure, using Equation (III-28). The rod breakage mode that involves an opening significantly larger than a pinhole has a failure probability of $5 \times 10^{-5}$, which is not quite an order of magnitude lower than the failure probability for a pinhole failure. This rod breakage failure mode has the highest probability of occurring at the end plate connection rather than the vicinity of fuel pellets. Furthermore, the deformed cross section of the rod at the break location is highly ovalized and nearly collapses. Thus, the maximum amount of fuel fragments that may be released is limited to less than the equivalent of one pellet.

For BWR assemblies, the highest failure probability (involving a pinhole or longitudinal slit failure only) occurs during an oblique end drop with slapdown. The resulting failure probability is roughly $8 \times 10^{-6}$ per rod per event. A pinhole-type failure is the most probable type of failure mechanism. Rod breakage that might involve fuel fragment discharge from fuel rods to the cask cavity is of minute risk for BWRs. However, it is a $10^{-5}$ event for PWRs, but with minimal additional release consequences.

\section{III.7 Summary and Conclusions}

This appendix describes a detailed methodology that has been developed to determine the likelihood of fuel rod failure under regulatory transport loading conditions. All loading mechanisms are considered, including fire, shock and vibration, and impact loading. The most critical loading mechanism is the regulatory accident $9.0-\mathrm{m}$ drop condition. 
The methodology can be divided into three parts:

1. Computational modeling,

2. Deterministic analysis, and

3. Probabilistic failure evaluation.

The computational models use the finite element method to model the assembly, and cask geometric and material properties. Many assumptions are required to idealize the complex physical system and evaluate fuel rod response. Each assumption is carefully examined for validity. Although a complete methodology is provided, parametric analyses are needed to further determine the sensitivity of fuel rod failure probability to the most influential and uncertain modeling assumptions.

Deterministic analyses of the computational models are performed for regulatory drop loading conditions using time history dynamic analysis procedures. The loading deceleration time history for each cask is based on the specific limiter design of a cask; the design is an important input parameter into the computational analysis.

The probabilistic fuel rod failure evaluation is based on deterministic analysis results (cladding strains and rod-to-rod pinch forces) and a probabilistic distribution of fuel rod initial conditions. These conditions include the cladding ductility limit, material fracture toughness, and the number, size, and distribution of part-wall cracks. Additional parameters considered by the failure evaluation model include the irradiation and thermal environments of the fuel rods.

Depending on the drop orientation, two types of fuel rod response models are analyzed. For end drops and the initial impact phase of corner drops, single-rod models are analyzed because the predominant load path for all rods is the same. The single-rod model is based on the conservative assumption of no rod-to-rod interaction (lateral displacement limitations are imposed based on the assembly and basket geometries). For side drops and the slapdown phase of corner drops, a two-dimensional assembly slice model is used to simulate the predominant rod-to-rod load path. Several slice models of each assembly are needed to include the effects of different tie- or control-rod configurations.

Both the single-rod and the two-dimensional slice models are composed of a large number of finite elements. The fuel rods are modeled with a minimum of 30 two-dimensional beam elements per rod. Nonlinear spring elements are used to model the fuel rod/spacer grid interaction. The force-displacement relation for these spring elements is developed through detailed analyses of individual spacer grid frames under fuel rod loading conditions. Nonlinear spring elements are also used to simulate rod-to-rod contact (between spacer grids) and to provide limitations on lateral displacement for the assembly two-dimensional slice and single-rod models. The assembly tie plates are treated as rigid members.

The regulatory free-drop loading condition is modeled as follows: the impact velocity of a cask is applied as an initial condition to all assembly components. This initial velocity is reacted by a retarding force 
based on the stiffness of the cask impact limiter and is applied at the support points of the model. Casks and baskets are assumed to be much stiffer than the assemblies and impact limiters and thus are treated as rigid links between the assembly and limiters. Further investigation into variations in basket design is required to verify this assumption. If a gap exists between an assembly and the cask/basket, the force time history is modified to model the effects of this secondary impact. This modification results in an increase in the peak retarding force. However, the net load applied to the assembly remains the same.

For end drops, only the portion of fuel mass directly attached to the cladding is modeled, whereas the effects of remaining mass are assumed to be transferred through pellet-to-pellet loading. The example analyses assume 10 and 758 of total fuel mass is attached to the cladding for BWR and PWR rods. This is an important assumption in determining the fuel rod failure probability and thus requires further quantification through experimental methods or analytical sensitivity analysis.

The impact analyses are performed using a temporally implicit solver with virtually no damping. Physical damping mechanisms, such as friction, intermittent cladding-pellet contact, and spacer grid plastic deformations, are conservatively neglected. Only a small amount of numerical damping is used to improve the solver performance. The total energy in the system, monitored throughout the solution time, shows that the energy loss due to numerical damping is minimal. Further quantification of the effects of structural damping on the impact event can be obtained from sensitivity analyses and experimentation.

The most probable modes of cladding failure are ductile tearing as a result of excessive tensile strain and material fracture at a preexisting part-wall cladding crack. Fluctuations, such as the ductility limit and fracture toughness of cladding material properties, are based on statistical distributions. Both parameters are functions of temperature and irradiation. The ductility limit also depends on the biaxial stress state in the fuel rod (the ratio of transverse stress to axial stress). The number and size distributions of cladding part-wall cracks are determined probabilistically. These statistical data for cladding properties are utilized to determine the probability of fuel rod failure using the deterministically calculated tensile strains and rod-to-rod pinch forces. The current methodology provides a means of quantifying the probability of fuel rod failure; however, further investigations of the statistical portions of the failure criteria evaluation are required through future experimental data development or sensitivity analyses.

\section{III.8 References}

[AN79] "Light Water Reactor Fuel Performance," in Proceedings of American Nuclear Society Meeting, Portland, OR, April 29-May 3, 1979.

[AN85] "Light Water Reactor Fuel Performance," in Proceedings of American Nuclear Society Meeting, Orlando, FL, April 21-24, 1985. 
[BA77] Bauer, A. A., W. J. Gallagher, L. M. Lowry, and A. J. Markworth, Evaluating Strength and Ductility of Irradiated Zircaloy, BattelleColumbus Laboratories, Columbus, OH, BMI-NUREG-I985, October 1977.

[BA87] Barse11, A. W., "Non1inear Statistical Analysis of Zircaloy-2 Fracture Toughness Data," Internal Correspondence, GA Technologies, San Diego, CA, February 1987.

[BE80] Benjamin, A. S., and D. J. McCloskey, "Spent Fuel Heatup Following Loss of Water During Storage," Nuclear Technology, 49, 2 (July 1980).

[BU76] Buchalet, C. B., and W. H. Bamford, "Stress Intensity Factor Solutions for Continuous Surface Flaws in Reactor Pressure Vessels," Mechanics of Crack Growth, ASTM STP-590, American Society for Testing and Materials, pp. 385-402 (1976).

[BU85] Burian, R. J., K. D. Kok, R. DiSalvo, M. E. Balmert, R. F. Kelly, and A. W. Pentiman, Response of Spent LWR Fuel to Extreme Environments, Battelle Columbus Laboratories, Columbus, OH, SAND857213, August 1985.

[CL69] Clough, R. W., "Analysis of Structural Vibration and Dynamic Response," Japan-U.S. Seminar on Matrix Methods of Structural Analysis and Design, Tokyo, Japan, August 25-30, 1969.

[GA80] Garzarolli, F., D. Jorde, R. Manzel, G. W. Parry, and P. G. Smead, Review of PWR Fuel Rod Waterside Corrosion Behavior, Electric Power Research Institute, Palo Alto, CA, EPRI NP-1472, 1980.

[GE71] General Electric Co., Fuel Rod Bowing Study, NEDC-23719, 1971.

[GE80] General Electric Co., Final Safety Analysis Report, GE-IF-300 Spent Fue1 Shipping Cask, NEDO-10084-2, December 1980.

[HA80] Hagrman, D. L., et al., MATPRO-Version 11 (Revision 2): A Handbook of Material Properties for Use in the Analysis of Light Water Reactor Fuel Rod Behavior, EG\&G Idaho Inc., Idaho Falls, ID, ID, NUREG/CR-0479, TREE-1280, Rev. 2, August 1981.

[HE79] Heliot, J., R. C. Labbens, and A. Pellissier-Tanon, "SemiE11iptical Cracks in a Cylinder Subjected to Stress Gradients," Fracture Mechanics, C. W. Smith, ed., ASTM STP-677, American Society for Testing and Materials, pp. 341-364 (1979).

[HI76] Hillner, E., "Corrosion of Zirconium-Base Alloys: An Overview," in Zirconium in the Nuclear Industry, ASTM STP-633, p. 211, 1976.

[HI78] Hilber, H. M., T.J.R. Hughes, and R. L. Taylor, "Collocation, Dissipation," Engineering and Structural Dynamics, 6, pp. 99-117 (1978).

[HI88] Hibbitt, H. D., et al., ABAQUS User's Manual, 1988. 
[HK79] Hibbit \& Karlsson, Inc., Analysis of Pipe Whip, NP-1208 Research Project 1324-1, Providence, RI, 1979.

[IA87] "Improvements in Water Reactor Fuel Technology and Utilization," in Proceedings of an International Symposium on Improvements in Water Reactor Fuel Technology and Utilization Organized by the International Atomic Energy Agency, Stockholm, Sweden, September 15-19, 1986.

[LA76] Labbens, R., A. Pellissier-Tanon, and J. Helliot, "Practical Method for Calculating Stress-Intensity Factors Through Weight Functions," Mechanics of Crack Growth, ASTM STP-590, American Society for Testing and Materials, pp. 368-384 (1976).

[LE77] Lewis, E. E., Nuclear Power Reactor Safety (New York, NY: John Wiley \& Sons, 1977), p. 306.

[MG79] McGowan, J. J., and M. Raymund, "Stress Intensity Factor Solutions for Internal Longitudinal Semi-Elliptical Surface Flaws in a Cylinder Under Arbitrary Loadings," Fracture Mechanics, C. W. Smith, ed., ASTM STP-677, American Society for Testing and Materials, pp. 365-380 (1979).

[MI84] Miller, A. K., A. Tasooji, and R. E. Einziger, "Modeling of Zircaloy Stress-Corrosion Cracking: Texture Effects and Dry Storage Spent Fuel Behavior," American Society for Testing and Materials, Philadelphia, PA, 1984.

[MI85] Mills, W. J., Fracture Mechanics Evaluation of N-Rector Fringe Tubes, Westinghouse Hanford Co., UNI-3136, March 1985.

[NL76] PWR Fuel Basket 10/24 Rail Cask, National Lead Co., 1976.

[N087] Notz, K., Characteristics of Spent Fuel, High-Level Waste and Other Radioactive Wastes Which May Require Long-Term Isolation, U.S. Department of Energy, Office of Civilian Radioactive Waste Management, DOE/RW-0184, 1987.

[RA82] Raju, I. S., and J. C. Newman, "Stress-Intensity Factors for Internal and External Surface Cracks in Cylindrical Vessels," Journal of Pressure Vessel Technology (November 1982).

[RA86] Raju, I. S., and J. C. Newman, "Stress-Intensity Factors for Circumferential Surface Cracks in Pipes and Rods Under Tension and Bending Loads," Fracture Mechanics, 17, ASTM STP 905, American Society for Testing and Materials, Philadelphia, PA, pp. 789-805 (1986).

[RA87] Rashid, Y. R., A. J. Zangari, and R. S. Dunham, FREY-01: Fue1 Rod Evaluation System, 1-4, EPRI NP-3277-CCM, August 1987.

[ZI71] Zienkiewicz, 0. C., The Finite Element Method in Engineering Science, (London: McGraw-Hil1, 1971). 
APPENDIX IV

RADIONUCLIDE RELEASE MODELS

IV - I 
This page is intentionally left blank.

IV - 2 


\section{IV.1 Introduction}

The largest source of radioactive material contained within a spentfuel shipping cask is the fuel itself. This appendix describes models developed to estimate the extent to which radioactivity associated with the fuel can be released into the cask cavity.

At temperatures up to approximately $1000^{\circ} \mathrm{C}$ in an inert of chemically reducing atmosphere, only material associated with the interconnected voids of primarily the fuel-cladding gap and plena will be released from a fuel rod when its cladding integrity has been violated. Because fuel temperatures will not exceed $530^{\circ} \mathrm{C}$ under regulatory-imposed accident conditions, only releases of material in the void spaces are of concern in shipping cask accident assessments. This is not correct if oxidation of the fuel occurs because of air ingress into the cask. The likelihood of that occurrence is also considered in this appendix.

Once the cladding integrity has been breached, three types of releases are possible: (1) gaseous species from the rods, (2) volatile species, and (3) particulates.

\section{IV.2 Release of Gaseous and Volatile Species}

Although the gap inventories of the cesium isotopes, long-lived noble gases, and (possibly) tellurium isotopes appear to be identical, the way these classes of fission products are distributed differs significantly. The noble gases are uniformly distributed throughout the interconnected void regions and are gaseous at all temperatures. Elemental cesium, on the other hand, boils at $669^{\circ} \mathrm{C}$; therefore only that quantity of cesium vapor required to maintain equilibrium with its condensed phase at any given temperature will be in the gaseous phase. Hence as the temperature is varied, the relative amounts of gas-phase and condensed-phase cesium also vary. (The vapor pressure of elemental cesium within a fuel rod is probably determined by the equilibrium state of cesium compounds rather than condensed elemental cesium, but that does not alter this description.)

Elemental tellurium, which boils at $1390^{\circ} \mathrm{C}$, would be expected to display characteristics similar to those of cesium, except that its vapor pressure at any temperature would be correspondingly lower. However, tellurium released to the fuel-cladding gap is observed to alloy with the Zircaloy cladding and then to be released as SnTe after a significant fraction of the Zircaloy has been oxidized [CO87].

Tritium, like krypton and xenon, is gaseous at all temperatures and thus is ordinarily distributed within the interconnected voids in the fuel rod in a manner similar to the noble gases, but in greater concentration because of its higher mobility in the $\mathrm{UO}_{2}$ lattice. Because of its solubility characteristics, however, much of the tritium that escapes the fuel enters the Zircaloy cladding. Measurements indicate that for intact rods, about 408 of the tritium produced in pressurized water reactor (PWR) fuel rods is located in the Zircaloy, and up to 608 has been observed in the cladding of a boiling water reactor (BWR) fuel rod [G080]. Like 
tellurium, the tritium contained in the cladding is released (as tritiated water, HTO, or $\mathrm{T}_{2} \mathrm{O}$ ) when the cladding is oxidized.

If the fuel rod cladding lost its integrity, virtually the entire gap inventory of the noble gases and tritium would be released, regardless of the location or size of the failure or of the temperature at which cladding failure occurred. No significant release of tellurium would be expected without a high temperature (greater than about $700^{\circ} \mathrm{C}$ ) and extensive oxidation of the cladding. This would result in additional tritium release as well.

The situation is more complicated for cesium, however. At the instant of cladding failure, the fill and fission product gases purge the void regions of gas-phase cesium; however, as stated earlier, this quantity is sensitive to temperature and the volume of purge gas. This phase represents the burst release component. After the purge gases are released from the rod so that internal and external pressures become equal, the remaining cesium associated with the interconnected void volume can exit the rod by an evaporation (or chemical decomposition) and diffusion process. Except for very long time periods or exposure of large surface areas of fuel, this diffusional release is dwarfed compared to burst release at temperatures of interest for cask safety assessment. For reasonably small cladding-failure sizes, approximately a few inches, both burst and diffusional release may be regarded as independent of failure location and size. An exception is cladding failure in the gas plenum region. Here the model that is described in this appendix will overestimate cesium release.

The cesium mass that escapes by the burst release mechanism can be estimated by [LO79]

$$
M_{B}=3.49 V_{B}\left(M_{O} / A\right)^{0.8} \exp [-7420 / T],
$$

where

$$
\begin{aligned}
\mathrm{M}_{\mathrm{B}}= & \text { total mass of the particular cesium nuclide released in the } \\
& \text { burst in } \mathrm{g} \\
\mathrm{V}_{\mathrm{B}}= & \text { volume in } \mathrm{cm}^{3} \text { of } \mathrm{fill} \text { and fission gases vented at } 0^{\circ} \mathrm{C} \text { and at } \\
& \text { system cask pressure } \\
\mathrm{M}_{\mathrm{O}}= & \text { inventory in } \mathrm{g} \text { of cesium nuclides in the fuel-cladding gap } \\
& \text { around the fuel } \\
\mathrm{A}= & \text { internal area in } \mathrm{cm}^{3} \text { of the cladding associated with } \mathrm{M}_{\mathrm{O}} \\
& \text { (excludes plenum space) } \\
\mathrm{T}= & \text { temperature in } \mathrm{K} \text { at the rupture location. }
\end{aligned}
$$

The numerical constants, whose values depend on the units for the other parameters, were evaluated from experimental burst release data over the temperature range 700 to $900^{\circ} \mathrm{C}$. This equation is applied outside the range of validity, as stated by Lorenz et al. [LO79]; it may be in error by no more than a factor of 10 at lower temperatures.

Diffusional release of cesium isotopes is modeled using a simple depletion equation. Over the temperature range 500 to $1200^{\circ} \mathrm{C}$, the equation takes the form [LO79] 


$$
M_{D}=M_{0}\left(1-\exp \left[-\left(R_{0} t / M_{0}\right)\right]\right),
$$

where

$$
\begin{aligned}
M_{D}= & \text { mass in } g \text { of the particular cesium nuclide released by } \\
& \text { diffusion } \\
t= & \text { time in hr at diffusion temperature } \\
R_{0}= & \text { initial rate in } g / h r \text { of release by diffusion. }
\end{aligned}
$$

The initial rate of release, $R_{0}$, is given by

$$
R_{0}=1900(W / P)\left(M_{o} / A\right) 0.8 \exp [-19,800 / T] \text {, }
$$

where

$$
\begin{aligned}
& \mathrm{W}=\text { width in } \mu \mathrm{m} \text { of the radial gap } \\
& \mathrm{P}=\text { system pressure in } \mathrm{MPa} \text {. }
\end{aligned}
$$

Sample calculations of ${ }^{137}$ Cs release by the burst and diffusional modes of escape are presented in Table IV-1 for a PWR fuel rod that is irradiated to $38.2 \mathrm{MWD} / \mathrm{kg} \mathrm{U}$ burnup after a 5 -yr period from reactor shutdown. The calculated gap inventory was taken from Table 4, the rod geometrical characteristics from Table I-14, and the total inventory from Table I-13. The results in Table IV-1 show that diffusional release over $24-\mathrm{hr}$ periods can be ignored at release temperatures up to $-600^{\circ} \mathrm{C}$, when

\begin{tabular}{|c|c|c|c|c|}
\hline \multirow[b]{2}{*}{$\begin{array}{c}\text { Temperature } \\
\left({ }^{\circ} \mathrm{C}\right)\end{array}$} & \multicolumn{2}{|c|}{ Burst Release } & \multicolumn{2}{|c|}{$\begin{array}{r}\text { 24-hr Diffusional Release } \\
\text { Through Rupture Opening }\end{array}$} \\
\hline & $\begin{array}{c}\text { Total Inventory } \\
(8) \\
\end{array}$ & $(\mathrm{Ci})$ & $\begin{array}{c}\text { Total Inventory } \\
(8)\end{array}$ & $(\mathrm{Ci})$ \\
\hline 100 & $4.42 \times 10^{-8}$ & $1.02 \times 10^{-7}$ & $2.36 \times 10-17$ & $5.45 \times 10^{-17}$ \\
\hline 200 & $2.97 \times 10^{-6}$ & $6.86 \times 10^{-6}$ & $1.12 \times 10-12$ & $5.29 \times 10^{-12}$ \\
\hline 300 & $4.58 \times 10^{-5}$ & $1.06 \times 10^{-4}$ & $1.25 \times 10^{-9}$ & $2.87 \times 10^{-9}$ \\
\hline 400 & $3.14 \times 10^{-4}$ & $7.25 \times 10^{-4}$ & $1.72 \times 10^{-7}$ & $3.97 \times 10^{-7}$ \\
\hline 530 & $1.87 \times 10^{-3}$ & $4.32 \times 10^{-3}$ & $1.66 \times 10^{-5}$ & $3.83 \times 10^{-5}$ \\
\hline 600 & $3.92 \times 10^{-3}$ & $9.06 \times 10^{-3}$ & $1.11 \times 10^{-4}$ & $2.55 \times 10^{-4}$ \\
\hline 800 & $1.91 \times 10^{-2}$ & $4.41 \times 10^{-2}$ & $6.38 \times 10^{-3}$ & $1.47 \times 10^{-3}$ \\
\hline 1000 & $5.67 \times 10^{-2}$ & $1.31 \times 10^{-1}$ & $9.81 \times 10^{-2}$ & $2.26 \times 10^{-1}$ \\
\hline
\end{tabular}
only the usual rupture opening is available for escape. A special case of diffusional release would occur if the cladding were stripped away from part of the fuel rod, as might occur with brittle-cladding failure.

\section{Table IV-1}

Comparison of the Releases of $137 \mathrm{Cs}$ from

a PWR Fuel Rod Irradiated to $38.2 \mathrm{MWD} / \mathrm{kg} \mathrm{U}$ Burnup After 5-Yr Decay 
The regulatory-specified accident conditions typically result in a maximum cladding temperature of $530^{\circ} \mathrm{C}$ (see Appendix I). Hence, diffusional release of cesium radionuclides can be ignored for all shipping cask accident analyses, except for a rod break or other cladding failure that exposes a large amount of fuel directly to the cask environment.

Furthermore, although the burst release component, Equation (IV-1), must be applied at temperatures below its stated range of validity, the error is judged to be less than a factor of 10 .

At temperatures in excess of $1200^{\circ} \mathrm{C}$, additional releases of the noble gases and cesium nuclides trapped within the $\mathrm{UO}_{2}$ lattice will occur.

Although some models describe these releases [NR81], such extreme temperatures are deemed incredible relative to transport accident considerations.

Additional tritium releases from the fuel matrix occur at temperatures considerably less than $1200^{\circ} \mathrm{C}$, but no database exists on which to build a corresponding release model. However, neglect of the additional tritium releases from the $\mathrm{UO}_{2}$ matrix introduces little error in the calculations because -408 of the tritium generated in a light water reactor (LWR) fuel rod is trapped in the cladding [G080]. Also, $50 \%$ of the total tritium inventory will be contained in the gap region at the time of cladding failure.

\section{3 Escape of Nonvolatile Species}

PWR fuel rods typically contain helium fill gas at a pressure of $-3.04 \mathrm{MPa}$; the fission product gases added to this gap inventory generally increase the internal pressure by only a few percentage points. BWR fuel rods, on the other hand, are pressurized to only $\sim 0.30 \mathrm{MPa}$ with helium, and the fission product gases in the gap region of BWR fuel rods can exceed the helium inventory significantly. PWR and BWR fuel rods of very early vintage were not pressurized.

As stated above, these fill and fission product gases serve to purge moderately volatile species from the gap region at the time of fuelcladding failure. The purge stream will also dislodge fuel fines and their attendant radionuclides. But experimental data on this purge stream are sparse. Six tests have been conducted with PWR fuel [L080, BU85] and two with BWR fuel [L081]. In each experiment, 90 to 998 of the fuel fines ejected from the test specimen were deposited within a few centimeters of the rupture point. Also, the resulting rupture hole sizes were estimated to be $-2 \mathrm{~mm}^{2}$ in area and less than $1 \mathrm{~cm}$ in length. Thus, although the experiments were conducted with $30.5-\mathrm{cm}-1$ long sections of irradiated commercial fuel rods, there is good reason to believe that the same amount of fines would be ejected regardless of the length of the fuel rod or the rupture location. Again, probably fewer fines would be released if the rupture occurred in the gas plenum region of a fuel rod.

Experiments conducted at Battelle [BU85] indicate that less than $0.001 \%$ of fuel in a rod would be ejected in the form of fuel fines at the time of rupture, whereas experiments conducted at Oak Ridge National Laboratory (ORNL) [L080, L081] suggest a value somewhat less than 0.0038 . 
There is no obvious correlation between the amount of fines ejected and burst temperature or pressure. Hence, for shipping cask safety assess. ments, it is recommended that $0.003 \%$ of fuel in a rod should be considered released from the rod during cladding failure, regardless of the location or temperature.

Unfortunately, there is no clear indication of the particle size distribution of the ejected fines. In the experimental investigations, 908 of the fines ejected from the rods at rupture were deposited only a short distance from the rupture point; the remainder were transported through the apparatus and collected for size classification. This transportable material had an average geometric diameter of $\sim 10 \mu \mathrm{m}$ [L080] and an aerodynamic diameter of less than $4 \mu \mathrm{m}$ [BU85]. Thus, for safety assessment purposes, it is recommended that only $10 \%$ of the fuel fines ejected from the fuel rod be assumed to remain airborne, but no further aerosol depletion mechanisms be considered.

Radiochemical analysis of the fuel fines ejected in the PWR fuel tests indicates identical isotope distributions in the fines and in the bulk of the fuel [L080]. For the BWR fuel, however, the ejected fuel fines contained five to seven times more cesium isotopes than the bulk fuel [L081]. This undoubtedly reflects the much higher cesium gap inventory of the BWR fuel employed in the tests $(\simeq 128$ of the total inventory) relative to that of the PWR fuel ( $\simeq 0.38$ of the total inventory). For that reason it is appropriate to regard the fines as consisting of the same isotopic composition as the bulk fuel for all radionuclides except cesium nuclides. If $134 \mathrm{Cs}$ and $137 \mathrm{Cs}$ gap inventories are greater than 18 of their total inventories, concentrations in the fines should be enhanced relative to their respective concentrations in the bulk fuel by a factor equal to the gap inventory, expressed as a percentage. For example, for a fuel with a $137 \mathrm{Cs}$ gap inventory that is 78 of its total inventory,

$$
\mathrm{C}_{137 \mathrm{Cs}}^{\text {fines }} / \mathrm{C}_{137}^{\text {bulk }}=7
$$

in which $\mathrm{C}_{\text {fines }}$ represents the concentration of $137 \mathrm{Cs}$ associated with ejected fines (in gram per gram of $\mathrm{UO}_{2}$ ) and $\mathrm{Cbulk}^{\mathrm{bu}}$ is the corresponding (average) ${ }^{137} \mathrm{Cs}$ value for the fuel. This $137 \mathrm{Cs}$ value is calculated first using ORIGEN2 [CR80], the gap inventory is subtracted, and then this amount is divided by the total amount of $\mathrm{UO}_{2}$ in the fuel rod.

Table IV-2 presents sample calculation results of radioactive material released as fuel fines from a PWR fuel rod that was irradiated to 38.2 MWD $/ \mathrm{kg} \mathrm{U}$ burnup after a 5 -yr decay period. It is assumed in these results that 0.0038 of the fuel is ejected from the rod as fines and that the composition of the fines is identical to that of the bulk fuel. For comparison, corresponding $A_{2}$ values are also tabulated as in 10 CFR 71 [US90]. (The default value $\mathrm{A}_{2}=0.4$ was used for the remainder of the nuclides that would be contained in the fines, but that would have been tabulated individually.) It is clear from the tabulation that the actinides will dominate shipping cask containment considerations relative to the potential escape of fuel fines. 
Table IV -2

Radioactive Material Release from

a PWR Fuel Rod as Fuel Finesa

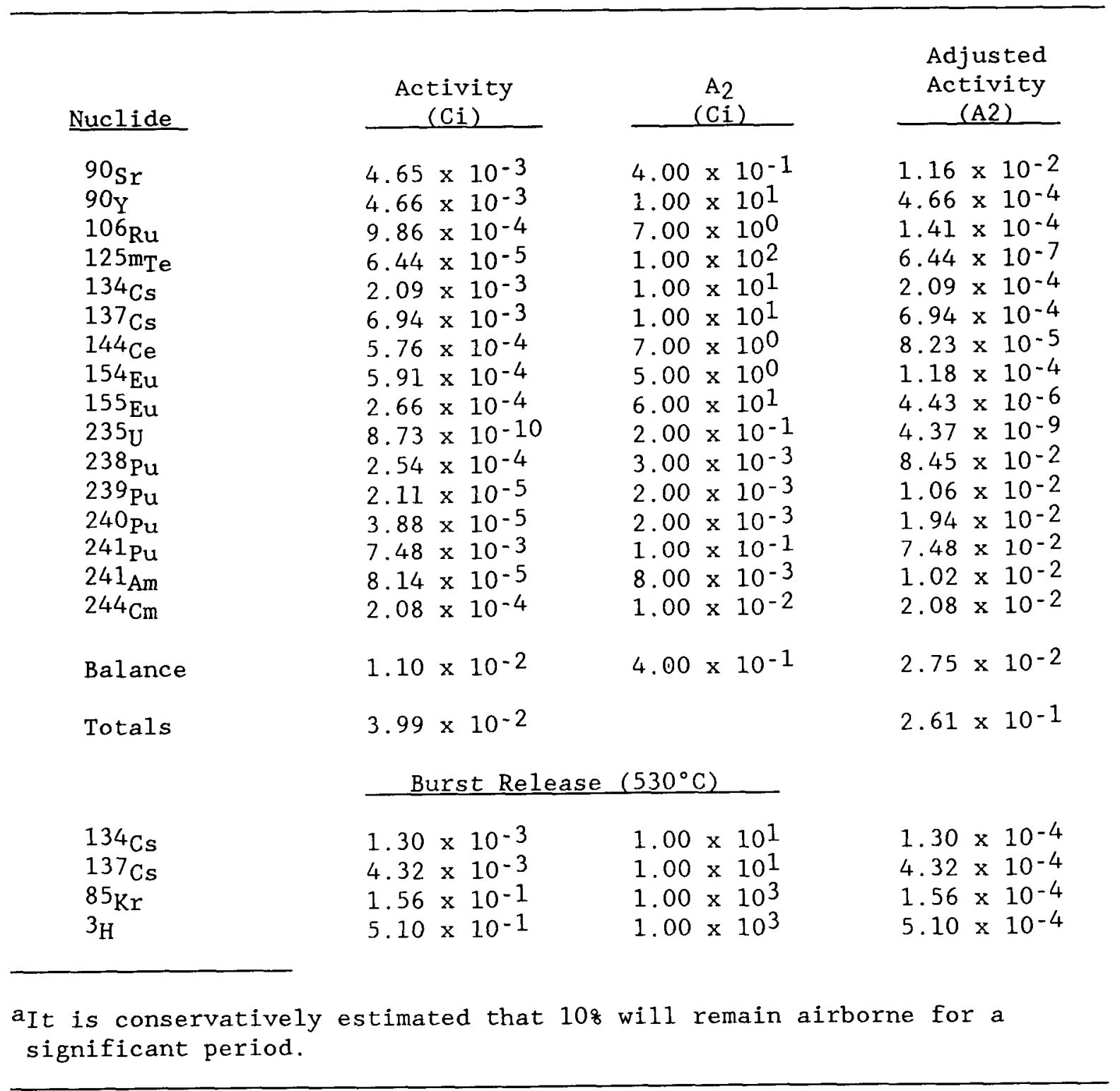

Gaseous release values at $530^{\circ} \mathrm{C}$ are also given in Table IV-2. When compared in terms of $\mathrm{A}_{2}$, the burst release contribution is small relative to the fuel fine release. If only $10 \%$ of the fuel fines persist as suspended material, the difference in magnitude between the fines and the burst release mechanisms is reduced corresponding to the estimated amount of suspended material, but the release component of the fines remains dominant. 


\section{IV.4 Generation of Aerosol from Fracturing Fue1}

The data presented in Section 5.0 and Appendix I show that 5-yr-old 40 GWD/MTU BWR fuel may contain roughly $5 \times 10^{3}$ equivalent $A_{2}$ of radioactive isotopes in a single fuel rod containing $3.3 \mathrm{~kg}$ of $\mathrm{U}_{2}$. Sandoval et al. [SA91] show that the maximum likely CRUD deposit on such a rod is $0.17 \mathrm{~A}_{2}$. The database on the radioactive contamination of cask interior surfaces (other than that of fuel rods) during transport is too sparse to allow a definitive statement, but the available evidence suggests that the level of this contamination is much smaller than the maximum CRUD $A_{2}$ value. Fuel, therefore, has the potential to dominate the radioactive source term in a spent-fuel shipping cask by at least six orders of magnitude.

The degree to which fuel dominates the source term is a function of both the amount of fuel that can escape the cladding barrier during a fuel rod failure and the fraction of this released material that becomes aerosolized. It is also a strong function of particle size distribution of the aerosol because for even relatively large hypothetical cask leak rates $\left(-1 \mathrm{~cm}^{3} / \mathrm{s}\right)$, aerosol residence times are so long $(-24 \mathrm{hr})$ that a large fraction of the aerosol may deposit inside the cask. This fraction is a strong function of the particle size distribution of the aerosol.

Measurements have been made [L080, L081, BU85] of the release of fuel fines from segments of spent-fuel rods undergoing cladding failure as a result of pressure bursting at elevated temperatures. Such a release, calculated as a fraction of the total fuel inventory of a full-length rod, was determined to be up to $3 \times 10^{-5}$. Approximately 108 of this release was captured on filters as airborne material. Thus, $3 \times 10^{-6}$ of a full-length rod was aerosolized. The release occurred through cladding fissures less than $1 \mathrm{~mm}$ wide. The size distribution of the released fines was not measured, but is obviously bounded by the size of the opening in the cladding.

The above analysis estimates the aerosol quantity that might be generated from preexisting fines on fuel release during a catastrophic fuel rod failure. Additional fines will, however, be generated if the fuel fragments themselves fracture as a result of the stresses imposed on them during such a failure. Several experimental investigations relating to this source of fines have been performed. These studies are concerned with fines released because of repository accidents, such as drops of brittle waste fuel forms down mine shafts, and fines released from shipping casks penetrated by explosives (sabotage). The major findings of these studies, as they apply here, are reviewed in the following paragraphs.

Schmidt, Sandoval, and Alvarez investigated the environmental release of fuel fines as a result of an explosive breach of a spent-fuel shipping cask [SC82, SA83, AL82]. The measurements were necessarily made on complex systems and restricted to global results. They are thus probably very specific to those systems and not readily transferable to other situations. The nature of the impact of the explosive disruptions may also differ from that of the mechanical disruptions of interest here (high temperatures and partial melting are a concern during tests with explosives). Nevertheless, 
one can view the measured yields of fines in these events as upper bounds on the quantity of fines that might be generated by the mechanical disruptions that are plausible for normal and accident transport environments.

Schmidt et al. performed a series of four tests on a model shipping cask in which a shaped charge was used to breach the cask. The cask contained five segments of H. B. Robinson (PWR) fuel that had cooled for $6.5 \mathrm{yr}$. Although the energy of the charge was not reported, it was sufficient to sever some of the pin segments. Aerosol consisting of fuel fines that escaped the cask was contained by a small sampling chamber. Both the concentration and size distribution of the sampling chamber were measured using filter and impactor samples. The quantity of disrupted fuel was also measured. Aerosol released to the inside of the model cask was not measured. It was probably the force of the disruption that led to significant impactive retention of fuel fines in the cask. Approximately half of the disrupted fuel was found there, although most of the disrupted fuel was in fragments that were generally larger than fines. Nevertheless, the total aerosol mass deduced from concentration measurements in the sampling chambers can be considered an order-of-magnitude estimate of total fines production in the disruptive event. The highest airborne mass measured in these tests was $0.103 \mathrm{~g} \mathrm{U}$, or $0.117 \mathrm{~g} \mathrm{UO}_{2}$. The amount of disrupted fuel was $-100 \mathrm{~g}$ for each test; the average amount was $119 \mathrm{~g}$. of the disrupted fuel, therefore, $9.8 \times 10^{-4}$ was measured as aerosolized. The mass median diameter of this aerosol ranged from 1.5 to $3.6 \mu \mathrm{m}$, with logarithmic standard deviations remarkably constant, at 2.5. These aerosol size-distribution parameters may reflect that the aerosol contained components other than $\mathrm{UO}_{2}$, because both the steel cask and the Zircaloy cladding were disrupted. These experiments included tests for cesium enrichment as a function of particle size, and it was found that cesium on 0.25 - to 0.5- $\mu \mathrm{m}$ particles is enriched by a factor of 10 to 17 relative to cesium on particles greater than $4 \mu \mathrm{m}$. Again, the high temperatures associated with the disruption may have influenced this result.

Sandoval et a1. [SA83] explosively breached a 22.7-tonne truck cask containing a single PWR-like assembly of unirradiated, depleted $\mathrm{UO}_{2}$ fuel rods ( $15 \times 15$ array, $1.2-\mathrm{m}$-long) and a quarter-scale cask containing a $5 \times 5$ array of $0.9-\mathrm{m}-1$ long depleted $\mathrm{UO}_{2}$ fuel rods. The quarter-scale cask was tested dry as well as filled with water. Only the dry quarter-scale cask results along with the full-scale cask results are of interest. Again, filter and impactor samples were taken for aerosol concentration and size measurements. In each case, the aerosol leaving the cask was contained in a chamber. The energy of the explosive charge was not given.

For the quarter-scale test, $179.5 \mathrm{~g}$ of $\mathrm{UO}_{2}$ were disrupted by the explosive blast and $0.78 \mathrm{~g}$ of $\mathrm{UO}_{2}$ were measured as airborne. This gives an aerosolization fraction of $4.4 \times 10^{-3}$, referenced to the amount of disrupted fuel. For the full-scale test, $5.460 \mathrm{~kg}$ of $\mathrm{UO}_{2}$ were disrupted and $2.94 \mathrm{~g}$ were aerosolized; hence, the aerosolization fraction for this test is $5.4 \times 10^{-4}$. The aerosol size distribution (UO 2 plus other components) had a mass median diameter of $-2 \mu \mathrm{m}$ and $\sigma-6$ for the full-scale 
test, and $-3.4 \mu \mathrm{m}$ and $\sigma-2.6$ for the quarter-scale test. Debris $\left(\mathrm{UO}_{2}\right.$ and other components) in both the quarter-scale and full-scale tests had a mass median diameter of $\sim 250 \mu \mathrm{m}$, as determined by sieving.

Alvarez et a1. [AL82] performed a series of impact experiments on single pellets of $\mathrm{clad} \mathrm{UO}_{2}$ that were both depleted and spent. This study was performed in a program supplementary to the experiments of Sandoval et al. [SA83] previously discussed. The aim of these experiments was to compare the fracturing behavior of depleted and irradiated $\mathrm{UO}_{2}$ ( $\mathrm{H}$. $\mathrm{B}$. Robinson, 22 GWD/t, 7-yr-old) under scaled disruptive impacts of the type investigated by Sandoval et al. Particulates resulting from the impacts were sized by sieving of deposits and cascade impactor sampling of material that became airborne in the sampling chamber. Sieving was performed with a nonpolar solvent down to a mesh size of $5 \mu \mathrm{m}$. The impact was caused by either a shaped charge or an explosively driven flyer plate directed radially against the clad pellet. (The fuel pellet was held in place by equivalent aluminum pellets at each end.) The impact energies were not given.

One shaped-charge run was performed with depleted $\mathrm{UO}_{2}$. For this experiment, the sieve analysis gave a mass median diameter of $78 \mu \mathrm{m}$ and a logarithmic standard deviation of 2.3. One flyer plate and two shapedcharge experiments were performed with the irradiated fuel. The sieve analyses for the two shaped-charge cases gave essentially identical results: a mass median diameter to $-30 \mu \mathrm{m}$. For the flying plate, the mass median diameter was found to be $-100 \mu \mathrm{m}$. All three of these experiments resulted in similarly shaped bimodal distributions with modes intersecting at $-30 \mu \mathrm{m}$. Particles below this diameter had a logarithmic standard deviation of $\sim 2$, and those above $\sim 8$.

The fraction of material made airborne by the impacts was apparently not measured. However, this fraction can be estimated from the sieve results by considering it equivalent to the fraction of particles with sieve diameters less than $10 \mu \mathrm{m}$. Both the flyer plate and the shapedcharge impacts on spent fuel yielded $2.5 \%$ particulates with diameters less than $10 \mu \mathrm{m}$. The equivalent percentage for depleted $\mathrm{UO}_{2}$ was less than 18 .

Alvarez et al. [AL82] also measured the $137 \mathrm{Cs}$ enrichment in the fines and found it to be independent of the impact type, negligible for $4-\mu \mathrm{m}$ particles, a few percent for $2-\mu \mathrm{m}$ particles, and 1008 or more for particles with diameters less than $1 \mu \mathrm{m}$, suggesting surface adsorption of gap inventory.

In summary, experiments with explosively disrupted fuel yielded the following values for the mass fraction of the disrupted fuel that became aerosolized:

- Schmidt et al. [SC82]: $9.8 \times 10^{-4}$

- Sandoval et al. [SA83]: $5.4 \times 10^{-4}$

$4.4 \times 10^{-3}$ 
- Alvarez et al. [AL82]: $\begin{array}{r}2.5 \times 10^{-2} \\ <1 \times 10^{-2}\end{array}$

The Alvarez data are estimated from sieve measurements rather than from precise measurements of airborne material (as are Schmidt et al. [SC82] and Sandoval et al. [SA83]).

These values may be considered bounding values for the mechanical disruptions that may occur in shipping-cask accidents. They can be compared to the estimated fraction of aerosolized fuel fines $\left(3 \times 10^{-6}\right)$, as discussed earlier.

More probable, rather than bounding values for aerosolized mass fractions may be deduced from experiments conducted to evaluate the potential effects of repository accidents. The impact fracture behavior of various simulated brittle waste-fuel forms has been examined [ME81]. Materials evaluated include $\sim 2 \mathrm{~cm}$ cylinders made of crystalline quartz, fused quartz, Pyrex, nepheline syenite, sandstone, MACOR glass ceramic, and three end-to-end, depleted $\mathrm{UO}_{2}$ pellets. The impact was supplied by a weight falling primarily against the sides of the cylinders and was therefore well characterized. The fragments were contained in a small vessel and their size distribution determined by separating particles smaller than $90 \mu \mathrm{m}$ by wet sieving in water and then drying the larger fraction and dry sieving it. The smaller fraction size distribution was determined down to $-2 \mu \mathrm{m}$ by Coulter counter analysis. Figure IV- 1

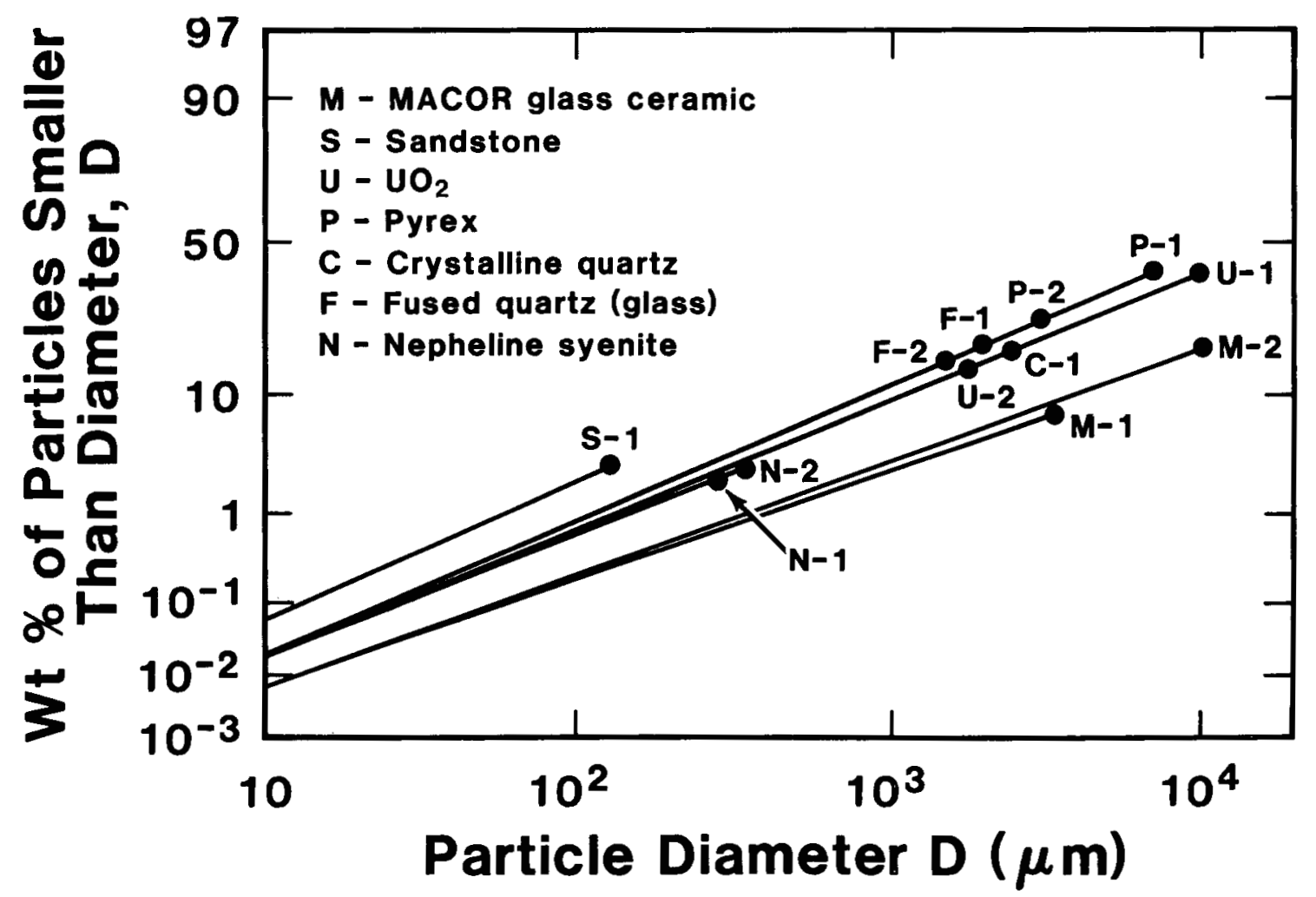

Figure IV-1. Lognormal Plot of Particle-Size Data for Fracture Particulates of Seven Materials [ME81] 
plots the measured size distributions (duplicate runs except for sandstone and crystalline quartz) [ME81]. The dots on the lines indicate the cutoff diameter, above which too few particles existed to permit statistical sampling; the distributions artificially veered from the lognormal. All cases involve an impact energy density of $1.2 \mathrm{~J} / \mathrm{cm}^{3}$. For comparison, a fall from $10 \mathrm{~m}$ results in an energy input of $0.1 \mathrm{~J} / \mathrm{g}$ or for $\mathrm{UO}_{2}, 1.1 \mathrm{~J} / \mathrm{cm}^{3}$.

Note the fragement sizes and standard deviations of the curves in Figure IV-1. First, all fragmentations appear to have resulted in lognormally distributed fragment sizes, regardless of the material. The sieve and Coulter-counter analyses show a smooth transition; their size distributions, plotted on $10 \mathrm{~g}$ probability paper, lie on the same straight line. Second, the logarithmic standard deviations of the curves (their slopes) are all approximately the same.

The data for depleted $\mathrm{UO}_{2}$ are plotted in Figure IV-2 [ME81]. Note that in Figure IV-2 the curve does not show a change in slope at the diameter corresponding to the fuel grain size, $8.8 \mu \mathrm{m}$; the fragmentation is unimpeded across grain boundaries (intragranular cracking occurs readily). The mass median diameter of the distribution is $-18 \mathrm{~mm}$ and the logarithmic standard deviation is 6.4 .

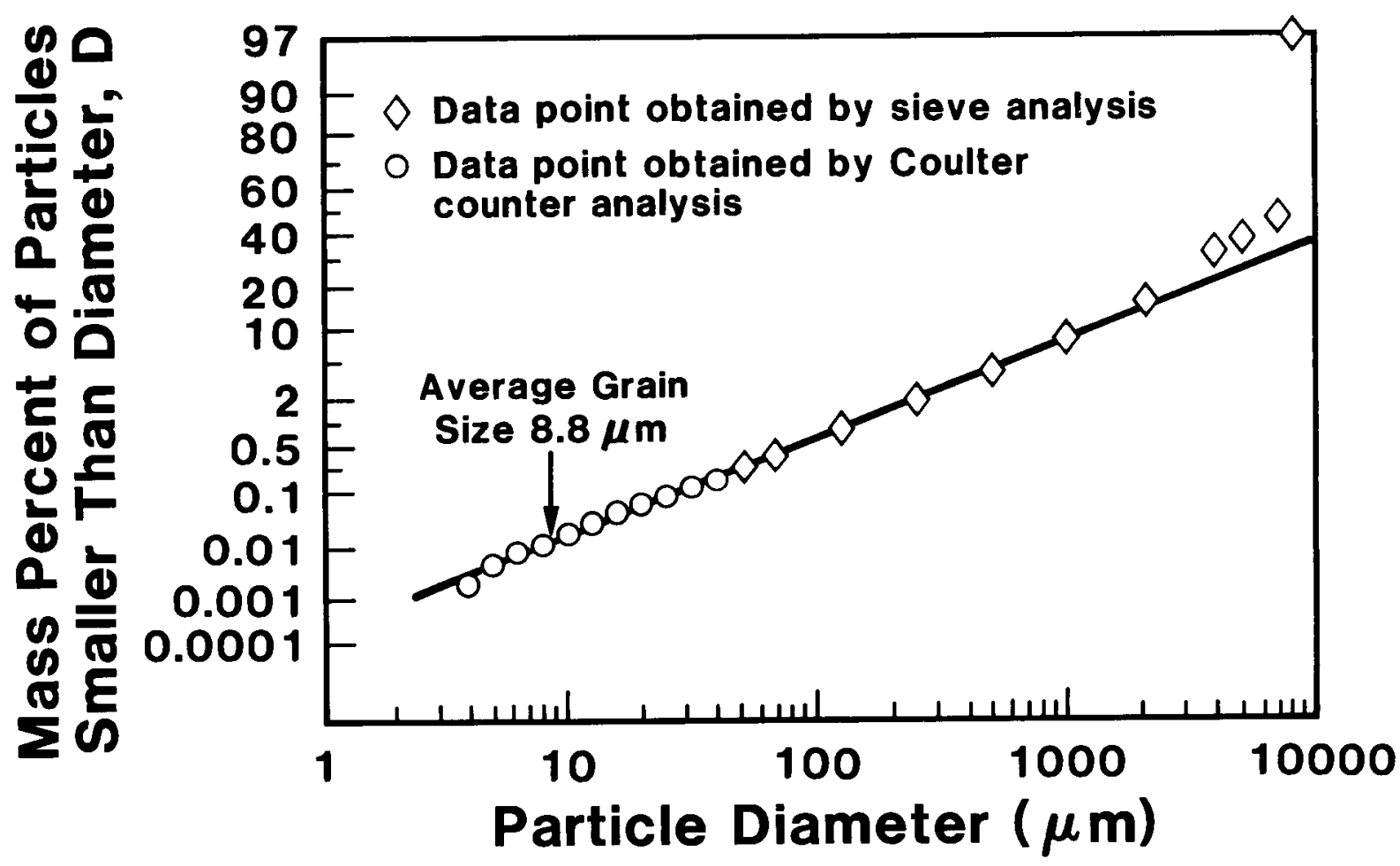

Figure IV-2. Fracture Particle Size Distribution for Depleted UO 2 [ME81] 
Mecham et al. [ME81] attempted to correlate experimental data by invoking earlier work of Piret et al. [AL82, KE61, ZE62]. Piret et al. performed small-scale fracture tests on Pyrex and quartz samples. In these tests, the energy, $W_{F}$, dissipated by the fracturing process was measured calorimetrically, and the increase in surface area, AF, was measured by the Becker-Emmet-Teller (BET) gas adsorption technique. Their findings are summarized in Figure IV-3. The important point to note in this figure is that while the slow-compression tests show a high degree of scatter, the impact tests indicate that the energy absorbed in fracture-per-unit of new surface area (specific surface energy), $\gamma_{F}=W_{F} / A_{F}$, is a weak, single-value function of the energy density, $W_{F} / V_{O}$, where $V_{O}$ is the volume of the body that was fractured. Based on this finding, Mecham et al. developed a correlation of their data in terms of the two parameters, $\alpha \gamma_{F} / \varepsilon$ and $W_{i} / V_{0}$. Here $W_{i}$ is the impact energy, which is related to the fracture energy, $W_{F}$, by $W_{F}=\varepsilon W_{i}$. The shape factor $\alpha$ corrects for nonsphericity of the fracture particles. For glassy materials, measurements of surface area by gas absorption and particle size give $\alpha-21$ [ME81]. For spherical particles, $\alpha=6$. The fracture particle size analysis was used to analytically determine the fracture surface area, $A_{F}$, based on assumed spherical particles distributed lognormally. For Pyrex cylinders and energy densities between 0.41 and $2.4 \mathrm{~J} / \mathrm{cm}^{3}$, fracture surface area and energy are well correlated by the constant

$$
\gamma_{\mathrm{F}} /\left(\mathrm{W}_{\mathrm{F}} / \mathrm{V}_{\mathrm{O}}\right)^{1 / 2}-2.5
$$

Here $\alpha \gamma F / \varepsilon$ is in units of $\mathrm{J} / \mathrm{m}^{2}$ and $\mathrm{W}_{\mathrm{i}} / \mathrm{V}_{\mathrm{O}}$ is in units of $\mathrm{J} / \mathrm{m}^{3}$. To achieve approximate agreement between this expression and the results of Figure IV-3 would require that $\varepsilon \sim 658$, a value in the range measured by Piret for this parameter [ME81] ( $\alpha$ is taken to be 21, as measured).

Ruhmann et al. [RU80] performed we11-defined fracture experiments on fragments of spent (22 GWD/MTU and 33 GWD/MTU) and depleted PWR UO 2 pellets using impact energy densities from 10 to $1000 \mathrm{~J} / \mathrm{g}$. Various impact energies (falling mass) were used. The fragments were contained in a small vessel and wet sieved in chloroform down to a mesh size of $5 \mu \mathrm{m}$. Sieving particles smaller than $10 \mu \mathrm{m}$ is very difficult. Unfortunately, unlike Mecham et al., Ruhmann et al. did not use an alternative method such as Coulter counter to determine the smaller particle sizes and so may not have accounted for them properly. From the size distribution plots, Ruhmann et al. believe that the fuel fragmented down to its grain size only; that is, they observed a sharp drop-off for diameters below $10 \mu \mathrm{m}$ on distributions plotted on log probability paper. This drop-off is suggestive because the grain diameter of Ruhmann et al. samples is also $\sim 10 \mu \mathrm{m}(6$ to $8 \mu \mathrm{m})$. In view of the data from Mecham et al., however, it is likely that the observed drop-off is an artifact of the sieving technique in that it is probable that fines remained associated with the larger fragments. Alvarez et al. [AL82] observed such an effect on micrographs of fragmented fuel. It appears reasonable to assume, therefore, that the measured fragment sizes are lognormally distributed like those of Mecham et al. and that the data can be linearly extrapolated to particles with diameters less than the grain size. The major results of Ruhmann's fracture experiments can be summarized as follows. 


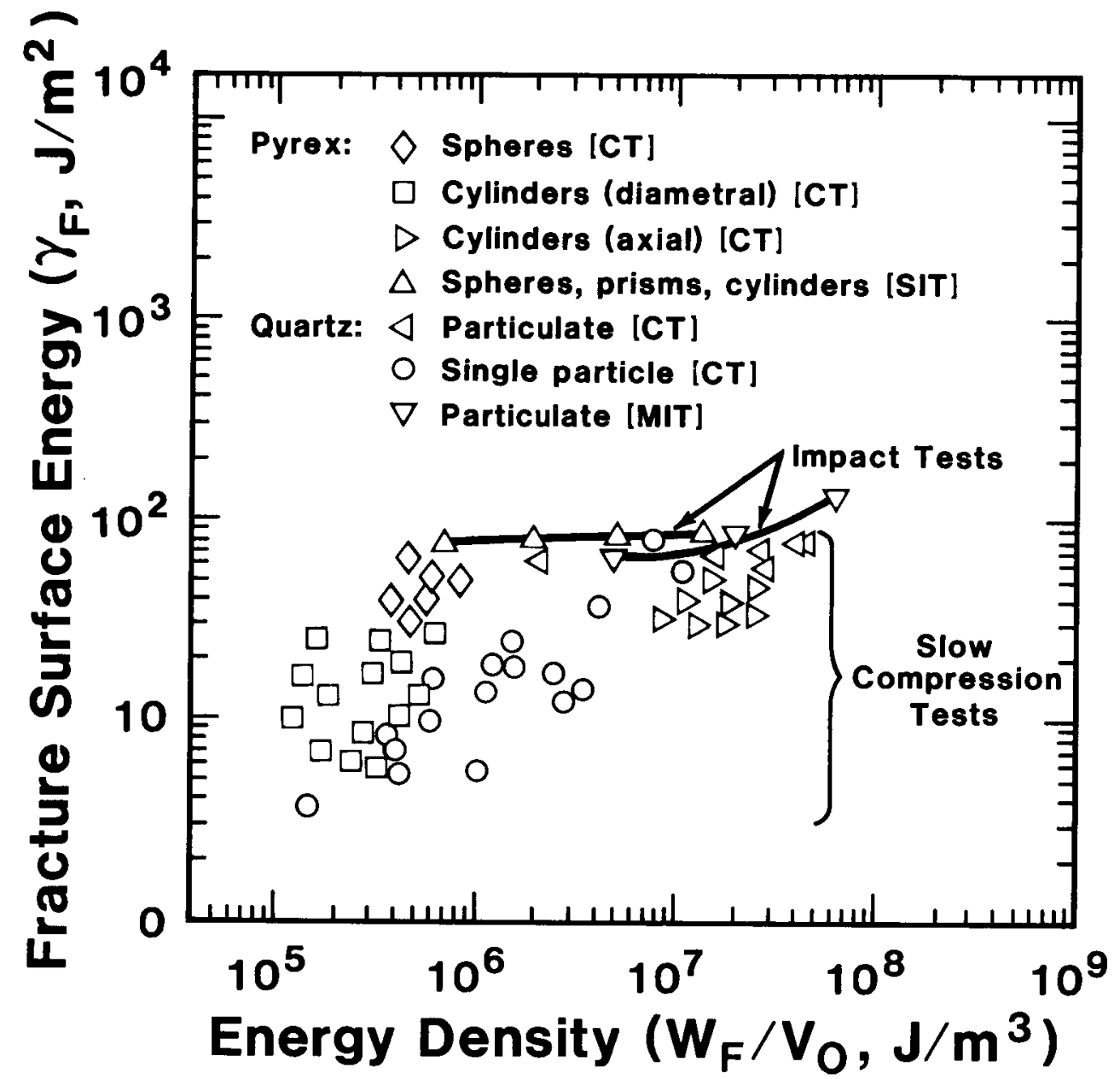

Figure IV-3. Summary of Fracture-Surface Energy Data from Impact Tests and Slow-Compression Tests $\left(W_{F} / V_{O}\right.$ is equal to the energy absorbed per unit volume of brittle material) [ME81].

- Higher impact energy densities lead to finer particles.

- Substituting two $\mathrm{UO}_{2}$ fragments for one in which the mass equals the sum of the two fragments' masses does not change the resultant particle distribution, provided the impact energy density (based on the two fragments) is the same for both.

- For a given impact energy, the higher burnup fuel yields a higher percentage of small particles.

More detail from Ruhmann et al. is presented in Figures IV-4 through IV-6 [RU80]. Figure IV-4 gives cumulative size distribution plots for depleted $\mathrm{UO}_{2}$ fragments resulting from a range of impact energies. 


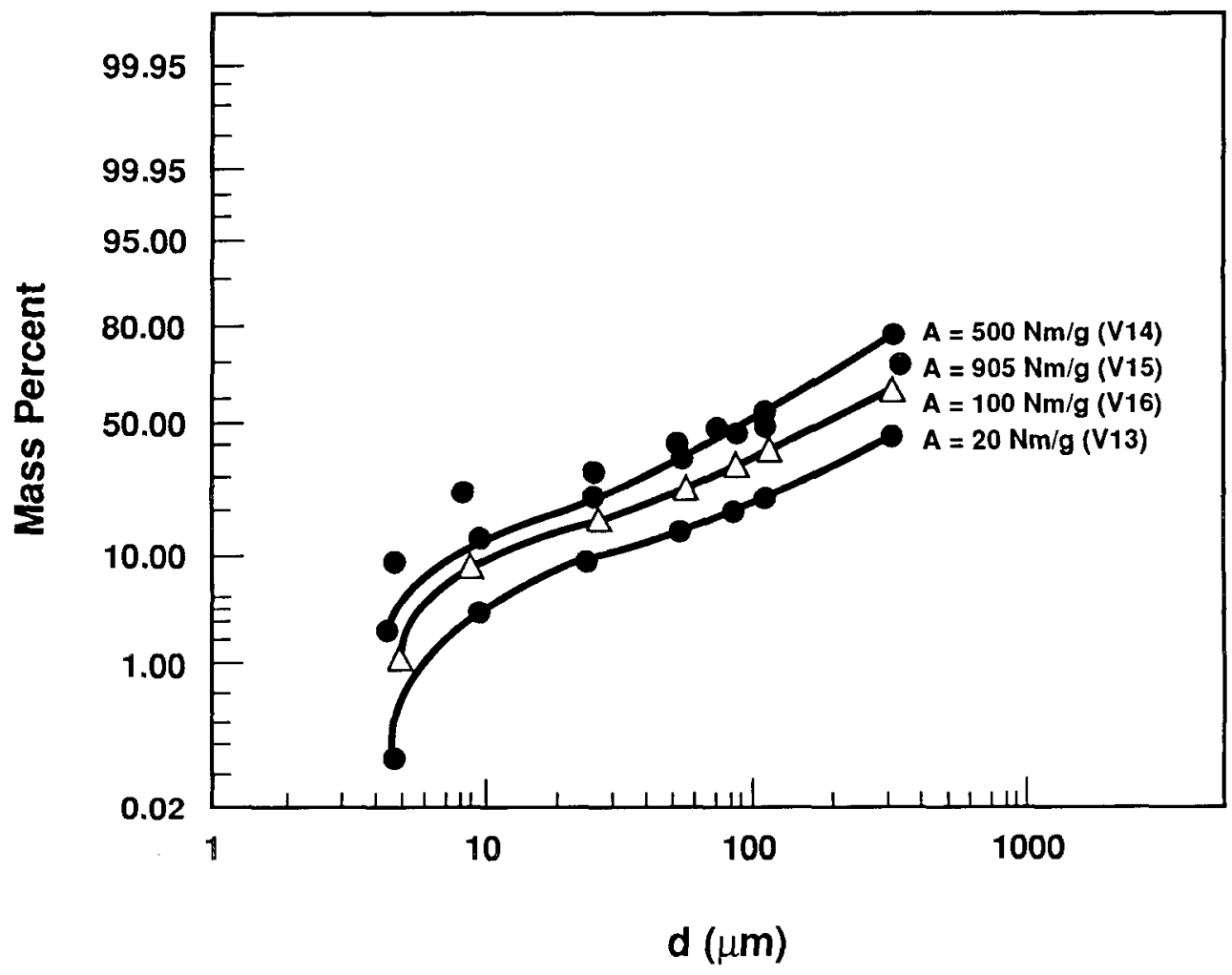

Figure IV-4. Size Distribution of Depleted UO 2 Fragments After Impact with Various Energy Densities [RU80]

Figure IV-5 gives the corresponding distributions for 33 GWD/MTU fuel. Figure IV-6 shows the total specific surface area of the fragments of 33 GWD/MTU fuel as a function of impact energy density. This surface area was calculated based on the assumption of spherical fragments and RosinRammler form of the distribution density. Ruhmann et al. consider this functional form of the distribution density to be a better fit of the data than the lognormal distribution, which would yield a straight line in Figures IV -4 and IV -5 .

\section{5 Impact Energy Correlation Function}

It is useful to correlate the data of Ruhmann et al. with the parameters corresponding to the fracture data of Mecham et al. previously discussed. To perform this correlation, straight lines were fit through the graphical presentations of the experimental data, which were assumed to correlate lognormally. Given the resolution of the reported figures, this correlation could be drawn only approximately and the results are therefore somewhat uncertain. 


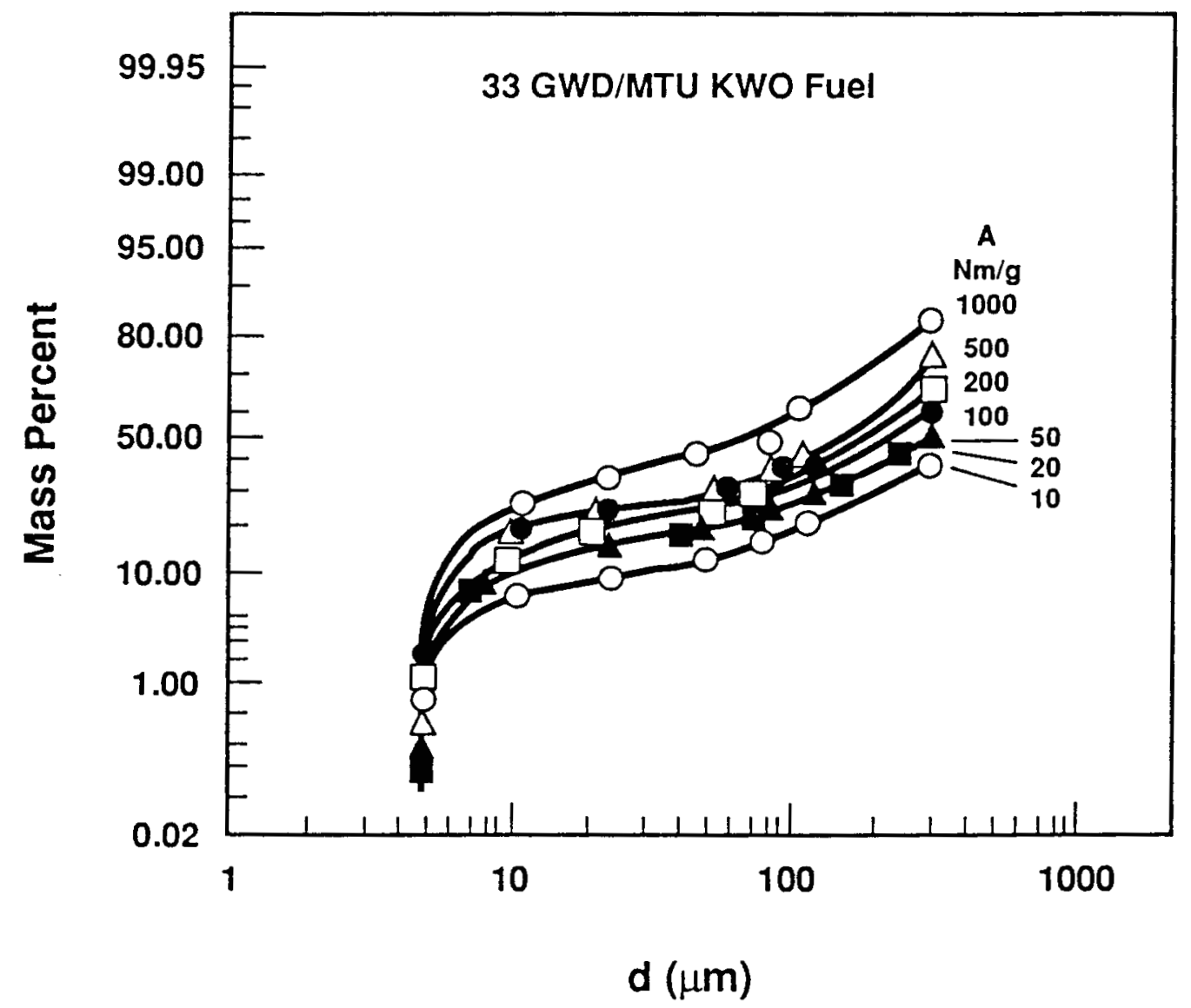

Figure IV-5. Size Distribution of 33 GWD/MTU KWU Fuel Fragments After Impact with Various Energy Densities [RU80]

The correlating parameters are the volume of the body, $V_{0}$, the impact energy, $W_{i}$, the fraction of that energy, $\varepsilon$, absorbed by the body $\left(W_{F}=\right.$ $\left.\varepsilon W_{i}\right)$, and the total fracture surface area, AF. From the graphs, one can determine the mass median diameter, $\mathrm{d}_{50}$, and the logarithmic standard deviation, $\sigma$ ( $\sigma$ is the ratio of the 508 diameter to the $16 \%$ diameter). Then, from the known properties of the lognormal distribution, the number geometeric mean diameter, $d_{g}$, is given by

$$
d_{g}=d_{50} e^{-31 n^{2} \sigma},
$$

and the nth moment of the distribution, $\left\langle\mathrm{d}^{\mathrm{n}}\right\rangle$, is given by

$$
\left.<d^{n}\right\rangle=d_{g}^{n} e^{n^{2} / 2} \ln ^{2} \sigma
$$




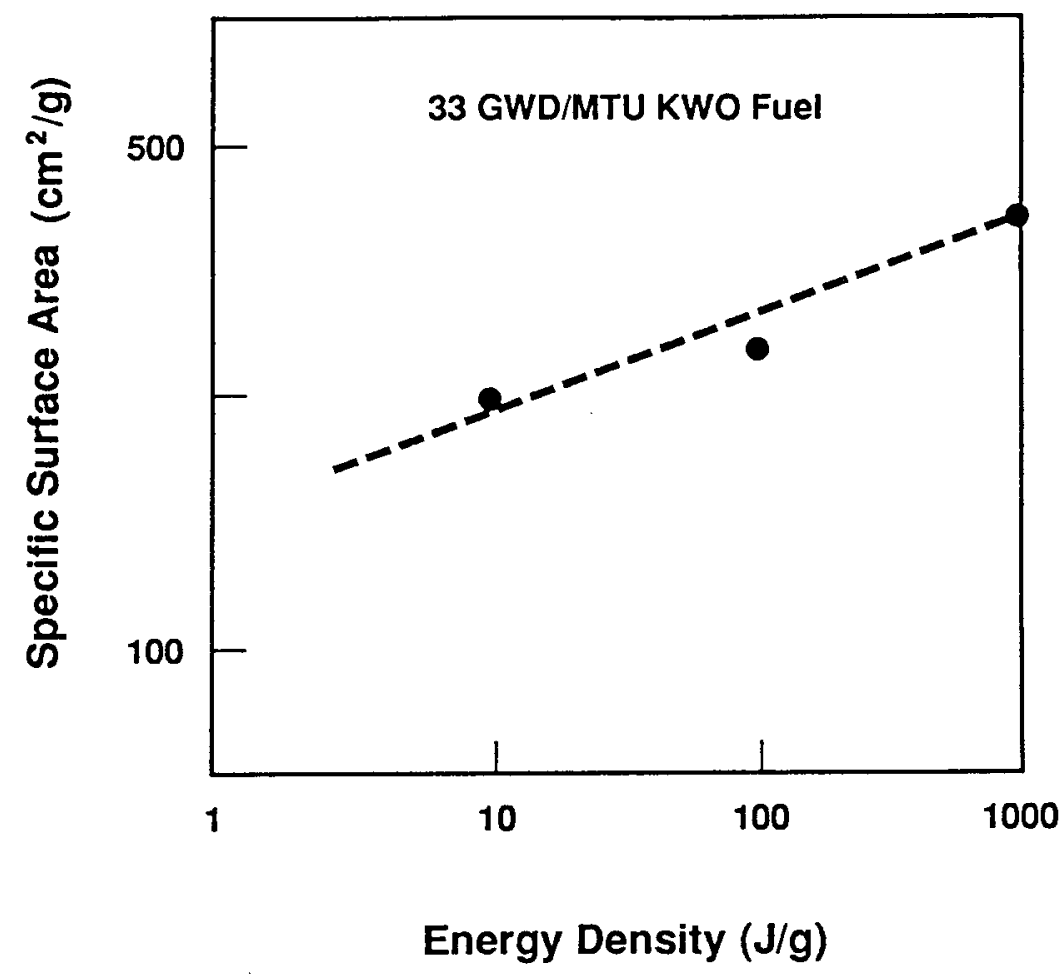

Figure IV-6. Specific Surface Area of 33 GWD/MTU Fuel Fragments After Impact with Various Energy Densities [RU80]

The total surface area of the fragments, assuming these are spherical, is

$$
A_{F}=\pi N<d^{2}>=\pi N d_{g}^{2} e^{2} \ln ^{2} \sigma
$$

and the total volume of the fragments is

$$
\mathrm{V}_{\mathrm{F}}=\frac{\pi}{6} \mathrm{~N}\left\langle\mathrm{~d}^{3}\right\rangle=\frac{\pi}{6} \mathrm{~N} \mathrm{~d}_{\mathrm{g}}^{3} \mathrm{e}^{4.5} \ln ^{2} \sigma
$$

where $\mathrm{N}$ is the total number of fragments. Elimination of $\mathrm{N}$ between these two expressions and with $V_{F}=V_{O}$, the volume of the body before fracture, gives

$$
\begin{aligned}
& A=\frac{6 V_{0}}{d_{g}} e^{2.5} \ln ^{2} \sigma \\
& A=\frac{6 V_{0}}{d_{50}} e^{0.5 \ln ^{2} \sigma} .
\end{aligned}
$$


It is difficult to obtain an accurate value of $\sigma$ from the reported plots of the size distributions. An estimate based on these plots gives approximately $\sigma=7$ for depleted $\mathrm{UO}_{2}, \sigma=15$ for $22 \mathrm{GWD} / \mathrm{MTU}$ fue1, and $\sigma=$ 19 for 33 GWD/MTU fuel, independent of impact energy. These values signify extremely broad distributions. The Mecham et al. value for $\sigma$ was similar to the value of Ruhmann et al., despite the fact that the impact energy density for Mecham's study was $0.11 \mathrm{~J} / \mathrm{g}$ and for Ruhmann's, a range of 10 to $1000 \mathrm{~J} / \mathrm{g}$. Using these values for $\sigma$ and the $\mathrm{d}_{50}$ values that can be extracted from the plots gives the tabulated values in Tables IV- 3 through IV-5 for the correlation parameters. (The curve for $905 \mathrm{~J} / \mathrm{g}$ in Figure IV-4 appears to be anomalous and is therefore not analyzed here.)

Several observations can be made about the tabulated values. First, the impact energy, $W_{\mathfrak{i}}$, used in the correlation is the kinetic energy of mass at impact, not necessarily the energy, $W_{F}$, absorbed by the impacted body, as desired. This occurs because $\varepsilon$ was assumed to be 1 . There is probably an uncertainty factor of 2 in this value. Second, the values of the distribution parameters, particularly $\sigma$, are uncertain. Picking $\sigma=15$ for the $33 \mathrm{GWD} / \mathrm{MTU}, 10 \mathrm{~J} / \mathrm{g}$ case, for example, yields

$$
\frac{\frac{\alpha \gamma_{F}}{\varepsilon}}{\left(W_{i} / V_{o}\right)^{1 / 2}}=0.24
$$

instead of 0.12 . Given these uncertainties, however, it is remarkable that the parameter

$$
\frac{\frac{\alpha \gamma_{F}}{\varepsilon}}{\left(\mathrm{W}_{i} / \mathrm{V}_{0}\right)^{1 / 2}}
$$

is roughly a constant for a given fuel type over the impact energy range investigated. The data of Ruhmann et al. thus appear to support the correlation model devised by Mecham et al., which shows promise as a means of determining the fragment size distribution resulting from a given impact on spent-fuel rods. The model clearly needs further experimental corroboration and possible refinement beyond the scope of currently reported data. In particular, more data are needed for lower impact energy densities, because those densities employed by Ruhmann are probably far in excess of the impact energy densities of spent fuel in transport.

Assuming the above correlation model devised by Mecham et al. holds, i.e., that

$$
\frac{\frac{\alpha \gamma_{F}}{\varepsilon}}{\left(W_{i} / V_{o}\right)^{1 / 2}}=K
$$


Table IV-3

Correlation Parameter Values for Depleted $\mathrm{UO}_{2}$

\begin{tabular}{|c|c|c|c|c|}
\hline \multirow[b]{2}{*}{ Parameter } & \multirow[b]{2}{*}{ Units } & \multicolumn{3}{|c|}{ Impact Energy Density } \\
\hline & & $\begin{array}{l}0.11 \mathrm{~J} / \mathrm{g} \\
\text { (Mecham) }\end{array}$ & $\begin{array}{c}20 \mathrm{~J} / \mathrm{g} \\
\text { (Ruhmann) } \\
\end{array}$ & $\begin{array}{c}500 \mathrm{~J} / \mathrm{g} \\
\text { (Ruhmann) } \\
\end{array}$ \\
\hline$v_{0}$ & $\mathrm{~cm}^{3}$ & 6.0 & 0.126 & 0.0843 \\
\hline $\mathrm{w}_{\mathrm{i}}$ & $\mathrm{J}$ & 7.2 & 27.2 & 455 \\
\hline $\mathrm{w}_{\mathrm{i}} / \mathrm{V}_{\mathrm{o}}$ & $\mathrm{J} / \mathrm{m}^{3}$ & $1.20 \times 10^{6}$ & $2.16 \times 10^{8}$ & $5.40 \times 10^{9}$ \\
\hline $\mathrm{d}_{50}$ & $\mu \mathrm{m}$ & 18,000 & 280 & 78 \\
\hline$\sigma$ & - & 6.4 & 7 & 7 \\
\hline $\mathrm{A}_{F} / \alpha$ & $\mathrm{m}^{2}$ & $1.87 \times 10^{-3}$ & $2.99 \times 10^{-3}$ & $7.18 \times 10^{-3}$ \\
\hline$\frac{\alpha \gamma}{\varepsilon}$ & $\mathrm{J} / \mathrm{m}^{2}$ & $3.85 \times 10^{3}$ & $9.10 \times 10^{3}$ & $6.34 \times 10^{4}$ \\
\hline$\left.\frac{\alpha \gamma}{\varepsilon} /{ }^{\left(W_{i}\right.} / \mathrm{V}_{0}\right)^{1 / 2}$ & $\mathrm{~J}^{1 / 2} / \mathrm{m}^{1 / 2}$ & 3.8 & 0.62 & 0.86 \\
\hline
\end{tabular}

Table IV-4

Correlation Parameter Values for

Spent $\mathrm{UO}_{2}$ (22 GWD/MTU) [RU80]

\begin{tabular}{|c|c|c|c|}
\hline \multirow[b]{2}{*}{ Parameter } & \multirow[b]{2}{*}{ Units } & \multicolumn{2}{|c|}{ Impact Energy Density } \\
\hline & & $20 \mathrm{~J} / \mathrm{g}$ & $100 \mathrm{~J} / \mathrm{g}$ \\
\hline$v_{0}$ & $\mathrm{~cm}^{3}$ & $6.48 \times 10^{-2}$ & 0.105 \\
\hline $\mathrm{w}_{\mathrm{i}}$ & $J$ & 14.0 & 113 \\
\hline $\mathrm{w}_{\mathrm{i}} / \mathrm{V}_{\mathrm{o}}$ & $\mathrm{J} / \mathrm{m}^{3}$ & $2.16 \times 10^{8}$ & $1.08 \times 10^{9}$ \\
\hline$d_{50}$ & $\mu \mathrm{m}$ & 600 & 240 \\
\hline$\sigma$ & - & 15 & 15 \\
\hline $\mathrm{A}_{\mathrm{F}} / \alpha$ & $\mathrm{m}^{2}$ & $4.23 \times 10^{-3}$ & $1.71 \times 10^{-2}$ \\
\hline$\frac{\alpha \gamma}{\varepsilon}$ & $\mathrm{J} / \mathrm{m}^{2}$ & $3.31 \times 10^{3}$ & $6.61 \times 10^{3}$ \\
\hline$\frac{\alpha \gamma}{\varepsilon} /\left(\mathrm{W}_{\mathrm{i}} / \mathrm{V}_{\mathrm{o}}\right)^{1 / 2}$ & $\mathrm{~J}^{1 / 2} / \mathrm{m}^{1 / 2}$ & 0.23 & 0.20 \\
\hline
\end{tabular}


Table IV-5

Correlation Parameter Values for

Spent $\mathrm{UO}_{2}$ (33 GWD/MTU)

\begin{tabular}{|c|c|c|c|c|}
\hline \multirow[b]{2}{*}{ Parameter } & \multirow[b]{2}{*}{ Units } & \multicolumn{3}{|c|}{ Impact Energy Density } \\
\hline & & $10 \mathrm{~J} / \mathrm{g}$ & $100 \mathrm{~J} / \mathrm{g}$ & $1000 \mathrm{~J} / \mathrm{g}$ \\
\hline \multirow[t]{2}{*}{$v_{0}$} & $\mathrm{~cm}^{3}$ & $5.83 \times 10^{-2}$ & $7.31 \times 10^{-2}$ & $5.83 \times 10^{-2}$ \\
\hline & & & $5.74 \times 10^{-2}$ & $6.76 \times 10^{-2}$ \\
\hline $\mathrm{W}_{\mathbf{i}}$ & $\mathrm{J}$ & 6.30 & 70.5 & 680 \\
\hline $\mathrm{w}_{\mathrm{i}} / \mathrm{v}_{\mathrm{o}}$ & $\mathrm{J} / \mathrm{m}^{3}$ & $1.08 \times 10^{8}$ & $1.08 \times 10^{9}$ & $1.08 \times 10^{10}$ \\
\hline$d_{50}$ & $\mu \mathrm{m}$ & 900 & 200 & 70 \\
\hline$\sigma$ & - & 19 & 19 & 19 \\
\hline $\mathrm{A}_{F} / \alpha$ & $\mathrm{m}^{2}$ & $4.94 \times 10^{-3}$ & $2.49 \times 10^{3}$ & $9.91 \times 10^{3}$ \\
\hline \multicolumn{5}{|c|}{ 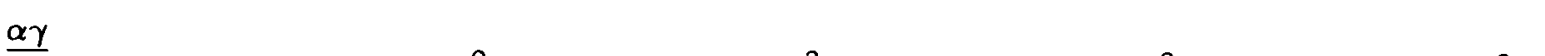 } \\
\hline$\varepsilon$ & $\mathrm{J} / \mathrm{m}^{2}$ & $1.28 \times 10^{3}$ & $2.83 \times 10^{3}$ & $9.91 \times 10^{3}$ \\
\hline$\left.\frac{\alpha \gamma}{\varepsilon} /{ }^{\left(W_{i}\right.} / \mathrm{V}_{o}\right)^{1 / 2}$ & $\mathrm{~J}^{1 / 2} / \mathrm{m}^{1 / 2}$ & 0.12 & 0.086 & 0.095 \\
\hline
\end{tabular}

where $\mathrm{K}$ is a constant that only depends on the fuel type, one can determine the fragment size distribution parameters for a given impact energy, $W_{i}$. The impact energy, $W_{i}$, and the volume of the fuel, $V_{0}$, affected by the impact are parameters that can be estimated from the fuel behavior calculations of the model discussed in section IV-3. The parameter $\mathrm{K}$ and the logarithmic standard deviation, $\sigma$, are derived from the experimental data. They appear to be relatively independent of impact energy for a given fuel type. The fraction of the emplaced fuel, $F_{A}$, that fragments to particle sizes less than $10 \mu \mathrm{m}$ is of interest (i.e., roughly the fraction that is aerosolized) and can be found by integration of the distribution function:

$$
\mathrm{F}_{\mathrm{A}}=\frac{1}{2}\left[1+\operatorname{erf}\left(\frac{\ln \mathrm{d}_{\mathrm{c}} / \mathrm{d}_{50}}{\sqrt{2} \ln \sigma}\right)\right] \text {, }
$$

where $d_{c}=10 \mu \mathrm{m}$. This expression can be conservatively maximized by choosing a constant value of $\sigma$ that envelopes the experimentally determined values. 
It is instructive to apply this model to a single fuel pellet falling from a height of $10 \mathrm{~m}$ onto an unyielding surface. If no bounce is assumed, or $\varepsilon=1$, then

$$
\frac{\mathrm{W}_{\mathrm{i}}}{\mathrm{V}_{\mathrm{o}}}=\frac{\mathrm{g} \rho \mathrm{h}_{\mathrm{g}} \mathrm{V}_{\mathrm{o}}}{\mathrm{V}_{\mathrm{o}}}=\rho \mathrm{gh},
$$

where $\rho$ is the density of the pellet (e.g., $10,800 \mathrm{~kg} / \mathrm{m}^{3}$ ). Inserting $\mathrm{g}=$ $9.81 \mathrm{~m} / \mathrm{s}^{2}$ and $\mathrm{h}=10 \mathrm{~m}$ into this expression gives $1.059 \times 10^{6} \mathrm{~J} / \mathrm{m}^{3}$, which is two orders of magnitude less than the energy insertions considered by Ruhmann et al.

If a 33 GWD/MTU burnup is assumed, $\mathrm{K}=0.02$ and, with $\sigma=19$, the mass median diameter is

$$
\mathrm{d}_{50}=7416 \mu \mathrm{m} .
$$

Using this value in the expression for the aerosolized mass fraction gives

$$
F_{\mathrm{A}}=1.28
$$

\section{6 cladding}

There may be instances in which fuel assemblies that contain rods having breached claddings are loaded for shipment. This situation can involve two types of breaches: one that results in the rods becoming waterlogged and the other in which the cladding defect and operational history are such that water has been prevented from entering the rod.

In either case, burst release occurs prior to preparing the assembly for shipment and cesium nuclides predominate. In the event that water does not penetrate the fuel rod, subsequent cesium release (during shipment) will occur by the diffusional process described earlier, and its magnitude can be calculated with Equation (IV-2). (Note that in Equation [IV-2] it is assumed that the gap inventory, $M_{O}$, remains constant. Equation [IV-2] thus necessarily overestimates the release for time periods that significantly deplete the gap inventory.)

When the temperature of a waterlogged rod is increased, water vaporization within the rod occurs and water is transported from the rod into the cask cavity until the partial pressure of the water vapor in the rod and the cask is equal, or until all water is vaporized. This transport has the same effect on cesium in the gap that fill- and fission-product gases do in the burst release mode. Thus, although the database is nonexistent, an argument can be made to justify application of a modified form of Equation (IV-1) to describe cesium release from a water-laden fuel rod. A recommended procedure is to apply Equation (IV-1) over a time period for which the cesium release does not decrease $M_{O}$ by more than $10 \%$, then to repeat the calculation after modifying $M_{O}$ for the next time period, and so on. 


\section{7 Air Oxidation of Rods Having Defected Claddings}

Uranium dioxide $\left(\mathrm{UO}_{2}\right)$ can be oxidized rapidly in air at elevated temperatures (greater than $-250^{\circ} \mathrm{C}$ ) [HA85] to produce a higher oxide $\left(\mathrm{U}_{3} \mathrm{O}_{8}\right)$. Because $\mathrm{U}_{3} \mathrm{O}_{8}$ is considerably less dense than $\mathrm{UO}_{2}$, the oxidation process involves significant changes to the $\mathrm{UO}_{2}$ crystal lattice, and thus opportunity for additional radionuclide escape from the fuel. Although studies of fission product release from $\mathrm{UO}_{2}$ under oxidizing conditions have been made [PA73], most of this work has been conducted on unclad fuel at temperatures in excess of $800^{\circ} \mathrm{C}$. As a consequence, the results are of limited use in safety assessments of spent-fuel transport.

Apparently only four experiments have been performed to investigate the release of fission products from fuel rods with defected claddings in an air atmosphere [HA85, C088]. At $200^{\circ} \mathrm{C}$, no oxidation of $\mathrm{UO}_{2}$ was detected after exposure for $24 \mathrm{hr}$; approximately $1.4 \times 10^{-4} \%$ of the $85 \mathrm{Kr}$ inventory of the rod was released over the $24 \mathrm{hr}$ period, and no release of $106 \mathrm{Ru}$, $134 \mathrm{CS}$, or $137 \mathrm{Cs}$ was detected [HA85]. At $400^{\circ} \mathrm{C}$, local oxidation of $\mathrm{UO}_{2}$ to $\mathrm{U}_{3} \mathrm{O}_{8}$ was noted on exposure over a $24 \mathrm{hr}$ period and $85 \mathrm{Kr}$ release was estimated to be $1.8 \times 10^{-4} \%$ of inventory, but again, no release of other nuclides was detected [HA85]. At $500^{\circ} \mathrm{C}$, cesium nuclide release on exposure of a fuel rod to air was observed to be almost identical to that determined in a steam atmosphere, whereas at $700^{\circ} \mathrm{C}$, about 58 times more cesium was released in dry air than in steam but the release rate was only $2.9 \times 10^{-4}$ o of total inventory per hr [CO88].

Additionally, some experiments were conducted in which irradiated fuel was deliberatelly oxidized to $\mathrm{U}_{3} \mathrm{O}_{8}$ using air or oxygen at $480^{\circ} \mathrm{C}$ [G080]. The experiments indicated complete release of the tritium, but only 4 to 68 release of the $85 \mathrm{Kr}$ inventory and virtually no release $(0.1$ to 0.28 of inventory) of ruthenium, cesium, and cerium. Oxidation does result in significant comminution of the fuel, however, and this can lead to an enhancement of the fission product source term.

All of these releases can occur only if air is already present in the cask; that is, the cask is inadvertently filled with air rather than an inert gas during preparation of the package for shipment. In contrast, in the event of an accident involving air in-leakage into the cask, oxidation of the $\mathrm{UO}_{2}$ will be severely limited by the accessibility to air. This can be readily demonstrated by the following example.

Assume that under accident conditions (as determined with air at $25^{\circ} \mathrm{C}$ ) the maximum permissible leak rate for a cask is $14 \mathrm{~cm}^{3} / \mathrm{s}$. From Table B2 of the ANSI standard ANSI N14.5-1987 [AN87], this leak rate corresponds to a leak path that is $0.03 \mathrm{~cm}$ in diameter and $1 \mathrm{~cm}$ in length. Also assume that a hypothetical impact environment causes a breach in this cask equal to the maximum permissible leak rate, that there is failure of some of the fuel rod claddings, and that internal temperatures are $-1000^{\circ} \mathrm{C}$ [BU85]. The cask is assumed to be maintained in this condition for two days. by [MA83]

The molar flux of any gaseous species $i$ in or out of the cask is given 


$$
J_{i}=\frac{-p D_{i j}}{R T} \frac{\partial x_{i}}{\partial z}+x_{i} J
$$

where

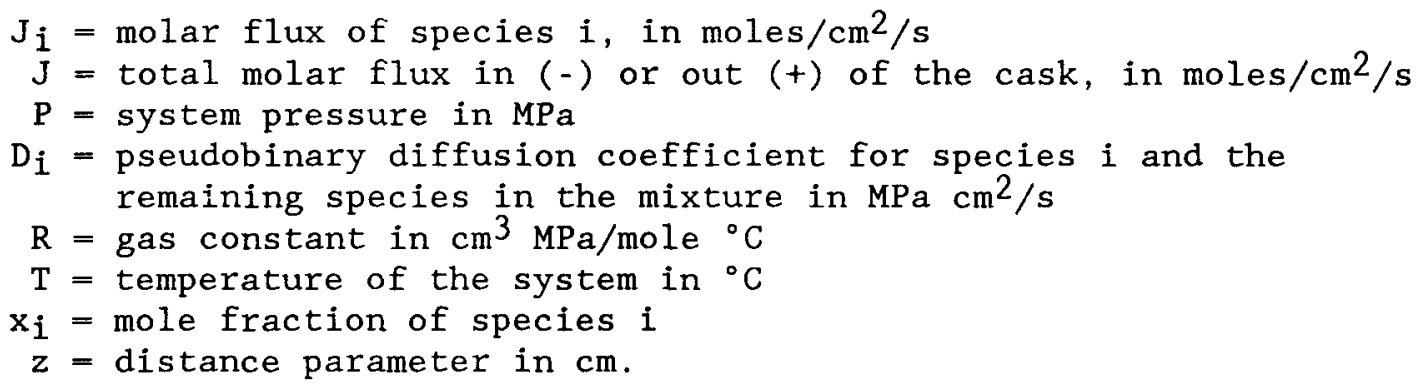

For materials to leak from the cask in significant quantity, $J$ must be positive, whereas for oxygen to leak into the cask, $\mathrm{J}_{\mathrm{O}_{2}}$ must be negative.

Thus, the calculation of oxygen ingress will yield maximal values if the net flux ( $\left.x_{j} J\right)$ term in Equation (IV-13) is ignored. Similarly, if all of the oxygen is immediately consumed as it enters the cask, $\mathrm{J}_{\mathrm{O}_{2}}$ is a constant, and the gradient expression becomes

$$
\left(\partial \mathrm{x}_{\mathrm{O}_{2}} / \partial z\right) \rightarrow\left(\mathrm{x}_{\mathrm{O}_{2}} / 1\right)
$$

where $\mathrm{x}_{2}$ is the mole fraction of oxygen in air (0.209) and 1 is the breach length in the cask $(1 \mathrm{~cm})$. Finally, the pseudobinary diffusion coefficient is taken to be that between helium and air; at $1000^{\circ} \mathrm{C}, \mathrm{p}$ (helium-air) $=$ $0.894 \mathrm{MPa} \mathrm{cm} / \mathrm{s}$ [MA70]. The molar flux of oxygen into the cask at $1000^{\circ} \mathrm{C}$ is thus

$$
\mathrm{J}_{2}=\frac{0.894 \mathrm{MPa} \mathrm{cm} / \mathrm{s}(0.209 / 1 \mathrm{~cm})}{\left(8.31 \mathrm{~cm}^{3} \mathrm{MPa} /{ }^{\circ} \mathrm{C} \mathrm{mole}\right)\left(1000^{\circ} \mathrm{C}\right)}=1.76 \times 10^{-5} \mathrm{~mol} / \mathrm{cm}^{2} \mathrm{~s} \text {, }
$$

or the rate of in-leakage through the $0.03-\mathrm{cm}$-diameter hole is

$$
\mathrm{Q}_{2}=\mathrm{J}_{\mathrm{O}_{2}}\left(\pi \mathrm{D}^{2} / 4\right)=1.25 \times 10^{-8} \mathrm{~mol} / \mathrm{s}
$$

Hence, in a period of 48 hours, $2.16 \times 10^{-3} \mathrm{~mol}$ of oxygen can enter the cask. If this entire amount reacts with $\mathrm{UO}_{2}$ to form $\mathrm{U}_{3} \mathrm{O}_{8}, 0.582 \mathrm{~g}$ of fuel can be oxidized. For the example PWR fuel rod described earlier, this amounts to $0.26 \%$ of the fuel, and $0.015 \%$ for the BWR fuel rod.

If the fuel temperature does not exceed $350^{\circ} \mathrm{C}$, additional releases from the oxidized fuel will be inconsequential [HA85]. Furthermore, 
although communication of the oxidized fuel will result, the absence of a driving force, as is present when a fuel rod first suffers cladding failure, will severely limit the amount of airborne fines produced. In view of the conservative assumptions made to investigate the effects of oxidation, it is therefore believed that air ingress scenarios involve additional radioactive material releases not much greater in magnitude than fuel fine releases that will occur as the claddings initially lose integrity.

\section{8 Containment Requirements}

As described earlier in Section IV.2, radionuclide release from 5-yr-old irradiated fuel rods undergoing loss of cladding integrity is of three types: (1) gaseous release, (2) volatile radionuclides, and (3) nonvolatile species (particulate) release. Gaseous release involves primarily tritium and $85 \mathrm{Kr}$, and it is simply assumed in the release model that the entire gap inventories of these nuclides escape during cladding failure. Releases of other volatile species involve ${ }^{134} \mathrm{Cs}$ and $137 \mathrm{Cs}$; these releases are described by the burst release mode1, Equation (IV-1). Furthermore, it has been demonstrated that a companion model that describes diffusional release can usually be ignored. The third model concerns radionuclide release in the form of fuel fines. The corresponding model simply states that the release is independent of temperature and equal to $0.0003 \%$ of the total fuel amount of a rod when its cladding fails. Impact-generated fines are a sensitivity case to be treated in future work. All three models yield release values that are assumed to be independent of the location and size of the point of cladding failure.

All gaseous species--once released from a fuel rod--are assumed to become uniformly distributed throughout a shipping cask. Pending more definitive data regarding particle size distributions of fuel fines ejected from rods during cladding failure, this work assumes, based on available experimental data, that only 10 of of the total amount of fines remains airborne, and that this airborne fraction does become uniformly distributed throughout the cask. A summary of radioactive material available for release, assuming no comminution of fuel as a result of impact, is tabulated for an illustrative PWR case in Table IV- 6.

An examination of the data presented in Table IV- 6 indicates that the radiotoxicity (in terms of $\mathrm{A}_{2}$ ) is dominated by the actinides. Also, for this example shipping cask, the total concentration of radioactive species available for release into the environment is $1.62 \times 10^{-7} \mathrm{~A}_{2}\left(\mathrm{Ci} / \mathrm{cm}^{3}\right)$ for every rod cladding failure during shipment.

Under normal transport conditions, ANSI N14.5 allows a release rate of not more than $10^{-6} \mathrm{~A}_{2}$ (Ci) in $1 \mathrm{hr}$ for adequate containment [AN87]. Because it appears unlikely under normal transport conditions that more than a single failure will occur, this translates directly into a leak rate of $6.19 \mathrm{~cm}^{3} / \mathrm{hr}$, or $1.72 \times 10^{-3} \mathrm{~cm}^{3} / \mathrm{s}$. Similarly, this translates to $6.17 \times 10^{6} \mathrm{~cm}^{3} / \mathrm{wk}$, or $10.2 \mathrm{~cm}^{3} / \mathrm{s}$, if it is assumed that only one rod fails during an accident. In the event an entire $15 \times 15$ assembly failed (204 rods/assembly), the criterion becomes $8.4 \times 10^{-6} \mathrm{~cm}^{3} / \mathrm{s}$ for normal conditions and $5.0 \times 10^{-2} \mathrm{~cm}^{3} / \mathrm{s}$ for regulatory accidents. 
Table IV-6

Concentrations of Airborne Radionuclides Resulting from the Failure of One PWR Fuel Rod in a Representative Truck Cask Volumea

\begin{tabular}{|c|c|c|}
\hline $\begin{array}{l}\text { Nuclides } \\
\left(\mathrm{A}_{2}\right) \\
\end{array}$ & $\begin{array}{l}\text { Number of } \mathrm{A}_{2} \\
\text { Equivalents Released } \\
\end{array}$ & $\begin{array}{l}\text { Airborne Concentration } \\
\text { (A2 Equivalents per } \mathrm{m}^{3} \text { ) }\end{array}$ \\
\hline $3_{\mathrm{H}}^{\mathrm{d}}$ & $5.10 \times 10^{-4}$ & $3.29 \times 10^{-3}$ \\
\hline $95 \mathrm{Kr}$ & $1.57 \times 10^{-4}$ & $1.01 \times 10^{-3}$ \\
\hline${ }^{90} \mathrm{Sr}$ & $1.16 \times 10^{-2}$ & $7.51 \times 10^{-3}$ \\
\hline $90 \mathrm{Y}$ & $4.66 \times 10^{-4}$ & $3.00 \times 10^{-4}$ \\
\hline $106_{\mathrm{Ru}}$ & $1.41 \times 10^{-4}$ & $9.08 \times 10^{-5}$ \\
\hline $125 \mathrm{~m} \mathrm{Te}$ & $6.44 \times 10^{-7}$ & $4.15 \times 10^{-7}$ \\
\hline $134 \mathrm{Cs}^{\mathrm{e}}$ & $3.38 \times 10^{-4}$ & $9.73 \times 10^{-4}$ \\
\hline $137 \mathrm{Cs}^{\mathrm{e}}$ & $1.13 \times 10^{-3}$ & $3.23 \times 10^{-3}$ \\
\hline $144 \mathrm{Ce}$ & $8.23 \times 10^{-5}$ & $5.31 \times 10^{-5}$ \\
\hline $154 \mathrm{Eu}$ & $1.18 \times 10^{-4}$ & $7.63 \times 10^{-5}$ \\
\hline $155_{\mathrm{Eu}}$ & $4.43 \times 10^{-6}$ & $2.86 \times 10^{-6}$ \\
\hline $235 U$ & $4.37 \times 10^{-9}$ & $2.82 \times 10^{-9}$ \\
\hline $238 \mathrm{Pu}$ & $8.45 \times 10^{-2}$ & $5.45 \times 10^{-2}$ \\
\hline${ }^{239} \mathrm{Pu}$ & $1.06 \times 10^{-2}$ & $6.81 \times 10^{-3}$ \\
\hline $240 \mathrm{Pu}$ & $1.94 \times 10^{-2}$ & $1.25 \times 10^{-3}$ \\
\hline${ }^{241} \mathrm{Pu}$ & $7.48 \times 10^{-2}$ & $4.83 \times 10^{-2}$ \\
\hline $241_{\mathrm{Am}}$ & $1.02 \times 10^{-2}$ & $6.56 \times 10^{-3}$ \\
\hline $244 \mathrm{~cm}$ & $2.08 \times 10^{-2}$ & $1.34 \times 10^{-2}$ \\
\hline Balance & $5.22 \times 10^{-3}$ & $3.37 \times 10^{-3}$ \\
\hline Totals & $2.40 \times 10^{-1}$ & $1.62 \times 10^{-1}$ \\
\hline \multicolumn{3}{|c|}{ 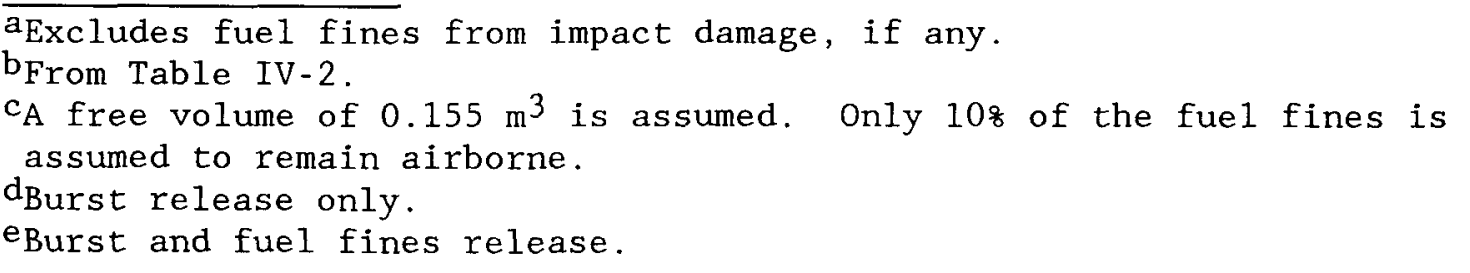 } \\
\hline
\end{tabular}

\section{IV.9 References}

[AL82] Alvarez, J. L., L. Isaacson, B. B. Kaiser, and V. J. Novick, Waste Forms Response Project Correlation Testing, EG\&G, Idaho Falls, ID, EGG-PR-5590, September 1982.

[AN87] ANSI (American National Standards Institute), American National Standard for Radioactive Materials--Leakage Tests on Packages for Shipment, ANSI Standard N14.5-1987, 1987. 
[BU85] Burian, R. J., K. D. Kok, R. Disalvo, M. E. Balmert, K. R. Freeman, and A. W. Pentiman, Response of Spent LWR Fuel to Extreme

Environments, Sandia National Laboratories, Albuquerque, NM, SAND85-7213, August 1985.

[C087] Collins, J. L., M. F. Osborne, and R. A. Lorenz, "Fission Product Tellurium Behavior Under Severe Light Water Reactor Accident Conditions," Nuclear Technology, 77, 18 (1987).

[C088] Collins, J. L., M. F. Osborne, R. A. Lorenz, and A. P. Malinauskas, "Fission Product Iodine and Cesium Release Behavior Under Light Water Reactor Accident Conditions," Nuclear Technology, 81, 78 (1988).

[CR80] Croff, A. G., "ORIGEN2--A Revised and Updated Version of the Oak Ridge Isotope Generation and Depletion Code," Oak Ridge National Laboratory, Oak Ridge, TN ORNL-5621, 1980.

[G080] Goode, J. H., R. G. Stacy, and V. C. Vaugen, Comparison Studies of Head-End Reprocessing Using Three LWR Fuels, Oak Ridge National Laboratory, Oak Ridge, TN, ORNL/TM-7103, 1980.

[HA85] Hastings, I. J., D. R. McCracken, E. Mizzan, R. D. Barrand, J. R. Kelm, K. E. Nash, and J. Novak, "Postirradiation Dimensional Stability and Fission Product Behavior of Deliberately Defected $\mathrm{UO}_{2}$ Fuel at $200^{\circ}$ and $400^{\circ} \mathrm{C}, "$ Nuclear Technology, 70, 268 (1985).

[KE61] Kenny, W. J., and E. L. Piret, "Slow Compression Crushing of Single Particles of Glass," AIChE Journal, 7,199 (1961).

[L079] Lorenz, R. A., J. L. Collins, and A. P. Malinauskas, "Fission Product Source Terms for the Light Water Reactor Loss-of-Coolant Accident," Nuclear Technology, 46, 404 (1979).

[L080] Lorenz, R. A., J. L. Collins, and A. P. Malinauskas, Fission Product Release from Highly Irradiated LWR Fuel, Oak Ridge National Laboratory, Oak Ridge, TN, NUREG/CR-0722, February 1980.

[L081] Lorenz, R. A., J. L. Collins, M. F. Osborne, R. L. Towns, and A. P. Malinauskas, Fission Product Release from BWR Fuel Under LOCA Conditions, Oak Ridge National Laboratory, Oak Ridge, TN, NUREG/CR1773, July 1981.

[MA70] Mason, E. A., and T. R. Merrero, "The Diffusion of Atoms and Molecules," Advances in Atomic and Molecular Physics, $\underline{6}, 155$ (1970).

[MA83] Mason, E. A., and A. P. Malinauskas, Gas Transport in Porous Media: The Dusty-Gas Model (New York: Elsevier, 1983).

[ME81] Mecham, W. J., et al., Interim Report of Brittle-Fracture Impact Studies: Development of Methodology, Argonne National Laboratory, Chicago, IL, ANL-81-27, October 1981. 
[NR81] Technical Bases for Estimating Fission Product Behavior During LWR Accidents, U.S. Nuclear Regulatory Commission, Washington, D.C., NUREG-0772, 1981.

[PA73] Parker, G. W., and C. J. Barton, "Fission Product Release," in The Technology of Nuclear Reactor Safety, T. J. Thompson and J. G. Beckerley, eds. (Cambridge, MA: M.I.T. Press, 1973), pp. 525-618.

[RU80] Ruhmann, H., A. Bleier, G. Kasper, G. Hofmann, H. Loscher, and M. Peehs, Untersuchungsprogramm zum Verhalten abgebrannter Brennelemente bei Straken mechanischem Einwirkungen, KWU Report, R917/8J/002, March 1980.

[SA83] Sandoval, R. P., et al., An Assessment of the Safety of Spent Fuel Transportation in Urban Environs, Sandia National Laboratories, Albuquerque, NM, SAND82-2365, June 1983.

[SA91] Sandova1, R. P., et a1., Estimate of CRUD Contribution to Shipping Cask Containment Requirements, Sandia National Laboratories, Albuquerque, NM, SAND88-1358, January 1991.

[SC82] Schmidt, E. W., M. A. Walters, B. D. Trott, and J. A. Gieseke, Final Report on Shipping CAsk Sabotage Source Term Investigation, Battelle Columbus Division Report, Columbus, OH, NUREG/CR-2472, VMI-2095, October 1982.

[US90] CFR (U.S. Code of Federal Regulations), Title 10, "Packaging and Transportation of Radioactive Material," in United States Code of Federal Regulations, Part 71, rev. ed. 1990.

[ZE62] Zeleny, R. A., and E. L. Piret, "Description of Energy in SingleParticle Crushing," Ind. Eng. Chem. Process Des. Dev., 1, pp. 37-41 (1962). 
No. of

Copies

175 U.S. Department of Energy Office of Scientific and

Technical Information

Attn: DOE/OSTI-4500-R74 UC-820

Oak Ridge, TN 37830

9 U.S. Department of Energy

office of Civilian

Radioactive Waste Management

Attn: R. Milner, RW-40

J. Williams, RW-411

J. Carlson, RW-43

C. Kouts, RW-431

W. Lake (5), RW-431

Routing RW-40

1000 Independence SW

Washington, DC 20585

2 U.S. Department of Energy

Naval Reactors

Attn: R. Kulbitskas, NE-60

B. K. Miles, NE-60

Routing NE-60

1000 Independence SW

Washington, DC 20585

1 U.S. Department of Energy

Northwestern On-Site

Remediation Branch

Attn: J. C. Lehr, EM-442

Mail Stop EM-442

B - 205

19901 Germantown Road

Germantown, MD 20585

1 U.S. Department of Energy Office of Waste Operations

Attn: S. P. Cowen, EM-30

Mail Stop EM-30

A - 214A

19901 Germantown Road

Germantown, MD 20858
No. of

Copies

1 U.S. Department of Energy

Office of Environmental

Restoration

Attn: R. P. Whitfield, EM-40

Mai1 Stop EM-40

7A- 049

1000 Independence SW

Washington, DC 20585

1 U.S. Department of Energy

Division of Demonstration

Testing and Evaluation

Attn: C. Cooley, EM-55

Mail Stop EM- 55

7A- 049

1000 Independence SW

Washington, DC 20585

1 U.S. Department of Energy

Office of Waste Operations

Attn: J. Lytle, EM-30

Mail Stop EM-30

7A-049

1000 Independence SW

Washington, DC 20585

1 U.S. Department of Energy

Division of Waste Management Projects

Attn: M. Frei, EM-34

Mail Stop EM-34

A- 233

19901 Germantown Road

Germantown, MD 20585

1 U.S. Department of Energy

Attn: K. Chacey, EM-343

Mail Stop EM-343

19901 Germantown Road

Germantown, MD 20585 
No. of

Copies

1 U.S. Department of Energy

Office of Environmental

Restoration and Waste

Management

Attn: Leo P. Duffy, Director, EM-1

Mail Stop EM-1

7A-049

1000 Independence SW

Washington, DC 20585

1 U.S. Department of Energy

Office of Technology

Development

Attn: Clyde Frank, Associate

7A-049

1000 Independence SW

Washington, DC 20585

6 U.S. Department of Energy

Attn: G. Boyd, EM-50.1

S. Denny, EM-50.1

F. Falci, EM-50.1

R. Garrison, EM-50.1

C. Miller, EM-50.1

K. Sorenson, EM-50.1

Routing EM-50.1

Washington, DC 20545

2 U.S. Department of Energy

Attn: L. Harmon, EM-53

D. Alexander, EM-53

Routing EM-53

Washington, DC 20545

1 U.S. Department of Energy

Nevada Operations office

Attn: W. Dixon

P.O. Box 14100

Las Vegas, NV 89114-4100
No. of

Copies

3 U.S. Department of Energy Albuquerque Operations Office Albuquerque Headquarters

Attn: J. Bickel

K. G. Golliher

P. A. Saxman

P.0. Box 5400

Albuquerque, NM 87115

9 U.S. Department of Energy

Idaho Operations office

Attn: M. Fisher

W. Mings (3)

K. Svinicki (5)

785 DOE Place

Idaho Falls, ID 83402

1 U.S. Department of Energy Oak Ridge Operations Office Attn: M. Heiskell

P.O. Box E

Oak Ridge, TN 37831

1 U.S. Department of Energy

Richland Operations office

Attn: J. Peterson

P.0. Box 550

Richland, WA 99352

1 U.S. Department of Energy

Office of Security Regulations

Defense Programs EM-321

Attn: J. Read

Washington, DC 20545

1 AEA Winfrith Technology

Reactor Physics Division

Attn: M. Burgess

205/B71 Winfrith Technology

Center Dorchester

Dorset

UNITED KINGDOM DT28DH 
No. of

Copies

1 American Nuclear Insurers

Attn: B. Einst

270 Farmington Ave.

Farmington, CT 06032

5 ANATECH Research Corporation

Attn: Y. R. Rashid

P. R. Barrett (4)

P.0. Box 9165

LaJolla, CA 92038

1 David Andress \& Associates

Inc.

Attn: D. Andress

11008 Harriet Lane

Kensington, MD 20895

1 ANEFCO, Inc.

Attn: J. Murphy

904 Ethan Allen Hwy

P.O. Box 433

Ridgefield, CT 06877

1 APTEK INC.

Attn: S. Sutherland

2860 So. Circle Drive

South B1dg., Suite 346

Colorado Springs, CO 80906

1 Argonne National Laboratory

Attn: G. Popper

9700 South Cass Ave.

Argonne, IL 60439

1 Babcock \& Wilcox

Nuclear Equipment Division

Attn: T. Stevens

91 Stirling Avenue

Barberton, OH 44203

2 Babcock \& Wilcox

Attn: G. Vames

R. Fisher

P.O. Box 10935

Lynchburg, VA 24506-0935
No. of

Copies

3 Battelle

Attn: T. I. McSweeney

V. Pasupathi (2)

505 King Ave.

Columbus, $\mathrm{OH}$ 43201-2693

20 Battelle Pacific Northwest

Laboratories

Attn: R. E. Einziger

P.O. Box 999

Richland, WA 99352

1 Bechtel National, Inc.

Attn: B. Hopkins

15740 Shady Grove Rd.

Gaithersburg, MD 20877-1454

1 Bettis Atomic Power Labs

Attn: 0. Rieley

P.O. Box 79

West Mifflin, PA 15122

1 British Nuclear Fuels, Ltd.

Attn: D. Snedeker

2926 92nd Ave. E.

Puyallup, WA 98371

1 British Nuclear Fuels, Ltd.

Attn: D. Boyer

Risley Fleming House

Warrington, Cheshire

UNITED KINGDOM WA36AS

1 Carolina Power \& Light Co.

Attn: R. Kunita

P.0. Box 1551

Raleigh, NC 27602

2 Center for Energy Studies

The University of Texas

Attn: D. Klein

S. Burns

10100 Burnet Rd.

Austin, TX 78758 
No. of

Copies

1 Central Electricity Generating Board

Nuclear Fuel Cycle Department Sudbury House

Attn: G. Mummery

15 Newgate St.

London

UNITED KINGDOM EC1A7AU

2 Central Research Institute of Electrical Power Industry

Attn: S. Fukuda

T. Saegusa

1-6-1 Ohtemachi

Chiyodo-Ku, Tokyo

JAPAN 100

1 Chem Nuclear Systems, Inc.

Attn: R. Anderson

220 Stoneridge $\mathrm{Dr}$.

Columbia, SC 29210

1 COGEMA Service Technico-

Commercial Branche

Retraitement

Attn: H. Bernard

2 Rue Paul Dautier

Velizy-Villacoublay

Cedex

FRANCE BP4.78141

1 Combustion Engineering

CE Power Systems

Attn: M. Falzarano

P.O. Box 500

Winsdor, CT 06095-0500

1 Commissariat a 1'Energie Atomique

Department des Etudes Mecaniques et Thermiques

Service d'Etudes des Systemes

Attn: C. Vallepin

Demt Syst. CEN-Saclay

Gif-sur-Yvette

Cedex

FRANCE 91191
No. of

Copies

1 Thomas A. Duffey

P.O. Box 1239

Tijeras, NM 87059

1 Duke Power Co.

Attn: B. Rasmussen

P.O. Box 33189

Charlotte, NC 28242

1 Edison Electric Institute

Attn: C. Henke1

1111 19th Street NW

Washington, DC 20036

1 Edlow International Company

Attn: J. Edlow

1666 Connecticut Ave.

Suite 500

Washington, DC 20009

7 EG\&G Idaho, Inc.

Attn: R. Chapman

B. DaBell

K. Henry (4)

H. Worle

P.O. Box 1625, MS 9109

Idaho Falls, ID 83415-9109

5 EG\&G Rocky Flats Inc.

Rocky Flats Plant

Attn: H. Jordan

P.O. Box 464

Golden, CO 80402-0464

2 Electric Power Research Institute

Attn: R. Lambert

$$
\text { R. Williams }
$$

P.0. Box 10412

Palo Alto, CA 94303

1 Florida Power \& Light Co.

Attn: D. Brodnick

P.0. Box 14000

Juno Beach, FL 33408 
No. of

Copies

2 General Atomics

Attn: R. Grenier

A. Zimmer

P. O. Box 85608

San Diego, CA 92138

1 General Nuclear Systems, Inc.

Attn: P. Pacquin

220 Stoneridge Dr.

Columbia, SC 29210

2 Gesellschaft f $\mathrm{r}$ Nuklear-

Service mbH (GNS)

Attn: H. Geiser

$\mathrm{K}$. Janberg

Goethestr. 88

D-4300 Essen 1

GERMANY

7 GRAM, Inc.

Attn: P. C. Reardon (5)

P. E. McConne 11

R. W. Herman

8500 Menaul B1vd. NE

Suite B-370

Albuquerque, NM 87112

2 Institut de Protection et de Surete Nucleaire

Departmente d'Analyses de Surete

Commissariat a 1'Energies

Atomique

Attn: D. Devillers

L. Tanguy

CEN/FAR - BP No. 6

FRANCE F-92260

1 International Atomic Energy Agency

Division of Publications

Attn: R. Kelleher

Wagramerstrasse 5

P.0. Box 100

Vienna

AUSTRIA A-1400
No. of

Copies

1 E. R. Johnson Associates, Inc.

Attn: B. Teer

10461 White Granite Dr.

Suite 204

Oakton, VA 22124

1 JNT, Inc.

Attn: R. Jones

P.O. Box 1510

Los Gatos, CA 95031-1510

1 Korea Advanced Energy Research Institute

Attn: P. Han

P.0. Box 7, Daeduk-Danji

Chung-Nam

KOREA 300-31

1 Lawrence Livermore National Laboratory

University of California

Attn: L. Fischer

P.O. Box 808

Livermore, CA 94550

1 C. Morotta

1504 Columbia Ave.

Rockville, MD 20850

1 National Conference of State Legislatures

Energy, Science, and Natural Resources

Attn: B. Foster

105017 th St., Suite 2100

Denver, CO 80265

1 National Congress of American Indians

Attn: G. Chehak

804 D Street NE

Washington, DC 20002 
No. of

Copies

1 Northern States Power Co.

Attn: L. McCarten

414 Nicollet Mall

Minneapolis, MN 55401

1 Nuclear Assurance Corp.

Attn: P. Aucoin

6251 Crooked Creek Rd.

Norcross, GA 30092

1 Nuclear Energy Department

University of Missouri-Rolla

Attn: N. Tsulfanidis

Rolla, MO 65401

1 Nuclear Packaging, Inc.

Attn: R. Doman

1010 S. 336th St.

Suite 220

Federal Way, WA 98003

1 NUMARC

Attn: L. Fairobent

1776 Eye Street NW

Suite 300

Washington, DC 20006

1 NUMATEC, Inc.

Attn: P. Saverot

2550 M. Street NW

Suite 400

Washington, DC 20037

1 NUTEC

Attn: J. Rollins

1301 Hightower Trail

Suite 205

Atlanta, GA 30350

1 NUTECH Engineers

Attn: B. Thomas

145 Martinvale Lane

San Jose, CA 95119
No. of

Copies

3 Oak Ridge National Laboratory

Attn: A. Malinauskas (2)

R. Pope

P.O. Box 2008

Oak Ridge, TN 37831-6370

I R. Odegaarden

Attn: R. Odegaarden

1263 E. 2nd Place

Mesa, AZ 85203

1 Ontario Hydro

Research Division

Attn: J. Boag

800 Kipling Avenue

Toronto, Ontario

CANADA M8Z 5S4

1 The Ralph M. Parsons Co.

Attn: N. Ketzlach

$100 \mathrm{~W}$. Walnut St.

Pasadena, CA 91124

1 Pickard, Low, \& Garrick Inc. (PLG)

Attn: P. Marsico

1615 M Street NW

Suite 730

Washington, DC 20036

1 Public Service Electric \& Gas Co.

Attn: S. Baker

P.O. Box 236, MC N20

Hancocks Bridge, NJ 08038

1 Science Applications

International Corp.

Nevada Nuclear Waste Storage

Investigations

Attn: W. Andrews

Valley Bank Center

101 Convention Center Dr.

Las Vegas, NV 89109 
No. of

Copies

1 Science Applications

International Corp.

Attn: T. Albert

311 Park Place Blvd. Suite 360

Clearwater, FL 34619

1 Science Applications

International Corp.

Attn: L. Danese

P.O. Box 2501

800 Oak Ridge Turnpike

Oak Ridge, TN 37831

1 Science Applications

International Corp.

Attn: J. Stokley

1710 Goodridge Dr.

McLean, VA 22102

1 Science Applications

International Corp.

Attn: T. Hill

P. O. Box 2501

165 Mitche11 Road

Oak Ridge, TN 37830

1 Siemens AG, KWU Group

Dept. U PK 13

Attn: 0 . Wildgruber

Hammerbacher Str. $12+14$

Erlangen

FEDERAL REPUBLIC OF GERMANY

D -8520

1 Southern California Edison Co.

Attn: J. Ladesich

2244 Walnut Grove Ave.

Rosemead, CA 91770

1 Southern Company Services

Attn: K. Folk

P.0. Box 2625

Birmingham, AL 35202
No. of

Copies

1 Southern States Energy Board

Attn: K. Nemeth

3091 Governors Lakes Dr.

Suite 400

Norcross, GA 30071

1 Stone \& Webster Energy Corp.

Attn: S. Bhatnagar

3 Executive Campus

Cherry Hill, NJ 08034

1 Svensk Karnleranslehantering $A B$

Transport and AFR Storage Division

Attn: B. Gustafsson

P.O. Box 5864

Stockholm

SWEDEN S-102 48

1 Southwest Engineering

Associates

Attn: M. Huerta

3616 Derick

E1 Paso, TX 79925

1 TESS Incorporated

One Federal Systems

Park Drive

Attn: B. Teer

Fairfax, VA 22023

1 Transnuclear, Inc.

Attn: M. Mason

2 Skyline Dr.

Hawthorn, NY 10532-2120

1 Union Electric

Attn: A. Passwater

P.O. Box 149 , Code 470

St. Louis, MO 63166

1 Union Electric

Callaway Plant

Attn: G. Hughes

P.0. Box 620

Fulton, MO 65251 
No. of

Copies

3 U.S. Council for Energy Awareness

Attn: F. Killar

G. Russ

J. Siegel

1776 I Street, NW

Suite 400

Washington, DC 20006-2495

1 U.S. Department of Transportation

Federal Rail Administration RRS - 32

Attn: J. Pena

4007 th Street SW

Washington, DC 20590

1 U.S. Department of

Transportation

office of Materials

Transportation

Attn: K. Smith

400 Seventh Street SW

Washington, DC 20590

10 U.S. Nuclear Regulatory Commission

Office of Nuclear Materials Safety and Safeguards

Attn: R. Chappell

E. Easton

G. Gardes

A. Gre1la

D. Huang

H. Lee

C. MacDonald

N. Osgood

L. Wang

C. Withee

Washington, DC 20555
No. of

Copies

1 U.S. Nuclear Regulatory Commission

Office of Nuclear Regulatory Research

Attn: W. Lahs

NL/S - 139

Washington, DC 20555

1 U.S. Nuclear Regulatory Commission

Attn: F. Cardile

Washington, DC 20555

2 U.S. Nuclear Regulatory Commission

Attn: G. Bidinger

L. Kopp

NMSS Mail Stop WF1-6H3

Washington, DC 20555

1 U.S. Nuclear Regulatory Commission

Attn: C. Peterson

Mail Stop 4H3

Washington, DC 20555

1 Utility Nuclear Waste Management Group

Attn: J. Davis

1111 19th Street NW

Washington, DC 20036

1 Virginia Power Co.

Attn: M. Smith

5000 Dominion

Glen Allen, VA 23060

1 Western Interstate Energy Board

Attn: L. Friel

333 Quebec St.

Denver, CO 80207 
No. of

Copies

1 Westinghouse Electric Corp.

Waste Technology Services

Division

Attn: B. Nair

P.O. Box 286

Madison, PA 15663-0286

1 Westinghouse Hanford Co.

Attn: C. Brown

P.O. Box 1970/MNIS T5-07

Richland, WA 99352

2 Roy $F$. Weston, Inc.

Attn: P. Bolton

M. Rahimi

955 L'Enfant Plaza SW

8 th Floor

Washington, DC 20024

1 Wisconsin Electric Power Co.

Attn: H. Shimon

$231 \mathrm{~W}$. Michigan St.

Milwaukee, WI 53201

2 Yankee Atomic Electric Co.

Attn: D. Napolitano

V. Pareto

580 Main Street

Bolton, MA 01740 
No. of

Copies

\begin{tabular}{rlll}
1 & 1832 & R. J. Salzbrenner \\
1 & 6000 & D. L. Hartley \\
1 & 6300 & D. E. Miller \\
1 & 6400 & D. J. McCloskey \\
1 & 6600 & J. B. Woodard \\
1 & 6603 & R. E. Luna \\
50 & 6603 & TTC Library \\
& & Attn: TTC Master File \\
1 & 6641 & R. P. Sandova1 \\
1 & 6641 & G. S. Brown \\
1 & 6641 & N. A. Russel1 \\
1 & 6642 & G. F. Hohnstreiter \\
1 & 6642 & D. J. Ammerman \\
1 & 6642 & R. E. Glass \\
1 & 6642 & W. L. Uncapher \\
1 & 6642 & H. R. Yoshimura \\
10 & 6643 & T. L. Sanders \\
1 & 6643 & P. C. Bennett \\
1 & 6643 & M. C. Brady \\
1 & 6643 & R. I. Ewing \\
1 & 6643 & S. E. Gianoulakis \\
10 & 6643 & K. D. Seager \\
1 & 6900 & T. O. Hunter \\
1 & $8523-2$ & Central Technical Files \\
5 & 7141 & Technical Library \\
1 & 7151 & Technical Publications \\
10 & $7613-2$ & Document Processing for \\
& \multicolumn{3}{l}{ DOE/OSTI }
\end{tabular}




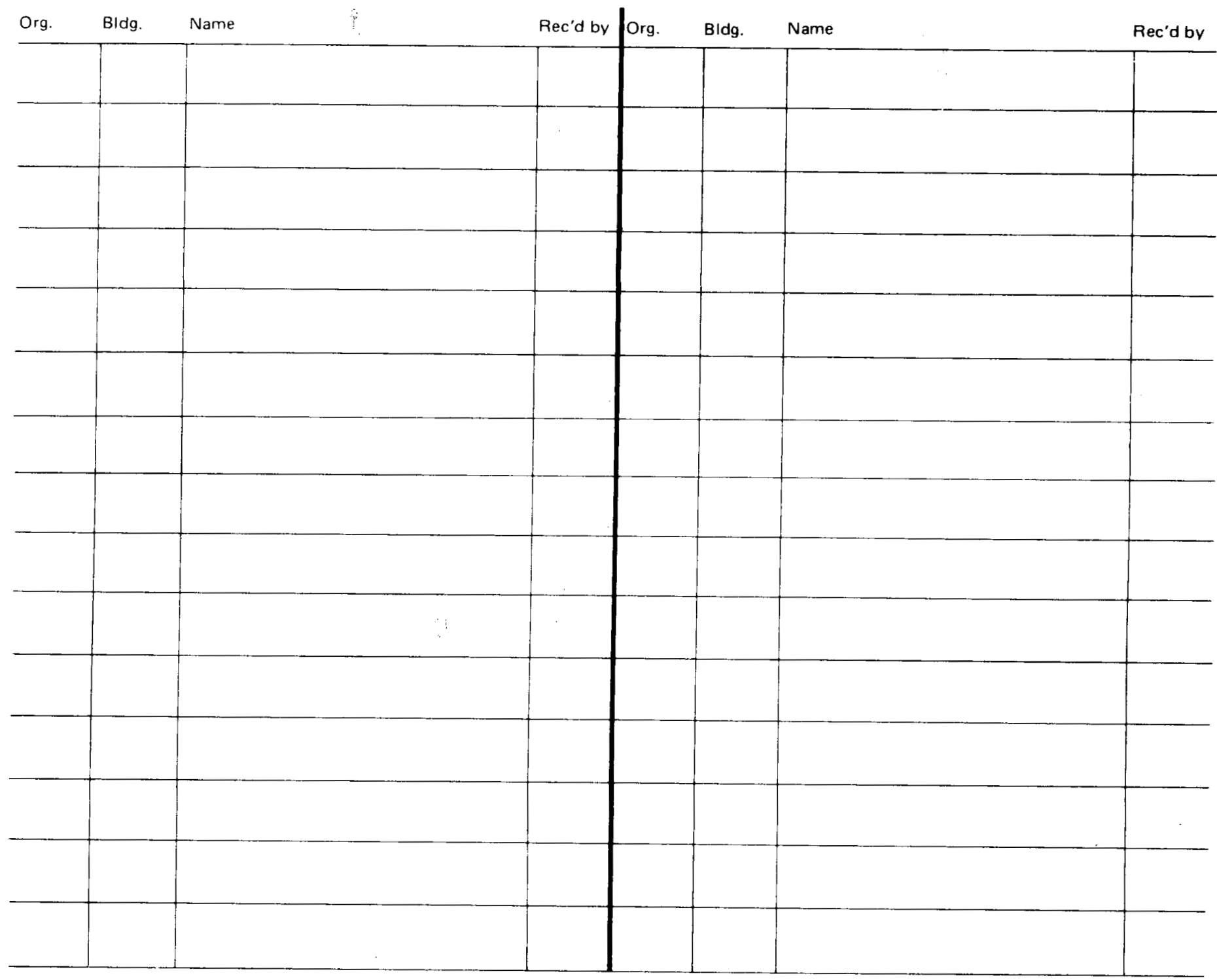

